DOE/CE/23810-110

(JMC/ARTI-0009A - RDBA950)

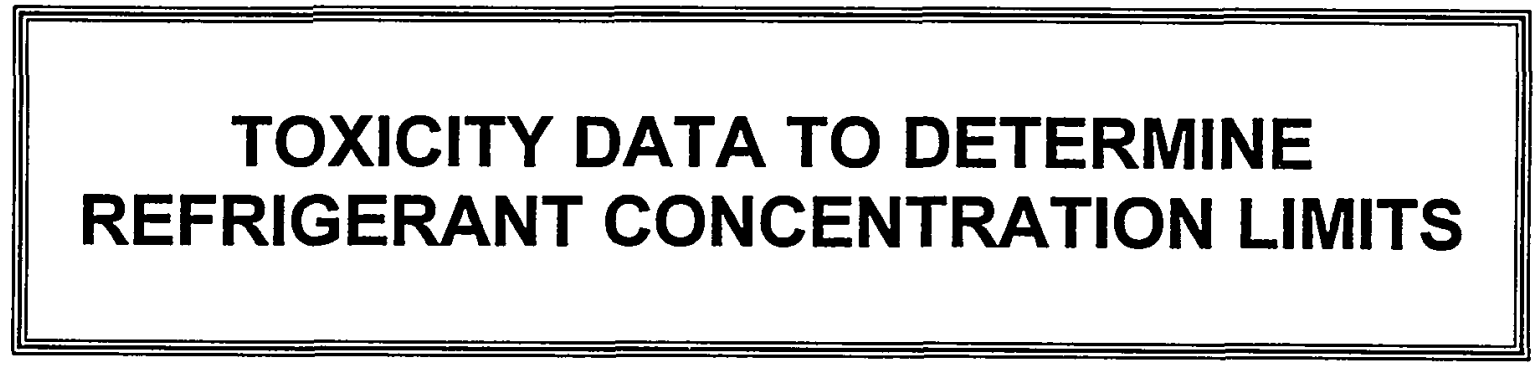

September 2000

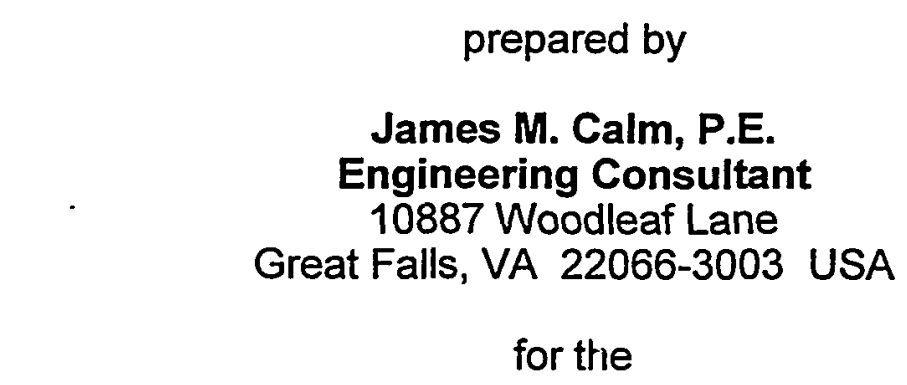

Air-Conditioning and Refrigeration Technology Institute 4301 North Fairfax Drive, Suite 425

Arlington, VA 22203-1627 USA 
Please refer questions or comments on this document to:

James M. Calm, P.E.

Engineering Consultant

10887 Woodleaf Lane

Great Falls, VA 22066-3003 USA

phone:

$703 / 450-4313$

fax:

707/516-0552

e-mail:

jmc@spectrum-internet.com

Glenn C. Hourahan, P.E.

Vice President

Air-Conditioning and Refrigeration Technology Institute

4301 North Fairfax Drive, Suite 425

Arlington, VA 22203-1627 USA

phone:

$703 / 524-8800$

fax:

703/522-2349

e-mail:

hourahan@ari.org

Neither James M. Calm, the Air-Conditioning and Refrigeration Technology Institute (ARTI), nor any person acting on behalf of them: (a) makes any warranty, expressed or implied, with respect to the accuracy, completeness, or usefulness of any information, apparatus, material, method, or process disclosed in this document or that such use may not infringe privately owned rights; or (b) assumes any liabilities with respect to use of, or damages resulting from use of, any information, apparatus, material, method, or process disclosed in this document. Reference herein to any specific commercial product, process, or service by trade name, trademark, manufacturer, or otherwise, does not necessarily constitute or imply its endorsement, recommendation, or favoring. 


\section{DISCLAIMER}

This report was prepared as an account of work sponsored by an agency of the United States Government. Neither the United States Government nor any agency thereof, nor any of their employees, make any warranty, express or implied, or assumes any legal liability or responsibility for the accuracy, completeness, or usefulness of any information, apparatus, product, or process disclosed, or represents that its use would not infringe privately owned rights. Reference herein to any specific commercial product, process, or service by trade name, trademark, manufacturer, or otherwise does not necessarily constitute or imply its endorsement, recommendation, or favoring by the United States Government or any agency thereof. The views and opinions of authors expressed herein do not necessarily state or reflect those of the United States Government or any agency thereof. 


\section{DISCLAIMER}

Portions of this document may be illegible in electronic image products. Images are produced from the best available original document. 


\title{
TOXICITY DATA TO DETERMINE REFRIGERANT CONCENTRATION LIMITS \\ OSTI
}

prepared by

James M. Calm, P.E.

Engineering Consultant

for the

\section{Air-Conditioning and Refrigeration Technology Institute}

\begin{abstract}
This report reviews toxicity data, identifies sources for them, and presents resulting exposure limits for refrigerants for consideration by qualified parties in developing safety guides, standards, codes, and regulations. It outlines a method to calculate an acute toxicity exposure limit (ATEL) and from it a recommended refrigerant concentration limit (RCL) for emergency exposures. The report focuses on acute toxicity with particular attention to lethality, cardiac sensitization, anesthetic and central nervous system (CNS) effects, and other escapeimpairing effects. It addresses R-11, R-12, R-22, R-23, R-32, R-113, R-114, R-116, R-123, R124, R-125, R-134, R-134a, R-E134, R-141b, R-142b, R-143a, R-152a, R-218, R-227ea, R236fa, R-245ca, R-245fa, R-290, R-500, R-502, R-600a, R-717, and R-744. It summarizes additional data for R-14, R-115, R-170 (ethane), R-C318, R-600 (n-butane), and R-1270 (propylene) to enable calculation of limits for blends incorporating them. The report summarizes the data and related safety information, including classifications and flammability data. It also presents a series of tables with proposed ATEL and RCL concentrations - in dimensionless form and the latter also in both metric (SI) and inch-pound (IP) units of measure - for both the cited refrigerants and 66 zeotropic and azeotropic blends. They include common refrigerants, such as R-404A, R-407C, R-410A, and R-507A, as well as others in commercial or developmental status. Appendices provide profiles for the cited single-compound refrigerants and for R-500 and R-502 as well as narrative toxicity summaries for common refrigerants. The report includes an extensive set of references.
\end{abstract}


Toxicity Data to Determine Refrigerant Concentration Limits James M. Calm, Engineering Consultant 


\section{TABLE OF CONTENTS}

TOXICITY DATA TO DETERMINE REFRIGERANT CONCENTRATION LIMITS....................1

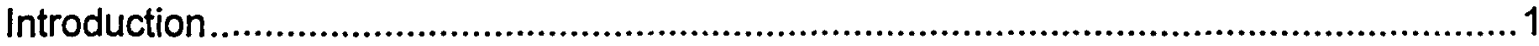

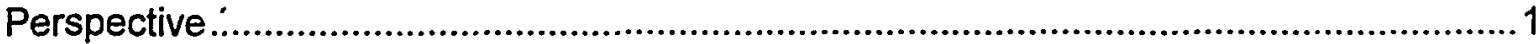

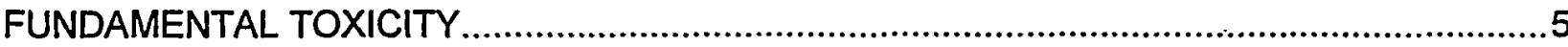

EXPOSURE CONCENTRATION LIMITS ................................................................

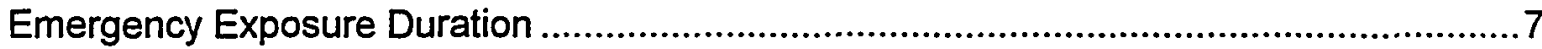

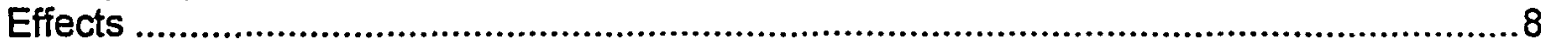

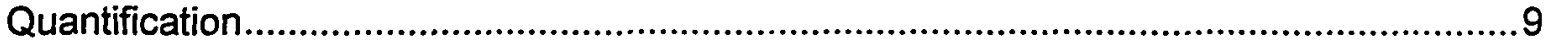

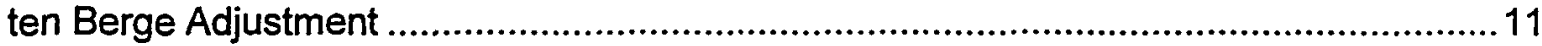

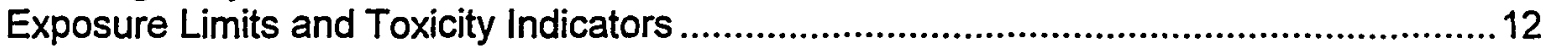

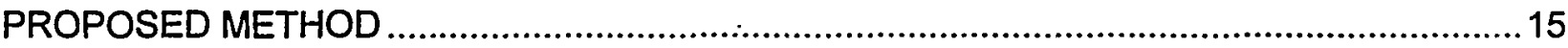

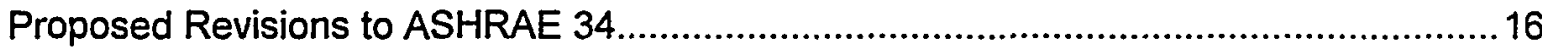

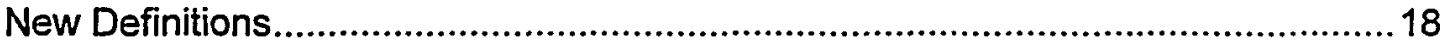

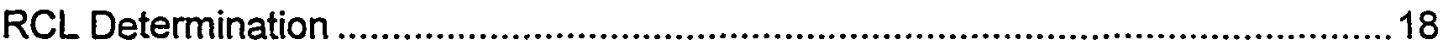

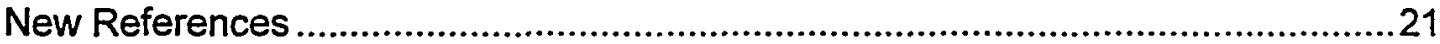

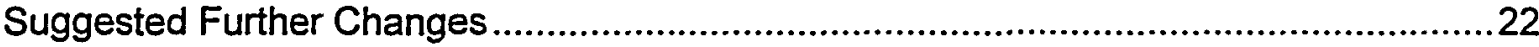

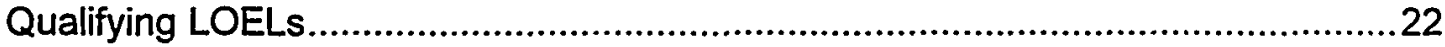

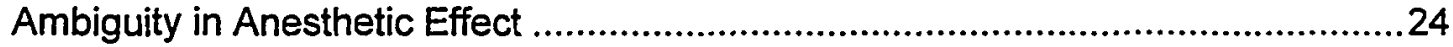

Safety Margin for Other Escape Impairing Effects or Permanent Injury...................25

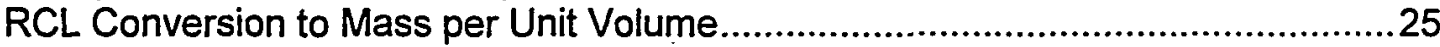

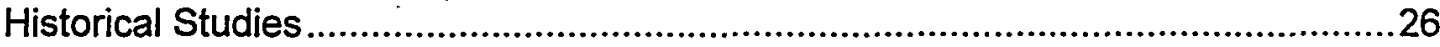

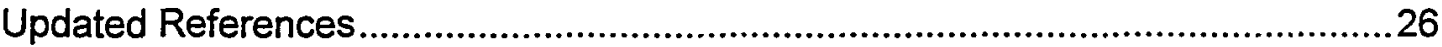

Revised Text of Method with Proposed Changes......................................................26

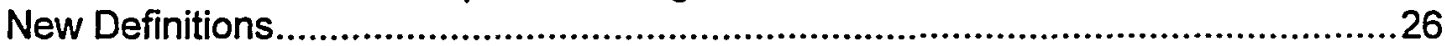

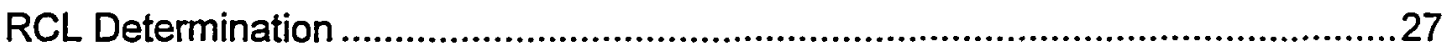

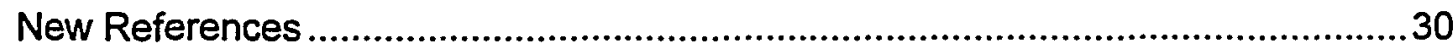

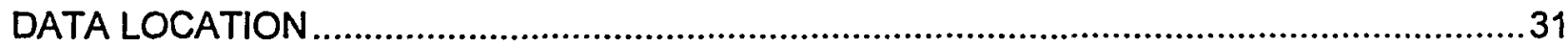

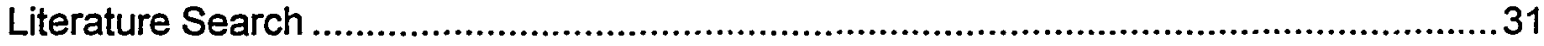

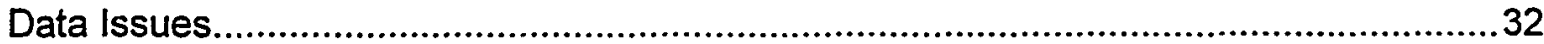

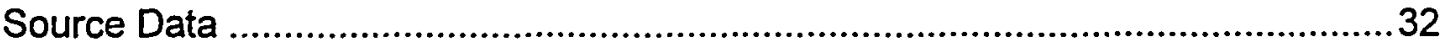

Nominal and Actual Concentrations ............................................................. 32

RECOMMENDED DATA TO DETERMINE ATELS .......................................................33

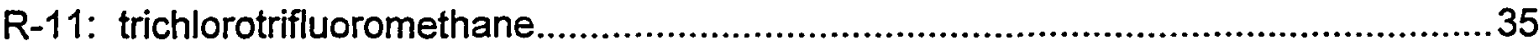

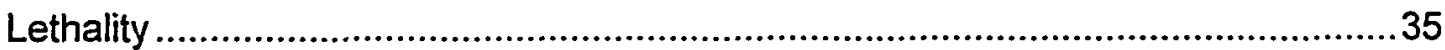

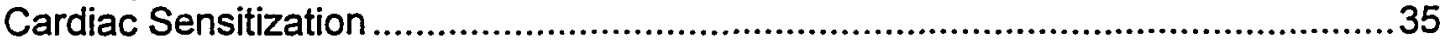

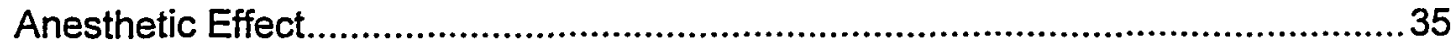


Other Escape-Impairing Effects and Permanent Injury ................................ 36

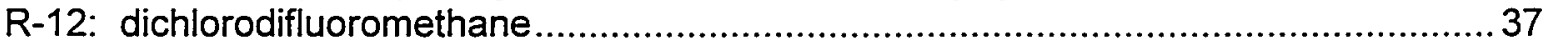

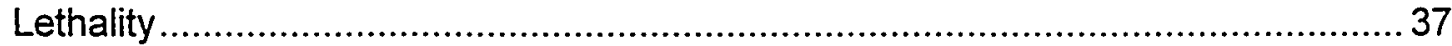

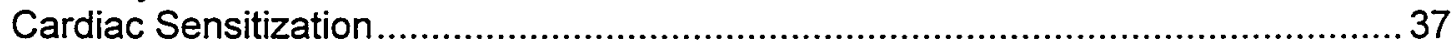

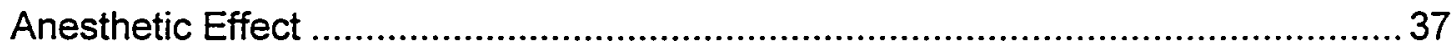

Other Escape-Impairing Effects and Permanent Injury .................................... 38

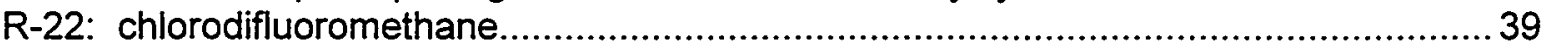

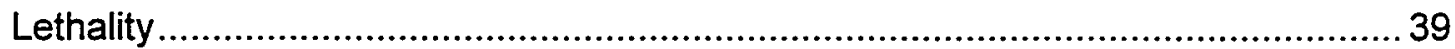

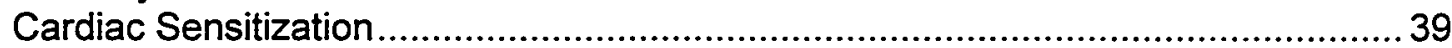

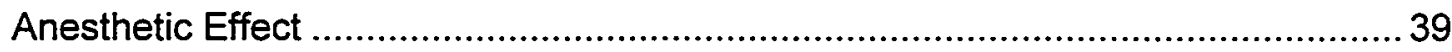

Other Escape-Impairing Effects and Permanent Injury ................................... 39

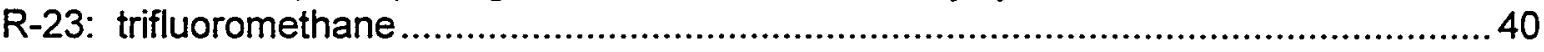

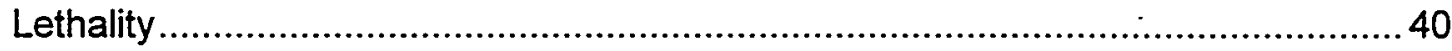

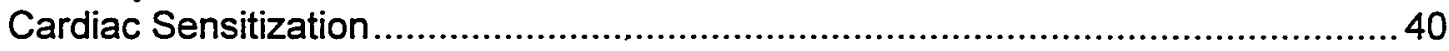

Anesthetic Effect .................................................................................. 40

Other Escape-Impairing Effects and Permanent Injury ................................... 40

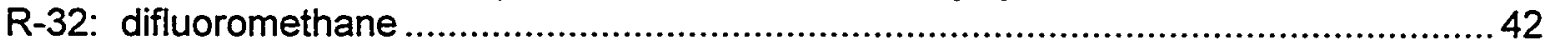

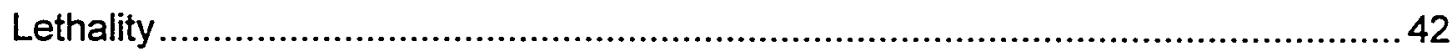

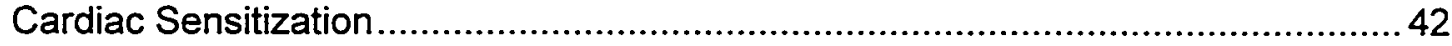

Anesthetic Effect ...................................................................................... 42

Other Escape-Impairing Effects and Permanent Injury .................................. 42

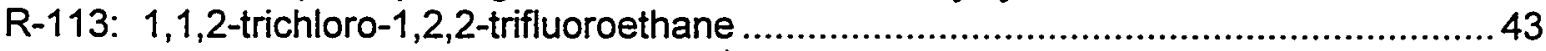

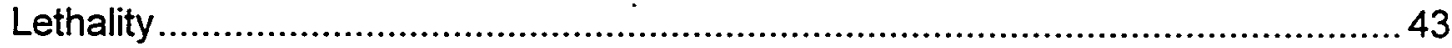

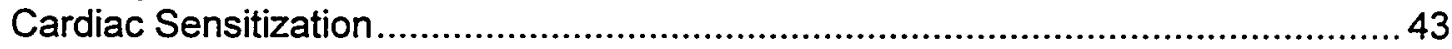

Anesthetic Effect ......................................................................................... 43

Other Escape-Impairing Effects and Permanent Injury .................................... 43

R-114: 1,2-dichloro-1, 1,2,2-tetrafluoroethane .................................................... 44

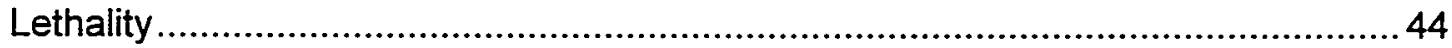

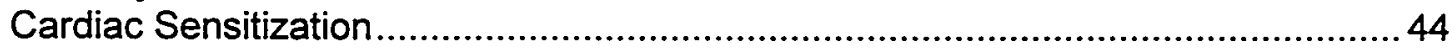

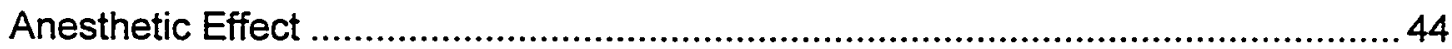

Other Escape-Impairing Effects and Permanent Injury .................................. 44

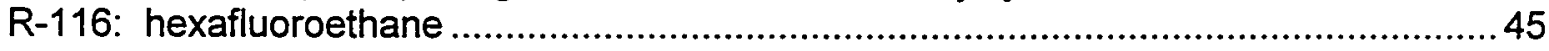

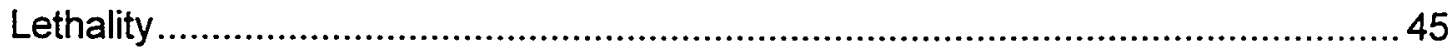

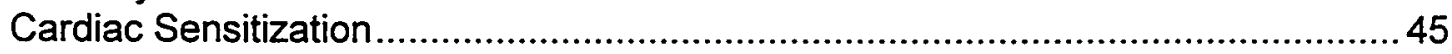

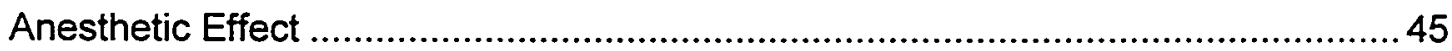

Other Escape-Impairing Effects and Permanent Injury .................................. 45

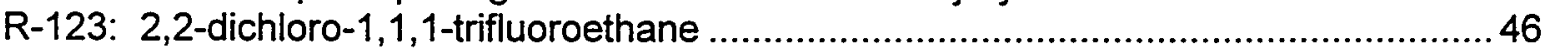

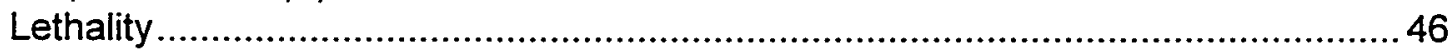

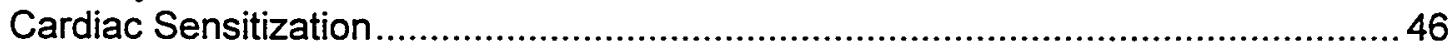

Anesthtic Effect ...................................................................................... 46

Other Escape-Impairing Effects and Permanent Injury ...................................4 47

R-124: 2-chloro-1,1,1,2-tetrafluoroethane ......................................................... 48

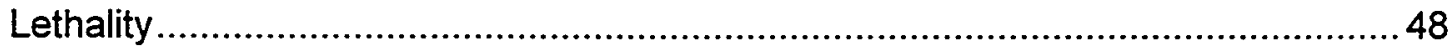

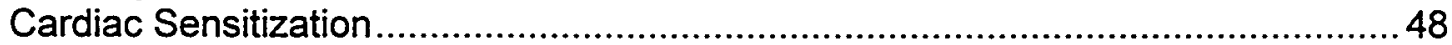

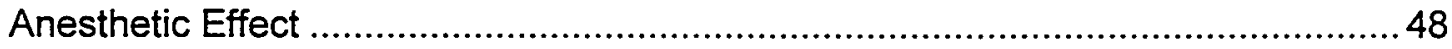

Other Escape-Impairing Effects and Permanent Injury .................................. 49

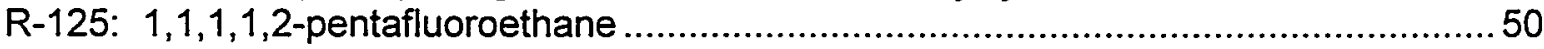

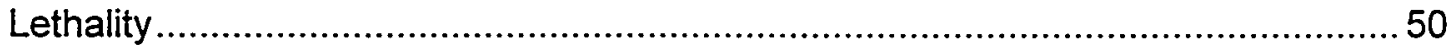

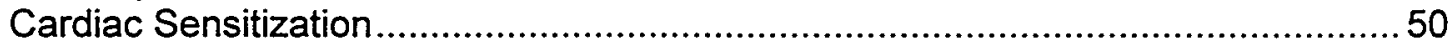

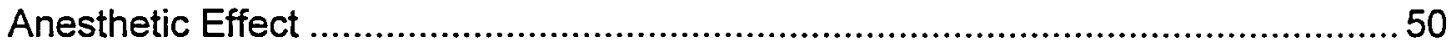

Other Escape-Impairing Effects and Permanent Injury ................................ 50 


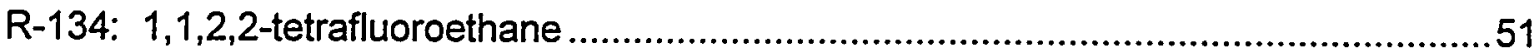

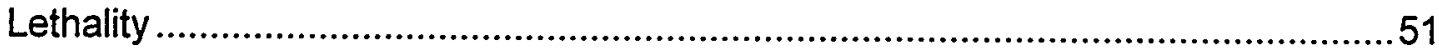

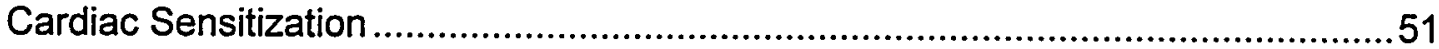

Anesthetic Effect.......................................................................................

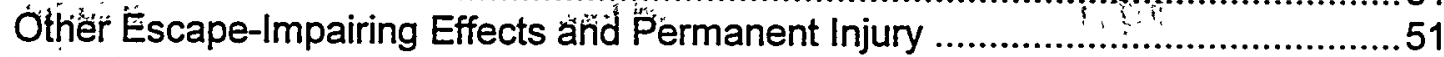

R-134a: 1,1,1,2-tetrafluoroethane ........................................................................5

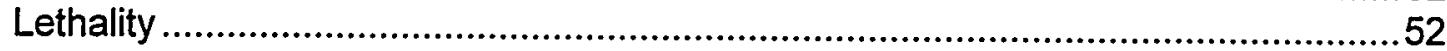

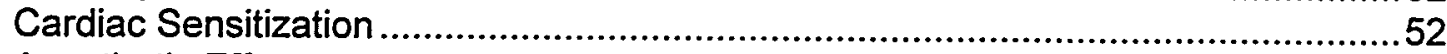

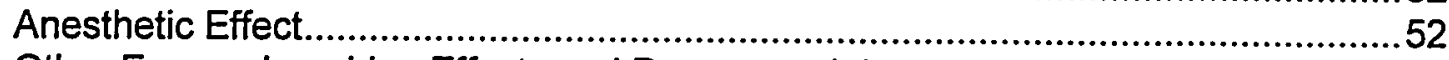

Other Escape-Impairing Effects and Permanent Injury .........................................53

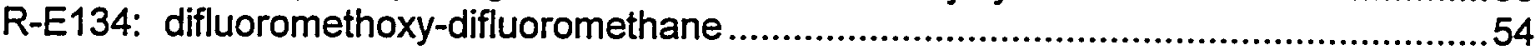

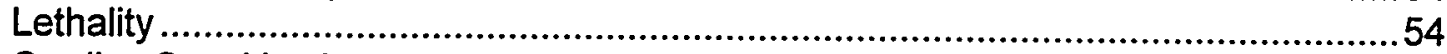

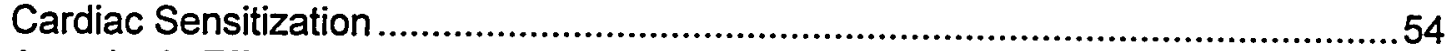

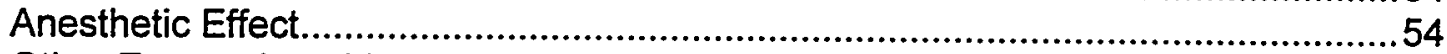

Other Escape-Impairing Effects and Permanent Injury ...........................................55

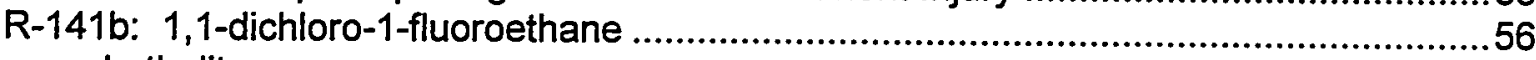

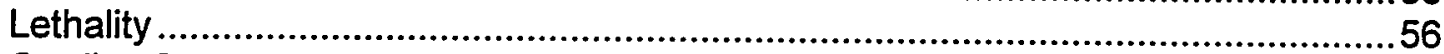

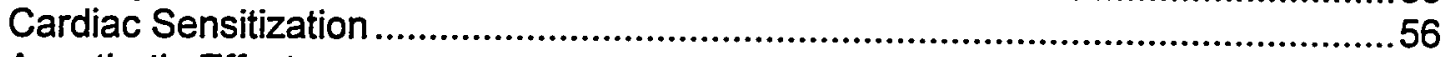

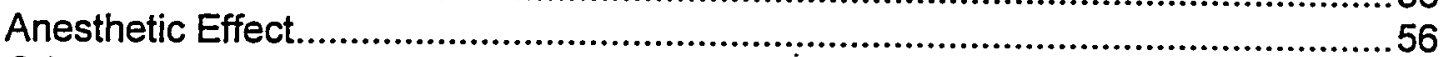

Other Escape-Impairing Effects and Permanent Injury .............................................5

R-142b: 1-chloro-1,1-difluoroethane ........................................................................5

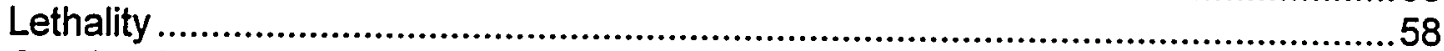

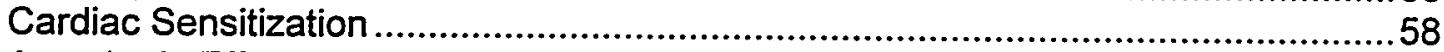

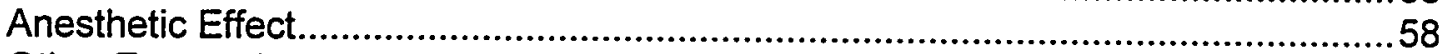

Other Escape-Impairing Effects and Permanent Injury ........................................59

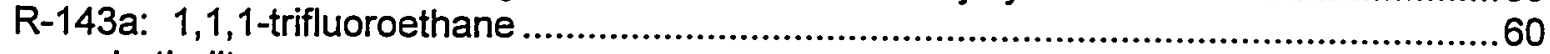

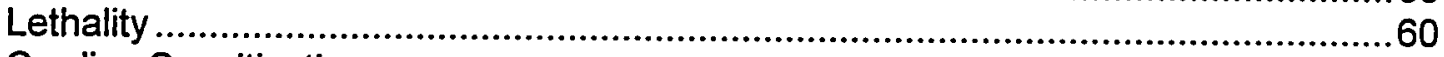

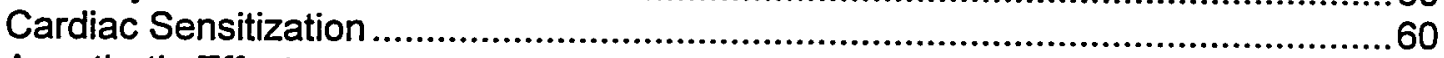

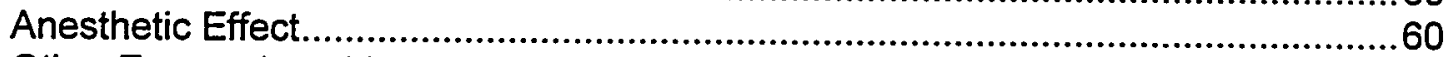

Other Escape-Impairing Effects and Permanent Injury ........................................60

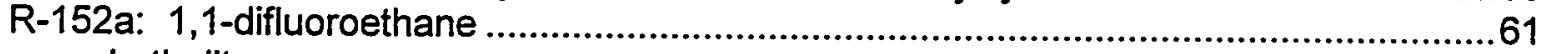

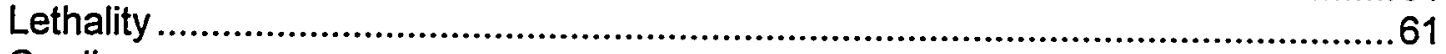

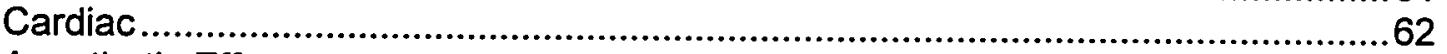

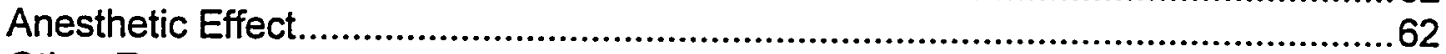

Other Escape-Impairing Effects and Permanent Injury ......................................62

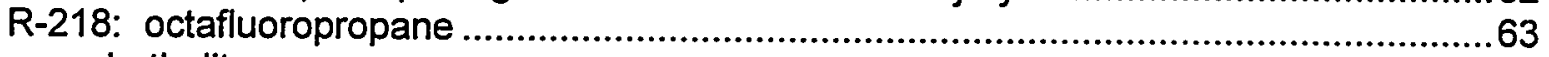

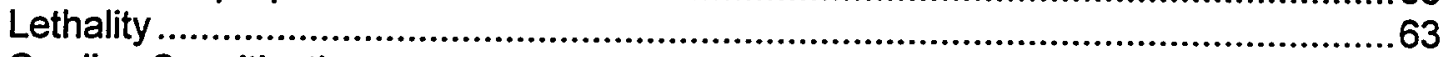

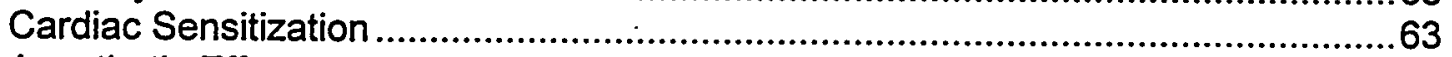

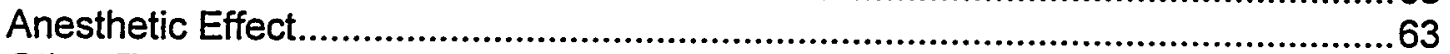

Other Escape-Impairing Effects and Permanent Injury .........................................63

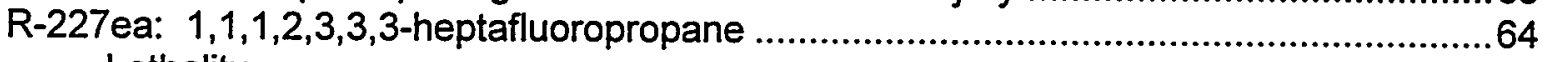

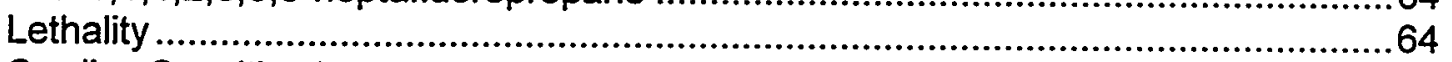

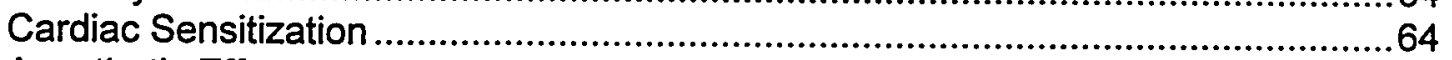

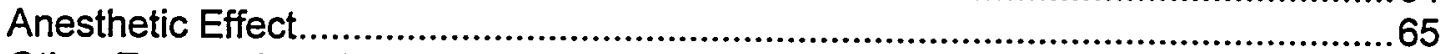

Other Escape-Impairing Effects and Permanent Injury ...........................................65

R-236fa: 1,1,1,3,3,3-hexafluoropropane ...................................................................66

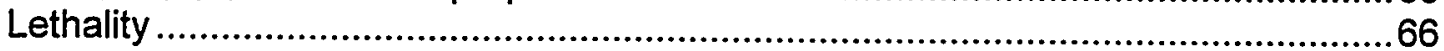

Cardiac Sensitization .............................................................................66

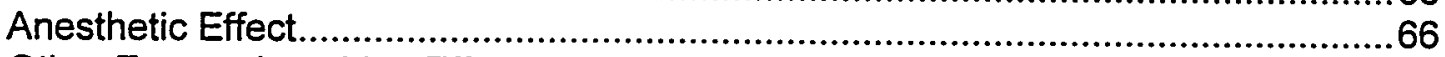

Other Escape-Impairing Effects and Permanent Injury ..........................................67

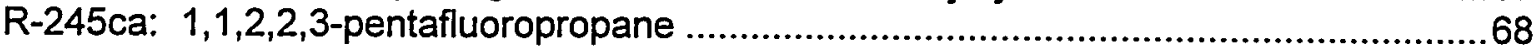




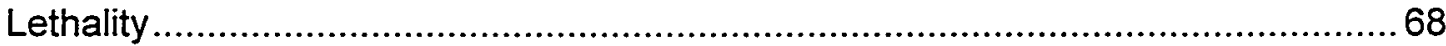

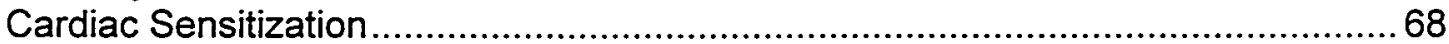

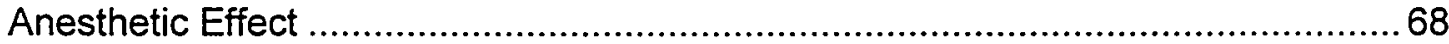

Other Escape-Impairing Effects and Permanent Injury .........................................68

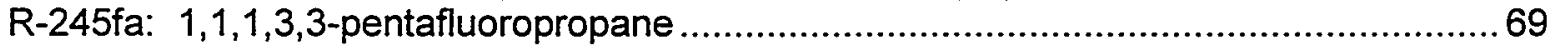

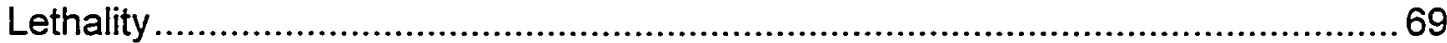

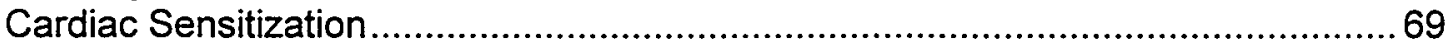

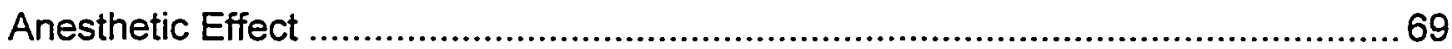

Other Escape-Impairing Effects and Permanent Injury ..........................................70

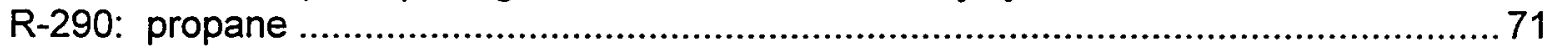

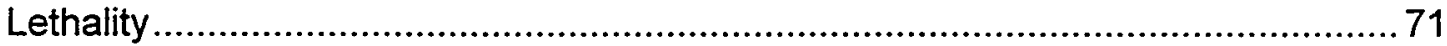

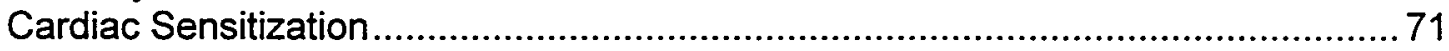

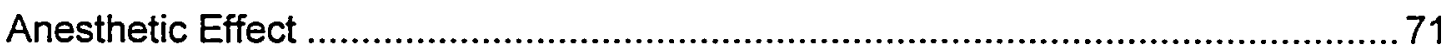

Other Escape-Impairing Effects and Permanent Injury ........................................72

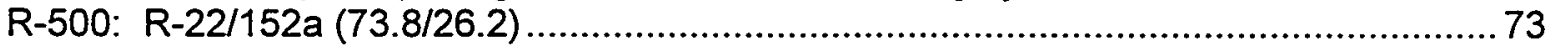

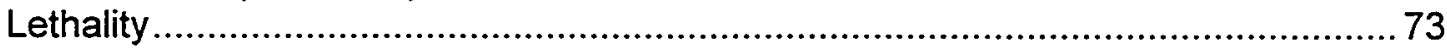

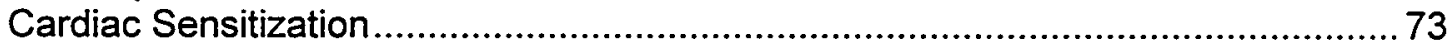

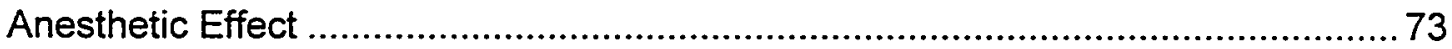

Other Escape-Impairing Effects and Permanent Injury .......................................73

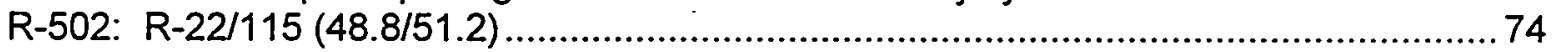

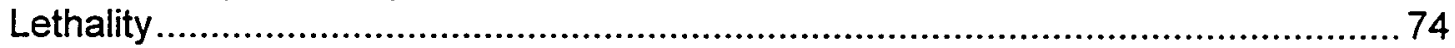

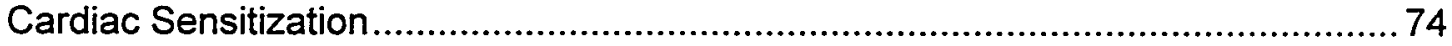

Anesthetic Effect ..................................................................................... 74

Other Escape-Impairing Effects and Permanent Injury ......................................75

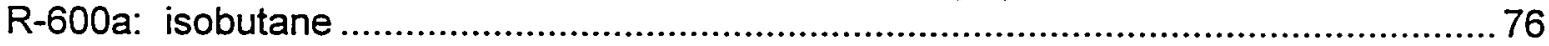

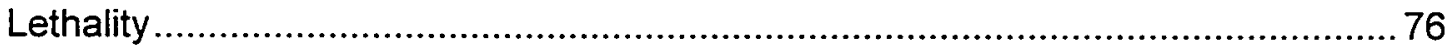

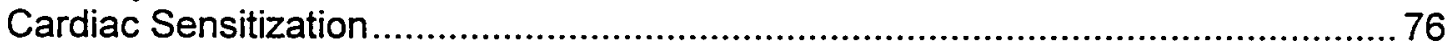

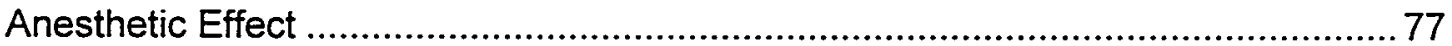

Other Escape-Impairing Effects and Permanent Injury ......................................77

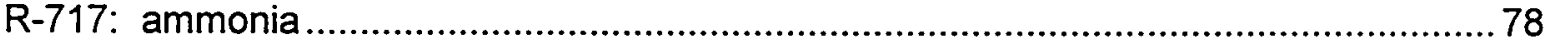

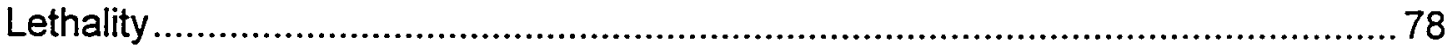

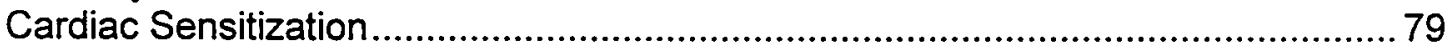

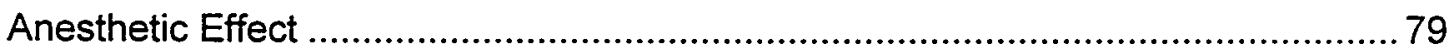

Other Escape-Impairing Effects and Permanent Injury .......................................... 80

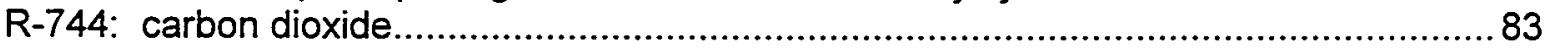

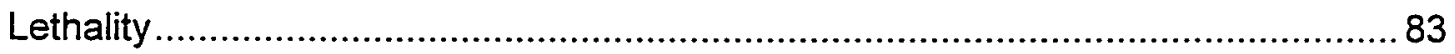

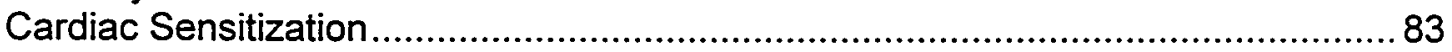

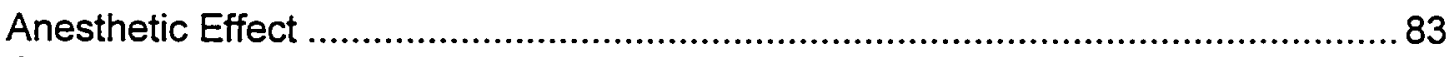

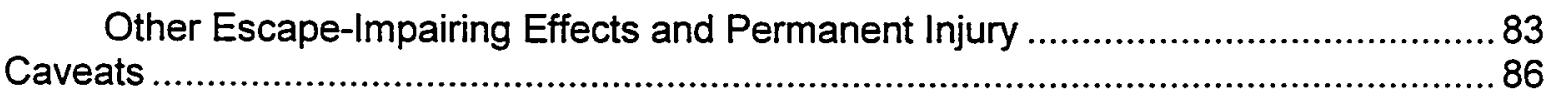

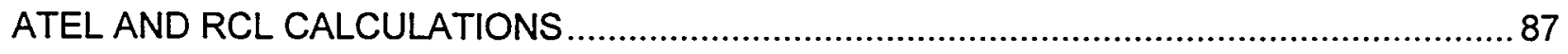

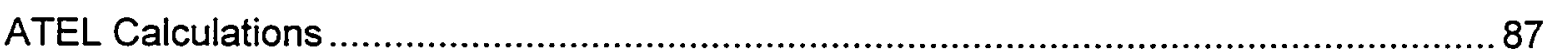

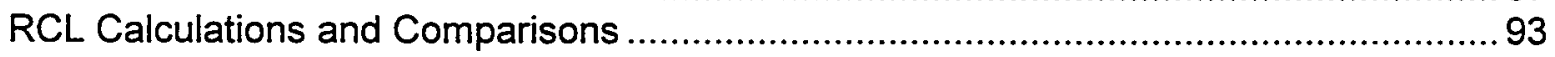

Proposed RCL Concentrations in Dimensional Form .................................................... 94

Sensitivity Analyses for the Flammability Data......................................................... 98

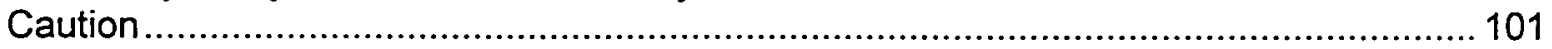

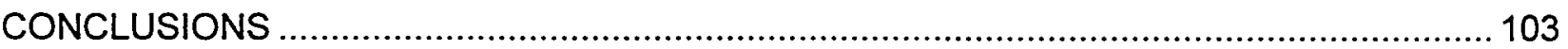

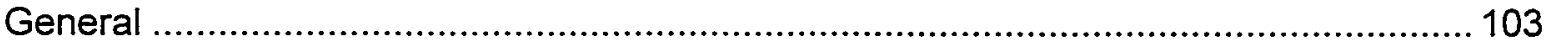




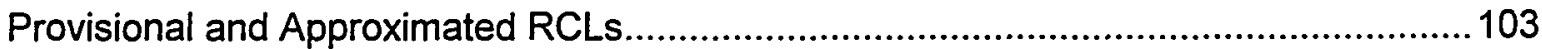

Toxicity Classification................................................................................... 103

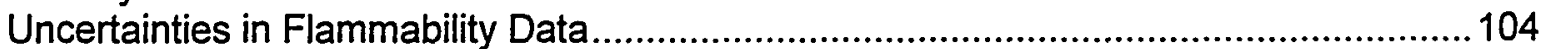

Oxygen Deprivation Limit............................................................................... 104

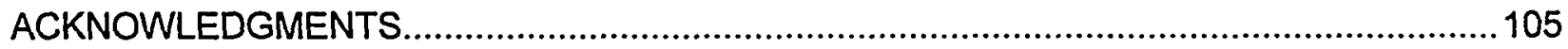

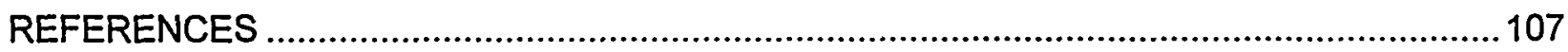

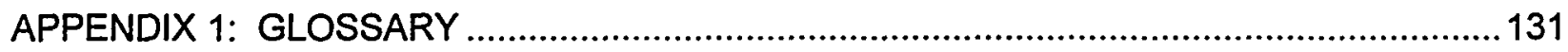

APPENDIX 2: REFRIGERANT PROFILES ......................................................... 135

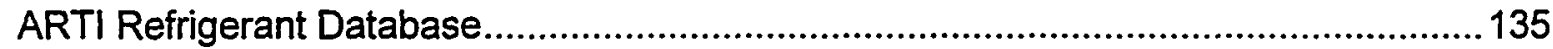

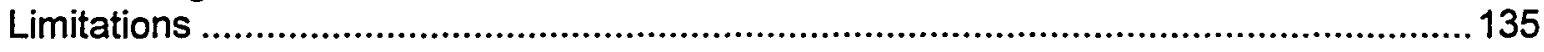

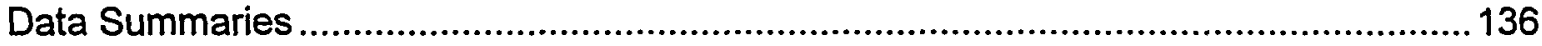

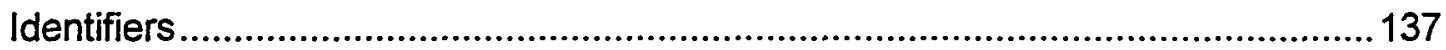

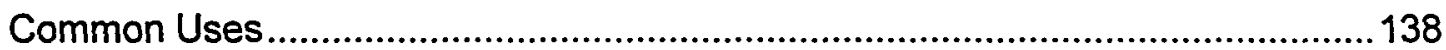

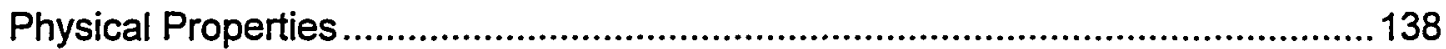

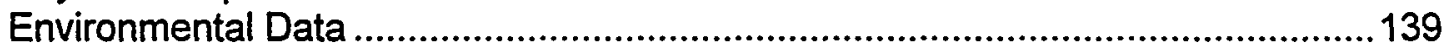

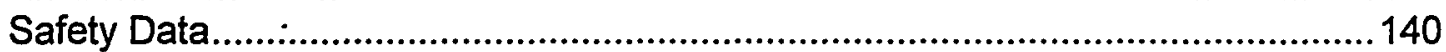

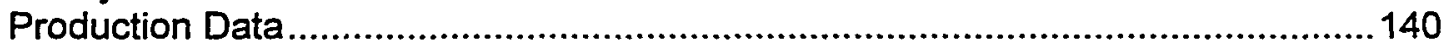

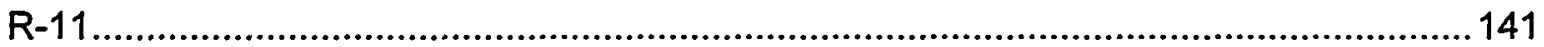

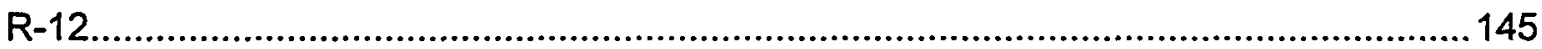

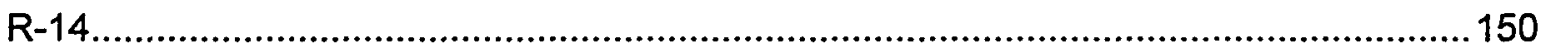

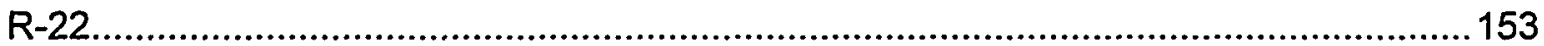

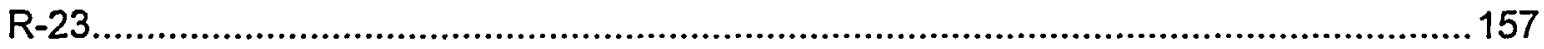

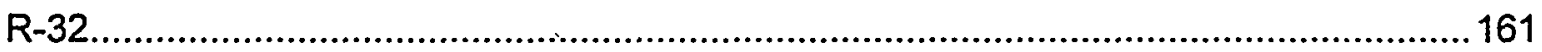

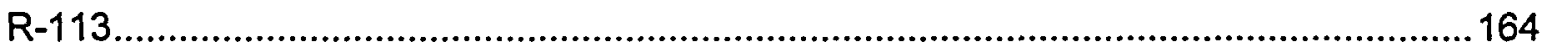

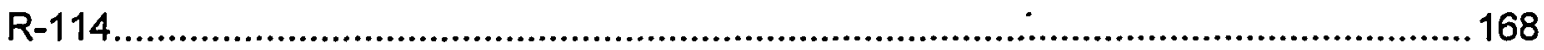

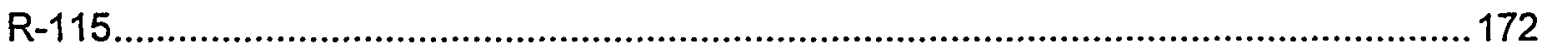

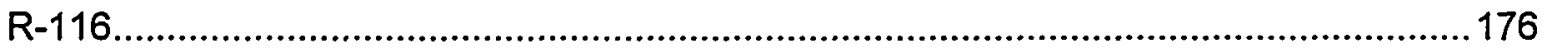

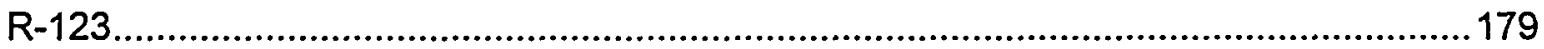

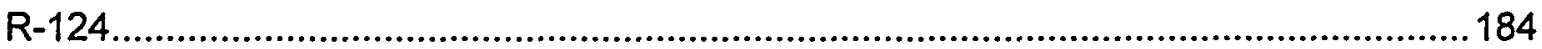

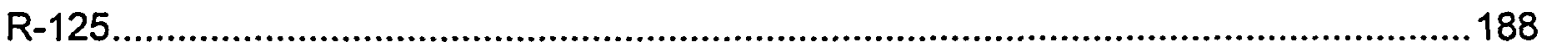

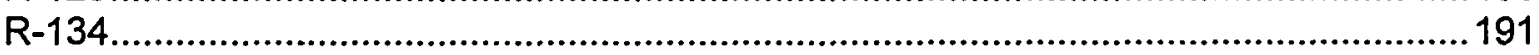

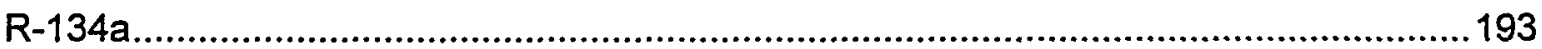

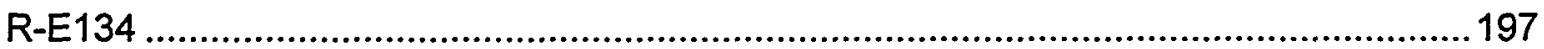

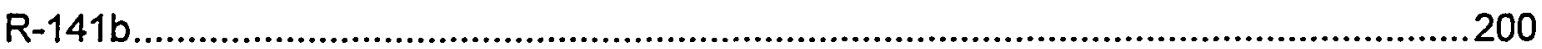

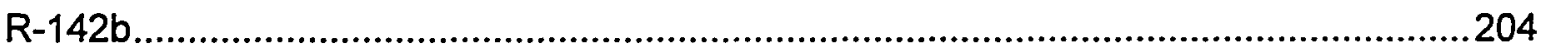

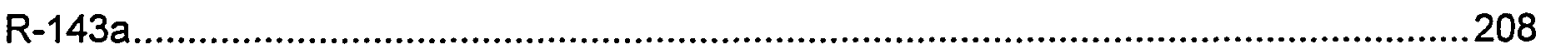

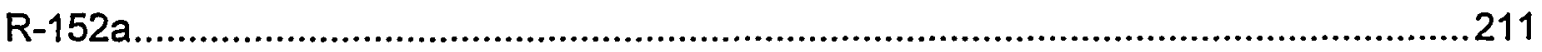

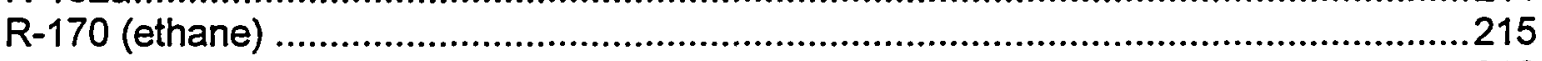

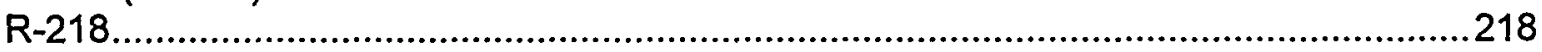

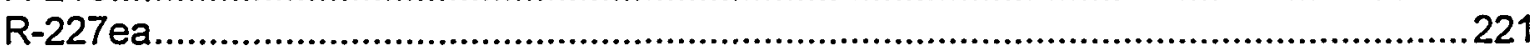

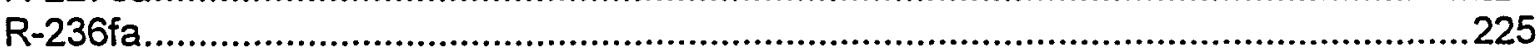

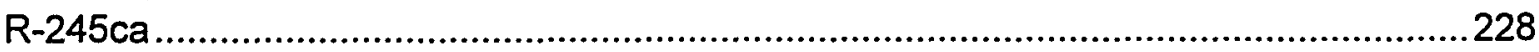

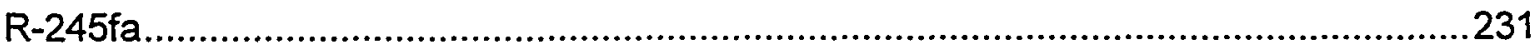

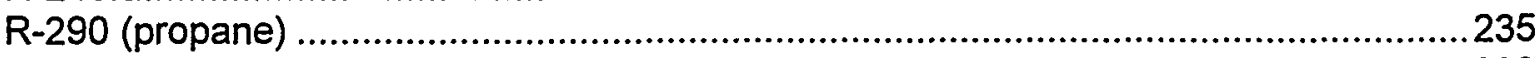

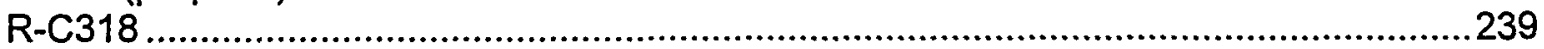


R-502 (n-butane)

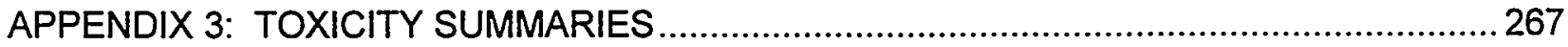

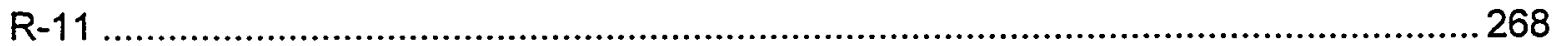

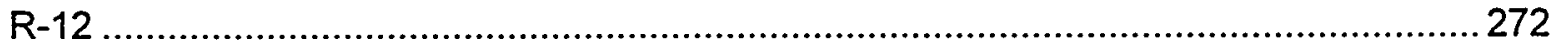

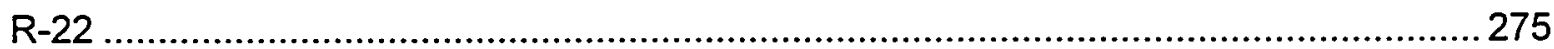

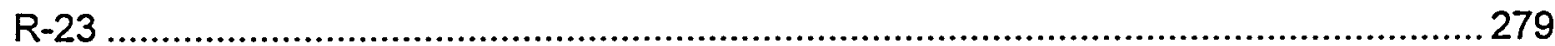

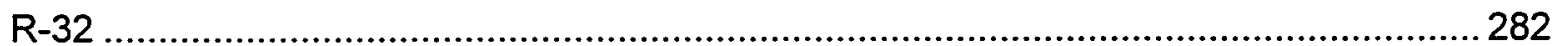

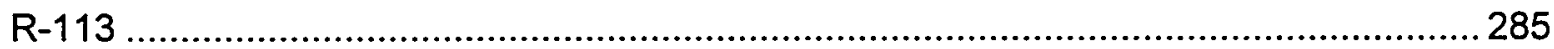

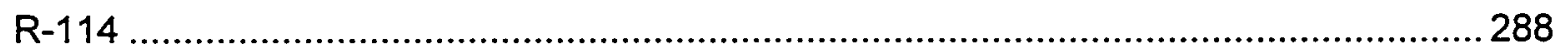

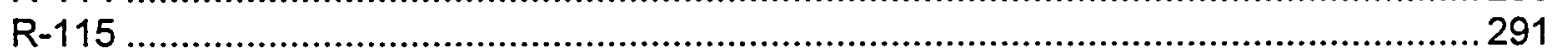

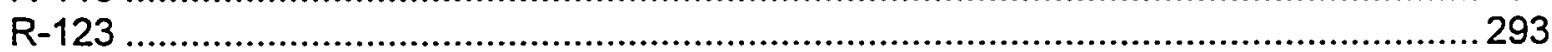

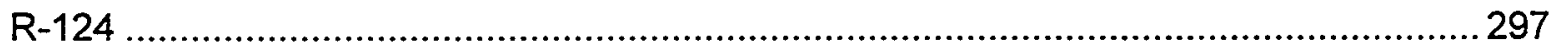

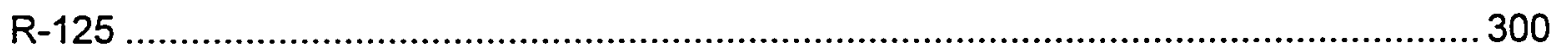

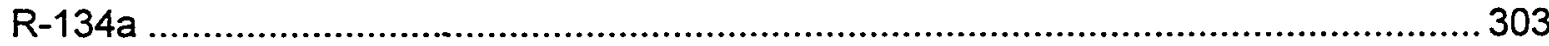

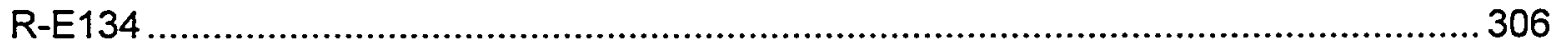

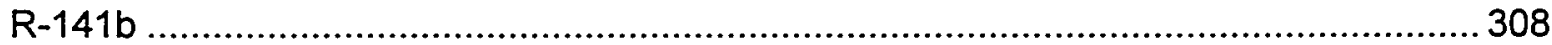

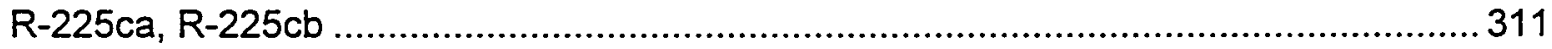

R-290 (propane) ................................................................................................... 313

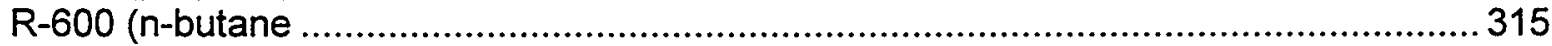

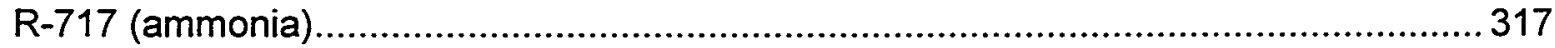

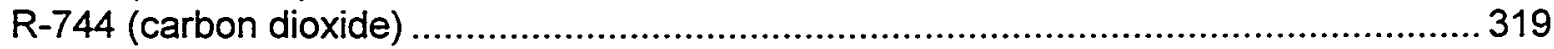

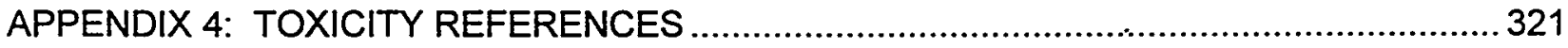

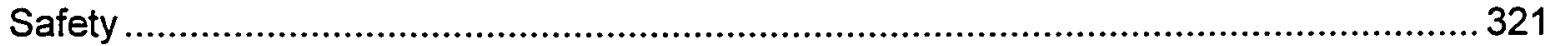

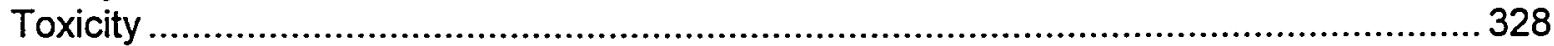

R-10

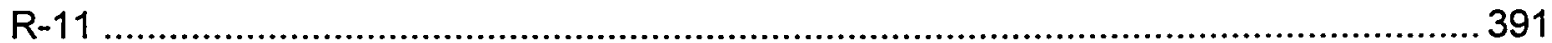

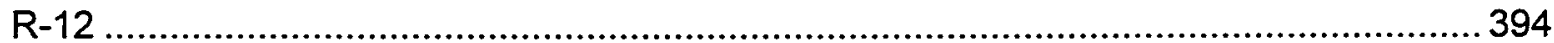

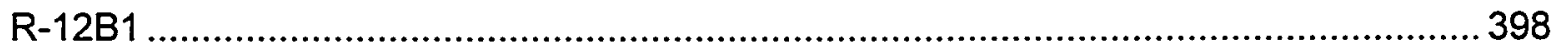

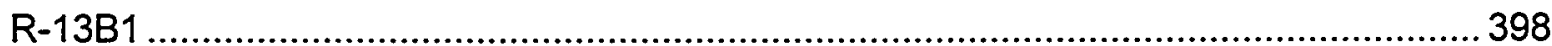

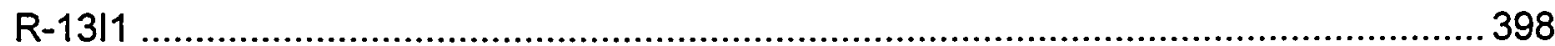

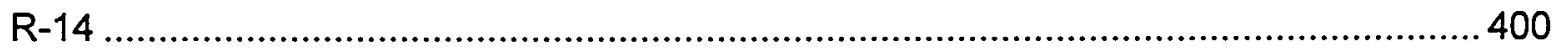

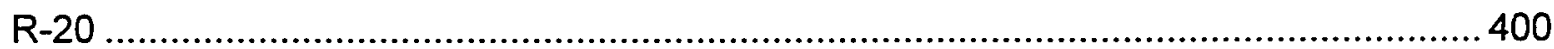

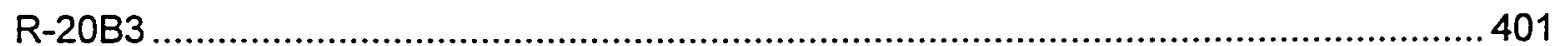

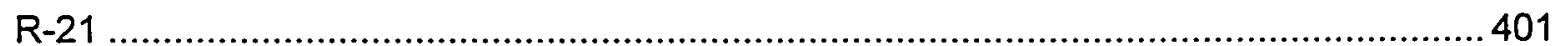

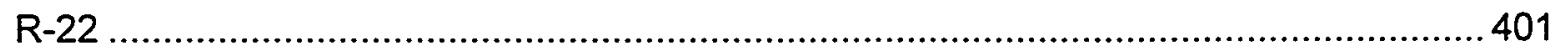

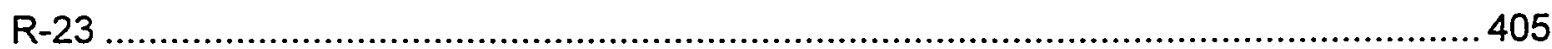

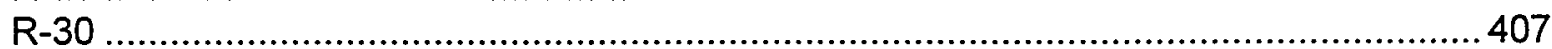

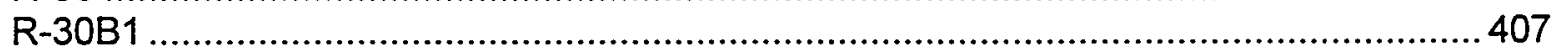

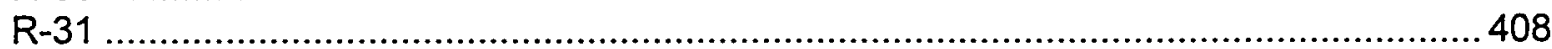

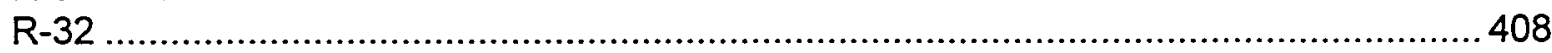

R-40

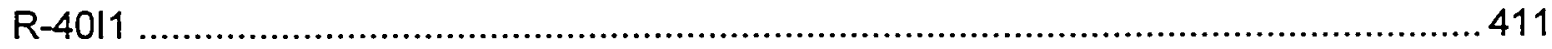




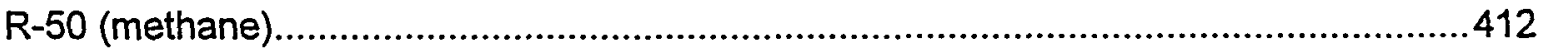

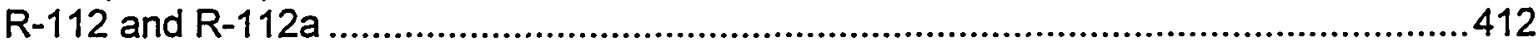

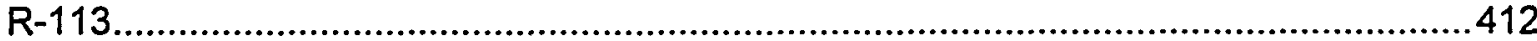

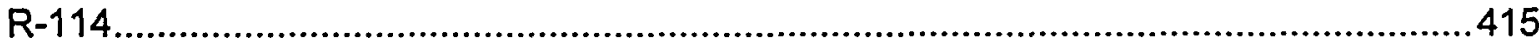

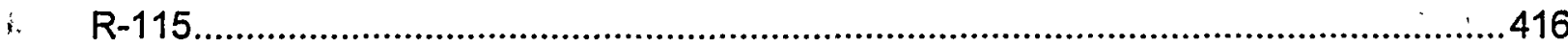

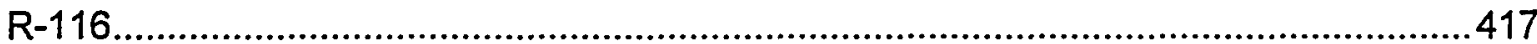

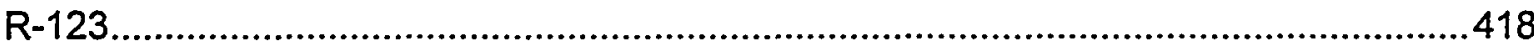

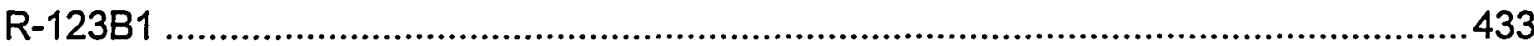

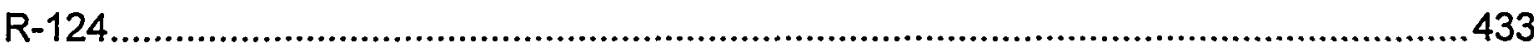

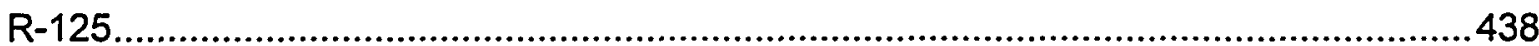

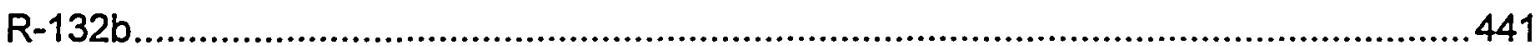

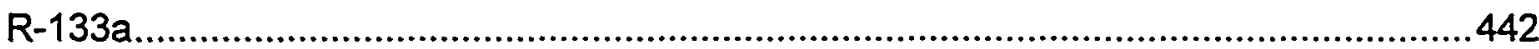

R-134

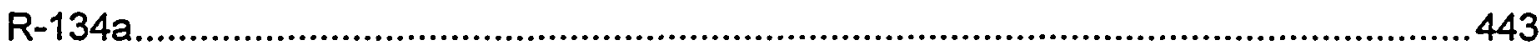

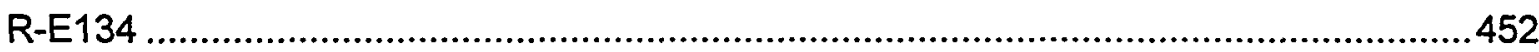

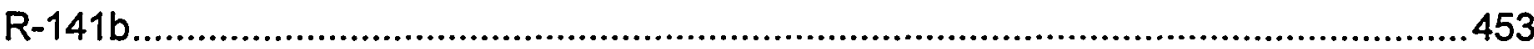

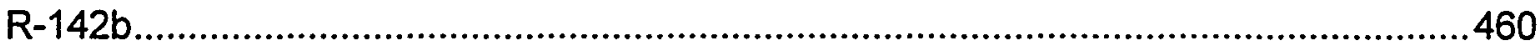

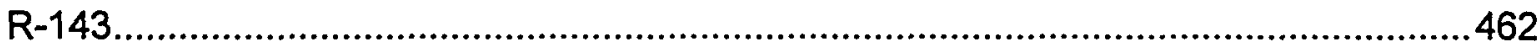

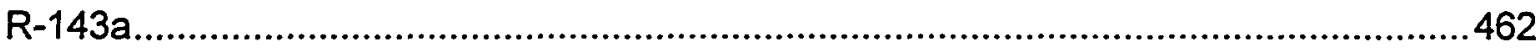

R-152a

R-160

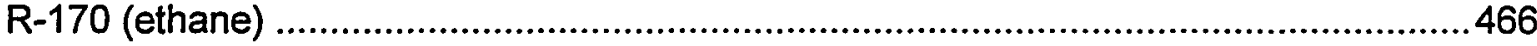

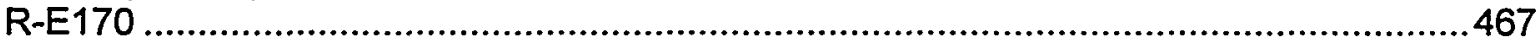

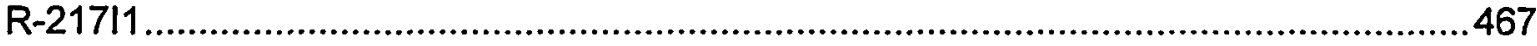

R-218

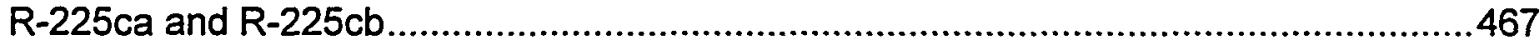

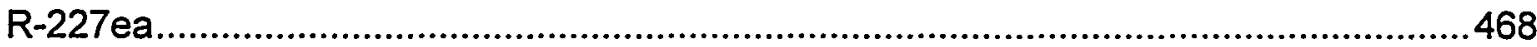

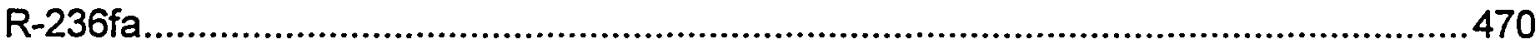

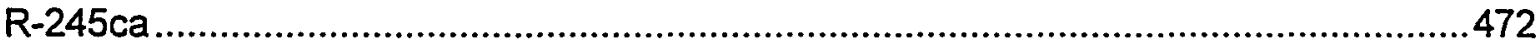

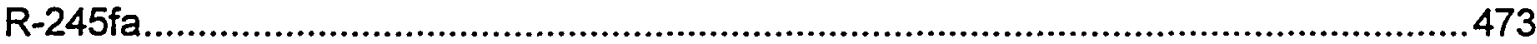

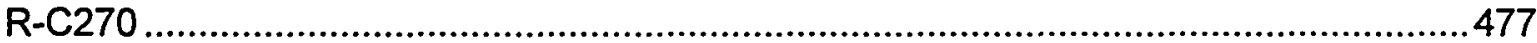

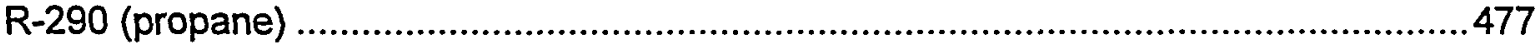

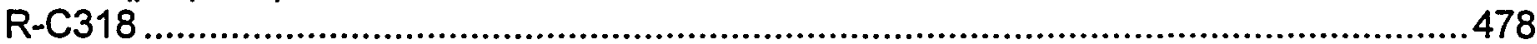

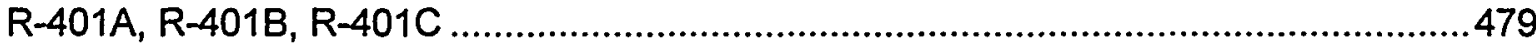

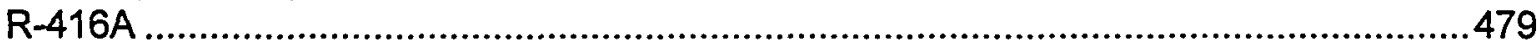

R-502

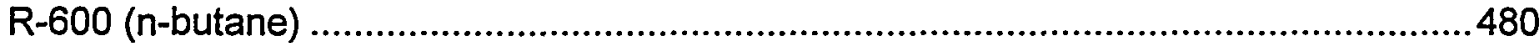

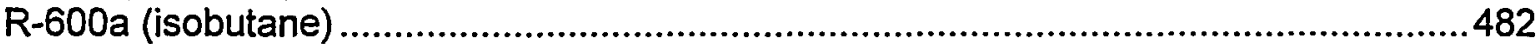

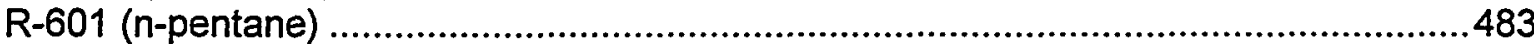

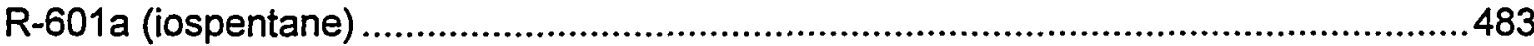

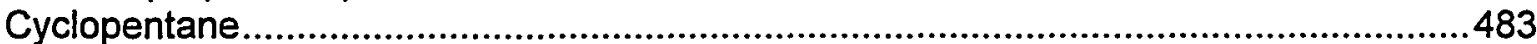

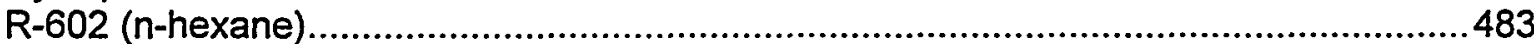

R-630...

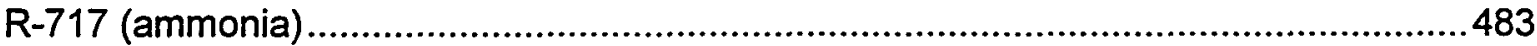

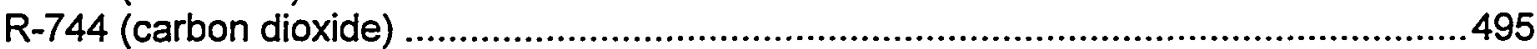

R-744a

R-764

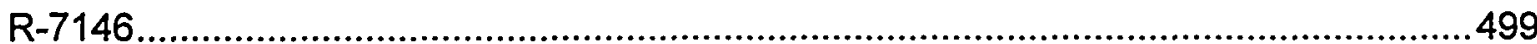

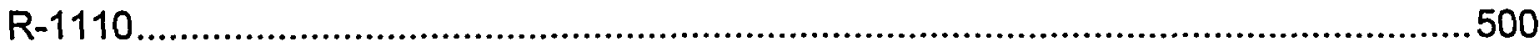

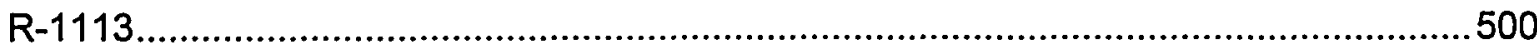

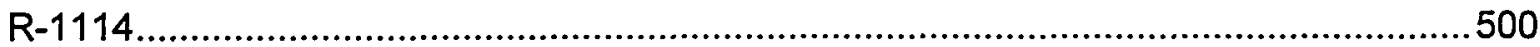


$\mathrm{R}-1120$ 500

$\mathrm{R}-1140$ 501

$\mathrm{R}-1150$

$\mathrm{R}-1216$ 501

$\mathrm{R}-1270$ 501

$\mathrm{R}-1316$ 501

hexafluoropropene oxide (HFPO). 505

APPENDIX 5: BACKGROUND PAPER 505 507

\section{LIST OF TABLES}

Table 1: Refrigerant Toxicity Data (ppm v/v)

Table 2: Refrigerant Toxicity Data with Revisions to ATEL/RCL Method (ppm v/v) 90

Table 3: Refrigerant Classifications and Exposure Limits 92

Table 4: RCL Dimensional Unit Conversions 95

Table 5: RCL Dimensional Unit Conversions for Zeotropic Blends with Designations 96

Table 6: RCL Dimensional Unit Conversions for Azeotropic Blends with Designations

Table 7: RCL Dimensional Unit Conversions for Additional Blends without Designations

Table 8: Parametric Analyses for Flammability

98 Appendix 6 


\section{TOXICITY DATA TO DETERMINE REFRIGERANT CONCENTRATION LIMITS}

\section{INTRODUCTION}

This report reviews toxicity data, identifies sources for them, and presents resulting exposure limits for refrigerants. The data primarily address those pertinent to acute inhalation exposures from emergency releases rather than chronic exposures. Acute toxicity refers to the adverse health effects of a short-term, single exposure. Chronic toxicity refers to those from long-term or repeated exposures. Emergency signifies unplanned and unintended releases with a view toward mitigating risks for the general public. In contrast, occupational exposure limits assume that potentially affected personnel should be cognizant of the potential for exposures, have special awareness or training, and may employ protective measures or devices.

The specific data addressed are those pertinent to a proposed method to set recommended concentration limits, in turn to improve refrigeration safety. This report does not address how those limits should be applied, for example adjustments for specific building occupancies or circumstances. It also does not address measures to assure that the recommended limits are not exceeded, the combinatorial influences of other chemicals that may be present or released in an emergency, or the decomposition products from refrigerant breakdown in fires or other chemical reactions. Based on differences in individual sensitivities to chemical hazards, some people may experience adverse or even life-threatening affects from exposures below the recommended concentration limits.

The scope of this compilation is data gathering and analysis. This report does not present new test results, verify prior findings, or imply endorsement of the identified data. The compilation is intended to assist qualified safety professionals; it is not meant for use by individuals lacking training and experience in control of chemical hazards and refrigeration safety.

The refrigerants covered include those in common use for the last decade, those used as components in common blends, and selected candidates for future replacements.

The data and recommendations are not the result of a consensus determination. Rather this report documents input for consideration by qualified parties in developing guides, standards, codes, and regulations. The primary intended use of the data is to assist the project committees for ASHRAE Standard 34, Designation and Safety Classification of Refrigerants, ${ }^{1}$ and in turn ASHRAE Standard 15, Safety Code for Mechanical Refrigeration (soon to be Safety Code for Refrigeration). ${ }^{2}$ Indeed, some of the recommended data differ from working and review drafts, prepared by those committees, based on preliminary data from this effort and other sources.

\section{PERSPECTIVE}

Most of the dominant refrigerants for the past fifty years have been or are being replaced, to protect the stratospheric ozone layer and to reduce contributions to global warming. Much to 
the credit of the air-conditioning and refrigeration industry, both chemical and equipment manufacturers have resisted compromise to either safety or performance in developing replacements. None of the alternative refrigerants commercialized are highly toxic or even toxic as classified by federal regulations. ${ }^{3}$ Scrutiny of the new refrigerants shows them to be as safe or safer than those they replace. ${ }^{4}$ Most safety concerns with the new refrigerants stem from lack of familiarity and necessary information rather than increased hazard levels.

The majority of early refrigerants - those applied before 1930 - were flammable, toxic, or both. The advent of fluorochemicals* ushered in a new era of safety, as illustrated by the dramatic demonstration by Thomas Midgley in April 1930. In announcing the development of fluorochemicals to the American Chemical Society, he inhaled R-12 and blew out a candle with it. ${ }^{5}$ Although this dramatic stunt suggested that the new refrigerant was deemed safe for toxicity and flammability, it would clearly violate current safety practices.

As subsequent testing established the low toxicity of the new refrigerants, recognition emerged that the primary safety risks were the pressure hazards inherent to any compressed gas, asphyxiation from possible displacement of air, and frostbite with skin contact at low temperatures. These concerns were, however, common to the volatile compounds used before fluorochemicals. As the level of safety improved, so did expectations. Rules evolved to also address acute exposure hazards under emergency conditions, for example potential decomposition in fires into carbonyl halides as well as hydrochloric and hydrofluoric acids. New safety provisions also address the potential for cardiac sensitization and the effects of chronic exposures for both technicians and building occupants. These standards and regulations restrict the use of refrigerants, set quantity limits in occupied areas, impose isolation requirements for refrigerant-containing components and machinery rooms, and prescribe a range of detection, ventilation, pressure relief, emergency discharge, and other safety provisions.

Recent focus on the effects of refrigerants on the environment spawned three significant safety measures, namely system tightening, modification of service practices to reduce venting, and use of electronic refrigerant detectors. Although motivated by environmental protection, to curtail avoidable emissions, the results also lower both the likelihood and the concentrations of refrigerant exposures. The advent of reliable leak detectors affords a mechanism to warn of leaks before harmful concentrations are reached.

The combination of safer chemicals, system tightening, improved service practices, and use of leak detection has greatly reduced the hazard of refrigerant use. In contrast to safety risks that obstructed wide use of refrigerators 70 years ago, consumers presume such appliances and even much larger systems to pose negligible or at least acceptable safety risks. While very infrequent accidents and deaths from refrigerants still occur, most now result from failure to comply with safety requirements and recommended practices. The most dangerous part of working on refrigeration systems today is getting to and from the job site. The chance of death from a refrigerant exposure, excluding intentional abuse, now is more than twenty times less than of being killed by lightning. ${ }^{\dagger}$ Unlike lightning, refrigeration saves far more - perhaps several magnitudes more - lives each year than are lost in refrigerant-related accidents. The lives saved

- compounds consisting of carbon, fluorine, and possibly hydrogen, other halogens such as chlorine, or other elements

$\dagger$ based on known incidents and published statistics for the United States from 1985-1999 
and enhanced result from preservation of foods and pharmaceuticals, conditioning of indoor climates to counter severe heat, and enabling of essential technologies.

While the safety record should improve with new products and compliance with updated safety standards and codes, there still is room for further progress. This report addresses one facet of the problem, to recommend concentration limits as the basis for safety standards and codes to protect the public from unplanned exposures.

A brief review of the fundamental toxicity basis precedes that topic. 
Toxicity Data to Determine Refrigerant Concentration Limits James M. Calm, Engineering Consultant

$-4-$ 


\section{FUNDAMENTAL TOXICITY}

Dosis solo facit venenum.*

* The dose alone makes the poison, a fundamental tenet of toxicology attributed to Paracelsus in the 16 th century ${ }^{6}$ 
Toxicity Data to Determine Refrigerant Concentration Limits James M. Calm, Engineering Consultant

Toxicity Data to Determine Refrigerant Concentration Limits James M. Calm, Engineering Consultant 


\section{EXPOSURE CONCENTRATION LIMITS}

The principle from Paracelsus, dosis solo facit venenum (the dose alone makes the poison), is axiomatic to the objectives of this report. It suggests that exposures to refrigerants, or other chemicals, will not cause harm if kept to a safe level. The goal here is to determine that level as a concentration - for a specified period of time, for common refrigerants, for the majority of people. It does not address exposure limits for people with rare conditions or taking special medications that may make them more sensitive or vulnerable.

Many steps can be taken to isolate refrigerants, to minimize the risks of human (and animal) exposures; they are a separate subject. The issue here is to quantify the concentration to which nearly all people can be exposed without harm. More specifically, the focus is on emergency exposures - those that may occur in unanticipated circumstances such as piping ruptures in occupied spaces. The goal is to determine objectively the highest concentration that will not harm people while they escape or until the refrigerant is removed (for example by ventilation or dissipation of the refrigerant to outside the space).

Such limits differ from occupational exposure limits, in which repeated exposures or potential exposures of worker are anticipated. Beyond using refrigerants with low toxicity, measures such as training, confinement, ventilation, special garments, or even self-contained-breathing apparatus may offer protection as appropriate to the refrigerants, tasks, and locations involved for occupational needs.

Emergency limits for public exposures are different. Most people are not trained or equipped for exposures and do not expect them. Indeed, exposures are infrequent and unlikely for most people.

The objective of this report is to propose data for and to calculate recommended concentration limits for unplanned releases. No practical limit assures complete safety. Differences in individual sensitivities, nonuniform mixing of releases, direct contact, abuse, occurrence of multiple catastrophic events, and similar additional factors may lead to injuries or death despite the best precautions. The method addressed herein builds on that embodied in ASHRAE 15, Safety Code for Mechanical Refrigeration, ${ }^{2}$ which has provided a very high degree of protection.

\section{EMERGENCY EXPOSURE DURATION}

Consistent with ASHRAE 15 and underlying measures to calculate its refrigerant limits in unprotected spaces, the basis in the present work is a maximum exposure duration of 30 minutes. This length is admittedly somewhat arbitrary, but experience suggests that it is ample or even conservative in nearly all cases. Most buildings can be evacuated in less time; institutional oc- 
cupancies* may warrant adjustments to the resulting limits, to compensate for longer evacuation times.

It is difficult to sustain high concentrations of refrigerants. Low-pressure refrigerants (typically those with atmospheric boiling points near or exceeding room temperature) are of low volatility and do not reach high concentrations under the conditions normally maintained in buildings. Indeed, with operation at subatmospheric pressures, air typically is sucked in rather than refrigerant escaping in the event of a leak. Even a catastrophic spill remains a liquid and yields comparatively low airborne concentrations. High-pressure refrigerants (typically those with atmospheric boiling points below or well below room temperature) quickly diffuse into the surrounding space, but then escape out of building openings - also quickly. As a result, they reach high concentrations, but generally only for short periods. The controlling "magic" in both cases is refrigerant volatility, an essential selection criterion for common refrigeration cycles.

Building ventilation helps, but cannot be assumed to function under all emergency conditions. Conversely, catastrophic emergencies - such as earthquakes and explosions - often create new openings by shattering windows, skylights, or even structural holes, thereby increasing ventilation.

Neither low nor high volatility assures safety. Elevated temperatures may increase concentrations or concentration spikes. Sealed areas may sustain them. The 30-min criterion is no more than a planning measure, not a guarantee. Other safety measures as specified in ASHRAE 15; similar safety standards; in building, fire, and mechanical codes and regulations; and incorporated into refrigeration equipment and systems provide further - but not absolute - protection.

As discussed below (see pages 9 and 19), some of the toxicity measures are based on exposure tests for shorter intervals. Some toxic effects develop quickly and then stay level or even decrease with time. Others can be extrapolated for selected intervals as reflected in the methodology proposed. Still others are intentionally longer to be conservative, based on the severity of consequences or inadequacy of samples.

\section{EFFECTS}

The method proposed concentrates on toxicity, oxygen deprivation (asphyxiation), and flammability. Within toxicity, it focuses on lethality, cardiac sensitization, anesthetic effects, and a catch-all category of other escape-impairing and or permanently injuring effects. The first three types of toxicity are deemed those most pertinent for protection against acute, emergency exposures and the fourth category allows latitude for special cases, as follows:

- Lethality (or mortality) indicates the likelihood of toxic fatalities from exposure, without regard to the mechanism except to exclude those from dismemberment, crushing, burning, or similar physical harm. Review of the toxicity studies cited below shows that the causes of death found in animal exposures varied in the affected organs or biological systems. The pertinent path is by inhalation, based on the premise that neither normal use nor emergency release of refrigerants is likely to result in sufficient dermal (skin) contact or ingestion (oral) to be issues. Some of the refriger-

* Examples of institutional occupancies include those for care of disabled people such as hospitals and convalescent homes, care or education of infants such as nurseries, and housing of confined people such as prisons. 
ants addressed herein (see page 35) either are or previously have been used for direct-contact food freezing, food propellants, or foam blowing agents for food packaging. Likewise, some either are or previously have been used as propellants in cosmetic, pharmaceutical, or other aerosol products. Some either are or previously have been used as topical, dental, or surgical anesthetic agents or as solvents in consumer and industrial products. Some either are or previously have been used as fire suppressants or in a range of specialty applications such as expansion gases for retinal eye surgery. The relevant toxicities for those applications, including both acute and chronic effects, go beyond the present scope.

- Cardiac sensitization refers to an acute effect in which the heart is rendered more sensitive to the body's own catecholamine compounds* or administered drugs, such as epinephrine, possibly resulting in irregular heart beat (cardiac arrhythmia) that could be fatal.

- Anesthetic effect refers to dulling of the senses, as manifested in loss of the ability to perceive pain and other sensory stimulation. Some toxicologists include impairment of judgement or mental ability to respond while others deem it to include only analgesia (insensibility to pain without loss of consciousness) or induced loss of consciousness. Central nervous system (CNS) effects include depression, distraction, stimulation, or other behavioral modification suggesting temporary or permanent changes to control by the brain. As addressed herein, anesthetic and central nervous system effects exclude those from intentional abuse (deliberate refrigerant inhalation seeking either a narcotic effect from either chemical induction or oxygen deprivation), sometimes referred to as "sniffing," for which the only safe limit is zero.

- Other escape impairing effects or permanent injuries include any effect that is likely to impede the ability to escape (such as distraction, blinding, tearing of the eyes sufficiently to reduce vision, or severe sensory irritation) or to cause other irreversible health effects (such as damage to or lesions in skin or other tissues that is not selfhealing).

There are many other forms of toxicity, such as carcinogenicity and reproductive effects. They warrant consideration in selection or regulation to protect exposed technicians. The effects addressed herein are those deemed pertinent to acute inhalation exposures from emergency releases rather than chronic exposures.

\section{QUANTIFICATION}

Toxicologists refer to the concentrations, determined by toxicity studies, that bracket the threshold (or trigger) effect level for specified exposure periods based on the number of subjects of the sample tested that show no or some treatment-related response. These concentration levels are the dose that makes the poison for specified exposure durations, biological species, and

* Catecholamines include adrenaline or adrenaline like chemicals that consist of benzene rings with attached groups of nitrogen and halogen atoms or nitrogen and halocarbon or hydrocarbon radicals. They function in the body as hormones, neurotransmitters, or both: The body's adrenal glands release them under conditions of stress or strong emotion such as fear or anger. Medical injections of epinephrine as a stimulant in cardiac arrest, as a vasoconstrictor in shock, and as a bronchodilator and antispasmodic in bronchial asthma have similar effects. 
conditions. The species tested, typically laboratory animals such as mice, rats, dogs, or monkeys but sometimes bacteria or plants, are selected for their suitability as models of specific toxic effects in humans or the environment.

The three effect levels used in the method described herein are the:

- no observed effect level (NOEL) - the highest concentration of a material, a refrigerant in the present context, at which no effect has been observed in even one tested animal.

- lowest observed effect level (LOEL) - the concentration of a material, a refrigerant in the present context, that has caused an observed effect to even one tested animal.

- median effective concentration $\left(E C_{50}\right)$ - the concentration of a material, a refrigerant in the present context, that has caused a biological effect to half the animals tested. $E_{50} s$ generally are calculated estimates from regression of the test data based on accepted methods.

Some toxicologists prefer to cite no obsenved adverse effect level (NOAEL) or the lowest observed adverse effect level (LOAEL) to exclude effects deemed of no toxicological import. Similarly, some toxicologist prefer no or lowest observable rather than no observed. Except as specifically noted, subsequent references in this report to NOEL and LOEL concentrations make no distinction between NOEL and NOAEL, between $\angle O E L$ and $L O A E L$, or observed and $o b$ servable.

Toxicologists sometimes refer to specific forms of the NOEL, LOEL, and $\mathrm{EC}_{50}$ concentrations, namely the:

lethality

- median lethal concentration ( $\left.L C_{50}\right)$ - an $\mathrm{EC}_{50}$ for lethality. It is the concentration that is fatal to half of exposed animals for an identified species and exposure duration either during the exposure or during a subsequent recovery and observation period. The $\mathrm{LC}_{50}$ is a primary measure of acute toxicity by inhalation of gases. It most commonly is measured with rats for exposures of four hours. A number of federal regulations in the USA (for example reference 1) and most building, fire, and mechanical codes deem substances to be toxic for a one-hour $\mathrm{LC}_{50}$ of $200-2,000 \mathrm{ppm} \mathrm{v} / \mathrm{v}$ and highly toxic for less than $200 \mathrm{ppm}$ v/v. Based on adjustments discussed on page 11, typical $L C_{50}$ concentrations for four hours are approximately equivalent in effect to half of those for one hour. This extrapolation suggests corresponding criteria for toxic substances of a 4-hr $L_{50}$ of $100-1,000 \mathrm{ppm}$ v/v and highly toxic substances for less than $100 \mathrm{ppm} v / \mathrm{v}$.

- median lethal dose $\left(L D_{50}\right)$ - an $\mathrm{EC}_{50}$ for lethality. The $\mathrm{LD}_{50}$ generally is used to quantify exposures in dermal (skin) and ingestion (oral) toxicity studies, but some old papers also use $L D_{50}$ in place of $L C_{50}$ for inhalation studies.

- approximate lethal concentration (ALC) - a LOEL for lethality. It is the lowest concentration that is fatal to one or more exposed animals for an identified species and exposure duration. It most commonly is measured with rats for inhalation exposures of four hours. The ALC is now more widely used to minimize the number of animals killed in testing, since further tests at higher concentrations and the larger sample sizes needed to determine an $L_{50}$ can be avoided. The ALC is a conservative ap- 
proximation to the $\mathrm{LC}_{50}$ as long as the ALC was fatal to less than half the animals exposed. It is a close approximation, but not necessarily conservative, when fatal to exactly half the animals tested.

- approximate lethal dose (ALD) - a LOEL for lethality. The ALD generally is used to quantify exposures in dermal and ingestion toxicity studies, but some old papers also use ALD in place of ALC for inhalation studies.

- minimal lethal concentration or minimum lethal concentration (MLC) - alternative descriptors for ALC.

cardiac sensitization

- median cardiac sensitization concentration $\left(C_{50}\right)$ - an $\mathrm{EC}_{50}$ for cardiac sensitization. It is the concentration that sensitizes the hearts of half of animals exposed by inhalation and given an epinephrine (or similar) challenge by injection for an identified species and exposure duration. It most commonly is measured with beagle dogs based on their aptitude for training for this test and their suitability as a model for human cardiovascular responses. Most studies simply refer to an $\mathrm{EC}_{50}$ for cardiac sensitization rather than a $\mathrm{CS}_{50}$.

\section{anesthetic or central nervous system (CNS) effects}

- anesthetic concentration $\left(A C_{50}\right)$ - an $\mathrm{EC}_{50}$ for anesthetic or CNS effects. It is the concentration that caused an anesthetic or CNS effect in half of test animals, normally measured for 10 minute exposures of mice or rodents. Most studies simply refer to an $\mathrm{EC}_{50}$ for anesthetic effect rather than an $\mathrm{AC}_{50}$.

- approximate anesthetic concentration (AAC) or approximate anesthetic dose (AAD) - a LOEL for anesthetic or CNS effects. It is the concentration that caused an anesthetic or CNS effect in one or more test animals. Most studies simply refer to a LOEL for anesthetic effect rather than an AAC or AAD.

\section{other}

- median rate decrease $\left(R D_{50}\right)$ for respiration - the concentration that resulted in $50 \%$ decrease in respiratory rate, normally measured in mice. A maximal effect generally occurs in less than 30 minutes; the response to R-717 (ammonia), as an example, takes approximately two minutes (see page 80 ).

\section{TEN BERGE ADJUSTMENT}

The exposure time used to test the lethality of chemicals differs between studies. The norm is 30 min or less for most old studies and in those explicitly gauging the effects of brief exposures. Most recent tests, particularly those of chemicals with low acute inhalation toxicity, are for intervals of 4 hours or longer to increase reliability. The Programme for Alternative Fluorocarbon Toxicity Testing (PAFT) studies, drawn on herein for some data for new refrigerants, used fourhour tests. ${ }^{7.8}$

There are several ways to estimate consistent $L_{5_{0}} S$ or ALCs determined for different time periods. Haber's law, a tenet of toxicology, states that different concentrations have the same lethal effect when the product of the exposure concentration and duration - c.t (where $c$ is concentration and $t$ is time) - is constant for small differences in time, ${ }^{6,9}$ hence: 


$$
\begin{aligned}
& L C_{50 \text { for } T}=L C_{50 \text { for } t} \cdot(t / T) \\
& \text { or } \\
& A L C_{T}=A L C_{t} \cdot(t / T)
\end{aligned}
$$

where:

$T$ is the desired lethality indication time, and

$t$ is the exposure time from the study being adjusted.

A widely cited study by ten Berge et al. found that treating $c^{n} \cdot t$ as constant - with the added exponent $n-$ affords better prediction. ${ }^{10}$ They found that $n$ ranged from 0.8 to 3.5 , with an average of 1.86 , for 20 different chemicals. That finding suggests that:

$$
\begin{aligned}
& L C_{50 \text { for } T}=L C_{50 \text { for } t} \cdot(t / T)^{1 / n} \\
& \text { or } \\
& A L C_{T}=A L C_{t} \cdot(t / T)^{1 / n}
\end{aligned}
$$

where:

$\mathrm{n}$ is the ten Berge et al. exponent.

Inspection shows that Haber's law and the ten Berge adjustment are the same for $n=1$. In determining the NIOSH IDLH concentrations (discussed below), Ludwig et al. use the coefficients for the chemicals studied by ten Berge et al. and a coefficient of $n=3.0$ for other substances. ${ }^{11}$ The International Fire Code, ${ }^{12}$ International Maritime Dangerous Goods Code, ${ }^{13}$ and Uniform Fire Code ${ }^{14}$ use $n=2.0$, which is the coefficient that ten Berge et al. report for five chemicals including HF (hydrofluoric acid) and R-717 (ammonia). ${ }^{10}$ The indication by Ludwig et al. that $n=3.0$ is "a conservative estimate" usually holds true as they used it, namely to estimate 30minute lethal concentrations most often from studies for longer periods. ${ }^{11}$ Whereas $n=2.0$ is more conservative than $n=3.0$ to estimate four-hour lethality from studies for shorter periods, and since $n=2.0$ is widely accepted in codes as noted, the exponent $n=2.0$ is used for the remainder for this report except where indicated.

\section{EXPOSURE LIMITS AND TOXICITY INDICATORS}

The following paragraphs explain several related terms that are cited in the discussion of toxicity data to arrive at the concentration limits or of those limits:

immediately dangerous to life and health (IDLH) concentration - a measure developed as a criterion for respirator selection. Some safety standards as well as building, fire, and mechanical codes subsequently applied IDLH concentrations to approximate the threshold level for unacceptable toxicity or flammability risk.* The U.S. National Institute for Occu-

* Although widespread, these uses beg controversy as inconsistent with the IDLH definition and for clouding of occupational and emergency risk criteria. Nevertheless, most fire codes in the USA use the IDLH, typically reduced by a safety factor, as a criterion for ventilation rates and emergency discharge treatment for refrigerants and other hazardous materials. ASHRAE 15 also uses the SCP IDLH as one of several criteria to determine allowed refrigerant quantities for occupied areas. 
pational Safety and Health (NIOSH) and U.S. Occupational Safety and Health Administration (OSHA) jointly promulgated the original IDLH values in the mid-1970s as part of the Standards Completion Program (SCP). The SCP definition for IDLH was "the maximum concentration from which, in the event of a respirator failure, one could escape within 30 minutes without a respirator and without experiencing any escape-impairing (e.g., severe eye irritation) or irreversible health effects." A NIOSH revision in 1994 defines the IDLH concentration as one "that poses a threat of exposure to airborne contaminants when that exposure is likely to cause death or immediate or delayed permanent adverse health effects or prevent escape from such an environment." The new IDLHs reflect additional toxicity criteria and data as well as a more restrictive multiplier for flammability, reduced from $100 \%$ to $10 \%$ of the lower-flammability limit (LFL). Of the 387 IDLH concentrations, the revision lowered all but 56 and raised none.

permissible exposure limit (PEL) - the occupational concentration limit established by OSHA. Without qualification, the PEL implies a time-weighted average (TWA) for an eight-hour work shift in a 40-hour work week. Consistent data include similarly-defined limits set by manufacturers, the Recommended Exposure Limit (REL) set by $\mathrm{NIOSH}^{15}$ the Threshold Limit Value (TLV) adopted by the American Conference of Governmental Industrial Hygienists (ACGIH), ${ }^{16}$ and the Workplace Environmental Exposure Level (WEEL) guides developed by the American Industrial Hygiene Association (AlHA). ${ }^{9}$ Several countries also publish similar measures, of which the German Maximale Arbeitsplatz Konzentration (MAK, maximum workplace concentration) is the most commonly cited. ${ }^{17.18}$ Where designated as a PEL-C (for example for R-11), the PEL is a ceiling concentration that shall not be exceeded. Most codes specify either the PEL or TLV-TWA values and consistent measures as the maximum activation levels for refrigerant leak-detector alarms. While the PEL and TLV-TWA are similarly defined, and the original PELs were based on TLVs, the PEL values have not been revised since 1971. More protective limits published in 1989 were vacated by a court order in 1992. ACGIH publishes annual TLV updates. Still, neither PELs nor TLVs have been set for most alternative refrigerants, for which the primary recourse is use of WEELs or other consistent measures. Some manufacturers identify their consistent or modified limits for refrigerants and other chemicals as a PEL while others use the terms allowable exposure limit (AEL), industrial exposure limit (IEL), or occupational exposure limit (OEL).

ASHRAE 34 safety group - This classification consists of a letter ( $A$ or $B$ ), which indicates the toxicity class, followed by a number $(1,2$, or 3 ), which indicates the flammability class. ${ }^{1}$ Toxicity classes $A$ and $B$ signify refrigerants with lower toxicity and higher toxicity, respectively, based on prescribed measures of chronic (long-term, repeated exposures) toxicity.* Flammability class 1 indicates refrigerants that do not show flame propagation in air when tested by prescribed methods at specified conditions. Classes 2 and 3 signify refrigerants with lower flammability and higher flammability, respectively; the distinction depends on both the LFL and heat of combustion (HOC). Some of the mechanical codes written before 1993 used an older safety classification system from earlier editions of ASHRAE 34. ${ }^{19}$ They included groups 1 (no flame propagation and low degree of toxicity), 2 (TLV-TWA less than 400 ppm), 3a (flammable with low LFL or high HOC), 3b (flammable with high LFL and low HOC), $4 a$ (mixtures of groups 1 and $3 a$ that are nonflammable

* The distinction is based on the data used to determine the TLV-TWA or consistent measures. Those with no identified toxicity at $400 \mathrm{ppm} v / \mathrm{v}$ or less are assigned to Class $A$, and those with identified toxicity at $400 \mathrm{ppm}$ v/v or less are assigned to Class B. 
as formulated, but could become flammable upon fractionation), and $4 b$ (mixtures of groups 1 and $3 \mathrm{~b}$ that are nonflammable as formulated, but could become flammable upon fractionation). Excluding the group 2 refrigerants, the ranked order from the lowest to highest flammability hazard was $1,4 b, 4 a, 3 b$, and $3 a$. One motive for the current classification system, introduced in 1992, was to provide a more rational system.

quantity limits for occupied areas - the primary criterion to determine whether refrigeration systems, or refrigerant-containing components, are allowed in occupied areas of buildings. The limits are set by ASHRAE 15 and by building, fire, and mechanical codes. ${ }^{2}$ Nearly all limits set in the codes are transcribed from ASHRAE 15 (or earlier versions of this standard), though a few intended and unintended revisions are included.

UL group - the Underwriters Laboratories classification reflects the comparative life hazard of refrigerants in the absence of flames or surfaces at high temperatures. Group 1 is the most toxic (for example R-764, sulfur dioxide) and group 6 the least. ${ }^{20}$ This measure is used to classify refrigerants in older codes, still in effect in some jurisdictions. 


\section{PROPOSED METHOD}

The method developed to determine a recommended refrigerant concentration limit $(R C L)$ for each refrigerant, on a consistent basis, is rooted in the "quantity of refrigerant per occupied space" from ASHRAE Standard 15, Safety Code for Mechanical Refrigeration. ${ }^{2}$ The standard provides that it shall be "the maximum amount of refrigerant that will leak into the occupied space at any one time, based on a leak from any single independent refrigerant circuit." Although referred to as a "quantity" in ASHRAE 15 and most safety code provisions derived from that standard, the limit actually is a concentration. The quantity limit is the product of this concentration and the calculated volume of the space. The concentration limit (or quantity limit) may be exceeded in certain circumstances with adherence to identified provisions, most commonly isolation of the portions of the system containing refrigerant in a refrigeration machinery room that meets prescribed safety requirements. Use of refrigeration machinery rooms is standard practice for large systems and sometimes opted even when not required. Neither ASHRAE nor most codes place a limit on the amount of refrigerant allowed in a refrigeration machinery room except in the case of flammable refrigerants.

Many of the specific requirements in ASHRAE 15 are based on safety classifications ${ }^{*}$ from ASHRAE 34. ${ }^{1}$ The latter standard is the definitive source for assignment of refrigerant number designations. It also provides a safety classification system and assigned classifications.

The RCL determination method ${ }^{\dagger}$ was first proposed to the separate project committees developing ASHRAE 15 and 34 on 1995.09 .04 for incorporation into these consensus standards, which are the basis for most refrigerant safety regulations both in the USA and internationally. By agreement of the two standards committees, the one for ASHRAE 34 and initially its Designations and Nomenclature Subcommittee and now its Toxicity Subcommittee are taking the lead to incorporate the method into a consensus standard. The subcommittee addressing Safety of Refrigeration Systems of the International Standards Organization (ISO) Technical Committee

* The key distinctions in ASHRAE 15 and code requirements relate to the flammability component of the safety group. As discussed on page 105, the toxicity component of the classification is of minor consequence.

$\dagger$ The initial draft identified the RCL as the Recommended Quantity Limit (RQL) in keeping with ASHRAE 15, but SSPC 34 recommended revision to reflect its quantification as a concentration rather than an absolute amount. The text shown starting on page 19 reflects minor revisions subsequently proposed to and/or recommended by SSPC 34 .

The draft method was placed in the public domain to avoid copyright complications and to facilitate its adoption in standards and codes. The following note was included in the original proposal and subsequent revisions: "As the author of part or all of [the Proposed Revision to ANSIIASHRAE Standard 34-1992], James M. Calm, Engineering Consultant, is placing the original portion in the public domain. The author grants to ASHRAE and to all other parties nonexclusive and royalty-free permission to publish, reproduce, sell, or reprint it, in whole or in part and in its present form or with modifications. No warranty is implied that portions of this document have not been copyrighted previously by other parties." 
on Refrigeration and Air Conditioning - "ISO TC86/SC1MG1" and "ISO TC86/SC1MG5" are drafting amendments to the pertinent international standards to incorporate the method, possibly with changes still being debated.

\section{PROPOSED REVISIONS TO ASHRAE 34}

The RCL is the lowest of three independently calculated limits for toxicity, oxygen deprivation, and flammability. The toxicity component is identified as the acute toxicity exposure limit (ATEL), based on the most restrictive of four effects addressed above (see page 8 ). The method is currently pending a third public review, previously identified as proposed addendum 34aa to 34-1992 and now as proposed addendum 34u to 34-1997, for revision of ASHRAE 34.

For context, the following terms already are defined in ASHRAE $34:^{1}$

acute toxicity: the adverse health effect(s) from a single, short-term exposure, as might occur during an accidental release of refrigerants.

cardiac sensitization: an acute effect in which the heart is rendered more sensitive to the body's own catecholamine compounds or administered drugs, such as epinephrine, possibly resulting in irregular heartbeat (cardiac arrhythmia), which could be fatal.

ceiling: an exposure level, permissible exposure level-ceiling (PEL-C) or threshold limit value-ceiling (TLV-C), that should not be exceeded during any part of the day.

chronic toxicity. adverse health effect(s) from long-term, repeated exposures. This information is used, in part, to establish a TLV-TWA, PEL, or consistent indices.

$L C_{50}$ : a measure of acute, inhalation toxicity representing a lethal concentration for $50 \%$ of exposed test animals for a specified time interval and species of animal.

ppm: parts per million

permissible exposure limit (PEL): the time-weighted average concentration (set by OSHA, the U.S. Occupational Safety and Health Administration) for a normal 8-hour work day and a 40-hour work week to which nearly all workers can be repeatedly exposed without adverse effect. Chemical manufacturers publish similar recommendations (e.g., acceptable exposure level, AEL; industrial exposure limit, IEL; or occupational exposure limit, OEL; depending on the company), generally for substances for which PEL has not been established.

refrigerant: the fluid used for heat transfer in a refrigerating system; the refrigerant absorbs heat and transfers it at a higher temperature and a higher pressure, usually with a phase change

short-term exposure limit (STEL): typically a 15-minute time-weighted average (TWA) exposure that should not be exceeded any time during a work day.

threshold limit values (TLVS): refer to airborne concentrations of substances and represent conditions under which it is believed that nearly all workers may be repeatedly exposed day after day without adverse health effects. Because of wide variation in individual susceptibility, however, a small percentage of workers may experience discomfort 
from some substances at concentrations at or below the threshold limit; a smaller percentage may be affected more seriously by aggravation of a pre-existing condition or by development of an occupational illness. Smoking of tobacco is harmful for several reasons. Smoking may act to enhance the biological effects of chemicals encountered in the workplace and may reduce the body's defense mechanisms against toxic substances.

Individuals may also be hypersusceptible or otherwise unusually responsive to some industrial chemicals because of genetic factors, age, personal habits (smoking, use of alcohol or other drugs), medication, or previous exposure. Such workers may not be adequately protected from adverse health effects from certain chemicals at or below the threshold limits. An occupational physician should evaluate the extent to which such workers require additional protection.

TLVs are based on the best available information from industrial experience, from experimental human and animal studies, and, when possible, from a combination of the three. The basis on which the values are established may differ from substance to substance; protection against impairment of health may be a guiding factor for some, whereas reasonable freedom from irritation, narcosis, nuisance, or other forms of stress may form the basis for others. *

threshold limit value-time-weighted average (TLV-TWA): the time-weighted average concentration for a normal 8-hour workday and a 40-hour workweek, to which nearly all workers may be repeatedly exposed, day after day, without adverse effect.*

toxicity: the ability of a refrigerant to be harmful or lethal due to acute or chronic exposure by contact, inhalation, or ingestion: The effects of concern include, but are not limited to, those of carcinogens, poisons, reproductive toxins, irritants, corrosives, sensitizers, hepatoxins [hepatotoxins], nephrotoxins, neurotoxins, agents that act on the hematopoietic system, and agents that damage the lungs, skin, eyes, or mucous membranes. For this Standard, temporary discomfort at a level that is not impairing is excluded.

WEEL: workplace environmental exposure limit (set by the American Industrial Hygiene Association, AlHA)

* The definition in ASHRAE 34 is reprinted from ACGIH. ${ }^{21}$ An ACGIH update adds: ${ }^{16}$

"The amount and nature of the information available for establishing a TLV varies from substance to substance; consequently, the precision of the estimated TLV is also subject to variation and the latest TLV Documentation should be consulted in order to assess the extent of the data available for a given substance.

"These limits are intended for use in the practice of industrial hygiene as guidelines or recommendations in the control of potential workplace health hazards and for no other use, e.g., in the evaluation or control of community air pollution nuisances; in estimating the toxic potential of continuous, uninterrupted exposures or other extended working periods; as proof or disproof of an exiting disease or physical condition; or adoption or use by countries whose working conditions or cultures differ from those in the United States of America and where substances and processes differ. These limits are not fine lines between safe and dangerous concentrations, nor are they a relative index of toxicity. They should not be used by anyone untrained in the discipline of industrial hygiene.

"The TLVs, as issued by ACGIH, are recommendations and should be used as guidelines for good practices. In spite of the fact that serious adverse health effects are not believed likely as a result of exposures to the threshold limit concentrations, the best practice is to maintain concentrations of all atmospheric concentrations as low as is practical." 
The section numbers in the text that follows refer to sections of ASHRAE $34:^{1}$

\section{New Definitions}

Add new definitions to ASHRAE 34 section 3 as follows:

acute-toxicity exposure limit (ATEL): the refrigerant concentration limit determined in accordance with this Standard and intended to reduce the risks of acute toxicity hazards in normally occupied, enclosed spaces. ATEL values are similar to the Immediately Dangerous to Life or Health (IDLH) concentrations, set by the National Institute of Occupational Safety and Health (NIOSH). ATELs include explicit, additional components for cardiac sensitization and anesthetic effects, but do not address flammability. The lowest of the ATEL, $50,000 \mathrm{ppm} v / \mathrm{v}$, or $10 \%$ of the LFL, therefore, provides a conservative approximation to IDLH concentrations, when needed for refrigerants without adopted IDLH values.

approximate lethal concentration (ALC): the concentration of a substance, a refrigerant in this Standard, that was lethal to even a single test animal when tested by the same conditions as for an $L C_{50}$ test.

anesthetic effect or anesthesia: loss of the ability to perceive pain and other sensory stimulation.

$E C_{50}$ : effective concentration $50 \%$, the concentration of a material, a refrigerant in this Standard, that has caused a biological effect to $50 \%$ of test animals.

flammable concentration limit (FCL): the refrigerant concentration limit, in air, determined in accordance with this Standard and intended to reduce the risk of fire or explosion in normally occupied, enclosed spaces.

lowest observed effect level (LOEL): the concentration of a material, a refrigerant in this Standard, that has caused any observed effect to even one test animal.

no-observed effect level (NOEL): the highest concentration of a material, a refrigerant in this Standard, at which no effect has been observed in even one test animal.

oxygen deprivation limit (ODL): the concentration of a refrigerant or other gas that results in insufficient oxygen for normal breathing. For this Standard, the ODL is the concentration that reduces the oxygen content in normal air to below $19.5 \%$, assuming uniform mixing.

refrigerant concentration limit ( $R C L)$ : the refrigerant concentration limit, in air, determined in accordance with this Standard and intended to reduce the risks of acute toxicity, asphyxiation, and flammability hazards in normally occupied, enclosed spaces.

\section{RCL Determination}

Also, add a new section to ASHRAE 34 as follows: 


\section{REFRIGERANT CONCENTRATION LIMIT (RCL)}

7.1 Single-Compound Refrigerants. The RCL for each refrigerant shall be the lowest of the quantities calculated in accordance with 7.1.1,7.1.2, and 7.1.3, using data as indicated in 7.3, and adjusted in accordance with 7.4. Determination shall assume full vaporization; no removal by ventilation, dissolution, reaction, or decomposition; and complete mixing of the refrigerant in the space to which it is released.

7.1.1 Acute-Toxicity Exposure Limit (ATEL). The ATEL shall be the lowest of items (a)-(d) as follows:

(a) Mortality: $28.3 \%$ of the 4 -hour $\mathrm{LC}_{50}$ for rats. If not determined, $28.3 \%$ of the 4 hour ALC for rats provided that it did not result in mortality for more than half the exposed animals. If neither has been determined, $0 \mathrm{ppm}$. The following equations shall be used to adjust $L_{50}$ or ALC values that were determined with 15-minute to 8-hour tests, for refrigerants for which 4-hour test data are not available:

$$
\begin{aligned}
& L C_{50 \text { for } T}=L C_{50 \text { for } t} \cdot(t / T)^{1 / 2} \\
& \text { or } \\
& A L C_{T}=A L C_{t} \cdot(t / T)^{1 / 2}
\end{aligned}
$$

where:

$T$ is 4 hours, and

$t$ is the test duration expressed in hours, $0.25-8$

(b) Cardiac Sensitization: $100 \%$ of the NOEL for cardiac sensitization in unanesthetized dogs. If not determined, $80 \%$ of the LOEL for cardiac sensitization in dogs provided that the LOEL did not induce sensitization in more than half the exposed animals. If neither has been determined, $0 \mathrm{ppm}$. The cardiac sensitization term is omitted from ATEL determination if the $L C_{50}$ or ALC in (a) is less than 10,000 ppm $\mathrm{v} / \mathrm{v}$ or if the refrigerant is found, by toxicological review, to not cause cardiac sensitization.

(c) Anesthetic Effect: $50 \%$ of the 10-minute $\mathrm{EC}_{50}$ for anesthesia in mice or rats. If not determined, $50 \%$ of the LOEL for signs of anesthesia in rats during acute toxicity studies, provided that the LOEL did not induce an anesthetic effect for more than half the exposed animals. If neither has been determined, $80 \%$ of the NOEL for signs of anesthesia in rats during an acute, subchronic, or chronic toxicity study in which clinical signs were documented.

(d) Other Escape-Impairing Effects and Permanent Injury: The lowest concentration, for human exposures of $\mathbf{3 0}$ minutes, that is likely to impair ability to escape or to cause either irreversible health effects or severe sensory irritation.

\subsubsection{Oxygen Deprivation Limit (ODL). The ODL shall be 69,100 ppm v/v.}

7.1.3 Flammable Concentration Limit (FCL). The FCL shall be calculated as $25 \%$ of the LFL determined in accordance with 6.1.3. 
7.2 Blends. The RCL for refrigerants comprising multiple compounds shall be determined by the method in 7.1 except that individual parameter values in 7.1 .1 (a) though (d) shall be calculated as the mole-weighted average, by composition of the nominal formulation, of the values for the components.

7.3 Data for Calculations. The data used to calculate the RCL shall be taken from scientific and engineering studies or published safety assessments by governmental agencies or expert panels. Source studies for toxicity data must comply with Good Laboratory Practices (GLP) in accordance with reference 6, 7, 8, or 9. Data from peer-reviewed publications, including journal articles and reports, also are allowed.

7.3.1 Alternative Data. Data from studies that have not been published, from studies that have not been peer reviewed, or from studies involving species other than indicated in 7.1 .1 (a)-(d), shall be submitted to the authority having jurisdiction (AHJ) for approval. For RCL values to be published in addenda or revisions to this Standard, the AHJ shall be the Committee. Submissions shall include an evaluation of the experimental and analytical methods used, data from alternative sources, and the extent of the data search. The submissions shall summarize the qualifications of the person or persons providing the evaluation.

7.3.2 Consistent Measures. Use of data that are determined in a consistent manner, or by methods that consistently yield a lower RCL for the same effects, is allowed for the parameters identified in 7.1 .

7.3.3 No-Effect Data. Where no treatment-related effect was observed in animal tests for items 7.1 .1 (a)-(d), the ATEL calculation required by 7.1 .1 shall use the highest concentration tested in lieu of the specified effect or no-effect level.

7.3.4 Conservative Data. Where multiple data values have been published, the values used shall be those resulting in the lowest RCL.

\section{EXCEPTIONS:}

1. Where subsequent, peer-reviewed studies explicitly document flaws in or refinements to previously published data, the newer values shall be used.

2. For the cardiac sensitization and anesthetic effect NOEL in 7.1.1 (b) and (c), respectively, the highest-published NOEL not exceeding a published LOEL, for any fraction of tested animals, shall be used. Both the NOEL and LOEL must conform to 7.3 or 7.3 .1 for this exception.

\subsection{Units Conversion}

7.4.1 Mass per Unit Volume. The following equation shall be used to convert the $\mathrm{RCL}$ from a volumetric ratio, $\mathrm{ppm} v / \mathrm{v}$, to mass per unit volume, $\mathrm{kg} / \mathrm{m}^{3}$ (lb/Mcf): 


$$
\mathrm{rCl}=\mathrm{RCL} \cdot \mathrm{a} \cdot \mathrm{M}
$$

where:

$$
\begin{aligned}
& \mathrm{rcl}=\text { the } \mathrm{RCL} \text { expressed as } \mathrm{kg} / \mathrm{m}^{3}(\mathrm{lb} / \mathrm{Mcf}) \\
& \mathrm{RCL}=\text { the } \mathrm{RCL} \text { expressed as } \mathrm{ppm} \mathrm{v} / \mathrm{\textrm {v }} \\
& \mathrm{a}=4.096 \cdot 10^{-8} \text { for } \mathrm{kg} / \mathrm{m}^{3}\left(2.557 \cdot 10^{-6} \text { for } \mathrm{lb} / \mathrm{Mcf}\right)^{*} \\
& \mathrm{M}=\text { the molecular mass of the refrigerant }
\end{aligned}
$$

7.4.2 Adjustment for Altitude. The RCL shall be adjusted for altitude, when expressed as mass per unit volume, $\mathrm{kg} / \mathrm{m}^{3}$ (lb/Mcf), for locations above sea level. The $\mathrm{RCL}$ shall not be adjusted when expressed as a volumetric ratio in $\mathrm{ppm}$.

$$
\mathrm{rcl}_{\mathrm{a}}=\mathrm{rcl} \cdot(1-(\mathrm{b} \cdot \mathrm{h}))
$$

where:

$$
\begin{array}{ll}
\mathrm{rcl}_{\mathrm{a}} & =\text { the adjusted } \mathrm{rcl} \\
\mathrm{b} & =7.94 \cdot 10^{-5} \text { for } \mathrm{m}\left(2.42 \cdot 10^{-5} \text { for } \mathrm{ft}\right) \\
\mathrm{h} & =\text { altitude above sea level in } \mathrm{m}(\mathrm{ft})
\end{array}
$$

7.5 RCL Values. Refrigerants are assigned the RCLs indicated in Tables 1 and 2 . $^{\dagger}$ RCLs for other refrigerants shall be determined in accordance with this Standard and submitted to the authority having jurisdiction (AHJ) for approval. Submissions shall include an evaluation of the experimental and analytical methods used, data from alternative sources, and an indication of the extent of the data search. The submission shall summarize the qualifications of the person or persons that determined the recommended RCLs.

\section{New References}

Also, add references in ASHRAE 34 section 10 as follows:

6 OECD Principles of Good Laboratory Practice, Annex 2 of Decision C(81)30(Final), Organization for Economic Cooperation and Development (OECD), Paris, France, 13 May 1981

7 Good Laboratory Practice for Nonclinical Laboratory Studies, Food and Drug Administration (FDA), 21 CFR Chapter 1 Part 58, Subparts A-K, Government Printing Office, Washington, DC, 1996

* The factor shown to convert the RCL from a volumetric ratio in ppm v/v to mass per unit volume in lb/Mcf, $2.557 \cdot 10^{-6}$ for lb/Mcf, actually is incorrect and should be $1.160 \cdot 10^{-3}$ for lb/Mcf. The conversion is shown in its present form for calculation with mixed units, namely with the molecular mass in $\mathrm{g} / \mathrm{mol}$, rather than $\mathrm{lb} / \mathrm{mol}$, even for inch-pound (lb/Mcf) calculations.

$\dagger$ These tables are not included in this report. The language citing them is a recommendation for incorporation into ASHRAE 34, with expectation that the RCL values recommended later in this report (see pages 97-100), as modified through the consensus standard development process, will be added to existing Tables 1 and 2 of ASHRAE 34. 
8 Good Laboratory Practice Standards, Environmental Protection Agency, 40 CFR Part 792, Subparts A-J, Government Printing Office, Washington, DC, 1996

- GLP for Industrial Chemicals, Kikyoku [Basic Industries Bureau] Dispatch 85, Ministry of International Trade and Industry (MITI), and Kanpogyo [Planning and Coordination Bureau] Dispatch 39, Environmental Agency, Tokyo, Japan, 31 March 1984

\section{SUGGESTED FURTHER CHANGES}

Several suggested revisions to improve the RCL method follow. The conventions used to highlight changes are strikethroughs (e.g., delete) to show deletions, underscores (e.g., add) to show additions, and double strikethroughs or underscores (e.g., or added) to show deletions or additions from a companion change already addressed.

\section{Qualifying LOELS}

\$7.1.1(a), (b), and (c) provide for use of an ALC when an $L_{50}$ was not determined, for use of a LOEL if a NOEL was not determined for cardiac sensitization, and for use of a LOEL when a 10minute $E_{50}$ was not determined for anesthetic effects, respectively. These sections stipulate that the ALC and LOELs must not have caused death or affected more than half the exposed animals. The intent was to require data that is approximately equal to or more conservative than a median effective concentration. However, in disqualifying some data, the provision as currently written may allow use of less conservative data.

To illustrate this point, consider the hypothetical case in which:

- One study reports $20,000 \mathrm{ppm} v / \mathrm{v}$ was fatal to four of six rats.

- A second indicates that $30,000 \mathrm{ppm} v / \mathrm{v}$ was fatal to three of six rats for the same exposure duration.*

- Both studies found increasing mortality fractions at higher concentrations, but neither determined an $\mathrm{LC}_{50}$.

The $20,000 \mathrm{ppm}$ v/V ALC would be disqualified on the basis of $67 \%$ mortality (more than half the animals). The ALC of $30,000 \mathrm{ppm} v / \mathrm{v}$ from the second study would then qualify even though the first study indicates that the lethality threshold is less than $20,000 \mathrm{ppm} \mathrm{v} / \mathrm{v}$. The $50 \%$ mortality criterion, therefore, forces use of less conservative data in this hypothetical but plausible case.

The following changes address this problem. The key changes insert a new provision, as $\$ 7.3 .4$, and delete the qualification criteria from $\$ 7.1 .1$ (a)-(c). The revision also modifies the sequence of $\$ 7.3 .2-7.3 .5$ to make the expanded text more cohesive.

* $30,000 \mathrm{ppm} v / \mathrm{v}$ should yield more deaths than $20,000 \mathrm{ppm} v / \mathrm{v}$ for treatment-related lethality, but incongruities such as the one postulated here are common for separate studies and sometimes within a single study. 
7.1.1 Acute-Toxicity Exposure Limit (ATEL). The ATEL shall be the lowest of items (a)-(d) as follows:

(a) Mortality: $28.3 \%$ of the 4-hour $L C_{50}$ for rats. If not determined, $28.3 \%$ of the 4hour ALC for rats provided that it did not result in morality for more than half the exposedanimals. If neither has been determined, $0 \mathrm{ppm}$. The following equations shall be used to adjust $L_{5}$ or ALC values that were determined with 15-minute to 8-hour tests, for refrigerants for which 4-hour test data are not available:

$$
\begin{aligned}
& L C_{50 \text { for } T}=L C_{50 \text { for } t} \cdot(t / T)^{1 / 2} \\
& \text { or } \\
& A L C_{T}=A L C_{t} \cdot(t / T)^{1 / 2}
\end{aligned}
$$

where:

$T$ is 4 hours, and

$t$ is the test duration expressed in hours, $0.25-8$

(b) Cardiac Sensitization: $100 \%$ of the NOEL for cardiac sensitization in unanesthetized dogs. If not determined, $80 \%$ of the LOEL for cardiac sensitization in dogs provided that the LOEL did not-induce-sensitization in more than half tho exposed animals. If neither has been determined, $0 \mathrm{ppm}$. The cardiac sensitization term is omitted from ATEL determination if the $L C_{50}$ or ALC in (a) is less than $10,000 \mathrm{ppm}$ $\mathrm{v} / \mathrm{V}$ or if the refrigerant is found, by toxicological review, to not cause cardiac sensitization.

(c) Anesthetic Effect: $50 \%$ of the 10 -minute $\mathrm{EC}_{50}$ for anesthesia in mice or rats. If not determined, $50 \%$ of the LOEL for signs of anesthesia in rats during acute toxicity studies, provided that the LOEL did not induce-an anesthetic offect for more than half the exposed animals. If neither has been determined, $80 \%$ of the NOEL for signs of anesthesia in rats during an acute, subchronic, or chronic toxicity study in which clinical signs were documented.

\section{:}

7.3 Data for Calculations. The data used to calculate the RCL shall be taken from scientific and engineering studies or published safety assessments by governmental agencies or expert panels. Source studies for toxicity data must comply with Good Laboratory Practices (GLP) in accordance with reference 6, 7, 8, or 9. Data from peer-reviewed publications, including journal articles and reports, also are allowed.

7.3.1 Alternative Data. Data from studies that have not been published, from studies that have not been peer reviewed, or from studies involving species other than indicated in 7.1 .1 (a)-(d), shall be submitted to the authority having jurisdiction (AHJ) for approval. For RCL values to be published in addenda or revisions to this Standard, the AHJ shall be the Committee. Submissions shall include an evaluation of the experimental and analytical methods used, data from alternative sources, and the extent of the data search. The submissions shall summarize the qualifications of the person or persons providing the evaluation. 
7.3.2 7.3.4-Conservative Data. Where multiple data values have been published, the values used shall be those resulting in the lowest $\mathrm{RCL}$.

\section{EXCEPTIONS:}

1. Where subsequent, peer-reviewed studies explicitly document flaws in or refinements to previously published data, the newer values shall be used.

2. For the cardiac sensitization and anesthetic effect NOEL in 7.1.1 (b) and (c), respectively, the highest-published NOEL not exceeding a published LOEL, for any fraction of tested animals, shall be used. Both the NOEL and LOEL must conform to 7.3 or 7.3 .1 for this exception.

7.3.3 No-Effect Data. Where no treatment-related effect was observed in animal tests for items 7.1 .1 (a)-(d), the ATEL calculation required by 7.1.1 shall use the highest concentration tested in lieu of the specified effect or no-effect level.

7.3.4 ALC and LOEL Qualification. No ALC or LOEL shall be used for items 7.1.1 (a)(c) if it resulted in the effect measured (mortality, cardiac sensitization, or anesthetic effect) in more than half the animals exposed at that concentration or if there is a lower ALC or LOEL for any fraction of tested animals.

7.3.5 7.3.2-Consistent Measures. Use of data that are determined in a consistent manner, or by methods that consistently yield a lower RCL for the same effects, is allowed for the parameters identified in 7.1.

Note that the change could result in no qualifying data for the hypothetical case, the same result if both studies had found lethality in more than half the animals tested at the lowest concentration each tested. As implied, the testing was insufficient.

\section{Ambiguity in Anesthetic Effect}

The language in $\$ 7.1 .1(\mathrm{c})$ is inconsistent and, therefore, invites interpretation. The intent was to include all signs of anesthetic or central nervous system (CNS) effect on the premise that they suggest impairment in perception, judgement, or ability to respond to the circumstances for which the RCL pertains, namely a potential refrigerant release or an emergency causing it. Use of the words "for anesthesia" suggests a more severe impact. Moreover, the current $\$ 7.1 .1(\mathrm{c})$ language is vague. The following changes to the definitions and to $\$ 7.1 .1$ (c) address these concerns:

anesthetic effect-or anesthesia: Loss of the ability to perceive pain and other sensory stimulation.

central nervous system (CNS) effect. Treatment-related depression, distraction, stimulation, or other behavioral modification suggesting temporary or permanent changes to control by the brain.

(c) Anesthetic or Central Nervous System Effects: $50 \%$ of the 10-minute $E_{50}$ for anesthesia in mice or rats for loss of righting ability in a rotating apparatus. If not determined, $50 \%$ of the LOEL for signs of anesthesia-any anesthetic or CNS effect 
in rats during acute toxicity studies thets If neither has been determined, $80 \%$ of the NOEL for signs of anesthesia-any anesthetic or CNS effect in rats during an acute, subchronic, or chronic toxicity study in which clinical signs were documented.

\section{Safety Margin for Other Escape Impairing Effects or Permanent Injury}

Of the four toxic effects used to calculate the ATEL, the first three are guidance levels - effect levels reduced by multipliers to provide a margin of safety for differences in both inter-and intraspecies sensitivities. The fourth term, other escape impairing effects or permanent injury, is the "lowest concentration, for human exposures of 30 minutes, that is likely to impair ability to escape or to cause either irreversible health effects or severe sensory irritation." The result is that the ATEL and RCL will be at a concentration likely to cause harm when this term governs.

The following revision to $\$ 7.1 .1(\mathrm{~d})$ changes the term to a guidance level using the same multiplier as for the cardiac sensitization LOEL:

(d) Other Escape-Impairing Effects and Permanent Injury: The-80\% of the lowest concentration, for human exposures of 30 minutes, that is likely to impair ability to escape or to cause either irreversible health effects or severe sensory irritation.

As indicated on pages 92 and 94, this change would make the ATEL for R-744 (carbon dioxide) match the revised 1994 IDLH for it and reduce the difference between the ATEL and the 1994 IDLH for R-12 and R-717 (ammonia).

\section{RCL Conversion to Mass per Unit Volume}

The factor shown to convert the RCL from a volumetric ratio in ppm v/v to mass per unit volume in $\mathrm{lb} / \mathrm{Mcf}, 2.557 \cdot 10^{-6}$ for $\mathrm{lb} / \mathrm{Mcf}$, is incorrect for consistent use of inch-pound units. The conversion shown assumes mercurial calculation with mixed units, namely with the molecular mass in $\mathrm{g} / \mathrm{mol}$ rather than $\mathrm{lb} / \mathrm{mol}$. The following changes make the units consistent and reduce the likelihood of someone incorrectly applying the conversion now shown with molecular mass in $\mathrm{lb} / \mathrm{mol}$.

7.4.1 Mass per Unit Volume. The following equation shall be used to convert the RCL from a volumetric ratio, $\mathrm{ppm} v / \mathrm{v}$, to mass per unit volume, $\mathrm{kg} / \mathrm{m}^{3}$ (lb/Mcf):

$$
\mathrm{rCl}=\mathrm{RCL} \cdot \mathrm{a} \cdot \mathrm{M}
$$

where:

$$
\begin{aligned}
& \mathrm{rcl}=\text { the } \mathrm{RCL} \text { expressed as } \mathrm{kg} / \mathrm{m}^{3} \text { (lb/Mcf) } \\
& \mathrm{RCL}=\text { the } \mathrm{RCL} \text { expressed as } \mathrm{ppm} \mathrm{v} / \mathrm{v} \\
& \mathrm{a}=4.096 \cdot 10^{-8} \text { for } \mathrm{kg} / \mathrm{m}^{3}\left(2.557 \cdot 10^{-6} 1.160 \cdot 10^{-3} \text { for } \mathrm{lb} / \mathrm{Mcf}\right) \\
& \mathrm{M}=\text { the molecular mass of the refrigerant in } \mathrm{g} / \mathrm{mol}(\mathrm{lb} / \mathrm{mol})
\end{aligned}
$$




\section{Historical Studies}

The ASHRAE 34u draft (see page 16) calls for toxicity data from studies that indicate compliance with Good Laboratory Practices (GLP). The referenced regulations changed with time, and investigators could not have foreseen future regulatory changes reliably. The following modification provides a recourse for studies performed under earlier GLP formulations:

7.3 Data for Calculations. The data used to calculate the RCL shall be taken from scientific and engineering studies or published safety assessments by governmental agencies or expert panels. Source studies for toxicity data must indicate compliance with Good Laboratory Practices (GLP) in accordance with reference $7,8,9$, or 10 or earlier editions of these references in effect when the studies were performed. Data from peer-reviewed publications, including journal articles and reports, also are allowed.

\section{Updated References}

Some of the references cited in the ASHRAE 34u draft (see page 16) are no longer current. The following changes cite updates:

6 OECD Principles of Good Laboratory Practice, Annex 2 of Decision C(81)30(Final), Organization for Economic Cooperation and Development (OECD), Paris, France, 13 May 1981 as revised through 1999

7 Good Laboratory Practice for Nonclinical Laboratory Studies, Food and Drug Administration (FDA), Department of Health and Human Services, 21 CFR Chapter 1 Part 58, Subparts A-K, Government Printing Office, Washington, DC, 1866-1 April 2000

8 Good Laboratory Practice Standards, Environmental Protection Agency, 40 CFR Part 792, Subparts A-J, Government Printing Office, Washington, DC, 1866-1 July 2000

9 GLP for Industrial Chemicals, Kikyoku [Basic Industries Bureau] Dispatch 85, Ministry of International Trade and Industry (MITI), and Kanpogyo [Planning and Coordination Bureau] Dispatch 39, Environmental Agency, Tokyo, Japan, 31 March 1984

\section{REVISED TEXT OF METHOD WITH PROPOSED CHANGES}

The following version of the method incorporates all of the suggested changes, to facilitate reading without interspersed explanations and highlighting:

\section{New Definitions}

Add new definitions in ASHRAE 34 section 3 as follows:

acute-toxicity exposure limit (ATEL): the refrigerant concentration limit determined in accordance with this Standard and intended to reduce the risks of acute toxicity hazards in normally occupied, enclosed spaces. ATEL values are similar to the Immediately Dangerous to Life or Health (IDLH) concentrations, set by the National Institute of Occupational Safety and Health (NIOSH). ATELs include explicit, additional components for car- 
diac sensitization and anesthetic effects, but do not address flammability. The lowest of the ATEL, $50,000 \mathrm{ppm} \mathrm{v/v}$, or $10 \%$ of the LFL, therefore, provides a conservative approximation to IDLH concentrations, when needed for refrigerants without adopted IDLH values.

approximate lethal concentration (ALC): the concentration of a substance, a refrigerant in this Standard, that was lethal to even a single test animal when tested by the same conditions as for an $\mathrm{LC}_{50}$ test.

anesthetic effect. loss of the ability to perceive pain and other sensory stimulation.

central nervous system (CNS) effect: treatment-related depression, distraction, stimulation, or other behavioral modification suggesting temporary or permanent changes to control by the brain.

$E C_{50}$ : effective concentration $50 \%$, the concentration of a material, a refrigerant in this Standard, that has caused a biological effect to $50 \%$ of test animals.

flammable concentration limit (FCL): the refrigerant concentration limit, in air, determined in accordance with this Standard and intended to reduce the risk of fire or explosion in normally occupied, enclosed spaces.

lowest observed effect level (LOEL): the concentration of a material, a refrigerant in this Standard, that has caused any observed effect to even one test animal.

no-observed effect level (NOEL): the highest concentration of a material, a refrigerant in this Standard, at which no effect has been observed in even one test animal.

oxygen deprivation limit $(O D L)$ : the concentration of a refrigerant or other gas that results in insufficient oxygen for normal breathing. For this Standard, the ODL is the concentration that reduces the oxygen content in normal air to below $19.5 \%$, assuming uniform mixing.

refrigerant concentration limit ( $R C L)$ : the refrigerant concentration limit, in air, determined in accordance with this Standard and intended to reduce the risks of acute toxicity, asphyxiation, and flammability hazards in normally occupied, enclosed spaces.

\section{RCL Determination}

Also, add a new section to ASHRAE 34 as follows:

\section{REFRIGERANT CONCENTRATION LIMIT (RCL)}

7.1 Single-Compound Refrigerants. The $\mathrm{RCL}$ for each refrigerant shall be the lowest of the quantities calculated in accordance with 7.1.1,7.1.2, and 7.1.3, using data as indicated in 7.3, and adjusted in accordance with 7.4. Determination shall assume full vaporization; no removal by ventilation, dissolution, reaction, or decomposition; and complete mixing of the refrigerant in the space to which it is released.

7.1.1 Acute-Toxicity Exposure Limit (ATEL). The ATEL shall be the lowest of items (a)-(d) as follows: 
(a) Mortality: $28.3 \%$ of the 4-hour $L_{50}$ for rats. If not determined, $28.3 \%$ of the 4 hour ALC for rats. If neither has been determined, $0 \mathrm{ppm}$. The following equations shall be used to adjust $L_{50}$ or ALC values that were determined with 15-minute to 8-hour tests, for refrigerants for which 4-hour test data are not available:

$$
\begin{aligned}
& L C_{50 \text { for } T}=L C_{50 \text { for } t} \cdot(t / T)^{1 / 2} \\
& \text { or } \\
& A L C_{T}=A L C_{t} \cdot(t / T)^{1 / 2}
\end{aligned}
$$

where:

$T$ is 4 hours, and

$t$ is the test duration expressed in hours, 0.25-8

(b) Cardiac Sensitization: $100 \%$ of the NOEL for cardiac sensitization in unanesthetized dogs. If not determined, $80 \%$ of the LOEL for cardiac sensitization in dogs. If neither has been determined, 0 ppm. The cardiac sensitization term is omitted from ATEL determination if the $L C_{50}$ or ALC in (a) is less than $10,000 \mathrm{ppm} v / \mathrm{v}$ or if the refrigerant is found, by toxicological review, to not cause cardiac sensitization.

(c) Anesthetic or Central Nervous System Effects: $50 \%$ of the 10-minute $\mathrm{EC}_{50}$ in mice or rats for loss of righting ability in a rotating apparatus. If not determined, $50 \%$ of the LOEL for signs of any anesthetic or CNS effect in rats during acute toxicity studies. If neither has been determined, $80 \%$ of the NOEL for signs of any anesthetic or CNS effect in rats during an acute, subchronic, or chronic toxicity study in which clinical signs were documented.

(d) Other Escape-Impairing Effects and Permanent Injury: $80 \%$ of the lowest concentration, for human exposures of 30 minutes, that is likely to impair ability to escape or to cause either irreversible health effects or severe sensory irritation.

\subsubsection{Oxygen Deprivation Limit (ODL). The ODL shall be $69,100 \mathrm{ppm} v / \mathrm{v}$.}

7.1.3 Flammable Concentration Limit (FCL). The FCL shall be calculated as $25 \%$ of the $L F L$ determined in accordance with 6.1.3.

7.2 Blends. The RCL for refrigerants comprising multiple compounds shall be determined by the method in 7.1 except that individual parameter values in 7.1 .1 (a) though (d) shall be calculated as the mole-weighted average, by composition of the nominal formulation, of the values for the components.

7.3 Data for Calculations. The data used to calculate the RCL shall be taken from scientific and engineering studies or published safety assessments by governmental agencies or expert panels. Source studies for toxicity data must comply with Good Laboratory Practices (GLP) in accordance with reference $6,7,8$, or 9 . Data from peer-reviewed publications, including journal articles and reports, also are allowed.

7.3.1 Alternative Data. Data from studies that have not been published, from studies that have not been peer reviewed, or from studies involving species other than indicated in 7.1 .1 (a)-(d), shall be submitted to the authority having jurisdiction (AHJ) for approval. For RCL values to be published in addenda or revisions to this Standard, the AHJ shall be 
the Committee. Submissions shall include an evaluation of the experimental and analytical methods used, data from alternative sources, and the extent of the data search. The submissions shall summarize the qualifications of the person or persons providing the evaluation.

7.3.2 Conservative Data. Where multiple data values have been published, the values used shall be those resulting in the lowest $\mathrm{RCL}$.

\section{EXCEPTIONS:}

1. Where subsequent, peer-reviewed studies explicitly document flaws in or refinements to previously published data, the newer values shall be used.

2. For the cardiac sensitization and anesthetic effect NOEL in 7.1.1 (b) and (c), respectively, the highest-published NOEL not exceeding a published LOEL, for any fraction of tested animals, shall be used. Both the NOEL and LOEL must conform to 7.3 or 7.3 .1 for this exception.

7.3.3 No-Effect Data. Where no treatment-related effect was observed in animal tests for items 7.1.1 (a)-(d), the ATEL calculation required by 7.1.1 shall use the highest concentration tested in lieu of the specified effect or no-effect level.

7.3.4 ALC and LOEL Qualification. No ALC or LOEL shall be used for items 7.1.1 (a)(c) if it resulted in the effect measured (mortality, cardiac sensitization, or anesthetic effect) in more than half the animals exposed at that concentration or if there is a lower ALC or LOEL for any fraction of tested animals.

7.3.5 Consistent Measures. Use of data that are determined in a consistent manner, or by methods that consistently yield a lower RCL for the same effects, is allowed for the parameters identified in 7.1 .

\subsection{Units Conversion}

7.4.1 Mass per Unit Volume. The following equation shall be used to convert the RCL from a volumetric ratio, $\mathrm{ppm} v / \mathrm{v}$, to mass per unit volume, $\mathrm{kg} / \mathrm{m}^{3}$ (lb/Mcf):

$$
\mathrm{rCl}=\mathrm{RCL} \cdot \mathrm{a} \cdot \mathrm{M}
$$

where:

$$
\begin{aligned}
& \mathrm{rCl}=\text { the } \mathrm{RCL} \text { expressed as } \mathrm{kg} / \mathrm{m}^{3} \text { (lb/Mcf) } \\
& \mathrm{RCL}=\text { the } \mathrm{RCL} \text { expressed as } \mathrm{ppm} \mathrm{v} / \mathrm{v} \\
& \mathrm{a}=4.096 \cdot 10^{-8} \text { for } \mathrm{kg} / \mathrm{m}^{3}\left(1.160 \cdot 10^{-3} \text { for } \mathrm{lb} / \mathrm{Mcf}\right) \\
& \mathrm{M}=\text { the molecular mass of the refrigerant in } \mathrm{g} / \mathrm{mol}(\mathrm{lb} / \mathrm{mol})
\end{aligned}
$$

7.4.2 Adjustment for Altitude. The RCL shall be adjusted for altitude, when expressed as mass per unit volume, $\mathrm{kg} / \mathrm{m}^{3}$ (lb/Mcf), for locations above sea level. The RCL shall not be adjusted when expressed as a volumetric ratio in ppm. 


$$
\mathrm{rcl}_{\mathrm{a}}=\mathrm{rcl} \cdot(1-(\mathrm{b} \cdot \mathrm{h}))
$$

where:

$$
\begin{array}{ll}
\mathrm{rcl}_{\mathrm{a}} & =\text { the adjusted } \mathrm{rcl} \\
\mathrm{b} & =7.94 \cdot 10^{-5} \text { for } \mathrm{m}\left(2.42 \cdot 10^{-5} \text { for } \mathrm{ft}\right) \\
\mathrm{h} & =\text { altitude above sea level in } \mathrm{m}(\mathrm{ft})
\end{array}
$$

7.5 RCL Values. Refrigerants are assigned the RCLs indicated in Tables 1 and 2. RCLs for other refrigerants shall be determined in accordance with this Standard and submitted to the authority having jurisdiction (AHJ) for approval. Submissions shall include an evaluation of the experimental and analytical methods used, data from alternative sources, and an indication of the extent of the data search. The submission shall summarize the qualifications of the person or persons that determined the recommended RCLs.

\section{New References}

Also, add references in ASHRAE 34 section 10 as follows:

6 OECD Principles of Good Laboratory Practice, Annex 2 of Decision C(81)30(Final), Organization for Economic Cooperation and Development (OECD), Paris, France, 13 May 1981 as revised through 1999

7 Good Laboratory Practice for Nonclinical Laboratory Studies, Food and Drug Administration (FDA), Department of Health and Human Services, 21 CFR Chapter 1 Part 58, Subparts A-K, Government Printing Office, Washington, DC, 1 April 2000

8 Good Laboratory Practice Standards, Environmental Protection Agency, 40 CFR Part 792, Subparts A-J, Government Printing Office, Washington, DC, 1 July 2000

9 GLP for Industrial Chemicals, Kikyoku [Basic Industries Bureau] Dispatch 85, Ministry of International Trade and Industry (MITI), and Kanpogyo [Planning and Coordination Bureau] Dispatch 39, Environmental Agency, Tokyo, Japan, 31 March 1984 


\section{DATA LOCATION}

The preceding sections of this report explain the objectives, focal toxicological effects, relevant measures for them, and the proposed method to calculate recommended concentration limits. This section identifies the resources used to locate the data presented in the following section, with emphasis on the toxicity data.

\section{LITERATURE SEARCH}

Data were located through systematic searches of several databases including:

- ARTI Refrigerant Database 22

- National Library of Medicine (NLM) Medline

- National Library of Medicine (NLM) Toxline

- NIOSH Occupational Safety and Health Database (NIOSHTIC)

- NIOSH Registry of Toxic Effects of Chemical Substances (RTECS) ${ }^{23}$

- National Toxicology Program (NTP) Chemical Hazard Information System (CHEMHAZIS)

- NTP Health and Safety Database

- U.K. Royal Society of Chemistry Chemical Safety Newsbase

Selective searches also were made in:

- Chemical Abstracts Service (CAS) Chemical Abstracts

- several major library catalogs including that of the U.S. Library of Congress and the National Technical Information Service

The following summaries and assessments were examined for references to relevant studies:

- published and to the extent obtainable unpublished assessments, summaries, and exposure limit or hygiene standard documentation from chemical manufacturers

- ACGIH documentation of Threshold Limit Values (TLVs) ${ }^{24}$

- AlHA Workplace Environmental Exposure Limit (WEEL) Guides for R-32, R-123, R124, R-125, R-134a, R-141b, R-142b, R-143a, R-152a, and R-236fa

- Air-Conditioning and Refrigeration Institute (ARI) Refrigerant Safety Data - Recommendations of the Air-Conditioning and Refrigeration Industry ${ }^{25}$

- Department of Health and Human Services and Department of Labor occupational safety and health guidelines ${ }^{26}$

- Environmental Protection Agency published toxicity assessments ${ }^{27-30}$ 
- European Chemical Industry Ecotoxicology and Toxicology Centre (ECETOC) Joint Assessment of Commodity Chemicals (JACC) reports for R-22, R-32, R-123, R-124, R-125, R-134a, R-141b, and R-142b

- International Institute of Ammonia Refrigeration (IIAR) reference book on R-717 (ammonia) $^{31}$ as well as assessments of ammonia toxicity

- National Fire Protection Association (NFPA) references on hazardous materials ${ }^{32}$

- National Research Council (NRC) exposure limit assessments ${ }^{33-43}$

- NIOSH criteria documents for recommended exposure standards ${ }^{44-46}$

- NIOSH documentation for Immediately Dangerous to Life or Health (IDLH) concentrations $^{11}$

- applications submitted for ASHRAE 34 designations and safety classifications

- chemical and hazardous materials handbooks, the most pertinent of which are cited in the discussion of the data

The following sources - usually lacking study citations for included data - were examined for relevant data. Their preparers were contacted to determine the data sources when not apparent from the prior steps:

- chemical manufacturer material safety data sheets (MSDSs), safety data sheets (SDSs), and hygiene standard recommendations

- Program for Alternative Fluorocarbon Toxicity Testing (PAFT) summaries ${ }^{7}$

Most of the data searches were conducted in 1995 and 1996 and summarized, together with background information on the approach, in a paper (see appendix 5 beginning on page 507). ${ }^{47}$

\section{DATA ISSUES}

\section{Source Data}

Wherever possible, the data shown in the following sections reflect "source data," that is data as presented or as converted from the original source, or as close to the original reports as could be located. While reliance on assessments, handbooks, and similar compilations is simpler, these "secondary" sources typically omit pertinent qualifiers and details. Moreover, the data integrity degrade with multiple quotations if rounded or converted. Toxicologists differ in their preferences for units. One study reported an effect at $204,000 \mathrm{ppm} \mathrm{v} / \mathrm{v}$. It was quoted first as $20 \%$ in a paper, then converted to $474 \mathrm{mg} / \mathrm{L}$ in a compilation. That value was cited later as $195,000 \mathrm{ppm} v / \mathrm{v}$, which might be mistaken as a corroborating rather than the same study. Such differences grow with successive conversions.

\section{Nominal and Actual Concentrations}

Summaries and compilations often do not distinguish between nominal (or target) concentrations for toxicity studies and those actually determined by measurement and analysis. Such differences may exceed $20 \%$. The data cited herein are measured rather than target data, since they are more indicative of the levels at which effects or absence of effects were found in tests. 


\section{RECOMMENDED DATA TO DETERMINE ATELS}

This section addresses the data needed to calculate ATEL concentrations. It identifies pertinent findings from referenced toxicity studies for 29 refrigerants. The refrigerants were selected by the project sponsor, ARTI, based on those in use as single compounds and blend components at inception of the project as well as two added in the course of the work. They include:

R-11: trichlorotrifluoromethane

R-12: dichlorodifluoromethane

R-22: chlorodifluoromethane

R-23: trifluoromethane

R-32: difluoromethane

R-113: 1,1,2-trichloro-1,2,2-trifluoroethane

R-114: 1,2-dichloro-1,1,2,2-tetrafluoroethane

R-116: hexafluoroethane

R-123: 2,2-dichloro-1,1,1-trifluoroethane

R-124: 2-chloro-1,1,1,2-tetrafluoroethane

R-125: 1,1,1,1,2-pentafluoroethane

R-134: 1,1,2,2-tetrafluoroethane

R-134a: 1,1,1,2-tetrafluoroethane

R-E134: difluoromethoxy-difluoromethane [also identified as bis(difluoromethyl)ether]

R-141b: 1,1-dichloro-1-fluoroethane

R-142b: 1-chloro-1,1-difluoroethane

R-143a: 1,1,1-trifluoroethane

R-152a: 1,1-difluoroethane

R-218: octafluoropropane

R-227ea: 1,1,1,2,3,3,3-heptafluoropropane

R-236fa: 1,1,1,3,3,3-hexafluoropropane

R-245ca: 1,1,2,2,3-pentafluoropropane

R-245fa: 1,1,1,3,3-pentafluoropropane

R-290: propane

R-500: R-22/152a (73.8/26.2)

R-502: R-22/115 (48.8/51.2)

$\mathrm{R}-600 \mathrm{a}$ : isobutane

R-717: ammonia

R-744: carbon dioxide

Their selection does not imply a preference or recommendation, and data also are available for other refrigerants. Indeed, several refrigerants introduced since inception of the work are included in tables in subsequent sections of this report even though not addressed here.

The data for each of the 29 refrigerants addresses four toxicity concerns, namely:

- lethality,

- cardiac sensitization,

- anesthetic effect, and 
- other escape-impairing effects and permanent injury

The data presented are not exhaustive either in terms of the toxicity endpoints (measures) addressed or in terms of all known data for each endpoint. Rather, the presentation covers the data needed to calculate ATEL values and examples of additional data that corroborate or disqualify cited data. The specific data selection criteria used, and referred to in the following discussion, are those defined by the proposed procedure for ATEL determination as qualified by the provisions on Data for Calculations (see text beginning on pages 19 and 20, respectively).

In the absence of corroborating data, the presentation cites representative toxicity assessments, manufacturer material safety data sheets (MSDSs), and similar documentation to show acceptance of specific data by others. Each subsection concludes with the author's recommendation based on the data selection criteria. 


\section{R-11: TRICHLOROTRIFLUOROMETHANE}

\section{Lethality}

A number of studies report 4-hr ALCs and $L^{2} C_{50} s$ in rats with a range of $26,000-66,000 \mathrm{ppm} \mathrm{v/v}$. Morrison reports no deaths at $35,600 \mathrm{ppm} v / \mathrm{v}$ and death of one of four rats $(25 \%)$ at 66,000 $\mathrm{ppm} \mathrm{v/v} \mathrm{as} \mathrm{found} \mathrm{in} \mathrm{a} \mathrm{4-hr} \mathrm{study.}{ }^{48}$ In a later study, Barras indicates a 4-hr LC L $_{50}$ in rats of $26,200 \mathrm{ppm} \mathrm{v/v.}{ }^{49}$ DuPont cites an $L_{50}$ of $26,000 \mathrm{ppm} \mathrm{v/v}$ in a bulletin summarizing PAFT findings. ${ }^{50}$ This value appears to have been rounded from $26,200 \mathrm{ppm}$ v/v since no source study was found to support it and DuPont cites $26,200 \mathrm{ppm} \mathrm{v} / \mathrm{v}$ in its MSDS for R-11. ${ }^{51}$ These data are reasonably consistent with $L_{5}$ findings of $120,000-130,000 \mathrm{ppm} v / \mathrm{v}$ for $10-30 \mathrm{~min}$ utes. $^{52-54}$ Applying the ten Berge (see page 11) extrapolation to them suggests 4-hr $L_{50} s$ of approximately $24,500-50,000 \mathrm{ppm} \mathrm{v} / \mathrm{v}$.

recommendation: $4-\mathrm{hr} L C_{50}$ rat $=26,200 \mathrm{ppm} \mathrm{v} / \mathrm{v}$

\section{Cardiac Sensitization}

Hardy et al. report NOELs of 9,000 and $15,000 \mathrm{ppm} v / \mathrm{v}$ in two separate studies, ${ }^{55,56}$ but an earlier and widely cited study by Reinhardt et al.. indicates the measured range of $900-1,300 \mathrm{ppm}$ $\mathrm{v} / \mathrm{v}$, with a midpoint of $1,100 \mathrm{ppm} \mathrm{v} / \mathrm{v}$, as a NOEL. ${ }^{57}$ The same study indicates a LOEL for one of twelve dogs in a measured range of $3,500-6,100 \mathrm{ppm} \mathrm{v/v}$, with a midpoint of $4,800 \mathrm{ppm} v / \mathrm{v}$.

Two later studies, also by Hardy and colleagues, indicate LOELs for one of two dogs at 20,000 $\mathrm{ppm} \mathrm{v/v,}{ }^{58,59}$ and other investigators report $\mathrm{EC}_{50} \mathrm{~s}$ of $12,400-12,500 \mathrm{ppm} \mathrm{v/v}{ }^{53,60,61}$

While it appears that the early Reinhardt et al. finding is lower than more recent data and that the NOEL would have been more than four times higher except for the response of one dog of twelve, it is the most conservative NOEL found and there is no basis to reject it.

recommendation: NOEL $\operatorname{dog}=1,100 \mathrm{ppm} \mathrm{v/v}$

\section{Anesthetic Effect}

Wada reports a $10-\mathrm{min} \mathrm{EC}_{50}$ in rats of $54,000 \mathrm{ppm} \mathrm{v/v.}{ }^{52}$ Weigand reports a $2-\mathrm{hr} \mathrm{EC}_{50}$ in rats of $60,000 \mathrm{ppm} v / \mathrm{v}^{62}$ which gives credence to the Wada finding, but Clark and Tinston report a more conservative $10-\mathrm{min} \mathrm{EC}_{50}$ in rats of $35,000 \mathrm{ppm} \mathrm{v/v} .^{53}$ That study appears to be the source for similar indications by others. ${ }^{63,64}$ Weigand also reports a 2-hr LOEL of 35,000 ppm $v / v$ based on tests of four rats, but does not indicate the fraction of animals showing effects. ${ }^{62}$ The same study reports a NOEL for 2 -hr exposures of four rats at $12,500 \mathrm{ppm} \mathrm{v/v.}{ }^{62}$ Lester and Greenberg report a $30-\mathrm{min}$ NOEL and LOEL for 30-min exposures of individual rats to 50,000 and $60,000 \mathrm{ppm} v / \mathrm{v}$ respectively. ${ }^{65}$

recommendation: $10-\mathrm{min} \mathrm{EC}_{50}$ rat $=35,000 \mathrm{ppm} \mathrm{v/v}$ 


\section{Other Escape-Impairing Effects and Permanent Injury}

Stewart et al. report that eight male volunteers repetitively exposed to $1,000 \mathrm{ppm} v / \mathrm{v}$ for $1-10$ $\mathrm{hr} / \mathrm{d}, 5 \mathrm{~d} / \mathrm{wk}$, for 2-4 wk showed minor decrements in several cognitive tests, but no change in pulmonary function or alteration of in cardiac rhythm. ${ }^{66,67}$ They note, however, that acute exposures of groups of eight male volunteers to 250,500 , or $1000 \mathrm{ppm} \mathrm{v/v} \mathrm{for} 1 \mathrm{~min}$ to $8 \mathrm{hr}$ "did not produce any untoward physiological effects. ${ }^{\text {66,67 }}$

recommendation: none 


\section{R-12: DICHLORODIFLUOROMETHANE}

\section{Lethality}

Lester and Greenberg report no deaths in 4-hr and 6-hr exposures of "a few" rats to 800,000 $\mathrm{ppm} \mathrm{v} / \mathrm{v}$, suggesting an $\mathrm{LC}_{50}$ of $>800,000 \mathrm{ppm} \mathrm{v} / \mathrm{v}{ }^{65}$ The 4-hr finding was corroborated by Clark and Tinston. ${ }^{53}$ Several secondary sources cite an $L C_{50}$ of 760,000 or $>760,000 \mathrm{ppm} \mathrm{v/v,}{ }^{50,68}$ but no basis was found for them.

Shugaev indicates a $3-\mathrm{hr} L \mathrm{LC}_{50}$ rat of $620,000 .^{69}$ This indication is echoed in an NRC examination of data. ${ }^{38}$ Applying the ten Berge extrapolation (see page proximately $540,000 \mathrm{ppm} v / \mathrm{v}$. Even without time adjustment, the Shugaev finding challenges an $\mathrm{LC}_{50}$ of $>800,000 \mathrm{ppm} \mathrm{v/v}$.

Absent the ability to examine the Shugaev data carefully, recognizing the possibility that the test sample used may have been contaminated, recognizing that Lester and Greenberg repeated their finding in separate 4- and 6-hr tests, recognizing corroboration by Clark and Tinston, and absent a strict criterion to use the 3-hr or time-adjusted 3-hr data with available 4-hr data, the 4$\mathrm{hr} \mathrm{LC}_{50}$ of $>800,000 \mathrm{ppm}$ v/v seems to meet the data selection criteria (see page 19). In any case, the lethality term is not the most restrictive one for the R-12 exposure limits.

\section{recommendation: $4-\mathrm{hr} L C_{50}$ rat $>800,000 \mathrm{ppm}$ v/v}

\section{Cardiac Sensitization}

Reinhardt et al. indicate a NOEL from tests of twelve dogs exposed to $25,000 \mathrm{ppm} \mathrm{v} / \mathrm{v}$ as well as a LOEL for five of twelve dogs at $50,000 \mathrm{ppm} \mathrm{v/v.^{57,70 }}$

Hardy et al. report no marked responses in groups of five dogs at both 40,000 and 80,000 ppm $\mathrm{v} / \mathrm{v},{ }^{56}$ the latter of which is disqualified by the data selection criteria (see page 19) based on the $50,000 \mathrm{ppm}$ v/V LOEL.

These data appear consistent with reported $\mathrm{EC}_{50} \mathrm{~s}$ of $77,000-120,000 \mathrm{ppm}$ v/v. ${ }^{53,60,71}$

The Hardy et al. NOEL of $40,000 \mathrm{ppm}$ v/v is the highest NOEL found that does not exceed a LOEL.

recommendation: NOEL dog $=40,000 \mathrm{ppm} \mathrm{v/v}$

\section{Anesthetic Effect}

Clark and Tinston report a $10-\mathrm{min} \mathrm{EC}_{50}$ in rats of $250,000 \mathrm{ppm} \mathrm{v/v.}{ }^{53}$ Weigand reports a 2-hr

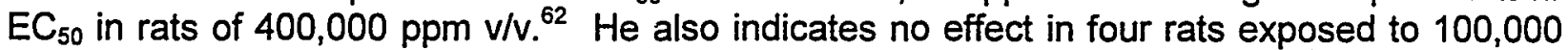
$\mathrm{ppm} \mathrm{v} / \mathrm{v}$ for $2 \mathrm{hr}$. Scholz reports a higher 2 -hr NOEL for rats of $200,000 \mathrm{ppm} v / \mathrm{v} .^{72}$

recommendation: $10-\mathrm{min} \mathrm{EC}_{50}$ rat $=250,000 \mathrm{ppm} \mathrm{v/v}$ 


\section{Other Escape-Impairing Effects and Permanent Injury}

Stewart et al. summarize acute and subchronic toxicity tests in healthy human volunteers by whole-body exposures. ${ }^{67,73}$ They indicate that acute exposures to 250,500 , or $1000 \mathrm{ppm} \mathrm{v} / \mathrm{v}$ for $1 \mathrm{~min}$ to $8 \mathrm{hr}$ "did not produce any untoward physiological effects" as determined by observation, routine hematology, clinical chemistry, EKG, and pulmonary function as well as by cognitive skill and other neurological measurements. ${ }^{6,73}$ They further note that repetitive exposures to $1,000 \mathrm{ppm} v / \mathrm{v}$ for $1-10 \mathrm{hr} / \mathrm{d}, 5 \mathrm{~d} / \mathrm{wk}$, for $2-4$ wk also showed no adverse effects. ${ }^{67,73}$ Reinhold and Rusch report the same conclusion in a review of the findings. ${ }^{74}$

Azar et al. report acute inhalation tests in two human volunteers. The study found that exposures to $10,000 \mathrm{ppm}$ v/v nominal $\left(10,173 \mathrm{ppm}\right.$ v/v average) for $2 \frac{1}{2} \mathrm{hr}$ evoked a $7 \%$ reduction in standardized psychomotor test scores. It notes that this finding is not indicative of a serious health threat and that breath samples showed that $R-12$ is quickly eliminated from the lungs. ${ }^{75}$ Adjusting the concentration of $10,173 \mathrm{ppm} v / \mathrm{v}$ from $2 \frac{1}{2} \mathrm{hr}$ to $30 \mathrm{~min}$ using the ten Berge approach yields a concentration of $22,700 \mathrm{ppm} v / \mathrm{v} .{ }^{*}$

recommendation: $L O E L=22,700 \mathrm{ppm} v / \mathrm{v}$

* The ten Berge approach (see page 11) is not generally applicable to anesthetic effects. The present use was carefully considered following examination of the Azar data and conclusions, recognition that there may have been no relevant effect for a shorter exposure, and examination of the data for anesthetic effect cited herein, in a detailed survey by Lee and Graham, ${ }^{76}$ and in the ARTI Refrigerant Database. ${ }^{22}$ 


\section{R-22: CHLORODIFLUOROMETHANE}

\section{Lethality}

Tinston found the 4-hr $\mathrm{LC}_{50}$ rat to be $220,000 \mathrm{ppm} v / \mathrm{v},{ }^{77}$ as reported by Litchfield and Longstaff and by an ECETOC JACC assessment. ${ }^{78,79}$ Weigand reports a 2-hr ALC in rats of 300,000 ppm $\mathrm{v} / \mathrm{v}$, based on death of one of four animals. ${ }^{62}$ This finding suggests a 4-hr ALC of approximately $210,000 \mathrm{ppm} v / \mathrm{v}$, with the ten Berge adjustment (see page 11), and therefore corroborates the Tinston finding.

recommendation: $4-\mathrm{hr} \mathrm{LC}_{50}$ rat $=220,000 \mathrm{ppm} \mathrm{v} / \mathrm{v}$

\section{Cardiac Sensitization}

Reinhardt et al. indicate a NOEL from tests of twelve dogs exposed to $50,000 \mathrm{ppm} \mathrm{v} / \mathrm{v}$ as well as a LOEL for marked responses in two of twelve dogs at 25,000 ppm v/v. ${ }^{5,70}$ Clark and Tinston report a consistent $E_{50}$ in dogs of $140,000 \mathrm{ppm} \mathrm{v/v.}$

$$
\text { recommendation: NOEL } \operatorname{dog}=25,000 \mathrm{ppm} v / \mathrm{v}
$$

\section{Anesthetic Effect}

Clark and Tinston report a $10-\mathrm{min} \mathrm{EC}_{50}$ in rats of $140,000 \mathrm{ppm}$ v/v based on action as a central nervous system (CNS) depressant. ${ }^{53}$ Weigand reports a LOEL of $50,000 \mathrm{ppm}$ v/v in rats for 2hr exposures in a subchronic inhalation toxicity study, but he does not indicate the number of animals affected of the four tested. ${ }^{62}$

recommendation: $10-\mathrm{min} \mathrm{EC}_{50}$ rat $=140,000 \mathrm{ppm} \mathrm{v/v}$

\section{Other Escape-Impairing Effects and Permanent Iniury}

None identified.

recommendation: none 


\section{R-23: TRIFLUOROMETHANE}

\section{Lethality}

Nash indicates that the 4-hr ALC rat (and therefore the $\mathrm{LC}_{50}$ ) for R-23 exceeds $663,000 \mathrm{ppm}$ $\mathrm{v} / \mathrm{v}$, which was the highest concentration tested, since there were no fatalities. ${ }^{80}$ The refrigerant-air mixture was enriched with oxygen to maintain 19.7-21\% levels. Skaggs and Moore cite this finding. ${ }^{81}$

recommendation: $4-\mathrm{hr}$ LC $_{50}$ rat $>663,000 \mathrm{ppm}$ v/v

\section{Cardiac Sensitization}

Hopkins and Krantz found no sensitization in dogs tested at 800,000 ppm v/v R-23 with oxygen. ${ }^{82}$ Hardy et al. indicate that the sensitization $E_{50}$ exceeds $500,000 \mathrm{ppm} \mathrm{v/v}$, but give no indication of specific test findings. ${ }^{71}$ The $800,000 \mathrm{ppm} \mathrm{v} / \mathrm{v}$ NOEL is cited in manufacturer MSDSs. $^{83,84}$

recommendation: NOEL dog $=800,000 \mathrm{ppm} \mathrm{v} / \mathrm{v}$

\section{Anesthetic Effect}

No $\mathrm{EC}_{50}$ was found. Van Poznak and Artusio report an anesthetic effect at $800,000 \mathrm{ppm} \mathrm{v} / \mathrm{v}$ in two of two dogs in 10-90 min, but neither the species nor the fraction affected satisfy the data selection criteria (see page 19). ${ }^{85}$ Nash reports that rats showed reduced response to sound during 4-hr exposures at 186,000 ppm v/v, but does not indicate how many of the six animals tested did so. ${ }^{80}$ Leuschner et al. indicate no adverse effects in rats exposed to $10,000 \mathrm{ppm} \mathrm{v} / \mathrm{V}$ in a subchronic inhalation toxicity study, ${ }^{86}$ as summarized by Krentz et al. and by Skaggs and Moore. ${ }^{81,87,88}$ Nash indicates that no behavioral changes were observed during exposures of six rats at $18,900 \mathrm{ppm} \mathrm{v} / \mathrm{v}$, so this concentration could be taken as a NOEL for anesthetic/CNS effect. $^{80}$

Munley reports that no compound-related effects were observed for clinical signs at $51,000 \mathrm{ppm}$ $\mathrm{v} / \mathrm{v}$ in an inhalation developmental toxicity study in rats exposed for $6 \mathrm{hr} / \mathrm{d}$ on days 7-21 of gestation. ${ }^{89}$ While interference might give false indications of an anesthetic/CNS effect during gestational studies, it is unlikely that gestation would suppress such an effect.

recommendation: NOEL rat $=51,000 \mathrm{ppm} \mathrm{v} / \mathrm{v}$

\section{Other Escape-Impairing Effects and Permanent Injury}

Fagan et al. indicate that four human volunteers experienced slight anesthetic effects, light headedness, tingling and numbness of the extremities, and hyperacusis* at $300,000 \mathrm{ppm} \mathrm{v/v}$, discomfort by one subject at $400,000 \mathrm{ppm}$ v/v, and significant anesthetic effect ("becoming lethargic") by one subject at $600,000 \mathrm{ppm} \mathrm{v/v} .{ }^{90}$ They adopted $300,000 \mathrm{ppm} \mathrm{v} / \mathrm{v}$ as the "maximum

* increased hearing 
tolerated concentration." Van Poznak and Artusio report that self-testing by one of the authors resulted in "definite analgesia and impairment of consciousness" at $800,000 \mathrm{ppm} \mathrm{v/v.}$

recommendation: anesthetic effect in humans at $300,000 \mathrm{ppm} \mathrm{v} / \mathrm{v}$ 


\section{R-32: DIFLUOROMETHANE}

\section{Lethality}

Parr-Dobrzanski indicates that there were no fatalities in 4-hr, nose-only, inhalation tests of rats exposed to 520,000 ppm v/v R-32 in air with oxygen-enrichment to avoid asphyxiation. ${ }^{91}$ Moore found no mortality in rats exposed for $4-\mathrm{hr}$ to $760,000 \mathrm{ppm} \mathrm{v/ \textrm {v } ^ { 9 2 }}$ This finding is cited by both Krentz et al. and a PAFT summary. ${ }^{93,94}$

\section{recommendation: $4-\mathrm{hr} \mathrm{LC}_{50}$ rat $>760,000 \mathrm{ppm} \mathrm{v} / \mathrm{v}$}

\section{Cardiac Sensitization}

Mullin and Trochimowicz report a marked response in one of twelve dogs at $250,000 \mathrm{ppm} \mathrm{v} / \mathrm{v}$, but no sensitization in the affected dog when tested several days later at $200,000 \mathrm{ppm} v / \mathrm{v}^{95}$ They note that two dogs tested at $400,000 \mathrm{ppm}$ v/v showed no response. ${ }^{95}$ Hardy et al. report an $\mathrm{EC}_{50}$ in dogs of $>350,000 \mathrm{ppm} \mathrm{v/v}{ }^{71}$ This finding is cited in a PAFT summary. ${ }^{7}$

recommendation: NOEL $\mathrm{dog}=200,000 \mathrm{ppm}$ v/v

\section{Anesthetic Effect}

Parr-Dobrzanski indicates no effect in rats in 4-hr exposures at 7,510 ppm v/v, but notes an anesthetic effect in ten of ten rats at $85,900 \mathrm{ppm} v / \mathrm{v} .{ }^{91}$ In a separate study, Parr-Dobrzanski found no behavioral changes in rats at $49,500 \mathrm{ppm} v / \mathrm{v}$, the highest concentration tested, by whole body exposures for $6 \mathrm{hr} / \mathrm{d}, 5 \mathrm{~d} / \mathrm{wk}$, for $4 \mathrm{wk} .{ }^{96}$ Moore found an anesthetic effect in six of six rats exposed at $111,000 \mathrm{ppm}$ v/v for $4-\mathrm{hr}^{92,93}$

$$
\text { recommendation: NOEL rat }=49,500 \mathrm{ppm} \mathrm{v/v}
$$

\section{Other Escape-Impairing Effects and Permanent Injury}

None identified. 


\section{R-113: 1,1,2-TRICHLORO-1,2,2-TRIFLUOROETHANE}

\section{Lethality}

ACGIH indicates a range of 4-hr $\mathrm{LC}_{50}$ rat values of $52,000-56,000 \mathrm{ppm} \mathrm{v/v.}{ }^{24}$ Of them, Sarver's indication of $52,500 \mathrm{ppm} v / \mathrm{V}$ appears to be the most conservative (the difference between it and the concentration identified in the ACGIH range is due to rounding). ${ }^{97}$ Sarver also indicates the lowest ALC identified, namely $45,000 \mathrm{ppm}$ v/v for one of six tested rats. Clayton shows higher 4- and 6-hr ALCs, but does not give sufficient information to determine data qualification. ${ }^{98,99}$

$$
\text { recommendation: } 4-\mathrm{hr} L \mathrm{C}_{50} \text { rat }=52,500 \mathrm{ppm} \mathrm{v} / \mathrm{v}
$$

\section{Cardiac Sensitization}

Reinhardt et al. indicate no marked responses in twelve dogs at 2,600 ppm v/v and marked re-

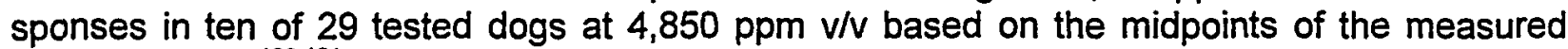
concentrations. ${ }^{100,101}$ These data are not inconsistent with $\mathrm{EC}_{50} \mathrm{~s}$ of 7,000 and $10,000 \mathrm{ppm} \mathrm{v} / \mathrm{v}$, both reported by Clark and Tinston. ${ }^{53,60}$

recommendation: NOEL $\operatorname{dog}=2,600 \mathrm{ppm} v / \mathrm{v}$

\section{Anesthetic Effect}

Raventós and Lemon indicate a $30-\mathrm{min} \mathrm{EC}_{50}$ mouse of $57,000 \mathrm{ppm} \mathrm{v} / \mathrm{v} .^{102}$ Clark and Tinston report a more conservative $10-\mathrm{min} \mathrm{EC}_{50}$ rat of $28,000 \mathrm{ppm} \mathrm{v} / \mathrm{v}$, based on action as a CNS stimulant. ${ }^{53}$ Weigand reports a NOEL and LOEL of 25,000 and $80,000 \mathrm{ppm} \mathrm{v} / \mathrm{v}$, respectively, in rats for 2-hr exposures in a subchronic inhalation toxicity study, but he does not indicate the number of animals affected. ${ }^{62}$

Burn (with compound identification in a later paper by Burn et al.) indicates that one of ten, nine of ten, and fifteen of fifteen mice were anesthetized in $15 \mathrm{~min}$ at 50,000,60,000, and 80,000 $\mathrm{ppm} v / \mathrm{v}$, respectively. ${ }^{103,104}$

recommendation: $10-\mathrm{min} \mathrm{EC}_{50}$ rat $=28,000 \mathrm{ppm} \mathrm{v/v}$

\section{Other Escape-Impairing Effects and Permanent Iniury}

None identified. 


\section{R-114: 1,2-DICHLORO-1,1,2,2-TETRAFLUOROETHANE}

\section{Lethality}

No lethality data were found for 4-hr exposures. Paulet reports a 30-min LC L $_{50}$ rat of 720,000 $\mathrm{ppm} v / \mathrm{v}^{105}$ as cited by Ludwig et al. ${ }^{11}$ Scholz indicates that the 2-hr $\mathrm{LC}_{50}$ rat exceeds 600,000 $\mathrm{ppm} v / \mathrm{v} .{ }^{72}$ Waritz cites the Scholz finding in a data compilation. ${ }^{106}$ DuPont indicates the former value in its MSDS while Honeywell cites the latter. ${ }^{107,108}$ Based on the ten Berge adjustment

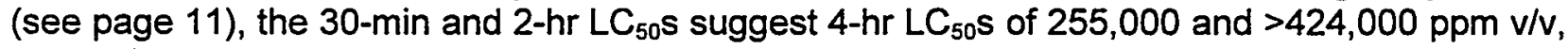
respectively, of which the former is more conservative.

recommendation: $4-\mathrm{hr} \mathrm{LC}_{50}$ rat $=255,000 \mathrm{ppm} \mathrm{v} / \mathrm{v}$

\section{Cardiac Sensitization}

No NOELs were found. Reinhardt et al. indicate a LOEL based on a marked response in one of twelve dogs for an exposure of $25,000 \mathrm{ppm} v / \mathrm{v}^{57,70}$ Hardy et al. report an $\mathrm{EC}_{50}$ in dogs of $46,000-100,000 \mathrm{ppm} \mathrm{v/v.^{71 }}$

recommendation: $L O E L$ dog $=25,000 \mathrm{ppm} \mathrm{v} / \mathrm{v}$

\section{Anesthetic Effect}

Montgomery et al. cite an unpublished $10-\mathrm{min} \mathrm{EC}_{50}$ rat of $250,000 \mathrm{ppm}$ v/v from D. G. Clark (ICI). ${ }^{109}$ Weigand reports a 2-hr LOEL rat of $300,000 \mathrm{ppm} \mathrm{v/v}$, but does not indicate the fraction of animals showing effects, as well as a NOEL of $100,000 \mathrm{ppm} \mathrm{v/v.}{ }^{62}$ The Weigand data corroborate the unpublished Clark data, even though efforts to obtain the source-study were unsuccessful.

recommendation: $10-\mathrm{min} \mathrm{EC}_{50}$ rat $=250,000 \mathrm{ppm} \mathrm{v/v}$

\section{Other Escape-Impairing Effects and Permanent Iniury}

The IPCS reports significant reduction in ventilatory lung capacity, bradycardia, ${ }^{*}$ and increased variability in heart rate following exposures to $2,300-21,400 \mathrm{ppm} v / \mathrm{v} R-114$ for 15,45 , or 60 seconds. ${ }^{110}$ Ludwig et al. review these data in documenting the NIOSH IDLH, but based the IDLH of $15000 \mathrm{ppm} \mathrm{v} / \mathrm{v}$ on cardiac sensitization data. ${ }^{11}$ That determination indicates that the effects cited by the IPCS were not considered to be either escape impairing or permanent injury. No case histories corroborating the IPCS indication were found despite wide historic use of R114 an aerosol propellant for cosmetics and pharmaceutical propellant in metered-dose inhalers (MDIs), both of which resulted in frequent human exposures.

recommendation: none

- slow heart rate 


\section{R-116: HEXAFLUOROETHANE}

\section{Lethality}

Morrison indicates that there were no deaths among four rats exposed to $800,000 \mathrm{ppm}$ V/v R116 in oxygen for $4 \mathrm{hr}^{111}$ Clayton and Waritz both present the Morrison finding. ${ }^{99,106}$ Hiddemen, contributing to a broader study by McHale, corroborates this finding with no deaths in a 1-hr ALC rat study at $800,000 \mathrm{ppm} \mathrm{v} / \mathrm{v}^{112,113}$

recommendation: $4-\mathrm{hr} \mathrm{LC}_{50}$ rat $>800,000 \mathrm{ppm} \mathrm{v} / \mathrm{v}$

\section{Cardiac Sensitization}

Mullin and Trochimowicz indicate a NOEL at $200,000 \mathrm{ppm} \mathrm{v} / \mathrm{v}$ from tests of twelve dogs. ${ }^{114}$ Wills et al. report a similar finding, but their data do not qualify since the tests were conducted on anesthetized dogs. ${ }^{115}$ Hughes suggests a NOEL in dogs of $600,000 \mathrm{ppm} \mathrm{v} / \mathrm{v}^{116}$ but the summary of it by Lee and Graham does not indicate whether an epinephrine challenge was administered. ${ }^{117}$ Brock examined the Hughes data, but also could not determine the basis. ${ }^{118}$

$$
\text { recommendation: } \text { NOEL } \operatorname{dog}=200,000 \mathrm{ppm} \text { v/v }
$$

\section{Anesthetic Effect}

No $\mathrm{EC}_{50}$ was found. Morrison notes that rats exposed to $200,000 \mathrm{ppm}$ v/v showed marginal signs of intoxication, but does not indicate how many of four rats were affected. ${ }^{111}$ Hiddemen notes an anesthetic effect for all ten rats exposed to $780,000 \mathrm{ppm} v / \mathrm{v}$ for $1 \mathrm{hr}$, but indicates normal behavior for 20 rats in a 10-day, continuous exposure study at $121,000 \mathrm{ppm} \mathrm{v/v} .^{112}$

recommendation: 10 -day NOEL rat $=121,000 \mathrm{ppm} \mathrm{v/v}$

\section{Other Escape-Impairing Effects and Permanent Injury}

None identified.

recommendation: none 


\section{R-123: 2,2-DICHLORO-1,1,1-TRIFLUOROETHANE}

\section{Lethality}

Waritz and Clayton indicate a 4-hr $\mathrm{LC}_{50}$ rat of $35,000 \mathrm{ppm} \mathrm{v} / \mathrm{v}^{119,120}$ Hall and Moore obtained a similar finding, but slightly lower finding. ${ }^{121}$ They report that 4-hr exposures of 20,700,32,000, $33,700,42,100,52,500$, and $55,000 \mathrm{ppm} v / \mathrm{v}$ were fatal to zero, three, three, four, six, and six rats, respectively, in groups of six, ${ }^{121}$ indicating an ALC of $32,000 \mathrm{ppm} \mathrm{v} / \mathrm{v}$. They conclude that R-123 has a 4-hr $L_{50}$ rat of approximately $32,000 \mathrm{ppm} \mathrm{v/v,}{ }^{121}$ which a PAFT summary cites. ${ }^{94}$ Coate reports a third, still later study with a somewhat higher $6-\mathrm{hr} \mathrm{LC}_{50}$ rat of $52,540 \mathrm{ppm} \mathrm{v/v}{ }^{122}$ which is cited by both an ECETOC JACC assessment and IPCS survey. ${ }^{123,124}$ The Hall and Moore finding is the most conservative.

recommendation: $4-\mathrm{hr} L C_{50}$ rat $=32,000 \mathrm{ppm} \mathrm{v/v}$

\section{Cardiac Sensitization}

Trochimowicz and Mullin report a NOEL for three dogs tested at $10,300 \mathrm{ppm} \mathrm{v/v}$, a LOEL for

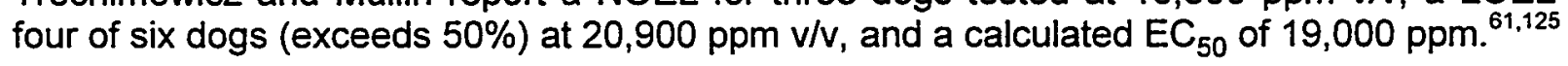

recommendation: NOEL dog $=10,300 \mathrm{ppm}$ v/v

\section{Anesthetic Effect}

Tests by Robbins indicate a $10-\mathrm{min} \mathrm{EC}_{50}$ of $27,000 \mathrm{ppm} \mathrm{v/v}$ for mice by a standard rotadrum (rotating bottle) method. ${ }^{126}$

Raventós and Lemon measured a $30-$ min $\mathrm{EC}_{50}$ mouse of $24,000 \mathrm{ppm} \mathrm{v/v}{ }^{102}$ This finding is cited in the NRC assessment to determine exposure guidance levels (CEGL, EGL, and EEGL) for special military applications on ships and aircraft. ${ }^{39}$ Mullin reports behavioral test data for R123 by show that the effective concentration decreases with increasing exposure duration (the effective concentration levels are lower at $30 \mathrm{~min}$ than at $15 \mathrm{~min}$, and lower still at 60 min than at $30 \mathrm{~min}) .{ }^{127}$ The slightly lower 30 -min $\mathrm{EC}_{50}$ from Raventós and Lemon therefore corroborates the 10-min $\mathrm{EC}_{50}$ finding by Robbins.

Wada indicates the 10-min approximate anesthetic concentration (AAC) to be $130,000 \mathrm{ppm}$ $\mathrm{v} / \mathrm{v}^{128}$ The $\mathrm{AAC}$ appears to be an $\mathrm{EC}_{50}$, but it is more conservative if it refers to a threshold concentration level.

The Mullin study also found "occasional failures in pinna reflex" after $60 \mathrm{~min}$ in one of six rats at $0 \mathrm{ppm}$ v/v (untreated control group) and in unspecified numbers of rats among six tested at 975 and 2,697 ppm v/v. ${ }^{127}$ That indicates a possible effect as low as $975 \mathrm{ppm} v / \mathrm{v}$, but it is not clear if it was exposure related, since a control animal $(0 \mathrm{ppm} \mathrm{v/v)}$ also failed the same criterion. Mullin indicates a $15-\mathrm{min} \mathrm{EC}_{50}$ of $7,080 \mathrm{ppm} \mathrm{v} / \mathrm{v}$ as the lowest in a range $(7,080-9,710 \mathrm{ppm} \mathrm{v} / \mathrm{v})$ for a suite of behavioral measures including unconditioned reflexes, simple behavioral patterns,

* characteristic response of flattening an ear touched lightly with a small brush or probe 
modified locomotor activity (grip, ${ }^{*}$ lift, $^{\dagger}$ vertical bar, ${ }^{\ddagger}$ ataxia, ${ }^{\S}$ and pinna tactile ${ }^{\star \star}$ ), and observation of unusual signs or symptoms. ${ }^{127.129}$ The resulting $E C_{50}$ is far more stringent than by the standard rotadrum test and is for 15-min rather than 10-minute tests.

Burns et al. report a LOEL of $40,000 \mathrm{ppm}$ v/v. ${ }^{130}$ Hall and Moore noted central nervous system (CNS) effects in rats at $20,700 \mathrm{ppm}$ v/v for $4 \mathrm{hr}$ exposures. ${ }^{121}$ Trochimowicz reports a NOEL of $2,500 \mathrm{ppm} \mathrm{v} / \mathrm{v} .^{131}$ Magda indicates a NOEL of $5,000 \mathrm{ppm} \mathrm{v} / \mathrm{v}$ in acute and subchronic inhalation studies. ${ }^{132}$ The PAFT toxicity summary for R-123 notes that anesthetic-like effects are observed at concentrations exceeding $5,000 \mathrm{ppm} \mathrm{v} / \mathrm{v} .^{7}$

The 10-min $\mathrm{EC}_{50}$ of $27,000 \mathrm{ppm}$ v/v from Robbins meets the data selection criteria (see page $19)$ and is the most conservative of similar test findings.

recommendation: $10-\mathrm{min} \mathrm{EC}_{50}$ rat $=27,000 \mathrm{ppm} \mathrm{v/v}$

\section{Other Escape-Impairing Effects and Permanent Injury}

There is anecdotal evidence of hepatotoxicty (liver toxicity) from chronic exposures well above the Workplace Environmental Exposure Level (WEEL) guide developed by the American Industrial Hygiene Association (AlHA) for R-123, but these accounts do not suggest any escapeimpairing effects. Moreover, they indicate that the effects were reversible (not permanent). ${ }^{133-136}$

recommendation: none

* ability to grasp a horizontal rod for support when swung gently by the tail toward the rod

$\dagger$ ability to raise the hind legs onto a horizontal rod, and remain there, while hanging from the rod by the forepaws

$\ddagger$ ability to grasp a vertical rod with both front and hind feet when swung gently by tail toward the rod

$\S$ inability to coordinate voluntary muscular movements determined by observation of gait on a non-slip surface

** characteristic response of flattening an ear touched lightly with a small brush or probe 


\section{R-124: 2-CHLORO-1,1,1,2-TETRAFLUOROETHANE}

\section{Lethality}

Gleason and Coate indicate no mortality from 6-hr rat exposures to $360,000 \mathrm{ppm} \mathrm{v/v.}{ }^{137}$ In a PAFT data summary, Finegan and Rusch indicate that the 4-hr LC $_{50}$ rat equals $360,000 \mathrm{ppm}$ $\mathrm{v} / \mathrm{v}^{138}$ In a separate summary of the PAFT data, Magda indicates that the ${ }^{13} \mathrm{C}_{50}$ rat exceeds $360,000 \mathrm{ppm} v / \mathrm{v}^{132}$ While he does not explicitly identify the duration of the tests, other data in the same table reflect 4-hr values. Honeywell's MSDS indicates that the 4-hr LC 50 rat equals or exceeds $360,000 \mathrm{ppm} v / \mathrm{v}^{139}$

Trochimowicz reports a $4-\mathrm{hr} \mathrm{LC}_{50}$ rat of $262,500 \mathrm{ppm} \mathrm{v} / \mathrm{v}$ determined from tests by Kelly. ${ }^{125,140}$ Kelly indicates 4-hr exposures to $230,000 \mathrm{ppm}$ v/v yielded no deaths, but six of six rats died at $300,000 \mathrm{ppm}$ v/v. ${ }^{140}$ DuPont's MSDS reflects the Kelly finding with indication that the 4-hr ALC is $230,000-300,000 \mathrm{ppm} v / \mathrm{v}^{141}$

The ECETOC JACC report for R-124 summarizes both the Gleason and Coate and the Kelly studies, but indicates that the $4-\mathrm{hr} \mathrm{LC}_{50}$ rat is $230,000-300,000 \mathrm{ppm} \mathrm{v} / \mathrm{v}$ in the summary and conclusions. ${ }^{142}$

recommendation: $4-\mathrm{hr} \mathrm{LC}_{50}$ rat $=262,500 \mathrm{ppm}$ v/v, 4-hr ALC rat $>230,000 \mathrm{ppm}$ v/v

\section{Cardiac Sensitization}

Mullin indicates a NOEL for zero of ten dogs at 10,100 ppm v/v and a LOEL for four of ten dogs at $25,000 \mathrm{ppm} \mathrm{v} / \mathrm{v}$. Kenny and Hardy cite an $\mathrm{EC}_{50}$ of $40,000 \mathrm{ppm} \mathrm{v} / \mathrm{v}$ from an EPA draft with an incomplete reference. ${ }^{143}$ An earlier external review draft obtained from EPA does not mention this $\mathrm{EC}_{50}{ }^{144}$

recommendation: NOEL dog $=10,100 \mathrm{ppm}$ v/v

\section{Anesthetic Effect}

The PAFT summary for R-124 indicates a $10-\mathrm{min}^{\mathrm{E}} \mathrm{EC}_{50}$ in animals of $140,000 \mathrm{ppm}$ v/v for nervous system effects, but it neither identifies the species tested nor references the specific study. ${ }^{7}$ Honeywell's MSDS also cites this $\mathrm{EC}_{50 .}{ }^{139}$ Wada indicates a calculated "approximate anesthetic concentration (AAC)" that appears to be an $E_{50}$ of $150,000 \mathrm{ppm} \mathrm{v} / \mathrm{v}$ from a 10 -min test of rats. $^{52}$ Van Poznak and Artusio report an effect in dogs exposed to R-124 at $400,000 \mathrm{ppm} \mathrm{v} / \mathrm{v}$, but do not indicated the fraction of responses among six subjects. ${ }^{85}$ Gleason and Coate indicate an effect for an unspecified quantity of the six rats at $100,000 \mathrm{ppm} \mathrm{v} / \mathrm{v}$ in the first $30 \mathrm{~min}$ of a $4 \mathrm{hr}$, acute inhalation study. ${ }^{137}$ Kelly indicates a NOEL from a 4-hr test of six rats at 48,000 $\mathrm{ppm} v / \mathrm{v}^{140}$ Barring discovery of a source for and verification of the PAFT indication, the Wada data appears to best fit the data selection criteria (see page 19).

recommendation: $10-\mathrm{min} \mathrm{EC}_{50}$ rat $=150,000 \mathrm{ppm} \mathrm{v/v}$ 
Other Escape-Impairing Effects and Permanent Injury

None identified.

recommendation: none 


\section{R-125: 1,1,1,1,2-PENTAFLUOROETHANE}

\section{Lethality}

Nakayama et al. concluded that the 4-hr ALC rat, and therefore also the $\mathrm{LC}_{50}$, exceeds 769,000 $\mathrm{ppm} \mathrm{v/v.}{ }^{145}$ This result is cited in many MSDSs but often as $800,000 \mathrm{ppm} \mathrm{v} / \mathrm{v}$ based on the target concentration. An earlier study by Panepinto found that the 4-hr ALC exceeds 709,000 ppm $\mathrm{V} / \mathrm{V}$ based on no deaths in six tested rats. ${ }^{146}$ Given that zero deaths is akin to a "NOEL" for scoring mortality and that Panepinto's open-ended finding does not contradict Nakayama's, the latter finding meets the selection criteria (see page 19).

\section{recommendation: $4-\mathrm{hr} \mathrm{LC}_{50}$ rat $>769,000 \mathrm{ppm} \mathrm{v/v}$}

\section{Cardiac Sensitization}

Hardy et al. report no and one marked responses among six dogs tested at 75,000 and 100,000 $\mathrm{ppm} \mathrm{v} / \mathrm{v}$, respectively. ${ }^{147}$ The same study reported an $E C_{50}$ of $139,000 \mathrm{ppm} \mathrm{v} / \mathrm{v}$ while a later compilation by the same authors indicates an $E_{50}$ of $170,000 \mathrm{ppm} \mathrm{v/v.1147}$ While the $E_{50}$ selection is unclear in this case, the NOEL is not.

recommendation: NOEL $\operatorname{dog}=75,000 \mathrm{ppm} v / \mathrm{v}$

\section{Anesthetic Effect}

No 10-min $E_{50}$ in mice or rats were found. But several 4-hr acute inhalation studies provide NOEL and LOEL data. Nakayama et al. indicate an effect in more than half the rats exposed to $769,000 \mathrm{ppm} \mathrm{v} / \mathrm{v} .^{145}$ Burns et al. report no anesthetic effect in mice at $930,000 \mathrm{ppm} \mathrm{v} / \mathrm{v},{ }^{130}$ but this indication warrants verification since the animals should have experienced oxygen deprivation at that concentration even with a mixture of $R-125$ and pure oxygen. Moreover, this finding fails the data selection criteria (see page 19) for a NOEL since it exceeds the LOEL reported by Nakayama et al. ${ }^{145}$ Panepinto indicates no effect for five rats at $709,000 \mathrm{ppm}$ v/v. ${ }^{146}$ Nakayama et al., in a separate study, report no effect at $50,113 \mathrm{ppm} \mathrm{v} / \mathrm{v}$ among 40 rats in a subchronic study. ${ }^{148}$ The Panepinto NOEL meets the selection criteria.

recommendation: $10-\mathrm{min} \mathrm{EC}_{50}$ rat $=709,000 \mathrm{ppm} \mathrm{v} / \mathrm{v}$

\section{Other Escape-Impairing Effects and Permanent Iniury}

None identified.

recommendation: none 


\section{R-134: $1,1,2,2-$ TETRAFLUOROETHANE}

\section{Lethality}

Warheit found no mortality in 4-hr exposures of rats at concentrations as high as 460,000 ppm $\mathrm{v} / \mathrm{V}$ in air (with oxygen enrichment to maintain $20-22 \%$ levels), which indicates that the 4-hr ALC and $E_{50}$ rat exceed $460,000 \mathrm{ppm} \mathrm{v/v.}{ }^{149}$ No other acute inhalation lethality data were located, but the DuPont MSDS shows the Warheit finding. ${ }^{150}$

recommendation: $4-\mathrm{hr} \mathrm{LC}_{50}$ rat $>460,000 \mathrm{ppm} \mathrm{v} / \mathrm{v}$

\section{Cardiac Sensitization}

A Huntingdon Research Centre (HRC) study found a NOEL at 75,000 ppm v/v and a LOEL for marked responses in $33 \%$ of tested dogs at $100,000 \mathrm{ppm} \mathrm{v/v.}{ }^{151}$

recommendation: NOEL dog $=75,000 \mathrm{ppm}$ v/v

\section{Anesthetic Effect}

The Warheit ALC study states that no clinical signs were observed at $120,000 \mathrm{ppm} v / \mathrm{v}$, but that an unspecified number of rats exposed to $200,000 \mathrm{ppm} v / \mathrm{v}$ showed no response to tapping on the chamber. ${ }^{149}$

recommendation: $4-\mathrm{hr}$ NOEL rat $=120,000 \mathrm{ppm}$ v/v

\section{Other Escape-Impairing Effects and Permanent Injury}

None identified.

recommendation: none 


\section{R-134a: 1,1,1,2-TETRAFLUOROETHANE}

The International Pharmaceutical Aerosol Consortium for Toxicology Testing (IPACT) and independent pharmaceutical companies have conducted extensive studies on the toxicity and tolerance of R-134a, to qualify its use as a pharmaceutical aerosol propellant for metered dose inhalers (MDIs), but declined to release those studies.

\section{Lethality}

Several sources cite a 4-hr ALC of $566,700 \mathrm{ppm} v / \mathrm{v}^{152,153}$ This ALC derives from a study by Silber, but it does not meet the data selection criteria (see page 19) since this concentration was fatal to more than half of the animals tested, namely five of six rats. ${ }^{154}$ Silber also indicates no mortality in six rats exposed to $359,300 \mathrm{ppm}$ v/v for $4 \mathrm{hr} .{ }^{154}$

Other sources cite a 4-hr $\mathrm{LC}_{50}$ rat of $>500,000 \mathrm{ppm} \mathrm{v/v.}{ }^{153,155-157}$ Several PAFT summaries also cite it. ${ }^{7125,132,138}$ An ECETOC JACC report attributes it to unpublished ICI data. ${ }^{158,159}$ Efforts to locate it through the identified source found only a citation by Ferguson-Smith that attributes the $\mathrm{LC}_{50}$ to an internal ICl communication that could not be retrieved. ${ }^{160,161}$

Rissolo found a $30-\mathrm{min}$ ALC rat of $750,000 \mathrm{ppm}$ v/v based on two deaths among four exposed rats. ${ }^{162}$

The 4-hr ALC of $>359,300 \mathrm{ppm}$ v/v from Silber, which implies a 4-hr $\mathrm{LC}_{50}$ of $>359,300 \mathrm{ppm} \mathrm{v} / \mathrm{v}$, meets the data selection criteria. The cited $4-h r L_{50}$ rat of $>500,000 \mathrm{ppm}$ v/v might meet the criteria if adequate documentation can be obtained. The $\mathrm{LC}_{50}$ will not impact the ATEL for R$134 a$ or common blends that include it, but might influence the ATEL for some other blends.

recommendation: $4-\mathrm{hr} \mathrm{LC}_{50}$ rat $>359,300 \mathrm{ppm} \mathrm{v} / \mathrm{v}$

\section{Cardiac Sensitization}

Mullin reports no sensitization in ten dogs at $49,800 \mathrm{ppm} v / \mathrm{v}$, marked response in two of ten dogs at $75,200 \mathrm{ppm} \mathrm{v} / \mathrm{v}$, and in two of four dogs with one death at $101,900 \mathrm{ppm} \mathrm{v/v}$. ${ }^{163}$ Hardy et al. report later tests with no sensitization at $40,000 \mathrm{ppm} v / \mathrm{v}$ as well as marked responses in two of six dogs at $80,000 \mathrm{ppm} \mathrm{v} / \mathrm{v}^{56}$ Kenny and Hardy as well as Hardy et al. indicate an $\mathrm{EC}_{50}$ of $205,000 \mathrm{ppm} \mathrm{v/v}$. $^{71,114}$

recommendation: NOEL dog $=49,800 \mathrm{ppm} \mathrm{v} / \mathrm{v}$

\section{Anesthetic Effect}

Shulman and Sadove report an $\mathrm{EC}_{50}$ level in mice of $270,000 \mathrm{ppm} \mathrm{v} / \mathrm{V}$ based on loss of righting reflex. ${ }^{164}$ They do not indicate the test duration except to note the anesthetic "action is rapid," but they note that induction times with concentrations of $500,000 \mathrm{ppm} v / \mathrm{v}$ or greater is under 30 seconds. ${ }^{164}$ Ferguson-Smith attributes a $10-\mathrm{min} \mathrm{EC}_{50}$ rat of $280,000 \mathrm{ppm} \mathrm{v} / \mathrm{v}$ to an internal $\mathrm{ICl}$ communication. ${ }^{160,161}$ 
Silber reports a LOEL of $205,200 \mathrm{ppm} v / \mathrm{v}$ from a 4-hr rat study, but does not indicate the number of affected rats, and a NOEL at $81,000 \mathrm{ppm} v / \mathrm{v}^{154}$ In a later study, Alexander found no evidence of acute toxicity or clinical reactions in rats or mice exposed for $1 \mathrm{hr}$ to $810,000 \mathrm{ppm} \mathrm{v} / \mathrm{v}$ with oxygen supplementation. ${ }^{165}$

Neither of the two $\mathrm{EC}_{50}$ values meet the letter of the data selection criteria, but they reinforce each other and appear consistent with the NOEL and LOEL data. The effect described by Shulman and Sadove as "rapid" probably was less than $10 \mathrm{~min}$, especially considering the very short induction time indicated for higher concentrations. If in error, use of a 10-min $E_{50}$ rat of $270,000 \mathrm{ppm} \mathrm{v/v}$ is still slightly more conservative than the $10-\mathrm{min} \mathrm{EC}_{50}$ rat of $280,000 \mathrm{ppm} \mathrm{v/v}$ indicated by Ferguson-Smith.

recommendation: $10-\min \mathrm{EC}_{50}$ rat $=270,000 \mathrm{ppm} \mathrm{v/v}$

\section{Other Escape-Impairing Effects and Permanent Injury}

Vinegar et al. describe tests in human volunteers, to validate a physiologically-based pharmacokinetic (PBPK) model, in which tests with R-134a had to be terminated when the subject lost consciousness and experienced reduced pulse and blood pressure at $4,000 \mathrm{ppm} \mathrm{v} / \mathrm{v} .{ }^{166}$ The report notes that a second subject experienced a rapid rise in blood pressure and pulse in sequential exposures at 4,000 and $2,000 \mathrm{ppm}$ v/v exposure as well as anomalous results with R227 ea (see page 65). ${ }^{166}$ Subsequent investigation indicates that the reported responses probably were not due to the chemical exposures, and that no adverse effects were seen in other human exposures at lower or higher concentrations. ${ }^{167,168}$

recommendation: none 


\section{R-E134: DIFLUOROMETHOXY-DIFLUOROMETHANE}

\section{Lethality}

Keller et al. with elaboration by Graham and Lardear indicate no fatalities among three rats exposed to $95,000 \mathrm{ppm} v / \mathrm{v}$ for $4 \mathrm{hr}^{169,170}$

Simons et al. summarize toxicity findings in a patent on dimethyl ethers. ${ }^{171}$ They indicate no deaths in ten mice exposed to slightly more than $200,000 \mathrm{ppm} v / \mathrm{v}$ for $2 \mathrm{hr}$ and in an unspecified quantity of rats exposed to $50,000 \mathrm{ppm} \mathrm{v/v}$ for $3 \mathrm{hr} / \mathrm{d}$ for 5 days. ${ }^{171}$ These findings suggest 4-hr $A L C s$ and $L C_{50} s$ of $>141,000$ and $>43,300 \mathrm{ppm} v / v$ with the ten Berge adjustment (see page 11).

In an unpublished presentation, O'Neill (one of the inventors in the Simons patent) indicates no deaths in inhalation exposures of unspecified numbers of rats to $50,000 \mathrm{ppm} v / \mathrm{v}$ for $3 \mathrm{hr}$, $200,000 \mathrm{ppm} v / \mathrm{v}$ for $2 \mathrm{hr}$, and $300,000 \mathrm{ppm} v / \mathrm{v}$ for $30 \mathrm{~min}^{172}$ These data suggest 4-hr ALCs and $L C_{50} s$ of $>43,300,>141,000$, and $>106,000 \mathrm{ppm} v / v$ with the ten Berge adjustment. While these unpublished and incomplete findings do not meet the data selection criteria (see page 19), they expand on the indications and corroborate the Simons et al. data.

recommendation: 4-hr $\mathrm{LC}_{50}$ rat $>141,000 \mathrm{ppm}$ v/v

\section{Cardiac Sensitization}

O'Neill summarizes a comparative study of $R-11, R-12, R-21, R-22$, and $R-E 134 a$ in monkeys for 30 -min exposures to $50,000 \mathrm{ppm}$ v/v mixed with oxygen. ${ }^{172}$ He reports that all of these chemicals predisposed the monkeys to cardiac arrhythmia and sensitized them to epinephrine challenges, but with only a limited sensitization for R-12. He notes that R-E134 behaved more like R-11 than the other compounds tested. O'Neill does not indicate either a NOEL or the fraction of monkeys with marked responses at $50,000 \mathrm{ppm} v / \mathrm{v}$, but he makes clear that R-E134 is a cardiac sensitizer. ${ }^{172}$ Moreover, the data are for monkeys rather than dogs, though monkeys usually are a good animal model for human toxicity.

recommendation: not determined

\section{Anesthetic Effect}

No $\mathrm{EC}_{50}$ was found.

O'Neill indicates that mice exposed to $100,000,130,000,182,500$, or $307,500 \mathrm{ppm} v / \mathrm{v}$ showed anesthetic effects in 60,30,5, and 2 min respectively. ${ }^{172}$ Simons et al. (including O'Neill) indicate anesthetic effects in ten mice exposed to slightly more than $200,000 \mathrm{ppm} v / \mathrm{v}$ for $2 \mathrm{hr} .{ }^{171}$ These data do not enable determination of a qualifying LOEL or NOEL.

Keller et al. indicate that rats exposed to $45,000 \mathrm{ppm} v / \mathrm{v}$ for $4 \mathrm{hr}$ appeared uncoordinated and responded sluggishly to sound. ${ }^{169,170}$ They further indicate that rats exposed to $18,000 \mathrm{ppm} \mathrm{v} / \mathrm{v}$ showed no signs of clinical toxicity. ${ }^{169,170}$ 


\section{Other Escape-Impairing Effects and Permanent Injury}

None identified.

recommendation: none 


\section{R-141b: 1,1-DICHLORO-1-FLUOROETHANE}

\section{Lethality}

Hardy et al. indicate 4-hr $L_{50}$ s of $58,931 \mathrm{ppm} \mathrm{v} / \mathrm{v}$ from tests of five male rats, $64,991 \mathrm{ppm} \mathrm{v} / \mathrm{v}$ for five females, and $61,647 \mathrm{ppm}$ v/v overall. ${ }^{173}$ These results also are summarized in an article by Seckar, who rounds the $L C_{50}$ to $62,000 \mathrm{ppm} \mathrm{v/v.}{ }^{174}$ The $L C_{50}$ finding is supported by an earlier 6-hr acute inhalation study in rats for which Doleba-Crowe reports an ALC of 50,200 ppm $\mathrm{v} / \mathrm{v},{ }^{175}$ cited by the IPCS and by Lee et al. ${ }^{124,176}$ Janssen reports a separate 6 -hr acute inhalation study that found the 6-hr ALC rat to exceed 11,096 ppm v/v. ${ }^{177}$

recommendation: $4-\mathrm{hr} \mathrm{LC}_{50}$ rat $=61,647 \mathrm{ppm} \mathrm{v} / \mathrm{v}$

\section{Cardiac Sensitization}

Mullin reports a NOEL and LOEL based on marked responses in zero and one of ten dogs at 2,600 and $5,200 \mathrm{ppm} v / \mathrm{v}$, respectively. ${ }^{178}$ These data suggest that $\mathrm{R}-141 \mathrm{~b}$ is a strong cardiac sensitizer.

In a later study, Hardy et al. document a marked response in one of two dogs tested at 9,000 $\mathrm{ppm} \mathrm{v} / \mathrm{v}$ (the lowest concentration tested), a mild response at $3,000 \mathrm{ppm} v / \mathrm{v}$ in monkeys, and both a marked response in the same animal and a questionable response in a second monkey at $5,000 \mathrm{ppm} \mathrm{v/v.55}$

In a third still later study, Kenny found neither marked responses nor abnormal clinical signs at $10,000 \mathrm{ppm} \mathrm{v/v,} \mathrm{but} \mathrm{a} \mathrm{marked} \mathrm{response} \mathrm{in} \mathrm{one} \mathrm{of} \mathrm{six} \mathrm{dogs} \mathrm{tested} \mathrm{at} 20,000 \mathrm{ppm} \mathrm{v/v.}{ }^{179}$

Brock et al. note the sensitization level as $9,000-19,000 \mathrm{ppm}$ v/v in dogs as well as at 5,000 and $10,000 \mathrm{ppm} \mathrm{v/v} \mathrm{in} \mathrm{monkeys.}{ }^{180}$ They conclude that "HCFC-141b is able to induce a cardiac sensitization response at $5000 \mathrm{ppm}$ and above, a similar response to that observed with CFC$11 . " 180$

The Mullin data appears to be the most conservative, though the data dispersion suggests some uncertainty.

\section{recommendation: NOEL dog $=2,600 \mathrm{ppm} \mathrm{v/v}$}

\section{Anesthetic Effect}

Robbins indicates a $10-\mathrm{min} \mathrm{EC}_{50}$ rat of $25,000 \mathrm{ppm} \mathrm{v/v} .^{126}$

Hardy et al. indicate that the rats in all exposure groups, of which the lowest was $29,674 \mathrm{ppm}$ $v / v$, showed "reduced motor activity, restless behavior, and abnormal body carriage" among other symptoms. ${ }^{173}$ Summarizing that study, Brock et al. indicate that anesthesia was noted above $29,000 \mathrm{ppm} v / \mathrm{v}^{180}$ Seckar indicates behavior typical of CNS depression above 30,000 ppm v/v in an unspecified number of rats. ${ }^{174}$ Janssen and Millischer et al. note NOELs at 11,096 in ten rats and 19,959 ppm v/v in 160 rats, respectively, in 6-hr exposures in acute and 
chronic inhalation toxicity studies, again respectively. ${ }^{177,181}$ Doleba-Crowe reports mild anesthesia within the first hour of $6-\mathrm{hr}$ exposures of six rats at $31,730 \mathrm{ppm} \mathrm{v/v.}{ }^{175}$

These data are reasonably consistent and support the Robbins $\mathrm{EC}_{50}$.

\section{recommendation: $10-\mathrm{min} \mathrm{EC}_{50}$ rat $=25,000 \mathrm{ppm} \mathrm{v/v}$}

\section{Other Escape-Impairing Effects and Permanent Injury}

None identified.

recommendation: none 


\section{R-142b: 1-CHLORO-1,1-DIFLUOROETHANE}

\section{Lethality}

Davies et al. indicate a $30-\mathrm{min} \mathrm{LC}_{50}$ mouse of $300,000 \mathrm{ppm} \mathrm{v/v},{ }^{182}$ as reported in an ECETOC JACC report. ${ }^{183}$ Applying the ten Berge extrapolation (see page 11) suggests a 4-hr $\mathrm{LC}_{50}$ of approximately $106,000 \mathrm{ppm} \mathrm{v/v.}$

Carpenter et al. indicate that $128,000 \mathrm{ppm} v / \mathrm{v}$ for $4 \mathrm{hr}$ kills two to four of six rats. ${ }^{184}$ Four of six would exceed the $50 \%$ criterion for an ALC.

Mecler and Knapinsky indicate a $6-\mathrm{hr}$ ALC rat of $400,000 \mathrm{ppm}$ with $20 \%$ mortality, ${ }^{185}$ but this finding conflicts with those of Davies et al. (with or without the ten Berge adjustment) and Carpenter et al.

Lester and Greenberg report that "all animals died within 9 exposures; 6 died in 7 days, 2 in 8 days, and 2 in 9 days" for exposures at $100,000 \mathrm{ppm} \mathrm{v/v}$ for $16 \mathrm{hr} / \mathrm{d}^{65}$ This wording implies that no deaths occurred in the first day of the 9-day rat study, which suggests a 16-hr ALC of $>100,000 \mathrm{ppm} \mathrm{v} / \mathrm{v}$. Applying the ten Berge adjustment suggests a 4-hr ALC of approximately $200,000 \mathrm{ppm} v / \mathrm{v}$, but the full adjustment may not be appropriate for a no-effect rather than an effect level.

Further testing of R-142b is unlikely with its scheduled phaseout pursuant to the Montreal Protocol.

While no 4-hr $\mathrm{LC}_{50}$ or ALC was found there is reasonable agreement between the findings of Davies et al. (though in a mouse rather than a rat), Carpenter et al., and Lester and Greenberg. Use of the most conservative of them, namely the adjusted data from Davies et al., should be appropriate.

recommendation: $4-\mathrm{hr} \mathrm{LC}_{50}$ mouse $=106,000 \mathrm{ppm} \mathrm{v/v}$

\section{Cardiac Sensitization}

Mullin indicates marked responses in zero of six, five of twelve, and twelve of twelve dogs tested at $25,000,50,000$, and $100,000 \mathrm{ppm} \mathrm{v} / \mathrm{v}$, respectively. ${ }^{178,186}$ Reinhardt et al. (including Mullin) explain the test procedure and findings. ${ }^{57}$

\section{recommendation: NOEL dog $=25,000 \mathrm{ppm} \mathrm{v} / \mathrm{v}$}

\section{Anesthetic Effect}

Robbins reports a $10-\mathrm{min} \mathrm{EC}_{50}$ mouse of $250,000 \mathrm{ppm} \mathrm{v/v.}{ }^{126}$

Lester and Greenberg state that a rat exhibited "slight intoxication" at $150,000 \mathrm{ppm} v / \mathrm{v}$ (the low-

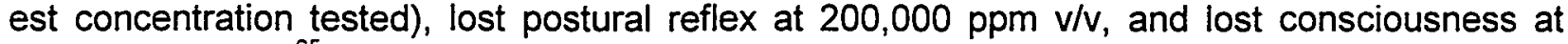
$300,000 \mathrm{ppm} \mathrm{v/v.}{ }^{65}$ The goal of their study was to screen fluorocarbons as anesthetic agents, so the authors were seeking levels inducing deep anesthesia. While "slight intoxication" fits the 
criterion of "any observed effect to even one test animal" in the definition of a LOEL, the tests involved only a single animal and therefore an effect in more than $50 \%$ of tested animals.

$$
\text { recommendation: } 10-\mathrm{min} \mathrm{EC}_{50} \text { rat }=250,000 \mathrm{ppm} \mathrm{v/v}
$$

\section{Other Escape-Impairing Effects and Permanent Iniury}

None identified.

recommendation: none 


\section{R-143a: 1,1,1-TRIFLUOROETHANE}

\section{Lethality}

Kelly indicates no mortality in six rats exposed to $540,000 \mathrm{ppm} v / \mathrm{v}$ for $4 \mathrm{hr} .^{187}$ In a later study, Cracknell indicates a similar result for ten rats at $591,000 \mathrm{ppm} \mathrm{v/v}{ }^{188}$ These results are reinforced by Nikijenko and Tolgskaya with no deaths in an acute inhalation test of mice at 540,000 ppm v/v. ${ }^{189}$

\section{recommendation: $4-\mathrm{hr} \mathrm{LC}_{50}$ rat $>591,000 \mathrm{ppm} \mathrm{v/v}$}

\section{Cardiac Sensitization}

Hardy and Sharman report no marked responses in six dogs at concentrations as high as $250,000 \mathrm{ppm} \mathrm{v} / \mathrm{v}$ in air, but marked response in two of five dogs at $300,000 \mathrm{ppm} \mathrm{v} / \mathrm{v}$. ${ }^{190}$ Brock et al. summarize the Hardy and Sharman study. ${ }^{191}$ Hardy et al. indicate the $E_{50}$ to be 310,000 ppm v/v. ${ }^{71}$

recommendation: NOEL $\operatorname{dog}=250,000 \mathrm{ppm} \mathrm{v} / \mathrm{v}$

\section{Anesthetic Effect}

Robbins indicates a $10-\mathrm{min} \mathrm{EC}_{50}$ range of $500,000-600,000 \mathrm{ppm} \mathrm{v} / \mathrm{v}$ in mice. ${ }^{126}$ The absence of any clinical signs of anesthetic effects in two acute inhalation toxicity studies, by Kelly at $540,000 \mathrm{ppm}$ v/v and by Cracknell at 591,000 ppm v/v, support the Robbins finding. ${ }^{187,188}$ A review of acute, subchronic, and chronic exposure studies by Brock et al. repeatedly notes the absence of adverse clinical signs. ${ }^{191}$ Based on the conservative end of the Robbins range, the $\mathrm{EC}_{50}$ is at least $500,000 \mathrm{ppm} \mathrm{v} / \mathrm{v}$.

$$
\text { recommendation: } 10-\mathrm{min} \mathrm{EC}_{50} \text { rat }=500,000 \mathrm{ppm} \mathrm{v/v}
$$

\section{Other Escape-Impairing Effects and Permanent Injury}

None identified. 


\section{R-152a: 1,1-DIFLUOROETHANE}

\section{Lethality}

Moore reports zero, one, and two deaths among groups of six rats exposed to 319,000 , 383,000 , or $437,500 \mathrm{ppm} \mathrm{v} / \mathrm{v}$, respectively, for $4 \mathrm{hr}^{192}$ While Moore concludes that the ALC is $383,000 \mathrm{ppm} \mathrm{v/v},{ }^{192}$ the data suggest that the $4-\mathrm{hr} \mathrm{LC}_{50}$ might exceed $437,500 \mathrm{ppm} \mathrm{v} / \mathrm{v}$.

An early study by Carpenter et al. suggest a contrary conclusion. A second by Lester and Greenberg does likewise, but also presents conflicting findings that are more consistent with the Moore data. A third early study by Limperos also offers consistent data, though insufficient to corroborate the Moore and Keller findings.

The study by Carpenter et al., published in 1949, indicates an ALC based on deaths of two to four of six rats at $64,000 \mathrm{ppm} v / \mathrm{v}$ in $4 \mathrm{hr}$. $^{184}$ Based on the uncertain number of deaths, this result might not meet the data selection criteria (see page 19).

A study by Lester and Greenberg, published in 1950, indicates that a $30-\mathrm{min}$ exposure to $500,000 \mathrm{ppm} \mathrm{v} / \mathrm{v}$ was fatal to the single rat tested. ${ }^{65}$ Applying the ten Berge extrapolation (see page 11) suggests that a 4-hr exposure to $177,000 \mathrm{ppm}$ v/v or a $16-\mathrm{hr}$ exposure to $88,400 \mathrm{ppm}$ v/v should be lethal. However, the same paper by Lester and Greenberg also reports a chronic exposure study of eight rats exposed to a concentration of $100,000 \mathrm{ppm}$ for $16 \mathrm{hr} /$ day for two months with no adverse clinical signs and only mild, microscopic effects in pathology at necropsy. ${ }^{65}$ Applying the ten Berge extrapolation suggests comparability to a 4-hr ALC that exceeds $200,000 \mathrm{ppm}$ v/v.

A 1951 study by Limperos reports occasional trembling and loss of coordination, but no deaths in pairs of rats at 100,000 and $200,000 \mathrm{ppm}$ v/v for $2 \mathrm{hr}^{193}$ Applying the ten Berge extrapolation suggests comparability to a 4-hr ALC that exceeds $141,000 \mathrm{ppm} \mathrm{v} / \mathrm{v}$.

Keller et al. interpret the Lester and Greenberg, Limperos, and Moore studies - the last two of which are Haskell studies as was the one by Keller et al. - as indicating a 4-hr LC $_{50}$ rat exceeding 400,000 ppm v/v. ${ }^{194}$

The documentation for the R-152a WEEL cites a 4-hr ALC of $383,000 \mathrm{ppm}$ v/v based on the Moore finding, a 3-min ALC exceeding 500,000.ppm v/v based on Lester and Greenberg, ${ }^{*}$ and a $2-\mathrm{hr} \mathrm{LC}_{50}$ mouse of $369,000 \mathrm{ppm}$ v/v based on Izmerov et al. ${ }^{195,196}$ Applying the ten Berge extrapolation to the Izmerov finding suggests a 4-hr LC ${ }_{50}$ mouse of $261,000 \mathrm{ppm} v / \mathrm{v}$.

Several toxicity assessments document additional testing, including for use as an aerosol propellant in consumer products. ${ }^{23,195,197-201}$ Manufacturer MSDSs show no consensus, variously citing the 4-hr ALC rat of 64,000 ppm v/v from Carpenter et al., ${ }^{202}$ the 4-hr ALC of 383,000 ppm $\mathrm{v} / \mathrm{v}$ from Moore, ${ }^{203}$ the same concentration as a 4-hr $\mathrm{LC}_{50}{ }^{204}$ and the same concentration as a 2$\mathrm{hr} \mathrm{LC}_{50}$ rat. $^{205}$ Based on the present data selection criteria (see page 19), the ALC and values

* The "3-min" indication appears to be an error. The intent may have been "30-min" based on the protocol description by Lester and Greenberg, but they state that "exposures to 50 and 55 per cent were lethal in from 10 to 25 minutes" for the R-152a tests. $^{65}$ 
other than for 4-hr rat studies come into play only if there is no 4-hr $\mathrm{LC}_{50}$ rat. On that basis, the Keller et al. 4-hr $\mathrm{LC}_{50}$ rat of $>400,000 \mathrm{ppm}$ v/v qualifies. But the cited data conflicts and preference for conservative data beg further study of this item.

recommendation: $4-\mathrm{hr} \mathrm{LC}_{50}$ rat $>400,000 \mathrm{ppm}$ v/v subject to further review

\section{$\underline{\text { Cardiac }}$}

Mullin indicates marked responses in zero of twelve and three of twelve dogs tested at 50,000 and $150,000 \mathrm{ppm} \mathrm{v/v}$, respectively. ${ }^{186}$ Although the results indicate a NOEL of $50,000 \mathrm{ppm} \mathrm{v} / \mathrm{v}$ for cardiac sensitization, she notes that the compound was not well tolerated. Reinhardt et al. (including Mullin) explain the test procedure and findings. ${ }^{57}$ Aviado examines the cardiotoxicity of R-152a in several papers, but draws on the Reinhardt et al. data for cardiac sensitization. ${ }^{206,207}$

recommendation: NOEL dog $=50,000 \mathrm{ppm}$ v/v

\section{Anesthetic Effect}

No $\mathrm{EC}_{50}$ data were found. Lester and Greenberg indicate an effect in the single rat tested at $200,000 \mathrm{ppm}$ for $30 \mathrm{~min}$, but no effect in a single rat tested at $100,000 \mathrm{ppm}$ v/v. ${ }^{65}$ Limperos reports occasional trembling and loss of coordination in two rats (probably in both, but not clearly indicated) exposed at $100,000 \mathrm{ppm} \mathrm{v} / \mathrm{v}$ for $2 \mathrm{hr}$, but no toxic effects at $74,000 \mathrm{ppm} \mathrm{v} / \mathrm{v}$ for 105 min. Neither of the LOELs meet the data selection criteria (see page 19) since both were for $100 \%$ of the tested animals.

recommendation: $30-\mathrm{min}$ NOEL rat $=100,000 \mathrm{ppm} \mathrm{v} / \mathrm{v}$

\section{Other Escape-Impairing Effects and Permanent Injury}

Van Poznak and Artusio report that several volunteers who inhaled $500,000 \mathrm{ppm}$ v/v "briefly" noted "good analgesia and impending loss of consciousness".

recommendation: anesthetic effect in humans at $500,00 \mathrm{ppm} v / \mathrm{v}$ 


\section{R-218: OCTAFLUOROPROPANE}

\section{Lethality}

Hiddeman reports no mortality either in ten rats exposed to $800,000 \mathrm{ppm}$ v/v with oxygen for 1 $\mathrm{hr}$ or in twenty rats exposed to $113,000 \mathrm{ppm} \mathrm{v} / \mathrm{v}$ continuously for 10 days. ${ }^{208}$ Two of twenty guinea pigs also exposed in the extended study became ill as did one control animal, but the cause was not attributed to exposures. ${ }^{208}$ A Huntingdon Research Centre (HRC) study found no deaths in ten rats exposed to $110,000 \mathrm{ppm}$ v/v for $4 \mathrm{hr},{ }^{209}$ as summarized by $3 \mathrm{M}^{210}$ Applying the ten Berge extrapolation to the acute exposure data from Hiddeman suggests that the 4-hr $\mathrm{LC}_{50}$ rat should exceed $400,000 \mathrm{ppm} \mathrm{v} / \mathrm{v}$.

\section{recommendation: $4-\mathrm{hr} \mathrm{LC}_{50}$ rat $>400,000 \mathrm{ppm} \mathrm{v/v}$}

\section{Cardiac Sensitization}

As summarized by $3 M_{1}{ }^{210}$ an HRC study found no responses in six dogs tested at 300,000 .ppm $\mathrm{v} / \mathrm{v}$, but a marked response in one dog and a questionable response in a second among eight dogs tested at $400,000 \mathrm{ppm} \mathrm{v/v^{211 }}$ Hardy et al. support the summary with an indication that the $\mathrm{EC}_{50}$ for dogs exceeds $400,000 \mathrm{ppm} \mathrm{v/v} .^{71}$

$$
\text { recommendation: NOEL } \operatorname{dog}=300,000 \mathrm{ppm} \text { v/v }
$$

\section{Anesthetic Effect}

No $\mathrm{EC}_{50}$ data were found. Hiddeman reports that all ten rats exposed to $800,000 \mathrm{ppm}$ v/v for 1 $\mathrm{hr}$ showed anesthetic effects, but that 20 rats and 20 guinea pigs exposed to $113,000 \mathrm{ppm} \mathrm{v} / \mathrm{V}$ continuously for 10 days showed normal behavior (except as noted above for two of the guinea pigs). ${ }^{208} 800,000 \mathrm{ppm}$ v/v does - not meet the data selection criteria (see page 19) for a LOEL since more than half of the animals showed an effect.

recommendation: subchronic NOEL rat $=113,000 \mathrm{ppm} \mathrm{v/v}$

\section{Other Escape-Impairing Effects and Permanent Injury}

None identified.

recommendation: none 


\section{R-227ea: $1,1,1,2,3,3,3$-HEPTAFLUOROPROPANE}

Both the International Pharmaceutical Aerosol Consortium for Toxicology Testing (IPACT) and independent pharmaceutical companies have conducted extensive studies of the toxicity and tolerance of R-227ea, to qualify its use as a pharmaceutical aerosol propellant for metered dose inhalers (MDIs). However, they declined to release those studies.

\section{Lethality}

Bury notes that no deaths occurred at $110,000 \mathrm{ppm} v / \mathrm{v}$, but does not give details. ${ }^{212}$ Hoechst's safety data sheet cites the same concentration as a $4-h r L_{50}$ rat. $^{213}$ Bury notes that higher concentrations were not tested because the animals breathed irregularly and became uncoordinated at $110,000 \mathrm{ppm} \mathrm{v/ \textrm {v } ^ { 2 1 2 }}$

WIL Research Laboratories reports no deaths in groups of six rats exposed to 121,267 ppm v/V for $4 \mathrm{hr}$ or $241,188 \mathrm{ppm}$ v/v for $4 \frac{1}{2} \mathrm{hr}$, the latter with a $19 \mathrm{~min}$ break during the exposure period, ${ }^{214}$ as summarized Great Lakes Chemical (GLC) and by the National Industrial Chemicals Notification and Assessment Scheme (NICNAS) of Worksafe Australia. ${ }^{215,216}$ WIL also reports no deaths in ten rats exposed to $788,696 \mathrm{ppm} \mathrm{v} / \mathrm{v}$ in oxygen and concludes that the 4-hr $\mathrm{LC}_{50}$ rat exceeds this concentration, ${ }^{217}$ again as summarized by GLC and NICNAS. ${ }^{215,216}$

Skaggs and Moore indicate that the $4-\mathrm{hr} \mathrm{LC}_{50}$ rat exceeds $800,000 \mathrm{ppm} \mathrm{v} / \mathrm{v}$, but it is based on the WIL data with rounding. ${ }^{218}$ The UK Halon Alternatives Group (HAG) also shows the 4-hr $L C_{50}$ rat as exceeding $800,000 \mathrm{ppm} v / \mathrm{v}$, but gives no source for it. ${ }^{219}$

The WIL 4-hr LC $_{50}$ rat appears to meet the data selection criteria (see page 19) except that the studies were not published or provided for examination. The recommendation relies on the NICNAS assessment of the WIL finding.

\section{recommendation: $4-\mathrm{hr} L_{50}$ rat $=788,696 \mathrm{ppm} \mathrm{v/v}$}

\section{Cardiac Sensitization}

Bury indicates no effect at $50,000 \mathrm{ppm} v / \mathrm{v}$ in dogs, but does not give details. ${ }^{212}$ WIL indicates no responses in dogs at $70,000 \mathrm{ppm} \mathrm{v/v,}{ }^{220}$ as summarized by NICNAS. ${ }^{216}$ WIL further indicates marked responses in one of ten, five of ten, and six of ten dogs at 90,000,105,000, and $140,000 \mathrm{ppm} v / \mathrm{v}$, respectively, as summarized by NICNAS. ${ }^{216,220}$ However, the GLC summary for the same study reports the NOEL and LOEL to be 90,000 and $105,000 \mathrm{ppm} \mathrm{v} / \mathrm{v}$, respectively, ${ }^{215}$ which implies a different interpretation of the results at $90,000 \mathrm{ppm} \mathrm{v} / \mathrm{v}$.

The more conservative WIL NOEL appears to meet the data selection criteria (see page 19) except that the study was not published or provided for examination. The recommendation relies again on the NICNAS assessment of it, but is qualified based on the uncertainty raised by the GLC summary.

recommendation: NOEL dog $=70,000 \mathrm{ppm}$ v/v subject to further review 


\section{Anesthetic Effect}

No $\mathrm{EC}_{50}$ data were found. Bury indicates that animals became uncoordinated at $110,000 \mathrm{ppm}$ $\mathrm{v} / \mathrm{v}^{212}$ Hoechst's safety data sheet indicates the animals as rats and exposure duration as $4 \mathrm{hr}$, but does not indicate the number of rats tested or showing effects. ${ }^{213}$ Bury also notes lethargy, sedation, and listlessness in mice and rats exposed to $150,000 \mathrm{ppm} v / \mathrm{v}$ for $1 \mathrm{hr}$, from a test to determine the maximum tolerable concentration. ${ }^{212}$ Bury reports a third study in which four dogs showed no clinical signs at $50,000 \mathrm{ppm} v / \mathrm{v}$, but exhibited lethargy, sedation, and listlessness at higher concentrations. ${ }^{212}$

WIL indicates that all six rats exposed to $121,267 \mathrm{ppm}$ v/v exhibited temporary anesthesia, hypoactivity, ${ }^{*}$ bradypnea, ${ }^{\dagger}$ and/or ataxia ${ }^{\ddagger}$ during treatment, ${ }^{214}$ as summarized by NICNAS. ${ }^{216}$ The summaries of other WIL studies do not provide sufficient information to determine a NOEL for anesthetic effects, though the full reports might provide such information. ${ }^{216,217}$

The GLC summary of toxicity studies describes a 14-day inhalation toxicity screening study not cited in the NICNAS assesment. ${ }^{215,216}$ It indicates that "there were no test-material related clinical findings" and that the exposures "did not cause any noticeable adverse effects" for tests at concentrations as high as $100,000 \mathrm{ppm} \mathrm{v/v}$ for $6 \mathrm{hr} / \mathrm{d}^{215}$ It similarly indicates "no treatmentrelated chemical signs" and "no test article related clinical signs" at concentrations as high as $105,000 \mathrm{ppm} \mathrm{v} / \mathrm{v}$ in 24 rats for $6 \mathrm{hr} / \mathrm{d}$ in 14- and 90-day inhalation studies, respectively. ${ }^{215}$ This summary does not meet the criteria for data sources, and attempts to obtain the study were unsuccessful.

The UK Halon Alternatives Group (HAG) indicates a NOEL and LOEL of 150,000 and 300,000 $\mathrm{ppm} \mathrm{v} / \mathrm{v}$ in dogs, respectively. ${ }^{219}$ No source is given, but the data appear to be from Bury. ${ }^{212}$ In any case, the Bury finding at $110,000 \mathrm{ppm} v / \mathrm{v}^{212,213}$ and WIL finding at $121,267 \mathrm{ppm} v / \mathrm{v}$ disqualify them. ${ }^{214,216}$

\section{recommendation: $6 \mathrm{hr}$ NOEL rat $=105,000 \mathrm{ppm}$ v/v subject to further review}

\section{Other Escape-Impairing Effects and Permanent Iniury}

Vinegar et al. describe a study with human exposures to R-134a and R-227ea to validate a physiologically-based pharmacokinetic (PBPK) model). ${ }^{166}$ Tests with R-227ea were terminated when a subject suffered rapid rise in pulse $3 \mathrm{~min}$ into an exposure to $6,400 \mathrm{ppm} \mathrm{v} / \mathrm{v}$, following anomalous results in another volunteer with $\mathrm{R}-134 \mathrm{a}$ (see page 53). ${ }^{166}$ Subsequent investigation indicates that the responses probably were not due to the chemical exposures, and that no adverse effects were seen in other human exposures at lower or higher concentrations. ${ }^{167,168}$

* less than normal activity

$\dagger$ slow breathing

$\ddagger$ inability to coordinate voluntary muscular movements 


\section{R-236fa: $1,1,1,3,3,3$-HEXAFLUOROPROPANE}

\section{Lethality}

WIL reports the 4-hr $\mathrm{LC}_{50}$ rat as $>457,000 \mathrm{ppm}$ based on a single, whole-body exposure of ten rats. $^{221}$

An earlier study by Robbins indicates an inconsistent $10-\min L_{50}$ mouse of $440,000 \mathrm{ppm}$ v/v. ${ }^{126}$ Applying the ten Berge extrapolation (see page 11) suggests an equivalent $4-h r L_{50}$ of approximately $90,000 \mathrm{ppm} v / \mathrm{v}$.

Findings by Keller refute the Robbins $L C_{50}$ and lend support to the WIL finding. Keller indicates a 4-hr ALC rat exceeding 189,275 ppm v/v from tests in groups of four rats. ${ }^{222}$ He also found no deaths in 4 rats exposed to $133,939 \mathrm{ppm} v / \mathrm{v}$ for $4 \mathrm{hr}^{222}$

Hilarski found no mortality in ten rats exposed to $148,600 \mathrm{ppm}$ for $4 \mathrm{hr}^{223}$

The documentation for the R-236fa WEEL notes that R-236fa is low in acute toxicity with a 4-hr $L_{50}$ rat of $>450,000 \mathrm{ppm} v / \mathrm{v}^{224}$

recommendation: $4-\mathrm{hr} \mathrm{LC}_{50}$ rat $>457,000 \mathrm{ppm}$ v/v

\section{Cardiac Sensitization}

Hardy et al. report a NOEL at $100,000 \mathrm{ppm} v / \mathrm{v}$ for six dogs and a LOEL for positive responses in two of six dogs at 150,000 ppm v/v. ${ }^{225}$ The WEEL documentation and an article by Valentine et al. both cite the Hardy et al. finding. ${ }^{224,226}$

recommendation: NOEL dog $=100,000 \mathrm{ppm} v / \mathrm{v}$

\section{Anesthetic Effect}

Robbins indicates a $10-\mathrm{min} \mathrm{EC}_{50}$ rat of $110,000 \mathrm{ppm} \mathrm{v/v.}{ }^{126}$

A Haskell Lab study by Keller reports symptoms of narcosis in an unspecified number of the four rats tested at $189,275 \mathrm{ppm} \mathrm{v} / \mathrm{v}$, but not in four rats exposed at $133,939 \mathrm{ppm} \mathrm{v} / \mathrm{v}^{222}$ The symptoms are identified as lack of response to sound in a 4-hr acute inhalation toxicity. ${ }^{222}$

A later Haskell study indicates that exposed rats seem to develop a tolerance for R-236fa. ${ }^{227}$ It notes that nearly all (six to eight of ten) rats exposed to $50,300 \mathrm{ppm} v / \mathrm{v}$ had a diminished response to an alerting stimulus on the first day of exposures, but that the number of rats so affected dropped to one to three during the first two weeks of the study. ${ }^{227}$ All of the rats exhibited normal responses to the sudden auditory alerting stimulus by day 18 as did other rats exposed to 4,980 and $20,000 \mathrm{ppm} \mathrm{v/v.^{227 }}$

In a third Haskell study, Valentine also suggests that rats develop a tolerance for R-236fa. He notes that all rats either did not respond or exhibited a diminished alerting response for the first two days of $50,000 \mathrm{ppm} v / \mathrm{v}$ exposures. ${ }^{228}$ He further notes that four to five of ten rats had no 
alerting response at some point during the 6-hr exposures, that the majority of rats showed normal responses on day three, and all did on day four, but some showed transient diminished responses on days five and nine. ${ }^{228}$ Valentine notes that all ten rats exposed to 5,020 and $20,000 \mathrm{ppm} \mathrm{v} / \mathrm{v}$ exhibited normal alerting response except for a transient, diminished response on test days two and three at $20,000 \mathrm{ppm} v / \mathrm{v}^{228}$

WIL reports hyperactivity and then prostration in ten of ten rats exposed to $457,000 \mathrm{ppm} v / \mathrm{v}$ for $4 \mathrm{hr}^{220}$

The metric used by Robbins (loss of righting reflex in a rotadrum test) differs from that used in the cited Haskell studies (diminished alerting response) and WIL study (hyperactivity and then prostration). Based solely on the finding during the initial exposure at each concentration of the cited studies, Robbins offers a qualifying $10-\mathrm{min} \mathrm{EC}_{50}$ of $110,000 \mathrm{ppm} \mathrm{v} / \mathrm{v}$, none of the studies offer a qualifying LOEL, and two of the Haskell studies suggest a NOEL at 20,000 ppm v/v.

recommendation: $10-\mathrm{min} \mathrm{EC}_{50}$ rat $=110,000 \mathrm{ppm} \mathrm{v} / \mathrm{v}$

\section{Other Escape-Impairing Effects and Permanent Injury}

None identified.

recommendation: none 


\section{R-245ca: 1,1,2,2,3-PENTAFLUOROPROPANE}

Only limited toxicity data were found for R-245ca. The extent of testing is inadequate.

\section{Lethality}

Bagnall indicates a $30-\mathrm{min}$ ALC mouse of $240,000 \mathrm{ppm} \mathrm{v} / \mathrm{v}$ in oxygen, found by successively testing individual mice to find the minimal lethal concentration. ${ }^{229}$ Applying the ten Berge extrapolation (see page 11) suggests a 4-hr ALC mouse of approximately $84,900 \mathrm{ppm}$ v/v. Grube references this study. ${ }^{230}$ The data selection criteria (see page 19) call for tests in rats, but the Bagnall finding is indicative of the magnitude of the lethal concentration.

Smith et al. report a 4-hr acute inhalation study in rats at $993 \mathrm{ppm}$ v/v that found no adverse effects, but note that more extensive tests will require larger quantities of the chemical than available then. ${ }^{231,232}$

recommendation: 4-hr ALC mouse $=84,900 \mathrm{ppm}$ v/v subject to further review

\section{Cardiac Sensitization}

No data found.

recommendation: not determined

\section{Anesthetic Effect}

No $\mathrm{EC}_{50}$ data were found.

A Haskell Lab study found an anesthetic effect in mice exposed to $100,000 \mathrm{ppm} v / \mathrm{v}$ for 5 or 10 min in a rotadrum (rotating apparatus) test, but no effect at $50,000 \mathrm{ppm} \mathrm{v/v}$, ${ }^{233}$ as reported by Grube. $^{230}$

Bagnall indicates a 30-min LOEL mouse of $100,000 \mathrm{ppm}$ v/v in oxygen, found by successively testing individual mice to find the minimum anesthetic concentration. ${ }^{229}$ The data selection criteria call for tests in rats (rats or mice for the 10-min $E C_{50}$ ), but the Bagnall finding is indicative of the magnitude of the lethal concentration.

Smith et al. found no adverse effects in rats at $993 \mathrm{ppm} v / \mathrm{v}$ in a 4-hr acute inhalation study. ${ }^{231,232}$ recommendation: $30-\mathrm{min}$ LOEL mouse $=100,000 \mathrm{ppm} \mathrm{v} / \mathrm{v}$ subject to further review

\section{Other Escape-Impairing Effects and Permanent Injury}

The cited Haskell Lab study found severe, but transient effects on respiration in mice exposed to 120,000 and $150,000 \mathrm{ppm} v / \mathrm{v}$ for $30 \mathrm{~min}^{233}$ as reported by Grube. ${ }^{230}$ The finding suggests further testing to determine if humans would incur a similar effect.

recommendation: respiratory effect in mice at $120,000 \mathrm{ppm}$ v/v subject to further review 


\section{R-245fa: $1,1,1,3,3$-PENTAFLUOROPROPANE}

\section{Lethality}

Jackson reports no deaths in groups of ten rats exposed to $116,000,143,000$, and 203,000 ppm $\mathrm{v} / \mathrm{v}^{234,235}$ He concludes that 4-hr LC $_{50}$ rat exceeds $203,000 \mathrm{ppm}$ v/v, but notes that occasional clonic $^{*}$ convulsions were seen in three of ten rats during exposures at $143,000 \mathrm{ppm} \mathrm{v/v.} .^{234}$ Rusch et al. also cite and summarize these findings. ${ }^{236}$

recommendation: $4-\mathrm{hr} \mathrm{LC}_{50}$ rat $>203,000 \mathrm{ppm}$ v/v

\section{Cardiac Sensitization}

Kenny indicates that six dogs showed no signs of cardiac sensitization in tests at 10,000 and $20,000 \mathrm{ppm} \mathrm{v/v.}{ }^{237}$

Rusch et al. summarize further tests in dogs with a fatal ventricular fibrillation ${ }^{\dagger}$ in the only dog tested at $73,000 \mathrm{ppm} \mathrm{v} / \mathrm{v}$, positive responses in one of four dogs at $44,000 \mathrm{ppm} \mathrm{v} / \mathrm{v}$, and no positive responses either in three dogs at $54,100 \mathrm{ppm} v / \mathrm{v}$ (the dog that showed a response at $44,000 \mathrm{ppm} v / \mathrm{v}$ was excluded) or in four dogs at $34,100 \mathrm{ppm} v / \mathrm{v}^{236}$

The $54,100 \mathrm{ppm}$ v/v level does not qualify as a NOEL since there was a lower qualifying LOEL. Likewise, the dog excluded based on its positive response at $44,000 \mathrm{ppm} \mathrm{v} / \mathrm{v}$ presumably would have shown a similar or more severe response if tested at $54,100 \mathrm{ppm} v / \mathrm{v}$. The recommended NOEL therefore is $34,100 \mathrm{ppm}$ v/v.

recommendation: NOEL dog $=34,100 \mathrm{ppm}$ v/v

\section{Anesthetic Effect}

No $\mathrm{EC}_{50}$ data were found.

Jackson indicates that all ten rats exposed to 143,000 and $203,000 \mathrm{ppm}$ v/v adopted an abnormal posture and exhibited reduced response to external stimuli during exposures. ${ }^{234}$ He concludes that there was evidence of an anesthetic effect. ${ }^{234}$ Jackson also indicates that all ten rats exposed to $116,000 \mathrm{ppm}$ v/v exhibited irregular respiration, restless behavior and intermittent muscular contractions. ${ }^{235}$ Rusch et al. characterize these symptoms as "central nervous system depression" and note that they "were more pronounced at the highest exposure level group but were seen to some degree at all levels." 236

Rusch et al. also summarize an inhalation toxicity study in mice. They note that ten mice given a single, snout-only exposure to $101,300 \mathrm{ppm}$ v/v for $4 \mathrm{hr}$ "all showed signs of reduced activity" that "abated almost immediately upon cessation of the exposure." 236 The Honeywell (formerly

* spasms of alternating contractions and partial relaxations

$\dagger$ very rapid and irregular contractions of the ventricles of the heart resulting in loss of synchronization between the heartbeat and pulse beat 
AlliedSignal) MSDS for R-245fa describes the observation as "evidence of transient underactivity during exposure." ${ }^{238}$ The data selection criteria (see page 19) allow mice data only for the $10-$ min $\mathrm{EC}_{50}$, but specify data from rats for the LOEL and NOEL.

Rusch et al. describe extended inhalation studies in rats exposed for $6 \mathrm{hr} / \mathrm{d}$ to concentrations as high as $50,400 \mathrm{ppm} \mathrm{v/v}$ for $2 \mathrm{wk}, 49,600 \mathrm{ppm} \mathrm{v} / \mathrm{v}$ for $4 \mathrm{wk}$, and $50,600 \mathrm{ppm} \mathrm{v/v}$ for $13 \mathrm{wk}$. $^{236}$ They indicate "absence of noticeable adverse effects of any kind" and further note that "no clinical signs were observed that appeared related to the treatment." 236

The observed "signs of reduced activity" or "transient underactivity" at 101,300 ppm v/v and of "central nervous system depression" at 116,000 ppm v/v described by Rusch et al. constitute an observed effect - even if mild and/or quickly reversible - rather than no effect, as used to define LOEL and NOEL, respectively. The $116,000 \mathrm{ppm} v / \mathrm{v}$ finding does not satisfy the data selection criteria (see page 19) as a LOEL since Jackson notes that all ten exposed rats were affected. The NOEL from the 13-wk study fits the data selection criteria.

recommendation: $6-\mathrm{hr}$ NOEL rat $=50,600 \mathrm{ppm} \mathrm{v/v}$

\section{Other Escape-Impairing Effects and Permanent Iniury}

Rusch et al. note that myocarditis* was seen in pathological studies of rats exposed to 10,000 and $50,000 \mathrm{ppm} \mathrm{v/v}$ and in one animal exposed to $2,000 \mathrm{ppm} \mathrm{v/v.}{ }^{236}$ They indicate that this effect did not compromise the overall health of the rats, but "could have progressed if the exposure had continued past three months. ${ }^{\text {236 }}$ The Honeywell (AlliedSignal) MSDS notes the severity as "trace to moderate." 238 The description and absence of related observations in acute studies suggest that this effect is not a concern for acute exposures.

recommendation: none

* inflammation of the heart muscle 


\section{R-290: PROPANE}

As is typical of hydrocarbons, toxicity data are sparse for exposures above the lower flammability limit (LFL). The risks inherent to testing flammable mixtures and historical presumption that application exposures will be kept below the LFL both mitigate against testing higher concentrations. Kirwin notes that the toxic properties of paraffinic hydrocarbons such as propane are not manifest until levels exceed fire hazard concentrations. ${ }^{239}$

\section{Lethality}

Phillips Petroleum indicates that no deaths occurred in ten rats exposed to $12,190 \mathrm{ppm}$ v/v for 4 $\mathrm{hr}^{240}$

Clark and Tinston report that the $15-\mathrm{min} \mathrm{LC}_{50}$ rat exceeds $800,000 \mathrm{ppm} \mathrm{v/v.}{ }^{53}$ Applying the ten Berge extrapolation (see page 11) suggests that the $4-\mathrm{hr} \mathrm{LC}_{50}$ may exceed $200,000 \mathrm{ppm} \mathrm{v} / \mathrm{v}$.

recommendation: $4-\mathrm{hr} \mathrm{LC}_{50}$ rat $>200,000 \mathrm{ppm}$ v/v

\section{Cardiac Sensitization}

Mullin indicates marked responses in zero of six, two of twelve, and seven of twelve dogs tested at $50,000,100,000$, and $200,000 \mathrm{ppm} \mathrm{v} / \mathrm{v}$, respectively. ${ }^{186}$ The results indicate a NOEL of $50,000 \mathrm{ppm} v / \mathrm{v}$ for cardiac sensitization. Reinhardt et al. (including Mullin) explain the test procedure and findings. ${ }^{57}$

Krantz et al. report that three of three dogs were sensitized at concentrations of 100,000 250,000 ppm. ${ }^{241}$ Based on the wide range of uncertainty in the actual concentration tested, this finding could be consistent with, and does not necessarily challenge, that of Reinhardt et al. Aviado and Belej indicate sensitization in mice at 100,000 ppm v/v. ${ }^{206,242}$ Clark and Tinston report the $\mathrm{EC}_{50}$ dog as 180,000 and $200,000 \mathrm{ppm} v / \mathrm{v}^{53,60}$

The data from these sources are relatively consistent.

recommendation: $N O E L$ dog $=50,000 \mathrm{ppm} \mathrm{v/v}$

\section{Anesthetic Effect}

Clark and Tinston note that R-290 causes central nervous system (CNS) depression and report the $10-\mathrm{min} \mathrm{EC}_{50}$ rat as $280,000 \mathrm{ppm} \mathrm{v/v.}{ }^{53}$

Kirwin, echoed by Sanders, notes that R-290 produces dizziness in a few minutes at 100,000 $\mathrm{ppm} \mathrm{v} / \mathrm{v}$, but not in ten minutes at $10,000 \mathrm{ppm}$ v/v. ${ }^{239,243}$ He does not specify the species possibly humans - for these findings. ${ }^{239,243}$ He cites data from Brown and Henderson that show that R-290 produces analgesia and deep anesthesia in cats at 600,000 and 930,000 ppm $\mathrm{v} / \mathrm{V}$, respectively, in oxygen. ${ }^{244}$

recommendation: $10-\mathrm{min} \mathrm{EC}_{50}$ rat $=280,000 \mathrm{ppm} \mathrm{v/v}$ 


\section{Other Escape-Impairing Effects and Permanent Injury}

Stewart et al. summarize acute and subchronic toxicity tests in healthy human volunteers by whole-body exposures. ${ }^{67.245}$ They indicate that acute exposures to 250,500 , or $1000 \mathrm{ppm} \mathrm{v} / \mathrm{v}$ for 1 min to 8 hr "did not produce any untoward physiological effects" as determined by observation, routine hematology, clinical chemistry, EKG, and pulmonary function as well as by cognitive skill and other neurological measurements. They further note that repetitive exposures to $1,000 \mathrm{ppm} \mathrm{v} / \mathrm{v}$ for $1-10 \mathrm{hr} / \mathrm{d}, 5 \mathrm{~d} / \mathrm{wk}$, for $2-4 \mathrm{wk}$ also showed no adverse effects.

recommendation: none 


\section{R-500: R-22/152A (73.8/26.2)}

No toxicity findings were found for tests of R-500. Manufacturers base their safety recommendations on the components. ${ }^{246-248}$

Lethality

None identified.

recommendation: base on data for $\mathrm{R}-22$ and $\mathrm{R}-152 \mathrm{a}$

\section{Cardiac Sensitization}

None identified.

recommendation: base on data for $R-22$ and $R-152 a$

\section{Anesthetic Effect}

None identified.

recommendation: base on data for $R-22$ and $R-152 a$

\section{Other Escape-Impairing Effects and Permanent Injury}

None identified.

recommendation: base on data for $\mathrm{R}-22$ and $\mathrm{R}-152 \mathrm{a}$ 


\section{R-502: R-22/115 (48.8/51.2)}

\section{Lethality}

Clayton concludes that the 2-hr ALC rat exceeds $200,000 \mathrm{ppm}$ v/v based on no deaths in a 2-hr exposure of four rats. ${ }^{249.250}$ Applying the ten Berge extrapolation (see page 11) suggests that the 4-hr ALC may exceed $141,000 \mathrm{ppm} v / \mathrm{v}$. This finding is consistent with and understates expectations based on the 4-hr LC ${ }_{50}$ of R-22 and the 4-hr ALC of R-115. The lower of them is a 4$\mathrm{hr} \mathrm{LC}_{50}$ of $220,000 \mathrm{ppm} \mathrm{v} / \mathrm{v}$ for R-22 (see page 39), which suggests that the 4-hr LC $\mathrm{L}_{50}$ blend should be higher in the absence of synergistic effects. Such effects are not evident in Clayton's reports. ${ }^{249,250}$

Honeywell indicates that the 4-hr $\mathrm{LC}_{50}$ rat exceeds $300,000 \mathrm{ppm} \mathrm{v} / \mathrm{v}$, but does not indicate the basis for it. ${ }^{251}$ Once again, it is consistent with expectations based on data for R-22 and R-115.

While the Honeywell MSDS does not qualify as a source based on a rigid reading of the eligible data, the 4-hr $\mathrm{LC}_{50}$ cited is conservative relative to a mole-weighted average of the component data.

\section{recommendation: $4-\mathrm{hr} L C_{50}$ rat $>300,000 \mathrm{ppm}$ v/v subject to further review}

\section{Cardiac Sensitization}

Mullin indicates marked responses in zero of six, five of twelve, and twelve of twelve dogs tested at 50,000, 100,000, and $200,000 \mathrm{ppm} v / \mathrm{v}$, respectively. ${ }^{186}$ Reinhardt et al. (including Mullin) explain the test procedure and findings. ${ }^{57}$

\section{recommendation: $N O E L$ dog $=50,000 \mathrm{ppm} \mathrm{v/v}$}

\section{Anesthetic Effect}

Clayton reports that the rats showed discomfort, tremors, and rapid respiration in an acute inhalation toxicity study at $200,000 \mathrm{ppm}$ v/v, but does not note signs of anesthesia during the 2-hr exposures. $^{249.250}$

Underwriters Laboratories (UL) indicates similar effects in all three guinea pigs exposed to $100,000 \mathrm{ppm} \mathrm{v/v}{ }^{252}$ as summarized by Lee. ${ }^{253}$ UL also reports signs of anesthesia including reduced activity, difficulty standing, loss of equilibrium, and difficulty walking in all three guinea pigs at $200,000 \mathrm{ppm} \mathrm{v} / \mathrm{v}$. While the data selection criteria (see page 19) call for data from rats (mice or rats for the 10-min $E C_{50}$ ), the UL finding is consistent with the Weigand report of a LOEL of $50,000 \mathrm{ppm} \mathrm{v} / \mathrm{v}$ in rats for $2-\mathrm{hr}$ exposures to R-22 (see page 39 ). ${ }^{62}$ The Weigand finding is from a subchronic inhalation toxicity study, but he does not indicate the number of animals affected of the four tested. ${ }^{62}$

The UL findings suggest a more conservative limit than those of Clayton, but the $200,000 \mathrm{ppm}$ $v / v$ effects found by UL do not qualify as a LOEL since all three (100\%) animals were affected and do not qualify here based on the species tested. 
recommendation: $2-\mathrm{hr}$ NOEL rat $=200,000 \mathrm{ppm} v / \mathrm{v}$, but base on data for $\mathrm{R}-22$ and $\mathrm{R}-115$

\section{Other Escape-Impairing Effects and Permanent Injury}

Valic et al. conducted experimental exposures to R-502; neither a summary nor the document was obtained. ${ }^{254}$

recommendation: none subject to further review 


\section{R-600a: ISOBUTANE}

As is typical of hydrocarbons, toxicity data are sparse for exposures above the lower flammability limit (LFL). The risks inherent to testing flammable mixtures and historical presumption that application exposures will be kept below the LFL both mitigate against testing higher concentrations. Kirwin notes that the toxic properties of paraffinic hydrocarbons such as isobutane are not manifest until levels exceed fire hazard concentrations. ${ }^{239}$

\section{LETHALITY}

Phillips Petroleum indicates no deaths in 10 rats exposed to $13,023 \mathrm{ppm}$ v/v for $4 \mathrm{hr}$ and a resulting conclusion that the $4-\mathrm{hr} \mathrm{LC}_{50}$ rat exceeds $13,023 \mathrm{ppm} \mathrm{v/v.}{ }^{255}$

Clark and Tinston report a 15-min $\mathrm{LC}_{50}$ rat of $570,000 \mathrm{ppm}$ v/v. ${ }^{53}$ Applying the ten Berge extrapolation (see page 11) suggests a $4-\mathrm{hr} \mathrm{LC}_{50}$ of approximately $142,500 \mathrm{ppm} \mathrm{v} / \mathrm{v}$.

Stoughton and Lamson provide supporting data from tests in mice. ${ }^{256}$ They found zero, six, and ten deaths in groups of ten mice for 2-hr exposures to $350,000,410,000$, and $520,000 \mathrm{ppm} \mathrm{v/v}$, respectively. ${ }^{256}$ Applying the ten Berge extrapolation suggests that the 4-hr ALC should exceed $247,000 \mathrm{ppm} \mathrm{v} / \mathrm{v}$, but that $290,000 \mathrm{ppm} v / \mathrm{v}$ for $4 \mathrm{hr}$ should be fatal to more than half the mice.

Aviado et al. indicate a $2-\mathrm{hr} \mathrm{LC}_{50}$ mouse of $520,000 \mathrm{ppm} \mathrm{v/v}{ }^{257}$ as identified by Bingham. ${ }^{258}$ Applying the ten Berge extrapolation suggests a 4-hr LC Lo $_{50}$ mouse of $368,000 \mathrm{ppm} \mathrm{v} / \mathrm{v}$. ACGIH and Sandmeyer indicate the Aviado finding to be a 1-hr $L^{-C_{50}}$ mouse. ${ }^{24,259}$ On that basis, the ten Berge extrapolation suggests a 4-hr $L C_{50}$ mouse of $260,000 \mathrm{ppm} v / \mathrm{v}$, which is consistent with Stoughton and Lamson finding. These data suggest lower lethality than that found by Clark and Tinston, but they do not meet the data selection criteria (see page 19) based on the species tested.

RTECS cites the Clark and Tinston finding as does the only MSDS found for R-600a that cites an $L_{5}$, from Rhône-Poulenc Chemicals. ${ }^{23,260}$ Based on the data noted above, the 4-hr $L C_{50}$ of approximately $142,500 \mathrm{ppm}$ v/v derived from the Clark and Tinston 15-min LC $_{50}$ rat meets the data selection criteria (see page 19).

recommendation: $4-\mathrm{hr} \mathrm{LC}_{50}$ rat $=142,500 \mathrm{ppm} \mathrm{v} / \mathrm{v}$

\section{Cardiac Sensitization}

Reinhardt, as confirmed in Reinhardt et al., ${ }^{57}$ indicate marked responses in zero of twelve dogs

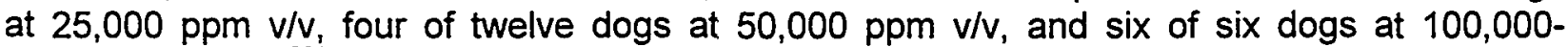
$200,000 \mathrm{ppm} \mathrm{v/v.}{ }^{261}$ Krantz et al. report that two of two dogs were sensitized at concentrations of $100,000-250,000 \mathrm{ppm} v / \mathrm{v}^{241}$ Clark and Tinston report the $\mathrm{EC}_{50} \mathrm{dog}$ as $70,000 \mathrm{ppm} \mathrm{v/v} .^{53}$

The data from Krantz et al. and from Clark and Tinston are consistent with the Reinhardt findings, leading to confidence in the NOEL and LOEL of 25,000 and $50,000 \mathrm{ppm} v / \mathrm{v}$, respectively.

recommendation: NOEL dog $=25,000 \mathrm{ppm}$ v/v 


\section{Anesthetic Effect}

Clark and Tinston note that R-600a causes central nervous system (CNS) stimulation and report the $10-\mathrm{min} \mathrm{EC}_{50}$ rat as $200,000 \mathrm{ppm} \mathrm{v/v.}{ }^{53}$

Stoughton and Lamson report that light anesthesia (taken as loss of ability to maintain an upright position in a rotating bottle) was evident in groups of six mice in an average of 60 min at $150,000 \mathrm{ppm} v / \mathrm{v}, 17 \mathrm{~min}$ at $200,000 \mathrm{ppm} \mathrm{v} / \mathrm{v}$, and $26 \mathrm{~min}$ at $230,000 \mathrm{ppm} \mathrm{v/v},{ }^{256}$ but these data do not suggest dose-dependence. Stoughton and Lamson also indicate that groups of ten mice showed loss of posture in a shaken and then stationary bottle in $25 \mathrm{~min}$ at $350,000 \mathrm{ppm} \mathrm{v} / \mathrm{v}, 3$ min at $410,000 \mathrm{ppm} \mathrm{v/v}$, and $2 \mathrm{~min}$ at $520,000 \mathrm{ppm} \mathrm{v/v.}{ }^{256}$ They report that it was difficult to induce good anesthesia in dogs since the concentration needed, $450,000 \mathrm{ppm} \mathrm{v/v}$, is close to a lethal level of $550,000 \mathrm{ppm} \mathrm{v/v.} .^{256}$ Kirwin cites the $450,000 \mathrm{ppm} \mathrm{v/v}$ finding for dogs from Stoughton and Lamson. ${ }^{239}$

recommendation: $10-\mathrm{min} \mathrm{EC}_{50}$ rat $=200,000 \mathrm{ppm} \mathrm{v} / \mathrm{v}$

\section{Other Escape-Impairing Effects and Permanent Injury}

Stewart et al. summarize acute and subchronic toxicity tests in healthy human volunteers by whole-body exposures. ${ }^{67,245,262}$ They indicate that acute exposures to 250,500 , or $1000 \mathrm{ppm} \mathrm{v} / \mathrm{v}$ for $1 \mathrm{~min}$ to $8 \mathrm{hr}$ "did not produce any untoward physiological effects" as determined by observation, routine hematology, clinical chemistry, EKG, and pulmonary function as well as by cognitive skill and other neurological measurements. They further note that repetitive exposures to $500 \mathrm{ppm} v / \mathrm{v}$ for 1,2 , or $8 \mathrm{hr} / \mathrm{d}, 5 \mathrm{~d} / \mathrm{wk}$, for 2 wk also showed no adverse effects.

recommendation: none 


\section{R-717: AMMONIA}

\section{Lethality}

Carpenter et al. indicates that a 4-hr exposure to $2,000 \mathrm{ppm}$ v/v was fatal to two, three, or four of six rats. ${ }^{184}$ The ambiguous fraction may exceed $50 \%$. Deichmann and Gerarde cite the $2,000 \mathrm{ppm} v / \mathrm{v}$ result, but describe it as a $4-\mathrm{hr} \mathrm{LC}_{50}$ rat. $^{263}$

The NIOSH/OSHA "Occupational Safety and Health Guideline for Ammonia" cites the 4-hr LC L $_{50}$ rat as 2,000 ppm. $^{264}$ and references it to RTECS. ${ }^{23}$ RTECS indicates $L C_{50}$ data for a number of species including rats, for which it indicates a $4-\mathrm{hr} L \mathrm{LC}_{50}$ of $2,000 \mathrm{ppm}$ referenced to Deichmann and Gerarde. ${ }^{263}$

As summarized by the Major Hazards Assessment Panel of the UK Institution of Chemical Engineers (ICE), ${ }^{265}$ Kimmerle reviews data from prior studies on the inhalation toxicity of R-717 (ammonia) in animals. ${ }^{266} \mathrm{He}$ indicates that a 4-hr exposure of rats to 2,000 ppm v/v was not fatal while another at $4,000 \mathrm{ppm} v / \mathrm{v}$ was, but does not indicate the quantities of animals involved. ${ }^{265,266}$ Kimmerle also notes reports of $2-h r L_{50}$ s of 3,420,4,760, and 10,930 ppm v/v in mice, mice, and rats respectively. ${ }^{265,266}$ Applying the ten Berge extrapolation (see page 11) suggests 4-hr LC $\mathrm{LO}_{50}$ s of approximately $2,420,3,370$, and $7,730 \mathrm{ppm} \mathrm{v} / \mathrm{v}$.

The 1988 ICE monograph examines twelve studies in mice and rats described as "all known ${ }^{L C} C_{50}$ results. ${ }^{265}$ Of them, the Carpenter et al. and the Kimmerle studies are the only ones involving 4-hr exposures. Applying the ten Berge extrapolation, the $\mathrm{LC}_{50} \mathrm{~S}$ adjusted to 4-hr exposures range from 2,000 to $8,240 \mathrm{ppm} v / \mathrm{v}$ with a mean of $3,960 \mathrm{ppm} \mathrm{v} / \mathrm{v}$. The range for the rat studies alone is the same, but the mean increases to $5,400 \mathrm{ppm} v / \mathrm{v}$.

Ludwig et al. cite additional lethality studies for diverse mammals in the NIOSH documentation of the IDLH concentration for ammonia. ${ }^{11}$ The added studies include rat data from Alarie and from Back et al. ${ }^{267,268}$ Applying the ten Berge extrapolation to them to approximate 4-hr $\mathrm{LC}_{50} \mathrm{~S}$, they cover a nearly identical range of $2,000-8,230 \mathrm{ppm} v / \mathrm{v}$, excluding two citations from 1928 and 1933 for a single human fatality and for an unspecified mammal (probably the same human incident). The mean for the rat data increases to $6,040 \mathrm{ppm} \mathrm{v/v}$.

Clement Associates documents a detailed assessment of the toxicity of ammonia, prepared for The Fertilizer Institute, that examines 19 studies of the acute inhalation lethality of ammonia in animals. ${ }^{269}$ They note the lowest $1-\mathrm{hr} \mathrm{LC}_{50}$ values to be $3,796 \mathrm{ppm} \mathrm{v} / \mathrm{v}$ in mice and $6,586 \mathrm{ppm}$ $\mathrm{v} / \mathrm{v}^{*}$ in rats. ${ }^{269}$ These data correspond to 1,900 and $3,300 \mathrm{ppm} \mathrm{v} / \mathrm{v}$ with the ten Berge adjustment to $4-\mathrm{hr} \mathrm{LC}_{50} \mathrm{~S}$.

The Agency for Toxic Substances and Disease Registry (ATSDR), in a toxicity profile of ammonia prepared by Syracuse Research Corporation under contract to Clement Associates, lists two rat studies among nine studies of lethality by acute exposure to ammonia. ${ }^{270}$ They include a 15 -

* The cited report is very helpful and well documented, but it is difficult to follow in places due to use of inconsistent factors to convert $\mathrm{mg} / \mathrm{m}^{3}$ to $\mathrm{ppm} \mathrm{v} / \mathrm{v}$ (e.g., 0.7 on page 89 versus 0.78 on page 94 versus 0.71 in other references) and occasional typographical errors (e.g., page 94 shows $5,137 \mathrm{mg} / \mathrm{m}^{3}$ as both 6,568 and $6,586 \mathrm{ppm} \mathrm{v} / \mathrm{v}$ ). 
min $L C_{50}$ rat of $17,401 \mathrm{ppm} v / \mathrm{v}$ from Propkop'eva et al. ${ }^{271}$ and a $16-\mathrm{hr} L C_{50}$ rat of $1,000 \mathrm{ppm} \mathrm{v} / \mathrm{v}$ from Weedon et al., ${ }^{272}$ which correspond to 4,350 and $2,000 \mathrm{ppm} v / \mathrm{v}$, respectively, with the ten Berge adjustment to 4-hr.

The U.S. Environmental Protection Agency (EPA) lists eight 10-min and 60-min $\mathrm{LC}_{50} \mathrm{~S}$ in its assessment. ${ }^{27}$ It cites the $1-\mathrm{hr} L \mathrm{LC}_{50}$ as $4,230 \mathrm{ppm} \mathrm{v} / \mathrm{v}$ in mice, $14,140 \mathrm{ppm} \mathrm{v} / \mathrm{v}$ in male rats, and $19,770 \mathrm{v} / \mathrm{V}$ in female rats in a summary. These data correspond to $2,120,7,070$, and $9,890 \mathrm{ppm}$ $\mathrm{v} / \mathrm{v}$, respectively, with the ten Berge adjustment to 4-hr $L C_{50} S$.

Much of the toxicity data for R-717 predates practical laboratory methods for precise and reliable determination of concentrations and purity. Accordingly, there is considerable dispersion in the data. Appelman et al. document inconsistencies between very early and more recent data. $^{273}$

Tabulae Biologicae indicates the lowest concentration reported as lethal to humans as 5,000 for 5 min $_{1}{ }^{274}$ as reported by Ludwig et al. ${ }^{11}$ and RTECS. ${ }^{23}$

The lowest 4-hr LC $\mathrm{LC}_{50}$ identified is $2,000 \mathrm{ppm}$ v/v extrapolated from Weedon et al. It is supported by findings from Carpenter et al. and citation by Deichmann and Gerarde, but challenged by a separate finding by Kimmerle. ATSDR, ICE, NIOSH, OSHA, and RTECS cite it, but other agencies such as EPA choose other data (though EPA cites an $\mathrm{LC}_{50}$ only slightly higher for mice).

Based on the conflicting finding from Kimmerle and the number of higher $\mathrm{LC}_{50}$ findings in more recent studies (notably from Appelman et al. ${ }^{273}$ and from Propkop'eva et al. ${ }^{271}$ ) than the 1940 Weedon et al. and 1949 Carpenter et al. data, it may be appropriate to choose the 4-hr LC $_{50}$ of $3,300 \mathrm{ppm} \mathrm{v/v}$, derived from the lowest $L C_{50}$ found in the detailed Clement Associates assessment. This alternative is still conservative compared to the average of reported $L_{50} s$ and ALCs.

recommendation: $4-\mathrm{hr} \mathrm{LC}_{50}$ rat $=2,000$ or $3,300 \mathrm{ppm}$ v/v subject to further review

\section{Cardiac Sensitization}

No indication was found to suggest that R-717 causes cardiac sensitization at concentrations less than the 4-hr $\mathrm{LC}_{50}$.

recommendation: exclude from ATEL

\section{Anesthetic Effect}

None of the acute inhalation studies that report clinical observations during exposures suggest that ammonia causes an anesthetic effect. In contrast, its sharp odor and irritating effects are prone to cause anyone exposed to attempt quick escape. Appelman et al. report that exposed rats were restless, showed both eye and nasal irritation (e.g., eyes closed with discharges, wet noses), and exhibited dyspnoea (e.g., labored breathing through the mouth). ${ }^{273}$ They do not mention any observations indicative of an anesthetic effect for concentrations as high as $38,900,27,200,22,700$, or 16,100 for $10,20,40$, or 60 min, respectively. ${ }^{273}$

recommendation: $10-\mathrm{min}$ NOEL $=38,900 \mathrm{ppm} \mathrm{v} / \mathrm{v}$ 


\section{Other Escape-Impairing Effects and Permanent Injury}

There are numerous anecdotal accounts of injuries and fatalities from exposures, but there also are accounts of tolerance of low concentrations by technicians accustomed to working with ammonia. $^{275,276}$

Schadeberg et al. conclude that concentrations of $50 \mathrm{ppm}$ v/v or less caused only dryness of the nose in one or two subjects, but did not cause irritation or discomfort in a panel of ten human volunteers exposed (head-only) to ammonia for $5 \mathrm{~min}^{277}$ They indicate that three or more subjects reported annoyance to the eyes, nose, mouth, throat, or chest at 72 and $134 \mathrm{ppm}$ v/v with an increase in effects at the higher concentration. ${ }^{277}$

Wallace summarizes subjective observations by seven human volunteers accustomed to working with ammonia to informal exposures for $1-3 \mathrm{~min}^{278}$ as summarized by Stoecker. ${ }^{279}$ Wallace indicates that concentrations of $150-200 \mathrm{ppm}$ v/v affected their eyes in approximately $1 \mathrm{~min}$, but did not seriously impair their vision and did not affect their breathing. ${ }^{278,279} 440 \mathrm{ppm} \mathrm{v} / \mathrm{v}$ affected their eyes more quickly, but not sufficiently to impair vision, while $600 \mathrm{ppm}$ v/v caused their eyes to stream after 30 seconds, but was still breathable. ${ }^{278,279} \quad 1,000 \mathrm{ppm} \mathrm{v} / \mathrm{v}$ caused their eyes to streamed instantly, impaired their vision, made breathing intolerable for most of those exposed, and caused skin irritation. $1,500 \mathrm{ppm}$ v/v induced an instant reaction to get out of the area. ${ }^{278,279}$

Verberk indicates that 16 subjects exposed to $50-140 \mathrm{ppm} v / \mathrm{v}$ for $2 \mathrm{hr}$ experienced nasal irritation, but no changes to their ventilation capacity, ${ }^{280}$ as reported by AlHA. ${ }^{281}$ Eight of the subjects found $140 \mathrm{ppm} v / \mathrm{v}$ to be intolerable. ${ }^{280,281}$

Johnston et al. report that volunteers experienced decreased respiratory tidal volume in exposures to 205-335 ppm v/v for $30 \mathrm{~min}$ and temporary changes in respiratory physiology at 500 $\mathrm{ppm}$ v/v for $30 \mathrm{~min}^{282}$ as summarized by AlHA. ${ }^{281}$

The International Institute of Ammonia Refrigeration (IIAR) indicates that while the data are not in agreement, accepted response levels include $400 \mathrm{ppm} \mathrm{v/v} \mathrm{for} \mathrm{immediate} \mathrm{throat} \mathrm{irritation,}$ $1,700 \mathrm{ppm} v / \mathrm{v}$ for cough, $2,400 \mathrm{ppm} \mathrm{v} / \mathrm{v}$ for a threat to life in $30 \mathrm{~min}$, and $5,000-10,000 \mathrm{ppm} \mathrm{v} / \mathrm{v}$ for a high likelihood of mortality for short exposure. ${ }^{283}$

Barrow et al. determined the $\mathrm{RD}_{50}{ }^{*}$ mouse to be of $303 \mathrm{ppm} \mathrm{v/v}$ and note that the maximal response for ammonia was achieved within the first two minutes of exposure. ${ }^{284}$ Alarie categorizes typical effects at multiples of the $\mathrm{RD}_{50}$ as severe injury and possible mortality at 10 , not tolerable by humans at 1 , some sensory irritation at 0.1 , no sensory irritation at 0.01 , and no effect of any kind on the respiratory system at $0.001 .^{267}$ Zissu reports that no pathological changes were observed from ammonia at $257 \mathrm{ppm}\left(85 \%\right.$ of the $\left.\mathrm{RD}_{50}\right)$, but that it induced lesions in the respiratory epithelium following exposures at $711 \mathrm{ppm}\left(235 \%\right.$ of the $\left.\mathrm{RD}_{50}\right){ }^{285} \mathrm{He}$ notes that the intensity of lesions became more severe with further exposures, progressing from moderate for 4 days, to severe for 9 days, and to very severe for 14 days based on exposures of $6 \mathrm{hr} / \mathrm{d}$ for $5 \mathrm{~d} / \mathrm{wk}^{285}$ Buckley et al. found moderate inflammation to the respiratory epithelium with ammonia exposures, but comment that its ionization in water is comparatively limited resulting in less damage than for other chemicals for which the $\mathrm{RD}_{50}$ and water solubility are similar. ${ }^{286}$ They conclude that "the value of the mouse sensory irritant model for setting occupa-

* The median respiratory rate decrease, or $\mathrm{RD}_{50}$, is the concentration that results in $50 \%$ decrease in respiratory rate, normally measured in mice. 
tional guidelines to sensory irritants is strengthened in that these studies demonstrate the $\mathrm{RD}_{50}$ concentration is associated with respiratory tract damage and is not an acceptable concentration for occupational exposures. ${ }^{\text {286 }}$ Kane et al. presents a model to predict human sensory irritation responses based on sensory irritation data from animal tests; they conclude that the emergency exposure limit (EEL) should be set at $30 \%$ of the $\mathrm{RD}_{50 .}{ }^{287}$

Ludwig et al. cite the $\mathrm{RD}_{50}$, but also indicate two limiting concentrations from Silverman et al. and from Henderson and Haggard in the NIOSH documentation for the 1994 IDLH concentration reduction. ${ }^{11}$

Silverman et al. report changes in respiration rate and moderate to severe irritation in humans at rest exposed to $500 \mathrm{ppm} \mathrm{v/v}$, six for $30 \mathrm{~min}$ and a seventh for $15 \mathrm{~min}^{288}$ They report that the male volunteers exhibited hyperventilation* with cyclic variation during exposures and transient hypoventilation ${ }^{\dagger}$ during recovery following the exposures. ${ }^{288}$ They indicate that one subject showed a slight increase in blood pressure and pulse rate. ${ }^{288}$ Silverman et al. also report irritation of the nose and throat, hypo-esthesia ${ }^{\ddagger}$ of the exposed skin, lacrimation, ${ }^{\S}$ and complaints of respiratory irritation. ${ }^{288}$ They note that these subjective reactions varied considerably, with some effects persisting for 24 hours after exposures, but were present in all subjects and severe in two. ${ }^{288}$

Henderson and Haggard report that the maximum short exposure tolerance is $300-500 \mathrm{ppm} v / \mathrm{v}$ for 30-60 minutes. ${ }^{289}$ The American Industrial Hygiene Association (AlHA) Hygienic Guide for anhydrous ammonia notes that a concentration of $300-500 \mathrm{ppm} v / \mathrm{v}$ is the maximum shortexposure tolerance for $30-60$ minutes, $2,500-6000 \mathrm{ppm}$ is considered dangerous to life, and $5,000-10,000 \mathrm{ppm}$ is reported to be fatal. ${ }^{290}$

The Clement Associates assessment concludes that "acute studies in animals have revealed that levels above $280 \mathrm{mg} / \mathrm{m}^{3}$ (400 ppm) can cause severe damage to the eyes, nose, throat, and respiratory tract." 269

Among short-term occupational (not emergency) limits, the NIOSH IDLH is $300 \mathrm{ppm} v / \mathrm{v}$ (reduced in 1994 from $500 \mathrm{ppm}$ v/v), ${ }^{15}$ the NIOSH STEL and the ACGIH TLV-STEL are both 35 $\mathrm{ppm}$ v/v TWA for $15 \mathrm{~min},{ }^{15,291}$ and the German maximum workplace concentration (MAK) for short-term excursions is $40 \mathrm{ppm} \mathrm{v/v}$ for up to $5 \mathrm{~min} .^{292}$ The OSHA short-term exposure limit (STEL) set in 1989, but vacated in 1992 with all changes since 1971, was 50 ppm v/v TWA for 15 min. $^{293}$ Among recognized emergency exposure limits, the NRC Emergency Exposure Guidance Level (EEGL) for single exposures for both 1 and $24 \mathrm{hr}$ is a ceiling concentration of

* abnormally high breathing rate

+ abnormally low breathing rate

$\ddagger$ reduced capacity for sensation and feeling

$\S$ abnormal or excessive secretion of tears

** a concentration limit that should not be exceeded at any time 
$100 \mathrm{ppm} \mathrm{v/v.} .^{39}$ The AIHA ERPG-1, ERPG-2, and ERPG-3* are 25, 200, and 1,000 ppm v/v, respectively, each for $1 \mathrm{hr} .{ }^{281.294}$ The ERPG-2 of $200 \mathrm{ppm} \mathrm{v/v}$ is the most pertinent of the three levels for present consideration.

Based on the indications from Schadeberg et al., it appears that concentrations below $100 \mathrm{ppm}$ $\mathrm{v} / \mathrm{v}$ may cause discomfort and temporary effects, but not escape-impairing effects or permanent injury. Verberk indicates that eight of 16 subjects found $140 \mathrm{ppm} \mathrm{v/v} \mathrm{to} \mathrm{be} \mathrm{intolerable.} \mathrm{By} \mathrm{Ala-}$ rie's criteria, the Barrow findings and resulting $\mathrm{RD}_{50}$ at $303 \mathrm{ppm} \mathrm{v} / \mathrm{v}$ constitute sensory irritation that is "intolerable to humans," but Henderson and Haggard and the AlHA indicate that the maximum short exposure tolerance for $300-500 \mathrm{ppm} v / \mathrm{v}$ is $30-60$ minutes. Silverman et al. document moderate to severe sensory irritation at $500 \mathrm{ppm} \mathrm{v/v} \mathrm{for} 30 \mathrm{~min}$. Barrow et al., and Buckley et al. document effects at concentrations as low as $300 \mathrm{ppm} v / \mathrm{v}$ that border on escapeimpairing or permanent. The finding by Clement Associates that levels above $400 \mathrm{ppm} v / \mathrm{v}$ can cause severe damage to the eyes, nose, throat, and respiratory tract is unambiguous.

The NRC EEGL of a ceiling of $100 \mathrm{ppm}$ v/v and AlHA ERPG-2 of $200 \mathrm{ppm} \mathrm{v} / \mathrm{v}$ set a strong precedent, while the Kane suggestion that the EELs should be set at $30 \%$ of the $R_{50}$ yields a similar result. Although they constitute occupational rather than emergency criteria, the NIOSH IDLH of $300 \mathrm{ppm} \mathrm{v/v}$ and several lower STELs reinforce them. All of them suggest that an emergency exposure limit less than $300 \mathrm{ppm}$ is appropriate, but the lowest concentration clearly causing permanent injury appears to be $400 \mathrm{ppm} v / \mathrm{v}$ while that causing severe sensory irritation in 30 min appears to be in the $300-500$ range.

recommendation: $400 \mathrm{ppm}$ v/v subject to further review

- The Emergency Response Planning Guideline (ERPG) concentrations are intended to "assist emergency response personnel planning for catastrophic chemical releases to the community." The ERPG values are "the maximum airborne concentration below which it is believed nearly all individuals could be exposed for 1 hour" without three specified response levels. The ERPG-1 addresses "mild transient adverse health effects or ... perceiving a clearly defined objectionable odor." The ERPG-2 addresses "irreversible or other serious health effects or symptoms that could impair an individual's ability to take protective action." The ERPG-3 addresses "life-threatening health effects." 


\section{R-744: CARBON DIOXIDE}

\section{Lethality}

No $L C_{50}$ or ALC studies in rats were found.

Tabulae Biologicae indicates the lowest concentration reported as lethal to humans as 90,000 ppm v/v for 5 min, ${ }^{274}$ as reported by Ludwig et al. ${ }^{11}$ and RTECS. ${ }^{23}$

ACGIH considers R-744 (carbon dioxide) to be a simple asphyxiant. ${ }^{16,24}$

recommendation: exclude from ATEL

\section{Cardiac Sensitization}

No cardiac sensitization studies or evidence that carbon dioxide causes it were found.

recommendation: exclude from ATEL

\section{Anesthetic Effect}

No qualifying animal data were found, but NIOSH notes that carbon dioxide acts as a central nervous system (CNS) depressant and that narcosis may occur when high concentrations of carbon dioxide are inhaled, potentially resulting in respiratory arrest and death. ${ }^{44}$ These concerns are further addressed below, based on findings in humans, as other escape-impairing effects.

recommendation: no qualifying animal data

\section{Other Escape-Impairing Effects and Permanent Injury}

$\mathrm{BOC}$ indicates that carbon dioxide is the most powerful cerebral vasodilator ${ }^{*}$ known. $^{295}$

Brown and Miller indicate that 15 of 17 dogs exposed to $400,000 \mathrm{ppm}$ v/v carbon dioxide for $2 \mathrm{hr}$ or more died upon sudden re-exposure to air, ${ }^{296}$ as summarized by NIOSH. ${ }^{44}$ Petty et al. indicate that rats exposed to concentrations of carbon dioxide exceeding $400,000 \mathrm{ppm} \mathrm{v} / \mathrm{v}$ for 20 min experienced arrhythmia $10-55 \mathrm{sec}$ after sudden withdrawal, ${ }^{297}$ also as summarized by $\mathrm{NIOSH}^{44}$ Caujolle notes that there are cases of instant death at concentrations exceeding 400,000 ppm v/v. ${ }^{298}$

Mukherjee and Singh indicate that 10 male mice exposed to $350,000 \mathrm{ppm} \mathrm{v/v}$ for $6 \mathrm{hr}$ experienced changes in their sperm development that resulted in smaller than normal litters, ${ }^{299}$ also cited by $\mathrm{NIOSH} .{ }^{44}$ This finding suggests reproductive toxicity.

* widening of the blood vessels of the brain 
The Aero Medical Association, Friedlander and Hill, Lambertsen, and Sechzer et al. report rapid loss of consciousness by humans at concentrations of $100,000-300,000 \mathrm{ppm} \mathrm{v/v},{ }^{300-303}$ as reported by $\mathrm{NIOSH}^{44}$

Dripps and Comroe report that one of 42 subjects who breathed $76,000 \mathrm{ppm}$ v/v for an average of 7.4 min lost consciousness while nine indicated dizziness. ${ }^{304}$ They also indicate that three of 31 subjects who breathed $104,000 \mathrm{ppm} v / \mathrm{v}$ for an average of $3.8 \mathrm{~min}$ lost consciousness while seven indicated faintness and 13 indicated dizziness (each subject may have reported more than one symptom). ${ }^{304}$

Schaefer reports a range of CNS effects in 42 human volunteers exposed to $75,000 \mathrm{ppm} \mathrm{v} / \mathrm{v}$ carbon dioxide for 15 minutes including restlessness for ten subjects, loss of balance in seven, loss of limb control for four, and mental disorientation for two, ${ }^{305}$ as reported by $\mathrm{NIOSH}^{44} \mathrm{He}$ concludes that exposure to $33,000 \mathrm{v} / \mathrm{v}$ and higher depressed the autonomic nervous system. ${ }^{\star 305,44}$

Brown reports that concentrations of $55,000-124,000 \mathrm{ppm} v / \mathrm{v}$ were tolerable to seven male subjects for only short periods of time, ranging from $22 \mathrm{~min}$ for $60,000 \mathrm{ppm} \mathrm{v} / \mathrm{v}$ to $30 \mathrm{sec}$ for $124,000 \mathrm{ppm} \mathrm{v/v} .^{306}$ He notes that exposed subjects showed increases in respiratory rate, pulse rate, and blood pressure as well as subjective symptoms, including dyspnea, $\uparrow$ dizziness, feeling of stupefaction, apprehension of impending collapse, and limited to moderately severe headache. Brown indicates that none of the subjects could have withstood $100,000 \mathrm{ppm} v / \mathrm{v}$ for more than 10 min without loss of consciousness, and that increasing the oxygen concentration did not alleviate the toxic effects of high carbon dioxide concentrations. ${ }^{306}$

Storm and Giannetta report that exposures to $40,000 \mathrm{ppm}$ v/v for 2 wk did not impair psychomotor performance in six healthy male volunteers based on tests of complex tracking performance, eye-hand coordination, and problem-solving ability, ${ }^{307}$ as reported by $\mathrm{NIOSH}^{44}$

Weybrew describes a test in a male volunteer to investigate the effect of exposure on psychological function, ${ }^{308}$ as reported by $\mathrm{NIOSH}^{44}$ He reports that an exposure increased from 300 $30,000 \mathrm{ppm} v / \mathrm{v}$ in $15 \mathrm{hr}$ for 6 days did not show any remarkable treatment-related changes. ${ }^{308,44}$

King tabulates 28 studies of human tolerance to various concentrations of carbon dioxide, with division by the severity of effects. ${ }^{309}$ He then plots these responses as functions of exposure duration and concentration to create a response map. For exposures of 10-30 min, it shows concentrations up to approximately $20,000 \mathrm{ppm} \mathrm{v} / \mathrm{v}$ as "bodily compensatory reactions, below level of perception," up to $40,000 \mathrm{ppm} v / \mathrm{v}$ as the "level for perception, distraction, bodily compensatory reactions," and up to $60,000 \mathrm{ppm}$ v/v as "distracting discomfort, bodily compensatory reactions." He further shows regions with failure of compensatory reactions, marked deterioration, and inability to take steps for self preservation at higher concentrations.

* the part of the nervous system that controls involuntary actions such as breathing and heartbeat

$\dagger$ difficult or labored breathing

$\ddagger$ reduced sensation, perception, and understanding 
Ludwig et al. ${ }^{11}$ indicate that the $1994 \mathrm{NIOSH}$ IDLH concentration of $40,000 \mathrm{ppm} \mathrm{v} / \mathrm{v}$ is based on acute inhalation toxicity data from humans from the Aero Medical Association, Flury and Zernik, and Schaefer.

The Aero Medical Association reference, on the toxicity of chemicals used on aircraft, indicates that concentrations of $50,000-100,000 \mathrm{ppm} \mathrm{v} / \mathrm{v}$ and higher act as a narcotic and that signs of intoxication may occur after $30 \mathrm{~min}$ at $50,000 \mathrm{ppm} \mathrm{v/v.}{ }^{300}$ It attributes these conclusions to an article from the Council on Pharmacy and Chemistry of the American Medical Association (AMA), but also mentions other sources without identifying them ("and by others"). The AMA report is less specific; it cautions those administering oxygen-carbon dioxide therapy at 50,000 $\mathrm{ppm} \mathrm{v} / \mathrm{v}$ carbon dioxide for longer than $30 \mathrm{~min}$ to look for signs of intoxication. ${ }^{310}$ it further recommends careful observation from the start for concentrations of $100,000 \mathrm{ppm} \mathrm{v} / \mathrm{V}$ and notes that carbon dioxide toxicity must be kept in mind after 10-15 min. ${ }^{310}$ By implication, the AMA cautions must have been predicated on incidents in which the anesthetic and other toxic affects suggested were observed. The findings discussed above from Schaefer and from Dripps and Comroe support this implication, but they cannot be the source since they were published later.

As reported by Ludwig et al. ${ }^{11}$ and further discussed above, Flury and Zernik indicate that exposure to $70,000-100,000 \mathrm{ppm}$ v/v produces unconsciousness in humans, ${ }^{311}$ while Schaefer reports that submarine personnel exposed continuously at $30,000 \mathrm{ppm}$ were only slightly affected, provided the oxygen content of the air was maintained at normal concentrations. ${ }^{312}$

Based on the Schaefer indication of only slight effect at $30,000 \mathrm{ppm} \mathrm{v} / \mathrm{v}$ for continuous (longterm) exposures, Storm and Giannetta finding that $40,000 \mathrm{ppm}$ v/v for 2 wk did not impair psychomotor performance, the Aero indication of narcotic effects as low as $50,000 \mathrm{ppm} v / \mathrm{v}$, and the supporting indications from AMA and from Flury and Zernik, it appears that narcotic effects may begin in humans at 50,000 ppm v/v in $30 \mathrm{~min}$ exposures. The Brown findings at 55,000 $124,000 \mathrm{ppm} \mathrm{v/v}$, the Schaefer report of CNS effects in humans at 75,000 ppm v/v for $15 \mathrm{~min}-$ utes, and the Dripps and Comroe findings at $76,000 \mathrm{ppm} v / \mathrm{v}$ for $7.4 \mathrm{~min}$ and $104,000 \mathrm{ppm} \mathrm{v} / \mathrm{v}$ for 3.8 minutes show that the effect severities increase in an approximately dose-dependent pattern with increasing concentration. These findings are roughly consistent with the King response map, which shows $40,000-60,000 \mathrm{ppm}$ v/v as distracting in less than $30 \mathrm{~min}$.

recommendation: $50,000 \mathrm{ppm}$ v/v 


\section{CAVEATS}

The cited data recommendations are not universal, but are limited in relevance to ATEL determination. Other uses may warrant consideration or use of additional or other data.

The data are not complete. The selections and recommendations are based on specific criteria for ATEL determination (see page 19). Redundant and non-qualifying data generally were excluded except to explain or reinforce the recommendations. Appendix 2 (see page 135) tabulates some additional toxicity data.

The data and recommendations are not the result of a consensus determination. Rather this report documents input for consideration by qualified parties in developing guides, standards, codes, and regulations. The primary intended use of the data is to assist the project committees for ASHRAE Standard 34, Designation and Safety Classification of Refrigerants, and in turn ASHRAE Standard 15, Safety Code for Mechanical Refrigeration (soon to be Safety Code for Refrigeration). Indeed, some of the recommended data differ from working and review drafts, prepared by those committees, based on preliminary data from this effort and other sources.

The ARTI Refrigerant Database ${ }^{22}$ identifies additional data and data sources for both the refrigerants addressed herein and others. Both this report and the database are intended to assist in locating information on refrigerants, but they are:

- neither a comprehensive nor authoritative reference source,

- not a substitute for independent data collection by users,

- not a substitute for examination of the data, information on how they were arrived at, underlying assumptions, associated caveats, flaws, and omissions in the cited documents, and

- not an endorsement of suitability or accuracy of referenced data and publications.

Safety considerations and other characteristics affecting suitability or desirability may be influenced by a number of factors. Among them are specific application conditions, additives, impurities, catalytic interactions with other materials used, and changes in compounding between one source or batch and another. Similarly, new findings or corrections may supersede previously published data. Neither the report nor the database should be viewed as the source of data for research, design, analysis, or other purposes. 


\section{ATEL AND RCL CALCULATIONS}

\section{ATEL CALCULATIONS}

Table 1 summarizes the toxicity data reviewed on pages 35-85. The sequence of the data columns follows the method as outlined on pages 15-22, with the order of parameters matching the hierarchy for each toxic effect. The last column is the resulting acute toxicity exposure limit (ATEL) as defined on page 18 and calculated as indicated on page 19. The governing parameter (the most restrictive effect when multiplied by the corresponding factor in the calculation method) is underlined to highlight the limiting toxic effect.

As shown in the table, cardiac sensitization is the limiting effect for 17 of the 29 singlecompound refrigerants, excluding R-E134, R-245ca, R-600 (n-butane), and R-1270 (propylene) - for which additional data are needed - and also excluding the two blends (R-500 and R502). Lethality or anesthetic/CNS effect govern for approximately one sixth of the refrigerants. This tally suggests that the ATEL formulation is conservative, since the governing effect is not necessarily lethal or even permanently injuring - even without the built-in diversity and safety factors - for the majority of refrigerants.

Table 1 includes six refrigerants, shown in italics and further noted with a bullet $(\bullet)$ following the refrigerant number, that are not examined on pages 35-85. The values indicated for them are collateral data, that is data found during searches performed for the targeted refrigerants (see page 33). Most (not all due to time constraints) of these serendipitous data, for example from toxicity studies examining or comparing multiple chemicals, were cataloged in the ARTI Refrigerant Database ${ }^{22}$ (see appendix 2 beginning on page 135) to facilitate future efforts.

Although the present project addressed only 29 refrigerants including two blends (see page 33), some or all of the data needed to calculate ATELs - and from them RCLs - were found for more than 200 others. These data were entered into the ARTI Refrigerant Database. The ATELs and RCLs from the collateral data for these additional refrigerants should be viewed as provisional, warranting extra caution in use. Dedicated data searches almost certainly would locate more data, which could raise or lower the provisional ATELs and RCLs. Supplementary NOEL data, if higher for governing effects, could increase the corresponding ATELs and RCLs. Lower LOELs or $E_{50}$ s could decrease them or disqualify some NOELs. Likewise, targeted searches might disclose other findings pertinent to escape-impairing effects or permanent injuries.

The keyed notes following table 1 recap the parameter definitions and flag estimated or substituted data, for example if based on differing test durations or species. Pages 35-85 provide more complete explanations.

Neither this report nor the tables included in it should be viewed as the source for toxicity data. They summarize data from the referenced studies, which should be examined for suitability if further use is made of the data. 
Table 1: Refrigerant Toxicity Data ${ }^{a}$ (ppm v/v)

\begin{tabular}{|c|c|c|c|c|c|c|c|c|c|}
\hline \multirow{2}{*}{$\begin{array}{l}\text { refrig- } \\
\text { erant }^{b}\end{array}$} & \multicolumn{2}{|c|}{ lethality } & \multicolumn{2}{|c|}{ cardiac sensitization } & \multicolumn{3}{|c|}{ anesthetic/CNS effect } & \multirow[b]{2}{*}{ other $^{i}$} & \multirow[b]{2}{*}{ ATEL } \\
\hline & $\mathrm{LC}_{50}{ }^{\mathrm{c}}$ & $A L C^{d}$ & $\mathrm{NOEL}^{e}$ & LOEL $^{e}$ & $E C_{50}{ }^{\dagger}$ & $\mathrm{LOEL}^{9}$ & NOEL $^{h}$ & & \\
\hline $\begin{array}{l}11 \\
12 \\
14 \\
22 \\
23\end{array}$ & $\begin{array}{r}26200 \\
>800000 \\
>390000 \\
220000 \\
>663000\end{array}$ & $\begin{array}{r}>800000 \\
>390000 \\
>663000\end{array}$ & $\begin{array}{r}\frac{1100}{40000} \\
200000 \\
25000 \\
800000\end{array}$ & $\begin{array}{r}4800 \\
50000 \\
-- \\
50000 \\
-\end{array}$ & $\begin{array}{r}35000 \\
250000 \\
140000 \\
-\end{array}$ & $\begin{array}{l}- \\
\overline{-} \\
-\end{array}$ & $\begin{array}{r}12500 \\
200000 \\
226000 \\
-\overline{51000} \\
\end{array}$ & $\begin{array}{r}2270 \overline{0} \\
\overline{-} \\
300000\end{array}$ & $\begin{array}{r}1100 \\
23000 \\
110000 j \\
25000 \\
41000\end{array}$ \\
\hline $\begin{array}{l}32 \\
113 \\
114 \\
115 \\
116\end{array}$ & $\begin{array}{r}>760000 \\
52500 \\
255000 \\
>800000 \\
>800000\end{array}$ & $\begin{array}{r}>760000 \\
45000 \\
- \\
>800000 \\
>800000\end{array}$ & $\begin{array}{r}200000 \\
2600 \\
- \\
\overline{-} \\
200000\end{array}$ & $\begin{array}{r}250000 \\
4850 \\
25000 \\
150000 \\
-\end{array}$ & $\begin{array}{r}28000 \\
250000 \\
- \\
-\end{array}$ & $\begin{array}{l}\overline{-} \\
\overline{-} \\
-\end{array}$ & $\begin{array}{r}49500 \\
25.000 \\
100000 \\
800000 \\
121000 \\
\end{array}$ & $\begin{array}{l}- \\
\overline{-} \\
-\end{array}$ & $\begin{array}{c}40000 \\
2600 \\
20000 \mathrm{k} \\
120000 \mathrm{j} \\
97000\end{array}$ \\
\hline $\begin{array}{l}123 \\
124 \\
125 \\
134 \\
134 a\end{array}$ & $\begin{array}{r}\frac{32000}{262500} \\
>769000 \\
>460000 \\
>359300\end{array}$ & $\begin{array}{r}32000 \\
>230000 \\
>769000 \\
>460000 \\
>359300\end{array}$ & $\begin{array}{l}10300 \\
10100 \\
75000 \\
75000 \\
49800 \\
\end{array}$ & $\begin{array}{r}2500 \overline{0} \\
100000 \\
100000 \\
75200\end{array}$ & $\begin{array}{r}27000 \\
150000 \\
- \\
280000\end{array}$ & $\begin{array}{l}- \\
\overline{-} \\
-\end{array}$ & $\begin{array}{r}2500 \\
48000 \\
709000 \\
120000 \\
81000\end{array}$ & $\begin{array}{l}- \\
\overline{-} \\
\overline{-}\end{array}$ & $\begin{array}{r}9100 \\
10000 \\
75000 \\
75000 \\
50000\end{array}$ \\
\hline $\begin{array}{c}E 134 \\
141 b \\
142 b \\
143 a \\
152 a\end{array}$ & $\begin{array}{r}>141000 \\
61647 \\
106000 \\
>591000 \\
>400000\end{array}$ & $\begin{array}{r}>141000 \\
>100000 \\
>591000 \\
383000\end{array}$ & $\begin{array}{r}\frac{2600}{25000} \\
250000 \\
50000\end{array}$ & $\begin{array}{r}520 \overline{\overline{0}} \\
50000 \\
300000 \\
150000\end{array}$ & $\begin{array}{r}25000 \\
250000 \\
500000 \\
-\end{array}$ & $\begin{array}{l}- \\
\overline{-} \\
-\end{array}$ & $\begin{array}{r}18000 \\
19959 \\
- \\
591000 \\
100000\end{array}$ & $\overline{-}$ & $\begin{array}{r}0 \\
2600 \\
25000 \\
170000 \\
50000\end{array}$ \\
\hline $\begin{array}{l}170 \bullet \\
218 \\
227 \mathrm{ea} \\
236 \mathrm{fa} \\
245 \mathrm{ca}\end{array}$ & $\begin{array}{r}>24838 \\
>400000 \\
>788696 \\
>457000 \\
-\end{array}$ & $\begin{array}{r}>24838 \\
>400000 \\
>788696 \\
>457000 \\
84900\end{array}$ & $\begin{array}{r}300000 \\
\frac{70000}{100000} \\
-\end{array}$ & $\begin{array}{r}100000 \\
400000 \\
90000 \\
150000 \\
=\end{array}$ & $\begin{array}{r}\overline{-} \\
110000 \\
-\end{array}$ & $\begin{array}{r}\overline{-} \\
\overline{-} \\
100000\end{array}$ & $\begin{array}{r}24838 \\
113000 \\
105000 \\
20000 \\
50000\end{array}$ & $\begin{array}{r}\overline{-} \\
\overline{-} \\
120000\end{array}$ & $\begin{array}{r}7000 \mathrm{j} \\
90000 \mathrm{~m} \\
70000 \\
55000 \\
0 \mathrm{n}\end{array}$ \\
\hline $\begin{array}{c}245 f a \\
290 \\
C 318 \bullet \\
500 \\
502\end{array}$ & $\begin{array}{r}>203000 \\
>200000 \\
>800000 \\
>\end{array}$ & $\begin{array}{r}>203000 \\
>800000 \\
>300000\end{array}$ & $\begin{array}{r}\frac{34100}{50000} \\
\overline{-} \\
50000\end{array}$ & $\begin{array}{r}44000 \\
100000 \\
100000 \\
100000\end{array}$ & $\begin{array}{r}280000 \\
>800000 \\
-\end{array}$ & $\begin{array}{l}- \\
\overline{-} \\
-\end{array}$ & $\begin{array}{r}50600 \\
800000 \\
200000\end{array}$ & $\begin{array}{l}- \\
\overline{-} \\
\overline{-}\end{array}$ & $\begin{array}{l}34000 \\
50000 \mathrm{o} \\
80000 \mathrm{j} \\
36000 \mathrm{p} \\
35000 \mathrm{pq}\end{array}$ \\
\hline $\begin{array}{l}600 \bullet \\
600 a \\
717 \\
744 \\
1270 \bullet\end{array}$ & $\begin{array}{r}272000 \\
142500 \\
3300 \\
-\mathrm{u} \\
-\end{array}$ & $\begin{array}{r}\overline{-} \\
\overline{-} \\
490000\end{array}$ & $\frac{25000}{-}$ & $\begin{array}{r}5000 \overline{\overline{0}} \\
-5 \\
-5 \\
=\end{array}$ & $\begin{array}{r}200000 \\
- \\
- \\
-\end{array}$ & $\begin{array}{l}\bar{z} \\
\bar{z} \\
-\end{array}$ & $\begin{array}{r}\overline{-} \\
38900 \\
1000 \overline{0}\end{array}$ & $\begin{array}{r}100000 \\
\frac{400}{000} \\
7200\end{array}$ & $\begin{array}{r}0 j \\
25000 \mathrm{r} \\
400 \mathrm{t} \\
50000 \\
0 j, v\end{array}$ \\
\hline
\end{tabular}

a Data and limitations should be verified in the sources identified on pages 33-86 (or reference 22 ) before use. The limiting effect is underlined for each refrigerant. Italicized data are provisional (see page 87).

b from ANSI/ASHRAE Standard $34^{1}$

c 4-hr $L C_{50}$ rat; federal and fire code toxicity classifications are based on a 1-hr $L C_{50}$ rat

d 4-hr ALC rat $e$ in dogs with epinephrine injection

f 10 -min EC $_{50}$ mouse or rat

$g$ lowest anesthetic/CNS LOEL rat during ALC, $\mathrm{LC}_{50}$, or other acute toxicity studies

$h$ highest anesthetic/CNS NOEL rat in any toxicity study not exceeding an acute LOEL

$i$ other escape-impairing or permanently injuring effects, including severe sensory irritation, for short exposures 
j data shown for R-14, R-115, R-170, R-C318, R600 , and $R-1270$ are collateral data; resulting ATELs and RCLs are provisional (see page 87)

k R-114 30-min LC 50 rat $=720000$ ppm v/v, 2-hr $L C_{50}$ rat $>600000 \mathrm{ppm} \mathrm{v/v}$

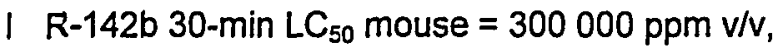
$4-\mathrm{hr} A L C \approx 128000 \mathrm{ppm}$ v/v for $2-4$ of 6 rats

m R-218 1-hr ALC rat > 800000 ppm v/v

n R-245ca 30-min ALC mouse $=240000 \mathrm{ppm} \mathrm{v/v}$

- R-290 15-min LC L $_{50}$ rat $>800000$ ppm v/v

p R-500 and R-502 ATEL based on blend calculation for components

q R-502 $L_{50}$ and ALC documentation is inadequate, but data are conservative for components r R-600a 15-min LC $L_{50}$ rat $=570000 \mathrm{ppm} \mathrm{v/V}$

$s$ no data, believed to exceed $L_{50}$ and $A L C$

t R-717 published $L_{50}$ rat $=6586-19671$ ppm $\mathrm{v} / \mathrm{v}$ for $1 \mathrm{hr}$ and $2000-4067$ for $4 \mathrm{hr}$; conversion of the lowest 1-hr $L_{50}$ rat to 4 -hr yields 3300 , approximately the midpoint of the 4-hr values

u R-744 treated as simple asphyxiant, 5-min LC Lo human $=90000 \mathrm{ppm} \mathrm{v/v}$

v R-1270 6-hr ALC > $400000 \mathrm{ppm}$ v/v; CS in 2/2 dogs at $100000 \mathrm{ppm} v / \mathrm{v} ; R_{50}=7200 \mathrm{ppm} \mathrm{v} / \mathrm{v}$

- provisional, see page 87

The input (toxicity) data are shown to the numerical precision found in the source studies to facilitate comparisons with the source studies or with their discussion on pages 35-85. Such precision is artificial in some cases, since it exceeds the accuracy to which concentrations can be controlled or reliably measured during test exposures. The calculated ATEL concentrations are consistently rounded to two significant digits, even though the implied accuracy of $1 \%$ exceeds the quantification certainty.

Four of the ATELs and resulting RCLs are zero, namely for R-E134 and R-245ca, neither of which is believed to be in known commercial use as a refrigerant, and for R-600 (n-butane) and R-1270 (propylene). The ATEL and RCL of zero do not imply either an extreme toxicity hazard for them or that these refrigerants cannot or should not be used, either as single-compound refrigerants or as blend components. Instead, it indicates that necessary data to determine their ATEL concentrations could not be located and possibly or probably do not exist. That in turn suggests that any party promoting their use as refrigerants should provide the missing data, which may require further toxicity testing. The inherent philosophy differs from the legal precept of "innocent until proven guilty." The axiom for potentially hazardous substances is "restrict until proven safe." The ATEL and resulting RCL of zero, if adopted in governing codes or regulations that are consistent with ASHRAE 15, generally would force isolation in a refrigeration machinery room with specified safety provisions.*

* $\quad$ ASHRAE 15 stipulates a number of alternative options for specific circumstances. ${ }^{2}$ 
Table 2: Refrigerant Toxicity Data with Revisions to ATEL/RCL Method ${ }^{a}$ (ppm v/v)

\begin{tabular}{|c|c|c|c|c|c|c|c|c|c|}
\hline \multirow{2}{*}{$\begin{array}{l}\text { refrig- } \\
\text { erant }^{b}\end{array}$} & \multicolumn{2}{|c|}{ lethality } & \multicolumn{2}{|c|}{ cardiac sensitization } & \multicolumn{3}{|c|}{ anesthetic/CNS effect } & \multirow[b]{2}{*}{ other ${ }^{i}$} & \multirow[b]{2}{*}{ ATEL } \\
\hline & $L C_{50}{ }^{c}$ & $A L C^{d}$ & $\overline{\text { NOEL }}{ }^{\varepsilon}$ & $\operatorname{LOEL}^{e}$ & $E C_{50}{ }^{f}$ & LOEL $^{g}$ & NOEL $^{h}$ & & \\
\hline $\begin{array}{l}11 \\
12 \\
14 \\
22 \\
23\end{array}$ & $\begin{array}{r}26200 \\
>800000 \\
>390000 \\
220000 \\
>663000\end{array}$ & $\begin{array}{r}>800000 \\
>390000 \\
>66300 \overline{0}\end{array}$ & $\begin{array}{r}\frac{1100}{40000} \\
200000 \\
25000 \\
800000\end{array}$ & $\begin{array}{r}4800 \\
50000 \\
- \\
50000 \\
-\end{array}$ & $\begin{array}{r}35000 \\
250000 \\
140000\end{array}$ & $\begin{array}{l}- \\
- \\
-\end{array}$ & $\begin{array}{r}12500 \\
200000 \\
226000 \\
51000 \\
\end{array}$ & $\begin{array}{r}2270 \overline{0} \\
\overline{-} \\
300000\end{array}$ & $\begin{array}{r}1100 \\
18000 \\
110000 j \\
25000 \\
41000\end{array}$ \\
\hline $\begin{array}{l}32 \\
113 \\
114 \\
115 \\
116\end{array}$ & $\begin{array}{r}>760000 \\
52500 \\
255000 \\
>800000 \\
>800000\end{array}$ & $\begin{array}{r}>760000 \\
45000 \\
- \\
>800000 \\
>800000\end{array}$ & $\begin{array}{r}200000 \\
\underline{2600} \\
- \\
200000\end{array}$ & $\begin{array}{r}250000 \\
4850 \\
25000 \\
150000 \\
\end{array}$ & $\begin{array}{r}28000 \\
250000 \\
-\end{array}$ & $\begin{array}{l}- \\
- \\
-\end{array}$ & $\begin{array}{r}49500 \\
25000 \\
100000 \\
800000 \\
121000 \\
\end{array}$ & $\begin{array}{l}- \\
- \\
- \\
-\end{array}$ & $\begin{array}{c}40000 \\
2600 \\
20000 \mathrm{k} \\
120000 \mathrm{j} \\
97000\end{array}$ \\
\hline $\begin{array}{l}123 \\
124 \\
125 \\
134 \\
134 a\end{array}$ & $\begin{array}{r}\frac{32000}{262500} \\
>769000 \\
>460000 \\
>359300\end{array}$ & $\begin{array}{r}32000 \\
>230000 \\
>769000 \\
>460000 \\
>359300\end{array}$ & $\begin{array}{l}10300 \\
10100 \\
75000 \\
75000 \\
49800 \\
\end{array}$ & $\begin{array}{r}2500 \overline{-} \\
100000 \\
100000 \\
75200\end{array}$ & $\begin{array}{r}27000 \\
150000 \\
- \\
280000\end{array}$ & $\begin{array}{l}- \\
- \\
-\end{array}$ & $\begin{array}{r}2500 \\
48000 \\
709000 \\
120000 \\
81000\end{array}$ & $\begin{array}{l}- \\
\overline{-} \\
\overline{-}\end{array}$ & $\begin{array}{r}9100 \\
10000 \\
75000 \\
75000 \\
50000\end{array}$ \\
\hline $\begin{array}{c}E 134 \\
141 b \\
142 b \\
143 a \\
152 a\end{array}$ & $\begin{array}{r}>141000 \\
61647 \\
106000 \\
>591000 \\
>400000\end{array}$ & $\begin{array}{r}>141000 \\
>100000 \\
>591000 \\
383000\end{array}$ & $\begin{array}{r}\frac{2600}{25000} \\
250000 \\
50000\end{array}$ & $\begin{array}{r}520 \overline{\overline{0}} \\
50000 \\
300000 \\
150000\end{array}$ & $\begin{array}{r}25000 \\
250000 \\
500000\end{array}$ & $\begin{array}{l}- \\
- \\
- \\
-\end{array}$ & $\begin{array}{r}18000 \\
19959 \\
- \\
591000 \\
100000\end{array}$ & $\begin{array}{r}\overline{-} \\
\overline{-} \\
500000\end{array}$ & $\begin{array}{r}0 \\
2600 \\
25000 \\
170000 \\
50000\end{array}$ \\
\hline $\begin{array}{l}170 \bullet \\
218 \\
227 \mathrm{ea} \\
236 \mathrm{fa} \\
245 \mathrm{ca}\end{array}$ & $\begin{array}{r}>24838 \\
>400000 \\
>788696 \\
>457000 \\
-\end{array}$ & $\begin{array}{r}>24838 \\
>400000 \\
>788696 \\
>457000 \\
84900\end{array}$ & $\begin{array}{r}300000 \\
70000 \\
100000\end{array}$ & $\begin{array}{r}100000 \\
400000 \\
90000 \\
150000 \\
=\end{array}$ & $\begin{array}{r}\overline{-} \\
110000 \\
\end{array}$ & $\begin{array}{r}\overline{-} \\
\overline{-} \\
0\end{array}$ & $\begin{array}{r}24838 \\
113000 \\
105000 \\
20000 \\
50000\end{array}$ & $\begin{array}{r}\overline{-} \\
\overline{-} \\
120000\end{array}$ & $\begin{array}{r}7000 j \\
90000 \mathrm{~m} \\
70000 \\
55000 \\
0 \mathrm{n}\end{array}$ \\
\hline $\begin{array}{c}245 \mathrm{fa} \\
290 \\
\text { C318 • } \\
500 \\
502\end{array}$ & $\begin{array}{r}>203000 \\
>200000 \\
>800000 \\
>300000\end{array}$ & $\begin{array}{r}>203000 \\
>800000 \\
>300000\end{array}$ & $\begin{array}{r}\frac{34100}{50000} \\
\overline{-} \\
50000\end{array}$ & $\begin{array}{r}44000 \\
100000 \\
100000 \\
100000\end{array}$ & $\begin{array}{r}280000 \\
>800000 \\
-\end{array}$ & $\begin{array}{l}- \\
\overline{-} \\
-\end{array}$ & $\begin{array}{r}50600 \\
800000 \\
200000\end{array}$ & $\begin{array}{l}- \\
\overline{-} \\
-\end{array}$ & $\begin{array}{l}34000 \\
50000 \mathrm{o} \\
80000 \mathrm{j} \\
29000 \mathrm{p} \\
35000 \mathrm{pq}\end{array}$ \\
\hline $\begin{array}{c}600 \bullet \\
600 \mathrm{a} \\
717 \\
744 \\
1270\end{array}$ & $\begin{array}{r}272000 \\
142500 \\
3300 \\
-u \\
-\end{array}$ & $\begin{array}{r}\overline{-} \\
\overline{-} \\
\overline{-} \\
490000\end{array}$ & $\frac{25000}{-}$ & $\begin{array}{r}5000 \overline{\overline{0}} \\
-5 \\
-5 \\
=\end{array}$ & $\begin{array}{r}200000 \\
- \\
- \\
-\end{array}$ & $\begin{array}{l}= \\
\overline{-} \\
\overline{-}\end{array}$ & $\begin{array}{r}\overline{-} \\
3890 \overline{0} \\
1000 \overline{0}\end{array}$ & $\begin{array}{r}100000 \\
\frac{400}{00} \\
72000\end{array}$ & $\begin{array}{r}0 j \\
25000 \mathrm{r} \\
320 \mathrm{t} \\
40000 \\
0 j, v\end{array}$ \\
\hline
\end{tabular}

a Data and limitations should be verified in the sources identified on pages 33-86 (or reference 22 ) before use. The limiting effect is underlined for each refrigerant. Italicized data are provisional (see page 87) Differences with table 1 are shown in bold type.

b from ANSI/ASHRAE Standard $34^{1}$

c 4-hr $\mathrm{LC}_{50}$ rat; federal and fire code toxicity classifications are based on a 1-hr $\mathrm{LC}_{50}$ rat

d 4-hr ALC rat $e$ in dogs with epinephrine injection

f 10 -min $\mathrm{EC}_{50}$ mouse or rat

g lowest anesthetic/CNS LOEL rat during ALC, $L_{50}$, or other acute toxicity studies

$\mathrm{h}$ highest anesthetic/CNS NOEL rat in any toxicity study not exceeding an acute LOEL

$i$ other escape-impairing or permanently injuring effects, including severe sensory irritation, for short exposures 
j data shown for R-14, R-115, R-170, R-C318, R600 , and $R-1270$ are collateral data; resulting ATELs and RCLs are provisional (see page 87)

k R-114 30-min LC 50 rat $=720000 \mathrm{ppm} \mathrm{v/v,} \mathrm{2-hr}$ $L C_{50}$ rat $>600000 \mathrm{ppm}$ v/v

1 R-142b 30-min LC 50 mouse $=300000 \mathrm{ppm}$ v/v, $4-\mathrm{hr} A L C \approx 128000 \mathrm{ppm}$ v/v for $2-4$ of 6 rats

$m$ R-218 1-hr ALC rat $>800000 \mathrm{ppm} v / \mathrm{v}$

n R-245ca 30-min ALC mouse $=240000 \mathrm{ppm}$ v/v

- R-290 15-min LC 50 rat $>800000 \mathrm{ppm} \mathrm{v/v}$

p R-500 and R-502 ATEL based on blend calculation for components

q R-502 LC $L_{50}$ and ALC documentation is inadequate, but data are conservative for components r R-600a 15-min $L_{50}$ rat $=570000 \mathrm{ppm} v / \mathrm{v}$

$s$ no data, believed to exceed $L_{50}$ and $A L C$

t R-717 published $L C_{50}$ rat $=6586-19671 \mathrm{ppm}$ $\mathrm{v} / \mathrm{v}$ for $1 \mathrm{hr}$ and $2000-4067$ for $4 \mathrm{hr}$; conversion of the lowest 1 -hr $L C_{50}$ rat to 4 -hr yields 3300 , approximately the midpoint of the $4-\mathrm{hr}$ values

u R-744 treated as simple asphyxiant, 5-min $L_{\mathrm{LO}}$ human $=90000 \mathrm{ppm} \mathrm{v} / \mathrm{v}$

v R-1270 6-hr ALC > $400000 \mathrm{ppm}$ v/v; CS in $2 / 2$ dogs at $100000 \mathrm{ppm} v / \mathrm{v} ; R_{50}=7200 \mathrm{ppm} \mathrm{v/v}$

- provisional, see page 87

Table 2 repeats the data summary with one change, namely ATEL calculation following the several changes presented pages 22-26. The key change is inclusion of a factor to convert the term for other escape-impairing effects and permanent injury-\$7.1.1(d)-from an effect level to a guidance level. The consequences of this change are highlighted in bold type.

As indicated in table 2 and directly compared in table 3, this change makes the ATEL for R-744 (carbon dioxide) match the revised 1994 IDLH for it and reduces the difference between the ATEL and the 1994 IDLH for R-12 and R-717 (ammonia).

Subsequent tables in this report are based on the data and ATEL values shown in table 2. 
Table 3: Refrigerant Classifications and Exposure Limits ${ }^{a}$

\begin{tabular}{|c|c|c|c|c|c|c|c|c|c|c|}
\hline \multirow[b]{2}{*}{$\begin{array}{l}\text { refrig- } \\
\text { erant }\end{array}$} & \multirow{2}{*}{$\begin{array}{l}\text { OSHA } \\
\text { fire code } \\
\text { toxic or } \\
\text { highly } \\
\text { toxic? }^{c} \\
\end{array}$} & \multirow[b]{2}{*}{$\begin{array}{c}\text { former } \\
U L \\
\text { group }\end{array}$} & \multirow[b]{2}{*}{$\begin{array}{l}\mathrm{LFL}^{\mathrm{e}} \\
(\%)\end{array}$} & \multirow{2}{*}{$\begin{array}{l}\text { ASHRAE } \\
34 \\
\text { safety } \\
\text { group }\end{array}$} & \multicolumn{2}{|c|}{$\mathrm{IDLH}^{\prime}$} & \multirow[b]{2}{*}{$\begin{array}{r}\text { ATEL } \\
(\mathrm{ppm} v / v)\end{array}$} & \multirow{2}{*}{$\begin{array}{r}\mathrm{RCL} \\
(\mathrm{ppm} \mathrm{v} / \mathrm{v})\end{array}$} & \multirow{2}{*}{$\begin{array}{c}\text { change to } \\
\text { ASHRAE } \\
15^{9} \\
\text { amount } \\
(\%)\end{array}$} & \multirow{2}{*}{$\begin{array}{c}\mathrm{PEL}^{\mathrm{h}} \\
\text { or } \sim \\
(\mathrm{ppm} v / \mathrm{v})\end{array}$} \\
\hline & & & & & $\begin{array}{r}\text { SCP } \\
(\mathrm{ppm} v / v)\end{array}$ & $\begin{array}{r}1994 \\
(\mathrm{ppm} v / \mathrm{v})\end{array}$ & & & & \\
\hline 11 & no & 5(a) & $n f$ & A1 & $10000 i$ & 2000 & 1100 & 1100 & -73 & C1 $000 j$ \\
\hline 12 & no & 6 & nf & A1 & 50000 & 15000 & 18000 & 18000 & -55 & 1000 \\
\hline 14 & no & 6 & $n f$ & A1 & - & - & 111000 & 69000 & +3 & - \\
\hline 22 & no & $5(a)$ & nf & A1 & $50000 \mathrm{k}$ & - & 25000 & 25000 & -40 & 10001 \\
\hline 23 & no & -6 & $n f$ & A1 & - & - & 41000 & 41000 & new & $1000 \mathrm{k}$ \\
\hline 32 & no & - & 12.7 & A2 & - & - & 40000 & 32000 & new & $1000 \mathrm{~m}$ \\
\hline 113 & no & $4-5$ & $\mathrm{nf}$ & $\mathrm{A} 1$ & 4500 & 2000 & 2600 & 2600 & -35 & 1000 \\
\hline 114 & no & 6 & $n f$ & A1 & 50000 & 15000 & 20000 & 20000 & -5 & 1000 \\
\hline 115 . & no & 6 & $n f$ & $A 1$ & - & - & 120000 & 69000 & +3 & $1000 j$ \\
\hline 116 & no & -6 & $n f$ & A1 & - & - & 97000 & 69000 & new & $1000 \mathrm{k}$ \\
\hline 123 & no & - & nf & B1 & $4000 \mathrm{k}$ & - & 9100 & 9100 & +810 & $50 \mathrm{~m}$ \\
\hline 124 & no & 6 & $\mathrm{nf}$ & A1 & - & - & 10000 & 10000 & new & $1000 \mathrm{~m}$ \\
\hline 125 & no & - & nf & A1 & - & - & 75000 & 69000 & new & $1000 \mathrm{~m}$ \\
\hline 134 & no & - & $\mathrm{nf}$ & - & - & - & 75000 & 69000 & new & $1000 \mathrm{k}$ \\
\hline $134 a$ & no & - & nf & A1 & $50000 k$ & - & 50000 & 50000 & -17 & $1000 \mathrm{~m}$ \\
\hline E134 & no & - & $\mathrm{nf}$ & - & - & - & 0 & 0 & new & - \\
\hline $141 b$ & no & - & 5.8 & - & - & - & 2600 & 2600 & new & $500 \mathrm{~m}$ \\
\hline $142 b$ & no & - & 6.0 & $\mathrm{~A} 2$ & - & - & 25000 & 15000 & new & $1000 \mathrm{~m}$ \\
\hline $143 a$ & no & - & 7.0 & $A 2$ & - & - & 170000 & 18000 & new & $1000 \mathrm{~m}$ \\
\hline $152 a$ & no & - & 3.7 & $\mathrm{~A} 2$ & - & - & 50000 & 9300 & +33 & $1000 \mathrm{~m}$ \\
\hline $170 \bullet$ & no & $5(b)$ & 2.9 & A3 & - & - & 7000 & 7000 & +9 & 1000 \\
\hline 218 & no & - & nf & A1 & - & - & 90000 & 69000 & new & $1000 \mathrm{k}$ \\
\hline 227ea & no & - & nf & - & - & - & 70000 & 69000 & new & $1000 \mathrm{k}$ \\
\hline $236 f a$ & no & - & nf & A1 & - & - & 55000 & 55000 & new & $1000 \mathrm{~m}$ \\
\hline $245 c a$ & no & - & 7.1 & - & - & - & 0 & 0 & new & - \\
\hline $245 f a$ & no & - & - & $B 1 n$ & - & - & 34000 & 34000 & new & $300 \mathrm{~m}$ \\
\hline 290 & no & $5(b)$ & 2.0 & $\mathrm{~A} 3$ & 20000 & 2100 & 50000 & 5000 & +14 & 2500 \\
\hline C318 • & no & -6 & $n f$ & A10 & - & - & 80000 & 69000 & +3 & \\
\hline 500 & no & $5(a)$ & nf & $\mathrm{A} 1$ & $50000 k$ & - & 29000 & $29000 p$ & -49 & 1000 \\
\hline 502 & no & $5(a)$ & $n f$ & A1 & $50000 \mathrm{k}$ & - & 35000 & $35000 p$ & -46 & 1000 \\
\hline 600 & no & $5(b)$ & 1.5 & $A 3$ & - & - & 0 & 0 & -100 & 8001 \\
\hline $600 \mathrm{a}$ & no & $5(b)$ & 1.0 & A3 & -- & - & 25000 & 2500 & -26 & $800 q$ \\
\hline 717 & no & 2 & 15.0 & B2 & 500 & 300 & 320 & 320 & -36 & $50 r$ \\
\hline 744 & no & $5(a)$ & nf & A1 & 50000 & 40000 & 40000 & 40000 & -20 & 5000 \\
\hline $1270 \bullet$ & no & - & 2.0 & $A 3$ & - & - & 0 & 0 & -100 & $375 k$ \\
\hline \multirow{2}{*}{\multicolumn{6}{|c|}{$\begin{array}{l}\text { Data and limitations should be verified in the } \\
\text { sources identified on pages } 33-86 \text { (or reference } \\
22 \text { ) before use. Concentrations shown in this } \\
\text { table as \% or ppm are v/v in air. Italicized data } \\
\text { are provisional (see page } 87 \text { ). }\end{array}$}} & \multicolumn{5}{|c|}{$\begin{array}{l}\text { c } 29 \text { CFR } 1910^{3} \text { and most model fire codes in- } \\
\text { cluding the IFC, }{ }^{12} \text { NFPC, SFPC, and UFC }\end{array}$} \\
\hline & & & & & & \multicolumn{5}{|c|}{$\begin{array}{l}\text { comparative life hazard where group } 1 \text { is the } \\
\text { most toxic and group } 6 \text { the least }\end{array}$} \\
\hline \multicolumn{11}{|c|}{ b from ANSI/ASHRAE Standard $34^{1}$} \\
\hline
\end{tabular}


e determined in accordance with ASHRAE 34 $\S 6.1 .3^{1}$; the values shown are taken from the referenced sources in the ARTI Refrigerant Database 22 based on the lowest cited there to be conservative (see §7.3.4 on page 20)

$f$ from the Standards Completion Program (SCP) and 1994 NIOSH IDLH revision ${ }^{11,15}$

$g$ from ASHRAE 15 table $1^{2}$

h PEL or consistent occupational exposure limit on a time-weighted average (TWA) basis (unless noted $C$ for ceiling) for an $8 \mathrm{hr} / \mathrm{d}$ and $40 \mathrm{hr} / \mathrm{wk}$

$i$ The SCP and 1994 IDL L Hs are 10000 and 2000 ppm $V / V$, respectively; ${ }^{11,15}$ ARI recommended reduction of the SCP IDLH to $5000 \mathrm{ppm} \mathrm{v/v.}{ }^{25}$

j The OSHA PEL is $1000 \mathrm{ppm} \mathrm{v/v;} ;{ }^{293}$ ARI recommends $C 1000 \mathrm{ppm}$ v/v based on the cardiac sensitization potential. ${ }^{25}$

$k$ industry or manufacturer recommendation
I ACGIH Threshold Limit Value - Time-Weighted Average (TLV-TWA)

$m$ AlHA Workplace Environmental Exposure Limit (WEEL)

n currently classified $A 1$ provisional, ${ }^{1}$ but a change to $B 1$ is pending

o not currently classified based on withdrawal of an earlier classification as $A 1,{ }^{1}$ but a change to reinstate the A1 classification is pending

p ATEL and RCL shown for R-500 and R-502 are based on blend calculations for their respective components (see table 4)

q NIOSH Recommended Exposure Limit (REL) ${ }^{15}$

r ACGIH Threshold Limit Value - Time-Weighted Average (TLV-TWA) $=25 \mathrm{ppm} \mathrm{v/ \textrm {v } ^ { 1 6 }}$

- provisional, see page 87

\section{RCL CALCULATIONS AND COMPARISONS}

Table 3 compares the ATEL and RCL concentrations to other guideline data. As previously noted, none of the refrigerants shown meet the federal definitions for toxic or highly toxic chemicals (see page 10). Indeed, most of the refrigerants classified into the former UL groups (see page 14) and that are still of interest fall into groups $4-6$, indicating comparatively low toxicity; the exception is R-717 (ammonia).

The majority are nonflammable as indicated in the lower-flammability limit (LFL) column. The LFLs shown are the lowest identified, as summarized in appendix 2 and in the ARTI Refrigerant Database Reference. ${ }^{22}$ Page 98 discusses them further, and table 8 summarizes sensitivity analyses for dispersion in the LFL data. Of the flammable refrigerants, most are primarily used as blend components or in niche or industrial applications. The hydrocarbons - most notably $\mathrm{R}-600 \mathrm{a}$ (isobutane) - and R-717 (ammonia) are exceptions, but their net use is still small particularly in the USA. Appendix 2 and the Database provide further information on the specific and candidate uses of these refrigerants.

Table 3 shows that the proposed ATEL values are similar to the 1994 IDLHs with two exceptions. First, the comparisons fail for flammable refrigerants, since ATELS address only flammability while IDLHs reflect both toxicity and flammability. Second, NIOSH caps IDLHs at 50,000 $\mathrm{ppm}$ v/v on the premise that occupational exposure should not or need not exceed that value without use of respirators. These two distinctions are noted in the ATEL definition (see page 18), to suggest a way to adjust ATELs as surrogates for IDLHs when needed for code compliance for refrigerants lacking IDLH values. 
The RCL values indicated were calculated following the revised method (see pages 26-30) using the ATELs shown in table 2 (and repeated in table 3 for convenience), the oxygen deprivation limit (ODL) of $69,100 \mathrm{ppm} v / \mathrm{v}$ (see page 28), and the flammable concentration limit (FCL) of $25 \%$ of the LFLs (see page 28). Like the ATEL concentrations, the RCLs shown here are consistently rounded to two significant digits, even though the implied accuracy of $1 \%$ exceeds the quantification certainty.

The column following the RCLs in table 3 compares the RCLs to the concentrations used in ASHRAE 15 to determine the allowed refrigerant amounts in occupied spaces (see page 15). This comparison does not reflect any changes that may result in the way ASHRAE 15 and/or codes apply the proposed RCL values. The RCLs generally are lower than the ASHRAE 15 concentrations except for flammable refrigerants. That distinction arises from a difference in the LFL multiplier used, namely $20 \%$ in ASHRAE 15 (see note e of table 1 in reference 2) and 25\% in the RCL method (see page 28) as well as in most newer fire codes (see reference 12 as an example). The differences for nonflammable refrigerants generally follow from the toxicity data used, more specific criteria for the RCL, inconsistencies in the ASHRAE 15 values, or a small increase in the ODL from 67,000 to $69,100 \mathrm{ppm}$ v/v (see page 28). The RCL method is deemed more rigorous based on explicit criteria to address anesthetic/CNS effects, escape-impairing effects, concentrations causing permanent injury, and for some parameters more conservative multipliers in line with current industrial hygiene practices.

The PEL or consistent measures are included in table 3 for reader convenience, but are not comparable to the RCLs since the former limits address chronic occupational exposures while the latter address acute, emergency exposures (see page 1).

\section{PROPOSED RCL CONCENTRATIONS IN DIMENSIONAL FORM}

Tables 4-7 present the proposed RCLs in dimensionless form and in both metric (SI) and inchpound (IP) units of measure, all rounded to two significant digits. They include the chemical name or blend composition, the proposed ATEL concentrations from table 2, and the comparisons to ASHRAE 15 amounts from table 3 for reference.

Table 4 addresses the single-compound refrigerants from table 2 including those with provisional ATELs. Tables 5 and 6 respectively address zeotropic and azeotropic blends - R-4nn and R-5nn series - with designations in ASHRAE $34^{1}$ plus a few developmental variants in the same series. Table 7 addresses additional blends with pending applications for designations in ASHRAE 34, that have been discussed in deliberations on the committees responsible for ASHRAE 15 and 34 to examine the RCL approach, and several others used to develop or check the calculation method. 
Table 4: RCL Dimensional Unit Conversions ${ }^{a}$

\begin{tabular}{|c|c|c|c|c|c|c|}
\hline \multicolumn{2}{|c|}{ refrigerant ${ }^{b}$} & \multirow[b]{2}{*}{$\begin{array}{c}\text { ATEL } \\
(\mathrm{ppm} v / \mathrm{v})\end{array}$} & \multirow[b]{2}{*}{$\begin{array}{c}\mathrm{RCL} \\
(\mathrm{ppm} \mathrm{v} / \mathrm{v})\end{array}$} & \multirow{2}{*}{$\begin{array}{l}\text { change to } \\
\text { ASHRAE } \\
15^{\mathrm{e}} \\
\text { amount } \\
\text { (\%) }\end{array}$} & \multirow[b]{2}{*}{$\begin{array}{l}\mathrm{RCL} \\
\left(\mathrm{g} / \mathrm{m}^{3}\right)\end{array}$} & \multirow[b]{2}{*}{$\begin{array}{c}\mathrm{RCL} \\
\text { (Ib/Mct) }\end{array}$} \\
\hline $\begin{array}{l}\text { desig- } \\
\text { nation }\end{array}$ & chemical name & & & & & \\
\hline $\begin{array}{l}11 \\
12 \\
14 \\
22 \\
23\end{array}$ & $\begin{array}{l}\text { trichlorofluoromethane } \\
\text { dichlorodifluoromethane } \\
\text { chlorodifluoromethane } \\
\text { trifluoromethane }\end{array}$ & $\begin{array}{r}1100 \\
18000 \\
110000 \\
25000 \\
41000\end{array}$ & $\begin{array}{r}1100 \\
18000 \\
69000 \\
25000 \\
41000\end{array}$ & $\begin{array}{l}-73 \\
-55 \\
+3 \\
-40 \\
-39\end{array}$ & $\begin{array}{r}6.2 \\
90 \\
250 \\
89 \\
120\end{array}$ & $\begin{array}{l}0.39 \\
5.6 \\
16 \\
5.5 \\
7.3\end{array}$ \\
\hline $\begin{array}{l}32 \\
113 \\
114 \\
115 \\
116\end{array}$ & $\begin{array}{l}\text { difluoromethane } \\
\text { 1,1,2-trichloro-1,2,2-trifluoroethane } \\
\text { 1,2-dichloro-1,1,2,2-tetrafluoroethane } \\
\text { chloropentafluoroethane } \\
\text { hexafluoroethane }\end{array}$ & $\begin{array}{r}40000 \\
2600 \\
20000 \\
120000 \\
97000\end{array}$ & $\begin{array}{r}32000 \\
2600 \\
20000 \\
69000 \\
69000\end{array}$ & $\begin{array}{r}\text { new } \\
-35 \\
-5 \\
+3 \\
\text { new }\end{array}$ & $\begin{array}{r}68 \\
20 \\
140 \\
440 \\
390\end{array}$ & $\begin{array}{r}4.2 \\
1.2 \\
8.7 \\
27 \\
24\end{array}$ \\
\hline $\begin{array}{l}123 \\
124 \\
125 \\
134 \\
134 \mathrm{a}\end{array}$ & $\begin{array}{l}\text { 2,2-dichloro-1,1,1-trifluoroethane } \\
\text { 2-chloro-1,1,1,2-tetrafluoroethane } \\
\text { pentafluoroethane } \\
1,1,2,2 \text {-tetrafluoroethane } \\
1,1,1,2 \text {-tetrafluoroethane }\end{array}$ & $\begin{array}{r}9100 \\
10000 \\
75000 \\
75000 \\
50000\end{array}$ & $\begin{array}{r}9100 \\
10000 \\
69000 \\
69000 \\
50000\end{array}$ & $\begin{array}{r}+810 \\
\text { new } \\
\text { new } \\
\text { new } \\
-17\end{array}$ & $\begin{array}{r}57 \\
56 \\
340 \\
290 \\
210\end{array}$ & $\begin{array}{l}3.5 \\
3.5 \\
21 \\
18 \\
13\end{array}$ \\
\hline $\begin{array}{l}E 134 \\
141 b \\
142 b \\
143 a \\
152 a\end{array}$ & $\begin{array}{l}\text { bis(difluoromethyl) ether } \\
\text { 1,1-dichloro-1-fluoroethane } \\
\text { 1-chloro-1,1-difluoroethane } \\
\text { 1,1,1-trifluoroethane } \\
\text { 1,1-difluoroethane }\end{array}$ & $\begin{array}{r}2600 \\
25000 \\
170000 \\
50000\end{array}$ & $\begin{array}{r}0 \\
2600 \\
15000 \\
18000 \\
9300\end{array}$ & $\begin{array}{l}\text { new } \\
\text { new } \\
\text { new } \\
\text { new } \\
+33\end{array}$ & $\begin{array}{r}0 \\
12 \\
62 \\
60 \\
25\end{array}$ & $\begin{array}{l}0 \\
0.78 \\
3.9 \\
3.8 \\
1.6\end{array}$ \\
\hline $\begin{array}{l}170 \\
218 \\
227 \mathrm{ea} \\
236 \mathrm{fa} \\
245 \mathrm{ca}\end{array}$ & $\begin{array}{l}\text { ethane } \\
\text { octafluoropropane } \\
1,1,1,2,3,3,3 \text {-heptafluoropropane } \\
1,1,1,3,3,3 \text {-hexafluoropropane } \\
1,1,2,2,3 \text {-pentafluoropropane }\end{array}$ & $\begin{array}{r}7000 \\
90000 \\
70000 \\
55000 \\
0\end{array}$ & $\begin{array}{r}7000 \\
69000 \\
69000 \\
55000 \\
0\end{array}$ & $\begin{array}{r}+9 \\
\text { new } \\
\text { new } \\
+162 * \\
-100^{*}\end{array}$ & $\begin{array}{l}8.7 \\
530 \\
480 \\
340 \\
0\end{array}$ & $\begin{array}{l}0.54 \\
33 \\
30 \\
21 \\
0\end{array}$ \\
\hline $\begin{array}{c}245 \mathrm{fa} \\
290 \\
C 318 \\
500 \\
502\end{array}$ & $\begin{array}{l}\text { 1,1,1,3,3-pentafluoropropane } \\
\text { propane } \\
\text { octafluorocyclobutane } \\
\text { (calculated as blend in table 6) } \\
\text { (calculated as blend in table 6) }\end{array}$ & $\begin{array}{l}34000 \\
50000 \\
80000\end{array}$ & $\begin{array}{r}34000 \\
5000 \\
69000\end{array}$ & $\begin{array}{r}\text { new } \\
+14 \\
+3\end{array}$ & $\begin{array}{l}190 \\
9.0 \\
570\end{array}$ & $\begin{array}{l}12 \\
0.56 \\
35\end{array}$ \\
\hline $\begin{array}{l}600 \\
600 a \\
717 \\
744 \\
1270\end{array}$ & $\begin{array}{l}\text { n-butane } \\
\text { isobutane } \\
\text { ammonia } \\
\text { carbon dioxide } \\
\text { propene (propylene) }\end{array}$ & $\begin{array}{r}0 \\
25000 \\
320 \\
40000 \\
0\end{array}$ & $\begin{array}{r}0 \\
2500 \\
320 \\
40000 \\
0\end{array}$ & $\begin{array}{r}-100 \\
-26 \\
-36 \\
-20 \\
-100\end{array}$ & $\begin{array}{l}0 \\
6.0 \\
0.22 \\
72 \\
0\end{array}$ & $\begin{array}{l}0 \\
0.37 \\
0.014 \\
4.5 \\
0\end{array}$ \\
\hline \multicolumn{2}{|c|}{$\begin{array}{l}\text { a ATEL and RCL concentrations shown in this } \\
\text { table are in air. RCLs shown as } \mathrm{g} / \mathrm{m}^{3} \text { and } \mathrm{lb} / \mathrm{Mcf} \\
\text { are at sea level. Italicized data are provisional } \\
\text { (see page } 87 \text { ). }\end{array}$} & \multicolumn{5}{|c|}{$\begin{array}{l}\text { C from ASHRAE } 15 \text { table } 1:^{2} \text { Where flagged with } \\
\text { an asterisk }(\star) \text { the reference values from } \\
\text { ASHRAE } 15 \text { are for the refrigerant that this re- } \\
\text { frigerant replaces in its primary application, so } \\
\text { flagged entries in this table differ from the corre- } \\
\text { sponding entries in table } 3 \text {. }\end{array}$} \\
\hline
\end{tabular}


Table 5: RCL Dimensional Unit Conversions for Zeotropic Blends with Designations ${ }^{\text {a }}$

\begin{tabular}{|c|c|c|c|c|c|c|}
\hline \multicolumn{2}{|c|}{ refrigerant ${ }^{\mathrm{b}}$} & \multirow[b]{2}{*}{$\begin{array}{c}\text { ATEL } \\
(\mathrm{ppm} v / v)\end{array}$} & \multirow[b]{2}{*}{$\begin{array}{c}\mathrm{RCL} \\
(\mathrm{ppm} \text { v/v) }\end{array}$} & \multirow{2}{*}{$\begin{array}{l}\text { change to } \\
\text { ASHRAE } \\
15^{\mathrm{C}} \\
\text { amount } \\
\text { (\%) }\end{array}$} & \multirow[b]{2}{*}{$\begin{array}{l}\mathrm{RCL} \\
\left(\mathrm{g} / \mathrm{m}^{3}\right)\end{array}$} & \multirow[b]{2}{*}{$\begin{array}{c}\mathrm{RCL} \\
\text { (Ib/Mct) }\end{array}$} \\
\hline $\begin{array}{l}\text { desig- } \\
\text { nation }\end{array}$ & composition & & & & & \\
\hline $\begin{array}{l}400 \\
400 \\
401 \mathrm{~A} \\
401 \mathrm{~B} \\
401 \mathrm{C}\end{array}$ & $\begin{array}{l}\text { R-12/114 (50.0/50.0) } \\
\text { R-12/114 (60.0/40.0) } \\
\text { R-22/152a/124 (53.0/13.0/34.0) } \\
\text { R-22/152a/124 (61.0/11.0/28.0) } \\
\text { R-22/152a/124 (33.0/15.0/52.0) }\end{array}$ & $\begin{array}{l}28000 \\
26000 \\
20000 \\
21000 \\
17000\end{array}$ & $\begin{array}{l}28000 \\
26000 \\
20000 \\
21000 \\
17000\end{array}$ & $\begin{array}{l}\text { new } \\
\text { new } \\
-50^{\circ} \\
-48^{*} \\
-58^{*}\end{array}$ & $\begin{array}{r}160 \\
150 \\
77 \\
79 \\
71\end{array}$ & $\begin{array}{c}10 \\
9.3 \\
4.8 \\
4.9 \\
4.4\end{array}$ \\
\hline $\begin{array}{l}402 A \\
402 B \\
403 A \\
403 B \\
404 A\end{array}$ & $\begin{array}{l}\mathrm{R}-125 / 290 / 22(60.0 / 2.0 / 38.0) \\
\mathrm{R}-125 / 290 / 22(38.0 / 2.0 / 60.0) \\
\mathrm{R}-290 / 22 / 218(5.0 / 75.0 / 20.0) \\
\mathrm{R}-290 / 22 / 218(5.0 / 56.0 / 39.0) \\
\mathrm{R}-125 / 143 a / 134 a(44.0 / 52.0 / 4.0)\end{array}$ & $\begin{array}{r}39000 \\
32000 \\
29000 \\
34000 \\
130000\end{array}$ & $\begin{array}{l}39000 \\
32000 \\
29000 \\
34000 \\
69000\end{array}$ & $\begin{array}{l}-40^{\star} \\
-51^{\star} \\
-55^{\star} \\
-48^{\star} \\
+6\end{array}$ & $\begin{array}{l}160 \\
120 \\
110 \\
140 \\
280\end{array}$ & $\begin{array}{c}10 \\
7.8 \\
6.9 \\
8.9 \\
17\end{array}$ \\
\hline \multicolumn{2}{|c|}{$\begin{array}{ll}405 \mathrm{~A} & \mathrm{R}-22 / 152 \mathrm{a} / 142 \mathrm{~b} / \mathrm{C} 318 \bullet(45 / 7 / 5.5 / 42.5) \\
406 \mathrm{~A} & \mathrm{R}-22 / 600 \mathrm{a} / 142 \mathrm{~b}(55.0 / 4.0 / 41.0) \\
& \mathrm{R}-22 / 600 \mathrm{a} / 142 \mathrm{~b}(65.0 / 4.0 / 31.0) \\
407 \mathrm{~A} & \mathrm{R}-32 / 125 / 134 \mathrm{a}(20.0 / 40.0 / 40.0) \\
407 \mathrm{~B} & \mathrm{R}-32 / 125 / 134 \mathrm{a}(10.0 / 70.0 / 20.0)\end{array}$} & $\begin{array}{l}32000 \\
25000 \\
25000 \\
78000 \\
77000\end{array}$ & $\begin{array}{l}32000 \\
25000 \\
25000 \\
69000 \\
69000\end{array}$ & $\begin{array}{l}-20^{*} \\
-38^{\circ} \\
-38 \\
+6 \\
+6\end{array}$ & $\begin{array}{r}150 \\
92 \\
91 \\
260 \\
290\end{array}$ & $\begin{array}{l}9.2 \\
5.7 \\
5.7 \\
16 \\
18\end{array}$ \\
\hline $\begin{array}{l}407 C \\
407 D \\
407 E \\
408 \mathrm{~A}\end{array}$ & $\begin{array}{l}\text { R-32/125/134a }(23.0 / 25.0 / 52.0) \\
\text { R-32/125/134a (15.0/15.0/70.0) } \\
\text { R-32/125/134a (25.0/15.0/60.0) } \\
\text { R-32/125/134a }(30.0 / 10.0 / 60.0) \\
\text { R-125/143a/22 }(7.0 / 46.0 / 47.0)\end{array}$ & $\begin{array}{l}76000 \\
65000 \\
72000 \\
66000 \\
47000\end{array}$ & $\begin{array}{l}69000 \\
65000 \\
69000 \\
66000 \\
47000\end{array}$ & $\begin{array}{l}+64^{*} \\
+38 * \\
+64^{*} \\
+57^{*} \\
+0^{*}\end{array}$ & $\begin{array}{l}240 \\
240 \\
240 \\
220 \\
170\end{array}$ & $\begin{array}{l}15 \\
15 \\
15 \\
14 \\
10\end{array}$ \\
\hline $\begin{array}{l}409 A \\
409 B \\
410 A \\
410 B\end{array}$ & $\begin{array}{l}\mathrm{R}-22 / 124 / 142 \mathrm{~b}(60.0 / 25.0 / 15.0) \\
\mathrm{R}-22 / 124 / 142 \mathrm{~b}(65.0 / 25.0 / 10.0) \\
\mathrm{R}-32 / 125(50.0 / 50.0) \\
\mathrm{R}-32 / 125(45.0 / 55.0) \\
\mathrm{R}-32 / 125(32.0 / 68.0)\end{array}$ & $\begin{array}{l}20000 \\
20000 \\
55000 \\
58000 \\
71000\end{array}$ & $\begin{array}{l}20000 \\
20000 \\
55000 \\
58000 \\
69000\end{array}$ & $\begin{array}{l}-50^{*} \\
-50^{\bullet} \\
+31^{\bullet} \\
+38^{\bullet} \\
+21^{\bullet}\end{array}$ & $\begin{array}{r}79 \\
78 \\
160 \\
180 \\
240\end{array}$ & $\begin{array}{l}4.9 \\
4.9 \\
10 \\
11 \\
15\end{array}$ \\
\hline $\begin{array}{l}411 \mathrm{~A} \\
411 \mathrm{~B} \\
412 \mathrm{~A}\end{array}$ & $\begin{array}{l}\text { R-32/125 (48.0/52.0) } \\
\text { R-1270/22/152a (1.5/87.5/11.0) } \\
\text { R-1270/22/152a (3.0/94.0/3.0) } \\
\text { R-1270/22/152a (3/95.5/1.5) } \\
\text { R-22/218/142b (70.0/5.0/25.0) }\end{array}$ & $\begin{aligned} & 56000 \\
\sim & 26000 \\
\sim & 23000 \\
\sim & 23000 \\
& 26000\end{aligned}$ & $\begin{aligned} & 56000 \\
\sim & 26000 \\
\sim & 23000 \\
\sim & 23000 \\
& 26000\end{aligned}$ & $\begin{array}{r}+33 \\
-38 \% \\
-65 \% \\
-43 \% \\
-45 *\end{array}$ & $\begin{array}{r}170 \\
86 \\
80 \\
79 \\
97\end{array}$ & $\begin{array}{r}11 \\
5.4 \\
5.0 \\
4.9 \\
6.0\end{array}$ \\
\hline \multicolumn{2}{|c|}{$\begin{array}{ll}413 \mathrm{~A} & \mathrm{R}-218 / 134 \mathrm{a} / 600 \mathrm{a}(9.0 / 88.0 / 3.0) \\
414 \mathrm{~A} & \mathrm{R}-22 / 124 / 600 \mathrm{a} / 142 \mathrm{~b}(51 / 28.5 / 4.0 / 16.5) \\
414 \mathrm{~B} & \mathrm{R}-22 / 124 / 600 \mathrm{a} / 142 \mathrm{~b}(50.0 / 39.0 / 1.5 / 9.5) \\
416 \mathrm{~A} \cdot \mathrm{R}-134 \mathrm{a} / 124 / 600 \bullet(59.0 / 39.5 / 1.5) \\
417 A \bullet R-125 / 134 a / 600 \bullet(46.6 / 50.0 / 3.4)\end{array}$} & $\begin{array}{r}49000 \\
19000 \\
18000 \\
\sim 21000 \\
\sim 45000\end{array}$ & $\begin{array}{r}49000 \\
19000 \\
18000 \\
\sim 21000 \\
\sim 45000\end{array}$ & $\begin{array}{l}+23 * \\
-533^{\star} \\
-55^{\star} \\
-48 \% \\
+7 \%\end{array}$ & $\begin{array}{r}210 \\
76 \\
73 \\
96 \\
200\end{array}$ & $\begin{array}{r}13 \\
4.8 \\
4.5 \\
6.0 \\
12\end{array}$ \\
\hline \multicolumn{2}{|c|}{$\begin{array}{l}\text { a ATEL and RCL concentrations shown in this } \\
\text { table are in air. RCLs shown as } \mathrm{g} / \mathrm{m}^{3} \text { and } \mathrm{Ib} / \mathrm{Mcf} \\
\text { are at sea level. Italicized data are provisional } \\
\text { (see page } 87) \text {. ATEL and RCL data preceded } \\
\text { by a tilda }(\sim) \text { are approximations since toxicity } \\
\text { data needed to calculate the ATEL are missing } \\
\text { for one or more blend components or because } \\
\text { adequate data on the blend flammability to cal- } \\
\text { culate the RCL are not available. }\end{array}$} & \multicolumn{5}{|c|}{$\begin{array}{l}\text { C from ASHRAE } 15 \text { table } 1:{ }^{2} \text { Where flagged with } \\
\text { an asterisk (*) the reference values from } \\
\text { ASHRAE } 15 \text { are for the refrigerant that this re- } \\
\text { frigerant replaces in its primary application, so } \\
\text { flagged entries in this table differ from the corre- } \\
\text { sponding entries in table } 3 \text {. }\end{array}$} \\
\hline
\end{tabular}


Table 6: RCL Dimensional Unit Conversions for Azeotropic Blends with Designations a

\begin{tabular}{|c|c|c|c|c|c|c|}
\hline \multicolumn{2}{|c|}{ refrigerant ${ }^{\mathrm{b}}$} & \multirow[b]{2}{*}{$\begin{array}{c}\text { ATEL } \\
(\mathrm{ppm} v / \mathrm{v})\end{array}$} & \multirow[b]{2}{*}{$\underset{(p p m v / v)}{R C L}$} & \multirow{2}{*}{$\begin{array}{c}\text { change to } \\
\text { ASHRAE } \\
15^{\mathrm{C}} \\
\text { amount } \\
(\%)\end{array}$} & \multirow[b]{2}{*}{$\begin{array}{l}\mathrm{RCL} \\
\left(\mathrm{g} / \mathrm{m}^{3}\right)\end{array}$} & \multirow[b]{2}{*}{$\begin{array}{c}\mathrm{RCL} \\
\text { (Ib/MCf) }\end{array}$} \\
\hline $\begin{array}{l}\text { desig- } \\
\text { nation }\end{array}$ & composition & & & & & \\
\hline $\begin{array}{l}500 \\
501 \\
502 \\
503 \\
504\end{array}$ & $\begin{array}{l}\mathrm{R}-12 / 152 a(73.8 / 26.2) \\
\mathrm{R}-22 / 12(75.0 / 25.0) \\
\mathrm{R}-22 / 115(48.8 / 51.2) \\
\mathrm{R}-23 / 13 \bullet(40.1 / 59.9) \\
R-32 / 115 \bullet(48.2 / 51.8)\end{array}$ & $\begin{array}{r}29000 \\
27000 \\
35000 \\
0 \\
53000\end{array}$ & $\begin{array}{r}29000 \\
27000 \\
35000 \\
0 \\
41000\end{array}$ & $\begin{array}{r}-49 \\
\text { new } \\
-46 \\
0 \\
\text { new }\end{array}$ & $\begin{array}{r}120 \\
100 \\
160 \\
0 \\
130\end{array}$ & $\begin{array}{c}7.4 \\
6.4 \\
10 \\
0 \\
8.4\end{array}$ \\
\hline $\begin{array}{l}505 \\
506 \\
507 A \\
508 A \\
508 B\end{array}$ & $\begin{array}{l}R-12 / 31 \cdot(78.0 / 22.0) \\
R-310 / 114(55.1 / 44.9) \\
\text { R-125/143a (50.0/50.0) } \\
\text { R-23/116 (39.0/61.0) } \\
\text { R-23/116 (46.0/54.0) }\end{array}$ & $\begin{array}{r}0 \\
0 \\
130000 \\
55000 \\
52000\end{array}$ & $\begin{array}{r}0 \\
0 \\
69000 \\
55000 \\
52000\end{array}$ & $\begin{array}{l}\overline{-} \\
+6^{*} \\
-18^{*} \\
-22\end{array}$ & $\begin{array}{r}0 \\
0 \\
280 \\
220 \\
200\end{array}$ & $\begin{array}{r}0 \\
0 \\
17 \\
14 \\
13\end{array}$ \\
\hline $509 A$ & $\mathrm{R}-22 / 218(44.0 / 56.0)$ & 38000 & 38000 & $-42 *$ & 190 & 12 \\
\hline
\end{tabular}

a ATEL and RCL concentrations shown in this table are in air. RCLs shown as $\mathrm{g} / \mathrm{m}^{3}$ and $\mathrm{lb} / \mathrm{Mcf}$ are at sea level. Italicized data are provisional (see page 87). ATEL and RCL data preceded by a tilda $(\sim)$ are approximations since toxicity data needed to calculate the ATEL are missing for one or more blend components or because adequate data on the blend flammability to calculate the RCL are not available. b from ANSI/ASHRAE Standard $34^{1}$ and the ARTI Refrigerant Database ${ }^{22}$

c from ASHRAE 15 table $1:{ }^{2}$ Where flagged with an asterisk $(\star)$ the reference values from ASHRAE 15 are for the refrigerant that this refrigerant replaces in its primary application, so flagged entries in this table differ from the corresponding entries in table 3 .

- provisional, see page 87 
Table 7: RCL Dimensional Unit Conversions for Additional Blends without Designations ${ }^{\text {a }}$

\begin{tabular}{|c|c|c|c|c|c|c|}
\hline \multicolumn{2}{|c|}{ refrigerant $^{\mathrm{b}}$} & \multirow[b]{2}{*}{$\begin{array}{c}\text { ATEL } \\
(\mathrm{ppm} \text { v/v) }\end{array}$} & \multirow[b]{2}{*}{$\begin{array}{c}\mathrm{RCL} \\
\text { (ppm v/v) }\end{array}$} & \multirow{2}{*}{$\begin{array}{l}\text { change to } \\
\text { ASHRAE } \\
15^{\mathrm{C}} \\
\text { amount } \\
(\%)\end{array}$} & \multirow[b]{2}{*}{$\begin{array}{l}\mathrm{RCL} \\
\left(\mathrm{g} / \mathrm{m}^{3}\right)\end{array}$} & \multirow[b]{2}{*}{$\begin{array}{c}\mathrm{RCL} \\
\text { (lb/Mct) }\end{array}$} \\
\hline $\begin{array}{l}\text { desig- } \\
\text { nation }\end{array}$ & composition & & & & & \\
\hline & $\begin{array}{l}\text { R-22/152a }(52.0 / 48.0) \\
\text { R-22/152a }(60.0 / 40.0) \\
\text { R-23/22/152a (5.0/90.0/5.0) } \\
\text { R-23/22/152a (5.0/80.0/15.0) } \\
\text { R-23/32/134a (4.5/21.5/74) }\end{array}$ & $\begin{array}{l}34000 \\
33000 \\
27000 \\
29000 \\
70000\end{array}$ & $\begin{array}{l}\sim 16000 \\
\sim 19000 \\
\sim 27000 \\
\sim 29000 \\
\sim 69000\end{array}$ & $\begin{array}{l}-60^{*} \\
-53^{*} \\
-58 * \\
-31^{*} \\
+64^{*}\end{array}$ & $\begin{array}{r}\sim 51 \\
\sim 60 \\
\sim 95 \\
\sim 98 \\
\sim 240\end{array}$ & $\begin{aligned} & \sim 3.2 \\
& \sim 3.7 \\
& \sim 5.9 \\
& \sim 6.1 \\
\sim & 15\end{aligned}$ \\
\hline & $\begin{array}{l}\text { R-32/125/143a }(10.0 / 45.0 / 45.0) \\
\text { R-32/134a }(25.0 / 75.0) \\
\text { R-32/134a (30.0/70.0) } \\
\text { R-32/134a (33.8/66.2) } \\
\text { R-32/125/143a/134a }(10.0 / 33.0 / 36.0 / 21.0)\end{array}$ & $\begin{array}{r}140000 \\
70000 \\
65000 \\
62000 \\
110000\end{array}$ & $\begin{array}{l}\sim 29000 \\
\sim 67000 \\
\sim 60000 \\
\sim 56000 \\
\sim 34000\end{array}$ & $\begin{array}{l}-55^{*} \\
+60^{\bullet} \\
+43^{*} \\
+33^{\bullet} \\
-19^{*}\end{array}$ & $\begin{array}{l}\sim 110 \\
\sim 230 \\
\sim 200 \\
\sim 180 \\
\sim 130\end{array}$ & $\begin{aligned} & \sim 6.7 \\
\sim & 14 \\
\sim & 12 \\
\sim & 11 \\
\sim & 7.9\end{aligned}$ \\
\hline \multirow[t]{2}{*}{ : } & $\begin{array}{l}R-32 / 600 \bullet(90.0 / 10.0) \\
R-32 / 600 \cdot(95.0 / 5.0) \\
R-32 / 600 \mathrm{a}(90.0 / 10.0) \\
R-32 / 600 \mathrm{a}(95.0 / 5.0) \\
\mathrm{R}-125 / 143 \mathrm{a} / 290 / 22(42.0 / 6.0 / 2.0 / 50.0)\end{array}$ & $\begin{array}{r}\sim 31000 \\
\sim 35000 \\
42000 \\
41000 \\
36000\end{array}$ & $\begin{array}{l}\sim 19000 \\
\sim 24000 \\
\sim 15000 \\
\sim 21000 \\
\sim 36000\end{array}$ & $\begin{array}{l}-55^{\bullet} \\
-43^{\star} \\
-64^{\star} \\
-50^{\bullet} \\
-45^{\star}\end{array}$ & $\begin{aligned} \sim 41 & \sim 51 \\
\sim & \sim 33 \\
\sim & 45 \\
\sim & 140\end{aligned}$ & $\begin{array}{l}\sim 2.5 \\
\sim 3.2 \\
\sim 2.1 \\
\sim 2.8 \\
\sim 8.8\end{array}$ \\
\hline & $\begin{array}{l}\text { R-134a/142b (80.0/20.0) } \\
\text { R-134a/600a (80.0/20.0) } \\
\text { R-218/152a (50.0/50.0) } \\
\text { R-290/124/123(3.0/40.0/57.0) } \\
\text { R-290/600a }(50.0 / 50.0)\end{array}$ & $\begin{array}{l}41000 \\
38000 \\
64000 \\
11000 \\
35000\end{array}$ & $\begin{array}{r}\sim 41000 \\
\sim 8000 \\
\sim 12000 \\
\sim 11000 \\
\sim 3500\end{array}$ & $\begin{array}{l}+3 \\
-80^{*} \\
-71^{*} \\
-48^{*} \\
-91^{*}\end{array}$ & $\begin{aligned} & \sim 170 \\
& \sim 29 \\
& \sim 49 \\
& \sim 62 \\
& \sim 7.2\end{aligned}$ & $\begin{array}{l}\sim 11 \\
\sim 1.8 \\
\sim 3.1 \\
\sim 3.8 \\
\sim 0.45\end{array}$ \\
\hline
\end{tabular}

a ATEL and RCL concentrations shown in this table are in air. RCLs shown as $\mathrm{g} / \mathrm{m}^{3}$ and $\mathrm{Ib} / \mathrm{Mcf}$ are at sea level. Italicized data are provisional (see page 87). ATEL and RCL data preceded by a tilda $(\sim)$ are approximations since toxicity data needed to calculate the ATEL are missing for one or more blend components or because adequate data on the blend flammability to calculate the RCL are not available. b from ANSI/ASHRAE Standard $34^{1}$ and the ARTI Refrigerant Database 22

c from ASHRAE 15 table $1:^{2}$ Where flagged with an asterisk $(*)$ the reference values from ASHRAE 15 are for the refrigerant that this refrigerant replaces in its primary application, so flagged entries in this table differ from the corresponding entries in table 3.

- provisional, see page 87

\section{SENSITIVITY ANALYSES FOR THE FLAMMABILITY DATA}

Table 8 summarizes sensitivity analyses for the flammability data used to calculate the flammable concentration limit ( $F C L$, see page 28) and in turn the RCL. There is wide dispersion in reported lower-flammability limit (LFL) values for some refrigerants, as can be seen in Appendix 2 (which begins on page 135). Table 8 shows a single LFL for flammable refrigerants with minimal or no LFL dispersion such as R-1270 (see page 266). The table shows both low and high LFLs for refrigerants with clustering at one of them, such as R-32, R-152a, and R-245ca (from pages 163,214 , and 229 , respectively). 
Table 8: Parametric Analyses for Flammability ${ }^{a}$

\begin{tabular}{|c|c|c|c|c|c|c|c|c|c|c|}
\hline \multirow[b]{2}{*}{$\begin{array}{l}\text { refrig- } \\
\text { erant }^{b}\end{array}$} & \multirow{2}{*}{$\begin{array}{l}\text { OSHA } \\
\text { fire code } \\
\text { toxic or } \\
\text { highly } \\
\text { toxic? }^{c}\end{array}$} & \multirow[b]{2}{*}{$\begin{array}{l}\text { former } \\
U L \\
\text { group }^{d}\end{array}$} & \multirow[b]{2}{*}{$\frac{\operatorname{LFL}^{\mathrm{e}}}{(\%)}$} & \multirow{2}{*}{$\begin{array}{c}\text { ASHRAE } \\
34 \\
\text { safety } \\
\text { group }\end{array}$} & \multicolumn{2}{|c|}{$\mathrm{IDLH}^{\mathrm{f}}$} & \multirow[b]{2}{*}{$\underset{(\mathrm{ppm} v / v)}{\mathrm{ATEL}}$} & \multirow[b]{2}{*}{$\begin{array}{r}R C L \\
(p p m v / v)\end{array}$} & \multirow{2}{*}{$\begin{array}{c}\text { change to } \\
\text { ASHRAE } \\
15^{9} \\
\text { amount } \\
(\%)\end{array}$} & \multirow{2}{*}{$\begin{array}{c}\text { PEL } \\
\text { or } \sim \\
(\mathrm{ppm} v / v)\end{array}$} \\
\hline & & & & & $\begin{array}{r}\mathrm{SCP} \\
(\mathrm{ppm} v / v)\end{array}$ & $\begin{array}{r}1994 \\
\text { (ppm v/v) }\end{array}$ & & & & \\
\hline 32 & no & - & $\begin{array}{l}12.7 \\
14.0\end{array}$ & $\mathrm{~A} 2$ & - & - & $\begin{array}{l}40000 \\
40000\end{array}$ & $\begin{array}{l}32000 \\
35000\end{array}$ & $\begin{array}{l}\text { new } \\
\text { new }\end{array}$ & $1000 \mathrm{i}$ \\
\hline $141 b$ & no & - & $\begin{array}{l}5.8 \\
7.2 \\
7.6\end{array}$ & - & - & - & $\begin{array}{l}2600 \\
2600 \\
2600\end{array}$ & $\begin{array}{l}2600 \\
2600 \\
2600\end{array}$ & $\begin{array}{l}\text { new } \\
\text { new } \\
\text { new }\end{array}$ & $500 i$ \\
\hline $142 b$ & no & - & $\begin{array}{l}6.0 \\
6.7 \\
9.0\end{array}$ & $\mathrm{~A} 2$ & - & - & $\begin{array}{l}25000 \\
25000 \\
25000\end{array}$ & $\begin{array}{l}15000 \\
17000 \\
23000\end{array}$ & $\begin{array}{l}\text { new } \\
\text { new } \\
\text { new }\end{array}$ & $1000 i$ \\
\hline $143 a$ & no & - & $\begin{array}{c}7.0 \\
7.1 \\
13.0\end{array}$ & A2 & - & - & $\begin{array}{l}170000 \\
170000 \\
170000\end{array}$ & $\begin{array}{l}18000 \\
18000 \\
33000\end{array}$ & $\begin{array}{l}\text { new } \\
\text { new } \\
\text { new }\end{array}$ & $1000 i$ \\
\hline $152 a$ & no & - & $\begin{array}{l}3.7 \\
5.1\end{array}$ & $\mathrm{~A} 2$ & - & - & $\begin{array}{l}50000 \\
50000\end{array}$ & $\begin{array}{r}9300 \\
13300\end{array}$ & $\begin{array}{l}+33 \\
+86\end{array}$ & $1000 i$ \\
\hline $170 \bullet$ & no & $5(b)$ & $\begin{array}{l}2.9 \\
3.0 \\
3.3\end{array}$ & A3 & - & - & $\begin{array}{l}7000 \\
7000 \\
7000\end{array}$ & $\begin{array}{l}7000 \\
7000 \\
7000\end{array}$ & $\begin{array}{l}+9 \\
+9 \\
+9\end{array}$ & 1000 \\
\hline $245 \mathrm{ca}$ & no & - & $\begin{array}{l}7.1 \\
8.3\end{array}$ & - & - & - & $\begin{array}{l}0 \\
0\end{array}$ & $\begin{array}{l}0 \\
0\end{array}$ & $\begin{array}{l}\text { new } \\
\text { new }\end{array}$ & - \\
\hline 290 & no & $5(b)$ & $\begin{array}{l}2.0 \\
2.1 \\
2.37\end{array}$ & A3 & 20000 & 2100 & $\begin{array}{l}50000 \\
50000 \\
50000\end{array}$ & $\begin{array}{l}5000 \\
5300 \\
5900\end{array}$ & $\begin{array}{l}+14 \\
+20 \\
+34\end{array}$ & 2500 \\
\hline 600 & no & $5(b)$ & $\begin{array}{l}1.5 \\
1.6 \\
1.9\end{array}$ & $A 3$ & - & - & $\begin{array}{l}0 \\
0 \\
0\end{array}$ & $\begin{array}{l}0 \\
0 \\
0\end{array}$ & $\begin{array}{l}-100 \\
-100 \\
-100\end{array}$ & $800 j$ \\
\hline $600 a$ & no & $5(b)$ & $\begin{array}{l}1.0 \\
1.8 \\
1.9\end{array}$ & A3 & - & - & $\begin{array}{l}25000 \\
25000 \\
25000\end{array}$ & $\begin{array}{l}2500 \\
4500 \\
4800\end{array}$ & $\begin{array}{l}-26 \\
+32 \\
+41\end{array}$ & $800 \mathrm{k}$ \\
\hline 717 & no & 2 & $\begin{array}{l}15.0 \\
15.1 \\
16.0\end{array}$ & B2 & 500 & 300 & $\begin{array}{l}320 \\
320 \\
320\end{array}$ & $\begin{array}{l}320 \\
320 \\
320\end{array}$ & $\begin{array}{l}-36 \\
-36 \\
-36\end{array}$ & 501 \\
\hline 1270 & no & - & 2.0 & $A 3$ & - & - & 0 & 0 & -100 & $375 \mathrm{~m}$ \\
\hline $\begin{array}{l}\text { a Data } \\
\text { sourc } \\
\text { 22) be } \\
\text { table } \\
\text { are } p r\end{array}$ & $\begin{array}{l}\text { d limitat } \\
\text { identifi } \\
\text { re use. }\end{array}$ & $\begin{array}{l}\text { ns sh } \\
\text { on pe }\end{array}$ & $\begin{array}{l}\text { Id be } \\
\text { les } 33 \\
\text { ration } \\
\text { in ail }\end{array}$ & $\begin{array}{l}\text { rified in th } \\
3 \text { (or refer } \\
\text { hown in th }\end{array}$ & $\begin{array}{l}\text { e } \\
\text { ence } \\
\text { nis } \\
\text { data }\end{array}$ & $\begin{array}{l}\text { from AN } \\
29 \text { CFR } \\
\text { cluding } t\end{array}$ & $\begin{array}{l}\text { I/ASHRAE } \\
910^{3} \text { and } \\
\text { e IFC, }{ }^{12} \mathrm{~N}\end{array}$ & Standar & $\begin{array}{l}34^{1} \\
\text { el fire cod } \\
C \text {, and UF }\end{array}$ & $\begin{array}{l}\text { Jes in- } \\
\text { FC }\end{array}$ \\
\hline
\end{tabular}


d comparative life hazard where group 1 is the most toxic and group 6 the least ${ }^{20}$

e determined in accordance with ASHRAE 34 $\$ 6.1 .3^{i}$; the values shown are taken from the referenced sources in the ARTI Refrigerant Database $^{22}$ based on the lowest cited there to be conservative (see $\$ 7.3 .4$ on page 20 )

f from the Standards Completion Program (SCP) and 1994 NIOSH IDLH revision ${ }^{11,15}$

$g$ from ASHRAE 15 table $1^{2}$

$\mathrm{h}$ PEL or consistent occupational exposure limit on a time-weighted average (TWA) basis (unless noted $\mathrm{C}$ for ceiling) for an $8 \mathrm{hr} / \mathrm{d}$ and $40 \mathrm{hr} / \mathrm{wk}$ i AlHA Workplace Environmental Exposure Limit (WEEL) ${ }^{9}$

j ACGIH Threshold Limit Value - Time-Weighted Average (TLV-TWA)

k NIOSH Recommencied Exposure Limit (REL) ${ }^{15}$

1 ACGIH Threshold Limit Value - Time-Weighted Average (TLV-TWA) $=25 \mathrm{ppm} \mathrm{v/ \textrm {v } ^ { 1 6 }}$

$m$ industry or manufacturer recommendation

- provisional, see page 87

Table 8 shows a third LFL for some refrigerants. The three values reflect the lowest LFL reported, the most commonly cited value, and the highest LFL reported. They exclude LFLs determined - if known - by methods other than as stipulated in ASHRAE $34 \$ 6.1 .3^{1}$ or determined by that method at elevated temperatures or with high-energy ignition sources. Some of the data still may not conform, since most material safety data sheets (MSDSs) and some publications do not identify the test method. In some cases, the lowest and/or highest LFLs represent suspicious values that depart from clustering in data from other reports. The tail is low for R-600a (isobutane), with an isolated indication $1.0 \% \mathrm{v} / \mathrm{v}$, but nearly all other reports in the range of $1.6-1.8 \% \mathrm{v} / \mathrm{v}$ (as shown on page 256). It is high for R-143a, for which most sources indicate $7.1 \% \mathrm{v} / \mathrm{v}$ or a value in the range of $7.0-7.4 \% \mathrm{v} / \mathrm{v}$ (see page 210 ), but the documentation for one exposure limit indicates $13.0 \% \mathrm{v} / \mathrm{v}^{313} \mathrm{R}-142 \mathrm{~b}$ has outliers at both ends; most sources indicate LFLs of $6.7-7.2 \% \mathrm{v} / \mathrm{v}$ versus a full range $6.0-9.0 \% \mathrm{v} / \mathrm{v}$ (as shown on page 207 ).

Unlike the toxicity data (see pages 35-85), many of the LFL values shown were taken from MSDSs prepared by manufacturers. The LFL dispersion would be even higher except that several manufacturers revised their MSDSs, in response to preliminary data and RCL summaries distributed in the course of this project and the supported deliberations in the ASHRAE 34 and ISO committees. The modified MSDSs reflect corrections and newer test results that narrow the LFL spread, but the LFL dispersion still is high.

Consistent with the proposed method for RCL determination (see page 15-22 and specifically the provision for conservative data on page 20), tables 3-7 show the RCL based on the lowest reported LFL other than those known to have been determined by nonconforming methods.

As shown in table 8, the LFL dispersion is not an issue for single-compound refrigerants for which the ATEL is more restrictive, for example R-141b and R-717 (ammonia). It still could influence blends of them, though none are known to be in commercial use for these two refrigerants. The ATEL also governs for R-170 ethane using collateral toxicity data (see page 87 ), but the LFL is likely to become the most restrictive parameter since further toxicity data probably will 
raise the ATEL. This refrigerant was not targeted in the data gathering for this project (as explained on page 33). The impact of LFL uncertainty is particularly significant for R-152a and R600a (isobutane).

\section{CAUTION}

Most of the qualifications on data sources, variations in individual susceptibility, aggravating factors, newer data, and guideline misuse indicated for TLVs (see page 16) are applicable to other exposure limits including the ATEL and RCL.

Safety considerations and other characteristics affecting suitability or desirability may be influenced by a number of factors. Among them are specific application conditions, additives, impurities, catalytic interactions with other materials used, and changes in compounding between one source or batch and another. Similarly, new findings or corrections may supersede previously published data. Neither this report nor the Refrigerant Database should be viewed as the source of data, as contrasted to a means to help locate them. 
Toxicity Data to Determine Refrigerant Concentration Limits James M. Calm, Engineering Consultant 


\section{CONCLUSIONS}

\section{GENERAL}

The toxicity data examined herein provide a consistent basis to set emergency exposure limits to promote safety in use of refrigerants. The report recommends a calculation method, specific data, and proposed limits for common refrigerants for consideration by the committees responsible for ASHRAE 15 and 34 and, in turn, by those developing codes and regulations governing refrigerant use. While the data generally show the alternative refrigerants to be comparable or lower in toxicity than those they replace, and especially so for acute effects, safe use depends on adherence to proper application, handling, and service procedures. The report presents the proposed refrigerant concentration limits (RCLs) in dimensionless form (in ppm v/v) as well as in metric (SI) and inch-pound (IP) units of measure for a total of 99 single-compound refrigerants and zeotropic and azeotropic blends of them.

\section{PROVISIONAL AND APPROXIMATED RCLS}

The recommended limits for six of the compounds, included as components in eight of the blends, are provisional. While the scope for this project did not include them, data searches for the targeted refrigerants also found sufficient data to calculate provisional limits for the extra refrigerants. Likewise, these searches also found some or all of the data needed to calculate RCLs for more than $\mathbf{2 0 0}$ other refrigerants and candidate refrigerants. Recognizing that the limits calculated with provisional data could increase or decrease with additional data from focused searches, the provisional limits should be used with caution and warrant reduction by a safety factor of approximately $20 \%$ for interim use.

The report also flags seven of the blends as approximations based on estimations in light of insufficient toxicity data, for one or more components for one or more effects included in the ATEL. Only four of these blends have designations and ASHRAE 34 safety classifications. The report also flags 18 blends as approximations based on estimates of their flammability; the flammability data needed may be included in future classification applications. These estimated limits should be used with caution and warrant reduction by a safety factor of approximately $50 \%$ for interim use.

The suggested factors of 20 and $50 \%$ for the provisional and approximated data are intentionally conservative to protect the public and to provide an incentive for parties interested in those refrigerants to supply the missing information. They are indicative of uncertainty rather than known or suspected increases in hazard. No radical changes to the provisional and approximated RCLs, or at least the majority of them, are likely with the added data.

\section{TOXICITY CLASSIFICATION}

Neither ASHRAE 15 nor current model building, fire, and mechanical codes use the toxicity component of the ASHRAE 34 safety classification except to prohibit refrigerants in the $B$ toxic- 
ity class from use in "high probability" air-conditioning systems for human comfort. "High" and "low probability" distinguish systems for which leaked refrigerant from a failure is likely or unlikely, respectively, to enter an occupied space, generally that served by the refrigeration system involved. Determination of the cited prohibition by an index of chronic - rather than acute toxicity is illogical since the underlying concern for this purpose is emergency exposures from failures and catastrophic causes. Indeed, ASHRAE15 \$7.4.1 and most codes provide exclusions to this restriction for specified systems with small charges (not exceeding $3 \mathrm{~kg}, 6.6 \mathrm{lb}$ ) in listed equipment. ${ }^{2}$

Both the restriction and exclusions are arbitrary. There is no compelling reason to prohibit use of "high probability" systems using refrigerants in the B toxicity class if loss of the full charge would not exceed the RCL. Likewise, the exciusion amount provides no assurance that a highly toxic refrigerant, if used, would not exceed the RCL in some cases.

Accordingly, the author recommends deletion of the toxicity classification from ASHRAE 34, deletion of the restriction based on chronic toxicity for comfort-conditioning systems from ASHRAE 15, and focus instead - in both standards - on the proposed RCLs to minimize risks of acute toxicity, oxygen deprivation, and flammability.

This conclusion is reinforced by noting that the flammability class included in the ASHRAE 34 safety group is inherently "acute" in nature, that is from a single event rather than a continuing or repeated event. ASHRAE 34 determined the toxicity classification from acute toxicity data until the 1989 revision. It determined classifications by lethality or "serious injury" to guinea pigs from a 2-hr exposure at $25,000 \mathrm{ppm} v / v^{314}$ presumably patterned after the early UL toxicity tests and classifications. ${ }^{20}$

\section{UNCERTAINTIES IN FLAMMABILITY DATA}

Although also beyond the scope of the project, the report presents sensitivity analyses to examine the impact of uncertainty in some of the flammability data used to calculate the RCLs. The findings show no or small effect for the majority of flammable refrigerants, but they also reveal two with significant consequence. The recommended limits use the most restrictive lowest LFLs identified to be conservative, but these limits may warrant some relaxation with more investigation of the flammability data used.

The author is aware of an ongoing study to measure the flammability of selected refrigerants by a proposed new method ("34p"). The further testing will add to the information base, but not refute the validity of the current data. The new data may increase the data dispersion for some refrigerants, but cannot reduce it. Accordingly, the author recommends critical review of the current data to examine the reasons for the wide variation, to dismiss data from noncomplying methods, and to document consistent data for use in calculating the flammability component of RCLs.

\section{OXYGEN DEPRIVATION LIMIT}

Review of the acute inhalation toxicity studies suggests that the currently used ODL warrants examination. Many studies found for this project report tests in atmospheres exceeding 69,000 $\mathrm{ppm} v / \mathrm{v}$ refrigerant, for four hours or longer, with no clinical signs. The author recommends careful determination of a justified ODL. 


\section{ACKNOWLEDGMENTS}

Many people provided data for report. William J. Brock (with DuPont Fluoroproducts at the start of the effort and now with Unilever) warrants special recognition for assistance in locating data, resolving data conflicts, and providing many explanations sought in the course of the work. Bill helped restore the image of toxicology to a science at times when murky and contradictory data challenged that view. His colleagues at DuPont Haskell Laboratory also assisted, notably Gary W. Jepson who succeeded Bill, both in his role at DuPont and as a valued resource in locating and interpreting data for this effort.

Bill Brock and Paul $\mathrm{H}$. Dugard (then with $\mathrm{ICl} \mathrm{Klea}$ ) deserve credit for assistance in identifying appropriate parameters and multipliers for the toxicity effects included in the RCL method. Thomas E. Watson (McQuay International and a coconspirator in the ASHRAE 15 and 34 committees) mediated some of the early discussions to formulate a consistent approach.

Robert G. Doerr (The Trane Company) joined the ASHRAE 34 effort in the latter stages of the work, but provided both useful feedback on some of the proposals and a very helpful review of the draft report.

Glenn C. Hourahan of the Air-Conditioning and Refrigeration Technology Institute (ARTI) was especially helpful in defining the project at its inception and providing motivation for its continuation in turbulent times. Glenn also provided valued suggestions on both the draft report and an earlier interim report first published as a conference paper.

Numerous companies and individuals contributed to the underlying assembly or interpretation of safety data including:

- 3M: Paul H. Lieder and Phillip E. Tuma

- American Conference of Governmental Industrial Hygienists (ACGIH): Kim Stewart, William D. Wagner, and Sharon E. Ziegler

- American Industrial Hygiene Association (AIHA): Faythe Benson

- Ausimont: Douglas Kelly and Giuseppe Malinverno

- BNFL Fluorochemicals: Neil Kenyon

- Calor Gas: Terence J. Ritter

- Deutsche Forschungsgemeinschaft: Helmut Greim

- Daikin Industries: Osami Kataoka and Kenichi Taguchi

- DuPont Fluoroproducts: William J. Brock (now with Unilever), Rebecca L. Brown, Gary W. Jepson, Scott Johnson, C. Curtis Lawson, Kathy McGown (no longer with DuPont), Henry J. Trochimowicz (now an independent consultant), and Clem L. Warrick (now retired)

- Elf Atofina: Allen Karpman, Sandra R. Murphy, and Gustavo D. Rolotti 
- Environmental Protection Agency (EPA): Reva Rubenstein

- European Centre for Ecotoxicology and Toxicology of Chemicals (ECETOC): Francis M. Carpanini and D. A. Stringer

- Exxon Chemical Company: Kay H. Wheeler

- Great Lakes Chemical: Christopher P. Chengelis

- Hampshire Chemical Corporation: Gerald J. O'Neill

- Hoechst: Rainer Henrici and Ewald Preisegger (now with Solvay)

- Honeywell Intemational: Cathy E. Beck, Claire J. Matlon, and George M. Rusch

- ICI Klea: Michael A. Collins (no longer with ICI), Bruce E. Gilbert, and Paul H. Dugard (now with HSIA and a consultant)

- International Institute of Ammonia Refrigeration (IIAR): M. Kent Anderson

- National Institute for Occupational Safety and Health (NIOSH): Susan G. Cairelli, Howard R. Ludwig, and Elaine Mann

- National Research Council (NRC): Kulbir S. Bakshi

- New Mexico Engineering Research Institute (NMERI): Stephanie R. Skaggs (later with Pacific Scientific) and Robert E. Tapscott (now with GlobeTech Incorporated)

- National Industrial Chemicals Notification and Assessment Scheme (NICNAS): Megan Smith

- Phillips Chemical Company: Vickie Catlin and Barbara Mitchell

- Rhodia Organique Fine Limited: Pauline A. Bingham and Christopher C. Mulliss

- Solvay: Kenneth B. Neugebauer, Christianus G. de Rooij, and Bruno Schmit

The danger of any list of this type is the inevitable omission of someone, typically the most helpful individual; to that person, thanks too.

In addition to the cited reviewers, Melissa C. Prince helped with proofing the report in its final preparation.

This report was prepared as an account of work supported in part by the U.S. Department of Energy under grant number DE-FG02-91CE23810, Materials Compatibility and Lubricant Research (MCLR) on CFC-Refrigerant Substitutes, managed by ARTI. Additional program funding was provided by air-conditioning and refrigeration manufacturers through the Air-Conditioning and Refrigeration Institute (ARI). The ARTI Project Manager guiding the toxicity data project was Mr. Hourahan. Support by the cited parties does not constitute an endorsement, warranty, or assumption of liability for the data and views expressed herein. 


\section{REFERENCES}

These citations are deliberately expanded to show the affiliation of the authors (investigators), if different from the publishers, and to identify the document number (the letters RDB followed by four letters and/or numbers) in the ARTI Refrigerant Database (see reference 4). The authors' affiliations may be helpful in assessing the data. The database number may be useful in searching the database for further information, although the author names or other information also may be used.

1 Designation and Safety Classification of Refrigerants, ANSI/ASHRAE Standard 341997, American Society of Heating, Refrigerating, and Air-Conditioning Engineers (ASHRAE), Atlanta, GA, 1997 (RDB8601)

Addenda to Number Designation and Safety Classification of Refrigerants, Addenda 34a, 34b, 34c, 34d, and 34f, ASHRAE, 1999 (RDB9710)

Addenda to Number Designation and Safety Classification of Refrigerants, Addenda 34h, 34j, and 34k, ASHRAE, 2000 (RDBA674)

2 Safety Code for Mechanical Refrigeration, ASHRAE Standard 15-1994, ASHRAE, August 1994 (RDB4903)

Emergency Interim Standards Action for ANSI/ASHRAE Standard 15-1994, ASHRAE, May 1998 (RDB8617)

Addendum to Safety Code for Mechanical Refrigeration, ASHRAE, Addendum 15c, 2000 (RDBA675)

3 Occupational Safety and Health Administration (OSHA), U.S. Department of Labor, Health Hazard Definitions in Air Contaminants, 29 CFR $\$ 1910.1200$, Appendix A, Government Printing Office, Washington, DC, 1 July 2000

4 J. M. Calm (Engineering Consultant), Refrigerant Safety, ASHRAE Joumal, American Society of Heating, Refrigerating, and Air-Conditioning Engineers (ASHRAE), Atlanta, GA, 36(7):17-26, July 1994, with erratum 36(8), August 1994 (RDB4766)

5 R. C. Downing (Consultant, formerly DuPont Chemicals), Fluorocarbon Refrigerants Handbook, Prentice Hall, Englewood Cliffs, NJ, 1988 (RDB3960)

6 Casarett and Doull's Toxicology: The Basic Science of Poisons (fourth edition), edited by C. Klassen, Pergamon Press, New York, NY, 1991

7 Programme for Alternative Fluorocarbon Toxicity Testing (PAFT) Toxicology Summaries, PAFT, Washington, DC, September 1995 (RDB5C39)

8 Testing to Extremes - Industry's Cooperative Effort to Test the Health and Safety of Selected Fluorocarbon Alternatives to CFCs, Programme for Alternative Fluorocarbon Toxicity Testing (PAFT), Washington, DC, undated circa 1996 (RDB65E9)

9 The AlHA 2000 Emergency Response Planning Guidelines and Workplace Environmental Exposure Level Guides Handbook, document 358-EA-00, American Industrial Hygiene Association (AlHA), Fairfax, VA, 2000 (RDBA948) 
10 W. F. ten Berge (DSM, The Netherlands), A. Zwart (TNO-CIVO Toxicology and Nutrition Institute, The Netherlands), and L. M. Appelman (TNO-CIVO), Concentration-Time Mortality Response Relationship of Irritant and Systematically Acting Vapours and Gases, Journal of Hazardous Materials, 13:301-309, 1986 (RDB6278)

11 H. R. Ludwig, S. G. Cairelli, and J. J. Whalen, Documentation for Immediately Dangerous to Life or Health Concentrations (IDLHs), National Institute for Occupational Safety and Health (NIOSH), U.S. Department of Health and Human Services, Cincinnati, OH, May 1994 (RDB5340)

12 International Fire Code; International Code Council (ICC), Falls Church, VA; for the Building Officials and Code Administrators (BOCA) International, Country Club Hills, IL; International Conference of Building Officials (ICBO), Whittier, CA; and Southern Building Code Congress International (SBCCI), Birmingham, AL; table E103.1.3.1, page 369, 1997

13 Class 6.1 - Poisons, International Maritime Dangerous Goods Code, International Maritime Organization, London, \$2.1.5.2.2, page 6007, 1986

14 Uniform Fire Code, International Fire Code Institute (IFCI), Whittier, CA, table A-VI-A-1, page 1-319, 1997

15 NIOSH Pocket Guide to Chemical Hazards, publication 99-115, National Institute for Occupational Safety and Health (NIOSH), U.S. Department of Health and Human Services, Cincinnati, OH, April 1999 (RDBA670)

162000 Threshold Limit Values for Chemical Substances in the Work Environment, $2000 \mathrm{TLVs}^{\circledR}$ and BE/s${ }^{\circledR}$ : Threshold Limit Values for Chemical Substances and Physical Agents and Biological Exposure Indices, publication 0100, American Conference of Governmental Industrial Hygienists (ACGIH), Cincinnati, OH, 2000 (RDBA936)

17 Commission for the Investigation of Health Hazards of Chemical Compounds in the Work Area, List of MAK and BAT Values 1996: Maximum Concentrations and Biological Tolerance Values at the Workplace, report 32, Deutsche Forschungsgemeinschaft [German Research Association] (DFG), Bonn, Germany; VCH Verlagsgesellschaft mbH, Weinheim, Germany, 1996 (RDB7101)

18 Commission for the Investigation of Health Hazards of Chemical Compounds in the Work Area, Occupational Toxicants: Critical Data Evaluation for MAK Values and Classification of Carcinogens, edited by D. Henschler, Deutsche Forschungsgemeinschaft [German Research Association] (DFG), Bonn, Germany; VCH Verlagsgesellschaft mbH, Weinheim, Germany; volume 1, September 1990; volume 2, July 1991; volume 3, September 1992; volume 4, July 1992; volume 5, July 1993; volume 6, August 1994; and volume 7 , April 1996 (RDB7102)

19 Designation and Safety Classification of Refrigerants, ANSI/ASHRAE Standard 341989, American Society of Heating, Refrigerating, and Air-Conditioning Engineers (ASHRAE), Atlanta, GA (then in New York, NY), 1989

20 A. H. Nuckolls, The Comparative Life, Fire, and Explosion Hazards of Common Refrigerants, Miscellaneous Hazard Report Number 2375, Underwriters' Laboratories Incorporated, Chicago, IL, pages 106-107, 13 November 1933 (RDB4B64)

211996 Threshold Limit Values for Chemical Substances in the Work Environment, 1996 TLVs $^{\circledR}$ and BEls ${ }^{\circledR}$. Threshold Limit Values for Chemical Substances and Physical 
Agents and Biological Exposure Indices, American Conference of Governmental Industrial Hygienists (ACGIH), Cincinnati, OH, 1996 (RDB6901)

22 J. M. Calm, ARTI Refrigerant Database, Air-Conditioning and Refrigeration Technology Institute (ARTI), Arlington, VA, July 2000: Some of the data cited herein are from an update being prepared for release on October 2000. Please see www.arti-21cr.org/db on the Internet for database and update information.

23 Registry of Toxic Effects of Chemical Substances (RTECS ${ }^{\circledR}$ ). computerized database, National Institute of Occupational Safety and Health (NIOSH), U.S. Department of Health and Human Services, Cincinnati, $O H$, updated quarterly since June 1971 (RDB4450)

24 Documentation of the Threshold Limit Values and Biological Exposure Indices (sixth edition), publication 0206, American Conference of Governmental Industrial Hygienists (ACGIH), Cincinnati, OH, 1991 (RDB5141)

25 J. M. Calm (Engineering Consultant), Refrigerant Safety Data - Recommendations of the Air-Conditioning and Refrigeration Industry, Air-Conditioning and Refrigeration Institute (ARI), Arlington, VA, October 1993 (RDB3A15)

26 Occupational Safety and Health Guideline for Chemical Hazards (Supplement IVOHG), U.S. Department of Health and Human Services and U.S. Department of Labor, Washington, DC, 1995

27 Hydrofluorocarbons and Hydrochlorofluorocarbons - Interim Report, external review draft, U.S. Environmental Protection Agency (EPA), Washington, DC, 15 November 1990 (RDB7234)

28 Meridian Research, Incorporated, Assessment of Occupational Exposures to Halon Substitutes Used for Fire Protection, U.S. Environmental Protection Agency (EPA), Washington, DC, 1992 (RDB6B54)

29 Summary Review of Health Effects Associated with Ammonia: Health Issue Assessment, report EPA 600/8-89/052F, Office of Health and Environmental Assessment, U.S. Environmental Protection Agency (EPA), Washington, DC, June 1989 (RDB5155)

30 R. Carchman and M. M. Greenberg, Summary Review of Health Effects Associated with 1,1,2-Trichloro-1,2,2-trifluoroethane (Chlorofluorocarbon 113): Health Issue Assessment, report EPA 600/8-82-002F, Office of Health and Environmental Assessment, U.S. Environmental Protection Agency (EPA), Washington, DC, January 1983 (RDB6494)

31 Ammonia Data Book, International Institute of Ammonia Refrigeration (IIAR), Washington, DC, 1993 (RDB3635)

32 Fire Protection Guide to Hazardous Materials (11th edition), National Fire Protection Association (NFPA), Quincy, MA, 1994 (RDB4C56) - includes NFPA 49-1994 (Hazardous Chemicals Data), NFPA 325-1994 (Guide to Fire Hazard Properties of Flammable Liquids, Gases, and Volatile Solids), NFPA 491M-1991 (Manual of Hazardous Chemical Reactions), and NFPA 704-1990 (Standard System for the Identification of Fire Hazards of Materials)

33 H. F. Smith, T. F. Hatch, K. H. Jacobson, M. L. Keplinger, and F. Princi (National Research Council (NRC) Ad Hoc Committee) with revisions by A. J. Lehman, W. G. Fredrick, H. W. Gerarde, H. E. Stokinger, and J. A. Zapp, Jr. (NRC Committee on Toxicology), Basis for Establishing Emergency Inhalation Exposure Limits Applicable to Military and Space Chemicals, National Academy of Sciences (NAS), National Academy Press, Washington, DC, 1964 (RDB5A19) 
34 National Research Council (NRC) Committee on Toxicology, Basis for Establishing Guides for Short-Term Exposures of the Public to Air Pollutants, National Academy Press, Washington, DC, 1971 (RDB7411)

35 National Research Council (NRC) Committee on Toxicology, Criteria for Short-Term Exposures to Air Pollutants, National Academy Press, Washington, DC, 1979 (RDB7412)

36 National Research Council (NRC) Committee on Medical and Biological Effects of Environmental Pollutants, Ammonia, University Park Press, Baltimore, MD, 1979 (RDB5771)

37 National Research Council (NRC) Committee on Toxicology, Emergency and Continuous Exposure Limits for Selected Airborne Contaminants, National Academy Press, Washington, DC, volume 1, April 1984 (RDB7413)

38 National Research Council (NRC) Committee on Toxicology, Emergency and Continuous Exposure Limits for Selected Airborne Contaminants, National Academy Press, Washington, DC, volume 2, October 1984 (RDB7414)

39 National Research Council (NRC) Committee on Toxicology, Emergency and Continuous Exposure Limits for Selected Airborne Contaminants, National Academy Press, Washington, DC, volume 3, October 1984 (RDB7415)

40 National Research Council (NRC) Committee on Toxicology, Guideline for Short Term Exposures of the Public to Air Pollutants, IV, Guide for Ammonia, National Academy Press, Washington, DC, 1987 (RDB5154)

41 K. L. Wong, Ammonia, Maximum Allowable Concentrations for Selected Airborne Contaminants in Spacecraft, National Research Council (NRC), Committee on Toxicology, 3959, 1995 (RDB6A32)

42 National Research Council (NRC) Committee on Toxicology, Toxicity of Alternatives to Chlorofluorocarbons: HFC-134a and HCFC-123, National Academy Press, Washington, DC, 1996 (RDB6A01)

43 K. S. Bakshi (National Research Council, NRC), Toxicity of Alternatives to Chlorofluorocarbons: HFC-134a and HCFC-123, Inhalation Toxicity, 10(10):963-967, October 1998 (RDB9127)

44 Criteria for a Recommended Standard - Occupational Exposure to Alkanes (C1-C8), publication 77-151, National Institute of Occupational Safety and Health (NIOSH), U.S. Department of Health and Human Services (DHS, then the U.S. Department of Health, Education, Welfare, DHEW), Cincinnati, OH, March 1977 (RDBA539)

45 Criteria for a Recommended Standard - Occupational Exposure to Ammonia, publication 74-136, National Institute of Occupational Safety and Health (NIOSH), U.S. Department of Health and Human Services, Cincinnati, OH, 1974 (RDB5369)

46 Criteria for a Recommended Standard - Occupational Exposure to Carbon Dioxide, publication 76-194, National Institute of Occupational Safety and Health (NIOSH), U.S. Department of Health and Human Services, Cincinnati, OH, August 1976 (RDB5787)

$47 \mathrm{~J}$. M. Calm (Engineering Consultant), The Toxicity of Refrigerants, Proceedings of the 1996 International Refrigeration Conference (23-26 July 1996), edited by J. E. Braun and E. A. Groll, Purdue University, West Lafayette, IN, 157-162, July 1996; republished as report DOE/CE/23810-72E, Air-Conditioning and Refrigeration Technology Institute (ARTI), Arlington, VA, 1996 (RDB6832) 
48 S. D. Morrison, Inhalation Toxicity of "Freon 11 " and of "Freon 11" $+0.3 \% \mathrm{Ni}-$ tromethane, report 53-61, Haskell Laboratory for Toxicology and Industrial Medicine, E. I. duPont de Nemours and Company, Incorporated, Newark, DE, 25 October 1961 (RDB$65 \mathrm{H} 3$ )

49 C. E. Barras, Acute Inhalation Test [of Trichloromonofluoromethane (Freon ${ }^{\circledast 11}$ )], report 648-74, Haskell Laboratory for Toxicology and Industrial Medicine, E. I. duPont de Nemours and Company, Incorporated, Newark, DE, 29 October 1974 (RDB5C53)

50 PAFT Update, document H-45950, DuPont Chemicals, Wilmington, DE, November 1992 (RDB3909)

51 Freon $^{\circledR}$ 11, Material Safety Data Sheet DU000026 (2090FR), DuPont Chemicals, Wilmington, DE, 8 January 1998

$52 \mathrm{H}$. Wada, Anesthetic Activity and Fatality (F-123, 123a, 124, and 11), attachment to 19 May 1977 correspondence to M. B. Berenbaum (Allied Chemical Corporation), Daikin Kogyu Company Limited Chemical Division, Settsu-shi, Osaka, Japan, 16 May 1977 (RDBA309)

53 D. G. Clark and D. J. Tinston (Imperial Chemical Industries PLC, ICl, UK), Acute Inhalation Toxicity of Some Halogenated and Nonhalogenated Hydrocarbons, Human Toxicology, 1(3):239-247, 1982 (RDB6110)

54 Toxicity Summary - Genetron ${ }^{\circledR} 11$ (Trichloromonofluoromethane), Acute Toxicity and Cardiotoxicity, Allied Corporation, Morristown, NJ, March 1978 (RDB5144)

55 C. J. Hardy, I. J. Sharman, and D. O. Chanter (Huntingdon Research Centre Limited, HRC, UK), Assessment of Cardiac Sensitization Potential in Dogs and Monkeys: Comparison of I-141b and F11, report PWT 86/89437, Pennwalt Corporation, King of Prussia, PA 8 September 1989 (RDB5C63)

56 C. J. Hardy, I. J. Sharman, and G. C. Clark (Huntingdon Research Centre Limited, HRC, UK), Assessment of Cardiac Sensitization Potential in Dogs: Comparison of HFA 134a and A12, report CTL/C/2521 (also identified as ISN 250/91169) for the Program for Alternative Fluorocarbon Toxicity Testing (PAFT), Central Toxicology Laboratory, Imperial Chemical Industries Limited (ICl), Cheshire, UK, 11 September 1991 (RDB5B42)

57 C. F. Reinhardt, A. Azar, M. E. Maxfield, P. E. Smith, Jr., and L. S. Mullin (E. I. duPont de Nemours and Company, Incorporated), Cardiac Arrhythmias and Aerosol "Sniffing", Archives of Environmental Health, 22(2):265-279, February 1971 (RDB5644)

58 C. J. Hardy, P. C. Kieran, I. J. Sharman, and G. C. Clark (Huntingdon Research Centre Limited, HRC, UK), Assessment of Cardiac Sensitization Potential in Dogs: Comparison of HFC 125 and Halon 13B1, report MA-RR-92-1845 (also identified as ALS 11/920116) for the Program for Alternative Fluorocarbon Toxicity Testing (PAFT), AlliedSignal Incorporated, Morristown, NJ, 10 June 1992 (RDB5755)

59 T. J. Kenny and C. J. Hardy (Huntingdon Research Centre Limited, HRC, UK), FRIGC ${ }^{\text {tw }}$ FR-12 ${ }^{\mathrm{Tu}}$ : Assessment of Cardiac Sensitization Potential in Dogs, report HZL 6/942384, Hazleton Washington, Incorporated, Vienna, VA, 31 May 1994 (RDB7407)

60 D. G. Clark and D. J. Tinston, Correlation of the Cardiac Sensitizing Potential of Halogenated Hydrocarbons with Their Physiochemical Properties, British Journal of Pharmacology, 49(2):355-357, 1973 (RDB6109) 
$61 \mathrm{H}$. J. Trochimowicz and L. S. Mullin, Cardiac Sensitization Potential $\left(\right.$ EC $\left._{50}\right)$ of Trifluorodichloroethane, report 132-73, Haskell Laboratory for Toxicology and Industrial Medicine, E. I. duPont de Nemours and Company, Incorporated, Newark, DE, 30 March 1973 (RDB6146)

$62 \mathrm{~W}$. Weigand, Untersuchungen über die Inhalationstoxizität von Fluoroderivaten des Methan, Äthan, und Cyclobutan [Studies of the Inhalation Toxicity of Fluorinated Derivatives of Methane, Ethane and Cyclobutane], Zentralblatt für Arbeitsmedizin und Arbeitsschutz [Occupational Medicine and Safety], 21(5):149-156, 1971 (in German, RDB5365)

63 W. J. Brock, Toxicology Summary for CFC's HCFC's and HFC's, Haskell Laboratory for Toxicology and Industrial Medicine, E. I. duPont de Nemours and Company, Incorporated, Newark, DE, undated circa 1996 (RDB6452)

64 Genetron $^{\circledR}$ 11, MSDS GTRN-0017, Honeywell Incorporated, Morristown, NJ, January 2000

65 D. Lester and L. A. Greenberg (Yale University), Acute and Chronic Toxicity of Some Halogenated Derivatives of Methane and Ethane, Archives of Industrial Hygiene and Occupational Medicine, 2:335-344, 1950 (RDB5147)

66 R. D. Stewart et al. (Medical College of Wisconsin), Acute and Repetitive Human Exposure to Fluorotrichloromethane, report PB-279203/LLC, National Technical Information Service (NTIS), Springfield, VA, 1 December 1975 (RDB6598 from summary)

67 R. D. Stewart, P. E. Newton, E. D. Baretta, A. A. Herrmann, H. V. Forster, and R. J. Soto (Medical College of Wisconsin), Physiological Response to Aerosol Propellants, Environmental Health Perspectives, 26:275-285, October 1978 (RDB6115)

68 Genetron $^{\circledR}$ 12, MSDS GTRN-0018, Honeywell Incorporated, Morristown, NJ, January 2000

69 V. A. Shugaev, The Toxicology of Freon-12, Gigiena i Sanitariya, Russia (then USSR), 28(6):95-97, 1963 (in Russian, RDB7434 from summary)

70 C. F. Reinhardt, Cardiac Sensitization, report 52-69, Haskell Laboratory for Toxicology and Industrial Medicine, E. I. duPont de Nemours and Company, Incorporated, Newark, DE, 20 March 1969 (RDB6121)

71 C. J. Hardy, P. C. Kieran, and I. J. Sharman (Huntingdon Research Centre Limited, HRC, UK), Assessment of Cardiac Sensitization Potential (CSP) of a Range of Halogenated Materials, poster presentation (Spring Meeting, Baltimore, MD, USA), Society of Toxicology (SOT), 1994 (RDB6684)

72 J. Scholz (Farbwerke Hoechst AG, Germany), New Toxicological Investigations of Certain Types of Freons Used as Propellants for Aerosols and Sprays, Fortschritte der biologischen Aerosol-Forschung [Progress in Biological Research of Aerosols], 4:420-429, 1962 (in German, RDB5355)

73 R. D. Stewart, A. A. Herrmann, E. D. Baretta, H. V. Forster, J. H. Crespo, P. E. Newton, and R. J. Soto (Medical College of Wisconsin), Acute and Repetitive Human Exposure to Difluorodichloromethane, report PB-279204/LLC, National Technical Information Service (NTIS), Springfield, VA, Aprii 1976 (RDB6486 from summary)

74 R. W. Reinhold and G. M. Rusch, Review of the Toxicity of Ten Selected Fluorocarbons, Department of Toxicology Report MA-127-80-2, AlliedSignal Incorporated, Morristown, NJ, 22 January 1986 (RDB5143) 
75 A. Azar, C. F. Reinhardt, M. E. Maxfield, P. E. Smith, Jr., and L. S. Mullin (E. I. duPont de Nemours and Company, Incorporated), Experimental Human Exposure to Fluorocardon [sic, Fluorocarbon] 12 (Dichlorodifluoromethane), AlHA Journal, 33(4):207-216, April 1972 (RDB5358)

76 M. A. Lee and R. C. Graham, untitled toxicity review for Freon ${ }^{\circledR} 12$ (methane, dichiorodifluoro-), Haskell Laboratory for Toxicology and Industrial Medicine, E. I. duPont de Nemours and Company, Incorporated, Newark, DE, 19 February 1992 (RDB7525)

77 D. J. Tinston, unpublished data, Central Toxicology Laboratory, Imperial Chemical Industries Limited (ICl), Cheshire, UK, 1976 (RDB6127 from summary)

$78 \mathrm{M}$. H. Litchfield and E. Longstaff (Imperial Chemical Industries, ICI, plc, UK), The Toxicological Evaluation of Chlorofluorocarbon 22 (CFC 22) [sic, HCFC-22], Fundamentals of Chemical Toxicology, 22(6):465-475, 1984 (RDB5923)

79 Chlorodifluoromethane (CAS No. 75-45-6), Joint Assessment of Commodity Chemicals (JACC) report 9, European Chemical Industry Ecology and Toxicology Centre (ECETOC), Brussels, Belgium, 19 October 1989 (RDB3718)

80 S. D. Nash, Inhalation Approximate Lethal Concentration (ALC) of Trifluoromethane, report 641-80, Haskell Laboratory for Toxicology and Industrial Medicine, E. I. duPont de Nemours and Company, Incorporated, Newark, DE, 30 September 1980 (RDB6513)

81 S. R. Skaggs and T. A. Moore (New Mexico Engineering Research Institute, NMERI), Toxicological Properties of Halon Replacements, Halon Replacements, Technology and Science (208th National Meeting, Washington, DC, 21-25 August 1994), ACS Symposium Series 611, American Chemical Society (ACS), Washington, DC, chapter 10, 99-109, 1994 (RDB5526)

82 R. M. Hopkins and J. C. Krantz (Huntingdon Research Center, HRC, USA), Jr., Anesthesia LXXV. Relative Effects of Haloforms and Epinephrine on Cardiac Automaticity, Anesthesia and Analgesia, 47(1):56-67, January-February 1968 (RDB5136)

83 Genetron $^{\circledR}$ 23, MSDS GTRN-0030 V2.94, AlliedSignal Incorporated, Morristown, NJ, January 1997

84 Freon $^{\circledR} 23$ Refrigerant, MSDS DU001064 (2025FR), DuPont Chemicals, Wilmington, DE, 8 January 1998

85 A. Van Poznak and J. F. Artusio, Jr. (New York Hospital and Cornell University Medical College), Anesthetic Properties of a Series of Fluorinated Compounds I. Fluorinated Hydrocarbons, Toxicology and Applied Pharmacology (TAP), 2(4):363-373, July 1960 (RDB5828)

86 F. Leuschner, B. W. Neumann, and F. Hübscher, Report on Subacute Toxicological Studies with Several Fluorocarbons in Rats and Dogs by Inhalation, Fortschritte der Arzneimittelforschung [Progress in Drug Research], 33/ll(10):1475-1476, 1983 (RDB5607 from summary)

87 D. M. Krentz, M. A. Lee, and R. C. Graham, untitled toxicity review for trifluoromethane, Haskell Laboratory for Toxicology and Industrial Medicine, E. I. duPont de Nemours and Company, Incorporated, Newark, DE, 3 August 1992 (RDB5604)

88 S. R. Skaggs (Pacific Scientific), private communication to identify sources of tabulated data, September 1996 
89 S. M. Munley, An Inhalation Developmental Toxicity Study in Rats, report 995-96, Haskell Laboratory for Toxicology and Industrial Medicine, E. I. duPont de Nemours and Company, Incorporated, Newark, DE, 27 February 1997 (RDB7631)

90 S. C. Fagan, A. A. Rahill, G. Balakrishnan, J. R. Ewing, C. A. Branch, and G. G. Brown (Henry Ford Hospital), Neurobehavioral and Physiological Effects of Trifluoromethane in Humans, Journal of Toxicology and Environmental Health, 44:101-109, 1995 (RDB5737)

91 R. J. Parr-Dobrzanski, HFC 32: 4-Hour Acute Inhalation Toxicity Study in the Rat, report CTL/P/3456 for the Program for Alternative Fluorocarbon Toxicity Testing (PAFT), Central Toxicology Laboratory, $\mathrm{ICl}$ Chemicals and Polymers Limited, Cheshire, UK, 5 June 1992 (RDB5740)

92 B. L. Moore, Acute Inhalation Toxicity (FC-32), report 698-75, Haskell Laboratory for Toxicology and Industrial Medicine, E. I. duPont de Nemours and Company, Incorporated, Newark, DE, 18 November 1975 (RDB6171)

93 D. M. Krentz, M. A. Lee, and R. C. Graham, untitled toxicity review for difluoromethane, Haskell Laboratory for Toxicology and Industrial Medicine, E. I. duPont de Nemours and Company, Incorporated, Newark, DE, 15 July 1992 (RDB5862)

94 Programme for Alternative Fluorocarbon Toxicity Testing (PAFT) Toxicology Summaries, PAFT, Bristol, United Kingdom, April 1994 (RDB4A66)

95 L. S. Mullin and H. J. Trochimowicz, Cardiac Sensitization Potential of Difluoromethane, report 257-73, Haskell Laboratory for Toxicology and Industrial Medicine, E. I. duPont de Nemours and Company, Incorporated, Newark, DE, 8 June 1973 (RDB6170)

96 R. J. Parr-Dobrzanski, HFC-32: 28-Day Subacute Inhalation Toxicity Study in the Rat, report CTL/P/3746, ICl Central Toxicology Laboratory, ICl Chemicals and Polymers Limited, Cheshire, UK, 24 August 1992 (RDB5741)

97 J. W. Sarver, 1,1,2-Trifluoro-1,2,2-trichloroethane: Four-Hour Inhalation Toxicity, report 104-71, Haskell Laboratory for Toxicology and Industrial Medicine, E. I. duPont de Nemours and Company, incorporated, Newark, DE, 7 April 1971 (RDB6804)

98 J. W. Clayton, Jr. (E. I. duPont de Nemours and Company, Incorporated), The Toxicity of Fluorocarbons with Special Reference to Chemical Constitution, Journal of Occupational Medicine, 4(5):262-273, May 1962 (RDB5169)

99 J. W. Clayton, Jr. (E. I. duPont de Nemours and Company, Incorporated), Fluorocarbon Toxicity: Past, Present, and Future, Journal of the Society of Cosmetic Chemists, 18:333-350, 27 May 1967 (RDB5C41)

100 Cardiac Arrhythmias Induced by Epinephrine During Inhalation of Certain Halogenated Hydrocarbons: Cardiac Sensitization, report 14-69, Haskell Laboratory for Toxicology and Industrial Medicine, E. I. duPont de Nemours and Company, Incorporated, Newark, DE, 1969 (RDB6A04 from summary)

101 C. F. Reinhardt, L. S. Mullin, and M. E. Maxfield (E. I. duPont de Nemours and Company, Incorporated), Epinephrine-Induced Cardiac Arrhythmia Potential of Some Common Industrial Solvents, Journal of Occupational Medicine, 15(12):953-955, December 1973 (RDB65A0) 
102 J. Raventós and P. G. Lemon (Imperial Chemical Industries Limited, UK), The Impurities of Fluoroethane: Their Biological Properties, British Journal of Anaesthesia, UK, 37(10):716-737, 1965 (RDB6165)

103 J. H. Burn (University of Oxford, UK), Pharmacological Testing of Anaesthetics, Proceedings of the Royal Society of Medicine (meeting of the Section of Anesthetics, 7 November 1958), UK, 52(2):95-98, February 1959 (RDB6587)

104 J. H. Burn, H. G. Epstein, and P. J. Goodford (University of Oxford, UK), The Properties of the Anaesthetic Substance 1:1:2-Trifluoro-1:2-dichloroethane, British Journal of Anaesthesia, 31:518-529, 1959 (RDB6586)

105 G. Paulet, Les Fluorocarbones en Question [Fluorocarbons at Issue], European Journal of Toxicology and Environmental Hygiene, 9(supplement to 7):385-407, 1976 (in French, RDB5179 from summary)

106 R. S. Waritz (E. I. duPont de Nemours and Company, Incorporated), The Toxicology of Some Commercial Fluorocarbons, paper 7, Proceedings of the 2nd Annual Conference on Environmental Toxicology (31 August - 2 September 1971), report AMRL-TR-71-120, Aerospace Medical Research Laboratory, U.S. Air Force, Wright-Patterson Air Force Base, $\mathrm{OH}, 85-99$, December 1971 (RDB65H1)

107 Freon 114, MSDS DU001061 (3038FR), DuPont Chemicals, Wilmington, DE, 8 January 1998

108 Genetron $^{\circledR}$ 114, MSDS GTRN-0028, Honeywell Incorporated, Morristown, NJ, January 2000

109 R. R. Montgomery, M. A. Lee, R. C. Graham, and P. J. Lardear, untitled toxicity review for 1,2-Dichloro-1,1,2,2-tetrafluoroethane, Haskell Laboratory for Toxicology and Industrial Medicine, E. I. duPont de Nemours and Company, Incorporated, Newark, DE, 11 June 1992 (RDB5855)

110 International Programme on Chemical Safety (IPCS), Fully Halogenated Chlorofluorocarbons, Environmental Health Criteria (EHC) report 113, World Health Organization (WHO), Geneva, Switzerland, 1990 (RDB5367)

111 S. D. Morrison, Freon-115 Chloropentafluoroethane (99.91\%) and Freon-116 Hexafluoroethane (99.39\%): Acute Inhalation Toxicity Studies, report 41-62, Haskell Laboratory for Toxicology and Industrial Medicine, E. I. duPont de Nemours and Company, Incorporated, Newark, DE, 19 June 1962 (RDB7616)

112 J. W. Hiddemen (Hazleton Washington, Incorporated, Vienna, VA), Acute and Ten-Day Inhalation Pyrolysis, and Cardiac Sensitization Studies with Freons 14, 116, 218 and 1301, report on project 264-107 for Atlantic Research Corporation, included in report for contract DAHC 19-71-C-0026, U.S. Army Research Office, Arlington, VA, 28 February 1972 (RDB7550)

113 E. T. Mctiale (Atlantic Research Corporation), Habitable Atmospheres Which Do Not Support Combustion, in report for contract DAHC 19-71-C-0026, U.S. Army Research Office, Arlington, VA, 15 March 1972 (RDB5C79)

114 L. S. Mullin and H. J. Trochimowicz, A. Cardiac Sensitization Studies of Hexafluoroethane (F-116), Sulfur Hexafluoride $\left(\mathrm{SF}_{6}\right)$, and Chloropentafluoroethane (F-115); $B$. Literature Review: Toxicity and Areas of Use (F-116 and SF $)$, report 150-72, Haskell 
Laboratory for Toxicology and Industrial Medicine, E. I. duPont de Nemours and Company, incorporated, Newark, DE, 20 April 1972 (RDB-6120)

115 J. H. Wills, P. Bradley, H. Kao, H. Grace, W. Hull, T. B. Griffin, F. Coulston, and E. S. Harris (Albany Medical College and NASA Manned-Spacecraft Center), Sensitization of the Heart to Catecholamine-Induced Arrhythmia by Haloalkanes, abstract 82 (11th Annual Meeting of the Society of Toxicology, SOT), Toxicology and Applied Pharmacology (TAP), 22:305-306, 1972 (RDB6118)

116 M. Hughes, unpublished data, Atlantic Research Corporation, 1973 (RDB6119 from summary)

117 M. A. Lee and R. C. Graham, untitled toxicity review for hexafluoroethane, Haskell Laboratory for Toxicology and Industrial Medicine, E. I. duPont de Nemours and Company, Incorporated, Newark, DE, 26 July 1991 (RDB5605)

118 W. J. Brock, private communication by letter, Haskell Laboratory for Toxicology and Industrial Medicine, E. I. duPont de Nemours and Company, Incorporated, Newark, DE, 21 May 1996

119 R. S. Waritz and J. W. Clayton, Acute Inhalation Toxicity on 1,1-Dichloro-2,2,2trifluoroethane (FC-123, Commercial Grade), report 16-66, Haskell Laboratory for Toxicology and Industrial Medicine, E. I. duPont de Nemours and Company, Incorporated, Newark, DE, 4 February 1966 with correction dated 12 May 1966 (RDB6150)

120 J. W. Clayton, Jr. (E. I. duPont de Nemours and Company, Incorporated), Fluorocarbon Toxicity and Biological Action, Fluorine Chemistry Reviews, 1:197-252, 1967 (RDB5C42)

121 G. T. Hall and B. L. Moore, 1,1-Dichloro-2,2,2-trifluoroethane: Acute Inhalation Toxicity, report 426-75, Haskell Laboratory for Toxicology and Industrial Medicine, E. I. duPont de Nemours and Company, Incorporated, Newark, DE, 16 July 1975 (RDB6139)

122 W. B. Coate (Hazleton Laboratories America, Incorporated), LC S0 $_{0}$ of G123 in Rats, report M165-162 (FYI report OTS-06890695), Allied Chemical Company, Morristown, NJ, 4 October 1976 (RDB6137 from summary)

123 1,1-Dichloro-2,2,2-trifluoromethane (HCFC-123, CAS No. 306-83-2), Joint Assessment of Commodity Chemicals (JACC) report 33, European Chemical Industry Ecotoxicology and Toxicology Centre (ECETOC), Brussels, Belgium, February 1996 (RDB65E8)

124 International Programme on Chemical Safety (IPCS), Partially Halogenated Chlorofluorocarbons (Ethane Derivatives), Environmental Health Criteria (EHC) report 139, World Health Organization (WHO), Geneva, Switzerland, 1992 (RDB5371)

$125 \mathrm{H}$. J. Trochimowicz (E. I. duPont de Nemours and Company, Incorporated), Industrial Research on Alternative Fluorocarbons, Toxicology Letters, 68(1-2):25-30, 1993 (RDB5170)

126 B. H. Robbins (Vanderbilt University School of Medicine), Preliminary Studies of the Anesthetic Activity of Fluorinated Hydrocarbons, Journal of Pharmacology and Experimental Therapeutics, 86:197-204, 1946 (RDB5980)

127 L. S. Mullin, Fluorocarbon 123 (2,2-Dichloro-1,1,1-trifluoroethane): Behavioral Toxicity Testing, report 941-76, Haskell Laboratory for Toxicology and Industrial Medicine, E. I. duPont de Nemours and Company, Incorporated, Newark, DE, 10 December 1976 (RDB6153) 
$128 \mathrm{H}$. Wada, Anesthetic Activity and Fatality (F-123, 123a, 124, and 11), attachment to 19 May 1977 correspondence to M. B. Berenbaum (Allied Chemical Corporation), Daikin Kogyu Company Chemical Division, Settsu-shi, Osaka, Japan, 16 May 1977 (RDBA309)

129 L. S. Mullin, Methods in Behavioral Toxicology, Part I: Testing of Five Industrial Chemicals, report 776-76, Haskell Laboratory for Toxicology and Industrial Medicine, E. I. duPont de Nemours and Company, Incorporated, Newark, DE, 19 October 1976 (RDBA945)

130 T. H. S. Burns (St. Thomas's Hospital and the Royal Northern Hospital, UK), J. M. Hall (Guy's Hospital, UK), A. Bracken, and G. Gouldstone (British Oxygen Company, UK), Fluorine Compounds in Anaesthesia (5): Examination of Six Heavily Halogenated Aliphatic Compounds, Anaesthesia, 17(3):337-343, July 1962 (RDB5939)

$131 \mathrm{H}$. J. Trochimowicz (E. I. duPont de Nemours and Company, Incorporated), The Toxicology of HCFC-123 (2,2-Dichloro-1,1,1-Trifluoroethane), Proceedings of the European Meeting of the Toxicology Forum (Toulouse, France, 18-22 September 1989), Sociéte Française de Toxicologie, France, 182-190, 1989 (RDB6145)

132 S. Magda (Solvay Pharmaceuticals, Germany), Fluorocarbons, Toxicology (edited by $\mathrm{H}$. Marquardt), Academic Press, San Diego, CA, 659-662, 1999 (RDBA526)

133 P. Hoet (Catholic University of Louvain, CUL, Belgium), M. L. Graf, M. Bourdi, L. R. Pohl (National Heart, Lung, and Blood Institute, UK), P. H. Duray (National Cancer Institute, UK), W. Chen, R. M. Peter, S. D. Nelson (University of Washington, USA), N. Verlinden (Beerse, Belgium), D. Lison (CUL), Epidemic of Liver Disease Caused by Hydrochlorofluorocarbons Used as Ozone-Sparing Substitutes of Chlorofluorocarbons, Lancet, 350(9077):556-559, 23 August 1997 (RDB7C70)

134 W. Johnson, Unsafe Application of HCFC-123, -124 Blamed for Liver Damage in 9 Workers, The Air Conditioning, Heating, and Refrigeration News, 1-2 and 19, 1 September 1997 (RDB7C74)

135 P. S. Zurer, Liver Damage Tied to CFC Substitute, Chemical and Engineering News, 25 August 1997 (RDB7C72)

136 T. Takebayashi (Keio University School of Medicine, Japan), I. Kabe, Y. Endo, S. Tanaka (Kitasato University, Japan), H. Miyauchi, K. Nozi, S. Imamiya, K. Takahashi, and K. Omae, Exposure to 2,2-Dichloro-1,1,1-trifluoroethane (HCFC-123) and Acute Liver Dysfunction: A Causal Inference, Journal of Occupational Health, Japanese Society of Occupational Health, Tokyo, Japan, 40(4):334-338, October 1998 (RDBA927)

137 Gleason and W. B. Coate (Hazleton Laboratories America, Incorporated), LC So $_{50}$ of 124 in Rats, report MA-RR-89-1450 (also identified as project 165-163), Allied Chemical Company, Morristown, NJ, 4 October 1976 (RDB5806)

138 C. E. Finegan and G. M. Rusch (AlliedSignal Incorporated), Update: Program for Alternative Fluorocarbon Toxicity Testing, Stratospheric Ozone Protection for the 90's (proceedings of the International CFC and Halon Alternatives Conference, Washington, DC), Alliance for Responsible CFC Policy, Arlington, VA, 895-904, October 1993 (RDB3A53)

139 Genetron $^{\circledast}$ 124, MSDS GTRN-0012, Honeywell Incorporated, Morristown, NJ, January 2000 
140 D. P. Kelly, Four-Hour Inhalation Approximate Lethal Concentration (ALC) of HCFC124 in Rats, report 71-90, Haskell Laboratory for Toxicology and Industrial Medicine, E. I. duPont de Nemours and Company, Incorporated, Newark, DE, 19 June 1990 (RDB6180)

141 Suva $^{\circledR}$ 124, MSDS DU002790 (2200FR), DuPont Chemicals, Wilmington, DE, 8 January 1998

142 1-Chloro-1,2,2,2-tetrafluoroethane (HCFC 124, CAS No. 2837-89-0), Joint Assessment of Commodity Chemicals (JACC) report 25, European Chemical Industry Ecotoxicology and Toxicology Centre (ECETOC), Brussels, Belgium, July 1994 (RDB4C02)

143 T. J. Kenny and C. J. Hardy (Huntingdon Research Centre Limited, HRC, UK), FRIGC ${ }^{\text {Tu }}$ FR-12 ${ }^{\mathrm{Tm}}$ : Assessment of Cardiac Sensitization Potential in Dogs, report HZL 6/942384, Hazleton Washington, Incorporated, Vienna, VA, 31 May 1994 (RDB7407)

144 Hydrofluorocarbons and Hydrochlorofluorocarbons, external review draft of interim report, U.S. Environmental Protection Agency, Washington, DC, 15 November 1990

145 E. Nakayama, K. Nagano, M. Chrishi, S. Katagiri, and O. Motegi (Japan Bioassay Laboratory, JBL, Japan), Acute Inhalation Toxicity Study of 1,1,1,2,2-Pentafluoroethane (HFC-125) in Rats, report on study 0184 (also identified as MA-RR-92-1801) for the Program for Alternative Fluorocarbon Toxicity Testing (PAFT), Japan Industrial Safety and Health Association, Kanagawa, Japan, 18 March 1992 (RDB5754)

146 A. S. Panepinto, Four-Hour Inhalation Approximate Lethal Concentration (ALC) of HFC-125 in Rats, report 582-90, Haskell Laboratory for Toxicology and Industrial Medicine, E. I. duPont de Nemours and Company, Incorporated, Newark, DE, 4 December 1990 (RDB6457)

147 C. J. Hardy, P. C. Kieran, I. J. Sharman, and G. C. Clark (Huntingdon Research Centre Limited, HRC, UK), Assessment of Cardiac Sensitization Potential in Dogs: Comparison of HFC 125 and Halon 13B1, report MA-RR-92-1845 (also identified as ALS 11/920116) for the Program for Alternative Fluorocarbon Toxicity Testing (PAFT), AlliedSignal Incorporated, Morristown, NJ, 10 June 1992 (RDB5755)

148 E. Nakayama, K. Nagano, M. Ohnishi, and O. Motegi (Japan Bioassay Laboratory, JBL, Japan), Thirteen-Week Inhalation Toxicity Study of 1,1,1,2,2-Pentafluoroethane (HFC125) in Rats, report 0197 for the Program for Alternative Fluorocarbon Toxicity Testing (PAFT), Japan Industrial Safety and Health Association, Kanagawa, Japan, 31 August 1993 (RDB5761)

149 D. B. Warheit, Four-Hour Inhalation Approximate Lethal Concentration (ALC) of HFC134 in Rats, report 452-91, Haskell Laboratory for Toxicology and Industrial Medicine, E. I. duPont de Nemours and Company, Incorporated, Newark, DE, 22 July 1991 revised 7 August 1991 (RDB6188)

150 HFC-124, MSDS DU008051 (6043FR), DuPont Chemicals, Wilmington, DE, 18 June 1995

151 Huntingdon Research Centre Limited (HRC), HFC 134: Assessment of Cardiac Sensitization Potential in Dogs, report DPT 300/932252, Haskell Laboratory for Toxicology and Industrial Medicine, E. I. duPont de Nemours and Company, Incorporated, Newark, DE, 21 February 1994 (RDB6802)

152 Suva ${ }^{\circledR 4}$ 134, MSDS DU000693 (2187FR), DuPont Chemicals, Wilmington, DE, 8 January 1998 
153 Fluorocarbon Gas HFC-134a, MSDS E(J)G-3200 00116, Daikin Industries Limited Chemical Division, Osaka, Japan, 6 July 1998

154 L. S. Silber, Acute Inhalation Toxicity Study of Tetrafluoroethane, report 422-79, Haskell Laboratory for Toxicoiogy and Industrial Medicine, E. I. duPont de Nemours and Company, Incorporated, Newark, DE, 3 August 1979 (RDB6529)

155 Meforex ${ }^{\circledR}$ 134a, Safety Data Sheet sym/ss101, Ausimont S.p.A., Bollate, Italy, 9 April 1996

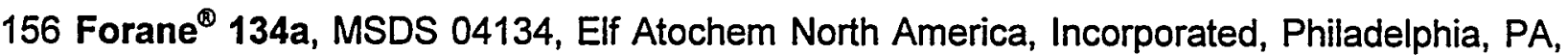
17 July 1999

157 Genetron $^{\circledR}$ 134a, MSDS GTRN-0011, Honeywell Incorporated, Morristown, NJ, January 2000

158 1,1,1,2-Tetrafluoroethane (HFC-134a, CAS No. 811-97-2), Joint Assessment of Commodity Chemicals (JACC) report 31, European Chemical Industry Ecology and Toxicology Centre (ECETOC), Brussels, Belgium, February 1995 (RDB6533)

159 M. A. Collins, HFC 134a: Acute Toxicity in Rats to Tetrafluoroethane, unpublished data, ICl Chemicals and Polymers Limited, Cheshire, UK, 1984

$160 \mathrm{~J}$. Ferguson-Smith, Hygiene Standard Documentation - HFC 134a, document HSP/93/02, ICl Chemicals and Polymers Limited, Cheshire, UK, 1993 (RDB5A79)

$161 \mathrm{D}$. J. Tinston, letter to T. D. Browne on Alternative Arctons, ICI Chemicals and Polymers Limited, Cheshire, UK, December 1976

162 S. B. Rissolo, 1,1,1,2-Tetrafluoroethane (Freon ${ }^{\circledR}$ 134a): Acute Inhalation Toxicity, report 190-67, Haskell Laboratory for Toxicology and Industrial Medicine, E. I. duPont de Nemours and Company, Incorporated, Newark, DE, 2 November 1967 (RDB6525)

163 L. S. Mullin, Ethane, 1,1,1,2-tetrafluoro-: Cardiac Sensitization, report 42-79, Haskell Laboratory for Toxicology and Industrial Medicine, E. I. duPont de Nemours and Company, Incorporated, Newark, DE, 26 January 1979 (RDB6527)

164 M. Shulman and M. S. Sadove (University of Illinois Research and Education Hospitals), 1,1,1,2-Tetrafluoroethane. An Inhalation Anesthetic Agent of Intermediate Potency, Anesthesia and Analgesia, 46(5):629-635, September-October 1967 (RDB7274)

165 D. J. Alexander (Glaxo Research and Development Limited, UK), Safety of Propellants, Journal of Aerosol Medicine, 8(supplement 1):S29-S34, 1995 (RDB8111)

166 A. Vinegar (ManTech Environmental Technology, Incorporated), R. Cook (U.S. Air Force, USAF), J. D. McCafferty (USAF), M. C. Caracci (Geocenters Incorporated), and G. W. Jepson (ManTech), Human Inhalation of Halon 1301, HFC-134a, and HFC-227ea for Collection of Pharmacokinetic Data, Armstrong Laboratory, U.S. Air Force, Wright-Patterson Air Force Base, OH, 19 August 1997 (RDB7C80)

167 PAFT Statement on Wright Patterson/Armstrong Laboratory Study Involving HFC134a and HFC-227ea, press release, Programme for Alternative Fluorocarbon Toxicity Testing (PAFT), Washington, DC, 24 September 1997 (RDB7C81)

168 T. A. Mahoney, Test Subject Faints After Inhaling HFC-134a in Botched Air Force Exposure Experiments, The Air Conditioning, Heating, and Refrigeration News, 202(8):1-2, 20 October 1997 (RDB7C82) 
169 D. A. Keller et al., HFOC-134E, report for project MR-9563-1, Haskell Laboratory for Toxicology and Industrial Medicine, E. I. duPont de Nemours and Company, Incorporated, Newark, DE, 25 March 1994 (RDB6207)

170 R. C. Graham and P. J. Lardear, untitled review of toxicity literature for bis(difluoromethyl) ether, Haskell Laboratory for Toxicology and Industrial Medicine, E. I. duPont de Nemours and Company, Incorporated, Newark, DE, 15 April 1994 (RDB6206)

171 C. W. Simons, G. J. O'Neill, and J. A. Gribens, New Aerosol Propellants for Personal Products, U.S. patent 4,041,148, 9 August 1977 (RDBA944)

172 G. J. O'Neill (Hampshire Chemicals Corporation), Development of a New Alternative: E134, unpublished presentation (The Global Business Outlook for CFC Alternatives, Boston, MA, 15-16 June 1993), Hampshire Chemicals Corporation, Nashua, NH, June 1993 (RDB5550)

173 C. J. Hardy, G. C. Jackson, R. S. Rao, D. J. Lewis, and C. Gopinath (Huntingdon Research Centre Limited, HRC, UK), HCFC 141b Acute Inhalation Toxicity Study in Rats 4-Hour Exposure, report PWT 95/881676, Pennwalt Corporation, King of Prussia, PA, 17 July 1989 (RDB5995)

174 J. A. Seckar (Pennwalt Corporation), The Toxicology of HCFC-141b (1,1-Dichloro-1fluoroethane), Journal of the American College of Toxicology, 8(6):1221, 1989 (RDB7278)

175 C. Doleba-Crowe, Acute Inhalation Toxicity - 6 Hours (FC 141b), report 61-77, Haskell Laboratory for Toxicology and Industrial Medicine, E. I. duPont de Nemours and Company, Incorporated, Newark, DE, 17 February 1977 (RDB6465)

176 M. A. Lee, D. M. Krentz, and R. C. Graham, untitled toxicity review for ethane, 1,1-dichloro1-fluoro-, Haskell Laboratory for Toxicology and Industrial Medicine, E. I. duPont de Nemours and Company, Incorporated, Newark, DE, 24 November 1993 (RDB5868)

177 P. J. M. Janssen (Duphar B.V., The Netherlands), Acute Inhalation Toxicity Studies on FC 141 b in Rats, report S.8820 (also identified as 55645/61/88), Solvay et Cie S.A., Brussels, Belgium, October 1988 with June 1989 supplement (RDB6463)

178 L. S. Mullin, Fluorocarbon 141b (Ethane, 1,1-dichloro-1-fluoro): Cardiac Sensitization, report 957-77, Haskell Laboratory for Toxicology and Industrial Medicine, E. I. duPont de Nemours and Company, Incorporated, Newark, DE, 23 November 1977 (RDB6467)

179 R. J. Kenny (Huntingdon Research Centre Limited, HRC, UK), HCFC-1717: Assessment of Cardiac Sensitization Potential in Dogs, report ALS 57/942811 (also identified as MARR-95-2156), AlliedSignal Incorporated, Morristown, NJ, USA, 22 December 1994 (RDB7206)

180 W. J. Brock (E. I. duPont de Nemours and Company, USA), H. J. Trochimowicz (DuPont), R-J. Millischer (Elf Atochem S.A., France), C. H. Farr (Elf Atochem North America, USA), T. Kawano (Daikin Industries, Japan), and G. M. Rusch (AlliedSignal Incorporated, USA), Acute and Subchronic Toxicity of 1,1-Dichloro-1-fluoroethane (HCFC-141b), Food and Chemical Toxicology, 33(6):483-490, June 1995 (RDB65A4)

181 R-J. Millischer (Elf Atochem S.A., France), C. H. de Rooij (Solvay S.A., Belgium), G. M. Rusch (AlliedSignal Incorporated), C. H. Farr (Elf Atochem North America, USA), R. BenDyke (Ben-Dyke Associates Incorporated, USA), C. J. Hardy (Huntingdon Research Centre Limited, HRC, UK), D. J. Lewis (HRC), and G. Hudson-Walker (Pharmaco:LSR, UK), Evaluation of the Genotoxicity and Chronic Inhalation Toxicity of 1,1-Dichloro-1- 
Fluoroethane (HCFC-141b), Food and Chemical Toxicology, 33(6):491-500, June 1995 (RDB65A8)

182 R. H. Davies, R. D. Bagnall, W. Bell, and W. G. M. Jones, The Hydrogen Bond Proton Donor Properties of Volatile Halogenated Hydrocarbons and Ethers and their Mode of Action in Anesthesia, International Journal of Quantum Chemistry: Quantum Biology Symposium, 3:171-185, 1976 (RDB6555)

183 Chlorodifluoroethane (1-Chloro-1,1-difluoroethane; HFA-142b, CAS: 75-68-3), Joint Assessment of Commodity Chemicals (JACC) report 17, European Chemical Industry Ecology and Toxicology Centre (ECETOC), Brussels, Belgium, February 1991 (RDB3721)

184 C. P. Carpenter, H. F. Smyth, Jr., and U. C. Pozzani (Mellon Institute, University of Pittsburgh), The Assay of Acute Vapor Toxicity and the Grading and Interpretation of Results on 96 Chemicals, Journal of Industrial Hygiene and Occupational Medicine, 31(6):343-346, 1949 (RDB5643)

185 F. J. Mecler and P. J. Knapinsky, Acute Inhalation Toxicity Study in Rats. Isotron 142b, report for project 20888, Litton Bionetics Incorporated (LBI), Kensington, MD, 1978 (RDB59A8)

186 L. S. Mullin, Cardiac Sensitization, report 354-69, Haskell Laboratory for Toxicology and Industrial Medicine, E. I. duPont de Nemours and Company, Incorporated, Newark, DE, 1969 (RDB6274)

187 D. P. Kelly, Four-Hour Acute Inhalation Toxicity Study with FC-143a in Rats, report 283-90, Haskell Laboratory for Toxicology and Industrial Medicine, E. I. duPont de $\mathrm{Ne}$ mours and Company, Incorporated, Newark, DE, 6 August 1990 (RDB5C17)

188 S. Cracknell (Life Science Research Limited, LSR, UK), Forane 143a: Acute Inhalation Toxicity in the Rat, report 91/ATH007/1159 (also identified as 91/1159), Elf Atochem S.A., Paris - La Défense, France, 4 February 1992 (RDB7450)

189 T. K. Nikijenko and M. S. Tolgskaya (Institute for Hygiene at Work and Occupational IIInesses of the AMS, USSR), On the Toxico-Pharmamorphological Changes in Animals Under the Effect of Freon ${ }^{\circledR} \mathrm{S} 141,142$, and 143 and the Intermediates Used in Their Production, Gigiena Truda i Professional'nye Zabolevaniya [Labor Hygiene and Occupational Diseases], Moscow, USSR, 9(10):37-44, 1965 (in Russian, RDB5852 from summary)

190 C. J. Hardy and I. J. Sharman (Huntingdon Research Centre Limited, HRC, UK), HFC 143a: Assessment of Cardiac Sensitization Potential in Dogs, report DPT 281/930477 Haskell Laboratory for Toxicology and Industrial Medicine, E. I. duPont de Nemours and Company, Incorporated, Newark, DE, 3 June 1993 (RDB5C26)

191 W. J. Brock, H. J. Trochimowicz (E. I. duPont de Nemours and Company, USA), C. H. Farr (Elf Atochem North America, USA), R-J. Millischer (Elf Atochem S.A., France), and G. M. Rusch (AlliedSignal Incorporated, USA), article 0092, Acute, Subchronic, and Developmental Toxicity and Genotoxicity of 1,1,1-Trifluoroethane (HFC-143a), Fundamental and Applied Toxicology, 31(2):200-209, June 1996 (RDB6597)

192 B. L. Moore, Difluoroethane: Acute Inhalation Toxicity, report 699-75, Haskell Laboratory for Toxicology and Industrial Medicine, E. I. duPont de Nemours and Company, Incorporated, Newark, DE, 18 November 1975 (RDB5C85) 
193 G. Limperos, Inhalation Toxicity Studies of Various Freon Compounds, report 2-52, Haskell Laboratory for Toxicology and Industrial Medicine, E. I. duPont de Nemours and Company, Incorporated, Newark, DE, 26 December 1951 (RDB7737)

194 D. A. Keller, D. C. Roe, and P. H. Lieder (E. I. duPont de Nemours and Company), Fluoroacetate-Mediated Toxicity of Fluorinated Ethanes, article 0058, Fundamental and Applied Toxicology, 30(2):213-219, April 1996 (RDBA648)

195 Workplace Environmental Exposure Level Guide - 1,1-Difluoroethane, American Industrial Hygiene Association (AlHA), Fairfax, VA, 1994 (RDB4B90)

196 N. F. Izmerov, I. V. Sanotsky, and K. K. Siderov, Toxicometric Parameters of Industrial Toxic Chemicals Under Single Exposure, USSR State Committee for Science and Technology, Moscow, USSR, 1982 (in Russian, RDB5C91 from summary)

197 D. M. Aviado (University of Pennsylvania School of Medicine), Toxicity of Propellants, Fortschritte der Arzneimittelforschung [Progress in Drug Research], 18:365-397, 1974 (RDB6593)

198 D. M. Aviado (University of Pennsylvania School of Medicine), Toxicity of Aerosol Propellants in the Respiratory and Circulatory Systems, $X$. Proposed Classification, Toxicology, 3(3):321-332, 1975 (RDB5920)

199 J. C. Edwards, Arcton 152a - Documentation for Hygiene Standard, ICl Chemicals and Polymers Limited, Cheshire, UK, 17 June 1985 (RDB5A81)

200 M. A. Lee, P. W. Grube, and R. C. Graham, untitled toxicity review for 1,1-difluoroethane, Haskell Laboratory for Toxicology and Industrial Medicine, E. I. duPont de Nemours and Company, Incorporated, Newark, DE, 4 May 1990 (RDB5857)

201 1,1-Difluoroethane (HFC 152a) Health Effects Information, CAS\# 75-37-6, Elf Atochem North America, Incorporated, Philadelphia, PA, undated circa 1995 (RDB5B59)

202 Daiflon 152a, MSDS E(J)G-3200 00117, Daikin Industries Limited Chemical Division, Osaka, Japan, 9 March 1998

203 HFC-152a, MSDS DU005704 (1058FR), DuPont Chemicals, Wilmington, DE, 29 June 1998

204 Forane ${ }^{\circledR}$ 152a, MSDS for product 04105, Elf Atochem North America, Incorporated, Philadelphia, PA, 8 May 2000

205 Genetron $^{\circledR}$ 152a, Material Safety Data Sheet (MSDS) GTRN-0035, Honeywell Incorporated, Morristown, NJ, January 2000

206 D. M. Aviado, Toxicity of Aerosol Propellants in the Respiratory and Circulatory Systems, X. Proposed Classification, Toxicology, 3(3):321-332, 1975 (RDB5920)

207 D. M. Aviado (University of Pennsylvania School of Medicine), Comparative Cardiotoxicity of Fluorocarbons, Cardiac Toxicology, edited by T. Balazs, CRC Press, Incorporated, Boca Raton, FL, II:213-222, 1981 (RDB6477)

208 J. W. Hiddeman (Hazleton Washington, Incorporated, Vienna, VA), Acute and Ten-Day Inhalation, Pyrolysis, and Cardiac Sensitization Studies with Freons 14, 116, 218, and 1301, report on Hazleton project 264-107 (inciuded in report for contract DAHC 19-71-C0026), U.S. Army Research Office, Arlington, VA, 18 February 1972 (RDB7550) 
209 Huntingdon Research Centre Limited (HRC, UK), report MIN 68/930457, 3M, St. Paul, MN, May 1993 (RDB65F8 from summary)

210 Product Toxicity Summary Sheet: PF-5030 3M Brand Performance Fluid, summary 25937, 3M, St. Paul, MN, 22 October 1993 (RDB5703)

211 Huntingdon Research Centre Limited (HRC, UK), report MIN 101/930623, 3M, St. Paul, MN, 23 June 1993 (RDB65F9)

212 Dr. Bury, ARW-Begründung für HFA-227 [Documentation for Exposure Limit for HFA227], Hoechst Aktiengesellschaft, Frankfurt am Main, Germany, 26 August 1992 (in German, RDB7116)

213 EC Safety Data Sheet - Reclin 227, SDS for substance 45257, Hoechst Aktiengesellschaft, Frankfurt am Main, Germany, 2 November 1995

214 WIL Research Laboratories, Incorporated, Acute Inhalational Toxicity Screen in Albino Rats with FM-200 ${ }^{\text {TM }}$, report on project WIL-12226, Great Lakes Chemical Corporation, West Lafayette, IN, 1991 (RDBA617 from summary)

$215 \mathrm{FM}-200^{\mathrm{TM}}$ Toxicological Study Summaries, Great Lakes Chemical Corporation, West Lafayette, IN, undated, circa 1995 (RDB65F2)

216 Heptafluoropropane (HFC-227ea) - Priority Existing Chemical 4 Secondary Notification Assessment, full public report, file EX/5 (NA/64), National Industrial Chemicals Notification and Assessment Scheme (NICNAS), Worksafe Australia, Sidney Australia, 30 July 1999 (RDBA612)

217 WIL Research Laboratories, Incorporated, An Acute Inhalational Toxicity Screen of FM$200^{\text {nd }}$ in Rats, report on project WIL-12248, Great Lakes Chemical Corporation, West Lafayette, IN, 1992 (RDBA616 from summary)

218 S. R. Skaggs and T. A. Moore (New Mexico Engineering Research Institute, NMERI), Toxicological Properties of Halon Replacements, Halon Replacements, Technology and Science (208th National Meeting, Washington, DC, 21-25 August 1994), ACS Symposium Series 611, American Chemical Society (ACS), Washington, DC, chapter 10, 99-109, 1994 (RDB5526)

219 Halon Alternatives Group (HAG), A Review of the Toxic and Asphyxiating Hazards of Clean Agent Replacements for Halon 1301, Loss Prevention Council, London, UK, February 1995 (RDBA668)

220 WIL Research Laboratories, Incorporated, Acute Cardiac Sensitization Study in Dogs with FM-200 ${ }^{\text {th }}$ by Inhalation, report on project WIL-12265, Great Lakes Chemical Corporation, West Lafayette, IN, 1993 (RDBA615 from summary)

221 Acute Inhalation Toxicity Study of HFC-236fa in Albino Rats, report WIL-189022, WIL Research Laboratories, Incorporated, circa 1996 (RDB6A94 from summary)

222 D. A. Keller, Acute Inhalation Toxicity of HFC-236fa and HFC-236ea in the Rat, report 761-93, Haskell Laboratory for Toxicology and Industrial Medicine, E. I. duPont de $\mathrm{Ne}-$ mours and Company, Incorporated, Newark, DE, 21 November 1994 (RDB6803)

223 R. J. Hilarski (International Research and Development Corporation, IR\&D), Nose Only Acute Inhalation Toxicity Evaluation on T-6195 in Rats, report 137-178, 3M Company, St. Paul, MN, 27 September 1995 (RDB6692) 
224 Workplace Environmental Exposure Level Guide - 1,1,1,3,3,3-Hexafluoropropane, American Industrial Hygiene Association (AlHA), Fairfax, VA, 1998 (RDB8612)

225 C. J. Hardy, P. C. Kieran, and I. J. Sharman (Huntingdon Research Centre Limited, UK), HFC 236fa: Assessment of Cardiac Sensitization Potential in Dogs, report DPT 293/931308, Haskell Laboratory for Toxicology and Industrial Medicine, E. I. duPont de Nemours and Company, Incorporated, Newark, DE, 3 February 1994 (RDB65A5)

226 R. Valentine, D. A. Keller, J. C. Cook, L. B. Biegel, G. S. Elliott, T. W. Slone, S. M. Munley, K. S. Bentley, W. J. Brock (E. I. duPont de Nemours and Company, Incorporated), and G. B. Briggs (Geo-Centers, Incorporated), Toxicologic Profile of the Alternative Refrigerant HFC-236fa, Fundamental and Applied Toxicology, 30(1/2):291, March 1996 (RDB6A96)

227 90-Day Inhalation Toxicity: 90-Day Study with HFC-236fa in Rats, report 211-95, Haskell Laboratory for Toxicology and Industrial Medicine, E. I. duPont de Nemours and Company, Incorporated, Newark, DE, 12 February 1996 (RDBA306)

228 R. Valentine, Two-Week Inhalation Toxicity Study with HFC-236fa in Rats, report 59694, Haskell Laboratory for Toxicology and Industrial Medicine, E. I. duPont de Nemours and Company, Incorporated, Newark, DE, 20 June 1995 (RDB6A93)

229 R. D. Bagnall R. D. Bagnall, W. Bell, and K. Pearson (Imperial Chemical Industries Limited, UK), New Inhalation Anaesthetics: IV - Fluorinated Propanes, Journal of Fluorine Chemistry, 13(3):209-223, 1979 (RDB5C76)

230 P. W. Grube, untitled toxicity review for 1,1,2,2,3-pentafluoropropane, Haskell Laboratory for Toxicology and Industrial Medicine, E. I. duPont de Nemours and Company, Incorporated, Newark, DE, 24 July 1991 (RDB5859)

23.1 N. D. Smith (U.S. Environmental Protection Agency, EPA), K. Ratanaphruks, M. W. Tufts, and $\mathrm{A}$. S. Ng (Acurex Environmental Corporation), R-245ca: A Potential Far-Term Alternative for R-11, ASHRAE Journal, 35(2):19-23; February 1993 (RDB3301)

232 N. D. Smith (U.S. Environmental Protection Agency, EPA), A. S. Ng, M. W. Tufts, A. M. Drago, and K. Ratanaphruks (Acurex Environmental Corporation), Evaluation of HFC245fa as a Potential Alternative for R-11 in Low-Pressure Chillers, Stratospheric Ozone Protection for the 90's (proceedings of the International CFC and Halon Alternatives Conference, Washington, DC, 24-26 October 1994), Alliance for Responsible Atmospheric Policy, Arlington, VA, 894-901, October 1994 (RDB5805)

233 Report 33-60, Haskell Laboratory for Toxicology and Industrial Medicine, E. I. duPont de Nemours and Company, Incorporated, Newark, DE, date 1960 (RDB5C77 from summary)

234 G. C. Jackson (Huntingdon Life Sciences Limited, HLS, UK), HFC 245fa (493-95A): Acute Inhalation Toxicity in Rats 4-Hour Exposure, report ALS 104/951746 (also identified as MA-RR-96-2270), AlliedSignal Incorporated, Morristown, NJ, USA, 22 February 1996 (RDB7111)

235 G. C. Jackson (Huntingdon Life Sciences Limited, HLS, UK), HFC 245fa (513-96A): Acute Inhalation Toxicity in Rats (4-Hour Exposure), report ALS 125/961468 (also identified as MA-RR-97-2342), AlliedSignal Incorporated, Morristown, NJ, USA, 27 January 1997 (RDB7115 from summary)

236 G. M. Rusch (AlliedSignal Incorporated, USA), D. W. Coombs, and C. J. Hardy (Huntingdon Life Sciences Limited, HLS, UK), The Acute, Genetic, Developmental, and Inhala- 
tion Toxicity of 1,1,1,3,3-Pentafluoropropane, Toxicological Sciences, 52(2):289-301, December 1999 (RDB9707)

237 T. J. Kenny (Huntingdon Research Centre Limited, HRC, UK), HFC 245fa: Assessment of Cardiac Sensitization Potential in Dogs, report ALS 52/942695 (also identified as MARR-94-2151), AlliedSignal Incorporated, Morristown, NJ, USA, 29 November 1994 (RDB7112 from summary)

238 HFC-245fa, Material Safety Data Sheet (MSDS) GTRN-0040, Allied Signal Incorporated (with a sticker indicating a name change to Honeywell), Morristown, NJ, January 1999

239 C. J. Kirwin, Toxicological Review of Propane, Butane, Isobutane, Pentane, and Isopentane, Phillips Petroleum Company, Bartlesville, OK, November 1976 (RDBA623)

240 Toxicity Study Summary: Propane (Hydrocarbon Propellant A-108), Phillips Petroleum Company, Bartlesville, OK, February 1990 (RDB7624)

241 J. C. Krantz, Jr., C. J. Carr, and J. F. Vitcha (University of Maryland), Anesthesia XXXI. A Study of Cyclic and Noncyclic Hydrocarbons on Cardiac Automaticity, Journal of Pharmacology and Experimental Therapeutics, 94:315-318, 1948 (RDB6192)

242 D. M. Aviado and M. A. Belej (University of Pennsylvania School of Medicine), Toxicity of Aerosol Propellants on the Respiratory and Circulatory Systems, I. Cardiac Arrhythmia in the Mouse, Toxicology, 2(1):31-42, 1974 (RDB5854)

243 P. A. Sanders, Toxicity of Hydrocarbon Propellants, Aerosol Age, 24(1):24-27,44 1979 (RDBA538)

244 W. E. Brown and V. E. Henderson (University of Toronto, Canada), Experiments with Anesthetic Gases, Anesthesia and Analgesia, 27(1):1-8, 1925 (RDB6108 from summary)

245 R. D. Stewart et al. (Medical College of Wisconsin), Acute and Repetitive Human Exposure to Isobutane and Propane, report IASS-CFTA-MCOW-ENVM-BP-77-1, Medical College of Wisconsin and Milwaukee, Milwaukee, WI, April 1977 (RDB6599 from summary)

246 Freon ${ }^{\circledast} 500$ Refrigerant, material safety data sheet DU001075 (2012FR), DuPont Chemicals, Wilmington, DE, 8 January 1998

247 Forane ${ }^{\circledR} 500$, material safety data sheet for product 04008 (revision 1), Elf Atochem North America, Incorporated, Philadelphia, PA, 17 July 1999

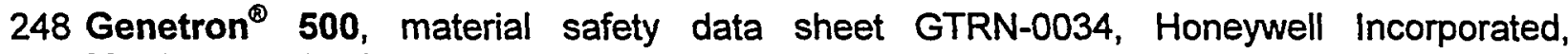
Morristown, NJ, January 2000

249 J. W. Clayton, Jr., Freon-502 Refrigerant (Azeotrope of F-22, 48.8\% and F-115, 51.2\%): Acute Testing, report 91-62, Haskell Laboratory for Toxicology and Industrial Medicine, E. I. duPont de Nemours and Company, Incorporated, Newark, DE, 13 December 1962 (RDB6272)

250 J. W. Clayton, Jr., Freon-502 Refrigerant (Azeotrope of F-22, 48.8\% and F-115, 51.2\%): Acute Testing, report 156-63, Haskell Laboratory for Toxicology and Industrial Medicine, E. I. duPont de Nemours and Company, Incorporated, Newark, DE, 27 December 1963 (RDB6273)

251 Genetron $^{\otimes}$ 502, material safety data sheet GTRN-0031, Honeywell Incorporated, Morristown, NJ, January 2000 
252 J. F. Breen and J. E. Wallis, Report on Refrigerant, Underwriters Laboratories Incorporated (UL), Northbrook, IL (then Chicago, IL), 19 November 1963·(RDB7654)

253 M. A. Lee, untitled toxicity review for Freon ${ }^{\circledR} 502$, Haskell Laboratory for Toxicology and Industrial Medicine, E. I. duPont de Nemours and Company, Incorporated, Newark, DE, 22 May 1992 (RDB5866)

254 V. Valic, Z. Skuric, and E. Zuskin, Experimental Exposures to Fluorochemicals 12, 22, and 502, Rad Jazu, Yugoslavia, 402(18):229-243, 1982 (RDB8183 not reviewed)

255 Toxicity Study Summary: Isobutane (A-31 Hydrocarbon Propellant), Phillips Petroleum Company, Bartlesville, OK, February 1990 (RDB7625)

256 R. W. Stoughton and P. D. Lamson (Vanderbilt University School of Medicine), The Relative Anesthetic Activity of the Butanes and Pentanes, Journal of Pharmacology and Experimental Therapeutics, 58:74-77, 1936 (RDB6197)

257 D. M. Aviado, S. Zakhari, and T. Watanabe (University of Pennsylvania School of Medicine), Non-fluorinated Propellants and Solvents for Aerosols, edited by L. Golberg, CRC Press, Cleveland, OH, 1977 (RDB6569 from summary)

258 P. A. Bingham, Toxicity Data on Refrigerants, private communication by letters, RhônePoulenc Chemicals, 7 and 8 May 1996

259 E. E. Sandmeyer, Aliphatic Hydrocarbons, Patty's Industrial Hygiene and Toxicology (third revised edition), edited by G. D. Clayton and F. E. Clayton, John Wiley and Sons, Incorporated, New York, NY, 2B:3175-3220, 1981 (RDB6290)

260 Isobutane, safety data sheet, Rhône-Poulenc Chemicals, Avonmouth, Bristol, UK, December 1993

261 C. F. Reinhardt, Cardiac Sensitization, report 52-69, Haskell Laboratory for Toxicology and Industrial Medicine, E. I. duPont de Nemours and Company, Incorporated, Newark, DE, 1969 (RDB6121)

262 R. D. Stewart, A. A. Herrmann, E. D. Baretta, H. V. Forster, J. J. Sikora, and R. J. Soto (Medical College of Wisconsin and S. C. Johnson \& Son, Incorporated), Acute and Repetitive Human Exposure to Isobutane, Scandinavian Journal of Work Environment and Health, 1977(3):234-243, 1977 (RDB5364)

263 W. B. Deichmann and H. W. Gerarde, Toxicology of Drugs and Chemicals, Academic Press, New York, NY, 1969 (RDB5266)

264 Occupational Safety and Health Guideline for Ammonia; National Institute of Occupational Safety and Health (NIOSH), U.S. Department of Health and Human Services, Cincinnati, OH; Occupational Safety and Health Administration (OSHA), U.S. Department of Labor, Washington, DC; 1992 (RDB5C46)

265 Ammonia Toxicity Monograph, The Institution of Chemical Engineers, Warwickshire, UK, 1988 (RDB5151)

266 A. Kimmerle, JFF/Combustion Technology, 1:4-51, 1974 (RDB6250 from summary)

267 Y. Alarie (University of Pittsburgh), Dose-Response Analysis in Animal Studies: Prediction of Human Responses, Environmental Health Perspectives (EHP), 42:9-13, 1981 (RDB6279) 
268 K. C. Back, A. A. Thomas, and J. D. MacEwen, Reclassification of Materials Listed as Transportation Health Hazards, report TSA-20-72-3, Aerospace Medical Research Laboratory (AMRL), U.S. Air Force, Wright-Patterson Air Force Base, OH, 1972 (RDB7298)

269 Clement Associates, Incorporated, Health Effects Assessment for Ammonia, The Fertilizer Institute, Washington, DC, February 1990 (RDB1106)

270 Syracuse Research Corporation, Toxicological Profile for Ammonia, report TP-90-03, Agency for Toxic Substances and Disease Registry (ATSDR), Public Health Service, U.S. Department of Health and Human Services, Washington, DC, December 1990 (RDB5156)

271 A. S. Propkop'eva, G. C. Yushkov, and 1. O. Ubasheev, Materials for a Toxicological Characteristic of the Long-Term Effect of Ammonia on the Organisms of Animals After Brief Exposure, Gigiena Truda i Professional'nye Zabolevaniya [Labor Hygiene and Occupational Diseases], USSR, 6:56-57, 1973 (RDB6229 from summary)

272 F. R. Weedon, A. Hartzell, and C. Setterstrom, Toxicity of Ammonia, Chlorine, Hydrogen Cyanide, Hydrogen Sulfide, and Sulfur Dioxide. V. Animals, Contrib. Boyce Thompson Institute, 11:475-482, 1940 (RDB6256 from summary)

273 L. M. Appelman (TNO-CIVO Toxicology and Nutrition Institute, The Netherlands), W. F. ten Berge (DSM, The Netherlands), and P. G. J. Reuzel (TNO-CIVO), Acute Inhalation Toxicology Study of Ammonia in Rats with Variable Exposure Periods, AlHA Journal, 43(9):662-665, September 1982 (RDB5152)

274 Tabulae Biologicae Per., 3:231-296, 1933 (in German, RDB6283 from summary)

275 Ammonia (CAS 7664-41-7), chemical hazard summary 37, report C88-1E, Canadian Centre for Occupational Health and Safety (CCOHS), Hamilton, ON, Canada, 1988 (RDB6270)

276 W. S. Ferguson, W. C. Koch, L. B. Webster, and J. R. Gould, Human Physiological Response and Adaptation to Ammonia, Journal of Occupational Medicine (JOM), 19:319326, 1977 (RDB6237 from summary)

277 K. J. Schadeberg, J. W. Goode, M. L. Keplinger, and J. C. Calandra (Industrial Bio-Test Laboratories, Incorporated), Irritation Threshold Evaluation Study with Ammonia, report IBT 663-03161, International Institute of Ammonia Refrigeration (IIAR), Washington, DC, 23 March 1973 (RDB6A12)

278 D. P. Wallace, Atmospheric Emissions and Control, Ammonia Plant Safety, 21:51-56, 1979 (RDBA672 from summary)

279 W. F. Stoecker (University of Illinois at Urbana-Champaign), Growing Opportunities for Ammonia Refrigeration, Technical Papers of the 11th Annual Meeting (12-15 March 1989, Austin, TX), International Institute of Ammonia Refrigeration (IIAR), Washington, DC, March 1989 (RDB4236)

280 M. M. Verberk, Effect of Ammonia in Volunteers, International Archives of Occupational and Environmental Health, Germany, 39:73-81, 1977 (RDB6A29 from summary)

281 Emergency Response Planning Guidelines - Ammonia, American Industrial Hygiene Association (AlHA), Fairfax, VA, 1988 (RDB4B76)

282 J. Johnston, L. Legters, and R. Dailey (Enviro Control, Incorporated), Biological Effects of Short, High-Level Exposure to Gases: Ammonia, draft final report under contract DMD 17-79-C-9086, U.S. Army Medical Research and Development Command, Fort Detrick, MD, 1979 (RDB6262 from summary) 
283 Ammonia Data Book, International Institute of Ammonia Refrigeration (IIAR), Washington, DC, 1993 (RDB3635)

284 C. S. Barrow, Y. Alarie, and M. F. Stock (University of Pittsburgh), Sensory Irritation and Incapacitation Evoked by Thermal Decomposition Products of Polymers and Comparisons with Known Sensory Irritants, Archives of Environmental Health, 33(2):79-88, March-April 1978 (RDB6231)

285 D. Zissu (Institut National de Recherche et de Sécurité, France), Histopathological Changes in the Respiratory Tract of Mice Exposed to Ten Families of Airborne Chemicals, Journal of Applied Toxicology, 15(3):207-213, 1995 (RDB6A33)

286 L. A. Buckley, X. Z. Jiang, R. A. James, K. T. Morgan, and C. S. Barrow (Chemical Industry Institute of Toxicology, CIIT), Respiratory Tract Lesions Induced by Sensory Irritants at the $\mathrm{RD}_{50}$ Concentration, Toxicology and Applied Pharmacology, 74(3):417-429, 1984 (RDB6227)

287 L. E. Kane, C. S. Barrow, and Y. Alarie (University of Pittsburgh), A Short-Term Test to Predict Acceptable Levels of Exposure to Airborne Sensory Irritants, AlHA Journal, 40:207-229, March 1979 (RDB6249)

288 L. Silverman, J. L. Whittenberger, and J. Muller (Harvard School of Public Health), Physiological Response of Man to Ammonia in Low Concentrations, Joumal of Industrial Hygiene and Toxicology, 31(2):74-78, March 1949 (RDB6226)

289 Y. Henderson and H. W. Haggard, Noxious Gases and the Principles of Respiration Influencing their Action (second edition), Reinhold Publishing Corporation, New York, NY, 1943 (RDB72A0)

290 Hygienic Guide Series - Anhydrous Ammonia, American Industrial Hygiene Association (AlHA), AlHA Journal, 32:139-142, 1971 (RDB5775)

2912000 Threshold Limit Values for Chemical Substances in the Work Environment, 2000 TLVs $^{\circledR}$ and BEIs ${ }^{\circledR}$ : Threshold Limit Values for Chemical Substances and Physical Agents; Biological Exposure Indices, publication 0100, American Conference of Governmental Industrial Hygienists (ACGIH), Cincinnati, OH, 2000 (RDBA936)

292 Commission for the Investigation of Health Hazards of Chemical Compounds in the Work Area, List of MAK and BAT Values 1996: Maximum Concentrations and Biological Tolerance Values at the Workplace, report 32, Deutsche Forschungsgemeinschaft [German Research Association] (DFG), Bonn, Germany; VCH Verlagsgesellschaft mbH, Weinheim, Germany, 1996 (RDB7101)

293 Occupational Safety and Health Administration (OSHA) of the U.S. Department of Labor, Air Contaminants; Rule, 58 FR 124, Federal Register, U.S. Government Printing Office, Washington, DC, 35338-35351, 30 June 1993 (RDB3904)

294 The AlHA 1999 Emergency Response Planning Guidelines and Workplace Environmental Exposure Level Guides Handbook, document 358-EA-99, American Industrial Hygiene Association (AlHA), Fairfax, VA, 1999 (RDB9503)

295 Carbon Dioxide, Gas, material safety data sheet G-8, BOC Gases Division of the BOC Group, Incorporated, Murray Hill, NJ, 7 June 1996 
296 E. B. Brown Jr. and F. Miller, Ventricular Fibrillation Following a Rapid Fall in Alveolar Carbon Dioxide Concentration, American Journal of Physiology, 169:56-60, 1952 (RDBA936 from summary)

297 W. C. Petty and T. S. Sulkowski, Narcosis in the Rat - II. Effects on the ECG, Aerospace Medicine, 42:553-558, 1971 (RDBA937 from summary)

298 F. Caujolle (Centre de Recherches sur les Toxicités du Centre National de la Recherche Scientifique, CNRS, France), Comparative Toxicity of Refrigerants / Toxicité Comparée des Fluides Frigorigènes, topical study 13131, Bulletin of the International Institute of Refrigeration (IIR), 44(1):20-55, 1964 (RDB7424)

299 D. P. Mukherjee and S. P. Singh, Effect of Increased Carbon Dioxide in Inspired Air on the Morphology of Spermatozoa and Fertility of Mice, Joumal of Reproduction and Fertility, 13:165-167, 1967 (RDBA938 from summary)

300 Committee on Aviation Toxicology, Aero Medical Association, Aviation Toxicology, The Blakiston Company, New York NY and Toronto ON, 1953 (RDB72A5)

301 W. J. Friedlander and T. Hill, EEG Changes During Administration of Carbon Dioxide, Diseases of the Nervous System, 15:71-75, 1954 (RDBA663 from summary)

302 C. J. Lambertsen, Therapeutic Gases - Oxygen, Carbon Dioxide, and Helium, Drill's Pharmacology in Medicine (fourth edition), McGraw-Hill Book Company, New York, NY, chapter 55, 1971 (RDBA664 from summary)

303 P. H. Sechzer, L. D. Egbert, H. W. Linde, D. Y. Cooper, R. D. Dripps, and H. L. Price, Effect of $\mathrm{CO}_{2}$ Inhalation on Arterial Pressure, ECG and Plasma Catecholamines and 17OH Corticosteroids in Normal Man, Journal of Applied Physiology, 13:454-458, 1960 (RDBA941 from summary)

304 R. D. Dripps and J. H. Comroe, Jr. (University of Pennsylvania and the Hospital of the University of Pennsylvania), The Respiratory and Circulatory Response of Normal Man to Inhalation of 7.6 and 10.4 Per Cent $\mathrm{CO}_{2}$ with a Comparison to the Maximal Ventilation Produced by Severe Muscular Exercise, Inhalation of $\mathrm{CO}_{2}$, and Maximal Voluntary Hyperventilation, American Journal of Physiology, 149:43-51, 1947 (RDBA658)

305 K. E. Schaefer, The Effects of $\mathrm{CO}_{2}$ and Electrolyte Shifts on the Central Nervous System, Selective Vulnerability of the Brain in Hypoxemia, edited by J. P. Schade and W. H. McMehemy, Blackwell Scientific Publications, Oxford, UK, 101-123, 1963 (RDBA939 from summary)

306 E. W. Brown. (Edgewood Arsenal Medical Corps), The Physiological Effects of High Concentrations of Carbon Dioxide, U.S. Navy Medical Bulletin, 28:721-734, 1930 (RDBA655)

307 W. Storm and C. L. Giannetta, Effects of Hypercapnia and Bedrest on Psychomotor Performance, Aerospace Medicine, 45:431-433, 1974 (RDBA943 from summary)

308 E. B. Weybrew, An Exploratory Study of the Psychological Effects of Intermittent Exposure to Elevated Carbon Dioxide Levels, report 647, Submarine Medical Research Laboratory, Bureau of Medicine and Surgery, U.S. Navy, Groton, CT, 1970 (RDBA942)

309 B. G. King (Medical Service, Civil Aeronautics Administration), High Concentration-Short Time Exposures and Toxicity, Joumal of Industrial Hygiene and Occupational Medicine, 31(6):365-375, November 1949 (RDBA660) 
310 Council on Pharmacy and Chemistry of the American Medical Association (AMA), Reports of the Council: Oxygen-Carbon Dioxide Mixtures, Journal of the American Medical Association, 114(12):1077-1079, 23 March 1940 (RDBA657)

311 F. Flury and F. Zernik, Schädliche gase, dämpfe, nebel, rauch- und staubarten [Dangerous Gases, Fumes, Mists, Smokes, and Dusts], Springer-Verlag (then Verlag von Julius Springer), Berlin, Germany, 1931 (in German, RDB72A6 from summary)

312 K. E. Schaefer, Studies of Carbon Dioxide Toxicity, report 181, Submarine Medical Research Laboratory, Bureau of Medicine and Surgery, U.S. Department of the Navy, New London, CT, 10:156-189, 1951 (RDB72A8 from summary)

313 Workplace Environmental Exposure Level Guide - 1,1,1-Trifluoroethane, American Industrial Hygiene Association (AlHA), Fairfax, VA, 1996 (RDB5C16)

314 Number Designation of Refrigerants, ANSI/ASHRAE Standard 34-78, American Society of Heating, Refrigerating, and Air-Conditioning Engineers (ASHRAE), Atlanta, GA (then in New York, NY), 1978 
acute toxicity

ALC

ACGIH

AlHA

anesthetic effect

ASHRAE

ASHRAE 15

ASHRAE 34

ASHRAE 34 group

ATEL

cardiac sensitization

chronic toxicity

collateral data

CNS

$E C_{50}$

EEGL

EEL

emergency exposure limit

\section{APPENDIX 1: GLOSSARY}

adverse health effects from a short-term, single exposure

approximate lethal concentration (see page 18)

American Conference of Governmental Industrial Hygienists

American Industrial Hygiene Association

dulling of the senses, as manifested in loss of the ability to perceive pain and other sensory stimulation, impairment of judgement or mental ability to respond (see page 9)

American Society of Heating, Refrigerating, and Air-Conditioning Engineers

Safety Code for Mechanical Refrigeration (see reference 2)

Designation and Safety Classification of Refrigerants (see reference 1)

refrigerant safety classification (see page13)

acute toxicity exposure limit (see page 18)

an acute toxicity effect in which the heart is rendered more sensitive to catecholamine-like compounds, possibly resulting in irregular heart beat (cardiac arrhythmia) that could be fatal (see page 9)

adverse health effects from long-term or repeated exposures

data found during searches performed for other, targeted refrigerants (see page 87)

central nervous system (see page 9)

median effective concentration (see page 10)

emergency exposure guidance level set by the NRC for special military purposes

emergency exposure limit

guidance to limit exposures for unplanned and unintended releases (contrast to occupational exposure limit) 
FCL

Haber's law

IDLH

IEL

lethality

$\mathrm{LC}_{50}$

$\mathrm{LC}_{\mathrm{LO}}$

LFL

LOEL

MAK

Mcf

mortality

MSDS

$\mathrm{NIOSH}$

NOEL

NRC

NTP

occupational exposure limit flammable concentration limit (see page 28)

toxic effect of the product of the exposure concentration and duration is constant (see page 18)

immediately dangerous to life and health (see page 12)

industrial exposure limit (see page 13)

potential to cause death (see page 8)

median lethal concentration (see page 10)

lowest lethal concentration: the lowest dose of a chemical reported to fatal to animals or humans

lower-flammability limit

lowest observed effect level (see page 10)

Maximale Arbeitsplatz Konzentration (maximum workplace concentration) set by the Deutsche Forschungsgemeinschaft (DFG, the German Research Association)

thousand cubic feet

potential to cause death (see page 8)

material safety data sheet

National Institute for Occupational Safety and Health (USA)

no observed effect level (see page 10)

National Research Council

National Toxicology Program

guidance to limit exposures for work related use of refrigerants (or other chemicals), which assume that potentially affected personnel should be cognizant of the potential for exposures, have special awareness or training, and may employ protective measures or devices (contrast to emergency exposure limit)

ODL

oxygen deprivation limit (see page 28)

OEL

occupational exposure limit (see page 13)

OSHA

Occupational Safety and Health Administration (USA)

PEL permissible exposure limit (see page 13) 
ppm

ppm v/v

provisional ATEL or RCL

$\mathrm{RCL}$

$\mathrm{RD}_{50}$

REL

SCP

SDS

STEL

subacute

subchronic

TLV

TWA

UL group

$v / v$

WEEL parts per million

parts per million by volume

ATELs and RCLs determined from collateral data and warranting extra caution in use (see page 87 )

refrigerant concentration limit (see pages 15 and 16)

median rate decrease, typically refers to concentration resulting in a $50 \%$ respiratory rate decrease in mice (see page 11 )

recommended exposure limit set by NIOSH (see page REL)

Standards Completion Program (see page 13)

safety data sheet

short term exposure limit

between acute and chronic, typically in reference to a toxicity study of five days to two weeks by some definitions or anything longer than a single exposure or one day up to six months by others: Some toxicologists avoid the term and include such studies in "subchronic."

between acute and chronic, typically in reference to a toxicity study of 15 days to 6 months by some definitions and several days to six months by others

threshold limit value set by ACGIH (see page 13)

time-weighted average

former Underwriters Laboratories refrigerant safety classification (see page 14)

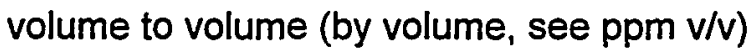

workplace environmental exposure level guides set by AIHA 
Toxicity Data to Determine Refrigerant Concentration Limits James M. Calm, Engineering Consultant 


\section{APPENDIX 2: REFRIGERANT PROFILES}

This appendix presents data summaries from the ARTI Refrigerant Database ${ }^{22}$ for the 35 refrigerants included in Table 1, namely those addressed on pages $35-85$ plus $R-14, R-115, R-170$ (ethane), R-C318, R-600 (n-butane), and R-1270 (propylene).

\section{ARTI REFRIGERANT DATABASE}

The Refrigerant Database is an information system on alternative refrigerants, associated lubricants, and their use in air conditioning and refrigeration. It consolidates and facilitates access to property, compatibility, environmental, safety, application, and other information. It provides corresponding information on older refrigerants, to assist manufacturers and those using alternative refrigerants to make comparisons and determine differences. The underlying purpose is to accelerate phase out of chemical compounds of environmental concern.

The database identifies sources of specific information on R-22, R-23, R-32, R-41, R-116, R123, R-124, R-125, R-134, R-134a, R-141b, R-142b, R-143a, R-152a, R-218, R-227ea, R236fa, R-245ca, R-245fa, R-290 (propane), R-C318, R-717 (ammonia), R-718 (water), R-744 (carbon dioxide), R-1270 (propylene). ethers, and others as well as azeotropic and zeotropic blends of these fluids. These blends include R-400, R-401A, R-401B, R-401C, R-402A, R402B, R-403A, R-403B, R-404A, R-405A, R-406A, R-407A, R-407B, R-407C, R-407D, R-408A, $R-409 A$, R-409B, R-410A, R-410B, R-411A, R-411B, R-412A, R-413A, R-414A, R-414B, R415A, R-416A, R-500, R-501, R-502, R-503, R-504, R-505, R-506, R-507A, R-508A, R-508B, $R-509 A$, and others for which information is available even though standard designations may not have been assigned yet. It addresses lubricants including alkylbenzene, polyalkylene glycol, polyolester, and other synthetics as well as mineral oils. It also references documents addressing compatibility of refrigerants and lubricants with metals, plastics, elastomers, motor insulation, and other materials used in refrigerant circuits.

The database provides bibliographic citations and abstracts for publications that may be useful in research and design of air-conditioning and refrigeration equipment. The complete documents are not included, though some may be added at a later date. Incomplete citations or abstracts are provided for some documents. They are included to accelerate availability of the information and will be completed or replaced in future updates.

\section{LIMITATIONS}

The Refrigerant Database is intended as a means to assist users in locating sources of information on alternative refrigerants. But, the database is:

- neither a comprehensive nor authoritative reference source,

- not a substitute for independent data collection by users,

- not a substitute for examination of the data, information on how they were arrived at, assumptions, and caveats in the cited documents, and 
- not an endorsement of suitability or accuracy of the referenced publications.

The information in the database was obtained from published and unpublished sources, or calculated from them, without verification. Some of the data may be imprecise or incorrect, as manifested - in some cases - by inclusion of conflicting data based on disagreement among identified sources. Similarly, errors may have occurred in assembling and processing the database. Users are cautioned to check the data and associated limitations and caveats in the referenced documents and other sources before use, particularly if such use might risk harm to life or property. Newer or more complete data may be available from refrigerant suppliers or elsewhere.

Materials compatibility, properties, safety considerations, and other characteristics affecting suitability or desirability may be influenced by a number of factors. Among them are specific application conditions, preparation such as drying before use, additives including fillers, impurities, catalytic interactions with other materials used, and changes in compounding between one source or batch and another. Similarly, new findings or corrections may supersede previously published data. The database is an aid in locating data that may be pertinent; it is not and should not be viewed as the source of data for research, design, analysis, or other purposes.

\section{DATA SUMMARIES}

The parameter descriptions that follow summarize the information included in data summaries for refrigerants, also referred to as refrigerant profiles. Each entry consists of the following parts:

- a label to identify and/or explain the data,

- a data value, typically rounded to common representation or limits of precision, generally expressed metric units or dimensionless form (metric units conform to the International System, SI, modified to use the Celsius scale for temperatures in place of the Kelvin scale),

- units of measure if applicable,

- converted data and units in the inch-pound (IP) system for data expressed in metric units,

- qualifying information on the data such as the animal species, exposure duration, and fraction of responses for toxicity test results, exposure periods, or manufacturer identity where the source is a safety data sheet, and

- four-digit alphanumeric Refrigerant Database ("RDB”) number (discussed below).

The specific data included for each single-compound refrigerant or blend depend upon availability. Multiple values are cited for the same or related parameters in some cases, for example when conflicting data were published by multiple credible sources. The purpose of the database is to assist users in locating data rather than to endorse or verify specific data or to resolve inconsistencies and conflicts. Older data generally are deleted for simplification when the original source reports later results or when scientific consensus is reported, but that does not suggest endorsement of the newer data. The data values shown are included to assist users, but the primary information for each entry is the four alphanumeric digit "RDB" number in the rightmost column, which indicates the document or other source from which the data were taken. 
Those sources may be located by searching for the number prefixed by "RDB" (for example $R D B 9901)$ in the database.

\section{Identifiers}

The refrigerant number shown in the heading is the standard designation based on those assigned by or recommended for addition to ASHRAE 34, Designation and Safety Classification of Refrigerants, ${ }^{1}$ as well as pending addenda and common industry extensions thereto. These familiar designations are used almost universally, usually preceded by "R-", "R", the word "Refrigerant", composition-designating prefixes (for example "CFC-", "HCFC-", "HFC-", or "HC-"), or manufacturer trade names. Nonstandard and pending designations generally are flagged as such or identified in notes included with the common uses.

The chemical formula indicates the molecular makeup of the single-compound refrigerants, namely those consisting of a single chemical substance. The blend composition is substituted for refrigerant blends, namely those consisting of two or more chemicals that are mixed to obtain desired characteristics. The composition consists of two parts. The first identifies the components, in order of increasing normal boiling points and separated by slashes. The second part, which is enclosed in parentheses, indicates the mass fractions (as percentages) of those components in the same order. The profiles also indicate alternative chemical names, the common and historic names, index numbers for common reference databases, empirical and structural chemical formulae, and standard container colors. Among the identifiers shown are the:

- standard designation following ASHRAE 34 as well as addenda, pending addenda (flagged), and common industry extensions to it (flagged),

- variants using the "R-", "R", "R ", composition-designating prefixes (for example "CFC-", "HCFC-", or "HFC-"),

- common fluorochemical number and variants,

- halon number for chemicals also used or considered as fire suppressants,

- chemical name following the International Union of Pure and Applied Chemistry (IUPAC) convention,

- chemical name following other common conventions,

- common names,

- structural formula following the IUPAC convention,

- structural formula following other common conventions,

- empirical formula following the Hill convention,

- other formulae including some flagged as "not recommended" to enable location by them

- Chemical Abstracts Service (CAS) registry number,

- Beilstein registry number,

- European Inventory of Existing Chemical Substances (EINECS) number,

- Merck index (volume and number), 
- National Institute of Occupational Safety and Health (NIOSH) Registry of Toxic Effects of Chemical Substances (RTECS) index number,

- trade name(s),

- historical name(s),

- names used for rule making notices for proprietary refrigerants in U.S. Environmental Protection Agency (EPA) Significant New Alternatives Program (SNAP) notices,

- standard container color name and Pantone number as assigned by Air-Conditioning and Refrigeration Institute (ARI) Guideline N (Assignment of Refrigeration Container Colors),

- composition for blends

- both mass and mole formulations for blends, and

- standard mass formulation tolerances for blends with ASHRAE safety classifications.

In some cases, primarily for proprietary blends, additional identifying information is included in the description of common uses.

\section{Common Uses}

The uses focus on application as refrigerants followed, if applicable, by terse indications of other applications if known. Limiting considerations such as toxicity, flammability, reactivity, or environmental concerns are cited for some substances.

This section also includes notes on pending changes in standard designations and safety classifications.

\section{Physical Properties}

The refrigerant profiles indicate key physical, thermodynamic, and transport properties at representative conditions, including the normal boiling point, $20^{\circ} \mathrm{C}\left(68^{\circ} \mathrm{F}\right), 60^{\circ} \mathrm{C}\left(140^{\circ} \mathrm{F}\right)$, and the critical point.

The molecular mass is a calculated value based on the atomic weights recognized by International Union of Pure and Applied Chemists (IUPAC). It indicates the mass in grams of a mole of the refrigerant or, for blends, the mass-weighted average of a mole of the mixture.

The normal boiling point (NBP) is the temperature at which liquid refrigerant will boil at standard atmospheric pressure, namely $101.325 \mathrm{kPa}$ (14.6959 psia). The NBP and most dimensional units in the tables are shown in both metric (SI) and inch-pound units of measure. Both the bubble point (temperature at which a bubble first appears, hence the temperature at which boiling begins, for a blend) and dew point typically are shown for blends.

The critical temperature $\left(T_{c}\right)$ is the temperature at the critical point of the refrigerant. The $T_{c}$ values shown for blends are the mass weighted averages of the component $T_{c} s$ unless actual values have been determined.

The critical pressure $\left(P_{c}\right)$ is the pressure at the critical point. 
The NBP and critical properties suggest the application range for which an individual refrigerant might be suitable. Those with extremely low NBP lend themselves to ultra-low temperature refrigeration, for example in cryogenic applications. Those with high NBPs generally are limited to high-temperature applications, such as for use in chillers or high-temperature heat pumps. Both capacity and efficiency decline in a typical vapor-compression (reverse Rankine) cycle, the one most commonly used, when condensing temperatures approach the $T_{c}$. The $P_{c}$ will exceed the operating pressure, except in transcritical cycles, which are uncommon except for R-744 (carbon dioxide).

\section{Environmental Data}

The atmospheric lifetime $\left(\tau_{\mathrm{atm}}\right)$ is an indication of the average persistence of the refrigerant, if released into the atmosphere, until it decomposes or reacts with other chemicals. The values shown are composite atmospheric lifetimes. Separate lifetimes also are shown if known for the tropospheric (lower atmosphere where we live), stratospheric (next layer where global depletion of ozone is a concern), and higher layers, since the atmospheric chemistry changes between layers.

The ozone depletion potential (ODP) is a normalized indicator, based on a value of 1.000 for $R$ 11 , of the ability of refrigerants (and other chemicals) to destroy stratospheric ozone molecules. The data shown are the modeled values adopted by the international scientific assessment. The ODPs shown for blends are mass-weighted averages. The values shown typically are modeled ODP values, the most indicative of environmental impacts. Semi-empirical ODP and regulatory values adopted in the Montreal Protocol also are indicated for some refrigerants.

The semi-empirical ODPs are calculated values that incorporate adjustments for observed atmospheric measurements. The concept is conceptually more accurate, but it is difficult to measure the data needed for representative adjustments accurately.

The regulatory values generally are required for specific purposes, but may not be updated with newer findings after adoption. The ODP values listed in the annexes to the Montreal Protocol, for example, have not been updated since 1987 for chlorofluorocarbons (CFCs) and 1992 for hydrochlorofluorocarbons (HCFCs). A note in the Protocol indicates that the values "are estimates based on existing knowledge and will be reviewed and revised periodically."

The global warming potential (GWP) is a similar indicator of the potency to warm the planet by action as a greenhouse gas. The values shown are relative to carbon dioxide $\left(\mathrm{CO}_{2}\right)$ for an integration period of $100 \mathrm{yr}$. Both the ODP and GWP are calculated from the $\tau_{\mathrm{atm}}$, measured chemical properties, and other atmospheric data. The GWPs shown for blends are massweighted averages.

The $\tau_{\mathrm{atm}}, \mathrm{ODP}$, and GWP values indicated for blends were calculated for the nominal blend compositions.

The database also indicates halocarbon global warming potential (HGWP) and photochemical reactivity at ground level if known. 


\section{Safety Data}

The safety section is subdivided into classifications, recommended exposure limits, acute (short-term, single exposure) and chronic (long-term, repeated exposure) toxicity data, flammability data, and detection (appearance and odor) information. The exposure limits are further separated into short-term occupational, long-term occupational, and emergency exposures. Depending on the refrigerant, more than 100 parameters - some with differing values for species or exposure durations in toxicity tests - are reported. The following brief summary addresses only the chronic toxicity and flammability indices used to determine standard refrigerant safety classifications.

The first value is an occupational exposure limit, namely the Threshold Limit Value - Time Weighted Average (TLV-TWA) or a consistent measure. It is an indication of chronic (long-term, repeat exposure) toxicity of the refrigerant. Some of the consistent toxicity indices are the workplace Environmental Exposure Level (WEEL) guides and the Permissible Exposure Limit (PEL). These measures indicate adopted limits for workplace exposures for trained personnel for typical workdays and work weeks.

The Lower Flammability Limit (LFL) is the lowest concentration at which the refrigerant will burn in air under prescribed test conditions. It is an indication of flammability. The absence of an LFL or even an indication of nonflammable does not assure that a substance will not burn or exacerbate an existing fire under some conditions, such as when mixed with other fuels (such as lubricants) or at elevated pressures and temperatures.

The Heat of Combustion (HOC) is an indicator of how much energy the refrigerant will release when it burns in air, assuming complete reaction to the most stable products in their vapor state. Negative values indicate endothermic reactions (those that require heat to proceed) while positive values indicate exothermic reactions (those that liberate heat).

The ASHRAE Standard 34 safety group is an assigned classification that is based on the TLVTWA (or consistent measure), LFL, and HOC. It comprises a letter (A or B) that indicates relative toxicity followed by a number $(1,2$, or 3$)$ that indicates relative flammability. These classifications are widely used in mechanical and fire construction codes, to determine requirements to promote safe use. Most of these code provisions are based on ASHRAE 15, Safety Code for Mechanical Refrigeration. ${ }^{2}$

\section{Production Data}

The final section of the refrigerant profiles indicates initial commercialization if known and the last year production is allowed in developed countries under the Montreal Protocol. 


\section{R-11}

Trichlorofluoromethane DATA SUMMARY
CCI3F CAS number 75-69-4

\section{IDENTIFIERS}

common name(s): R-1I; RII; R 11

CFC-11

chTorof7uorocarbon 11

f7uorocarbon 11

fluorochemica7 11; FC 11

halocarbon 11

halochemical 11

chemical name (by IUPAC convention):

trichlorofluoromethane

alternative chemical names/formulae:

methane, trich7orofluoro-

fluorotrichloromethane

trichloromonofluoromethane

CCl3F

not recommended: CFC13; C13FC

CAS number: 75-69-4 (Chemica7 Abstracts

Service registry number)

BeiTstein registry number: 1732469

EINECS number: 200-892-3 (European Inventory

of Existing Chemical

Substances)

Merck Index (volume-number): 12-9770

NIOSH RTECS number: PB6125000 (Registry of Toxic

Effects of Chemical

Substances)

trade name(s): Asahi Glass Fron AF-11

BOC Gases Halocarbon 11 MSDS

Daikin Daiffon (R) 11

DuPont Freon(R) 11

Elf Atochem Forane(R) 11

Hoechst Frigen $(R)$ 11

Honeywe 11 Genetron(R) 11

ICI Arcton (R) 11

Pennwait/Atochem Isotron(R) 11

historical name(s): A]1jed Corp refrigerant 43

Altiedsignal Genetron $(R) 11$

Carrier corporation Carrene 2

DuPont "Zeron"

Hoechst Frigen(R) 9

Kinetic \#11; also K11

Montecatini (I) Algofrene $11 \quad 7601$

SIC (Italy) Edifren $11 \quad 7601$

ARI container color / Pantone number: orange/ 021 6601

MSDS

MSDS

MSDS

MSDS

MSDS

PHYSICAL

- properties

molar mass:

$137.3672032 \mathrm{~g} / \mathrm{mot}(0.302843$

8820

7b/mo T)

normal freezing/melting/triple point:

- normal boiling point - temperature:

$-110.5^{\circ} \mathrm{C}\left(-166.8^{\circ} \mathrm{F}\right)$

8401

$23.7^{\circ} \mathrm{C}\left(74.7^{\circ} \mathrm{F}\right)$

8401 
density, saturated liquid: density, saturated vapor: specific volume, saturated liquid: specific volume, saturated vapor: heat of vaporization: velocity of sound, saturated 7iquid: velocity of sound, saturated vapor: viscosity, saturated vapor: viscosity, saturated 7iquid: thermal conductivity, Tiquid:

therma1 conductivity, vapor:

- normal pressure, $20{ }^{\circ} \mathrm{C}\left(68^{\circ} \mathrm{F}\right)$----

- normal pressure, $21.1{ }^{\circ} \mathrm{C}\left(70^{\circ} \mathrm{F}\right)$

- $20^{\circ} \mathrm{C}\left(68^{\circ} \mathrm{F}\right)$ - density, 1iquid: pressure, saturated vapor: density, saturated Tiquid: density, saturated vapor:

specific volume, saturated liquid: specific volume, saturated vapor: velocity of sound, saturated 1iquid: velocity of sound, saturated vapor: viscosity, saturated 7iquid: viscosity, saturated vapor: thermal conductivity, saturatd liquid:

thermal conductivity, saturated vapor:

- $60{ }^{\circ} \mathrm{C}\left(140{ }^{\circ} \mathrm{F}\right)$

pressure, saturated vapor: heat of vaporization:

- critical point -re pressure: density: specific volume:
$1479 \mathrm{~kg} / \mathrm{m} 3(92.35 \mathrm{lb} / \mathrm{cf}) \quad 8401$

$5.85 \mathrm{~kg} / \mathrm{m} 3(0.365 \mathrm{1b} / \mathrm{cf}) \quad 8401$

$0.676 \mathrm{~L} / \mathrm{kg}(0.0108 \mathrm{cf} / 7 \mathrm{~b}) \quad 8401$

$170.9 \mathrm{~L} / \mathrm{kg}(2.7369 \mathrm{cf} / 7 \mathrm{~b}) \quad 8401$

$181.4 \mathrm{~kJ} / \mathrm{kg}(78.0 \mathrm{Btu} / 1 \mathrm{~b}) \quad 8401$

$736 \mathrm{~m} / \mathrm{s}(2415 \mathrm{ft} / \mathrm{s}) \quad 8401$

$138 \mathrm{~m} / \mathrm{s}(452 \mathrm{ft} / \mathrm{s}) \quad 8401$

$10.14 \mu \mathrm{Pa} \cdot \mathrm{s}(0.01014 \mathrm{cp}) \quad 8401$

$410 \mu \mathrm{Pa} \cdot \mathrm{s}(0.410 \mathrm{cp}) \quad 8401$

$0.0872 \mathrm{~W} / \mathrm{m} \cdot \mathrm{K}(0.0504 \quad 8401$

$\left.\mathrm{Btu} / \mathrm{hr} \cdot \mathrm{ft}^{\circ} \mathrm{F}\right)$

$0.0084 \mathrm{~W} / \mathrm{m} \cdot \mathrm{K}(0.0049$

$\mathrm{Btu} / \mathrm{hr} \cdot \mathrm{ft}^{\circ} \mathrm{F}$ )

8401

$1488 \mathrm{~kg} / \mathrm{m} 3(12.42 \mathrm{lb} / \mathrm{ga1}) \quad 8401$

$1486 \mathrm{~kg} / \mathrm{m} 3(12.40 \mathrm{lb} / \mathrm{gal}) \quad 8401$

$88.7 \mathrm{kPa}$ (12.86 psja) 8401

$1488 \mathrm{~kg} / \mathrm{m} 3(92.907 \mathrm{~b} / \mathrm{cf}) \quad 8401$

$5.17 \mathrm{~kg} / \mathrm{m} 3(0.323 \mathrm{ib} / \mathrm{cf}) \quad 8401$

$0.672 \mathrm{~L} / \mathrm{kg}(0.0108 \mathrm{cf} / 7 \mathrm{~b}) \quad 8401$

$193.4 \mathrm{~L} / \mathrm{kg}(3.0983 \mathrm{cf} / 7 \mathrm{~b}) \quad 8401$

$749 \mathrm{~m} / \mathrm{s}(2456 \mathrm{ft} / \mathrm{s}) \quad 8401$

$137 \mathrm{~m} / \mathrm{s}(450 \mathrm{ft} / \mathrm{s}) \quad 8401$

$425 \mu \mathrm{Pa} \cdot \mathrm{s}(0.425 \mathrm{cp}) \quad 8401$

$10.0 \mu \mathrm{Pa} \cdot \mathrm{s}(0.0100 \mathrm{cp}) \quad 8401$

$0.0882 \mathrm{~W} / \mathrm{m} \cdot \mathrm{K}(0.0510 \quad 8401$

$\mathrm{Btu} / \mathrm{hr} \bullet \mathrm{ft}^{\circ} \mathrm{F}$ )

$0.00824 \mathrm{~W} / \mathrm{m} \cdot \mathrm{K}(0.00476 \quad 8401$

$\mathrm{Btu} / \mathrm{hr} \cdot \mathrm{ft}^{\circ} \mathrm{F}$ )

$313 \mathrm{kPa}$ (45.4 psia)

8401

$166.9 \mathrm{~kJ} / \mathrm{kg}(71.8 \mathrm{Btu} / 7 \mathrm{~b}) \quad 8401$

$198.0^{\circ} \mathrm{C}\left(388.3^{\circ} \mathrm{F}\right) \quad 8401$

$4408 \mathrm{kPa}(639.3 \mathrm{psia}) \quad 8401$

$554 \mathrm{~kg} / \mathrm{m} 3(34.6 \mathrm{~Tb} / \mathrm{cf}) \quad 8401$

$1.81 \mathrm{~L} / \mathrm{kg}(0.0289 \mathrm{cf} / 7 \mathrm{~b}) \quad 8401$

ENVIRONMENTAL

average atmospheric 1jfetime (Tatm): ODP (ozone depletion potential):

GWP (global warming potential):

HGWP (halocarbon GWP):
$45 \mathrm{yr}$

1.000 (model-derived relative to $\mathrm{R} 11$ )

1.000 (semi-empirical relative 9501 to $R$ 11)

1.0 (estimate used for the Montreal Protocol)

6904

4600 relative to $\mathrm{CO} 2$ for 1009501 yr integration

1.000 relative to $R 11$ for

infinite integration period

SAFETY

- classification

safety group (ASHRAE Standard 34): NFPA 704 degrees of hazard $(H-F-R-S)$ :

NPCA HMIS hazard ratings (H-F-R):

A1

ARI recommendation: $2-0-0^{\circ} \quad 3 A 15$

BOC Gases: 1-0-0 MSDS

Honeywe 11: 2-0-0 MSDS

health-flammabi 7 ity-reactivity

[-special]: $0=$ no, $4=$ severe

BOC Gases: 1-0-0

DuPont: $1-0-1$

Honeywe 11: 1-0-0
MSDS

MSDS

MSDS 
UL Comparative Hazard to Life Group: ACGIH carcinogenicity category:

DFG pregnancy risk group: - short-term occupational 1imit ----NIOSH IDLH (immediately dangerous): ARI "IDLH" recommendation: NIOSH SCP IDLH (immediately dangerous: - occupational exposure limit -.--.-NIOSH REL-C (exposure ceiling): ACGIH TLV-C (exposure ceiTing): MAK (maximum workplace concentration): - Tong-term occupational limit ------OSHA PEL (permissibie exposure 7imit): exposure limit consistent to OSHA PEL: MAK (maximum workplace concentration):

- emergency exposure 7imit NRC EEGL (emergency exposure level):

Acute Toxicity Exposure Limit (ATEL):

Refrigerant concentration Limit ( $R C L)$ :

- specia7-purpose exposure control --NRC CEGL (continuous exposure 1eve1):

- acute (short-term) toxicity -----LC50 (7etha7 concentration, 50\%):

ALC (approximate Tethal concentration: hea]th-flammability-reactivity $0=$ insignificant, $4=$ extreme

5 (a) in absence of flame or hot objects

A4, not Classifiable as a 9504

uman carcinogen

C (no risk fear below MAK/BAT) 5561

$2,000 \mathrm{ppm} \mathrm{v} / \mathrm{v}$

$5,000 \mathrm{ppm} v / \mathrm{v}$ for $30 \mathrm{~min} \quad 3204$

$10,000 \mathrm{ppm} \mathrm{v/v}$ for $30 \mathrm{~min} \quad 3903$

$1,000 \mathrm{ppm} v / \mathrm{v}$ (must not 3903

exceed)

$1,000 \mathrm{ppm} v / v$ (must not

exceed)

IV: 2,000 ppm $\mathrm{v} / \mathrm{v}$ momentary 605561 min

$1,000 \mathrm{ppm} v / \mathrm{v}$ TWA for $8 \mathrm{hr} /$ day 3904 and $40 \mathrm{hr} / \mathrm{wk}$

ARI PEL-Cei7ing: $\mathrm{C1}, 000 \mathrm{ppm}$

$v / v$ TWA for $8 \mathrm{hr} / \mathrm{day}$ and 40

$3 \mathrm{~A} 15$ $\mathrm{hr} / \mathrm{wk}$

$1,000 \mathrm{ppm} v / \mathrm{v}$ TWA for $8 \mathrm{hr} /$ day 5561 40 (or 42) hr/wk

$1 \mathrm{hr}: 1,500 \mathrm{ppm} \mathrm{v} / \mathrm{v}$ ceiling guidance level for single emergency exposures

$24 \mathrm{hr}: 500 \mathrm{ppm} \mathrm{v} / \mathrm{v}$ ceiling guidance level for single emergency exposures

$1,100 \mathrm{ppm} v / \mathrm{v}$ (preliminary

based on ASHRAE 34u draft)

$1,100 \mathrm{ppm} v / \mathrm{v}$ (preliminary

based on ASHRAE 34u draft)

1,100 ppm v/v (preTiminary

based on ASHRAE 34u draft)

$1,100 \mathrm{ppm} v / \mathrm{v}$ (preliminary

based on ASHRAE $34 \mathrm{u}$ draft)

7414

7414

$34 u$

A950

$34 \mathrm{u}$

A950

90 day: $100 \mathrm{ppm} v / \mathrm{v}$ cejling

guidance for prolonged

exposure in closed

envi ronments

rat, 10 min: $120,000 \mathrm{ppm}$

(fatal concentration by inhalation for half of test animals)

rat, 15 min: 130,000 ppm

(fata) concentration by

inhalation for half of test anima7s)

rat, 4 hr: 26, 200 ppm (fatal

concentration by inhalation

for half of test animals)

rat, $30 \mathrm{~min}, 1 / 1$ : 100,000 ppm

(lowest exposure tested with

one or more deaths by

inhalation)

rat, $4 \mathrm{hr}, 0 / 4$ : $>35,600 \mathrm{ppm}$

(7owest exposure tested with 
oral LD50 (7ethal dosage, 50\%):

cardiac sensitization (CS) EC50:

cardiac sensitization threshold/LOEL:

cardiac sensitization (CS) NOEL: anesthetic/CNS effect EC50: anesthetic/CNS effect LOEL: anesthetic/CNS effect NOEL:

- flammability

LFL-UFL (flammability limits in air): heat of combustion (by ASHRAE 34-92): flash point:

autoignition temperature: autodecomposition temperature: former UL Classification:

- detection

appearance: odor:

odor sensing, lower threshold: one or more deaths by

inhalation)

rat, $4 \mathrm{hr}, 1 / 4$ : $66,000 \mathrm{ppm}$

(lowest exposure tested with

$65 \mathrm{H} 3$

one or more deaths by

inhalation)

rat, $4 \mathrm{hr}, 3 / 6$ : 24,900 ppm

(lowest exposure tested with

one or more deaths by

inhalation)

rat, Elf Atochem: $>3,725 \mathrm{mg} / \mathrm{kg}$ MSDS

(fatal dose by ingestion for

half of test animals)

rat: $3,752 \mathrm{mg} / \mathrm{kg}$ (fatal dose

by ingestion for half of test animals)

dog, 5 min: $12,400 \mathrm{ppm} \mathrm{v/v}$

(effective concentration in

half of test animals)

dog, 5 min: $12,500 \mathrm{ppm} \mathrm{v/v}$

(effective concentration in

half of test animals)

$5 C 53$

dog 1/12: 3500-6100ppm, $\div 4,800$

$\mathrm{ppm} \mathrm{v} / \mathrm{v}$ (lowest observed effect level in test animals) dog: $5,000 \mathrm{ppm} v / \mathrm{v}$ (lowest observed effect leve 1 in test anima7s)

dog, 5min: 900-1300 ppm, $\div 1100$

6595

$\mathrm{ppm} \mathrm{v} / \mathrm{v}$ (no observed effect

level in test anima7s)

rat, 10 min, stimulant: 35,000

$\mathrm{ppm} v / \mathrm{v}$ (effective

concentration in half of test anima7s)

rat, $10 \mathrm{~min}: 54,000 \mathrm{ppm} \mathrm{v/v}$

(effective concentration in

half of test animals)

rat, $2 \mathrm{hr}$, ?/4: 35,000 ppm v/v 5365

(lowest observed effect leve 1

in ALC or LC50 studies)

rat, $2 \mathrm{hr}, 0 / 4: 12,500 \mathrm{ppm} v / \mathrm{v} 5365$

(no observed effect Tevel in

ALC or LC50 studies)

6146

6110

5644

$4 A 66$

5644

6110

A309

none (nonflammable as tested) 2525

$0.9 \mathrm{MJ} / \mathrm{kg}$ (387 Btu/7b)

ICI: does not fiash

2318

$>7500^{\circ} \mathrm{C}\left(>1382^{\circ} \mathrm{F}\right)$

5931

DuPont: $>593{ }^{\circ} \mathrm{C}\left(>1099{ }^{\circ} \mathrm{F}\right)$ MSDS

nonflammable (wi thdrawn for 6938

revision of the classification

system, category SBQT2)

colorless

$\begin{array}{lr}\text { ICI: nearly odorless } & \text { MSDS } \\ \text { faint ethereal } & 5367\end{array}$

$5 \mathrm{ppm} \mathrm{v} / \mathrm{v}$

PRODUCTION

first commercial use as a refrigerant:

last year production allowed:
1932 by $T$. Midgley, associates 5 C39 1995 in developed countries 8C01 under the Montreal Protocol 


\section{R-12}

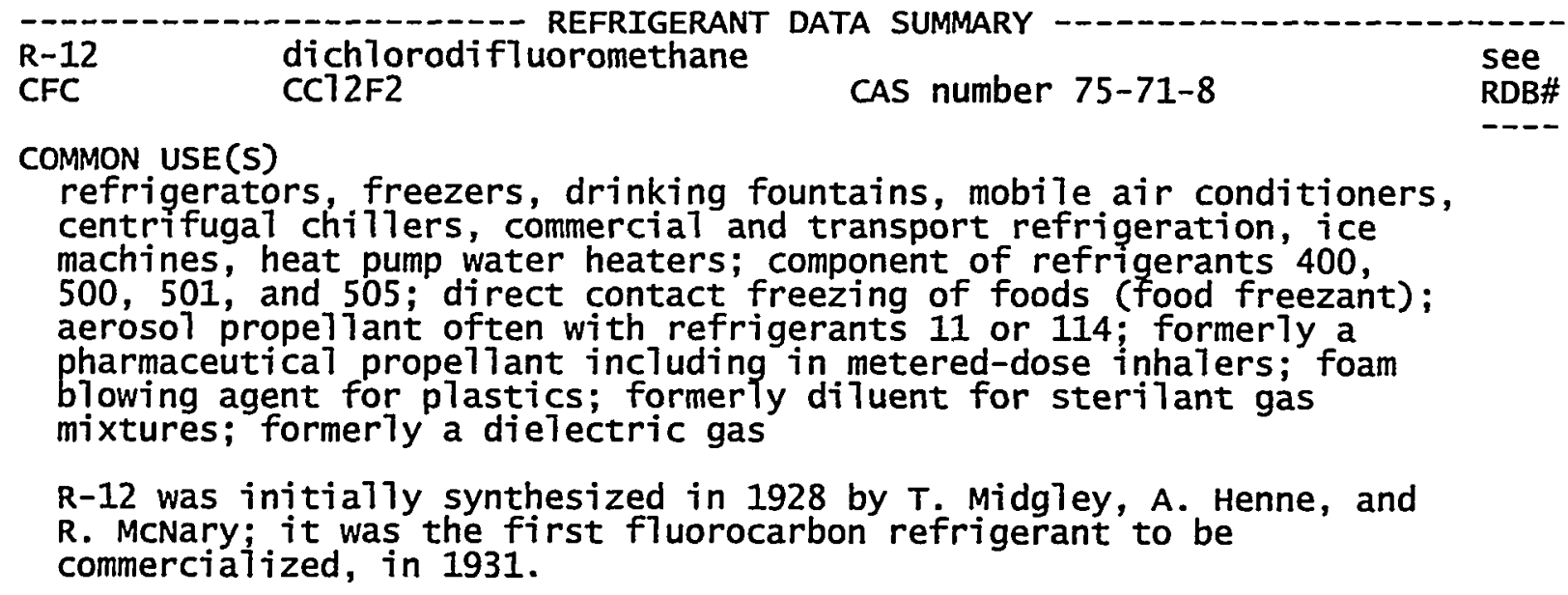

IDENTIFIERS

common name(s): R-12; R12; R 12

CFC-12

chlorofluorocarbon 12

f7uorocarbon 12

fluorochemical 12; FC 12

halocarbon 12

halochemical 12

halon 122

chemical name (by IUPAC convention): dichlorodifluoromethane

a]ternative chemical names/formulae:

methane, dichlorodifiuoro-

difluorodichloromethane

CCT2F2

not recommended: $\mathrm{CF} 2 \mathrm{C} 12$

CAS number: 75-71-8 (Chemical Abstracts

Service registry number)

EINECS number: 200-893-9 (European Inventory

of Existing Chemical

Substances)

NIOSH RTECS number: PA8200000 (Registry of Toxic

Effects of Chemical

Substances)

trade name(s): Asahi Glass Fron AF-12

Daikin Daiflon(R) 12

DuPont Freon $(R) 12$

Elf Atochem Forane(R) 12

Honeywe 11 Genetron(R) 12

ICI $\operatorname{Arcton}(R) 12$

Solvay Solkane(R) 12

MSDS

MSDS

MSDS

MSDS

historical name(s): Allied Corp refrigerant 142

A 1 iedsignal Genetron(R) 12 MSDS

Elf Atochem Racon(R) $12 \quad 6938$

Eskimon (USSR)

Hoechst Frigen (R) 12

ICI Arcton $(R) 6$

Kinetic \#12; a1so $\mathrm{K} 12$

Kinetic Chemica7s "Freon"

Montecatini (I) Algofrene $12 \quad 7601$

Nitto (Japan) Co7-Flon 12

Pennwalt/Atochem Isotron(R) 12

SIC (Ita7y) Edifren 12

Unichemie (NL) Fresane 12 
ARI container color / Pantone number:

PHYSICAL

- properties

molar mass:

normal freezing/melting/triple point:

- normal boiling point -ro density, saturated liquid: density, saturated vapor: specific volume, saturated 7iquid: specific volume, saturated vapor: heat of vaporization: velocity of sound, saturated liquid: velocity of sound, saturated vapor: viscosity, saturated liquid: viscosity, saturated vapor: thermal conductivity, 1iquid:

therma1 conductivity, vapor:

- norma1 pressure, $20^{\circ} \mathrm{C}\left(68^{\circ} \mathrm{F}\right)$-.--

- normal pressure, $21.1{ }^{\circ} \mathrm{C}\left(70^{\circ} \mathrm{F}\right)$ vapor:

- normal pressure, $21.1{ }^{\circ} \mathrm{C}\left(70{ }^{\circ} \mathrm{F}\right)---$

- $20^{\circ} \mathrm{C}\left(68^{\circ} \mathrm{F}\right)$

pressure, saturated vapor: density, saturated 1iquid: density, saturated vapor: specific volume, saturated liquid: specific volume, saturated vapor: velocity of sound, saturated 1iquid: velocity of sound, saturated vapor: viscosity, saturated liquid: viscosity, saturated vapor: thermal conductivity, saturatd liquid: thermal conductivity, saturated vapor: - $60^{\circ} \mathrm{C}\left(140{ }^{\circ} \mathrm{F}\right)$ pressure, saturated vapor: heat of vaporization:

- critical point

temperature: pressure: density: specific volume:

ENVIRONMENTAL

average atmospheric Tifetime (Tatm): average tropospheric lifetime (Ttrop): ODP (ozone depletion potential):

GWP (global warming potential): HGWP (halocarbon GWP):
Union Carbide Ucon(R) 12 VVB Dresden Frigedohn 12 white / none

7601

7601

6601

$120.9129064 \mathrm{~g} / \mathrm{mol}(0.266567 \quad 8820$

1b/mol)

$-157.0^{\circ} \mathrm{C}\left(-250.7^{\circ} \mathrm{F}\right) \quad 8401$

$-29.8^{\circ} \mathrm{C}\left(-21.6^{\circ} \mathrm{F}\right) \quad 8401$

$1487 \mathrm{~kg} / \mathrm{m} 3(92.83 \mathrm{lb} / \mathrm{cf}) \quad 8401$

$6.29 \mathrm{~kg} / \mathrm{m} 3(0.393 \mathrm{ib} / \mathrm{cf}) \quad 8401$

$0.672 \mathrm{~L} / \mathrm{kg}(0.0108 \mathrm{cf} / \mathrm{lb}) \quad 8401$

$159.0 \mathrm{~L} / \mathrm{kg}(2.5469 \mathrm{cf} / 7 \mathrm{~b}) \quad 8401$

$166.2 \mathrm{~kJ} / \mathrm{kg}(71.4 \mathrm{Btu} / \mathrm{lb}) \quad 8401$

$732 \mathrm{~m} / \mathrm{s}(2403 \mathrm{ft} / \mathrm{s}) \quad 8401$

$135 \mathrm{~m} / \mathrm{s}(441 \mathrm{ft} / \mathrm{s}) \quad 8401$

$345 \mu \mathrm{Pa} \cdot \mathrm{s}(0.345 \mathrm{cp})$

$9.56 \mu \mathrm{Pa} \cdot \mathrm{s}(0.00956 \mathrm{cp})$

$0.0868 \mathrm{~W} / \mathrm{m} \cdot \mathrm{K}(0.0501$

$\mathrm{Btu} / \mathrm{hr} \bullet \mathrm{ft}^{\circ} \mathrm{F}$ )

$0.0073 \mathrm{~W} / \mathrm{m} \bullet \mathrm{K}(0.0042$

$\mathrm{Btu} / \mathrm{hr} \cdot \mathrm{ft}^{\circ} \mathrm{F}$ )

8401

8401

8401

8401

$5.130 \mathrm{~kg} / \mathrm{m} 3(0.3203 \mathrm{lb} / \mathrm{cf}) \quad 8401$

$5.109 \mathrm{~kg} / \mathrm{m} 3(0.3190 \mathrm{1b} / \mathrm{cf}) \quad 8401$

$566.4 \mathrm{kPa}(82.15 \mathrm{psia}) \quad 8401$

$1329 \mathrm{~kg} / \mathrm{m} 3(82.96 \mathrm{~Tb} / \mathrm{cf}) \quad 8401$

$32.14 \mathrm{~kg} / \mathrm{m} 3(2.006 \mathrm{lb} / \mathrm{cf}) \quad 8401$

$0.753 \mathrm{~L} / \mathrm{kg}(0.0121 \mathrm{cf} / \mathrm{lb}) \quad 8401$

$31.1 \mathrm{~L} / \mathrm{kg}(0.4985 \mathrm{cf} / 7 \mathrm{~b}) \quad 8401$

$532 \mathrm{~m} / \mathrm{s}(1744 \mathrm{ft} / \mathrm{s}) \quad 8401$

$136 \mathrm{~m} / \mathrm{s}(446 \mathrm{ft} / \mathrm{s}) \quad 8401$

$201 \mu \mathrm{Pa} \cdot \mathrm{s}(0.201 \mathrm{cp}) \quad 8401$

$11.6 \mu \mathrm{Pa} \cdot \mathrm{s}(0.0116 \mathrm{cp}) \quad 8401$

$0.0689 \mathrm{~W} / \mathrm{m} \cdot \mathrm{K}(0.0398 \quad 8401$

$\mathrm{Btu} / \mathrm{hr} \bullet \mathrm{ft}^{\circ} \mathrm{F}$ )

$0.01308 \mathrm{~W} / \mathrm{m} \cdot \mathrm{K}(0.00756 \quad 8401$

$\mathrm{Btu} / \mathrm{hr} \bullet \mathrm{ft}^{\circ} \mathrm{F}$ )

$1522 \mathrm{kPa}$ (220.7 psia)

$114.5 \mathrm{~kJ} / \mathrm{kg}(49.2 \mathrm{Btu} / 7 \mathrm{~b})$

8401

8401

$112.0^{\circ} \mathrm{C}\left(233.5^{\circ} \mathrm{F}\right)$

$4136 \mathrm{kPa}$ (599.9 psia)

$565 \mathrm{~kg} / \mathrm{m} 3$ (35.3 $\mathrm{ib} / \mathrm{cf})$

$1.77 \mathrm{~L} / \mathrm{kg}(0.0284 \mathrm{cf} / 7 \mathrm{~b})$

8401

8401

8401

8401

$100 \mathrm{yr}$

900,000 yr

0.82 (mode 7 -derived relative

9501

to $R$ 11)

0.9 (semi-empirical relative to $\mathrm{R}$ 11)

1.0 (estimate used for the Montreal Protocol)

10,600 relative to $\mathrm{CO} 2$ for 100 yr integration

3.1 relative to $R 11$ for

infinite integration period
$9 \mathrm{~B} 04$

9501

9501

6904

9501

5964 
SAFETY

- classification safety group (ASHRAE Standard 34): NFPA 704 degrees of hazard $(H-F-R-S)$ :

NPCA HMIS hazard ratings $(H-F-R)$ :

A1

ARI recommendation:

BOC Gases:

Honeywe17:

health-flammability-reactivity

[-special]: $0=$ no, 4=severe

BOC Gases: $1-0-0$

DuPont: $\quad 1-0-1$

Honeywe 11: $1-0-0$

health-flammability-reactivity

$0=$ insignificant, $4=$ extreme

UL Comparative Hazard to Life Group: ACGIH carcinogenicity category:

DFG pregnancy risk group:

- short-term occupational 7imit -----

NIOSH IDLH (immediately dangerous): NIOSH SCP IDLH (immediately dangerous:

- occupational exposure Timit

MAK (maximum workplace concentration):

- Tong-term occupational 7imit ------OSHA PEL (permissible exposure limit):

NIOSH REL (recommendd exposure Timit):

ACGIH TLV-TWA (time-weighted average): MAK (maximum workplace concentration):

- emergency exposure 7imit

NRC EEGL (emergency exposure level):

Acute Toxicity Exposure Limit (ATEL):

Refrigerant concentration Limit ( $R C L)$ :

- special-purpose exposure control --NRC CEGL (continuous exposure level):

- acute (short-term) toxicity

ALC (approximate lethal concentration:

derma1 LD50 (letha1 dosage, 50\%):
6 in absence of flame or hot $4 B 64$ objects

A4, not classifiable as a 9504 human carcinogen

C (no risk fear below MAK/BAT) 5561

$15,000 \mathrm{ppm} \mathrm{v} / \mathrm{v}$

$50,000 \mathrm{ppm} / \mathrm{v}$ for $30 \mathrm{~min} \quad 3903$

IV: $2,000 \mathrm{ppm} \mathrm{v} / \mathrm{v}$ momentary 605561 min

$1,000 \mathrm{ppm} \mathrm{v} / \mathrm{v}$ TWA for $8 \mathrm{hr} /$ day 3904 and $40 \mathrm{hr} / \mathrm{wk}$

$1,000 \mathrm{ppm} v / \mathrm{V}$ TWA for $10 \quad 3903$

$\mathrm{hr} /$ day and $40 \mathrm{hr} / \mathrm{wk}$

$1,000 \mathrm{ppm} \mathrm{v} / \mathrm{v}$ TWA for $8 \mathrm{hr} /$ day 9504 and $40 \mathrm{hr} / \mathrm{wk}$

$1,000 \mathrm{ppm} \mathrm{v/v}$ TWA for $8 \mathrm{hr} /$ day 5561 40 (or 42) $\mathrm{hr} / \mathrm{wk}$

$1 \mathrm{hr}: 10,000 \mathrm{ppm} \mathrm{v} / \mathrm{v}$ ceiling 7414

guidance level for single

emergency exposures

$24 \mathrm{hr}: 1,000 \mathrm{ppm} v / \mathrm{v}$ ceiling 7414

guidance level for single

emergency exposures

$18,000 \mathrm{ppm} v / \mathrm{v}$ (preliminary

based on ASHRAE $34 \mathrm{u}$ draft)

$22,000 \mathrm{ppm} \mathrm{V} / \mathrm{v}$ (preliminary

based on ASHRAE 34u draft)

A950

$18,000 \mathrm{ppm} v / \mathrm{v}$ (preliminary

$22,000 \mathrm{ppm} \mathrm{v} / \mathrm{v}$ (pre7iminary

based on ASHRAE 34u draft)

90 day: $100 \mathrm{ppm} v / \mathrm{v}$ ceiling

7414

guidance for prolonged

exposure in closed

environments

rat, $3 \mathrm{hr}: 620,000 \mathrm{ppm}$ (lowest 7434

exposure tested with one or

more deaths by inhalation)

rat, $4 \mathrm{hr}, 0 /$ few: $>800,000$ ppm 5147

Clowest exposure tested with

one or more deaths by

inhalation)

rat: $>2,000 \mathrm{mg} / \mathrm{kg}$ (fata) dose 5368

by skin contact for half of based on ASHRAE $34 \mathrm{u}$ draft) 
oral LD50 (1ethal dosage, 50\%): cardiac sensitization (CS) EC50:

cardiac sensitization threshold/LOEL: cardiac sensitization (CS) NOEL: anesthetic/CNS effect EC50: anesthetic/CNS effect LOEL: anesthetic/CNS effect NOEL:

- flammability

LFL-UFL (flammability 7imits in air): heat of combustion (by ASHRAE 34-92): flash point: autoignition temperature: former UL Classification: test animaTs)

rat: $>1,000 \mathrm{mg} / \mathrm{kg}$ (fata) dose by ingestion for half of test animais)

dog, 5 min: $77,000 \mathrm{ppm} v / \mathrm{v}$ (effective concentration in half of test anima7s) dog, $10 \mathrm{~min}: 120,000 \mathrm{ppm} \mathrm{v} / \mathrm{v}$ (effective concentration in half of test anima7s)

dog, $5 \mathrm{~min}, 5 / 12$ : $50,000 \mathrm{ppm}$ $v / v$ (lowest observed effect Tevel in test animals)

dog, 5 min, 0/12: 25,000 ppm $V / V$ (no observed effect leve 7 in test animals)

dog, 5 min, 0/12: 25,000 ppm $v / v$ (no observed effect level in test anima Ts)

dog, $10 \mathrm{~min}, 0 / 5$ : 40,000 ppm $v / v$ (no observed effect leve? in test animais)

rat, 10 min: stimulant 250,000 6110 ppm $v / v$ Ceffective concentration in half of test anima $7 \mathrm{~s}$ )

rat, $2 \mathrm{hr}: 400,000 \mathrm{ppm} \mathrm{v/v}$ (effective concentration in half of test animals)

human, $14 \mathrm{~min}, 1 / 2$ : $40,000 \mathrm{ppm} 5 \mathrm{C45}$ $\mathrm{v} / \mathrm{v}$ (lowest observed effect level)

human, 80 min 1/1: 20000-40000 5C45 $\mathrm{ppm} v / \mathrm{v}$ (lowest observed effect 1eve T) human, $1 / 2 \mathrm{hr}, 2 / 2$ : 9855-10,173 5136 $\mathrm{ppm} \mathrm{v} / \mathrm{v}$ (lowest observed effect 1eve1)

human, min, ?/?: 50,000 ppm 5271 $\mathrm{v} / \mathrm{v}$ (jowest observed effect Teve1)

rat, 2 hr: $200,000 \mathrm{ppm} v / \mathrm{v}$ (no 5355 observed effect leve 1 in test animals)

human, $1 \mathrm{hr}, 0 / 8$ : 3,913 ppm $v / v$ (no observed effect level) human, $2 \frac{1}{2} \mathrm{hr}, 0 / 2$ : 1,000-1027 $\mathrm{ppm} \mathrm{v} / \mathrm{v}$ (no observed effect leve1)

none (nonflammable as tested)

$-0.8 \mathrm{MJ} / \mathrm{kg}(-344 \mathrm{Btu} / 1 \mathrm{~b})$

0036

none (nonflammable as tested) 3903 $>750^{\circ} \mathrm{C}\left(>1382^{\circ} \mathrm{F}\right)$

BOC Gases: none 5931 MSDS nonflammable (withdrawn for 6938 revision of the classification system, category SBQT2)

appearance: colorless odor: 
PRODUCTION

first commercial use as a refrigerant: 1931 by T. Midgley, associates 5c39

last year production allowed: 1995 in developed countries 8C01 under the Montreal Protocol 


\section{R-14}

COMMN USE(S)
CF
extremely low temperature refrigeration, often in the lowest stage of
a multistage, cascaded system; industrial solvent in electronics
manufacturing; plasma etchant

\section{IDENTIFIERS}

common name(s): $\mathrm{R}-14$; R14; R 14

FC-14

carbon tetrafluoride

fluorocarbon 14

fluorochemical 14; FC 14

halocarbon 14

halochemical 14

perfluorocarbon 14; "PFC-14"

chemical name (by IUPAC convention):

tetrafluoromethane

alternative chemical names/formulae:

methane, tetrafluoro-

perfluoromethane

tetrafluorocarbon

CF4

CAS number: 75-73-0 (Chemical Abstracts

Service registry number)

EINECS number: 200-896-5 (European Inventory

of Existing Chemical

Substances)

NIOSH RTECS number: FG4920000 (Registry of Toxic Effects of Chemical

Substances)

$\begin{array}{lll}\text { trade name (S): } & \text { BOC Gases Halocarbon } 14 \\ & \text { Daikin Daiflon (R) 14 } & \text { MSDS } \\ & \text { MSDS }\end{array}$

Dupont Freon $(R) 14 \quad$ MSDS

historical name(s): $\begin{aligned} & \text { Hoechst Frigen(R) } 14 \\ & \text { Alljed Corp refrigerant } 340\end{aligned}$

carbon fluoride

ARI container color / Pantone number: yellow-brown (mustard) / 1246601

PHYSICAL

- properties

molar mass: $88.0043128 \mathrm{~g} / \mathrm{mol}(0.194016$

8820

$7 \mathrm{~b} / \mathrm{mo} 7)$

normal freezing/melting/triple point:

$-183.6^{\circ} \mathrm{C}\left(-298.5^{\circ} \mathrm{F}\right)$

8401

- normal boiling point -re density, saturated Tiquid: density, saturated vapor: specific volume, saturated Tiquid: specific volume, saturated vapor: heat of vaporization: velocity of sound, saturated liquid: velocity of sound, saturated vapor: viscosity, saturated Tiquid: viscosity, saturated vapor: thermal conductivity, Tiquid:

thermal conductivity, vapor:

$-128.0{ }^{\circ} \mathrm{C}\left(-198.5^{\circ} \mathrm{F}\right)$

8401

$1603 \mathrm{~kg} / \mathrm{m} 3(100.08 \mathrm{~Tb} / \mathrm{cf})$

8401

$7.66 \mathrm{~kg} / \mathrm{m} 3 \quad(0.478 \mathrm{~Tb} / \mathrm{cf})$

8401

$0.624 \mathrm{~L} / \mathrm{kg}(0.0100 \mathrm{cf} / 7 \mathrm{~b}) \quad 8401$

$130.6 \mathrm{~L} / \mathrm{kg}(2.0915 \mathrm{cf} / 7 \mathrm{~b}) \quad 8401$

$134.4 \mathrm{~kJ} / \mathrm{kg}(57.8 \mathrm{Btu} / 7 \mathrm{~b}) \quad 8401$

$702 \mathrm{~m} / \mathrm{s}(2304 \mathrm{ft} / \mathrm{s}) \quad 8401$

$129 \mathrm{~m} / \mathrm{s}(422 \mathrm{ft} / \mathrm{s})$

$307 \mu \mathrm{Pa} \cdot \mathrm{s}(0.307 \mathrm{cp})$

8401

$8.38 \mu \mathrm{Pa} \cdot \mathrm{s}(0.00838 \mathrm{cP}) \quad 8401$

$0.0973 \mathrm{~W} / \mathrm{m} \cdot \mathrm{K}(0.0562 \quad 8401$

$\left.\mathrm{Btu} / \mathrm{hr} \bullet \mathrm{ft}^{\circ} \mathrm{F}\right)$

$0.0054 \mathrm{~W} / \mathrm{m} \cdot \mathrm{K}(0.0031$

8401 
- normal pressure, $20{ }^{\circ} \mathrm{C}\left(68^{\circ} \mathrm{F}\right)$

- normal pressure, $21.1{ }^{\circ} \mathrm{C}\left(70{ }^{\circ} \mathrm{F}\right)$---

$3.673 \mathrm{~kg} / \mathrm{m} 3(0.2293 \mathrm{lb} / \mathrm{cf}) \quad 8401$

- critical point density, vapor:

$3.659 \mathrm{~kg} / \mathrm{m} 3(0.2284 \mathrm{lb} / \mathrm{cf})$

8401

temperature:

pressure:

density:

specific volume:

$-45.6{ }^{\circ} \mathrm{C}\left(-50.2^{\circ} \mathrm{F}\right)$

8401

$3750 \mathrm{kPa}(543.9$ psia) $\quad 8401$

$626 \mathrm{~kg} / \mathrm{m3}(39.1 \mathrm{lb} / \mathrm{cf}) \quad 8401$

$1.60 \mathrm{~L} / \mathrm{kg}(0.0256 \mathrm{cf} / \mathrm{Tb}) \quad 8401$

ENVIRONMENTAL

average atmospheric Tifetime (Tatm): ODP (ozone depletion potential):

$50,000 \mathrm{yr}$

0.000 (model-derived relative

6694

GWP (global warming potential): to $R$ 11)

5700 relative to $\mathrm{CO} 2$ for 100

yr integration

HGWP (halocarbon GWP):

700 relative to

infinite integration period

9501

DW

SAFETY

- Classification

safety group (ASHRAE Standard 34): NFPA 704 degrees of hazard (H-F-R-S):

NPCA HMIS hazard ratings $(H-F-R)$ :

AI

BOC Gases: 1-0-0

8601

Matheson: 1-0-0

MSDS

hea] th-fiammability-reactivity

[-special]: $0=$ no, $4=$ severe

MSDS

BOC Gases: $1-0-0$

DuPont: $1-0-1$

health-f7ammabi 1ity-reactivity

$0=i n s i g n i f i c a n t, 4=e x t r e m e$

UL Comparative Hazard to Life Group:

6 in absence of flame or hot objects

- emergency exposure limit

Acute Toxicity Exposure Limit (ATEL):

Refrigerant concentration Limit ( $R C L)$ :

provisiona7: $110,000 \mathrm{ppm} \mathrm{v} / \mathrm{v}$

(preliminary based on ASHRAE

$34 \mathrm{u}$ draft)

provisiona1: $69,000 \mathrm{ppm} \mathrm{v} / \mathrm{v}$

(preliminary based on ASHRAE

$34 \mathrm{u}$ draft)

- acute (short-term) toxicity -------

ALC (approximate lethal concentration:

rat, 15 min, DuPont: 895,000

ppm (lowest exposure tested

with one or more deaths by

inhalation)

rat, $h r, 0 / 10:>780,000 \mathrm{ppm} 7550$

(Towest exposure tested with

one or more deaths by

inhalation)

cardiac sensitization threshold/LOEL:

dog, 10 min, 1?/6: 200,000 ppm 7550

$v / v$ (lowest observed effect

level in test animals)

dog, $10 \mathrm{~min}, 3 / 6$ : 600,000 ppm 7550

$v / v$ (lowest observed effect

leve 7 in test anima 75 )

cardiac sensitization (CS) NOEL:

dog, $10 \mathrm{~min}, 0 / 5$ : 200,000 ppm

$v / v$ (no observed effect level

in test animals)

anesthetic/CNS effect LOEL: $\mathrm{rat}, 1 \mathrm{hr}, 10 / 10: 780,000 \mathrm{ppm}$

MSDS

MSDS

0036

A950

A950

$v / v$ (lowest observed effect

Teve 1 in ALC or LC50 studies)

anesthetic/CNS effect NOEL: rat, 10 day, 0/20: $226,000 \mathrm{pp}$

MSDS

in subchronic or chronic study)

7550

7550

7550 
- flammability LFL-UFL (flammability limits in air): none (nonflammable as tested)
flash point: DuPont: will not burn autoignition temperature: BOC Gases: none

0036

MSDS

- detection DuPont: $>1,100^{\circ} \mathrm{C}\left(>2,000{ }^{\circ} \mathrm{F}\right)$

MSDS

MSDS

appearance: DuPont: clear, colorless
odor: Daikin: faint ethereal odor

MSDS

DuPont: none

Matheson: odorless

MSDS

MSDS

MSDS

PRODUCTION

first commercial use as a refrigerant: 1955

Tast year production allowed: unrestricted 


\section{R-22}

\section{$\mathrm{R}-22$ \\ HCFC \\ REFRIGERANT chlorodifluoromethane CHC1F2}

DATA SUMMARY

CAS number 75-45-6

COMMON USE(S)

most-widely used refrigerant: air conditioners, heat pumps, applied systems, chillers (with piston, scrol1, screw, and centrifugal compressors), commercial refrigeration, dehumidifiers; component of refrigerants 401A, 401B, 401C, 402A, 402B, 403A, 403B, 405A, 406A, $409 \mathrm{~A}, 409 \mathrm{~B}, 411 \mathrm{~A}, 411 \mathrm{~B}, 412 \mathrm{~A}, 414 \mathrm{~A}, 414 \mathrm{~B}, 501,502,509 \mathrm{~A}$, and others; foam blowing agent especially for polystyrenes, polyethylenes, polyurethanes, polyisocyanurates, and phenolics; aerosol propellant for non-food uses; inert ingredient for medical sterilant gases using ethylene oxide (e.g., Alliedsignal oxyfume(R) 2002); fire suppressant; intermediate to manufacture fiuoropolymers

\section{IDENTIFIERS}

common name(s): $\mathrm{R}-22 ; \mathrm{R} 22 ; \mathrm{R} 22$

HCFC- 22

not recommended: HFA- 22

f7uorocarbon 22

fiuorochemical 22; FC 22

haTocarbon 22

hatochemical 22

chemical name (by IUPAC convention):

hydroch 7orofluorocarbon 22

alternative chemical names/formulae:

chlorodifluoromethane

methane, chlorodifluoro-

difluorochloromethane

difluoromonochloromethane

monoch lorodifluoromethane

CHCTF2

not recommended:

C7F2H; CHF2C1

CAS number: 75-45-6 (Chemical Abstracts Service registry number)

Beilstein registry number:

EINECS number: 200-871-9 (European Inventory

of Existing Chemical

Substances)

NIOSH RTECS number: PA6390000 (Registry of Toxic

Effects of Chemical

substances)

trade name(s): Asahi Glass Fron AF-22

Ausimont Algofrene(R) 22

Ausimont Meforex(R) 22

Daikin Daiflon (R) HCFC-22

DuPont Freon(R) 22

Elf Atochem Forane(R) 22

HRP (UK) $\operatorname{HARP}(R) 22$

Honeywe 71 Genetron(R) 22

ICI Arcton(R) 22

Pennwalt/Atochem Isotron(R) 22

Solvay Solkane(R) 22

historical name(s): Aljied Corp refrigerant 141

Alliedsignal Genetron(R) 22 MSDS Hoechst Frigen $(R) 22$

$\operatorname{ICI} \operatorname{Arcton}(R) 4$

Kinetic Chemicals Freon 22

Montecatini (I) Algofrene 22

Nitto (Japan) Co7-Fion 22 
ARI container color / Pantone number:
Union Carbide Ucon(R) 22

VVB Dresden Frigedohn 22

Tight green / 352
7601

7601

6601

PHYSICAL

- properties

molar mass:

normal freezing/melting/triple point:

- normal boiling point

temperature density, saturated 7iquid: density, saturated vapor:

specific volume, saturated 7 iquid: specific volume, saturated vapor: heat of vaporization:

velocity of sound, saturated 7iquid: velocity of sound, saturated vapor: viscosity, saturated 1iquid: viscosity, saturated vapor: thermal conductivity, 1iquid:

thermal conductivity, vapor:

- norma 7 pressure, $20{ }^{\circ} \mathrm{C}\left(68{ }^{\circ} \mathrm{F}\right)$

- normal pressure, $21.1^{\circ} \mathrm{C}\left(70^{\circ} \mathrm{F}\right)$

- $20^{\circ} \mathrm{C}\left(68^{\circ} \mathrm{F}\right)$ density, vapor

pressure, saturated vapor: density, saturated liquid: density, saturated vapor: specific volume, saturated 7 iquid: velocity of sound, saturated liquid: velocity of sound, saturated vapor: viscosity, saturated 7iquid: viscosity, saturated vapor: thermal conductivity, saturatd liquid:

thermal conductivity, saturated vapor:

- $60{ }^{\circ} \mathrm{C}\left(140{ }^{\circ} \mathrm{F}\right)$ pressure, saturated vapor:
heat of vaporization:

- critical point pressure: density: specific volume:

ENVIRONMENTAL

average atmospheric 1ifetime (Tatm): average stratospheric 1ifetime (Tstr): ODP (ozone depletion potential):

GWP (global warming potential): HGWP (haTocarbon GWP):
$86.4681464 \mathrm{~g} / \mathrm{mol}(0.190630$

8820

$\mathrm{lb} / \mathrm{mol}$ )

$-157.4{ }^{\circ} \mathrm{C}\left(-251.4^{\circ} \mathrm{F}\right)$

8401

$-40.8^{\circ} \mathrm{C}\left(-41.5^{\circ} \mathrm{F}\right)$

$1409 \mathrm{~kg} / \mathrm{m} 3(87.97 \mathrm{lb} / \mathrm{cf})$

$4.70 \mathrm{~kg} / \mathrm{m} 3(0.2947 \mathrm{~b} / \mathrm{cf})$

$0.710 \mathrm{~L} / \mathrm{kg}(0.0114 \mathrm{cf} / \mathrm{lb})$

$212.6 \mathrm{~L} / \mathrm{kg}(3.4055 \mathrm{cf} / 7 \mathrm{~b})$

$233.8 \mathrm{~kJ} / \mathrm{kg}(100.5 \mathrm{Btu} / \mathrm{lb})$

$851 \mathrm{~m} / \mathrm{s}(2791 \mathrm{ft} / \mathrm{s})$

$160 \mathrm{~m} / \mathrm{s}(525 \mathrm{ft} / \mathrm{s})$

$346 \mu \mathrm{Pa} \cdot \mathrm{s}(0.346 \mathrm{cp})$

$9.75 \mu \mathrm{Pa} \cdot \mathrm{s}(0.00975 \mathrm{cp})$

$\mathrm{Btu} / \mathrm{hr} \cdot \mathrm{ft}^{\circ} \mathrm{F}$ )

$0.0070 \mathrm{~W} / \mathrm{m} \cdot \mathrm{K}(0.0041$

$\mathrm{Btu} / \mathrm{hr} \cdot \mathrm{ft}^{\circ} \mathrm{F}$ )

$3.651 \mathrm{~kg} / \mathrm{m} 3(0.2279 \mathrm{~Tb} / \mathrm{cf}) \quad 8401$

$3.637 \mathrm{~kg} / \mathrm{m} 3(0.2270 \mathrm{~Tb} / \mathrm{cf})$

8401

$910.0 \mathrm{kPa}$ (131.99 psia) 8401

$1210 \mathrm{~kg} / \mathrm{m} 3(75.53 \mathrm{lb} / \mathrm{cf}) \quad 8401$

$38.48 \mathrm{~kg} / \mathrm{m} 3(2.402 \mathrm{~Tb} / \mathrm{cf}) \quad 8401$

$0.827 \mathrm{~L} / \mathrm{kg}(0.0132 \mathrm{cf} / 7 \mathrm{~b}) \quad 8401$

$565 \mathrm{~m} / \mathrm{s}(1854 \mathrm{ft} / \mathrm{s})$

$161 \mathrm{~m} / \mathrm{s}(529 \mathrm{ft} / \mathrm{s})$

$175 \mu \mathrm{Pa} \cdot \mathrm{s}(0.175 \mathrm{cp})$

$12.4 \mu \mathrm{Pa} \cdot \mathrm{s}(0.0124 \mathrm{cp})$

$0.0859 \mathrm{~W} / \mathrm{m} \cdot \mathrm{K}(0.0496$

$\mathrm{Btu} / \mathrm{hr} \cdot \mathrm{ft}^{\circ} \mathrm{F}$ )

$0.01095 \mathrm{~W} / \mathrm{m} \cdot \mathrm{K}(0.00632$

$\mathrm{Btu} / \mathrm{hr} \cdot \mathrm{ft}^{\circ} \mathrm{F}$ )

$2428 \mathrm{kPa}$ (352.1 psia) 8401 $139.9 \mathrm{~kJ} / \mathrm{kg}(60.2 \mathrm{Btu} / 7 \mathrm{~b}) \quad 8401$

$96.2{ }^{\circ} \mathrm{C}\left(205.1^{\circ} \mathrm{F}\right)$

$4990 \mathrm{kPa}$ (723.7 psia)

$524 \mathrm{~kg} / \mathrm{m3}(32.7 \mathrm{lb} / \mathrm{cf})$

$1.91 \mathrm{~L} / \mathrm{kg}(0.0306 \mathrm{cf} / 7 \mathrm{~b})$

8401

8401

8401

8401

8401

8401

$11.8 \mathrm{yr}$

9501

$210 \mathrm{yr}$

0.034 (model-derived relative 5301

to $R$ i1)

0.05 (semi-empirical relative to $R$ 11)

0.055 (estimate used for the Montreal Protocol)

1900 relative to $\mathrm{CO} 2$ for 100 $\mathrm{yr}$ integration

0.345 relative to $R 11$ for infinite integration period

0.35 relative to $R 11$ for
7713

0906

8401

8401
9501

6904

9501

5964

$6 \mathrm{~B} 35$
$0.1135 \mathrm{~W} / \mathrm{m} \bullet \mathrm{K}(0.0656$ 
photochemical reactivity (grnd leve1):

SAFETY

- classification

safety group (ASHRAE Standard 34): NFPA 704 degrees of hazard (H-F-R-S):

NPCA HMIS hazard ratings (H-F-R):

UL Comparative Hazard to Life Group:

IARC/CIRC human carcinogicity group: ACGIH carcinogenicity category:

DFG pregnancy risk group:

- short-term occupational Timit -...-

ARI "IDLH" recommendation:

NIOSH STEL (short-term exposur Timit):

- occupational exposure limit -....--

MAK (maximum workplace concentration):

- Tong-term occupational 7imit ------

NIOSH REL (recommendd exposure limit):

ACGIH TLV-TWA (time-weighted average):

exposure Timit consistent to OSHA PEL:

MAK (maximum workplace concentration):

- emergency exposure 7imit ---

Acute Toxicity Exposure Limit (ATEL):

Refrigerant Concentration Limit (RCL):

- acute (short-term) toxicity

LC50 (lethal concentration, $50 \%$ ):

ALC (approximate lethal concentration: infinite integration period

0.37 relative to $R 11$ for

infinite integration period

$4 \mathrm{~B} 16$

4511

A1

ARI recommendation: 2-0-0

Ausimont:

BOC Gases:

Honeywe 17:

2-0-0

$1-0-0$

2-1-0

hea th-flammability-reactivity

[-special]: $0=$ no, $4=$ severe

BOC Gases: $1-0-0$

DuPont: $1-0-1$

Honeywe 11: $\quad 1-1-0$

hea 7 th-flammability-reactivity

$0=i n s i g n i f i c a n t, 4=e x t r e m e$

5 (a) in absence of flame or

hot objects

3 , not classifiable

A4, not classifiable as a uman carcinogen

$C$ (no risk fear below MAK/BAT)

8601

3A15

MSDS

MSDS

MSDS

$50,000 \mathrm{ppm} v / \mathrm{v}$ for $30 \mathrm{~min}$

$1,250 \mathrm{ppm} v / \mathrm{v}$ TWA for $15 \mathrm{~min}$

5175

IV: $1,000 \mathrm{ppm} \mathrm{v} / \mathrm{v}$ momentary 60 $\min$

$1,000 \mathrm{ppm} v / \mathrm{v}$ TWA for 10 $\mathrm{hr} /$ day and $40 \mathrm{hr} / \mathrm{wk}$

$1,000 \mathrm{ppm} \mathrm{V} / \mathrm{v}$ TWA for $8 \mathrm{hr} /$ day 9504 and $40 \mathrm{hr} / \mathrm{wk}$

ARI: $1,000 \mathrm{ppm} v / \mathrm{v}$ TWA for $83 \mathrm{~A} 15$

$\mathrm{hr} /$ day and $40 \mathrm{hr} / \mathrm{wk}$

$500 \mathrm{ppm} \mathrm{v/v}$ TWA for $8 \mathrm{hr} /$ day 5561

40 (or 42) hr/wk

applies only to pure substance 5561

see note on R-31 contamination 5561

$25,000 \mathrm{ppm} v / \mathrm{v}$ (pretiminary

based on ASHRAE $34 u$ draft)

$25,000 \mathrm{ppm} v / \mathrm{v}$ (preliminary

based on ASHRAE $34 \mathrm{u}$ draft)

8420

9504

5561

3 A15

5204

5561

5204

25,000 ppm v/v (preliminary

based on ASHRAE $34 \mathrm{u}$ draft)

$25,000 \mathrm{ppm} v / \mathrm{V}$ (preliminary

based on ASHRAE $34 u$ draft)

$34 \mathrm{u}$

A950

$34 \mathrm{u}$

A950

rat, $15 \mathrm{~min}: 350,000 \mathrm{ppm}$

(fatal concentration by inhalation for half of test anima7s)

rat, 4 hr: 220,000 ppm (fata)

concentration by inhalation

for half of test animals)

rat, 4 hr: 220,000 ppm (fatal

concentration by inhalation

for half of test animals)

rat, $2 \mathrm{hr}, 1 / 4: 300,000 \mathrm{ppm}$

(lowest exposure tested with
6110

3718

6127

5365 


\section{cardiac sensitization (CS) EC50:}

cardiac sensitization threshold/LOEL:

cardiac sensitization (CS) NOEL:

anesthetic/CNS effect EC50:

anesthetic/CNS effect LOEL:

- flammability

LFL-UFL (flammability limits in air):

heat of combustion (by ASHRAE 34-92):

flash point:

autoignition temperature:

autodecomposition temperature:

former UL Classification:

- detection

appearance:

odor:

\section{PRODUCTION}

first commercial use as a refrigerant: last year production allowed: one or more deaths by

inhalation)

dog, 5 min: 140,000 ppm v/v

(effective concentration in

half of test animals)

dog, 5 min, 2/12: 50,000 ppm

$v / v$ (lowest observed effect

level in test anima 7s)

dog, $5 \mathrm{~min}, 0 / 12: 25,000 \mathrm{ppm}$

$v / v$ (no observed effect level

in test anima $7 \mathrm{~s}$ )

dog, 5 min, 0/12: 25,000 ppm

$v / v$ (no observed effect level

in test anima $7 \mathrm{~s}$ )

rat, 10min: depressant 140,000 6110

ppm $\mathrm{v} / \mathrm{v}$ (effective

concentration in half of test anima7s)

6110

5644

5644

6121

rat 2 hr

(lowest observed effect level

in ALC or LC50 studies)

5365

$\begin{array}{ll}\text { none (nonflammable as tested) } & 2525 \\ \text { none (nonflammable as tested) } & 5648\end{array}$

$2.2 \mathrm{MJ} / \mathrm{kg}(946 \mathrm{Btu} / \mathrm{7b}) \quad 2318$

ICI: does not f7ash MSDS

none (nonflammable as tested) 5204

$632{ }^{\circ} \mathrm{C}\left(1170{ }^{\circ} \mathrm{F}\right) \quad 5175$

BOC Gases: none

Solvay: $635^{\circ} \mathrm{C}\left(1175{ }^{\circ} \mathrm{F}\right) \quad$ MSDS

Dupont: $632^{\circ} \mathrm{C}\left(1170^{\circ} \mathrm{F}\right)$ MSDS

Elf Atochem: $480^{\circ} \mathrm{C}\left(896^{\circ} \mathrm{F}\right)$ SDS

practically nonflammable 6938

(withdrawn for revision of the

classification system,

category SBQT2)

Honeywe 17: clear, colorless MSDS

Elf Atochem: faint ether odor MSDS

1936 by T. Midgley, associates

2029 in developed countries $8 \mathrm{C01}$ under the Montreal Protocol 


\section{R-23}

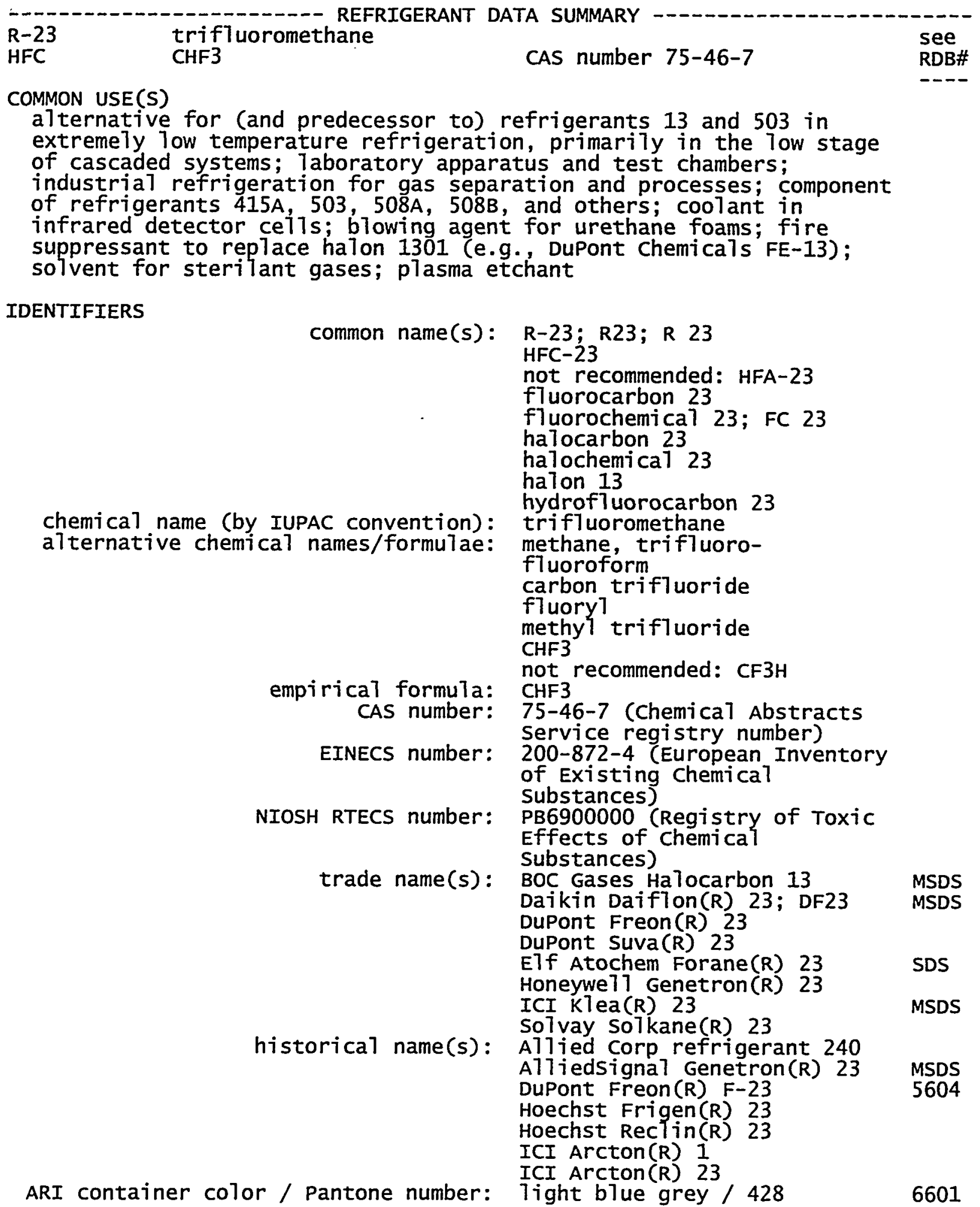


PHYSICAL

- properties

molar mass:

$70.0138496 \mathrm{~g} / \mathrm{mol}(0.154354$

8820

normal freezing/melting/triple point:

$\mathrm{Tb} / \mathrm{mo} 1)$

- normal boiling point -rorat

density, saturated Tiquid:

density, saturated vapor:

specific volume, saturated 7iquid: specific volume, saturated vapor: heat of vaporization: velocity of sound, saturated 7iquid: velocity of sound, saturated vapor: viscosity, saturated 7iquid: viscosity, saturated vapor: thermal conductivity, 7 iquid:

thermal conductivity, vapor:

- norma 7 pressure, $20^{\circ} \mathrm{C}\left(68^{\circ} \mathrm{F}\right)----$

- normal pressure, $21.1{ }^{\circ} \mathrm{C}\left(70^{\circ} \mathrm{F}\right)$--density, vapor:

- $20^{\circ} \mathrm{C}(68$

pressure, saturated vapor: density, saturated 1iquid: density, saturated vapor: specific volume, saturated $7 i q u i d:$ specific volume, saturated vapor: velocity of sound, saturated liquid: velocity of sound, saturated vapor: viscosity, saturated liquid: viscosity, saturated vapor: thermal conductivity, saturatd liquid: thermal conductivity, saturated vapor:

- critical point

temperature: pressure: density: specific volume:

$-155.2^{\circ} \mathrm{C}\left(-247.3^{\circ} \mathrm{F}\right)$

8401

$-82.1^{\circ} \mathrm{C}\left(-115.8^{\circ} \mathrm{F}\right) \quad 8401$

$1443 \mathrm{~kg} / \mathrm{m} 3(90.09 \mathrm{7b} / \mathrm{cf}) \quad 8401$

$4.65 \mathrm{~kg} / \mathrm{m} 3(0.2901 \mathrm{~b} / \mathrm{cf}) \quad 8401$

$0.693 \mathrm{~L} / \mathrm{kg}(0.0111 \mathrm{cf} / \mathrm{lb}) \quad 8401$

$215.0 \mathrm{~L} / \mathrm{kg}(3.4444 \mathrm{cf} / 7 \mathrm{~b}) \quad 8401$

$240.7 \mathrm{~kJ} / \mathrm{kg}$ (103.5 Btu/7b) 8401

$806 \mathrm{~m} / \mathrm{s}(2645 \mathrm{ft} / \mathrm{s}) \quad 8401$

$165 \mathrm{~m} / \mathrm{s}(542 \mathrm{ft} / \mathrm{s}) \quad 8401$

$392 \mu \mathrm{Pa} \cdot \mathrm{s}(0.392 \mathrm{cp}) \quad 8401$

$8.91 \mu \mathrm{Pa} \cdot \mathrm{s}(0.00891 \mathrm{cp}) \quad 8401$

$0.1357 \mathrm{~W} / \mathrm{m} \cdot \mathrm{K}(0.0784 \quad 8401$

$\left.\mathrm{Btu} / \mathrm{hr} \cdot \mathrm{ft}^{\circ} \mathrm{F}\right)$

$0.0065 \mathrm{~W} / \mathrm{m} \cdot \mathrm{K}(0.0037$

$\mathrm{Btu} / \mathrm{hr} \bullet f \mathrm{t}^{\circ} \mathrm{F}$ )

8401

$2.934 \mathrm{~kg} / \mathrm{m} 3(0.1832 \mathrm{~Tb} / \mathrm{cf}) \quad 8401$

$2.923 \mathrm{~kg} / \mathrm{m} 3(0.1825 \mathrm{~Tb} / \mathrm{cf}) \quad 8401$

$4201.9 \mathrm{kPa}$ (609.44 psia) 8401

$776 \mathrm{~kg} / \mathrm{m3}$ (48.47 $\mathrm{Tb} / \mathrm{cf}$ )

8401

$285.13 \mathrm{~kg} / \mathrm{m} 3 \quad(17.800 \mathrm{lb} / \mathrm{cf}) \quad 8401$

$1.288 \mathrm{~L} / \mathrm{kg}(0.0206 \mathrm{cf} / \mathrm{lb}) \quad 8401$

$3.5 \mathrm{~L} / \mathrm{kg}(0.0562 \mathrm{cf} / \mathrm{lb}) \quad 8401$

$162 \mathrm{~m} / \mathrm{s}(530 \mathrm{ft} / \mathrm{s}) \quad 8401$

$131 \mathrm{~m} / \mathrm{s}(428 \mathrm{ft} / \mathrm{s}) \quad 8401$

$58 \mu \mathrm{Pa} \cdot \mathrm{s}(0.058 \mathrm{cp}) \quad 8401$

$19.1 \mu \mathrm{Pa} \cdot \mathrm{s}(0.0191 \mathrm{cp}) \quad 8401$

$0.0642 \mathrm{~W} / \mathrm{m} \cdot \mathrm{K}(0.0371 \quad 8401$

$\mathrm{Btu} / \mathrm{hr} \bullet \mathrm{ft}^{\circ} \mathrm{F}$ )

$0.04668 \mathrm{~W} / \mathrm{m} \cdot \mathrm{K}(0.02697$

$\mathrm{Btu} / \mathrm{hr} \cdot \mathrm{ft}^{\circ} \mathrm{F}$ )

8401

$25.9{ }^{\circ} \mathrm{C}\left(78.7^{\circ} \mathrm{F}\right)$

$4836 \mathrm{kPa}$ (701.4 psia)

$525 \mathrm{~kg} / \mathrm{m} 3$ (32.8 ib/cf)

$1.90 \mathrm{~L} / \mathrm{kg}(0.0305 \mathrm{cf} / 7 \mathrm{~b})$

8401

8401

8401

8401

ENVIRONMENTAL

average atmospheric lifetime (Tatm): ODP (ozone depletion potentia1):

GWP (global warming potential):

HGWP (halocarbon GWP):

$243 \mathrm{yr}$

9501

$<0.0004$ (mode]-derived

relative to $R$ 11)

14,800 relative to $\mathrm{CO} 2$ for 1009501

yr integration

6 relative to $\mathrm{R} 11$ for

infinite integration period

8.32 relative to $R 11$ for

infinite integration period

DW

SAFETY

- Classification

safety group (ASHRAE standard 34): NFPA 704 degrees of hazard (H-F-R-S):

NPCA HMIS hazard ratings $(H-F-R)$

A1

Al7iedsignal: $1-0-1$

BOC Gases: $1-0-0$

Elf Atochem: 2-0-0

health-flammability-reactivity

[-specia]]: $0=$ no, $4=$ severe

MSDS 
UL Comparative Hazard to Life Group:

- 1ong-term occupational Timit exposure jimit consistent to OSHA PEL:

- emergency exposure Timit Acute Toxicity Exposure Limit (ATEL):

Refrigerant Concentration Limit (RCL):

- acute (short-term) toxicity ------ALC (approximate TethaT concentration:

cardiac sensitization (CS) EC50: cardiac sensitization (CS) NOEL: anesthetic/CNS effect LOEL: anesthetic/CNS effect NOEL:

- flammability

LFL-UFL (flammability limits in air): heat of combustion (by ASHRAE 34-92): flash point: autoignition temperature: autodecomposition temperature: former UL Classification:
BOC Gases: $1-0-0$

DuPont: $\quad 1-0-1$

hea 7 th-f] ammabi7ity-reactivity

$0=$ insignificant, $4=e x t r e m e$

MSDS

6 by estimate (not UL test) in 5906 absence of flame or hot objects

A77iedsignal PEL: 1,000 ppm $\mathrm{v} / \mathrm{v}$ TWA for $8 \mathrm{hr} /$ day and 40 $\mathrm{hr} / \mathrm{wk}$

DuPont AEL: $1,000 \mathrm{ppm} \mathrm{v/V} \mathrm{TWA} 5604$ for $8 \mathrm{hr} /$ day and $40 \mathrm{hr} / \mathrm{wk}$

ICI OEL: $1,000 \mathrm{ppm} \mathrm{V} / \mathrm{V}$ TWA for 5168 $8 \mathrm{hr} /$ day and $40 \mathrm{hr} / \mathrm{wk}$

41,000 ppm $v / v$ (preliminary based on ASHRAE $34 \mathrm{u}$ draft) $41,000 \mathrm{ppm} v / \mathrm{v}$ (preliminary based on ASHRAE 34u draft)

MSDS

$41,000 \mathrm{ppm} v / v$ (preliminary based on ASHRAE $34 u$ draft) $41,000 \mathrm{ppm} v / \mathrm{v}$ (preTiminary based on ASHRAE $34 \mathrm{u}$ draft)

$34 \mathrm{u}$

A950

$34 \mathrm{u}$

A950

rat, $4 \mathrm{hr}, 0 / 6$ : $>663,000 \mathrm{ppm}$ (lowest exposure tested with one or more deaths by inhalation)

dog, 10 min: $>500,000 \mathrm{ppm} v / \mathrm{v}$ (effective concentration in half of test animals)

dog, 5-10 min, 0/5: 800,000 $\mathrm{ppm} \mathrm{v} / \mathrm{v}$ (no observed effect Tevel in test animals) dog: $500,000 \mathrm{ppm} v / \mathrm{v}$ (no observed effect level in test anima $7 s$ )

dog, 2/2: ?analgesia 800,000

$\mathrm{ppm} v / \mathrm{v}$ (lowest observed effect leve 7 in test animals) rat, $4 \mathrm{hr}$, ?/6: 186,000 ppm $v / v$ (lowest observed effect Teve 1 in ALC or LC50 studies) human, 1/1: analgesia 800,000 $\mathrm{ppm} \mathrm{v} / \mathrm{v}$ (Towest observed effect Teve 1) human, 4/4: anesthetic 300,000 5737 $\mathrm{ppm} v / \mathrm{v}$ (lowest observed effect level)

rat, $6 \mathrm{hr}, 0 / 25$ : 51,000 ppm $v / v$ (no observed effect leve? in ALC or LC50 studies)

6513

6684

none (nonflammable as tested) none (nonflammable as tested) $-12.5 \mathrm{MJ} / \mathrm{kg}(-5374 \mathrm{Btu} / \mathrm{lb})$ A71iedsigna7: no flash point TOC, DuPont: will not burn $765^{\circ} \mathrm{C}\left(1409^{\circ} \mathrm{F}\right)$ $>750{ }^{\circ} \mathrm{C}\left(>1382^{\circ} \mathrm{F}\right)$ BOC Gases: none

7631

Elf Atochem: $1150{ }^{\circ} \mathrm{C}\left(2102^{\circ} \mathrm{F}\right)$ nonflammable (withdrawn for
4511

5648

2318

MSDS

MSDS

3960

5931

MSDS

SDS 
revision of the classification system, category SBQT2)

- detection

appearance: odor:
DuPont: clear, colorless DuPont: slight ethereal ICI: odorless
MSDS

MSDS

CSDS

PRODUCTION

$8 \mathrm{C} 01$ 


\section{R-32}

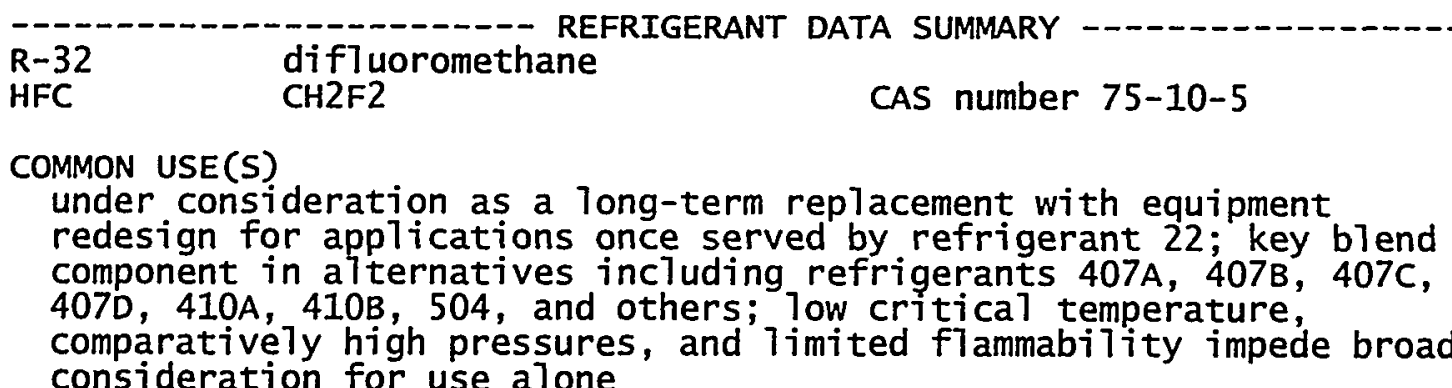

\section{IDENTIFIERS}

$$
\text { common name(s): } \begin{aligned}
& R-32 ; R 32 ; R 32 \\
& H F C-32 \\
& \text { f Juorocarbon } 32
\end{aligned}
$$

fluorochemical 32; FC 32

haTocarbon 32

ha7ochemica7 32

hydrofluorocarbon 32

not recommended: HFA-32

chemical name (by IUPAC convention):

difluoromethane

alternative chemical names/formulae:

methane, difluoro-

methylene difluoride

methylene fluoride

carbon fluoride hydride

$\mathrm{CH} 2 \mathrm{~F} 2$

not recommended:

$\mathrm{F} 2 \mathrm{H} 2$; H2F2C; $\mathrm{F} 2 \mathrm{H} 2 \mathrm{C}$

CAS number: 75-10-5 (Chemical Abstracts

Service registry number)

EINECS number: 200-839-4 (European Inventory

of Existing Chemical

Substances).

trade name(s): Ausimont Meforex(R) 32

Daikin fluorocarbon HFC-32 MSDS

DuPont HFC-32

Elf Atochem Forane(R) 32

Honeywe 77 Genetron(R) 32

ICI Klea(R) 32

Solvay Solkane(R) 32

ZCIRI Kehua (PRC) HFC -32

historical name(s): ATlied Corp refrigerant 140

Alliedsignal Genetron(R) 32

DuPont Freon(R) 32

2909

ARI container color / Pantone number:

$$
\text { ith red/ } 185 \text { band }
$$

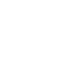


velocity of sound, saturated 1iquid: velocity of sound, saturated vapor: viscosity, saturated 7iquid: viscosity, saturated vapor: thermal conductivity, liquid:

thermal conductivity, vapor:

- normal pressure, $20{ }^{\circ} \mathrm{C}\left(68^{\circ} \mathrm{F}\right)$-----

- normal pressure, $21.1{ }^{\circ} \mathrm{C}\left(70^{\circ} \mathrm{F}\right)$.--

- $20{ }^{\circ} \mathrm{C}\left(68^{\circ} \mathrm{F}\right)$ - density, vapor

pressure, saturated vapor: density, saturated 7iquid: density, saturated vapor:

specific volume, saturated liquid: specific volume, saturated vapor: velocity of sound, saturated liquid: velocity of sound, saturated vapor: viscosity, saturated 7iquid: viscosity, saturated vapor: thermal conductivity, saturatd liquid: thermal conductivity, saturated vapor:

- $60{ }^{\circ} \mathrm{C}\left(140{ }^{\circ} \mathrm{F}\right)$-...-. pressure, saturated vapor:

- critical point heat of vaporization:

temperature:

pressure: density:

specific volume:

ENVIRONMENTAL

average atmospheric 1ifetime (Tatm): ODP (ozone depletion potential):

GWP (global warming potential): HGWP (halocarbon GWP):

$971 \mathrm{~m} / \mathrm{s}(3185 \mathrm{ft} / \mathrm{s}) \quad 8401$

$207 \mathrm{~m} / \mathrm{s}(680 \mathrm{ft} / \mathrm{s}) \quad 8401$

$281 \mu \mathrm{Pa} \cdot \mathrm{s}(0.281 \mathrm{cp}) \quad 8401$

$9.25 \mu \mathrm{Pa} \cdot \mathrm{s}(0.00925 \mathrm{cp}) \quad 8401$

$0.1901 \mathrm{~W} / \mathrm{m} \cdot \mathrm{K}(0.1098 \quad 8401$

$\left.\mathrm{Btu} / \mathrm{hr} \cdot \mathrm{ft}^{\circ} \mathrm{F}\right)$

$0.0083 \mathrm{~W} / \mathrm{m} \cdot \mathrm{K}(0.0048 \quad 8401$

$\mathrm{Btu} / \mathrm{hr} \cdot \mathrm{ft}^{\circ} \mathrm{F}$ )

$2.192 \mathrm{~kg} / \mathrm{m} 3(0.1368 \mathrm{lb} / \mathrm{cf}) \quad 8401$

$2.183 \mathrm{~kg} / \mathrm{m} 3(0.1363 \mathrm{lb} / \mathrm{cf}) \quad 8401$

$1474.6 \mathrm{kPa}$ (213.87 psia) 8401

$981 \mathrm{~kg} / \mathrm{m3}(61.27 \mathrm{ib} / \mathrm{cf}) \quad 8401$

$40.86 \mathrm{~kg} / \mathrm{m} 3 \quad(2.551 \mathrm{7b} / \mathrm{cf}) \quad 8401$

$1.019 \mathrm{~L} / \mathrm{kg}(0.0163 \mathrm{cf} / 7 \mathrm{~b}) \quad 8401$

$24.5 \mathrm{~L} / \mathrm{kg}(0.3921 \mathrm{cf} / 7 \mathrm{~b}) \quad 8401$

$584 \mathrm{~m} / \mathrm{s}(1915 \mathrm{ft} / \mathrm{s}) \quad 8401$

$206 \mathrm{~m} / \mathrm{s}(675 \mathrm{ft} / \mathrm{s}) \quad 8401$

$125 \mu \mathrm{Pa} \cdot \mathrm{s}(0.125 \mathrm{cp}) \quad 8401$

$12.7 \mu \mathrm{Pa} \cdot \mathrm{s}(0.0127 \mathrm{Cp}) \quad 8401$

$0.1390 \mathrm{~W} / \mathrm{m} \bullet \mathrm{K}(0.0803 \quad 8401$

$\mathrm{Btu} / \mathrm{hr} \cdot \mathrm{ft}^{\circ} \mathrm{F}$ )

$0.01430 \mathrm{~W} / \mathrm{m} \cdot \mathrm{K}(0.00826 \quad 8401$

$\mathrm{Btu} / \mathrm{hr} \bullet \mathrm{ft}^{\circ} \mathrm{F}$ )

$3933 \mathrm{kPa}$ (570.5 psia) 8401

$175.5 \mathrm{~kJ} / \mathrm{kg}(75.5 \mathrm{Btu} / \mathrm{7b}) \quad 8401$

$78.1{ }^{\circ} \mathrm{C}\left(172.6^{\circ} \mathrm{F}\right) \quad 8970$

$78.2{ }^{\circ} \mathrm{C}\left(172.7^{\circ} \mathrm{F}\right) \quad 9125$

$5782 \mathrm{kPa}(838.6$ psia) $\quad 8401$

$424 \mathrm{~kg} / \mathrm{m} 3(26.5 \mathrm{lb} / \mathrm{cf}) \quad 8401$

$429 \mathrm{~kg} / \mathrm{m} 3(26.8 \mathrm{lb} / \mathrm{cf}) \quad 9125$

$2.36 \mathrm{~L} / \mathrm{kg}(0.0378 \mathrm{cf} / \mathrm{Tb}) \quad 8401$

$5.6 \sim 1.4 \mathrm{yr} \quad 6695$

0.000 (model-derived relative $3 \mathrm{~B} 12$ to R 11)

880 relative to $\mathrm{CO} 2$ for 100 yr 9501 integration

0.11 relative to $R 11$ for infinite integration period 0.13 relative to $R 11$ for infinite integration period 0.14 relative to $R 11$ for infinite integration period

ICI: 0.15 relative to $R 11$ for CSDS infinite integration period

4511

3730

$6 \mathrm{~B} 35$

\section{SAFETY}

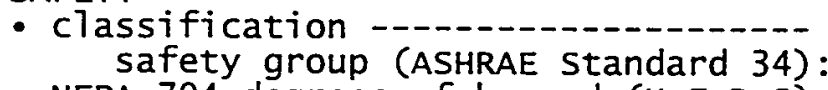
NFPA 704 degrees of hazard $(H-F-R-S)$ :

NPCA HMIS hazard ratings (H-F-R):

A2

Honeywe 11: 2-2-0

health-fl ammability-reactivity [-special]: $0=$ no, $4=$ severe

Al7iedsigna7: 2-4-0

DuPont: $1-4-1$

Honeywe 11: 1-2-0

health-flammability-reactivity

$0=$ insignificant, $4=e x t r e m e$
8601

MSDS

97.01 MSDS

MSDS

MSDS 
- long-term occupational 7imit -...-.-. AIHA WEEL (workplace env1 exp 1imit):

- emergency exposure 7imit Acute Toxicity Exposure Limit (ATEL)

Refrigerant Concentration Limit (RCL):

- acute (short-term) toxicity -.---.-LC50 ( 7 ethal concentration, 50\%):

ALC (approximate letha] concentration: cardiac sensitization (CS) EC50: cardiac sensitization threshold/LOEL: cardiac sensitization (CS) NOEL: anesthetic/CNS effect LOEL: anesthetic/CNS effect NOEL:

- flammability

LFL-UFL (f7ammability 7imits, $95^{\circ} \mathrm{C}$ ): LFL-UFL (with spark ignition):

LFL-UFL (with hot'wire ignition): flash point:

autoignition temperature:

autodecomposition temperature:

- detection

appearance:

odor:
LFL-UFL (flammability limits in air): heat of combustion (by ASHRAE 34-92):

$1,000 \mathrm{ppm} v / \mathrm{v}$ TWA for $8 \mathrm{hr} /$ day $5 \mathrm{C} 14$ and $40 \mathrm{hr} / \mathrm{wk}$

40,000 ppm v/v (preliminary based on ASHRAE $34 \mathrm{u}$ draft) 40,000 ppm v/v (preliminary based on ASHRAE $34 u$ draft) $32,000 \mathrm{ppm} v / \mathrm{v}$ (preliminary based on ASHRAE 34u draft) $32,000 \mathrm{ppm} v / \mathrm{v}$ (pretiminary based on ASHRAE $34 u$ draft)

$34 u$

A950

rat, $4 \mathrm{hr}, 0 / 10:>520,000 \mathrm{ppm}$

(fata) concentration by inhalation for half of test animals)

rat, $4 \mathrm{hr}, 0 / 6$ : $>760,000 \mathrm{ppm}$ (lowest exposure tested with one or more deaths by inhalation)

dog, $10 \mathrm{~min}:>350,000 \mathrm{ppm} \mathrm{v} / \mathrm{v}$ (effective concentration in half of test animals)

dog, $10 \mathrm{~min}, 1 / 12$ : 250,000 ppm 6170 $\mathrm{v} / \mathrm{v}$ (Towest observed effect Teve 7 in test anima $7 \mathrm{~s}$ )

dog, $10 \mathrm{~min}$, 0/1: 200,000 ppm $v / v$ (no observed effect Tevel in test animals)

rat, $4 \mathrm{hr}, 10 / 10: 85,900 \mathrm{ppm}$ $v / v$ (lowest observed effect Tevel in ALC or LC50 studies) rat, $6 \mathrm{hr}, 0 / 10: 49,500 \mathrm{ppm}$ $v / v$ (no observed effect level in subchronic or chronic study)

$12.7-33.5 \% \mathrm{v} / \mathrm{v}$ $14-31 \% \mathrm{v} / \mathrm{v}$

Daikin: 13.3-29.3\% v/v Elf Atochem: 12.7-33.4\% v/v Honeywe 17: $12.7-33.4 \% \mathrm{v} / \mathrm{v}$

6170

5740

5741

$11.4-33.8 \% \mathrm{v} / \mathrm{v}$

$13.3-29.3 \% \mathrm{v} / \mathrm{v}$

$13.5-31.4 \% \mathrm{v} / \mathrm{v}$

$14.2-28.9 \% \mathrm{v} / \mathrm{v}$

$9.4 \mathrm{MJ} / \mathrm{kg}$ (4034 Btu/7b)

Elf Atochem: 1iquified $R-32$ is "extremely flammable" Honeywe 17: gas, not applicable $648^{\circ} \mathrm{C}\left(1198^{\circ} \mathrm{F}\right)$ $685^{\circ} \mathrm{C}\left(1265^{\circ} \mathrm{F}\right)$ Honeywe 17: $>7500^{\circ} \mathrm{C}\left(>1382^{\circ} \mathrm{F}\right)$ Honeywe 17: $>250^{\circ} \mathrm{C}\left(>482^{\circ} \mathrm{F}\right)$

SDS SDS

MSDS

3960

$5 \mathrm{C} 14$

MSDS

MSDS

colorless

slight etherea]

$5 \mathrm{C} 14$

5 C14

PRODUCTION

last year production allowed: unrestricted

$8 \mathrm{CO} 1$ 


\section{R-113}

\begin{tabular}{|c|c|c|}
\hline $\begin{array}{l}R-113 \\
\text { CFC }\end{array}$ & $\begin{array}{l}1,1,2-\text { trichloro-1,2,2-trifluoroethane } \\
\text { CCT2FCCTF2 CAS number 76-13-1 }\end{array}$ & $\begin{array}{l}\text { see } \\
\text { RDB\# }\end{array}$ \\
\hline $\begin{array}{l}\text { COMmON } \\
\text { limi } \\
\text { high } \\
\text { refr } \\
\text { clea } \\
\text { clea }\end{array}$ & $\begin{array}{l}\text { in centrifugal chillers; very limited use in } \\
\text { ature heat pumps; aerosol solvent usually prope } \\
12 \text {; widely used as an industrial solvent part } \\
\text { ectronic circuit boards and, historical7y, for } \\
\text { lowing agent }\end{array}$ & \\
\hline
\end{tabular}

IDENTIFIERS

$$
\text { common name(s): R-113; R113; R } 113
$$

CFC-113

TTE; TCTFE

fluorocarbon 113

fluorochemical 113; FC 113

ha]ocarbon 113

chemical name (by IUPAC convention): 1,1,2-trichloro-1,2,2-

rifluoroethane

a7ternative chemical names/formulae: ethane, 1,1,2-trichloro-1,2,2rifluoro-

1,1,2-trichlorotrifluoroethane

trichlorotrifluoroethane

CCT2FCCTF2; CCT2F-CCTF2

not recommended:

F2C1CFC12; CF2C1-CFC12

FC72CF2C1; CFC12-CF2C1

$12 \mathrm{FCCC} 7 \mathrm{~F} 2$; C $72 \mathrm{FC}-\mathrm{CC} 7 \mathrm{~F} 2$

2CTCCFC12; F2C1C-CFC72

empirical formula: C2C13F3

C12CCF2C1; FC12C-CF2C1

\section{CAS number:}

76-13-1 (Chemical abstracts

Service registry number)

EINECS number: 200-936-1 (European Inventory

of Existing Chemical

Substances)

NIOSH RTECS number: KJ4000000 (Registry of Toxic

Effects of Chemical

Substances)

trade name(s): Asahi GTass Fron AF-113

Daikin Daiflon(R) 113

DuPont Freon(R) 113

DuPont Freon(R) TF

Elf Atochem Forane(R) 113

Hoechst Frigen $(R) 113$

historical name(s):

Honeywe 71 Genetron(R) 113

A77ied Corp refrigerant 226

A77iedsigna 7 Genetron(R) 113 MSDS

Carrier Corporation Carrene 3

ICI Arcton(R) 113

ICI Arcton(R) 63

Union Carbide Ucon(R) 113

VVB Dresden Frigedohn 113

ARI container color / Pantone number:

dark purple (violet) / 266

MSDS

MSDS

MSDS

MSDS

7601

7601

6601

PHYSICAL

- properties

molar mass:

$187.3747096 \mathrm{~g} / \mathrm{mol} \quad(0.413091$ 
normal freezing/melting/triple point:

- normat boiting point

temperature:

density, saturated 7iquid: density, saturated vapor:

specific volume, saturated liquid:

specific volume, saturated vapor: heat of vaporization:

velocity of sound, saturated liquid: velocity of sound, saturated vapor: viscosity, saturated vapor: viscosity, saturated liquid: thermal conductivity, Tiquid:

thermal conductivity, vapor:

- normal pressure, $20^{\circ} \mathrm{C}\left(68^{\circ} \mathrm{F}\right)$

- normal pressure, $21.1{ }^{\circ} \mathrm{C}\left(70^{\circ} \mathrm{F}\right)$---

- $20^{\circ} \mathrm{C}\left(68^{\circ} \mathrm{F}\right)$ density, Tiquid:

pressure, saturated vapor: density, saturated 7iquid: density, saturated vapor:

specific volume, saturated 7iquid: specific volume, saturated vapor: velocity of sound, saturated Tiquid: velocity of sound, saturated vapor: viscosity, saturated 7iquid: viscosity, saturated vapor: thermal conductivity, saturatd Tiquid: thermal conductivity, saturated vapor: - $60{ }^{\circ} \mathrm{C}\left(140{ }^{\circ} \mathrm{F}\right)$ pressure, saturated vapor: heat of vaporization:

- critical point

temperature: pressure: density: specific volume:

ENVIRONMENTAL

average atmospheric lifetime (Tatm): ODP (ozone depletion potential):

GWP (global warming potential): HGWP (haTocarbon GWP): density, Tiquid:

$-36.2^{\circ} \mathrm{C}\left(-33.2^{\circ} \mathrm{F}\right)$

8401

$47.6{ }^{\circ} \mathrm{C}\left(117.7^{\circ} \mathrm{F}\right)$

$1508 \mathrm{~kg} / \mathrm{m} 3(94.157 \mathrm{~b} / \mathrm{cf})$

$7.42 \mathrm{~kg} / \mathrm{m} 3(0.463 \mathrm{~Tb} / \mathrm{cf})$

$0.663 \mathrm{~L} / \mathrm{kg}(0.0106 \mathrm{cf} / 1 \mathrm{~b})$

$134.7 \mathrm{~L} / \mathrm{kg}(2.1575 \mathrm{cf} / 1 \mathrm{~b})$

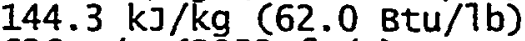

$626 \mathrm{~m} / \mathrm{s}(2053 \mathrm{ft} / \mathrm{s})$

$119 \mathrm{~m} / \mathrm{s}(390 \mathrm{ft} / \mathrm{s})$

$10.31 \mu \mathrm{Pa} \cdot \mathrm{s}(0.01031 \mathrm{cp})$

$500 \mu \mathrm{Pa} \cdot \mathrm{s}(0.500 \mathrm{cp})$

$0.0636 \mathrm{~W} / \mathrm{m} \bullet \mathrm{K}(0.0368$

$\mathrm{Btu} / \mathrm{hr} \cdot \mathrm{ft}^{\circ} \mathrm{F}$ )

$0.0095 \mathrm{~W} / \mathrm{m} \cdot \mathrm{K}(0.0055$

$\mathrm{Btu} / \mathrm{hr} \bullet \mathrm{ft}^{\circ} \mathrm{F}$ )

8401

8401

8401

8401

8401

8401

8401

8401

8401

8401

8401

8401

$1575 \mathrm{~kg} / \mathrm{m} 3$ (13.14 7b/gal)

8401

$1573 \mathrm{~kg} / \mathrm{m} 3$ (13.12 1b/gal)

8401

$36.7 \mathrm{kPa}$ (5.32 psia)

$1575 \mathrm{~kg} / \mathrm{m3}(98.32 \mathrm{lb} / \mathrm{cf})$

$2.88 \mathrm{~kg} / \mathrm{m} 3(0.180 \mathrm{1b} / \mathrm{cf})$

$0.635 \mathrm{~L} / \mathrm{kg}(0.0102 \mathrm{cf} / 1 \mathrm{~b})$

$34.8 \mathrm{~L} / \mathrm{kg}(0.5570 \mathrm{cf} / 7 \mathrm{~b})$

$716 \mathrm{~m} / \mathrm{s}(2348 \mathrm{ft} / \mathrm{s})$

$116 \mathrm{~m} / \mathrm{s}(381 \mathrm{ft} / \mathrm{s})$

$709 \mu \mathrm{Pa} \cdot \mathrm{s}(0.709 \mathrm{cp})$

$9.5 \mu \mathrm{Pa} \cdot \mathrm{s}(0.0095 \mathrm{cp})$

$0.0693 \mathrm{~W} / \mathrm{m} \cdot \mathrm{K}(0.0400$

$\mathrm{Btu} / \mathrm{hr} \cdot \mathrm{ft}^{\circ} \mathrm{F}$ )

$0.00827 \mathrm{~W} / \mathrm{m} \cdot \mathrm{K}(0.00478$

$\mathrm{Btu} / \mathrm{hr} \bullet \mathrm{ft}^{\circ} \mathrm{F}$ )

8401

8401

8401

8401

8401

8401

8401

8401

8401

8401

8401

$150 \mathrm{kPa}$ (21.8 psia)

$140.2 \mathrm{~kJ} / \mathrm{kg}(60.3 \mathrm{Btu} / 7 \mathrm{~b})$

8401

$214.1{ }^{\circ} \mathrm{C}\left(417.3^{\circ} \mathrm{F}\right)$

$3392 \mathrm{kPa}$ (492.0 psia)

$576 \mathrm{~kg} / \mathrm{m} 3$ (36.0 $\mathrm{lb} / \mathrm{cf})$

$1.79 \mathrm{~L} / \mathrm{kg}(0.0286 \mathrm{cf} / 1 \mathrm{~b})$

8401

8401

8401

0036

8401

$85 \mathrm{yr}$

0.90 (model-derived relative 9501

to $R$ II)

0.9 (semi-empirical relative to $\mathrm{R}$ 11)

0.8 (estimate used for the

Montreal Protocol)

9501

6000 relative to $\mathrm{CO} 2$ for 100

yr integration

1.35 relative to $R 11$ for

infinite integration period

6904

9501

5964

SAFETY

- classification

safety group (ASHRAE Standard 34 ): NFPA 704 degrees of hazard $(H-F-R-S)$ :
A1

ARI recommendation: 2-0-0

BOC Gases:

Honeywe 11:

$1-0-0$

2-0-0

health-flammability-reactivity

[-special]: $0=$ no, $4=$ severe
8601

3 A15

MSDS

MSDS 
NPCA HMIS hazard ratings $(H-F-R)$ :

UL Comparative Hazard to Life Group: ACGIH carcinogenicity category:

- short-term occupational 1imit

NIOSH IDLH (immediately dangerous): NIOSH SCP IDLH (immediately dangerous: NIOSH STEL (short-term exposur limit): ACGIH TLV-STEL (short-term exp Timit):

- occupational exposure limit -

MAK (maximum workplace concentration):

- Tong-term occupational Timit

OSHA PEL (permissible exposure limit):

NIOSH REL (recommendd exposure Timit):

ACGIH TLV-TWA (time-weighted average):

MAK (maximum workplace concentration):

- emergency exposure Timit

NRC EEGL (emergency exposure leve1):

Acute Toxicity Exposure Limit (ATEL):

Refrigerant concentration Limit ( $R C L)$ :

- special-purpose exposure control --NRC CEGL (continuous exposure Teve T):

- acute (short-term) toxicity --.----. LC50 (lethal concentration, 50\%):

ALC (approximate 1etha1 concentration:
BOC Gases: 1-0-0 MSDS DuPont: $1-0-1$ MSDS Honeywe 17: 1-0-0 MSDS heal th-flammability-reactivity $0=i n s i g n i f i c a n t, 4=e x t r e m e$

between 4 and 5 in absence of 5174 flame or hot objects

A4, not classifiable as a 9504 uman carcinogen

$2,000 \mathrm{ppm} \mathrm{v} / \mathrm{V} \quad 5204$

$4,500 \mathrm{ppm} v / v$ for $30 \mathrm{~min} \quad 3903$

$1,250 \mathrm{ppm} v / v$ TWA for $15 \mathrm{~min} 3903$

$1,250 \mathrm{ppm} \mathrm{v} / \mathrm{v}$ TWA for $15 \mathrm{~min} 9504$

IV: $1,000 \mathrm{ppm} \mathrm{v} / \mathrm{v}$ momentary 607101 min

$1,000 \mathrm{ppm} v / \mathrm{v}$ TWA for $8 \mathrm{hr} /$ day 3904 and $40 \mathrm{hr} / \mathrm{wk}$

$1,000 \mathrm{ppm} v / \mathrm{v}$ TWA for 10

3903

$\mathrm{hr} /$ day and $40 \mathrm{hr} / \mathrm{wk}$

$1,000 \mathrm{ppm} v / \mathrm{v}$ TWA for $8 \mathrm{hr} /$ day 9504 and $40 \mathrm{hr} / \mathrm{wk}$

$500 \mathrm{ppm} v / \mathrm{v}$ TWA for $8 \mathrm{hr} /$ day 40 (or 42) hr/wk

7101

$1 \mathrm{hr}: 1,500 \mathrm{ppm} \mathrm{v} / \mathrm{v}$ ceiTing guidance level for single emergency exposures

$24 \mathrm{hr}: 500 \mathrm{ppm} \mathrm{v/v}$ cejling

guidance level for single

emergency exposures

2,600 ppm $v / v$ (prełiminary

based on ASHRAE 34u draft)

2,600 ppm $\mathrm{v} / \mathrm{V}$ (preliminary

based on ASHRAE 34u draft)

$2,600 \mathrm{ppm} \mathrm{V} / \mathrm{v}$ (preliminary

based on ASHRAE 34u draft)

2,600 ppm v/v (preliminary

based on ASHRAE $34 u$ draft)

7414

7414

$34 u$

A950

$34 \mathrm{u}$

A950

90 day: $100 \mathrm{ppm} v / \mathrm{v}$ ceiling

7414

guidance for prolonged

exposure in closed

envi ronments

mouse, $30 \mathrm{~min}, 0 / 10:>100,000$

6165

ppm (fatal concentration by

inhalation for half of test animals)

rat, 15 min: $130,000 \mathrm{ppm}$

(fatal concentration by

inhalation for half of test anima7s)

rat, $4 \mathrm{hr}: 52,500 \mathrm{ppm}$ (fatal 6804

concentration by inhalation

for half of test animals)

mouse, $15 \mathrm{~min}, 2 / 10$ : 110,000

ppm (lowest exposure tested

with one or more deaths by

inhalation)

rat, 2 hr: 110,000 ppm (1)west 6B56

exposure tested with one or 
derma7 LD50 (7etha7 dosage, 50\%):

ora1 LD50 (lethal dosage, 50\%):

cardiac sensitization (CS) EC50:

cardiac sensitization threshold/LOEL:

cardiac sensitization (CS) NOEL:

anesthetic/CNS effect EC50:

anesthetic/CNS effect LOEL:

anesthetic/CNS effect NOEL:

- flammability -......-LFL-UFL (flammabi7ity Timits in air): heat of combustion (by ASHRAE 34-92): flash point:

autoignition temperature:

autodecomposition temperature: former UL Classification:

- detection

appearance:

odor:

PRODUCTION

first commercial use as a refrigerant: Tast year production allowed: more deaths by inhalation)

rat, $4 \mathrm{hr}, 1 / 6$ : $45,000 \mathrm{ppm}$

(lowest exposure tested with one or more deaths by inhalation)

rabbit: $>11,000 \mathrm{mg} / \mathrm{kg}$ (fatal dose by skin contact for half of test anima7s)

rat: $43,000 \mathrm{mg} / \mathrm{kg}$ (fatal dose by ingestion for half of test animais)

dog, 5 min: $7,000 \mathrm{ppm} v / \mathrm{v}$ (effective concentration in half of test animals)

dog, $10 \mathrm{~min}$; 10/29: 4,850 ppm $v / v$ (lowest observed effect level in test animals)

dog, 10 min, 0/12: 2,600 ppm $v / v$ (no observed effect 7 eve 7 in test anima7s)

mouse, 30 min: $57,000 \mathrm{ppm} \mathrm{v} / \mathrm{v}$ (effective concentration in ha7f of test animals)

rat, 10 min: stimulant 28,000 ppm $v / v$ (effective concentration in half of test anima7s)

mouse, $15 \mathrm{~min}, 1 / 10: 50,000$

$\mathrm{ppm} v / \mathrm{v}$ (lowest observed effect leve 7 in test animals) mouse, $15 \mathrm{~min}, 9 / 10$ : 60,000 $\mathrm{ppm} v / \mathrm{v}$ (lowest observed effect level in test animals) 6804 7541 7441 6110 $65 \mathrm{AO}$ $65 A 0$ 6165

6110 observed effect level in ALC or LC50 studies)

none (nonflammable as tested)

$0.1 \mathrm{MJ} / \mathrm{kg}$ (43 Btu/7b)

2525

DuPont: wi 17 not burn

2318

Honeywe 71: none

ICI: does not flash

MSDS

$680^{\circ} \mathrm{C}\left(1256^{\circ} \mathrm{F}\right)$

MSDS

BOC Gases: none

5174

MSDS

Honeywe 11: $770^{\circ} \mathrm{C}\left(1418^{\circ} \mathrm{F}\right)$ MSDS

DuPont: $300^{\circ} \mathrm{C}\left(572^{\circ} \mathrm{F}\right)$ MSDS

practically nonflammable 5931

(withdrawn for revision of the classification system,

category SBQT2)

BOC Gases: water-white liquid Honeywe 17: colorless

colortess

DuPont: s7ight etherea]

Honeywe 77: faint etherea 7 and Honeywe11: s7ight7y sweetish nearly odorless

MSDS

MSDS

7414

MSDS

MSDS

MSDS

5367

1934 by T. Midgley, associates 5C39 1995 in developed countries 8C01 under the Montreal Protocol 


\section{R-114}

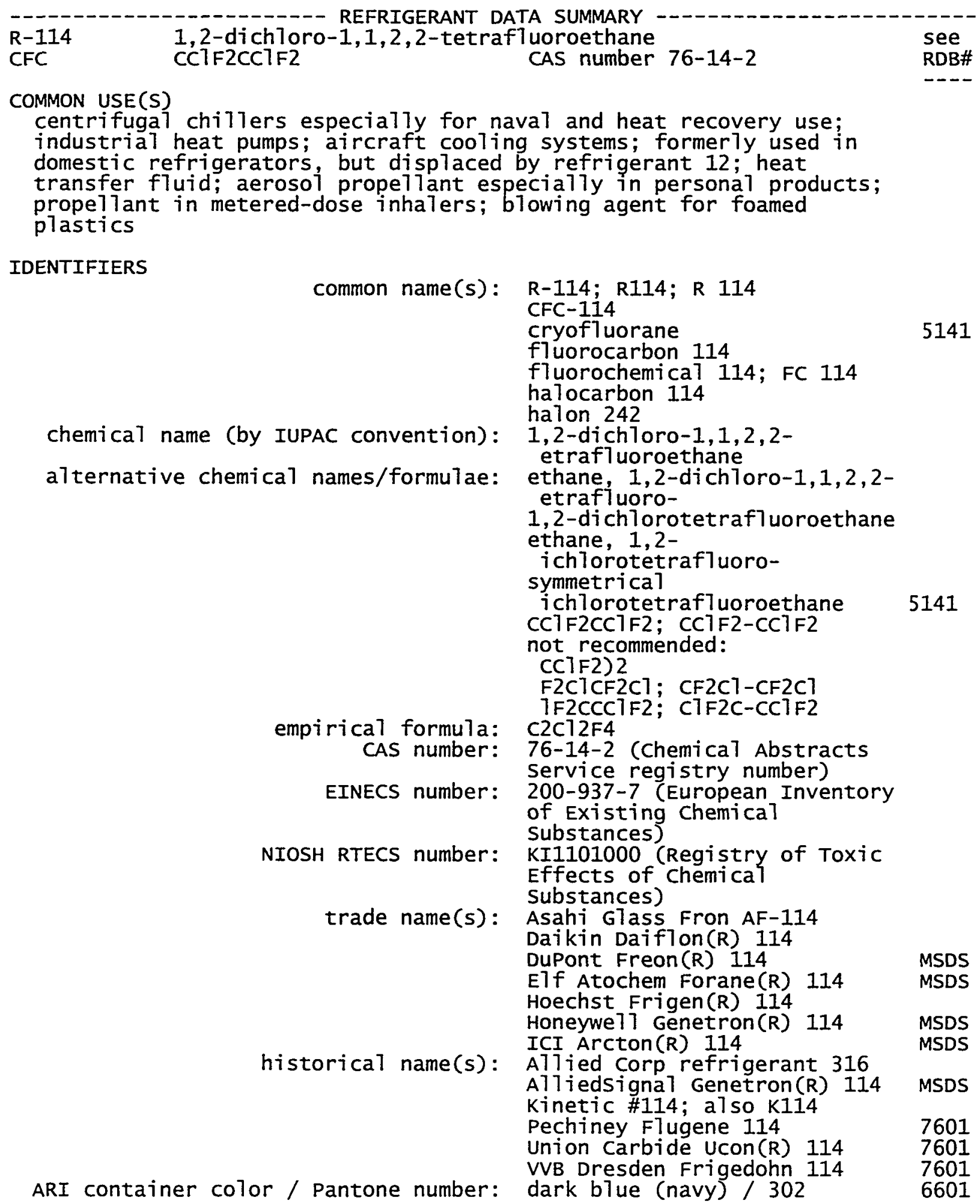


PHYSICAL

- properties

molar mass:

normal freezing/melting/triple point:

- normal boiling point

temperature: density, saturated 7 iquid: density, saturated vapor: specific volume, saturated vapor: heat of vaporization: velocity of sound, saturated vapor: viscosity, saturated vapor: viscosity, saturated 7 iquid: thermal conductivity, Tiquid:

thermal conductivity, vapor:

- normal pressure, $20^{\circ} \mathrm{C}\left(68^{\circ} \mathrm{F}\right)$

- normal pressure, $21.1{ }^{\circ} \mathrm{C}\left(70^{\circ} \mathrm{F}\right)$ density, vapor

pressure, saturated vapor: density, saturated liquid: density, saturated vapor:

specific volume, saturated liquid: specific volume, saturated vapor: velocity of sound, saturated 1iquid: velocity of sound, saturated vapor: viscosity, saturated 7iquid: viscosity, saturated vapor: thermal conductivity, saturatd 7iquid: thermal conductivity, saturated vapor:

- $60^{\circ} \mathrm{C}\left(140^{\circ} \mathrm{F}\right)$ pressure, saturated vapor: heat of vaporization:

- critical point

temperature: pressure: density: specific volume: specific volume, saturated Tiquid: velocity of sound, saturated liquid:

$170.9204128 \mathrm{~g} / \mathrm{mol}(0.376815$ $1 \mathrm{~b} / \mathrm{mo}$ )

$-181.3^{\circ} \mathrm{C}\left(-294.3^{\circ} \mathrm{F}\right)$

$-94.1{ }^{\circ} \mathrm{C}\left(-137.5^{\circ} \mathrm{F}\right)$

$3.6^{\circ} \mathrm{C}\left(38.5^{\circ} \mathrm{F}\right)$

$1518 \mathrm{~kg} / \mathrm{m}^{3}(94.77 \mathrm{lb} / \mathrm{cf})$

$7.83 \mathrm{~kg} / \mathrm{m} 3(0.489 \mathrm{~Tb} / \mathrm{cf})$

$0.659 \mathrm{~L} / \mathrm{kg}(0.0106 \mathrm{cf} / 7 \mathrm{~b})$

$127.7 \mathrm{~L} / \mathrm{kg}(2.0457 \mathrm{cf} / 7 \mathrm{~b})$

$135.9 \mathrm{~kJ} / \mathrm{kg}(58.4 \mathrm{Btu} / 7 \mathrm{~b})$

$630 \mathrm{~m} / \mathrm{s}(2066 \mathrm{ft} / \mathrm{s})$

$116 \mathrm{~m} / \mathrm{s}(382 \mathrm{ft} / \mathrm{s})$

$10.03 \mu \mathrm{Pa} \cdot \mathrm{s}(0.01003 \mathrm{cp})$

$357 \mu \mathrm{Pa} \cdot \mathrm{s}(0.357 \mathrm{cp})$

$0.0656 \mathrm{~W} / \mathrm{m} \cdot \mathrm{K}(0.0379$

$\left.\mathrm{Btu} / \mathrm{hr} \bullet \mathrm{ft}{ }^{\circ} \mathrm{F}\right)$

$0.0091 \mathrm{~W} / \mathrm{m} \cdot \mathrm{K}(0.0053$

$\mathrm{Btu} / \mathrm{hr} \cdot f \mathrm{t}^{\circ} \mathrm{F}$ )

$7.342 \mathrm{~kg} / \mathrm{m} 3(0.4584 \mathrm{~Tb} / \mathrm{cf})$

$7.312 \mathrm{~kg} / \mathrm{m} 3(0.4564 \mathrm{~Tb} / \mathrm{cf})$

8401

$182.0 \mathrm{kPa}$ (26.40 psia)

$1470 \mathrm{~kg} / \mathrm{m} 3$ ( $91.76 \mathrm{lb} / \mathrm{cf}$ )

$13.59 \mathrm{~kg} / \mathrm{m} 3(0.848 \mathrm{~Tb} / \mathrm{cf})$

$0.680 \mathrm{~L} / \mathrm{kg}(0.0109 \mathrm{cf} / 7 \mathrm{~b})$

$16.0 \mathrm{~L} / \mathrm{kg}(0.2560 \mathrm{cf} / 7 \mathrm{~b})$

$570 \mathrm{~m} / \mathrm{s}(1870 \mathrm{ft} / \mathrm{s})$

$117 \mathrm{~m} / \mathrm{s}$ (385 ft/s)

$295 \mu \mathrm{Pa} \cdot \mathrm{s}(0.295 \mathrm{cp})$

$10.6 \mu \mathrm{Pa} \cdot \mathrm{s}(0.0106 \mathrm{cp})$

$0.0620 \mathrm{w} / \mathrm{m} \cdot \mathrm{K}(0.0358$

$\left.\mathrm{Btu} / \mathrm{hr} \bullet \mathrm{ft}{ }^{\circ} \mathrm{F}\right)$

$0.00999 \mathrm{w} / \mathrm{m} \cdot \mathrm{K}(0.00577$

$\mathrm{Btu} / \mathrm{hr} \cdot f \mathrm{t}^{\circ} \mathrm{F}$ )

$580 \mathrm{kPa}$ (84.1 psia)

$112.7 \mathrm{~kJ} / \mathrm{kg}(48.5 \mathrm{Btu} / 7 \mathrm{~b})$

$145.7^{\circ} \mathrm{C}\left(294.2^{\circ} \mathrm{F}\right)$

$3257 \mathrm{kPa}$ (472.4 psia)

$580 \mathrm{~kg} / \mathrm{m} 3$ (36.2 $\mathrm{ib} / \mathrm{cf})$

$1.72 \mathrm{~L} / \mathrm{kg}(0.0276 \mathrm{cf} / 7 \mathrm{~b})$

8820

MSDS

8401

8401

8401

8401

8401

8401

8401

8401

8401

8401

8401

8401

8401

8401

8401

8401

8401

8401

8401

8401

8401

8401

8401

8401

8401

8401

8401

8401

8401

8401

8401

ENVIRONMENTAL average atmospheric 7 ifetime (Tatm): ODP (ozone depletion potentia7):

GWP (global warming potential): HGWP (hatocarbon GWP):
$300 \mathrm{yr}$

0.85 (model-derived relative to $R$ 11)

1.0 (estimate used for the Montreal Protocol)

9800 relative to $\mathrm{CO} 2$ for 100 yr integration

7.20 relative to $R 11$ for infinite integration period
6695

5301

6904

9501

DW

SAFETY

- classification

safety group (ASHRAE Standard 34): 
NFPA 704 degrees of hazard $(H-F-R-S)$ : NPCA HMIS hazard ratings $(H-F-R)$ :

UL Comparative Hazard to Life Group: ACGIH carcinogenicity category:

- short-term occupational 7imit ------

NIOSH IDLH (immediately dangerous): NIOSH SCP IDLH (immediately dangerous: - occupational exposure limit -.-.-.--

MAK (maximum workplace concentration):

- Tong-term occupational 7imit -...-.OSHA PEL (permissible exposure Timit): NIOSH REL (recommendd exposure Timit): ACGIH TLV-TWA (time-weighted average): MAK (maximum workplace concentration):

- emergency exposure Timit NRC EEGL (emergency exposure leve1):

Acute Toxicity Exposure Limit (ATEL):

Refrigerant concentration Limit ( $R C L)$ :

- specia7-purpose exposure control --NRC CEGL (continuous exposure level):

- acute (short-term) toxicity -.-.--LC50 (letha7 concentration, $50 \%$ ):

oral LD50 (letha1 dosage, 50\%):
ARI recommendation: 2-0-0 3A15

BOC Gases: $1-0-0$ MSDS

Honeywe 17: 2-0-0 MSDS

hea 7 th-flammability-reactivity

[-special]: $0=$ no, $4=$ severe

BOC Gases: 1-0-0 MSDS

DuPont: $1-0-1 \quad$ MSDS

Honeywe 77: 1-0-0 MSDS

health-flammability-reactivity

$0=$ insignificant, $4=$ extreme

6 in absence of flame or hot 4B64

objects

A4, not classifiable as a 9504 uman carcinogen

$15,000 \mathrm{ppm} v / \mathrm{v} \quad 5204$

$50,000 \mathrm{ppm} v / \mathrm{v}$ for $30 \mathrm{~min} \quad 3903$

IV: 2,000 ppm $\mathrm{v} / \mathrm{v}$ momentary 605561 $\min$

1,000 ppm $v / \mathrm{v}$ TWA for $8 \mathrm{hr} /$ day 3904 and $40 \mathrm{hr} / \mathrm{wk}$

$1,000 \mathrm{ppm} v / \mathrm{V}$ TWA for $10 \quad 3903$

$\mathrm{hr} /$ day and $40 \mathrm{hr} / \mathrm{wk}$

$1,000 \mathrm{ppm} v / \mathrm{v}$ TWA for $8 \mathrm{hr} /$ day 9504 and $40 \mathrm{hr} / \mathrm{wk}$

$1,000 \mathrm{ppm} v / \mathrm{v}$ TWA for $8 \mathrm{hr} /$ day 5561 40 (or 42) hr/wk

being examined for changes 7101

I hr: $10,000 \mathrm{ppm} \mathrm{v} / \mathrm{v}$ ceiling 7414

guidance level for single

emergency exposures

$24 \mathrm{hr}$ : 1,000 ppm $\mathrm{v} / \mathrm{v}$ ceiling 7414

guidance level for single

emergency exposures

20,000 ppm v/v (preliminary

based on ASHRAE $34 \mathrm{u}$ draft)

$20,000 \mathrm{ppm} v / \mathrm{v}$ (preTiminary

$34 u$

based on ASHRAE 34u draft)

A950

$20,000 \mathrm{ppm} \mathrm{V} / \mathrm{v}$ (preliminary

$34 \mathrm{u}$

based on ASHRAE $34 u$ draft)

$20,000 \mathrm{ppm} \mathrm{V} / \mathrm{v}$ (pretiminary

A950

based on ASHRAE $34 \mathrm{u}$ draft)

7414

guidance for prolonged

exposure in closed

envi ronments

rat, 30 min: 720,000 ppm

(fatal concentration by inhalation for half of test animals)

rat, hr: $>600,000 \mathrm{ppm}$ (fatal 5355

concentration by inhalation

for half of test animals)

rat, hr, ElfAtochem: 600,000 MSDS

ppm (fatal concentration by

inhalation for half of test animals)

rat: $>2,250 \mathrm{mg} / \mathrm{kg}$ (fata) dose by ingestion for half of test 
Cardiac sensitization (CS) EC50: $\begin{aligned} & \operatorname{anima} 7 \mathrm{~s}) \\ & \operatorname{dog}, 10\end{aligned}$

Cardiac sensitization (CS) EC50:

dog, 10 min: 46,000-100,000

6684

$\mathrm{ppm} v / \mathrm{v}$ (effective

concentration in half of test

anima7s)

dog, 5 min: 25,000-50,000 ppm 5644

$\mathrm{v} / \mathrm{v}$ (effective concentration

in half of test animals)

cardiac sensitization threshold/LOEL:

dog, 5 min, 1/12: 25,000 ppm

$v / v$ (lowest observed effect

level in test anima7s)

anesthetic/CNS effect EC50: rat, $10 \mathrm{~min}: 250,000 \mathrm{ppm} \mathrm{v/v}$

(effective concentration in

half of test animals)

anesthetic/CNS effect NOEL:

rat, 2 hr: $100,000 \mathrm{ppm} v / \mathrm{v}$ (no 5365

observed effect Tevel in ALC or LC50 studies)

- flammability

LFL-UFL (flammability limits in air):

heat of combustion (by ASHRAE 34-92):

flash point: autoignition temperature:

autodecomposition temperature: former UL Classification:

- detection

appearance:
odor:

none (nonflammable as tested)

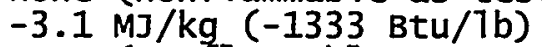

0036

2318

none (nonflammable as tested) 3903

$>750^{\circ} \mathrm{C}\left(>1382^{\circ} \mathrm{F}\right)$

5931

DuPont: $593^{\circ} \mathrm{C}\left(1099^{\circ} \mathrm{F}\right) \quad$ MSDS

nonflammable (withdrawn for 5931

revision of the classification

system, category SBQT2)

$\begin{array}{ll}\text { colorless } & 7414 \\ \text { Dupont: s7ight ethereal } & \text { MSDS } \\ \text { nearly odorless } & 5367\end{array}$

PRODUCTION

first commercial use as a refrigerant: last year production allowed:

1933 by T. Midgley, associates 5 C39

1995 in developed countries 8C01 under the Montreal Protocol 


\section{R-115}

Chloropentafluoroethane DATA SUMMARY
CFC CAS number 76-15-3
COMMON USE(S)
Component of refrigerant 502; Timited use as a single-compound
refrigerant for refrigerated display cases in Japan; industrial
refrigeration with centrifugal (turbo) compressors); dielectric
fluid; formerly used, until phased out pursuant to the Montreal
Protocol, as an aerosol propel7ant for foods and other uses, as an
aerating agent for foamed or sprayed food products and as a food
additive for vapor depression; also formerly used as a plasma etchant

IDENTIFIERS

common name(s): R-115; R115; R 115

CFC -115

CPFE

f7uorocarbon 115

see

RDB\#

- - -

fluorochemica7 115; FC 115

chemical name (by IUPAC convention):

ha7ocarbon 115

a)ternative chemical names/formulae:

chloropentafluoroethane

1-chloro-1, 1, 2, 2,2-

entafluoroethane

ethane, chloropentafluoro-

ethane, 1-ch Toro-1,1,2,2,2-

entafiuoro-

monoch loropentafluoroethane

CC1F2CF3; CC1F2-CF3

not recommended:

F3CC1F2; CF3-CC1F2

$1 F 2 C C F 3 ; C 7 F 2 C-C F 3$

empirical formula: 3CCCTF2; F3C-CCTF2

CAS number: 76-15-3 (Chemical Abstracts

Service registry number)

EINECS number: 200-938-2 (European Inventory

of Existing Chemica]

Substances)

NIOSH RTECS number: KH7877500 (Registry of Toxic

Effects of Chemical

Substances)

trade name(s): Asahi G1ass Fron AF-115

Daikin Daiflon(R) 115

DuPont Freon(R) 115

Elf Atochem Forane(R) 115

Honeywe 11 Genetron(R) 115

ICI ArCton (R) 115

$5 C 41$

historical name(s): $\begin{array}{ll}\text { Allied Corp refrigerant } 410 \\ \text { Altiedsignal Genetron(R) } 115\end{array}$ Hoechst Frigen(R) 115

ARI container color / Pantone number: none, use light green grey/413 6601

PHYSICAL

- properties

molar mass:

$154.466116 \mathrm{~g} / \mathrm{mo} 7(0.340539$

8820

$7 \mathrm{~b} / \mathrm{mo} 7$ )

normal freezing/melting/triple point:

- normal boiling point -.r.-

$-99.4{ }^{\circ} \mathrm{C}\left(-146.9^{\circ} \mathrm{F}\right)$

8401

density, saturated liquid:

$-38.9^{\circ} \mathrm{C}\left(-38.1^{\circ} \mathrm{F}\right)$

8401

$1558 \mathrm{~kg} / \mathrm{m} 3(97.28 \mathrm{lb} / \mathrm{cf}) \quad 8401$ 
density, saturated vapor: specific volume, saturated 1iquid: specific volume, saturated vapor: heat of vaporization: velocity of sound, saturated Tiquid: velocity of sound, saturated vapor: viscosity, saturated liquid: viscosity, saturated vapor: thermal conductivity, liquid:

thermal conductivity, vapor:

- normat pressure, $20{ }^{\circ} \mathrm{C}\left(68^{\circ} \mathrm{F}\right)$-----

- normar pressure, $21.1{ }^{\circ} \mathrm{C}\left(70^{\circ} \mathrm{F}\right)$-.-

- $20^{\circ} \mathrm{C}\left(68^{\circ} \mathrm{F}\right)$-_ensity, vapor density, vapor

pressure, saturated vapor: density, saturated liquid: density, saturated vapor:

specific volume, saturated liquid: specific volume, saturated vapor: velocity of sound, saturated liquid: velocity of sound, saturated vapor: viscosity, saturated liquid: viscosity, saturated vapor: thermal conductivity, saturated vapor: - $60{ }^{\circ} \mathrm{C}\left(140^{\circ} \mathrm{F}\right)$

- critical point pressure, saturated vapor: heat of vaporization:

temperature: pressure: density: specific volume: thermal conductivity,. saturatd Tiquid:

$8.41 \mathrm{~kg} / \mathrm{m} 3(0.525 \mathrm{lb} / \mathrm{cf})$

$0.642 \mathrm{~L} / \mathrm{kg}(0.0103 \mathrm{cf} / \mathrm{lb})$

$119.0 \mathrm{~L} / \mathrm{kg}(1.9054 \mathrm{cf} / 7 \mathrm{~b})$

$125.4 \mathrm{~kJ} / \mathrm{kg}(53.9 \mathrm{Btu} / 7 \mathrm{~b})$

$610 \mathrm{~m} / \mathrm{s}(2002 \mathrm{ft} / \mathrm{s})$

$113 \mathrm{~m} / \mathrm{s}(372 \mathrm{ft} / \mathrm{s})$

$336 \mu \mathrm{Pa} \cdot \mathrm{s}(0.336 \mathrm{cp})$

$9.72 \mu \mathrm{Pa} \cdot \mathrm{s}(0.00972 \mathrm{cp})$

$0.0712 \mathrm{~W} / \mathrm{m} \cdot \mathrm{K}(0.0411$

$\mathrm{Btu} / \mathrm{hr} \cdot \mathrm{ft}^{\circ} \mathrm{F}$ )

$0.0082 \mathrm{~W} / \mathrm{m} \cdot \mathrm{K}(0.0047$

$\mathrm{Btu} / \mathrm{hr} \bullet \mathrm{ft}{ }^{\circ} \mathrm{F}$ )

$6.555 \mathrm{~kg} / \mathrm{m} 3(0.4092 \mathrm{7b} / \mathrm{cf})$

$6.529 \mathrm{~kg} / \mathrm{m} 3(0.4076 \mathrm{~Tb} / \mathrm{cf})$

$789.3 \mathrm{kPa}$ (114.48 psia)

$1315 \mathrm{~kg} / \mathrm{m} 3(82.07 \mathrm{lb} / \mathrm{cf})$

$61.82 \mathrm{~kg} / \mathrm{m3}(3.859 \mathrm{~Tb} / \mathrm{cf})$

$0.761 \mathrm{~L} / \mathrm{kg}(0.0122 \mathrm{cf} / \mathrm{lb})$

$16.2 \mathrm{~L} / \mathrm{kg}(0.2591 \mathrm{cf} / 7 \mathrm{~b})$

$359 \mathrm{~m} / \mathrm{s}(1177 \mathrm{ft} / \mathrm{s})$

$109 \mathrm{~m} / \mathrm{s}(357 \mathrm{ft} / \mathrm{s})$

$153 \mu \mathrm{Pa} \cdot \mathrm{s}(0.153 \mathrm{cp})$

$12.3 \mu \mathrm{Pa} \cdot \mathrm{s}(0.0123 \mathrm{cp})$

$0.0525 \mathrm{~W} / \mathrm{m} \cdot \mathrm{K}(0.0303$

$\mathrm{Btu} / \mathrm{hr} \cdot \mathrm{ft}^{\circ} \mathrm{F}$ )

$0.01789 \mathrm{~W} / \mathrm{m} \cdot \mathrm{K}(0.01034$

$\mathrm{Btu} / \mathrm{hr} \cdot \mathrm{ft}^{\circ} \mathrm{F}$ )

$2078 \mathrm{kPa}$ (301.3 psia)

$62.9 \mathrm{~kJ} / \mathrm{kg}(27.1 \mathrm{Btu} / 7 \mathrm{~b})$

$80.0^{\circ} \mathrm{C}\left(175.9^{\circ} \mathrm{F}\right)$

$3120 \mathrm{kPa}$ (452.5 psia)

$613 \mathrm{~kg} / \mathrm{m} 3$ (38.3 lb/cf)

$1.63 \mathrm{~L} / \mathrm{kg}(0.0261 \mathrm{cf} / \mathrm{lb})$

8401

8401

8401

8401

8401

8401

8401

8401

8401

8401

8401

8401

8401

8401

8401

8401

8401

8401

8401

8401

8401

8401

8401

8401

8401

8401

8401

8401

8401

ENVIRONMENTAL average atmospheric 7ifetime (Tatm): ODP (ozone depletion potential):

GWP (global warming potential): HGWP (halocarbon GWP):
$1700 \mathrm{yr}$

0.40 (model-derived relative to $R$ 11)

0.6 (estimate used for the Montreal Protocol)

10,300 relative to $\mathrm{CO} 2$ for 1009501 $y r$ integration

36.0 relative to $R 11$ for infinite integration period

6695

9501

6904

DW
SAFETY

- classification

safety group (ASHRAE standard 34):

NFPA 704 degrees of hazard (H-F-R-S):

NPCA HMIS hazard ratings $(H-F-R)$ :

UL Comparative Hazard to Life Group:
A1

8601

BOC Gases: $1-0-0$

Elf Atochem: 2-0-0 hea th-f1 ammabi1ity-reactivity [-specia] ]: $0=$ no, $4=$ severe

BOC Gases: 1-0-0

DuPont: $1-0-1$

hea 1th-f1 ammability-reactivity $0=$ insignificant, $4=e x t r e m e$

6 in absence of flame or hot objects
MSDS MSDS

MSDS MSDS

5931 
- Tong-term occupational limit -....NIOSH REL (recommendd exposure limit): ACGIH TLV-TWA (time-weighted average): exposure limit consistent to OSHA PEL:

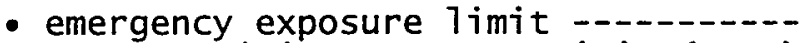
Acute Toxicity Exposure Limit (ATEL):

Refrigerant Concentration Limit ( $R C L)$ :

- acute (short-term) toxicity ALC (approximate lethal concentration:

oral LD50 (7etha7 dosage, 50\%):

cardiac sensitization (CS) EC50:

cardiac sensitization threshold/LOEL: anesthetic/CNS effect EC50:

anesthetic/CNS effect NOEL:

- flammability LFL-UFL (flammability limits in air): heat of combustion (by ASHRAE 34-92): flash point: autoignition temperature: autodecomposition temperature: former UL Classification:
$1,000 \mathrm{ppm} \mathrm{v} / \mathrm{v}$ TWA for 10

5204

$\mathrm{hr} /$ day and $40 \mathrm{hr} / \mathrm{wk}$

9504 and $40 \mathrm{hr} / \mathrm{wk}$

ICI LTEL: $1,000 \mathrm{ppm} \mathrm{v} / \mathrm{v}$ TWA

for $8 \mathrm{hr} /$ day and $40 \mathrm{hr} / \mathrm{wk}$

$120,000 \mathrm{ppm} v / \mathrm{v}$ (preliminary based on ASHRAE 34u draft) provisional: $120,000 \mathrm{ppm} \mathrm{V/V}$ (preliminary based on ASHRAE $34 \mathrm{u}$ draft)

$69,000 \mathrm{ppm} v / \mathrm{v}$ (preliminary based on ASHRAE $34 \mathrm{u}$ draft) provisiona7: $69,000 \mathrm{ppm} \mathrm{v/v}$ (preliminary based on ASHRAE $34 u$ draft)

rat, $2 \mathrm{hr}, 0 / 4$ : $>600,000 \mathrm{ppm}$ (lowest exposure tested with one or more deaths by inhalation) rat, $4 \mathrm{hr}, 0 / 4:>800,000 \mathrm{ppm}$ (lowest exposure tested with one or more deaths by inhalation)

rat, $4 \mathrm{hr}, 0 / 4$ : $>800,000 \mathrm{ppm}$ (lowest exposure tested with one or more deaths by

inhalation)

dog: $>1,200 \mathrm{mg} / \mathrm{kg}$ (fatal dose by ingestion for half of test animals)

rat, DuPont: $>1,200 \mathrm{mg} / \mathrm{kg}$ (fatal dose by ingestion for half of test animals)

CSDS

$34 u$

A950

$34 \mathrm{u}$

A950

5365

7616

7617

7239

MSDS

dog, 10 min: $320,000 \mathrm{ppm} \mathrm{v/v}$

(effective concentration in

half of test animals)

dog, 5 min, 1/13: 150,000 ppm

$v / v$ (lowest observed effect

level in test animals)

rat, 10 min: estimated 250,000 A622 ppm $v / v$ (effective

concentration in half of test animals)

rat, $2 \mathrm{hr}, 0 / 4$ : 600,000 ppm

$v / v$ (no observed effect level

in ALC or LC50 studies)

rat, $3 \frac{1}{2}-4 \mathrm{hr}, 0 / 4$ : 800,000 ppm 7616 $\mathrm{v} / \mathrm{v}$ (no observed effect leve 1 in ALC or LC50 studies)

none (nonflammable as tested)

$-2.1 \mathrm{MJ} / \mathrm{kg}$ ( $-903 \mathrm{Btu} / \mathrm{7b})$

2250

880 (nonflammable as tested) 5204

Dupont: $>700{ }^{\circ} \mathrm{C}\left(>1292^{\circ} \mathrm{F}\right)$ MSDS

nonflammable (withdrawn for 5931

revision of the classification

system, category SBQT2) 
- detection

appearance: DuPont: clear, colorless

MSDS odor: A77iedsignal: faint ethereal MSDS

PRODUCTION

Tast year production allowed: 1995 in developed countries

$8 \mathrm{CO1}$ under the Montreal Protocol 


\section{R-116}

hexaf7uoroethane
CF3CF3

IDENTIFIERS

common name(s): $\mathrm{R}-116$; R116; $\mathrm{R} 116$

FC- 116

fluorocarbon 116

fluorochemica7 116; FC 116

halocarbon 116

halochemical 116

perfiuorocarbon 116; "PFC-116"

chemical name (by IUPAC convention):

hexafluoroethane

alternative chemical names/formulae:

empirical formula: ethane, hexafluoro-

perf7uoroethane

$1,1,1,2,2,2$-hexafluoroethane

ethane, $1,1,1,2,2,2$-hexafluoro

ethane, perfluoro-

CF3CF3; CF3-CF3

not recommended: $(C F 3) 2$;

3CCF3; F3C-CF3

CAS number: 76-16-4 (Chemica) Abstracts

Service registry number)

Beilstein registry number: 1740328

EINECS number: 200-939-8 (European Inventory

of Existing Chemical

Substances)

NIOSH RTECS number: KI4100000 (Registry of Toxic

Effects of Chemical

Substances)

trade name(s): BOC Gases Halocarbon 116

MSDS

DuPont Freon(R) $116 \quad 5605$

historical name(s): Allied corp refrigerant 500

ARI container color / Pantone number: dark grey (battleship) / 4246601

PHYSICAL

- properties

molar mass: $138.0118192 \mathrm{~g} / \mathrm{mol}(0.304264$

1b/mol)

normal freezing/melting/triple point:

- normal boiling point -

$-97.1{ }^{\circ} \mathrm{C}\left(-142.9^{\circ} \mathrm{F}\right)$

8820

$-78.2^{\circ} \mathrm{C}\left(-108.7^{\circ} \mathrm{F}\right)$

8401

density, saturated liquid:

density, saturated vapor:

specific volume, saturated liquid:

$1604 \mathrm{~kg} / \mathrm{m} 3(100.13 \mathrm{~Tb} / \mathrm{cf})$

8401

$9.09 \mathrm{~kg} / \mathrm{m} 3(0.568 \mathrm{7b} / \mathrm{cf}) \quad 8401$

$0.623 \mathrm{~L} / \mathrm{kg}(0.0100 \mathrm{cf} / 7 \mathrm{~b}) \quad 8401$

$110.0 \mathrm{~L} / \mathrm{kg}(1.7620 \mathrm{cf} / 7 \mathrm{~b}) \quad 8401$

specific volume, saturated vapor:

heat of vaporization:

velocity of sound, saturated liquid:

velocity of sound, saturated vapor:

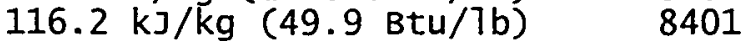

$522 \mathrm{~m} / \mathrm{s}(1712 \mathrm{ft} / \mathrm{s}) \quad 8401$

$110 \mathrm{~m} / \mathrm{s}(361 \mathrm{ft} / \mathrm{s}) \quad 8401$

viscosity, saturated liquid:

viscosity, saturated vapor:

$314 \mu \mathrm{Pa} \cdot \mathrm{s}(0.314 \mathrm{cp})$

$9.18 \mu \mathrm{pa} \cdot \mathrm{s}(0.00918 \mathrm{cP}) \quad 8401$

$0.0691 \mathrm{~W} / \mathrm{m} \cdot \mathrm{K}(0.0399 \mathrm{P}) 8401$

$\mathrm{Btu} / \mathrm{hr} \cdot \mathrm{ft}^{\circ} \mathrm{F}$ )

thermal conductivity, vapor:

$0.0075 \mathrm{~W} / \mathrm{m} \cdot \mathrm{K}(0.0043$

8401 
- normal pressure, $20^{\circ} \mathrm{C}\left(68^{\circ} \mathrm{F}\right)$ - normal pressure, $21.1{ }^{\circ} \mathrm{C}\left(70^{\circ} \mathrm{F}\right)$--

critical point density, vapor:

temperature: pressure: density: specific volume:
$\left.B t u / h r \cdot f t^{\circ} \mathrm{F}\right)$

$5.796 \mathrm{~kg} / \mathrm{m} 3(0.3619 \mathrm{lb} / \mathrm{cf}) \quad 8401$

$5.774 \mathrm{~kg} / \mathrm{m} 3(0.3604 \mathrm{lb} / \mathrm{cf}) \quad 8401$

$19.9^{\circ} \mathrm{C}\left(67.8^{\circ} \mathrm{F}\right) \quad 8401$

$3042 \mathrm{kPa}$ (441.2 psia) 8401

$622 \mathrm{~kg} / \mathrm{m} 3$ (38.8 $\mathrm{lb} / \mathrm{cf}) \quad 8401$

$1.61 \mathrm{~L} / \mathrm{kg}(0.0258 \mathrm{cf} / 7 \mathrm{~b}) \quad 8401$

ENVIRONMENTAL

average atmospheric 1ifetime (Tatm): ODP (ozone depletion potential):

GWP (global warming potential):

$10,000 \mathrm{yr}$

0.000 (model-derived relative

6694 to $\mathrm{R}$ 1I)

HGWP (halocarbon GWP):

11,400 relative to $\mathrm{CO} 2$ for 1009501

$y r$ integration

205 relative to $R 11$ for

infinite integration period

DW

SAFETY

- classification

safety group (ASHRAE Standard 34):

NFPA 704 degrees of hazard $(H-F-R-S)$ :

NPCA HMIS hazard ratings $(H-F-R)$ :

A1

BOC Gases: 1-0-0

8601

health-flammability-reactivity

[-special]: $0=$ no, $4=$ severe

BOC Gases: 1-0-0

DuPont: $1-0-1$

hea 7 th-f7 ammabi 7ity-reactivity

MSDS

$0=$ insignificant, $4=e x t r e m e$

UL Comparative Hazard to Life Group: 6 by estimate (not UL test) in 5906 absence of flame or hot

objects

- Tong-term occupational Timit

exposure Timit consistent to OSHA PEL:

- emergency exposure limit

Acute Toxicity Exposure Limit (ATEL):

DuPont AEL: $1,000 \mathrm{ppm} v / \mathrm{v}$ TWA

for $8 \mathrm{hr} /$ day and $40 \mathrm{hr} / \mathrm{wk}$

ICI LTEL: $1,000 \mathrm{ppm} v / \mathrm{v}$ TWA

for $8 \mathrm{hr} /$ day and $40 \mathrm{hr} / \mathrm{wk}$

5605

97,000 ppm v/v (pre]iminary

based on ASHRAE $34 \mathrm{u}$ draft)

$97,000 \mathrm{ppm} v / \mathrm{V}$ (preliminary

based on ASHRAE $34 \mathrm{u}$ draft)

Refrigerant Concentration Limit $(R C L)$ :

69,000 ppm V/v (pretiminary

based on ASHRAE $34 \mathrm{u}$ draft)

$69,000 \mathrm{ppm} v / v$ (preliminary

based on ASHRAE $34 \mathrm{u}$ draft)

CSDS

- acute (short-term) toxicity

ALC (approximate Tethal concentration:

rat, $1 \mathrm{hr}, 0 / 10:>800,000 \mathrm{ppm}$

(lowest exposure tested with

one or more deaths by

inhalation)

rat, $4 \mathrm{hr}, 0 / 4$ : $>800,000 \mathrm{ppm}$

(lowest exposure tested with

one or more deaths by

inhalation)

cardiac sensitization threshold/LOEL:

dog: >200,000 ppm v/v Clowest observed effect Tevel in test anima $7 s$ )

cardiac sensitization (CS) NOEL:

dog: 200,000 ppm $v / v$ (no

observed effect level in test

$34 u$

A950

$34 u$

A950

anima 1s)

7550

7616

6120

6120 
anesthetic/CNS effect LOEL: rat, $1 \mathrm{hr}, 10 / 10: 780,000 \mathrm{ppm} 7550$ $v / v$ (Towest observed effect level in test animals)

rat, $2 \mathrm{hr}$, ?/4: 200,000 ppm $v / v$ (Towest observed effect Teve 7 in ALC or LC50 studies)

anesthetic/CNS effect NOEL: rat, 10 day, 0/20: 121,000 ppm 7550 $v / v$ (no observed effect level in subchronic or chronic

- flammability study)

LFL-UFL (flammability limits in air): flash point:

none (nonflammable as tested) 2A06 autoignition temperature:

none (nonflammable as tested)

$870^{\circ} \mathrm{C}\left(1598^{\circ} \mathrm{F}\right)$

BOC Gases: none

MSDS

- detection

DuPont: $>870^{\circ} \mathrm{C}\left(>1598^{\circ} \mathrm{F}\right)$

MSDS

appearance: odor:

DuPont: clear, colorless

BOC Gases: odorless

MSDS

DuPont: siight ethereal

MSDS

MSDS

PRODUCTION

last year production allowed: unrestricted 8C01 


\section{R-123}

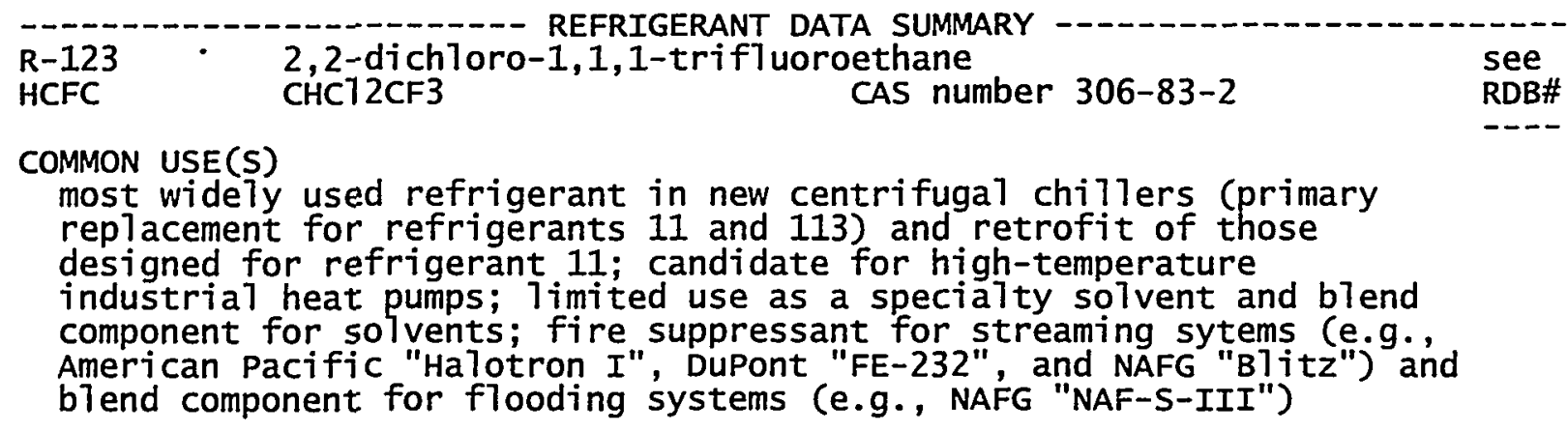

IDENTIFIERS

$$
\text { common name(s): } R-123 ; R 123 ; R 123
$$

HCFC- 123

not recommended: HFA-123

f7uorocarbon 123

fluorochemical 123; FC 123

haTocarbon 123

halochemical 123

halon 232

chemical name (by IUPAC convention): $\begin{aligned} & \text { hydrochlorofluorocarbon } 123 \\ & 2,2 \text {-dichloro-1,1,1-trifluoro- } \\ & \text { ethane }\end{aligned}$

alternative chemical names/formulae: 1,1-dichloro-2,2,2-trifluoro-

ethane (not recommended)

ethane, 2,2-dichloro-1,1,1trifluoro-

dichlorotrifluoroethane

trifluorodichloroethane

dichloro(trifluoromethyl)-

methane

CHC12CF3; CHC12-CF3

not recommended:

CF3CHC72; CF3-CHC12

C12CHCF3; C12CH-CF3

C12HCCF3; C72HC-CF3

HCL 2CCF3; HC12C-CF3

empirical formula: C2HC12F3

CAS number: 306-83-2 (Chemical Abstracts

Service registry number)

Beilstein registry number:

1736763

EINECS number: 206-190-3 (European Inventory

of Existing Chemical

Substances)

NIOSH RTECS number: KII108000 (Registry of Toxic

Effects of Chemical

Substances)

trade name(s): Asahi Glass Asahiklin AK-123

Ausimont Meforex(R) 123

Daikin fluorocarbon HCFC-123

DuPont Suva(R) 123

Elf Atochem Forane(R) 123

Honeywe 11 Genetron(R) 123

Solvay Solkane(R) 123

ZCIRI Kehua (PRC) HCFC-123

historical name(s): Allied Corp refrigerant 220

A 1 liedsignal Genetron( $R$ ) $123 \quad 3452$

DuPont Suva(R) Centri-LP 4504 
PHYSICAL

- properties

motar mass:

normal freezing/melting/triple point:

- normal boiling point

temperature:

density, saturated liquid:

density, saturated vapor:

specific volume, saturated 1iquid:

specific volume, saturated vapor: heat of vaporization:

velocity of sound, saturated 7 iquid:

velocity of sound, saturated vapor: viscosity, saturated vapor: viscosity, saturated 7iquid: thermal conductivity, 1iquid:

thermal conductivity, vapor:

- normal pressure, $20^{\circ}{ }^{\circ}\left(68^{\circ} \mathrm{F}\right)$ -

- normal pressure, $21.1^{\circ} \mathrm{C}\left(70^{\circ} \mathrm{F}\right)$ -

- $20^{\circ} \mathrm{C}\left(68^{\circ} \mathrm{F}\right)$ - density, Tiquid:

pressure, saturated vapor: density, saturated 7iquid: density, saturated vapor:

specific volume, saturated liquid: specific volume, saturated vapor: velocity of sound, saturated 7iquid: velocity of sound, saturated vapor: viscosity, saturated 1iquid:

viscosity, saturated vapor:

thermal conductivity, saturatd liquid:

thermal conductivity, saturated vapor:

- $60{ }^{\circ} \mathrm{C}\left(140{ }^{\circ} \mathrm{F}\right)$-.-.-.

pressure, saturated vapor:

heat of vaporization:

temperature:

pressure:

density:

specific volume:

$152.9299496 \mathrm{~g} / \mathrm{mol}(0.337153$

8820

$1 \mathrm{~b} / \mathrm{mol}$ )

$-107.1^{\circ} \mathrm{C}\left(-160.9^{\circ} \mathrm{F}\right)$

4319

$27.8{ }^{\circ} \mathrm{C}\left(82.1^{\circ} \mathrm{F}\right)$

7607

$1457 \mathrm{~kg} / \mathrm{m} 3(90.93 \mathrm{~Tb} / \mathrm{cf}) \quad 7607$

$6.47 \mathrm{~kg} / \mathrm{m} 3(0.404 \mathrm{lb} / \mathrm{cf}) \quad 7607$

$0.687 \mathrm{~L} / \mathrm{kg}(0.0110 \mathrm{cf} / 7 \mathrm{~b}) \quad 8401$

$154.5 \mathrm{~L} / \mathrm{kg}(2.4753 \mathrm{cf} / 7 \mathrm{~b}) \quad 8401$

$170.2 \mathrm{~kJ} / \mathrm{kg}(73.2 \mathrm{Btu} / \mathrm{lb}) \quad 7607$

$693 \mathrm{~m} / \mathrm{s}(2273 \mathrm{ft} / \mathrm{s}) \quad 7607$

$129 \mathrm{~m} / \mathrm{s}(422 \mathrm{ft} / \mathrm{s}) \quad 7607$

$10.84 \mu \mathrm{Pa} \cdot \mathrm{s}(0.01084 \mathrm{cp}) \quad 8401$

$404 \mu \mathrm{Pa} \cdot \mathrm{s}(0.404 \mathrm{cp}) \quad 8401$

$0.0756 \mathrm{~W} / \mathrm{m} \cdot \mathrm{K}(0.0437 \quad 8401$

$\left.\mathrm{Btu} / \mathrm{hr} \cdot \mathrm{ft}^{\circ} \mathrm{F}\right)$

$0.0093 \mathrm{~W} / \mathrm{m} \cdot \mathrm{K}(0.0054 \quad 8401$

$\mathrm{Btu} / \mathrm{hr} \cdot \mathrm{ft}^{\circ} \mathrm{F}$ )

$1477 \mathrm{~kg} / \mathrm{m} 3(12.32 \mathrm{lb} / \mathrm{ga}) \quad 7607$

$1474 \mathrm{~kg} / \mathrm{m} 3(12.30 \mathrm{lb} / \mathrm{gaT}) \quad 7607$

$75.6 \mathrm{kPa}(10.97 \mathrm{psia}) \quad 7607$

$1477 \mathrm{~kg} / \mathrm{m} 3(92.18 \mathrm{lb} / \mathrm{cf}) \quad 7607$

$4.92 \mathrm{~kg} / \mathrm{m} 3(0.307 \mathrm{1b} / \mathrm{cf}) \quad 7607$

$0.677 \mathrm{~L} / \mathrm{kg}(0.0108 \mathrm{cf} / \mathrm{lb}) \quad 8401$

$203.4 \mathrm{~L} / \mathrm{kg}(3.2578 \mathrm{cf} / 7 \mathrm{~b}) \quad 8401$

$723 \mathrm{~m} / \mathrm{s}(2371 \mathrm{ft} / \mathrm{s}) \quad 7607$

$128 \mathrm{~m} / \mathrm{s}(420 \mathrm{ft} / \mathrm{s}) \quad 7607$

$443 \mu \mathrm{Pa} \cdot \mathrm{s}(0.443 \mathrm{cp}) \quad 8401$

$10.6 \mu \mathrm{Pa} \cdot \mathrm{s}(0.0106 \mathrm{cp}) \quad 8401$

$0.0778 \mathrm{~W} / \mathrm{m} \cdot \mathrm{K}(0.0450 \quad 8401$

$\mathrm{Btu} / \mathrm{hr} \cdot \mathrm{ft}^{\circ} \mathrm{F}$ )

$0.00889 \mathrm{~W} / \mathrm{m} \cdot \mathrm{K}(0.00514 \quad 8401$

$\mathrm{Btu} / \mathrm{hr} \cdot \mathrm{ft}^{\circ} \mathrm{F}$ )

$286 \mathrm{kPa}$ (41.5 psia) 7607

$155.7 \mathrm{~kJ} / \mathrm{kg}(67.0 \mathrm{Btu} / 7 \mathrm{~b}) \quad 8401$

$183.7^{\circ} \mathrm{C}\left(362.6^{\circ} \mathrm{F}\right) \quad 7607$

$183.8^{\circ} \mathrm{C}\left(362.8^{\circ} \mathrm{F}\right) \quad 7713$

$3662 \mathrm{kPa}$ (531.1 psia) 3711

$550 \mathrm{~kg} / \mathrm{m} 3(34.3 \mathrm{lb} / \mathrm{cf}) \quad 0915$

$1.82 \mathrm{~L} / \mathrm{kg}(0.0291 \mathrm{cf} / 7 \mathrm{~b}) \quad 0915$

ENVIRONMENTAL

average atmospheric lifetime (Tatm): average stratospheric 1 ifetime (Tstr): ODP (ozone depletion potential):

$1.4 \mathrm{n} 0.4 \mathrm{yr}$

6695

$47 \mathrm{yr}$

0.012 (model-derived relative 9501

to $R$ 11)

0.02 (semi-empirical relative 9501 to $R$ 11)

0.02 (estimate used for the 6904

GWP (global warming potential): Montrea 7 Protoco 1 )

120 relative to $\mathrm{CO} 2$ for $100 \mathrm{yr} 9501$ integration

HGWP (halocarbon GWP):

$0.0185 \pm 0.0015$ relative to $R \quad 5964$

11 for infinite integration period 
photochemical reactivity (grnd 7 eve1): 4.8 relative to methane

4511

SAFETY

- Classification --safety group (ASHRAE standard 34): B1 NFPA 704 degrees of hazard $(H-F-R-S)$ :

NPCA HMIS hazard ratings $(H-F-R)$ :

ARI recommendation: Ausimont: Honeywe 11:

Honeywe 11: 2-1-0

DuPont: $\quad 1-0-1$

$2-0-0$

8601

3 A15

MSDS

MSDS

health-flammability-reactivity

MSDS

MSDS

$0=$ insignificant, $4=$ extreme

DFG carcinogicity class: IIIB: suspect, to be eva7uated 5561

- short-term occupational limit --.---

ARI "IDLH" recommendation: $4,000 \mathrm{ppm} \mathrm{v} / \mathrm{v}$ for $30 \mathrm{~min}$

$3 A 15$

recommended short-term exposure limit:

DuPont EEL: $1,000 \mathrm{ppm}(1 \mathrm{hr}) \quad 4 C 50$

- Tong-term occupationaT limit --.----

DuPont EEL: $2,500 \mathrm{ppm}$ (1 min) 4C50

AIHA WEEL (workplace envi exp 1imit):

exposure limit consistent to OSHA PEL:

$50 \mathrm{ppm} \mathrm{v} / \mathrm{v}$ TWA for $8 \mathrm{hr} /$ day

8611 and $40 \mathrm{hr} / \mathrm{wk}$

DuPont AEL: $50 \mathrm{ppm} v / \mathrm{v}$ TWA for MSDS $8 \mathrm{hr} /$ day and $40 \mathrm{hr} / \mathrm{wk}$

Honeywe 11 PEL: $10 \mathrm{ppm} \mathrm{V/V}$ TWA MSDS

for $8 \mathrm{hr} /$ day and $40 \mathrm{hr} / \mathrm{wk}$

- emergency exposure 1imit
NRC EEGL (emergency exposure 1evel):

1 min: $1,900 \mathrm{ppm} v / v$ cejling 6A01 guidance level for single emergency exposures

Acute Toxicity Exposure Limit (ATEL):

$9,100 \mathrm{ppm} v / \mathrm{v}$ (preliminary

based on ASHRAE $34 \mathrm{u}$ draft)

9,100 ppm v/v (preliminary

based on ASHRAE 34u draft)

Refrigerant Concentration Limit (RCL):

9,100 ppm v/v (preliminary

based on ASHRAE 34u draft)

9,100 ppm v/v (preliminary

based on ASHRAE 34u draft)

$34 u$

A950

$34 u$

A950

- acute (short-term) toxicity ------LC50 (7ethal concentration, 50\%):

ALC (approximate Tetha] concentration:

mouse, $30 \mathrm{~min}: 74,000 \mathrm{ppm}$

6165

(fatal concentration by

inhalation for half of test

animals)

rat, 10 min: $90,000 \mathrm{ppm}$ (fata] A309

concentration by inhatation

for half of test animals)

rat, $4 \mathrm{hr}$ : 32,000 ppm (fata)

concentration by inhalation

for half of test animals)

rat, $10 \mathrm{~min}, 1 / 4: 72,500 \mathrm{ppm}$

(lowest exposure tested with

one or more deaths by

inhalation)

rat, $4 \mathrm{hr}, 3 / 6: 32,000 \mathrm{ppm}$

(lowest exposure tested with one or more deaths by

inhalation)

derma] LD50 (letha] dosage, 50\%):

rabbit, $24 \mathrm{hr}, 0 / 10$ : >2,000

$\mathrm{mg} / \mathrm{kg}$ (fata) dose by skin

contact for half of test

animals)

$\mathrm{rat}, 24 \mathrm{hr}, 0 / 5:>2,000 \mathrm{mg} / \mathrm{kg}$

(fata 1 dose by skin contact

6139

for half of test animals)

A309

6139

6148

6147 
oral LD50 (1ethal dosage, 50\%): rat, $1 / 1: 9,000 \mathrm{mg} / \mathrm{kg}$ (fatal dose by ingestion for half of test animals)

cardiac sensitization (CS) EC50: dog, $5 \mathrm{~min}$ : 19,000 ppm v/v (effective concentration in half of test animals)

cardiac sensitization threshold/LOEL: dog, 5 min, 4/6: 20,900 ppm $v / v$ (lowest observed effect level in test animals)

cardiac sensitization (CS) NOEL: dog, 5 min, 0/3: 10,300 ppm $v / v$ (no observed effect leve 1 in test animals)

anesthetic/CNS effect EC50:

mouse, $10 \mathrm{~min}$ : $27,000 \mathrm{ppm} \mathrm{v} / \mathrm{v}$

(effective concentration in

half of test animals)

rat, $10 \mathrm{~min}: 130,000 \mathrm{ppm} \mathrm{v/v}$

(effective concentration in

half of test animals)

rat, $10 \mathrm{~min}: 40,000 \mathrm{ppm} \mathrm{v} / \mathrm{v}$

(effective concentration in

half of test animals)

rat, 15 min behaviora $7: 7,080$

$\mathrm{ppm} \mathrm{v} / \mathrm{v}$ (effective

concentration in half of test animals)

anesthetic/CNS effect LOEL:

g.pig, 10-15 min, ?/10: 10,000

$\mathrm{ppm} \mathrm{v} / \mathrm{v}$ (lowest observed

effect leve 7 in test animals)

rat, $15 \mathrm{~min}$, ?/6: 5,237 $\mathrm{ppm}$

$\mathrm{v} / \mathrm{v}$ (lowest observed effect

Tevel in test anima Ts)

rat, $60 \mathrm{~min}, 1 / 6$ : 0 (control)

ppm $v / v$ (lowest observed

effect level in test anima 75 )

rat, $60 \mathrm{~min}, ? / 6: 975 \mathrm{ppm} \mathrm{v} / \mathrm{v}$

( lowest observed effect level

in test animals)

rat, subchronic, ?/?: 5,000

ppm $v / v$ (lowest observed

effect Tevel in ALC or LC50

studies)

anesthetic/CNS effect NOEL: rat, subchronic: $>5,000 \mathrm{ppm}$

$v / v$ (no observed effect Teve?

in ALC or LC50 studies)

- flammability --LFL-UFL (flammability limits in air):
heat of combustion (by ASHRAE 34-92): flash point: autoignition temperature:

autodecomposition temperature: former UL Classification:

- detection

appearance: odor: none (nonflammable as tested) 2525

$2.1 \mathrm{MJ} / \mathrm{kg}(903 \mathrm{Btu} / 7 \mathrm{~b}) \quad 2318$

DuPont, TOC: none MSDS

$730^{\circ} \mathrm{C}\left(1346^{\circ} \mathrm{F}\right) \quad 5 \mathrm{C} 10$

DuPont Suva(R): $730^{\circ} \mathrm{C}\left(1346^{\circ} \mathrm{F}\right) 5931$

Elf Atochem: $\quad 714^{\circ} \mathrm{C}\left(1317^{\circ} \mathrm{F}\right) 6938$

Honeywe 17: $770{ }^{\circ} \mathrm{C}\left(1418^{\circ} \mathrm{F}\right)$ MSDS

Honeywe $71:>250^{\circ} \mathrm{C}\left(>482^{\circ} \mathrm{F}\right)$ MSDS

practicaliy nonflammable 6938

(withdrawn for revision of the classification system,

category SBQT2)

Dupont: clear, colorless

MSDS

Honeywe 17: faint ethereal and MSDS sweetish odor

MSDS 
PRODUCTION

first commercial use as a refrigerant: 1989

last year production allowed: 2029 in developed countries under the Montreal Protocol

8001 


\section{R-124}

2-ChToro-1,1,1,2-tetraf7uoroethane
CHCIFCF3

IDENTIFIERS

common name(s): R-124; RI24; R 124

HCFC-124

f7uorocarbon 124

fluorochemica7 124; FC 124

halocarbon 124

halochemical 124

halon 241

hydrochlorof7uorocarbon 124

chemical name (by IUPAC convention): 2-chloro-1,1,1,2-tetrafluoroethane

alternative chemical names/formulae: 1-chloro-1,2,2,2-tetrafluoro-

ethane (not recommended)

ethane, 2-chloro-1,1,1,2-

tetrafluoro-

chlorotetrafluoroethane

tetrafluoromonochloroethane

CHC 7FCF3; CHC IF-CF3

not recommended:

CC1FHCF3; CCIFH-CF3

CF3CC7FH; CF3-CCTFH

CF3CHCTF; CF3-CHClF

HCTFCCF3; HCIFC-CF3

empirical formula: $\mathrm{C} 2 \mathrm{HC} 7 \mathrm{~F} 4$

CAS number: 2837-89-0 (Chemical Abstracts

Service registry number)

EINECS number: 220-629-6 (European Inventory

of Existing Chemical

Substances)

trade name(s): Asahi Glass Asahiklin AK-124

Ausimont Meforex(R) 124

Daikin fluorocarbon HCFC-124

DuPont Suva(R) 124

Elf Atochem Forane(R) 124

Honeywe 11 Genetron(R) 124

historical name(s): Allied Corp refrigerant 310

A 71 iedSigna 7 Genetron(R) 124

Kinetic Chemica7s Freon 124

ARI container color / Pantone number: deep green (DOT green) / 335 see

RDB\# 
PHYSICAL

- properties

molar mass:

normal freezing/melting/triple point:

- normal boiling point -

density, saturated 7iquid: density, saturated vapor: specific volume, saturated liquid: specific volume, saturated vapor: heat of vaporization:

velocity of sound, saturated liquid: velocity of sound, saturated vapor: viscosity, saturated vapor: viscosity, saturated liquid: thermal conductivity, Tiquid:

thermal conductivity, vapor:

- normal pressure, $20{ }^{\circ} \mathrm{C}\left(68{ }^{\circ} \mathrm{F}\right)-\ldots$

- normal pressure, $21.1{ }^{\circ} \mathrm{C}\left(70^{\circ} \mathrm{F}\right)$---

- $20^{\circ} \mathrm{C}\left(68^{\circ} \mathrm{F}\right)$-- density, vapor: density, vapor:

pressure, saturated vapor: density, saturated 7iquid: density, saturated vapor:

specific volume, saturated liquid: specific volume, saturated vapor: velocity of sound, saturated 1iquid: velocity of sound, saturated vapor: viscosity, saturated 7 iquid: viscosity, saturated vapor: thermal conductivity, saturatd liquid: thermal conductivity, saturated vapor: - $60{ }^{\circ} \mathrm{C}\left(140{ }^{\circ} \mathrm{F}\right)$ pressure, saturated vapor:

- critical point heat of vaporization:

specific volume:

$$
\begin{array}{r}
\text { temperature: } \\
\text { pressure: } \\
\text { density: }
\end{array}
$$

\section{pressure: density:}

ENVIRONMENTAL

average atmospheric lifetime (Tatm): average stratospheric lifetime (Tstr): ODP (ozone depletion potentia1):

GWP (global warming potential):

$136.4756528 \mathrm{~g} / \mathrm{mol} \quad(0.300877$

8820

$7 \mathrm{~b} / \mathrm{mo} 7)$

$-199.1{ }^{\circ} \mathrm{C}\left(-326.5^{\circ} \mathrm{F}\right)$

8401

$-12.0^{\circ} \mathrm{C}\left(10.5^{\circ} \mathrm{F}\right)$

8401

$1474 \mathrm{~kg} / \mathrm{m} 3(91.99 \mathrm{~Tb} / \mathrm{cf})$

$6.66 \mathrm{~kg} / \mathrm{m} 3(0.415 \mathrm{lb} / \mathrm{cf})$

$0.679 \mathrm{~L} / \mathrm{kg}(0.0109 \mathrm{cf} / \mathrm{lb})$

$150.3 \mathrm{~L} / \mathrm{kg}(2.4069 \mathrm{cf} / 7 \mathrm{~b})$

$165.8 \mathrm{~kJ} / \mathrm{kg}(71.3 \mathrm{Btu} / 7 \mathrm{~b})$

$673 \mathrm{~m} / \mathrm{s}(2209 \mathrm{ft} / \mathrm{s})$

$128 \mathrm{~m} / \mathrm{s}(419 \mathrm{ft} / \mathrm{s})$

$10.08 \mu \mathrm{Pa} \cdot \mathrm{s}(0.01008 \mathrm{cp})$

$371 \mu \mathrm{Pa} \cdot \mathrm{s}(0.371 \mathrm{cp})$

$0.0800 \mathrm{~W} / \mathrm{m} \cdot \mathrm{K}(0.0462$

$\left.\mathrm{Btu} / \mathrm{hr} \cdot \mathrm{ft}^{\circ} \mathrm{F}\right)$

$0.0093 \mathrm{~W} / \mathrm{m} \cdot \mathrm{K}(0.0054$

$\mathrm{Btu} / \mathrm{hr} \bullet f \mathrm{t}^{\circ} \mathrm{F}$ )

$5.836 \mathrm{~kg} / \mathrm{m} 3(0.3643 \mathrm{~Tb} / \mathrm{cf}) \quad 8401$

$5.812 \mathrm{~kg} / \mathrm{m} 3(0.3628 \mathrm{lb} / \mathrm{cf}) \quad 8401$

$326.9 \mathrm{kPa}$ (47.42 psia)

$1372 \mathrm{~kg} / \mathrm{m} 3(85.68 \mathrm{ib} / \mathrm{cf})$

$20.28 \mathrm{~kg} / \mathrm{m} 3(1.266 \mathrm{~Tb} / \mathrm{cf})$

$0.729 \mathrm{~L} / \mathrm{kg}(0.0117 \mathrm{cf} / 7 \mathrm{~b})$

$49.3 \mathrm{~L} / \mathrm{kg}(0.7900 \mathrm{cf} / 7 \mathrm{~b})$

$545 \mathrm{~m} / \mathrm{s}(1787 \mathrm{ft} / \mathrm{s})$

$128 \mathrm{~m} / \mathrm{s}(422 \mathrm{ft} / \mathrm{s})$

$250 \mu \mathrm{Pa} \cdot \mathrm{s}(0.250 \mathrm{cp})$

$11.3 \mu \mathrm{Pa} \cdot \mathrm{s}(0.0113 \mathrm{cp})$

$0.0697 \mathrm{~W} / \mathrm{m} \cdot \mathrm{K}(0.0403$

$\mathrm{Btu} / \mathrm{hr} \bullet \mathrm{ft}^{\circ} \mathrm{F}$ )

$0.01132 \mathrm{~W} / \mathrm{m} \cdot \mathrm{K} \quad(0.00654$

$\mathrm{Btu} / \mathrm{hr} \cdot \mathrm{ft}^{\circ} \mathrm{F}$ )

$997 \mathrm{kPa}$ (144.6 psia)

8401

8401

8401

8401

8401

8401

8401

8401

8401

8401

8401

$123.1 \mathrm{~kJ} / \mathrm{kg}(52.9 \mathrm{Btu} / 7 \mathrm{~b})$

$122.2^{\circ} \mathrm{C}\left(252.0^{\circ} \mathrm{F}\right)$

$122.3^{\circ} \mathrm{C}\left(252.1^{\circ} \mathrm{F}\right)$

$3624 \mathrm{kPa}(525.6$ psia)

$559 \mathrm{~kg} / \mathrm{m} 3$ (34.9 ib/cf)

$560 \mathrm{~kg} / \mathrm{m} 3(34.9 \mathrm{lb} / \mathrm{cf})$

$560 \mathrm{~kg} / \mathrm{m} 3(35.0 \mathrm{lb} / \mathrm{cf})$

$560 \mathrm{~kg} / \mathrm{m} 3(35.0 \mathrm{~Tb} / \mathrm{cf})$

8401

8401

8401

8401

8401

8401

8401

8401

8401

8401

8401

$1.79 \mathrm{~L} / \mathrm{kg}(0.0286 \mathrm{cf} / 7 \mathrm{~b})$

8401

9125

7714

8401

7714

7713

8401

9125

8401

$6.1 \pm 1.5 \mathrm{yr}$

6695

$130 \mathrm{yr}$

0.026 (mode)-derived relative 9501 to $R$ 11)

0.022 (estimate used for the 6904 Montrea 7 Protoco 1)

620 relative to $\mathrm{CO} 2$ for $100 \mathrm{yr} 9501$ integration

(halocarbon GWP): 0.10 relative to $R 11$ for

infinite integration period

photochemical reactivity (grnd level):

1.2 relative to methane 
SAFETY

- Classification
safety group (ASHRAE Standard 34): NFPA 704 degrees of hazard $(H-F-R-S)$ :

NPCA HMIS hazard ratings (H-F-R):

UL Comparative Hazard to Life Group:

- 1ong-term occupational 7imit --.-.-AIHA WEEL (workplace env1 exp 7imit):

- emergency exposure Timit Acute Toxicity Exposure Limit (ATEL):

Refrigerant Concentration Limit ( $R C L)$ :

- acute (short-term) toxicity LC50 (Tethat concentration, $50 \%$ ):

ALC (approximate lethal concentration:

cardiac sensitization (CS) EC50:

cardiac sensitization threshold/LOEL:

cardiac sensitization (CS) NOEL: anesthetic/CNS effect EC50:

Al

Ausimont: $2-0-0$

Honeywe 17: 2-1-0

hea th-flammability-reactivity

[-special]: $0=$ no, $4=$ severe

DuPont: $1-0-1$

Honeywe 11: 1-1-0

health-flammability-reactivity

$0=$ insignificant, $4=$ extreme

6 in absence of flame or hot 5176 objects

$1,000 \mathrm{ppm} v / \mathrm{v}$ TWA for $8 \mathrm{hr} /$ day $4 \mathrm{~B} 88$ and $40 \mathrm{hr} / \mathrm{wk}$

$10,000 \mathrm{ppm} v / \mathrm{v}$ (pretiminary $34 \mathrm{u}$

based on ASHRAE $34 \mathrm{u}$ draft)

$10,000 \mathrm{ppm} v / \mathrm{v}$ (preliminary

based on ASHRAE $34 \mathrm{u}$ draft)

A950

$10,000 \mathrm{ppm} v / \mathrm{V}$ (preliminary

based on ASHRAE $34 \mathrm{u}$ draft)

$10,000 \mathrm{ppm} \mathrm{V} / \mathrm{v}$ (preliminary

based on ASHRAE $34 \mathrm{u}$ draft)

$34 \mathrm{u}$

A950

rat, 10 min: 44,000 ppm (fatal A309

concentration by inhalation

for half of test animals)

rat, 4 hr: $262,500 \mathrm{ppm}$ (fata)

concentration by inhalation

for half of test animals)

rat, $10 \mathrm{~min}, 0 / 4:>39,900 \mathrm{ppm}$

(lowest exposure tested with

one or more deaths by

inhalation)

rat, $10 \mathrm{~min}, 1 / 4: 42,500 \mathrm{ppm}$

Clowest exposure tested with one or more deaths by

inhalation)

rat, $4 \mathrm{hr}, 0 / 6$ : 230,000 ppm

(lowest exposure tested with

one or more deaths by

inhalation)

rat, $4 \mathrm{hr}, 6 / 6$ : 300,000 ppm

(lowest exposure tested with one or more deaths by

inhalation)

dog, (source unknown): 40,000

$\mathrm{ppm} v / \mathrm{v}$ (effective

concentration in half of test animals)

dog, $10 \mathrm{~min}, 4 / 10: 25,000 \mathrm{ppm}$

$v / v$ (lowest observed effect

Tevel in test animals)

dog, $10 \mathrm{~min}$, 0/10: 10,100 ppm

$v / v$ (no observed effect leve

in test animals)

$10 \mathrm{~min}: 140,000 \mathrm{ppm} \mathrm{v} / \mathrm{v}$

(effective concentration in

half of test animals)

rat, 10 min: $150,000 \mathrm{ppm} \mathrm{v/v}$

(effective concentration in

half of test animals)

5170

A309

A309

6180

6180

7407

5808

5808

$5 C 39$

A309 
anesthetic/CNS effect LOEL: dog, 10-90 min, ?/6: 400,000 5828

$\mathrm{ppm} v / \mathrm{v}$ (lowest observed

effect level in test animals)

rat, $30 \mathrm{~min}$, ?/10: $100,000 \mathrm{ppm} 5806$

$v / v$ (lowest observed effect

level in test animals)

rat, $4 \mathrm{hr}$, ?/6: $160,000 \mathrm{ppm}$

$v / v$ (lowest observed effect

level in test animals)

anesthetic/CNS effect NOEL: rat, $4 \mathrm{hr}, 0 / 6: 48,000 \mathrm{ppm} v / \mathrm{v} 6180$ (no observed effect level in

- flammability test animals)

LFL-UFL (flammability 7imits in air): heat of combustion (by ASHRAE 34-92):

flash point:

none (nonflammable as tested)

2525

$0.9 \mathrm{MJ} / \mathrm{kg}$ (387 Btu/7b)

2318

if Atochem: none as tested SDS Honeywe 17: gas, not applicable MSDS nonflammable

$637^{\circ} \mathrm{C}\left(11799^{\circ} \mathrm{F}\right)$ $4 \mathrm{~B} 88$

autoignition temperature: Dupont: $715^{\circ} \mathrm{C}\left(1319^{\circ} \mathrm{F}\right)$ MSDS

autodecomposition temperature: Honeywe $71:>250^{\circ} \mathrm{C}\left(>482^{\circ} \mathrm{F}\right) \quad$ MSDS

- detection

appearance: odor:

Honeywe 77: clear, colorless Daikin: faint sweet odor faint ethereal odor

PRODUCTION

first commercial use as a refrigerant: 1992

Tast year production allowed: 2029 in developed countries under the Montreal Protocol

$8 C 01$ 


\section{R-125}

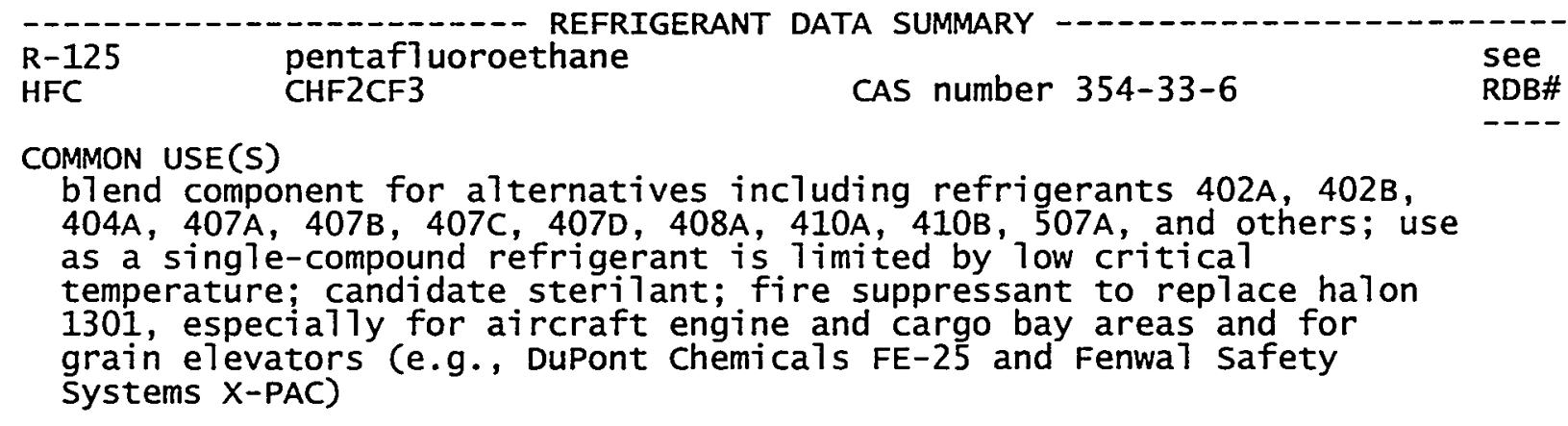

\section{IDENTIFIERS}

common name(s): R-125; RI25; R 125

HFC- 125

fluorocarbon 125

fluorochemica7 125; FC 125

halocarbon 125

halochemical 125

halon 25

hydrofluorocarbon 125

not recommended: HFA-125

chemical name (by IUPAC convention): alternative chemical names/formulae:

empirical formula:

pentafluoroethane

1,1,1,2,2-pentafluoroethane

ethane, pentafiuoro-

ethane, 1,1,1,2,2-pentafluoro-

CHF2CF3; CHF2-CF3

not recommended:

CF2HCF3; CF2H-CF3

CF3CF2H; CF3-CF2H

CF3CHF2; CF3-CHF2

HF2CCF3; HF2C-CF3

CAS number: 354-33-6 (Chemical Abstracts

Service registry number)

EINECS number: 206-557-8 (European Inventory

of Existing Chemical

Substances)

trade name(s): A17iedsignal Genetron(R) 125

Asahi Glass AsahikTin AK-125

Ausimont Meforex(R) 125

Daikin f7uorocarbon HFC-125 MSDS

DuPont Suva(R) 125 Refrigerant MSDS

Elf Atochem Forane(R) 1.25 MSDS

ICI Klea(R) 125 MSDS

Solvay Solkane(R) 125 MSDS

ZCIRI Kehua (PRC) HFC- 125

historical name(s): Allied Corp refrigerant 400

DuPont Freon(R) 125

ARI container color / Pantone number: medium brown (tan) / 465

PHYSICAL

- properties

molar mass:

normal freezing/melting/triple point:

$120.021356 \mathrm{~g} / \mathrm{mol}(0.264602$

8820

$\mathrm{lb} / \mathrm{mo}$ )

$-100.6{ }^{\circ} \mathrm{C}\left(-149.1{ }^{\circ} \mathrm{F}\right)$

8401

- normal boiling point - temperature: density, saturated Tiquid:

$-48.1^{\circ}{ }^{\circ} \mathrm{C}\left(-54.7^{\circ} \mathrm{F}\right) \mathrm{kg} / \mathrm{m} 3(94.58 \mathrm{bb} / \mathrm{cf})$ 
density, saturated vapor: specific volume, saturated liquid: specific volume, saturated vapor: heat of vaporization: velocity of sound, saturated liquid: velocity of sound, saturated vapor: viscosity, saturated 7iquid: viscosity, saturated vapor: thermal conductivity, 1iquid:

thermal conductivity, vapor:

- normal pressure, $20^{\circ} \mathrm{C}\left(68^{\circ} \mathrm{F}\right)$-----

- normal pressure, $21.1{ }^{\circ} \mathrm{C}\left(70^{\circ} \mathrm{F}\right)$---

- $20{ }^{\circ} \mathrm{C}\left(68^{\circ} \mathrm{F}\right)$ density, vapor:

pressure, saturated vapor: density, saturated 7iquid: density, saturated vapor: specific volume, saturated liquid: specific volume, saturated vapor: velocity of sound, saturated liquid: velocity of sound, saturated vapor: viscosity, saturated liquid: viscosity, saturated vapor: thermal conductivity, saturatd Tiquid: thermal conductivity, saturated vapor: - $60{ }^{\circ} \mathrm{C}\left(140{ }^{\circ} \mathrm{F}\right)$ pressure, saturated vapor: heat of vaporization:

- critical point

temperature: pressure: density: specific volume:

ENVIRONMENTAL

average atmospheric lifetime (Tatm): average stratospheric lifetime (Tstr): ODP (ozone depletion potential):

GWP (global warming potential): HGWP (haTocarbon GWP):

photochemical reactivity (grnd level):
$6.80 \mathrm{~kg} / \mathrm{m} 3(0.424 \mathrm{~Tb} / \mathrm{cf})$

$0.660 \mathrm{~L} / \mathrm{kg}(0.0106 \mathrm{cf} / 7 \mathrm{~b})$

$147.2 \mathrm{~L} / \mathrm{kg}(2.3573 \mathrm{cf} / 7 \mathrm{~b})$

$164.0 \mathrm{~kJ} / \mathrm{kg}(70.5 \mathrm{Btu} / 7 \mathrm{~b})$

$698 \mathrm{~m} / \mathrm{s}(2289 \mathrm{ft} / \mathrm{s})$

$128 \mathrm{~m} / \mathrm{s}(419 \mathrm{ft} / \mathrm{s})$

$406 \mu \mathrm{Pa} \cdot \mathrm{s}(0.406 \mathrm{cp})$

$9.64 \mu \mathrm{Pa} \cdot \mathrm{s}(0.00964 \mathrm{cp})$

$0.0924 \mathrm{~W} / \mathrm{m} \cdot \mathrm{K}(0.0534$

$\left.\mathrm{Btu} / \mathrm{hr} \cdot \mathrm{ft}^{\circ} \mathrm{F}\right)$

$0.0089 \mathrm{~W} / \mathrm{m} \cdot \mathrm{K}(0.0051$

$\mathrm{Btu} / \mathrm{hr} \cdot \mathrm{ft}^{\circ} \mathrm{F}$ )

$5.072 \mathrm{~kg} / \mathrm{m} 3(0.3167 \mathrm{lb} / \mathrm{cf}) \quad 8401$

$5.052 \mathrm{~kg} / \mathrm{m} 3(0.3154 \mathrm{lb} / \mathrm{cf}) \quad 8401$

$1204.5 \mathrm{kPa}$ (174.70 psia) 8401

$1219 \mathrm{~kg} / \mathrm{m3}(76.08 \mathrm{1b} / \mathrm{cf}) \quad 8401$

$77.83 \mathrm{~kg} / \mathrm{m} 3$ (4.859 1b/cf) 8401

$0.821 \mathrm{~L} / \mathrm{kg}(0.0131 \mathrm{cf} / 7 \mathrm{~b}) \quad 8401$

$12.8 \mathrm{~L} / \mathrm{kg}(0.2058 \mathrm{cf} / 7 \mathrm{~b}) \quad 8401$

$355 \mathrm{~m} / \mathrm{s}(1166 \mathrm{ft} / \mathrm{s}) \quad 8401$

$119 \mathrm{~m} / \mathrm{s}(392 \mathrm{ft} / \mathrm{s}) \quad 8401$

$150 \mu \mathrm{Pa} \cdot \mathrm{s}(0.150 \mathrm{cp}) \quad 8401$

$13.1 \mu \mathrm{Pa} \cdot \mathrm{s}(0.0131 \mathrm{cp}) \quad 8401$

$0.0619 \mathrm{~W} / \mathrm{m} \cdot \mathrm{K}(0.0358 \quad 8401$

$\mathrm{Btu} / \mathrm{hr} \cdot \mathrm{ft}^{\circ} \mathrm{F}$ )

$0.01472 \mathrm{~W} / \mathrm{m} \cdot \mathrm{K}(0.00851$

$\mathrm{Btu} / \mathrm{hr} \cdot \mathrm{ft}^{\circ} \mathrm{F}$ )

$3167 \mathrm{kPa}$ (459.4 psia)

$52.5 \mathrm{~kJ} / \mathrm{kg}(22.6 \mathrm{Btu} / 7 \mathrm{~b})$

8401

$66.2{ }^{\circ} \mathrm{C}\left(151.1{ }^{\circ} \mathrm{F}\right)$

$3629 \mathrm{kPa}(526.3$ psia)

$571 \mathrm{~kg} / \mathrm{m} 3$ (35.7 lb/cf)

$1.75 \mathrm{~L} / \mathrm{kg}(0.0280 \mathrm{cf} / 7 \mathrm{~b})$

8401

$32.6 \tilde{n} 11.4 \mathrm{yr}$

$400 \mathrm{yr}$

$<0.00003$ (model-derived

relative to $R$ 11)

3800 relative to $\mathrm{CO} 2$ for 100

$\mathrm{yr}$ integration

$0.58 \pm 0.07$ relative to $R 11$

for infinite integration period

0.65 relative to $R 11$ for

infinite integration period

0.84 relative to $R 11$ for

infinite integration period

0.90 relative to $R 11$ for

infinite integration period

ICI: 0.70 relative to $R 11$ for CSDS

infinite integration period

4511

6695

5508

9501

9501

5964

DW

4511

$6 \mathrm{~B} 35$

0.3 relative to methane

8601

AI

Honeywe $17:$ 2-1-0

MSDS safety group (ASHRAE standard 34): NFPA 704 degrees of hazard $(H-F-R-S)$ : 
[-specia]]: $0=$ no, $4=$ severe

NPCA HMIS hazard ratings $(H-F-R)$ :

DuPont: $1-0-1$ MSDS

Honeywe 17: 1-1-0 MSDS

health-flammability-reactivity

$0=$ insignificant, $4=$ extreme

- Tong-term occupational limit ------AIHA WEEL (workplace env1 exp 7imit):

$1,000 \mathrm{ppm} v / \mathrm{V}$ TWA for $8 \mathrm{hr} /$ day $5 \mathrm{Cl}$ and $40 \mathrm{hr} / \mathrm{wk}$

exposure Timit consistent to OSHA PEL:

Elf Atochem VME: $1000 \mathrm{ppm} \mathrm{v} / \mathrm{v}$ SDS

- emergency exposure limit

TWA for $8 \mathrm{hr} /$ day and $40 \mathrm{hr} / \mathrm{wk}$

Acute Toxicity Exposure Limit (ATEL):

Refrigerant Concentration Limit (RCL):

$75,000 \mathrm{ppm} v / \mathrm{v}$ (preliminary

based on ASHRAE $34 \mathrm{u}$ draft)

$69,000 \mathrm{ppm} v / \mathrm{v}$ (preliminary

based on ASHRAE $34 \mathrm{u}$ draft)

$69,000 \mathrm{ppm} v / \mathrm{v}$ (preliminary

based on ASHRAE $34 \mathrm{u}$ draft)

$34 u$

$34 \mathrm{u}$

A950

- acute (short-term) toxicity

LC50 (7ethal concentration, 50\%):

ALC (approximate Tethal concentration:

rat, $4 \mathrm{hr}:>769,000 \mathrm{ppm}$ (fatal

5754

concentration by inhalation

for half of test animals)

rat, $4 \mathrm{hr}, 0 / 10$ : >769,000 ppm

5754

(lowest exposure tested with

one or more deaths by

inhalation)

cardiac sensitization (CS) EC50: dog, $10 \mathrm{~min}$ : $139,000 \mathrm{ppm} \mathrm{v} / \mathrm{v}$

5755

(effective concentration in

half of test animals)

cardiac sensitization threshold/LOEL: dog, $10 \mathrm{~min}, 1 / 6: 100,000 \mathrm{ppm}$

$v / v$ (lowest observed effect

Tevel in test animals)

cardiac sensitization (CS) NOEL: $\operatorname{dog}, 10 \mathrm{~min}, 0 / 6: 75,000 \mathrm{ppm}$

$v / v$ (no observed effect level

in test animals)

anesthetic/CNS effect LOEL: rat, $4 \mathrm{hr},>5 / 10: 769,000 \mathrm{ppm}$

$v / v$ (lowest observed effect

level in ALC or LC50 studies)

anesthetic/CNS effect NOEL: rat, $4 \mathrm{hr}, 0 / 5: 709,000 \mathrm{ppm}$

$v / v$ (no observed effect level

in test animals)

- flammability

LFL-UFL (flammability limits in air):

heat of combustion (by ASHRAE 34-92):

flash point:

autoignition temperature:

autodecomposition temperature:

former UL Classification:

- detection

appearance: colorless

odor: very faint ethereal odor

5755

5755

5754

6457

none (nonflammable as tested)

$-1.5 \mathrm{MJ} / \mathrm{kg}(-645 \mathrm{Btu} / \mathrm{Tb})$

2525

Elf Atochem: none as tested SDS nonflammable

$733{ }^{\circ} \mathrm{C}\left(1351{ }^{\circ} \mathrm{F}\right)$

5 C15

Honeywe 17: $>750^{\circ} \mathrm{C}\left(>1382^{\circ} \mathrm{F}\right)$ MSDS

Honeywe 11: $>250^{\circ} \mathrm{C}\left(>482^{\circ} \mathrm{F}\right)$ MSDS

practicaliy nonflammable 5931

(withdrawn for revision of the

classification system,

category SBQT2)

$4 \mathrm{C} 01$

$5 \mathrm{C} 15$

PRODUCTION

last year production allowed: unrestricted

$8 \mathrm{C} 01$ 


\section{R-134}

R-134
HFC
COMMON USE(S)
considered circa $1990-1996$ both as a blend component and use as an
alternative

\section{IDENTIFIERS}

common name(s): R-134; R134; R 134

HFC- 134

f7uorocarbon 134

f7uorochemica7 134; FC 134

halocarbon 134

haTochemica 1134

hydrofluorocarbon 134

chemical name (by IUPAC convention): alternative chemical names/formulae:

1,1,2,2-tetrafluoroethane

ethane, 1,1,2,2-tetrafluoro-

symmetricaך tetrafluoroethane

CHF2CHF2; CHF2-CHF2

not recommended:

CF2HCF2H; CF2H-CF2H

empirical formula: $\mathrm{C} 2 \mathrm{H} 2 \mathrm{~F} 4$

HF2CCHF2; HF2C-CHF2

CAS number: 359-35-3 (Chemical Abstracts

EINECS number: $206-628-3$ (European Inventory

of Existing Chemical

Substances)

trade name (s):

DuPont Freon(R) 134

historical name(s):

ARI container color / Pantone number:

A7tied corp refrigerant 305

none, use light green grey/413 6601

PHYSICAL

- properties

molar mass:

$102.0308928 \mathrm{~g} / \mathrm{mol} \quad 0.224940$

$1 \mathrm{~b} / \mathrm{mo} 7)$
$-89.0{ }^{\circ} \mathrm{C}\left(-128.2^{\circ} \mathrm{F}\right)$

8820

normal freezing/melting/triple point:

- normal boiling point

temperature:

$-19.7^{\circ} \mathrm{C}\left(-3.5^{\circ} \mathrm{F}\right)$

7601

$-19.8^{\circ} \mathrm{C}\left(-3.6^{\circ} \mathrm{F}\right)$

$-23.0^{\circ} \mathrm{C}\left(-9.4^{\circ} \mathrm{F}\right)$

2A06

3627

2250

- normal pressure, $20^{\circ} \mathrm{C}\left(68^{\circ}{ }^{\circ} \mathrm{F}\right)-\overline{---}$

$$
4.343 \mathrm{~kg} / \mathrm{m} 3(0.2711 \mathrm{~Tb} / \mathrm{cf}) \quad 4101
$$

- critical point

temperature: pressure:

$119.0^{\circ} \mathrm{C}\left(246.1^{\circ} \mathrm{F}\right)$

7713

$4562 \mathrm{kPa}$ (661.7 psia)

2834

$4615 \mathrm{kPa}$ (669.4 psia)

density:

$535 \mathrm{~kg} / \mathrm{m} 3$ (33.4 ib/cf)

0918

$539 \mathrm{~kg} / \mathrm{m} 3$ ( $33.6 \mathrm{tb} / \mathrm{cf})$

0918

specific volume:

$1.87 \mathrm{~L} / \mathrm{kg}(0.0299 \mathrm{cf} / \mathrm{Tb})$

7713

0918

ENVIRONMENTAL

average atmospheric lifetime (Tatm): ODP (ozone depletion potentia7):

$10.6+21.2,-10.6 \mathrm{yr}$

6695

GWP (global warming potential):

0.000 (mode $7-$ derived relative to $R$ 11)

1200 relative to $\mathrm{CO} 2$ for 100 yr integration

9501 
SAFETY

- CTassification

safety group (ASHRAE standard 34): NPCA HMIS hazard ratings (H-F-R):

none (no application pending) 8601

DuPont: $1-0-2$

- 1ong-term occupational limit

health-fiammability-reactivity exposure limit consistent to OSHA PEL:

$0=i n s i g n i f i c a n t, \quad 4=e x t r e m e$

- emergency exposure limit

DuPont AEL: $1,000 \mathrm{ppm} \mathrm{V} / \mathrm{V}$ TWA MSDS

for $8 \mathrm{hr} /$ day and $40 \mathrm{hr} / \mathrm{wk}$ Acute Toxicity Exposure Limit (ATEL):

Refrigerant Concentration Limit ( $R C L)$ :

$75,000 \mathrm{ppm} \mathrm{v/v} \mathrm{(pre7iminary}$

based on ASHRAE $34 u$ draft)

$69,000 \mathrm{ppm} v / \mathrm{v}$ (pretiminary

based on ASHRAE $34 \mathrm{u}$ draft)

A950

- acute (short-term) toxicity --.-----

rat, $4 \mathrm{hr}, 0 / 6$ : $>460,000 \mathrm{ppm}$

(lowest exposure tested with

one or more deaths by

inhalation)

cardiac sensitization threshold/LOEL: dog, 33\%: 100,000 ppm v/v

(lowest observed effect level

in test animals)

cardiac sensitization (CS) NOEL:

dog, 0\%: 75,000 ppm $v / v$ (no

observed effect leve 7 in test

animals)

anesthetic/CNS effect LOEL:

rat, $4 \mathrm{hr}, ? / 6: 200,000 \mathrm{ppm}$

$v / v$ (lowest observed effect

Teve 1 in ALC or LC50 studies)

anesthetic/CNS effect NOEL:

- flammability

rat, $4 \mathrm{hr}, 0 / 6$ : 120,000 ppm

$\mathrm{v} / \mathrm{V}$ (no observed effect level

A950

in ALC or LC50 studies)

LFL-UFL (flammability Timits in air):

heat of combustion (by ASHRAE 34-92):

none (nonflammable as tested)

2525

$4.3 \mathrm{MJ} / \mathrm{kg}$ (1849 Btu/7b)

2318 autoignition temperature:

- detection

appearance:

$630^{\circ} \mathrm{C}\left(1166^{\circ} \mathrm{F}\right)$

3960

odor: slight ethereal odor 5864

PRODUCTION

first commercial use as a refrigerant: last year production allowed:

not known to be commercialized unrestricted 


\section{R-134a}

\begin{tabular}{|c|c|}
\hline $\begin{array}{l}1,1,1,2 \text {-tetrafluoroethane } \\
\text { CH2FCF3 }\end{array}$ & CAS number $811-97-2$ \\
\hline $\begin{array}{l}\text { COMMON USE(S) } \\
\text { domestic and commercial refrigeration, } \\
\text { transport refrigeration, centrifugal c } \\
\text { for refrigerants } 12 \text { and } 500 ; \text { blend com } \\
\text { including refrigerants } 404 A \text {, } 407 A, 407 \\
\text { others; foam blowing agent especialiy } \\
\text { polyurethanes, polyisocyanurates, and } \\
\text { pharmaceutical propellant for metered } \\
\text { suppressant, usually as a blend compon } \\
\text { (e.g., NAFG "NAF P-III") }\end{array}$ & $\begin{array}{l}\text { automobile air conditioners, } \\
\text { hillers; primary alternative } \\
\text { ponent for alternatives } \\
\text { B, 407C, } 4070,413 \mathrm{~A}, 416 \mathrm{~A} \text {, and } \\
\text { for polystyrenes, } \\
\text { phenolics; aerosol propellant; } \\
\text { dose inhalers; fire } \\
\text { ent, for streaming systems }\end{array}$ \\
\hline
\end{tabular}

IDENTIFIERS

common name(s): R-134a; R134a; R 134a

HFC $-134 a$

TFE

7274

f7uorocarbon $134 a$

fluorochemica1 134a; FC 134a

halocarbon $134 a$

halochemical $134 a$

hydrofluorocarbon $134 a$

not recommended: HFA-134a

chemical name (by IUPAC convention): alternative chemical names/formulae:

empirical formula:

1,1,1,2-tetrafiuoroethane

ethane, 1,1,1,2-tetrafluoro-

$\mathrm{CH} 2 \mathrm{FCF} 3$; $\mathrm{CH} 2 \mathrm{~F}-\mathrm{CF} 3$

not recommended:

CF3CFH2; CF $3-\mathrm{CFH} 2$

$\mathrm{CF} 3 \mathrm{CH} 2 \mathrm{~F} ; \mathrm{CF} 3-\mathrm{CH} 2 \mathrm{~F}$

CFH 2CF3; $\mathrm{CFH} 2-\mathrm{CF} 3$

$\mathrm{F} 3 \mathrm{CCFH} 2 ; \mathrm{F} 3 \mathrm{C}-\mathrm{CFH} 2$

$\mathrm{F} 3 \mathrm{CCH} 2 \mathrm{~F} ; \mathrm{F} 3 \mathrm{C}-\mathrm{CH} 2 \mathrm{~F}$

$\mathrm{FCH} 2 \mathrm{CF} 3 ; \mathrm{FCH} 2-\mathrm{CF} 3$

H2FCCF3; H2FC-CF3

CAS number: 811-97-2 (Chemical Abstracts

Service registry number)

EINECS number: 212-377-0 (European Inventory

of Existing Chemical

Substances)

trade name(s): Asahi Glass AsahikTin AK-134a

Ausimont Meforex(R) 134a

Daikin fluorocarbon HFC-134a

DuPont Suva(R) $134 a$

Elf Atochem Forane(R) $134 a$

HRP (UK) HARP (R) 134a

Honeywe 17 Genetron(R) 134a MSDS

ICI Klea(R) 134a 6302

RGI Quick Change (with MO/PAG)

Rhodia Isceon 134a

Solvay Solkane(R) $134 a$

LPCR

Allied corp refrigerant 300

MSDS

MSDS

6739

4768

A11jedsignal Genetron(R) 134a

MSDS

historical name(s):

DuPont Suva(R) Cold-MP

DuPont Suva(R) Trans-AC

Hoechst Reclin(R) $134 a$

Rhône-PouTenc Isceon 134a

ARI container color / Pantone number: Tight blue (sky) / 2975 
PHYSICAL

- properties

molar mass:

normal freezing/melting/triple point:

normal boiling point temperature:
rated 7 iquid: density, saturated Tiquid:
density, saturated vapor: specific volume, saturated 7 iquid: specific volume, saturated vapor: heat of vaporization: velocity of sound, saturated 7iquid: velocity of sound, saturated vapor: viscosity, saturated 7iquid:

viscosity, saturated vapor: thermal conductivity, liquid:

thermal conductivity, vapor:

- normal pressure, $20^{\circ} \mathrm{C}\left(68^{\circ} \mathrm{F}\right)$

- normar pressure, $21.1^{\circ} \mathrm{density,} \mathrm{vapor:}$

- 20 density, vapor: density, vapor

pressure, saturated vapor: density, saturated 7iquid: density, saturated vapor:

specific volume, saturated liquid: specific volume, saturated vapor: velocity of sound, saturated liquid: velocity of sound, saturated vapor: viscosity, saturated 7iquid: viscosity, saturated vapor: thermal conductivity, saturatd 7 iquid: thermal conductivity, saturated vapor:

- $60^{\circ} \mathrm{C}\left(140{ }^{\circ} \mathrm{F}\right)$ pressure, saturated vapor:

- critical point heat of vaporization:

temperature: pressure: density: specific volume:

$102.0308928 \mathrm{~g} / \mathrm{moT}(0.224940$

8820

$7 \mathrm{~b} / \mathrm{mo}$ )

$-103.3^{\circ} \mathrm{C}\left(-153.9^{\circ} \mathrm{F}\right)$

8401

$-26.1{ }^{\circ} \mathrm{C}\left(-14.9^{\circ} \mathrm{F}\right) \quad 8401$

$1377^{1} \mathrm{~kg} / \mathrm{m3}(85.94 \mathrm{~Tb} / \mathrm{cf}) \quad 8401$

$5.26 \mathrm{~kg} / \mathrm{m} 3(0.328 \mathrm{1b} / \mathrm{cf}) \quad 8401$

$0.726 \mathrm{~L} / \mathrm{kg}(0.0116 \mathrm{cf} / 7 \mathrm{~b}) \quad 8401$

$190.2 \mathrm{~L} / \mathrm{kg}(3.0464 \mathrm{cf} / 7 \mathrm{~b}) \quad 8401$

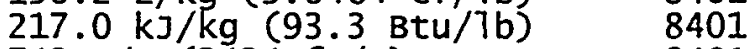

$742 \mathrm{~m} / \mathrm{s}(2434 \mathrm{ft} / \mathrm{s})$

$146 \mathrm{~m} / \mathrm{s}(478 \mathrm{ft} / \mathrm{s})$

$384 \mu \mathrm{Pa} \cdot \mathrm{s}(0.384 \mathrm{cp})$

$9.68 \mu \mathrm{Pa} \cdot \mathrm{s}(0.00968 \mathrm{cp})$

$0.1039 \mathrm{~W} / \mathrm{m} \cdot \mathrm{K}(0.0600$

$\mathrm{Btu} / \mathrm{hr} \bullet \mathrm{ft}^{\circ} \mathrm{F}$ )

$0.0093 \mathrm{~W} / \mathrm{m} \bullet \mathrm{K}(0.0054$

$\mathrm{Btu} / \mathrm{hr} \bullet f t^{\circ} \mathrm{F}$ )

8401

8401

8401

8401

8401

$4.336 \mathrm{~kg} / \mathrm{m} 3(0.2707 \mathrm{lb} / \mathrm{cf}) \quad 8401$

$4.319 \mathrm{~kg} / \mathrm{m} 3(0.2696 \mathrm{~Tb} / \mathrm{cf}) \quad 8401$

$571.5 \mathrm{kPa}(82.89 \mathrm{psia}) \quad 8401$

$1225 \mathrm{~kg} / \mathrm{m} 3(76.49 \mathrm{7b} / \mathrm{cf}) \quad 8401$

$27.78 \mathrm{~kg} / \mathrm{m} 3(1.734 \mathrm{7b} / \mathrm{cf}) \quad 8401$

$0.816 \mathrm{~L} / \mathrm{kg}(0.0131 \mathrm{cf} / \mathrm{Tb}) \quad 8401$

$36.0 \mathrm{~L} / \mathrm{kg}(0.5766 \mathrm{cf} / \mathrm{/b}) \quad 8401$

$530 \mathrm{~m} / \mathrm{s}(1738 \mathrm{ft} / \mathrm{s}) \quad 8401$

$145 \mathrm{~m} / \mathrm{s}(476 \mathrm{ft} / \mathrm{s}) \quad 8401$

$211 \mu \mathrm{Pa} \cdot \mathrm{s}(0.211 \mathrm{cp}) \quad 8401$

$11.6 \mu \mathrm{Pa} \cdot \mathrm{s}(0.0116 \mathrm{cP}) \quad 8401$

$0.0833 \mathrm{~W} / \mathrm{m} \cdot \mathrm{K}(0.0481$

8401

$\mathrm{Btu} / \mathrm{hr} \cdot \mathrm{ft}^{\circ} \mathrm{F}$ )

$0.01333 \mathrm{~W} / \mathrm{m} \cdot \mathrm{K}(0.00770 \quad 8401$

$\left.\mathrm{Btu} / \mathrm{hr} \cdot \mathrm{ft}^{\circ} \mathrm{F}\right)$

$1682 \mathrm{kPa}$ (243.9 psia)

$139.1 \mathrm{~kJ} / \mathrm{kg}(59.8 \mathrm{Btu} / \mathrm{lb})$

8401

$101.1^{\circ} \mathrm{C}\left(213.9^{\circ} \mathrm{F}\right)$

$4059 \mathrm{kPa}(588.7 \mathrm{psia})$

$512 \mathrm{~kg} / \mathrm{m} 3(32.0 \mathrm{lb} / \mathrm{cf})$

$1.95 \mathrm{~L} / \mathrm{kg}(0.0313 \mathrm{cf} / \mathrm{Tb})$

8401

8401

8401

8401

8401

ENVIRONMENTAL

average atmospheric lifetime (Tatm): average stratospheric lifetime (Tstr): ODP (ozone depletion potential):

GWP (global warming potential):

HGWP (halocarbon GWP):

photochemical reactivity (grnd level):

$13.6 \mathrm{yr}$

9501

$210 \mathrm{yr}$

5508

$<0.000015$ (model-derived 9501

relative to $R$ 11)

$<0.0005$ (semi-empirica 1

9501

relative to $R$ 11)

1600 relative to $\mathrm{CO}$ for 1009501

yr integration

0.28 relative to $R 11$ for

infinite integration period

ICI: 0.30 relative to $R 11$ for CSDS

infinite integration period

6739

0.6 relative to methane

4511 
SAFETY

- Classification

safety group (ASHRAE standard 34):

NFPA 704 degrees of hazard $(H-F-R-S)$ :

NPCA HMIS hazard ratings (H-F-R):

A1

8601

ARI recommendation: 2-0-0 3A15

Honeywe 17: 2-1-0 MSDS

health-flammability-reactivity

[-specia1]: $0=$ no, $4=$ severe

Honeywe 17: 1-1-0 MSDS

DuPont: $1-0-1 \quad$ MSDS

hea 1th-flammability-reactivity

$0=$ insignificant, $4=$ extreme

DFG pregnancy risk group: C (no risk fear below MAK/BAT) 7101

- short-term occupational Timit --.--

ARI "IDLH" recommendation: $50,000 \mathrm{ppm} \mathrm{v} / \mathrm{v}$ for $30 \mathrm{~min}$

$3 \mathrm{~A} 15$

- occupational exposure 7 imit -..----

MAK (maximum workplace concentration):

- Tong-term occupational Timit ------AIHA WEEL (workplace envi exp Timit):

exposure Timit consistent to OSHA PEL:

MAK (maximum workplace concentration):

IV: $2,000 \mathrm{ppm} \mathrm{v} / \mathrm{V}$ momentary 607101 $\min$

$1,000 \mathrm{ppm} \mathrm{v} / \mathrm{v}$ TWA for $8 \mathrm{hr} /$ day $4 \mathrm{~B} 86$ and $40 \mathrm{hr} / \mathrm{wk}$

ARI: $1,000 \mathrm{ppm} v / \mathrm{v}$ TWA for 83 3A15

$\mathrm{hr} /$ day and $40 \mathrm{hr} / \mathrm{wk}$

$1,000 \mathrm{ppm} v / \mathrm{v}$ TWA for $8 \mathrm{hr} /$ day 7101 40 (or 42) $\mathrm{hr} / \mathrm{wk}$

- emergency exposure limit

NRC EEGL (emergency exposure level):

Acute Toxicity Exposure Limit (ATEL):

$1 \mathrm{hr}: 4,000 \mathrm{ppm} v / \mathrm{v}$ ceiling guidance level for single emergency exposures

$24 \mathrm{hr}: 1,000 \mathrm{ppm} v / \mathrm{v}$ ceiling $6 \mathrm{A01}$

guidance level for single

emergency exposures

$50,000 \mathrm{ppm} v / \mathrm{v}$ (preliminary

based on ASHRAE $34 \mathrm{u}$ draft)

$50,000 \mathrm{ppm} \mathrm{v} / \mathrm{v}$ (pre7iminary

based on ASHRAE 34u draft)

Refrigerant Concentration Limit ( $R C L$ ):

$50,000 \mathrm{ppm} v / v$ (preliminary

based on ASHRAE 34u draft)

$50,000 \mathrm{ppm} v / \mathrm{v}$ (pre7iminary

based on ASHRAE 34u draft)

- special-purpose exposure control ---

90 day: $1,000 \mathrm{ppm} v / \mathrm{v}$ ceiling

guidance for prolonged

exposure in closed

envi ronments

- acute (short-term) toxicity

LC50 (lethal concentration, 50\%) :

rat, 15 min: $>800,000 \mathrm{ppm}$

$5 A 79$

(fatal concentration by

inhalation for half of test

anima7s)

ALC (approximate lethat concentration:

rat, 30 min, 2/4: 750,000 ppm

(7owest exposure tested with

one or more deaths by

inhalation)

rat, $4 \mathrm{hr}, 0 / 6: 359,300 \mathrm{ppm}$

(7owest exposure tested with

one or more deaths by

inhalation)

rat, $4 \mathrm{hr}, 5 / 6: 566,700 \mathrm{ppm}$

(7owest exposure tested with

one or more deaths by

inhalation)

cardiac sensitization (CS) EC50: dog, $10 \mathrm{~min}$ : 205,000 ppm v/v

A950

$34 u$

$34 \mathrm{u}$

A950

(effective concentration in

6A01

half of test animals)

6525

6529

6684 
cardiac sensitization threshold/LOEL: dog, $10 \mathrm{~min}, 2 / 10: 75,200 \mathrm{ppm} 6527$

$\mathrm{v} / \mathrm{v}$ (lowest observed effect

level in test animals)

cardiac sensitization (CS) NOEL: dog, $10 \mathrm{~min}, 0 / 10: 49,800 \mathrm{ppm}$

$\checkmark / V$ (no observed effect 7 eve $T$

in test animals)

anesthetic/CNS effect EC50:

mouse, rapid: $270,000 \mathrm{ppm} v / \mathrm{v}$

(effective concentration in

half of test animals)

rat, $10 \mathrm{~min}: 280,000 \mathrm{ppm} \mathrm{v} / \mathrm{v}$

(effective concentration in

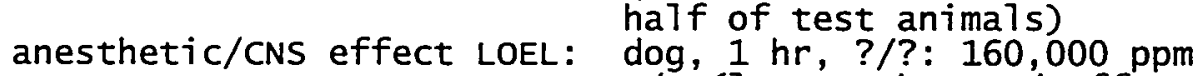

$v / v$ (Towest observed effect

level in ALC or LC50 studies)

rat, $4 \mathrm{hr}$, ?/6: 205,200 ppm

$v / v$ (lowest observed effect

level in ALC or LC5O studies)

anesthetic/CNS effect NOEL: dog, $1 \mathrm{hr}, 0 / ?$ : $80,000 \mathrm{ppm} \mathrm{V} / \mathrm{v} 8111$

(no observed effect level in

ALC or LC50 studies)

rat, $4 \mathrm{hr}, 0 / 6$ : 81,000 ppm v/v 6529

(no observed effect level in

ALC or LC50 studies)

human, $1 \mathrm{hr}, 0 / 8: 7,600 \mathrm{ppm}$

$v / v$ (no obsérved effect level)

- flammability

LFL-UFL (flammability limits in air):

none (nonflammable as tested)

none (nonflammable as tested)

heat of combustion (by ASHRAE 34-92):

flash point: autoignition temperature:

$4.2 \mathrm{MJ} / \mathrm{kg}$ (1806 Btu/7b)

6527

7274

$5 A 79$

8111

6529

none (nonflammable as tested)

$743{ }^{\circ} \mathrm{C}\left(1369^{\circ} \mathrm{F}\right)$

$770{ }^{\circ} \mathrm{C}\left(1418{ }^{\circ} \mathrm{F}\right)$

Dupont: $>743{ }^{\circ} \mathrm{C}\left(>1369^{\circ} \mathrm{F}\right) \quad 4682$

former UL Classification:

practically nonflammable

(withdrawn for revision of the

classification system,

category SBQT2)

- detection

appearance: clear, colorless gas

odor: faint ethereal odor

A535

2525

5648

2318

4682

6938

6938

4940

$6 B 26$

PRODUCTION

first commercial use as a refrigerant: 1990

last year production allowed: unrestricted

$8 \mathrm{C} 01$ 


\section{R-E134}

\begin{tabular}{|c|c|c|}
\hline $\begin{array}{l}\text { R-E134 } \\
\text { HFE }\end{array}$ & $\begin{array}{l}\text { dif7uoromethoxy-difluoromethane } \\
\text { CHF2-0-CHF2 CAS number 1691-17-4 }\end{array}$ & $\begin{array}{l}\text { see } \\
\text { RDB\# }\end{array}$ \\
\hline $\begin{array}{l}\text { OMMON } \\
\text { candi } \\
\text { compo } \\
\text { app } 1 \text { i } \\
\text { syste } \\
\text { prope } \\
\text { anest } \\
\text { refri }\end{array}$ & $\begin{array}{l}\text { Tternative for refrigerant } 114 \text {, alone and as a bl } \\
\text { though believed to have been rejected as such for } \\
\text { is based on compatibjlity tests with air purificat } \\
\text { samined in early } 1990 \text { as as a foam-blowing agent or } \\
\text { but not commercialized; originally identified as } \\
\text { to replace halothane, but found to be unsuitable; } \\
\text { : may be covered by U.S. patent } 4,961,321\end{array}$ & \\
\hline
\end{tabular}

IDENTIFIERS

common name(s): R-E134; RE134; R E134

HFE-E134; HFOC-E134

f7uorochemica] E134; FC E134

fluoroether E134; E-134

chemical name (by IUPAC convention): halochemical E134

alternative chemical names/formulae:

difluoromethoxy-

difluoromethane

methane, difluoromethoxy-

diffuoro-

bis(difluoromethyl) ether

di (difluoromethyl) ether

1,1,3,3-tetrafluorodimethy 1

ether

CHF2OCHF2; CHF2-O-CHF2

not recommended:

CF2HOCF2H; CF2H-O-CF2H

empirical formula:

HF2COCHF2; HF2C-O-CHF2

CAS number:

$\mathrm{C} 2 \mathrm{H} 2 \mathrm{~F} 40$

1691-17-4 (Chemical Abstracts

Service registry number)

PHYSICAL

- properties

molar mass: $118.0302928 \mathrm{~g} / \mathrm{mol}(0.260212$

8820

$\mathrm{Tb} / \mathrm{mo}$ )

normal freezing/melting/triple point:

- normal boiling point

temperature:

density, saturated 7iquid:

$-151.1^{\circ} \mathrm{C}\left(-240.0^{\circ} \mathrm{F}\right)$

$4.7^{\circ} \mathrm{C}\left(40.4^{\circ} \mathrm{F}\right)$

$5.2^{\circ} \mathrm{C}\left(41.3^{\circ} \mathrm{F}\right)$

9318

$6.2{ }^{\circ} \mathrm{C}\left(43.1{ }^{\circ} \mathrm{F}\right)$

8703

3327

$1425 \mathrm{~kg} / \mathrm{m} 3(88.96 \mathrm{7b} / \mathrm{cf}) \quad 4101$

density, saturated vapor:

$5.42 \mathrm{~kg} / \mathrm{m} 3(0.338 \mathrm{~Tb} / \mathrm{cf})$

4101

specific volume, saturated Tiquid:

$0.702 \mathrm{~L} / \mathrm{kg}(0.0112 \mathrm{cf} / \mathrm{lb})$

4101

specific volume, saturated vapor: velocity of sound, saturated 7iquid:

velocity of sound, saturated vapor: viscosity, saturated Tiquid:

- normal pressure, $20{ }^{\circ} \mathrm{C}\left(68^{\circ} \mathrm{F}\right)$-..--

- $20^{\circ} \mathrm{C}\left(68^{\circ} \mathrm{F}\right)$ density, vapor:

pressure, saturated vapor: density, saturated 1iquid: density, saturated vapor:

specific volume, saturated $7 i q u i d:$ $184.6 \mathrm{~L} / \mathrm{kg}(2.9570 \mathrm{cf} / 7 \mathrm{~b})$

$734 \mathrm{~m} / \mathrm{s}(2409 \mathrm{ft} / \mathrm{s})$

$142 \mathrm{~m} / \mathrm{s}(466 \mathrm{ft} / \mathrm{s})$

$395 \mu \mathrm{Pa} \cdot \mathrm{s}(0.395 \mathrm{cP})$

4101

4101

4101

4101

$5.084 \mathrm{~kg} / \mathrm{m} 3$ (0.3174 7b/cf)

4101

$174.9 \mathrm{kPa}$ (25.37 psia)

4101

$1388 \mathrm{~kg} / \mathrm{m} 3(86.65 \mathrm{7b} / \mathrm{cf})$

4101

$9.03 \mathrm{~kg} / \mathrm{m} 3(0.564 \mathrm{ib} / \mathrm{cf})$

4101

$0.721 \mathrm{~L} / \mathrm{kg}(0.0115 \mathrm{cf} / \mathrm{lb})$

4101

specific volume, saturated vapor:

velocity of sound, saturated liquid:

$110.8 \mathrm{~L} / \mathrm{kg}(1.7748 \mathrm{cf} / 1 \mathrm{~b})$

4101

$675 \mathrm{~m} / \mathrm{s}(2215 \mathrm{ft} / \mathrm{s})$

4101 
velocity of sound, saturated vapor: viscosity, saturated 7 iquid: viscosity, saturated vapor: thermal conductivity, saturatd liquid: thermal conductivity, saturated vapor:

- $60{ }^{\circ} \mathrm{C}\left(140{ }^{\circ} \mathrm{F}\right)$ -

- critical point -re

pressure:

density:

specific volume:
$143 \mathrm{~m} / \mathrm{s}(469 \mathrm{ft} / \mathrm{s})$

$334 \mu \mathrm{Pa} \cdot \mathrm{s}(0.334 \mathrm{cp})$

$11.0 \mu \mathrm{Pa} \cdot \mathrm{s}(0.0110 \mathrm{cp})$

$0.0952 \mathrm{~W} / \mathrm{m} \cdot \mathrm{K}(0.0550$

$\mathrm{Btu} / \mathrm{hr} \cdot \mathrm{ft}^{\circ} \mathrm{F}$ )

$0.01227 \mathrm{~W} / \mathrm{m} \cdot \mathrm{K}(0.00709$

$\mathrm{Btu} / \mathrm{hr} \bullet \mathrm{ft}^{\circ} \mathrm{F}$ )

$624 \mathrm{kPa}(90.5 \mathrm{psia})$

4101

1133

3327

9318

3407

$4 A 58$

9318

4101

3327

3327

ENVIRONMENTAL

average atmospheric lifetime (Tatm):

ODP (ozone depletion potential):

GWP (global warming potential):

$19 \mathrm{yr}$

9413

$29.7 \mathrm{yr}$

0.000 (model-derived relative 9318 to $\mathrm{R}$ 11)

6900 relative to $\mathrm{CO} 2$ for 100 $y r$ integration

9501

SAFETY

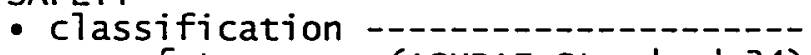

safety group (ASHRAE Standard 34):

- emergency exposure limit -...--

none (no application pending)

8601

Acute Toxicity Exposure Limit (ATEL):

Refrigerant Concentration Limit (RCL):

$0 \mathrm{ppm} \mathrm{v} / \mathrm{v}$ (preliminary based

A950

on ASHRAE $34 \mathrm{u}$ draft)

- acute (short-term) toxicity -------on ASHRAE $34 u$ draft)

A950

ALC (approximate lethal concentration:

cardiac sensitization threshold/LOEL:

rat, $30 \mathrm{~min}, 0 /$ ?: $>300,000$ ppm 5550

(lowest exposure tested with

one or more deaths by

inhalation)

rat, 2 hr, 0/10: >200,000 ppm A944

(lowest exposure tested with

one or more deaths by

inhalation)

rat, $2 \mathrm{hr}, 0 /$ ?: $>200,000$ ppm 5550

(lowest exposure tested with

one or more deaths by

inhalation)

rat, $3 \mathrm{hr}, 0 /$ ?: $>50,000 \mathrm{ppm}$

(lowest exposure tested with

one or more deaths by

inhalation)

rat, $3 \mathrm{hr}, 0 /$ ?: $>50,000 \mathrm{ppm}$

(7owest exposure tested with

one or more deaths by

inhalation)

rat, $4 \mathrm{hr}, 0 / 3$ : $>95,000 \mathrm{ppm}$

(lowest exposure tested with

5550

one or more deaths by

inhalation)

monkey, 30min: $\sim R 11$ at 50,000

A944

$\mathrm{ppm} \mathrm{v} / \mathrm{v}$ (lowest observed

effect leve 7 in test animals) 
anesthetic concentration: mouse, $2 \mathrm{~min}, ? / ?: 307,500 \quad 5550$

$\mathrm{ppm} \mathrm{v} / \mathrm{v}$

mouse, 5 min, ?/?: $182,500 \quad 5550$

ppm $v / v$

mouse, $30 \mathrm{~min}$, ?/?: 130,000 5550

ppm $\mathrm{v} / \mathrm{v}$

mouse, $60 \mathrm{~min}$, ?/?: 100,000 5550

anesthetic/CNS effect LOEL:

ppm $\mathrm{v} / \mathrm{v}$

$\mathrm{ppm} v / \mathrm{v}$
rat, $4 \mathrm{hr}, ? / 3: 45,000 \mathrm{ppm} v / \mathrm{v} 6207$
(lowest observed effect level

anesthetic/CNS effect NOEL:

in ALC or LC50 studies)

rat, $4 \mathrm{hr}, 0 / 3: 18,000 \mathrm{ppm} \mathrm{v/v} 6207$

(no observed effect level in

- flammabiTity ALC or LC50 studies)

LFL-UFL (flammability limits in air): none (nonflammable as tested) 5550 autodecomposition temperature: less than critical temperature 8703

PRODUCTION

first commercial use as a refrigerant: not known to be commercialized

last year production allowed: unrestricted $8 \mathrm{C01}$ 


\section{R-141b}

R-I41b CHC CAS number 1717-00-6
COMMON USE(S)
COnsidered but not selected as a replacement for refrigerant 11 ;
widely used as a foam blowing agent especially for polyurethanes,
flexible polystyrenes, polyisocyanurates, and phenolics; solvent and
blend component for solvents; aerosol propellant

IDENTIFIERS

common name (s): R-141b; R141b; R 141b

HCFC $-141 b$

not recommended: HFA-141b

f7uorocarbon 141b

f7uorochemica $7141 b$; FC 141b

halocarbon $141 b$

halochemical $141 b$

chemical name (by IUPAC convention):

1,1-dich7oro-1-f7uoroethane

alternative chemical names/formulae:

ethane, 1,1-dichloro-1-fluoro-

methy 7 dichlorofluoromethy?

$\mathrm{CH} 3 \mathrm{CC} 72 \mathrm{~F} ; \mathrm{CH} 3-\mathrm{CC} 12 \mathrm{~F}$

not recommended:

$\mathrm{CC} 12 \mathrm{FCH} 3 ; \mathrm{CC} 72 \mathrm{~F}-\mathrm{CH} 3$

empirical formula:

$\mathrm{H} 3 \mathrm{CCC} 72 \mathrm{~F} ; \mathrm{H} 3 \mathrm{C}-\mathrm{CC} 72 \mathrm{~F}$

CAS number: 1717-00-6 (Chemical abstracts

Service registry number)

EINECS number: 404-080-1 (European Inventory

of Existing Chemical

Substances)

NIOSH RTECS number: KI0997000 (Registry of Toxic

Effects of Chemica?

Substances)

trade name(s): Asahi G1ass Asahik7in AK-141b

Ausimont Meforex(R) 141b

DuPont HCFC-141b

Elf Atochem Forane (R) $141 b$

Honeywe 11 Genetron(R) 141b

ICI Arcton $(R) 141 b$

LaRoche Chemicals R-141b

Pennwa7t Isotron( $R$ ) $141 \mathrm{~b}$

ZCIRI Kehua (PRC) HCFC-141b

historical name(s): A17ied corp refrigerant 2

Alliedsignal Genetron(R) 141b $5 \mathrm{~A} 33$

DuPont Freon(R) 141b 5868

ARI container color / Pantone number: none, use light green grey/413 6601

PHYSICAL

- properties

molar mass:

normal freezing/melting/triple point:

- normal boiling point

temperature:

density, saturated Tiquid:

density, saturated vapor:

specific volume, saturated liquid:

specific volume, saturated vapor:

heat of vaporization:

$\begin{array}{ll}116.9490232 \mathrm{~g} / \mathrm{mol}(0.257828 & 8820 \\ 1 \mathrm{~b} / \mathrm{mol}){ }^{\circ} \mathrm{C}\left(-153.9{ }^{\circ} \mathrm{F}\right) & 8401 \\ -103.3^{\circ} & 8401 \\ 32.0{ }^{\circ} \mathrm{C}\left(89.7{ }^{\circ} \mathrm{F}\right) & 8401 \\ 1220 \mathrm{~kg} / \mathrm{m} 3(76.17 \mathrm{lb} / \mathrm{cf}) & 8401 \\ 4.85 \mathrm{~kg} / \mathrm{m} 3(0.303 \mathrm{lb} / \mathrm{cf}) & 8401 \\ 0.820 \mathrm{~L} / \mathrm{kg}(0.0131 \mathrm{cf} / \mathrm{b}) & 8401 \\ 206.2 \mathrm{~L} / \mathrm{kg}(3.3024 \mathrm{cf} / \mathrm{bb}) & 8401 \\ 223.0 \mathrm{~kJ} / \mathrm{kg}(95.9 \mathrm{Btu} / \mathrm{lb}) & \end{array}$


velocity of sound, saturated liquid: velocity of sound, saturated vapor: viscosity, saturated 7iquid: viscosity, saturated vapor: thermal conductivity, Tiquid:

thermal conductivity, vapor:

- normal pressure, $20{ }^{\circ} \mathrm{C}\left(68^{\circ} \mathrm{F}\right)$ density, jiquid:

- normat pressure, $21.1^{\circ} \mathrm{C}\left(70^{\circ} \mathrm{F}\right)$-.-

pressure, saturated vapor: density, saturated 1iquid: density, saturated vapor:

specific volume, saturated liquid: specific volume, saturated vapor: velocity of sound, saturated Tiquid: velocity of sound, saturated vapor: viscosity, saturated 1iquid: viscosity, saturated vapor: thermal conductivity, saturatd 1iquid: thermal conductivity, saturated vapor: - $60{ }^{\circ} \mathrm{C}\left(140{ }^{\circ} \mathrm{F}\right)$ pressure, saturated vapor: - critical point heat of vaporization: pressure: density: specific volume: temperature:
$797 \mathrm{~m} / \mathrm{s}(2616 \mathrm{ft} / \mathrm{s})$

$150 \mathrm{~m} / \mathrm{s}(492 \mathrm{ft} / \mathrm{s})$

$378 \mu \mathrm{Pa} \cdot \mathrm{s}(0.378 \mathrm{cp})$

$9.45 \mu \mathrm{Pa} \cdot \mathrm{s}(0.00945 \mathrm{cp})$

$0.0920 \mathrm{~W} / \mathrm{m} \cdot \mathrm{K}(0.0532$

$\mathrm{Btu} / \mathrm{hr} \bullet \mathrm{ft}^{\circ} \mathrm{F}$ )

$0.0099 \mathrm{~W} / \mathrm{m} \cdot \mathrm{K}(0.0057$

$\mathrm{Btu} / \mathrm{hr} \cdot \mathrm{ft}^{\circ} \mathrm{F}$ )

$1244 \mathrm{~kg} / \mathrm{m} 3$ (10.38 7b/gaT)

$1242 \mathrm{~kg} / \mathrm{m} 3$ (10.36 7b/gal)

$65.0 \mathrm{kPa}(9.43 \mathrm{psia})$

$1244 \mathrm{~kg} / \mathrm{m} 3(77.66 \mathrm{lb} / \mathrm{cf})$

$3.21 \mathrm{~kg} / \mathrm{m} 3(0.2007 \mathrm{~b} / \mathrm{cf})$

$0.804 \mathrm{~L} / \mathrm{kg}(0.0129 \mathrm{cf} / 7 \mathrm{~b})$

$311.7 \mathrm{~L} / \mathrm{kg}(4.9933 \mathrm{cf} / 7 \mathrm{~b})$

$841 \mathrm{~m} / \mathrm{s}(2760 \mathrm{ft} / \mathrm{s})$

$149 \mathrm{~m} / \mathrm{s}(487 \mathrm{ft} / \mathrm{s})$

$432 \mu \mathrm{Pa} \cdot \mathrm{s}(0.432 \mathrm{cp})$

$9.1 \mu \mathrm{Pa} \cdot \mathrm{s}(0.0091 \mathrm{cp})$

$0.0958 \mathrm{~W} / \mathrm{m} \cdot \mathrm{K}(0.0554$

$\mathrm{Btu} / \mathrm{hr} \cdot \mathrm{ft}^{\circ} \mathrm{F}$ )

$0.00930 \mathrm{~W} / \mathrm{m} \cdot \mathrm{K} \quad(0.00537$

$\mathrm{Btu} / \mathrm{hr} \cap \mathrm{ft}^{\circ} \mathrm{F}$ )

$246 \mathrm{kPa}$ (35.7 psia)

$208.9 \mathrm{~kJ} / \mathrm{kg}(89.8 \mathrm{Btu} / 7 \mathrm{~b})$

$4250 \mathrm{kPa}$ (6I6.4 psia)

$460 \mathrm{~kg} / \mathrm{m} 3(28.7 \mathrm{lb} / \mathrm{cf})$

$2.17 \mathrm{~L} / \mathrm{kg}(0.0348 \mathrm{cf} / \mathrm{lb})$
$204.2{ }^{\circ} \mathrm{C}\left(399.6^{\circ} \mathrm{F}\right)$

8401

8401

8401

8401

8401

8401

8401

8401

8401

8401

8401

8401

8401

8401

8401

8401

8401

8401

8401

8401

8401

8401

8401

8401

8401

$9.2 \mathrm{yr}$

9501

$\begin{array}{ll}76 \text { yr } & \\ 0.086 & 508 \\ & \end{array}$

to $\mathrm{R}$ 11)

0.1 (semi-empirical relative 9501 to $R$ 11)

0.11 (estimate used for the 6904 Montreal Protocol)

GWP (global warming potential):

HGWP (haTocarbon GWP):

700 relative to $\mathrm{CO2}$ for $100 \mathrm{yr} 9501$ integration

$0.0905 \pm 0.0065$ relative to $R \quad 5964$ 11 for infinite integration period

0.15 relative to $R 11$ for infinite integration period 0.16 relative to $R 11$ for infinite integration period

4511

5B15

SAFETY

- classification safety group (ASHRAE Standard 34)

NPCA HMIS hazard ratings $(H-F-R)$ :

none (no application pending) Ausimont: $\quad 2-0-0$ Honeywe 71: $\quad 2-1-0$ hea th-flammability-reactivity [-special]: $0=$ no, $4=$ severe

DuPont: 2-1-0 Honeywe 77: $1-1-0$ health-flammability-reactivity
8601

MSDS

MSDS

MSDS MSDS 
- Tong-term occupational Timit ---.--AIHA WEEL (workplace env1 exp 7imit):

- emergency exposure Timit Acute Toxicity Exposure Limit (ATEL):

Refrigerant Concentration Limit (RCL):

- acute (short-term) toxicity ---.---LC50 (7ethal concentration, $50 \%$ ):

ALC (approximate lethal concentration:

derma1 LD50 (7etha1 dosage, 50\%):

oral LD50 (1etha1 dosage, 50\%):

cardiac sensitization (CS) EC50:

cardiac sensitization threshold/LOEL:

cardiac sensitization (CS) NOEL:

anesthetic/CNS effect EC50:
$0=$ insignificant, $4=$ extreme

$500 \mathrm{ppm} \mathrm{v} / \mathrm{v}$ TWA for $8 \mathrm{hr} /$ day and $40 \mathrm{hr} / \mathrm{wk}$

2,600 ppm v/v (preliminary based on ASHRAE $34 \mathrm{u}$ draft) 2,600 ppm $\mathrm{v} / \mathrm{v}$ (preliminary based on ASHRAE 34u draft) 2,600 ppm v/v (preliminary based on ASHRAE $34 \mathrm{u}$ draft) 2,600 ppm v/v (preliminary based on ASHRAE $34 u$ draft)

$34 u$

A950

$34 u$

A950

mouse, $10 \mathrm{~min}: 50,000 \mathrm{ppm}$

(fatal concentration by inhalation for half of test animals)

rat, 4 hr: 61,647 ppm (fata) concentration by inhalation for half of test animals)

rat, $6 \mathrm{hr}, 0 / 6$ : $>42,800 \mathrm{ppm}$ (lowest exposure tested with one or more deaths by inhalation)

rat, $6 \mathrm{hr}, 1 / 6: 50,200 \mathrm{ppm}$ (Towest exposure tested with one or more deaths by inhalation)

rabbit: $>2,000 \mathrm{mg} / \mathrm{kg}$ (fatal dose by skin contact for half of test animals)

rat, 0/10: >2,000 mg/kg (fata) 6460 dose by skin contact for half of test animals)

5980

5995

6465

rat, 0/10: >5,000 mg/kg (fatal 5988 dose by ingestion for half of test animais)

dog, $10 \mathrm{~min}: 20,000 \mathrm{ppm} \mathrm{v} / \mathrm{v}$ (effective concentration in half of test animals)

dog, 10 min, 1/10: 5,200 ppm 6467 $v / v$ (lowest observed effect level in test anima7s) dog, $10 \mathrm{~min}, 1 / 2: 9,000 \mathrm{ppm}$ $v / v$ (lowest observed effect leve 1 in test anima $7 \mathrm{~s}$ ) dog, $10 \mathrm{~min}, 1 / 6: 20,000 \mathrm{ppm}$ $v / v$ (lowest observed effect level in test anima $7 \mathrm{~s}$ )

monkey, $10 \mathrm{~min}, 1 / 2: 5,000 \mathrm{ppm} 5 \mathrm{C63}$ $v / v$ (lowest observed effect level in test animals)

monkey, $10 \mathrm{~min}, 1 \mathrm{mi} 1 \mathrm{~d} / 1: 3,0005 \mathrm{c63}$ $\mathrm{ppm} \mathrm{v} / \mathrm{v}$ (lowest observed effect level in test anima1s)

dog, $10 \mathrm{~min}, 0 / 10$ : 2,600 ppm 6467 $v / v$ (no observed effect level in test animals) dog, $10 \mathrm{~min}, 0 / 6$ : $10,000 \mathrm{ppm}$ $v / v$ (no observed effect 7 evel in test animals)

mouse, $10 \mathrm{~min}: 25,000 \mathrm{ppm} \mathrm{v} / \mathrm{v}$ (effective concentration in
6684

$5 C 63$

7206

7206

5980 
half of test anima7s)

rat, 1-hr, ?/6: 31,730 ppm v/v 6465

(7owest observed effect level

in ALC or LC50 studies)

rat, 4-hr, ?/10: $29,674 \mathrm{ppm}$

$v / v$ (lowest observed effect

Tevel in ALC or LC50 studies)

rat, ?/? depression: 30,000

ppm $v / v$ (lowest observed

effect level in ALC or LC50 studies)

anesthetic/CNS effect NOEL: rat, 6-hr, 0/10: 11,096 ppm

$v / v$ (no observed effect leve]

in ALC or LC50 studies)

rat, 6-hr, 0/160: 19,959 ppm

$v / v$ (no observed effect level

in subchronic or chronic

study)

- flammability

LFL-UFL (flammability limits in air):

$5.8-17.7 \% \mathrm{v} / \mathrm{v}$

$7.2-18.0 \% \mathrm{v} / \mathrm{v}$

$7.3-16.0 \% \mathrm{v} / \mathrm{v}$

$7.4-15.5 \% \mathrm{v} / \mathrm{v}$

$7.6-17.7 \% \mathrm{v} / \mathrm{v}$

Ausimont: $7.4-15.5 \% \mathrm{v} / \mathrm{v}$

Dupont: $6.4-15.1 \% \mathrm{v} / \mathrm{v}$

Elf Atochem: $5.6-17.7 \%$ with a

Elf Atochem: powerfut source

Elf Atochem: of energy

Honeywe 11: nonflammable for

Honeywe 17: spark ignition

LFL-UFL (with spark ignition): $9.0-15.4 \% \mathrm{v} / \mathrm{v}$

heat of combustion (by ASHRAE 34-92):

flash point:

autoignition temperature:

autodecomposition temperature:

- detection

appearance:

odor:

$8.6 \mathrm{MJ} / \mathrm{kg}$ (3697 Btu/7b)

CC, DuPont: none

TCC, Elf Atochem: none

TCC, ICI : $>82^{\circ} \mathrm{C}\left(>180^{\circ} \mathrm{F}\right)$

none

$550{ }^{\circ} \mathrm{C}\left(1022^{\circ} \mathrm{F}\right)$

Dupont: $530^{\circ} \mathrm{C}\left(986^{\circ} \mathrm{F}\right)$

Eif Atochem: $532^{\circ} \mathrm{C}\left(990^{\circ} \mathrm{F}\right)$

Honeywe 77: $550^{\circ} \mathrm{C}\left(1022^{\circ} \mathrm{F}\right)$

Honeywe 17: $>250^{\circ} \mathrm{C}\left(>482^{\circ} \mathrm{F}\right)$

clear, colorless Tiquid

faint ethereal odor

5995

7278

6463

65A8

$5 \mathrm{~B} 15$

2525

4511

0521

5 A33

MSDS

MSDS

SDS

SDS

SDS

MSDS

MSDS

5648

2318

MSDS

MSDS

MSDS

$5 A 30$

5A30

MSDS

SDS

MSDS

MSDS

$5 A 33$

$5 A 33$

PRODUCTION

first commercial use as a refrigerant: last year production allowed: blowing agent: December 1990 2029 in developed countries under the Montreal Protocol 


\section{R-142b}

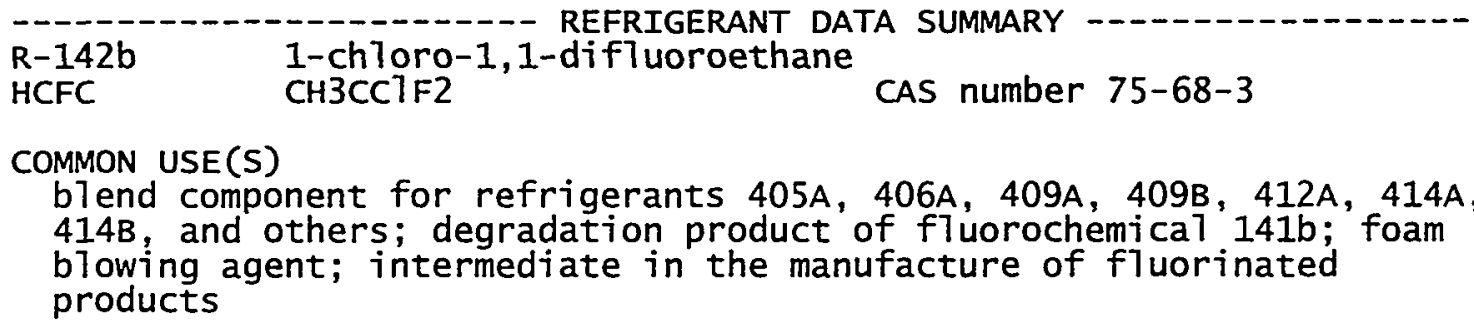

\section{IDENTIFIERS}

common name(s): R-142b; R142b; R 142b

HCFC-142b

CDFE

fluorocarbon $142 b$

fluorochemical $142 b$; FC $142 b$

halocarbon $142 b$

halochemical 142b

chemical name (by IUPAC convention): alternative chemical names/formulae:

empirical formula:

1-chloro-1,1-difluoroethane

2-ch7oro-2,2-diffuoroethane ethane, 1-chloro-1,1-difluorodifluorochloroethane

diffuoromonochloroethane alphachloroethylidene fluoride CH3CC1F2; $\mathrm{CH} 3-\mathrm{CC} 7 \mathrm{~F} 2$ not recommended:

CCIF2CH3; CC7F2-CH3

$\mathrm{CF} 2 \mathrm{C} 7 \mathrm{CH} 3$; $\mathrm{CF} 2 \mathrm{Cl}-\mathrm{CH} 3$

$\mathrm{CH} 3 \mathrm{CF}_{2} \mathrm{C} 7$; $\mathrm{CH} 3-\mathrm{CF} 2 \mathrm{Cl}$

CAS number: 75-68-3 (Chemical Abstracts Service registry number)

Beilstein registry number: 1731584

EINECS number: 200-891-8 (European Inventory of Existing Chemical Substances)

NIOSH RTECS number: KH7650000 (Registry of ToxiC Effects of Chemical Substances)

trade name(s): Asahi Glass AsahikTin AK-142b

Ausimont Meforex(R) $142 b$

Daikin Daiflon(R) $142 \mathrm{~b}$

DuPont HCFC-142b

Elf Atochem Forane(R) 142b Honeywe 77 Genetron(R) 142b

historical name(s): $\begin{aligned} & \text { Allied Corp refrigerant } 101 \\ & \text { Alliedsignal Genetron(R) } 142 \mathrm{~b}\end{aligned}$ DuPont Freon( $R$ ) $142 \mathrm{~b}$ Elf Atochem Isotron(R) 142b Pennwalt Isotron(R) $142 b$

ARI container color / Pantone number: none, use light green grey/413 6601 -. with red / 185 band

PHYSICAL

- properties

molar mass:

$100.4947264 \mathrm{~g} / \mathrm{mol} \quad(0.221553$

$1 \mathrm{~b} / \mathrm{mo} 1)$

$-131.1^{\circ} \mathrm{C}\left(-204.1^{\circ} \mathrm{F}\right)$

normal freezing/melting/triple point:

- normal boiling point 
density, saturated 7iquid: density, saturated vapor: specific volume, saturated liquid: specific volume, saturated vapor: heat of vaporization: velocity of sound, saturated Tiquid: velocity of sound, saturated vapor: viscosity, saturated Tiquid: viscosity, saturated vapor: thermal conductivity, liquid:

thermat conductivity, vapor:

- normat pressure, $20^{\circ} \mathrm{C}\left(68^{\circ} \mathrm{F}\right)$

- normal pressure, $21.1{ }^{\text {density, }} \mathrm{C}\left(70^{\circ} \mathrm{F}\right)$ vapor:

density, vapor:

pressure, saturated vapor: density, saturated liquid: density, saturated vapor: specific volume, saturated liquid: specific volume, saturated vapor: velocity of sound, saturated 1iquid: velocity of sound, saturated vapor: viscosity, saturated liquid: viscosity, saturated vapor: thermal conductivity, saturatd liquid: thermal conductivity, saturated vapor: - $60^{\circ} \mathrm{C}\left(140^{\circ} \mathrm{F}\right)$ pressure, saturated vapor: - critical point heat of vaporization:

\section{temperature: \\ pressure: \\ density:}

specific volume:

ENVIRONMENTAL

average atmospheric lifetime (Tatm): average stratospheric Tifetime (Tstr): average tropospheric lifetime (Ttrop): ODP (ozone depletion potential):

GWP (global warming potential): HGWP (ha7ocarbon GWP):

photochemical reactivity (grnd Teve1):
$1192 \mathrm{~kg} / \mathrm{m} 3 \quad(74.407 \mathrm{~b} / \mathrm{cf})$

8401

$4.85 \mathrm{~kg} / \mathrm{m} 3(0.303 \mathrm{~Tb} / \mathrm{cf})$
$0.839 \mathrm{~L} / \mathrm{kg}(0.0134 \mathrm{cf} / 7 \mathrm{~b})$

$206.4 \mathrm{~L} / \mathrm{kg}(3.3056 \mathrm{cf} / 7 \mathrm{~b})$

$222.7 \mathrm{~kJ} / \mathrm{kg}(95.8 \mathrm{Btu} / 7 \mathrm{~b})$

$769 \mathrm{~m} / \mathrm{s}(2523 \mathrm{ft} / \mathrm{s})$

$151 \mathrm{~m} / \mathrm{s}(497 \mathrm{ft} / \mathrm{s})$

$354 \mu \mathrm{Pa} \cdot \mathrm{s}(0.354 \mathrm{cp})$

$8.74 \mu \mathrm{Pa} \cdot \mathrm{s}(0.00874 \mathrm{cp})$

$0.0943 \mathrm{~W} / \mathrm{m} \cdot \mathrm{K}(0.0545$

$\mathrm{Btu} / \mathrm{hr} \cdot \mathrm{ft}^{\circ} \mathrm{F}$ )

$0.0093 \mathrm{~W} / \mathrm{m} \cdot \mathrm{K}(0.0054$

$\mathrm{Btu} / \mathrm{hr} \cdot \mathrm{ft}^{\circ} \mathrm{F}$ )

$4.303 \mathrm{~kg} / \mathrm{m} 3(0.2686 \mathrm{~Tb} / \mathrm{cf}) \quad 8401$

$4.285 \mathrm{~kg} / \mathrm{m} 3(0.2675 \mathrm{~Tb} / \mathrm{cf}) \quad 8401$

$288.0 \mathrm{kPa}$ (41.77 psia) 4801

$1125 \mathrm{~kg} / \mathrm{m} 3(70.22 \mathrm{7b} / \mathrm{cf}) \quad 8401$

$13.01 \mathrm{~kg} / \mathrm{m} 3(0.812 \mathrm{~Tb} / \mathrm{cf}) \quad 8401$

$0.889 \mathrm{~L} / \mathrm{kg}(0.0142 \mathrm{cf} / 7 \mathrm{~b}) \quad 8401$

$76.9 \mathrm{~L} / \mathrm{kg}(1.2314 \mathrm{cf} / 7 \mathrm{~b}) \quad 8401$

$653 \mathrm{~m} / \mathrm{s}(2142 \mathrm{ft} / \mathrm{s}) \quad 8401$

$153 \mathrm{~m} / \mathrm{s}(502 \mathrm{ft} / \mathrm{s}) \quad 8401$

$252 \mu \mathrm{Pa} \cdot \mathrm{s}(0.252 \mathrm{cp}) \quad 8401$

$9.7 \mu \mathrm{Pa} \cdot \mathrm{s}(0.0097 \mathrm{cp}) \quad 8401$

$0.0832 \mathrm{~W} / \mathrm{m} \bullet \mathrm{K}(0.0481 \quad 8401$

$\mathrm{Btu} / \mathrm{hr} \cdot \mathrm{ft}^{\circ} \mathrm{F}$ )

$0.01117 \mathrm{~W} / \mathrm{m} \cdot \mathrm{K}(0.00646 \quad 8401$

$\mathrm{Btu} / \mathrm{hr} \bullet \mathrm{ft}^{\circ} \mathrm{F}$ )

$879 \mathrm{kPa}$ (127.5 psia)

8401

$175.5 \mathrm{~kJ} / \mathrm{kg}(75.4 \mathrm{Btu} / 7 \mathrm{~b}) \quad 8401$

$137.1^{\circ} \mathrm{C}\left(278.8^{\circ} \mathrm{F}\right)$

$137.2^{\circ} \mathrm{C}\left(278.9^{\circ} \mathrm{F}\right)$

$4048 \mathrm{kPa}(587.1 \mathrm{psia})$

$4123 \mathrm{kPa}(598.0 \mathrm{psia})$

$435 \mathrm{~kg} / \mathrm{m} 3(27.2 \mathrm{lb} / \mathrm{cf})$

$444 \mathrm{~kg} / \mathrm{m} 3(27.7 \mathrm{lb} / \mathrm{cf})$

$2.21 \mathrm{~L} / \mathrm{kg}(0.0354 \mathrm{cf} / \mathrm{jb}) \quad 8401$

$2.25 \mathrm{~L} / \mathrm{kg}(0.0361 \mathrm{cf} / 7 \mathrm{~b}) \quad 8322$

8401

8322

8322

8401

8401

8401

$18.5 \mathrm{yr} \quad 9501$

$220 \mathrm{yr} \quad 5508$

$24.8 \mathrm{yr} 9 \mathrm{~B} 04$

0.043 (model-derived relative 9501 to $\mathrm{R}$ 11)

0.066 (semi-empirical relative 9501 to $\mathrm{R}$ 11)

0.065 (estimate used for the 6904

Montreal Protoco 7 )

2300 relative to $\mathrm{CO} 2$ for 1009501

yr integration

0.36 relative to $R 11$ for

infinite integration period

$0.365 \pm 0.025$ relative to $R$ I1 5964

for infinite integration

period

0.43 relative to $\mathrm{R} 11$ for

infinite integration period

4136

0.5 relative to methane 
SAFETY

- classification ---

safety group (ASHRAE standard 34): NFPA 704 degrees of hazard $(H-F-R-S)$ :

A2

8601

Ausimont: $2-4-0 \quad$ MSDS

Honeywe 11: 2-2-0 MSDS

hea 7 th-flammabi 7 ity-reactivity

NPCA HMIS hazard ratings $(H-F \sim R)$ :

[-special]: 0=no, 4=severe

1-4-1

Honeywe 11: 1-2-0 MSDS

hea 7 th-flammabi 7 ity-reactivity

- occupational exposure Timit

$0=$ insignificant, $4=e x t r e m e$

MAK (maximum workplace concentration)

IV: $2,000 \mathrm{ppm} \mathrm{v/v} \mathrm{momentary} 607101$ $\min$

- Tong-term occupational Timit

AIHA WEEL (workplace envi exp 7 imit):

MAK (maximum workplace concentration):

- emergency exposure limit

1,000 ppm $v / v$ TWA for $8 \mathrm{hr} /$ day $4 \mathrm{~B} 89$ and $40 \mathrm{hr} / \mathrm{wk}$

$1,000 \mathrm{ppm} v / \mathrm{v}$ TWA for $8 \mathrm{hr} /$ day 7101 40 (or 42) $\mathrm{hr} / \mathrm{wk}$

Acute Toxicity Exposure Limit (ATEL):

Refrigerant concentration Limit (RCL):

25,000 ppm v/v (preliminary

$34 u$

based on ASHRAE $34 u$ draft)

25,000 ppm $v / v$ (preliminary

A950

based on ASHRAE $34 u$ draft)

A950

based on ASHRAE $34 u$ draft)

$17,000 \mathrm{ppm} \mathrm{v} / \mathrm{v}$ (preliminary

based on ASHRAE $34 \mathrm{u}$ draft)

AIHA ERPG-3 (1ife-threatening):

$25,000 \mathrm{ppm} \mathrm{v} / \mathrm{v}$ for $1 \mathrm{hr}$

$34 u$

$15,000 \mathrm{ppm} \mathrm{v} / \mathrm{v}$ for $1 \mathrm{hr}$

AIHA ERPG-2 (injurious or impairing):
AIHA ERPG-1 (odor or mild effects):

- acute (short-term) toxicity -------

LC50 (7etha] concentration, 50\%):

ALC (approximate lethal concentration:

$10,000 \mathrm{ppm} \mathrm{v} / \mathrm{v}$ for $1 \mathrm{hr}$

9503

9503

9503

rat, $2 \mathrm{hr}$ Elf Atochem: 368,000 MSDS ppm (fatal concentration by inhalation for half of test anima 15 )

rat, 4 hr, Honeywe 17: >128,000 MSDS ppm (fataj concentration by inhalation for half of test anima7s)

rat, $30 \mathrm{~min}, 1 / 1$ : $500,000 \mathrm{ppm} 5147$

( Towest exposure tested with

one or more deaths by

inhalation)

rat, $2 \mathrm{hr}, ? / ?:$ 400,000 ppm

(7owest exposure tested with

one or more deaths by

inhalation)

rat, $4 \mathrm{hr},(2-4) / 6: 128,000$

ppm (7owest exposure tested

with one or more deaths by

inhalation)

rat, $6 \mathrm{hr}, 20 \%$ : 400,000 ppm

(7owest exposure tested with

one or more deaths by

inhalation)

rat, 16 hr 0/10 day 1: 100,000

$\mathrm{A} 622$

ppm (lowest exposure tested

with one or more deaths by

inhalation)

cardiac sensitization (CS) EC50: dog, $10 \mathrm{~min}$ : 73,000 ppm v/v

dog, 10 min: $73,000 \mathrm{ppm} \mathrm{v} / \mathrm{v}$

5643

half of test animals) 
cardiac sensitization threshold/LOEL: dog, $5 \mathrm{~min}$, 5/12: 50,000 ppm

cardiac sensitization (CS) NOEL: dog, 5 min, 0/6: 25,000 ppm

anesthetic/CNS effect EC50:

anesthetic/CNS effect LOEL:

- f7ammability -.....-. LFL-UFL (flammability limits in air):

LFL-UFL (with spark ignition):

LFL-UFL (with hot-wire ignition): LFL (with hot-wire ignition): heat of combustion (by ASHRAE 34-92): flash point: autoignition temperature: autodecomposition temperature: - detection appearance: odor:

PRODUCTION last year production allowed: $v / v$ (lowest observed effect Teve 1 in test animals) dog, $5 \mathrm{~min}, 5 / 12$ : 50,000 ppm $v / v$ (lowest observed effect level in test animals) $v / v$ (no observed effect level in test animals)

dog, 5 min, 0/6: 25,000 ppm $v / v$ (no observed effect level in test anima7s)

mouse, $10 \mathrm{~min}: 250,000 \mathrm{ppm} \mathrm{v} / \mathrm{v} 5980$ (effective concentration in half of test animals)

5644

6274

5644

6274

5147 $v / v$ (lowest observed effect level in test animals)

\begin{tabular}{|c|c|}
\hline 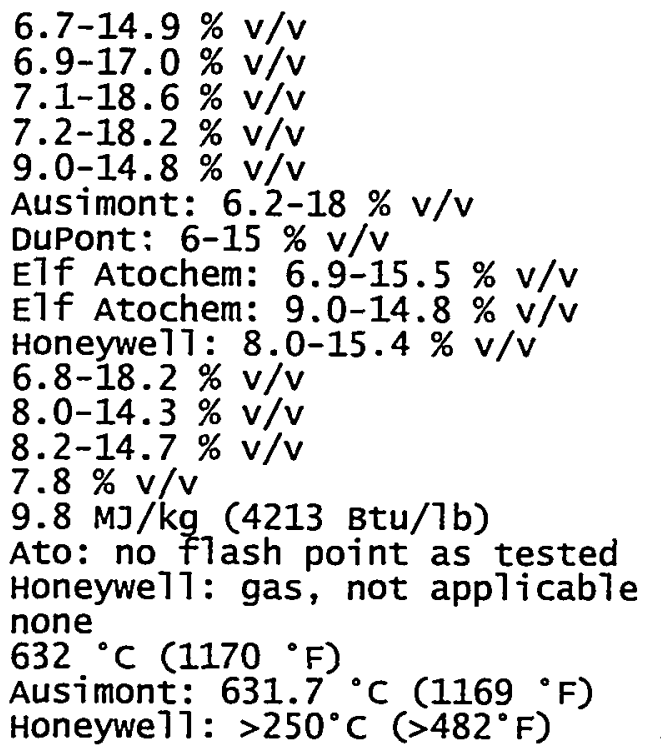 & $\begin{array}{l}3 A 58 \\
2525 \\
4136 \\
\text { MSDS } \\
\text { MSDS } \\
\text { MSDS } \\
\text { SDS } \\
\text { MSDS } \\
5648 \\
3 A 58 \\
3 A 58 \\
0525 \\
2318 \\
\text { SDS } \\
\text { MSDS } \\
3 A 58 \\
\text { 3A58 } \\
\text { MSDS } \\
\text { MSDS }\end{array}$ \\
\hline $5 / 7 g r$ & \\
\hline
\end{tabular}

$8 C 01$ 


\section{R-143a}

R-143a CHC
COMMON USE(S)
blend component particularly for a7ternatives to refrigerant 502
including refrigerants 404A, 408A, 507A, and others; degradation
product of fluorochemica7 141b

IDENTIFIERS

common name(s): R-143a; R143a; R 143a

HFC-143a

f7uorocarbon $143 a$

fluorochemica1 143a; FC 143a

halocarbon $143 a$

halochemical 143a

hydrofluorocarbon $143 a$

chemical name (by IUPAC convention): not recommended: HFA-143a

alternative chemical names/formulae:

empirical formula:

1,1,1-trifiuoroethane

ethane, 1,1,1-trifluoro-

methy 1 fluoroform

CH3CF3; CH3-CF3

not recommended:

$\mathrm{CF} 3 \mathrm{CH} 3 ; \mathrm{CF}_{3}-\mathrm{CH} 3$

$\mathrm{F} 3 \mathrm{CCH} 3 ; \mathrm{F} 3 \mathrm{C}-\mathrm{CH} 3$

$\mathrm{H} 3 \mathrm{CCF} 3 ; \mathrm{H} 3 \mathrm{C}-\mathrm{CF} 3$

CAS number: 420-46-2 (Chemical Abstracts

420-46-2 (Chemical Abstrac

EINECS number: 206-996-5 (European Inventory

of Existing Chemical

Substances)

NIOSH RTECS number: KJ4110000 (Registry of Toxic

Effects of Chemical

Substances)

trade name(s): Ausimont Meforex(R) 143a

Daikin fluorocarbon HFC-143a

DuPont HFC-143a

Elf Atochem Forane(R) $143 a$

Honeywe 11 Genetron(R) $143 a$

Solvay solkane(R) $143 a$

ZCIRI Kehua (PRC) HFC-143a

historical name(s): Allied corp refrigerant $200 \quad 3975$

MSDS

MSDS

MSDS

MSDS

MSDS

MSDS

A 71 iedsignal Genetron(R) 143a MSDS

ARI container color / Pantone number: ICI Arcton(R) $143 a$

none, use light green grey/413 6601 with red / 185 band

PHYSICAL

- properties

molar mass:

$84.0404296 \mathrm{~g} / \mathrm{mol}(0.185277$

8820

$\mathrm{lb} / \mathrm{mol})$

normal freezing/me]ting/triple point:

$-111.8^{\circ} \mathrm{C}\left(-169.3^{\circ} \mathrm{F}\right)$

8401

- normal boiting point -rore

density, saturated 7iquid:

density, saturated vapor:

specific volume, saturated liquid:

$-47.2{ }^{\circ} \mathrm{C}\left(-53.0^{\circ} \mathrm{F}\right)$

8401

$1168 \mathrm{~kg} / \mathrm{m} 3(72.90 \mathrm{lb} / \mathrm{cf})$

$4.76 \mathrm{~kg} / \mathrm{m} 3(0.297 \mathrm{lb} / \mathrm{cf})$

8401

$0.856 \mathrm{~L} / \mathrm{kg}(0.0137 \mathrm{cf} / 7 \mathrm{~b})$

$210.1 \mathrm{~L} / \mathrm{kg}(3.3652 \mathrm{cf} / 7 \mathrm{~b})$

8401

specific volume, saturated vapor:

heat of vaporization:

$227.1 \mathrm{~kJ} / \mathrm{kg}$ (97.6 Btu/7b)

8401

8401 


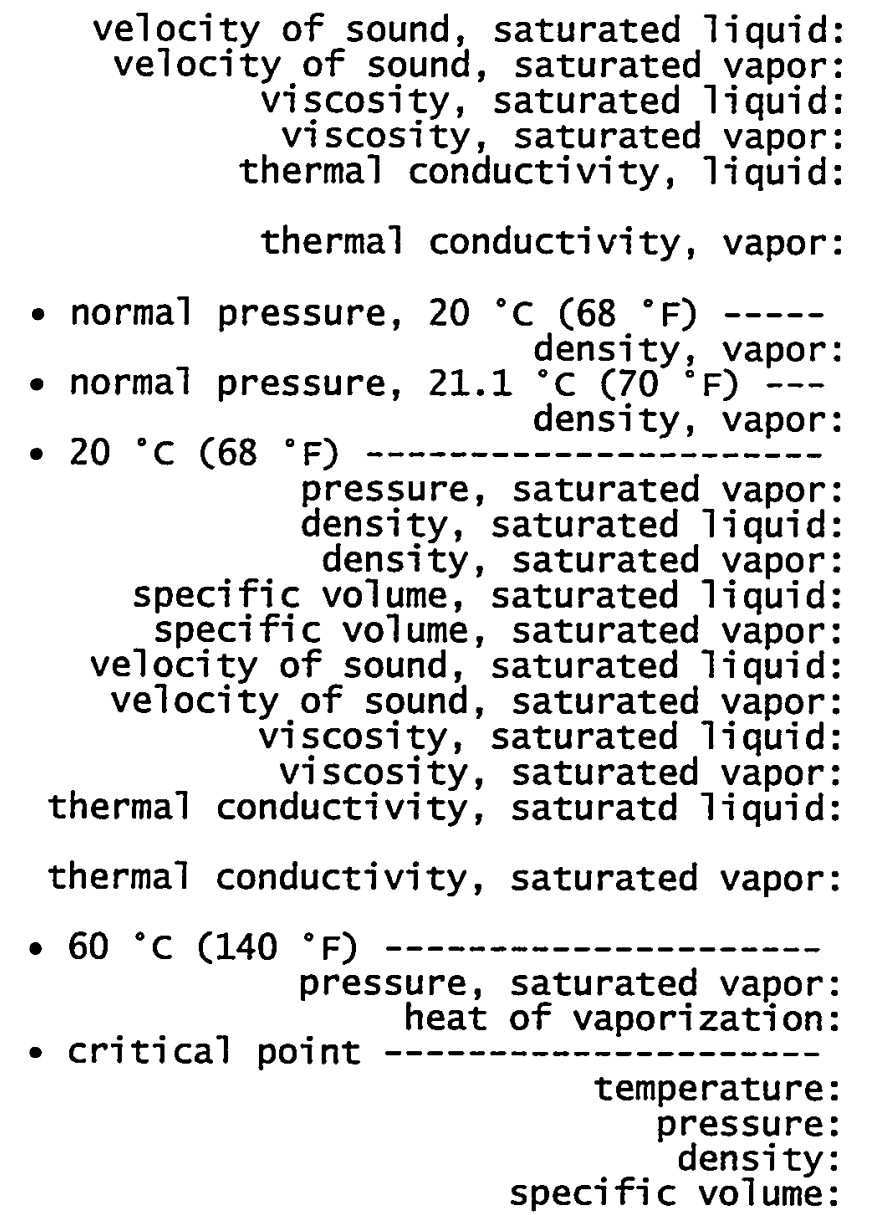

ENVIRONMENTAL

average atmospheric lifetime (Tatm): ODP (ozone depletion potential):

GWP (global warming potential): HGWP (ha7ocarbon GWP):

photochemical reactivity (grnd level):

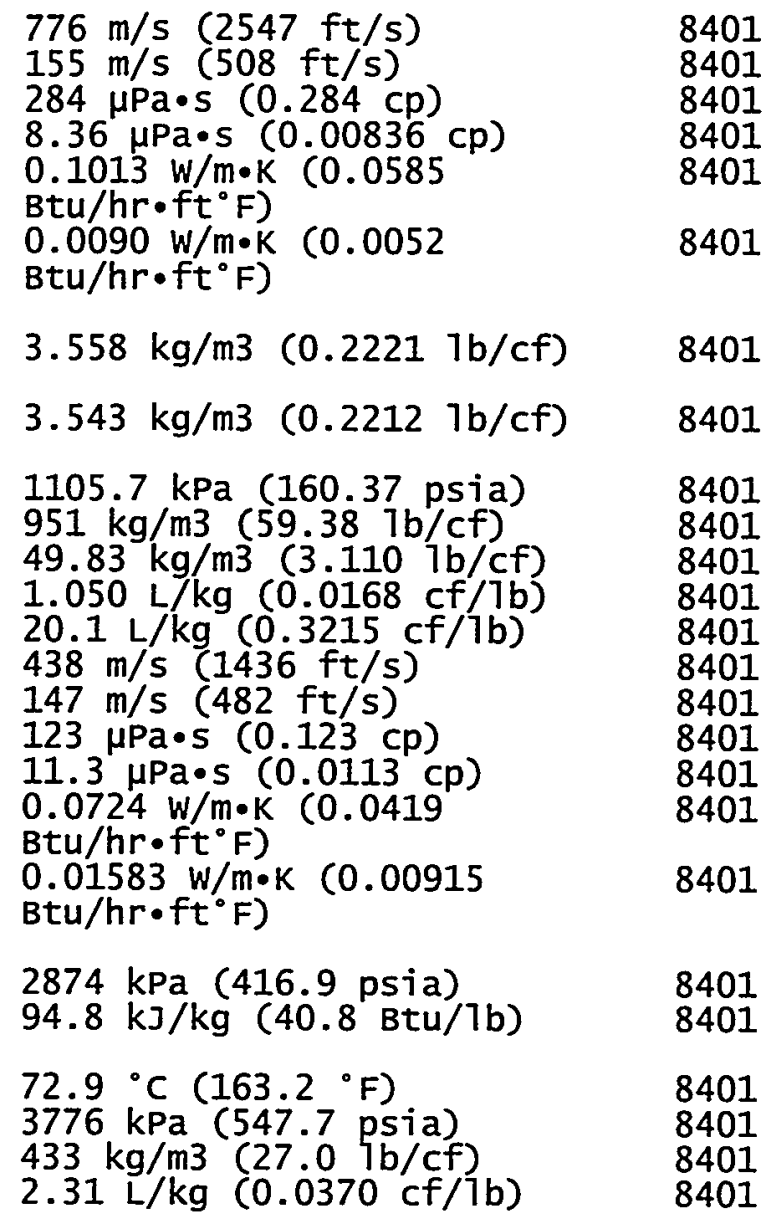

$53.5 \mathrm{yr}$ 0.000 (mode1-derived relative $3 \mathrm{~B} 1.2$ to $R$ 11)

5400 relative to CO2 for 1009501 yr integration

$0.74 \pm 0.02$ relative to $R 11 \quad 5964$

for infinite integration period

0.97 relative to $R 11$ for infinite integration period 1.I relative to $R 11$ for infinite integration period 0.2 relative to methane

DW

4511

4511

\section{SAFETY}

- classification

safety group (ASHRAE Standard 34 ): NFPA 704 degrees of hazard $(H-F-R-S)$ :

NPCA HMIS hazard ratings $(H-F-R)$ :

- Tong-term occupational limit ------AIHA WEEL (workplace envi exp 1imit):
A2

Ausimont: $2-4-0$ MSDS hea th-flammability-reactivity [-special]: $0=$ no, $4=$ severe Honeywe 11: 1-2-0 MSDS health-flammability-reactivity $0=$ insignificant, $4=e x t r e m e$

$1,000 \mathrm{ppm} v / \mathrm{v}$ TWA for $8 \mathrm{hr} /$ day $5 \mathrm{Cl} 6$ and $40 \mathrm{hr} / \mathrm{wk}$ Honeywe 11: 2-2-0 MSDS DuPont: 1-4-0 
- emergency exposure Timit -...-..--

Acute Toxicity Exposure Limit (ATEL): $170,000 \mathrm{ppm} \mathrm{v/v} \mathrm{(preliminary}$

$34 \mathrm{u}$

based on ASHRAE $34 \mathrm{u}$ draft)

$170,000 \mathrm{ppm} \mathrm{V} / \mathrm{v}$ (preliminary

based on ASHRAE $34 \mathrm{u}$ draft)

A950

Refrigerant concentration Limit (RCL): $18,000 \mathrm{ppm} \mathrm{V} / \mathrm{V}$ (preliminary

based on ASHRAE $34 \mathrm{u}$ draft)

$18,000 \mathrm{ppm} v / \mathrm{V}$ (preliminary

A950

acute (short-term) toxicity

based on ASHRAE $34 \mathrm{u}$ draft)

ALC (approximate lethal concentration:

rat, $4 \mathrm{hr}, 0 / 10:>591,000 \mathrm{ppm}$

7450

(lowest exposure tested with one or more deaths by

cardiac sensitization (CS) EC50: inhalation)

dog, $10 \mathrm{~min}: 310,000 \mathrm{ppm} \mathrm{v} / \mathrm{v}$

(effective concentration in

half of test animals)

cardiac sensitization threshold/LOEL: dog, $10 \mathrm{~min}, 2 / 5: 300,000 \mathrm{ppm}$

$\mathrm{v} / \mathrm{V}$ ( lowest observed effect level in test anima 75 )

cardiac sensitization (CS) NOEL: dog, $10 \mathrm{~min}, 0 / 6: 250,000 \mathrm{ppm}$

$\mathrm{v} / \mathrm{v}$ (no observed effect level in test animals)

anesthetic/CNS effect EC50:

mouse, 10 min: $500,000-600,000$

6684

$\mathrm{ppm} \mathrm{v} / \mathrm{v}$ (effective

concentration in half of test anima 75 )

anesthetic/CNS effect NOEL: $\mathrm{rat}, 4 \mathrm{hr}, 0 / 10: 591,000 \mathrm{ppm}$

- flammabiTity

$\checkmark / v$ (no observed effect level in ALC or LC50 studies)

LFL-UFL (flammability limits in air):

$13-17.4 \% \mathrm{v} / \mathrm{v}$

$7.0-19.0 \% \mathrm{v} / \mathrm{v}$

$7.4-18.8 \% \mathrm{v} / \mathrm{v}$

DuPont: $7.7-17.4 \% \mathrm{v} / \mathrm{v}$

Elf Atochem: $7.1-16.1 \% \mathrm{v} / \mathrm{v}$

Elf Atochem: 7.1-16.1\% v/v

Solvay: $7.1-16.1 \% \mathrm{v} / \mathrm{v}$

heat of combustion (by ASHRAE 34-92):

flash point:

autoignition temperature:

$10.3 \mathrm{MJ} / \mathrm{kg}$ (4428 Btu/7b)

$5 C 26$

6597

5980

DuPont: $-90{ }^{\circ} \mathrm{C}\left(-130^{\circ} \mathrm{F}\right)$ Honeywe 11: gas, not applicable MSDS

no flash point (as tested) SDS

Elf Atochem: $750^{\circ} \mathrm{C}\left(1382^{\circ} \mathrm{F}\right)$ SDS

- detection

Honeywe11: $>750^{\circ} \mathrm{C}\left(>1382^{\circ} \mathrm{F}\right)$

MSDS

appearance: DuPont: clear, colorless

odor: Daikin: faint sweet odor

Honeywe 11: faint etherea]

MSDS

MSDS

MSDS

PRODUCTION

last year production allowed: unrestricted

$8 \mathrm{COI}$ 


\section{R-152a}

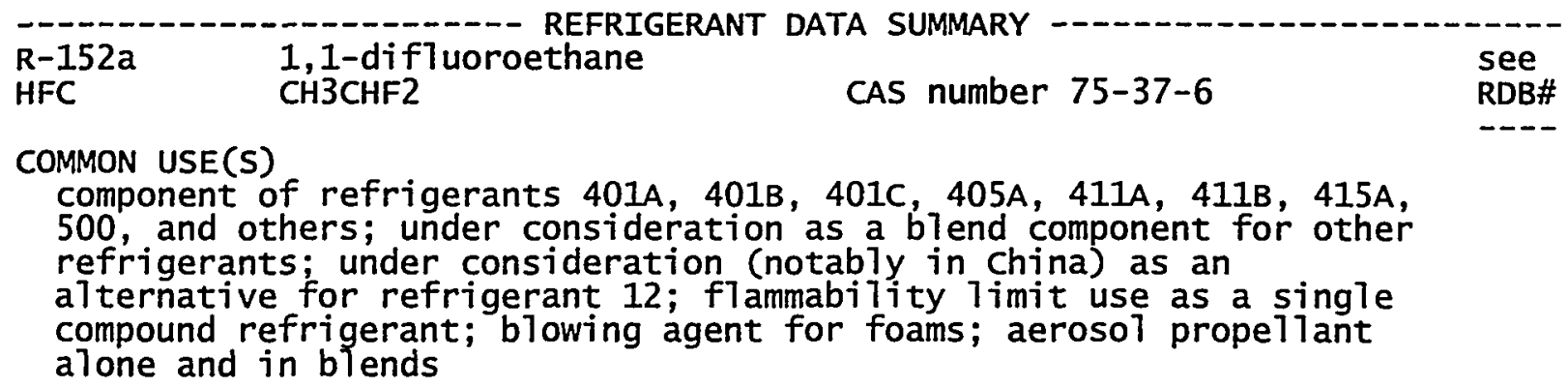

IDENTIFIERS

common name(s): $\begin{aligned} & \text { R-152a; R152a; R 152a } \\ & \text { HFC-152a }\end{aligned}$

DFE

f7uorocarbon $152 \mathrm{a}$

fluorochemica] 152a; FC 152a

halocarbon $152 \mathrm{a}$

halochemical 152a

hydrofluorocarbon $152 a$

chemical name (by IUPAC convention):

1,1-difluoroethane

alternative chemical names/formulae:

empirical formula:

ethane, 1,1-difluoro-

ethylidene difluoride

ethylidene fluoride

"ethylene fluoride"

CH3CHF2; $\mathrm{CH} 3-\mathrm{CHF} 2$

not recommended:

$\mathrm{CF} 2 \mathrm{HCH} 3$; $\mathrm{CF} 2 \mathrm{H}-\mathrm{CH} 3$

$\mathrm{CH} 3 \mathrm{CF} 2 \mathrm{H}$; $\mathrm{CH} 3-\mathrm{CF} 2 \mathrm{H}$

$\mathrm{CHF} 2 \mathrm{CH} 3$; $\mathrm{CHF} 2-\mathrm{CH} 3$

H3CCHF2; H3C-CHF2

CAS number: 75-37-6 (Chemical Abstracts

75-37-6 (Chemical Abstract

EINECS number: 200-866-1 (European Inventory

of Existing Chemical

Substances)

NIOSH RTECS number: KII410000 (Registry of Toxic

Effects of Chemical

Substances)

trade name(s): Asahi Glass Asahiklin AK-152a

DuPont HFC-152a

Elf Atochem Forane(R) 152a MSDS

Honeywe 71 Genetron(R) 152a MSDS

ICI Klea(R) $152 \mathrm{a}$

ZCIRI Kehua (PRC) HFC-152a

CSDS

historical name(s): Al7jed Corp refrigerant 100

A7liedsignal Genetron(R)

DuPont FCD-711

DuPont Freon(R) 152a

ARI container color / Pantone number: none, use light green grey/413 6601 with red/ 185 band

PHYSICAL

- properties

molar mass:

$66.0499664 \mathrm{~g} / \mathrm{mol} \quad(0.145615$

8820

1b/mol)

normal freezing/me7ting/triple point:

$-118.6^{\circ} \mathrm{C}\left(-181.5^{\circ} \mathrm{F}\right)$

8401 
- normal boiling point

temperature: density, saturated liquid: density, saturated vapor: specific volume, saturated 7iquid: specific volume, saturated vapor: heat of vaporization:

velocity of sound, saturated Tiquid: velocity of sound, saturated vapor: viscosity, saturated 7iquid: viscosity, saturated vapor: thermal conductivity, 7 iquid:

thermal conductivity, vapor:

- normal pressure, $20{ }^{\circ} \mathrm{C}\left(68^{\circ} \mathrm{F}\right)$-.---

- normal pressure, $21.1{ }^{\circ} \mathrm{C}\left(70^{\circ} \mathrm{F}\right)$---

- 20 density, vapor pressure, saturated vapor: density, saturated liquid: density, saturated vapor:

specific volume, saturated liquid: specific volume, saturated vapor: velocity of sound, saturated liquid: velocity of sound, saturated vapor: viscosity, saturated liquid: viscosity, saturated vapor: thermal conductivity, saturatd liquid: thermal conductivity, saturated vapor:

- $60{ }^{\circ} \mathrm{C}\left(140{ }^{\circ} \mathrm{F}\right)$-..... pressure, saturated vapor:

- critical point heat of vaporization: temperature:

\section{pressure: \\ density: \\ specific volume:}

ENVIRONMENTAL

average atmospheric lifetime (Tatm): average stratospheric lifetime (TStr): ODP (ozone depletion potential):

GWP (global warming potential): HGWP (halocarbon GWP):

photochemical reactivity (grnd level):

SAFETY

- classification

safety group (ASHRAE standard 34): NFPA 704 degrees of hazard (H-F-R-S):

NPCA HMIS hazard ratings $(H-F-R)$ :
$-24.0{ }^{\circ} \mathrm{C}\left(-11.2^{\circ} \mathrm{F}\right) \quad 8401$

$1011 \mathrm{~kg} / \mathrm{m} 3(63.13 \mathrm{lb} / \mathrm{cf}) \quad 8401$

$3.38 \mathrm{~kg} / \mathrm{m} 3(0.211 \mathrm{lb} / \mathrm{cf}) \quad 8401$

$0.989 \mathrm{~L} / \mathrm{kg}(0.0158 \mathrm{cf} / 7 \mathrm{~b}) \quad 8401$

$296.2 \mathrm{~L} / \mathrm{kg}(4.7450 \mathrm{cf} / 7 \mathrm{~b}) \quad 8401$

$329.9 \mathrm{~kJ} / \mathrm{kg}(141.8 \mathrm{Btu} / 7 \mathrm{~b}) \quad 8401$

$889 \mathrm{~m} / \mathrm{s}(2918 \mathrm{ft} / \mathrm{s})$

$185 \mathrm{~m} / \mathrm{s}(608 \mathrm{ft} / \mathrm{s})$

$301 \mu \mathrm{Pa} \cdot \mathrm{s}(0.301 \mathrm{cp})$

$8.44 \mu \mathrm{Pa} \cdot \mathrm{s}(0.00844 \mathrm{cp})$

$0.1202 \mathrm{~W} / \mathrm{m} \cdot \mathrm{K}(0.0694$

$\mathrm{Btu} / \mathrm{hr} \cdot \mathrm{ft}^{\circ} \mathrm{F}$ )

$0.0094 \mathrm{~W} / \mathrm{m} \cdot \mathrm{K}(0.0054$

$\left.\mathrm{Btu} / \mathrm{hr} \bullet \mathrm{ft}^{\circ} \mathrm{F}\right)$

8401

8401

8401

8401

8401

8401

$2.810 \mathrm{~kg} / \mathrm{m} 3(0.1754 \mathrm{lb} / \mathrm{cf}) \quad 8401$

$2.799 \mathrm{~kg} / \mathrm{m} 3(0.1747 \mathrm{lb} / \mathrm{cf}) \quad 8401$

$512.9 \mathrm{kPa}$ (74.39 psia) 8401

$912 \mathrm{~kg} / \mathrm{m3}$ (56.93 lb/cf) 8401

$15.91 \mathrm{~kg} / \mathrm{m} 3(0.993 \mathrm{7b} / \mathrm{cf}) \quad 8401$

$1.097 \mathrm{~L} / \mathrm{kg}(0.0176 \mathrm{cf} / \mathrm{/b}) \quad 8401$

$62.9 \mathrm{~L} / \mathrm{kg}(1.0068 \mathrm{cf} / 7 \mathrm{~b}) \quad 8401$

$673 \mathrm{~m} / \mathrm{s}(2208 \mathrm{ft} / \mathrm{s})$

$186 \mathrm{~m} / \mathrm{s}(611 \mathrm{ft} / \mathrm{s})$

$172 \mu \mathrm{Pa} \cdot \mathrm{s}(0.172 \mathrm{cp})$

$10.1 \mu \mathrm{Pa} \cdot \mathrm{s}(0.0101 \mathrm{cp})$

$0.1002 \mathrm{~W} / \mathrm{m} \cdot \mathrm{K}(0.0579$

$\mathrm{Btu} / \mathrm{hr} \cdot \mathrm{ft}^{\circ} \mathrm{F}$ )

$0.01424 \mathrm{~W} / \mathrm{m} \cdot \mathrm{K} \quad(0.00823$

$\mathrm{Btu} / \mathrm{hr} \bullet \mathrm{ft}^{\circ} \mathrm{F}$ )

8401

8401

8401

8401

8401

8401

$1501 \mathrm{kPa}$ (217.7 psia) 8401

$229.4 \mathrm{~kJ} / \mathrm{kg}(98.6 \mathrm{Btu} / \mathrm{7b}) \quad 8401$

$113.2^{\circ} \mathrm{C}\left(235.7^{\circ} \mathrm{F}\right) \quad 9125$

$113.3^{\circ} \mathrm{C}\left(235.9^{\circ} \mathrm{F}\right) \quad 4121$

$4515 \mathrm{kPa}$ (654.8 psia) 4121

$4517 \mathrm{kPa}$ (655.1 psia) 4B09

$368 \mathrm{~kg} / \mathrm{m} 3$ (23.0 ib/cf) 4B09

$369 \mathrm{~kg} / \mathrm{m} 3(23.07 \mathrm{~b} / \mathrm{cf}) \quad 9125$

$2.72 \mathrm{~L} / \mathrm{kg}(0.0435 \mathrm{cf} / \mathrm{lb}) \quad 4 \mathrm{~B} 09$

$1.5 \pm 0.4 \mathrm{yr} \quad 6695$

$61 \mathrm{yr} \quad 5508$

0.000 (model-derived relative $3 \mathrm{~B} 12$ to $\mathrm{R} 11$ )

190 relative to $\mathrm{CO} 2$ for $100 \mathrm{yr} 9501$ integration

$0.0295 \pm 0.0035$ relative to $R 5964$

11 for infinite integration

period

4.8 relative to methane

4511

A2

hea th-flammability-reactivity

[-special]: $0=$ no, $4=$ severe

DuPont: $1-4-1$

MSDS 
- 1ong-term occupational 1imit ------AIHA WEEL (workplace envi exp 1imit):

- emergency exposure limit Acute Toxicity Exposure Limit (ATEL):

Refrigerant Concentration Limit ( $R C L)$ :

- acute (short-term) toxicity ------LC50 (1etha 7 concentration, 50\%):

ALC (approximate lethal concentration:

ora7 LD50 (7ethaT dosage, 50\%):

cardiac sensitization threshold/LOEL:

cardiac sensitization (CS) NOEL:

anesthetic/CNS effect LOEL:
Honeywe $77:$ 1-2-0

health-fiammability-reactivity

$0=$ insignificant, $4=e x t r e m e$

MSDS

$1,000 \mathrm{ppm} v / \mathrm{v}$ TWA for $8 \mathrm{hr} /$ day 4890 and $40 \mathrm{hr} / \mathrm{wk}$

$50,000 \mathrm{ppm} \mathrm{v/v} \mathrm{(preliminary}$ based on ASHRAE $34 \mathrm{u}$ draft)

$50,000 \mathrm{ppm} v / \mathrm{V}$ (preliminary based on ASHRAE $34 \mathrm{u}$ draft)

9,300 ppm V/v (preTiminary

based on ASHRAE $34 \mathrm{u}$ draft)

9,300 ppm v/v (preliminary

based on ASHRAE $34 \mathrm{u}$ draft)

$34 u$

A950

$34 \mathrm{u}$

A950

rat, $4 \mathrm{hr}:>400,000 \mathrm{ppm}$ (fatal A648

concentration by inhalation

for half of test animals)

rat, 10-25min, 1/1: 500,000

ppm (lowest exposure tested

with one or more deaths by

inhalation)

rat, 10-25min, 1/1: 550,000

$\mathrm{ppm}$ (7)

with one or more deaths by

inhalation)

rat, 10-25min: 500,000-550,000

7424

ppm (lowest exposure tested

with one or more deaths by

inhalation)

rat, $2 \mathrm{hr}, 0 / 2$ : 200,000 ppm

(7owest exposure tested with

7737

one or more deaths by

inhalation)

rat, $4 \mathrm{hr}, 1 / 6$ : $383,000 \mathrm{ppm}$

(7owest exposure tested with

one or more deaths by

inhalation)

rat, $4 \mathrm{hr}, 2-4 / 6$ : 64,000 ppm

(lowest exposure tested with one or more deaths by

inhalation)

rat, $4 \mathrm{hr}, 2 / 6: 437,500 \mathrm{ppm}$

(lowest exposure tested with one or more deaths by

inhalation)

rat: $>1,500 \mathrm{mg} / \mathrm{kg}$ (fata) dose by ingestion for half of test animais)

dog, $5 \mathrm{~min}, 3 / 12: 150,000 \mathrm{ppm}$

$v / v$ (lowest observed effect

Teve 7 in test animals)

dog, 5 min, 3/12: 150,000 ppm

$v / v$ (Towest observed effect

leve 1 in test anima $7 \mathrm{~s}$ )

dog, 5 min, 0/12: 50,000 ppm

$v / v$ (no observed effect level

in test animals)

dog, 5 min, 0/12: 50,000 ppm

$v / V$ (no observed effect level

$5 c 85$

in test animals)

5643

$5 C 85$

$5 C 84$

5644

6274

5644

6274

rat, 30 min, 1/1: 200,000 ppm 5147

$v / v$ (lowest observed effect 
Tevel in test anima $1 \mathrm{~s}$ )

rat, $2 \mathrm{hr}$, ?/2: $100,000 \mathrm{ppm}$

$v / v$ (lowest observed effect

level in ALC or LC50 studies)

anesthetic/CNS effect NOEL:

rat, 30 min, 0/1: 100,000 ppm 5147

$v / v$ (no observed effect leve 1

in test animals)

- flammability

LFL-UFL (flammability limits in air):

LFL-UFL (flammability Timits, $\left.95{ }^{\circ} \mathrm{C}\right):$
LFL-UFL (with spark ignition):

$3.9-16.9 \% \mathrm{v} / \mathrm{v}$

$4.5-19.8 \% \mathrm{v} / \mathrm{v}$

4511

$4.8-20.2 \% \mathrm{v} / \mathrm{v}$

Daikin: $4.0-19.6 \% \mathrm{v} / \mathrm{v}$

E]f Atochem: $3.7-16.9 \% \mathrm{v} / \mathrm{v}$

Elf Atochem: 3.7-18.0\% v/V Honeywe 17: $3.7-18.0 \% \mathrm{v} / \mathrm{V}$

LFL-UFL (with hot-wire ignition):

heat of combustion (by ASHRAE 34-92):

flash point:

autoignition temperature:

autodecomposition temperature:

- detection

appearance: colorless gas

odor: slight ethereal odor

(0. $4 \%$ v $/ v$

$4.0-19.6 \% \mathrm{v} / \mathrm{v}$

$4.5-19.8 \% \mathrm{v} / \mathrm{v}$

$4.8-18.8 \% \mathrm{v} / \mathrm{V}$

$16.9 \mathrm{MJ} / \mathrm{kg}$ (7266 Btu/7b)

$17.4 \mathrm{MJ} / \mathrm{kg}$ (7472 Btu/7b)

Honeywe 17: not applicable

TOC: $<-50^{\circ} \mathrm{C}\left(<-58^{\circ} \mathrm{F}\right)$

$455^{\circ} \mathrm{C}\left(851^{\circ} \mathrm{F}\right)$

DuPont: $454{ }^{\circ} \mathrm{C}\left(849^{\circ} \mathrm{F}\right)$

4785

2525

MSDS

MSDS

SDS

MSDS

2525

5648

2525

2525

2318

4785

MSDS

4B90

3960

MSDS

$4 \mathrm{~B} 90$

$4 B 90$

PRODUCTION

7ast year production allowed: unrestricted

$8 C 01$ 


\section{R-170 (ETHANE)}

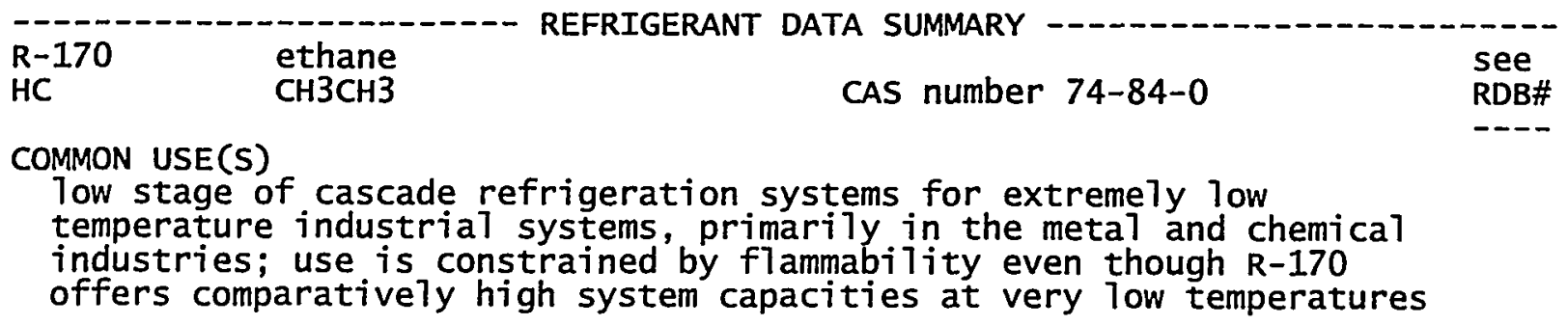

IDENTIFIERS

common name(s): $\mathrm{R}-170 ; \mathrm{R} 170 ; \mathrm{R} 170$

$\mathrm{HC}-170$

bimethy 7

dimethy 1

hydrocarbon 170

chemical name (by IUPAC convention): $\mathrm{n}-\mathrm{C} 2 ; \mathrm{n}-\mathrm{C} 2 \mathrm{H} 6$

alternative chemical names/formulae:

ethane

methy 7 methane

methy Imethane

ethyl hydride

$\mathrm{CH} 3 \mathrm{CH} 3 ; \mathrm{CH} 3-\mathrm{CH} 3$

empirical formula: $\mathrm{C} 2 \mathrm{H} 6$

not recommended: $(\mathrm{CH} 3) 2$

CAS number: 74-84-0 (Chemical Abstracts

Beilstein registry number: 1730716

Service registry number)

EINECS number:

Merck Index (volume-number):

200-814-8 (European Inventory

of Existing Chemical

Substances)

NIOSH RTECS number: KH3800000 (Registry of ToxiC

Effects of Chemical

Substances)

ARI container color / Pantone number:

none, use 7ight green grey/413 6601

with red / 185 band

PHYSICAL

- properties

molar mass:

normal freezing/melting/triple point:

- normal boiling point -rorature

density, saturated 7iquid: density, saturated vapor:

specific volume, saturated Tiquid: specific volume, saturated vapor:

heat of vaporization:

velocity of sound, saturated 1iquid:

velocity of sound, saturated vapor: viscosity, saturated 7iquid: viscosity, saturated vapor: thermal conductivity, 1iquid:

thermal conductivity, vapor:

- normal pressure, $20{ }^{\circ} \mathrm{C}\left(68{ }^{\circ} \mathrm{F}\right)----$

$30.06904 \mathrm{~g} / \mathrm{mo} 7(0.066291$

8820

$1 \mathrm{~b} / \mathrm{mo} 1)$

$-182.8^{\circ} \mathrm{C}\left(-297.0^{\circ} \mathrm{F}\right) \quad 8401$

$-88.6^{\circ} \mathrm{C}\left(-127.5^{\circ} \mathrm{F}\right) \quad 8401$

$544 \mathrm{~kg} / \mathrm{m} 3(33.96 \mathrm{lb} / \mathrm{cf}) \quad 8401$

$2.05 \mathrm{~kg} / \mathrm{m} 3(0.128 \mathrm{7b} / \mathrm{cf}) \quad 8401$

$1.838 \mathrm{~L} / \mathrm{kg}(0.0294 \mathrm{cf} / \mathrm{lb}) \quad 8401$

$487.0 \mathrm{~L} / \mathrm{kg}(7.8007 \mathrm{cf} / 7 \mathrm{~b}) \quad 8401$

$489.5 \mathrm{~kJ} / \mathrm{kg}$ (210.4 Btu/7b) 8401

$1313 \mathrm{~m} / \mathrm{s}(4308 \mathrm{ft} / \mathrm{s}) \quad 8401$

$249 \mathrm{~m} / \mathrm{s}(815 \mathrm{ft} / \mathrm{s}) \quad 8401$

$162 \mu \mathrm{Pa} \cdot \mathrm{s}(0.162 \mathrm{cp}) \quad 8401$

$5.86 \mu \mathrm{Pa} \cdot \mathrm{s}(0.00586 \mathrm{cp}) \quad 8401$

$0.1931 \mathrm{~W} / \mathrm{m} \cdot \mathrm{K}(0.1116 \quad 8401$

$\left.\mathrm{Btu} / \mathrm{hr} \bullet \mathrm{ft}^{\circ} \mathrm{F}\right)$

$0.0102 \mathrm{~W} / \mathrm{m} \cdot \mathrm{K}$ (0.0059

8401

$B t u / h r \cdot f t^{\circ} F$ )

$1.260 \mathrm{~kg} / \mathrm{m} 3(0.0787 \mathrm{lb} / \mathrm{cf})$

8401 
- normal pressure, $21.1{ }^{\circ} \mathrm{C}\left(70^{\circ} \mathrm{F}\right)$---

- $20^{\circ} \mathrm{C}\left(68^{\circ} \mathrm{F}\right)$ density, vapor

$1.255 \mathrm{~kg} / \mathrm{m} 3(0.0784 \mathrm{~Tb} / \mathrm{cf}) \quad 8401$

pressure, saturated vapor: density, saturated liquid: density, saturated vapor:

specific volume, saturated 7 iquid: specific volume, saturated vapor: velocity of sound, saturated 1iquid: velocity of sound, saturated vapor: viscosity, saturated 7iquid: viscosity, saturated vapor:

thermal conductivity, saturatd Tiquid:

thermal conductivity, saturated vapor:

$3766.0 \mathrm{kPa}(546.21 \mathrm{psia})$

8401

$339 \mathrm{~kg} / \mathrm{m3}$ (21.18 1b/cf) 8401

$86.39 \mathrm{~kg} / \mathrm{m} 3(5.393 \mathrm{~Tb} / \mathrm{cf}) \quad 8401$

$2.947 \mathrm{~L} / \mathrm{kg}(0.0472 \mathrm{cf} / \mathrm{lb}) \quad 8401$

$11.6 \mathrm{~L} / \mathrm{kg}(0.1854 \mathrm{cf} / 7 \mathrm{~b}) \quad 8401$

$371 \mathrm{~m} / \mathrm{s}(1219 \mathrm{ft} / \mathrm{s})$

$211 \mathrm{~m} / \mathrm{s}(693 \mathrm{ft} / \mathrm{s})$

$41 \mu \mathrm{Pa} \cdot \mathrm{S}(0.041 \mathrm{cp})$

$11.6 \mu \mathrm{Pa} \cdot \mathrm{s}(0.0116 \mathrm{cp})$

$0.0958 \mathrm{~W} / \mathrm{m} \cdot \mathrm{K}(0.0554$

$\mathrm{Btu} / \mathrm{hr} \cdot \mathrm{ft}^{\circ} \mathrm{F}$ )

$0.03795 \mathrm{~W} / \mathrm{m} \cdot \mathrm{K}(0.02193$

$\mathrm{Btu} / \mathrm{hr} \bullet \mathrm{ft}^{\circ} \mathrm{F}$ )

8401

8401

8401

8401

8401

- critical point

temperature:

pressure: density:

specific volume:

$32.2^{\circ} \mathrm{C}\left(89.9^{\circ} \mathrm{F}\right)$

$4872 \mathrm{kPa}(706.6 \mathrm{psia})$

$207 \mathrm{~kg} / \mathrm{m} 3(12.9 \mathrm{lb} / \mathrm{cf})$

8401

$4.84 \mathrm{~L} / \mathrm{kg}(0.0775 \mathrm{cf} / 7 \mathrm{~b})$

8401

8401

8401

8401

ENVIRONMENTAL

ODP (ozone depletion potential):

GWP (global warming potential):

photochemical reactivity (grnd level):

0.000 (model-derived relative

to $\mathrm{R}$ 11)

unknown, but very low: $\sim 20$

relative to $\mathrm{CO} 2$ for $100 \mathrm{yr}$

integration

SAFETY

- classification

safety group (ASHRAE Standard 34): NFPA 704 degrees of hazard (H-F-R-S):

NPCA HMIS hazard ratings $(H-F-R)$ :

37 relative to methane

4511

A3

BOC Gases: $0-4-0$

8601

Texaco: 1-4-0

MSDS

health-flammabi 1ity-reactivity

[-special]: $0=$ no, $4=$ severe

BOC Gases: $0-4-0$

Texaco: 1-4-0

health-flammabi 7ity-reactivity

$0=$ insignificant, $4=$ extreme

UL Comparative Hazard to Life Group:

5 (b) in absence of flame or hot objects

- occupationat exposure warnings --.-ACGIH caution:

- Tong-term occupational 7 imit -

simple asphyxiant

MSDS

exposure limit consistent to OSHA PEL:

- emergency exposure limit --- - - - - -

Acute Toxicity Exposure Limit (ATEL):

Refrigerant concentration Limit (RCL):

Exxon: 1,000 ppm $V / V$ TWA for 8 MSDS $\mathrm{hr} /$ day and $40 \mathrm{hr} / \mathrm{wk}$

Pennzoi 1, C1-C4 assumed: 1,000 MSDS $\mathrm{ppm} v / \mathrm{v}$ TWA for $8 \mathrm{hr} /$ day and $40 \mathrm{hr} / \mathrm{wk}$

Phillips: 1,000 ppm $v / v$ TWA MSDS

for $8 \mathrm{hr} /$ day and $40 \mathrm{hr} / \mathrm{wk}$

MSDS

MSDS

$7,000 \mathrm{ppm} \mathrm{v/v} \mathrm{(preliminary} 34 \mathrm{u}$

based on ASHRAE $34 \mathrm{u}$ draft)

provisional: 7,000 ppm $v / v$

(preliminary based on ASHRAE

$34 \mathrm{u}$ draft)

$7,000 \mathrm{ppm} v / \mathrm{v}$ (preliminary

based on ASHRAE $34 u$ draft)

provisiona $7: 7,000 \mathrm{ppm} v / \mathrm{V}$ (preliminary based on ASHRAE

$4 B 64$

$34 \mathrm{u}$ draft) 
- acute (short-term) toxicity

Alc (approximate 7 ethat concentration:

ALc (approximate lethal concentration.

cardiac sensitization threshold/LOEL:

rat, $4 \mathrm{hr}, 0 / 10:>24,838 \mathrm{ppm}$

7623

(lowest exposure tested with

one or more deaths by

inhalation)

dog 10min 2/4: 100,000-250,000 6192

ppm $v / v$ Clowest observed

effect level in test animals)

anesthetic/CNS effect NOEL: rat, $4 \mathrm{hr}, 0 / 10: 24,838 \mathrm{ppm}$

7623

$v / v$ (no observed effect tevel

in ALC or LC50 studies)

- flammabi 1 ity -

\begin{tabular}{|c|c|}
\hline $\begin{array}{l}3.2-12.45 \% \mathrm{v} / \mathrm{v} \\
3.3-10.6 \% \mathrm{v} / \mathrm{v} \\
\text { BOC Gases: } 3.0-12.4 \% \mathrm{v} / \mathrm{v} \\
\text { Exxon: } 3.0-12.5 \% \mathrm{v} / \mathrm{v} \\
\text { Phi17ips: } 2.9-13.0 \% \mathrm{v} / \mathrm{v} \\
\text { Texaco: } 2.9-13.0 \% \mathrm{v} / \mathrm{v} \\
\text { Union Carbide: } 3.2-12.5 \% \mathrm{v} / \mathrm{v} \\
2.6-13.9 \% \mathrm{v} / \mathrm{v} \\
-135{ }^{\circ} \mathrm{C}\left(-21{ }^{\circ} \mathrm{F}\right) \\
\text { CC, Exxon: }-152{ }^{\circ} \mathrm{C}\left(-242{ }^{\circ} \mathrm{F}\right) \\
510 \mathrm{C}\left(950^{\circ} \mathrm{F}\right) \\
\text { BOC Gases: } 472{ }^{\circ} \mathrm{C}\left(882{ }^{\circ} \mathrm{F}\right) \\
\text { Exxon: } 515{ }^{\circ} \mathrm{C}\left(959^{\circ} \mathrm{F}\right) \\
\text { Texaco: } 515{ }^{\circ} \mathrm{C}\left(959^{\circ} \mathrm{F}\right)\end{array}$ & $\begin{array}{l}6290 \\
0036 \\
\text { MSDS } \\
\text { MSDS } \\
\text { MSDS } \\
\text { MSDS } \\
\text { MSDS } \\
5648 \\
6290 \\
\text { MSDS } \\
4 \text { B64 } \\
\text { MSDS } \\
\text { MSDS } \\
\text { MSDS }\end{array}$ \\
\hline & \\
\hline
\end{tabular}

- detection

LFL-UFL (with spark ignition):

flash point:

autoignition temperature:

$510^{\circ} \mathrm{C}\left(950^{\circ} \mathrm{F}\right)$

Exxon: $515^{\circ} \mathrm{C}\left(959^{\circ} \mathrm{F}\right)$

$\begin{array}{rlr}\text { appearance: } & \text { Phil1ips: colorless } & \text { MSDS } \\ \text { odor: Union Carbide: odorless } & \text { MSDS }\end{array}$

PRODUCTION

last year production a77owed: unrestricted $8 \mathrm{C01}$ 


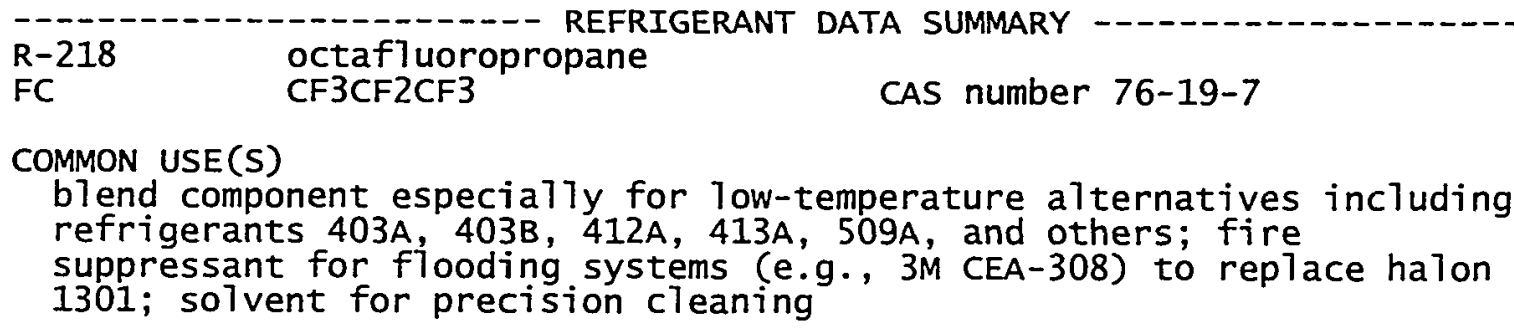

\section{IDENTIFIERS}

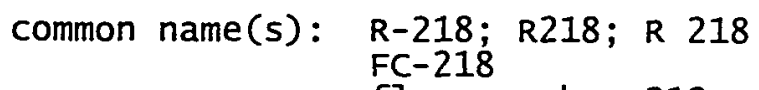

fTuorocarbon 218

fluorochemica7 218; FC 218

halocarbon 218

halochemical 218

chemical name (by IUPAC convention): alternative chemical names/formulae: perf]uorocarbon 218; "PFC-218" octaf7uoropropane perfluoropropane propane, octafluoroCF3CF2CF3; CF3-CF2-CF3 not recommended:

empirical formula: CF2(CF3) $2 ; \mathrm{F} 2 \mathrm{C}(\mathrm{CF} 3) 2$

CAS number: 76-19-7 (Chemical Abstracts 5ervice registry number) EINECS number: 200-941-9 (European Inventory of Existing Chemical Substances)

NIOSH RTECS number: TZ5255000 (Registry of Toxic Effects of Chemical Substances)

trade name(s): 3 M Performance Fluid PF-5030 Al 7 iedsigna 7 Genetron(R) 218 BNFL Flutec(R) 218

BOC Gases $\mathrm{H}-218$ BOC Gases Octaf7uoropropane DuPont Freon(R) 218

ICI PFC 218

Rhodia Isceon 218

historical name(s): Allied Corp refrigerant 700 Rhône-Poulenc Isceon 218

ARI container color / Pantone number: none, use light green grey/413 6601

PHYSICAL

- properties

molar mass:

$188.0193256 \mathrm{~g} / \mathrm{mol}(0.414512$

8820

normal freezing/melting/triple point:

$7 \mathrm{~b} / \mathrm{mo}$ )

- normal boiling point -density, saturated 7iquid: density, saturated vapor: specific volume, saturated 7iquid: specific volume, saturated vapor: heat of vaporization:

velocity of sound, saturated liquid:

$-160.1^{\circ} \mathrm{C}\left(-256.3^{\circ} \mathrm{F}\right)$

$-183.0^{\circ} \mathrm{C}\left(-297.4^{\circ} \mathrm{F}\right) \quad 3331$

$-36.6^{\circ} \mathrm{C}\left(-33.9^{\circ} \mathrm{F}\right)$

$1611 \mathrm{~kg} / \mathrm{m} 3(100.55 \mathrm{lb} / \mathrm{cf})$

$10.20 \mathrm{~kg} / \mathrm{m} 3(0.637 \mathrm{~Tb} / \mathrm{cf})$

8814

$0.621 \mathrm{~L} / \mathrm{kg}(0.0099 \mathrm{cf} / 1 \mathrm{~b})$

$98.1 \mathrm{~L} / \mathrm{kg}(1.5708 \mathrm{cf} / 7 \mathrm{~b})$

8814

$105.3 \mathrm{~kJ} / \mathrm{kg}$ (45.3 Btu/7b)

8814

$106.5 \mathrm{~kJ} / \mathrm{kg}(45.8 \mathrm{Btu} / 7 \mathrm{~b})$

3331

8814

8814 
velocity of sound, saturated vapor: viscosity, saturated 7iquid: viscosity, saturated vapor: thermal conductivity, 1iquid:

thermal conductivity, vapor:

- normal pressure, $20{ }^{\circ} \mathrm{C}\left(68^{\circ} \mathrm{F}\right)$

- normal pressure, $21.1{ }^{\circ} \mathrm{C}\left(70^{\circ} \mathrm{F}\right)$---

- $20{ }^{\circ} \mathrm{C}\left(68^{\circ} \mathrm{F}\right)$ density, vapor: pressure, saturated vapor: density, saturated 7iquid: density, saturated vapor: specific volume, saturated Tiquid: specific volume, saturated vapor: velocity of sound, saturated vapor:

viscosity, saturated 7iquid: viscosity, saturated vapor:

thermal conductivity, saturated vapor:

- $60^{\circ} \mathrm{C}\left(140{ }^{\circ} \mathrm{F}\right)$

pressure, saturated vapor: heat of vaporization:

- critical point

temperature: pressure: density:

specific volume:

ENVIRONMENTAL

average atmospheric 1ifetime (Tatm): ODP (ozone depletion potentia7):

GWP (global warming potential): HGWP (halocarbon GWP) : thermal conductivity, saturatd liquid:

$101 \mathrm{~m} / \mathrm{s}(332 \mathrm{ft} / \mathrm{s})$

8814

$317 \mu \mathrm{Pa} \cdot \mathrm{s}(0.317 \mathrm{cp})$

$9.80 \mu \mathrm{Pa} \cdot \mathrm{s}(0.00980 \mathrm{cp})$

$0.0556 \mathrm{~W} / \mathrm{m} \cdot \mathrm{K}(0.0321$

$\mathrm{Btu} / \mathrm{hr} \cdot \mathrm{ft}^{\circ} \mathrm{F}$ )

$0.0091 \mathrm{~W} / \mathrm{m} \cdot \mathrm{K}(0.0052$

$\mathrm{Btu} / \mathrm{hr} \cdot \mathrm{ft}^{\circ} \mathrm{F}$ )

$7.996 \mathrm{~kg} / \mathrm{m} 3 \quad(0.4992 \mathrm{lb} / \mathrm{cf})$

8814

$7.963 \mathrm{~kg} / \mathrm{m} 3(0.4971 \mathrm{lb} / \mathrm{cf})$

8814

$758.5 \mathrm{kPa}$ (110.01 psia)

$1354 \mathrm{~kg} / \mathrm{m} 3(84.51 \mathrm{lb} / \mathrm{cf})$

$73.45 \mathrm{~kg} / \mathrm{m3}$ (4.585 Tb/cf)

$0.739 \mathrm{~L} / \mathrm{kg}(0.0118 \mathrm{cf} / 1 \mathrm{~b})$

$13.6 \mathrm{~L} / \mathrm{kg}(0.2181 \mathrm{cf} / 7 \mathrm{~b})$

$307 \mathrm{~m} / \mathrm{s}(1008 \mathrm{ft} / \mathrm{s})$

$95 \mathrm{~m} / \mathrm{s}(312 \mathrm{ft} / \mathrm{s})$

$142 \mu \mathrm{Pa} \cdot \mathrm{s}(0.142 \mathrm{cp})$

$12.3 \mu \mathrm{Pa} \cdot \mathrm{s}(0.0123 \mathrm{cp})$

$0.0424 \mathrm{~W} / \mathrm{m} \cdot \mathrm{K}(0.0245$

$\mathrm{Btu} / \mathrm{hr} \cdot \mathrm{ft}^{\circ} \mathrm{F}$ )

$0.01336 \mathrm{~W} / \mathrm{m} \cdot \mathrm{K} \quad(0.00772$

$\mathrm{Btu} / \mathrm{h} \dot{\mathrm{r}} \cdot \mathrm{ft}^{\circ} \mathrm{F}$ )

$2021 \mathrm{kPa}$ (293.2 psia)

$45.2 \mathrm{~kJ} / \mathrm{kg}$ (19.4 Btu/7b)

8814

$71.9^{\circ} \mathrm{C}\left(161.4^{\circ} \mathrm{F}\right)$

$2680 \mathrm{kPa}$ (388.7 psia)

$627 \mathrm{~kg} / \mathrm{m} 3(39.1 \mathrm{lb} / \mathrm{cf})$

$628 \mathrm{~kg} / \mathrm{m} 3(39.2 \mathrm{lb} / \mathrm{cf})$

$1.59 \mathrm{~L} / \mathrm{kg}(0.0255 \mathrm{cf} / 7 \mathrm{~b})$

8814

8814

8814

8814

8814

8814

8814

8814

8814

8814

8814

8814

8814

7713

8814

8814

$2600 \mathrm{yr}$

0.000 (mode]-derived relative

6694

to $R$ 11)

8600 relative to $\mathrm{CO} 2$ for 100

yr integration

40.9 relative to $R 11$ for

infinite integration period

9501

DW

SAFETY

- classification

safety group (ASHRAE standard 34): NFPA 704 degrees of hazard $(H-F-R-S)$ :

NPCA HMIS hazard ratings $(H-F-R)$ :

- Tong-term occupational 1imit ------exposure Timit consistent to OSHA PEL:

- emergency exposure Timit ------Acute Toxicity Exposure Limit (ATEL):

Refrigerant Concentration Limit ( $R C L)$ :
A1

3M: $\begin{array}{r}1-0-0 \\ 1-0-0\end{array}$

8601

MSDS

MSDS

[-special]: $0=$ no, $4=$ severe

3M: $0-0-0$

BOC Gases: 1-0-0

hea 7 th-flammabi lity-reactivity

$0=$ insignificant, $4=e x t r e m e$

Rhône-Poulenc: 1,000 ppm $\mathrm{v} / \mathrm{v}$

TWA for $8 \mathrm{hr} /$ day and $40 \mathrm{hr} / \mathrm{wk}$

MSDS

MSDS

$90,000 \mathrm{ppm} v / \mathrm{v}$ (preliminary based on ASHRAE $34 \mathrm{u}$ draft) $90,000 \mathrm{ppm} v / \mathrm{v}$ (preliminary based on ASHRAE 34u draft)

$1 \mathrm{tr}$

$34 u$

A950

$34 u$ hea 7 th-f7 ammabi 7 ity-reactivity 
- acute (short-term) toxicity LC50 (lethal concentration, 50\%):

cardiac sensitization (CS) EC50:

cardiac sensitization threshold/LOEL:

cardiac sensitization (CS) NOEL:

anesthetic/CNS effect LOEL:

anesthetic/CNS effect NOEL:

- flammabi Tity

LFL-UFL (flammability limits in air):

flash point:

autoignition temperature:

- detection based on ASHRAE 34u draft)

$69,000 \mathrm{ppm} v / \mathrm{V}$ (preliminary

based on ASHRAE $34 \mathrm{u}$ draft)

A950

rat, $1 \mathrm{hr}, 0 / 10:>800,000 \mathrm{ppm} 7550$

(fata 1 concentration by

inhalation for half of test anima 75 )

rat, $4 \mathrm{hr}, 0 / 10$ : >110,000 ppm

(fatal concentration by

inhalation for half of test

anima 75 )

rat, 10 day, 0/20: >113,000

ppm (fatal concentration by

inhalation for half of test animals)

dog, $10 \mathrm{~min}:>400,000 \mathrm{ppm} \mathrm{v} / \mathrm{v}$

(effective concentration in

half of test animals)

dog, 2/8: 400,000 ppm v/v

(lowest observed effect leve]

in test animals)

dog, 0/6: 300,000 ppm v/v (no

observed effect level in test animals)

rat, $1 \mathrm{hr}, 10 / 10: 800,000$ ppm 7550

$v / v$ (Towest observed effect

Tevel in ALC or LC50 studies)

7550

6684

$65 F 9$

$65 F 9$

rat, 10 day, 0/20: 113,000 ppm 7550

$v / v$ (no observed effect level

in subchronic or chronic

study)

none (nonflammable as tested)

BOC Gases: none

BOC Gases: none

2525

MSDS

MSDS

appearance:

odor:

Rhône-Poulenc: colorless

MSDS

BNFL Fluorochemicals: odorless MSDS

BOC Gases: faintly sweet odor MSDS

PRODUCTION

last year production allowed: unrestricted

$8 C 01$ 


\section{R-227ea}

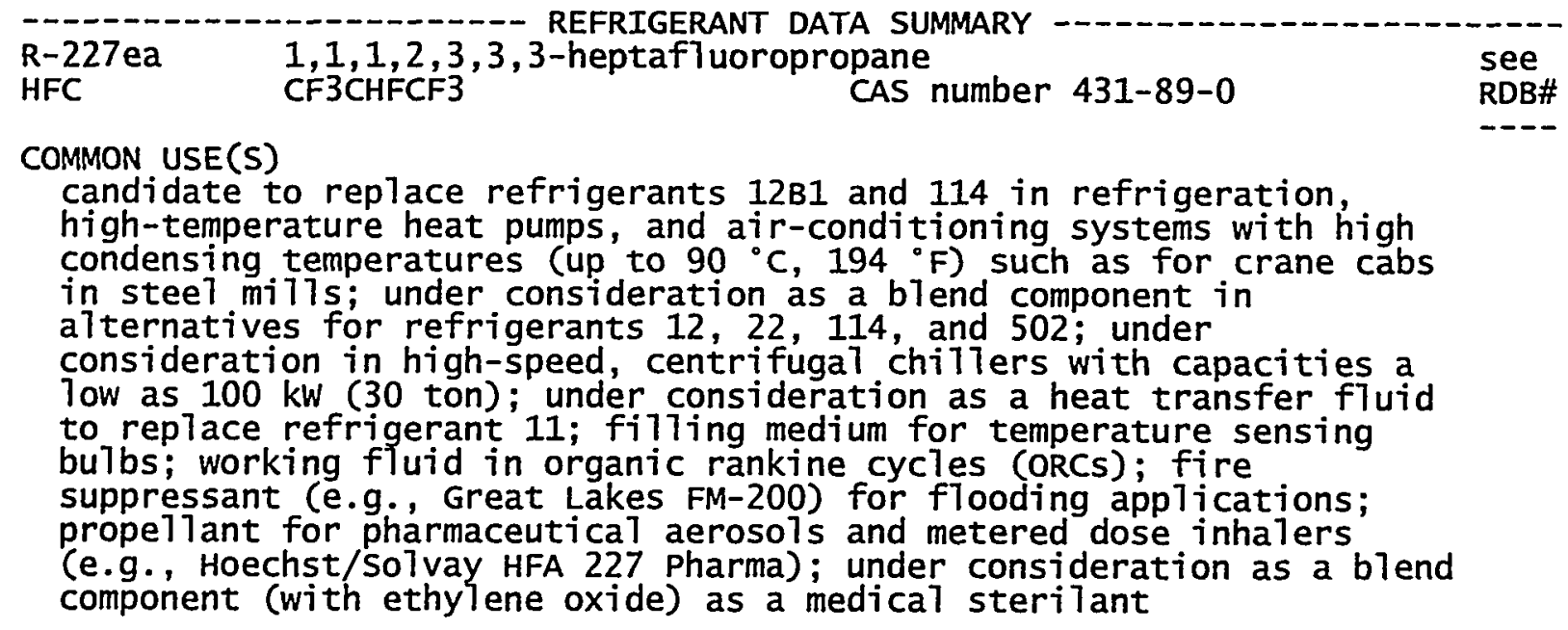

IDENTIFIERS

common name(s): R-227ea; R227ea; R 227ea

HFC-227ea

not recommended: HFA-227ea

fluorochemical 227ea; FC 227ea halon 37

chemical name (by IUPAC convention):

$1,1,1,2,3,3,3$

a]ternative chemical names/formulae:

heptafluoropropane

propane, $1,1,1,2,3,3,3-$

heptafiuoro-

$2 \mathrm{H}$-heptafluoropropane

CF3CHFCF 3 ; CF3-CHF-CF3

not recommended:

empirical formula: C3HF7

CF3CFHCF 3; CF3-CFH-CF3

CAS number: 431-89-0 (Chemical Abstracts

Service registry number)

EINECS number: 207-079-2 (European Inventory

of Existing Chemical

Substances)

trade name(s): Great Lakes FM-200

Solvay Reclin(R) 227

Solvay Solkane(R) 227 ea

ZCIRI Kehua (PRC) HFC-227ea

historical name(s):

Hoechst Reclin(R) 227

Hoechst Refrigerant R 227

A612

none, use light green grey/413 6601

ARI container color / Pantone number:

PHYSICAL

- properties

molar mass:

$170.0288624 \mathrm{~g} / \mathrm{mol}$ ( 0.374849

8820

$\mathrm{Tb} / \mathrm{mo})$

normal freezing/melting/triple point:

$-126.8^{\circ} \mathrm{C}\left(-196.2^{\circ} \mathrm{F}\right)$

3408

$-131.1^{\circ} \mathrm{C}\left(-204.1^{\circ} \mathrm{F}\right) \quad 3728$

- normal boiling point -re-

$-15.6^{\circ} \mathrm{C}\left(3.9^{\circ} \mathrm{F}\right)$

8401

density, saturated liquid:

$1541 \mathrm{~kg} / \mathrm{m} 3(96.21 \mathrm{lb} / \mathrm{cf})$

density, saturated vapor:

specific volume, saturated liquid:

$8.47 \mathrm{~kg} / \mathrm{m} 3 \quad(0.528 \mathrm{7b} / \mathrm{cf})$

$0.649 \mathrm{~L} / \mathrm{kg}(0.0104 \mathrm{cf} / 1 \mathrm{~b}) \quad 8401$

8401

$118.1 \mathrm{~L} / \mathrm{kg}(1.8923 \mathrm{cf} / 1 \mathrm{~b}) \quad 8401$

specific volume, saturated vapor:

$135.9 \mathrm{~kJ} / \mathrm{kg}$ (58.4 Btu/lb)

8401 
velocity of sound, saturated 7iquid: velocity of sound, saturated vapor: viscosity, saturated 7iquid: viscosity, saturated vapor: thermal conductivity, Tiquid:

thermal conductivity, vapor:

- normal pressure, $20{ }^{\circ} \mathrm{C}\left(68{ }^{\circ} \mathrm{F}\right)$-.---

- density, vapor:

- normal pressure, $21.1{ }^{\circ} \mathrm{C}\left(70{ }^{\circ} \mathrm{F}\right)---$

- $20^{\circ} \mathrm{C}\left(68^{\circ} \mathrm{F}\right)$

pressure, saturated vapor: density, saturated liquid: density, saturated vapor: specific volume, saturated 7 iquid: specific volume, saturated vapor: velocity of sound, saturated liquid: velocity of sound, saturated vapor: viscosity, saturated 1 iquid: viscosity, saturated vapor: thermal conductivity, saturatd Tiquid: therma 7 conductivity, saturated vapor: - $60{ }^{\circ} \mathrm{C}\left(140{ }^{\circ} \mathrm{F}\right)$ pressure, saturated vapor: - critical point heat of vaporization:

$$
\begin{array}{r}
\text { temperature: } \\
\text { pressure: } \\
\text { density: } \\
\text { specific volume: }
\end{array}
$$

average atmospheric lifetime (Tatm): average tropospheric 1 ifetime (Ttrop):

ODP (ozone depletion potential): GWP (global warming potential): HGWP (hatocarbon GWP):
ENVIRONMENTAL

$552 \mathrm{~m} / \mathrm{s}(1811 \mathrm{ft} / \mathrm{s}) \quad 8401$

$111 \mathrm{~m} / \mathrm{s}(365 \mathrm{ft} / \mathrm{s}) \quad 8401$

$441 \mu \mathrm{Pa} \cdot \mathrm{s}(0.441 \mathrm{cp}) \quad 8401$

$9.83 \mu \mathrm{Pa} \cdot \mathrm{s}(0.00983 \mathrm{cp}) \quad 8401$

$0.0641 \mathrm{~W} / \mathrm{m} \cdot \mathrm{K}(0.0370 \quad 8401$

$\mathrm{Btu} / \mathrm{hr} \cdot \mathrm{ft}^{\circ} \mathrm{F}$ )

$0.0098 \mathrm{~W} / \mathrm{m} \cdot \mathrm{K}(0.0057$

$\left.\mathrm{Btu} / \mathrm{hr} \bullet \mathrm{ft}^{\circ} \mathrm{F}\right)$

$7.284 \mathrm{~kg} / \mathrm{m} 3(0.4547 \mathrm{~Tb} / \mathrm{cf}) \quad 8401$

$7.253 \mathrm{~kg} / \mathrm{m} 3(0.4528 \mathrm{~Tb} / \mathrm{cf}) \quad 8401$

$388.8 \mathrm{kPa}$ (56.39 psia) 8401

$1407 \mathrm{~kg} / \mathrm{m} 3(87.83 \mathrm{7b} / \mathrm{cf}) \quad 8401$

$31.04 \mathrm{~kg} / \mathrm{m3}(1.938 \mathrm{7b} / \mathrm{cf}) \quad 8401$

$0.711 \mathrm{~L} / \mathrm{kg}(0.0114 \mathrm{cf} / 7 \mathrm{~b}) \quad 8401$

$32.2 \mathrm{~L} / \mathrm{kg}(0.5161 \mathrm{cf} / 7 \mathrm{~b}) \quad 8401$

$417 \mathrm{~m} / \mathrm{s}(1368 \mathrm{ft} / \mathrm{s}) \quad 8401$

$110 \mathrm{~m} / \mathrm{s}(361 \mathrm{ft} / \mathrm{s}) \quad 8401$

$262 \mu \mathrm{Pa} \cdot \mathrm{s}(0.262 \mathrm{cp}) \quad 8401$

$11.2 \mu \mathrm{Pa} \cdot \mathrm{s}(0.0112 \mathrm{cp}) \quad 8401$

$0.0546 \mathrm{~W} / \mathrm{m} \cdot \mathrm{K}(0.0315 \quad 8401$

$\left.\mathrm{Btu} / \mathrm{hr} \cdot \mathrm{ft}^{\circ} \mathrm{F}\right)$

$0.01228 \mathrm{~W} / \mathrm{m} \cdot \mathrm{K}(0.00710 \quad 8401$

$\mathrm{Btu} / \mathrm{hr} \bullet \mathrm{ft}{ }^{\circ} \mathrm{F}$ )

$1182 \mathrm{kPa}$ (171.5 psia)

$86.9 \mathrm{~kJ} / \mathrm{kg}(37.4 \mathrm{Btu} / 7 \mathrm{~b})$

8401

8401

$102.8^{\circ} \mathrm{C}\left(217.0^{\circ} \mathrm{F}\right)$

$2980 \mathrm{kPa}$ (432.2 psia)

7713

8401

$\begin{array}{lll}580 \mathrm{~kg} / \mathrm{m} 3 & (36.2 \mathrm{~Tb} / \mathrm{cf}) & 3408 \\ 1.72 \mathrm{~L} / \mathrm{kg}(0.0276 \mathrm{cf} / 1 \mathrm{~b}) & 3408\end{array}$

$36.5 \pm 7.3 \mathrm{yr}$

6695

$40 \mathrm{yr}$

estimated: $63-72 \mathrm{yr}$

0.000 (mode 7 -derived relative to $R$ 11)

3800 relative to $\mathrm{CO} 2$ for 100

yr integration

0.69 relative to $R 11$ for

infinite integration period

5804

8312

9501

8101

none (no application pending) 8601

Hoechst internal guide: 1,000 $\mathrm{mfr}$ $\mathrm{ppm} v / \mathrm{v}$ TWA for $8 \mathrm{hr} /$ day and $40 \mathrm{hr} / \mathrm{wk}$

$70,000 \mathrm{ppm} v / v$ (preliminary based on ASHRAE $34 \mathrm{u}$ draft)

69,000 ppm v/v (preliminary based on ASHRAE $34 u$ draft)

A950

Acute Toxicity Exposure Limit (ATEL):

Refrigerant concentration Limit ( $R C L$ ):

- acute (short-term) toxicity --.----LC50 (lethal concentration, 50\%): rat, $4 \mathrm{hr}, 0 \%:>110,000 \mathrm{ppm}$

(fatal concentration by

inhalation for half of test

animals)

rat, $4 \mathrm{hr}, 0 / 10$ : >788,696 ppm 4616 
cardiac sensitization (CS) EC50: cardiac sensitization threshold/LOEL: cardiac sensitization (CS) NOEL:

anesthetic/CNS effect LOEL:

- flammability -.-.-LFL-UFL (flammability limits in air): heat of combustion (by ASHRAE 34-92): flash point: autodecomposition temperature: - detection

anesthetic/CNS effect NOEL: appearance: odor: (fatal concentration by inhalation for half of test anima7s)

rat, $4 \mathrm{hr}, 0 / 6$ : >121,267 ppm A617

(fatal concentration by inhalation for half of test anima 75 )

rat, 4 hr: $>788,696 \mathrm{ppm}$ (fatal 65F2

concentration by inhalation

for half of test anima7s)

rat, $4 \mathrm{hr}$ : $>800,000$ (rounded) 5526

ppm (fatai concentration by inhalation for half of test anima1s)

rat, 4 hr: $>800,000$ (rounded)

ppm (fatal concentration by inhalation for half of test animals)

rat, 4\%/2hr w break 0\%: >241,188 A617

ppm (fatal concentration by inhalation for half of test anima 75 )

dog, 30 min: 140,000 ppm v/v (effective concentration in half of test animals)

dog, 30 min, 1/10: 90,000 ppm A615 $\mathrm{v} / \mathrm{v}$ (lowest observed effect level in test animals)

dog, 30 min, 0/10: 70,000 ppm A615 $v / v$ (no observed effect leve? in test animals)

dog, 30 min, 0/10: 90,000 ppm 65F2 $v / v$ (no observed effect 1 eve $T$ in test animals)

dog,? epinephrine: 50,000 ppm 7116 $v / v$ (no observed effect level in test anima7s)

$\operatorname{dog}, 1 \mathrm{hr}, ? / 4: 150,000 \mathrm{ppm}$ $v / v$ ( lowest observed effect level in ALC or LC50 studies) rat, $4 \mathrm{hr}, 6 / 6$ : 121,267 ppm $\mathrm{V} / \mathrm{V}$ (lowest observed effect Tevel in ALC or LC50 studies) rat, $4 \mathrm{hr}$, ?/?: $110,000 \mathrm{ppm}$ $v / v$ (lowest observed effect Teve1 in ALC or LC50 studies)

dog, $1 \mathrm{hr}, 0 / 4$ : 50,000 ppm v/v 7116

(no observed effect level in ALC or LC50 studies)

rat, $6 \mathrm{hr}$, 0/10: 105, 000 ppm $v / v$ (no observed effect level in subchronic or chronic study)

none (nonflammab7e as tested) $3.3 \mathrm{~mJ} / \mathrm{kg}$ (1398 Btu/7b) Great Lakes: nonflammable gas $>700^{\circ} \mathrm{C}\left(>1292^{\circ} \mathrm{F}\right)$

7116

colorless gas

Great Lakes: odorless

Hoechst: ether-like odorless 
Toxicity Data to Determine Refrigerant Concentration Limits James M. Calm, Engineering Consultant

\section{PRODUCTION}

first commercial use as a refrigerant: January 1996

last year production allowed: unrestricted 


\section{R-236fa}

R-236fa
HFC

IDENTIFIERS

common name(s): R-236fa; R236fa; R 236fa

$\mathrm{HFC}-236 \mathrm{fa}$

not recommended: HFA-236fa

f7uorocarbon $236 \mathrm{fa}$

fluorochemical $236 \mathrm{fa}$; FC $236 \mathrm{fa}$

halocarbon $236 \mathrm{fa}$

halochemical $236 \mathrm{fa}$

hexafluoropropane

hydrofluorocarbon $236 \mathrm{fa}$

chemica7 name (by IUPAC convention): a7ternative chemical names/formulae:

empirical formula:

1,1,1, 3, 3,3-hexaf7uoropropane

propane, $1,1,1,3,3,3-$

hexaf7uoro-

bis (trifluoromethy 1 ) methane

bistrifluoromethylmethane

2,2-dihydroperfluoropropane

CF 3 CH2CF3; CF3-CH2-CF3

CAS number: 690-39-1 (Chemical Abstracts

Service registry number)

NIOSH RTECS number: TZ4043332 (Registry of Toxic

Effects of Chemical

Substances)

trade name (s): $3 M(T M)$ HFG-3236

MSDS

3M(TM) HFG-3236fa 6B05

A77iedsigna1 Genetron(R) 236fa MSDS

DuPont Suva(R) $236 \mathrm{fa}$ MSDS

ARI container color / Pantone number: none, use light green grey/413 6601

PHYSICAL

- properties

molar mass:

normal freezing/melting/triple point:

- normat boiling point -romperature:

density, saturated Tiquid:

density, saturated vapor:

specific volume, saturated 7iquid: specific volume, saturated vapor:

heat of vaporization:

velocity of sound, saturated liquid:

velocity of sound, saturated vapor:

viscosity, saturated liquid:

viscosity, saturated vapor:

therma 7 conductivity, 7 iquid:

$152.0383992 \mathrm{~g} / \mathrm{mol}(0.335187 \quad 8820$

$1 \mathrm{~b} / \mathrm{mol}$ ?

$-93.6{ }^{\circ} \mathrm{C}\left(-136.5^{\circ} \mathrm{F}\right)$

8401

$-1.4^{\circ} \mathrm{C}\left(29.4^{\circ} \mathrm{F}\right) \quad 8401$

$1445 \mathrm{~kg} / \mathrm{m3}(90.19 \mathrm{7b} / \mathrm{cf}) \quad 8401$

$7.14 \mathrm{~kg} / \mathrm{m3}(0.446 \mathrm{ib} / \mathrm{cf}) \quad 8401$

$0.692 \mathrm{~L} / \mathrm{kg}(0.0111 \mathrm{cf} / \mathrm{fb}) \quad 8401$

$140.0 \mathrm{~L} / \mathrm{kg}(2.2423 \mathrm{cf} / \mathrm{jb}) \quad 8401$

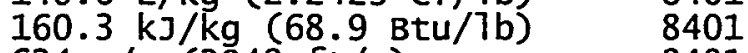

$624 \mathrm{~m} / \mathrm{s}(2048 \mathrm{ft} / \mathrm{s}) \quad 8401$

$122 \mathrm{~m} / \mathrm{s}(399 \mathrm{ft} / \mathrm{s}) \quad 8401$

$356 \mu \mathrm{Pa} \cdot \mathrm{s}(0.356 \mathrm{cp}) \quad 8401$

$9.96 \mu \mathrm{Pa} \cdot \mathrm{s}(0.00996 \mathrm{cp}) \quad 8401$

$0.0724 \mathrm{~W} / \mathrm{m} \cdot \mathrm{K}(0.0418 \quad 8401$

$\mathrm{Btu} / \mathrm{hr} \cdot \mathrm{ft}{ }^{\circ} \mathrm{F}$ ) 
thermal conductivity, vapor:

- normal pressure, $20^{\circ} \mathrm{C}\left(68{ }^{\circ} \mathrm{F}\right)$----density, vapor:

- normal pressure, $21.1{ }^{\circ} \mathrm{C}\left(70^{\circ} \mathrm{F}\right)$--density, vapor:

- $20{ }^{\circ} \mathrm{C}(68$

p)
density, saturated 7 vaquid: density, saturated vapor:

specific volume, saturated liquid: specific volume, saturated vapor: velocity of sound, saturated 1iquid: velocity of sound, saturated vapor: viscosity, saturated 7iquid: viscosity, saturated vapor: thermal conductivity, saturated vapor: heat of vaporization:

- critical point temperature: pressure: density: specific volume:

ENVIRONMENTAL

average atmospheric lifetime (Tatm): average tropospheric lifetime (Ttrop): ODP (ozone depletion potential):

GWP (global warming potential): HGWP (halocarbon GWP): thermal conductivity, saturatd 7iquid:

$0.0106 \mathrm{~W} / \mathrm{m} \cdot \mathrm{K}(0.0061$ $\mathrm{Btu} / \mathrm{hr} \cdot \mathrm{ft}^{\circ} \mathrm{F}$ )

$6.542 \mathrm{~kg} / \mathrm{m} 3(0.4084 \mathrm{lb} / \mathrm{cf}) \quad 8401$

$6.514 \mathrm{~kg} / \mathrm{m} 3(0.4066 \mathrm{lb} / \mathrm{cf}) \quad 8401$

$229.6 \mathrm{kPa}$ (33.31 psia) 6B05

$1376 \mathrm{~kg} / \mathrm{m} 3(85.93 \mathrm{1b} / \mathrm{cf}) \quad 8401$

$15.58 \mathrm{~kg} / \mathrm{m} 3(0.973 \mathrm{~Tb} / \mathrm{cf}) \quad 8401$

$0.727 \mathrm{~L} / \mathrm{kg}(0.0116 \mathrm{cf} / \mathrm{lb}) \quad 8401$

$64.2 \mathrm{~L} / \mathrm{kg}$ (1.0278 cf/1b) 8401

$545 \mathrm{~m} / \mathrm{s}(1789 \mathrm{ft} / \mathrm{s}) \quad 8401$

$122 \mathrm{~m} / \mathrm{s}(400 \mathrm{ft} / \mathrm{s}) \quad 8401$

$270 \mu \mathrm{Pa} \cdot \mathrm{s}(0.270 \mathrm{cp}) \quad 8401$

$10.7 \mu \mathrm{Pa} \cdot \mathrm{s}(0.0107 \mathrm{cp}) \quad 8401$

$0.0662 \mathrm{~W} / \mathrm{m} \bullet \mathrm{K}(0.0382 \quad 8401$

$\left.\mathrm{Btu} / \mathrm{hr} \bullet \mathrm{ft}^{\circ} \mathrm{F}\right)$

$0.01198 \mathrm{~W} / \mathrm{m} \cdot \mathrm{K}(0.00692 \quad 8401$

$\mathrm{Btu} / \mathrm{hr} \cdot \mathrm{ft}^{\circ} \mathrm{F}$ )

$765 \mathrm{kPa}$ (110.9 psia) 8401

$122.6 \mathrm{~kJ} / \mathrm{kg}(52.7 \mathrm{Btu} / 7 \mathrm{~b}) \quad 8401$

$124.9^{\circ} \mathrm{C}\left(256.9^{\circ} \mathrm{F}\right) \quad 7714$

$3200 \mathrm{kPa}(464.1 \mathrm{psia}) \quad 8401$

$551 \mathrm{~kg} / \mathrm{m} 3(34.4 \mathrm{ib} / \mathrm{cf}) \quad 8401$

$1.81 \mathrm{~L} / \mathrm{kg}(0.0291 \mathrm{cf} / 7 \mathrm{~b}) \quad 8401$

226 yr 9501

estimated: 77-218 yr 8312

0.000 (model-derived relative to R 11)

9400 relative to $\mathrm{CO} 2$ for 1009501

yr integration

3.79 relative to $R 11$ for

infinite integration period

4.2 relative to $R 11$ for

infinite integration period

8101

6805

SAFETY

- classification safety group (ASHRAE Standard 34): NFPA 704 degrees of hazard $(H-F-R-S)$ :

NPCA HMIS hazard ratings $(H-F-R)$ :

- 1ong-term occupational 7imit -...-.AIHA WEEL (workplace env1 exp Timit): exposure limit consistent to OSHA PEL:

- emergency exposure limit Acute Toxicity Exposure Limit (ATEL):

Refrigerant Concentration Limit (RCL):
A1 7 iedsigna $1: 2-0-0$ health-flammability-reactivity [-specia7]: $0=$ no, $4=$ severe

A77iedsigna $7: 2-0-0$ DuPont: $1-0-1$

health-flammability-reactivity $0=$ insignificant, $4=$ extreme

$1,000 \mathrm{ppm} \mathrm{v} / \mathrm{v}$ TWA for $8 \mathrm{hr} /$ day 8612 and $40 \mathrm{hr} / \mathrm{wk}$

DuPont AEL: $1,000 \mathrm{ppm} \mathrm{V/V} \mathrm{TWA} \mathrm{MSDS}$ for $8 \mathrm{hr} /$ day and $40 \mathrm{hr} / \mathrm{wk}$

$100,000 \mathrm{ppm} \mathrm{v} / \mathrm{v}$ (preliminary $34 \mathrm{u}$ based on ASHRAE $34 \mathrm{u}$ draft) $55,000 \mathrm{ppm} v / \mathrm{v}$ (preliminary based on ASHRAE 34u draft)

$55,000 \mathrm{ppm} V / \mathrm{V}$ (preliminary based on ASHRAE $34 \mathrm{u}$ draft) $69,000 \mathrm{ppm} v / \mathrm{v}$ (preliminary
$34 \mathrm{e}$ MSDS

MSDS MSDS A950 A950 $34 u$ 
- acute (short-term) toxicity -LC50 (lethal concentration, 50\%):

cardiac sensitization threshold/LOEL: cardiac sensitization (CS) NOEL: anesthetic/CNS effect EC50: anesthetic/CNS effect LOEL: anesthetic/CNS effect NOEL:

- flammability LFL-UFL (flammability limits in air): flash point: autodecomposition temperature:

- detection based on ASHRAE 34u draft)

mouse, 10 min: 440,000 ppm

(fatal concentration by inhalation for half of test animals)

rat, 4 hr: $>457,000$ ppm (fatal 6A94 concentration by inhalation for half of test animals)

dog, $10 \mathrm{~min}, 2 / 6: 150,000 \mathrm{ppm}$ 65A5 $v / v$ (lowest observed effect leve 7 in test anima7s)

dog, $10 \mathrm{~min}, 0 / 6$ : 100,000 ppm $v / v$ (no observed effect 7 eve 7 in test anima7s)

mouse, $10 \mathrm{~min}$ : $110,000 \mathrm{ppm} \mathrm{v} / \mathrm{v} 5980$ (effective concentration in half of test animals)

rat, $4 \mathrm{hr}$, ?/4: 189,275 ppm $v / v$ (lowest observed effect Teve 1 in ALC or LC50 studies)

rat, $6 \mathrm{~h} / \mathrm{d}$ 14wk 6-8/10: 50,300 A306 $\mathrm{ppm} v / \mathrm{v}$ (Towest observed effect leve 7 in ALC or LC50 studies)

rat, $4 \mathrm{hr}, 0 / 4: 133,939 \mathrm{ppm}$

6803

$v / v$ (no observed effect level

in ALC or LC50 studies)

rat, 2-week, 0/4: 5,020 ppm

$v / v$ (no observed effect level

in subchronic or chronic study)

rat, $6 \mathrm{~h} / \mathrm{d} 14$ wk, 0/20: 20,000 A306

$\mathrm{ppm} v / \mathrm{v}$ (no observed effect

level in subchronic or chronic study)

rat, day 1 of 2wk, 0/4: 20,000 6A93 $\mathrm{ppm} v / \mathrm{v}$ (no observed effect level in subchronic or chronic study)

none (nonflammable as tested) 3M: none

Alliedsigna $7:>250^{\circ} \mathrm{C}\left(>482^{\circ} \mathrm{F}\right)$

6B05

MSDS

MSDS

appearance: DuPont: colorless

odor: 3M: faint ethereal

MSDS

MSDS

PRODUCTION

first commercial use as a refrigerant: October 1996

last year production allowed: 


\section{R-245ca}

R-245ca CHFC
COMMON USE(S)
COnsidered as an a7ternative for refrigerants 11 and 123 circa 1992 ,
constrained by marginal flammability; also considered for use as a
foam blowing agent

IDENTIFIERS

common name(s): R-245ca; R245ca; R 245ca

$\mathrm{HFC}-245 \mathrm{Ca}$

fluorocarbon $245 \mathrm{ca}$

fiuorochemical $245 \mathrm{Ca}$; FC $245 \mathrm{ca}$

halocarbon $245 \mathrm{ca}$

halochemica7 $245 \mathrm{ca}$

hydrof7uorocarbon $245 \mathrm{ca}$

chemical name (by IUPAC convention): a7ternative chemical names/formulae:

empirical formula: 1,1,2, 2,3-pentaf7uoropropane propane,
$1,1,2,2,3$-pentafluoro-
CH2FCF2CHF2; CH2F-CF2-CHF2
not recommended:
CF2HCF2CFH2; CF2H-CF2-CFH2
CFH2CF2CF2H; $\mathrm{CFH} 2-\mathrm{CF} 2-\mathrm{CF} 2 \mathrm{H}$
CHF2CF2CH2F; $\mathrm{CHF} 2-\mathrm{CF} 2-\mathrm{CH} 2 \mathrm{~F}$
FCH2CF2CHF2; FCH2-CF2-CHF2

\section{C $3 \mathrm{H} 3 \mathrm{~F} 5$}
679-86-7 (Chemical Abstracts
Service registry number)

CAS number:

PHYSICAL

- properties

molar mass:

normal freezing/melting/triple point:

- normal boiling point

temperature:

density, saturated 7iquid: density, saturated vapor:

specific volume, saturated liquid: specific volume, saturated vapor: heat of vaporization: velocity of sound, saturated liquid: velocity of sound, saturated vapor:

viscosity, saturated vapor: viscosity, saturated 1 iquid:

thermal conductivity, 1iquid:

thermal conductivity, vapor:

- normal pressure, $20^{\circ} \mathrm{C}\left(68^{\circ} \mathrm{F}\right)$ -

$134.047936 \mathrm{~g} / \mathrm{mol}(0.295525$

8820

$7 \mathrm{~b} / \mathrm{mo} 7)$

$-73.4^{\circ} \mathrm{C}\left(-100.1^{\circ} \mathrm{F}\right)$

3301

$25.1^{\circ} \mathrm{C}\left(77.2^{\circ} \mathrm{F}\right)$

$1386 \mathrm{~kg} / \mathrm{m} 3(86.53 \mathrm{7b} / \mathrm{cf})$

$5.74 \mathrm{~kg} / \mathrm{m} 3(0.358 \mathrm{7b} / \mathrm{cf})$

$0.722 \mathrm{~L} / \mathrm{kg}(0.0116 \mathrm{cf} / 7 \mathrm{~b})$

$174.3 \mathrm{~L} / \mathrm{kg}(2.7922 \mathrm{cf} / 1 \mathrm{~b})$

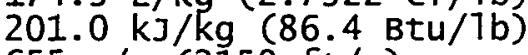

$655 \mathrm{~m} / \mathrm{s}(2150 \mathrm{ft} / \mathrm{s})$

$136 \mathrm{~m} / \mathrm{s}(446 \mathrm{ft} / \mathrm{s})$

$10.16 \mu \mathrm{Pa} \cdot \mathrm{s}(0.01016 \mathrm{cp})$

$542 \mu \mathrm{Pa} \cdot \mathrm{s}(0.542 \mathrm{cp})$

$0.0873 \mathrm{~W} / \mathrm{m} \cdot \mathrm{K}(0.0505$

$\mathrm{Btu} / \mathrm{hr} \cdot \mathrm{ft}^{\circ} \mathrm{F}$ )

$0.0123 \mathrm{~W} / \mathrm{m} \cdot \mathrm{K}(0.0071$

$\mathrm{Btu} / \mathrm{hr} \bullet \mathrm{ft}^{\circ} \mathrm{F}$ )

8401

8401

8401

8401

8401

8401

8401

8401

8401

8401

8401

8401

$1399 \mathrm{~kg} / \mathrm{m} 3$ (11.67 1b/gal) 8401

- normal pressure, $21.1{ }^{\circ} \mathrm{C}\left(70^{\circ} \mathrm{F}\right)$-.-

- $20{ }^{\circ} \mathrm{C}\left(68^{\circ} \mathrm{F}\right)$-... density, Tiquid:

pressure, saturated vapor: density, saturated 7 iquid: density, saturated vapor:

specific volume, saturated 7 iquid:

$1396 \mathrm{~kg} / \mathrm{m} 3$ (11.65 7b/ga7)

8401

$82.9 \mathrm{kPa}$ (12.02 psia)

$1399 \mathrm{~kg} / \mathrm{m} 3(87.32 \mathrm{ib} / \mathrm{cf})$

8401

$4.75 \mathrm{~kg} / \mathrm{m} 3$ (0.296 ib/cf)

8401

$0.715 \mathrm{~L} / \mathrm{kg}(0.0115 \mathrm{cf} / \mathrm{lb})$

8401

8401 specific volume, saturated vapor:

$210.7 \mathrm{~L} / \mathrm{kg}$ ( $3.3744 \mathrm{cf} / 7 \mathrm{~b})$

8401 
velocity of sound, saturated Tiquid: velocity of sound, saturated vapor: viscosity, saturated 7iquid: viscosity, saturated vapor: thermal conductivity, saturatd 1iquid: thermal conductivity, saturated vapor: - $60{ }^{\circ} \mathrm{C}(140$

- critical point pressure, saturated vapor: heat of vaporization:

temperature: pressure: density: specific volume:
$674 \mathrm{~m} / \mathrm{s}(2210 \mathrm{ft} / \mathrm{s})$

8401

$135 \mathrm{~m} / \mathrm{s}(444 \mathrm{ft} / \mathrm{s})$

$584 \mu \mathrm{Pa} \cdot \mathrm{s}(0.584 \mathrm{cp})$

$10.0 \mu \mathrm{Pa} \cdot \mathrm{s}(0.0100 \mathrm{cp})$

$0.0889 \mathrm{~W} / \mathrm{m} \cdot \mathrm{K}(0.0513$

$\mathrm{Btu} / \mathrm{hr} \cdot \mathrm{ft}^{\circ} \mathrm{F}$ )

$0.01187 \mathrm{~W} / \mathrm{m} \cdot \mathrm{K}(0.00686$

$\mathrm{Btu} / \mathrm{hr} \cdot \mathrm{ft}^{\circ} \mathrm{F}$ )

$327 \mathrm{kPa}$ (47.5 psia)

$180.6 \mathrm{~kJ} / \mathrm{kg}$ (77.6 Btu/7b)

8401

8401

$174.4^{\circ} \mathrm{C}\left(346.0^{\circ} \mathrm{F}\right)$

7714

$3935 \mathrm{kPa}$ (570.7 psia)

8401

7714

7714
8401

8401

8401

8401
6695

5804

8312

estimated: 1.3-3 yr

0.000 (mode 7 -derived relative to $R$ 11)

720 relative to CO2 for 100 yr 9501 integration

0.13 relative to $R 11$ for

infinite integration period

8101

SAFETY

- classification

safety group (ASHRAE Standard 34):

- emergency exposure Timit

Acute Toxicity Exposure Limit (ATEL):

Refrigerant concentration Limit (RCL):

- acute (short-term) toxicity

ALC (approximate lethal concentration:

none (no application pending)

8601

0 ppm $v / v$ (preliminary based A950

on ASHRAE $34 \mathrm{u}$ draft)

0 ppm $v / v$ (preliminary based

on ASHRAE $34 u$ draft)

A950

mouse, $30 \mathrm{~min}, 1 />2: 240,000$

ppm (lowest exposure tested

with one or more deaths by

inhalation)

mouse, $30 \mathrm{~min}, 1 /$ ?: 240,000

ppm (lowest exposure tested

with one or more deaths by

inhalation)

rat, $4 \mathrm{hr}$ : >993 ppm (lowest

exposure tested with one or

more deaths by inhalation)

anesthetic/CNS effect LOEL:

mouse, $30 \mathrm{~min}, 1 />2$ : 100,000

$\mathrm{ppm} \mathrm{v/v} \mathrm{(lowest} \mathrm{observed}$

effect level in test animals)

mouse, 5or10 min, ?/?: 100,000 5C77

$\mathrm{ppm} \mathrm{v} / \mathrm{v}$ (Towest observed

effect level in test anima7s)

anesthetic/CNS effect NOEL: mouse, 50 r10 min, $0 / ?: 50,000$

$5 C 77$

$\mathrm{ppm} v / \mathrm{v}$ (no observed effect

leve 7 in test anima7s)

- flammability

LFL-UFL (flammability Timits in air):

$7.1-14.8 \% \mathrm{v} / \mathrm{v}$

4785

$8.3-12.8 \% \mathrm{v} / \mathrm{v}$

$5 \mathrm{~B} 15$

heat of combustion (by ASHRAE 34-92):

none (nonflammable as tested) $2 \mathrm{AO1}$

$7.1 \mathrm{MJ} / \mathrm{kg}$ (3052 Btu/7b) 6501

$8.4 \mathrm{~mJ} / \mathrm{kg}$ (3616 Btu/7b) 4785

flash point: none

$5 B 15$ 
Toxicity Data to Determine Refrigerant Concentration Limits James M. Calm, Engineering Consultant

\section{PRODUCTION}

first commercial use as a refrigerant: not known to be commercialized

last year production allowed: unrestricted

$8 \mathrm{CO1}$ 


\section{R-245fa}

\begin{tabular}{|c|c|c|}
\hline $\begin{array}{l}R-245 \text { fa } \\
H F C\end{array}$ & $\begin{array}{l}1,1,1,3,3 \text {-pentafiuoropropane } \\
\text { CHF2CH2CF3 }\end{array}$ & CAS number $460-73-1$ \\
\hline
\end{tabular}

COMMON USE(S)

under consideration as an a7ternative for refrigerants 11 and 123 for use in centrifugal chillers; constrained by higher pressure (stil) low pressure, but sufficient to require compliance with requirements for pressure vesse7s) and - particulariy with single-stage compressors and in typical capacities - lower efficiency; candidate to replace fluorochemical $141 \mathrm{~b}$ as a foam blowing agent, alone and in azeotropic mixtures with hydrocarbons

Note: ASHRAE SSPC 34 proposed revision of the safety classification from "AI provisional" to B1 on 2000.02.06 in 7ight of the new WEEL of $300 \mathrm{ppm} \mathrm{V} / \mathrm{v}$, adopted by AIHA in January 2000. The revision is subject to a review and approval procedure now underway; actual assignment wi71 not occur unti published in an addendum or revision to ANSI/ASHRAE Standard 34-1997.

\section{IDENTIFIERS}

$$
\text { common name(s): R-245fa; R245fa; R 245fa }
$$

$\mathrm{HFC}-245 \mathrm{fa}$

f7uorocarbon $245 \mathrm{fa}$

f7uorochemica7 245fa; FC $245 \mathrm{fa}$

halocarbon $245 \mathrm{fa}$

ha7ochemical $245 \mathrm{fa}$

hydrof1uorocarbon $245 \mathrm{fa}$

chemical name (by IUPAC convention): alternative chemical names/formulae:

empirical formula: CAS number:

trade name (s):

historical name(s):

ARI container color / Pantone number: $1,1,1,3,3$-pentafluoropropane

propane, $1,1,1,3,3-$

pentafiuoro-

CHF2CH2CF3; $\mathrm{CHF}_{2}-\mathrm{CH}_{2}-\mathrm{CF}_{3}$

not recommended:

CF2HCH2CF3; CF2H-CH2-CF3

$\mathrm{CF} 3 \mathrm{CH}_{2} \mathrm{CF} 2 \mathrm{H}$; $\mathrm{CF} 3-\mathrm{CH} 2-\mathrm{CF} 2 \mathrm{H}$

CF3CH2CHF2; $\mathrm{CF} 3-\mathrm{CH}_{2}-\mathrm{CHF} 2$

C3H3F5

460-73-1 (Chemica) Abstracts

Service registry number)

Honeywe 17 HFC-245fa

A17jedsignal Genetron(R) $245 \mathrm{fa} \mathrm{mfr}$

A7liedsignal HFC-245fa

none, use light green grey/413 MSDS

PHYSICAL

- properties

molar mass:

$134.047936 \mathrm{~g} / \mathrm{mo}$ ( 0.295525

8820

normal freezing/melting/triple point:

7b/mol)

$-102.1^{\circ} \mathrm{C}\left(-151.8^{\circ} \mathrm{F}\right)$

$-80.0^{\circ} \mathrm{C}\left(-112.0^{\circ} \mathrm{F}\right)$

3408

$<-160.0^{\circ} \mathrm{C}\left(<-256^{\circ} \mathrm{F}\right)$

MSDS

- normal boiling point

temperature:

$14.9{ }^{\circ} \mathrm{C}\left(58.8^{\circ} \mathrm{F}\right)$

$15.1{ }^{\circ} \mathrm{C}\left(59.2{ }^{\circ} \mathrm{F}\right)$

$1366 \mathrm{~kg} / \mathrm{m} 3(85.26 \mathrm{lb} / \mathrm{cf})$

$5.92 \mathrm{~kg} / \mathrm{m} 3(0.3701 \mathrm{~b} / \mathrm{cf})$

$0.732 \mathrm{~L} / \mathrm{kg}(0.0117 \mathrm{cf} / 7 \mathrm{~b})$

$5 A 34$

density, saturated 7iquid:

density, saturated vapor:

specific volume, saturated 7iquid:

specific volume, saturated vapor:

heat of vaporization:

$16.9 \mathrm{~L} / \mathrm{kg}(0.2705 \mathrm{cf} / 7 \mathrm{~b})$

$196.7 \mathrm{~kJ} / \mathrm{kg}(84.6 \mathrm{Btu} / 7 \mathrm{~b})$ 
velocity of sound, saturated 7iquid: velocity of sound, saturated vapor: viscosity, saturated 7iquid: viscosity, saturated vapor: therma 1 conductivity, 1iquid:

thermal conductivity, vapor:

- $20{ }^{\circ} \mathrm{C}(68$

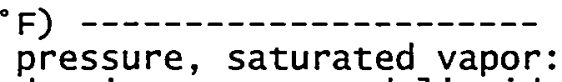

density, saturated 7 iquid: density, saturated vapor:

specific volume, saturated Tiquid: specific volume, saturated vapor: velocity of sound, saturated liquid: velocity of sound, saturated vapor: viscosity, saturated 7iquid: viscosity, saturated vapor: thermal conductivity, saturatd Tiquid:

thermal conductivity, saturated vapor:

- $60^{\circ} \mathrm{C}\left(140{ }^{\circ} \mathrm{F}\right)$

pressure, saturated vapor: heat of vaporization:

- critical point ---

temperature:

pressure:

density:

specific volume:
$715 \mathrm{~m} / \mathrm{s}(2346 \mathrm{ft} / \mathrm{s})$

$134 \mathrm{~m} / \mathrm{s}(439 \mathrm{ft} / \mathrm{s})$

$464 \mu \mathrm{Pa} \cdot \mathrm{s}(0.464 \mathrm{cp})$

$9.99 \mu \mathrm{Pa} \cdot \mathrm{s}(0.00999 \mathrm{cp})$

$0.0841 \mathrm{~W} / \mathrm{m} \cdot \mathrm{K}(0.0486$

$\left.\mathrm{Btu} / \mathrm{hr} \cdot \mathrm{ft}{ }^{\circ} \mathrm{F}\right)$

$0.0117 \mathrm{~W} / \mathrm{m} \cdot \mathrm{K}(0.0067$

$\mathrm{Btu} / \mathrm{hr} \cdot \mathrm{ft}^{\circ} \mathrm{F}$ )

$123.8 \mathrm{kPa}$ (17.96 psia)

$1352 \mathrm{~kg} / \mathrm{m} 3(84.42 \mathrm{~Tb} / \mathrm{cf})$

$7.16 \mathrm{~kg} / \mathrm{m} 3(0.447 \mathrm{lb} / \mathrm{cf})$

$0.740 \mathrm{~L} / \mathrm{kg}(0.0118 \mathrm{cf} / 7 \mathrm{~b})$

$14.0 \mathrm{~L} / \mathrm{kg}(0.2238 \mathrm{cf} / 7 \mathrm{~b})$

$693 \mathrm{~m} / \mathrm{s}(2272 \mathrm{ft} / \mathrm{s})$

$134 \mathrm{~m} / \mathrm{s}(441 \mathrm{ft} / \mathrm{s})$

$432 \mu \mathrm{Pa} \cdot \mathrm{s}(0.432 \mathrm{cp})$

$10.2 \mu \mathrm{Pa} \cdot \mathrm{s}(0.0102 \mathrm{cp})$

$0.0826 \mathrm{~W} / \mathrm{m} \bullet \mathrm{K}(0.0477$

$\mathrm{Btu} / \mathrm{hr} \cdot \mathrm{ft}^{\circ} \mathrm{F}$ )

$0.01208 \mathrm{~W} / \mathrm{m} \cdot \mathrm{K}(0.00698$

$\mathrm{Btu} / \mathrm{hr} \cdot \mathrm{ft}^{\circ} \mathrm{F}$ )

$464 \mathrm{kPa}$ (67.2 psia)

$168.4 \mathrm{~kJ} / \mathrm{kg}(72.4 \mathrm{Btu} / 1 \mathrm{~b})$

8401

8401

7714

8401

8324

7714

7714

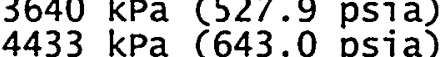

$517 \mathrm{~kg} / \mathrm{m} 3(32.3 \mathrm{lb} / \mathrm{cf})$

$1.93 \mathrm{~L} / \mathrm{kg}(0.0310 \mathrm{cf} / 7 \mathrm{~b})$

9709

ODP (ozone depletion potential):

GWP (global warming potential): $\begin{array}{ll}8.4 \mathrm{yr} & 8323 \\ 8.8 \mathrm{yr} & 5442 \\ 8.6 \mathrm{yr} & 5805 \\ \text { estimated: } 7-11 \mathrm{yr} & 8312\end{array}$

$\begin{array}{ll}8.4 \mathrm{yr} & 8323 \\ 8.8 \mathrm{yr} & 5442 \\ 8.6 \mathrm{yr} & 5805 \\ \text { estimated: } 7-11 \mathrm{yr} & 8312\end{array}$

$\begin{array}{ll}8.4 \mathrm{yr} & 8323 \\ 8.8 \mathrm{yr} & 5442 \\ 8.6 \mathrm{yr} & 5805 \\ \text { estimated: } 7-11 \mathrm{yr} & 8312\end{array}$

$\begin{array}{ll}8.4 \mathrm{yr} & 8323 \\ 8.8 \mathrm{yr} & 5442 \\ 8.6 \mathrm{yr} & 5805 \\ \text { estimated: } 7-11 \mathrm{yr} & 8312\end{array}$

0.000 (mode1-derived relative to $\mathrm{R}$ 11)

760 relative to $\mathrm{CO} 2$ for 100 yr 9709 integration

820 relative to $\mathrm{CO} 2$ for 100 yr 8101 integration

990 relative to $\mathrm{CO} 2$ for 100 yr ETF integration

0.18 relative to $R 11$ for

infinite integration period

0.24 relative to $R 11$ for

infinite integration period

8101

$5 B 15$
HGWP (halocarbon GWP):

\section{SAFETY}

Classification
safety group (ASHRAE standard 34):

NFPA 704 degrees of hazard (H-F-R-S):

NPCA HMIS hazard ratings (H-F-R)

- Tong-term occupational limit ---AIHA WEEL (workplace envi exp 7imit):
AI provisional

BI proposed as a revision on 2000.06.25

A7 Tiedsigna $7:$ 2-0-1 hea th-flammability-reactivity [-special]: $0=$ no, $4=$ severe

A] Tiedsignal : 2-0-1

health-flammability-reactivity

$0=$ insignificant, $4=$ extreme

\section{$34 \mathrm{~h}$}

$34 a m$

34am

MSDS

$300 \mathrm{ppm} \mathrm{v} / \mathrm{v}$ TWA for $8 \mathrm{hr} /$ day 
- emergency exposure Timit Acute Toxicity Exposure Limit (ATEL):

Refrigerant Concentration Limit (RCL):

acute (short-term) toxicity -.----ALC (approximate Tethal concentration:

derma 1 LD50 (7etha1 dosage, 50\%):

cardiac sensitization threshold/LOEL: cardiac sensitization (CS) NOEL:

anesthetic/CNS effect LOEL:

anesthetic/CNS effect NOEL:

- flammability

LFL-UFL (flammability limits in air): heat of combustion (by ASHRAE 34-92): autoignition temperature:

autodecomposition temperature: former UL Classification:

- detection and $40 \mathrm{hr} / \mathrm{wk}$

$34,000 \mathrm{ppm} \mathrm{v} / \mathrm{v}$ (preliminary based on ASHRAE $34 \mathrm{u}$ draft) 34,000 ppm v/v (preliminary based on ASHRAE $34 \mathrm{u}$ draft)

$34 u$

A950

$34,000 \mathrm{ppm} v / \mathrm{v}$ (preliminary

based on ASHRAE $34 \mathrm{u}$ draft)

$34,000 \mathrm{ppm} v / v$ (preliminary

based on ASHRAE $34 u$ draft)

$34 \mathrm{u}$

A950

mouse, $4 \mathrm{hr}, 0 / 10:>101,300$

ppm (lowest exposure tested

9707 with one or more deaths by inhalation)

rat, $4 \mathrm{hr}, 0 / 10:>203,000 \mathrm{ppm}$ (lowest exposure tested with one or more deaths by inhalation)

rabbit: $>2,600 \mathrm{mg} / \mathrm{kg}$ (fatal dose by skin contact for half of test animals)

dog, $10 \mathrm{~min}, 1 / 4: 44,000 \mathrm{ppm}$

$v / v$ (lowest observed effect level in test animals)

dog, 0/6: 20,000 ppm v/v (no observed effect 7 evel in test anima1s)

dog, $10 \mathrm{~min}, 0 / 3: 54,100 \mathrm{ppm}$ $v / v$ (no observed effect leve in test animals)

dog, $10 \mathrm{~min}, 0 / 4$ : 34,100 ppm $v / v$ (no observed effect Teve 7 in test animals)

mouse, $4 \mathrm{hr}, 10 / 10$ : 101, 300

$\mathrm{ppm} \mathrm{v} / \mathrm{v}$ (Towest observed

effect leve 1 in ALC or LC50 studies)

rat, $4 \mathrm{hr}, 10 / 10: 116,000 \mathrm{ppm}$

$v / v$ (Towest observed effect Teve 1 in ALC or LC50 studies)

rat, $4 \mathrm{hr}$, 10/10: 143,000 ppm

$v / v$ (lowest observed effect

Tevel in ALC or LC50 studies)

rat, $6 \mathrm{hr}, 0 / 20: 50,600 \mathrm{ppm}$

$v / v$ (no observed effect level

in subchronic or chronic

study)

none (not flammable as tested) 6820 none (not flammable as tested) 7114 $6.1 \mathrm{MJ} / \mathrm{kg}$ (2618 Btu/Tb) UL none

$704{ }^{\circ} \mathrm{C}\left(1299^{\circ} \mathrm{F}\right)$

Alliedsignal: $412{ }^{\circ} \mathrm{C}\left(774{ }^{\circ} \mathrm{F}\right)$

for 500 and $160 \mathrm{~m} 7$ flasks

A7tiedsigna7: $>250^{\circ} \mathrm{C}\left(>482^{\circ} \mathrm{F}\right)$

practical7y nonflammable

(withdrawn for revision of the classification system, category SBQT2)

appearance:

MSDS

Altiedsignal: colorless

9707

7112

9707

9707

9707

7115

7111

9707

UR 20

UL

MSDS

MSDS

MSDS

UL volatile Tiquid 

$\begin{array}{cc}\text { odor: A17iedsignal: faint ethereal } & \text { MSDS } \\ \text { and sweetish odor } & \text { MSDS }\end{array}$

PRODUCTION

first commercial use as a refrigerant: projected: 2001-2003

last year production allowed: unrestricted 


\section{R-290 (PROPANE)}

$\begin{array}{ll}\mathrm{R}-290 & \text { propane } \\ \mathrm{HC} & \mathrm{CH} 3 \mathrm{CH} 2 \mathrm{CH} 3\end{array}$

REFRIGERANT DATA SUMMARY

CAS number 74-98-6

\section{COMMON USE(S)}

blend component for alternatives to refrigerants 12,22 , and 502 including 402A, 402B, 403A, and 403B; limited use in industrial refrigeration; primary use is as a fuel as a principal component of Tiquified petoleum gas (LPG); feedstock for organic synthesis of other compounds; thermobuTb fi77 for temperature and pressure control sensors; selective solvent for removal of anphaltic components from high-boiting fractions of crude oil

Natural propane contains approximately $96 \%$ actual propane and $4 \%$ other hydrocarbons and contaminants. Some commercial grades of propane include 0-50 ppm $\mathrm{V} / \mathrm{v}$ oderants such as methy 7 mercaptan and thiopane.

\section{IDENTIFIERS}

$$
\text { common name (s): }
$$

chemical name (by IUPAC convention): alternative chemical names/formulae:

empirical formula: CAS number: EINECS number:

NIOSH RTECS number: trade name(s):

ARI container color / Pantone number:
$R-290 ; R 290 ; R 290$
$\mathrm{HC}-290$
bottled gas
hydrocarbon 290
$\mathrm{n}-\mathrm{C} 3 ; \mathrm{n}-\mathrm{C} 3 \mathrm{H} 8$

n-propane; normal-propane

propane

dimethy 7 methane

dimethylmethane; $\mathrm{CH} 2(\mathrm{CH} 3)_{2}$ propy 7 hydride

$\mathrm{CH} 3 \mathrm{CH} 2 \mathrm{CH}_{3} ; \mathrm{CH}_{3}-\mathrm{CH} 2-\mathrm{CH} 3$

\section{$\mathrm{C} 3 \mathrm{H} 8$}

74-98-6 (Chemical Abstracts

Service registry number)

200-827-9 (European Inventory

of Existing Chemical

Substances)

TX2275000 (Registry of Toxic Effects of Chemical Substances)

\section{Calor Gas (UK) CARE 40}

Ecozone BV (NL) ECOOT-PRO

none, use Tight green grey/413 6601

with red / 185 band

PHYSICAL

- properties

molar mass:

normal freezing/melting/triple point:

$44.09562 \mathrm{~g} / \mathrm{mol}(0.097214$

$\mathrm{ib} / \mathrm{mol}$ )

$-187.3^{\circ} \mathrm{C}\left(-305.1{ }^{\circ} \mathrm{F}\right)$

8820

normal boiling point

temperature:

density, saturated liquid:

$-42.1{ }^{\circ} \mathrm{C}\left(-43.8^{\circ} \mathrm{F}\right)$

8401

density, saturated vapor:

specific volume, saturated liquid:

$581 \mathrm{~kg} / \mathrm{m} 3(36.29 \mathrm{lb} / \mathrm{cf})$

$2.42 \mathrm{~kg} / \mathrm{m} 3(0.151 \mathrm{lb} / \mathrm{cf})$

$1.721 \mathrm{~L} / \mathrm{kg}(0.0276 \mathrm{cf} / \mathrm{lb})$

$413.4 \mathrm{~L} / \mathrm{kg}(6.6225 \mathrm{cf} / 1 \mathrm{~b})$

heat of vaporization:

velocity of sound, saturated liquid:

$425.4 \mathrm{~kJ} / \mathrm{kg}(182.9 \mathrm{Btu} / 7 \mathrm{~b})$

$1158 \mathrm{~m} / \mathrm{s}(3800 \mathrm{ft} / \mathrm{s})$

$218 \mathrm{~m} / \mathrm{s}(716 \mathrm{ft} / \mathrm{s})$

$197 \mu \mathrm{Pa} \cdot \mathrm{s}(0.197 \mathrm{cp})$

$6.47 \mu \mathrm{Pa} \cdot \mathrm{s}(0.00647 \mathrm{cp})$

8401

8401

8401

8401

8401

8401

8401

8401

viscosity, saturated liquid:

viscosity, saturated vapor:

8401

8401 
thermal conductivity, Tiquid:

thermal conductivity, vapor:

- normal pressure, $20{ }^{\circ} \mathrm{C}\left(68^{\circ} \mathrm{F}\right)$-.--density, vapor: - normal pressure, $21.1{ }^{\circ} \mathrm{C}\left(70^{\circ} \mathrm{F}\right)---$

- $20^{\circ} \mathrm{C}(68$

pressure, saturated vapor: density, saturated Tiquid: density, saturated vapor: specific volume, saturated liquid: specific volume, saturated vapor: velocity of sound, saturated 7iquid: velocity of sound, saturated vapor: viscosity, saturated liquid: viscosity, saturated vapor: thermal conductivity, saturatd 1iquid: thermal conductivity, saturated vapor: heat of vaporization:

- critical point

temperature: pressure: density: specific volume:
$0.1294 \mathrm{~W} / \mathrm{m} \cdot \mathrm{K} \quad(0.0747$

8401

$\mathrm{Btu} / \mathrm{hr} \bullet \mathrm{ft}^{\circ} \mathrm{F}$ )

$0.0114 \mathrm{~W} / \mathrm{m} \cdot \mathrm{K}(0.0066$

$\mathrm{Btu} / \mathrm{hr} \bullet \mathrm{ft}^{\circ} \mathrm{F}$ )

$1.865 \mathrm{~kg} / \mathrm{m} 3(0.1164 \mathrm{lb} / \mathrm{cf})$

8401

$1.858 \mathrm{~kg} / \mathrm{m} 3(0.1160 \mathrm{~Tb} / \mathrm{cf})$

8401

$836.2 \mathrm{kPa}$ (121.29 psia)

$500 \mathrm{~kg} / \mathrm{m} 3$ (31.23 7b/cf)

$18.10 \mathrm{~kg} / \mathrm{m} 3 \quad(1.130 \mathrm{~Tb} / \mathrm{cf})$

$1.999 \mathrm{~L} / \mathrm{kg}(0.0320 \mathrm{cf} / 7 \mathrm{~b})$

$55.2 \mathrm{~L} / \mathrm{kg}(0.8850 \mathrm{cf} / 7 \mathrm{~b})$

$754 \mathrm{~m} / \mathrm{s}(2473 \mathrm{ft} / \mathrm{s})$

$217 \mathrm{~m} / \mathrm{s}(711 \mathrm{ft} / \mathrm{s})$

$102 \mu \mathrm{Pa} \cdot \mathrm{s}(0.102 \mathrm{cp})$

$8.5 \mu \mathrm{Pa} \cdot \mathrm{s}(0.0085 \mathrm{cp})$

$0.0954 \mathrm{~W} / \mathrm{m} \cdot \mathrm{K}(0.0551$

$\mathrm{Btu} / \mathrm{hr} \cdot \mathrm{ft}^{\circ} \mathrm{F}$ )

$0.01838 \mathrm{~W} / \mathrm{m} \cdot \mathrm{K}(0.01062$

$\mathrm{Btu} / \mathrm{hr} \bullet \mathrm{ft}^{\circ} \mathrm{F}$ )

8401

8401

8401

8401

8401

8401

8401

8401

8401

8401

8401

$2116 \mathrm{kPa}$ (306.9 psia)

8401

$258.4 \mathrm{~kJ} / \mathrm{kg}$ (111.1 Btu/7b)

8401

$96.7^{\circ} \mathrm{C}\left(206.1^{\circ} \mathrm{F}\right)$

7713

$4248 \mathrm{kPa}$ (616.1 psia)

8401

7713

7713

$4.54 \mathrm{~L} / \mathrm{kg}(0.0726 \mathrm{cf} / 7 \mathrm{~b})$

ENVIRONMENTAL

ODP (ozone depletion potential):

GWP (global warming potential):

0.000 (mode1-derived relative

to $R$ 11)

unknown, but very low: $\sim 20$

relative to $\mathrm{CO} 2$ for $100 \mathrm{yr}$

integration

HGWP (ha7ocarbon GWP):

$\sim 0$ relative to $\mathrm{R} 11$ for

infinite integration period

photochemical reactivity (grnd level):

220 relative to methane

4511

SAFETY

- classification

safety group (ASHRAE Standard 34): NFPA 704 degrees of hazard $(H-F-R-S)$ :

NPCA HMIS hazard ratings $(H-F-R)$ :

UL Comparative Hazard to Life Group:

- short-term occupational limit

NIOSH IDLH (immediately dangerous):

NIOSH SCP IDLH (immediately dangerous:

- occupational exposure limit .......

MAK (maximum workplace concentration):

- Tong-term occupational Timit -------

A3

BOC Gases: 1-4-0

8601

Texaco: 1-4-0

health-f1 ammabi 7 ity-reactivity

[-special]: $0=$ no, $4=$ severe

BOC Gases: 1-4-0

Texaco: 1-4-0

health-flammabi 1ity-reactivity

$0=$ insignificant, $4=e x t r e m e$

5 (b) in absence of flame or 4864 hot objects

MSDS

MSDS

$2,100 \mathrm{ppm} \mathrm{v} / \mathrm{v}$ based on $10 \%$ of 5204

LEL

$20,000 \mathrm{ppm} \mathrm{v} / \mathrm{v}$ for $30 \mathrm{~min}$

3903

IV: 2,000 ppm $\mathrm{v} / \mathrm{v}$ momentary 605561 min

OSHA PEL (permissible exposure limit):

$1,000 \mathrm{ppm} \mathrm{v} / \mathrm{v}$ TWA for $8 \mathrm{hr} /$ day 3904 and $40 \mathrm{hr} / \mathrm{wk}$ 
NIOSH REL (recommendd exposure Timit): $1,000 \mathrm{ppm} \mathrm{V} / \mathrm{V}$ TWA for 10

ACGIH TLV-TWA (time-weighted average):

$\mathrm{hr} /$ day and $40 \mathrm{hr} / \mathrm{wk}$

9504 $2,500 \mathrm{ppm} \mathrm{v/v}$

exposure Timit consistent to OSHA PEL:

Exxon: $1,000 \mathrm{ppm} \mathrm{V} / \mathrm{V}$ TWA for 8 MSDS $\mathrm{hr} /$ day and $40 \mathrm{hr} / \mathrm{wk}$

Texaco: 1,000 ppm $\mathrm{V} / \mathrm{v}$ TWA for MSDS

$8 \mathrm{hr} /$ day and $40 \mathrm{hr} / \mathrm{wk}$

\section{MAK (maximum workplace concentration): $\begin{aligned} 1,000 \mathrm{ppm} v / \mathrm{v} \text { TWA } \\ 40 \text { (or } 42 \text { ) } \mathrm{hr} / \mathrm{wk}\end{aligned}$} 40 (or 42) $\mathrm{hr} / \mathrm{wk}$ being examined for changes

7101

- emergency exposure Timit Acute Toxicity Exposure Limit (ATEL):

$50,000 \mathrm{ppm} v / \mathrm{v}$ (preTiminary based on ASHRAE $34 \mathrm{u}$ draft) $50,000 \mathrm{ppm} v / \mathrm{V}$ (pretiminary
based on ASHRAE 34u draft)

$34 \mathrm{u}$

Refrigerant Concentration Limit ( $R C L$ ): 5,000 ppm v/v (preliminary based on ASHRAE $34 \mathrm{u}$ draft) $5,000 \mathrm{ppm} v / v$ (preliminary based on ASHRAE $34 \mathrm{u}$ draft)

- acute (short-term) toxicity LC50 (lethal concentration, 50\%):

ALC (approximate lethal concentration:

rat, $15 \mathrm{~min}:>800,000 \mathrm{ppm}$

(fata 7 concentration by inhalation for half of test animals)

$34 \mathrm{u}$

A950

rat, $4 \mathrm{hr}, 0 / 10$ : >12,190 ppm

(lowest exposure tested with one or more deaths by inhalation)

derma7 LD50 (7etha7 dosage, 50\%): $>100,000 \mathrm{mg} / \mathrm{kg}$ (fatal dose by skin contact for half of test anima 75 )

cardiac sensitization (CS) EC50:

dog, 5 min: $180,000 \mathrm{ppm} \mathrm{v/v}$ (effective concentration in half of test animals)

cardiac sensitization thresho7d/LOEL: dog, 5 min, 2/12: 100,000 ppm $v / v$ (lowest observed effect leve 7 in test animals) cardiac sensitization (CS) NOEL: dog, 5 min, 0/6: 50,000 ppm $v / v$ (no observed effect leve] in test animals)

dog, $5 \mathrm{~min}, 0 / 6: 50,000 \mathrm{ppm}$ $v / v$ ' (no observed effect level in test anima7s)

anesthetic/CNS effect EC50: rat, 10min: depressant 280,000 $\mathrm{ppm} v / \mathrm{v}$ (effective concentration in half of test anima 1s)

anesthetic/CNS effect LOEL: animal?, few min, ?/?: 100,000 A623 ppm $v / v$ (lowest observed effect level in test animals) anesthetic/CNS effect NOEL: animaT?, "brief", ?/?: 10,000 $\mathrm{ppm} v / \mathrm{v}$ (no observed effect Tevel in test animals) anima7?, $10 \mathrm{~min}, 0 /$ ?: 10,000 $\mathrm{ppm} \mathrm{v} / \mathrm{v}$ (no observed effect Teve] in test animals)

- flammability LFL-UFL (flammability limits in air):

$\begin{array}{ll}2.1-9.5 \% \mathrm{v} / \mathrm{v} & \\ 2.1-9.6 \% \mathrm{v} / \mathrm{v} & 5204 \\ 2.2-9.5 \% \mathrm{v} / \mathrm{v} & 2525 \\ 2.37-9.5 \% \mathrm{v} / \mathrm{v} & \mathrm{A} 38 \\ \text { Ecozone: } 2.0-10.0 \% \mathrm{v} / \mathrm{v} & 6290 \\ \text { Texaco: } 2.3-9.5 \% \mathrm{v} / \mathrm{v} & \mathrm{mfr} \\ & \text { MSDS }\end{array}$




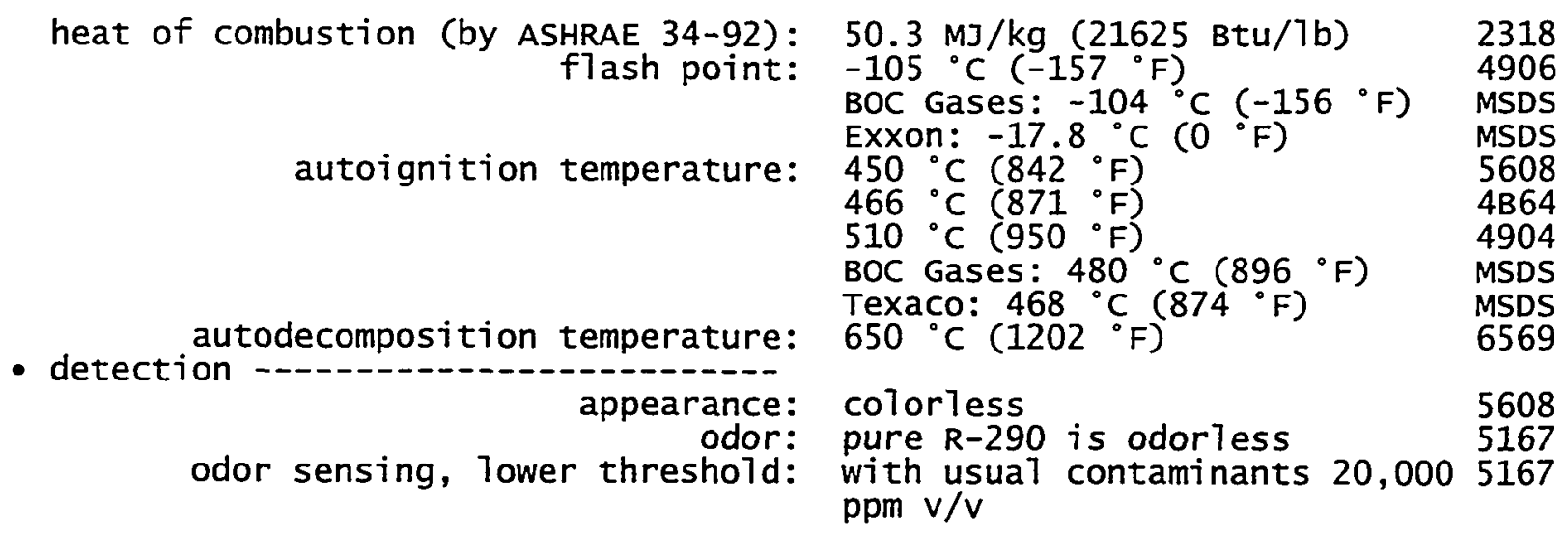

Tast year production allowed: unrestricted $\quad 8 \mathrm{CO}$ 


\section{R-C318}

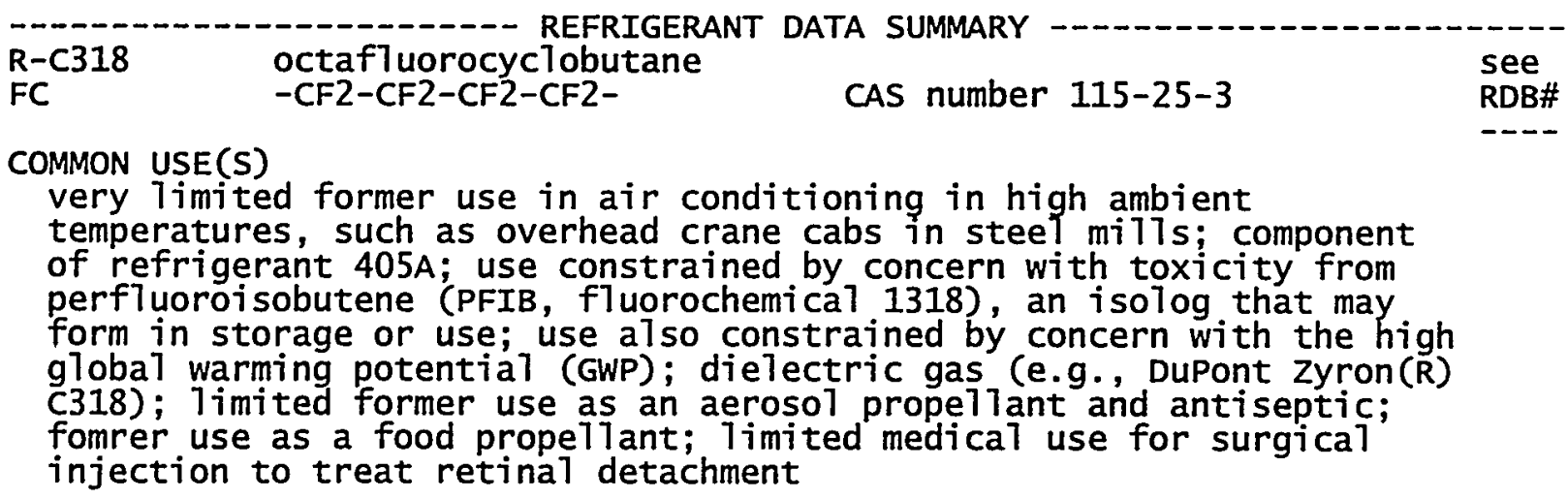

Note: Based on an application submitted in May 1998 and a revision submitted in May 1999, SSPC 34 proposed a safety classification of A1 on 20 June 1999. The proposed classification is subject to a review and approva 1 procedure now underway; actua 7 assignment wi 71 not occur until published in an addendum or revision to ANSI/ASHRAE Standard 34-1997.

IDENTIFIERS

Common name $(s): R-C 318 ; R C 318 ; R$ C318

$\mathrm{FC}-318$

"PFC-C318"

OFCB

f7uorocarbon $\mathrm{C} 318$

f7uorochemica 7 C318; FC C318

halochemical $\mathrm{C} 318$

prope 7 Tant C318

chemical name (by IUPAC convention): octafluoroyclobutane

perfluoroyclobutane

alternative chemical names/formulae:

empirical formula:

cyclobutane, octafiuoro-

perfluorocyc7obutane

cyclooctafluorobutane

octafluoroycloalkane

perfluorocycloalkane

-CF2-CF2-CF2-CF2-

-CF2CF2CF2CF2-

C-CF2-CF2-CF2-CF2-

CyC10-CF2-CF2-CF2-CF2-

not recommended:

C-C4F8; CyC7ic C4F8; - (CF2)4-

CAS number: 115-25-3 (Chemical Abstracts

Service registry number)

NIOSH RTECS number: GU1779500 (Registry of Toxic

Effects of Chemical

Substances)

trade name(s): BNFL Flutec(R) "R-318"

Daikin Daiflon $(R)$ C318

DuPont Freon $(R) C-318$

Hoechst Frigen(R) C318

MSDS

ARI container color / Pantone number: none, use light green grey/413 6601

PHYSICAL

- properties

molar mass: $200.0300256 \mathrm{~g} / \mathrm{mo}$ ( 0.440991

8820 
normal freezing/melting/triple point:

- normal boiling point -rerat density, saturated 7 iquid: density, saturated vapor: specific volume, saturated liquid: specific volume, saturated vapor: heat of vaporization: velocity of sound, saturated liquid: velocity of sound, saturated vapor: viscosity, saturated vapor: viscosity, saturated 1iquid: thermal conductivity, 1iquid:

thermal conductivity, vapor:

- normal pressure, $20^{\circ} \mathrm{C}\left(68^{\circ} \mathrm{F}\right)$

- normal pressure, $21.1{ }^{\circ} \mathrm{C}\left(70^{\circ} \mathrm{F}\right)$

- $20^{\circ} \mathrm{C}\left(68^{\circ} \mathrm{F}\right)$ density, vapor pressure, saturated vapor: density, saturated 1 iquid: density, saturated vapor:

specific volume, saturated 1 iquid: specific volume, saturated vapor: velocity of sound, saturated liquid: velocity of sound, saturated vapor: viscosity, saturated liquid: viscosity, saturated vapor: thermal conductivity, saturatd 1 iquid:

thermal conductivity, saturated vapor:

- $60^{\circ} \mathrm{C}\left(140{ }^{\circ} \mathrm{F}\right)$ pressure, saturated vapor: heat of vaporization:

- critical point -

temperature: pressure: density: specific volume: $-39.8^{\circ} \mathrm{C}\left(-39.6^{\circ} \mathrm{F}\right)$

8401

$-6.0^{\circ} \mathrm{C}\left(21.2^{\circ} \mathrm{F}\right)$

$1615 \mathrm{~kg} / \mathrm{m} 3(100.83 \mathrm{lb} / \mathrm{cf})$

$9.54 \mathrm{~kg} / \mathrm{m} 3(0.596 \mathrm{lb} / \mathrm{cf})$

$0.619 \mathrm{~L} / \mathrm{kg}(0.0099 \mathrm{cf} / 7 \mathrm{~b})$

$104.8 \mathrm{~L} / \mathrm{kg}(1.6788 \mathrm{cf} / 7 \mathrm{~b})$

$116.8 \mathrm{~kJ} / \mathrm{kg}(50.2 \mathrm{Btu} / \mathrm{lb})$

$553 \mathrm{~m} / \mathrm{s}(1814 \mathrm{ft} / \mathrm{s})$

$104 \mathrm{~m} / \mathrm{s}(342 \mathrm{ft} / \mathrm{s})$

$10.22 \mu \mathrm{pa} \cdot \mathrm{s}(0.01022 \mathrm{cp})$

$341 \mu \mathrm{Pa} \cdot \mathrm{s}(0.341 \mathrm{cp})$

$0.0567 \mathrm{~W} / \mathrm{m} \cdot \mathrm{K}(0.0328$

$\left.\mathrm{Btu} / \mathrm{hr} \cdot \mathrm{ft}{ }^{\circ} \mathrm{F}\right)$

$0.0102 \mathrm{~W} / \mathrm{m} \cdot \mathrm{K}(0.0059$

$\left.\mathrm{Btu} / \mathrm{hr} \bullet \mathrm{ft}^{\circ} \mathrm{F}\right)$

$8.588 \mathrm{~kg} / \mathrm{m} 3(0.5362 \mathrm{~Tb} / \mathrm{cf}) \quad 8401$

$8.552 \mathrm{~kg} / \mathrm{m} 3(0.5339 \mathrm{1b} / \mathrm{cf})$

8401

$265.5 \mathrm{kPa}(38.51 \mathrm{psia})$

$1518 \mathrm{~kg} / \mathrm{m} 3(94.78 \mathrm{~Tb} / \mathrm{cf})$

$23.93 \mathrm{~kg} / \mathrm{m} 3(1.494 \mathrm{~Tb} / \mathrm{cf})$

$0.659 \mathrm{~L} / \mathrm{kg}(0.0106 \mathrm{cf} / 7 \mathrm{~b})$

$41.8 \mathrm{~L} / \mathrm{kg}(0.6695 \mathrm{cf} / 7 \mathrm{~b})$

$456 \mathrm{~m} / \mathrm{s}(1495 \mathrm{ft} / \mathrm{s})$

$104 \mathrm{~m} / \mathrm{s}(342 \mathrm{ft} / \mathrm{s})$

$243 \mu \mathrm{Pa} \cdot \mathrm{s}(0.243 \mathrm{cp})$

$11.2 \mu \mathrm{Pa} \cdot \mathrm{s}(0.0112 \mathrm{cp})$

$0.0512 \mathrm{~W} / \mathrm{m} \cdot \mathrm{K}(0.0296$

$\mathrm{Btu} / \mathrm{hr} \cdot \mathrm{ft}^{\circ} \mathrm{F}$ )

$0.01191 \mathrm{~W} / \mathrm{m} \cdot \mathrm{K}(0.00688$

$\left.B t u / h r \cdot f t^{\circ} F\right)$

$839 \mathrm{kPa}$ (121.7 psia) 8401

$86.0 \mathrm{~kJ} / \mathrm{kg}(37.0 \mathrm{Btu} / \mathrm{lb}) \quad 840 \mathrm{I}$

$115.2{ }^{\circ} \mathrm{C}\left(239.4^{\circ} \mathrm{F}\right)$

$2778 \mathrm{kPa}(402.9 \mathrm{psia})$

$620 \mathrm{~kg} / \mathrm{m} 3(38.7 \mathrm{ib} / \mathrm{cf})$

8401

8401

$1.61 \mathrm{~L} / \mathrm{kg}(0.0258 \mathrm{cf} / 7 \mathrm{~b}) \quad 8401$

$3200 \mathrm{yr}$

0.000 (model-derived relative

6694

to $\mathrm{R}$ 1I)

11,200 relative to $\mathrm{CO} 2$ for 1009501

GWP (global warming potential): yr integration

SAFETY

- Classification -

safety group (ASHRAE standard 34):

NFPA 704 degrees of hazard (H-F-R-S):

NPCA HMIS hazard ratings $(H-F-R)$ :

none (application pending)

$34 \mathrm{c}$

Al proposed 1999.06.20

BOC Gases: 0-0-0

$34 q$

hea 7 th-flammability-reactivity [-specia1]: $0=$ no, $4=$ severe

BOC Gases: $0-0-0$

DuPont: $1-0-1$

hea 1th-flammability-reactivity

$0=$ insignificant, $4=$ extreme

UL Comparative Hazard to Life Group:

6 by estimate (not tested) in 5906 absence of flame or hot

objects 
- Tong-term occupational limit exposure Timit consistent to OSHA PEL:

- emergency exposure limit Acute Toxicity Exposure Limit (ATEL):

Refrigerant concentration Limit (RCL):

- acute (short-term) toxicity LC50 (lethal concentration, $50 \%$ ):

ALC (approximate Tethal concentration:

LCLo (Tetha] concentration, Towest):

cardiac sensitization (CS) EC50:

cardiac sensitization threshold/LOEL:

anesthetic/CNS effect EC50:

anesthetic/CNS effect NOEL:

- flammability

LFL-UFL (flammability Timits in air): flash point: autoignition temperature:

- detection -autoignition temperature: odor:
DuPont AEL: $1,000 \mathrm{ppm} v / \mathrm{V}$ TWA MSDS for $8 \mathrm{hr} /$ day and $40 \mathrm{hr} / \mathrm{wk}$

$80,000 \mathrm{ppm} \mathrm{v/v} \mathrm{(preliminary} 34 \mathrm{u}$ based on ASHRAE $34 \mathrm{u}$ draft) $80,000 \mathrm{ppm} \mathrm{V} / \mathrm{V}$ (preliminary based on ASHRAE 34u draft) A950

$69,000 \mathrm{ppm} v / \mathrm{v}$ (preliminary based on ASHRAE $34 u$ draft) $69,000 \mathrm{ppm} \mathrm{v} / \mathrm{v}$ (preliminary based on ASHRAE $34 u$ draft)

$34 \mathrm{u}$

A950

rat, $4 \mathrm{hr}:>800,000$ ppm (fatal 5169

concentration by inhalation

for half of test animals)

rat, $4 \mathrm{hr}, 0 / 2$ : 792000-804000

7736

ppm (lowest exposure tested with one or more deaths by inhalation)

rat, $4 \mathrm{hr}, 0 / 6:>800,000 \mathrm{ppm}$ (lowest exposure tested with one or more deaths by inhalation)

mouse, $2 \mathrm{hr}$, BOC Gases: 780,000 MSDS ppm (fatal concentration by inhalation for even one test anima1)

dog, 5 min: $250,000-500,000$

5644

ppm $v / v$ (effective

concentration in half of test anima7s)

dog; 5 min, 1/12: 100,000 ppm 5644

$v / v$ (lowest observed effect

level in test anima7s)

dog, 5 min, 1/12: 100,000 ppm

$v / v$ (lowest observed effect

level in test animals)

dog, 5 min, 1/6: 150,000 ppm $v / v$ (lowest observed effect

Teve 1 in test anima 15 )

mouse, $10 \mathrm{~min}, 0 / 6$ : $>800,000$

ppm $v / v$ (effective

concentration in half of test anima7s)

mouse, $10 \mathrm{~min}, 0 / 6$ : > 798,000

ppm $v / v$ (effective

concentration in half of test anima 7s)

rat, $4 \mathrm{hr}, 0 / 6: 800,000 \mathrm{ppm}$

$v / v$ (no observed effect level in ALC or LC50 studies)

6121

$5 A 25$

7736

none (nonflammable as tested)

BOC Gases: none

TOC DuPont: will not burn

BOC Gases: none MSDS

DuPont: clear colorless gas MSDS

BOC Gases: odorless

DuPont: none

Liquid Carbonic: slight7y MSDS

ethereal 
Toxicity Data to Determine Refrigerant Concentration Limits James M. Calm, Engineering Consultant

\section{PRODUCTION}

first commercial use as a refrigerant: 1961

last year production allowed: unrestricted

$5 C 39$

$8 \mathrm{CO}$

$-242-$ 


\section{R-500}

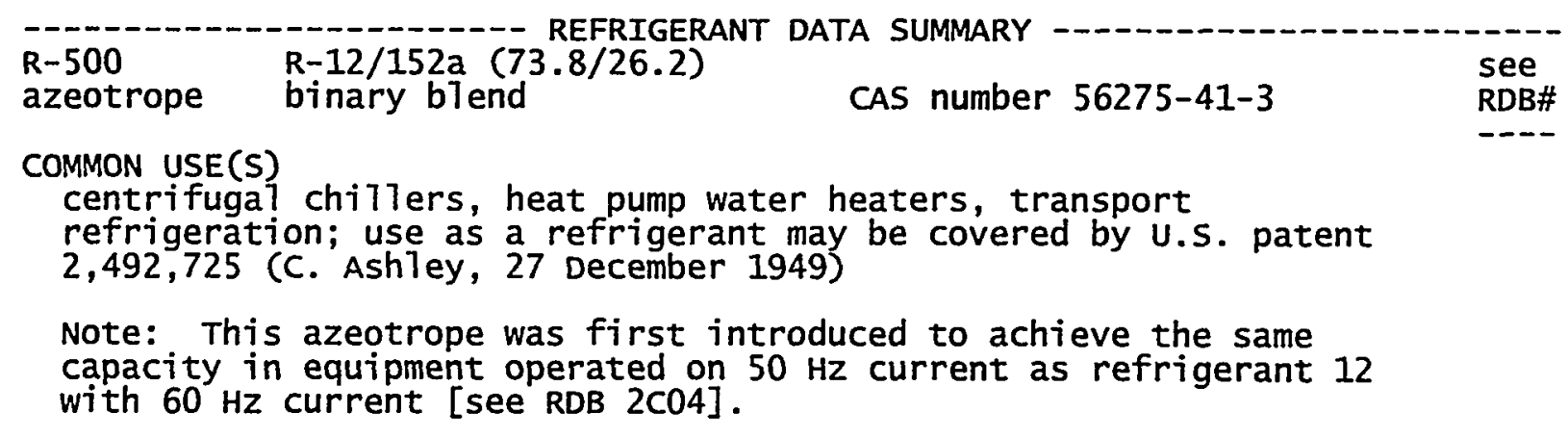

IDENTIFIERS

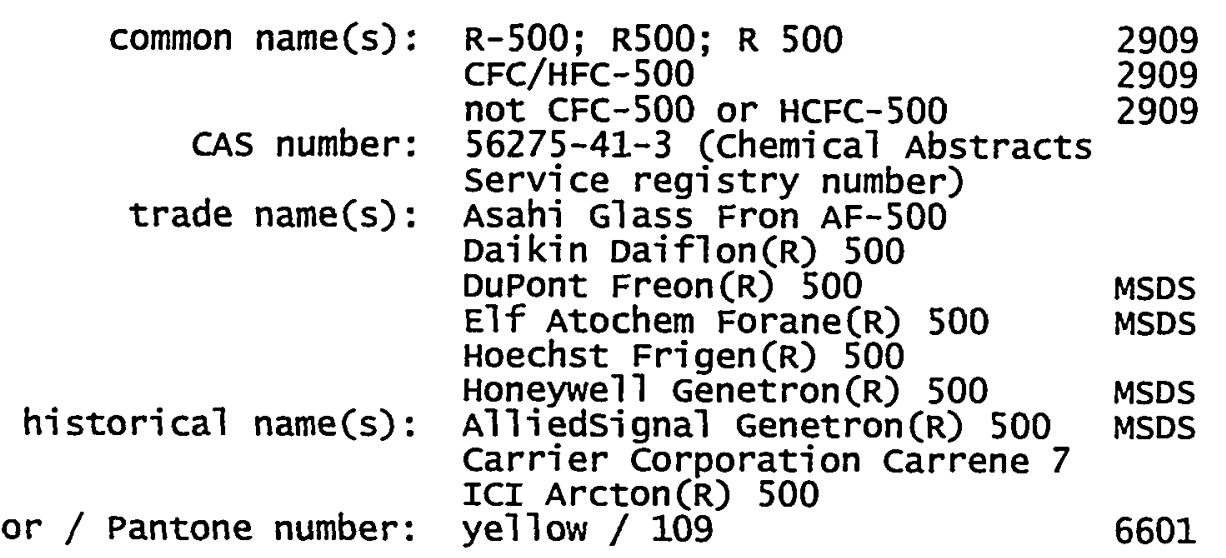

ARI container color / Pantone number: yellow / 109 6601

PHYSICAL

- nominal blend formulation

composition: component weight fractions: component mole fractions:

- properties azeotropic temperature:

$\mathrm{R}-12 / 152 \mathrm{a}$

$73.8 / 26.2 \%$

$60.610 / 39.390 \%$

$0.0^{\circ} \mathrm{C}\left(32.0^{\circ} \mathrm{F}\right)$

$99.30230 \mathrm{~g} / \mathrm{mol} \quad(0.218924$

7b/mo T)

$-158.9^{\circ} \mathrm{C}\left(-254.0^{\circ} \mathrm{F}\right)$

$-33.6^{\circ} \mathrm{C}\left(-28.5^{\circ} \mathrm{F}\right)$

$-33.6^{\circ} \mathrm{C}\left(-28.5{ }^{\circ} \mathrm{F}\right)$

$0.00^{\circ} \mathrm{C}\left(0.0^{\circ} \mathrm{F}\right)$

$1319 \mathrm{~kg} / \mathrm{m3}(82.35 \mathrm{jb} / \mathrm{cf})$

$5.27 \mathrm{~kg} / \mathrm{m} 3$ (0.329 $\mathrm{ib} / \mathrm{cf})$

$0.758 \mathrm{~L} / \mathrm{kg}(0.0121 \mathrm{cf} / \mathrm{lb})$

$189.8 \mathrm{~L} / \mathrm{kg}(3.0400 \mathrm{cf} / 7 \mathrm{~b})$

$202.5 \mathrm{~kJ} / \mathrm{kg}(87.0 \mathrm{Btu} / \mathrm{lb})$

$762 \mathrm{~m} / \mathrm{s}(2502 \mathrm{ft} / \mathrm{s})$

$148 \mathrm{~m} / \mathrm{s}(484 \mathrm{ft} / \mathrm{s})$

$319 \mu \mathrm{Pa} \cdot \mathrm{s}(0.319 \mathrm{cp})$

$9.21 \mu \mathrm{Pa} \cdot \mathrm{s}(0.00921 \mathrm{cp})$

$0.0951 \mathrm{~W} / \mathrm{m} \cdot \mathrm{K}(0.0549$

$\left.\mathrm{Btu} / \mathrm{hr} \cdot \mathrm{ft}^{\circ} \mathrm{F}\right)$

$0.0076 \mathrm{~W} / \mathrm{m} \bullet \mathrm{K}(0.0044$

$\mathrm{Btu} / \mathrm{hr} \bullet \mathrm{ft}^{\circ} \mathrm{F}$ )

2909

2909

8820

2909

8820

0036

8401

8401

8401

8401

8401

8401

8401

8401

8401

8401

8401

8401

8401

8401

$4.213 \mathrm{~kg} / \mathrm{m} 3(0.2630 \mathrm{~Tb} / \mathrm{cf}) \quad 8401$ 
- normal pressure, $21.1{ }^{\circ} \mathrm{C}\left(70^{\circ} \mathrm{F}\right)$--pressure, Tiquid (bubble point): pressure, vapor (dew point): density, saturated Tiquid: density, saturated vapor: specific volume, saturated liquid: specific volume, saturated vapor: velocity of sound, saturated liquid: velocity of sound, saturated vapor: viscosity, saturated 1iquid: viscosity, saturated vapor: thermal conductivity, saturatd liquid:

thermal conductivity, saturated vapor:

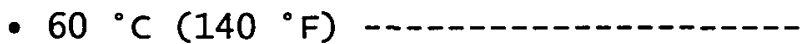
pressure, liquid (bubble point): pressure, vapor (dew point): heat of vaporization:

- critical point

temperature: pressure: density: specific volume:

ENVIRONMENTAL

ODP (ozone depletion potential):

GWP (global warming potential): HGWP (halocarbon GWP):
- $20^{\circ} \mathrm{C}\left(68^{\circ} \mathrm{F}\right)$ - density, vapor:

$4.196 \mathrm{~kg} / \mathrm{m} 3(0.2619 \mathrm{7b} / \mathrm{cf})$

8401

$670.6 \mathrm{kPa}$ (97.26 psia)

$668.8 \mathrm{kPa}(97.00 \mathrm{psia})$

$1158 \mathrm{~kg} / \mathrm{m} 3(72.32 \mathrm{7b} / \mathrm{cf})$

$32.02 \mathrm{~kg} / \mathrm{m} 3$ (1.999 $\mathrm{Tb} / \mathrm{cf})$

$0.863 \mathrm{~L} / \mathrm{kg}(0.0138 \mathrm{cf} / \mathrm{lb})$

$31.2 \mathrm{~L} / \mathrm{kg}(0.5003 \mathrm{cf} / 1 \mathrm{~b})$

$528 \mathrm{~m} / \mathrm{s}(1732 \mathrm{ft} / \mathrm{s})$

$148 \mathrm{~m} / \mathrm{s}(485 \mathrm{ft} / \mathrm{s})$

$172 \mu \mathrm{Pa} \cdot 5(0.172 \mathrm{cp})$

$11.4 \mu \mathrm{Pa} \cdot \mathrm{s}(0.0114 \mathrm{cp})$

$0.0744 \mathrm{~W} / \mathrm{m} \cdot \mathrm{K}(0.0430$

$\mathrm{Btu} / \mathrm{hr} \cdot \mathrm{ft}^{\circ} \mathrm{F}$ )

$0.01178 \mathrm{~W} / \mathrm{m} \cdot \mathrm{K} \quad(0.00681$

$\mathrm{Btu} / \mathrm{hr} \cdot \mathrm{ft}^{\circ} \mathrm{F}$ )

$1809 \mathrm{kPa}$ (262.4 psia)

$1801 \mathrm{kPa}$ (261.2 psia)

$129.7 \mathrm{~kJ} / \mathrm{kg}$ for Tiquid and

vapor both at nominal

composition (55.8 Btu/7b)

$127.1 \mathrm{~kJ} / \mathrm{kg}$ coexisting 7 iquid and vapor at bubble-point

pressure ( $54.6 \mathrm{Btu} / 7 \mathrm{~b})$

$102.1{ }^{\circ} \mathrm{C}\left(215.8^{\circ} \mathrm{F}\right)$

$4173 \mathrm{kPa}(605.2 \mathrm{psia})$

$492 \mathrm{~kg} / \mathrm{m} 3$ (30.7 ib/cf)

$2.03 \mathrm{~L} / \mathrm{kg}(0.0326 \mathrm{cf} / 7 \mathrm{~b})$

8401

8401

8401

8401

0.605 mass-weighted average (model-derived relative to $R$ 11)

0.664 mass-weighted average (semi-empirical relative to $R$ 11)

7870 mass-weighted average relative to $\mathrm{CO} 2$ for $100 \mathrm{yr}$ integration

2.0 mass-weighted average relative to $\mathrm{R} 11$ for infinite integration period 2.2 mass-weighted average relative to $R 11$ for infinite integration period

9501

9501

9501

7214

DW

A1

ARI recommendation: 2-0-0 3A15 Honeywe 77: 2-1-0 MSDS health-flammability-reactivity [-specia]]: $0=$ no, $4=$ severe

NPCA HMIS hazard ratings (H-F-R): DuPont: $1-0-1$

MSDS Honeywe 17: 1-1-0 hea 7 th-flammabi 1ity-reactivity $0=$ insignificant, $4=$ extreme

UL Comparative Hazard to Life Group: 5 (a) in absence of flame or hot objects

- short-term occupational Timit ARI "IDLH" recommendation:
$50,000 \mathrm{ppm} \mathrm{v} / \mathrm{v}$ for $30 \mathrm{~min}$ 
- Tong-term occupational Timit -.---exposure Timit consistent to OSHA PEL:

ARI: $1,000 \mathrm{ppm} \mathrm{v} / \mathrm{V}$ TWA for 83 A 315 $\mathrm{hr} /$ day and $40 \mathrm{hr} / \mathrm{wk}$

components are PEL $1,000 / 3904$

WEEL $1,000 \mathrm{ppm} \mathrm{V} / \mathrm{V}$ TWA for $84 \mathrm{~B} 90$

- emergency exposure Timit $\mathrm{hr} /$ day and $40 \mathrm{hr} / \mathrm{wk}$

Acute Toxicity Exposure Limit (ATEL): $29,000 \mathrm{ppm} \mathrm{v} / \mathrm{v}$ (preliminary

A950

Refrigerant Concentration Limit ( $R C L$ ): based on ASHRAE 34u draft)

29,000 ppm v/v (preliminary based on ASHRAE 34u draft)

$36,000 \mathrm{ppm} v / \mathrm{v}$ (preliminary based on ASHRAE $34 u$ draft)

A950

$34 u$

- flammability

LFL-UFL (flammability limits in air):

none (nonflammable as tested) 0036

none (nonflammable as tested) 5648

flash point:

DuPont: wi 77 not burn

MSDS

autoignition temperature: Honeywe 17: gas, not applicable MSDS $386^{\circ} \mathrm{C}\left(727^{\circ} \mathrm{F}\right)$ autodecomposition temperature: former UL Classification:

DuPont: $<445^{\circ} \mathrm{C}\left(<833^{\circ} \mathrm{F}\right) \quad$ MSDS

practica7ly nonflammable 6938

(withdrawn for revision of the classification system, category SBQT2)

- detection

appearance:

odor:

DuPont: clear, colorless

Honeywe 71: faint ethereal

MSDS

MSDS

PRODUCTION

first commercial use as a refrigerant:

last year production allowed:

circa 1950

1995 based on refrigerant $12 \quad 8 \mathrm{CO}$ in developed countries under the Montreal Protocol 


\section{R-502}

R-502
azeotrope binary b7end $\quad$ CAS number $39432-81-0$

IDENTIFIERS

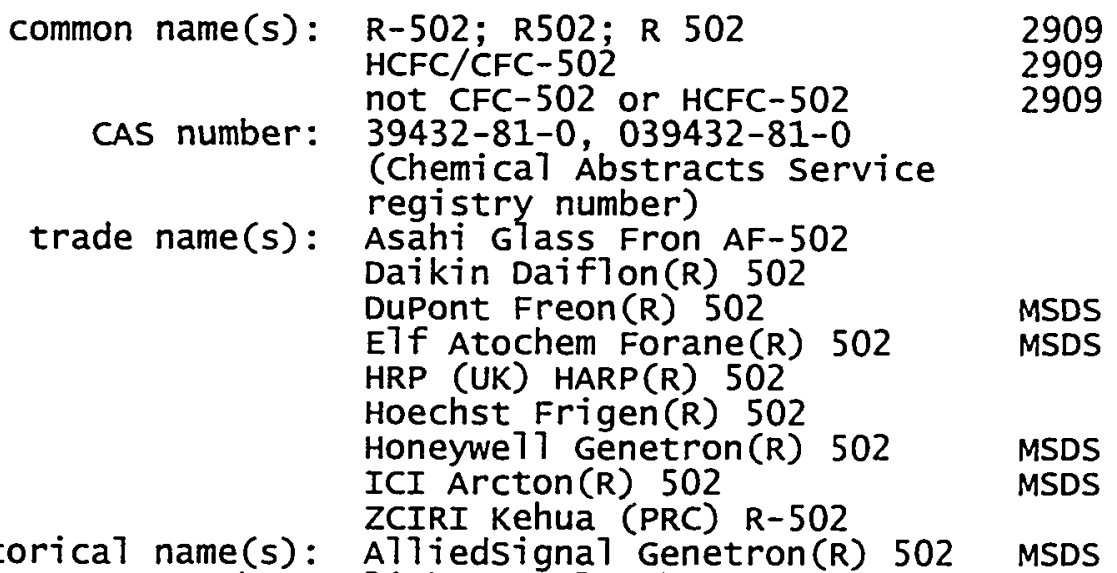

historical name(s): Al7iedsignal Genetron(R) 502 MSDS ARI container color / Pantone number: Tight purple (lavender) / 2516601

PHYSICAL

- nominal blend formutation

composition: component weight fractions: component mole fractions: azeotropic temperature:

- properties

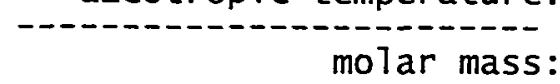

- normal boiling point -------bubble point temperature: dew point temperature: maximum temperature glide: density, saturated 7iquid: density, saturated vapor: specific volume, saturated Tiquid: specific volume, saturated vapor: heat of vaporization: velocity of sound, saturated 7iquid: velocity of sound, saturated vapor: viscosity, saturated Tiquid: viscosity, saturated vapor: thermal conductivity, Jiquid:

thermal conductivity, vapor:

- normal pressure, $20^{\circ} \mathrm{C}\left(68^{\circ} \mathrm{F}\right)----$

- normal pressure, $21.1{ }^{\circ} \mathrm{C}\left(70^{\circ} \mathrm{F}\right)$. density, vapor:
$\mathrm{R}-22 / 115$

$48.8 / 51.2 \%$

$62.999 / 37.001 \%$

$19.0{ }^{\circ} \mathrm{C}\left(66.2{ }^{\circ} \mathrm{F}\right)$

$111.62783 \mathrm{~g} / \mathrm{mo}$ ( 0.246097 $\mathrm{Tb} / \mathrm{mo} \mathrm{T})$

$-45.3^{\circ} \mathrm{C}\left(-49.5^{\circ} \mathrm{F}\right)$

$-45.0^{\circ} \mathrm{C}\left(-49.0^{\circ} \mathrm{F}\right)$

$0.04^{\circ} \mathrm{C}\left(0.1^{\circ} \mathrm{F}\right)$

$1485 \mathrm{~kg} / \mathrm{m} 3(92.72 \mathrm{1b} / \mathrm{cf})$

$6.21 \mathrm{~kg} / \mathrm{m} 3(0.388 \mathrm{1b} / \mathrm{cf})$

$0.673 \mathrm{~L} / \mathrm{kg}(0.0108 \mathrm{cf} / \mathrm{lb})$

$161.0 \mathrm{~L} / \mathrm{kg}(2.5795 \mathrm{cf} / 7 \mathrm{~b})$

$173.3 \mathrm{~kJ} / \mathrm{kg} \mathrm{(74.5 \textrm {Btu } / 7 \mathrm { b } )}$

$728 \mathrm{~m} / \mathrm{s}(2387 \mathrm{ft} / \mathrm{s})$

$136 \mathrm{~m} / \mathrm{s}(445 \mathrm{ft} / \mathrm{s})$

$340 \mu \mathrm{Pa} \cdot \mathrm{s}(0.340 \mathrm{cp})$

$9.73 \mu \mathrm{Pa} \cdot \mathrm{s}(0.00973 \mathrm{cp})$

$0.0906 \mathrm{~W} / \mathrm{m} \cdot \mathrm{K}(0.0523$

$\left.\mathrm{Btu} / \mathrm{hr} \cdot \mathrm{ft}^{\circ} \mathrm{F}\right)$

$0.0070 \mathrm{~W} / \mathrm{m} \cdot \mathrm{K} \quad(0.0041$

$\left.\mathrm{Btu} / \mathrm{hr} \cdot \mathrm{ft}^{\circ} \mathrm{F}\right)$

$4.718 \mathrm{~kg} / \mathrm{m} 3(0.2945 \mathrm{~Tb} / \mathrm{cf})$

$4.699 \mathrm{~kg} / \mathrm{m} 3(0.2934 \mathrm{lb} / \mathrm{cf})$
8401

2909

2909

8820

2909

8820

8401

8401

8401

8401

8401

8401

8401

8401

8401

8401

8401

8401

8401

8401

8401 

pressure, Tiquid (bubble point): pressure, vapor (dew point): density, saturated liquid: density, saturated vapor: specific volume, saturated liquid: specific volume, saturated vapor: velocity of sound, saturated liquid: velocity of sound, saturated vapor: viscosity, saturated liquid: viscosity, saturated vapor: thermal conductivity, saturatd Tiquid: thermal conductivity, saturated vapor: - $60{ }^{\circ} \mathrm{C}\left(140{ }^{\circ} \mathrm{F}\right)$ pressure, liquid (bubble point): pressure, vapor (dew point): heat of vaporization:
- critical point

temperature: pressure: density: specific volume:

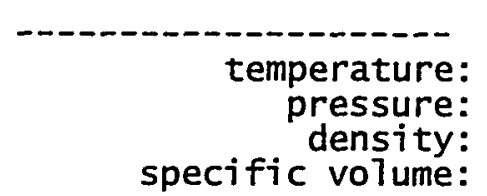

ENVIRONMENTAL

ODP (ozone depletion potential):

GWP (global warming potential): HGWP (halocarbon GWP):
1015.1 kPa (147.23 psia)

$1014.6 \mathrm{kPa}$ (147.16 psia)

$1237 \mathrm{~kg} / \mathrm{m} 3$ ( $77.20 \mathrm{lb} / \mathrm{cf})$

$57.93 \mathrm{~kg} / \mathrm{m3}(3.616 \mathrm{lb} / \mathrm{cf})$

$0.809 \mathrm{~L} / \mathrm{kg}(0.0130 \mathrm{cf} / 1 \mathrm{~b})$

$17.3 \mathrm{~L} / \mathrm{kg}(0.2765 \mathrm{cf} / 7 \mathrm{~b})$

$429 \mathrm{~m} / \mathrm{s}(1406 \mathrm{ft} / \mathrm{s})$

$132 \mathrm{~m} / \mathrm{s}(433 \mathrm{ft} / \mathrm{s})$

$152 \mu \mathrm{Pa} \cdot \mathrm{s}(0.152 \mathrm{cp})$

$12.7 \mu \mathrm{Pa} \cdot \mathrm{s}(0.0127 \mathrm{cp})$

$0.0649 \mathrm{~W} / \mathrm{m} \cdot \mathrm{K}(0.0375$

$\mathrm{Btu} / \mathrm{hr} \cdot \mathrm{ft}^{\circ} \mathrm{F}$ )

$0.01150 \mathrm{~W} / \mathrm{m} \cdot \mathrm{K}(0.00665$

$B t u / h r \cdot f t^{\circ} F$ )

$2630 \mathrm{kPa}$ (381.4 psia)

$2630 \mathrm{kPa}$ (381.4 psia)

$86.0 \mathrm{~kJ} / \mathrm{kg}$ for 7 iquid and vapor both at nominal composition (37.0 Btu/lb)

$86.2 \mathrm{~kJ} / \mathrm{kg}$ coexisting 7 iquid and vapor at bubble-point pressure ( $37.0 \mathrm{Btu} / \mathrm{/b})$

$80.7^{\circ} \mathrm{C}\left(177.3^{\circ} \mathrm{F}\right)$

$4018 \mathrm{kPa}(582.8$ psia)

$569 \mathrm{~kg} / \mathrm{m3}(35.5 \mathrm{ib} / \mathrm{cf})$

$1.76 \mathrm{~L} / \mathrm{kg}(0.0282 \mathrm{cf} / \mathrm{lb})$
8401

8401

8401

8401

8401

8401

8401

8401

8401

8401

8401

8401

8401

8401

8401

8401

8401

8401

8401

8401

0.221 mass-weighted average (model-derived relative to $R$ 11)

0.229 mass-weighted average (semi-empirical relative to $R$ 11)

6200 mass-weighted average relative to $\mathrm{CO} 2$ for $100 \mathrm{yr}$ integration

19 mass-weighted average relative to $R 11$ for infinite integration period

3.75 relative to $R 11$ for infinite integration period

9501

9501

9501

DW

4510

8601

A1

ARI recommendation: $2-0-0$ 3A15 Honeywe 17: 2-1-0 MSDS hea th-flammability-reactivity [-specia1]: $0=$ no, $4=$ severe

NPCA HMIS hazard ratings $(H-F-R)$ :

UL Comparative Hazard to Life Group:

- short-term occupational limit ARI "IDLH" recommendation:

- long-term occupational Timit -...... Dupont: 1-0-1

Honeywe 17: 1-1-0 health-f1 ammability-reactivity $0=$ insignificant, $4=$ extreme

5 (a) in absence of flame or 0036 hot objects

$50,000 \mathrm{ppm} v / \mathrm{v}$ for $30 \mathrm{~min}$

$3 \mathrm{~A} 15$

none, both components 1,000

5204

MSDS MSDS ppm v/v TWA for $10 \mathrm{hr} / \mathrm{day}$ and 
ACGIH TLV-TWA (time-weighted average):

exposure Timit consistent to OSHA PEL:

- emergency exposure limit Acute Toxicity Exposure Limit (ATEL):

Refrigerant Concentration Limit ( $R C L)$ :

- acute (short-term) toxicity LC50 (1 ethal concentration, 50\%):

ALC (approximate lethal concentration:

cardiac sensitization threshold/LOEL: cardiac sensitization (CS) NOEL:

- flammability

LFL-UFL (flammability limits in air):

flash point:

autoignition temperature: former UL Classification:

- detection

appearance: odor:

PRODUCTION

first commercial use as a refrigerant: last year production allowed:
$40 \mathrm{hr} / \mathrm{wk}$

none, both components 1,000

9504

$\mathrm{ppm} \mathrm{V} / \mathrm{v}$ TWA for $8 \mathrm{hr} /$ day and $40 \mathrm{hr} / \mathrm{wk}$

ARI: $1,000 \mathrm{ppm} v / \mathrm{v}$ TWA for 8

$\mathrm{hr} /$ day and $40 \mathrm{hr} / \mathrm{wk}$

35,000 ppm $v / v$ (preliminary

based on ASHRAE $34 \mathrm{u}$ draft)

$35,000 \mathrm{ppm} v / \mathrm{v}$ (preTiminary

based on ASHRAE $34 \mathrm{u}$ draft)

$35,000 \mathrm{ppm} \mathrm{V} / \mathrm{V}$ (preliminary

based on ASHRAE $34 u$ draft)

$3 A 15$

A950

$34 \mathrm{u}$

A950

rat, $4 \mathrm{hr}$, Honeywe 11: >300,000 MSDS

ppm (fatal concentration by

inhalation for half of test animals)

rat, $2 \mathrm{hr}, 0 / 4$ : $>200,000 \mathrm{ppm}$

6272

(lowest exposure tested with

one or more deaths by

inhalation)

dog, 5 min, 5/12: 100,000 ppm

$v / v$ (lowest observed effect

Tevel in test animals)

dog, 5 min, 5/12: 100,000 ppm

$v / v$ (lowest observed effect

Tevel in test animals)

dog, $10 \mathrm{~min}, 0 / 6$ : 50,000 ppm

$v / v$ (no observed effect level

in test anima $7 \mathrm{~s}$ )

dog, $10 \mathrm{~min}, 0 / 6$ : $50,000 \mathrm{ppm}$

$v / v$ (no observed effect level

in test animals)

5644

6274

5644

6274

none (nonflammable as tested) 2525

none (nonflammable as tested) 5648

Honeywe 17: gas, not applicable MSDS

ICI: does not f7ash MSDS

$704{ }^{\circ} \mathrm{C}\left(1299^{\circ} \mathrm{F}\right) \quad 3960$

practical7y nonflammable 5931

(withdrawn for revision of the classification system, category SBQT2)

DuPont: clear, colorless MSDS

Honeywe 11: faint ethereal MSDS

1961

$5 C 39$

1995 based on refrigerant 115 8C01 in developed countries under the Montreal Protocol 


\section{R-600 (n-BUTANE)}

\begin{tabular}{|c|c|c|}
\hline $\begin{array}{l}\text { n-butane } \\
\mathrm{CH} 3-\mathrm{CH}-\mathrm{CH} 2-\mathrm{CH} 3\end{array}$ & CAS number 106-97-8 & $\begin{array}{l}\text { see } \\
\text { RDB\# }\end{array}$ \\
\hline \multicolumn{3}{|c|}{$\begin{array}{l}\text { COMMON USE(S) } \\
\text { blend component with fluorocarbons and other hydrocarbons as } \\
\text { alternatives to refrigerants } 12 \text { and } 500 \text {, among them refrigerants } 416 \mathrm{~A} \\
\text { and others; refrigerant in hydraulic refrigeration systems; use is } \\
\text { constrained by flammabitty concerns; primary uses are as a fuel, } \\
\text { aerosol propellant, and feedstock for solvents, rubbers, and plastics }\end{array}$} \\
\hline $\begin{array}{l}\text { chemical name (by IUPAC convention): } \\
\text { alternative chemical names/formulae: }\end{array}$ & $\begin{array}{l}\text { R-600; R600; R } 600 \\
\text { HC-600 } \\
\text { "R-3-11-0" (in Russia) } \\
\text { hydrocarbon } 600 \\
\text { n-C4; n-C4H10 } \\
\text { n-butane; norma]-butane } \\
\text { butane } \\
\text { 1-butane } \\
\text { diethy } 7 \\
\text { ethy1-ethy } 7 \\
\text { methylethy Tmethane } \\
\text { buty hydride } \\
\text { CH3CH2CH2CH3; CH3-CH2-CH2-CH3; } \\
\text { CH3(CH2) } 2 \mathrm{CH} 3 ; \mathrm{CH} 3-(\mathrm{CH} 2) 2-\mathrm{CH} 3 \\
\text { C4H10 } \\
\text { 106-97-8 (Chemical Abstracts } \\
\text { Service registry number) } \\
\text { EJ4200000 (Registry of Toxic } \\
\text { Effects of Chemical } \\
\text { Substances). }\end{array}$ & \\
\hline ARI container color / Pantone number: & $\begin{array}{l}\text { none, use light green grey/413 } \\
\text { with red / } 185 \text { band }\end{array}$ & 660 \\
\hline
\end{tabular}

PHYSICAL

- properties

molar mass:

$58.12220 \mathrm{~g} / \mathrm{mol} \quad(0.128138$

8820

normal freezing/melting/triple point: $\mathrm{Tb} / \mathrm{mo} 7)$

- normal boiling point heat of vaporization:

- normal pressure, $20{ }^{\circ} \mathrm{C}\left(68^{\circ} \mathrm{F}\right)$ -

$-138.3^{\circ} \mathrm{C}\left(-216.9^{\circ} \mathrm{F}\right) \quad 3209$

$-0.5^{\circ} \mathrm{C}\left(31.0^{\circ} \mathrm{F}\right) \quad 8401$

$385.8 \mathrm{~kJ} / \mathrm{kg}(165.9 \mathrm{Btu} / 7 \mathrm{~b}) \quad 8401$

- normal pressure, $21.1{ }^{\text {density, } \mathrm{C}\left(70^{\circ} \mathrm{F}\right) \text { vapor: }}$

$2.496 \mathrm{~kg} / \mathrm{m} 3(0.1558 \mathrm{~Tb} / \mathrm{cf}) \quad 8401$

- $20{ }^{\circ} \mathrm{C}\left(68^{\circ} \mathrm{F}\right)$-_ansity, vapor

$2.486 \mathrm{~kg} / \mathrm{m} 3(0.1552 \mathrm{lb} / \mathrm{cf}) \quad 8401$

pressure, saturated vapor: density, saturated liquid: density, saturated vapor:

specific volume, saturated 7 iquid: specific volume, saturated vapor: velocity of sound, saturated liquid: velocity of sound, saturated vapor: viscosity, saturated 1iquid: viscosity, saturated vapor: thermal conductivity, saturatd liquid:

thermal conductivity, saturated vapor: 
- $60^{\circ} \mathrm{C}\left(140^{\circ} \mathrm{F}\right)$

pressure, saturated vapor: heat of vaporization:

- critical point -.
$\left.\mathrm{Btu} / \mathrm{hr} \bullet f \mathrm{t}^{\circ} \mathrm{F}\right)$

$639 \mathrm{kPa}(92.7 \mathrm{psia}) \quad 8401$

$320.8 \mathrm{~kJ} / \mathrm{kg}(137.9 \mathrm{Btu} / 7 \mathrm{~b}) \quad 8401$

$152.0^{\circ} \mathrm{C}\left(305.6^{\circ} \mathrm{F}\right) \quad 8401$

$3796 \mathrm{kPa}(550.6 \mathrm{psia}) \quad 8401$

$228 \mathrm{~kg} / \mathrm{m} 3(14.2 \mathrm{lb} / \mathrm{cf}) \quad 8401$

$4.38 \mathrm{~L} / \mathrm{kg}(0.0702 \mathrm{cf} / 7 \mathrm{~b}) \quad 0036$

ENVIRONMENTAL

ODP (Ozone depletion potentia1):

GWP (global warming potential):

0.000 (mode1-derived relative to $R$ 11)

unknown, but very low: $\sim 20$

relative to $\mathrm{CO} 2$ for $100 \mathrm{yr}$

integration

HGWP (halocarbon GWP): $\sim 0$ relative to $\mathrm{R} 11$ for

infinite integration period

photochemical reactivity (grnd 1eve1): 320 relative to methane

4511

SAFETY

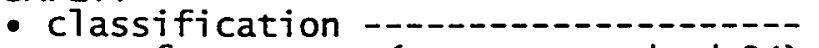

safety group (ASHRAE Standard 34):

NFPA 704 degrees of hazard $(H-F-R-S)$ :

NPCA HMIS hazard ratings $(H-F-R):$

A3 8601

BOC Gases: 1-4-0 MSDS

hea 7 th-flammabi 1 ity-reactivity

[-specia]]: $0=$ no, $4=$ severe

BOC Gases: 1-4-0 MSDS

hea 7 th-f Tammability-reactivity

$0=$ insignificant, $4=e x t r e m e$

UL Comparative Hazard to Life Group:

5 (b) in absence of flame or

hot objects

- occupational exposure Timit --------

MAK (maximum workplace concentration):

- Tong-term occupational Timit ------

NIOSH REL (recommendd exposure 1imit):

ACGIH TLV-TWA (time-weighted average):

IV: $2,000 \mathrm{ppm} \mathrm{V} / \mathrm{V}$ momentary 605561

min

$800 \mathrm{ppm} \mathrm{V} / \mathrm{v}$ TWA for $10 \mathrm{hr} /$ day 5204 and $40 \mathrm{hr} / \mathrm{wk}$

$800 \mathrm{ppm} \mathrm{v/v}$ TWA for $8 \mathrm{hr} /$ day 9504

MAK (maximum workplace concentration):

- emergency exposure Timit

and $40 \mathrm{hr} / \mathrm{wk}$

$1,000 \mathrm{ppm} v / \mathrm{v}$ TWA for $8 \mathrm{hr} /$ day 5561

40 (or 42) hr/wk

being examined for changes 7101

Acute Toxicity Exposure Limit (ATEL):

Refrigerant concentration Limit (RCL):

$0 \mathrm{ppm} v / \mathrm{v}$ (preliminary based

$34 \mathrm{u}$

on ASHRAE $34 u$ draft)

provisiona1: $0 \mathrm{ppm} \mathrm{V} / \mathrm{V}$

(preliminary based on ASHRAE

$34 u$ draft)

provisional: $0 \mathrm{ppm} \mathrm{v} / \mathrm{v}$

(preliminary based on ASHRAE

34u draft)

- acute (short-term) toxicity

LC50 (lethal concentration, 50\%):

mouse, $2 \mathrm{hr}$ : $280,000 \mathrm{ppm}$

(fatal concentration by

inhalation for half of test animals)

mouse, $2 \mathrm{hr}: 281,000 \mathrm{ppm}$

(fatal concentration by

inhalation for half of test animals)

rat, 2 hr: 280,000 ppm (fatal

concentration by inhalation

for half of test animals)

rat, 4 hr: $272,000 \mathrm{ppm}$ (fatal

A950

A950

A538

5363

A538

5363 
ALC (approximate 1etha1 concentration:

LCLo (Tetha1 concentration, Towest):

cardiac sensitization threshold/LOEL:

anesthetic/CNS effect LOEL:

- flammability

LFL-UFL (flammability limits in air):

heat of combustion (by ASHRAE 34-92):

flash point:

autoignition temperature:

autodecomposition temperature:

- detection

appearance:

odor:

odor sensing, lower threshold: concentration by inhalation for half of test animals)

rat, ? hr: $280,000 \mathrm{ppm}$ (fatal

A623

concentration by inhalation

for half of test animals)

mouse, $2 \mathrm{hr}, 0 / 10$ : $>220,000$

ppm (lowest exposure tested

with one or more deaths by

inhalation)

mouse, $2 \mathrm{hr}, 4 / 10$ : 270,000 ppm 6197

(lowest exposure tested with

one or more deaths by

inhalation)

dog, ? min, ?/?: 200000-250000

6197

ppm (fatal concentration by

inhalation for even one test

anima1)

dog 10min 2/2: 100,000-250,000 6192

ppm $v / v$ (lowest observed

effect level in test animals)

anima1?, $10 \mathrm{~min}$, ?/?: 100,000

A623

$\mathrm{ppm} \mathrm{v} / \mathrm{v}$ (7owest observed

effect level in test animals)

dog, ? min, ?/?: 200000-250000 6197

$\mathrm{ppm} v / \mathrm{v}$ (Towest observed

effect 7 eve 7 in test animals)

dog, ? min, ?/?: 250,000 ppm

A623

$v / v$ (lowest observed effect

leve 7 in test anima 15 )

mouse, Iminavg,10/10: 220,000 6197

$\mathrm{ppm} \mathrm{v} / \mathrm{v}$ (Towest observed

effect leve 7 in test anima 7 s)

mouse, 25minavg, 6/ 6: 130,000 6197

$\mathrm{ppm} v / \mathrm{v}$ (lowest observed

effect Teve 7 in test anima7s)

human, $10 \mathrm{~min}, ? / ?: 100,000$

6290

ppm $v / v$ (lowest observed

effect level)

$1.5-10.1 \% \mathrm{v} / \mathrm{v}$

$1.6-8.4 \% \mathrm{v} / \mathrm{v}$

$1.7-10.3 \% \mathrm{v} / \mathrm{v}$

BOC Gases: $1.8-8.4 \% \mathrm{v} / \mathrm{V}$

Exxon: $1.9-8.5 \% \mathrm{v} / \mathrm{v}$

Phil7ips: $1.9-8.5 \% \mathrm{v} / \mathrm{v}$

Scott: $1.9-8.5 \% \mathrm{v} / \mathrm{v}$

$49.5 \mathrm{MJ} / \mathrm{kg}(21281 \mathrm{Btu} / \mathrm{Tb}) \quad 2318$

CC, BOC Gases: $-73.9^{\circ} \mathrm{C}\left(-101^{\circ} \mathrm{F}\right)$ MSDS

$C C$, Exxon: $-60^{\circ} \mathrm{C}\left(-76^{\circ} \mathrm{F}\right)$ MSDS

not applicable for gas 5204

$430{ }^{\circ} \mathrm{C}\left(806^{\circ} \mathrm{F}\right) \quad 4 \mathrm{~B} 64$

$510{ }^{\circ} \mathrm{C}\left(950{ }^{\circ} \mathrm{F}\right) \quad 4906$

BOC Gases: $420^{\circ} \mathrm{C}\left(788^{\circ} \mathrm{F}\right)$ MSDS

Exxon: $405^{\circ} \mathrm{C}\left(761^{\circ} \mathrm{F}\right)$ MSDS

BOC Gases: $435^{\circ} \mathrm{C}\left(815^{\circ} \mathrm{F}\right)$ MSDS

Exxon: colorless gas MSDS

BOC Gases: odorless MSDS

Exxon: faint disagreeable odor MSDS

Phillips: none

scott: faint, sweet odor MSDS

$50,000 \mathrm{ppm} \mathrm{v/v} \quad 6290$ 
Toxicity Data to Defermine Refrigerant Concentration Limits

James M. Calm, Engineering Consultant

PRODUCTION

7ast year production a77owed: unrestricted

$8 \mathrm{C01}$ 


\section{R-600a (ISOBUTANE)}

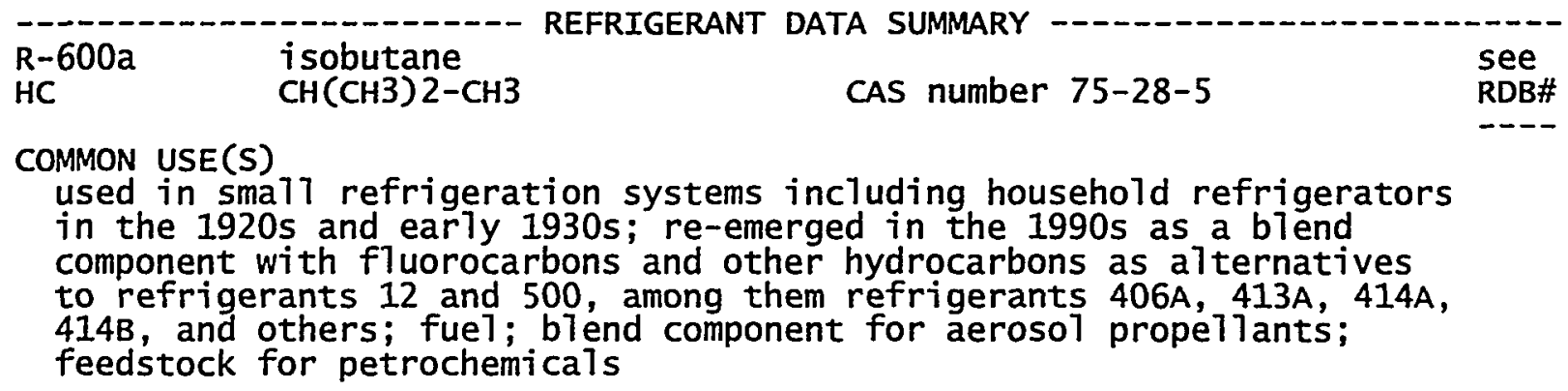

IDENTIFIERS

common name(s): R-600a; R600a; R 600a

HC-600a

hydrocarbon $600 \mathrm{a}$

i-C4; $\mathrm{i}-\mathrm{C} 4 \mathrm{H} 10$

iso-C4; iso-C $4 \mathrm{H} 10$

chemical name (by IUPAC convention): i sobutane

alternative chemical names/formulae:

propane, 2-methyl-

isomethylpropane

methylpropane, iso

2-methylpropane, (CH3) $2 \mathrm{CH}-\mathrm{CH} 3$

trimethylmethane, $\mathrm{CH}(\mathrm{CH} 3) 3$

$\mathrm{CH} 3 \mathrm{CH}(\mathrm{CH} 3) \mathrm{CH} 3, \mathrm{CH} 3-\mathrm{CH}(\mathrm{CH} 3)-\mathrm{CH} 3$

empirical formula: $\mathrm{C} 4 \mathrm{HIO}$

CAS number: 75-28-5 (Chemica1 Abstracts

Service registry number)

NIOSH RTECS number: TZ4300000 (Registry of Toxic

Effects of Chemical

Substances)

trade name(s): Calor Gas (UK) CARE 10

Diversified CPC Aeron A-31 MSDS

historical name(s): "R-601" (nonstandard number) 2113

"R601" (nonstandard number) 2113

Cope 7 and Products Freezo 12113

ARI container color / Pantone number: none, use light green grey/413 6601 with red / 185 band

PHYSICAL

- properties

molar mass:

$58.12220 \mathrm{~g} / \mathrm{mol} \quad(0.128138$

8820

$7 \mathrm{~b} / \mathrm{mo}$ )

normal freezing/melting/triple point:

$-159.6^{\circ} \mathrm{C}\left(-255.3^{\circ} \mathrm{F}\right)$

8401

- normal boiling point - temperature:

density, saturated liquid:

$-11.6^{\circ} \mathrm{C}\left(11.1{ }^{\circ} \mathrm{F}\right)$

$594 \mathrm{~kg} / \mathrm{m} 3(37.06 \mathrm{~Tb} / \mathrm{cf})$

$2.83 \mathrm{~kg} / \mathrm{m} 3(0.176 \mathrm{~Tb} / \mathrm{cf})$

8401

density, saturated vapor:

specific volume, saturated liquid:

$1.685 \mathrm{~L} / \mathrm{kg}(0.0270 \mathrm{cf} / 7 \mathrm{~b})$

8401

$353.7 \mathrm{~L} / \mathrm{kg}(5.6664 \mathrm{cf} / 7 \mathrm{~b}) \quad 8401$

$366.7 \mathrm{~kJ} / \mathrm{kg}$ (157.6 Btu/7b) 8401

heat of vaporization:

velocity of sound, saturated liquid:

$1008 \mathrm{~m} / \mathrm{s}(3308 \mathrm{ft} / \mathrm{s})$

$197 \mathrm{~m} / \mathrm{s}(645 \mathrm{ft} / \mathrm{s})$

$228 \mu \mathrm{Pa} \cdot \mathrm{s}(0.228 \mathrm{cP})$

$6.64 \mu \mathrm{Pa} \cdot \mathrm{s}(0.00664 \mathrm{cp})$

$0.1121 \mathrm{~W} / \mathrm{m} \cdot \mathrm{K}(0.0648$

$\left.\mathrm{Btu} / \mathrm{hr} \cdot \mathrm{ft}^{\circ} \mathrm{F}\right)$

$0.0126 \mathrm{~W} / \mathrm{m} \cdot \mathrm{K}(0.0073$

$\mathrm{Btu} / \mathrm{hr} \cdot \mathrm{ft}^{\circ} \mathrm{F}$ )

8401

8401

8401

8401

8401

thermal conductivity, vapor:

8401 
- normal pressure, $20{ }^{\circ} \mathrm{C}\left(68{ }^{\circ} \mathrm{F}\right)$

- normal pressure, $21.1{ }^{\circ} \mathrm{C}\left(70^{\circ} \mathrm{F}\right)$---

- $20^{\circ} \mathrm{C}\left(68^{\circ} \mathrm{F}\right)$ density, vapor

$2.485 \mathrm{~kg} / \mathrm{m} 3(0.1551 \mathrm{7b} / \mathrm{cf}) \quad 8401$

$2.475 \mathrm{~kg} / \mathrm{m} 3(0.1545 \mathrm{~Tb} / \mathrm{cf}) \quad 8401$

pressure, saturated vapor:

$301.8 \mathrm{kPa}(43.77 \mathrm{psia}) \quad 8401$ density, saturated 7 iquid:
density, saturated vapor: specific volume, saturated 7 iquid: specific volume, saturated vapor: velocity of sound, saturated 1iquid: velocity of sound, saturated vapor: viscosity, saturated 7 iquid: viscosity, saturated vapor: thermal conductivity, saturatd liquid: $557 \mathrm{~kg} / \mathrm{m} 3(34.77 \mathrm{1b} / \mathrm{cf}) \quad 8401$ $7.90 \mathrm{~kg} / \mathrm{m} 3(0.493 \mathrm{lb} / \mathrm{cf}) \quad 8401$ $1.796 \mathrm{~L} / \mathrm{kg}(0.0288 \mathrm{cf} / 7 \mathrm{~b}) \quad 8401$ $126.6 \mathrm{~L} / \mathrm{kg}(2.0284 \mathrm{cf} / 7 \mathrm{~b}) \quad 8401$ $838 \mathrm{~m} / \mathrm{s}(2749 \mathrm{ft} / \mathrm{s}) \quad 8401$ $198 \mathrm{~m} / \mathrm{s}(651 \mathrm{ft} / \mathrm{s}) \quad 8401$ $160 \mu \mathrm{Pa} \cdot 5(0.160 \mathrm{cp}) \quad 8401$ $7.6 \mu \mathrm{Pa} \cdot \mathrm{s}(0.0076 \mathrm{cp}) \quad 8401$ $0.0978 \mathrm{~W} / \mathrm{m} \cdot \mathrm{K}(0.0565 \quad 8401$

thermal conductivity, saturated vapor: $\left.\mathrm{Btu} / \mathrm{hr} \cdot \mathrm{ft}^{\circ} \mathrm{F}\right)$

- $60{ }^{\circ} \mathrm{C}\left(140{ }^{\circ} \mathrm{F}\right)$ pressure, saturated vapor:

$0.02110 \mathrm{~W} / \mathrm{m} \cdot \mathrm{K}(0.01219$

Btu/hr・ft $\left.{ }^{\circ} \mathrm{F}\right)$

8401

868 kPa (125.9 psia) 8401

$285.2 \mathrm{~kJ} / \mathrm{kg}(122.6 \mathrm{Btu} / 7 \mathrm{~b}) \quad 8401$

- critical point heat of vaporization:

temperature:

pressure:

density:

specific volume:

$134.7^{\circ} \mathrm{C}\left(274.5^{\circ} \mathrm{F}\right)$

$3640 \mathrm{kPa}(527.9 \mathrm{psia})$

$224 \mathrm{~kg} / \mathrm{m} 3(14.0 \mathrm{jb} / \mathrm{cf})$

$4.46 \mathrm{~L} / \mathrm{kg}(0.0714 \mathrm{cf} / 7 \mathrm{~b})$

8401

8401

8401

8401

ENVIRONMENTAL

ODP (ozone depletion potential):

GWP (global warming potential):

0.000 (model-derived relative to $R$ 11)

unknown, but very low: $\sim 20$

relative to $\mathrm{CO} 2$ for $100 \mathrm{yr}$

integration

HGWP (halocarbon GWP):

$\sim 0$ relative to $R 11$ for

infinite integration period

SAFETY

- classification
safety group (ASHRAE Standard 34) NFPA 704 degrees of hazard (H-F-R-S):

NPCA HMIS hazard ratings $(H-F-R)$ :

UL Comparative Hazard to Life Group:

- short-term occupational limit recommended short-term exposure limit:

- occupational exposure limit MAK (maximum workplace concentration):

- Tong-term occupational limit

A3 8601

Matheson: 1-4-0 MSDS

Pennzoi 1 : 0-4-0 MSDS

Texaco: 1-4-0 MSDS

health-flammabi 7 ity-reactivity

[-special]: $0=$ no, $4=$ severe

Texaco: 1-4-0 MSDS

health-flammability-reactivity

$0=$ insignificant, $4=$ extreme

5 (b) in absence of f7ame or 0036 hot objects

Rhône-PouTenc: 750 ppm(10 min) MSDS

IV: $2,000 \mathrm{ppm} \mathrm{v} / \mathrm{v}$ momentary 605561 min

$800 \mathrm{ppm} v / \mathrm{v}$ TWA for $10 \mathrm{hr} /$ day 5204 and $40 \mathrm{hr} / \mathrm{wk}$

Pennzoi 1, C1-C4 assumed: 1,000 MSDS $\mathrm{ppm} v / \mathrm{v}$ TWA for $8 \mathrm{hr} /$ day and $40 \mathrm{hr} / \mathrm{wk}$

Rhône-POuTenc OES: $600 \mathrm{ppm} \mathrm{V} / \mathrm{V}$ MSDS

TWA for $8 \mathrm{hr} /$ day and $40 \mathrm{hr} / \mathrm{wk}$

MAK (maximum workplace concentration):

$1,000 \mathrm{ppm} v / \mathrm{v}$ TWA for $8 \mathrm{hr} /$ day 5561 40 (or 42) hr/wk 
- emergency exposure limit

being examined for changes

7101

Acute Toxicity Exposure Limit (ATEL):

25,000 ppm $v / v$ (preliminary

$34 \mathrm{u}$

based on ASHRAE 34u draft)

25,000 ppm $v / v$ (pre7iminary

based on ASHRAE $34 \mathrm{u}$ draft)

Refrigerant Concentration Limit ( $R C L)$ :

2,500 ppm v/v (preliminary

A950

based on ASHRAE $34 \mathrm{u}$ draft)

4,300 $\mathrm{ppm} v / \mathrm{v}$ (preliminary

A950

based on ASHRAE $34 \mathrm{u}$ draft)

$34 \mathrm{u}$

- acute (short-term) toxicity LC50 (7ethal concentration, 50\%):

ALC (approximate Tethal concentration: mouse, $2 \mathrm{hr}, 0 / 10:>350,000$

mouse, $2 \mathrm{hr}$ : $410,000 \mathrm{ppm}$

A538

(fatal concentration by

inhalation for half of test anima7s)

mouse, $2 \mathrm{hr}: 520,000 \mathrm{ppm}$

(fata) concentration by

inhalation for half of test anima7s)

rat, 15 min: $570,000 \mathrm{ppm}$

(fatal concentration by

inhalation for half of test anima7s)

ppm (lowest exposure tested

with one or more deaths by

inhalation)

mouse, $2 \mathrm{hr}, 6 / 10$ : 410,000 ppm 6197

(lowest exposure tested with

one or more deaths by

inhalation)

LCLo (Tetha] concentration, Towest):

dog, ? min, ?/?: 550,000 ppm

6197

(fata) concentration by

inhalation for even one test anima?)

cardiac sensitization (CS) EC50: dog, 5 min: $70,000 \mathrm{ppm} v / \mathrm{v}$

(effective concentration in

half of test animals)

cardiac sensitization threshold/LOEL: dog, $5 \mathrm{~min}, 4 / 12: 50,000 \mathrm{ppm}$

5644

$v / v$ (lowest observed effect

leve 7 in test animals)

cardiac sensitization (CS) NOEL:

dog, 5 min, 0/12: 25,000 ppm

$\checkmark / V$ (no observed effect level

in test animals)

dog, 5 min, 0/12: 25,000 ppm

$\checkmark / v$ (no observed effect level

in test anima7s)

anesthetic/CNS effect EC50: rat, $10 \mathrm{~min}$ : stimulant 200,000 6110

ppm $v / v$ (effective

concentration in half of test

animals)

anesthetic/CNS effect LOEL:

dog,? min, ?/?: 450,000 ppm

$v / v$ (lowest observed effect

level in test animals)

dog, ? min, ?/?: 450,000 ppm

$v / v$ (lowest observed effect

level in test animals)

mouse, 17 minavg: 6/6: 200,000 6197

$\mathrm{ppm} v / \mathrm{v}$ (lowest observed

effect leve 7 in test anima 75 )

mouse, 26 minavg, 6/6: 230,000 6197

$\mathrm{ppm} v / \mathrm{v}$ (lowest observed

effect leve 7 in test anima1s)

mouse, 60 minavg, 6/6: 150,000 6197 
ppm $v / v$ (lowest observed
anesthetic/CNS effect NOEL:
human, $8 \mathrm{hr}, 0 / 8: 1,000 \mathrm{ppm}$

anesthetic/CNS effect NOEL: human, $8 \mathrm{hr}, 0 / 8: 1,000 \mathrm{ppm}$ :

5364

- flammability LFL-UFL (flammability limits in air):

heat of combustion (by ASHRAE 34-92):

$1.7-9.7 \% \mathrm{v} / \mathrm{v}$

$1.9-10.0 \% \mathrm{v} / \mathrm{v}$

2525

Ecozone: $1-8.5 \% \mathrm{v} / \mathrm{v}$

Pennzoi 1: $1.8-8.5 \% \mathrm{v} / \mathrm{V}$

PhiT7ips: $1.8-8.4 \% \mathrm{v} / \mathrm{V}$

Texaco: 1.8-8.4\% v/V

9516

$\mathrm{mfr}$

MSDS

MSDS

MSDS

9516

$49.4 \mathrm{MJ} / \mathrm{kg}(21238 \mathrm{Btu} / \mathrm{lb}) \quad 2318$

flash point: $-83^{\circ} \mathrm{C}\left(-117^{\circ} \mathrm{F}\right)$

6290

Matheson CC: $-88^{\circ} \mathrm{C}\left(-126^{\circ} \mathrm{F}\right)$ MSDS

Pennzoi 7: $-83^{\circ} \mathrm{C}\left(-117^{\circ} \mathrm{F}\right)$ MSDS

Phi77ips: $-83^{\circ} \mathrm{C}\left(-117^{\circ} \mathrm{F}\right)$ MSDS

Texaco: $-118{ }^{\circ} \mathrm{C}\left(-181{ }^{\circ} \mathrm{F}\right)$ MSDS

not applicable for gas $\quad 5204$

$\begin{array}{lll}\text { autoignition temperature: } & 530^{\circ} \mathrm{C}\left(9866^{\circ} \mathrm{F}\right){ }^{\circ} \mathrm{C}\left(864^{\circ} \mathrm{F}\right) & 4906 \\ & \text { Pennzoi } 1: 462.2{ }^{\circ} \mathrm{CSD}\end{array}$

Rhône-Poutenc: $420^{\circ} \mathrm{C}\left(788^{\circ} \mathrm{F}\right)$ MSDS

Texaco: $460.0^{\circ} \mathrm{C}\left(860^{\circ} \mathrm{F}\right)$ MSDS

former UL Classification: flammable (withdrawn for UL revision of the classification system, category SBQT2)

- detection

appearance: Phillips: colorless

MSDS

odor:

Matheson: slight petroleum

MSDS

Phi71ips: none

Rhône-Poulenc: virtuality

MSDS

odorless

MSDS

PRODUCTION

first commercial use as a refrigerant:

last year production allowed:

1920 s by $E$ Copeland, $H$ Edwards 1923 by J DeRemer 1923 by Savage Arms Company unrestricted

2113

6104

6104

$8 \mathrm{C} 01$ 


\section{R-717 (AMMONIA)}

R-717 REmic
inorganic NH3
COMMON USE(S) CAS number 7664-41-7
chillers, widely used in industrial refrigeration; wide use as a
fertilizer; intermediate to manufacture synthetic fibers, plastics,
and explosives; cleaning solvent; ammonia is found in water, soil,
and air - it is a natural decomposition product of decaying manure,
plants, and animals

IDENTIFIERS

common name(s): R-717; R717; R 717

anhydrous ammonia

nitro-sil

chemical name (by IUPAC convention): ammonia

alternative chemical names/formulae: CAS number:

NIOSH RTECS number:

historical name(s): ARI container color / Pantone number:

NH3; Tess commonty $\mathrm{H} 3 \mathrm{~N}$

7664-41-7 (Chemical Abstracts

Service registry number)

B00875000 (Registry of Toxic

Effects of Chemical

Substances)

spirit of hartshorn

none, use 1ight green grey/413 6601

with red / 185 band

PHYSICAL

- properties

motar mass:

normal freezing/melting/triple point:

- normal boiling point -rere:

density, saturated liquid:

density, saturated vapor:

specific volume, saturated liquid: specific volume, saturated vapor: heat of vaporization:

velocity of sound, saturated Tiquid: velocity of sound, saturated vapor: viscosity, saturated 7iquid: viscosity, saturated vapor: thermal conductivity, Tiquid:

thermal conductivity, vapor:

- normal pressure, $20{ }^{\circ} \mathrm{C}\left(68^{\circ} \mathrm{F}\right)$-----

- normal pressure, $21.1{ }^{\circ} \mathrm{C}\left(70^{\circ} \mathrm{F}\right)$---

- $20^{\circ} \mathrm{C}\left(68^{\circ} \mathrm{F}\right)$-.... density, vapor:

pressure, saturated vapor: density, saturated Tiquid: density, saturated vapor: specific volume, saturated liquid: specific volume, saturated vapor: velocity of sound, saturated liquid: velocity of sound, saturated vapor: viscosity, saturated 7iquid: viscosity, saturated vapor:

$17.03056 \mathrm{~g} / \mathrm{mo} 7 \quad 0.037546$

$7 \mathrm{~b} / \mathrm{mo} 7)$

$-77.7^{\circ} \mathrm{C}\left(-107.8^{\circ} \mathrm{F}\right)$

$-33.3^{\circ} \mathrm{C}\left(-28.0^{\circ} \mathrm{F}\right)$

$682 \mathrm{~kg} / \mathrm{m} 3(42.57 \mathrm{lb} / \mathrm{cf})$

$0.89 \mathrm{~kg} / \mathrm{m} 3(0.056 \mathrm{1b} / \mathrm{cf})$

$1.466 \mathrm{~L} / \mathrm{kg}(0.0235 \mathrm{cf} / \mathrm{lb})$

$1242.2 \mathrm{~L} / \mathrm{kg}(19.8981 \mathrm{cf} / \mathrm{ib})$

$1369.5 \mathrm{~kJ} / \mathrm{kg}(588.8 \mathrm{Btu} / 1 \mathrm{~b})$

$1768 \mathrm{~m} / \mathrm{s}(5801 \mathrm{ft} / \mathrm{s})$

$386 \mathrm{~m} / \mathrm{s}(1267 \mathrm{ft} / \mathrm{s})$

$255 \mu \mathrm{Pa} \cdot \mathrm{s}(0.255 \mathrm{cp})$

$8.05 \mu \mathrm{Pa} \cdot \mathrm{s}(0.00805 \mathrm{cp})$

$0.6666 \mathrm{~W} / \mathrm{m} \cdot \mathrm{K}(0.3851$

$\mathrm{Btu} / \mathrm{hr} \bullet \mathrm{ft} \mathrm{F}^{\circ} \mathrm{F}$ )

$0.0210 \mathrm{~W} / \mathrm{m} \cdot \mathrm{K}(0.0121$

$\mathrm{Btu} / \mathrm{hr} \cdot \mathrm{ft}^{\circ} \mathrm{F}$ )

$0.716 \mathrm{~kg} / \mathrm{m} 3(0.0447 \mathrm{~Tb} / \mathrm{cf}) \quad 8401$

$0.713 \mathrm{~kg} / \mathrm{m} 3(0.0445 \mathrm{lb} / \mathrm{cf}) \quad 8401$

$857.5 \mathrm{kPa}$ (124.37 psia)

$610 \mathrm{~kg} / \mathrm{m} 3(38.09 \mathrm{7b} / \mathrm{cf})$

$6.70 \mathrm{~kg} / \mathrm{m} 3(0.4181 \mathrm{~b} / \mathrm{cf})$

$1.639 \mathrm{~L} / \mathrm{kg}(0.0263 \mathrm{cf} / 7 \mathrm{~b})$

$149.2 \mathrm{~L} / \mathrm{kg}(2.3900 \mathrm{cf} / 7 \mathrm{~b})$

$1385 \mathrm{~m} / \mathrm{s}(4542 \mathrm{ft} / \mathrm{s})$

$405 \mathrm{~m} / \mathrm{s}(1328 \mathrm{ft} / \mathrm{s})$

$138 \mu \mathrm{Pa} \cdot \mathrm{s}(0.138 \mathrm{cp})$

$9.7 \mu \mathrm{Pa} \cdot \mathrm{s}(0.0097 \mathrm{cp})$
8820

8401

8401

8401

8401

8401

8401

8401

8401

8401

8401

8401

8401

8401

8401

8401

8401

8401

8401

8401

8401

8401

8401 
thermal conductivity, saturatd liquid: thermal conductivity, saturated vapor:

- $60{ }^{\circ} \mathrm{C}\left(140{ }^{\circ} \mathrm{F}\right)$

pressure, saturated vapor:

- critical point heat of vaporization:

temperature: pressure:

density:

specific volume:
$0.4999 \mathrm{~W} / \mathrm{m} \cdot \mathrm{K}(0.2888$

$\mathrm{Btu} / \mathrm{hr} \cdot \mathrm{ft}^{\circ} \mathrm{F}$ )

$0.02552 \mathrm{~W} / \mathrm{m} \cdot \mathrm{K}(0.01474$

$\mathrm{Btu} / \mathrm{hr} \cdot \mathrm{ft}^{\circ} \mathrm{F}$ )

$2616 \mathrm{kPa}$ (379.4 psia)

8401

$997.3 \mathrm{~kJ} / \mathrm{kg}(428.8 \mathrm{Btu} / \mathrm{lb})$

8401

$132.3^{\circ} \mathrm{C}\left(270.1{ }^{\circ} \mathrm{F}\right)$

$11333 \mathrm{kPa}$ (1643.7 psia)

$11336 \mathrm{kPa}$ (1644.2 psia)

$225 \mathrm{~kg} / \mathrm{m} 3(14.0 \mathrm{ib} / \mathrm{cf})$

$235 \mathrm{~kg} / \mathrm{m} 3$ (14.7 $7 \mathrm{~b} / \mathrm{cf})$

$4.26 \mathrm{~L} / \mathrm{kg}(0.0682 \mathrm{cf} / \mathrm{7b})$

$4.44 \mathrm{~L} / \mathrm{kg}(0.0712 \mathrm{cf} / 7 \mathrm{~b})$

8401

8401

9701

8401

9701

9701

8401

ENVIRONMENTAL

ODP (ozone depletion potential):

0.000 (model-derived relative to $R$ 11)

GWP (global warming potential):

unknown, but very low: $<1$

relative to $\mathrm{CO} 2$ for $100 \mathrm{yr}$

integration

SAFETY

- classification

safety group (ASHRAE Standard 34):

NFPA 704 degrees of hazard (H-F-R-S):

NPCA HMIS hazard ratings (H-F-R):

B2

8601

ARI recommendation: 3-1-0 3A15

hea 7 th-flammabi 7 ity-reactivity

[-special]: $0=$ no, $4=$ severe

BoC Gases: 3-1-0

health-flammability-reactivity

$0=i n s i g n i f i c a n t, 4=e x t r e m e$

UL Comparative Hazard to Life Group:

2 in absence of flame or hot

objects

DFG pregnancy risk group:

- short-term occupationa1 Jimit --

C (no risk fear below MAK/BAT) 7101

NIOSH IDLH (immediately dangerous): NIOSH SCP IDLH (immediately dangerous: OSHA STEL (short-term exposure limit):

NIOSH STEL (short-term exposur limit): ACGIH TLV-STEL (short-term exp Timit): recommended short-term exposure limit:

- occupational exposure Timit -..---.MAK (maximum workplace concentration):

- 7ong-term occupationa1 7imit

$300 \mathrm{ppm} \mathrm{v} / \mathrm{V}$

$500 \mathrm{ppm} \mathrm{v} / \mathrm{v}$ for $30 \mathrm{~min} \quad 3903$

$50 \mathrm{ppm} \mathrm{v} / \mathrm{V}$ TWA for $15 \mathrm{~min}$ (set 5204

in 1989, but vacated in 1992)

$35 \mathrm{ppm} \mathrm{V} / \mathrm{V}$ TWA for $15 \mathrm{~min}$

$35 \mathrm{ppm} v / v$ Twa for $15 \mathrm{~min}$

DuPont EEL: 500 ppm (<10 min) 9504

DuPont EEL: $300 \mathrm{ppm}(10-60 \mathrm{~min})$ MSDS

DuPont EEL: $500 \mathrm{ppm}$ (ceiling) MSDS

I: $40 \mathrm{ppm} \mathrm{v} / \mathrm{V}$ momentary for 57101 min

$50 \mathrm{ppm} \mathrm{V} / \mathrm{v}$ TWA for $8 \mathrm{hr} /$ day 3904

and $40 \mathrm{hr} / \mathrm{wk}$

$25 \mathrm{ppm} \mathrm{v} / \mathrm{v}$ TWA for $10 \mathrm{hr} /$ day 3903

NIOSH REL (recommendd exposure 1imit):

ACGIH TLV-TWA (time-weighted average):

exposure limit consistent to OSHA PEL:

and $40 \mathrm{hr} / \mathrm{wk}$

$25 \mathrm{ppm} \mathrm{v} / \mathrm{v}$ TWA for $8 \mathrm{hr} /$ day 9504

and $40 \mathrm{hr} / \mathrm{wk}$

DuPont AEL: $25 \mathrm{ppm} V / \mathrm{V}$ TWA for MSDS

$8 \mathrm{hr} /$ day and $40 \mathrm{hr} / \mathrm{wk}$

MAK (maximum workplace concentration):

$20 \mathrm{ppm} \mathrm{v/v} \mathrm{TWA} \mathrm{for} 8 \mathrm{hr} /$ day 407101 (or 42) $\mathrm{hr} / \mathrm{wk}$

- emergency exposure 1imit -.-.-----

$1 \mathrm{hr}: 100 \mathrm{ppm} \mathrm{v} / \mathrm{v}$ ceiling

guidance level for single

emergency exposures

$24 \mathrm{hr}: 100 \mathrm{ppm} v / \mathrm{v}$ ceiling

5154

5154 
Acute Toxicity Exposure Limit (ATEL):

Refrigerant Concentration Limit (RCL):

AIHA ERPG-3 (1 ife-threatening): AIHA ERPG-2 (injurious or impairing): AIHA ERPG- 1 (odor or mild effects): - acute (short-term) toxicity LC50 (TethaT concentration, $50 \%$ ):

LCL50 (7owest pub7ished LC50 va7ue): ALC (approximate Tethal concentration:

LCLo (letha] concentration, 1owest):

ora1 LD50 (7ethal dosage, 50\%): anesthetic/CNS effect NOEL: respiratory RD50 (response dose 50\%): guidance level for singte emergency exposures

$300 \mathrm{ppm} \mathrm{v} / \mathrm{v}$ (pre)iminary based $34 \mathrm{u}$ on ASHRAE $34 \mathrm{u}$ draft)

$320 \mathrm{ppm} \mathrm{V} / \mathrm{V}$ (preliminary based $A 950$ on ASHRAE 34u draft)

$300 \mathrm{ppm} v / \mathrm{v}$ (preliminary based $34 \mathrm{u}$ on ASHRAE $34 \mathrm{u}$ draft)

$320 \mathrm{ppm} \mathrm{V} / \mathrm{v}$ (preTiminary based $A 950$ on ASHRAE $34 \mathrm{u}$ draft)

$1,000 \mathrm{ppm} \mathrm{v} / \mathrm{v}$ for $1 \mathrm{hr} \quad 4 \mathrm{~B} 76$

$200 \mathrm{ppm} v / \mathrm{v}$ for $1 \mathrm{hr} \quad 4 \mathrm{~B} 76$

$25 \mathrm{ppm} v / \mathrm{v}$ for $1 \mathrm{hr} \quad 4 \mathrm{~B} 76$

rat, 30 min: 23,472 ppm (fata) 6278 concentration by inhalation for half of test animals)

rat, I hr: 7,338 ppm (fatal 6230 concentration by inhalation

for half of test animals)

rat, $1 \mathrm{hr}: 7,338-11,590$ ppm 5340 (fatal concentration by inhalation for half of test anima $7 s$ )

rat, $1 \mathrm{hr}: 11,342 \mathrm{ppm}$ (fatal 6229

concentration by inhalation

for half of test animals)

rat, $1 \mathrm{hr}: 16,600 \mathrm{ppm}$ (fatal 5152

concentration by inhalation

for half of test animals)

rat, $4 \mathrm{hr}: 2,000 \mathrm{ppm}$ (fatal 5266

concentration by inhalation

for half of test animals)

rat, $4 \mathrm{hr}:$ 2,000 ppm (fatal 5C46

concentration by inhalation

for half of test animals)

rat, $4 \mathrm{hr}: 4,067 \mathrm{ppm}$ (fata) 5151

concentration by inhalation

for half of test animals)

rat, $1 \mathrm{hr}: 6,586 \mathrm{ppm} \quad 1106$

rat, $4 \mathrm{hr}, 2-4 / 6$ : 2,000 ppm 5643

(lowest exposure tested with one or more deaths by

inhalation)

rat, $4 \mathrm{hr}$, ?/?: $>2,000 \mathrm{ppm} 6250$

(lowest exposure tested with

one or more deaths by

inhalation)

human, 5 min: 5,000 ppm

(published fataj concentration

to one or more humans)

human, rapid: 5,000-10,000 ppm 7424

(published fataj concentration

to one or more humans)

rat, using $\mathrm{NH} 4 \mathrm{OH}: 350 \mathrm{mg} / \mathrm{kg}$

6267

(fatal dose by ingestion for

half of test animals)

rat, $10 \mathrm{~min}, 0 / 10: 38,900 \mathrm{ppm}$

$v / v$ (no observed effect level

in test animals)

mouse, $30 \mathrm{~min}: 303 \mathrm{ppm} \mathrm{v} / \mathrm{v}$

(50\% decrease in respiratory

rate in test anima1s) 
- flammability LFL-UFL (flammability limits in air):

$\begin{array}{ll}15-16-25-28 \% \mathrm{v} / \mathrm{v} & 3635 \\ 15-28 \% \mathrm{v} / \mathrm{v} & 4908 \\ 16-25 \% \mathrm{v} / \mathrm{v} & 4757 \\ 16-25 \% \mathrm{v} / \mathrm{v} & 5 \mathrm{C} 46 \\ \text { at } 100 \% \mathrm{RH}: 15.95-26.55 \% \mathrm{v} / \mathrm{v} & 6350 \\ \text { in dry air: 15.15-27.35\% v/v } & 4 \mathrm{~B} 60 \\ \text { match ignition: } 14.8-33.4 \% & 2525 \\ \mathrm{v} / \mathrm{v} & \\ 15.1 \% \mathrm{v} / \mathrm{v} & 2525 \\ 15.1 \% \mathrm{v} / \mathrm{v} & 2525 \\ 22.5 \mathrm{MJ} / \mathrm{kg}(9673 \mathrm{Btu} / \mathrm{lb}) & 2318 \\ \text { none (nonflammable as tested) } & 3903 \\ 651 \mathrm{C}\left(1204{ }^{\circ} \mathrm{F}\right) & 4757 \\ \text { BOC Gases: } 6900^{\circ} \mathrm{C}\left(1274{ }^{\circ} \mathrm{F}\right) & \mathrm{MSDS} \\ \text { DuPont: } 400-500{ }^{\circ} \mathrm{C}\left(842-932^{\circ} \mathrm{F}\right) & \mathrm{MSDS} \\ & \\ \text { colorless } & 5 \mathrm{C} 46 \\ \text { penetrating and pungent odor } & 5 \mathrm{C} 46 \\ 5-50 \mathrm{ppm} \mathrm{v} / \mathrm{v} & 3635\end{array}$

LFL (with spark ignition): $15.1 \% \mathrm{v} / \mathrm{v}$ LFL (with hot-wire ignition): $15.1 \% \mathrm{v} / \mathrm{v}$ heat of combustion (by ASHRAE 34-92): flash point: autoignition temperature: autodecomposition temperature:

- detection appearance: odor sensing, Tower threshold: PRODUCTION first commercial use as a refrigerant: last year production allowed: 1869 by $C$. Linde (compression) 2113 unrestricted 


\section{R-744 (CARBON DIOXIDE)}

R-744 Carbon dioxide
inorganic CO2
COMmON USE(S) CAS number 124-38-9
industria1 refrigerant, first used in 1866 and still in use;
component of air (0.033 ño.001\%, 330 ppm, in dry atmospheric air);
frozen carbon dioxide (dry ice) is used as a coolant for temporary
storage at refrigerated conditions; propellant; fire suppressant;
carbonation of beverages

IDENTIFIERS

common name(s): R-744; R744; R 744

carbon dioxide; "carbon oxide"

chemical name (by IUPAC convention): dry ice (when frozen)

a)ternative chemical names/formulae:

carbon dioxide CAS number:

EINECS number:

$\mathrm{CO} 2$

124-38-9 (Chemical Abstracts

Service registry number)

204-696-9 (European Inventory

of Existing Chemica]

Substances)

NIOSH RTECS number:

FF6400000 (Registry of Toxic

Effects of Chemical

Substances)

trade name(s): Khladon 744

historical name(s): carbonic acid anhydride

carbonic acid gas

carbonic anhydride

5787

ARI container color / Pantone number: none, use light green grey/413 6601

PHYSICAL

- properties

molar mass: $44.0095 \mathrm{~g} / \mathrm{mol}$ (0.097024

$1 \mathrm{~b} / \mathrm{mo} 7$ )

$-56.6^{\circ} \mathrm{C}\left(-69.8^{\circ} \mathrm{F}\right)$

8820

normal freezing/melting/triple point:

- normat boiling point

temperature:

$-78.4{ }^{\circ} \mathrm{C}\left(-109.1{ }^{\circ} \mathrm{F}\right)$

3209

heat of vaporization:

- norma 7 pressure, $20{ }^{\circ} \mathrm{C}\left(68^{\circ} \mathrm{F}\right)$ - -

$572.9 \mathrm{~kJ} / \mathrm{kg}(246.3 \mathrm{Btu} / \mathrm{lb}) \quad 3401$

density, vapor:

$1.839 \mathrm{~kg} / \mathrm{m} 3(0.1148 \mathrm{~Tb} / \mathrm{cf}) \quad 8401$

- normat pressure, $21.1{ }^{\circ}{ }^{\mathrm{C}}\left(70^{\circ} \mathrm{F}\right)---$

- $20^{\circ} \mathrm{C}\left(68^{\circ} \mathrm{F}\right)$

$1.832 \mathrm{~kg} / \mathrm{m} 3(0.1144 \mathrm{lb} / \mathrm{cf}) \quad 8401$

pressure, saturated vapor: density, saturated 1iquid: density, saturated vapor: specific volume, saturated 1 iquid: specific volume, saturated vapor: velocity of sound, saturated liquid: velocity of sound, saturated vapor: viscosity, saturated 1iquid: viscosity, saturated vapor:

thermal conductivity, saturatd 1iquid:

$5721.6 \mathrm{kPa}$ (829.85 psia)

$774 \mathrm{~kg} / \mathrm{m3}\left(48.33^{7 \mathrm{~b} / \mathrm{cf})}\right.$

8401

$192.32 \mathrm{~kg} / \mathrm{m3}(12.006 \mathrm{lb} / \mathrm{cf}) \quad 8401$

$1.292 \mathrm{~L} / \mathrm{kg}(0.0207 \mathrm{cf} / \mathrm{1b}) \quad 8401$

$5.2 \mathrm{~L} / \mathrm{kg}(0.0833 \mathrm{cf} / 7 \mathrm{~b})$

$341 \mathrm{~m} / \mathrm{s}$ (1119 ft/s)

$198 \mathrm{~m} / \mathrm{s}(649 \mathrm{ft} / \mathrm{s})$

$67 \mu \mathrm{Pa} \cdot \mathrm{s}(0.067 \mathrm{cp})$

$17.3 \mu \mathrm{Pa} \cdot \mathrm{s}(0.0173 \mathrm{cp})$

$0.0712 \mathrm{~W} / \mathrm{m} \cdot \mathrm{K}(0.0412$

$\mathrm{Btu} / \mathrm{hr} \cdot \mathrm{ft}^{\circ} \mathrm{F}$ )

thermal conductivity, saturated vapor:

$0.01894 \mathrm{~W} / \mathrm{m} \cdot \mathrm{K}(0.01094$

$\mathrm{Btu} / \mathrm{hr} \cdot \mathrm{ft}^{\circ} \mathrm{F}$ )

8401

8401

8401

8401

8401

8401

- critical point

$31.1{ }^{\circ} \mathrm{C}\left(87.9^{\circ} \mathrm{F}\right)$

$7384 \mathrm{kPa}$ (1071.0 psia)

3209

8401 
ENVIRONMENTAL

average atmospheric lifetime (Tatm): ODP (ozone depletion potential):

variable: $>50$ yr

6694

GWP (global warming potential): 0.000 (model-derived relative to $\mathrm{R} 11$ )

1 relative to $\operatorname{co} 2$ for $100 \mathrm{yr}$

9501

HGWP (ha7ocarbon GWP): integration

0.00076 relative to $R 11$ for 6307

SAFETY

- Classification

infinite integration period

safety group (ASHRAE Standard 34): NFPA 704 degrees of hazard (H-F-R-S):

NPCA HMIS hazard ratings $(H-F-R)$ :

AI

ARI recommendation: 2-0-0 3A15

Amerex:

BOC Gases: 1-0-0

heal th-flammabi Tity-reactivity

[-specia]]: $0=$ no, $4=$ severe

Amerex: $1-0-0$

BOC Gases: $1-0-0$

DuPont: $1-0-0$

hea 7 th-f7 ammabi lity-reactivity

$0=$ insignificant, $4=$ extreme

UL Comparative Hazard to Life Group:

- short-term occupational Timit ------

NIOSH IDLH (immediately dangerous): NIOSH SCP IDLH (immediately dangerous: NIOSH STEL (short-term exposur limit): ACGIH TLV-STEL (short-term exp limit): - occupational exposure limit -..---MAK (maximum workplace concentration):

- Tong-term occupational limit

5 (a) in absence of flame or hot objects

$40,000 \mathrm{ppm} \mathrm{v} / \mathrm{v}$

$50,000 \mathrm{ppm} \mathrm{v} / \mathrm{v}$ for $30 \mathrm{~min}$

5204

$30,000 \mathrm{ppm} \mathrm{v} / \mathrm{v}$ TWA for $15 \mathrm{~min} 3903$

$30,000 \mathrm{ppm} \mathrm{v} / \mathrm{v}$ TWA for $15 \mathrm{~min} 9504$

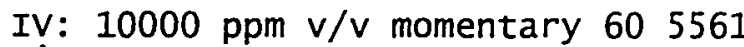
min

5,000 ppm $v / v$ TWA for $8 \mathrm{hr} /$ day 3904 and $40 \mathrm{hr} / \mathrm{wk}$

$5,000 \mathrm{ppm} v / \mathrm{V}$ TWA for $10 \quad 3903$

$\mathrm{hr} /$ day and $40 \mathrm{hr} / \mathrm{wk}$

$5,000 \mathrm{ppm} \mathrm{v} / \mathrm{v}$ TWA for $8 \mathrm{hr} /$ day 9504 and $40 \mathrm{hr} / \mathrm{wk}$

$5,000 \mathrm{ppm} \mathrm{V} / \mathrm{v}$ TWA for $8 \mathrm{hr} /$ day 5561 40 (or 42) hr/wk

being examined for changes 7101

- emergency exposure Timit - - - - - -

Acute Toxicity Exposure Limit (ATEL):

Refrigerant concentration Limit $(R C L)$ :

- acute (short-term) toxicity -------

$40,000 \mathrm{ppm} v / \mathrm{v}$ (preliminary $34 \mathrm{u}$

based on ASHRAE $34 \mathrm{u}$ draft)

$40,000 \mathrm{ppm} \mathrm{v} / \mathrm{v}$ (preliminary

A950

based on ASHRAE 34u draft)

40,000 ppm v/v (preliminary

based on ASHRAE $34 u$ draft)

40,000 ppm v/v (preliminary

based on ASHRAE $34 \mathrm{u}$ draft)

human, instant: $400,000 \mathrm{ppm} \quad 7424$

(published fatal concentration

to one or more humans)

human, 5.min: $90,000 \mathrm{ppm}$

(published fatal concentration

to one or more humans)

anesthetic/CNS effect LOEL: human, $3.8 \mathrm{~min}$ 13/31: 104,000 A658

$\mathrm{ppm} v / \mathrm{v}$ (lowest observed

effect 7 evel) 
- flammability LFL-UFL (flammability limits in air):
autoignition temperature:
autodecomposition temperature:

- detection appearance:
odor:

human, $5 \mathrm{~min}, ? / 3: 55,000 \mathrm{ppm}$ A657 $v / v$ (jowest observed effect Teve 1)

human, $7.4 \mathrm{~min}, 9+/ 42: 76,000$ A658 ppm $v / v$ (lowest observed

effect leve1) human, 10 min caution: 100,000 A657 $\mathrm{ppm} v / \mathrm{v}$ (Towest observed effect leve 7 ) human, 15 min, <22/42: 75,000 $\mathrm{ppm} v / \mathrm{v}$ (1owest observed effect leve1) human, 30 min caution: 50,000 $\mathrm{ppm} v / \mathrm{v}$ (lowest observed effect 1eve ?)

human, 30 min: $50,000-100,000$ $\mathrm{ppm} \mathrm{v} / \mathrm{v}$ (lowest observed effect Teve 7 ) human, $>50,000 \mathrm{ppm} \mathrm{v} / \mathrm{v}$ (7owest 7424 observed effect level)

none (nonflammable as tested) none (nonflammable as tested) BOC Gases: none

BOC Gases: $1700{ }^{\circ} \mathrm{C}\left(3092^{\circ} \mathrm{F}\right)$

colorless odorless
2525

3903

MSDS

MSDS

6280

5141

\section{PRODUCTION}

first commercial use as a refrigerant: 1869 by T. S. C. Lowe 


\section{R-1270 (PROPYLENE)}

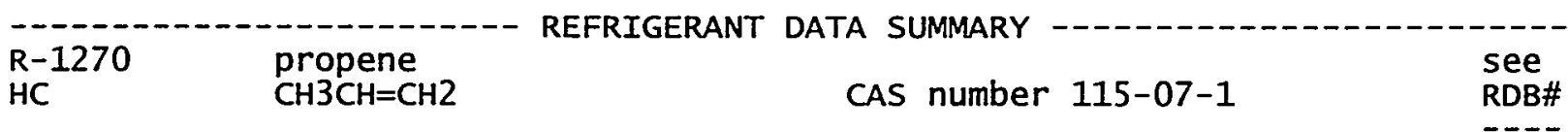

COMMON USE(S)

component of refrigerants $411 \mathrm{~A}$ and $411 \mathrm{~B}$; very 7 imited use as an industrial refrigerant; further use as a single-compound refrigerant is constrained by its flammability; aerosol propellant, alone or as a blend component; widely used feedstock to produce plastics, alcohols, gasoline, synthetic rubbers, and other chemical products; considered as an anesthetic agent in the 1920s, but not widely used due to depression of the heart function

Revision of the safety classification for this refrigerant was proposed on 1998.01.18. The revision is subject to a review and approval procedure; assignment wi7l not occur until published in an addendum or revision to ANSI/ASHRAE Standard 34-1997.

IDENTIFIERS

$$
\text { common name(s): } \begin{aligned}
& \text { R-1270; R1270; R } 1270 \\
& \text { HC-1270 }
\end{aligned}
$$

alternative chemical names/formulae: propene; propylene

1-propene; 1-propylene methylethene

methylethylene

$\mathrm{CH} 3 \mathrm{CH}=\mathrm{CH} 2 ; \mathrm{CH} 3-\mathrm{CH}=\mathrm{CH} 2$

not recommended:

$\mathrm{CH} 2=\mathrm{CHCH} 3 ; \mathrm{CH} 2=\mathrm{CH}-\mathrm{CH} 3$

$\mathrm{CH} 3 \mathrm{CHCH} 2 ; \mathrm{H} 3 \mathrm{CCH}=\mathrm{CH} 2$;

empirical formula: $\mathrm{C} 3 \mathrm{H} 6$

$\mathrm{H} 3 \mathrm{C}-\mathrm{CH}=\mathrm{CH} 2 ; \mathrm{H} 3 \mathrm{CCH}=\mathrm{CH} 2$

CAS number: 115-07-1 (Chemical Abstracts Service registry number)

UC6740000 (Registry of Toxic Effects of Chemical

NIOSH RTECS number:

ARI container color / Pantone number: Substances)

none, use light green grey/413 6601 with red / 185 band

\section{PHYSICAL}

- properties

molar mass:

$42.07974 \mathrm{~g} / \mathrm{mol}(0.092770$

$1 \mathrm{~b} / \mathrm{mo} 1)$

normal freezing/melting/triple point:

$-185.2^{\circ} \mathrm{C}\left(-301.4^{\circ} \mathrm{F}\right)$

normal boiling point -rere:

density, saturated 7iquid:

$-47.7^{\circ} \mathrm{C}\left(-53.8^{\circ} \mathrm{F}\right)$

$609 \mathrm{~kg} / \mathrm{m} 3(38.02 \mathrm{lb} / \mathrm{cf})$

$2.36 \mathrm{~kg} / \mathrm{m3}(0.147 \mathrm{7b} / \mathrm{cf})$

$1.642 \mathrm{~L} / \mathrm{kg}(0.0263 \mathrm{cf} / 7 \mathrm{~b})$

$424.2 \mathrm{~L} / \mathrm{kg}(6.7944 \mathrm{cf} / 7 \mathrm{~b})$

$439.2 \mathrm{~kJ} / \mathrm{kg}$ (188.8 Btu/7b)

$1169 \mathrm{~m} / \mathrm{s}(3835 \mathrm{ft} / \mathrm{s})$

$224 \mathrm{~m} / \mathrm{s}(735 \mathrm{ft} / \mathrm{s})$

$244 \mu \mathrm{Pa} \cdot \mathrm{s}(0.244 \mathrm{cp})$

$5.84 \mu \mathrm{Pa} \cdot \mathrm{s}(0.00584 \mathrm{cp})$

$0.1573 \mathrm{~W} / \mathrm{m} \cdot \mathrm{K} \quad(0.0909$

$\mathrm{Btu} / \mathrm{hr} \bullet \mathrm{ft}{ }^{\circ} \mathrm{F}$ )

$0.0104 \mathrm{~W} / \mathrm{m} \cdot \mathrm{K}(0.0060$

8401

8401

8401

8401

8401

8401

8401

8401

8401

8401

8401 thermal conductivity, liquid:

thermal conductivity, vapor:

$$
\mathrm{Btu} / \mathrm{hr} \cdot \mathrm{ft}^{\circ} \mathrm{F} \text { ) }
$$

8401 
- normal pressure, $20{ }^{\circ} \mathrm{C}\left(68{ }^{\circ} \mathrm{F}\right)$--.--

- normal pressure, $21.1{ }^{\circ} \mathrm{C}\left(70{ }^{\circ} \mathrm{F}\right)$---

- $20^{\circ} \mathrm{C}\left(68^{\circ} \mathrm{F}\right)$ - density, vapor: pressure, saturated vapor: density, saturated 7 iquid: density, saturated vapor: specific volume, saturated liquid: specific volume, saturated vapor: velocity of sound, saturated 7iquid: velocity of sound, saturated vapor: viscosity, saturated Tiquid: viscosity, saturated vapor: thermal conductivity, saturatd liquid:

thermal conductivity, saturated vapor:

- $60^{\circ} \mathrm{C}\left(140{ }^{\circ} \mathrm{F}\right)$

pressure, saturated vapor: heat of vaporization:

- critical point

temperature: pressure: density: specific volume:
$1.776 \mathrm{~kg} / \mathrm{m} 3(0.1109 \mathrm{~Tb} / \mathrm{cf})$

8401

$1.769 \mathrm{~kg} / \mathrm{m} 3(0.1104 \mathrm{~Tb} / \mathrm{cf})$

8401

$1019.9 \mathrm{kPa}$ (147.92 psia)

$513 \mathrm{~kg} / \mathrm{m} 3$ (32.03 $1 \mathrm{~b} / \mathrm{cf})$

$21.44 \mathrm{~kg} / \mathrm{m3}$ (1.338 Tb/cf)

$1.949 \mathrm{~L} / \mathrm{kg}(0.0312 \mathrm{cf} / 7 \mathrm{~b})$

$46.6 \mathrm{~L} / \mathrm{kg}(0.7471 \mathrm{cf} / 7 \mathrm{~b})$

$727 \mathrm{~m} / \mathrm{s}(2387 \mathrm{ft} / \mathrm{s})$

$223 \mathrm{~m} / \mathrm{s}(730 \mathrm{ft} / \mathrm{s})$

$106 \mu \mathrm{Pa} \cdot \mathrm{s}(0.106 \mathrm{cp})$

$7.7 \mu \mathrm{Pa} \cdot \mathrm{s}(0.0077 \mathrm{cp})$

$0.1113 \mathrm{w} / \mathrm{m} \cdot \mathrm{K}(0.0643$

$\mathrm{Btu} / \mathrm{hr} \cdot \mathrm{ft}^{\circ} \mathrm{F}$ )

$0.08639 \mathrm{~W} / \mathrm{m} \cdot \mathrm{K}(0.04991$

$\mathrm{Btu} / \mathrm{hr} \bullet \mathrm{ft}^{\circ} \mathrm{F}$ )

$2533 \mathrm{kPa}$ (367.3 psia)

$249.7 \mathrm{~kJ} / \mathrm{kg}$ (107.3 Btu/7b)

8401

$92.4^{\circ} \mathrm{C}\left(198.4^{\circ} \mathrm{F}\right)$

$4665 \mathrm{kPa}$ (676.6 psia)

$223 \mathrm{~kg} / \mathrm{m} 3(13.9 \mathrm{lb} / \mathrm{cf})$

$4.48 \mathrm{~L} / \mathrm{kg}(0.0717 \mathrm{cf} / 1 \mathrm{~b})$

8401

8401

8401

8401

8401

8401

8401

8401

8401

8401

8401

8401

8401

8401

8401

8401

ENVIRONMENTAL

ODP (ozone depletion potentia1):

0.000 (model-derived relative to $R$ 11)

SAFETY

- classification -...-. safety group (ASHRAE Standard 34):

NFPA 704 degrees of hazard (H-F-R-S):

NPCA HMIS hazard ratings $(H-F-R)$ :

IARC/CIRC human carcinogicity group: ACGIH carcinogenicity category:

DFG carcinogicity class:

- occupational exposure warnings --.--

- Tong-term occupationaT Timit Caution: exposure Timit consistent to OSHA PEL:

- emergency exposure Timit Acute Toxicity Exposure Limit (ATEL):

A3

Ashland: $1-4-1$.

BOC Gases: 1-4-1 MSDS

DuPont: 1-4-1 MSDS

Phillips: 1-4-1 MSDS

Texaco: 1-4-1 MSDS

hea 7 th-f7 ammabi 1ity-reactivity

[-specia]]: $0=$ no, $4=$ severe

BOC Gases: 0-4-0 MSDS

DuPont: $0-4-2$ MSDS

Texaco: 1-4-1 MSDS

hea 7 th-f7 ammabi 7 ity-reactivity

$0=i n s i g n i f i c a n t, 4=e x t r e m e$

3 , not classifiable 2428

A4, not classifiable as a 9504

human carcinogen

being examined for new entry 7101

simple asphyxiant 9504

Exxon: $1,000 \mathrm{ppm} \mathrm{v} / \mathrm{v}$ TWA for 8 MSDS $\mathrm{hr} /$ day and $40 \mathrm{hr} / \mathrm{wk}$

Greencoo 1: $375 \mathrm{ppm} v / \mathrm{v}$ TWA for MSDS $8 \mathrm{hr} /$ day and $40 \mathrm{hr} / \mathrm{wk}$

Phil7ips: 1,000 ppm $v / v$ TWA MSDS

for $8 \mathrm{hr} /$ day and $40 \mathrm{hr} / \mathrm{wk}$

$0 \mathrm{ppm} v / \mathrm{v}$ (preliminary based $34 \mathrm{u}$

on ASHRAE $34 \mathrm{u}$ draft)

provisional: $0 \mathrm{ppm} \mathrm{v} / \mathrm{v}$

A950 
Refrigerant concentration Limit (RCL):

ALC (approximate lethal concentration:

cardiac sensitization threshold/LOEL:

anesthetic concentration:

anesthetic/CNS effect LOEL:

anesthetic/CNS effect NOEL:

- flammability

LFL-UFL (flammability limits in air):

flash point:

autoignition temperature:

- detection (preliminary based on ASHRAE $34 \mathrm{u}$ draft)

provisiona1: $0 \mathrm{ppm} \mathrm{v} / \mathrm{V}$

(preliminary based on ASHRAE

$34 u$ draft)

rat, $2 \mathrm{hr}$, ?/?: $650,000 \mathrm{ppm}$

(lowest exposure tested with one or more deaths by

inhalation)

rat, $6 \mathrm{hr}, 0 / ?:>400,000 \mathrm{ppm}$

(lowest exposure tested with

one or more deaths by

inhalation)

dog 10min 2/2: 100,000-250,000

$\mathrm{ppm} \mathrm{v} / \mathrm{v}$ (lowest observed

effect leve1 in test anima1s)

human, $2.25 \mathrm{~min}: 64,000 \mathrm{ppm}$ $v / v$

mouse, 1 min, ?/?: 500,000 ppm 6A70

$v / v$ (lowest observed effect

leve 7 in test anima7s)

rat, 15-20 min, 1/1: 400,000

$\mathrm{ppm} v / \mathrm{v}$ (lowest observed

effect leve 1 in test animals)

mouse, $6 \mathrm{hr} / \mathrm{d}$ chronic: 9,957

$\mathrm{ppm} v / \mathrm{v}$ (no observed effect

level in test animals)

rat, $6 \mathrm{hr} / \mathrm{d}$ chronic: 9,891 ppm 7534

$v / v$ (no observed effect level

in test animals)

rat, $6 \mathrm{hr} / \mathrm{d}$ subchronic: 10,000 7534

$\mathrm{ppm} v / \mathrm{v}$ (no observed effect

level in test animals)

$2.0-11.0 \% \mathrm{v} / \mathrm{v}$

6290

Exxon: $2.0-11.1 \% \mathrm{v} / \mathrm{v}$

Phillips: $2.0-10 \% \mathrm{v} / \mathrm{v}$

$-108{ }^{\circ} \mathrm{C}\left(-162{ }^{\circ} \mathrm{F}\right)$

Ashland: $-40{ }^{\circ} \mathrm{C}\left(-40^{\circ} \mathrm{F}\right)$

$65 \mathrm{~B} 9$

7534

Dupont: $460^{\circ} \mathrm{C}\left(860^{\circ} \mathrm{F}\right)$

Exxon: $458{ }^{\circ} \mathrm{C}\left(856^{\circ} \mathrm{F}\right)$

Texaco: $497^{\circ} \mathrm{C}\left(927^{\circ} \mathrm{F}\right)$

MSDS

MSDS

6290

MSDS

MSDS

MSDS

MSDS

appearance: colorless

odor: BOC Gases: mild olefinic odor

Phillips: mild, sour odor

Texaco: odorless

practically odorless

odor sensing, lower threshold:

DuPont: 10-60 ppm v/v

6290

MSDS

MSDS

MSDS

6290

MSDS

PRODUCTION

last year production allowed: unrestricted

$8 C 01$ 


\section{APPENDIX 3: TOXICITY SUMMARIES}

This appendix presents toxicity summaries from the ARTI Refrigerant Database ${ }^{22}$ most of them prepared in 1995 with minor updates since then. 


\section{R-11}

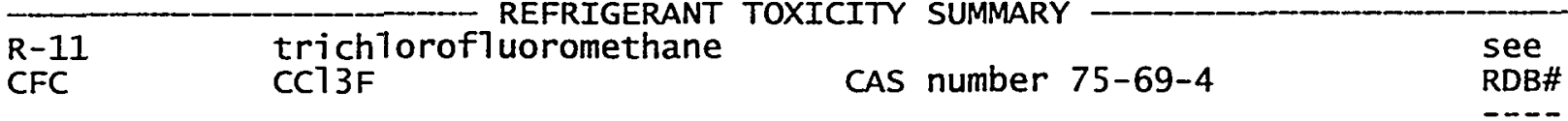

R-11 has extremely low toxicity by skin contact or ingestion, but 5873

it can cause frostbite if splashed on the skin, drying and redness 5847

with repeated or prolonged contact, and mild irritation to the eyes.

It has very low toxicity by inhalation and is neither carcinogenic nor mutagenic. Animal tests show no developmental or reproductive effects. R-11 is, however, a comparatively potent cardiac sensitizer to adrenaline and depresses the cardiopulmonary and central nervous systems with exposures at high concentrations. /1-9/

\section{OCCUPATIONAL EXPOSURE LIMITS}

The Occupational Safety and Health Administration (OSHA) promulgated a Permissible Exposure Limit (PEL) of 1,000 ppm for R-11. The National Institute for Occupational Health and Safety (NIOSH) and American Conference of Governmental Industrial Hygienists (ACGIH) adopted a Recommended Exposure Limit Ceiling (REL-C) and a Threshold Limit value - Ceiling (TLV-C), respectively, of $1,000 \mathrm{ppm}$. The AirConditioning and Refrigeration Institute (ARI) a1so recommends a ceiling concentration limit (PEL-C) of $1,000 \mathrm{ppm}$.

\section{GENERAL CAUTIONS}

More recent and/or more complete information may be found in Material Safety Data sheets (MSDSS), provided by the chemical supplier(s), and in other toxicology references. Inhalation of high concentrations of refrigerant vapors is harmful. Anesthetic effects including loss of consciousness, heart irregularities, or death may result. Intentional misuse or deliberate inhalation ("sniffing") may cause death without warning. Massive leaks or spi $71 \mathrm{~s}$ also may pose an asphyxiation hazard, by displacing air, in confined or inadequately ventilated locations. This refrigerant is heavier than air, and leakage may accumulate in low areas.

NATIONAL RESEARCH COUNCIL (NRC) COMMITTEE ON TOXICOLOGY SUMMARY OF TOXICITY INFORMATION (OCtOber 1984)

EFFECTS ON HUMANS: Inhalation of f7uorocarbons during the years 19601970 was a prominent cause of abusive death among teenagers. Severe cardiac arrhythmia - resulting from light plane anesthesia and intensified by hypercapnia, stress, or activity - was suggested as an explanation for 110 cases of sudden sniffing death (Bass, 1970). Typical7y, a person would spray the Freon [f7uorocarbon] into a paper bag from a commercial aerosol product and inhale it; after a few breaths and a short excitement period, death might occur. Fluorocarbons are thought to sensitize the heart to asphyxia-induced sinus bradycardia, atrioventricular block, and ventricular T-wave depression ( $\mathrm{Haj}$ et al., 1980).

Accidental ingestion of FC-11 occurred when a healthy man mistook a bottle in a refrigerator for a bottle of plain water. This resulted in freezing, tissue necrosis, and multiple perforations of the stomach. The patient recovered after surgery to remove the damaged tissue (Haj et a 1., 1980)

Labeled FC-11 administered to four healthy males by inhalation of a single breath held for $5 \mathrm{~s}$ was eliminated rapidly. Results in humans 
appeared to parallel those in rats in more detailed studies (Williams et a7., 1974). The investigators found rapid transfer of FC-11 to blood followed by distribution to fat, from which release was slow. Mergner et al. (1975) exposed a male and a female volunteer to radiolabeled $\mathrm{FC}-11$ at $1,000 \mathrm{ppm}$ for 7-17 min. Recovery of administered radioactivity in exhaled air was essential7y complete (99\% and $79 \%$ ). Errors in collection of rapidly eliminated gases account for the differences from $100 \%$. Only a very smal1 fraction of the administered radioactivity (1ess than $0.2 \%$ ) was exha7ed as [14] $\mathrm{CO} 2$ or excreted as nonvolatile urinary activity. The impurities in FC-11 - namely chloroform and carbon tetrachloride - known to be metabolized could account for a 77 the radioactivity found in urine and exhaled $\mathrm{CO2}$ after exposure to $\mathrm{FC}-11$.

Cardiac effects have been studied in healthy subjects and patients with bronchopulmonary disease. None of the subjects exhibited cardiotoxic effects (Fabel et al., 1972).

Human volunteers were exposed to FC-113 (similar to FC-11) at 500 or $1,000 \mathrm{ppm}$ for $6 \mathrm{~h} / \mathrm{d}, 5 \mathrm{~d} / \mathrm{wk}$ during a 2 -wk period. No adverse changes were seen in performance of complex menta] tasks, clinical status, or results of biochemical tests. Breath analysis did not reveal a significant buiTdup of FC-113 (Reinhardt et al., 1971).

EFFECTS ON ANIMALS: FC-11 has not shown appreciable oral toxicity in rats and dogs in either acute or chronic studies (Haske11 Laboratory, 1970; NCI, 1978). The chronic investigations include 1-mo, 90-d, and 2-yr studies. FC-11 was tested on the intact skin of mice. It was we 77 tolerated by the skin, but retarded the recovery of wounds and burns and regrowth of hair (Quevauvi 11er, 1960;

Quevauvi7ler et a7., 1963). Dermal application of FC-11 to rabbit skin did not produce any lesions (Scholz, 1962). Transient conjunctival irritation was observed after application of FC-11 solution to the rabbit eye. No irreversible eye damage was seen (Haske11 Laboratory, 1970; Kudo et a1., 1971).

The LC50 of FC-11 for rats in a 4-hour exposure is 26,200 ppm (Haske11 Laboratory, 1970). A 30-min exposure of rats at $50,000 \mathrm{ppm}$ caused no signs of intoxication. Similar exposure at higher concentrations caused clinical signs of central nervous system depression. Concentrations of $100,000 \mathrm{ppm}$ or more were fatal after less than $30 \mathrm{~min}$ (Lester and Greenberg, 1950). Acute exposure of other species of laboratory animals produced similar effects (caujolle, 1964; Haske17 Laboratory, 1970; Nuckol1s, 1933; Scholz, 1962).

Rats, guinea pigs, monkeys, and dogs were continuously ( $24 \mathrm{~h} / \mathrm{d})$ exposed to $\mathrm{FC}-11$ at approximately $1,000 \mathrm{ppm}$ for $90 \mathrm{~d}$. One monkey died on day 78 , but its death was not definitely linked to exposure to FC-11. No other animals were affected. No compound-related pathological changes were observed. Another group of animals was exposed at $10,250 \mathrm{ppm}, 8$ $\mathrm{h} / \mathrm{d}, 5 \mathrm{~d} / \mathrm{wk}$ for 6 wk without adverse effects (Jenkins et a), 1970). In another study, dogs, cats, guinea pigs, and rats were exposed to FC11 for $3.5 \mathrm{~h} / \mathrm{d}, 5 \mathrm{~d} / \mathrm{wk}$, for $4 \mathrm{wk}$; the dogs were exposed at $12,500 \mathrm{ppm}$, and the other animals at 25,000 ppm. No microscopic evidence of damage to the lungs, heart, spleen, liver, or kidneys was seen (scholz, 1962).

$5 C 53$

Rats and mice exposed to FC-11 at 1,000 or 5,000 ppm for 1ifetime showed no evidence of carcinogenicity or other adverse health effects (C. Maltoni, unpublished).

FC-11, Tike other chlorof7uorocarbons and hydrocarbons, was capable of sensitizing the beagle heart to exogenous epinephrine in standard 5-min cardiac sensitization screening studies. A 5 -min cardiac-sensitization 
screening test consists of a control intravenous injection of epinephrine at $8 \mu \mathrm{g} / \mathrm{kg}$, followed 7ater by a 5-min exposure to fluorocarbon and then a challenge with $8 \mu \mathrm{g} / \mathrm{kg}$ intravenous7y. Manifestation of arrhythmia (multiple consecutive ventricular beats), which is considered to pose a serious threat to life, or cardiac arrest (ventricular fibrillation) constitutes a positive test. The lowest concentration that elicited a marked response in exposed dogs was 5,000 ppm. A concentration of $1,000 \mathrm{ppm}$ was ineffective. Dogs exposed while running on a treadmi 17' (to increase their circulating epinephrine) were not sensitized at concentrations up to $10,000 \mathrm{ppm}$ (MuT7in et a1., 1972).

Azar et al. (1973) studied nonanesthetized dogs and reported that the average blood concentrations of FC-11 associated with cardiac sensitization were $28.6 \mu \mathrm{g} / \mathrm{L}$ in arterial and $19.7 \mu \mathrm{g} / \mathrm{L}$ in venous blood.

Belej and Aviado (1975) studied cardiopulmonary toxicity of prope 7 lants in anesthetized dogs. They concluded that FC-11, un 7 ike eight other halocarbon propellants studied, produced bronchodilation, rather than bronchoconstriction. It also reduced pulmonary compliance and respiratory minute volume. FC-11 had the greatest tachycardiac effect of a 11 compounds studied. Effects of FC-11 on the circulatory system were summarized by Aviado (1975, 1978).

In a bioassay supported by the National Cancer Institute (1978), oral FC-II was not carcinogenic in rats or mice. No significant increase in tumor formation was seen in a study that used subcutaneous injection (Epstein et a1., 1967). Additional7y, FC-11 has not been shown to be mutagenic in the Salmonella typhimurium reverse-mutation bioassay (C. F. Reinhardt, Haske11 Laboratory, personal communication). No embryotoxic fetotoxic, or teratogenic effect of FC-11 was shown in a study with pregnant rats and rabbits; the animals were exposed for $2 \mathrm{~h} / \mathrm{d}$ to $200,000 \mathrm{ppm}$ of a FC-11/FC-12 (1:9) mixture from day 4 to 16 of gestation for rats and from day 5 to 20 for rabbits (Paulet, 1976). Blake and Mergner (1974) investigation studied the biotransformation of 14C-7abelled FC-11 (8,000-12,000 ppm) in male and female beagles after a short (6-20 min) inhalation. Essentially all the inhaled fluorocarbon was recovered in the exhaied air within $1 \mathrm{~h}$. Oniy traces of radioactivity were found in urine or exhaled $\mathrm{CO} 2$. The investigators concluded that $\mathrm{FC}-11$ is relatively refractory to biotransformation after a short inhalation exposure and that it is rapidly exhaled chemically unaltered.

\section{OTHER}

Human volunteers exposed to $R-11$ at $2,800-27,000 \mathrm{ppm}$ for $15-60$ seconds exhibited acute reduction of ventilatory lung capacity, bradycardia, increased variability in heart rate, and effects on their electrocardiograms. /3/

\section{ADDITIONAL REFERENCES}

1. Freon(R) 11, Material Safety Data Sheet (MSDS) DU000026 (2090FR), DuPont Chemicals, wilmington, DE, 8 January 1998

2. Used Refrigerant 11, MSDS 3090FR, DuPont Chemicals, wilmington, DE, 21 January 1992

3. Forane(R) 11, MSDS 04011, E7f Atochem North America, Incorporated, Philadelphia, PA, 3 November 1999

4. Forane(R) 11 Reclaim, MSDS 04411.5, Elf Atochem North America, Incorporated, Philadelphia, PA, 15 October 1996

5. Fluorotrichloromethane, MSDS 309, Genium Pub7ishing Corporation, Schenectady, NY, February 1986

6. Genetron(R) 11, MSDS GTRN-0017, Honeywe 17 Incorporated, Morristown, NJ, January 2000

7. Arcton $(R) 11$, MSDS 232, ICI K1ea, wilmington, DE, 23 January 1998 
8. Arcton(R) 11, Chemical Safety Data sheet (CSDS) revision 04, ICI Kiea, Runcorn, Cheshire, UK, Apri 11997

9. Fluorotrichloromethane, MSDS OHS0990, occupational Hea7th Services, Incorporated (OHS), New York, NY, 14 July 1993 


\section{R-12}

$\begin{array}{llll}R-12 & \text { dichlorodif7uoromethane } & \text { SEe } \\ \text { CFC } & \text { CC12F2 } & \text { CAS number } 75-71-8 & \text { RDB\# }\end{array}$

R-12 has extremely low toxicity by skin contact or ingestion, but it can cause frostbite if splashed on the skin, drying and redness with repeated or prolonged contact, and mild irritation to the eyes. It has very low toxicity by inhalation and is neither carcinogenic nor mutagenic. Animal tests show no developmental or reproductive effects. R-12 is, however, a cardiac sensitizer to adrenaline and produces a rapid anesthetic effect at high concentrations. /1-8/ Tests in humans showed no $i 11$ effects at $1,000 \mathrm{ppm} v / \mathrm{V} R-12$ in air for up to eight hours; R-12 is quickly eliminated from the body in exhaled air.

\section{OCCUPATIONAL EXPOSURE LIMITS}

The Occupational Safety and Health Administration (OSHA), National Conference of Governmental Industrial Hygienists (ACGIH), and

have a 71 adopted recommended chronic exposure 7imits - the Permissible Exposure Limit (PEL), Recommended Exposure Limit (REL), Threshold Limit Value - Time-Weighted Average (TLV-TWA), and Maximale Arbeitsplatz Konzentration (MAK), respectively - of $1,000 \mathrm{ppm} \mathrm{v} / \mathrm{v}$ on a timeweighted-average basis for an 8-10 hour workday and 40-42 hour workweek.

\section{GENERAL CAUTIONS}

More recent and/or more complete information may be found in Material Safety Data Sheets (MSDSS), provided by the chemical supplier(s), and in other toxicology references. Inhalation of high concentrations of refrigerant vapors is harmful. Anesthetic effects including loss of consciousness, heart irregularities, or death may result. Intentional misuse or deliberate inhalation ("sniffing") may cause death without warning. Massive leaks or spil1s also may pose an asphyxiation hazard, by displacing air, in confined or inadequately ventilated locations. This refrigerant is heavier than air, and leakage may accumulate in low areas.

NATIONAL RESEARCH COUNCIL (NRC) COMMITTEE ON TOXICOLOGY SUMMARY OF TOXICITY INFORMATION (October 1984)

EFFECTS ON HUMANS: of 34 cases of sudden death reported to the medical examiner of Da77as, Texas, to be due to abusive, deliberate use of products that can be inhaled, 16 involved fluorocarbons (Garriott and Petty, 1980). The victims were mainly white males aged 13-30 (mean, 18.4). FC-11 and FC-114 were used in addition to FC-12, and many products contained mixtures of $F C-11$ and $F C-12$. The sequence of events has been summarized by Aviado (1978) as follows: sensitization of the heart to prearrhythmic effects of epinephrine, depression of myocardial contractility, reduction in cardiac output, and irritation of mucosa in upper and lower respiratory tract that causes an increase in sympathetic and vagal impulses to the heart.

Azar et a7. (1972) exposed two human volunteers to FC-12 at 1,000 5561 and $10,000 \mathrm{ppm}$ for $2.5 \mathrm{~h}$. Each volunteer was exposed twice at each concentration. Electrocardiographic monitoring and psychomotor performance tests revealed no adverse effects of exposure at 1,000 ppm. At $10,000 \mathrm{ppm}$, there was only a $7 \%$ reduction in psychomotor-test score. 
Rapid elimination from the lungs occurred when exposure stopped. The investigators concluded that a single brief exposure (up to $2.5 \mathrm{~h}$ ) to FC-12 at $10,000 \mathrm{ppm}$ could be tolerated without adverse health effects.

Kehoe (1943) reported that exposure to FC-12 at up to $60,000 \mathrm{ppm}$ $5 C 45$ was tolerated for $80 \mathrm{~min}$ by one subject. When exposed at 40,000 ppm for $14 \mathrm{~min}$ and then at $20,000 \mathrm{ppm}$ for $66 \mathrm{~min}$, a second subject developed EEG changes and had slurred speech and decreased scores in psychologic tests. At $110,000 \mathrm{ppm}$, amnesia and cardiac arrythmia occurred within about $10 \mathrm{~min}$.

Mergner et a1. (1975) exposed a male and female volunteer to radiolabelled FC-12 at 1,000 ppm for 7-17 min. Recovery of administered radioactivity in exhaled air was essentially complete. Radioactivity in urine and exha7ed CO2 together amounted to 1 ess than $0.2 \%$ of administered radioactivity.

EFFECTS ON ANIMALS: FC-12 has not shown appreciable oral toxicity in Taboratory animals in either acute or chronic exposures. These included 18-wk rat and dog feeding studies (fed at $160-379 \mathrm{mg} / \mathrm{kg}$ per day) and 2-yr rat feeding studies (up to $150 \mathrm{mg} / \mathrm{kg}$ per day) (Haske 11 Laboratory, 1955a; Hood, 1956; Sherman, 1974; Sherman and Barnes, 1966).

$65 \mathrm{H} 5$

7526

7433

$65 \mathrm{H} 8$

No skin irritation was observed after contact of the gas with the skin of rats, rabbits, or guinea pigs. Transient eye irritation followed application of a $50 \%$ solution to rabbit eyes. Treated eyes were norma1 $24 \mathrm{~h}$ 1ater. No eye irritation was seen after an aerosol mixture containing $\mathrm{FC}-12$ was sprayed into the eyes of rabbits (Haske11 Laboratory, 1955b; Hood, 1956).

The lethal concentration of $\mathrm{FC}-12$ for rats in a 3-h exposure was $620,000 \mathrm{ppm}$ (Shugaev, 1963). Two-hour exposure at 600,000 ppm was lethal to rats, but not to guinea pigs (scholz, 1962). Central nervous system effects were observed in a 71 exposed species. In rats exposed for $30 \mathrm{~min}$, there were no reactions at 200,000 ppm (Lester and Greenberg, 1950). At higher concentrations, the following effects were observed: muscular twitching and tremors at $300,000-400,000 \mathrm{ppm}$, loss of postural reflex at $500,000 \mathrm{ppm}$, and loss of righting reflex at $600,000 \mathrm{ppm}$. Guinea pigs were simi lariy affected at 200,000-300,000 ppm (Nuckol1s, 1933). Mice survived a 24-h exposure at 10,000 ppm, but microscopic examination revealed nonspecific lung changes (Quevauvi 7ler et a7., 1963).

Rats, guinea pigs, monkeys, rabbits, and dogs were exposed to FC-12 continuous $7 \mathrm{y}$ at $810 \mathrm{ppm}$ for $90 \mathrm{~d}$ (Prendergast et a7., 1967). A7though 2 of 15 rats and 1 of 15 guinea pigs died during exposure, there were no visible signs of toxicity. During the course of the experiment which involved tests of several other chemicals, such as trichloroethylene, carbon tetrachioride, and other fluorocarbons -7 of 304 control rats and 2 of 34 control guinea pigs died. Pathologic examination revealed focal necrosis in the 7 ivers of the guinea pigs. This change was thought to be due to the continuous nature of the exposure or the high degree of susceptibility of the guinea pig. No pathologic changes were seen in the tissues of the other four species. In another experiment, test animals were exposed at $840 \mathrm{ppm}$ for $8 \mathrm{~h} / \mathrm{d}, 5 \mathrm{~d} / \mathrm{wk}$, for 6 wk (Nuckol1s, 1933). No signs of toxicity were seen, and pathologic examination revealed changes similar to those seen in the continuous study (Nuckolis, 1933). At higher concentrations, toxic signs indicative of CNS effects were observed on repeated exposure (Sayers et a1., 1930; Scholz, 1962; watanabe and Aviado, 1975). In addition, rats' and mice exposed to FC-12 at 1,000 and $5,000 \mathrm{ppm}$ for 104 wk showed no evidence of carcinogenicity (C. Maltoni, unpub 7 ished). 
FC-12, Tike other chlorofluorocarbons and hydrocarbons, is capable of sensitizing the beagle heart to exogenous epinephrine in standard 5-min cardiac-sensitization screening studies. The concentration needed to elicit marked responses in $50 \%$ of exposed dogs is $80,000 \mathrm{ppm}$ (Clark and Tinston, 1973). Dogs exposed to FC-12 while running on a treadmi 77 to increase their own epinephrine concentration were sensitized at concentrations over 100,000 ppm (Mul7in, 1970; Mullin et a1., 1972).

Lessard et a7. (1978) reported that epinephrine perfusion induced

cardiac arrhythmia in rabbits and dogs breathing gas mixtures of $79 \%$ FC -12 and $21 \% 02$. After 5 min of inhalation, arterial blood contained FC-12 at $0.8 \mathrm{mg} / \mathrm{L}$ in rabbits and $0.7 \mathrm{mg} / \mathrm{L}$ in dogs.

Blake and Mergner (1974) studied the biotransformation and elimination of $\mathrm{FC}-12$ in beagles after exposure to radiolabeled $\mathrm{FC}-12(8,000-$ $12,000 \mathrm{ppm}, \mathrm{v} / \mathrm{v})$. Essential7y a77 the inhaled air was recovered in the exhaled air within $1 \mathrm{~h}$. Onty traces of radioactivity were found in urine or exhaled $\mathrm{C} 02$. A 71 tissues contained measurable concentrations of nonvolatile radioactivity $24 \mathrm{~h}$ after exposure, but together represented less than $1 \%$ of the administered dose. It was not possible to determine whether this radioactivity was associated with metabolites of FC-12 or with unavoidable radiolabeled impurities present in the administered gas mixture. The investigators concluded that $\mathrm{FC}-12$ is relatively refractory to biotransformation after a short inhalation exposure and that it is rapidly exhaled in its unaltered form.

\section{ADDITIONAL REFERENCES}

1. Freon(R) 12, Material Safety Data Sheet (MSDS) Du001065 (2022FR), DuPont Chemicals, wilmington, DE, 8 January 1998

2. Used Refrigerant 12, MSDS 3035FR, DuPont Chemicals, wilmington, DE, 8 October 1992

3. Forane(R) 12, MSDS 04012, Elf Atochem North America, Incorporated, Philadelphia, PA, 17 JuTy 1999

4. Forane(R) 12 Reclaim, MSDS 04412.5, Elf Atochem North America, Incorporated, Philadelphia, PA, 17 October 1996

5. Dichlorodifluoromethane, MSDS, Genium Publishing Corporation

Schenectady, NY, February 1986

6. Genetron(R) 12, MSDS GTRN-0018, Honeywe 11 Incorporated, Morristown, NJ, January 2000

7. Arcton(R) 12, MSDS 230, ICI K1ea, wilmington, DE, 23 January 1998

8. Arcton(R) 12, Chemical Safety Data sheet (CSDS) revision 04, ICI

KTea, Runcorn, Cheshire, UK, Apri 11997

9 . Dichlorodifluoromethane, MSDS 06880 , Occupational Health Services, Incorporated (OHS), New York, NY, 24 March 1993 


\section{R-22}

\begin{tabular}{llll}
\hline$R-22$ & Chlorodif7uoromethane & see \\
HCFC & CHCTF2 & CAS number $75-45-6$ & RDB\#
\end{tabular}

R-22 has an extremely low order of acute toxicity; its lethal concentration (4-hr LC50 rat) exceeds $200,000 \mathrm{ppm}$.

\section{OCCUPATIONAL EXPOSURE LIMITS}

The American Conference of Governmental Industrial Hygienists (ACGIH) adopted a Threshold Limit Value - Time-Weighted Average (TLV-TWA) of $1,000 \mathrm{ppm}$ in 1990 .

\section{GENERAL CAUTIONS}

More recent and/or more complete information may be found in Material Safety Data Sheets (MSDSS), provided by the chemical supplier(s), and in other toxicology references. Inhalation of high concentrations of refrigerant vapors is harmful. Anesthetic effects including loss of consciousness, heart irregularities, or death may result. Intentional misuse or deliberate inhalation ("sniffing") may cause death without warning. Massive leaks or spi11s also may pose an asphyxiation hazard, by displacing air, in confined or inadequately ventilated locations. This refrigerant is heavier than air, and leakage may accumulate in low areas.

\section{INHALATION}

$R-22$ is rapidly equilibrated in tissues; it is eliminated from the blood in expired air, with a half 7 ife of a few minutes. Inhalation and blood concentrations are Tinearly correlated over a wide range. Metabolism is limited if it occurs at a77, making toxicological activities from formation of reactive intermediates unlikely.

Lethal concentrations are documented for several animal species and exposure durations. Tests with rats for four hours indicate an LC50 of $220,000 \mathrm{ppm}$ and a minimum lethal concentration (MLC) of $250,000 \mathrm{ppm}$. A two hour exposure at $200,000 \mathrm{ppm}$ was not lethal. 90 minute tests with dogs showed concentrations of 395,000 and $690,000 \mathrm{ppm}$ to be not letha7 and letha1, respectively. Exposures at 200,000 for five minutes were not lethal to monkeys.

High concentrations of R-22 produce stimulation followed by depression of the central nervous system (CNS) and finally asphyxiation. The minimum concentration capable of altering the reflex response of rabbits is $11,000-20,000 \mathrm{ppm}$. Concentrations of 75,000-100,000 ppm for two hours produce excitation or changes in equilibrium in rats and guinea pigs. Narcosis results at $200,000 \mathrm{ppm}$, and mortality at 300,000 and $400,000 \mathrm{ppm}$. The minimum lethal concentration for mice is 370,000 $\mathrm{ppm}$ for two hour exposures.

Guinea pigs exposed to $180,000-226,000 \mathrm{ppm}$ showed occasional chewing movements, rapid breathing, convulsive tremors, slight lachrymation (eye tearing) and nasal discharge, and inability to stand or walk after five minutes. Stupor was evident at 30 minutes, and prostration and audible breathing at one hour. These effects intensified with exposures of up to two hours, but the animals survived. They recovered within one day, and appeared to be normal for the remainder of a tenday observation period. No pathological conditions were noted in one animal autopsied after two days. Similar tests at 10,000-11,000 ppm 
and 24,000-27,000 ppm resulted in minimal effects, such as occasional chewing movements and slight 7achrymation. The responses progressed with increased exposures of $51,000-117,000 \mathrm{ppm}$.

Guinea pigs exposed to concentrations of 10,000 and $25,000 \mathrm{ppm}$ in the presence of gas flames, to test the effects of decomposition products, showed increasing responses and were killed within 29 minutes. Similar tests at $51,000 \mathrm{ppm}$ and hot electric range units showed lesser

responses, but two of three animals died 12 minutes and 51 hours, respectively, after the test.

Animal tests for concentrations of 50,000-700,000 ppm indicate effects to the CNS, liver, Tungs, kidneys, and spleen as we 77 as cardiac sensitization, decreased gain in body weight, and partial anesthesia $11,2,10,11 /$ Chronic inhalation of $50,000 \mathrm{ppm}$ produced a sma11, but statisticaliy significant increase in fibrosarcomas (a malignant connective-tissue tumor) and zymbal gland tumors in male rats, but not in female rats or male or female mice $/ 1-3,8,10$. No carcinogenic effects were seen at 1,000 or $10,000 \mathrm{ppm}$ in either species $/ 1-3 /$. Rats given $R-22$ orally showed no increased incidence of tumors $/ 3 /, \quad R-22$ is mutagenic in bacterium salmonella cultures, but not in mammalian cell cultures or in animal assays with mice and rats $/ 1-3 /$.

Concentrations of $25,000 \mathrm{ppm}$ or more reduce heart efficiency.

Cardiac sensitization to epinephrine has been observed at

concentrations of $50,000 \mathrm{ppm} / 10,11 /$. R-22 also increases the

sensitivity of the heart to adrenalin, possibly resulting in rapid tachycardia (heartbeat), cardiac arrhythmias (irregular heartbeat), and depression of cardiac function $/ 3,6 /$. Individuals with preexisting CNS or cardiovascular diseases may have increased susceptibility to the toxicity of excessive exposures $/ 1-3 /$.

A slight, but significant increase in developmental toxicity (e.g., eye malformations and decreased fetal weights) occurs in the offspring of rats exposed to $50,000 \mathrm{ppm}$, a concentration that also is maternally toxic. No effects on the maternal rats or their offspring are seen at 100 or $1,000 \mathrm{ppm} . / 1-4,6 /$ Developmental toxicity studies in rabbits at $100,1,000$, and $50,000 \mathrm{ppm}$ were negative $/ 1,2 /$.

Few indications of adverse health effects to humans have been reported in more than 50 years of $R-22$ use. One group of surveyed hospital workers, who were chronically exposed to $300 \mathrm{ppm}$, indicated increased heart palpitations, a subjective symptom.

\section{SKIN OR EYE CONTACT}

R-22 is not a skin sensitizer, though contact with Tiquid $\mathrm{R}-22$ or escaping vapor may cause frostbite or local freezing /8,10,11/ and slight irritation to the skin and eyes /11/. It is unitikely that $R-22$ wi 11 be absorbed through the skin $/ 6 /$.

\section{INGESTION}

Ingestion also is unlikely due to the high volatility of the compound $/ 1-4,6,8-11 /$. Frostbite damage to the 7 ips, mouth, and mucous membranes as wel1 as nausea and vomiting may occur if swallowed $/ 8,10 /$.

NIOSH-OSHA OCCUPATIONAL SAFETY AND HEALTH GUIDELINE (OSHG, "OHG") TOXICOLOGY SUMMARY (1995)

EFFECTS ON ANIMALS: Chlorodifluoromethane causes depression of the nervous system, cardiac arrhythmias, and asphyxiation. The LC50 in the rat is 35 parts per hundred (pph) $(350,000 \mathrm{ppm})$ for a 15 -min exposure; acutely poisoned animals exhibited altered sleep times, stumbling, and 
respiratory depression before death. Mice exposed for $2-h r$ periods showed a maximum tolerated concentration of $320,000 \mathrm{ppm}$, and a minimum fatal Tevel of $370,000 \mathrm{ppm}$. Rats and pigs exposed by inhalation to airborne concentrations of 75,000 to $100,000 \mathrm{ppm}$ chlorodifluoromethane for 2-hr showed excitation and/or changes in equilibrium; narcosis occurred at $200,000 \mathrm{ppm}$ and death occurred at 300,000 and 400,000 ppm. Dogs exposed to a $50,000 \mathrm{ppm}$ concentration of chloro-

difluoromethane developed cardiac sensitization; exposure to $25,000 \mathrm{ppm}$ did not cause this effect. The minimum concentration capable of altering the reflex response in rabbits varied between 11,000 and $20,000 \mathrm{ppm}$. In 2-hr exposures to guinea pigs, the highest concentration tested $(200,000 \mathrm{ppm})$ did not cause death, while 50,000 ppm produced mild clinical changes, and minimal effects were noted at $25,000 \mathrm{ppm}$. In a 4-week study in which rats, gujnea pigs, dogs, and cats were exposed to a $50,000 \mathrm{ppm}$ concentration of chlorodifluoromethane for twenty $3.5 \mathrm{hr}$ episodes, no clinical, biochemical, or pathological effects were observed. Rats, mice, and rabbits were exposed for $6 \mathrm{hr} / \mathrm{day}, 6$ days/week for 10 months to $14,000 \mathrm{ppm}$ chlorodifiuoromethane. In another experiment, rats and mice were exposed to 2,000 ppm of this substance for the same period. No effects were noted in animals exposed at the lower concentration, but at 14,000 ppm alterations occurred in body weight, physiological endurance, and hematological characteristics; pathological changes were noted in the lungs, central nervous system, heart, iiver, kidneys, and spleen. In one inhalation bioassay in rats, males exposed to the highest concentration administered showed on $7 y$ a marginal increase in the incidence of subcutaneous fibrosarcomas and Zymbal-gland tumors, and negative results were obtained for females. Based on this study, the International Agency for Research on Cancer (IARC) has concluded that the evidence for chlorodifluoromethane's carcinogenicity in animals is limited.

EFFECTS ON HUMANS: Chlorodifluoromethane can effect the heart; at very high concentrations it is an asphyxiant. A study of hospital personnel exposed to $300 \mathrm{ppm}$ chlorodifluoromethane during tissue freezing procedures revealed a $3 \frac{1}{2}$-fold increase in the incidence of palpitations in exposed individuals over the incidence in nonexposed hospital personne1. An epidemiologic study involving workers exposed to chlorofluorocarbons, including chlorodifluoromethane, showed no increased mortality due to heart, circulatory, or malignant disorders. A small study of 539 refrigeration workers exposed to a mixture of chlorofluorocarbons, including chlorodifluoromethane, for at least 6 month and followed for up to 30 years was inconclusive, leading IARC to conclude that the evidence for the carcinogenicity of this substance in humans is inadequate.

\section{ADDITIONAL REFERENCES}

1. Freon(R) 22, Material Safety Data Sheet (MSDS) DU000025 (2008FR), DuPont Chemicals, Wilmington, DE, 8 January 1998

2. Used Refrigerant 22, MSDS, DuPont Chemicals, wilmington, DE, 8 October 1992

3. Forane(R) 22, MSDS 04022, Elf Atochem North America, Incorporated, Philadelphia, PA, 21 September 1999

4. Forane(R) 22 Reclaim, MSDS 04422, Elf Atochem North America, Incorporated, Philadelphia, PA, 30 December 1996

5. Forane(R) 22, Safety Data Sheet (SDS) 00055, Elf Atochem S.A., Paris La Défense, France, 17 August 1998

6. Chlorodifluoromethane, MSDS, Genium Publishing corporation, Schenectady, NY, February 1986

7. Genetron(R) 22, MSDS GTRN-0026, Honeywe 17 Incorporated, Morristown, NJ, January 2000

8. ArCton(R) 22, Chemical Safety Data sheet (CSDS), ICI Klea, Runcorn, Cheshire, UK, Apri† 1997 
Toxicity Data to Determine Refrigerant Concentration Limits

James M. Calm, Engineering Consultant

9. Arcton(R) 22, MSDS 254, ICI Americas Incorporated, wilmington, DE, 15 Apri1 1998

10. MSDS 04760, Occupational Health Services, Incorporated (OHS), New York, NY, 14 July 1993

11. Solkane(R) 22, MSDS, Solvay Fluor und Derivate GmbH, Hannover, Germany, 5 August 1996 


\section{R-23}

\begin{tabular}{llll}
\hline R-23 & trifluoromethane & See \\
HFC & CHF3 & CAS number $75-46-7$ & RDB\#
\end{tabular}

The toxicity of hydrofluorocarbons (HFCS) is generally lower than for corresponding brominated and chlorinated hydrocarbons, based in part on the higher stability of the carbon-fluorine bond. overal7, $\mathrm{R}-23$ has very low toxicity.

Alliedsigna7, DuPont, and ICI have adopted recommended occupational exposure 7 imits of $1000 \mathrm{ppm}$ for 8 and 12 hour, time-weighted average (TWA) exposures, identified by DuPont as an Acceptable Exposure Limit (AEL) $\mid 1-3,6-7 /$.

\section{GENERAL CAUTIONS}

More recent and/or more complete information may be found in Material Safety Data Sheets (MSDSS), provided by the chemical supplier(s), and in other toxicology references. Inhalation of high concentrations of refrigerant vapors is harmful. Anesthetic effects including loss of consciousness, heart irregularities, or death may result. Intentional misuse or deliberate inhalation ("sniffing") may cause death without warning. Massive Teaks or spil1s also may pose an asphyxiation hazard, by displacing air, in confined or inadequately ventilated locations. This refrigerant is heavier than air, and leakage may accumulate in low areas.

\section{INHALATION}

Inhalation studies for R-23 with baboons (papio anubis) identified a dose-response effect for respiratory rate, electroencephalogram, and cardiac sinus rate, which exhibited a stepwise decrease from 100,000 ppm. No spontaneous arrhythmias were noted, and arterial blood pressure remained unchanged at any Teve1. Intravenous epinephrine infusions induced transient cardiac arrhythmia in one animal at 700,000 ppm: R-23 appears to induce mild dose-related physiological changes, indicative of an anesthetic effect, at inspired concentrations of 300,000 or more. These data suggest that $\mathrm{R}-23$ may be safe to humans, without significant adverse acute effects, at concentrations up to $300,000 \mathrm{ppm}$.

No deaths occurred in rats exposed to concentrations of 18,900 , 186,000 , and $663,000 \mathrm{ppm}$ for four hours. No evidence of clinical toxicity were noted at $18,900 \mathrm{ppm}$. The animals showed reduced response to sound at $186,000 \mathrm{ppm}$, suggesting an anesthetic effect. The rats showed no response to sound, breathing difficulties, and sluggishness at $663,000 \mathrm{ppm}$; one exhibited compulsive gnawing on its basket. The animals showed mild weight loss after exposures, but recovered after one-two days.

No compound-related pathologic changes were found by histologic examination following exposures of rats to $10,000 \mathrm{ppm}$ or beagles at $5,000 \mathrm{ppm}$ for six hours per day over 90 days.

No deaths, clinical signs of toxicity, or changes revealed by pathological examinations were found in guinea pigs exposed to 200,000 $\mathrm{ppm}$ for two hours. Likewise, no signs of toxicity or pathological changes were observed for exposures of $30,000 \mathrm{ppm}$ for six hours. 
No cardiac sensitization was observed in female, mongrel dogs exposed to concentrations of $800,000 \mathrm{ppm}$ and $810,000 \mathrm{ppm}$ for 5-10 minutes followed by an epinephrine challenge. Tests on beagles receiving adrenaline injections showed no positive responses in concentrations up to $300,000 \mathrm{ppm}$ in air or 500,000 with auxiliary oxygen. A mixture of $\mathrm{R}-23 / 125$ at $36.5 / 63.5 \%$ volume in air was found to induce cardiac sensitization in one of six beagles, with fatal results following adrenatine injections.

DuPont indicates a 4-hour, lethal concentration (LC50) of $>663,000 \mathrm{ppm}$ in rats $/ 2 /$. ICI cites a 2-hour LC of $>200,000 \mathrm{ppm}$ for rats and an effective concentration (EC50) of $>500,000 \mathrm{ppm}$ for cardiac sensitization $/ 7 /$.

R-23 exposures at $900,000 \mathrm{ppm}$ cause distinct, but not complete, narcosis.

Unspecified, higher concentrations may cause central nervous system (CNS) depression with headache, dizziness, tremor, drowsiness, disorientation, incoordination, giddiness, lightheadedness, and unconsciousness $/ 7 /$. Shortness of breath, nausea, and vomiting also may occur $/ 7 \%$. No corroborating source studies were found for these indications; they may be generic to similar compounds rather than specific to $R-23$.

Like other fluorocarbons, R-23 may decompose into toxic fumes, with a sharp, acrid odor when exposed to flames or red-hot metals.

SKIN OR EYE CONTACT

As with al7 low boiling point liquids, skin contact may cause frostbite. Excessive contact with fluorocarbons also may cause defatting of the skin.

Skin contact also may cause blisters and numbness. Eye contact may cause biurred vision $/ 8 /$.

\section{INGESTION}

Ingestion is unlikely due to the high volatility of the compound. Freeze burns wi 77 result /5,9/. R-23 is expected to be total7y unreactive in biological systems and is not expected to be metabolized if it entered the bloodstream $/ 3 /$.

\section{OTHER}

Increased mutation rates were observed in progeny of flies (drosophilia melanogaster) exposed to $R-23$ in high concentration tests $(980,000$ ppm for ten minutes). Ames assays results were negative, suggesting but not conclusively indicating - that $R-23$ is not mutagenic $/ 1-3,5 \%$.

\section{ADDITIONAL REFERENCES}

1. Genetron(R) 23, Material Safety Data Sheet (MSDS) GTRN-0030 V2.94, A 77 iedSignal Incorporated, Morristown, NJ, January 1997

2. Freon(R) 23 Refrigerant, MSDS DU001064 (2025FR), DuPont Chemicals, Wi 7mington, $D E, 8$ January 1998

3. D. M. Krentz, M. A. Lee, and R. C. Graham, untitled toxicity review for trifluoromethane, Haskeil Laboratory for Toxicology and Industrial Medicine, E. I. duPont de Nemours and Company, Incorporated, Newark, DE, 3 August 1992

4. Forane(R) 23, Safety Data Sheet (SDS) 00451, Elf Atochem S.A., Paris La Défense, France, 18 August 1998 
5. Klea(R) 23, Chemical Safety Data sheet (CSDS), ICI Chemica7s and Polymers Limited, Runcorn, UK, December 1992

6. Klea(R) 23, CSDS revision 04, ICI Klea, Runcorn, cheshire, UK, Apri1 1997

7. Toxicology Summary for FC 23 (Trifluoromethane), ICI Chemicals and Polymers Limited, Runcorn, UK, 21 september 1994

8. MSDS 09970, Occupational Health Services, Incorporated (OHS), New York, NY, 24 March 1993

9. Solkane(R) 23, MSDS, Solvay Fluor und Derivate GmbH, Hannover, Germany, 31 January 1996 


\section{R-32}

R-32 Cifluoromethane CAS number 75-10-5
HFC CH2F2 TOXICITY SUMMARY -
The toxicity of hydrofluorocarbons (HFCS) is generally lower than for
Corresponding brominated and chlorinated hydrocarbons, based in part
on the higher stability of the carbon-fluorine bond. Test results
show that R-32 has very low acute and subchronic inhalation toxicity,
is not a developmental toxicant, and is not mutagenic.

OCCUPATIONAL EXPOSURE LIMITS

The American Industrial Hygiene Association adopted a workplace Environmental Exposure Level (WEEL) Guide of 1,000 ppm, for an 8-hour exposure based on a time-weighted average (TWA), in 1996.

\section{GENERAL CAUTIONS}

More recent and/or more complete information may be found in Material Safety Data Sheets (MSDSS), provided by the chemical supplier(s), and in other toxicology references. Inhalation of high concentrations of refrigerant vapors is harmful. Anesthetic effects including loss of consciousness, heart irregularities, or death may result. Intentional misuse or deliberate inhalation ("sniffing") may cause death wi thout warning. Massive leaks or spills also may pose an asphyxiation hazard, by displacing air, in confined or inadequately ventilated locations. This refrigerant is heavier than air, and leakage may accumulate in low areas.

PAFT TOXICOLOGY SUMMARY (September 1995) included with permission of PAFT

HFC-32 is one of a series of fluorocarbon a7ternatives being studied as part of the Programme for Alternative Fluorocarbon Toxicity Testing (PAFT). HFC-32 is being considered primarily as an alternative, in blends, for refrigeration systems.

HFC-32 has very low toxicity by inhalation. The lowest concentration that causes mortality in experimental animals - the approximate letha? concentration (ALC) - for a 4-hour exposure is greater than 760,000 ppm (76\%). Anaesthetic-7ike effects, such as 7ethargy and incoordination, are observed in rats at very high inhalation concentrations (greater than $110,000 \mathrm{ppm}$ ).

As with many other halocarbons and hydrocarbons, inhalation of HFC- 32 followed by intravenous injection of epinephrine, which simulates human stress reactions, results in a cardiac sensitization response in experimental screening studies with dogs. This cardiac sensitization response is observed at approximate 7 y $350,000 \mathrm{ppm}$ of $\mathrm{HFC}-32$, a leve 1 wel1 above expected exposures. By comparison, a cardiac sensitization response is observed with CFC-11 at approximate $7 y$ 5,000 ppm, and with CFC-12 at approximate $7 y$ 50,000 ppm.

In repeated inhalation exposure studies, the low toxicity of HFC-32 continues to be evident. No adverse effects were observed in rats exposed by inhalation at concentrations of up to $50,000 \mathrm{ppm}$ for up to 90 days.

Inhalation developmental toxicity studies with rats and rabbits have been completed. The results indicate that HFC-32 is not teratogenic, 
and does not cause fetal effects at inhalation concentrations of up to $50,000 \mathrm{ppm}$.

In genetic toxicity testing, HFC-32 was not mutagenic in an Ames assay, a Chinese Hamster Lung (CHL) assay, or a chromosomal aberration study with human Tymphocytes. These studies were in vitro assays. A7so, HFC-32 was not active in an in vivo mouse micronucleus study.

Metabolism studies with HFC-32 have indicated only very low levels of metabolism (less than $0.5 \%$ ).

The testing of HFC-32 under PAFT $V$ has been completed. The results are summarized below.

- HFC-32 has very low acute and subchronic inhalation toxicity.

- HFC-32 is not a developmental toxicant.

- HFC-32 is not mutagenic.

An exposure Timit of $1,000 \mathrm{ppm}$ (8-hour time-weighted average) for HFC32 has been recommended by the American Industria 7 Hygiene Association's Workplace Environmental Exposure Limit (WEEL) Committee.

As for all chemicals, PAFT recommends that exposures be kept to a practicable minimum.

ECETOC JOINT ASSESSMENT OF COMMODITY CHEMICALS (JACC) TOXICOLOGY SUMMARY (MaY 1995)

copyright ECETOC 1995, included with permission

In animals 1ess than $1 \%$ of inhaled difluoromethane is metabolized. The majority of inhaled material is exhaled unchanged. Metabolized diffuoromethane is eliminated as $\mathrm{CO} 2$ and fluoride. There is no significant formation of carboxyhaemoglobin indicating that carbon monoxide formation is not a metabolic pathway.

Acute inhalation toxicity is very low with no mortality seen in the rat after 4 hours exposure to $550,000 \mathrm{ppm}$. Signs of depression of the nervous system were apparent only at concentrations of $86,000 \mathrm{ppm}$ and above. In the dog there was no evidence of cardiac sensitization in response to exogenous epinephrine challenge at difluoromethane exposure concentrations up to $350,000 \mathrm{ppm}$, the highest concentration tested.

Inhalation studies in rats with difluoromethane for 4 and 13 weeks at concentrations up to $50,000 \mathrm{ppm}$ produced no toxic effects.

Difluoromethane was not teratogenic to the rat or rabbit. Evidence of minimal fetal and maternal toxicity in the rat, and maternal toxicity in the rabbit was seen at $50,000 \mathrm{ppm}$.

Difluoromethane was not mutagenic both in in vitro and in vivo studies using bacteria, mammalian cel7 lines and in the mouse micronucleus assay.

\section{ADDITIONAL REFERENCES}

1. HFC-32, MSDS DU005983 (6027FR), DuPont Chemica7s, wilmington, DE, 26 June 1998

2. Forane(R) 32 , MSDS 04132.3, Elf Atochem North America, Incorporated, Philadelphia, PA, 29 January 1998

3. Forane(R) 32, Safety Data Sheet (SDS) 01736, Elf Atochem S.A., Paris La Défense, France, 25 July 1996

4. Genetron(R) 32, Material Safety Data sheet (MSDS) GTRN-0025, 
Honeywe11 Incorporated, Morristown, NJ, January 2000

5. K1ea(R) 32, MSDS 259 revision D, ICI Klea, wilmington, DE, 21 October 1998

6 . Methylene Fluoride, MSDS OHS14937, Occupational Health Services, Incorporated (OHS), New York, NY, 24 March 1993

7. Solkane (R) 32, MSDS, Solvay Fluor und Derivate GmbH, Hannover, Germany, 4 october 1996 


\section{R-113}

\begin{tabular}{llll}
\hline R-113 & REFRIGERANT TOXICITY SUMMARY & see \\
CFC & CC72FCC7F2 & CAS number 76-13-1 & RDB\#
\end{tabular}

R-113 has very low toxicity by skin contact or ingestion, but it can cause frostbite if splashed on the skin, drying and redness with repeated or prolonged contact, and mild irritation to the eyes. It has very low toxicity by inhalation and is neither carcinogenic nor mutagenic. Animal tests show no developmental or reproductive effects. R-113 is, however, a comparatively potent cardiac sensitizer to adrenatine and depresses the cardiopulmonary and central nervous systems with exposures at high concentrations. /1-8/

\section{OCCUPATIONAL EXPOSURE LIMITS}

The occupational Safety and Health Administration (OSHA), National Institute for Occupational Health and Safety (NIOSH), and American Conference of Governmental Industrial Hygienists (ACGIH) have adopted recommended Chronic exposure limits - the Permissible Exposure Limit (PEL), Recommended Exposure Limit (REL), and Threshold Limit Value - Time-Weighted Average (TLV-TWA) respectively - of $1,000 \mathrm{ppm} \mathrm{v/v}$ on a time-weighted-average (TWA) basis for an 8-10 hour workday and 40 hour workweek. The Deutsche Forschungsgemeinschaft (DFG) [German Research Association] adopted a Maximale Arbeitsplatz Konzentration (MAK) of $500 \mathrm{ppm}$ basis for an 8-10 hour workday and 40-42 hour workweek. NIOSH and OSHA recommend a short-term exposure limit (STEL) of $1,250 \mathrm{ppm} \mathrm{V} / \mathrm{V}$ TWA for $15 \mathrm{~min}$.

7541

5874

5644

5141

3904

3903

6901

5561

3903

6901

\section{GENERAL CAUTIONS}

More recent and/or more complete information may be found in Material Safety Data Sheets (MSDSs), provided by the chemical supplier(s), and in other toxicology references. Inhalation of high concentrations of refrigerant vapors is harmful. Anesthetic effects including loss of consciousness, heart irregularities, or death may result. Intentional misuse or deliberate inhalation ("sniffing") may cause death without warning. Massive Teaks or spi71s also may pose an asphyxiation hazard, by displacing air, in confined or inadequately ventilated locations. This refrigerant is heavier than air, and leakage may accumulate in low areas.

NIOSH-OSHA OCCUPATIONAL HEALTH GUIDELINE (OHG) TOXICOLOGY SUMMARY (September 1978)

1,1,2-Trich7oro-1,2,2-trifluoroethane vapor is a narcotic. In guinea pigs exposed to $25,000 \mathrm{ppm}$, nasal irritation was rapidly apparent and incoordination occurred at $50,000 \mathrm{ppm}$ after 30 minutes; death occurred after one hour at this leve7. In dogs, cardiac sensitization to epinephrine occurred at concentrations of 5,000 to $10,000 \mathrm{ppm}$. The liquid produced no irritation on the abraded or intact skin of guinea pigs; in the eyes of rabbits it produced mild conjunctivitis and minimal corneal dullness, which were reversible. In experimental human studies, exposure to $4,500 \mathrm{ppm}$ for 30 to 100 minutes resulted in significant impairment in tests of manual dexterity and vigilance; subjects reported loss of concentration and a tendency to somnolence which disappeared 15 minutes after the exposure ended; at 1,500 ppm no effects were observed. More prolonged human exposures of 6 hours daily, 5 days per week for 2 weeks at concentrations of approximately 500 and $1,000 \mathrm{ppm}$ resulted in no adverse effects, with the exception of mild throat irritation on the first day; there was no decrement in 
performance of manual tasks. The Tiquid dissolves the natural oils of the skin, and dermatitis may occur as a result of repeated contact.

NATIONAL RESEARCH COUNCIL (NRC) COMMITTEE ON TOXICOLOGY SUMMARY OF TOXICITY INFORMATION (October 1984)

EFFECTS ON HUMANS: Exposure of the skin of human subjects to FC-113 resulted in Tocalized numbness followed by transient erythema after exposure stopped (Reinhardt and SchuTtze, 1968). End-tidal air samples demonstrated that some solvent had been absorbed through the skin.

Human exposure at $1,500 \mathrm{ppm}$ produced no effects after $2.75 \mathrm{~h}$ (Stopps and MCLaugh in, 1967). Signs indicative of CNS involvement were seen after 30 min of exposure at $2,500 \mathrm{ppm}$. These slight decreases in psychomotor performance were reversibie and disappeared by 15 min after exposure stopped.

Human volunteers were exposed at 500 or $1,000 \mathrm{ppm}$ for $6 \mathrm{~h} / \mathrm{d}, 5 \mathrm{~d} / \mathrm{wk}$, during a 2-wk period. No adverse changes were seen in performance of complex mental tasks, clinical status, or results of biochemical tests. Breath analysis did not reveal a significant buildup of FC-113

(Reinhardt et al., 1971).

EFFECTS ON ANIMALS: FC-113 has very low acute oral toxicity, with an LD50 in rats of $43 \mathrm{~g} / \mathrm{kg}$ (Michaelson and Huntsman, 1964). Its Tethal dose after application to the skin of rabbits is greater than $11 \mathrm{~g} / \mathrm{kg}$, the largest feasible dose. Mild irritation results from contact with the skin and eyes (Haske7l Laboratory, 1963; Reinke, 1962).

Exposure to $\mathrm{FC}-113$ at $50,000-60,000 \mathrm{ppm}$ has proved 7 ethal to rats after $4 \mathrm{~h}$. Signs of toxicity indicative of CNS involvement were incoordination, tremors, irregular respiration, and convulsions (Bodganowi CZ, 1973; Dashie11, 1971; Sarver, 1971).

Rats were exposed at an average concentration of $2,520 \mathrm{ppm}, 7 \mathrm{~h} / \mathrm{d}, 5$ $\mathrm{d} / \mathrm{wk}$, for 6 wk (Limperos, 1954). No signs of toxicity were seen throughout the experiment.

Other groups of laboratory animals were similarly exposed at up to 5,100 ppm without effect (Carter et a1., 1970; Philadelphia Naval shipyard, 1952; Steinberg et a7., 1969). In a Timited study involving six rats exposed at $12,000 \mathrm{ppm}$ for up to $24 \mathrm{mo}$, a slight sleepiness was observed and disappeared immediately after daily exposure stopped (Desoille et a7., 1968). Rats were exposed by inhalation for $6 \mathrm{hr}$ a day, $5 \mathrm{~d} / \mathrm{wk}$, for $104 \mathrm{wk}$. Exposures were at 0 , $2,000,10,000$, and $20,000 \mathrm{ppm}(\mathrm{v} / \mathrm{v})$. No significant toxic effects were observed, and no evidence of carcinogenicity was seen ( $C$. F. Reinhardt, personal communication).

FC-113, like other ch7orofluorocarbons and hydrocarbons, is capable of sensitizing the beagle heart to exogenous epinephrine in standard 5-min cardiac-sensitization screening studies. A concentration of 5,000 ppm can sensitize 25-35\% of exposed dogs; $2,500 \mathrm{ppm}$ is ineffective

(Clark and Tinston, 1973; Reinhardt et a7., 1973). However, dogs exposed while running on a treadmi 77 (to increase their own epinephrine concentration) were not sensitized at concentrations up to 20,000 ppm (Mul1in et a1., 1971; Trochimowicz et a1., 1974).

FC- 113 is analogous to FC-11 with regard to its pharmacokinetics and metabolism. It has a short half-life in the body, is not metabolized to any significant extent, and is rapid7y expelled through the 1ungs upon removal from exposure ( $C$. F. Reinhardt, personal communication). 


\section{ADDITIONAL REFERENCES}

1. Freon(R) 113, Material Safety Data Sheet (MSDS) DU000126 (2026FR), Dupont Chemicals, wilmington, DE, 8 January 1998

2. Used Refrigerant 113, MSDS 2106FR, DuPont Chemicals, wilmington, DE, 11 August 1992

3. Forane(R) 113, MSDS 04013, Elf Atochem North America, Incorporated, Philadelphia, PA, 3 November 1999

4. Forane(R) 113 Reclaim, MSDS 04413, Elf Atochem North America, Incorporated, Philadelphia, PA, 1 June 1995

5. Trichlorotrifluoroethane, MSDS 314, Genium Pub7ishing Corporation, Schenectady, NY, February 1986

6 . Genetron(R) 113 , MSDS GTRN-0027, Honeywe 17 Incorporated, Morristown, NJ, January 2000

7. Arcton(R) 113, MSDS 0133, ICI Americas Incorporated, wilmington, DE, 25 February 1994

8. 1,1,2-Trichloro-1,2,2-trifluoromethane, MSDS 26370, Occupational Health Services, Incorporated (OHS), New York, NY, 14 July 1993 


\section{R-114}

\begin{tabular}{|c|c|}
\hline $\begin{array}{l}\text { 1,2-dichloro-1,1,2,2-tetrafluoroethane } \\
\text { CClF2CC1F2 }\end{array}$ & $\begin{array}{l}\text { see } \\
\text { RDB\# }\end{array}$ \\
\hline $\begin{array}{l}\text { R-114 has extremely low toxicity by skin contact or ingestion, but } \\
\text { it can cause frostbite if splashed on the skin, drying and redness } \\
\text { with repeated or prolonged contact, and mild irritation to the } \\
\text { eyes. It has extremely low toxicity by inhalation and is neither } \\
\text { carcinogenic nor mutagenic. Animal tests show no developmental or } \\
\text { reproductive effects. R-114 is, however, a cardiac sensitizer to } \\
\text { adrenaline and produces an anesthetic effect at high concentrations. } \\
\text { /1-10/ Tests in humans showed no ill effects at } 20,000 \text { ppm v/v R-114 } \\
\text { in air and that most is excreted in exhaled air within minutes. }\end{array}$ & $\begin{array}{l}5855 \\
5 B 51 \\
7414\end{array}$ \\
\hline
\end{tabular}

\section{OCCUPATIONAL EXPOSURE LIMITS}

The Occupational Safety and Hea7th Administration (OSHA) Permissible Exposure Limit (PEL), National Institute for Occupational Health and Safety (NIOSH) Recommended Exposure Limit (REL), and American Conference of Governmental Industrial Hygienists (ACGIH) Threshold Limit value - Time-Weighted Average (TLV-TWA) for R-114 are 1,000 $\mathrm{ppm} v / \mathrm{v}$.

\section{GENERAL CAUTIONS}

More recent and/or more complete information may be found in Material Safety Data Sheets (MSDSs), provided by the chemical supplier(s), and in other toxicology references. Inhalation of high concentrations of refrigerant vapors is harmful. Anesthetic effects including loss of consciousness, heart irregularities, or death may result. Intentional misuse or deliberate inhalation ("sniffing") may cause death wi thout warning. Massive leaks or spi 11s also may pose an asphyxiation hazard, by displacing air, in confined or inadequately ventilated locations. This refrigerant is heavier than air, and leakage may accumulate in low areas.

NIOSH-OSHA OCCUPATIONAL HEALTH GUIDELINE (OHG) TOXICOLOGY SUMMARY (September 1978)

Refrigerant 114 vapor is a respiratory irritant and causes asphyxia at extremely high concentrations. Exposure to 200,000 ppm for 16 hours was fatal to dogs, while singie 8-hour exposures produced tremors and convulsions, but no fatalities. Repeated exposures at 140,000 to $160,000 \mathrm{ppm}$ for 8 hours caused incoordination, tremors, and occasional convulsions, but a77 dogs survived. At $47,000 \mathrm{ppm}$ guinea pigs developed respiratory irritation. Sniffing aerosols of fluorochlorinated hydrocarbons has caused sudden death by cardiac arrest, probably due to sensitization of the myocardium. The liquid spilied on the skin may produce frostbite.

\section{INHALATION}

An investigation of toxicity to mice and rats concluded that $\mathrm{R}-114$ has no acute toxicity or deleterious effect at concentrations of 100,000 $\mathrm{ppm}$ or 7ess. The lethal concentration was found to be approximately $700,000 \mathrm{ppm}$. Exposures at $100,000 \mathrm{ppm}$ for $2 \frac{1}{2}$ hours per day, five days per week, for two weeks found no change in weight, blood granules, or pulmonary lesions. STight weight loss, an increase in lymphocytes, and congestive and exudative puTmonary lesions were observed at 200,000 
ppm. No indications of chronic toxicity were found for $10,000 \mathrm{ppm}$ exposures for $2 \frac{1}{2}$ hours per day, five days per week, for two months.

NATIONAL RESEARCH COUNCIL (NRC) COMMITTEE ON TOXICOLOGY SUMMARY OF TOXICITY INFORMATION (October 1984)

EFFECTS ON HUMANS: Human exposures to FC-114 at up to $20,000 \mathrm{ppm}$ have shown it to be poorly absorbed and rapidly excreted. Warning signs of overexposure are dizziness, headache, and a tingling sensation (Dol7ery et a7., 1970; Morgan et a1., 1972a,b; Paulet, 1970; Pautet and Desbrousses, 1969; Paulet et a1., 1969).

7448

$5 \mathrm{C} 73$

7449

5172

5370

EFFECTS ON ANIMALS: FC-114 has not shown appreciable oral toxicity in Taboratory animals in either acute or subchronic studies (Griffith and Sherman, 1969; Haske11 Laboratory, 1955a; Quevauviller, 1965; Sherman, 1972). Administration of a foam propelled by FC-114 or of the gas itself to the skin or eyes of guinea pigs and rabbits has resulted in only mild transient irritation (Haskel1 Laboratory, 1955b; Hood, 1967; Quevauviller et a7., 1964).

$5 \mathrm{~A} 70$

$65 \mathrm{H} 5$

$5 A 72$

5 A71

7447

$65 \mathrm{H} 6$

5368

Underwriters' Laboratories, Inc., (1933) has placed FC-114 in 4B64

Category 6 of its classification of life hazards. This category contains the least toxic gases and vapors; i.e., they do not appear to produce injury or death in test animals as a result of exposures at $200,000 \mathrm{ppm}$ for about $2 \mathrm{~h}$.

The lethal concentration of $\mathrm{FC}-114$ for rats in a $2-\mathrm{h}$ exposure is greater than $600,000 \mathrm{ppm}$, and for guinea pigs, greater than $500,000 \mathrm{ppm}$ (Scholz, 1962). The LC50 in mice exposed for 30 min is $700,000 \mathrm{ppm}$ (Paulet and Desbrousses, 1969). A dog survived an 8-h exposure a $200,000 \mathrm{ppm}$, but a 16-h exposure was lethal to another dog (Yant et a 7., 1932). Exposure of dogs at $150,000 \mathrm{ppm}$ for $24 \mathrm{~h}$ was not Tethal and resulted only in a loss of appetite (Yant et a 7. , 1932). Exposure at $200,000 \mathrm{ppm}$ caused pupiliary dilation, convulsions, opisthotonus, and unconsciousness in dogs. However, exposure at $400,000-600,000 \mathrm{ppm}$ was necessary to cause mild CNS effects in rats and guinea pigs. Recovery was rapid and complete in all species.

FC-114, like other chlorofluorocarbons and hydrocarbons, is capable of sensitizing the beagle heart to exogenous epinephrine in standard 5-min cardiac-sensitization screening studies. The concentration needed to elicit marked responses in $50 \%$ of a group of beagle is $45,000 \mathrm{ppm}$ (Reinhardt et a7., 1971). However, dogs exposed to FC-114 while running on a treadmi 17 to increase their own epinephrine concentration were not sensitized until exposures reached $50,000-100,000 \mathrm{ppm}$. In another endogenous-epinephrine study, a concentration of $800,000 \mathrm{ppm}$ ( $80 \%$ FC-113:20\% oxygen) was shown to sensitize the beagle heart after its epinephrine concentration was increased by fright (Mullin et al., 1972).

Rats and mice were expostd- at $200,000 \mathrm{ppm}$ for $2.5 \mathrm{~h} / \mathrm{d}, 5 \mathrm{~d} / \mathrm{wk}$, for 2 wk (Paulet and Desbrousses, 1969). Slight blood and body-weight changes were seen, as well' as sijght evidence of Tung irritation. Exposure at $100,000 \mathrm{ppm}$ had no effect, even after 2 mo of exposure (Paulet and Desbrousses, 1969). Dogs and guinea pigs were exposed at $141,600 \mathrm{ppm}$ for $8 \mathrm{~h} / \mathrm{d}$ for $21 \mathrm{~d}$ (Yant, 1933). The severity of

the clinical signs attributab7e to CNS effects decreased during the 21 $d$ of exposure. In the dogs, slight hematologic changes were observed, but the measures in question returned to norma $15-17$ d after exposure; no gross pathologic changes were seen.

No effects were seen in dogs, cats, guinea pigs, or rats exposed at $100,000 \mathrm{ppm}$ for $3.5 \mathrm{~h}$ per exposure for 20 exposures (scholz, 1962). 
No effects were seen in rats and rabbits exposed at 10,000 Ppm for 2 h/d, $5 \mathrm{~d} / \mathrm{wk}$, during 8-9 mo (Desoille et a1., 1973).

\section{ADDITIONAL REFERENCES}

1. Freon(R) 114, Materia1 Safety Data Sheet (MSDS) DU001061 (3038FR), DuPont Chemicals, wilmington, DE, 8 January 1998

2 . Used Refrigerant 114 , MSDS $2112 \mathrm{FR}$, DuPont Chemicals, wi7mington, DE, 22 June 1992

3. Forane(R) 114, MSDS 04014, Elf Atochem North America, Incorporated, Phi Tadelphia, PA, 17 JuTy 1999

4. Forane(R) 114 Reclaim MSDS 04414, ETf Atochem North America, Incorporated, Philadelphia, PA, 28 July 1997

5 . Dich7orotetrafluoroethane, MSDS 572 , Genium Pub7ishing Corporation, Schenectady, NY, December 1985

6 . Arcton $(\dot{R}) 114$, MSDS 240 revision $G$, ICI Klea, wilmington, DE, 23 January 1998

7. Genetron(R) 114, MSDS GTRN-0028, Honeywe11 Incorporated, Morristown, NJ, January 2000

8. ArCton(R) 114, Chemical Safety Data sheet (CSDS) revision 04, ICI Kiea, Runcorn, Cheshire, UK, April 1997

9. i,2-Dichlorotetraf7uoroethane, MSDS 06980 , Occupational Health Services, Incorporated (OHS), New York, NY, 24 March 1993

10. Racon $(R)$ 114, MSDS, Racon Incorporated (now part of Elf Atochem North America, Incorporated), Wichita, KS, 7 Apri 1989 


\title{
R-115
}

\author{
R-115 1-chloro-1,1,2,2,2-pentafluoroethane \\ CFC CCIF2CF3 CAS number 76-15-3 \\ R-115 has an extremely low order of acute toxicity; its lethal \\ concentration (4-hr LC50 rat) exceeds $800,000 \mathrm{ppm}$.
}

see

$5 C 41$

\section{OCCUPATIONAL EXPOSURE LIMITS}

The Occupational Safety and Health Administration (OSHA) has not promulgated a Permissible Exposure Limit (PEL) for R-115. The The National Institute for Occupational Hea7th and Safety (NIOSH) has established a Recommended Exposure Limit (REL) of 1,000 ppm as a timeweighted average for up to a 10-hr work day and a $40 \mathrm{hr}$ work week. This limit is based on the risk of cardiac, skin, central nervous system, and respiratory effects associated with exposure to R-115. The American Conference of Governmental Industrial Hygienists (ACGIH) adopted a Threshold Limit value - Time-weighted Average (TLV-TWA) of $1,000 \mathrm{ppm}$ in 1981 .

\section{GENERAL CAUTIONS}

More recent and/or more complete information may be found in Material Safety Data Sheets (MSDSS), provided by the chemical supplier(s), and in other toxicology references. Inhalation of high concentrations of refrigerant vapors is harmful. Anesthetic effects including loss of consciousness, heart irregularities, or death may result. Intentional misuse or deliberate inhalation ("sniffing") may cause death without warning. Massive leaks or spil1s also may pose an asphyxiation hazard, by displacing air, in confined or jnadequately ventilated locations. This refrigerant is heavier than air, and leakage may accumulate in low areas.

\section{NIOSH-OSHA OCCUPATIONAL SAFETY AND HEALTH GUIDELINE (OSHG, "OHG")} TOXICOLOGY SUMMARY (1995)

EFFECTS ON ANIMALS: In animaTs, chloropentafluoroethane affects the heart and respiratory system at very high doses. Early studies in experimental animals showed that inhalation of a chloropentafluoroethane concentration of approximately $200,000 \mathrm{ppm}$ caused confusion, pulmonary irritation, tremors, and occasionaliy coma. At a concentration of $190,000 \mathrm{ppm}$, chloropentafluoroethane caused cessation of respiration (apnea) in rats exposed from 4 to 26 min. Rats exposed at $800,000 \mathrm{ppm}$ plus $20 \%$ oxygen for $4 \mathrm{hr}$ showed no clinical effects of histopathology. Rats and guinea pigs exposed at $600,000 \mathrm{ppm}$ chloropentafluoroethane in oxygen for $2 \mathrm{hr}$ and guinea pigs exposed at $200,000 \mathrm{ppm}$ chloropentafluoroethane in air for varying intervals up to $2 \mathrm{hr}$ showed no adverse clinical signs. Studies with anesthetized dogs, rats, and monkeys showed that, under certain conditions, exposures of 100,000 to $200,000 \mathrm{ppm}$ ch7oropentafiuoroethane may increase blood pressure, accelerate heart rate, cause myocardial depression, or change pulmonary mechanics. Species differences in sensitivity are great. Monkeys do not show respiratory or circulatory effects when exposed to a 200,000 ppm concentration of chloropentafluoroethane; dogs show no respiratory depression when exposed to $200,000 \mathrm{ppm}$ but do show bronchoconstriction, decreased compliance, sensitization of the heart to epinephrine, tachycardia, myocardial depression, and hypotension when inhaling 100,000 to $250,000 \mathrm{ppm}$ chloropentafluoroethane; rats show bronchospasm, decreased compliance, and respiratory stimulation after inhalation of $100,000 \mathrm{ppm}$ chloropentafluoroethane. 
Rats, mice, rabbits, and dogs that received 90 exposures, $6 \mathrm{hr}$ daity, to 100,000 ppm chloropentafluoroethane showed no adverse effects, and rats, guinea pigs, dogs, and cats exposed to $200,000 \mathrm{ppm}$ ch7oropentafluoroethane for $3 \frac{1}{2} \mathrm{hr} /$ day, 5 days/week for 4 weeks showed no adverse effects after cessation of exposure.

EFFECTS ON HUMANS: In humans, chloropentafluoroethane can affect the heart, central nervous system, and skin. The neurological effects of chlorofluorocarbon exposure were evaluated in 27 refrigeration repair workers and 14 aged-matched control workers from a local union of plumbers, pipefitters, and insulation workers. Personal air samples taken on two worker-participants over the course of a typical work shift showed average exposures of $1.4 \mathrm{ppm}$ chlorodifluoromethane [refrigerant 22] and $2.2 \mathrm{ppm}$ chloropentafluoroethane. No periphera] neuropathy was seen among the study subjects, and there was no significant difference in mean nerve conduction velocities between study and reference subjects; however, 1ightheadedness and palpitations were reported significantly more often by refrigeration workers than by controls. The defatting effects of fluorocarbons such as chloropentafluoroethane, when in prolonged or repeated contact with the skin, may cause dermatologic problems, and contact of the skin with liquified chloropentafluoroethane may cause frostbite.

ADDITIONAL REFERENCES

1. Genetron(R) 115 (Chloropentafluoroethane), Product Safety Data Sheet, A7liedsignal Incorporated, Morristown, NJ, May 1989

2. Freon( $R$ ) 115 Refrigerant, Material Safety Data sheet (MSDS)

DU002791 (2018FR), DuPont Chemicals, wilmington, DE, 8 January 1998

3 . Forane(R) 115, MSDS 04016, Elf Atochem North America, Incorporated, Philadelphia, PA, 1 June 1995

4. Arcton(R) 115, MSDS 276 revision G, ICI K7ea, wilmington, DE, 23 January 1998

5. Arcton(R) 115, Chemical Safety Data sheet (CSDS) revision 03, ICI Kiea, Runcorn, Cheshire, UK, April 1997

6. Chloropentafluoroethane, MSDS 04810, Occupational Hea7th Services, Incorporated (OHS), New York, NY, 14 Juity 1993 
This page cannot be converted.

Please view the native document

for the original page. 
response to $H C F C-123$, were observed in some studies, but these effects occurred at high concentrations (i.e., 10,000-20,000 ppm).

A two-year inhalation study with HCFC-123 has been completed. Exposure concentrations for rats were $0,300,1,000$, and $5,000 \mathrm{ppm}$. During the study, slight reductions in body weight were observed at the two higher concentrations, and decreases in cholesterol and triglyceride

concentrations were observed at al1 exposure levels. After one year of exposure, no changes in compound-related tissue pathology were observed at any of the concentrations tested.

At the end of the study, an increase in benign tumours was observed in the Tiver, pancreas, and testis (Leydig ce77s) of the treated rats. However, no malignant tumours attributab7e to exposure to HCFC-123 were observed and there was no increased mortality in the groups treated with HCFC-123. An independent review of the pathology findings supported this conclusion. None of the benign tumours observed were life-threatening, and all occurred near the end of the study. To understand the biological significance of these tumours, mechanistic studies were conducted to examine the possible relationship of certain biochemical processes to the development of the tumours. Preliminary results suggest that certain enzyme activity in the Tiver of the rat may partially explain the occurrence of liver tumours, and changes in certain hormone levels may be responsible for the development of testicular tumours. Studies investigating any biological relevance of the tumours to humans are being conducted.

Several genetic toxicity studies have also been completed with HCFC-123. These studies included the Ames assay, chromosomal aberration study with human 7ymphocytes, in vivo mouse micro-nucleus, in vivo chromosomal aberration study, and unscheduled DNA synthesis. Based on the weight of evidence from a11 in vivo and in vitro studies, HCFC-123 is not genotoxic. In on7y one study, which utilized an in vitro culture of human lymphocytes, did HCFC-123 cause changes in the genetic material. However, a similar study with rat 7ymphocytes in vivo did not show the same response. Therefore, the overal7 evidence from these genotoxicity studies and the results from long-term studies suggest that HCFC- 123 does not produce maTignant tumours in experimental animals and is not genotoxic.

The results from developmental toxicity studies with HCFC-123 show that this material does not have embryotoxic or teratogenic effects in rats or rabbits. At high concentrations (10,000 ppm and greater), anaesthetic-7ike effects and reduced body weights are observed in pregnant animals. At these high concentrations, slight fetal effects are also evident. Reproduction and neurotoxicity studies on rats have been completed. HCFC-123 was not neurotoxic nor did it cause adverse reproductive effects. In a two-generation reproduction study, rats were exposed to HCFC- 123 concentrations of $30,100,300$, or $1,000 \mathrm{ppm}$. No effects on reproduction were observed in rats at any exposure concentration. During the period of maternal milk production (lactation), a slight decrease in the pup body weight gain and increases in liver weight were observed at $30 \mathrm{ppm}$ or greater. This effect was only seen when the pups were being nursed and may be related to a depression of triglycerides.

HCFC-123 is oxidatively metabolized by the body following inhalation exposure, as suggested by a slight increase in urinary fluoride levels. Also, trifluoroacetic acid (TFA) was detected following HCFC-123 administration.

The testing of HCFC-123 under PAFT I has been completed. The results are summarized below. 
- HCFC-123 has low acute dermal and inhalation toxicity.

- HCFC-123 caused an increased incidence of benign, but not 1ifethreatening, tumors in animals following long-term exposure.

- HCFC-123 is not a developmental toxicant.

- HCFC-123 does not affect reproductive performance.

- HCFC-123 is not genotoxic.

Several PAFT companies have set occupational exposure Timits for HCFC-123, typically at 10 or $30 \mathrm{ppm}$ (8-hour time-weighted average). [DuPont and Elf Atochem North America have recommended exposure Timits to $50 \mathrm{ppm} \mathrm{V} / \mathrm{V} / 1,3 /$; Elf Atochem S.A. (France) and Honeywe 17 have recommended a limit of $10 \mathrm{ppm} / 4,5 /$. The American Industrial Hygiene Association (AIHA) has published a workplace Environmental Exposure Limit (WEEL) of $50 \mathrm{ppm} \mathrm{v} / \mathrm{v}$.

As for a11 chemicals, PAFT recommends that exposures be kept to a practicable minimum.

\section{ECETOC JOINT ASSESSMENT OF COMMODITY CHEMICALS (JACC) TOXICOLOGY} SUMMARY (February 1996)

copyright ECETOC 1996, included with permission

Dichlorotrifluoroethane would not be considered to be readily biodegradable. Studies conducted in algae, Daphnia, and rainbow trout indicated a moderate level of toxicity to aquatic organisms. The octanol/water partition coefficient (1og $P O W=2.0-2.8)$ is indicative of a Tow potential to bioaccumulate.

Studies in rodents indicate that dichlorotrifluoroethane is easily absorbed via the respiratory route and undergoes a biphasic uptake. It is distributed in a 71 organs and is more concentrated in the fiver. About $90 \%$ of unchanged dichlorotrifluoroethane was eliminated via the lungs. The rest was metabolised to trifluoroacetic acid which was excreted in the urine. Sma11 amounts of trifluoroacetylated proteins were detected in exposed rats.

Dichlorotrifluoroethane has a low order of acute toxicity. Its oral approximate lethal dose in rats was $9,000 \mathrm{mg} / \mathrm{kg}$; its 4-hour inhalation LC50 was $200,000 \mathrm{mg} / \mathrm{m} 3[32,000 \mathrm{ppm} \mathrm{v} / \mathrm{v}]$ in rats and $178,000 \mathrm{mg} / \mathrm{m} 3$ $[28,500 \mathrm{ppm}]$ in hamsters. Its derma 1 LD50 in rats and rabbits was greater than $2,000 \mathrm{mg} / \mathrm{kg}$. The primary effect was a rapidiy reversible central nervous depression. After a few minute's exposure it induced pre-narcotic effects at $31,300 \mathrm{mg} / \mathrm{m} 3[5,000 \mathrm{ppm}]$ and anaesthesia at $129,000 \mathrm{mg} / \mathrm{m} 3[20,600 \mathrm{ppm}]$ in rats.

Liquid dichlorotrifluoroethane did not induce skin irritation in rabbit or skin sensitization in guinea pigs but it caused moderate eye irritation in rabbits.

Repeated inhalation exposure studies in rats exposed $6 \mathrm{~h} / \mathrm{d}, 5 \mathrm{~d} / \mathrm{wk}$, for 4 to 13 weeks, to vapour 7 evels of up to $125,000 \mathrm{mg} / \mathrm{m} 3[20,000 \mathrm{ppm}]$, have resulted in body weight decreases and 1 iver weight increases but with minimal adverse histological findings. Dichlorotrifluoroethane was found to cause a marked drop in serum cholestero1, triglyceride and glucose 1evels at $1,880 \mathrm{mg} / \mathrm{m} 3$ [300 ppm] and above. Signs of CNS depressing effects were seen during exposure periods above $6,250 \mathrm{mg} / \mathrm{m} 3$ $[1,000 \mathrm{ppm}]$. However, there was no evidence of neurotoxicity when specific testing for neurobehavioral and nervous tissue histology were performed in a 13-week neurotoxicity study in rats.

Dichlorotrifluoroethane elicited cardiac sensitization to adrenaline in dogs acutely exposed by inhalation at a concentration of $119,000 \mathrm{mg} / \mathrm{m} 3$ $[19,000 \mathrm{ppm}]$. The no-effect- 7 eve 7 was $62,500 \mathrm{mg} / \mathrm{m} 3[10,000 \mathrm{ppm}]$. 
No clear effects were seen in serum steroid hormone and CCK levels in rats and guinea pigs exposed to dichlorotrifluoroethane. In rats dichlorotrifluoroethane produced minimal decreases in testicular luteinizing hormone and testosterone levels under stimulation conditions on 7y: Dichiorotrifluoroethane was found to induce Tiver peroxisome proliferation in rats. Its proliferating potential is weak and may be due to its metabolism to trifluoroacetic acid.

Dichlorotrifluoroethane was not teratogenic or embryotoxic to rats and rabbits exposed by inhalation at concentrations of 31,300 and 62,500 $\mathrm{mg} / \mathrm{m} 3[5,000$ and $10,000 \mathrm{ppm}]$ which induced slight maternal toxicity.

In a two-generation inhalation study conducted with dichlorotrifluoroethane in rats exposed $6 \mathrm{~h} / \mathrm{d}, 7 \mathrm{~d} / \mathrm{wk}$ at concentrations ranging from 188 to $6,250 \mathrm{mg} / \mathrm{m} 3$ [30 to $1,000 \mathrm{ppm}$, there were no effects on mating performance or offspring survival. Growth retardation was observed in pups during nursing at a11 test concentrations, but normal growth was restored in the post-weaning phase.

Dichlorotrifluoroethane was inactive in several in vitro studies including a series of Salmonella typhimurium assays, a saccharomyces cerevisiae assay and a cel1 transformation assay. It was active on $7 y$ in the human Tymphocyte chromosome aberration assay.

Dichlorotrifluoroethane was clearly inactive in a series of in vivo studies, including mouse micronucleus, rat chromosome aberration and rat unscheduled DNA synthesis. In conclusion, dichlorotrifluoroethane is not genotoxic in vivo and therefore does not have toxicologically significant genotoxicity.

Rats were exposed $6 \mathrm{~h} / \mathrm{d}, 5 \mathrm{~d} / \mathrm{wk}$ for 2 years by inhalation in a Tifetime study to concentrations of $1,875,6,250$, and $31,300 \mathrm{mg} / \mathrm{m} 3[300,1,000$, and $5,000 \mathrm{ppm}]$. Compared to controls, survival was significantly improved in an exposure related pattern in a 71 exposed groups.

significantly depressed body weights and levels of serum trigylcerides, glucose and cholesterol were observed. compound-related increased incidences of benign tumours were observed in the Jiver, testis, and pancreas. These tumours were a continuum from hyperplasia and occurred late in iife. It is thought that they are related with non-genotoxic mechanisms associated with the peroxisome proliferating potential of dich7orotrifluoroethane or with hormonal disturbances occurring in senescent rats. Considering they occurred late in 1 ife and were benign, the reievance of these findings is questionable in terms of carcinogenic risk to man.

\section{ADDITIONAL REFERENCES}

1. Suva(R) 123, Material Safety Data Sheet (MSDS) DU002798 (2190FR), DuPont Chemicals, wilmington, $D E, 29$ June 1998

2. Used Refrigerant 123, MSDS, DuPont Chemicals, wilmington, DE, 11 March 1992

3. Forane(R) 123, MSDS 04123, Elf Atochem North America, Incorporated, Philadelphia, PA, 3 November 1999

4. Forane(R) 123, Safety Data Sheet (SDS) 00940, Elf Atochem S.A., Paris La Défense, France, 23 Apri 1998

5. Genetron (R) 123, MSDS GTRN-0013, Honeywe 11 Incorporated, Morristown, NJ, January 2000

6. Arcton(R) 123, Chemical Safety Data sheet, ICI Chemicals \& Polymers, Runcorn, UK, Apri7 1990

7. 2,2-Dichloro-1,1,1-trifluoroethane, MSDS, occupational Health Services (OHS), New York, NY, Incorporated, 17 November, 1992

8. Solkane(R) 123, MSDS, Solvay Fluor und Derivate GmbH, Hannover, Germany, 8 August 1995 


\section{R-124}

\begin{tabular}{|c|c|c|}
\hline $\begin{array}{l}R-124 \\
\text { HCFC }\end{array}$ & $\begin{array}{l}\text { 2-chloro-1,1,1,2-tetrafluoroethane } \\
\text { CHCTFCF3 } \\
\text { CAS number } 2\end{array}$ & $2837-89-0$ \\
\hline
\end{tabular}

Test results show that $R-124$ has very low acute and subchronic inhalation toxicity. Lifetime exposures neither produced signs of chronic toxicity nor caused any tumors. R-124 is not a developmenta] toxicant nor is it genotoxic.

\section{OCCUPATIONAL EXPOSURE LIMITS}

The American Industrial Hygiene Association adopted a workplace Environmental Exposure Leve1 (WEEL). Guide of $1,000 \mathrm{ppm}$, for an 8-hour exposure based on a time-weighted average (TWA), in 1992.

\section{GENERAL CAUTIONS}

More recent and/or more complete information may be found in Material Safety Data Sheets (MSDSS), provided by the chemical supplier(s), and in other toxicology references. Inhalation of high concentrations of refrigerant vapors is harmful. Anesthetic effects including loss of consciousness, heart irregularities, or death may result. Intentional misuse or deliberate inhalation ("sniffing") may cause death without warning. Massive leaks or spi77s also may pose an asphyxiation hazard, by dispiacing air, in confined or inadequately ventilated locations. This refrigerant is heavier than air, and leakage may accumulate in low areas.

PAFT TOXICOLOGY SUMMARY (September 1995) included with permission of PAFT

HCFC-124 is one of a series of fluorocarbon a7ternatives being tested by the Programme for Alternative Fluorocarbon Toxicjty Testing (PAFT). HCFC-124 is considered primarily as an alternative in specialized refrigeration systems.

HCFC-124 has very low acute toxicity by inhalation. The lowest concentration that causes mortality in experimentai animals - the approximate lethal concentration (ALC) - for a 4-hour exposure is between 230,000 and $300,000 \mathrm{ppm}$ in rats. Similar exposure levels cause toxicity in other species, such as the guinea pig. During inhalation exposure to very high concentrations, anaesthetic-like effects, such as weakness and incoordination, are observed. The 10-minute exposure concentration that causes these effects (EC50 for nervous system effects) in experimental animals is about $140,000 \mathrm{ppm}$. Similar anaesthetic-like effects are observed with many other fluorocarbons in acute inhalation studies.

As with many other halocarbons and hydrocarbons, inhalation of HCFC-124, followed by intravenous injection of epinephrine, which simulates human stress reactions, results in a cardiac sensitization response in experimental screening studies with dogs. This cardiac sensitization response is observed at approximate 7 y $25,000 \mathrm{ppm}$ of HCFC-124, a leve7 we 71 above expected exposures. By comparison, a cardiac sensitization response is observed with CFC-11 at approximately $5,000 \mathrm{ppm}$.

Longer term studies of up to 90 days in duration have also been conducted with HCFC-124. On7y changes in clinical chemistry parameters (e.g., triglycerides) and anaesthetic-like effects were noted. These 
were observed on7y in the 90-day study. No other effects were evident in any of these studies at concentrations of up to $50,000 \mathrm{ppm}$. In a 90-day study with mice, inhalation exposures caused an increase in beta-oxidation (indicative of peroxisome protiferation) and a decrease in triglycerides. No other toxic effects were noted in this 90-day study.

A two-year inhalation study with HCFC-124 was conducted with exposure concentrations of $0,2,000,10,000$ and $50,000 \mathrm{ppm}$. No adverse effects were noted during the study, and no compound-related grossly visible or microscopic changes were observed at any HCFC-124 exposure 1eve1. The no-observed-adverse-effect level (NOAEL) was 50,000 ppm.

Several genetic toxicity studies have also been completed with HCFC-124. Studies conducted with HCFC-124 include the Ames assay, mouse micronucleus, and in vitro chromosomal aberration with human Tymphocytes. Based on the evidence from all in vitro and in vivo studies, HCFC-124 is not genotoxic.

The results from developmental toxicity studies with HCFC-124 show that this material does not have embryotoxic or teratogenic effects in rats or rabbits. At very high concentrations (e.g., 50,000 ppm), anaesthetic-like effects and reduced body weights were observed in pregnant anima7s. However, no fetal effects were observed at these high, maternally toxic concentrations.

HCFC-124 is oxidatively metabolized by the body following inhalation exposure, as suggested by a slight increase in urinary fluoride levels. Also, trifluoroacetic acid (TFA) has been detected following HCFC -124 administration. The rate of metabolism appears to be low.

The testing of HCFC-124 under PAFT III is now complete. The results are summarized below.

- HCFC-124 has very low acute and subchronic inhalation toxicity.

- HCFC-124 is not tumourigenic.

- HCFC-124 is not a developmental toxicant.

- HCFC-124 is not genotoxic.

An exposure limit of 1,000 ppm (8-hour time-weighted average) has been recommended by the American Industrial Hygiene Association, Workplace Environmental Exposure Limit (WEEL) Committee.

As for a11 chemicals, PAFT recommends that exposures be kept to a practicable minimum.

ADDITIONAL REFERENCES

1. Meforex(R) 124, Safety Data Sheet sym/ss76, Ausimont S.p.A., Bollate, Italy, 11 March 1997

2. Suva(R) 124, Material Safety Data sheet (MSDS) Du002790 (2200FR), DuPont Chemicals, wilmington, DE, 8 January 1998

3. Used Refrigerant 124, MSDS 6008FR, DuPont Chemicals, wilmington, DE, 23 February 1993

4. Forane(R) 124, MSDS 04124, E1f Atochem North America, Incorporated, Phi Tadelphia, PA, 28 July 1999

5. Forane(R) 124, Safety Data Sheet (SDS) 01446, Elf Atochem S.A., Paris La Défense, France, 19 December 1997

6 . Genetron (R) 124, MSDS GTRN-0012, Honeywe 17 Incorporated, Morristown, NJ, January 2000 
7. 1,1,1,2-Tetraf7uoro-2-ch7oroethane, MSDS OHS63246, Occupational Health Services, Incorporated (OHS), New York, NY, 24 March 1993 


\section{R-125}

\begin{tabular}{|c|c|c|}
\hline $\begin{array}{l}\mathrm{R}-125 \\
\mathrm{HFC}\end{array}$ & $\begin{array}{l}\text { pentaf7 uoroethane } \\
\text { CHF2CF3 }\end{array}$ & $\begin{array}{l}\text { see } \\
\text { RDB\# }\end{array}$ \\
\hline \multicolumn{3}{|c|}{$\begin{array}{l}\text { The toxicity of hydrofluorocarbons (HFCs) is generally lower than for } \\
\text { corresponding brominated and chlorinated hydrocarbons, based in part } \\
\text { on the higher stability of the carbon-fluorine bond. Toxicity test } \\
\text { results indicate that R-125 has negligible acute and subchronic } \\
\text { inhalation toxicity, is not a developmental toxicant, and is not } \\
\text { aenotoxic }\end{array}$} \\
\hline
\end{tabular}

OCCUPATIONAL EXPOSURE LIMITS

The American Industrial Hygiene Association adopted a workplace Environmental Exposure Level (WEEL) Guide of $1,000 \mathrm{ppm}$, for an 8-hour exposure based on a time-weighted average (TWA), in 1996.

GENERAL CAUTIONS

More recent and/or more complete information may be found in Material Safety Data Sheets (MSDSS), provided by the chemical supplier(s), and in other toxicology references. Inhalation of high concentrations of refrigerant vapors is harmful. Anesthetic effects including loss of consciousness, heart irregularities, or death may result. Intentional misuse or deliberate inhalation ("sniffing") may cause death wi thout warning. Massive leaks or spi71s also may pose an asphyxiation hazard, by dispiacing air, in confined or inadequately ventilated locations. This refrigerant is heavier than air, and leakage may accumulate in low areas.

PAFT TOXICOLOGY SUMMARY (September 1995)

included with permission of PAFT

HFC-125 is one of a series of fluorocarbon alternatives being tested by the Programme for Alternative Fluorocarbon Toxicity Testing (PAFT). HFC- 125 is considered primarily as an alternative for refrigeration systems.

HFC-125 has very low acute toxicity by inhalation. The lowest concentration that causes mortality in experimental animals - the approximate lethal concentration (ALC) - for a 4-hour exposure is greater than $800,000 \mathrm{ppm}(80 \%)$ with added oxygen. Even at these high inhalation concentrations, no clinical signs of toxicity are evident.

As with many other halocarbons and hydrocarbons, inhalation of HFC-125, followed by intravenous injection of epinephrine, which simulates human stress reactions, results in a cardiac sensitization response in experimental screening studies with dogs. This cardiac sensitization response is observed at approximately $100,000 \mathrm{ppm}$ of HFC-125, a leve 1 we 71 above expected exposures. By comparison, a cardiac sensitization response is observed with CFC-11 at approximately $5,000 \mathrm{ppm}$.

In repeated inhalation exposure studies, the low toxicity of HFC- 125 continued to be evident. No adverse effects were observed in rats exposed by inhalation at concentrations of up to $50,000 \mathrm{ppm}$ for up to 90 days.

Inhalation developmental toxicity studies with rats and rabbits have been completed. The results indicate that HFC- 125 is not teratogenic 
and does not cause fetal effects at inhalation concentrations up to $50,000 \mathrm{ppm}$.

In genetic toxicity testing, HFC-125 was not mutagenic in an Ames assay, Chinese Hamster ovary assay, or chromosomal aberration study with human lymphocytes. These studies were in vitro assays. A7so, HFC-125 was not active in an in vivo mouse micronucleus study.

Metabolism studies with HFC-125 have not detected any metabolism.

The testing of HFC-125 under PAFT III is now complete. The results are summarized below.

- HFC-125 has very low acute and subchronic inhalation toxicity.

- HFC-125 is not a developmental toxicant.

- HFC-125 is not genotoxic.

An exposure limit for HFC-125 of 1,000 ppm (8-hour time-weighted average) has been recommended by the American Industrial Hygiene Association, Workplace Environmental Exposure Limit (WEEL) Committee.

As for al1 chemicals, PAFT recommends that exposures be kept to a practicable minimum.

ECETOC JOINT ASSESSMENT OF COMMODITY CHEMICALS (JACC) TOXICOLOGY SUMMARY (MaY 1994)

copyright ECETOC 1994, included with permission

In Timited metabolic studies in vivo in the rat, the metabolism of pentafluoroethane was negligible. Toxicokinetic studies are in progress.

The acute inhalation toxicity of pentafluoroethane is very low. The 4hour LC50 in the rat is greater than $3,928,000 \mathrm{mg} / \mathrm{m} 3(800,000 \mathrm{ppm})$. During the exposure, the only clinical signs were ataxia, decrease of locomotor activity, and dyspnoea.

As with many other fluorocarbons, inhalation of high concentration of pentafluoroethane, followed by an intravenous epinephrine challenge to simulate stress, can induce a cardiac sensitization response in dogs. The threshold concentration for this effect was $491,000 \mathrm{mg} / \mathrm{m} 3(100,000$ $\mathrm{ppm}$ ) and the No Observed Effect Concentration (NOEC) was $368,250 \mathrm{mg} / \mathrm{m} 3$ $(75,000 \mathrm{ppm})$.

Exposures by inhalation up to $245,000 \mathrm{mg} / \mathrm{m} 3(50,000 \mathrm{ppm}) 5$ days a week for 4 to 13 consecutive weeks, did not induce any toxic effect and the NOEC was greater that $245,000 \mathrm{mg} / \mathrm{m} 3(50,000 \mathrm{ppm})$.

Developmental studies by inhalation route were carried out in both rats and rabbits. No evidence of embryotoxicity or teratogenicity was seen even at exposure leve1s as high as $245,000 \mathrm{mg} / \mathrm{m} 3(50,000 \mathrm{ppm})$.

Pentafluoroethane was not mutagenic both in vitro and in vivo studies using bacteria, mammalian ce 77 lines and in the mouse micronucleus assay.

There are no reported effects of pentafluoroethane in man.

An occupationa1 exposure 1imit (8-hour time-weighted average) of 1,000 ppm $(4,910 \mathrm{mg} / \mathrm{m} 3)$ is recommended by producers. 


\section{ADDITIONAL REFERENCES}

1. HFC-125, Material Safety Data sheet (MSDS) Du002868 (3020FR), DuPont Chemicals, wilmington, DE, 25 June 1998

2. Forane(R) 125 , MSDS 04387, Elf Atochem North America, Incorporated, Philadelphia, PA, 17 JuTy 1999

3. Forane(R) 125, Safety Data sheet (SDS) 01447, Elf Atochem S.A., Paris La Défense, France, 17 Apri 1996

4. Klea(R) 125 , MSDS 280 revision $F$, ICI Klea, wilmington, DE, 11 March 1999

5. Genetron(R) 125, MSDS GTRN-0024, Honeywe 11 Incorporated, Morristown, $N J$, January 2000

6. Klea(R) 125, Chemical Safety Data sheet (CSDS) revision 05, ICI Klea, Runcorn, Cheshire, UK, Apri 1997

7. Solkane(R) 125 , MSDS, Solvay Fluor und Derivate $\mathrm{GmbH}$, Hannover, Germany, 8 August 1995 


\section{R-134a}

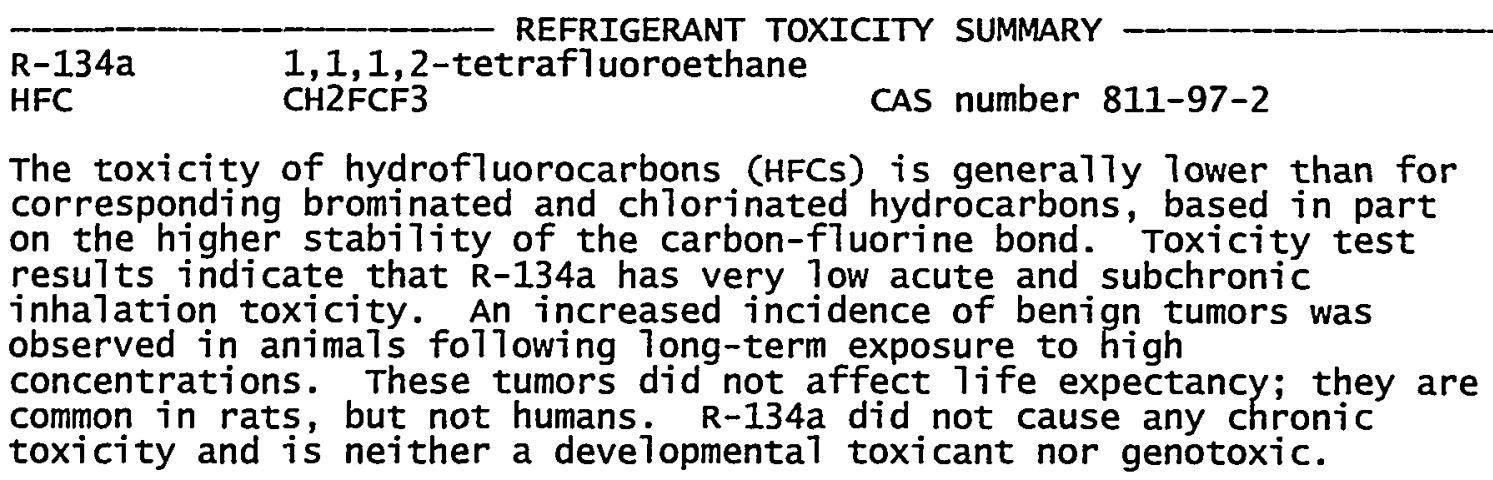
toxicity and is neither a developmental toxicant nor genotoxic.

\section{OCCUPATIONAL EXPOSURE LIMITS}

The American Industrial Hygiene Association adopted a workplace Environmental Exposure Level (WEEL) Guide of $500 \mathrm{ppm}$, for an 8-hour exposure based on a time-weighted average (TWA), in 1992.

GENERAL CAUTIONS

More recent and/or more complete information may be found in Material Safety Data sheets (MSDSs), provided by the chemical supplier(s), and in other toxicology references. Inhalation of high concentrations of refrigerant vapors is harmful. Anesthetic effects including loss of consciousness, heart irregularities, or death may result. Intentional misuse or deliberate inhalation ("sniffing") may cause death without warning. Massive leaks or spil1s also may pose an asphyxiation hazard, by displacing air, in confined or inadequately ventilated locations. This refrigerant is heavier than air, and leakage may accumulate in low areas.

PAFT TOXICOLOGY SUMMARY (September 1995) included with permission of PAFT

HFC-134a is one of a series of fluorocarbon alternatives being studied as part of the Programme for A7ternative Fluorocarbon Toxicity Testing (PAFT). HFC-134a is being considered as an a7ternative for refrigeration and air conditioning, medical dose delivery systems, and as a foam blowing agent.

HFC-134a has very low acute inhalation toxicity. The lowest concentration that causes mortality in rats - the approximate lethal concentration (ALC) - for a 4-hour exposure is greater than 500,000 $\mathrm{ppm}$. Anaesthetic-like effects, such as Tethargy and incoordination, are observed in rats at very high inhalation concentrations (greater than 200,000 ppm).

As with many other halocarbons and hydrocarbons, inhalation of HFC-134a followed by intravenous injection of epinephrine, which simulates human stress reactions, results in a cardiac sensitization response in experimental screening studies with dogs. This cardiac sensitization response is observed at approximately $75,000 \mathrm{ppm}$ of HFC-134a, a leve 1 we 11 above expected exposures. By comparison, a cardiac sensitization response is observed with CFC-12 at approximate7y 50,000 ppm.

Longer term studies have also been conducted with HFC-134a. No significant toxicological effects were observed in rats foilowing 
inhalation exposure for up to one year at concentrations up to 50,000 ppm.

At the end of the two-year inhalation study, no effects were observed in body weights, in-life measurements, clinical observations or clinical chemistry, or haematology. Except for the testis of male rats, no grossiy visible or microscopic changes were observed in the any of the HFC-134a exposed rats. At $50,000 \mathrm{ppm}$, an increased incidence of hyperplasia (cell growth) and benign tumours of Leydig cells was observed on microscopic examination of the testis. No malignant tumours attributable to exposure to HFC-134a were observed. An independent review of the pathology findings supported these conclusions. None of the benign tumours were life-threatening, and all occurred near the end of the study. No effects were observed at lower concentrations in this two-year study; the no-observed-effect level (NOEL) was $10,000 \mathrm{ppm}$.

Several genetic toxicity studies with HFC-134a have been completed. These included a bacterial reverse mutation (Ames) test, an in vitro chromosomal aberration study with human 7ymphocytes, and a cytogenetics assay with Chinese Hamster Lung Ce71 (CHL). In vivo studies included cytogenetics, mouse micronucleus, and a dominant lethal study in the mouse. Evidence from a7? in vitro and in vivo studies clearly indicates that HFC-134a is not genotoxic. Furthermore, these data and data obtained from the two-year inhalation study suggest that the increased incidence of benign tumours observed in the two-year inhalation study is not due to an effect on genetic material.

Results from inhalation developmental toxicity studies indicate that HFC-134a does not cause teratogenic effects in rats or rabbits. At inhalation concentrations of $300,000 \mathrm{ppm}$, slight maternal toxicity and embryotoxicity, evidenced by a decrease in fetal body weights, were observed in rats. Lower fetal body weights of rats and rabbits have been observed at 50,000 ppm with slight maternal toxicity; lower maternal body weights were also observed in rats at this concentration. In an additional study, no fetal effects were observed in rabbits at inhalation concentrations of up to $40,000 \mathrm{ppm}$.

A7though not metabolized to any significant extent in animals, HFC-134a is oxidatively metabolized following inhalation exposure, as suggested by a slight increase in urinary fluoride levels. However, the rate of metabolism of HFC-134a is very 1ow, and about $99 \%$ of an inhaled dose is eliminated unchanged.

The testing of HFC-134a under PAFT I has been completed. The results are summarized below.

- HFC-134a has very low acute and subchronic inhalation toxicity.

- HFC-134a caused an increased incidence of benign tumours in animals following long-term exposure to high concentrations.

- HFC-134a is not a developmental toxicant.

- HFC-134a is not genotoxic.

An exposure limit of $1,000 \mathrm{ppm}$ (8-hour time-weighted average) has been recommended by the American Industrial Hygiene Association, Workplace Environmental Exposure Limit (WEEL) Committee.

As for all chemicals, PAFT recommends that exposures be kept to a practicable minimum. 


\section{ADDITIONAL REFERENCES}

1. Meforex(R) 134a, Safety Data Sheet sym/ss101, Ausimont S.p.A., Bol7ate, Italy, 9 Apri 71996

2. Suva(R) 134a, Material Safety Data sheet (MSDS) DU000693 (2187FR), DuPont Chemicals, wi Tmington, DE, 8 January 1998

3. Used Refrigerant 134a, MSDS 3025FR, DuPont Chemicals, wilmington, DE, 23 February 1993

4. Forane(R) 134a, MSDS 04134, Elf Atochem North America, Incorporated, Philadelphia, PA, 17 July 1999

5. Forane(R) 134a - Reclaim, MSDS 04434, E7f Atochem North America, Incorporated, Philadelphia, PA, 29 January 1998

6. Forane(R) 134a, Safety Data sheet (SDS) 00941, Elf Atochem S.A., Paris La Défense, France, 19 october 1998

7. Genetron(R) 134a, MSDS GTRN-0011, Honeywe 17 Incorporated, Morristown, $N J$, January 2000

8. Klea(R) 134a, MSDS 274 revision I, ICI Americas, Incorporated, WiTmington, DE, 20 Apri 1998

9. KTea(R) 134a, Chemica7 Safety Data sheet (CSDS) revision 05, ICI Kiea, Runcorn, Cheshire, UK, April 1997

10. Isceon 134a, Safety Data Sheet, Rhône-Poulenc Chemicals Limited, Bristo1, UK, 15 Apri门 1994

11. Solkane $(R)$ 134a, MSDS, Solvay Fluor und Derivate GmbH, Hannover, Germany, 22 August 1995 


\section{R-E134}

$\begin{array}{llll} & & \\ \text { R-E134 } & \text { bis (difluoromethyT) ether } & \text { REFRIGERANT TOXICITY SUMMARY } & \text { See } \\ \text { HFE } & \text { CHF2-0-CHF2 } & \text { CAS number 1691-17-4 } & \text { RDB\# }\end{array}$

only limited testing, mostly to gauge its anesthetic value, has been performed for R-E134. Long-term testing is sti17 required. The results thus far suggest that R-E134 has very low toxicity.

OCCUPATIONAL EXPOSURE LIMITS

No recommended exposure Timits were found for R-E134.

GENERAL CAUTIONS

More recent and/or more complete information may be found in Material Safety Data Sheets (MSDSs), provided by the chemical supplier(s), and in other toxicology references. Inhalation of high concentrations of refrigerant vapors is harmful. Anesthetic effects including loss of consciousness, heart irregularities, or death may result. Intentional misuse or deliberate inhalation ("sniffing") may cause death without warning. Massive leaks or spil1s also may pose an asphyxiation hazard, by displacing air, in confined or inadequately ventilated locations. This refrigerant is heavier than air, and leakage may accumulate in low areas.

\section{INHALATION}

Exposure tests with unspecified animals found that R-E134 bare induced anesthesia after 30 minutes at $130,000 \mathrm{ppm}$. The animals were anesthetized after five minutes at $180,000 \mathrm{ppm}$, but not deep 7y; they regained consciousness in approximately two minutes. They were anesthetized after two minutes at $300,000 \mathrm{ppm}$, but recovered in approximately two minutes. No signs of distress that would indicate respiratory or other irritation or a convulsant effect were noted. Rapid recovery from high concentrations and deep anesthesia suggests low solubility in and quick removal from body tissues. The low residence time reduces the probability of metabolism, resultant formation of toxic products, or interference with metabolic pathways. The test animals showed no $i 71$ effects over a period of one week, and the autopsy results were negative. R-E134 was deemed unattractive as an anesthetic based on the high concentrations required to induce and maintain anesthesia.

Rats exposed to 50,000 ppm R-E134 and 950,000 ppm oxygen for one week showed no obvious adverse effects during or after the exposure. No gross abnormalities were found during necropsy.

Cardiac sensitization studies found R-E134 to be between $R-21$ and $R-22$ in arrythmogenic properties, but that it behaved more $1 i k e ~ R-11$ after epinephrine challenges. The tests were conducted with cynomolgus monkeys for 30 minute exposures at $50,000 \mathrm{ppm}$ with $950,000 \mathrm{ppm}$ oxygen. $R-12, R-21$, and $R-22$ caused irregular heart beats. A71 of the compounds except R-12 predisposed the monkeys to spontaneous cardiac sensitization and sensitized them to epinephrine challenges. Most of the specimens were not sensitized by $R-12$. 
SKIN OR EYE CONTACT

As with all low boiling point liquids, skin contact may cause frostbite. Excessive contact with fiuorocarbons also may cause defatting of the skin.

INGESTION

No information was found, but ingestion is unlikely with a normal boiling point of $6 \varnothing \mathrm{C}(43 \curvearrowleft \mathrm{F})$.

OTHER

Ames assays results were negative, suggesting - but not conclusively indicating - that R-E134 is not mutagenic. 


\title{
R-141b
}

\begin{abstract}
REFRIGERANT TOXICITY SUMMARY
HCFC $\mathrm{CH} 3 \mathrm{CC} 72 \mathrm{~F}$

CAS number 1717-00-6

Test results show that $R-141 \mathrm{~b}$ has $10 \mathrm{w}$ acute and subchronic inhalation toxicity. R-141b caused an increased incidence of benign tumors in animals following long-term exposure to high concentrations. These tumors did not affect 1 ife expectancy and are unique to rodents. $\mathrm{R}-141 \mathrm{~b}$ did not cause any chronic toxicity. It is not a developmental or reproductive toxicant, and it not genotoxic.
\end{abstract}

see

RDB\#

OCCUPATIONAL EXPOSURE LIMITS

The American Industrial Hygiene Association adopted a Workplace Environmental Exposure Level (WEEL) Guide of $500 \mathrm{ppm}$, for an 8-hour exposure based on a time-weighted average (TWA), in 1992.

\section{GENERAL CAUTIONS}

More recent and/or more complete information may be found in Material Safety Data Sheets (MSDSS), provided by the chemical supplier(s), and in other toxicology references. Inhalation of high concentrations of refrigerant vapors is harmful. Anesthetic effects including loss of consciousness, heart irregularities, or death may result. Intentional misuse or deliberate inhalation ("sniffing") may cause death without warning. Massive leaks or spills also may pose an asphyxiation hazard, by displacing air, in confined or inadequately ventilated locations. This refrigerant is heavier than air, and leakage may accumulate in low areas.

PAFT TOXICOLOGY SUMMARY (September 1995) included with permission of PAFT

HCFC-141b is one in a series of fluorocarbon alternatives being tested by the Programme for Alternative Fluorocarbon Toxicity Testing (PAFT). HCFC-147b is primarily an alternative to CFC-11 as a foam-blowing agent, but may also be used as a solvent in cleaning applications.

Data from acute toxicity studies demonstrated that HCFC-141b has very low acute toxicity. HCFC-141b is not a skin irritant and is only a mild eye irritant. Skin application of HCFC-141b at high doses $(2,000$ $\mathrm{mg} / \mathrm{kg}$ body weight) produces no adverse effects. Oral administration of HCFC-141b at high doses $(5,000 \mathrm{mg} / \mathrm{kg}$ body weight) does not cause any mortality. Therefore, the ora] LD50 is greater than $5,000 \mathrm{mg} / \mathrm{kg}$ body weight. HCFC-14Ib al so has very low acute inhalation toxicity as measured by the concentration that causes $50 \%$ mortality in experimental anima7s, the LC50. The 4-hour exposure LC50 for HCFC-141b is 62,000 $\mathrm{ppm}$ in rats. Anaesthetic-like effects are observed at high concentrations.

As with many other halocarbons and hydrocarbons, inhalation of HCFC-141b followed by intravenous injection of epinephrine, which simulates human stress reactions, results in a cardiac sensitization response in experimental screening studies with dogs. This cardiac sensitization response is observed with HCFC-141b at approximately $5,000 \mathrm{ppm}$, a level we 17 above expected exposures. Similarly, a cardiac sensitization response is observed with CFC-1I at approximate $1 y$ 5,000 ppm. 
Longer term studies of up to 90 days in duration have a7so been conducted with HCFC-141b. In these studies, on ty stight changes in clinical chemistry parameters (e.g., cholesterol) and slight anaesthetic-like effects were observed. No other effects were evident in any of these studies at concentrations of up to $20,000 \mathrm{ppm}$. The noobserved-effect level (NOEL) in these studies was about $8,000 \mathrm{ppm}$.

A two-year inhalation study with HFC-141b was conducted at exposure concentrations of $0,1,500,5,000$ and $20,000 \mathrm{ppm}$. During the study, no significant adverse effects were noted, although at the highest concentrations, slightly lower body weights were observed in male rats. Except for the testis of male rats, no grossly visibie or microscopic changes were noted in any of the HCFC-141b treated animals. In the testis, an increase in hyperplasia (cell growth) and benign tumours of Leydig ce71s was observed in the 5,000 and $20,000 \mathrm{ppm}$ groups. No malignant tumours attributable to HCFC-141b were observed. An independent review of the pathology findings supported these conclusions. None of the benign tumours were 1 ife-shortening, and 271 occurred near the end of the study. No effects were observed at Tower concentrations in this two-year study; the NOEL was 1,500 ppm.

Several genetic toxicity studies have also been completed with HCFC-141b. Based on the evidence from all in vitro and in vivo studies, HCFC-141b is not mutagenic. Studies conducted with HCFC-141b included Ames, in vitro chromosomal aberration with human 7ymphocytes, and mouse micronucleus. HCFC-141b was active only at very high exposures in the Chinese Hamster ovary chromosomal aberration study. However, the overa 71 evidence suggests that HCFC-141b is not genotoxic.

The results from developmental toxicity studies with HCFC-14Ib show that this material does not have embryotoxic effects in rats and rabbits at inhalation concentrations of $8,000 \mathrm{ppm}$ and $1,400 \mathrm{ppm}$, respectively. In studies conducted in rats (at levels up to 20,000 ppm) and in rabbits (at leve1s up to $12,600 \mathrm{ppm}$ ), no teratogenic effects were seen. In a reproduction study, results suggest that HCFC-141b caused a slight reduction in litter size and total iitter weight at 20,000 ppm. The NOEL was 8,000 ppm for the pups, and 2,000 $\mathrm{ppm}$ for the aduits.

Metabolism studies suggest that HCFC-141b is oxidatively metabolized, a) though the rate of metabolism appears to be low.

The testing of HCFC-14Ib under PAFT II has been completed. The results are summarized below.

- HCFC-141b has low acute and subchronic inhalation toxicity.

- HCFC-141b caused an increased incidence of benign, but not 7 ifethreatening, tumours in animals following long-term exposure to high concentrations.

- HCFC-141b is not a developmental toxicant.

- HCFC-141b does not affect reproductive performance.

- HCFC-141b is not genotoxic.

An exposure limit of $500 \mathrm{ppm}$ (8-hour time-weighted average) has been recommended by the American Industrial Hygiene Association, Workplace Environmental Exposure Limit (WEEL) Committee.

As for all chemicals, PAFT recommends that exposures be kept to a practicable minimum. 


\section{ADDITIONAL REFERENCES}

1. Meforex(R) 141b, Material Safety Data Sheet (MSDS), Ausimont USA, Incorporated, Thorofare, NJ, 2 November 1998

2. HCFC-141b, MSDS DU000694 (042114688), DuPont Chemicals, wilmington, DE, 29 June 1998

3. Forane(R) 141b, MSDS 04089, Elf Atochem North America,

Incorporated, Philadelphia, PA, 3 November 1999

4. Forane(R) 141b, Safety Data Sheet (SDS) 00995, Elf Atochem S.A., Paris La Défense, France, 19 December 1997

5. Genetron(R) 141b, MSDS GTRN-0015, Honeywe 11 Incorporated, Morristown, NJ, January 2000

6. Arcton(R) 141b, MSDS 6733, ICI Klea, wilmington, DE, 16 March 1995

7. Arcton(R) 141b, Chemical Safety Data Sheet (CSDS) revision 02-UK00, ICI Klea, Runcorn, Cheshire, UK, December 1995 


\section{R-225ca, R-225cb}

$\begin{array}{ll}\text { R-225Ca } & \text { 3,3-dich7oro-1,1,1,2,2-pentaf7uoropropane } \\ \text { HCFC } & \text { CHC12CF2CF3 }\end{array}$

$\mathrm{R}-225 \mathrm{cb}$

1,3-dich7oro-1,1,2, 2,3-pentafluoropropane

HCFC CHC1FCF2CCIF2

CAS number 507-55-1

see

RDB\#

Studies of $R-225 \mathrm{Ca}$ and $R-225 \mathrm{cb}$ indicate that these compounds have very low acute oral, derma 7 , and inhalation toxicity. Exposures in the range of $600-1,000 \mathrm{ppm}$ with $\mathrm{R}-225 \mathrm{ca}$ produced effects on the liver in rodents, but minor effects in a primate. Exposures in the range of $1,000-5,000 \mathrm{ppm}$ with $\mathrm{R}-225 \mathrm{cb}$ resulted in on $7 y$ marginal effects in rodents or in a primate. Neither isomer was found to be genotoxic.

GENERAL CAUTIONS

More recent and/or more complete information may be found in Material Safety Data Sheets (MSDSS), provided by the chemical supplier(s), and in other toxicology references. Inhalation of high concentrations of refrigerant vapors is harmful. Anesthetic effects including loss of consciousness, heart irregularities, or death may result. Intentional misuse or deliberate inhalation ("sniffing") may cause death without warning. Massive leaks or spi 17s also may pose an asphyxiation hazard, by displacing air, in confined or inadequately ventilated locations. $R-225 \mathrm{ca}$ and $R-225 \mathrm{cb}$ are heavier than air, and leakage may accumulate in low areas.

PAFT TOXICOLOGY SUMMARY (September 1995)

included with permission of PAFT

HCFC-225ca and HCFC-225cb (two isomers of dichloropentafluoropropane) are two of a series of fiuorocarbon alternatives being tested by the Programme for Alternative Fluorocarbon Toxicity Testing (PAFT). HCFC$225 \mathrm{ca}$ and $\mathrm{HCFC}-225 \mathrm{cb}$ are being considered primari7y as alternatives to CFC-113 as a solvent in cleaning applications. The isomers have been commercialized as a $45 / 55$ weight percent mixture of HCFC-225ca and HCFC- $225 \mathrm{cb}$. Testing has been conducted on the separate isomers to allow characterization of the toxicity of each isomer and of the mixed isomer products.

Data from acute toxicity studies demonstrate that HCFC-225Ca and HCFC$225 \mathrm{cb}$ have very low acute toxicity. Neither isomer causes eye irritation nor dermal toxicity in standardized tests; skin application of both isomers at high doses $(2,000 \mathrm{mg} / \mathrm{kg}$ body weight) produces no adverse effects. oral administration of either isomer at high doses $(5,000 \mathrm{mg} / \mathrm{kg}$ body weight) does not cause any mortality. Therefore, the ora 7 LD50s are greater than $5,000 \mathrm{mg} / \mathrm{kg}$ body weight. Both isomers also have very low acute inhalation toxicity as measured by the concentration that causes $50 \%$ mortality in experimental animals, the LC50. The 4-hour exposure LC50s for both isomers are approximately $37,000 \mathrm{ppm}$ in rats. Anaesthetic-like effects are observed in rats at high inhalation concentration (greater than 5,000 ppm).

As with many other halocarbons and hydrocarbons, inhalation of HCFC$225 \mathrm{Ca}$ and HCFC-225cb followed by intravenous injection of epinephrine, which simulates human stress reactions, results in a cardiac sensitization response in experimental screening studies with dogs. This cardiac sensitization response is observed at approximate $7 y 15,000$ $\mathrm{ppm}$ for the mixture of HCFC-225ca/ HCFC-225cb (45/55 weight percent) and $20,000 \mathrm{ppm}$ for HCFC-225cb, which are levels we11 above expected 
exposures. By comparison, a cardiac sensitization response is observed with $\mathrm{CFC}-113$ at approximately $5,000 \mathrm{ppm}$.

In 28-day inhalation studies with rats, the activity and responsiveness of the animals was reduced at exposures of 5,000 ppm or greater for each isomer. Toxicity was otherwise confined to the 1iver; liver enlargement and induction of peroxisomes was seen following treatment with either of the isomers. HCFC-225ca was more potent than HCFC $-225 \mathrm{cb}$ in eliciting these liver effects.

To investigate the biological relevance of the liver toxicity to humans, comparative repeated inhalation studies have been conducted with rats, hamsters, guinea pigs and marmosets. In 14-day exposure studies with rats, hamsters and guinea pigs, the liver effects were also observed in rodents, while no such effects were observed in guinea pigs. In the 28-day study with marmosets, exposure to HCFC-225ca at $1,000 \mathrm{ppm}$ caused effects on the liver, such as slight fat deposition associated with changes in serum biochemical parameters. In the same study, exposure to HCFC- $225 \mathrm{cb}$ at $5,000 \mathrm{ppm}$ caused somnolence during exposure and an increase of cytochrome $P-450$, indicative of an adaptive response to HCFC-225cb. However, no liver enlargement was seen and virtualiy no peroxisomal induction was observed with either isomer.

Several genetic studies have also been completed with both isomers. These studies included an Ames assay, in vitro chromosomal aberration with Chinese Hamster Lung (CHL) and human Tymphocyte, and in vivo unscheduled DNA synthesis assay. Based on the weight of evidence from al7 in vitro and in vivo studies, neither isomer is mutagenic. In only one study, which utilized an in vitro culture of human 7ymphocyte, did HCFC-225ca cause changes in the genetic materials while HCFC-225cb elicited a marginal response. However, the overal1 evidence from these studies implies that neither isomer is genotoxic.

Pharmacokinetic studies with rats indicated that either isomer found in blood is rapid7y eliminated on termination of exposure.

The testing of HCFC-225 Ca and HCFC-225cb under PAFT IV has been completed. The results are summarized below.

- HCFC-225ca and HCFC-225cb have low acute oral, dermal and inhalation toxicity.

- Exposure in the range of 650 to $1,000 \mathrm{ppm}$ with HCFC-225ca produced effects on the liver in rodents, but a minor effect in a primate.

- Exposures in the range of 1,000 to 5,000 ppm with HCFC-225cb resulted in only marginal effects in rodents or a primate.

- Neither isomer is genotoxic.

As for all chemicals, PAFT recommends that exposures be kept to a practicable minimum. 


\section{R-290 (PROPANE)}

\begin{tabular}{ll}
\hline $\mathrm{R}-290$ & propane \\
$\mathrm{HC}$ & $\mathrm{CH} 3 \mathrm{CH} 2 \mathrm{CH} 3$
\end{tabular}

REFRIGERANT TOXICITY SUMMARY

$\mathrm{HC} \quad \mathrm{CH} 3 \mathrm{CH} 2 \mathrm{CH} 3$

CAS number 74-98-6

see

RDB\#

R-290 (propane) is essential7y non-toxic in concentrations below 1,000 $\mathrm{ppm}$, and no symptoms result from brief exposures to $10,000 \mathrm{ppm}$. The primary hazard for propane is flammability.

5609

OCCUPATIONAL EXPOSURE LIMITS

The Occupational Safety and Health Administration (OSHA) has set a Permissible Exposure Limit (PEL) of $1,000 \mathrm{ppm}$, on a time-weighted average (TWA) basis for an 8 hour shift of a 40 hour workweek, for R-290. The American Conference of Governmental Industrial Hygienists (ACGIH) Classifies R-290 as a "simple asphyxiant." ACGIH adopted a Threshold Limit Value - Time-Weighted Average (TLV-TWA) of 1,000 ppm in 1966, but deleted it in 1970. ACGIH proposed a new TLV-TWA of 2,500 ppm for comment in 1996.

GENERAL CAUTIONS

More recent and/or more complete information may be found in Material Safety Data Sheets (MSDSs), provided by the chemical supplier(s), and in other toxicology references. Inhalation of high concentrations of refrigerant vapors is harmful. Anesthetic effects including loss of consciousness, heart irregularities, or death may result. Intentional misuse or deliberate inhalation ("sniffing") may cause death without warning. Massive leaks or spi77s also may pose an asphyxiation hazard, by displacing air in confined or inadequately ventilated locations. This refrigerant is heavier than air, and leakage may accumulate in low areas.

\section{INHALATION}

Concentrations exceeding $10,000 \mathrm{ppm}$ can cause headaches, 10ss of concentration, and tiredness due to central nervous system (CNS) depression. Breathing pure propane wi 17 cause immediate loss of consciousness and almost immediate death $/ 3 /$.

Guinea pigs exposed to concentrations of 22,000-29,000 ppm exhibited sniffing and chewing movements during 5 minute exposures, but recovered quickly. They also showed irregular rates of breathing during longer exposures, from 30 minutes to two hours. The same results were found at concentrations of 47,000-55,000 ppm, along with indications of mild stupor for 30 minute exposures and increased stupor for longer durations. The animals appeared to be normal during 7-10 day observations after exposures. An autopsy of one specimen, a week after the two hour exposure at 47,000-55,000 ppm, showed no pathologica 1 conditions to the vital organs.

Guinea pigs exposed to concentrations of up to $55,000 \mathrm{ppm}$ for 1-2 hours showed tremors after five minutes, then nausea, retching, and stupefaction. A7l survived, and no significant tissue damage was found by autopsy. Brief exposures to $10,000 \mathrm{ppm}$ caused no symptoms in human subjects; $100,000 \mathrm{ppm}$ produced slight dizziness in a few minutes of exposure, but was not noticeably irritating to the eyes, nose, or respiratory tract. Reports from accidental overexposures cite disorientation, excitation, excessive salivation, headache, and vomiting. 
Concentrations of $630,000 \mathrm{ppm}$ in air induced mild anesthesia in tested cats.

Propane exhibits some anesthetic action and is mildly irritating to the mucous membranes. Rapid respiration, dyspnea, ataxia, dimini shed alertness, emotional instability, rapid fatigue, nausea, vomiting, prostration, Toss of consciousness, and convulsions may occur depending on the duration of exposure and concentration. Deep coma may occur at concentrations near those that induce simple asphyxiation.

Effects at high concentrations include cardiac sensitization, analgesia, irregular respiration, and hypertension. No animal test reports for carcinogenic, developmental, or reproductive hazards are available $/ 5 /$.

SKIN OR EYE CONTACT

No effects are reported from gas exposures. As with all low boiling point liquids, skin contact may cause frostbite. Liquid splashes will cause serious cold burns to the eyes.

INGESTION

Ingestion is unlikely due to the high volatility of the compound.

OTHER

Tests in bacteria cel1 cultures were negative, suggesting - but not conclusively indicating - that $\mathrm{R}-290$ is not mutagenic $/ 5 \%$.

ADDITIONAL REFERENCES

1. Propane, Material Safety Data Sheet (MSDS) 93617000, Exxon Chemical Americas, Houston, TX, 1 March 1995

2. Propane (Refrigeration Grade), MSDS US057840, Phi11ips Chemical Company, Bartlesvilie, OK, 31 August 1993

3. Propane, Chemical Safety Data Sheet, Rhône-Poulenc chemicals, Avonmouth, Bristol, UK, undated (received 16 December 1992

4. Propane, MSDS 60001, Texaco Natural gas Plants and Liquids Division, TuTsa, OK, 5 JuTy 1995

5. "Suva" HP80 (R-402A), MSDS 6003FR, Dupont Chemica7s, wilmington, $D E, 1$ August 1994 [R-402A is a blend containing $R-290]$ 


\section{R-600 (n-BUTANE}

$\begin{aligned} & \text { R-600 } \\ & \text { HC } \\ & \text { CH3CH2CH2CH3 }\end{aligned}$
R-600 is comparatively nontoxic./1-3/ Like other simple hydrocarbons,
it can irritate the eyes, mucous membranes, and respiratory system at
high concentrations./1/ Inhalation of high concentrations may cause
dizziness, disorientation, incoordination, narcosis, nausea, or
narcotic effects. R-600 may displace oxygen, posing an asphyxiation hazard, if released in a confined space. The effects from oxygen deficiency may include rapid breathing, diminished mental alertness and judgement, impaired muscular coordination, depression of a 11 sensations, emotional instability, and fatigue. As asphyxiation progresses, nausea, vomiting, prostration, and loss of consciousness may result leading to convulsions, coma, and death./1/ oxygen deficiency during pregnancy has produced developmental abnormalities in humans and experimenta? animals./1/

\section{GENERAL CAUTIONS}

More recent and/or more complete information may be found in Material Safety Data Sheets (MSDSs), provided by the chemical supplier(s), and in other toxicology references. Inhalation of high concentrations of refrigerant vapors is harmful. Anesthetic effects including loss of consciousness, heart irregularities, or death may result. Intentional misuse or deliberate inhalation ("sniffing") may cause death without warning. Massive leaks or spi71s also may pose an asphyxiation hazard, by displacing air, in confined or inadequately ventilated locations. This refrigerant is heavier than air, and leakage may accumulate in low areas.

NIOSH-OSHA OCCUPATIONAL HEALTH GUIDELINE (OHG) TOXICOLOGY SUMMARY (1992)

EFFECTS ON ANIMALS: Contact with Tiquid n-butane can cause frostbite. This gas is also an anesthetic and asphyxiant in animals at very high concentrations. Although n-butane was mild7y to moderately irritating to rabbit skin, it did not produce ocular or respiratory tract irritation. However direct contact with 7 iquid n-butane can cause frostbite. Anesthetized dogs concomitantiy exposed to 5,000 ppm developed hermodynamic changes (decreased myocardial contractility, stroke volume, cardiac output, and left ventricular and aortic pressures). Dogs exposed to $10,000 \mathrm{ppm}$ for $2 \mathrm{hr}$ or $200,000 \mathrm{ppm}$ for 2 min were hypersensitized to epinephrine-induced ventricular fibritiation. Exposures of dogs to 150,000 to $900,000 \mathrm{ppm}$ for $10 \mathrm{~min}$ also caused heart sensitization to epinephrine-induced arrhythmias. The anesthetic concentration for $n$-butane is $250,000 \mathrm{ppm}$ in dogs and $220,000 \mathrm{ppm}$ for 1 min or 130,000 for $25 \mathrm{~min}$ in mice. The 30-min LC50 for mice is approximately $285,000 \mathrm{ppm}$, but the 15 min LC50 for rats is about $275,000 \mathrm{ppm}$. n-Butane was not mutagenic in the Salmonella typhimurium assay.

EFFECTS ON HUMANS: A transient blurring of vision resulted when $n-$ butane was accidental7y sprayed into the eyes from a cigarette lighter. However, contact of the eyes or skin with n-butane does not normaliy cause irritation, but contact with the liquified form may cause frostbite of the eyes or skin. Exposure to $10,000 \mathrm{ppm}$ for $10 \mathrm{~min}$ may lead to drowsiness, but does not appear to cause systemic effects, A 2-year-old girl developed seizures, hypotension, and recurrent ventricular tachycardia following inhalation of an aerosol spray from a 
can containing a mixture of $n$-butane, isobutane, and propane. Progressive bilateral pulmonary infiltrates consistent with hydrocarbon pneumonitis developed in a "fire breather" following inhalation of unignited fumes from a butane 1ighter. Exposures of volunteers to $1,000 \mathrm{ppm}$ for $8 \mathrm{hr}$ or $500 \mathrm{ppm}$ for $8 \mathrm{hr} /$ day, 5 days/week for 2 weeks did not induce toxicity. Intentional inhalation abuse of $n$-butane for 1 year by a 16-year-old girl caused her to suffer from visual hallucinations, increased irritability, and social withdrawal. Exposure to $n$-butane at very high concentrations can affect the central nervous system, causing narcosis and asphyxiation.

ADDITIONAL REFERENCES

1. N-Butane, Material Safety Data sheet (MSDS) G-17, BOC Gases Division of the BOC Group, Incorporated, Murray Hi77, NJ, 7 June 1996 2. Butane, Material Safety Data Sheet (MSDS), Exxon Chemical Americas, Houston, TX, 27 March 1995

3. n-Butane (Pure Grade), MSDs us003448, Phi77ips Chemical Company, Bart7esvi 17e, OK, 30 Apri 1993 


\section{R-717 (AMMONIA)}

\begin{tabular}{llll}
\hline$R-717$ & ammonia & REFRIGERANT TOXICITY SUMMARY & see \\
inorganic & $\mathrm{NH3}$ & CAS number $7664-41-7$ & RDB\#
\end{tabular}

\section{GENERAL CAUTIONS}

More recent and/or more complete information may be found in Material Safety Data Sheets (MSDSs), provided by the chemical supplier(s), and in other toxicology references. Inhalation of high concentrations of refrigerant vapors is harmful.

NIOSH-OSHA OCCUPATIONAL HEALTH GUIDELINE (OHG) TOXICOLOGY SUMMARY (1992)

EFFECTS ON ANIMALS: Ammonia is a severe irritant of the eyes, respiratory tract, and skin. Liquified ammonia can induce freezing, burns, and corrosion of tissues. Exposure of guinea pigs to 5,000 to $6,000 \mathrm{ppm}$ for 30 to 120 minute induced blindness. The approximate 1-hr LC50 for cats and rabbits was 9,900 ppm. This concentration induced severe bronchial damage, alveolar congestion, edema atelectasis, hemorrhage, and emphysema in the animals that died. The $4-\mathrm{hr}$ LC50 in rats is $2,000 \mathrm{ppm}$, and the ora7 LD50 is $350 \mathrm{mg} / \mathrm{kg}$. In mice, the RD50 (the concentration capable of reducing respiratory rate by 50\%) was approximately $300 \mathrm{ppm}$. Continued exposure for $6 \mathrm{hr} /$ day for 5 days produced nasal lesions including hypertrophy, hyperplasia, epithelial erosion, ulceration, and necrosis. continuous exposure to ammonia for severa 7 weeks at $658 \mathrm{ppm}$ produced marked eye irritation in dogs and rabbits and corneal opacity in rabbits. Rats similarly exposed developed interstitial pneumonitus with calcification of the bronchi and renal tubules, epithelial proliferation of the renal tubules, myocardial fibrosis, and fatty liver. Rats, guinea pigs, dogs, rabbits, and monkeys exposed to $1,100 \mathrm{ppm}$ for $8 \mathrm{hr} / \mathrm{day}$, 5 days/week for 6 weeks showed signs of moderate eye and nasa 7 irritation but no signs of systemic poisoning. Although al1 monkeys, dogs, and rabbits survived a continuous 90-day exposure to $680 \mathrm{ppm}$, this exposure was fatal to 13 of 15 rats and 4 of 15 guinea pigs. No clinically significant findings were detected at autopsy in rats continuous $7 y$ exposed to $180 \mathrm{ppm}$ for 90 days.

EFFECTS ON HUMANS: Ammonia gas is severely irritating to the eyes and to the moist skin and mucous membranes of humans. In contact with the eyes, Tiquid and anhydrous ammonia causes severe damage that may lead to biindness. When in contact with the skin, liquified ammonia can cause freezing and third-degree burns. Two volunteers exposed to ammonia at approximately $24 \mathrm{ppm}$ showed signs of mild nasal irritation on clinical examination. Five of six volunteers exposed to $30 \mathrm{or}$ $50 \mathrm{ppm}$ for $10 \mathrm{~min}$ reported mild to moderate upper respiratory tract irritation. At $130 \mathrm{ppm}$, however, volunteers experienced lacrimation and nose and throat irritation; one volunteer experienced pulmonary irritation. Exposure of humans to $500 \mathrm{ppm}$ for 30 min caused changes in respiratory rate, and exposure to 1,720 produced coughing in exposed individuals. Accidental exposure to ammonia gas or the vapors of ammonia at high concentrations (approximately 2,500 to 6,500 ppm) for up to $2 \mathrm{hr}$ induced chemical pneumonitus, burns (eyes, face, and mouth), severe local edema, dyspnea, progressive cyanosis, and death. Death is normally caused by either suffocation or pulmonary edema. In near-lethal cases, residual effects may include visual impairment, decreased pulmonary function, and hoarseness. 
NOTE: While the LD50 concentration indicated in the preceding NIOSHOSHA Toxicology Summary is widely cited in the literature, it was measured in tests using ammonium hydroxide (ammonia-water solution, $\mathrm{NH} 4 \mathrm{OH}$ ). A paper on the study notes that the LD50 was calculated for ammonia.

\section{ADDITIONAL REFERENCES}

1. Ammonia, Material Safety Data Sheet (MSDS) G-11, BOC Gases Division of the BOC Group, Incorporated, Murray Hi77, NJ, I Ju7y 1996

2 Ammonia, MSDS DU000002 (6007CR), DuPont Chemicals, wilmington, DE, 27 August 1993

3. Ammonia, MSDS, Hawkeye Chemical Company, 20 March 1992

4. Ammonia, Anhydrous, MSDS OHS01050, MDL Information Systems, Incorporated, San Leandro, CA, 28 June 1996

5. Ammonia, Anhydrous-Refrigeration Grade, MSDS, Unocal Corporation, Los Angeles, CA, 30 August 1995

6. Anhydrous Ammonia, MSDS, General Electric Company, November 1979

7. Anhydrous Ammonia, MSDS, Genium Publishing Corporation, Schenectady, NY, August 1985

8 . Anhydrous Ammonia, MSDS, Hill Brothers Chemical Company, 8 January 1991

9 Anhydrous Ammonia, MSDS, LaRoche Industries Incorporated, January 1991 


\section{R-744 (CARBON DIOXIDE)}

$\begin{array}{ll}\text { R-744 } & \text { carbon dioxide } \\ \text { inorganic } & \text { CO2 }\end{array}$

REFRIGERANT TOXICITY SUMMARY

While the toxicity of R-744 is comparatively low, its potential to displace air, inhalation effects, pressure ruptures and releases, and freezing from contact with its 1 iquid form pose hazards. Carbon dioxide is the most powerful cerebral vasoditator known./2/ It is a rapid acting, acute respiratory stimulant at low concentrations. succeedingly higher concentrations wil1, in addition, produce effects on the central nervous system. They include headache, dizziness, muscular spasm, and loss of consciousness. Brief exposures at a concentration of $30,000 \mathrm{ppm}$ do not appear to present a toxic hazard in terms of respiratory stimulant or central nervous system effects. This concentration is recommended as a ceiling limit for up to a 10-minute exposure. Prolonged exposure to carbon dioxide at a concentration of $10,000 \mathrm{ppm}$ results in physiological changes, limited to renal and respiratory compensatory mechanisms that do not result in any apparent adverse symptomatology. At higher concentrations, work-related exercise will exaggerate the respiratory stimulant as we 71 as other physiologic effects of carbon dioxide, resulting in undesirable side effects.

Carbon dioxide is present in the atmosphere at approximately $300 \mathrm{ppm}$. It is a normal body constituent and respiratory stimulant./3/

\section{OCCUPATIONAL EXPOSURE LIMITS}

The Occupational Safety and Health Administration (OSHA) Permissible Exposure Limit ( $P E L$ ) for $R-744$ is $5,000 \mathrm{ppm}$ on a time-weighted average (TWA) basis for an 8 hour shift of a 40 hour workweek. The American Conference of Governmental Industrial Hygienists (ACGIH) adopted a Threshold Limit Value - Time-Weighted Average (TLV-TWA) of $5,000 \mathrm{ppm}$ in 1948 and a Threshold Limit value - Short-Term Exposure Limit (TLV-STEL) of $30,000 \mathrm{ppm}$ in 1986.

\section{GENERAL CAUTIONS}

More recent and/or more complete information may be found in Material Safety Data Sheets (MSDSS), provided by the chemical supplier(s), and in other toxicology references. Inhalation of high concentrations of refrigerant vapors is harmful. Anesthetic effects including loss of consciousness, heart irregularities, or death may result. Intentional misuse or deliberate inhalation ("sniffing") may cause death without warning. Massive leaks or spi71s also may pose an asphyxiation hazard, by displacing air, in confined or inadequately ventilated locations. This refrigerant is heavier than air, and leakage may accumulate in low areas.

NIOSH-OSHA OCCUPATIONAL HEALTH GUIDELINE (OHG) TOXICOLOGY SUMMARY (September 1978)

Carbon dioxide gas is an asphyxiant, a potential respiratory stimulant, and both a stimulant and depressant of the CNS [Centra] Nervous System]. Respiratory volume is doubled at $4 \%[40,000 \mathrm{ppm}]$ carbon dioxide and redoubled at $5 \%[50,000 \mathrm{ppm}]$. Increases in heart rate and blood pressure have been noted at $7.6 \%[76,000 \mathrm{ppm}]$ and dyspnea, headache, dizziness, and sweating occur if exposure at that leve 1 is prolonged. At $10 \%[100,000 \mathrm{ppm}]$ and above, prolonged exposure can result in unconsciousness. Above $11 \%[110,000 \mathrm{ppm}$, unconsciousness 
occurs in 1 minute or 1ess. Numerous human fatalities have occurred after persons entered fermentation vats, wells, and silos where oxygen had been largely replaced by carbon dioxide. Exposure to very high concentrations, 25 to $30 \%$ [250,000 to $300,000 \mathrm{ppm}]$, may cause convulsions. Carbon dioxide at room temperature wi77 not injure the skin, but frostbite may result from the solid or 7iquid phases.

\section{RELATED TOXICOLOGICAL INFORMATION}

oxygen deficiency during pregnancy has produced developmental abnormalities in humans and experimental animals. Exposure of female rats to $60,000 \mathrm{ppm}(6 \%)$ carbon dioxide for $24 \mathrm{hr}$ has produced toxic effects to the embryo and fetus in pregnant rats. Toxic effects also have been observed in other mammalian species at similar concentrations./2/

\section{ADDITIONAL REFERENCES}

1. Carbon Dioxide, Material Safety Data sheet (MSDS), Amerex

Corporation, Trussville, AL, 1 February 1996

2. Carbon Dioxide, Gas, MSDS G-8, BOC Gases Division of the BOC Group, Incorporated, Murray Hi i , NJ, 7 June 1996

3. Carbon Dioxide, MSDS 6077CR, DuPont Chemicals, wilmington, DE, 29 August 1993

4. Carbon Dioxide, MSDS, Genium Pub7ishing Corporation, Schenectady, NY, Apri† 1986

5. Carbon Dioxide, MSDS, Union Carbide Corporation, 8 october 1991 


\section{APPENDIX 4: TOXICITY REFERENCES}

This appendix presents toxicity citations from the ARTI Refrigerant Database 22 that primarily address toxicity. The current version of Database provides more than 1400 citations addressing refrigerant toxicity.

\section{SAFETY}

P. J. Baldock (Imperial Chemical Industries PLC, ICl, UK), Accidental Releases of Ammonia: An Analysis of Reported Incidents, AlChE Loss Prevention Symposium, American Institute of Chemical Engineers (AIChE), New York, NY, 13:, 1980 (RDB7535)

R-717, safety, accidents involving human exposures

J. Berghmans and $\mathrm{H}$. Duprez (Katholieke Universiteit Leuven, Belgium), Safety Aspects of $\mathrm{CO}_{2}$ Heat Pumps, Heat Pumps - a Benefit for the Environment (proceedings of the Sixth International Energy Agency (IEA) Heat Pump Conference, Berlin, Germany, 31 May - 2 June 1999), Verlagsund Wirtschaftsgesellschaft der Elektrizitätswerke m.b.H. (WWEW-Verlag), Frankfurt am Main, Germany, posters tab 5, May 1999 (10 pages with 10 figures and 1 table, RDB9838)

analyzes the safety risks of R-744 (carbon dioxide) by quantitative risk analysis (QRA) and compares them to other refrigerant options; addresses toxicity, leakage scenarios, boiling liquid expanding vapor explosion (BLEVE), dynamic room concentrations, and pressure explosions; concludes that the risks related to R744 use in heat pumps are low and acceptable provided the charge is limited so concentrations upon full charge release are less than 50,000 $\mathrm{ppm} \mathrm{v} / \mathrm{v}$

T. Brown, D. M. Holder, and A. J. Gigiel (University of Bristol, UK), Safety Aspects of Air Cycle Refrigeration and Heat Pump Systems, Proceedings of the 19th International Congress of Refrigeration (The Hague, The Netherlands, 20-25 August 1995), International Institute of Refrigeration (IIR), Paris, France, IVb:742-747, 1995 (6 pages with 1 figure and 2 tables, RDB7915) factors that affect the operational safety of air (R-729) cycle systems including component or piping failure from pressure, failure of highspeed rotary components (turbine compressors or expanders), noise, and contamination; concludes that hazards are ordinary

J. M. Calm (Engineering Consultant), Refrigerant Safety, ASHRAE Joumal, 36(7):17-26, July 1994; erratum, 36(8), August 1994 (10 pages with 3 figures and 2 tables, available from JMC as RDB4766)

This article outlines safety concerns and summarizes safety data for refrigerants, with emphasis on those used in chiliers. It briefly reviews the history of refrigeration, noting that nearly all of the early refrigerants were flammable, toxic, or both, and that some also were highly reactive. It describes the discovery of organic fluorides as refrigerants, by Thomas Midgley in 1926, and the first toxicity tests. The article reviews the commercial introduction and growth of fluorocarbon refrigerants. It then describes the attributes of ideal refrigerants, noting that none exist or are likely to be found. The article summarizes toxicity concerns and several reasons for increased attention to them. A table provides a glossary of safety terminology including common indices used to quantify recommended exposure limits and concentrations that pose recognized risks. Among them are the Acceptable Exposure Limit (AEL), Immediately Dangerous to Life or Health (IDLH), Industrial Exposure Limit (IEL), Lethal Concentration for $50 \%$ of specimens $\left(L_{50}\right)$, No Observed Effect Level (NOEL), Occupational Exposure Limit (OEL), Permissible Exposure Level (PEL), Recommended Exposure Limit (REL), Short-Term Exposure Limit (STEL), Threshold Limit Value (TLV), and Workplace Environmental Exposure Level (WEEL). Flammability limits also are addressed includ- 
ing the lower and upper flammability limits (LFL and UFL, respectively). The article then summarizes toxicity data for $\mathrm{R}-123$ and $\mathrm{R}-134 \mathrm{a}$, and relates the R-123 data to measured concentrations in machinery rooms. It also explains other hazards, including the potential for irritation, corrosive effects, asphyxiation, narcosis, and other physiological effects. A table compares safety indicators for $R-11, R-12, R-$ 22, R-123, R-134a, and R-717 (ammonia) including acute, subchronic, and chronic toxicity, cardiac sensitization response level, mutagenicity, carcinogenicity, and teratogenicity. The paper then summarizes the safety classifications and the recommended installation, handling, and service procedures found in ASHRAE standards 34 and 15 , respectively. The article concludes that the new, alternative refrigerants can be used with comparable or higher safety than those they replace. It notes that the alternatives have been subjected to more stringent qualification criteria and testing. The article also indicates that more care is needed with all refrigerants.

T. Dierckx and J. Berghmans (Katholieke Universiteit Leuven, Belgium), Safety Aspects of Working Fluids, Energy Efficiency and Global Warming Impact (proceedings of the meetings of Commissions B1 and B2, Ghent, Belgium 12-14 May 1993), International Institute of Refrigeration (IIR), Paris, France, 293-300, 1993 (8 pages with 1 figure and 3 tables, RDB5323)

R-11, R-12, R-22, R-23, R-32, R-125, R-134a, R-142a (possibly intended to be R-143a), R290 (propane), and R-717 (ammonia) as potential replacements for R-12; R-124 (cited as $\mathrm{R}-124(\mathrm{a})$ ) and R-227 (probably R-227ea) as potential replacements for R-114; R-21, R-30, $R-123, R-132 a$, and $R-133$ as potential replacements for R-11; flammability; toxicity

R. E. Dufour and A. J. Perkins, The Comparative Life, Fire, and Explosion Hazards of Trifluorotrichloroethane ("Freon-113"), Miscellaneous Hazard Report 3072, Underwriters Laboratories Incorporated, Northbrook, IL (then Chicago, IL), 7 April 1941 (22 pages with 6 tables, RDB5174)

R-113, toxicity, flammability, stability

L. Fluer (Fluer, Incorporated, and HMEx, Incorporated), Hazardous Materials Classification Guide, edited by J. M. Shapiro, International Fire Code Institute (IFCI), Whittier, CA, 1993 (818 pages with 1 table, RDB3965)
This book provides guidance for classification of hazards for approximately 3,500 chemicals for use with building and fire safety codes. The volume also explains the fundamentals of hazard classification and provides a cross-reference between more than 7,000 chemical names and Chemical Abstract Service (CAS) registry numbers. A table, forming most of the book, lists the chemicals by CAS number (or pseudo-number for mixtures). It also identifies their chemical and/or trade names as well as common names for verification. Each entry provides the physical state (solid, liquid, or gaseous) at $20^{\circ} \mathrm{C}\left(68^{\circ} \mathrm{F}\right)$, concentration, indication of listing under the U.S. Environmental Protection Agency (EPA) List of Extremely Hazardous Substances (40 CFR $355 \S 302$ and $\S 304$ ), information for warning placards, and physical and health hazard categories. The placard information follows the National Fire Protection Agency Standard 704-1985 Standard System for the Identification of the Fire Hazards of Materials. It includes recommended hazard identification rating numbers for health, flammability, and reactivity as well as a designator for special hazards. The physical categories identified include aerosols, combustible dusts and fibers, flammable and oxidizing cryogenics, explosives, flammable and combustible liquids, flammable gases, organic peroxides, oxidizers, pyrophorics, unstable reactives, and water reactives. The health hazards addressed are carcinogenic, corrosive, irritant, other health hazard, radioactive, sensitizer, and toxic/highly toxic. An introductory section explains the criteria, data sources, classification approach, and assigned hazard levels for each. The guide covers R-11, R-12, R-12B2, R-13, R14, R-21, R-22, R-23, R-30, R-113, R-114, R115, R-116, R-123, R-134a, R-152a, R-290 RC318, R-500, R-503, R-717 (ammonia), and R744 (carbon dioxide), R-1132a, and a number of mineral oil lubricants among the other chemicals. [see RDB3966 for software version]

L. Fluer (Fluer, Incorporated, and HMEx, Incorporated), HMEx Assistant - The Hazardous Materials Expert, International Fire Code Institute (IFCl), Whittier, CA, version 1.02, 1993 (software package with 72 page user's manual, RDB3966)

This software provides guidance for classification of hazards for approximately 3,500 chemicals for use with building and fire safety codes. The software enables look-up by chemical, commercial, or common names; Chemical Abstract Service (CAS) registry numbers (or 
pseudo-number for mixtures); or Registry of Toxic Effects of Chemical Substances $\left(\right.$ RTECS $\left.^{\circledR}\right)$ codes. The entries indicate the physical state (solid, liquid, or gaseous) at 20 ${ }^{\circ} \mathrm{C}\left(68^{\circ} \mathrm{F}\right)$, concentration, indication of listing under the U.S. Environmental Protection Agency (EPA) List of Extremely Hazardous Substances (40 CFR $355 \$ 302$ and $\S 304$ ), information for warning placards, and physical and health hazard categories. The placard information follows the National Fire Protection Agency Standard 704-1985 Standard System for the Identification of the Fire Hazards of Materials. It includes recommended hazard identification rating numbers for health, flammability, and reactivity as well as a designator for special hazards. The physical categories identified include aerosols, combustible dusts and fibers, flammable and oxidizing cryogenics, explosives, flammable and combustible liquids, flammable gases, organic peroxides, oxidizers, pyrophorics, unstable reactives, and water reactives. The health hazards addressed are carcinogenic, corrosive, irritant, other health hazard, radioactive, sensitizer, and toxic/highly toxic. The entries also identify the classifier and reviewer for each chemical according to initials or codes. A companion user's manual provides installation instructions and explains the criteria, data sources, classification approach, and assigned hazard levels. The software covers R-11, R-12, R-12B2, R-13, R-14, R-21，R-22, R-23, R-30, R-113, R-114, R-115, R-116, R-123, R-134a, R-152a, R-290 R-C318, R-500, R-503, R-717 (ammonia), and R-744 (carbon dioxide), R-1132a, and a number of mineral oils among the other chemicals. [see RDB3965 for printed version]

K. J. M. Ham and J. Gansevoort (Netherlands Organization for Applied Scientific Research, TNO, The Netherlands), Risk Analysis for Ammonia Refrigeration Systems, Proceedings of the 19th International Congress of Refrigeration (The Hague, The Netherlands, 20-25 August 1995), International Institute of Refrigeration (IIR), Paris, France, IVa:678-686, 1995 (9 pages with 2 figures, RDB7911)

hazards associated with R-717 (ammonia) use; review of Dutch safety regulations for ammonia refrigerating installations; prior evaluations indicate that many existing systems do not meet these requirements, that many were never inspected or fully tested, and that complete replacement of the installation would be required to obtain approvals; requirements for a Quanti- fied Risk Assessment (QRA); proposed code of practice to obtain approvals for nonconforming systems

International Programme on Chemical Safety (IPCS), Partially Halogenated Chlorofluorocarbons (Ethane Derivatives), Environmental Health Criteria (EHC) report 139, World Health Organization (WHO), Geneva, Switzerland, 1992 (134 pages with 1 figure and 16 tables, RDb5371)

R-123, R-123, R-124, R-132b, R-133a, R-141b, $R-142 b$ : identification; summary properties; analytical methods; sources of human and environmental exposure; environmental transport, distribution, and transformation; environmental levels and human exposure; kinetics and metabolism; effects on laboratory mammals; in vitro tests; effects on humans; effects on other organisms in the laboratory and field; evaluation of human health risks and effects on the environment; recommendations for protection of human health and the environment

R. W. James, Safe Use of Refrigerants for Process Applications, Journal of Loss Prevention in the Process Industries, 7(6):492-500, 1994 (9 pages, RDBA666)

discusses safety issues related to refrigerant use in process operations with focus on hydrocarbons and R-717 (ammonia) as potential replacements for fluorochemicals; discusses hazards from accidental refrigerant releases including toxic decomposition products when refrigerant vapors contact flames or hot surfaces, suffocation resulting from refrigerants in confined spaces, narcotic and cardiac effects, fire, freezing of tissues, corrosive attack on sensitive tissues such as the eyes, or impact damage resulting from component or equipment failure; discusses safety standards and codes; examines refrigerant charge limits in equipment based room volume, occupancy, and maximum allowable concentrations; discusses safety precautions including restricting access to rooms where refrigerating equipment is installed, work practices for maintaining and repairing equipment, protecting against noise and vibration exposure, design criteria, pressure release during fires, ventilation, alarm systems, and safety checks

G. J. Koster (Grenco Refrigeration B.V., The Netherlands), A Way to Safe Use of Ammonia, Proceedings of the 19th International Congress of Refrigeration (The Hague, The Netherlands, 20-25 August 1995), International Institute of Refrigera- 
tion (IIR), Paris, France, IVa:641-645, 1995 (5 pages with 3 tables, RDB7908)

advocates use of R-717 (ammonia) as an environmentally-safe refrigerant; factors affecting application including regulations

R. J. Lewis, Sr., Hazardous Chemicals Desk Reference (fourth edition), VNR / John Wiley and Sons, Incorporated, New York, NY, 1997 (1,760 pages, RDB8361)

safety profiles and hazard ratings for chemicals: flammability, toxicity, and other safety data

A. Lindborg (Frigoscandia AB, Sweden), Ammonia the Obvious Refrigerant, Proceedings of the 19th International Congress of Refrigeration (The Hague, The Netherlands, 20-25 August 1995), International Institute of Refrigeration (IIR), Paris, France, IVa:646-651, 1995 (6 pages with 4 tables, RDB7909)

advocates use of R-717 (ammonia) as a "natural" refrigerant; physical and physiological effects of ammonia; public perceptions of ammonia

W. E. Stewart, R. W. Clark (University of MissouriKansas City), L. A. Stickler, and C. K. Saunders (Inter-Mountain Research), Gaseous Ammonia Dispersion: Comparison of Experimental Data with Two Predictive Models, paper 3924, Transactions (Annual Meeting, San Diego, CA, 24-28 June 1995), American Society of Heating, Refrigerating and Air-Conditioning Engineers (ASHRAE), Atlanta, GA, 101(2):497-501, 1995 (4 pages with 4 figures and 1 table, RDB6362)

R-717, safety, validation of dispersion models, DEGADIS (DEnse GAs DISpersion model), SLAB

R. J. M. van Gerwen (Netherlands Organization for Applied Scientific Research, TNO, The Netherlands), Safety Aspects of Natural Working Fluids, IEA Heat Pump Center Newsletter - CFC and HFC Replacement, International Energy Agency (IEA) Heat Pump Center (HPC), Sittard, The Netherlands, 13(1):19-21, March 1995 (3 pages with 1 figure, RDB5684)

$\mathrm{R}-717$, hydrocarbons, accidents, risk assessment

R. J. M. van Gerwen and C. M. A. Jansen, Risk Assessment of the Use of Flammable Refrigerants, report 8327-24191, Netherlands Organization for Applied Scientific Research (TNO), Apeldoorn, The Netherlands, June 1994 (RDB5686) safety, flammability, accidents

P. G. J. M. van Dijck, untitled report on the flammability of R-245fa, report 96D2/2344, Prins Maurits Laboratory, TNO [Netherlands Organization for Applied Scientific Research], Rijswijk, The Netherlands, 20 December 1996 (6 pages with 1 figure and 1 table, available from JMC as RDB7114)

investigation of the flammability of R-245fa in concentrations of $0-15 \%$ in air in a vertical, vapor-explosion burette using a $6.25 \mathrm{~J}(0.0059$ Btu) spark for ignition: concludes that R-245fa is nonflammable as tested; an annex describes the apparatus, test procedure, and deviations from the test prescribed in EC Directive 92/69/EEC

R. J. M. van Gerwen and H. Koffijberg (Netherlands Organization for Applied Scientific Research, TNO, The Netherlands), Natural Refrigerants: Applications and Risks, Proceedings of the 19th International Congress of Refrigeration (The Hague, The Netherlands, 20-25 August 1995), International Institute of Refrigeration (IIR), Paris, France, IVb:790-796, 1995 (7 pages with 1 figure and 3 tables, RDB7920)

status of R-717 (ammonia) use in the Netherlands, primarily in food processing with a $50 \%$ share ( $65 \%$ by refrigerant amount) with an average of $7.5 \mathrm{~kg} / \mathrm{kW}(4.8 \mathrm{lb} / \mathrm{ton})$ and ice skating rinks with a $75 \%$ share; use in commercial refrigeration and chillers is limited by safety regulations, but there are new indirect systems incorporating secondary heat transfer fluids; hydrocarbons have not been used for 40 years except R-290/600 (propane/butane) in domestic refrigerators; broader application of hydrocarbons, in almost all appliances starting in 1995 and transport refrigeration is expected based on quantified risk assessment (QRA) findings; review of ammonia accident data cites 37 accidents ( 5 in the Netherlands) with 152 injuries and 2 fatalities ( 1 in the Netherlands); 196 additional accidents with 185 injuries and 17 fatalities are noted for Norway and Japan; QRA methods and accepted individual and societal risk criteria are summarized for Australia, Hong Kong, the Netherlands, and the United Kingdom; discusses directives and standards for safety in the Netherlands and more broadly in Europe

Aard en omvang van het gebruik van ammoniak als koudemiddel in de Nederlandse Voedings- en Genotmiddelenindustrie, Netherlands Organization for Applied Scientific Research 
(TNO), Apeldoorn, The Netherlands, May 1992 (RDB5495)

accident statistics with use of ammonia (R-717) as a refrigerant in the Netherlands, safety

Addenda to Design and Safety Classifications of Refrigerants [the title of the revised standard actually is Designation and Safety Classification of Refrigerants, the word Design should be Designation and the word Classifications should be singular], ANSI/ASHRAE Addenda 34h-2000, 34j2000, and 34k-2000 (Addenda to ANSI/ASHRAE 34-1997), American Society of Heating, Refrigerating, and Air-Conditioning Engineers (ASHRAE), Atlanta, GA, 2000 [first distributed June 2000] (6 pages with 1 tables, RDBA674)

addendum $34 \mathrm{~h}$ adds a provisional safety classification of A1 for R-245fa and editorial changes to the table with refrigerant data and safety classifications; $34 \mathrm{j}$ modifies the submission requirements for toxicity information for designation and classification applications; 34k deletes the requirements for proof of commercialization for blends for such applications; these addenda revise ANSI/ASHRAE Standard 34-1997 [see RDB8601]

Addenda to Number Designation and Safety Classification of Refrigerants [the title of the revised standard actually is "Designation and Safety Classification of Refrigerants", the word "Number" was deleted in the 1997 revision], ANSI/ASHRAE Addenda 34a, 34b, 34c, 34d, and 34f (Addenda to ANSI/ASHRAE 34-1997), American Society of Heating, Refrigerating, and Air-Conditioning Engineers (ASHRAE), Atlanta, GA, 1999 -[first distributed June 1999] (8 pages with 2 tables, RDB9710)

Addendum 34 a deletes some refrigerants that were never or are no longer used to focus the standard on refrigerants in current use or expected to be used in the future. It corrects the chemical formulae and names cited for some refrigerants, though this information is indicated as informational only and not a part of the standard. Addendum $34 \mathrm{~b}$ adds R-413A, R$218 / 134 a / 600 a(9.0 / 88.0 / 3.0)$, and both a safety classification and tolerances for it. Addendum $34 \mathrm{c}$ deletes the safety classifications for RC318 and R-405A based on insufficient toxicity data. Addendum $34 d$ deletes a previous requirement for submission of recommended NFPA 704 Hazard Signals from applications for designation and classification. Addendum $34 f$ adds R-407E, R-32/125/134a (25.0/15.0/60.0), and both a safety classification and tolerances for it. Addendum 34 e adds a safety classifica- tion for R-236fa. These addenda revise ANSI/ASHRAE Standard 34-1997 [see RDB8601].

Designation and Safety Classification of Refrigerants, ANSI/ASHRAE Standard 34-1997, American Society of Heating, Refrigerating, and Air-Conditioning Engineers (ASHRAE), Atlanta, GA, 1997 [first distributed June 1998] (22 pages with 1 figure and 7 tables, RDB8601)

This voluntary, consensus standard describes a shorthand way of naming refrigerants and classifies them according to potential hazards. It is intended to establish a simple means of referring to common refrigerants, instead of using the chemical name, formula, or trade name. It also establishes a uniform system for assigning unambiguous reference numbers, compositiondesignating prefixes for refrigerants, and safety classifications based on toxicity and flammability, both as formulated and under worst-case of fractionation conditions. This standard is widely cited in construction codes and provides the classifications used in ASHRAE Standard 15. Safety Code for Mechanical Refrigeration, to specify safety requirements for refrigerant use. This version supersedes ANSI/ASHRAE Standard 34-1992 and earlier editions.

Equipment, Design, and Installation of Ammonia Mechanical Refrigerating Systems, ANSI/IIAR Standard 2-1992, International Institute of Ammonia Refrigeration (IIAR), Washington, DC, December 1992 (40 pages with 2 figures and 5 tables, RDB4757)

This voluntary, consensus standard is intended to serve as guide to the design, manufacture, installation, and use of mechanical refrigerating systems employing R-717 (anhydrous ammonia). It applies to closed mechanical refrigerating systems in industrial occupancies. Its provisions represent minimum requirements for the areas addressed, to minimize property damage or loss and risks to persons. The standard comprises a foreword, introduction, definitions, and sections on equipment, machinery room design, and installation. The equipment provisions cover compressors, evaporative condensers, pressure reliefs, shell-and-tube condensers, double-pipe condensers, pressure vessels, evaporators, refrigerant pumps, refrigerant valves, pressure-relief devices, piping, testing, materials, and ultimate strength requirements. Most of these topics identify specific design criteria, nameplate data, and testing. The provisions for machinery rooms cover room layout, open flames, ventilation, archi- 
tectural features, location, electrical, and piping. The installation topics include materials selection, piping, joints, pipe hangers and supports, pressure-relief protection, foundations and equipment supports, system testing, and ammonia handling and storage. The standard concludes with lists of references and their sources, an appendix on emergency discharge of ammonia refrigerant, and two drawings detailing emergency control stations. This version supersedes ANSI/IIAR Standard 2-1984 and earlier editions.

Genetron ${ }^{\circledR}$ AZ-20 (R-410A) Safety Overview, bulletin G525-084, AlliedSignal Incorporated, Morristown, NJ, December 1996 (2 pages with 1 table, limited copies available from JMC as RDB7110)

This bulletin complements the Material Safety Data Sheet (MSDS) with discussion of health and safety information for use of R-410A. A table shows the composition to be R-32/125 (50/50). Discussion of its toxicity notes that data for the components were developed under the Program for Alternative Fluorocarbon Toxicity Testing (PAFT), and that review of the results shows low toxicity in animals for both R32 and R-125. AlliedSignal recommends an occupational exposure limit (OEL) of $1,000 \mathrm{ppm}$ on an 8-hr time-weighted average (TWA) basis; the bulletin discusses concerns with oxygen displacement, asphyxiation, cardiac sensitization, and overexposure. Ingestion is noted as causing discomfort in the gastrointestinal tract, some of the effects of inhalation, and necrosis from freezing tissue. The vapor can irritate the skin and eyes while the liquid can freeze them on contact; suggestions are given for care in such cases. The bulletin discusses evacuation in response to leaks and recovery procedures. It identifies the ASHRAE Standard 34 safety classification as A1/A1, the Underwriter's Laboratory (UL) rating as practically nonflammable, and U.S. Department of Transportation (DOT) consideration as nonflammable (Green label). The bulletin cautions that R-410A can become combustible in air at elevated pressures and recommends against mixing or leak testing with air. It notes that $\mathrm{R}-410 \mathrm{~A}$ can decompose at high temperatures, such as those found in open flames and red- or white-hot metals, yielding toxic, corrosive, and irritating compounds as well as pungent and irritating vapors. The refrigerant is described as stable at normal operating conditions, but contact with freshly abraded aluminum and active metals should be avoided. The bulletin concludes with a discus- sion of pressures, noting that those for $R-410 \mathrm{~A}$ are approximately $60 \%$ higher than for R-22. Suggested ratings are given for hoses and equipment used with R-410A. AlliedSignal's product name for $R-410 A$ is Genetron ${ }^{\circledR} A Z-20$.

Guidelines for Ammonia Machinery Room Ventilation, IIAR bulletin 111, International Institute of Ammonia Refrigeration (IIAR), Washington, DC, October 1991 (24 pages with 1 figure, RDB4760)

This bulletin provides guidance for ventilation systems for machinery rooms using ammonia in industrial refrigeration facilities. The recommended design scheme and minimum ventilation levels are applicable to systems with large quantities of ammonia, exceeding several thousand pounds, but may not be appropriate for smaller systems or those for other applications. The bulletin identifies reference sources, including major codes and standards, used to prepare the guidelines. It then summarizes ventilation requirements to be classified as a "Non-Hazardous (Unclassified) Location" under the National Fire Protection Association (NFPA) Standard 70, National Electrical Code (NEC). It specifically addresses continuously operated mechanical exhaust, independent emergency ventilation, and minimum volume requirements, A section on recommended design schemes reviews the steps to determine code minimums and outlines the design process. The document also addresses operation and maintenance. A worksheet to facilitate calculations, system illustration, and design example are provided.

Hazardous Chemicals Data, publication 49, National Fire Protection Association (NFPA), Quincy, MA, 1994 (170 pages, RDB6709)

flammability, toxicity, reactivity, safety classifications

History of Accidents in Ammonia Refrigeration Facilities in Japan (1951-1990), High Pressure Gas Safety Institute of Japan, 1991 (RDB5451)

R-717 (ammonia), safety

Kirk-Othmer Encyclopedia of Chemical Technology (third edition), John Wiley and Sons, Incorporated, New York, NY, 1978-1984 (RDB5165)

reference for flammability, toxicity, reactivity, and other safety data

Ongevallen met Ammoniak Koelinstallaties [ACcidents with Ammonia Refrigeration Systems], Netherlands Organization for Applied Scientific 
Research (TNO), Apeldoorn, The Netherlands, 28 May 1991 (RDB5496)

R-717 (ammonia), safety

Proctor \& Hughes' Chemical Hazards of the Workplace (fourth edition), edited by G. J. Hathaway, N. H. Proctor, and J. P. Hughes, VNR I John Wiley and Sons, Incorporated, New York, NY, 1996 (720 pages, RDB8360)

widely cited reference on chemicals that pose occupational hazards: identifiers, acute and chronic toxicity, flammability, and other safety data

Refrigerant Safety Data - Recommendations of the Air-Conditioning and Refrigeration Industry, Air-Conditioning and Refrigeration Institute (ARI), Arlington, VA, October 1993 (32 pages with 6 tables, available from JMC as RDB3A15)

The report provides toxicity and flammability data on refrigerants as well as recommended hazard categories and safety data. This information is needed to meet requirements for refrigerant use under the Uniform Fire Code (UFC) and Uniform Mechanical Code (UMC). The refrigerants addressed include $R-11, R-12$, R-22, R-113, R-114, R-123, R-134a, R-500, R502, R-717 (ammonia), and R-744 (carbon dioxide). The information addressed includes Permissible Exposure Level (PEL), Immediately Dangerous to Life or Health (IDLH), and Lower Flammability Limit (LFL) values needed to set alarm and automatic shutdown levels. Where unavailable from sources prescribed by the UFC and/or UMC, surrogate values - established on a consistent basis - are offered. The report also provides hazard categories determined in accordance with the UFC and hazard signals determined in accordance with the UFC, UFC Standard 79-3, and National Fire Protection Association (NFPA) Standard 70490. The report summarizes data assembled by industry experts, critically examined at a meeting convened solely for this purpose, and subsequently reviewed by the participants and others. The report provides an extensive list of references used to assemble the data. It also identifies the participants, contributors, and reviewers.

Refrigerants (SLGV), Electrical Appliance and Utilization Equipment, Underwriters Laboratories Incorporated (UL), Northbrook, IL, 1023-1025, 1996 (3 pages, RDB6938)

These listings cover refrigerants intended for use in air-conditioning and refrigerating equip- ment. The refrigerants have been classified for flammability only; pressure hazards were not evaluated. The listings cover product versions of R-11, R-12, R-22, R-123, R-134a, R-404A, R-407A, R-407B, R-407C, R-408A, R-409A, R$410 A, R-500, R-507 A$, and R-508A. The listings indicate the UL flammability group (nonflammable, practically nonflammable, or flammable) following UL Standard 2182, Refrigerants and the ignition temperature if below 750 ${ }^{\circ} \mathrm{C}\left(1382{ }^{\circ} \mathrm{F}\right)$. They also indicate the chemical constituents, phase as shipped, and container types.

Addendum to Safety Code for Mechanical Refrigeration, ANSI/ASHRAE Addendum 15c-2000 (Addendum to ANSI/ASHRAE 15-1994), American Society of Heating, Refrigerating, and Air-Conditioning Engineers (ASHRAE), Atlanta, GA, 2000 [first distributed June 2000] (14 pages with 4 tables, RDBA675)

addendum $15 \mathrm{c}$ adds definitions, provisions, and data for pressure-relief valve piping for refrigerants; it also adds an appendix that documents a method to determine equivalent piping lengths; this addendum revises ANSI/ASHRAE Standard 15-1994 [see RDB4903]

Emergency Interim Standards Action for ANSI/ASHRAE Standard 15-1994, American Society of Heating, Refrigerating, and Air-Conditioning Engineers (ASHRAE), Atlanta, GA, June 1998 (1 page, RDB8617)

this Emergency Interim Standards Action (EISA) changes the allowed test gases for field pressure testing of refrigeration systems to avoid gases or mixtures of gases that may become flammable; this EISA revises ANSIIASHRAE Standard 15-1994 [see RDB4903]

Safety Code for Mechanical Refrigeration, ANSIIASHRAE Standard 15-1994, American Society of Heating, Refrigerating, and Air-Conditioning Engineers (ASHRAE), Atlanta, GA, August 1994 (RDB4903)

This voluntary, consensus standard specifies safety requirements for design, construction, installation, and operation of refrigerating systems, to establish safeguards for life, limb, health, and property. It applies to mechanical refrigerating systems and heat pumps used in identified occupancies. It also covers components added after adoption, refrigerant conversions, and replacement of parts and components with new ones that are not identical in function. This standard is widely incorporated 
into construction codes, either by reference or by transcription with amendments. It identifies occupancy, system, and refrigerant safety classifications, the last by reference to ANSI/ASHRAE Standard 34-1992. It then prescribes restrictions on refrigerant use, including the maximum quantities of individual refrigerants that may be used for specific occupancies and systems. It also prescribes safety standards for design and construction of equipment and systems, operation, testing, and general requirements. Minimum safety devices, labeling, and tests also are specified. Appendices address the maximum allowable concentrations for blends, guidelines for emergency discharge of refrigerants, worst case fractionation, and a method for calculating the discharge capacity of pressure-relief devices for positive displacement compressors. A final appendix provides a cross-reference between paragraph numbers in the previous and current versions of this standard. This version supersedes ANSI/ASHRAE Standard 15-1992 and earlier editions.

Safety of Suva Refrigerants, document AS-1 (H27530-3), DuPont Chemicals, Wilmington, DE, January 1994 (6 pages with 1 table, RDB4C48)

This bulletin reviews considerations for safe use of refrigerants by providing answers to common questions. It outlines the introduction of alternative refrigerants and associated testing. It briefly reviews the collaborative Programme for Alternative Fluorocarbon Toxicity Testing (PAFT) and individual programs and findings for specific refrigerants. The document discusses the flammability of R-123, R-124, R125 , and $R-134 a$, noting that they are neither flammable nor explosive, but can become so when mixed with gases that are. The document notes that mixtures of R-134a with more than $60 \%$ air (by volume) can become flammable at raised pressures and temperatures. It counsels against use of refrigerant-air mixtures for leak testing. The document then addresses inhalation toxicity and the potential for suffocation. It explains the DuPont Acceptable Exposure Limit (AEL), a time-weighted average (TWA) concentration for an 8 or 12 hour day or 40 hour week, to which nearly all workers may be repeatedly exposed without adverse effects. It also explains Emergency Exposure Limit (EEL) values as airborne concentrations of brief duration which should not result in permanent adverse effects or interfere with escape in the event of a major release or spill. The document discusses symptoms of exposure to high refrig- erant concentrations, cardiac sensitization, suffocation, safety measures for enclosed areas, dangers of deliberate inhalation, detection by odor, and guidance when a large spill or leak occurs. It goes on to address special requirements for R-123. The chemical formulae, boiling points, AELs, and EELs are tabulated for R-123, R-124, R-125, and R-134a. The bulletin provides general advice on skin and eye contact with refrigerants, frostbite, pressure hazards, handling and disposal of cylinders, brazing or welding of pipes in air-conditioning and refrigeration systems, and decomposition. It concludes with specific cautions. The document identifies related bulletins that may be helpful and recommends familiarization with the Material Safety Data Sheet (MSDS) provided for the refrigerant to be used. DuPont's product names are Suva ${ }^{\circledR}$ Centri-LP and Suva ${ }^{\circledR} 123$ for R-123, Suva ${ }^{\circledR} 124$ for R-124, and Suva ${ }^{\circledR} 125$ for R-125. It identifies R-134a as Suva ${ }^{\circledR}$ ColdMP, Suva ${ }^{\circledR}$ Trans $A / C$, and Suva ${ }^{\circledR} 134 a$.

Sax's Dangerous Properties of Industrial Materials (ninth edition), edited by R. J. Lewis, Sr. (revision of publication by N. I. Sax), VNR / John Wiley and Sons, Incorporated, New York, NY, 1996 ( 3 volumes totaling more than 2,454 pages, RDB6106)

widely cited reference for flammability, toxicity, and other safety data

Unfallverhütungsvorschrift UV 20, Kälteanlagen [Accident Prevention Regulations UW 20, Refrigerating Plants], Federal Republic of Germany, Köln, Germany (RDB4681)

refrigerant safety requirements

\section{TOXICITY}

B. E. Abreu (University of California Medical School), Auerbach, Thuringer, and Peoples, Journal of Pharmacology and Experimental Therapeutics (JPET), 80:139 ff, 1944 (RDB7290)

anesthetic effect of chlorine, bromine, and iodine derivatives of the methane and ethylene series of hydrocarbons, toxicity - as reported in RDB5980

B. E. Abreu (University of California Medical School), Anesthesiology, 2:393 ff, 1941 (RDB7291)

anesthetic effect of chlorine, bromine, and iodine derivatives of the methane and ethylene 
series of hydrocarbons, toxicity - as reported in RDB5980

J. Adir, D. A. Blake, and G. W. Mergner, Pharmacokinetics of Fluorocarbons 11 and 12 in Dogs and Humans, The Journal of Clinical Pharmacology, 15:760-770, 1975 (11 pages, RDB5C74)

inhalation toxicity of R-11 and R12; biochemistry; biotransformation; elimination

J. Adir, D. A. Blake, and G. W. Mergner (University of Maryland), Kinetics of Uptake and Elimination of Trichlorofluoromethane (F-11) and Dichlorodifluoromethane (F-12) in Beagles and Humans, abstract 171 (13th Annual Meeting of the Society of Toxicology (SOT), Washington, DC, 10-14 March 1974), Toxicology and Applied Pharmacology (TAP), 29(1):142-143, July 1974 (2 pages with no figures or tables, RDB59A9)

kinetics of uptake and elimination of R-11 and $\mathrm{R}-12$ based on blood concentration in 6 beagles and 2 humans; concludes that the kinetics of these chemicals are consistent with their blood solubilities, oil-water partition characteristics, and other physiologic considerations

Y. Alarie and J. E. Luo, Sensory Irritation by Airborne Chemicals: A Basis to Establish Acceptable Levels of Exposures, Toxicology of the $\mathrm{Na}$ sal Passages (based on the Seventh Chemical Industry Institute of Toxicology, CIIT, Conference on Toxicology, Raleigh, NC, 22-23 February 1984), edited by C. S. Barrow, Hemisphere Publishing Corporation, Washington, DC, 91-100, 1986 (10 pages with 5 figures and 1 table, RDB6285)

acute toxicity, sensory irritation, prediction of acceptable levels, TLV-TWA predictions based on $R D_{50}, R D_{50}$ values for 40 industrial chemicals, among which are $303 \mathrm{ppm}$ for R-717 (ammonia) and $117 \mathrm{ppm}$ for R-764 (sulfur dioxide)

Y. Alarie (University of Pittsburgh), Dose-Response Analysis in Animal Studies: Prediction of Human Responses, Environmental Health Perspectives (EHP), 42:9-13, 1981 (5 pages with 2 figures and 4 tables, RDB6279)

acute toxicity, dependence on exposure durations for irritation and lethal concentration measures, prediction of the levels and types of responses in humans at various multiples of the $R D_{50}$ value found in mice, regression to TLV$T W A, R D_{50}$ values for 25 airborne, sensory irritants: Among them are 303 ppm for R-717 (ammonia) and $117 \mathrm{ppm}$ for R-764 (sulfur dioxide). The effects indicated at multiples of the
$\mathrm{RD}_{50}$ are severe injury and possible mortality at 10 , intolerable to humans at 1 , some sensory irritation at 0.1 , no sensory irritation at 0.01 , and no effect of any kind on the respiratory system at 0.001

Y. Alarie (University of Pittsburgh), Bioassay for Evaluating the Potency of Airborne Sensory Irritants and Predicting Acceptable Levels of Exposure in Man, Food and Cosmetics Toxicology, 19:623-626, 1981 (RDB6557)

health effects, toxicity, $R D_{50}, T L V, R-717, R-$ 764

Y. Alarie, L. E. Kane, and C. S. Barrow (University of Pittsburgh), Sensory Irritation: A Basis to Establish Acceptable Exposure to Airborne Chemical Irritants, Principles and Practice of Industrial Toxicology, edited by A. Reeves, John Wiley and Sons, Incorporated, New York, NY, 1980 (RDB6504)

health effects, toxicity, $R_{50}$

Y. Alarie, Sensory Irritation by Airborne Chemicals, Critical Reviews in Toxicology, Chemical Rubber Company (CRC), Cleveland, $\mathrm{OH}, 2: 299$ 366, 1973 (RDB6503)

acute toxicity, sensory irritation

Y. Alarie, I. Wikisaka, and S. Oka, Sensory Irritation by Sulfur Dioxide and Chlorobenzylidene Malononitrile, Environmental Physiology and Biochemistry, 3:53-74, 1973 (22 pages, RDB6506)

acute toxicity of R-764 (sulfur dioxide); sensory irritation $\mathrm{RD}_{50}=117 \mathrm{ppm}$ - as reported in RDB6557

M. W. Anders (University of Rochester Medical Center), Metabolism and Toxicity of Hydrochlorofluorocarbons: Current Knowledge and Needs for the Future, Environmental Health Perspectives (EHP), 96:185-191, 1991 (7 pages, RDB5935)

toxicity of R-123, R-125, and others; mutagenicity

K. Andersson and B. Johansson, Measurement of Fluorocarbon (Freon) Contents in Different Working Environments, report IVL B743, Inst. Vatten Luftvardsforsk, Göteborg, Sweden, 1983 (RDB7227)

measurements of refrigerant concentration in field conditions; safety; toxicity

M. E. Andersen, M. L. Gargas, R. A. Jones, and L. J. Jenkins, Jr., Determination of the Kinetic 
Constants for Metabolism of Inhaled Toxicants In Vivo Using Gas Uptake Measurements, Toxicology and Applied Pharmacology (TAP), 54:100116, 1980 (17 pages, RDB7254)

toxicity of R-114 and others; teratology

M. Antti-Poika, J. Heikkila, and L. Saarinen, Cardiac Arrhythmias During Occupational Exposure to Fluorinated Hydrocarbons, British Journal of Industrial Medicine, 47:138-140, 1990 (3 pages, RDBA935)

cardiac sensitization; toxicity

C. Aranyi, W. J. O'Shea, C. A. Halder, C. E. Holdsworth, and B. Y. Cockrell, Absence of Hydrocarbon-Induced Nephropathy in Rats Exposed Subchronically to Volatile Hydrocarbon Mixtures Pertinent to Gasoline, Toxicology and Industrial Health, 2(1):85-98, 1986 (14 pages, RDB6194)

tests of subchronic inhalation toxicity by exposure of male and female F-344 rats to a 50/50 mixture by weight of R-600a (isobutane) and R$601 a$ (isopentane) - R-600a/601a (50/50) for 6 $\mathrm{hr} / \mathrm{d}, 5 \mathrm{~d} / \mathrm{wk}$, for $13 \mathrm{wk}$ : no significant, treatment-related effects were found and there was no evidence of nephropathy at the end of the study, though mild kidney effects were seen at an interim sacrifice point at 28 days - as reported in RDB7621

D. M. Aviado, Fluorine-Containing Organic Compounds, Patty's Industrial Hygiene and Toxicology (fourth edition), edited by G. D. Clayton and F. E. Clayton, John Wiley and Sons, Incorporated, New York, NY, 2B:1179-1204, 1994 (26 pages, RDB7231)

summary toxicity data and outline of toxicological considerations for fluorocarbons using R-11 as a prototype; also addresses comparative toxicity of R-12, R-21, R-22, R-113, R-114, R$115, R-142 b, R-152 a, R-C 318$; animal models for evaluation of fluorocarbon toxicity

D. M. Aviado (University of Pennsylvania School of Medicine), Comparative Cardiotoxicity of Fluorocarbons, Cardiac Toxicology, edited by T. Balazs, CRC Press, Incorporated, Boca Raton, FL, II:213-222, 1981 (10 pages with 1 figure and 3 tables, RDB6477)

detailed review of the cardiotoxicity of $\mathrm{R}-11$; comparisons for R-12, R-13, R-21, R-22, R-23, R-31, R-113, R-114, R-115, R-123, R-124, R132b, R-133a, R-142b, R-152a, R-1113, and R1114 , toxicity
D. M. Aviado (University of Pennsylvania School of Medicine), Effects of Fluorocarbons, Chlorinated Solvents, and Inosine on the Cardiopulmonary System, Environmental Health Perspectives (EHP), 26:207-215, 1978 (9 pages, RDB7423)

toxicity of R-11 and others

D. M. Aviado (University of Pennsylvania School of Medicine), Physiological and Biochemical Responses to a Specific Group of Inhalants: Concluding Remarks, Federation Proceedings, Federation of the American Society of Experimental Biologists, 37:2508-2509, 1978 (2 pages, RDB7430)

$\mathrm{R}-11, \mathrm{R}-12, \mathrm{R}-114$, mixtures of them, and others, health effects, toxicity: sequence of events in deaths from cardiac sensitization from abuse of fluorocarbon aerosols (many also used as refrigerants) in "sniffing;" sensitization of the heart to prearrhythmic effects of epinephrine, depression of myocardial contractility, reduction in cardiac output, and irritation of mucosa in the upper and lower respiratory tract that causes an increase in sympathetic and vagal impulses to the heart - as reported in RDB7414

D. M. Aviado, S. Zakhari, and T. Watanabe (University of Pennsylvania School of Medicine), Nonfluorinated Propellants and Solvents for Aerosols, edited by L. Golberg, CRC Press, Cleveland, $\mathrm{OH}, 1977$ (118 pages, Rdb6569)

health effects, interactions, and toxicity of $R-30$, R-140a, R-290 (propane), R-600 (n-butane), R600 a (isobutane), $R-1120$, ethanol, $A-46$ (R$290 / 600 a / 600(17.1 / 80.4 / 2.5)$ ), and others with comparative data for R-11, R-12, and R-114; cites inhalation toxicity data for R-600 of 2-hr $L C_{50}$ mouse $=680 \mathrm{mg} / \mathrm{l}[281,000 \mathrm{ppm} \mathrm{v} / \mathrm{v}]$ and 4 -hr $L C_{50}$ rat $=658 \mathrm{mg} / /[272,000 \mathrm{ppm} \mathrm{v/v}]$ from Shugaev [see RDB5363])

D. M. Aviado (University of Pennsylvania School of Medicine), Preclinical Pharmacology and Toxicology of Halogenated Solvents and Propellants, monograph 15, National Institute of Drug Abuse Research, chapter 10, 164-184, 1977 (21 pages, RDB7247)

toxicity of R-114 and others

D. M. Aviado, S. Zakhari (University of Pennsylvania School of Medicine, USA), J. A. Simaan (American University of Beirut, Lebanon), and A. G. Ulsamer (U.S. Consumer Products Safety Commission, CPSC, USA), Methyl Chloroform and Trichloroethylene in the Environment, ed- 
ited by L. Golberg, CRC Press, Cleveland, $\mathrm{OH}$, 1976 (RDB7292)

toxicity of R-140a and R-1120; comparative data to other chemicalss

D. M. Aviado and J. Drimal (University of Pennsylvania School of Medicine), Five Fluorocarbons for Administration of Aerosol Bronchodilators, Journal of Clinical Pharmacology, 15(1/2):116-128, 1975 (13 pages with 4 figures and 3 tables, RDB6122)

investigates separate toxic effects on the upper (nose, pharynx, and larynx) and lower (lungs) respiratory tracts for R-11, R-12, R-114, R-115, and R-C318 administered by tracheal cannulas inserted in anesthetized dogs; responses measured by pulmonary resistance and compliance, heart rate, and aortic blood pressure; concludes that the three fluorochemicals widely used in aerosols, namely R-11, R-12, and R114, elicit both bradycardia (slow heart rate) and tachycardia (relatively rapid heart rate) and induce bronchoconstriction or bronchodilation when inspired in large doses; also found that R115 and R-C318 do not elicit any change in heart rate and produce only bronchodilation; recommends further study of the $R-115$ and $R$ C318 as potential replacements if the other three prove to be linked to deaths from use and abuse of aerosols

D. M. Aviado and D. G. Smith (University of Pennsylvania School of Medicine), Toxicity of Aerosol Propellants in the Respiratory and Circulatory Systems, VIll. Respiration and Circulation in Primates, Toxicology, 3(2):241-252, 1975 (12 pages, RDB5735)

toxicity of R-11, R-12, R-23, R-115, R-C318, and others

D. M. Aviado (University of Pennsylvania School of Medicine), Toxicity of Aerosol Propellants in the Respiratory and Circulatory Systems, $X$. Proposed Classification, Toxicology, 3(3):321332,1975 (12 pages with 2 tables, RDB5920)

R-11, R-12, R-21, R-22, R-30, R-113, R-114, R-115, R-140 or R-140a, R-142b, R-152a, R290, R-C318, R-600a, and R-1140; toxicity: classifies propellants based on relative pressure and toxicity, the latter primarily effects on the respiratory and circulatory systems and the levels at which cardiac arrhythmias were produced; classifications include low-pressure propellants of high toxicity (R-11, R-21, R-30, $\mathrm{R}-113$, and $\mathrm{R}-140$ or R-140a), low-pressure propellants of intermediate toxicity (R-114, R- 142b, R-C318, and R-600a); high-pressure propellants of intermediate toxicity (R-12, R-22, $R-290$, and R-1140), and high-pressure propellants of low toxicity (R-115 and R-152a); discussion of animal models for evaluation of acute toxicity, noting that the unanesthetized dog with epinephrine injection is 5-10 times more sensitive to propellants (many of which also used as refrigerants) than the anesthetized dog without injection of epinephrine

D. M. Aviado and M. A. Belej (University of Pennsylvania School of Medicine), Toxicity of Aerosol Propellants in the Respiratory and Circulatory Systems, V. Ventricular Function in the Dog, Toxicology, 3:79-86, 1975 (8 pages with 2 figures and 2 tables, RDB5C80)

toxicity of R-11, R-113, R-115, R-152a, and RC318; concludes that "there is no available propellant for aerosols that is devoid of cardiodepressant action"

D. M. Aviado (University of Pennsylvania School of Medicine), Toxicity of Aerosols with Special Reference to the Role of Propellants in Asthmatic Deaths, The Journal of Clinical Pharmacology, 15:86-104, 1975 (19 pages, RDB65F6)

toxicity

D. M. Aviado (University of Pennsylvania School of Medicine), Toxicity of Propellants, Fortschritte der Arzneimittelforschung [Progress in Drug Research], Birkhäuser Verlag, Basel, Switzerland, 18:365-397, 1974 (35 pages, RDB6593)

toxicity of R-11, R-12, R-21, R-22, R-30, R-113, R-114 or R-114a, R-140a, R-142b, R-152a, R290, R-C318, R-600a, R-1140, and others; health effects; toxicity: review of available information on the toxicity of 15 aerosol propellants (many also used as refrigerants); respiratory and cardiovascular effects on animals

D. M. Aviado (University of Pennsylvania School of Medicine), Toxicity of Propellants, Proceedings of the Fourth Annual Conference on Environmental Toxicology, Elsevier/North Holland, Amsterdam, The Netherlands, 16-19 October 1974 (RDB6594)

toxicity of R-152a and others

D. M. Aviado and M. A. Belej (University of Pennsylvania School of Medicine), Toxicity of Aerosol Propellants on the Respiratory and Circulatory Systems, I. Cardiac Arrhythmia in the Mouse, Toxicology, 2(1):31-42, 1974 (12 pages with 2 figures and 3 tables, RDB5854) 
observes that when "aerosols were first introduced in the 1950s, the refrigerants were examined for possible use as propellants"; reports of fatalities from use or abuse of aerosols led to reexamination of their toxicity; cardiac sensitization, without and with epinephrine injection, in male mice anesthetized with sodium pentobarbitol: R-11, R-12, R-21, R-22, R-30, R-113, $\mathrm{R}-114, \mathrm{R}-115, \mathrm{R}-140$ or R-140a, R-142b, R152a, R-290, R-C318, R-600a, and R-1140; compares findings for mice to cardiac sensitization data for dogs based on published tests by others (see RDB5644) noting that $R-12$, R$142 b$, and $R-152 a$ sensitize dogs but not mice (no chemicals are identified that sensitize mice but not dogs); toxicity classifications for R-10, R-11, R-12, R-20, R-21, R-22, R-40, R-40B1, $\mathrm{R}-113, \mathrm{R}-114, \mathrm{R}-115, \mathrm{R}-140$ or R-140a, R142b, R-152a, R-160, R-160B1, R-290, RC318, R-600a, R-611, R-717, R-744, R-764, R1140 , and others

D. M. Aviado (University of Pennsylvania School of Medicine), Cardiopulmonary Effects of Fluorocarbon Compounds, paper 4, Proceedings of the 2nd Annual Conference on Environmental Toxicology (31 August - 2 September 1971), report AMRL-TR-71-120, Aerospace Medical Research Laboratory, U.S. Air Force, Wright-Patterson Air Force Base, OH, 31-39, December 1971 (9 pages with 4 figures, available from NTIS as document AD-751426, RDB6736)

$\mathrm{R}-11, \mathrm{R}-12$, and R-114; examines relation of cardiopulmonary toxicity to irritation of the sensory receptors in respiratory passages by tests on anesthetized dogs; identifies biological defense mechanisms to prevent entrance into and absorption of an irritant vapor by the lungs; observes that fluorocarbon compounds can cause depression of ventricular function

A. Azar, H. J. Trochimowicz, J. B. Terrill, and L. S. Mullin (E. I. duPont de Nemours and Company, incorporated), Blood Levels of Fluorocarbon Related to Cardiac Sensitization, AIHA Journal, 34(3):102-109, 1973 (8 pages, RDB5357)

\section{toxicity of R-11 and others}

A. Azar (E. I. duPont de Nemours and Company, Incorporated), Cardiac Sensitization of Fluorocarbon Propellants, presentation at the Conference of Toxic Hazards of Halocarbon Propellants, U.S. Food and Drug Administration (FDA), Washington, DC, 16 July 1971 (23 pages with 3 figures and 7 tables, RDB6596) toxicity of $R-11, R-12$, and $R-114$; cardiac sensitization

K. C. Back, A. A. Thomas, and J. D. MacEwen, Reciassification of Materials Listed as Transportation Health Hazards, report TSA-20-72-3, Aerospace Medical Research Laboratory (AMRL), U.S. Air Force, Wright-Patterson Air Force Base, OH, 1972 (RDB7298)

R-717 (ammonia) and others, health effects, toxicity: for ammonia, $1-\mathrm{hr} \mathrm{LC}_{50}$ mouse $=4,837$ $\mathrm{ppm} \mathrm{v/v}$ and $1-\mathrm{hr} L \mathrm{LC}_{50}$ rat $=7,338 \mathrm{ppm} \mathrm{v/v-}$ as reported in RDB5340

R. D. Bagnall, W. Bell, K. Pearson, and A. Jeater (Imperial Chemical Industries Limited, UK), New Inhalation Anaesthetics: III - Fluorinated Aliphatic Ethers, Journal of Fluorine Chemistry, 13:123-140, 1979 (8 pages, RDB8311)

toxicity of hydrofluoroethers; anesthetic effects of R-E245ca2 and others - as reported in RDB7B15

G. N. Bakhishev, Relation of the Toxicity of Some Halogenated Aliphatic Hydrocarbons to Their Electronic Structure, Gigiena Truda i Professional'nye Zabolevaniya [Labor Hygiene and Occupational Diseases], Moscow, Russia (then USSR), 1:39-40, 1980 (2 pages in Russian, RDB5820)

\section{toxicity of R-23 and others}

K. S. Bakshi (National Research Council, NRC), Toxicity of Alternatives to Chlorofluorocarbons: HFC-134a and HCFC-123, Inhalation Toxicity, 10(10):963-967, October 1998 (5 pages with 1 table, RDB9127)

summarizes a detailed assessment (see RDB$6 A 01)$ by the Board on Environmental Studies and Toxicology of the toxicities of R-123 and R134a: provides recommendations to set emergency exposure guidance level (EEGL) concentrations for both and a continuous exposure guidance level (CEGL) for R-134a; recommends a $1 \mathrm{hr}$ EEGL for R-134a of $4,000 \mathrm{ppm}$ $\mathrm{v} / \mathrm{v}$ based on the no-observed-adverse-effect level (NOAEL) in cardiac sensitization tests, divided by an uncertainty factor of 10 for interspecies variability; recommends a $24 \mathrm{hr}$ EEGL for R-134a of $1,000 \mathrm{ppm}$ based on a NOAEL of $10,000 \mathrm{ppm}$ for ferotoxicity effects (slight retardation of skeletal ossification) in rats divided by an uncertainty factor of 10 ; recommends a 90day CEGL for R-134a of $900 \mathrm{ppm}$ based on a NOAEL of $50,000 \mathrm{ppm}$ for a $2-y \mathrm{r}$ chronic toxicity study in rats divided by an uncertainty factor 
of 10, a factor of 4 for exposures of $24 \mathrm{hr} / \mathrm{d}$ instead of $6 \mathrm{hr} / \mathrm{d}$, and a factor of $5 / 7$ for exposures of $7 \mathrm{~d} / \mathrm{wk}$ instead of the tested $5 \mathrm{~d} / \mathrm{wk}$; recommends a 1-min EEGL for R-123 of 1,900 $\mathrm{ppm}$ based on a cardiac sensitization $\mathrm{EC}_{50}$ dog of $19,000 \mathrm{ppm}$ divided by an uncertainty factor of 10; differences in the time periods for the R123 and R-134a exposure levels addressed reflect proposed uses of R-134a as a refrigerant on submarines, versus $R-123$ as a fire suppressant to replace halon 12 in flight-line extinguishers with very quick discharges

R. E. Bales (Tracor Jitco, Incorporated), Fluorocarbons - Industrial Hygiene Survey, publication PB-297772, National Institute of Occupational Safety and Health (NIOSH), U.S. Department of Health, Education, and Welfare (DHEW), Cincinnati, May 1978; republished as Fluorocarbons An Industrial Hygiene Survey of Worker Exposure in Four Facilities, publication 79-101, NIOSH, Cincinnati, OH, October 1978 (50 pages with 2 figures and 8 tables, available from NTIS, RDB5824)

concentrations of R-10, R-11, R-12, R-13, R13B1, R-22, R-23, R-30, R-113, R-114, R-115, R-116, R-140a, R-142b, R-152a (possibly R152 , incorrectly identified in the report as " $\mathrm{CH}_{3} \mathrm{~F}-\mathrm{CH}_{3} \mathrm{~F}$ "), R-1110, R-1120, R-1132a, and others including toxic decomposition products in industrial sites: the plants surveyed (A) manufacture $R-11, R-12, R-115$, and $R-116$, package them, R-13B1, R-22, R-113, R-114, R$142 b, R-152 a, R-1132 a$, and other chemicals and intermediates, (B) produce R-11 and R-12 and package them and R-503 separately or with other gases, (C) use R-12 and R-113 to charge refrigeration compressors and degrease parts, respectively, and (D) produce R-764 and amines and package them with and other materials with $R-11$ and $R-12$ in aerosol spray cans for solvent, insecticide, and other uses; report shows worker exposure levels to be less than permissible levels on a time-weighted average basis for each of the sites, but identifies recommendations to minimize further exposures

S. M. Barlow and F. M. Sullivan, Fluorocarbons, Reproductive Hazards of Industrial Chemistry, Academic Press, London, UK, 326-333, 1982 (8 pages, RDB5171)

toxicity of R-11, R-12, R-21, R-22, R-31, R-112, $R-113, R-114, R-115$, and others

H. Bartsch, C. Malaveille, A.-M. Camus, G. MartelPlanche, G. Brun, A. Hautefeuille, N. Sabadie, A.
Barbin, T. Kuroki, C. Drevon, C. Piccoli, and P. Montesano, Validation and Comparative Studies of 180 Chemicals with SalmonellaTyphimurium Strains and V-79 Chinese Hamster, Mutation Research, 78(1):1-50 (possibly 76:1-50), 1980 (50 pages, RDB5922)

toxicity of R-22 and others

R. C. Baselt and R. H. Cravey, A Fatal Case Involving Trichlorofluoromethane and Dichlorodifluoromethane, Joumal of Forensic Science, 13(3):407-410, 1968 (4 pages, RDB6721)

toxicity of $R-11$ and $R-12$; accident involving human exposure - as reported in RDB6138

M. A. Belej and D. M. Aviado (University of Pennsylvania School of Medicine), Cardiopulmonary Toxicity of Propellants for Aerosols, presented at the Fifth International Pharmacological Congress (San Francisco, 23-28 July 1972); republished in The Journal of Clinical Pharmacology, 15(1/2):105-115, January 1975 (11 pages with 3 figures and 3 tables, RDB59A4)

R-11, R-12, R-21, R-114, R-115, R-123B1, R$142 b, R-152 a, R-C 318$, and R-600a as propellants; cardiopulmonary effects, bronchopulmonary and cardiovascular toxicity: concludes that all of the propellants cause acceleration of the heart rate; shows that R-11 has a significantly greater impact on blood pressure and heart rate in 30 anesthetized mongrel dogs while R-152a had the least effect

M. A. Belej, D. G. Smith, and D. M. Aviado (University of Pennsylvania School of Medicine), Toxicity of Aerosol Propellants in the Respiratory and Circulatory Systems, IV. Cardiotoxicity in the Monkey, Toxicology, 2(4):381-395, 1974 (15 pages, RDB5178)

toxicity of R-22, R-23, R-115, R-600a (isobutane), and others

M. A. Belej and D. M. Aviado (University of Pennsylvania School of Medicine), Federation Proceedings, Federation of the American Society of Experimental Biologists, 32:814, 1973 (1 page, RDB5C62)

toxicity of R-12 and others

R. Benigni, M. Cotta-Ramusino, and C. Andreoli, Relationship Between Chlorofluorocarbon Chemical Structure and Their Salmonella Mutagenicity, Journal of Toxicology and Environmental Health, 34(3):397-408, 1991 (12 pages, RDB5778) quantitative analysis of the relationship between chemical structure and Salmonella mu- 
tagenicity of R-32, R-114, R-115, R-124, R125, R-134a, R-143a, R-152a, and others: molecules were characterized by both molecular orbital and physical chemical parameters; results of the analysis indicate that mutagenicity is correlated with both the free energy of binding to biological receptors and the energy of the highest occupied molecular orbit; concludes that fluorochemical mutagenicity is determined more by the rate of initial activation than by the rate of DNA attack since the same two factors favor catalyzed metabolism by cytochrome $P-450$

A. G. Berends (Solvay Duphar, The Netherlands), C. G. de Rooij (Solvay S.A., Belgium), S. Shin-ya (Asahi Glass Company, Limited, Japan), and R. S. Thompson (Zeneca, UK), Biodegradation and Ecotoxicity of HFCs and HCFCs, Archives of Environmental Contamination Toxicology, 36(2):146151, February 1999 (6 pages, RDB9B44)

summarizes results of closed-bottle tests of the biodegradation of $R-32, R-123, R-124, R-125$, $\mathrm{R}-134 a, \mathrm{R}-141 \mathrm{~b}, \mathrm{R}-225 \mathrm{ca}$, and R-225cb; found that these chemicals are not readily biodegradable since less than $60 \%$ was removed after 28 days; summarizes standard acute toxicity tests with R-123, R-141b, and R-225ca using algae, water fleas, and fish revealed $E_{50}$ values in the range of $17-126 \mathrm{mg} / \mathrm{L}$; similar tests of R$134 a$ found $E C_{50}$ concentrations of $450-980$ $\mathrm{mg} / \mathrm{L}$; fish studies with R-141b and R-225ca revealed bioaccumulation factors of $<3$ and 1564, respectively; a study with plants revealed no effect from R-141b on seed germination and growth of wheat (Triticum aestivum), radish (Raphanus sativus), and cress (Lepidium sativum); concludes that hydrochlorofluorocarbons (HCFCs) and hydrofluorocarbons (HFCs) are not very toxic to aquatic organisms and terrestrial plants; also concludes that there was no evidence for any aerobic biodegradation for most of the HFCs and HCFCs

M. C. Bindal et al., Quantitative Correlation of Anesthetic Potencies of Halogenated Hydrocarbons with Boiling Points and Molecular Connectivity, Fortschritte der Arzneimittelforschung [Progress in Drug Research], Birkhäuser Verlag, Basel, Switzerland, 30(2):234-236, 1980 (3 pages, RDB6184)

toxicity of R-124 and others

D. A. Blake and G. W. Mergner (University of Maryland), Are Fluorocarbon Propellants Metabolized? Studies in ${ }^{14} \mathrm{C}$-Labeled Trichloro- fluoromethane (F-11) and Dichlorodifluoromethane (F-12) in Beagles and Humans, abstract 170 (13th Annual Meeting of the Society of Toxicology (SOT), Washington, DC, 14 March 1974), Toxicology and Applied Pharmacology (TAP), 29(1):142, July 1974 (1 page with no figures or tables, RDB59A7)

investigation of biotransformation of R-11 and $\mathrm{R}-12$ in 5 male and female beagles and 4 humans after a 7-20 minute inhalation; found that essentially all of the chemicals was recovered in unaltered form from exhaled air within one hour and, therefore, that $\mathrm{R}-11$ and $\mathrm{R}-12$ are refractory to biotransformation

H. M. Bolt (University of Dortmund, Germany) and J. T. Borlak (Fraunhofer Institute, Germany), Halogenated Hydrocarbons, Toxicology, edited by $\mathrm{H}$. Marquardt, Academic Press, San Diego, CA, 645-657, 1999 (13 pages with 8 figures and 1 table, RDBA525)

reviews the toxicity of common halocarbons with focus on those used as solvents; addresses R-10 (carbon tetrachloride), R-20 (chloroform), R-30 (methylene chloride), R-140 (1,1,2-trichloroethane), methyl chloroform), R140a (methyl chloroform), R-1110 (PER, perchloroethylene), R-1120 (TRI, trichloroethylene), R-1130a (vinylidene chloride), and R1140 (vinyl chloride); also briefly addresses $\mathrm{R}$ 11, R-13, R-21, R-22, R-31, R-112, R-113, R123B1 (halothane), R-130, R-142b, R-150, R$150 B 2$, and R-260B2; discussion emphasizes exposure limits, underlying metabolism of the chemicals, and chemical similarities

W. Braker and A. L. Mossman, Nontoxic Halogenated Aliphatic Hydrocarbons and Halogenated Fluorocarbons, Effects of Exposure to Toxic Gases - First Aid and Medical Treatment, edited by W. Braker and A. L. Mossman, 71-73, 1970 (3 pages, RDB7224)

toxicity fluorochemicals; safety

W. T. Brashear, M. M. Ketcha, D. L. Pollard, C. S. Godin, H. F. Leahy, P. P. Lu, E. R. Kinkead, and R. E. Wolfe (ManTech Environmental Technology, Incorporated), Metabolite Identification of Halon Replacement Compounds, report AL-TR-19920078, Armstrong Laboratory, U.S. Air Force, Wright-Patterson Air Force Base, OH, June 1992 (36 pages with 13 figures and 8 tables, available from JMC as RDB6B50)

metabolism of R-123, R-124, R-142b, R-51-14 (perfluorohexane) by groups of Fischer 344 and Sprague-Dawley rats from exposures at 10,000 
$\mathrm{ppm} v / \mathrm{v}$ in air for $2 \mathrm{hr}$ : biochemistry, health effects, toxicity

W. J. Brock and H. J. Trochimowicz (E. I. duPont de Nemours and Company), Use of Acute Inhalation Toxicity Data in Setting Emergency Exposure Limits, EUROTOX'97, Diversification in Toxicology: Man and Environment (proceedings of the XXXVI European Congress of Toxicology, EuroTox, Århus, Denmark, 25-28 June 1997), Nordic Pharmacological Society, Copenhagen Denmark, 1997; abstract P4-38 republished in Pharmacology and Toxicology, 80(supplement III):117, 1997 (1 page with no figures or tables, RDBA621)

describes an approach and data assembled to set recommended emergency escape concentrations (EECs), to ensure safe egress from situations where a large release has occurred; notes that acute toxicity data were obtained for $\mathrm{R}-32, \mathrm{R}-123, \mathrm{R}-125, \mathrm{R}-134 \mathrm{a}$, and $\mathrm{R}-141 \mathrm{~b}$ in the Program for Alternative Fluorocarbon Toxicity Testing (PAFT); all showed low inhalation toxicity with $L_{50} \mathrm{~S}$ of 32,000 to $>800,000 \mathrm{ppm} \mathrm{v} / \mathrm{v}$; indicates that these compounds, like many halocarbons and hydrocarbons, can sensitize the heart to an intravenous epinephrine challenge; the concentrations that induce cardiac sensitization in these compounds were 5,000-250,000 $\mathrm{ppm} \mathrm{V/v;}$; based on these data, the authors recommended EEC concentrations of 1,000$50,000 \mathrm{ppm} \mathrm{v/v}$

W. J. Brock and H. J. Trochimowicz, Use of Acute Inhalation Toxicity Data in Setting Emergency Exposure Limits, presentation at EUROTOX'97, Diversification in Toxicology: Man and Environment (XXXVI European Congress of Toxicology, EuroTox, Árhus, Denmark, 25-28 June 1997), Haskell Laboratory for Toxicology and Industrial Medicine, E. I. duPont de Nemours and Company, Incorporated, Newark, DE, 1997 (18 pages with 18 presentation charts, RDBA622)

presents data and criteria to set recommended emergency escape concentrations (EECs) to ensure safe egress from situations where a large release has occurred; notes that acute toxicity data were obtained for R-32, R-123, R125 , R-134a, and R-141b in the Program for Alternative Fluorocarbon Toxicity Testing (PAFT); all showed low inhalation toxicity with

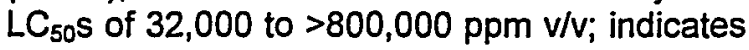
that these compounds, like many halocarbons and hydrocarbons, can sensitize the heart to an intravenous epinephrine challenge; the concentrations that induce cardiac sensitization in these compounds were $5,000-250,000 \mathrm{ppm} v / \mathrm{v}$; based on these data, the authors recommended EEC concentrations of 1,000-50,000 $\mathrm{ppm} v / \mathrm{v}$; tabulates the chemical formulae, normal boiling point (NBP) temperatures, median or approximate lethal concentrations $\left(L C_{50}\right.$ or ALC), cardiac sensitization threshold, and 10min median effective concentrations $\left(E C_{50}\right)$ for anesthetic effects for R-11, R-12, R-13, R13B1, R-22, R-23, R-32, R-113, R-114, R-115, R-123, R-124, R-125, R-134a, R-141b, R-142b, R-143a, R-152a, R-225cb, and R-236fa; outlines EEC criteria involving these data and other escape impairing effects; tabulates proposed EEC concentrations for these refrigerants

W. J. Brock, Toxicology Summary for CFC's HCFC's and HFC's, Haskell Laboratory for Toxicology and Industrial Medicine, E. I. duPont de $\mathrm{Ne}-$ mours and Company, Incorporated, Newark, DE, undated circa 1996 (RDB6452)

R-11, R-12, R-22, R-23, R-30, R-32, R-113, R114, R-115, R-123, R-123B1, R-124, R-125, R134a, R-140a, R-141b, R-143a, R-152a, R236fa, R-43-10, R-717, R-1110, R-1120; tabular summary of toxicity indicators including ALC/LC 50 , cardiac sensitization threshold, anesthetic concentration, subchronic NOELNOAEL, in vitro mutagenicity, in vivo mutagenicity, developmental toxicity, chronic and oncogenicity (carcinogenicity and tumors), and AEL/TLV

R. S. Brody, T. Watanabe, and D. M. Aviado (University of Pennsylvania School of Medicine), Toxicity of Aerosol Propellants on the Respiratory and Circulatory Systems, III. Influence of Bronchopulmonary Lesion on Cardiopulmonary Toxicity in the Mouse, Toxicology, 2:173-184, 1974 (12 pages, RDB5C66)

toxicity of R-11, R-12, R-152a, and others; cardiac sensitization

W. E. Brown (University of Toronto, Canada), Experiments with Anesthetic Gases Propylene, Methane, Dimethyl-Ether, Journal of Pharmacology and Experimental Therapeutics (JPET), 23:485-496, 1924 (8 pages with no figures or tables, RDB6A61)

experimental study in cats to evaluate the effectiveness R-50, R-E170, and R-1270, as anesthetic agents by inhalation; toxicity: R-50 produces general anesthesia, but only in concentrations exceeding $850,000 \mathrm{ppm} ; 900,000$ $\mathrm{ppm}$ caused irregular respiration; R-E170 produces general anesthesia with concentrations 
of $650,000 \mathrm{ppm}$, but is unpleasant to take; R1270 induces anesthesia in cats at 370,000 $500,000 \mathrm{ppm}$; effect is sustainable with 200,000-310,000 ppm; some toxic effects were noted above $400,000 \mathrm{ppm} ; 500,000 \mathrm{ppm}$ produced anesthesia in humans in 2 minutes; recovery was complete

W. E. Brown and V. E. Henderson (University of Toronto, Canada), Experiments with Anesthetic Gases, Anesthesia and Analgesia, 27(1):1-8, 1925 or Journal of Pharmacology and Experimental Therapeutics (JPET), 27:1 ff, 1925 (8/? pages, RDB6108)

anesthetic effect and toxicity of hydrocarbons including R-290 and others

R. M. Burgison, W. E. O'Malley, C. K. Heisse, J. W. Forest, and J. C. Krantz, Jr., Anesthesia. XLVI. Fluorinated Ethylenes and Cardiac Arrhythmias Induced by Epinephrine, Journal of Pharmacology and Experimental Therapeutics (JPET), 114:470-472, 1955 (3 pages, RDB6568)

R-1111, R-1113, R-1114, R-1122 or R-1122a, $\mathrm{R}-1130 \mathrm{c}, \mathrm{R}-1130 t, \mathrm{R}-1132$ or $\mathrm{R}-1132 \mathrm{a}$, and others, cardiac sensitization, toxicity as reported in RDB5644

J. H. Burn, H. G. Epstein, and P. J. Goodford (University of Oxford, UK), The Properties of the Anaesthetic Substance 1:1:2-Trifluoro-1:2-dichloroethane, British Journal of Anaesthesia, 31:518529,1959 (12 pages with 7 figures and 4 tables, RDB6586)

reports toxicity tests in mice and dogs to assess the potentials of R-123a, R-123B1 (halothane), and ether as medical anesthetic agents

J. H. Burn (University of Oxford, UK), Pharmacological Testing of Anaesthetics, Proceedings of the Royal Society of Medicine (meeting of the Section of Anesthetics, 7 November 1958), UK, 52(2):95-98, February 1959 (4 pages with 2 tables, RDB6587)

reports toxicity tests in mice to compare the potentials R-112, R-113, and R-123B1 (halothane) to ether as medical anesthetic agents [R-112 and R-113 are identified only as compounds " $A$ " and " $B$ " in this paper, but are revealed $R D B 6586$ ]

T. H. S. Burns (St. Thomas's Hospital and the Royal Northern Hospital, UK), J. M. Hall (Guy's Hospital, UK), A. Bracken, and G. Gouldstone (formerly British Oxygen Company Limitred), Fluorine Compounds in Anaesthesia (9): Examina- tion of Six Aliphatic Compounds and Four Ethers, Anaesthesia, 37:278-284, 1982 (7 pages with 1 table, RDB6B51)

R-123, R-133al1, R-227ca, R-318, RE347pce2, R-E356pee2, R-41-12, R-51-14, and others, anesthetic effect, toxicity: 6 mice smoothly and deeply anesthetized by 80,000 $\mathrm{ppm} \mathrm{v/v} \mathrm{in} 1 \mathrm{~min} 10 \mathrm{sec}, 2$ mice died in $16 \mathrm{~min}$ and 2 more died in $17 \mathrm{~min}$ when the concentration had fallen to $53,000 \mathrm{ppm} ; \mathrm{R}-318$ exposures resulted in muscle spasms and death to 1 of 4 mice at "high concentration" for 25 min

T. H. S. Burns (St. Thomas's Hospital and the Royal Northern Hospital, UK), J. M. Hall (Guy's Hospital, UK), A. Bracken, and G. Gouldstone (British Oxygen Company, UK), Fluorine Compounds in Anaesthesia (6): Examination of 14 Heavily Halogenated Ring Compounds, $A n-$ aesthesia, 19:167-176, 1964 (10 pages, RDB7560)

cyclic halocarbons possibly including R-C270 and R-C318, anesthetic effect, toxicity

T. H. S. Burns (St. Thomas's Hospital and the Royal Northern Hospital, UK), J. M. Hall (Guy's Hospital, UK), A. Bracken, and G. Gouldstone (British Oxygen Company, UK), Fluorine Compounds in Anaesthesia (5): Examination of Six Heavily Halogenated Aliphatic Compounds, Anaesthesia, 17(3):337-343, July 1962 (7 pages with 1 table, RDB5939)

anesthetic effect, toxicity: R-123 10-min anesthetic LOEL mouse $=40,000 \mathrm{ppm} \mathrm{v} / \mathrm{v}, 40-\mathrm{min}$ anesthetic LOEL mouse $=30,000 \mathrm{ppm} ; \mathrm{R}-125$ anesthetic NOEL mouse at $930,000 \mathrm{ppm}$; R235cb; R-235cbB1; R-1113

T. H. S. Burns (St. Thomas's Hospital and the Royal Northern Hospital, UK), J. M. Hall (Guy's Hospital, UK), A. Bracken, and G. Gouldstone (British Oxygen Company, UK), Fluorine Compounds in Anaesthesia (4): Examination of an Ethane and Four Ethers, Anaesthesia, 16:440444, 1961 (5 pages, RDB7559)

anesthetic effect, toxicity

T. H. S. Burns (St. Thomas's Hospital and the Royal Northern Hospital, UK), J. M. Hall (Guy's Hospital, UK), A. Bracken, G. Gouldstone (British Oxygen Company, UK), and D. S. Newland, An Investigation of New Fluorine Compounds in Anaesthesia (1), Anaesthesia, 16:3-18, 1961 (16 pages, RDB65B4)

anesthetic effect, toxicity 
J. M. Calm (Engineering Consultant), Toxicity Data to Determine Refrigerant Concentration Limits, report DOE/CE/23810-110, Air-Conditioning and Refrigeration Technology Institute (ARTI), Arlington, VA, September 2000 (534 pages with 9 tables, RDBA950)

This report reviews toxicity data, identifies sources for them, and presents resulting exposure limits for refrigerants for consideration by qualified parties in developing safety guides, standards, codes, and regulations. It outlines a method to calculate an acute toxicity exposure limit (ATEL) and from it a recommended concentration limit (RCL) for emergency exposures. The report focuses on acute toxicity with particular attention to lethality, cardiac sensitization, anesthetic and central nervous system (CNS) effects, and other escape-impairing effects. It addresses R-11, R-12, R-22, R-23, R32, R-113, R-114, R-116, R-123, R-124, R-125, R-134, R-134a, R-E134, R-141b, R-142b, R143a, R-152a, R-218, R-227ea, R-236fa, R245ca, R-245fa, R-290, R-500, R-502, R-600a, $\mathrm{R}-717$, and $\mathrm{R}-744$. It summarizes additional data for R-14, R-115, R-170 (ethane), R-C318, $R-600$ (n-butane), and R-1270 (propylene) to enable calculation of limits for blends incorporating them. The report summarizes the data and related safety information, including classifications and flammability data. It also presents a series of tables with proposed ATEL and RCL concentrations - in dimensionless form and the latter also in both metric (SI) and inchpound (IP) units of measure - for both the cited refrigerants and 66 zeotropic and azeotropic blends. They include common refrigerants such as R-404A, R-407C, R-410A, R$507 A$, and others. Appendices provide profiles for the cited single-compound refrigerants and for R-500 and R-502 as well as narrative toxicity summaries for common refrigerants. The primary intended use of the data is to assist the committees responsible for ASHRAE Standard 34, Designation and Safety Classification of Refrigerants, and in turn ASHRAE Standard 15, Safety Code for Mechanical Refrigeration. The report includes an extensive set of references. [see RDB6832 for background]

J. M. Calm (Engineering Consultant), The Toxicity of Refrigerants, Proceedings of the 1996 International Refrigeration Conference at Purdue (23-26 July 1996), edited by J. E. Braun and E. A. Groll, Purdue University, West Lafayette, IN, 157-162, July 1996; republished as report DOE/CE/23810$72 E$, Air-Conditioning and Refrigeration Technol- ogy institute (ARTI), Arlington, VA, 1996 (6 pages with 1 table, available from JMC as RDB6832)

This paper presents toxicity data and exposure limits for refrigerants. The data address both acute (short-term, single exposure) and chronic (long-term, repeated exposure) effects, with emphasis on the former. The refrigerants covered include those in common use for the last decade, those used as components in alternatives, and selected candidates for future replacements. Specifically addressed are $R-11$, R-12, R-22, R-23, R-32, R-113, R-114, R-115, R-116, R-123, R-124, R-125, R-134a, R-141b, R-142b，R-143a，R-152a，R-218，R-290，RC318, R-600, R-600a, R-717, R-744, and R1270. The paper also reviews the toxicity indicators used in both safety standards and building, mechanical, and fire codes. Data are tabulated for the $L C_{50}$, cardiac sensitization NOEL and LOEL, anesthetic/CNS $E_{50}$, respiratory $R_{50}, I D L H$ (both from the Standards Completion Program and from the 1994 revisions), PEL, UL group, and Standard 34 safety classifications. The paper outlines current classification methods for refrigerant safety and relates them to standard and code usage. [see RDBA950 for update]

D. D. Campbell, J. E. Lockey, N. Petajan, B. J. Gunter, and W. H. Rom, Health Effects Among Refrigeration Repair Workers Exposed to Fluorocarbons, British Joumal of Industrial Medicine, 43(2):107-111, 1986 (5 pages, RDB5946)

toxicity of R-12, R-502, and others in humans; clinical report of exposures; safety

R. D. Cane, N. Buchanan, and M. Miller, Pulmonary Oedema Associated with Hydrocarbon Inhalation, Intensive Care Medicine, 3:31-33, 1977 (3 pages, RDB8121)

toxicity of hydrocarbons in humans

C. P. Carpenter, H. F. Smyth, Jr., and U. C. Pozzani (Carnegie-Mellon Institute, then the Mellon Institute, University of Pittsburgh), The Assay of Acute Vapor Toxicity and the Grading and Interpretation of Results on 96 Chemicals, Journal of Industrial Hygiene and Occupational Medicine, 31(6):343-346, 1949 (4 pages with 2 tables, RDB5643)

R-130, R-140, R-142b, R-150, R-150a, R-152a, R-161, R-717, R-1112a, R-1113, R-1120, R$1130 a, R-1132 a$, and others: $L C_{50}$ (widely referenced) 
C. J. Carr, R. M. Burgison, J. F. Vitcha, and J. C. Krantz, Jr. (University of Maryland), Anesthesia XXXIV. Chemical Constitution of Hydrocarbons and Cardiac Automaticity, Journal of Pharmacology and Experimental Therapeutics (JPET), 97:1-3, 1949 (3 pages, RDB7404)

describes toxicity tests to determine the cardiac sensitization potential of hydrocarbons $(\mathrm{HC})$ mixed with oxygen in dogs with epinephrine hydrochloride injections before and approximately 10 minutes into exposures

W. H. Chambers et al., An Investigation of the Toxicity of Proposed Fire Extinguishing Fluids, report 23, Medical Division, Chemical Corps, U.S. Army Chemical Center, October 1950 (RDBA656)

toxicity of R-744 (carbon dioxide) and others

F. Caujolle (Centre de Recherches sur les Toxicités du Centre National de la Recherche Scientifique, CNRS, France), Comparative Toxicity of Refrigerants / Toxicité Comparée des Fluides Frigorigènes, topical study 13131, Bulletin of the International Institute of Refrigeration (IIR), 44(1):20-55, 1964 (36 pages with 5 figures and 1 table, in English and French, RDB7424)

comparative toxicity, concern levels, exposure symptoms, health effects, and other data for R11, R-12, R-12B1, R-13, R-13B1, R-14, R-20, R-21, R-22, R-23, R-30, R-40, R-112, R-113, R-11, R-114B2, R-115, R-116, R-140a, R-160, R-160B1, R-C318, R-600, R-600a, R-601, R601a, R-602, R-602a, R-744, R-717, R-764, R1113, R-1114, R-1130, R-1140, R-1216, and others

F. Cavender, Aliphatic Hydrocarbons, Patty's industrial Hygiene and Toxicology (fourth edition), edited by G. D. Clayton and F. E. Clayton, John Wiley and Sons, Incorporated, New York, NY, 2B:1221-1266, 1994 (46 pages, RDB7619)

summarizes toxicity data for R-600a (isobutane) and others

S. Chang, H. A. Lincoff, D. J. Coleman, W. Fuchs, and M. E. Farber, Perfluorocarbon Gases in Vitreous Surgery, Ophthalmology, 92(5):651-656, 1985 (6 pages, RDB7633

toxicity of R-116 and R-218: summarizes treatment of 56 patients with complicated retinal detachments by vitreous surgery using perfluorocarbon (PFC) gases, noted as capable of greater expansion and longevity compared to R-7146 (sulfur hexafluoride); the retinas of 31 patients $(55.4 \%)$ were attached at 6 months after the disappearance of the gas; complica- tions included increased intraocular pressure, which was usually transient, and gas-induced lens opacities

J. L. Chapel and G. Thomas, Aerosol Inhalation for "Kicks", Modern Medicine, 67:378 ff, 1970 (RDB7218)

"sniffing" and associated deaths, abuse

F. A. Charlesworth, The Fate of Fluorocarbons, Inhaled or Ingested, Food and Chemical Toxicology (FCT), 13(5):572-574, October 1975 (3 pages with 1 table, RDB5940)

compares the blood levels from metered-dose inhaler (MDI) and food aerosol use to the cardiac-sensitization (CS), inhalation concentrations of R-11, R-12, R-113, R-114, R-115: concludes that the blood concentrations from aerosol use are far below the CS levels; notes that while the average elimination half-life of the fluorochemicals is very short (13.7 seconds for $R-11$ and 14.8 seconds for $R-12$ ) is very short, the biological half-life is longer due to rapid distribution and absorption in tissues

M. B. Chenoweth and C. L. Hake (Dow Chemical Company), The Smaller Halogenated Aliphatic Hydrocarbons, publication unknown, 363-398, circa 1962 (36 pages with no figures or tables, RDB7128)

summarizes the toxicity of selected halogenated compounds with focus on potential as anesthetic agents; addresses R-10, R-20, R20B3, R-30, R-31, R-40, R-40B1, R-123B1, R130, R-140a, R-150, R-150a, R-150B2, R-160, R-E252cb1, R-1110, R-1120, R-1140, and others; summarizes literature review covering 1955-1961

M. B. Chenoweth (Edgewood Arsenal Medical Research Laboratory, U.S. Army), Ventricular Fibrillation Induced by Hydrocarbons and Epinephrine, Journal of Industrial Hygiene and Toxicology, 28(4):151-158, July 1946 (8 pages with 9 figures and 1 table, RDB65A2)

presents qualitative tests of cardiac sensitization to epinephrine in anesthetized dogs by exposures to R-50 (methane), R-600 (n-butane), R-602 (hexane), R-603 (heptane), benzene, $x y-$ lene, toluene, petroleum ether, and gasoline; found that heptane and benzene were the most potent and that gasoline and the cyclic compounds were nearly as effective, but all of these "solvents" produced cardiac sensitization; also presents rough quantitative tests for $\mathrm{R}-600$ and gasoline, with concentrations estimated by a 
"hot-wire analyzer" [see note below]; notes that R-C270 (cyclopropane), petroleum ether, benzene, R-10 (carbon tetrachloride), R-20 (chloroform), R-30 (methylene chloride), R-40 (methyl chloride), R-160 (ethyl chloride), bromine analogues of the cited chlorocarbons, DDT also are capable of cardiac sensitization based on other studies; toxicity [hot-wire analyzers were used to estimate concentrations of combustible gases, but were not precise instruments.]

W. L. Chiou and J. H. Hsiao, Fluorocarbon Aerosol Propellants V: Binding Interaction with Human Albumin, Journal of Pharmaceutical Sciences, 64:1052-1054, 1975 (3 pages, RDB7256)

toxicity of R-114 and others; teratology

W. L. Chiou and J. H. Hsiao, Thermodynamic Aspect of the Interaction of Bovine Albumin, Pharmacology, 13:128-136, 1975 (9 pages, RDB7257)

toxicity of R-114 and others; teratology

W. L. Chiou, Research Communications in Chemical Pathology and Pharmacology, 7(4):679-686, 1974 (8 pages, RDB7542)

toxicity of R-114 and others; biochemistry; decomposition

W. L. Chiou and S. Niazi, A Simple and UItraSensitive Head-Space Gas Chromatographic Method for the Assay of Fluorocarbon Propellants in Blood, Research Communications in Chemical Pathololgy and Pharmacology, 6(2):481498, 1973 (RDB8131)

describes a laboratory method to quantify fluorochemical propellant (and refrigerant) concentrations in blood

D. G. Clark and D. J. Tinston (Imperial Chemical Industries PLC, ICl, UK), Acute Inhalation Toxicity of Some Halogenated and Nonhalogenated Hydrocarbons, Human Toxicology, 1(3):239-247, 1982 (9 pages with 4 tables, RDB6110)

presents acute inhalation toxicity findings for $R$ 10, R-11, R-12, R-12B1, R-13, R-13B1, R-20, R-22, R-30, R-112, R-113, R-114B2, R-140a, $\mathrm{R}-290$ (propane), R-600a (isobutane), R-1120, and R-1140; addresses lethality, LC $_{50}$ data, central nervous system (CNS) effects, anesthetic $E_{50}$ data, cardiac sensitization (CS), and CS EC 50 data

D. G. Clark and D. J. Tinston (Imperial Chemical Industries Limited, ICI, UK), Correlation of the Cardiac Sensitizing Potential of Halogenated
Hydrocarbons with Their Physiochemical Properties, British Journal of Pharmacology, 49(2):355-357, 1973 (3 pages with 1 table, RDB6109)

the potencies for cardiac sensitization to adrenaline in dogs for $R-10, R-11, R-12, R-13$, R-13B1, R-21, R-30, R-112, R-113, R-114, R123B1, R-140a, $R-290$, and $R-1140$ were found to be directly related to their saturated vapor pressures: the ratio of the partial pressure at the $\mathrm{EC}_{50}$ for cardiac sensitization to the saturated vapor pressure at $37^{\circ} \mathrm{C}\left(98.6^{\circ} \mathrm{F}\right)$ was approximately constant (0.01-0.03), despite sensitization concentrations that differed 700 fold and values of partial pressures that differed 300-fold; findings suggest that cardiac sensitization is probably a structurally nonspecific action that is an example of physical toxicity; paper notes that none of the halogenated hydrocarbons studied produced cardiac arrhythmias on inhalation without adrenaline, but all sensitized the heart at sufficiently high concentrations; paper also notes that a post exposure injection of adrenaline never resulted in arrhythmias, even when it followed a challenge injection that had caused arrhythmias, as the chemicals are rapidly eliminated from the body; this observations indicates that sensitization is a transient phenomenon dependent on temporary interactions and not on structural damage to the heart; a table presents the sensitization $E_{50}$ and physical property data

D. G. Clark and D. J. Tinston (Imperial Chemical Industries PLC, ICl, UK), Cardiac Effects of Isoproternol, Hypoxia, Hypercapnia and Fluorocarbon Propellants and their Use in Asthma Inhalers, Annals of Allergy, 30(9):536-541, 1972, also RDB5367] (6 pages, RDB6724)

cardiac sensitization to epinephrine of R-11, R12, and others; indicates a LOEL for R-11 at $12,500 \mathrm{ppm} v / \mathrm{v}$ and a LOEL for R-12 at 70,000 $80,000 \mathrm{ppm} \mathrm{v/v} \mathrm{-} \mathrm{as} \mathrm{reported} \mathrm{in} \mathrm{RDB5143} \mathrm{and}$ RDB5367

D. G. Clark and D. J. Tinston (Imperial Chemical Industries Limited, $\mathrm{ICl}$, UK), The Influence of Fluorocarbon Propellants on the Arrhythmogenic Activities of Adrenaline and Isoprenaline, Proceedings of the XIIIth Meeting of the European Society for the Study of Drug Toxicology, 13:212217, 1972 (6 pages, RDB5A68)

$R-11, R-12, R-114$, and others, cardiac sensitization, toxicity as reported in RDB5141 and RDB7525 
D. G. Clark and D. J. Tinston (Imperial Chemical Industries Limited, ICl, UK), British Medical Journal, UK, III:113-114, 1971 (2 pages, RDB7543)

toxicity of R-114 and others

J. W. Clayton, Jr. (E. I. duPont de Nemours and Company, Incorporated), Highlights of Fluorocarbon Toxicology, Laboratory Diagnosis of Diseases Caused by Toxic Agents, edited by F. W. Sunderman, Warren H. Green, Incorporated, Saint Louis, MO, chapter 21A, 199-214, 1970 (6 pages, RDB8192)

toxicity of fluorochemical refrigerants

J. W. Clayton, Jr. (E. I. duPont de Nemours and Company, Incorporated), Fluorocarbon Toxicity: Past, Present, and Future, Journal of the Society of Cosmetic Chemists, 18:333-350, 27 May 1967 (18 pages with 8 tables, RDB5C41)

R-10, R-11, R-12, R-12B1, R-12B2, R-13, R13B1, R-14, R-20, R-21, R-22, R-23, R-30, R40, R-112, R-112a, R-113, R-114, R-115, R116, R-122, R-123, R-124a, R-125, R-133a, R133aB1, R-142, R-142B1, R-226cb, R226cbB1, R-234cb, R-235ca, R-235cc, RC318, toxicity, historical evolution of toxicity classification

J. W. Clayton, Jr. (E. I. duPont de Nemours and Company, Incorporated), Fluorocarbon Toxicity and Biological Action, Fluorine Chemistry Reviews, 1:197-252, 1967; republished in Toxicity of Anaesthetics, edited by Fink, Williams and Williams Publishers, 1968 (56 pages with 5 figures and 36 tables, RDB5C42)

$\mathrm{R}-10, \mathrm{R}-11, \mathrm{R}-12, \mathrm{R}-12 \mathrm{~B} 1, \mathrm{R}-12 \mathrm{~B} 2, \mathrm{R}-13, \mathrm{R}-$ 13B1, R-14, R-20, R-21, R-22, R-23, R-30, R40, R-112, R-112a, R-113, R-114, R-115, R116, R-122, R-123, R-124a, R-125, R-133a, R133aB1, R-142, R-142B1, R-226cb, R226cbB1, R-234cb, R-235ca, R-235cc, RC318, toxicity (acute inhalation, biological action, chronic, oral, skin, and eye), historical evolution of toxicity classification; comprehensive summary and outline of attempts to link toxicity to chemical structure; indicates that substitution of fluorine for hydrogen or other halogens produces a chemically more stable compound and reduces toxicity

J. W. Clayton, Jr. (E. I. duPont de Nemours and Company, Incorporated) The Mammalian Toxicology of Organic Compounds Containing Fluorine, chapter 9, Handbuch der experimentellen Pharmakologie [Handbook of Experimental Pharmacologyl, edited by $\mathrm{O}$. Eichler, A. Farah, $\mathrm{H}$.
Herken, and A. D. Welch, Springer-Verlag, Berlin, Germany, XX/1:459-500, 1966 (42 pages, RDB6590)

toxicity review of fluorine chemicals as reported in RDB5C42

J. W. Clayton, Jr. (E. I. duPont de Nemours and Company, Incorporated), The Toxicity of Fluorocarbons with Special Reference to Chemical Constitution, Journal of Occupational Medicine, 4(5):262-273, May 1962; republished as bulletin S22, E. l. duPont de Nemours and Company, Incorporated, Wilmington, DE, April 1965 (12 pages with 10 figures and 9 tables, RDB5169)

R-10, R-11, R-12, R-13, R-14, R-20, R-21, R22, R-23, R-30, R-40, R-112, R-113, R-114, R115, R-116, R-124a, R-133a, R-133aB1, R142, R-142B1, R-217ba, R-226cb, R-226cbB1, R-234cb, R-235ca, R-235cc, R-C318, R-752 $\left(\mathrm{SF}_{4}\right), \mathrm{R}-7146\left(\mathrm{SF}_{6}\right), \mathrm{R}-7122\left(\mathrm{~S}_{2} \mathrm{~F}_{10}\right), \mathrm{R}-1110$, R-1112, R-1113, R-1114, R-1120, R-1130, R1132a, R-1216, R-1318, fluoroelastomer (Viton $A$ and $B$ ), monofluoroacetic acid, polyfluoroalcohols, polytetrafluoroethylene (PTFE) resin, and others; decomposition, toxicity

M. A. Collins (ICl Chemicals and Polymers Limited, UK) and P. A. Bingham (Rhône-Poulenc Chemicals, now Rhodia Organique Fine Limited, UK), Overview of the Chronic Toxicity of Haloethanes, EUROTOX'97, Diversification in Toxico/ogy: Man and Environment (proceedings of the XXXVI European Congress of Toxicology, EuroTox, Árhus, Denmark, 25-28 June 1997), Nordic Pharmacological Society, Copenhagen Denmark, 1997; abstract P5-15, republished in Pharmacology and Toxicology, 80(supplement III):127-128, 1997 (2 pages with no figures or tables, RDBA644)

outlines a two-year study of the chronic toxicity of $R-123, R-124, R-134 a$, and $R-141 b$ in rats; notes that $R-124$ showed no effects even at the limit concentration of $50,000 \mathrm{ppm} v / \mathrm{v} ; \mathrm{R}-123$, $\mathrm{R}-134 \mathrm{a}$, and R-141b exposures resulted in increased incidences of Leydig cell hyperplasia and benign adenoma at $300,50,000$, and 5,000 $\mathrm{ppm} \mathrm{v} / \mathrm{v} ; \mathrm{R}-123$ also caused increased incidence of hepatic adenoma at $300 \mathrm{ppm}$ and pancreatic acinar cell adenoma at 1,000 ppm $\mathrm{v} / \mathrm{v}$; survival was not affected with any of the compounds and survival actually increased with increasing R-123 exposure concentrations; hypotheses suggested that these tumors types are of limited or no relevance to human risk assessment, spurring further mechanistic studies; while metabolic changes were found in cultured 
cells, the in vitro tests found no consistent pattern indicating the mechanism of Leydig cell

M. A. Collins ( $\mathrm{ICl}$ Chemicals and Polymers Limited, UK), D. J. Tinston (ICI), and C. J. Hardy (Huntingdon Research Centre Limited, HRC, UK), Studies on the Acute Toxicity of a Number of Hydrofluorocarbons, Proceedings of the Sixth International Congress of Toxicology (Rome, Italy, 28 June - 3 July 1992), International Union of Toxicology, June 1992 (RDB5B43)

toxicity of R-125 and other HFC refrigerants; cardiac sensitization

M. Combaz (Compagnie Française de Raffinage, France), Les intoxications par gaz en raffinerie de pétrole (1969 à 1974) [Gas Intoxications in a Petroleum Refinery (1969 to 1974)], Staub-Reinhalt. Luft., 36(1):15-19, January 1976 (5 pages with 1 figure and 1 table, in French, RDB6A62)

toxicity summary of 40 incidents of narcotic effects in humans by gases in a refinery during a five year period: of the accidents, exposures to R-1150 and R-1270 accounted for two of them; no acute lung edema or fatalities resulted in any of the incidents, but paper recommends use of respiratory masks for dangerous tasks

Committee on Aviation Toxicology, Aero Medical Association, Aviation Toxicology, The Blakiston Company, New York NY and Toronto ON, 1953 (RDB72A5)

reference on the toxicity of chemicals used on aircraft; for R-744 (carbon dioxide), indicates that concentrations of $50,000-100,000 \mathrm{ppm} v / \mathrm{v}$ and higher act as a narcotic in humans and that signs of intoxication may occur after $30 \mathrm{~min}$ at $50,000 \mathrm{ppm} v / \mathrm{v}$ [see RDBA657 for further information]

Commission for the Investigation of Health Hazards of Chemical Compounds in the Work Area, List of MAK and BAT Values 1996: Maximum Concentrations and Biological Tolerance Values at the Workplace, report 32, Deutsche Forschungsgemeinschaft [German Research Association] (DFG), Bonn, Germany; VCH Verlagsgesellschaft mbH, Weinheim, Germany, 1996 (208 pages with 3 figures and 12 tables, RDB7101)

English language version of the MAK- und BAT-Werte-Liste; maximale Arbeitsplatz Konzentration [maximum workplace concentration] and biologischer Arbeitsstoff-Toleranz-Wert [biological tolerance value]; German occupational exposure limits for approximately 600 chemicals including R-10, R-11, R-12, R-12B2,
$\mathrm{R}-13, \mathrm{R}-13 \mathrm{~B} 1, \mathrm{R}-20, \mathrm{R}-20 \mathrm{B3}, \mathrm{R}-21, \mathrm{R}-22$, R30, R-30B1, R-31, R-40, R-40B1, R-4011, R110, R-112, R-112a, R-113, R-114, R-123, R123B1, R-130, R-134a (new entry), R-140, R140a, R-140B2, R-142b, R-150, R-150a, R160, R-160B1, R-E170, R-270da, R-290, R600, R-600a, R-601, R-601a, R-601b, R-602, R-610, R-611 (revised), R-630, R-717 (revised), R-744, R-744A, R-746, R-764, R-7146, R-1110, R-1120, R-1130, R-1130a, R-1130c, $R-1130 t, R-1132 a, R-1140, R-1150$, and others; classification of carcinogenic, embryotoxicant, and sensitizing substances

Commission for the Investigation of Health Hazards of Chemical Compounds in the Work Area, Occupational Toxicants: Critical Data Evaluation for MAK Values and Classification of Carcinogens, edited by D. Henschler, Deutsche Forschungsgemeinschaft [German Research Association] (DFG), Bonn, Germany; VCH Verlagsgesellschaft $\mathrm{mbH}$, Weinheim, Germany; volume 1, September 1990 (400 pages with 15 figures and 95 tables); volume 2, July 1991 (349 pages with 42 figures and 65 tables); volume 3, September 1992 ( 379 pages with 12 figures and 82 tables); volume 4, July 1992 (385 pages with 11 figures and 95 tables); volume 5, July 1993 (389 pages with 8 figures and 77 tables); volume 6. August 1994 (368 pages with 10 figures and 69 tables); and volume 7. April 1996 (440 pages with 11 figures and 83 tables); (RDB7102)

analysis of available toxicity data used to set maximale Arbeitsplatz Konzentration [maximum workplace concentration] (MAK) values and carcinogenicity classifications; description of known effects, identification of inadequacies in the data, and recommendations for further research: volume 6 addresses R-13B1, R-717 (ammonia), and others; volume 7 addresses $\mathrm{R}$ 10B3, R-160, R-160B1, R-16011, R-630, and others

P. Conzen and $M$. Nuscheler, New Inhalation Anaesthetics, Anaesthesist, 45:674-693, 1996 (20 pages, RDB8313)

toxicity of hydrofluoroethers (HFE) used as anesthetics and potentially as refrigerants

W. A. Cook, Maximal Allowable Concentrations of Industrial Atmospheric Contaminants, Industrial Medicine, 14:936 ff, 1945 (RDB6168)

first exposure standards (Maximum Allowable Concentration, MAC) for fluoroalkanes, ranged from $5,000 \mathrm{ppm}$ for $R-11$ to $100,000 \mathrm{ppm}$ for $R$ 12 as reported in RDB5C41 
F. Coulston and T. B. Griffin, Physiological and Toxicological Aspects of Combustion (proceedings of international symposium), 151-164, 1976 (14 pages, RDB6576)

inhalation toxicity of R-116 and others

P. J. Cox, L. J. King, and D. V. Parke, A Comparison of the Interactions of Trichlorofluoromethane and Carbon Tetrachloride with Hepatic Cytochrome P-450, Biochemical Journal, 130:87P ff, 1972 (RDB8133)

toxicity of R-10 and R-11; biochemistry; metabolism, pharmacokinetics

J. R. Creech (Geocenters Incorporated), R. K. Black (Geocenters), B. L. Garrity (Geocenters), R. Abbas (Geocenters), L. Dong (Geocenters), R. J. Williams (U.S. Air Force Armstrong Laboratory, USAF-AL), and J. N. McDougal (USAF-AL), Inhalation Uptake and Metabolism of lodohalogenated Compounds $\mathrm{CF}_{3} \mathrm{l}, \mathrm{C}_{6} \mathrm{~F}_{13} \mathrm{l}$, and $\mathrm{C}_{3} \mathrm{~F}_{7} \mathrm{l}$, report AL/OE-TR-1995-0089, Armstrong Laboratory, U.S. Air Force, Wright-Patterson Air Force Base, $\mathrm{OH}$, 1995 (RDB8414)

inhalation toxicity, metabolism, and pharmacokinetics of R-13l1, R-217cal1, and R-51-13l1 (probably $\mathrm{R}-51-13 \mathrm{mccc} / 1$ ), which are candidates to replace halon $1301(\mathrm{R}-13 \mathrm{~B} 1)$ and also under consideration as refrigerants

J. R. Creech, R. K. Black, S. K. Neurath, M. C. Caracci (Geocenters Incorporated), R. J. Williams (U.S. Air Force Armstrong Laboratory, USAF-AL), and A. Vinegar (ManTech Environmental Technology, Incorporated), Inhalation Uptake and Metabolism of Proposed Halon 1301 Replacement: HFC-125, and FC-218 The Toxicologist, Society of Toxicology (SOT), 15(1):271 ff, 1995 (RDB7C83)

inhalation toxicity, metabolism, and pharmacokinetics of R-125 and R-218 as replacements for $R-13 B 1$ (also used as refrigerants)

J. R. Creech, R. K. Black, S. K. Neurath, M. C. Caracci (Geocenters Incorporated), R. J. Williams (U.S. Air Force Armstrong Laboratory, USAF-AL), G. W. Jepson, and A. Vinegar (ManTech Environmental Technology, Incorporated), Inhalation Uptake and Metabolism of Halon 1301 Replacement Candidates HFC-227ea, HFC-125, and FC218, report AL/OE-TR-1995-0022, Armstrong Laboratory, U.S. Air Force, Wright-Patterson Air Force Base, OH, 1995 (RDB7C84)

inhalation toxicity, metabolism, and pharmacokinetics of R-125, R-218, and R-227ea as re- placements for R-13B1 (also used as refrigerants)

R. Culik and D. P. Kelly, Embryotoxic and Teratogenic Studies in Rats with Inhaled Dichlorofluoromethane (Freon ${ }^{\circledR}$ 21) and 2,2-Dichloro1,1,1-Trifluoroethane (FC-123), report 227-76, Haskell Laboratory for Toxicology and Industrial Medicine, E. I. duPont de Nemours and Company, Incorporated, Newark, DE, 1976 (RDB6156)

R-21 and R-123, developmental and reproductive toxicity: tests of R-123 at $10,000 \mathrm{ppm}$ suggest a fetotoxic effect in the offspring of exposed female rats, but the results were not statistically significant and no other concentration was tested - as reported in RDB6B52

T. Dalhamn, Studies on $\mathrm{SO}_{2}, \mathrm{NO}_{2}$ and $\mathrm{NH}_{3}$ : Effect on Ciliary Activity in Rabbit Trachea of Single in Vitro Exposure and Resorption in Rabbit Nasal Cavity, Acta Physiologica Scandinavica, 58:287-291, 1963 (RDB6A47)

R-717 (ammonia), R-746 (nitrogen dioxide), and R-764 (sulfur dioxide), health effects, toxicity

T. Dalhamn, Freon som orsak till förgiftningsfall [Freon as a Cause of Toxicity], paper 11012, Nordisk Hygienisk Tidskrift, 39:165-169, 1958 (5 pages with 2 tables, RDB7205)

$\mathrm{R}-11, \mathrm{R}-12, \mathrm{R}-22, \mathrm{R}-113$, health effects, toxicity, and toxicity of decomposition products in open flames; cites two cases of alleged phosgene poisoning from use of $R-12$ as an aerosol propellant; one involved a leak in the presence of an acetylene flame, and the other, which was fatal, the use of an aerosol insecticide; see RDB5C40 for discussion of this paper

T. Dalhamn, Mucous Flow and Ciliary Activity in the Trachea of Healthy Rats and Rats Exposed to Respiratory Irritant Gases $\left(\mathrm{SO}_{2}, \mathrm{H}_{3} \mathrm{~N}\right.$, HCHO). VIII. The Reaction of the Tracheal Ciliary Activity to Single Exposure to Respiratory Irritant Gases and Studies of the pH, Acta Physiologica Scandinavica, 36(supplement 123):93-105, 1956 (13 pages, RDB6A46)

toxicity of R-717, R-764, and others

T. Dalhamn, Mucous Flow and Ciliary Activity in the Trachea of Healthy Rats and Rats Exposed to Respiratory Irritant Gases $\left(\mathrm{SO}_{2}, \mathrm{H}_{3} \mathrm{~N}\right.$, $\mathrm{HCHO}$ ). A Functional and Morphologic (Light Microscopic and Electron Microscopic) Study, with Special Reference to Technique, Acta 
Physiologica Scandinavica, 36(supplement 123):$161 \mathrm{ff}, 1956$ (RDB6A08)

toxicity of R-717, R-764, and others

R. H. Davies, R. D. Bagnall, W. Bell, and W. G. M. Jones (Imperial Chemical Industries Limited, UK), The Hydrogen Bond Proton Donor Properties of Volatile Halogenated Hydrocarbons and Ethers and Their Mode of Action in Anesthesia, International Journal of Quantum Chemistry: Quantum Biology Symposium, 3:171-185, 1976 (15 pages with 7 figures and 1 table, RDB6555)

toxicity of R-142b and others; indicates a 30$\min L_{50}$ mouse $=300,000 \mathrm{ppm}$ for $\mathrm{R}-142 \mathrm{~b}-$ as reported in RDB3721

R. H. Davies, R. D. Bagnall, and W. G. M. Jones (Imperial Chemical Industries Limited, UK), Quantitative Interpretation of Phase Effects in Anesthesia, International Journal of Quantum Chemistry: Quantum Biology Symposium, 1:201-212, 1974 (12 pages, RDB59A6)

toxicity of R-124, R-142b, and others; indicates and anesthetic effect, 30 -min $\mathrm{EC}_{50}$ mouse = $230,000 \mathrm{ppm}$ for R-142b - as reported in RDB5869

W. Dekant (Universität Würzburg, Germany), Toxicology of Chlorofluorocarbon Replacements, Environmental Health Perspectives (EHP), 104(supplement 1):75-83, March 1996 (9 pages with 5 figures and 2 tables, Rdb8123)

$R-123, R-124, R-125, R-134 a$, and R-141b show only a low potential for skin and eye irritation; R-123 affects the liver and R-134a and $\mathrm{R}-141 \mathrm{~b}$ the testes, including tumor formation, in long-term inhalation studies for very high concentrations; all of these alternatives are, to varying extents, biotransformed, mainly by cytochrome P450-catalyzed oxidation of carbonhydrogen bonds; the resulting acyl halides are hydrolyzed to yield excretable carboxylic acids; halogenated aldehydes that are formed may be further oxidized to halogenated carboxylic acids or alcohols, found as metabolites in the urine of exposed test rats; the chronic toxicity of these alternatives is unlikely to be of relevance for humans exposed during production and use of these chemicals; further research is required for a better understanding of the chronic toxicity of $R-123$ and the mechanisms involved

W. B. Deichmann and H. W. Gerarde, Toxicology of Drugs and Chemicals, Academic Press, New York, NY, 1969 (Rdb5266) general reference on toxicity; cites a 4-hr $\mathrm{LC}_{50}$ rat of 2,000 ppm for R-717 (ammonia)

C. G. de Rooij (misspelled "Ronij" in the document, Solvay S.A., Belgium), S. Shin-ya (Asahi Glass Company, Limited, Japan), R. S. Thompson (Zeneca, UK), and A. G. Berends (Solvay Duphar, The Netherlands), Environmental Toxicity Studies with HFCs and HCFCs, EUROTOX'97, Diversification in Toxicology: Man and Environment (proceedings of the XXXVI European Congress of Toxicology, EuroTox, Århus, Denmark, 25-28 June 1997), Nordic Pharmacological Society, Copenhagen Denmark, 1997; abstract P5-15, republished in Pharmacology and Toxicology, 80(supplement III):138, 1997 (1 page with no figures or tables, RDBA645)

biodegradation, ecotoxicity, and impacts on aquatic life of R-32, R-123, R-124, R-125, R134a, R-141b, R-225ca, and R-225cb

J. V. Dilley et al., Fluoride Ion Excretion by Male Rats After Inhalation of One of Several Fluoroethylenes or Hexafluoropropene Toxicology and Applied Pharmacology (TAP), 27(3):582-590, 1974 (RDB6579)

toxicity of R-116, R-1216, and others; male rats exposed to R-1216 in air showed increased urinary fluoride after exposures and necrosis of renal tubules; metabolism

T. DiPaolo et al., Molecular Connectivity Study of Halocarbon Anesthetics, Journal of Pharmaceutical Sciences, 68(1):39-42, 1979 (4 pages, RDB6185)

anesthetic effects of R-124 and others; toxicity of fluorocarbon refrigerants

K. T. Dishart, The Synthesis and Evaluation of Some New Fluorinated Inhalation Anesthetics, National Meeting (Chicago), American Chemical Society (ACS), Columbus, OH, September 1961 (RDB5907)

anesthetic effects of R-226cb, R-226cbB1, R226daB1, R-244ca, R-244caB1, R-245ca, and others; toxicity

E. C. Dittman and E. Etschenberg, Endoanesthetic and Narcotic Activity in Halogenated Methane Derivatives, European Journal of Pharmacology, 24(3):389-398, 1973 (10 pages, RDB5821)

anesthetic effects of R-23 and others; toxicity

D. E. Dodd, A. D. Ledbetter, and A. D. Mitchell (ManTech Environmental Technology, Incorpo- 
rated), Genotoxicity Testing of Halon Replacement Candidates Trifluoroiodomethane $\left(\mathrm{CF}_{3} \mathrm{I}\right)$ and 1,1,1,2,3,3,3-Heptafluoropropane (HFC227ea) Using the Salmonella Typhimurium and L5178Y Mouse Lymphoma Mutation Assays and Mouse Micronucleus Test, Inhalation Toxicology, 9:111-131, 1997 (21 pages, RDB8415)

toxicity tests of R-13!1 and R-227ea

D. E. Dodd, W. T. Brashear, and A. Vinegar (ManTech Environmental Technology, Incorporated), Metabolism and Pharmacokinetics of Selected Halon Replacement Candidates, Toxicology Letters, 68(1-2):37-47, May 1993 (11 pages, RDB6212)

toxicity tests of the metabolism of a series of chemicals and of the pharmacokinetics of R123: the metabolism tests addressed R-12B1, R-123, R-124, R-142b, R-51-14 in Fischer 344 and Srague-Dawley rats exposed for $2 \mathrm{hr}$; animals exposed to R-123 and R-124 excreted trifluoroacetic acid (TFA) in their urine; urinary bromide and fluoride levels increased for animals exposed to $R-12 B 1$ and $R-124$, respectively; small amounts of R-133a and $R-1122 a$ were detected in the livers of rats exposed to $R-123$; animals exposed to $R-142 b$ excreted chlorodifluoroacetic acid in their urine, but no volatile metabolytes were found in tissues; no metabolites were found in the urine or tissues of animals exposed to R-51-14; no remarkable differences were found between the two strains of rats; paper concludes that the results are consistent with proposed oxidative and reductive pathways of metabolism for these chemicals; pharmacokinetic tests of R-123 were based on inhalation exposures with rats at 100 , 1,000 , and $10,000 \mathrm{ppm}$; blood concentrations of R-123 fell sharply following exposures; TFA levels rose for approximately $5 \mathrm{hr}$ and then declined gradually; saturation of R-123 metabolism was estimated with a physiologically-based pharmacokinetic (PBPK) model to occur at approximately $2,000 \mathrm{ppm}$

R. E. Doherty and D. M. Aviado (University of Pennsylvania School of Medicine), Toxicity of Aerosol Propellants in the Respiratory and Circulatory Systems, VI. Influence of Cardiac and Pulmonary Vascular Lesions in the Rat, Toxicology, Elsevier/North Holland, Amsterdam, The Netherlands, 3(2):213-224, 1975 (12 pages, RDB5C81)

toxicity of R-12, R-152a, and others; cardiac sensitization
C. T. Dollery, Absorption and Fate of Isoproterenol and Fluorocarbons Inhaled from Pressurized Aerosols, Proceedings of the 28th Annual Congress (Dallas, TX, 8 March 1972), 1972 (RDB7243)

fluorochemical toxicity

C. T. Dollery, J. Gragg, and M. C. R. Path, Sniffing Syndrome, British Medical Journal, 2:334 ff, 1971 (RDB7297)

fluorocarbon abuse; potential for human fatalities

C. T. Dollery, G. H. Draffen, D. S. Davies, F. M. Williams, and M. E. Conolly, Blood Concentrations in Man of Fluorinated Hydrocarbons After Inhalation of Pressurized Aerosols, paper 7684, Lancet, UK, 2(7684):1164-1166, 1970 (6 pages, RDB6725)

effects of inhalation in humans of R-12, R-114, and others; toxicity: no adverse effects from R12 at aerosol bronchodilator doses in healthy adults or asthmatic patients - as reported in RDB5143

C. T. Dollery, F. M. Williams, G. H. Draffen, G. Wise, H. Sahyoun, J. W. Paterson, and S. R. Walker, Arterial Blood Levels of Fluorocarbons in Asthmatic Patients Following Use of Pressurized Aerosols, Clinical Pharmacology and Therapeutics, 15:59-66, 1973 (8 pages, RDB8119)

fluorochemical blood levels in humans; pharmacokinetics, toxicity; based on a study for use as metered-dose inhaler (MDI) aerosol propellants (compounds also are used as refrigerants)

R. C. Downing (Consultant, formerly DuPont Chemicals), Refrigerant Toxicology, Refrigerating Service and Contracting (RSC), 31-32, January 1987 ( 2 pages with 2 tables, RDB6850)

review of refrigerant inhalation risks for technicians; indicates that fluorocarbon refrigerants are low in toxicity, do not accumulate in the body, and are not metabolized or broken down to any extent; still recommends to avoid breathing them if possible; notes that the decomposition products of fluorocarbons are toxic, but there is no evidence of phosgene $\left(\mathrm{COCl}_{2}\right)$ formation in decomposition; identifies hydrochloric $(\mathrm{HCl})$ and hydrofiuoric acids (HF) as the primary decomposition products, sometimes with small amounts of chlorine gas $\left(\mathrm{Cl}_{2}\right)$; these acids have a very acrid, acidic odor at very low concentrations that serves as a warning of decomposition; reviews the historical as- 
sociation of phosgene with fluorocarbon decomposition, noting that other carbonyl halides, notably carbonyl fluoride $\left(\mathrm{COF}_{2}\right)$ and carbonyl chlorofluoride (COCIF), were misidentified as phosgene in early studies; explains that actual phosgene formation is doubtful based on relative bond strengths of carbon fluorine and carbon-chlorine bonds; explains and presents the ACGIH Threshold Limit Value (TLV) concentrations for $\mathrm{HCl}, \mathrm{HF}, \mathrm{COClF}$, and phosgene; reviews other studies on the decomposition of refrigerants by lit cigarettes that found miniscule decomposition of R-11 and R-22; concludes that these chemicals do not pose a greater health hazard to smokers than nonsmokers

R. C. Downing and D. Madinabeitia (E. I. duPont de Nemours and Company, Incorporated), The Toxicity of Fluorinated Hydrocarbon Aerosol Propellants, Aerosol Bulletin, Federation of European Aerosol Associations, February-April 1960; republished in Aerosol Age, 5(9):25 ff, September 1960; republished as bulletin A-52 (A-82784), E. I. duPont de Nemours and Company, Incorporated, Wilmington, DE, August 1972 (8 pages with 1 figure and 5 tables, RDB5C40)

$\mathrm{R}-11, \mathrm{R}-12, \mathrm{R}-22$, R-113, decomposition products (phosgene $/ \mathrm{COCl}_{2}$, hydrochloric acid / $\mathrm{HCl}$, hydrofluoric acid / $\mathrm{HF}$, chlorine $\mathrm{Cl}_{2}$, others), refrigerants, toxicity

H. N. Doyle and R. E. Bales (Tracor Jitco, Incorporated), Fluorocarbons - Industrial Hygiene Survey Report: E. I. duPont de Nemours and Co., Inc., Deepwater, New Jersey, National Institute of Occupational Safety and Health (NIOSH), U.S. Department of Health, Education, and Welfare (DHEW), Cincinnati, January 1977 (20 pages with 1 figure and 6 tables, available from NTIS, RDB7364)

concentrations of R-10, R-11, R-12, R-22, R30, R-113, R-114, R-115, R-116, R-142b, R152a (possibly $R-152$, incorrectly identified in the report as ${ }^{~} \mathrm{CH}_{3} \mathrm{~F}-\mathrm{CH}_{3} \mathrm{~F}$ "), R-1132a, and others at a chemical manufacturing site: plant (Chambers Works) manufactures diverse chemicals, among them R-11, R-12, R-115, and $R-116$; it also package these chemicals and R-22, R-113, R-114, R-142b, R-152a, and $R-1132 a$; report concludes that the predominant opportunities for exposures are associated with packaging operations; samples collected by personal samplers worn by plant operators show the concentrations of R-22 to be $0.1-0.3$ $\mathrm{ppm}$ and for all other fluorocarbons less than $20 \mathrm{ppm}$; appends a synopses and data tables prepared by $W$. E. Neeld, which concludes that there was no indication of increased morbidity or mortality among 22 fluorocarbon workers compared to 44 controls based on a 19-year retrospective study

L. M. Dzubow, Histologic and Temperature Alterations Induced by Skin Refrigerants, Journal of the American Academy of Dermatology, 12(5/1):796-809, 1985 (14 pages, RDB7259)

toxicity of R-114 and others

C. Edling, C. G. Olson, G. Ljungkvist, A. Oliv, and B. Soderholm, Cardiac Arrhythmia in Refrigerator Repairmen Exposed to Fluorocarbons, British Journal of Industrial Medicine, UK, 47(3):207212, 1990 (6 pages, RDB7226)

cardiac sensitization R-12 and others

C. Edling and C. G. Olson, Health Risks with Exposure to Fluorocarbons, University Hospital, Uppsala, Sweden, 1988 (in Swedish, RDB8141)

toxicity of fluorochemical refrigerants

C. Edling and P. Sderkvist, Criteria Group for Occupational Standards: Fluorocarboner, Arbete och HIsa, 26:1-38, 1982 (38 pages, RDB6B55)

toxicological review of R-11, R-12, R-22, and $\mathrm{R}-113$; metabolism, acute effects on the central nervous system and the heart; cardiac sensitization in the dog, monkey, mouse and rat; reports heart palpitations in humans after longterm exposure to R-22; liver effects (lipid droplets) found after chronic exposures to R-12, R22 , and $\mathrm{R}-113$

A. Edmonds, Toxicity of Vaporizing Liquids, $A n$ nals of Occupational Hygiene, 9:235 ff, 1966 (RDB8115)

toxicity of fluorochemicals

M. J. Ellenhorn and D. G. Barceloux, Aerosol Propellants, Medical Toxicology, Diagnosis and Treatment of Human Poisoning, Elsevier Publishing Company, Incorporated, New York, NY, 528 ff, 1988 (RDB5947)

toxicity

M. J. Ellenhorn and D. G. Barceloux, Fluorocarbons, Medical Toxicology, Diagnosis and Treatment of Human Poisoning, Elsevier Publishing Company, Incorporated, New York, NY, 884 ff, 1988 (RDB5948)

toxicity 
M. J. Ellenhorn and D. G. Barceloux, Inhalant Abuse, Medical Toxicology, Diagnosis and Treatment of Human Poisoning. Elsevier Publishing Company, Incorporated, New York, NY, $841 \mathrm{ff}$, 1988 (RDB5949)

\section{toxicity}

H. B. Elkins and L. Levine (Division of Occupational Hygiene, Massachusetts Department of Labor and Industries), Decomposition of Halogenated Hydrocarbon Vapors by Smoking, Journal of Industrial Hygiene and Toxicology, 21(6):221225, June 1939 (5 pages with 2 tables, RDB6773)

reports experiments to determine the extent of decomposition of halocarbons, including some used as refrigerants, passing through lighted cigarettes and cigars; describes the experimental and analytical methods used; tabulates tested concentrations and measured bromides and chlorides for R-10, R-12, R-160B1, R$1120, \mathrm{R}-1130 \mathrm{~B} 2$, and dichlorobenzene; addresses tolerable phosgene levels and influences of dusts and humidity; notes warning properties of hydrochloric acid, a primary decomposition product representing $80 \%$ of decomposition chlorides, at concentrations of 10 $\mathrm{ppm}$; concludes that the extent of decomposition of the cited compounds in smoking cigarettes or cigars in the presence of their vapors "is of a low order and does not constitute a health hazard"; toxicity

S. S. Epstein, E. Arnold, J. Andrea, W. Bass, and Y. Bishop, Toxicology and Applied Pharmacology (TAP), 23:288 ff, 1972 (RDBA671)

toxicity of hydrocarbons

S. S. Epstein, S. Joshi, J. Andrea, P. Clapp, H. Falk, and N. Mantel, Synergistic Toxicity and Carcinogenicity of 'Freons' and Piperonyl Butoxide, Nature, 214:526-528, 1967 (3 pages, RDB7425)

toxicity of R-11 and others; no significant increase in tumor formation was found with subcutaneous injection of R-11 - as reported in RDB7414

L. Eriksson, J. Johnsson, and R. Berglind, External Validation of a QSAR for the Acute Toxicity of Halogenated Aliphatic Hydrocarbons, Environmental Toxicology and Chemistry, 12:11851192, 1993 (8 pages, RDB7262)

teratology of R-114 and others; toxicity

L. Eriksson, J. Johnsson, M. Sjostrom, and S. Wold, A Strategy for Ranking Environmentally
Occurring Chemicals. Part V: The Development of Two Genotoxicity QSARs for halogenated Aliphatics, Environmental Toxicology and Chemistry, 10:585-596, 1991 (12 pages, RDB7263)

teratology of R-114 and others; toxicity

L. Eriksson, J. Johnsson, S. Hellberg, F. Lindoren, et al., A Strategy for Ranking Environmentally Occurring Chemicals. Part III: Multivariate Quantitative Structure-Activity Relationships for Halogenated Aliphatics, Environmental Toxicology and Chemistry, 9:, 1990 (RDB7264)

teratology of R-114 and others; toxicity

H. Fabel, R. Wettengel, and W. Hartmann, Myocardial Ischemia and Arrhythmias Due to the Use of Pressurized Aerosols in Man, Deutsch Med. Wochenschrift [German Medical Weekly], Germany, 97:428-431, 1972 (4 pages, RDB6726)

acute inhalation toxicity of R-12/11 (60/40) in humans; cardiac sensitization; no change in EKGs - as reported in RDB5143

L. T. Fairhall, Industrial Toxicology (second edition), Williams and Wilkins, Baltimore, MD, 1957 (RDBA629)

reference on toxicity; indicates that R-12 has little if any anesthetic or toxic action - as reported in RDB5358

L. T. Fairhall, Industrial Toxicology Williams and Wilkins, Baltimore, MD, 1949 (RDB5987)

reference on toxicity

Federation of the American Societies for Experimental Biology (FASEB), Evaluation of the Health Aspects of Nitrogen, Helium, Propane, n-Butane, iso-Butane, and Nitrous Oxide as Gases Used in Foods, Bureau of Food, U.S. Department of Health and Human Services (DHS, then the U.S. Department of Health, Education, Welfare, DHEW), Washington, DC, March 1979 (RDBA541)

toxicity of R-290 (propane), R-600 (n-butane), R-600a (isobutane), R-704 (helium), R-728 ínitrogen), and R-744A (nitrous oxide) for food uses, some of which also are used as refrigerants

A. T. Fidler, E. L. Baker, and R. Letz, The Neurobehavioral Effects of Occupational Exposure to Organic Solvents Among Construction Painters, British Journal of Industrial Medicine, 1:211228, 1987 (18 pages, RDB5838)

inhalation toxicity 
C. E. Finegan and G. M. Rusch (AlliedSignal Incorporated), Update: Program for Alternative Fluorocarbon Toxicity Testing, Stratospheric Ozone Protection for the 90's (proceedings of the International CFC and Halon Alternatives Conference, Washington, DC), Alliance for Responsible CFC Policy, Arlington, VA, 895-904, October 1993 (10 pages with 12 tables, available from JMC as RDB3A53)

summary of the status and findings of the Program for Alternative Fluorocarbon Toxicity Testing (PAFT); addresses the toxicity of R-32, R-123, R-124, R-125, R-141b, R-134a, R225ca, and R-225cb [see RDB7464 for update]

N. C. Flowers, R. C. Hand, and L. G. Horan (Helen Dwight Reid Educational Foundation), Concentrations of Fluoroalkanes Associated with Cardiac Conduction System Toxicity, Archives of Environmental Health, 30(7):353-360, 1975 (8 pages, RDB5C65)

toxicity of R-11, R-12, and others; cardiac effects and sensitization

F. Flury, Moderne gewerbliche Vergiftungen in pharmakologisch-toxikologischer Hinsight [Pharmacological-Toxicological Aspects of Intoxicants in Modern Industry], Archiv für Experimentelle Pathologie und Pharmakologie, 138:65-82, 1928 (18 pages, RDB7299)

general reference on toxicity; cites a 5-min $L_{L_{L}}$ mammal of $5,000 \mathrm{ppm} v / \mathrm{v}$ for $\mathrm{R}-717$ (ammonia) - as reported in RDB5340

S. Fogel, Sudden Death and Fluorocarbon-Containing Aerosols, Canadian Medical-Association Journal, Ottawa, Canada, 114:671-672, 1976 (2 pages, RDB7530)

R-12 and others, toxicity, accident involving human exposure

V. C. Foltz and R. Fuerst, Mutation Studies with Drosophila Malanogaster Exposed to Four Fluorinated Hydrocarbon Gases, Environmental Research, 7(3):275-285, 1974 (11 pages, RDB5139)

mutagenicity of R-23, R-152a, and others; toxicity - as reported in RTECS

R. B. Forney, Jr., and R. N. Harger, Reaction of Mice from Acute Exposure to Various Concentrations of Methane, Ethane, Propane, and Butane in Air, or in Oxygen, Proceedings of the Sixth International Meeting of Forensic Sciences (Edinburgh, Scotland), 1972 (12 pages, RDB7462)
$R-50, R-170, R-290, R-600$, health effects, toxicity: anesthetic properties of R-50 (methane) - as reported in $\mathrm{RDB7413}$

S. A. Friedman, M. Cammarato, and D. M. Aviado (University of Pennsylvania School of Medicine), Toxicity of Aerosol Propellants on the Respiratory and Circulatory Systems, II. Respiratory and Bronchopulmonary Effects in the Rat, Toxicology, 1(4):345-355, 1973 (11 pages with 2 figures and 4 tables, RDB6191)

toxicity of R-11, R-12, R-21, R-114, R-115, R$\mathrm{C} 318$, and $\mathrm{R}-600 \mathrm{a}$; classifies propellants (many of which also are used as refrigerants) into three classes, namely those that induce arrythmia and sensitize the heart to epinephrine (R-11 and R-21), those that sensitize the heart to epinephrine-induced arrythmia ( $R-114, R-$ 115, R-C318, and R-600a), and those that do not induce arrythmia or sensitize the heart to epinephrine (R-12) in anesthetized rats; recommends against continued use of R-11 and R-21 as propellants; exposures to R-12 at 360,000 $\mathrm{ppm} \mathrm{v} / \mathrm{v}$ produced apnea and finally death, but did not cause arrhythmias [RDB6138 notes that this death may have been due to the anesthetic (diallylbarbituric acid and urethane)]

R. Fuerst and S. Stephens, Effects of Gases and Gamma Irradiation on Neurospora Crassa, Develop. Ind. Microbiology, 11:301-310, 1970 (19 pages, RDB5C83)

toxicity of R-152a and others; mutagenicity; mutagenic potential

R: Fuerst and M. M. Landry, Develop. Ind. Microbiology, 8:305-312, 1966 (8 pages, RDB6130)

toxicity; mutagenicity; mutagenic potential

M. Furuya, Experimental Chlorofluorohydrocarbon Poisoning, Tokyo Jikeikai Ika Daigaku Zasshi, Japan, 94(6):1201-1214, 1979 (14 pages in Japanese, RDB8142)

health effects, toxicity

S. C. Gad et al., Correlation of Ocular and Dermal Irritancy of Industrial Chemicals, Journal of Toxicology Cutaneous Ocular Toxicol., 5(3):195214, 1986 (20 pages, RDB6133)

toxicity of R-152a and others

S. Garb and M. B. Chenoweth, Studies on Hydrocarbon-Epinephrine Induced Ventricular Fibrillation, Journal of Pharmacology and Experimental Therapeutics (JPET), 94:12-18, 1948 (7 pages, RDB8143) 
summarizes studies of cardiac sensitization by inhalation exposures; inducement of ventricular fibrillation (very rapid and irregular contractions of the ventricles of the heart resulting in loss of synchronization between the heartbeat and pulse beat); health effects; toxicity

M. L. Gargas, P. G. Seybold, and M. E. Andersen, Modeling the Tissue Solubilities and Metabolic Rate Constant $\left(\mathrm{V}_{\text {max }}\right)$ of Halogenated Methanes, Ethanes, and Ethylenes, 17th Conference on Toxicology (Dayton, $\mathrm{OH}, 3-5$ November 1987); republished IN Toxicology Letters, Elsevier/North-Holland Biomedical Press, Amsterdam, The Netherlands, 43(1-3):235-256, 1988 (22 pages, RDB5776)

R-30, R-32, R-1120, R-1130, R-1140, and others: toxicity

M. L. Gargas, H. J. Clewell III, and M. E. Andersen, Metabolism of Inhaled Dihalomethanes in Vivo: Differentiation of Kinetic Constants for Two Independent Pathways, Toxicology and Applied Pharmacology (TAP), 82(2):211-223, 1986 (13 pages, RDB5780)

R-32 and others, toxicity

S. Garrett and R. Fuerst, Sex-Linked Mutations in Drosophila after Exposure to Various Mixtures of Gas Atmospheres, Environmental Research, 7(3):286-293, 1974 (8 pages, RDB5819)

R-23, R-152a, and others; mutagenicity, inhalation toxicity

J. Garriott and C. S. Perry (Medical Examiner, Dallas TX), Death from Inhalant Abuse: Toxicological and Pathological Evaluation of $\mathbf{3 4}$ Cases, Clinical Toxicology, 16:305-315, 1980 (11 pages, RDB7431)

$\mathrm{R}-11, \mathrm{R}-12, \mathrm{R}-114$, mixtures of them, and others, health effects, toxicity: analysis of 34 deaths after cardiac sensitization from deliberate abuse of inhalants, 16 of which were attributed to fluorocarbon aerosols (many also used as refrigerants), by "sniffing" as reported in RDB7414

E. M. Gause and J. R. Rowlands, Effects of Fluorocarbon Inhalants upon Alveolar Macrophages, Proceedings of the Western Pharmacology Society, 19:373-380, 1976 (8 pages, RDB5822)

$r-12, R-23$, and others, health effects, toxicity

T. L. Giguz, Vliianie malykh kontsentratsii ammiaka i okislov azota na podrostkovipri proiz- vodstvennom obuchenii ikh na predpriiatiiakh khimicheskoi promyshlennosti [Effect of Low Concentrations of Ammonia and Nitrogen Oxides on Adolescents during Vocational Training at Plants in the Chemical Industry], Gigiena $i$ Sanitariya, Moscow, Russia (then USSR, 33(9):100102, September 1968; also cited by some authors as appearing in Hyg. Sanitariya, 7:431-434, 1968 (3/4 pages, RDB6A49)

R-717, R-746, and others, health effects, toxicity: study of chronic, occupational exposures of humans; 140 students aged 16-19 exposed to ammonia as well as nitrogen oxides in a fertilizer factory experienced an $11 \%$ increase in respiratory tract disorders and a $27 \%$ increase in xeroderma (disease of the skin characterized by dryness and roughness) in the first year, compared to 85 students in the same vocational school not exposed to ammonia or nitrogen oxides; diseases of the upper respiratory tract and skin increased by 24 and $36 \%$, respectively, in the second year; groups of 34 students with 3 hour shifts in the factory were then compared to a control group of 26 students; the exposed group showed increases in gamma globulin and decreases in serum albumen together with changes in fat metabolism; author suggested that there is stress on the hepatic functions that could be ascribed to low concentrations of ammonia as reported in RDB1106; limitations to the study, including unknown prior health of the subjects and possible causes other than ammonia are discussed in RDB1106

R. Gill, S. Hatchett, C. Broster, M. Osselton, J. Ramsey, $H$. Wilson, and A. Wilcox, The Response of Evidential Breath Alcohol Testing Instruments with Subjects Exposed to Organic Solvents and Gases. I. Toluene, 1,1,1Trichloroethane, and Butane, Sci. Law, 31:187200, 1991 (4 pages, RDB7B44)

R-140a, R-600, pharmacokinetics, elimination, inhalation toxicity in humans

J. H. Glover and H. W. Hodgson, An Investigation of New Fluorine Compounds in Anaesthesia (3), Anaesthesia, 16:333 ff, 1961 (RDB65B6)

anesthetic effect, toxicity

J. H. Glover and H. W. Hodgson, An Investigation of New Fluorine Compounds in Anaesthesia (2), Anaesthesia, 16:19 ff, 1961 (RDB65B5)

anesthetic effect, toxicity 
A. E. Goldsmith et al., Cancer Detection and Prevention, 4(1-4):109-115, 1981 (7 pages, RDB5784)

R-744 (carbon dioxide) and others, health effects, toxicity

S. Gosseye, M.-C. Golaire, and J.-C. Larroche, Cerebral, Renal, and Splenic Lesions Due to Fetal Anoxia and their Relationships to Malformations, Dev. Med. Child. Neurol., 24:510-518, 1982 (9 pages, RDB7B45)

R-600, reproductive toxicity, fetal death following an attempted suicide using butane, toxicity in humans

R. E. Gosselin, R. P. Smith, and H. C. Hodge, Fluorocarbon Refrigerants and Propellants, Clinical Toxicology of Commercial Products (fifth edition), Williams and Wilkins, Baltimore, MD, 11159, 1984 (148 pages, RDB5177)

reference for toxicity data

V. Graff-Lonnevig, Diurnal Expiratory Flow After Inhalation of Freons and Genoterol in Childhood Asthma, Journal of Allergy and Clinical Immunology, 64:534-538, 1979 (5 pages, RDB8144)

health effects, toxicity

W. M. Grant, Toxicology of the Eye (third edition), Charles C. Thomas Publishers, Springfield, IL, 1986 (RDB5C51)

refrigerants and other chemicals, toxicity as reported in RDB5C46

T. Green, The Metabolic Activation of Dichloromethane and Chlorofluoromethane in a Bacte-

rial Mutation Assay Using SalmonellaTyphimurium, Mutation Research, 118(4):277288, 1983 (12 pages, RDB6480)

R-30, R-31, and others, toxicity, mutagenicity

T. B. Griffin and F. Coulston, Study of Toxicological Evaluation of Fire Suppressants and Extinguishers, report CR-147658, National Aeronautics and Space Administration (NASA), Washington, DC, 31 March 1975 (RDB6571)

R-116 and others, toxicity: rabbits exposed to $50,000 \mathrm{ppm}$ atmospheres of R-116 showed low absorption which was quickly eliminated; 4-hr ALC rat $>800,000 \mathrm{ppm}$ - as reported in RDB6687

T. B. Griffin, J. L. Byard, and F. Coulston, Toxicological Responses to the Halogenated Hydrocarbons, Proceedings of the Symposium on Application of Halogenated Fire Extinguishing Agents
(Washington, DC, 11-12 April 1972), National Academy of Science (NAS), Washington, DC, 136147, 1972 (12 pages, RDB6578)

R-116, R-C318, and others, toxicity

R. Guillern et al., Effect of Chlorofluoromethanes on Ciliary Activity of the Respiratory Epithelium, Ann. Pharm. Français, France, 18:542-544, 1960 (3 pages in French, RDB7546)

R-114 and others, health effects, sensory irritation, toxicity

C. A. Halder et al., Gasoline Vapor Exposures. Part I., AIHA Journal, 47(3):164-172, 1986 (9 pages, RDB6189)

R-600, R-600a, R-601, R-601a, health effects, toxicity, inhalation studies in rats

C. A. Halder, G. S. Van Gorp, N. S. Hatoum, and t. M. Warne, Gasoline Vapor Exposures. Part II. Evaluation of the Nephrotoxicity of the Major C4/C5 Hydrocarbon Components, AIHA Journal, 47(3):173-175, 1986 (3 pages, RDB6190)

tests of subchronic inhalation toxicity by exposure of Sprague-Dawley rats to $4,437 \mathrm{ppm}$ of a blend of R-600 (n-butane), R-600a (isobutane), $R-601$ (n-pentane), and R-601a (isopentane) R-600a/600/601a/601 (25/25/25/25) - for 6 $\mathrm{hr} / \mathrm{d}, 5 \mathrm{~d} / \mathrm{wk}$, for $3 \mathrm{wk}$ : no clinical signs of distress or evidence of kidney lesions were found; hematology and clinical chemistry were within norm - as reported in RDB7621

G. T. Hall, Two-Week Inhalation Toxicity Study (HFA 132b and HFA 124), report 727-76, Haskell Laboratory for Toxicology and Industrial Medicine, E. I. duPont de Nemours and Company, Incorporated, Newark, DE, 1976 (RDB6179)

$R-124$ and $R-132 b$, health effects, toxicity, subchronic inhalation study: R-124 anesthetic LOEC rat $=100,000 \mathrm{ppm}$ as reported in RDB5863

Halon Alternatives Group, A Review of the Toxic and Asphyxiating Hazards of Clean Agent Replacements for Halon 1301, Loss Prevention Council, London, UK, February 1995 (24 pages with 4 tables, RDBA668)

compares the toxicity and asphyxiation potentials of six commercial fire suppressants (some also used as refrigerants) as alternatives for $R$ 13B1; they include R-23 (DuPont FE-13), R227ea (Great Lakes Chemical FM-200), R-3110 (3M CEA 410), R-728/740 (50/50) (Ginge Kerr Argonite), R-728/740/744 (52/40/8) (Ansul Inergen), and R-740 (Preussag Fire Protection 
Argon); discusses criteria for asphyxiation and maximum duration of oxygen diminished concentrations; tabulates the typical use concentrations, median lethal concentrations (4-hr $\mathrm{LC}_{50}$ for an unidentified species), central nervous system (CNS) effects, cardiac sensitization, respiratory sensitization, genotoxicity, and developmental toxicity; recommends "safe use criteria" (the maximum recommended concentration for systems on automatic control for occupied areas); indicates those recommendations as $60,000 \mathrm{ppm} v / \mathrm{v}$ for R-13B1, 170,000 $\mathrm{ppm} \mathrm{v} / \mathrm{v}$ (may be raised to $290,000 \mathrm{ppm} \mathrm{v} / \mathrm{v}$ with pending data) for R-23, 90,000 ppm v/v for R-227ea (Great Lakes Chemical FM-200), and 290,000 ppm v/v for R-31-10; discusses the criteria and terminology used for the assessment

A. Hamilton and H. L. Hardy, Industrial Toxicology (second edition), Paul B. Hoeber, Incorporated, New York, NY, 1949 (RDB5574)

toxicity of refrigerants

P. L. Hanst, J. W. Spence, And F. R. Clay (U.S. Environmental Protection Agency), Chlorofluoromethanes: Their Thermal Stability in Passing Through Cigarettes, AlHA Journal, 39:772-776, September 1978 (5 pages with 4 figures, RDB6775)

refrigerants, toxicity: test for decomposition of $\mathrm{R}-11$ at $2,000 \mathrm{ppm}$ and R-22 at 2,500 ppm on passing through lit cigarettes; no carbonyl chloride (phosgene), carbonyl fluoride, or carbonyl chlorofluoride was found at a sensitivity of $0.1 \%$ of the starting material; documents the reactions that might yield the toxic carbonyl halides, experimental methods, and measured infrared spectra; found no measurable decomposition of the chlorofluoromethane compounds with simulated smoking; concludes that chlorofluoromethane pollutants in a room do not pose a greater health hazard for smokers than nonsmokers

C. J. Hardy, P. C. Kieran, and I. J. Sharman (Huntingdon Research Centre Limited, HRC, UK), Assessment of Cardiac Sensitization Potential (CSP) of a Range of Halogenated Materials, poster presentation (Spring Meeting, Baltimore, MD, USA), Society of Toxicology (SOT), 1994 (10 pages with 4 figures and 1 table, RDB6684)

cardiac sensitization potential (CSP) in beagle dogs; method modification to predetermine appropriate adrenaline challenge for individual animals; presents [widely accepted] test regime for CSP measurement; 10-min $\mathrm{EC}_{50}$ dog was $10,000-20,000 \mathrm{ppm}$ for R-11, $120,000 \mathrm{ppm}$ for $\mathrm{R}-12,250,000$ for $\mathrm{R}-13 \mathrm{~B} 1,>500,000 \mathrm{ppm}$ for $\mathrm{R}-23,>350,000 \mathrm{ppm}$ for R-32, $170,000 \mathrm{ppm}$ for R-125, 205,000 ppm for R-134a, 20,000 ppm for R-141b, $\sim 310,000 \mathrm{ppm}$ for R-143a, $>400,000 \mathrm{ppm}$ for R-218, >400,000 ppm for R$31-10,>170,000 \mathrm{ppm}$ (maximum attainable vapor concentration) for R-51-14, and $>230,000$ (maximum attainable vapor concentration) for perfluoro- $\mathrm{N}$-methyl morpholine; examines relation to normal boiling point and to molecular composition with data from the current and prior studies; concludes that "although cardiac sensitization is likely to be an example of physical toxicity and is related to the physiochemical properties of a material, the [normal] boiling point alone cannot be used as a predictor"; also concludes that "halogenated alkanes in which $>75 \%$ of the halogens are fluorine are likely to be of low CSP and that halogenated alkanes in which $\geq 50 \%$ of the halogens are chlorine are likely to be of high CSP"; finally concludes that "knowledge of the molecular formula and the boiling point may, together, allow some indication of likely CSP ${ }^{n}$ as a guide for animal studies

C. J. Hardy et al., Halon 13B1, Freon 23, Mixture of Freon 23 and HFC 125: Assessment of Cardiac Sensitization Potential in Dogs, report DPT 273/921009, Huntingdon Research Centre Limited (HRC), Cambridgeshire, UK, 15 March 1993 (42 pages, RDB6458)

toxicity tests for cardiac sensitization in beagle dogs with administration of adrenaline by intravenous injection before and during inhalation; R-11 LOEL (100\%) at 20,000 ppm v/v; R-13B1 NOEL at $100,000 \mathrm{ppm}$, LOEL (17\%) at 150,000 ppm, $E_{50}$ at 250,000 ppm; R-23 NOEL at $500,000 \mathrm{ppm}$ with auxiliary oxygen; R-23/125 $(36.5 / 63.5 \mathrm{v} / \mathrm{v})$ LOEL $(1 / 6)$ at $100,000 \mathrm{ppm}$

C. J. Hardy, P. C. Kieran, I. J. Sharman, and G. C. Clark (Huntingdon Research Centre Limited, HRC, UK), Assessment of Cardiac Sensitization Potential in Dogs: Comparison of HFC 125 and Halon 13B1, report MA-RR-92-1845 (also identified as ALS 11/920116) for the Program for Alternative Fluorocarbon Toxicity Testing (PAFT), AlliedSignal Incorporated, Morristown, NJ, 10 June 1992 (44 pages, RDB5755)

toxicity tests to determine the cardiac sensitization potential in male beagle dogs with intravenous injection of adrenaline five minutes before and, with a challenge injection, midway into a 
10 minute inhalation exposure through a face mask: R-11 LOEL (1/2) at 20,000 ppm v/v; R$13 B 1$ NOEL $(0 / 6)$ at 150,000 ppm with a questionable response not deemed as positive, LOEL (2/6) at $200,000 \mathrm{ppm}$, and $\mathrm{EC}_{50}$ at $250,000 \mathrm{ppm} ; \mathrm{R}-125$ NOEL $(0 / 6)$ at 75,000 $\mathrm{ppm}, \operatorname{LOEL}(1 / 6)$ at $100,000 \mathrm{ppm}$, and $\mathrm{EC}_{50}$ at $139,000 \mathrm{ppm}$

C. J. Hardy, I. J. Sharman, and G. C. Clark (Huntingdon Research Centre Limited, HRC, UK), Assessment of Cardiac Sensitization Potential in Dogs: Comparison of HFA 134a and A12, report CTL/C/2521 (also identified as ISN 250/91169) for the Program for Alternative Fluorocarbon Toxicity Testing (PAFT), Central Toxicology Laboratory, Imperial Chemical Industries Limited (ICI), Cheshire, UK, 11 September 1991 (62 pages with 11 figures and 4 tables, Rdb5B42)

toxicity tests of cardiac sensitization in beagle dogs by inhalation with an intravenous administration of an epinephrine challenge; for R-11, NOEL $0 / 2=15,000$ and LOEL $2 / 2=20,000$

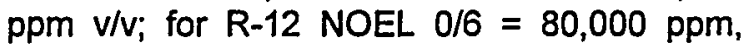
LOEL $3 / 3=160,000$ ( 3 dogs disqualified for struggling and convulsions), and $\mathrm{EC}_{50}>80,000$ and $<160,000 \mathrm{ppm} \mathrm{v/v}$; for R-134a NOEL $0 / 6=$ 40,000 , LOEL $2 / 6=80,000 \mathrm{ppm}$, and $E_{50}$ $>160,000$ and $<320,000 \mathrm{ppm}$; concentrations indicated are of the test chemical in air except that the air was enriched for the two highest concentrations to maintain $21 \%$ oxygen levels; report documents preliminary tests to determine appropriate levels of adrenaline doses for each dog and also scoring criteria since some ventricular tachycardia (a series or run of unifocal ectopic beats) were observed in tests with air and therefore not compound related; the criterion adopted for positive responses was appearance of five or more multifocal ventricular ectopic beats or ventricular fibrillation (very rapid and irregular contractions of the ventricles of the heart resulting in loss of synchronization between the heartbeat and pulse beat); report indicates measured blood levels measured during tests of up to $84.2 \mu \mathrm{g} / \mathrm{ml}$ for R-12 and up to $188.5 \mu \mathrm{g} / \mathrm{ml}$ for R-134a; concentrations were determined with an infrared gas analyzer; the report indicates Good Laboratory Practice (GLP) compliance

C. J. Hardy, I. J. Sharman, and D. O. Chanter (Huntingdon Research Centre Limited, HRC, UK), Assessment of Cardiac Sensitization Potential in Dogs and Monkeys: Comparison of $1-141 \mathrm{~b}$ and F11, report PWT 86/89437, Elf Atochem
North America (then Pennwalt Corporation), King of Prussia, PA, 8 September 1989 (50 pages with 7 figures and 7 tables, RDB5C63)

documents a cardiac sensitization study in beagle dogs and cynomolgus monkeys; $\mathrm{R}-11$ tests in dogs found a NOEL at $9,000 \mathrm{ppm} \mathrm{v} / \mathrm{v}$ and LOEL at $13,000 \mathrm{ppm} v / \mathrm{v} ; \mathrm{R}-141 \mathrm{~b}$ tests in dogs found a LOEL at $9,000 \mathrm{ppm} \mathrm{v/v}$ (the lowest concentration tested); R-11 tests in monkeys found a LOEL at $6,000 \mathrm{ppm}$ v/v based on one marked and one mild responses of the two animals tested; R-141b tests in monkeys found a mild response at $3,000 \mathrm{ppm} \mathrm{v/v}$ and a marked response in the same animal at $5,000 \mathrm{ppm} \mathrm{v} / \mathrm{v}$ as well as a questionable response in a second monkey at $5,000 \mathrm{ppm} v / \mathrm{v}$, but no response at $7,500 \mathrm{ppm}$ v/v; both monkeys showed marked responses at 10,000 ppm v/v; report deemed the LOEL monkey to be $5,000-10,000 \mathrm{ppm}$ v/V for both dogs and monkeys; study concluded that the potential to cause cardiac sensitization is similar for R-11 and R-141b; toxicity data

J. W. Harris, J. P. Jones, J. L. Martin, A. C. LaRosa, M. J. Olson (General Motors Research Laboratories), L. R. Pohl, and M. W. Anders (University of Rochester Medical Center), Pentahaloethane-Based Chlorofluorocarbon Substitutes and Halothane: Correlation of In Vivo Hepatic Protein Trifluoroacetylation and Urinary Trifluoroacetic Acid Excretion with Calculated Enthalpies of Activation, Chemical Research in Toxicology, 5(5):720-725, September 1992 (6 pages, RDB5942)

toxicity of R-123, R-123B1 (halothane), R-125, $\mathrm{R}-134 \mathrm{a}$, and others; metabolism; health effects

W. S. Harris, Aerosol Propellants are Toxic to the Heart, Journal of the American Medical Association (JAMA), 223:1508-1509, 1973 (2 pages, RDB8147)

toxicity of R-114 and others used as aerosol propellants (and also as refrigerants); cardiac sensitization; cardiotoxicity; health effects

W. S. Harris, Toxic Effects of Aerosol Propellants on the Heart, Annals of Internal Medicine, 131:162-166, 1973 (5 pages, RDB7248)

$\mathrm{R}-114$ and others used as aerosol propellants (and also as refrigerants), cardiac sensitization, health effects, toxicity

W. S. Harris, Cardiac Effects of Halogenated Hydrocarbons, Proceedings of the Symposium on Application of Halogenated Fire Extinguishing Agents (Washington, DC, 11-12 April 1972), Na- 
tional Academy of Science (NAS), Washington, DC, 114-126, 1972 (13 pages, available from NTIS as document AD-751426, RDB8148)

$\mathrm{R}-114$ and others used as fire suppressants (and also as refrigerants), cardiac sensitization, health effects, toxicity

W. S. Harris, Cardiac Toxicity of Aerosol Propellants, Proceedings of the 2nd Annual Conference on Environmental Toxicology (31 August - 2 September 1971), report AMRL-TR-71-120, Aerospace Medical Research Laboratory, U.S. Air Force, Wright-Patterson Air Force Base, $\mathrm{OH}_{1}$ 2329. December 1971 (7 pages, available from NTIS as document $A D-751426$, RDB7249)

R-114 and others used as aerosol propellants (and also as refrigerants), cardiac sensitization, health effects, toxicity

W. S. Harris, S. M. Kilen, G. J. Taylor, and S. Levitsky, Evidence from Animals and Man that Freon Depresses Myocardial Contractility, Circulation, 44(supplement II):119 ff, 1971 (RDB8149)

cardiac effects of aerosol propellants (and also as refrigerants), health effects, toxicity

J. K. Haseman, D. D. Crawford, J. E. Huff, G. A. Boorman, E. E. McConnell (National Institute of Environmental Health Sciences), Results From 86 Two-Year Carcinogenicity Studies Conducted by the National Toxicology Program, Journal of Toxicology and Environmental Health, 14(5-6):621-640, 1984 (20 pages with 5 tables, RDB6A80)

summary findings of toxicity tests for 86 chemicals to examine their carcinogenic potentials: majority were based on 2-yr feeding or gavage experiments involving groups of 50 male and female Fischer $344 / \mathrm{N}$ rats and $\mathrm{B} 6 \mathrm{C} 3 \mathrm{~F}$ mice; the liver was the most frequent site of cancer in the animals tested; males were more prone to neoplasia in rats while females were in mice; $50 \%$ of the 86 chemicals showed carcinogenic effects, including R-30B1, R-120, R-150B2, R260daB2, R-270da, and R-1120;6\% showed equivocal evidence of carcinogenicity; $42 \%$ gave no evidence of carcinogenicity including R-1130a and R-1270; and $2 \%$ of the studies were regarded as inadequate

H. W. Hays, Etiology of Cardiac Arrhythmias, Proceedings of the 3rd Annual Conference on Environmental Toxicology, report 7737166, National Technical Information Service (NTIS), Springfield,
VA, 173-183, 1972 (11 pages, available from NTIS, RDB8152)

fluorochemical health effects, cardiac sensitization, toxicity

J. Healy and O. W. Van Auken, Comparison of the Effects of Three Fluorocarbons on Certain Bacteria, Canadian Journal of Microbiology, 21(2):221-226, 1975 (6 pages, RDB5921)

R-22 and others, toxicity

H. Hermann and J. Vial, Nouvelles Syncopes Cardiaques par Association Toxique de l'Adrénaline et de Divers Produits Organiques Volatils [New Cardiac Sensitization by Toxic Effect of Adrenaline and Various Volatile Organic Compounds], Compte Rendu Société de Biol., Paris, France, 119:1316-1317, July 1935 (2 pages, RDB6564)

identifies R-10 as having intermediate potency as a cardiac sensitizer; identifies R-30, R-40, $\mathrm{R}-40 \mathrm{~B} 1, \mathrm{R}-4011, \mathrm{R}-150, \mathrm{R}-160 \mathrm{~B} 1, \mathrm{R}-16011$, and other chemicals as weak cardiac-sensitizing agents as reported in RDB5644

J. W. Hiddeman (Hazleton Washington, Incorporated, Vienna, VA), Acute and Ten-Day Inhalation, Pyrolysis, and Cardiac Sensitization Studies with Freons 14, 116, 218, and 1301, report on project 264-107 (included in report for contract DAHC 19-71-C-0026), U.S. Army Research Office, Arlington, VA, 18 February 1972 (103 pages with 1 figure and 27 tables, RDB7550)

reports tests of the acute inhalation toxicity in groups of ten male Sprague-Dawley albino rats for 1-hr; provides details of the tests for R-13B1 at 560,000 and $770,000 \mathrm{ppm} \mathrm{v/v,} \mathrm{R-14} \mathrm{at}$ $780,000 \mathrm{ppm} v / \mathrm{v}, \mathrm{R}-116$ at $800,000 \mathrm{ppm} \mathrm{v} / \mathrm{v}$, and $\mathrm{R}-218$ at $800,000 \mathrm{ppm}$ v/v all with oxygen plus a control group with air (R-729); indicates no mortality in any of the exposures, but changes in behavior of all treated animals; also reports tests of the subchronic inhalation toxicity in groups of ten male and ten female Sprague-Dawley albino rats and with 10 male and 10 female guinea pigs for $24-\mathrm{hr} / \mathrm{d}$ for 10 days; indicates the measured mean concentrations as $51,000 \mathrm{ppm} v / \mathrm{v}$ for R-13B1, 226,000 ppm $\mathrm{v} / \mathrm{v}$ for R-14, 121,000 ppm v/v for R-116, and $113,000 \mathrm{ppm}$ v/v for R-218 all with oxygen enrichment to maintain a $20 \%$ level and notes similar tests of a control group with air; states that behavior was normal and there were no manifestations of toxicity except that five guinea pigs appeared to be ill and were sacrificed for necropsy before completing the full 
term to avoid transmission of a suspected respiratory disease to other animals; of them one was in the control group, one in R-116, and two in R-218; indicates that these deaths were not considered to be compound-related; also reports pyrolysis studies to evaluate the toxicity of decomposition products from use of the subject gases to quench a fire; notes compoundspecific behavioral and hematological changes, but no mortalities or remarkable gross or histopathological changes; also reports a cardiac sensitization study for R-14 in six male beagles; concludes that R-14 did not cause arrhythmias at the tested concentrations of 200,000 and $600,000 \mathrm{ppm} v / \mathrm{v}$, but that it did sensitize 3 of 6 dogs at $600,000 \mathrm{ppm} v / \mathrm{v}$ as manifested by occasional preventricular contractions (OPVC) after the epinephrine challenges; notes that 1 of 6 dogs also experienced OPVC after the epinephrine challenge during the $200,000 \mathrm{ppm} v / \mathrm{v}$ exposure, but could no conclusively attribute it to sensitization since the same dog showed a similar response to epinephrine under control conditions without $R-14$ exposure

G. Hodson-Walker (Pharmaco-LSR Limited, UK), G. M. Rusch (AlliedSignal Incorporated, USA), and F. M. H. Debets (Akzo Chemicals International B.V. The Netherlands), Mutagenicity Testing of a Number of CFC Replacements. Methodology and Results, presented at the Sixth International Conference on Environmental Mutagens (Melbourne, Australia, February 1993); presented at the 24th Annual Meeting of the Environmental Mutagen Society (Norfolk, VA, April 1993); published in Environmental and Molecular. Mutagenesis, 21(supplement 22):28 ff, 1993 (4 pages with 8 figures and 1 table, Rdb65D3)

mutagenicity tests of R-123, R-124, R-125, R$141 \mathrm{~b}$, and R-1131a by Ames Assay, chromosome aberration studies, and - for R-125 mouse bone-marrow micronucleus tests by nose-only exposures: identifies concentrations resulting in toxicity and concludes that the five hydrochlorofluorocarbons (HCFCs) tested showed no evidence of mutagenic activity except that R-123 showed clastogenic (chromosome-damaging) activity in human lymphocytes, R-141b showed clastogenic activity in CHO-KI cells, and R-1131a was positive in the Ames test; presents a new method for testing gas- and water-insoluble, volatile liquids

P. Hoet (Catholic University of Louvain, CUL, Belgium), M. L. Graf (also shown as Graff), M. Bourdi, L. R. Pohl (National Heart, Lung, and Blood Insti- tute, UK), P. H. Duray (National Cancer Institute, UK), W. Chen, R. M. Peter, S. D. Nelson (University of Washington, USA), N. Verlinden (Beerse, Belgium), D. Lison (CUL), Epidemic of Liver Disease Caused by Hydrochlorofluorocarbons Used as Ozone-Sparing Substitutes of Chlorofluorocarbons, Lancet, UK, 350(9077):556-559, 23 August 1997 (4 pages with 3 figures, RDB7C70)

toxicity findings following accidental human exposures to a blend of R-290/124/123 (reported in this article as $R-124 / 123$ ) in an industrial plant: reports an investigation of liver disease in nine workers affected to various degrees following repeated exposures; metabolism of both $R-123$ and R-124 produces reactive trifluoroacetyl halide intermediates, which have been implicated in the hepatotoxicity of R-123B1 (halothane); a liver biopsy for one of the workers showed hepatocellular necrosis that was prominent in perivenular zone three and extended focally from portal tracts to portal tracts and centrilobular areas (bridging necrosis); immunohistochemical stainings in the same worker showed the presence of trifluoroacetyladducted proteins in surviving hepatocytes; autoantibodies that react with human liver cytochrome P450 2E1 and P58 protein disulphide isomerase isoform were detected by serum tests in five of six affected workers; concludes that repeated exposure of human beings to $R$ 123 and R-124 can result in serious liver injury and notes while that the exact mechanism of hepatotoxicity is unknown, the findings suggest involvement of trifluoroacetyl-altered liver proteins; the authors suggest that safer alternatives are needed [Subsequent investigation of the accident indicates that the refrigerant involved was $R-290 / 124 / 123$. It was used in a direct-expansion air conditioner serving the cab and controls of an overhead gantry crane. The system had been retrofitted from R-114 to the blend, but the old, incompatible hoses were not changed. The leak persisted for several months without repairs, despite repeated requirements for refrigerant recharge, and no leak detector was installed. The concentrations to which the affected workers, all crane operators, were exposed could not be determined, but calculations indicate that they far exceeded recommended exposure limits. The liver biopsy reported was performed on only one of the workers, for whom no prior medical history was available. See RDB7C71 - RDB7C75 for further information.] 
D. B. Hood, Skin and Eye Irritation Tests with Aerosol Hair Sprays, report 31-56, Haskell Laboratory for Toxicology and Industrial Medicine, E. I. duPont de Nemours and Company, Incorporated, Newark, DE, 1956 (RDB7526)

$\mathrm{R}-11$ and R-12, dermal contact toxicity for aerosol propellants [also used as refrigerants], irritation as reported in RDB7414 and RDB7525

B. K. Hoover and R. C. Graham, A Table of Acute Oral and Dermal $L_{50}$ 's and Inhalation $L C_{50}$ 's for Fluorocarbon-11, 12, 22, 113, 114, 115, and 152a, report OTS-0520424 (also numbered OTS0520425), Haskell Laboratory for Toxicology and Industrial Medicine, E. I. duPont de Nemours and Company, Incorporated, Newark, DE, 11 May 1978 (8 pages, RDB6595)

summary table of acute oral, dermal, and inhalation toxicity data for six fluorochemical refrigerants indicates: for $\mathrm{R}-11, \mathrm{LC}_{50}=26,200$ $\mathrm{ppm}, \mathrm{LD}_{50}$ oral rat $>3,752 \mathrm{mg} / \mathrm{kg}$; for $\mathrm{R}-12, \mathrm{LC}_{50}$ $>800,000 \mathrm{ppm}, L D_{50}$ oral rat $>1,000 \mathrm{mg} / \mathrm{kg}$; for $\mathrm{R}-22, \mathrm{ALC}=300,000 \mathrm{ppm}$; for $\mathrm{R}-113, \mathrm{LC}_{50}=$ $56,000-68,000 \mathrm{ppm}, \quad L_{50}$ dermal rabbit $>11,000 \mathrm{mg} / \mathrm{kg}, L D_{50}$ oral rat $=43,000 \mathrm{mg} / \mathrm{kg}$; for $R-114, L C_{50}=600,000 \mathrm{ppm}, L D_{50}$ oral rat $>2,250 \mathrm{mg} / \mathrm{kg}$; for R-115, $\mathrm{LC}_{50}>800,000 \mathrm{ppm}$; for $\mathrm{R}-152 \mathrm{a}, \mathrm{LC}_{50}=383,000 \mathrm{ppm}$; time period and test species for $L_{50}$ and ALC data is not indicated

D. K. Hoover, K. O. Yu, R. K. Black, G. W. Jepson (ManTech Environmental Technology, Incorporated), and J. W. Fisher (U.S. Air Force Armstrong Laboratory, USAF-AL), Kinetic Evaluation of Halon 1211, HCFC-123, HCFC-142b, and HCFC124 Using Gas Uptake Methods, abstract (SOT 31st Annual Meeting, February 1992), The Toxicologist, 12(1):353, 1992 (1 page, RDB7289)

R-12B1, R-123, R-142b, R-124, biochemistry, health effects, toxicity

R. M. Hopkins and J. C. Krantz, Jr. (Huntingdon Research Center, HRC, USA), Anesthesia LXXV. Relative Effects of Haloforms and Epinephrine on Cardiac Automaticity, presented at the 51st Annual Meeting of the Federation of American Society for Experimental Biology (Pharmacology Sessions, Chicago, 19 April 1967), 1967; republished in Anesthesia and Analgesia, 47(1):56-67, January-February 1968 (12 pages with 5 figures and 4 tables, RDB5136)

tests of the cardiac sensitization potential of R20 (chloroform), R-20B3 (bromoform), R2013 (iodoform), R-23 (fluoroform), and R-50 (methane); tests of R-23 and R-50 were conducted by inhalation at concentrations of 800,000 and $810,000 \mathrm{ppm} \mathrm{v} / \mathrm{v}$, respectively, in oxygen for 5 $10 \mathrm{~min}$ with an epinephrine challenge; reports that neither caused sensitization as tested despite a prior qualitative finding of sensitization for R-50 (see RDB65A2); also reports tests with intravenous administration of R-20, R-20B2, and R-2013 in anesthetized and unanesthetized dogs with epinephrine challenges; summarizes estimates of the mean molar concentrations evoking sensitization for these three compounds; toxicity tests

A. Inserra, M. Donpe, and V. Timpanaro, Blood Dyscrasia and Experimental Poisoning with Propane and Butane, Folia Med., Italy, 51:779 ff, 1968 (RDB7410)

$\mathrm{R}-290, \mathrm{R}-600, \mathrm{R}-290 / 600$, toxicity, as reported in RDB6569

N. F. Izmerov, I. V. Sanotsky, and K. K. Siderov, Toxicometric Parameters of Industrial Toxic Chemicals Under Single Exposure, USSR State Committee for Science and Technology, Moscow, USSR, 1982 (in Russian, RDB5C91)

toxicity of R-141b, R-152a, and others - as reported in RDB4450 and RDB5868; 2-hr LC LO $_{50}$ mouse for R-152a $=369,000 \mathrm{ppm} v / \mathrm{v}$ - as reported in RDB4450 and RDB4B90

D. Jack, Sniffing Syndrome, British Medical Journal, 2:708-709, 1971 (2 pages, RDB8153)

fluorocarbon abuse, potential for human fatalities

W. Johnson, Unsafe Application of HCFC-123, -124 Blamed for Liver Damage in 9 Workers, The Air Conditioning, Heating, and Refrigeration News, 1-2 and 19, 1 September 1997 (3 pages with no figures or tables, limited copies available from JMC as RDB7C74)

provides further information on the underlying incident and subsequent investigations in response to an article in The Lancet (see RDB7C70) on exposures of workers to R-124/123 [actually R-290/124/123]: summarizes the incident, points made in the article, and reactions from informed industry sources; quotes $E$. L. Smithart (The Trane Company, USA), R. Rubenstein (Environmental Protection Agency, EPA, USA), P. Hoet (Catholic University of Louvain, Belgium), G. R. Rusch (AlliedSignal Incorporated, USA), W. R. Brock (DuPont Chemicals, USA), and J. M. Calm (Engineering Consultant, USA); the information presented indicates that the application was not typical 
and did not conform to recognized safety standards; also notes that the exposures exceeded recommended limits and would not have occurred if accepted practices had been followed and attention paid to the required system recharges

R. T. Johnstone and S. E. Miller, Occupational Diseases and Industrial Medicine, W. B. Saunders Company, Philadelphia, PA, 1961 (RDB5C43)

fluorocarbon inhalation effects, oxygen deprivation, toxicity

B. C. Karpov, Lethal and Threshold Concentration of Freons, Tr. Leningrad Sanit. Gigien. Med. Inst., 241:128-137, 1963; English translation published in Industrial Hygiene and Occupational Pathology, U.S. Department of Commerce, Washington, DC, $118 \mathrm{ff}, 1965$ (10 pages in Russian, RDB5976)

$\mathrm{R}-141 \mathrm{~b}$ and others, toxicity as reported in RDB6365]

D. A. Keller, D. C. Roe, and P. H. Lieder (E. I. duPont de Nemours and Company), FluoroacetateMediated Toxicity of Fluorinated Ethanes, article 0058, Fundamental and Applied Toxicology, 30(2):213-219, April 1996 (7 pages with 4 figures and 1 table, RDBA648)

summarizes tests of the inhalation toxicity for 1 (di)halo-2-fluoroethanes including R-142a (1chloro-1,2-difluoroethane), R-151 (1-chloro-2fluoroethane), R-151B1 (1-bromo-2fluoroethane), and R-152 (1,2-difluoroethane); notes that these compounds are highly toxic by inhalation even though reported in the literature to be nontoxic or of low toxicity; all four compounds had 4-hr approximate lethal concentrations (ALCs) of $100 \mathrm{ppm}$ v/v or less in rats; exposed rats showed clinical signs of fluoroacetate toxicity (lethargy, hunched posture, and convulsions); R-142a, R-152, R-151, and R151B1 increased concentrations of citrate in serum and heart tissue, a hallmark of fluoroacetate intoxication; analyses confirmed the presence of fluoroacetate in the urine of exposed rats; analyses also found fluorocitrate, a condensation product of fluoroacetate and oxaloacetate, in the kidney and concentration-related elevation of serum and heart citrate in rats exposed to 0-1000 ppm v/v R-152 1,2difluoroethane; indicates that metabolism of 1,2-difluoroethane by cytochrome P450 (most likely CYP2E1) is suspected; experimental evidence indicates that the metabolism of the toxic fluoroethanes is initiated at the carbon-hydrogen bond, with metabolism to fluoroacetate via an aldehyde or an acyl fluoride; the results of these studies show that 1-(di)halo-2-fluoroethanes are highly toxic to rats and should be considered a hazard to humans unless demonstrated otherwise; the paper notes that R-152a (1,1-difluoroethane), in contrast, has very low acute toxicity with a 4-hr $L^{-} C_{50}$ of $>400,000 \mathrm{ppm}$ in rats

D. A. Keller, Acute Inhalation Toxicity of HFC236fa and HFC-236ea in the Rat, report 761-93, Haskell Laboratory for Toxicology and Industrial Medicine, E. I. duPont de Nemours and Company, Incorporated, Newark, DE, 21 November 1994 (9 pages with 2 tables, RDB6803)

toxicity of R-236ea and R-236fa by whole-body exposures of young adult male, Charles River rats at target concentrations of $15,000,25,000$, 50,000 , and $100,000 \mathrm{ppm}$ v/v R-236ea and at 150,000 and $200,000 \mathrm{ppm} \mathrm{v} / \mathrm{v}$ R-236fa (the means of actual concentrations determined by gas chromatography (GC) were 13,586, $23,588,45,813$, and $84,927 \mathrm{ppm}$ V/v for R236ea and 133,939 and 189,275 for R-236fa); oxygen level in the air was maintained at $21 \pm 3 \%$; concluded that both isomers can cause sleep induction (narcosis); for R-236ea, 4-hr ALC rat is $>85,000 \mathrm{ppm} \mathrm{v} / \mathrm{v}$ and NOEL and LOEL for narcosis are 14,000 and $24,000 \mathrm{ppm}$, respectively; for R-236fa, 4-hr ALC rat is $>189,000 \mathrm{ppm}$ and NOEL and LOEL for symptoms of narcosis (lack of response to sound) are 134,000 and $189,000 \mathrm{ppm}$, respectively; toxicity data

D. P. Kelly, R. Culik, H. J. Trochimowicz, and W. E. Fayerweather (E. I. duPont de Nemours and Company, Incorporated), Inhalation Teratology Studies on Three Fluorocarbons, abstract 170 (Seventeenth Annual Meeting of the Society of Toxicology, SOT), Toxicology and Applied Pharmacology (TAP), 45(1):293, 1978 (1 page with no figures or tables, RDB5827)

toxicity of R-123, R-142b, and others

D. P. Kelly, Two-Week Inhalation Toxicity Studies, report 149-76, Haskell Laboratory for Toxicology and Industrial Medicine, E. I. duPont de $\mathrm{Ne}-$ mours and Company, Incorporated, Newark, DE, 1 March 1976 (4 pages with no figures or tables, RDB6158)

reports subchronic inhalation toxicity tests of $R$ 21 and R-123; rats exposed to $10,000 \mathrm{ppm}$ v/v R-123 for $6 \mathrm{hr} / \mathrm{d}, 5 \mathrm{~d} / \mathrm{wk}$, for 2 weeks showed 
signs suggestive of anesthesia - as reported in RDB6153 and RDB6941; no adverse hematological, clinical chemical, urine analytical, or histopathological effects were found as reported in RDB6941

T. J. Kenny, C. K. Shepherd, and C. J. Hardy (Huntingdon Research Centre Limited, HRC, UK), lodotrifluoromethane and lodoheptafluoropropane: Assessment of Cardiac Sensitization Potential in Dogs, report AL/OE-TR-1995-0031, Armstrong Laboratory, U.S. Air Force, Wright-Patterson Air Force Base, OH, 1995 (RDB8418)

toxicity tests to determine the cardiac sensitization potential of R-13/1 and R-217bal1 in dogs

C. J. Kirwin (Phillips Petroleum Company), W. C. Thomas, and V. F. Simmon (Stanford Research Institute), In Vitro Microbiological Mutagenicity Studies of Hydrocarbon Propellants, Journal of the Society of Cosmetic Chemists, 31(7):367-370, December 1980 (4 pages, RDB6111)

summarizes tests of the mutagenic potential of hydrocarbon propellants [also used as refrigerants] by Ames assay in Salmonella typhimurium, with and without metabolic activation; addresses R-290 (propane), R-600 (n-butane), 2 samples of R-600a (isobutane), R-601 (n-pentane), and R-601a (isopentane); although some toxicity was observed with the higher molecular weight hydrocarbons, none of the five tested compounds was found to be mutagenic - as reported in RDB6A83 and RDB7621

C. J. Kirwin, Toxicological Review of Propane, Butane, Isobutane, Pentane, and Isopentane, Phillips Petroleum Company, Bartlesville, OK, November 1976 (10 pages with no figures or tables, RDBA623)

summarizes the toxicity of R-290 (propane), R600 (n-butane), R-600a (isobutane), R-601 (pentane), and R-601a (isopentane) as candidates to replace chlorofluorocarbons (CFCs) as aerosol propellants [and as refrigerants]; addresses skin and eye irritation, median lethal concentrations $\left(\mathrm{LC}_{50}\right)$, anesthetic potential, chronic exposure effects, pharmacologic effects, the toxicology of blends, Threshold Limit Values (TLV), authorization for food use, and mutagenicity; indicates that very high concentrations of paraffinic hydrocarbons are necessary to produce alterations in normal body functions and that no permanent alterations are induced by these chemicals; notes that the toxic properties of these chemicals are not manifest until levels that exceed fire hazard concentrations; also notes that these hydrocarbons can act as simple asphyxiants

A. E. Kozbakova, Comparative Toxicity of Chlorinated and Fluorinated Methane and Ethane Derivatives, Gigiena Truda i Professional'nye Zabolevaniya [Labor Hygiene and Occupational Diseases], Moscow, Russia (then USSR), 20(11):38-41, 1976 (4 pages in Russian, RDB7547)

$\mathrm{R}-21$ as reported in RDB5340; R-114 and others, health effects, toxicity

J. C. Krantz, Jr., and F. G. Rudo, The Fluorinated Anesthetics, chapter 10, Handbuch der experimentellen Pharmakologie [Handbook of Experimental Pharmacology], edited by O. Eichler, A. Farah, $H$. Herken, and A. D. Welch, Springer-Verlag, Berlin, Germany, XX(1):501-564, 1966 (64 pages, RDB6589)

R-113a, R-114, and others, toxicity review of fluorine chemicals: $R-113 a$ anesthetic concentration at $60,000 \mathrm{ppm}$ ) as reported in RDB5143

J. C. Krantz, Jr., The Rationale of the Use of Fluorinated Hydrocarbons and Ethers as Volatile Anesthetic Agents, Anesthesia and Analgesia, 44:260 ff, March-April 1965 (RDBA627)

anesthetic effects and toxicity of hydrocarbons, fluorochemicals, and fluoroethers

J. C. Krantz, Jr., C. J. Carr, and J. F. Vitcha (University of Maryland), Anesthesia XXXI. A Study of Cyclic and Noncyclic Hydrocarbons on Cardiac Automaticity, Journal of Pharmacology and Experimental Therapeutics (JPET), 94:315-318, 1948 (4 pages with 1 figure and 1 table, RDB6192)

toxicity tests to determine the cardiac sensitization potential of hydrocarbons mixed with oxygen in dogs with epinephrine hydrochloride injections before and approximately 10 minutes into exposures: the number of dogs sensitized at concentrations of $100,000-250,000 \mathrm{ppm}$ was 2/4 for R-170, 3/3 for R-290, 2/2 for R-C390, 2/2 for $R-600,2 / 2$ for $R-600 a, 0 / 12$ for $R-1150$, and $2 / 2$ for $R-1270$; the number sensitized at $150,000-900,000 \mathrm{ppm}$ was $2 / 3$ for R-C $41-10$ and $3 / 3$ for R-601a; data also are provided for cis-trans-butene-2, cyclobutene, and 2,2dimethylbutane; concludes that while R-1150 does not produce sensitization, the remaining 11 hydrocarbons tested do; also concludes that cyclobutane and cyclobutene produce excellent anesthetic syndromes, the other hydrocarbons tested are unsatisfactory anesthetic agents in the dog 
H. Kubler, Physiological Properties of Propellants, Journal of the Society of Cosmetic Chemists, 14:341-351, 1963 (11 pages, RDB6737)

\section{R-114 and others, toxicity}

K. Kudo, S. Toida, S. Matsuura, T. Sasaki, and H. Kawamura, Comparison of Freon-11S and Freon 11. Acute, Subacute Toxicity and Irritation of Mucous Membrane, Toho Igakkai Zasshi, Japan, 18(2):363-367, 1971 (5 pages, RDB7426)

$\mathrm{R}-11$, health effects, toxicity as reported in RDB7414

C. J. Lambertsen, Therapeutic Gases - Oxygen, Carbon Dioxide, and Helium, Drill's Pharmacology in Medicine (fourth edition), McGraw-Hill Book Company, New York, NY, chapter 55, 1971 (RDBA664)

toxicity and therapeutic uses of R-704 (helium), R-732 (oxygen), and R-744 (carbon dioxide); indicates that inhalation by humans of carbon dioxide at $100,000 \mathrm{ppm} \mathrm{v} / \mathrm{v}$ for $1.5 \mathrm{~min}$ causes neurologic effects including eye flickering, psychomotor excitation, and myoclonic twitches (involuntary contractions of muscles); also indicates increasing effects at higher concentrations including unconsciousness and convulsions at $300,000 \mathrm{ppm} \mathrm{v} / \mathrm{v}$ in oxygen in less than 2 minutes - as reported in RDB5787

M. M. Landry and R. Fuerst, Gas Ecology of Bacteria, Develop. Ind. Microbiology, 9:370-388, 1967 (19 pages, RDB6112)

R-116, R-152a, R-290, R-C318, R-1270, and others; mutagenic potential, toxicity; R-1270 is not mutagenic in Escherichia coli, but protects against mutations as reported in RDB6A59

W. R. Lee, S. Abrahamson, R. Valencia, E. S. von Halle, F. E. Wurgler, and S. Zimmering, The SexLinked Recessive Lethal Test for Mutagenesis in Drosophila Malanogaster Exposed to Four Fluorinated Hydrocarbon Gases (Report of the U.S. Environmental Protection Agency, EPA, Gene-Tox Program), Mutation Research, 123:183279, 1983 (97 pages, RDB6592)

\section{R-152a and others, mutagenicity, toxicity}

D. Lester and L. A. Greenberg (Yale University), Acute and Chronic Toxicity of Some Halogenated Derivatives of Methane and Ethane, Archives of Industrial Hygiene and Occupational Medicine, 2:335-344, 1950 (10 pages with 9 tables, RDB5147)

R-11, R-12, R-132bB2, R-142b, R-152a, R$1132 a$, and $R-1141$, toxicity: acute exposures of rats, for $30 \mathrm{~min}$, to concentrations of $0-80 \%$ by volume, dominant clinical response found to be narcosis evidenced by disappearance of various reflexes at increasing concentrations, death when occurring appeared to be due to respiratory failure, recommends maximum allowable concentrations; chronic exposures of rats for $16 \mathrm{hr} / \mathrm{d}$; provides data for ALC, complete anesthesia, recommended maximum allowable exposures; subchronic toxicity data for $R-142 b$ and R-152a exposures at 100,000 ppm $\mathrm{V} / \mathrm{V}$; attempts to correlate toxic effects to boiling points

G. Limperos, Inhalation Toxicity Studies of Various Freon Compounds, report 2-52, Haskell Laboratory for Toxicology and industrial Medicine, E. I. duPont de Nemours and Company, Incorporated, Newark, DE, 26 December 1951 (5 pages with 3 tables, RDB7737)

preliminary acute inhalation toxicity tests of $R$ 12B2, R-12B1, R-13B1, and R-152a (identified in the report as "Brom-12," "Chlorbrom-12," "Brom-13," and "Freon-152," respectively) in groups of two male albino rats by whole body exposures: for $\mathrm{R}-12 \mathrm{~B} 1,2 / 2$ rats died in $6-7 \mathrm{~min}$ at $100,000 \mathrm{ppm}$, severe convulsions but no deaths resulted at $57,000 \mathrm{ppm}$, violent trembling but no deaths resulted at $11,500 \mathrm{ppm}$ for $6 \mathrm{hr}, 17,000 \mathrm{ppm}$ for $2 \mathrm{hr}$, and $19,000 \mathrm{ppm}$ for 3 $\mathrm{hr}$, and no toxic effects were observed at $10,000 \mathrm{ppm}$ for $2 \mathrm{hr}$, for R-12B1, 2 of 2 rats died at $500,000 \mathrm{ppm}$ for $5 \mathrm{~min}$ (possibly with some asphyxiation effect), violent trembling and occasional convulsions but no deaths resulted at $140,000 \mathrm{ppm}$ for $1 \mathrm{hr}$, occasional nervous movements but not deaths resulted at 62,000 $\mathrm{ppm}$, and no toxic effects were observed at $31,000 \mathrm{ppm}$ for $1 \mathrm{hr}$, for R-13B1, no toxic effects, gross or microscopic pathology, or deaths resulted at either $200,000 \mathrm{ppm}$ for $2 \mathrm{hr}$ or $300,000 \mathrm{ppm}$ for $40 \mathrm{~min}$; for R-152a, occasional trembling and incoordination, but no gross or microscopic pathology or deaths resulted at either 100,000 or $200,000 \mathrm{ppm}$, and no toxic effects were observed at $74,000 \mathrm{ppm}$ for 1-3/4 hr; R-C316 and R-C317 produced no detectable cumulative toxic effects nor any pathology in groups of 10 rats exposed in subchronic studies to $10,000 \mathrm{ppm}$ for $6 \mathrm{hr}$ for at least 40 exposures

H. A. Lincoff and I. Kreissig, Intravitreal Behavior of Perfluorocarbons, Dev. Ophthalmology, 2:1723, 1981 (7 pages, RDB5C78) 
R-116, R-218, R-C318, and others; ophthalmological effects, toxicity

R. C. Lind, A. J. Gandolfi, and P. D. Hall (University of Arizona College of Medicine), Biotransformation and Hepatotoxicity of HCFC-123 in the Guinea Pig: Potentiation of Hepatic Injury by Prior Glutathione Depletion, Toxicology and Applied Pharmacology (TAP), 134(1):175-181, September 1995 (7 pages, RDB7C75)

acute toxicity of R-123: 4-hr tests in male outbred Hartley guinea pigs by inhalation exposures to $10,000 \mathrm{ppm} v / \mathrm{v} R-123$ in oxygen enriched $(400,000 \mathrm{ppm} \mathrm{v} / \mathrm{v})$ air; animals were injected $24 \mathrm{hr}$ before exposures with either a pretreatment to deplete hepatic glutathione or a control solution; R-123 caused minimal liver injury with only 1 of 8 control animals displaying confluent zone 3 necrosis; glutathione depletion potentiated injury producing submassive to massive liver necrosis in some animals; this potentiation was associated with a $36 \%$ increase in covalent binding of reactive R-123 intermediates to hepatic protein; plasma concentrations of the metabolites trifluoroacetic acid (TFA) and fluoride ion indicate that these results were not due to alterations in the biotransformation of R-123; R-123 also was found to cause a decrease in liver glutathione concentrations following exposure; concludes that R-123 inhalation [at high concentations] can cause acute hepatic injury in the guinea pig that is worsened by low hepatic glutathione concentrations

G. T. Linteris (National Institute of Standards and Technology, NIST), Acid Gas Production in Inhibited Propane-Air Diffusion Flames, Halon Replacements, Technology and Science (208th National Meeting, Washington, DC, 21-25 August 1994), ACS Symposium Series 611, American Chemical Society (ACS), Washington, DC, 225242, 1995 (8 pages, RDB6209)

R-13B1, R-22, R-116, R-124, R-134a, R-218, R-227ea, R-236fa, R-31-10, R-C318, R-410A, decomposition in flames, reaction kinetics, potential formation of corrosive byproducts containing hydrofluoric and hydrochloric acids (HF and $\mathrm{HCl}$ )

J. Little (Imperial Chemical Industries Limited (ICl), UK), The Formation of Phosgene by the Action of Hot Surfaces and Its Absence When Tobacco is Smoked in Atmospheres Containing Chlorinated Hydrocarbon Vapours, British Jour- nal of Industrial Medicine, 12:304-308, 1955 (5 pages, RDB7260)

refrigerants, toxicity: cites cautions of and disagreement by others on phosgene formation from inhaled gases in cigarette smoking and the secondary danger of formation around a glowing cigarette tip; presents an experimental study of smoking to detect phosgene at concentrations $0.05 \mathrm{ppm}$ (indicated as a tenth the tolerance limit): no phosgene was detected at a continuous smoking rate selected between the average and peak identified in other studies; moreover, phosgene was not detected in the effluent even when cigarettes were smoked in atmospheres that contained phosgene, attributed by further study to absorption by the tars in cigarette smoke; tests to detect phosgene in the air surrounding cigarettes found none to a detection level of $0.1 \mathrm{ppm}$ in atmospheres containing R-10, R-20, R-130, R-1110, $\mathrm{R}-1120$, or $0.2-20 \mathrm{ppm}$ phosgene; concludes that while phosgene does form when chlorinecontaining solvent vapors contact surfaces $>400^{\circ} \mathrm{C}\left(752^{\circ} \mathrm{F}\right)$, phosgene was not found in inhaled gases from cigarettes or the air surrounding glowing tips and that phosgene added to the atmosphere was destroyed by passage through the cigarette being smoked

F. Leuschner, B. W. Neumann, and F. Hübscher, Report on Subacute Toxicological Studies with Several Fluorocarbons in Rats and Dogs by Inhalation, Fortschritte der Arzneimittelforschung [Progress in Drug Research], 33/ll(10):1475-1476, 1983 (2 pages, RDB5607)

R-22, R-23 (subchronic NOAEL rat $=10,000$ $\mathrm{ppm}$, subchronic NOAEL dog $=5,000 \mathrm{ppm}$, as reported in RDB5604), and others, toxicity

E. Longstaff ( $\mathrm{ICl}$ Chemicals and Polymers, UK), Carcinogenic and Mutagenic Potential of Several Fluorocarbons, Living in a Chemical World: Occupational and Environmental Significance of Industrial Carcinogens, edited by $\mathrm{C}$. Maltoni and I. J. Selikoff, Annals of the New York Academy of Sciences, New York, NY, 534:283-298, 1988 (16 pages, RDB7225)

$\mathrm{R}-12$ and others, carcinogenicity, mutagenicity, toxicity

E. Longstaff, M. Robinson, C. Bradbrook, J. A. Styles, and I. F. H. Purchase ( $\mathrm{ICl}$ Chemicals and Polymers, UK), Genotoxicity and Carcinogenicity of Fluorocarbons: Assessment by ShortTerm in Vitro Tests and Chronic Exposure in 
Rats, Toxicology and Applied Pharmacology (TAP), 72(1):15-31, 1984 (32 pages, RDB5781)

R-32, R-123, R-124, R-134, R-134a, R-142b, $\mathrm{R}-143 a, \mathrm{R}-152 \mathrm{a}$, and others; negative carcinogenic findings, toxicity as reported in RDB4B90, RDB5367, RDB5862, RDB5870, RDB5C16, and RDB6686

E. Longstaff, M. Robinson, C. Bradbrook, J. A. Styles, and I. F. H. Purchase (ICl Chemicals and Polymers, UK), Carcinogenicity of Chlorofluorocarbons. Assessment by Short-Term in Vitro Tests and Chronic Exposure in Rats, The Toxicologist, 2:106 ff, 1982 (RDB6276)

toxicity

E. Longstaff, Fluorocarbons: A Summary Report of the Findings from Short-Term Predictive Tests and Long-Term Rat Carcinogenicity Studies, ICl Chemicals and Polymers, Macclesfield, Cheshire, UK, 1982 (RDB5B68)

toxicity

N. Loprieno and A. Abbondandolo, Comparative Mutagenic Evaluation of Some Industrial Compounds, Proceedings of the Symposium on ShortTerm Testing for Systematic Detection of Carcinogenicity, edited by K. H. Norpoth and R. C. Garner, Springer-Verlag, Berlin, Germany, 333-356, 1980 (24 pages, RDB5930)

R-22 and others, health effects, toxicity, mutagenicity: findings for $\mathrm{R}-22$ were negative as reported in RDB5923

R. G. Love et al., Annals of Occupational Hygiene, 22(1):43-54, 1979 (12 pages, RDB5785)

R-744 (carbon dioxide) and others, health effects, toxicity

G. Lu, S. L. Johnson, M. S. Ling, and J. C. Krantz, Jr., Anesthesia. XLI. The Anesthetic Properties of Certain Fluorinated Hydrocarbons and Ethers, Anesthesiology, 14:466-472, 1953 (7 pages, RDB65B7)

anesthetic effect, toxicity: found that some hydrogen content appears to be essential for anesthetic activity in fluorinated hydrocarbons as reported in RDB5828

G. H. W. Lucas, Study of the Fate and Toxicity of Bromine and Chlorine Containing Anesthetics, Journal of Pharmacology and Experimental Therapeutics (JPET), 34:223 ff, 1928 (RDBA628) indicates that some brominated hydrocarbons are readily decomposed in the body - as reported in RDB5136

H. R. Ludwig, S. G. Cairelli, and J. J. Whalen, Documentation for Immediately Dangerous to Life or Health Concentrations (IDLHs), National Institute for Occupational Safety and Health (NIOSH), U.S. Department of Health and Human Services, Cincinnati, OH, May 1994 (available from NTIS as document PB-94-195047, RDB5340)

This publication documents the criteria and information sources used to determine the $\mathrm{Na}$ tional Institute for Occupational Safety and Health (NIOSH) Immediately Dangerous to Life or Health (IDLH) concentrations. It addresses approximately 400 substances.

B. T. Luke et al., Theoretical Investigation of the Anaerobic Reduction of Halogenated Alkanes by Cytochrome P-450. 2. Vertical Electron Affinities of Chlorofluoromethanes as a Measure of Their Activity, Journal of the American Chemical Society, 110(11):3396-3400 (5 pages, RDB5777)

toxicity, fluorocarbons

A. Z. Lure and Z. I. Pleshkova, Comparative Characteristics of the Incidence of Upper Respiratory Tract Mucous Membrane Disease in Workers of Different Shops of a Fluorocarbon Production Plant, Zh. Ushn. Nos. Gorl. Bolezn., 3:47-50, 1977 (4 pages in Russian, RDB8162)

fluorochemical refrigerants and others, effects of human exposures, toxicity

S. Magda (Solvay Pharmaceuticals, Germany), Fluorocarbons, Toxicology, edited by $H$. Marquardt, Academic Press, San Diego, CA, 659-662, 1999 (4 pages with 2 figures and 1 table, RDBA526)

reviews the toxicity of fluorochemicals $R-32$, $R$ 123, R-124, R-125, R-134a, R-141b, R-225ca, and $R-225 \mathrm{cb}$ investigated in the Programme for Alternative Fluorocarbon Toxicity Testing (PAFT); tabulates the median lethal concentrations $\left(L C_{50}\right)$ and no observed effect levels (LOEL) from acute studies and the lowest observed adverse effect level (LOAEL, identified in the paper as the "lowest toxic concentration") and NOEL from subchronic and chronic studies; notes that fluorocarbons have very low acute toxicity by inhalation; also notes that the symptoms generally are reversible effects on the central nervous system (CNS); also indicates that the alternative fluorocarbons are 
prone to induce cardiac sensitization, but at levels well above expected human exposures; notes that increased benign tumors were observed in two-year animal studies following exposures to $R-123, R-134 a$, and $R-141 b$, but none were life threatening, all occurred late in life, and the overall evidence suggests that the incidence was not due to genotoxic effects; introduction briefly recaps concerns with chlorinated and brominated fluorochemicals for stratospheric ozone depletion; figures illustrate the catalytic chemical reactions involved and comparative ozone depletion potentials [based on data from 1987 and 1990 for chlorofluorocarbons (CFCs) and hydrochlorofluorocarbons (HCFCs) respectively]

G. Malinverno (Ausimont S.p.A., Italy), W. J. Brock (E. I. duPont de Nemours and Company, USA), and S. Magda (Solvay Pharmaceuticals, Germany), Reproduction Studies with HCFC Alternatives, EUROTOX'97, Diversification in Toxicology: Man and Environment (proceedings of the XXXVI European Congress of Toxicology, EuroTox, Århus, Denmark, 25-28 June 1997), Nordic Pharmacological Society, Copenhagen Denmark, 1997; abstract P4-38 republished in Pharmacology and Toxicology, 80(supplement III):139-140, 1997 (2 pages with no figures or tables, RDBA647)

summarizes studies of the developmental toxicities of R-32, R-123, R-124, R-125, R-134a, and $R-141 b$ along with reproductive toxicities of $\mathrm{R}-123$ and $\mathrm{R}-141 \mathrm{~b}$; indicates that no developmental toxicity was seen for any tested compound and that the no observed effect level (NOEL) for R-141b was determined to be 2000 $\mathrm{ppm} \mathrm{v} / \mathrm{v}$; also summarizes a two-generation reproduction study of R-123 in rats based on in-

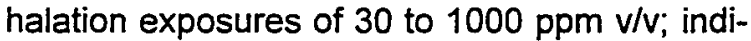
cates that no adverse effects on reproductive performance were observed; describes a crossfostering study in rats to investigate the mechanism for a decrease in body weight gain in offspring; attributes the effects to trifluoroacetic acid (TFA) content in milk from nursing rather than developmental toxicity; outlines a further study in Rhesus monkeys exposed to $1000 \mathrm{ppm} \mathrm{v/v}$ in which no effects were seen in mothers or offspring, though TFA was detected in both maternal and offspring blood

G. Malinverno and G. Bargigia (Ausimont S.p.A., Italy), Reproductive Toxicity and Teratogenicity of Alternatives to Current CFCs, Proceedings of the Sixth International Congress of Toxicology
(Rome, Italy, 28 June - 3 July 1992), International Union of Toxicology, June 1992 (RDB5B45)

toxicity of R-125 and others

C. Maltoni, G. Lefermine, D. Tivoli, and G. Perino, Long-Term Carcinogenicity Bioassays of Three Chlorofluorocarbons (Trichlorofluoromethane, FC11; Dichlorodifluoromethane, FC12; and Chlorodifluoromethane, FC22) Administered by Inhalation to Sprague-Dawley Rats and Swiss Mice, Annals of the New York Academy of Science (Living in a Chemical World), 534:261-282, 1988 (22 pages, RDB6570)

toxicity of R-11, R-12, and R-22; 104-week inhalation study in the rat and 78-week inhalation study in the mouse; findings negative

C. Maltoni, A. Ciliberti, and D. Carretti (Institute of Oncology F. Addarii, Italy), Experimental Contributions in Identifying Brain Potential Carcinogens in the Petrochemical Industry, Annals of the New York Academy of Science, 381:216-249, 1982 (34 pages with 29 tables, RDB7743)

inhalation toxicity tests to determine the incidence of brain tumors in Sprague-Dawley (and for R-1140 also Wistar) rats from exposures to selected chemicals, among them R-11 $(1,000$ and $5,000 \mathrm{ppm}$ for $4 \mathrm{hr} / \mathrm{d}, 5 \mathrm{~d} / \mathrm{wk}$, for $104 \mathrm{wk}$ ), $\mathrm{R}-12(1,000$ and $5,000 \mathrm{ppm}$ for $4 \mathrm{hr} / \mathrm{d}, 5 \mathrm{~d} / \mathrm{wk}$, for $104 \mathrm{wk}), \mathrm{R}-22(1,000$ and $5,000 \mathrm{ppm}$ for 4 $\mathrm{hr} / \mathrm{d}, 5 \mathrm{~d} / \mathrm{wk}$, for $104 \mathrm{wk}), \mathrm{R}-150, \mathrm{R}-1130 \mathrm{a}, \mathrm{R}-$ 1140 , and $R-1270(200,1,000$, and $5,000 \mathrm{ppm}$ for $7 \mathrm{hr} / \mathrm{d}, 5 \mathrm{~d} / \mathrm{wk}$, for $104 \mathrm{wk}$ ): of those listed above, only R-1140 was found to produce brain tumors, which were observed following extended exposures exceeding $500 \mathrm{ppm} \mathrm{v} / \mathrm{v}$; paper also reports ingestion tests for similar purposes with Sprague-Dawley rats for R-1120, R$1130 a, R-1132 a, R-1140$, and others; none of the compounds tested by ingestion seemed to produce brain tumors

G. Marier, G. H. MacFarland, and P. Dussalt, A Study of Blood Fluorocarbon Levels Following Exposure to a Variety of Household Aerosols, Household and Personal Products Industry, 10(12):68, 70, 92, and 99, 1973 (4 pages, RDB7540)

R-12 and others, health effects, toxicity: blood levels of R-12 were below detection limits in normal subjects using household aerosols as reported in RDB5141

T. Marti, Note sur l'intoxication par le freon [Note on the Narcotic Effect of Fluorochemicals], Ann. Med. Legale, Criminol., Police Sci., Med. So- 
ciale, Toxicol., 28:147-148, 1948 (2 pages, RDB7221)

$$
\text { toxicity of R-12; abuse; "sniffing" }
$$

J. L. Martin (National Institute of Health, NIH), J. W. Harris (Johns Hopkins Medical Institutions), A. C. LaRosa, M. J. Olson (General Motors Research Laboratories), M. W. Anders (University of Rochester Medical Center), and L. R. Pohl, Metabolism in Vivo of Halothane and the Chlorofluorocarbon Substitutes HCFC-123, HCFC124, and HFC-125 to Trifluoroacetylated Liver Protein Adducts, abstract 151, The Toxicologist, $12: 62,1992$ (1 page with no figures or tables, RDB6B53)

toxicity of R-123, R-123B1 (halothane), R-124, and R-125: rats exposed to $10,000 \mathrm{ppm}$ V/v R123 or R-123B1 produced nearly identical levels of trifluoroacetylated (TFA) liver proteins; much lower levels were found for similar exposures to R-124 or R-125; urinary trifluoroacetic acid excretion correlated with the postexposure levels of TFA adducts; concludes that R-124 and R-125 may be safer alternatives than R123 because they produce lower TFA neoantigen concentrations, since halothane hepatitis may be mediated by an immune response directed against TFA proteins in the livers of susceptible individuals

D. A. McClure, Failure of Fluorocarbon Propellants to Alter the Electrocardiogram of Mice and Dogs, Toxicology and Applied Pharmacology (TAP), 22(2):221-230, 1972 (10 pages, RDB7295)

cardiac sensitization of R-12 and others; toxicity

A. E. M. McLean, Chlorofluorocarbons - In the Can, In Man, and In the Environment, British Journal of Clinical Pharmacology, 4(6):663-666, 1977 (4 pages, RDB7265)

toxicity of R-114 and others; teratology

E. T. McHale (Atlantic Research Corporation), Habitable Atmospheres Which Do Not Support Combustion, report for contract DAHC 19-71-C0026 (also identified as ARC report 41-5521), U.S. Army Research Office, Arlington, VA, 15 March 1972 (154 pages with 5 figures and 37 tables, RDB5C79)

presents a study of use R-13B1, R-14, R-116, $\mathrm{R}-218$ in mixtures with air to evaluate atmospheres that do not support combustion of ordinary materials, but allow humans to live and function normally for extended periods; examines two modes, one with continuous use of the special atmospheres and one in which the agents were used to quench on-going fires in a situation where personnel must remain in the post-fire environment; concludes that the second mode probably is not feasible because of noxious decomposition products and smoke; indicates that flammability and toxicological studies offer favorable indications for the first mode; embeds a detailed report on toxicity tests of the four chemicals (see RDB7550 for further summary); notes that those tests address acute and ten-day, animal inhalation studies in which no adverse physiological effects were found and R-14 cardiac sensitization with only mild effects under severe conditions

W. D. McNally, A Case of Phosgene Poisoning, Industrial Medicine, 6:539 ff, 1937 (RDB6774)

refrigerants, toxicity: asserts that phosgene is formed when R-1120 (trichloroethylene) passes through burning tobacco in smoking

W. J. Meek, H. R. Hathaway, and O. S. Orth, The Effects of Ether, Chloroform, and Cyclopropane in Cardiac Automaticity, Journal of Pharmacology and Experimental Therapeutics (JPET), 61:240-252, 1937 (13 pages, RDB6562)

cardiac sensitization of R-20 (chloroform), RC270 (cyclopropane), and ether (probably R610); toxicity

J. Mendelhoff (possibly Mendeloff), Death After Repeated Exposures to Refrigerant Gases, Archives of Industrial Hygiene and Occupational Medicine, 6:518-524, 1952 (7 pages, RDB7222)

toxicity of R-12 and others; accidents; fatalities

G. W. Mergner (University of Maryland), D. A. Blake (Johns Hopkins University), and M. Helrich (University of Maryland), Biotransformation and Elimination of ${ }^{14} \mathrm{C}$-Trichlorofluoromethane (FC 11) and ${ }^{14} \mathrm{C}$-Dichlorodifluoromethane (FC 12) in Man, Anesthesiology, 42(3):345-351, March 1975 ( 7 pages with 4 figures and 1 table, RDB$5 \mathrm{C} 72)$

biochemistry and elimination tests of radiolabeled R-11 and R-12 in 2 human volunteers by inhalation of $1,000 \mathrm{ppm} \mathrm{v} / \mathrm{V}$ for 7-17 min: recovery in exhaled air in the female and male was 99.5 and $79.4 \%$, respectively, for of R-11 and 95.4 and $103.2 \%$ for R-12 (errors in collection of rapidly eliminated gases account for the differences from $100 \%$ ); only a very small fraction of the administered radioactivity (less than $0.2 \%$ ) was found in urine or exhaled carbon dioxide; total metabolites were less than $0.2 \%$ of 
the administered dose and insufficient to allow determination of possible metabolites; paper notes that the trace levels of metabolites could be products of impurities, namely chloroform and carbon tetrachloride

Meridian Research, Incorporated, Assessment of Occupational Exposures to Halon Substitutes Used for Fire Protection, U.S. Environmental Protection Agency (EPA), Washington, DC, 1992 (RDB6B54)

\section{toxicity of R-123 and others}

K. W. Miller, W. D. M. Paton, E. B. Smith, and R. A. Smith (Department of Pharmacology and Physical Chemistry Laboratory, UK), Physiochemical Approaches to the Mode of Action of General Anesthetics, Anesthesiology, 36(4):339351 . April 1972 (13 pages with 3 figures and 6 tables, RDB6129)

R-12, R-14, R-20, R-116, R-123B1, R-142B1, R-218, R-C270, R-744A (nitrous oxide), R-7146 (sulfur hexafluoride), and others, anesthetic effect, inhalation toxicity

R-J. Millischer (Elf Atochem S.A., France) and J. D. Wade $(\mathrm{ICl}$ Chemicals and Polymers, Limited, UK), Final Update of the Program for Alternative Fluorocarbon Toxicity Testing (PAFT), EUROTOX'97, Diversification in Toxicology: Man and Environment (proceedings of the XXXVI European Congress of Toxicology, EuroTox, Århus, Denmark, 25-28 June 1997), Nordic Pharmacological Society, Copenhagen Denmark, 1997; abstract P5-13 republished in Pharmacology and Toxicology, 80(supplement III):126-127, 1997 (2 pages with no figures or tables, RDBA643)

summarizes the 10-year Program for Alternative Fluorocarbon Toxicity Testing (PAFT) by 14 manufacturers of chlorofluorocarbons from Europe, America, and Asia to investigate the toxicity of alternatives including R-32, R-123, R124, R-125, R-141b, R-134a, R-225ca, and R225cb; discusses the cooperation needed; mentions that more that 200 reports were produced by 14 laboratories around the world covering acute, subacute, and chronic toxicity as well as genotoxicity, reproductive toxicity, carcinogenicity, and metabolism; notes that complementary research studies were designed to address additional questions that surfaced

R-J. Millischer (Elf Atochem S.A., France) and C. H. Farr (Elf Atochem North America, USA), Genetic Toxicity and Carcinogenicity of Fluorinated CFC Substitutes Studied within the Pro- gramme for Alternative Fluorocarbon Toxicity Testing. Proceedings of the Sixth International Congress of Toxicology (Rome, Italy, 28 June - 3 July 1992), International Union of Toxicology, June 1992; republished in Toxicology Letters Supplement, Elsevier/North-Holland Biomedical Press, Amsterdam, The Netherlands, 87-88, 1992 (RDB5B46)

genetic toxicity and carcinogenicity of R-32, R123, R-124, R-125, R-134a, R-141b, R-225ca, R-225cb

P. Montel, Toxicité des gas dans l'industrie et leur détection [Toxicity of Gases in Industry and their Detection], Archives des Maladies Professionelles de Médecine du Travail et de Securité Sociale, France, 33(9):493-496, 1972 (4 pages, in French, RDBA540)

toxicity; detection methods

R. R. Montgomery and C. F. Reinhardt, Toxicity of Fluorocarbon Propellants, Handbook of Aerosol Technology, edited by P. Saunders, Van Nostrand-Reinhold Publishers, New York, NY, 1979 (RDB65F7)

toxicity

A. F. Moore for the Cosmetic, Toiletry, and Fragrance Association (CTFA) Cosmetic Ingredient Review (CIR) Expert Panel, Safety Assessment of Isobutane, Isopentane, n-Butane, and Propane, Journal of the American College of Toxicology (JACT), 1(4):127-142, 1982 (26 pages with 5 tables, RDB6113)

summarizes a safety assessment of selected hydrocarbon $(\mathrm{HC})$ aerosol propellants that also are used as refrigerants; they include R-290 (propane), R-600 (n-butane), R-600a (isobutane), and R-601a (isopentane); discusses and tabulates summary chemical and physical properties; describes uses, derivations, common impurities, commercial purity grades, and standard containers for transport; tabulates concentrations in representative cosmetic formulations; summarizes toxicology data from a literature review and limited inputs from the CTFA membership; outlines data on mutagenicity concluding that none of the subject HCs was mutagenic by Ames assays with or without metabolic activation; discusses anesthetic effects from a published study [see RDB6197]; summarizes eye and skin irritation and tabulates primary dermal irritation data for the individual compounds and combinations of them; summarizes acute and chronic inhalation toxicity studies in animals; summarizes clinical 
acute inhalation studies in humans; summarizes recommended exposure limits; concludes that the four HCs examined are considered safe as cosmetic ingredients on the basis of the information presented and then present use; notes that $R-600$ causes drowsiness in humans at concentrations of $10,000 \mathrm{ppm}$ for $10 \mathrm{~min}$ as reported in RDB6A83

A. Morgan, A. Black, and D. R. Belcher, Studies on the Absorption of Halogenated Hydrocarbons and their Excretion in Breath Using ${ }^{38} \mathrm{Cl}$ Tracer Techniques, Annals of Occupational Hygiene, 15(2-4):273-283, 1972 (11 pages, RDB7448)

inhalation toxicity of $\mathrm{R}-11, \mathrm{R}-12, \mathrm{R}-114$, and others

A. Morgan, A. Black, M. Walsh, and D. R. Belcher, The Absorption and Retention of Inhaled Fluorinated Hydrocarbon Vapours, International Journal of Applied Radiat. Isotopes, 23(6):285291, 1972 (7 pages, RDB5C73)

inhalation toxicity of R-11, R-12, R-114, and others

M. Morita, A. Miki, H. Kazama, and M. Sakata, Case Reports of Deaths Caused by Freon Gas, Forensic Science, 10(3):253-260, 1977 (8 pages, RDB8151)

fatal human exposures involving fluorochemicals (some of which are used as refrigerants); health effects; toxicity

L. E. Morris, M. H. Noltensmeyer, J. M. White, Jr., Epinephrine Induced Cardiac Irregularities in the Dog During Anesthesia with Trichloroethylene, Cyclopropane, Ethyl Chloride, and Chloroform, Anesthesiology, 14:153-158, 1953 (6 pages, RDB6563)

toxicity tests for cardiac sensitization by $\mathrm{R}-20$ (chloroform), R-160 (ethyl chloride), R-C270 (cyclopropane), and R-1120 (1,1,2-trichloroethene

S. D. Morrison, Freon-115 Chloropentafluoroethane $(99.91 \%)$ and Freon-116 Hexafluoroethane (99.39\%): Acute Inhalation Toxicity Studies, report 41-62, Haskell Laboratory for Toxicology and Industrial Medicine, E. I. duPont de Nemours and Company, Incorporated, Newark, DE, 19 June 1962 (2 pages with 1 table, RDB7616)

tests of the acute inhalation toxicity of $R-115$ and R-116 in groups of 4 male ChR-CD rats exposed to $200,000 \mathrm{ppm} \mathrm{v/v} \mathrm{R-115} \mathrm{in} \mathrm{air} \mathrm{for} 2 \mathrm{hr}$,
$800,000 \mathrm{ppm} v / \mathrm{v} \mathrm{R}-115$ in oxygen for $3 \frac{1}{2}-4 \mathrm{hr}$, $200,000 \mathrm{ppm} v / \mathrm{v}$ R-116 in air for $2 \mathrm{hr}$, or $800,000 \mathrm{ppm}$ v/v R-116 in oxygen for $4 \mathrm{hr}$ : no deaths resulted; animals exposed to R-115 showed modified depth of respiration, but no other effect; no interference was found in body weight gain; exposed animals showed marginal signs of intoxication; animals exposed to R-116 showed deep and rapid breathing as well as at $200,000 \mathrm{ppm}$ - obvious discomfort and irregular breathing and at $800,000 \mathrm{ppm}$ cutaneous vasodilation; rats exposed to $200,000 \mathrm{ppm}$ failed to gain weight on the day after exposure while those exposed to $800,000 \mathrm{ppm}$ showed weight loss for one day after exposure; findings suggest a 31/2-4 hr ALC rat of $>800,000 \mathrm{ppm} \mathrm{v} / \mathrm{v}$ for R-115 and a $4 \mathrm{hr}$ ALC rat of $>800,000 \mathrm{ppm}$ $v / v$ for R-116

L. S. Mullin, Methods in Behavioral Toxicology, Part I: Testing of Five Industrial Chemicals, report 776-76, Haskell Laboratory for Toxicology and Industrial Medicine, E. I. duPont de Nemours and Company, Incorporated, Newark, DE, 19 October 1976 (33 pages with no figures or tables, RDBA945)

toxicity tests to determine the concentrations at which behavioral effects occur for whole-body exposures of groups of 6 male Charles River $C D$ rats; describes the test methods used for other studies pertinent to refrigerants

L. S. Mullin, A. Azar, C. F. Reinhardt, P. E. Smith, Jr., and E. F. Fabryka (E. I. duPont de Nemours and Company, Incorporated), Halogenated $\mathrm{Hy}$ drocarbon-Induced Cardiac Arrhythmias Associated with Release of Endogenous Epinephrine, American Industrial Hygiene Conference (Toronto, ON, Canada, May 1971); republished in AlHA Joumal, 33(6):389-396, June 1972 (8 pages with 3 figures and 3 tables, RDB5360)

$\mathrm{R}-11, \mathrm{R}-12, \mathrm{R}-114$, toxicity: compares cardiac abnormalities produced by endogenous epinephrine with those produced by exogenous epinephrine in beagle dogs; the results show that higher concentrations are needed to sensitize a dog's heart to the action of its own circulating level of epinephrine, raised by exercise, than by experimental injection; the observed responses were fewer and less severe; paper notes that the wide differences cannot be extrapolated directly to humans, but they suggest a sufficient margin of safety based on tests by epinephrine injection; paper also notes that humans could develop cardiac arrhythmias under conditions of stress, such as fear, anxiety, or 
exertion, by exposures to unusually high levels of propellant (many of which also are used as refrigerants) vapors

L. S. Mullin and H. J. Trochimowicz, A. Cardiac Sensitization Studies of Hexafluoroethane (F116), Sulfur Hexafluoride $\left(\mathrm{SF}_{6}\right)$, and Chloropentafluoroethane (F-115); B. Literature Review: Toxicity and Areas of Use (F-116 and $\mathrm{SF}_{6}$ ), report 150-72, Haskell Laboratory for Toxicology and Industrial Medicine, E. I. duPont de Nemours and Company, Incorporated, Newark, DE, 20 April 1972 (17 pages with 4 tables, RDB6120)

$\mathrm{R}-116$, cardiac sensitization, toxicity as reported in RDB5605

L. S. Mullin, A. Azar, C. F. Reinhardt, P. E. Smith, Jr., and E. F. Fabryka, Halogenated Hydrocarbon-Induced Cardiac Arrhythmias Associated with Release of Endogenous Epinephrine, report 279-71, Haskell Laboratory for Toxicology and Industrial Medicine, E. I. duPont de Nemours and Company, Incorporated, Newark, DE, 1971 (21 pages, RDB7442)

toxicity of R-11, R-12, R-113, R-114, and others; cardiac sensitization - as reported in RDB7414

L. S. Mullin, Cardiac Sensitization - Fright Exposures, report 81-70, Haskell Laboratory for Toxicology and Industrial Medicine, E. I. duPont de Nemours and Company, Incorporated, Newark, DE, 1970 (5 pages, RDB6522)

toxicity of R-12, R-142b, R-C318 and others; effect of frightening noise to stimulate the release of endogenous epinephrine in cardiac sensitization as reported in RDB5869 and RDB7215

L. S. Mullin, Cardiac Sensitization, report 354-69, Haskell Laboratory for Toxicology and Industrial Medicine, E. I. duPont de Nemours and Company, Incorporated, Newark, DE, 1969 (3 pages with 1 table, RDB6274)

reports toxity tests to determine cardiac sensitization levels in dogs for R-E170 (dimethyl ether), R-142b, R-152a, R-290 (propane), R502, R-1110 (perchloroethylene), and R-1140 (vinyl chloride) [see RDB5644 for further description of the test method and findings]

L. S. Mullin, Cardiac Sensitization: Fright/Treadmill Studies, report 325-69, Haskell Laboratory for Toxicology and Industrial Medicine, E. I. duPont de Nemours and Company, Incorporated, Newark, DE, 1969 (RDB6B46) cardiac sensitization, toxicity

W. Nastainczyk, H. J. Ahr, and V. Ullrich, The Mechanism of the Reductive Dehalogenation of Polyhalogenated Compounds by Microsomal Cytochrome P-450, Advances in Experimental Med. Biol., 136A:799-808, 1982 (10 pages, RDB8163)

fluorochemical refrigerants, biochemistry, health effects, metabolism, pharmacokinetics, toxicity

W. Nastainczyk, H. J. Ahr, and V. Ullrich, The Reductive Metabolism of Halogenated Alkanes by Liver Microsomal Cytochrome P-450, Biochemical Pharmacology, UK, 31(3):391-396, 1982 (6 pages, RDB8164)

fluorochemical refrigerants, biochemistry, health effects, mutagenicity, pharmacokinetics, teratogenicity, toxicity

National Research Council (NRC) Committee on Toxicology, Toxicity of Alternatives to Chlorofluorocarbons: HFC-134a and HCFC-123, National Academy of Sciences (NAS), National Academy Press, Washington, DC, 1996 (134 pages with 7 figures and 21 tables, RDB6A01)

$\mathrm{R}-123, \mathrm{R}-134 \mathrm{a}$, assessment by the Board on Environmental Studies and Toxicology: briefly reviews the history of "cardiac sensitization" concern arising from "sniffing" and industrial fatalities; examination of the standard dog cardiac sensitization test, using a sequence of start with face mask in place at 0 minutes, administer epinephrine injection at 2 minutes, begin test chemical exposure by inhalation at 7 minutes, administer challenge injection of epinephrine at 12 minutes, and stop at 17 minutes; concludes that the test is effective, but overpredicts the potential for a chemical to induce cardiac arrhythmia under normal or physiological levels of endogenous epinephrine; recommends that the mechanism of cardiac sensitization be determined and that a more sensitive test be developed; examines toxicity data for R123 and R-134a and sets recommended emergency exposure guidance level (EEGL) concentrations for both and a continuous exposure guidance level (CEGL) for the former; differences in the time periods for the R-123 and R$134 a$ exposure levels addressed reflect proposed uses of R-134a as a refrigerant on submarines, versus $R-123$ as a fire suppressant to replace halon 12 in flight-line extinguishers with very quick discharges; recommends a $1 \mathrm{hr}$ EEGL for R-134a of $4,000 \mathrm{ppm} v / \mathrm{v}$ based on 
the no-observed-adverse-effect level (NOAEL) in cardiac sensitization tests, divided by an uncertainty factor of 10 for interspecies variability; recommends a $24 \mathrm{hr}$ EEGL for R-134a of 1,000 ppm based on a NOAEL of $10,000 \mathrm{ppm}$ for ferotoxicity effects (slight retardation of skeletal ossification) in rats divided by an uncertainty factor of 10; recommends a 90-day CEGL for R-134a of $900 \mathrm{ppm}$ based on a NOAEL of $50,000 \mathrm{ppm}$ for a 2-yr chronic toxicity study in rats divided by an uncertainty factor of 10 , a factor of 4 for exposures of $24 \mathrm{hr} / \mathrm{d}$ instead of 6 $\mathrm{hr} / \mathrm{d}$, and a factor of $5 / 7$ for exposures of $7 \mathrm{~d} / \mathrm{wk}$ instead of the tested $5 \mathrm{~d} / \mathrm{wk}$; recommends a 1 min EEGL for R-123 of $1,900 \mathrm{ppm}$ based on a cardiac sensitization $E_{50}$ dog of $19,000 \mathrm{ppm}$ divided by an uncertainty factor of 10; appendices and attachments provide supporting documentation of toxicity data for R-123, R-123B1, and $\mathrm{R}-134 \mathrm{a}$

National Research Council (NRC) Committee on Toxicology, Emergency and Continuous Exposure Limits for Selected Airborne Contaminants, National Academy of Sciences (NAS), National Academy Press, Washington, DC, volume 8 , 1988 (78 pages with 1 figure and 6 tables, available from NTIS as document PB-89-174726, RDB7420)

examines the toxicity and other health effects of R-1120 and lithium chromate; recommends Emergency Exposure Guidance Level (EEGL) and Continuous Exposure Guidance Level (CEGL) concentrations

National Research Council (NRC) Committee on Toxicology, Emergency and Continuous Exposure Limits for Selected Airborne Contaminants, National Academy of Sciences (NAS), National Academy Press, Washington, DC, volume 7, 1987 (78 pages with 8 tables, RDB7419)

examines the toxicity and other health effects of R-717 (ammonia), hydrogen chloride, lithium bromide, and toluene; recommends Emergency Exposure Guidance Level (EEGL) and Continuous Exposure Guidance Level (CEGL) concentrations; the EEGL for ammonia is $100 \mathrm{ppm} \mathrm{v/v}$ for both $1 \mathrm{hr}$ and $24 \mathrm{hr}$

National Research Council (NRC) Committee on Toxicology, Emergency and Continuous Exposure Limits for Selected Airborne Contaminants, National Academy of Sciences (NAS), National Academy Press, Washington, DC, volume 6, 1986 (64 pages with 7 tables, available from NTIS as document PB-86-145158, RDB7418) examines the toxicity and other health effects of hydrazine, monomethylhydrazine, and 1,1dimethylhydrazine; recommends Emergency Exposure Guidance Level (EEGL) and Continuous Exposure Guidance Level (CEGL) concentrations; presents a method for cancer risk assessment for emergency exposures

National Research Council (NRC) Committee on Toxicology, Emergency and Continuous Exposure Limits for Selected Airborne Contaminants, National Academy of Sciences (NAS), National Academy Press, Washington, DC, volume 5, 1985 (64 pages with 7 tables, available from NTIS as document PB-86-145158, RDB7417)

examines the toxicity and other health effects of hydrazine, monomethylhydrazine, and 1,1-dimethylhydrazine; recommends Emergency Exposure Guidance Level (EEGL) and Continuous Exposure Guidance Level (CEGL) concentrations; presents a method for cancer risk assessment for emergency exposures

National Research Council (NRC) Committee on Toxicology, Emergency and Continuous Exposure Limits for Selected Airborne Contaminants, National Academy of Sciences (NAS), National Academy Press, Washington, DC, volume 4, 1985 (120 pages with 1 figure and 14 tables, available from NTIS as document PB-85-239630, RDB7416)

examines the toxicity and other health effects of R-728 (nitrogen), R-744A (nitrous oxide), R-746 (nitrogen dioxide), aluminum oxide, carbon monoxide, ethylene glycol, hydrogen sulfide, methanol, and tetroxide; announces a change in nomenclature from Emergency Exposure Limit (EEL) to Emergency Exposure Guidance Level (EEGL) and Continuous Exposure Limit (CEL) to Continuous Exposure Guidance Level (CEGL) to avoid confusion with limits set by regulatory agencies

National Research Council (NRC) Committee on Toxicology, Emergency and Continuous Exposure Limits for Selected Airborne Contaminants, National Academy of Sciences (NAS), National Academy Press, Washington, DC, volume 3, October 1984 (32 pages with 4 tables, RDB7415)

recommended Emergency Exposure Limit (EEL) and Continuous Exposure Limit (CEL) concentrations in enclosed environments, not intended for application in general industrial settings or as exposure limits for the general public; this volume addresses R-13B1; defines $E E L$ as "a ceiling limit for an unpredictable sin- 
gle exposure, usually lasting 60 minutes or less, and never more than $24 \mathrm{~h}$ - an occurrence expected to be rare in the lifetime of any person" addressing nonincapacitating, reversible effects; defines CEL as "a ceiling limit to avoid adverse health effects, either immediate or delayed, and to avoid degradation in crew performance that might endanger the objectives of a particular mission;" summarizes the physical and chemical properties, occurrence, use, acute and chronic toxicity information, inhalation exposure limits set by others, recommended exposure limits, recommended further research, and references for each chemical; prepared for the U.S. Department of Defense (DOD) and National Aeronautics and Space Administration (NASA) to update previous and to recommend new exposure limits for selected chemicals; EELS and CELs do not take into consideration the possible effects of exposure on hypersensitive people [EEL and CEL concentrations were subsequently renamed Emergency Exposure Guidance Level (EEGL) and Continuous Exposure Guidance Level (CEGL), respectively - see RDB7416]

National Research Council (NRC) Committee on Toxicology, Emergency and Continuous Exposure Limits for Selected Airborne Contaminants, National Academy of Sciences (NAS), National Academy Press, Washington, DC, volume 2, October 1984 (134 pages with 11 tables, RDB7414)

recommended Emergency Exposure Limit (EEL) and Continuous Exposure Limit (CEL) concentrations in enclosed environments, not intended for application in general industrial settings or as exposure limits for the general public; this volume addresses for chlorine, chlorine trifluoride, ethanolamine, R-11, R-12, R-21, $R-113, R-114$, isopropyl alcohol, phosgene, sodium hydroxide, R-764 (sulfur dioxide), R$1130 \mathrm{a}$ (vinylidene chloride), and xylene; defines EEL as "a ceiling limit for an unpredictable single exposure, usually lasting 60 minutes or less, and never more than $24 \mathrm{~h}-$ an occurrence expected to be rare in the lifetime of any person" addressing nonincapacitating, reversible effects; defines CEL as "a ceiling limit to avoid adverse health effects, either immediate or delayed, and to avoid degradation in crew performance that might endanger the objectives of a particular mission;" summarizes the physical and chemical properties, occurrence, use, acute and chronic toxicity information, inhalation exposure limits set by others, recom- mended exposure limits, recommended further research, and references for each chemical; prepared for the U.S. Department of Defense (DOD) and National Aeronautics and Space Administration (NASA) to update previous and to recommend new exposure limits for selected chemicals; EELS and CELs do not take into consideration the possible effects of exposure on hypersensitive people [EEL and CEL concentrations were subsequently renamed Emergency Exposure Guidance Level (EEGL) and Continuous Exposure Guidance Level (CEGL), respectively - see RDB7416]

National Research Council (NRC) Committee on Toxicology, Emergency and Continuous Exposure Limits for Selected Airborne Contaminants, National Academy of Sciences (NAS), National Academy Press, Washington, DC, volume 1, April 1984 (122 pages with 24 tables, available from NTIS as document AD-4142-13318, RDB7413)

recommended Emergency Exposure Limit (EEL) and Continuous Exposure Limit (CEL) concentrations in enclosed environments, not intended for application in general industrial settings or as exposure limits for the general public; this volume addresses for acetone, acrolein, arsine, carbon disulfide, R-20 (chloroform), fluorine, mercury vapor, R-50 (methane), R-748 (ozone), and sulfuric acid; defines EEL as "a ceiling limit for an unpredictable single exposure, usually lasting 60 minutes or less, and never more than $24 \mathrm{~h}$ - an occurrence expected to be rare in the lifetime of any person" addressing nonincapacitating, reversible effects; defines CEL as "a ceiling limit to avoid adverse health effects, either immediate or delayed, and to avoid degradation in crew performance that might endanger the objectives of a particular mission;" summarizes the physical and chemical properties, occurrence, use, acute and chronic toxicity information, inhalation exposure limits set by others, recommended exposure limits, recommended further research, and references for each chemical; prepared for the U.S. Department of Defense (DOD) and National Aeronautics and Space Administration (NASA) to update previous and to recommend new exposure limits for selected chemicals; EELs and CELs do not take into consideration the possible effects of exposure on hypersensitive people [EEL and CEL concentrations were subsequently renamed Emergency Exposure Guidance Level (EEGL) and 
Continuous Exposure Guidance Level (CEGL), respectively - see RDB7416]

National Research Council (NRC) Committee on Toxicology, Criteria for Short-Term Exposures to Air Pollutants, National Academy of Sciences (NAS), National Academy Press, Washington, DC, 1979 (15 pages, RDB7412)

definitions, applicability, principles, and criteria for recommended Emergency Exposure Limit (EEL), Short-Term Public Limit (STPL), and Short-Term Public Emergency Limit (SPEL) concentrations: R-11, R-12, R-113, R-114, R764 (sulfur dioxide), R-1130a (vinylidene chloride), and others

National Research Council (NRC) Committee on Toxicology, Basis for Establishing Guides for Short-Term Exposures of the Public to Air Pollutants, National Academy of Sciences (NAS), National Academy Press, Washington, DC, 1971 (15 pages, RDB7411)

definitions, applicability, and criteria for recommended Emergency Exposure Limit (EEL), Short-Term Public Limit (STPL), and Public Emergency Limit (PEL) [later renamed the Short-Term Public Emergency Limit (SPEL)] concentrations: R-11, R-12, R-113, R-114, R764 (sulfur dioxide), R-1130a (vinylidene chloride), and others

S. Niazi and W. L. Chiou, Fluorocarbon Aerosol Propellants VI: Interspecies Differences in Solubilities in Blood and Plasma and their Possible Implications in Toxicity Studies, Journal of Pharmaceutical Sciences, 64(9):1538-1541, 1975 (3 pages, RDB7258)

$\mathrm{R}-12, \mathrm{R}-114$, and others, health effects, toxicity

M. S. Nick, Freon-115 ${ }^{\circledR}$, Highest Purity $(99.99 \%)$, Freon-115 ${ }^{\circledR}$, Commercial Composite (99.98\%), and Freon-115 ${ }^{\circledR} /$ Freon-C $318^{\circledR}$ (20/80): Acute Inhalation Toxicity, report 11-65, Haskell Laboratory for Toxicology and Industrial Medicine, E. I. duPont de Nemours and Company, Incorporated, Newark, DE, 5 February 1965 (2 pages with 1 table, RDB7617)

tests of the acute toxicity of R-115 and R$115 /$ C318 $(20 / 80)$ by inhalation exposure of groups of 4 male ChR-CD rats to concentrations of $800,000 \mathrm{ppm} v / \mathrm{v}$ in oxygen for $4 \mathrm{hr}$ : no deaths resulted, but animals exposed to R-115 exhibited rapid and deep respiration and inactivity; animals exposed to the R-115/C318 blend exhibited deep respiration and slightly red extremities; recovery was immediate fol- lowing all exposures anno interference was found with body weight gain; no compoundrelated effects were found by microscopic pathological examinations at necropsy; findings suggest a $4 \mathrm{hr}$ ALC rat $>800,000 \mathrm{ppm} \mathrm{v} / \mathrm{v}$ for both R-115 and R-115/C318 (20/80)

T. K. Nikijenko and T. A. Kochetkova, Effect of Some Hydrochlorofluorocarbons on the Thyroid (Experimental Study), Toksikologiya Novykh Promyshlennykh Khimicheskikh Veshchestva [Toxicology of New Industrial Chemical Substances], Moscow, Russia (then USSR), 9:175182, 1967 (8 pages in Russian, RDB5853)

toxicity of R-141b, R-142b, and other HCFCs

T. K. Nikijenko and M. S. Tolgskaya (Institute for Hygiene at Work and Occupational Illnesses of the AMS, USSR), On the Toxico-Pharmamorphological Changes in Animals Under the Effect of Freon ${ }^{\circledR} S 141,142$, and 143 and the Intermediates Used in Their Production, Gigiena Truda $i$ Professional'nye Zabolevaniya [Labor Hygiene and Occupational Diseases], Moscow, Russia (then USSR), 9(10):37-44, 1965 (8 pages in Russian, RDB5852)

toxicity of R-141b, R-142b, and R-143a

Occupational Safety and Health Administration (OSHA) of the U.S. Department of Labor, Air Contaminants; Rule, 58 FR 124, Federal Register, U.S. Government Printing Office, Washington, DC, 35338-35351, 30 June 1993 (14 pages, available from JMC as RDB3904)

This rule revokes the Final Rule exposure limits in Table Z-1-A (Limits for Air Contaminants) of 29 CFR 1910.1000. It leaves the Permissible Exposure Limits (PELs) included in Table Z-1 before 1989, also published as Transitional Limits in table Z-1-A, in effect. Tables Z-2 and $Z-3$ (Mineral Dusts) also remain in effect. The rule reviews the court decision leading to this action and provides the three tables for enforcement of the action. A number of refrigerants are covered. PEL values are provided for R-10, R-11, R-12, R-12B2, R-13B1, R-20B3, R21, R-30, R-40, R-40B1, R-4011, R-110, R-112, R-112a, R-113, R-114, R-130, R-140, R-140a, R-160, R-160a, R-160B1, R-290, R-601, R602, R-610, R-611, R-630, R-717, R-744, R746, R-748, R-764, R-7146, R-1110, R-1120, and others. This rule is subject to revision and may have been amended or withdrawn.

Occupational Safety and Health Administration (OSHA) of the U.S. Department of Labor, Code of 
Federal Regulations, Air Contaminants, 29 CFR Part 1910, U.S. Government Printing Office, as published at 54 FR 2920, 19 January 1989 (RDB3905)

Permissible Exposure Limit (PEL) values: This regulation is subject to revision and may have been amended or withdrawn.

H. J. Oettel, Zentr. Gewerbehyg. Unfallverhutl., Germany, 28:131 ff., 1941 (RDB6944)

toxicity of R-290 (propane), R-600 (n-butane), R-600a (isobutane), R-601a (isopentane), and others; contact with the skin may result in frostbite; effects on skin and eyes - as reported in RDBA538

H. J. Oettel, Archiv für Experimentelle Pathologie und Pharmakologie, 183:641-696, 1936 (2 pages, RDB6195)

toxicity of R-290 (propane), R-600 (n-butane), R-600a (isobutane), R-601a (isopentane), and others; effects on skin and eyes - as reported in RDBA538

H. J. Oettel, Bericht 8 Intern. Kongr. Unfallmed. Bercifekrankh., Germany, 2:1228 ff, 1936 (RDB6A87)

toxicity of R-290 (propane), R-600 (n-butane), R-600a (isobutane), R-601a (isopentane), and others; effects on skin and eyes - as reported in RDBA538

L. Olander, A. Colmsjo, B. Holmberg, S. Krantz, and $U$. Landstrom, Occupational Implications of the Chlorofluorocarbon Ban in Sweden, American Journal of Industrial Medicine, 19(6):818-826, 1991 (9 pages, RDB6B58)

toxicity of chlorofluorocarbon (CFC) and alternative refrigerants; noise and vibration levels for refrigeration and freezing equipment; handling; additional problems including increased energy use both to manufacture the substitutes and for their use

R. S. Oremland (U.S. Geological Survey, USGS), D. J. Lonergan (University of Massachusetts), C. W. Culbertson (USGS), and D. R. Lovley (University of Massachusetts), Microbial Degradation of Hydrochlorofluorocarbons $\quad\left(\mathrm{CHCl}_{2} \mathrm{~F}\right.$ and $\mathrm{CHCl}_{2} \mathrm{CF}_{3}$ ) in Soils and Sediments, Applied and Environmental Microbiology, 62(5):1818-1821, May 1996 (4 pages with 4 figures, RDB7C69)

ecotoxicity of R-21 and R-123: examines the ability of microorganisms to degrade trace levels of these chemicals; for R-21, methanotrophlinked oxidation was observed in aerobic soils and anaerobic degradation in freshwater and salt marsh sediments; for R-123, microbial degradation was observed in anoxic freshwater and salt marsh sediments, and recovery of R133a indicates the involvement of reductive dechlorination; no degradation of R-123 was observed in aerobic soils; concludes that degradation at low (parts per billion) concentrations suggests that bacteria may remove hydrochlorofluorocarbons (HCFCs) from the atmosphere, but further study is needed to assess this ability and its global significance as a sink for HCFCs

H. Oujesky and L. Job, Effects of Five Gaseous Atmospheres on the Survival Rate of DNA Synthesis in Escherichia Coli, Develop. Ind. Microbiology, 18:451-456, 1977 (6 pages, RDB6514)

toxicity of R-12, R-23, and others; biochemical studies; mutagenicity

H. Oujesky and R. Fuerst, Effects of Freon ${ }^{\otimes_{S}}$ and Genetron ${ }^{\circledR} S$ on DNA Polymerase from E. Coli, Develop. Ind. Microbiology, 15:405-410, 1974 (6 pages, RDB5C82)

mutagenicity of R-23, R-152a, R-C318, and others; toxicity

H. Oujesky and I. Bhagat, Response of Staphylococcus Aureus to Atmospheres of Freon ${ }^{\circledR} \mathrm{S}$ and Genetron ${ }^{\circledR}$ S, Develop. Ind. Microbiology, 14:229237, 1973 (9 pages, RDB5823)

toxicity of R-23, R-152a, and others; mutagenicity

J. W. Patterson, M. F. Studlow, and S. R. Walker, Blood Levels of Fluorinated Hydrocarbons in Asthmatic Patients After Inhalation of Pressurized Aerosols, Lancet, UK, 2:565-568, 1971 (3 pages, RDB6728)

toxicity of R-12 and others; fluorochemical blood levels in humans; pharmacokinetics; based on a study for use as aerosol propellants (compounds also are used as refrigerants)

G. Paulet, Les Fluorocarbones en Question [Fluorocarbons at Issue], European Journal of Toxicology and Environmental Hygiene, 9(supplement to 7):385-407, 1976 (23 pages in French, RDB5179)

$\mathrm{R}-12, \mathrm{R}-114$, and others, toxicity: for R-114, $30-$ min $L_{50}$ rat $=720,000 \mathrm{ppm}$ as reported in RDB5340

G. Paulet, The Fluorocarbons at Issue, Aerosol Report, 16(2):44-49,52, 1977 (7 pages, RDB6126) 
$\mathrm{R}-12, \mathrm{R}-114$, and others, inhalation toxicity

G. Paulet, The Fluorocarbons at Issue, Aerosol Report, 16(1):22-30, 1977 (9 pages, RDB6125)

$\mathrm{R}-12, \mathrm{R}-114$, and others, inhalation toxicity

G. Paulet and Y. Lessard, Actions of Fluorocarbons 12 (Difluorodichloromethane) and 11 (Monofluorotrichloromethane) on Smooth Muscle, Compte Rendu des Séances de la Société Biol. [Proceedings of the Meeting of the Biological Society], Paris, France, 169(3):665-671, 1975 (7 pages, in French, RDB7531)

R-11 (trichlorofluoromethane), R-12 (dichlorodifluoromethane), health effects, toxicity

G. Paulet, G. Roncin, E. Vidal, P. Toulouse, and J. Dassonville, Fluorocarbon and General Metabolism in the Rat, Rabbit, and Dog, Toxicology and Applied Pharmacology (TAP), 34:197-203, 1975 (7 pages, RDB5142)

$\mathrm{R}-12$ and others, health effects, toxicity

G. Paulet, J. Lanoë, A. Thos, P. Toulouse, and J. Dassonville (Laboratoire de Physiologie Médicale, U.E.R. Médicales et Pharmaceutiques, France), Fate of Fluorocarbons in the Dog and Rabbit After Inhalation, Toxicology and Applied Pharmacology (TAP), 34(2):204-213, November 1975 (10 pages with 6 figures and 5 tables, RDB5C71)

R-11 and R-12, toxicity: inhalation of R-11 at 25,000 and $50,000 \mathrm{ppm}, \mathrm{R}-12$ at 200,000 and $500,000 \mathrm{ppm}$, and R-12/11 (90/10) at 250,000 and $900,000 \mathrm{ppm}$ by anesthetized dogs and rabbits through tracheal cannula with oxygen adjusted to 200,000 ppm; animals were tested for these chemicals in their exhaled air, blood, bile, cerobrospinal fluid, and urine to measure elimination; concluded that the chemicals diffuse quickly in the body fluids, but that elimination is completed in $20-50$ minutes, $98 \%$ of it through the pulmonary system

G. Paulet, S. Desbrousses, and E. Vidal, Absence d'Effet Teratogene des Fluorocarbons Chez le Rat et le Lapin [Absence of Teratogenic Effect in the Rat and Rabbit], Archives des Maladies Professionelles de Médecine du Travail et de Securité Sociale, France, 35:658 ff, 1974 (in French, RDB7539)

$R-11, R-12, R-12 / 11$ (90/10), and others, genetic and reproductive effects, teratogenicity, toxicity: no effect in rats and rabbits at 300,000 ppm R-12/11 (90/10) for 13-16 2-hr exposures as reported in RDB5141
G. Paulet, Action of Fluorohydrocarbons Used in Aerosols: Problems of Their Retention by the Organism After Inhalation, Trib. CEBE$D E A U, 23(324): 487-497,1970$ (11 pages, RDB7449)

R-114 and others, health effects, toxicity

G. Paulet, Aerosol Report, 8(12):612-622, 1969 (11 pages, RDB5148)

R-114 and others, health effects, toxicity

G. Paulet, De l'action des hydrocarbures chlorofluorés sur l'organisme [On the Effect of Chlorofluorocarbons on Organisms], Labo-Pharma. Probl. Tech., France, 180:74-78, 1969 (RDB8168)

CFC biochemistry, fluorocarbons, toxicity

G. Paulet, R. Chevrier, J. Paulet, M. Duchene, and J. Chappet, De la rétention des fréons par les poumons et les voies aériennes [On the Retention of Fluorocarbons by the Lungs and Air Passages], Archives des Maladies Professionelles de Médecine du Travail et de Securité Sociale, France, 30(3):101-120, 1969 (20 pages, in French, RDB5370)

$\mathrm{R}-12$ and others, biochemistry, fluorocarbons, toxicity

G. Paulet and R. Chevrier, Modalités de l'élimination par l'air expiré du fluorane 11 inhalé. Étude chez l'homme et chez l'animal [Mechanisms of Elimination of Inhaled R-11 by Expired Air. Human and Animal Study], Archives des Maladies Professionelles de Médecine du Travail et de Securité Sociale, France, 30(4-5):251-256, 1969 (in French, RDB7296)

biochemistry, fluorocarbons, toxicity

J. A. Prendergast, R. A. Jones, L. J. Jenkins, Jr., and J. Siegel (National Naval Medical Center, U.S. Navy), Effects on Experimental Animals of Long Term Inhalation of Trichloroethylene, Carbon Tetrachloride, 1,1,1-Trichloroethane, Dichlorodifluoromethane, and 1,1-Dichloroethylene, Toxicology and Applied Pharmacology (TAP), 10(2):270-289, March 1967 (20 pages with 1 figure and 4 tables, RDB5C44)

R-10, R-12, R-140a with 8\% inhibitor, R-1120, R-1130a, subchronic inhalation toxicity: effects of long-term inhalation exposure to develop data pertinent to human exposures in submarines; continuous exposures of rats, guinea pigs, monkeys and dogs for 90 days and repeated exposures for $8 \mathrm{hr} / \mathrm{d}, 5 \mathrm{~d} / \mathrm{wk}$, for 6 weeks; 2 of 15 rats and 1 guinea pig died during continuous exposures to R-12 at $840 \mathrm{ppm}$ 
$\left(3,997 \mathrm{mg} / \mathrm{m}^{3}\right) ; 1$ of 15 rats died in the repeated exposures to $\mathrm{R}-12$ at $4,136 \mathrm{mg} / \mathrm{m}^{3}$; no other signs of toxicity were observed for R-12, suggesting that the mortality was not treatment related

K. Pennington and R. Fuerst (Helen Dwight Reid Educational Foundation), Biochemical and Morphological Effects of Various Gases on Rabbit Erythrocytes, Archives of Environmental Health, 22(4):476-481, 1971 (6 pages, RDB6131)

R-50, R-152a and others; biochemical studies, toxicity

H. Peter, J. G. Filser, L. V. Sventpály, and H. J. Wiegand (Universität Dortmund, Germany), Different Pharmacokinetics of Dichlorodifluoromethane (CFC 21) [HCFC-21] and Chlorodifluoromethane (CFC 22) [HCFC-22], Archives of Toxicology, 58:282-283, 1986 (2 pages, RDBA533)

toxicity of R-21 and R-22; carcinogenicity; mutagenicity; health effects from exposures; animal studies

Z. Petrovic, Abuse of Inhalants, Solvents, and Volatile Substances, Psihijatr Danas, 16(34):273-280, 1984 (8 pages, RDB7549)

human exposures, sudden sniffing deaths from abusive use of aerosol products also used as refrigerants

A. Poklis, Determination of Fluorocarbon 11 and Fluorocarbon 12 in Postmortem Tissues: A Case Report, Forensic Science, 5(1):53-60, 1975 (8 pages, RDB6729)

fatal human exposures involving R-11 and R12 , sudden sniffing death from abusive use of aerosol products

C. L. Potter et al., Effects of Inhaled Chlorotrifluoroethylene and Hexafluoropropene on the Rat Kidney Toxicology and Applied Pharmacology (TAP), 59(3):431 ff, 1981 (RDB8125)

tests of acute inhalation toxicity of R-1113 and R-1216 by exposures of male Fischer 344 rats: rats exposed to concentrations of $380-1200$ ppm exhibited dose-related, proximal tubular necrosis, diuresis, increases in urinary fluoride, urinary lactic dehyrogenase (LDH) activity, serum creatinine, and blood urea nitrogen

I. F. H. Purchase, E. Longstaff, J. Ashby, J. A. Styles, D. Anderson, P. A. Lefevre, and F. R. Westwood ( $\mathrm{ICl}$ Chemicals and Polymers, UK), An Evaluation of Six Short-Term Tests for Detect- ing Organic Chemical Carcinogens, British Journal of Cancer, 37:873 ff, 1978 (RDB6277)

toxicity, carcinogenicity

A. Quevauviller, Hygiene et Securité des Pulseurs pour Aerosols Médicamentaux [Hygiene and Safety of Propellants for Medicated Aerosols], Prod. Probl. Pharm., 20(1):14-29, 1965 (16 pages in French, RDB5A72)

$\mathrm{R}-12, \mathrm{R}-114$, and others, toxicity, eye and skin irritation

A. Quevauviller, M. Schrenzel, and V. Ngoc Huyen, Tolérance Locale (Peau, Muqueuses, Plaies, Brûlures) Chez l'Animal aux Hydrocarbures Chlorofluores [Local Tolerance (Skin, Mucous Membranes, Sores, and Burns) of Animals to Chlorofluorohydrocarbons], Therapie, 19:247-263, 1964 (17 pages in French, RDB5368)

R-12, R-22, R-114, and others, toxicity, eye and skin irritation, hydrochlorofluorocarbons (HCFC): daily dose of R-12 tolerated by rats for a period of 23-33 days as reported in $\mathrm{RDB} 65 \mathrm{H} 1$

A. Quevauviller, J. Billon, J. Longuet, and R. Pierre, Tolerance of the Lungs of Mice to Fluorinated Hydrocarbons, Ann. Pharm. Français, France, 27(1):25-28, 1969 (4 pages, RDB7237)

$\mathrm{R}-12, \mathrm{R}-115$, and others, toxicity

A. Quevauviller, M. Chaigneau, and M. Schrenzel, Experimental Studies with Mice on Tolerance of the Lungs Mice to Chlorofluorinated Hydrocarbons, Ann. Pharm. Français, France, 21(11):727-734, 1963 (8 pages in French, RDB6128)

$\mathrm{R}-11, \mathrm{R}-12, \mathrm{R}-22$ and others, toxicity, hydrochlorofluorocarbons (HCFC)

A. Quevauviller, Skin Tolerance of Chlorofluoromethanes Used as Propellants in Cosmetology, Parfum. Cosmet. Savons, France, 3:228-230, 1960 (3 pages in French, RDB7428)

R-11 and others, health effects when used as an aerosol propellant, toxicity: well tolerated by the skin, but retarded recovery from wounds and burns as well as regrowth of hair as reported in RDB7414

J. Ramsey, H. R. Anderson, K. Bloor, and R. J. Flanagan, An Introduction to the Practice, Prevalence, and Chemical Toxicology of Volatile Substance Abuse, Human Toxicology, 8(4):261-269, 1989 (9 pages, RDB7455) 
reviews abuse of volatile substance by "sniffing", noting that it results in approximately 100 deaths per year in the UK and that the victims are mostly teenagers, come from all social classes, and are $90 \%$ males: $3 \frac{1}{2}-10 \%$ of young people in the UK have at least experimented and $0.5-1 \%$ are current users; many products are involved, but approximately 20 solvents, adhesives, fuels, and aerosol propellants (some of which also are used as refrigerants) are commonly encountered; specifically identifies toluene, chlorinated solvents such as R-140a (methyl chloroform), R-600 butane, and hydrocarbon and halogenated hydrocarbon propellants as abused substances; acute hazards varies with the compound, product, and mode of abuse; chronic toxicity is difficult to assess, partly because of the diversity of substances involved, but some long-term abusers suffer permanent damage to the central nervous system (CNS), heart, liver, and kidneys; preventive measures including guidelines for sale of the products involved, education, and legislation have had little impact

A. G. Rauws, M. Olling, and A. E. Wibowo, Determination of Fluorochlorocarbons in Air and Body Fluids, Journal of Pharmacy and Pharmacology, UK, 25(9):718-722, 1973 (5 pages, RDB7548)

R-114 and others, health effects, toxicity

J. Raventós and P. G. Lemon (Imperial Chemical Industries Limited, UK), The Impurities of Fluoroethane: Their Biological Properties, British Journal of Anaesthesia, UK, 37(10):716-737, 1965 (22 pages with 20 figures and 6 tables, RDB6165)

examines the anesthetic potential, lethality, and other toxicity effects of impurities found in R123B1 (halothane); tabulates the $30-\mathrm{min}^{2} \mathrm{LC}_{50}$ and $30-$ min $\mathrm{AC}_{50}$ (median anesthetic concentration) based on tests in groups of ten mice for R-11B1, R-20, R-113, R-113aB1, R-113aB2, R123, R-123B1, R-123B2, R-132b, R-133a, R133aB1, R-161, R-C326, R-1316, R-1326t, and $\mathrm{R}-1326 \mathrm{tB1}$; indicates the 30-min $\mathrm{LC}_{50}$ mouse and $30-$ min $A C_{50}$ (median anesthetic concentration) mouse in $\mathrm{ppm} v / \mathrm{v}$ as $>100,000$ and 57,000 for R-113 and 74,000 and 24,000 for R123 , respectively

H. Reichelt, Toxicity and Mode of Action of Practically Important Fluorocarbons, Prevention and Treatment of Exposures, Zeitschrift für die gesamte Hygiene und ihre Grenzgebiete, 30(4), 1984 (RDB7238)

R-115 and others, health effects, toxicity

C. F. Reinhardt, L. S. Mullin, and M. E. Maxfield (E. I. duPont de Nemours and Company, Incorporated). Epinephrine-Induced Cardiac Arrhythmia Potential of Some Common Industrial Solvents, Journal of Occupational Medicine, 15(12):953-955, December 1973 (3 pages with 1 figure and 1 table, RDB65A0)

cardiac sensitization in beagle dogs for 5minute exposures with epinephrine challenge provides NOEL, LOEL, and $E_{50}$ data; R-30 $0 / 12$ dogs sensitized at 20,000 nominal $(17,900$ mean of analytical) ppm v/v; R-113 $0 / 12$ at $2,500(2,600), 10 / 29$ at $5,000(4,850)$, and $3 / 4$ at $10,000(9,250) \mathrm{ppm} ; \mathrm{R}-140 \mathrm{a} 0 / 12$ at 2,500 $(2,300), 3 / 18$ at $5,000(5,100)$, and $12 / 12$ at $10,000(10,650) \mathrm{ppm} ; \mathrm{R}-1110 \mathrm{0} / 12$ at 10,000 $(10,400)$ ppm; R-1120 $1 / 12$ at $5,000(4,350)$ and $7 / 12$ at $10,000(9,750) \mathrm{ppm}$; central nervous system effects above $20,000(17,900) \mathrm{ppm}$ with R-30 and $10,000(10,400)$ ppm with R1110 precluded cardiac sensitization tests at higher concentrations; toxicity

C. F. Reinhardt, L. S. Mullin, and M. E. Maxfield (E. I. duPont de Nemours and Company, Incorporated), Halocarbon-Epinephrine-Induced Cardiac Arrhythmia Potential of Some Common Industrial Solvents, abstract 81 (11th Annual Meeting of the Society of Toxicology (SOT), Williamsburg, VA, 5-9 March 1972), Toxicology and Applied Pharmacology (TAP), 22(2):305, June 1972 (1 page with no figures or tables, RDB6558)

cardiac sensitization in beagle dogs with epinephrine challenge, toxicity; reports no sensitization at 20,000 ppm with R-30 or at 10,000 $\mathrm{ppm}$ with $\mathrm{R}-1110$; response at $5,000-10,000$ with R-113 (possibly R-113a), R-140a, and R1120; central nervous system effects above 20,000 ppm with R-30 and 10,000 ppm with R1110 precluded cardiac sensitization tests at higher concentrations

C. F. Reinhardt, A. Azar, M. E. Maxfield, P. E. Smith, Jr., and L. S. Mullin (E. I. duPont de Nemours and Company, Incorporated), Cardiac Arrhythmias and Aerosol 'Sniffing', Archives of Environmental Health, 22(2):265-279, February 1971 ( 15 pages with 4 figures and 7 tables, RDB5644)

toxicity tests to determine the cardiac sensitization potential in male beagle dogs with intravenous injection of epinephrine five minutes be- 
fore and, with a challenge injection, midway into a 10 minute exposure for $R-11, R-12, R-22$, R-114, R-115, R-142b, R-152a, R-E170, R290, R-C318, R-502, R-600a, R-1140, and others

C. F. Reinhardt, Cardiac Sensitization, report 5269, Haskell Laboratory for Toxicology and Industrial Medicine, E. I. duPont de Nemours and Company, Incorporated, Newark, DE, 1969 (3 pages with 1 table, RDB6121)

toxicity tests to determine the cardiac sensitization potential of R-12, R-22, R-114, R-115, R$140 a, R-C 318$, and R-600a in male beagle dogs with intravenous injection of epinephrine five minutes before and, with a challenge injection, midway into a 10 minute exposure; also describes a modified test of R-12 in which the oxygen concentration was reduced to a low level $(8.4 \% \mathrm{v} / \mathrm{v})$ deliberately as well as control case with reduced oxygen alone; concludes that the combination of hypoxia and the R-12 exposure probably increases sensitization to epinephrine while hypoxia alone does not, but notes that the number of animals tested was insufficient for a firm conclusion; discusses the R-C318 tests at $500,000 \mathrm{ppm}$ v/v noting that the oxygen concentration for it was slightly less than $10 \% \mathrm{v} / \mathrm{v}$, but notes a similar result $(83.3$ marked responses) for a repeat with oxygen enrichment to $20 \%$

R. W. Reinhold and G. M. Rusch, Review of the Toxicity of Ten Selected Fluorocarbons, Department of Toxicology Report MA-127-80-2, AlliedSignal Incorporated, Morristown, NJ, 22 January 1986 (RDB5143)

R-12, R-32, R-113, R-113a, R-114, R-114a, R123, R-123a, R-134a, R-142b, toxicity

B. R. Rennick, S. D. Malton, G. K. Moe, and M. H. Seevers, Induction of Idioventricular Rhythms by 1,1,1-Trichloroethane and Epinephrine, Federation Proceedings, Federation of the American Society of Experimental Biologists, 8:327 ff, March 1949 (RDB65A3)

examines the potential of R-140a to induce cardiac sensitization; health effects; toxicity

C. Reynolds (Tulane University of Louisiana), Propylene, Ethylene, Nitrous Oxid[e] and Ether: Some Comparative Investigations, Fifth Annual Meeting of the Southern Association of Anesthetists (Atlanta, GA, 16-17 November 1926); republished in Anesthesia and Analgesia, 6:121-124, June 1927 (4 pages with 2 figures, RDB6A73) examines the potential of R-744A (nitrous oxide), R-1150 (ethene, ethylene), R-1270 (propene, propylene), and ether (possibly R-610) as anesthetic agents; indicates the minimum anesthetic concentration (MAC) in mice for an unspecified time as $900,000 \mathrm{ppm} \mathrm{v} / \mathrm{v}$ for $\mathrm{R}$ 744A [SEE NOTE BELOW], $900,000 \mathrm{ppm} \mathrm{v} / \mathrm{v}$ for R-1150, and 400,000 ppm v/v for R-1270; indicates the minimum lethal concentration (MLC) in mice for an unspecified time as $2,700,000 \mathrm{ppm} v / \mathrm{v}$ for $\mathrm{R}-744 \mathrm{~A}, 1,460,000 \mathrm{ppm}$ $\mathrm{v} / \mathrm{v}$ for $\mathrm{R}-1150$, and $650,000 \mathrm{ppm} \mathrm{v} / \mathrm{v}$ for $\mathrm{R}$ 1270 ; indicates that $R-1270$ produces complete analgesia in white mice at $500,000 \mathrm{ppm} v / \mathrm{v}$ that can be maintained by $400,000 \mathrm{ppm} v / \mathrm{v} ; \mathrm{R}-1270$ causes a drop in blood pressure at 650,000 $\mathrm{ppm} \mathrm{v/v} \mathrm{and} \mathrm{death} \mathrm{at} 750,000-800,000 \mathrm{ppm} v / \mathrm{v}$ in mice as well as cardiac effects in dogs above $250,000 \mathrm{ppm} \mathrm{v/v}$ without mention of epinephrine challenges; inhalation toxicity [NOTE: The paper presents concentrations as percentages. Their meaning is unclear since it discusses and plots multiple concentrations exceeding 100\% and as high as $270 \%$, yet seems to recognize the need to maintain approximately $20 \%$ oxygen in some instances. Not only are concentrations exceeding $100 \%$ or $1,000,000 \mathrm{ppm}$ inherently contradictory, but exposures at $90 \%$ treatment concentrations should have resulted in asphyxiation.]

C. Reynolds (Tulane University of Louisiana), Comparative Studies of Propylene, Ethylene, Nitrous Oxide, and Ether, Journal of Pharmacology and Experimental Therapeutics (JPET), 27(2):93-99, 1926 (7 pages with 1 figure and 2 tables, RDB6A74)

compares the inhalation toxicity of R-1270 (propene, propylene) as a candidate anesthetic agent to that of R-744A (nitrous oxide), R-1150 (ethene, ethylene), and ether (possibly R-610); notes that the R-1270 samples for some tests were impure, but reasons that a pure sample probably probably would not be more toxic; tabulates prior test exposures and resulting liver effects (staining, fatty degeneration, and fatty infiltration) of the four gases in mice; describes the effects of R-1150 and R-1270 on frog and turtle hearts, virgin and pregnant guinea pig uteri, and duedenal tissue from rabbits

L. K. Riggs (E. R. Squibb and Sons), Anesthetic Properties of the Olefine Hydrocarbons, Ethylene, Propylene, Butylene, and Amylene, Journal of the American Pharmaceutical Association, 
14(5):380-387, May 1925 (8 pages with 1 figure and 3 tables, RDB65B9)

anesthetic effect in rats, toxicity: R-1150 results in light anesthesia in 1 of 1 rats at 900,000 $\mathrm{ppm} \mathrm{v} / \mathrm{v}$ in oxygen in 18-20 min and no other toxic symptoms in 3 hours, deep anesthesia [oxygen deprivation?] at $950,000 \mathrm{ppm}$ within 1 min; R-1270 produces light anesthesia in 1 of 1 rats at 400,000 ppm within $15 \mathrm{~min}$ and no other toxic symptoms in $6 \mathrm{hr}$ [implies 6 -hr ALC rat $0 /$ ? exceeds $400,000 \mathrm{ppm}$ ], deep anesthesia at $550,000 \mathrm{ppm}$ in 3-6 min, and death in rats at $650,000 \mathrm{ppm}$ v/v with $25 \%$ oxygen and $10 \%$ nitrogen in $2 \mathrm{hr}$ [implies 2-hr ALC rat ?/? = 650,000 ppm]; butylene; amylene

L. K. Riggs (E. R. Squibb and Sons), The Physiologic Properties of Some Unsaturated Hydrocarbons, Proceedings of the Society of Experimental Biology and Medicine, New York, NY, 22:269-270, 1924-1925 (2 pages, RDB7738)

R-1150, R-1270, and others, health effects, toxicity: examination of ethylene and propylene as general anesthetic agents

W. F. Riker, F. Depierre, J. Roberts, B. B. Roy, and J. Reilly, The Epinephrine and Hydrocarbon-Epinephrine Disturbances in the Cat, Journal of Pharmacology and Experimental Therapeutics (JPET), 114:1 ff, 1955 (RDBA946)

toxicity study of hydrocarbons $(\mathrm{HC})$ for cardiac sensitization

B. H. Robbins (Vanderbilt University School of Medicine), Preliminary Studies of the Anesthetic Activity of Fluorinated Hydrocarbons, Journal of Pharmacology and Experimental Therapeutics (JPET), 86:197-204, 1946 (8 pages with 3 tables, RDB5980)

tests for anesthetic effect in mice exposed to 46 fluorinated hydrocarbons for 10 minutes in a rotating bottle, including $\mathrm{R}-20, \mathrm{R}-123\left(\mathrm{EC}_{50}=\right.$ $27,000 \mathrm{ppm} v / \mathrm{v}), \quad \mathrm{R}-123 \mathrm{~B} 2, \mathrm{R}-132 \mathrm{~b}, \mathrm{R}-$ 132bB1ß, R-133a, R-133aB1, R-13al1, R-141b $\left(E C_{50}=25,000\right), R-142, R-142 b \quad\left(E C_{50}=\right.$ 250,000), R-142B1, R-143a $\left(E C_{50}=500,000-\right.$ $600,000), R-223 d a, R-236 f a\left(E C_{50}=110,000\right)$, R-243ab, R-243db, R-243fa, R-243dbB2, R252dc, R-252dcB1B, R-252fc, R-253dbB1, R253fb, R-261fc, R-262ca, R-262caB1, R-262db, R-262fc, R-263fb, R-E263fb1, R-365mfc, R$374, R-392, R-610, R-1243 a, R-1372$, and others; also tests for $10-$ min LC $_{50}$ mouse for most of them; also tests of 18 of them for effects on the blood pressure and heart rhythm in anesthetized dogs; recommends consideration of R-
123B2, R-243db, R-253dbB1, and R-262db as possible anesthetic agents

R. Rubenstein (U.S. Environmental Protection Agency, EPA), Human Health and Environmental Toxicity Issues for Evaluation of Halon Replacements, Toxicology Letters, 68(1-2):21-24, 1993 (4 pages with 3 tables, RDB5950)

R-22, R-22B1，R-23，R-32, R-123, R-124, R125, R-134a, R-227ea, R-31-10, R-51-14, R718 (water), R-744 (carbon dioxide), and others: brief summary of the approach used by the U.S. Environmental Protection Agency (EPA) for health hazard assessment of alternative fire suppressants (many of which also are used as refrigerants); tabulates criteria, qualitative suitability (acceptable, unacceptable, or pending) in occupied and unoccupied areas, and qualitative suitability for consumer, commercial, industrial, or military use

G. M. Rusch (Honeywell Incorporated), Toxicological Evaluations of Alternative Fluorocarbons, Drug and Chemical Toxicology, 23(1):2740, 2000 (14 pages, RDBA925)

inhalation, subchronic, and chronic toxicity of $\mathrm{R}-123, \mathrm{R}-141 \mathrm{~b}$, and others; also addresses metabolism, genotoxicity, teratology, gas-uptake pharmacokinetics, and reproductive toxicity

G. M. Rusch (Honeywell Incorporated, then AlliedSignal Incorporated) and H. J. Trochimowicz (E. I. duPont de Nemours and Company), Occupation Exposure Limits for Alternate Refrigerants, EUROTOX'97, Diversification in Toxicology: Man and Environment (proceedings of the XXXVI European Congress of Toxicology, EuroTox, Árhus, Denmark, 25-28 June 1997), Nordic Pharmacological Society, Copenhagen Denmark, 1997; abstract P4-38 republished in Pharmacology and Toxicology, 80(supplement III):138-139, 1997 (2 pages with no figures or tables, RDBA646)

discusses assessments of toxicity data to set occupational exposure limits for alternative fluorochemicals; notes suggested permissible exposure limits [Workplace Environmental Exposure Level (WEEL) Guides] of $1000 \mathrm{ppm}$ v/v for R-32, R-124, R-125, and R-142b, 500 ppm $\mathrm{v} / \mathrm{v}$ for R-141b; $250 \mathrm{ppm} v / \mathrm{v}$ for R-225cb, and $25 \mathrm{ppm} v / \mathrm{v}$ for R-225ca; indicates that the results for R-123 are still under review

G. M. Rusch (AlliedSignal Incorporated), Update: Program for Alternative Fluorocarbon Toxicity 
Testing, publication unknown, circa January 1996 (13 pages with 13 tables, RDB7464)

summary of the status and findings of the Program for Alternative Fluorocarbon Toxicity Testing (PAFT); addresses the toxicity of R-32, R-123, R-124, R-125, R-141b, R-134a, R$225 \mathrm{ca}$, and R-225cb

G. M. Rusch (AlliedSignal Incorporated), Toxicity Studies with New Refrigerant Gases, abstract 324, The Toxicologist, 11(1):102, February 1991

(1 page with no figures or tables, RDB6551)

summary of ongoing studies for the Program for Alternative Fluorocarbon Toxicity Testing (PAFT): reduced response to noise, probably indicating light anesthesia, was noted in a 4week inhalation study [species not indicated] with R-124 at concentrations up to $50,000 \mathrm{ppm}$, but this effect cleared within minutes following exposures; transient, depressed response to noise also was seen in an ongoing, 3-month inhalation study with exposures of up to 50,000 ppm R-124 [species not indicated]; R-124 was not active in the Ames, cytogenetics, or in vivo micronucleus assays; no developmental effects were found in teratology studies in the rat at concentrations up to $50,000 \mathrm{ppm}$; no treatmentrelated effects were observed in a 3-month inhalation study [species not indicated] with $R$ $134 \mathrm{a}$ even for exposures of $50,000 \mathrm{ppm}$; a chronic toxicity study is underway; $R-134$ a did not induce unscheduled DNA synthesis and was not active in the Ames, cytogenetics, or in vivo micronucleus assays; no developmental effects were found in teratology studies in the rabbit with R-134a at concentrations up to $40,000 \mathrm{ppm}$

G. M. Rusch (AlliedSignal Incorporated), Results from the Program for Alternative Fluorocarbon Toxicology Testing Activities, FCKW - Ausstieg wohin? [CFC - Phaseout Whereto?] (proceedings of the 8. DECHEMA-Fachgespräch Umweltschutz [Eighth DECHEMA Colloquium on Environmental Protection], Bonn, Germany, 2-3 May 1990), Deutsche Gesellschaft für Chemisches Apparatewesen (DECHEMA), Chemische Technik und Biotechnologie e.V., Frankfurt am Main, Germany, 245-261, 1990 (17 pages with text and 17 presentation charts, RDB65F4)

summary of the structure, tests, findings, and plans of the Program for Alternative Fluorocarbon Toxicity Testing (PAFT); addresses the toxicity of R-123, R-124, R-125, R-134a, R141b [see RDB3A53 for update]
A. G. Salmon, J. A. Nash, C. M. Walklin, and R. B. Freedman (Imperial Chemical Industries Limited, (Cl), Dechlorination of Halocarbons by Microsomes and Vesicular Reconstituted Cytochrome P-450 Systems Under Reductive Conditions, British Journal of Industrial Medicine, 42(5):305-311, 1981 (7 pages, RDB8171)

biochemistry, toxicity of chlorinated halochemical refrigerants

A. G. Salmon, R. B. Jones, and W. C. Mackrodt (Imperial Chemical Industries Limited, $\mathrm{ICl}$ ), Microsomal Dechlorination of Chloroethanes: Structure-Reactivity Relations, Xenobiotica, 11(11):723-734, 1981 (12 pages, RDB8172)

biochemistry, toxicity of chlorinated halochemical refrigerants

P. A. Sanders (Industrial Hydrocarbons, Incorporated), Toxicity of Hydrocarbon Propellants, Aerosol Age, 24(1):24-27,44, January 1979 (5 pages with 8 tables, RDBA538)

toxicity of R-290 (propane), R-600 (n-butane), R-600a (isobutane), R-601 (n-pentane), R-601a (isopentane) for propellant uses (some of them also are used as refrigerants)

E. E. Sandmeyer, Aliphatic Hydrocarbons, Patty's Industrial Hygiene and Toxicology (third revised edition), edited by G. D. Clayton and F. E. Clayton, John Wiley and Sons, Incorporated, New York, NY, 2B:3175-3220, 1981 (46 pages with 4 tables, RDB6290)

R-50 (methane), R-170 (ethane), R-290 (propane), R-600 (butane), R-600a (isobutane), R601 (pentane), R-601a (isopentane), R-601b (neopentane), R-602 (hexane), R-1150 (ethylene), R-1270 (propylene), and others; health effects, toxicity

E. Schaefer, Experientia, 38(10):1163-1168, 1982 (6 pages, RDB5786)

R-744 (carbon dioxide) and others, health effects, toxicity

R. E. Schroeder (Pharmaco-LSR, Incorporated), P. E. Newton, G. M. Rusch (AlliedSignal Incorporated), and H. J. Trochimowicz (E. I. duPont de Nemours and Company), Inhalation Developmental Toxicity Studies with HCFC-123 and HCFC-124 in the Rabbit, Teratology, 51(3):196, March 1995; presented at the joint 35th Annual Meeting of the Teratology Society (Newport Beach, CA, 24-29 June 1995) and the 19th Annual Meeting of the Neurobehavioral Teratology Society 
(New-port Beach, CA, 25-28 June 1995), 1995 (1 page, RDB7632)

developmental toxicity studies of R-123 and R124 in rabbits: groups of 20-24 female New Zealand White rabbits were naturally mated and exposed by whole-body inhalation to concentrations of $0,500,1,500$, or $5,000 \mathrm{ppm} v / \mathrm{v}$ $\mathrm{R}-123$ in air and to $0,5,000,15,000$, or 50,000

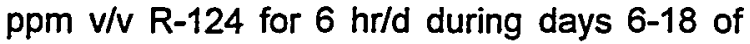
gestation; in-life observations included periodic measurements of body weight, food consumption, and clinical signs; animals were sacrificed on day 30 of gestation; maternal toxicity was evidenced by reduced weight gain and food consumption during the treatment period at all exposure levels for R-123, but no developmental toxicity was seen; no maternal or developmental toxicity was seen at the 5,000 and 15,000 ppm exposure levels for R-124; maternal toxicity was indicated from a decrease in inchamber activity and a slight reduction in food consumption during exposures to $50,000 \mathrm{ppm}$ $R-124$, but no developmental toxicity was seen; concludes that the NOEL for developmental toxicity was 5,000 and $50,000 \mathrm{ppm} \mathrm{v} / \mathrm{v}$ for R123 and R-124, respectively

J. Scholz and W. Weigand (Farbwerke Hoechst AG, Germany), Zentralblatt für Arbeitsmedizin, Arbeitsschutz [Occupational Medicine and Safety], 14:129 ff, 1964 (RDB6B42)

[Frigen ${ }^{\circledR}$ ] R-11, R-12, R-13B1, R-113, R-114, narcotic effect, toxicity as reported in RDB6365

J. Scholz (Farbwerke Hoechst AG, Germany), New Toxicological Investigations of Certain Types of Freons Used as Propellants for Aerosols and Sprays, Fortschritte der biologischen Aerosol-Forschung [Progress in Biological Research of Aerosols], 4:420-429, 1962 (10 pages with 10 figures and 2 tables, in German, Rdb5355)

[Frigen ${ }^{\circledR}$ ] R-11, R-12, R-113, R-114, narcotic effect, toxicity

J. Scholz (Farbwerke Hoechst AG, Germany), Progress in Biological Research of Aerosols 1957-1961, proceedings of the Aerosol Congress (Stuttgart, Germany, 1961), F. K. Schattaver Verlag, Germany, 1961 (in German, RDB8170)

toxicity of fuorochemicals used as aerosol propellants (and as refrigerants)

L. Shargel and R. Koss, Determination of Fluorinated Hydrocarbon Propellants in Blood of Dogs After Aerosol Administration, Journal of
Pharmaceutical Sciences, 61(9):1445-1449, 1972 (5 pages, RDB7242)

fluorocarbon elimination from the bloodstream, toxicity

F. E. Shipway (Guy's Hospital, UK), Acetylene, Ethylene, and Propylene, Lancet, UK, 1:11261130,30 May 1925 (5 pages with 3 tables, RDB6A82)

surgical tests of the effectiveness and toxicity of acetylene, R-1150, and R-1270 as anesthetic agents

B. B. Shugaev (Yaroslavl Medical Institute, USSR), Concentrations of Hydrocarbons in Tissues as a Measure of Toxicity, Archives of Environmental Health, 18:878-882, 1969 (5 pages with 2 figures and 6 tables, RDB5363)

toxicity of R-600 (n-butane), butadiene, hexene, isobutylene, isoprene, and styrene: 2-hr $L C_{50}$ mouse and 4-hr LC 50 rat data; for R-600, 2-hr $\mathrm{LC}_{50}$ mouse $=680 \mathrm{mg} / \mathrm{l}[281,000 \mathrm{ppm}$ v/v $] ; 4-\mathrm{hr}$ $\mathrm{LC}_{50}$ rat $=658 \mathrm{mg} / \mathrm{l}[272,000 \mathrm{ppm} \mathrm{v} / \mathrm{v}]$

B. B. Shugaev, Distribution and Toxicity of Aliphatic Hydrocarbons in Body Tissues, Farmakol. Toksikol., Russia (then USSR), 31(3):360363, 1968 (4 pages, RDB6A89)

$\mathrm{R}-600$, health effects, metabolism, toxicity

B. B. Shugaev, Combined Action of Aliphatic Hydrocarbons, Such as Butane and Isobutylene, with Reference to Their Effective Concentration in Brain Tissue, Farmakol. Toksikol., Russia (then USSR), 30(1):102-105, 1967 (4 pages, RDB6A90)

$\mathrm{R}-600$, health effects, toxicity: $2-\mathrm{hr} \mathrm{LC}_{50}$ mouse $=286,000 \mathrm{ppm} ; 4-\mathrm{hr} \mathrm{LC}_{50}$ rat $=277,000 \mathrm{ppm}$ as reported in RDB6A83

E. Siegel and S. Wason, Sudden Death Caused by Inhalation of Butane and Propane, New England Journal of Medicine, 323(6):1638 ff, December 1990 (RDB7451)

deaths of two children (ages 11 and 15) from inhalation of R-290 (propane) and R-600 (n-butane): concludes that although inhalation of butane and propane has been largely unreported in the United States, there is an urgent need for preventive efforts, directed at teenagers, with an emphasis on the risk of sudden death from "sniffing" hydrocarbons; abuse, health effects, toxicity

A. Silverglade, Cardiac Toxicity of Aerosol Propellants, Journal of the American Medical Associ- 
ation (JAMA), 222:827-828, 1972 (2 pages, RDB7403)

cardiac sensitization, toxicity

J. A. Simaan and D. M. Aviado (University of Pennsylvania School of Medicine), Hemodynamic Effects of Aerosol Propellants, III. Vascular Resistance in Canine Hind Limb, Toxicology, 5(3):287-295, 1976 (9 pages, RDB7251)

$\mathrm{R}-12, \mathrm{R}-114$, and others, health effects, toxicity

J. A. Simaan and D. M. Aviado (University of Pennsylvania School of Medicine), Hemodynamic Effects of Aerosol Propellants, I. Cardiac Depression in the Dog, Toxicology, 5(2):127-138, 1975 (12 pages with 1 figure and 4 tables, RDB6123)

$R-11, R-12, R-114, R-152 a$, and R-C318, cardiac sensitization in anesthetized mongrel dogs, health effects, toxicity: inhalation of fluorocarbons cased a depression of myocardial concontractility, aortic hypotension, decrease in cardiac output, and increase in pulmonary vascular resistance; LOEL that elicited these changes are $10,000 \mathrm{ppm}$ for R-11, $100,000 \mathrm{ppm}$ for R-12, and $25,000 \mathrm{ppm}$ for R114 ; concentrations of $200,000 \mathrm{ppm}$ did not influence these hemodynamic parameters for $R$ $152 a$ and R-C318

J. A. Simaan and D. M. Aviado (University of Pennsylvania School of Medicine), Hemodynamic Effects of Aerosol Propellants, II. Pulmonary Circulation in the Dog, Toxicology, 5(2):139-146, 1975 (8 pages, RDB6124)

$\mathrm{R}-12, \mathrm{R}-114$ and others, cardiac sensitization, health effects, toxicity

V. F. Simmon and S. Wiest (Stanford Research Institute), In Vitro Microbiological Studies Mutagenicity Studies of Phillips Petroleum Company Hydrocarbon Propellants and Aerosols, Phillips Petroleum Company, Bartlesville, OK, 13 May 1977 (RDBA310)

toxicity study of R-600a (isobutane), R-601 ( $\mathrm{n}$ pentane), R-601a (isopentane), and others

V. F. Simmon (Stanford Research Institute), Studies of Hydrocarbon Propellants, Phillips Petroleum Company, Bartlesville, OK, 14 February 1977 (RDBA669)

toxicity study of R-600a (isobutane), R-601 ( $n$ pentane), R-601a (isopentane), and others

S. R. Skaggs and T. A. Moore (New Mexico Engineering Research Institute, NMERI), Toxicologi- cal Properties of Halon Replacements, Halon Replacements, Technology and Science (208th National Meeting, Washington, DC, 21-25 August 1994), ACS Symposium Series 611, American Chemical Society (ACS), Washington, DC, chapter 10, 99-109, 1994 (11 pages with 3 tables, RDB5526)

toxicity: indicates a $15-\mathrm{min} \mathrm{LC}_{50}$ rat of 274,000 ppm for R-1311; 4-hr ALC rat concentrations of $663,000 \mathrm{ppm}$ for R-23, 230,000 ppm for R-124, $>800,000 \mathrm{ppm}$ for R-125, and $>800,000 \mathrm{ppm}$ for R-31-10 ("PFC-410"); 4-hr LC $\mathrm{LC}_{50}$ rat concentrations of $100,000-130,000 \mathrm{ppm}$ for R-12B1, $32,000 \mathrm{ppm}$ for $\mathrm{R}-123,>800,000 \mathrm{ppm}$ for $\mathrm{R}-$ $227 e a$, and $>800,000 \mathrm{ppm}$ for R-51-14; also indicates NOAEL levels for cardiac sensitization of 50,000-100,000 ppm for R-12B1, 70,000 $\mathrm{ppm}$ for R-13B1, 2,000 ppm for R-1311, $300,000 \mathrm{ppm}$ for R-23, $10,000 \mathrm{ppm}$ for R-123, $10,000 \mathrm{ppm}$ for R-124,75,000 ppm for R-125, $90,000 \mathrm{ppm}$ for R-227ea, $400,000 \mathrm{ppm}$ for R$31-10$, and $>400,000 \mathrm{ppm}$ for R-51-14; also tabulates developmental toxicity NOAEL and subchronic (13-week) inhalation toxicity NOAEL; data sources are not explicit

Z. Skuric, E. Zuskin, and V. Valic, Effects of Aerosols in Common Use on the Ventilatory Capacity of the Lung, Int. Arch. Arbeitsmedizin [International Archives of Occupational Medicine], 34:137-149, 1975 (13 pages, RDB8177)

health effects of fluorochemical aerosol propellants (also used as refrigerants); toxicity

T. E. Slater, A Note on the Relative Toxic Activities of Tetrachloromethane and Trichlorofluoromethane on the Rat, Biochemical Pharmacology, UK, 14(2):178-181, 1965 (3 pages, RDB$5 \mathrm{C} 52)$

$\mathrm{R}-10$ and $\mathrm{R}-11$, ingestion toxicity as reported in RDB5141, RDB5367, and RDB7296

F. A. Smith, W. L. Downs, H. C. Hodge, and E. A. Maynard (University of Rochester School of Medicine and Dentistry), Screening of FluorineContaining Compounds for Acute Toxicity, Toxicology and Applied Pharmacology (TAP), 2(1):54-58, January 1960 (5 pages, RDB5908)

R-51-12 and other fluorine-containing organic and inorganic compounds, health effects, acute toxicity

H. F. Smith, T. F. Hatch, K. H. Jacobson, M. L. Keplinger, and F. Princi (National Research Council (NRC) Ad Hoc Committee) with revisions by A. J. Lehman, W. G. Fredrick, H. W. Gerarde, H. E. 
Stokinger, and J. A. Zapp, Jr. (NRC Committee on Toxicology), Basis for Establishing Emergency Inhalation Exposure Limits Applicable to Military and Space Chemicals, National Academy of Sciences (NAS), National Academy Press, Washington, DC, 1964 (10 pages with no figures or tables, RDB5A19)

rationale for emergency inhalation exposure limits for new chemicals for military and space applications: notes that no authoritative source exists to establish emergency exposure levels; also notes that derivation of short-term exposure limits, based on a factor representing the ratio of exposure periods, from existing ACGIH Threshold Limit Values (TLVs), does not take into consideration conditions of exposure that differ from industrial patterns or the body systems involved; characterizes occupational exposures as predictable, controlled by quantity and distance limits, and allowing use of personal protective equipment; characterizes nonoccupational exposures as unavoidable, unpredictable, and rare; notes that the eventual occurrence of nonoccupational exposures is not a certainty and that there is justification for exposing individuals to some degree of risk; encourages promulgation of lists of Occupational and Nonoccupational Emergency Exposure Limits; defines an Emergency Exposure Limit $(E E L)$ "for short-term exposure to an airborne contaminant as a concentration which, when inhaled for a specified brief period, rare in the lifetime of any individual, is believed not to result in a period of disability or interfere with the performance of his assigned task;" notes that "sensory comfort of the individual is not a necessary concern" and that no selected value shall "produce danger from flammability of combustible aerosols, or result in substantial impairment of vision or visibility, or the ability to breath;" recommends procedures to avoid second exposures without medical consultation; recommends against incorporation of safety factors, suggesting that they be applied by the specialist using the limit guides and familiarity with the application rather than by toxicologists setting the limits; outlines the toxicological data required to promulgate EELs including identification of target organs and effects, doseduration response data extending (in both directions) beyond the time intervals for which limits are to be promulgated, and certain (unspecified) exposure data for orientation purposes
J. K. Smith and M. T. Case, Subacute and Chronic Toxicity Studies of Fluorocarbon Propellants in Mice, Rats, and Dogs, Toxicology and Applied Pharmacology (TAP), 26(3):438-443, 1973 (6 pages, RDB8176)

toxicity of fluorochemicals used as aerosol propellants (and also as refrigerants); health effects

S. L. Smith, W. R. Webb, L. W. Fabian, and V. D. Hagaman, Cardiac Excitability in Ether, Cyclopropane, and Halothane Anesthesia, Anesthesiology, 23:766 ff, 1962 (RDB5576)

toxicity tests for cardiac sensitization by $R$ 123B1 (halothane), R-C270 (cyclopropane), and ether

R. Snyder, Ethel Browning's Toxicity and Metabolism of Industrial Solvents (second edition), Elsevier, New York, NY, 1987 (RDBA534)

toxicity of solvents some of which also are used as refrigerants

F. E. Speizer, D. H. Wegman, and A. Ramirez, Palpitation Rates Associated with Fluorocarbon Exposure in a Hospital Setting, New England Journal of Medicine, 292:624-626, 1975 (3 pages, RDB5366)

fluorocarbons, toxicity

L. J. Standley and T. L. Bott, Trifluoroacetate, an Atmospheric Breakdown Product of Hydrofluorocarbon Refrigerants: Biomolecular Fate in Aquatic Organisms. Environmental Science and Technology, 32(4):469-475, 15 February 1998 (7 pages, RDBA926)

measurements of the xenobiotic trifluoroacetate incorporation into biomolecules for aquatic organisms, ranging from microbes to macroinvertebrates, and plants; biodegradation; ecotoxicity

S. Stephens et al., Phenotypic and Genetic Effects in Neurospora Crassa Produced by Selected Gases and Gases Mixed with Oxygen, Develop. Ind. Microbiology, 12:346-353, 1971 (8 pages, RDB6515)

R-23 and others; mutagenicity, toxicity, biochemical studies

R. D. Stewart, P. E. Newton, E. D. Baretta, A. A. Herrmann, H. V. Forster, and R. J. Soto (Medical College of Wisconsin), Physiological Response to Aerosol Propellants, Environmental Health Perspectives (EHP), 26:275-285, October 1978 (11 pages with 10 tables, RDB6115) 
summarizes acute and subchronic toxicity tests in healthy human volunteers by whole-body exposure to R-11, R-12, R-290 (propane), and R600 a (isobutane); acute exposures to 250,500 , or $1000 \mathrm{ppm} \mathrm{v} / \mathrm{v}$ for $1 \mathrm{~min}$ to $8 \mathrm{hr}$ "did not produce any untoward physiological effects" as determined by observation, routine hematology, clinical chemistry, EKG, and pulmonary function as well as by cognitive skill and other neurological measurements; repetitive exposures to $1,000 \mathrm{ppm} v / \mathrm{v}$ for $1-10 \mathrm{hr} / \mathrm{d}, 5 \mathrm{~d} / \mathrm{wk}$, for $2-4$ wk also showed no adverse effects except with $\mathrm{R}-11$; the R-11 exposures yielded minor decrements in several cognitive tests, but no change in pulmonary function or alteration of in cardiac rhythm

R. D. Stewart et al. (Medical College of Wisconsin), Acute and Repetitive Human Exposure to Isobutane and Propane, report IASS-CFTAMCOW-ENVM-BP-77-1, Department of Environmental Medicine, Medical College of Wisconsin and Milwaukee, Milwaukee, WI, April 1977 (available from NTIS as report PB-279205/LLC, RDB6599)

toxicity tests of aerosol propellants (some of which also are used as refrigerants) in human volunteers; addresses the health effects of R290 (propane) and R-600a (isobutane)

R. D. Stewart, A. A. Herrmann, E. D. Baretta, H. V. Forster, J. H. Crespo, P. E. Newton, and R. J. Soto (Medical College of Wisconsin), Human Exposure to Aerosol Propellants, Department of Environmental Medicine, Medical College of Wisconsin and Milwaukee, Milwaukee, WI, 17 May 1977 (available from NTIS, RDB7620)

toxicity tests of aerosol propellants (some of which also are used as refrigerants) in human volunteers; addresses the health effects of $R$ 12, R-290 (propane), R-600 (n-butane), R-600a (isobutane), and others

R. W. Stoughton and P. D. Lamson (Vanderbilt University School of Medicine), The Relative Anesthetic Activity of the Butanes and Pentanes, Journal of Pharmacology and Experimental Therapeutics (JPET), 58:74-77, 1936 (4 pages with 1 table, RDB6197)

summarizes tests of the average time to induce light anesthesia (taken as loss of ability to maintain an upright position in a bottle rotating at $14 \mathrm{rpm}$ ), complete anesthesia, and lethality in mice by acute inhalation of selected hydrocarbons (HCs) and one ether as a reference; complete anesthesia occurred with exposures to R-C270 (cyclopropane) in 3 minutes at $130,000 \mathrm{ppm} v / \mathrm{v}, 11 / 2 \mathrm{~min}$ at $150,000 \mathrm{ppm}$, and $11 / 4 \mathrm{~min}$ at $180,000 \mathrm{ppm}$; light anesthesia was evident with exposures to R-600 (n-butane) for $25 \mathrm{~min}$ at $130,000 \mathrm{ppm} \mathrm{v} / \mathrm{v}$ and $1 \mathrm{~min}$ at $220,000 \mathrm{ppm} v / \mathrm{v}$; notes that R-600 "required a concentration of about 20-25 per cent for relaxation, but this caused death after a short time"; light anesthesia was evident for exposures to R-600a (isobutane) for $60 \mathrm{~min}$ at $150,000 \mathrm{ppm} \mathrm{v} / \mathrm{v}, 17 \mathrm{~min}$ at $200,000 \mathrm{ppm} \mathrm{v} / \mathrm{v}$, and $26 \mathrm{~min}$ at $230,000 \mathrm{ppm}$ v/v [these data do not suggest a dose-dependent pattern or challenge the reliability of the study]; light anesthesia was evident with exposures to R-601 (npentane) for $10 \mathrm{~min}$ at $70,000 \mathrm{ppm} v / \mathrm{v}, 3 \mathrm{~min}$ at $80,000 \mathrm{ppm} \mathrm{v/v}$, and $1.3 \mathrm{~min}$ at $90,000 \mathrm{ppm} \mathrm{v/v}$; light anesthesia was evident with exposures to R-601a (isopentane) for $11.6 \mathrm{~min}$ at 90,000

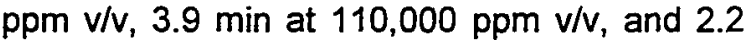

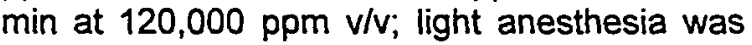
evident with exposures to R-601b (neopentane) for $30 \mathrm{~min}$ at 200,000 ppm v/v; light anesthesia was evident with exposures to "ether" (probably $\mathrm{R}-610$ ) for $3 \mathrm{~min}$ at $50,000 \mathrm{ppm} \mathrm{v} / \mathrm{v}$ but not at $35,000 \mathrm{ppm} \mathrm{V} / \mathrm{v}$; notes that all of these substances produced considerable excitement in mice during anesthesia, but that mice that did not die from the exposures recovered very quickly, usually in 1 or $2 \mathrm{~min}$; tests for anesthesia in dogs found that R-C270 induced deep anesthesia with ease, but that it was difficult to induce deep anesthesia with R-600, R-600a, or $\mathrm{R}-601 \mathrm{a}$; paper indicates the dog testing was insufficient for accuracy; toxicity

H. C. Struck and E. B. Plattner, Study of Pharmacological Properties of Certain Saturated Fluorocarbons, Journal of Pharmacology and Experimental Therapeutics (JPET), 68:217-219, 1940 (RDB7126)

R-31-10, R-C41-10, mixed depressant and excitant effects, anesthesia

M. Stupfel et al., Comparative Acute Toxicity, in Male and Female Mice, of Several Air Pollutants: Automobile Exhaust Gas, Nitrogen Oxides, Sulfur Dioxide, Ozone, Ammonia, and Carbon Dioxide, C. R. Soc. Biol., 165(9):18691872, 1971 (4 pages, RDB5817)

R-717, R-744, R-748, R-764, health effects, toxicity

T. Takano and Y. Miyazaki, Toxicological Sciences, 7(2):143-149, 1992 (7 pages, RDB5971) 
biochemical study of R-125, R-134, and others; toxicity

G. J. Taylor and W. S. Harris, Cardiac Toxicity of Aerosol Propellants, Journal of the American Medical Association (JAMA), 214(1):81-85, 1970 (5 pages, RDB6B43)

R-12 and others: initial linkage of cardiac sensitization to fatalities from use or abuse of fluoroalkane propellants also used as refrigerants (see RDB5854); nasal inhalation of aerosols by mice

W. F. ten Berge (DSM, The Netherlands), A. Zwart, and L. M. Appelman (TNO-CIVO Toxicology and Nutrition Institute, The Netherlands), Concentration-Time Mortality Response Relationship of Irritant and Systematically Acting Vapours and Gases, Journal of Hazardous Materials, 13:301-309, 1986 (9 pages with 7 figures and 4 tables, RDB6278)

This article presents an analysis of mortality data, to determine time-dependent relations for lethal acute concentrations in various species for 20 chemicals. The maximum likelihood estimates of regression coefficients, variances, and covariances and chi-square confidence predictor were determined by probit analysis of the raw data from previously published toxicity studies. The results show that the product of exposure and time $(c \cdot t)$ is not always a good parameter for predicting mortality response (Haber's rule). The product of exposure to the power of $\mathrm{n}$ and time predicts the response well, where $n$ ranges from 0.8 to 3.5 for 20 studied substances. Among them are $n=2.8$ for $R-10$, $\mathrm{n}=1.6$ for R-30B1, $\mathrm{n}=2.0$ for $\mathrm{R}-717$ (ammonia), $n=3.5$ for $R-746$ (nitrogen dioxide), $n=2.0$ for $R-$ 1110 , and $n=0.8$ for $R-1120$. These results estimate the $L C_{50}$ for a specified period from data taken for another exposure duration.

R. C. Terrell, L. Speers, A. J. Szur, T. Ucciardi, and J. F. Vitcha (Air Reduction Company, Incorporated, Airco), General Anesthetics. 3. Fluorinated Methyl Ethyl Ethers as Anesthetic Agents, Journal of Medicinal Chemistry, 15(6):604-606, 1972 (3 pages with 2 tables, RDB8318)

toxicity of fluoroether (FE), hydrofluoroether (HFE), and other halogenated ether candidates

R. C. Terrell, L. Speers, A. J. Szur, J. Treadwell, and T. R. Ucciardi (Air Reduction Company Incorporated), General Anesthetics. 1. Halogenated Methyl Ethyl Ethers as Anesthetic Agents,
Journal of Medicinal Chemistry, 14(6):517-519, 1971 (3 pages with 1 table, RDB7461)

toxicity tests of 51 halogenated ethers, including 36 newly-synthesized compounds, for anesthetic effects in mice: among those tested are R-236ca1 (light anesthesia at $125,000 \mathrm{ppm}$ $\mathrm{V} / \mathrm{V}$ ), R-E245fa1 (very weak anesthetic at $100,000 \mathrm{ppm} \mathrm{v/v}$ ), R-E254cb1 (convulsant at 25,000 ppm v/v), R-E254fa1 (very weak anesthetic at 50,000 ppm v/v), and R-E263fb1 (weak anesthetic properties by injection); concludes that those ethers having one hydrogen atom with at least two halogens other than fluorine or having two hydrogen with at least one bromine or one chlorine were the most potent as anesthetics [the reverse of which suggests that ethers containing only hydrogen and fluorine should have weaker anesthetic properties]

J. B. Terrill (E. I. duPont de Nemours and Company, Incorporated), Determination of Fluorocarbon Propellant in Blood and Animal Tissues, AlHA Journal, 33(11):736-744, November 1972 (9 pages, RDB7240)

$R-12, R-115$, and others, health effects, toxicity

B. Thiessen and O. F. Pedersen, Effect of Freon Inhalation on Maximal Expiratory Flows and Heart Rhythym after Treatment with Salbutamol and Ipratropium Bromide, European Journal of Respiratory Diseases, 61(3):156-161, 1980 (6 pages, RDB7405)

$\mathrm{R}-12$ and others, health effects, toxicity

H. J. Trochimowicz (E. I. duPont de Nemours and Company, Incorporated), Industrial Research on Alternative Fluorocarbons, Toxicology Letters, 68(1-2):25-30, 1993 (6 pages with 5 tables, RDB5170)

R-123, R-124, R-125, R-134a, R-141b, R-142b, R-152a, R-E170 (DME), R-225ca, R-225cb: brief review of candidate alternatives for refrigerants and other chlorofluorocarbons and halons; overview of the Programme for Alternative Fluorocarbons Toxicity Testing (PAFT); brief summaries of findings for $R-123, R-124, R-125$, and $\mathrm{R}-134 \mathrm{a}$ indicate $4-\mathrm{hr} \mathrm{LC}_{50}$ rat concentrations as $32,000,262,500,>800,000$, and $>500,000 \mathrm{ppm} \mathrm{v} / \mathrm{v}$, respectively, and 5-min cardiac sensitization thresholds in dogs at 20,000 , $25,000,100,000$, and $75,000 \mathrm{ppm}$, respectively

H. J. Trochimowicz (E. I. duPont de Nemours and Company, Incorporated) and T. Kawano (Daikin Industries, Limited), Subchronic Inhalation Toxicity Studies on Seven Alternative Fluorocar- 
bons, Proceedings of the Sixth International Congress of Toxicology (Rome, Italy, 28 June - 3 July 1992), International Union of Toxicology, June 1992 (RDB5B44)

$R-125$ and other refrigerants

H. J. Trochimowicz (E. I. duPont de Nemours and Company, Incorporated), The Toxicology of Certain Gaseous Fluorocarbons, Proceedings of the 2nd International Symposium on Gaseous Dielectrics, edited by L. G. Christophoran, Pergamon Press, Oxford, UK, 450 ff, 1980 (RDB6489)

R-22 and others, health effects, toxicity

H. J. Trochimowicz, J. P. Lyon, D. P. Kelly, and T. Chiu (E. I. duPont de Nemours and Company, Incorporated), Ninety-Day Inhalation Toxicity Studies on Two Fluorocarbons, abstract 164, Toxicology and Applied Pharmacology (TAP), 41:200, 1977 (1 page, RDB5915)

R-21 and $R-142 b$, health effects, toxicity

H. J. Trochimowicz, B. L. Moore, and T. Chiu (E. I. duPont de Nemours and Company, Incorporated), Subacute Inhalation Toxicity Studies on Eight Fluorocarbons, Toxicology and Applied Pharmacology (TAP), abstract 161, 41:198-199, 1977 (2 pages, RDB5A13)

$R-21, R-32, R-124$, and others, health effects, toxicity

H. J. Trochimowicz, C. F. Reinhardt, L. S. Mullin, A. Azar, and B. W. Karrh (E. I. duPont de Nemours and Company, incorporated). The Effect of Myocardial Sensitization Potential of Certain Halocarbons, Journal of Occupational Medicine, 18(1):26-30, January 1976 (5 pages with 3 figures and 3 tables, RDB65E7)

R-11, R-13B1 (halon 1301), R-140a (methyl chloroform), toxicity, cardiac sensitization NOEL and LOEL: summarizes tests on beagle dogs; "results showed no greater potential for cardiac sensitization among dogs having recovered from [induced] myocardial infarction as compared to normal healthy animals"

H. J. Trochimowicz, A. Azar, J. B. Terrill, and L. S. Mullin (E. I. duPont de Nemours and Company, incorporated), Blood Levels of Fluorocarbons Related to Cardiac Sensitization: Part II, AIHA Journal, 35(10):632-639, October 1974 (8 pages with 7 figures and 7 tables, RDB5361)

$\mathrm{R}-11, \mathrm{R}-12, \mathrm{R}-113, \mathrm{R}-114$, and R-115; health effects, toxicity
H. J. Trochimowicz (E. I. duPont de Nemours and Company, Incorporated), Blood Levels of Fluorocarbon During and After Acute Inhalation, paper 11, Proceedings of the Fourth Annual Conference on Environmental Toxicology (Fairborn, $\mathrm{OH}$, 16-18 October 1973), report AMRL-TR-73-125, Aerospace Medical Research Laboratory, U.S. Air Force, Wright-Patterson Air Force Base, $\mathrm{OH}, 137$ 149, December 1973 (13 pages with 7 figures and 7 tables including discussion, available from NTIS as document AD-781031, RDB65H2)

blood concentrations of R-11, R-12, R-113, R114 , and $R-115$ in unanesthetized beagle dogs based on tests using implanted catheters; no challenge epinephrine was administered; toxicity

S. Tsukamoto, S. Chiba, T. Muto, T. Ishikawa, and M. Shimamura, Study on the Metabolism of Volatile Hydrocarbons in Mice, Journal of Toxicological Science, Japan, 10(4):323-332, 1985 (10 pages, RDB6116)

$\mathrm{R}-290$, R-600a, and others; metabolism in mice, toxicity

H. Uehleke, T. Werner, H. Greim, and M. Kramer, Metabolic Activation of Haloalkanes and Tests in Vitro for Mutagenicity, Xenobiotica, 7(7):393400, 1977 (8 pages, RDB8182)

fluorochemical refrigerants, mutagenicity, toxicity

F. Valic and D. Beritic-Stahuljak (University of Zagreb, Croatia), Are Chlorine-Free Compounds a Solution for Health Problems Caused by Ozone-Depleting Substances?, Acta Medica Croatica, 50(2):61-63, 1996 (3 pages, RDB6A97)

reviews the environmental consequences of chlorofluorocarbons (CFCs), hydrochlorofluorocarbons (HCFCs), and hydrofluorocarbons (HFCs); concludes that CFCs and HCFCs are not acceptable based on their potentials for ozone depletion; examines the toxicity of HFC candidates; of those found to be nonflammable, the paper suggests that the toxicity of R-125, $\mathrm{R}-134 \mathrm{a}$, and R-227ea appear to be low, but have not been fully evaluated

F. Valic and D. Beritic-Stahuljak (University of Zagreb, Croatia), Substitutes for Dangerous Chlorofluorocarbons, A Challenge, Croatian Medical Journal, 34(2):108-114, 1992 (7 pages, RDB5967)

$\mathrm{R}-125$ and others, toxicity

V. Valic, Z. Skuric, and E. Zuskin, Experimental Exposures to Fluorochemicals 12, 22, and 502 , 
Rad Jazu, Yugoslavia, 402(18):229-243, 1982 (15 pages in Croatian with English summary, RDB8183)

$$
\mathrm{R}-12, \mathrm{R}-22, \mathrm{R}-502 \text {, health effects, toxicity }
$$

V. Valic, Z. Skuric, M. Bantic, M. Rudar, and M. Hecej, Effects of Fluorocarbon Propellants on Respiratory Flow and ECG, British Journal of Industrial Medicine, 34(2):130-136, 1977 (7 pages, RDB7245)

fluorochemical aerosol propellants (also used as refrigerants), health effects, toxicity

V. Valic, E. Zuskin, Z. Skuric, and M. Denich, Effects of Common Aerosols on the Ventilatory Lung Capacity, I. Change in FEV ${ }_{1.0}$ in Exposure to Hair Sprays, Acta Medica Yugoslavia, 28:231-246, 1974 (16 pages in Croatian with English summary, RDB8184)

fluorochemical aerosol propellants (also used as refrigerants), health effects, toxicity

O. W. Van Auken and R. H. Wilson, Halogenated Hydrocarbon Induced Reduction in Coupling Parameters of Rabbit Liver and Mung Bean Mitochondria, Naturwissenschaften [Natural Science], 60:259 ff, 1973 (RDB8191)

fluorochemical refrigerants, health effects, toxicity

A. Van Poznak and J. F. Artusio, Jr. (New York Hospital and Cornell University Medical College), Anesthetic Properties of a Series of Fluorinated Compounds I. Fluorinated Hydrocarbons, Toxicology and Applied Pharmacology (TAP), 2(4):363-373, July 1960 (11 pages with 1 table, RDB5828)

R-12, R-22, R-22B1, R-23, R-114, R-124, R124B1, R-125, R-133aB1, R-152a, R-226da, R243db, R-1233B1, and others; health effects, acute toxicity, and anesthetic effects in dogs; offers several generalizations, among them that completely halogenated hydrocarbons are likely to be convulsants or without anesthetic activity; based on findings by others and the new data, notes that anesthetic potency increases with halogenation or increases in the chain length (number of carbons for alkanes), either of which usually raises the boiling point as well as the molecular weight

A. Van Poznak and J. F. Artusio, Jr. (New York Hospital and Cornell University Medical College), Anesthetic Properties of a Series of Fluorinated Compounds II. Fluorinated Ethers, Toxicology and Applied Pharmacology (TAP),
2(4):374-378, July 1960 (5 pages with 1 table, RDB5872)

health effects, acute toxicity, anesthetic effects and cardiac sensitization in dogs

K. Verschueren, Handbook of Environmental Data on Organic Chemicals (second edition), Van Nostrand Reinhold Company, New York, NY, 1983 (3 pages, Rdb6211)

$\mathrm{R}-601 \mathrm{a}, \mathrm{R}-717$, and others, toxicity: cites $\mathrm{RD}_{50}$ mouse $=303 \mathrm{ppm}$ for $\mathrm{R}-\mathbf{7 1 7}$

A. Vinegar (ManTech Environmental Technology, Incorporated), R. Cook (U.S. Air Force, USAF), J. D. McCafferty (USAF), M. C. Caracci (Geocenters Incorporated), and G. W. Jepson (ManTech), Human Inhalation of Halon 1301, HFC-134a, and HFC-227ea for Collection of Pharmacokinetic Data, posted on the Internet (http://voyager.wpafb.af.mil, since removed), Armstrong Laboratory, U.S. Air Force, WrightPatterson Air Force Base, OH, 19 August 1997 (18 pages with 4 figures, RDB7C80)

inhalation toxicity of R-13B1, R-134a, and R227ea: describes tests in human volunteers to validate a physiologically-based pharmacokinetic (PBPK) model for estimating chemical biodistribution; seven male subjects were exposed in a medical facility via face masks for up to 30 minutes; inhaled and end alveolar expired concentrations were measured and periodic blood samples were drawn through an inserted venous cannula; subjects were monitored via ECG, blood pressure, and pulse-rate measurements; although the tests were conducted at concentrations well below published lowest observable adverse effect levels (LOAELs), the tests with R-134a and R-227ea had to be terminated following unexpected responses; one volunteer exposed to $4,000 \mathrm{ppm} v / \mathrm{v}$ R-134a in air displayed a rapid increase in blood concentration at $21 / 2$ minutes into the exposure; he lost consciousness and both his pulse and blood pressure dropped to zero at $4 \mathrm{~min}$, upon which the test was terminated and medical personnel intervened; a second subject exposed to 4,000 $\mathrm{ppm} v / \mathrm{V}$ R-134a in air experienced a rapid rise in blood pressure and pulse before he signalled for termination; he was exposed again after recovery, to 2,000 ppm v/v, but his blood pressure and pulse again rose rapidly after $2 \frac{1}{2} \mathrm{~min}$; both subjects recovered rapidly but reported dizziness and balance problems 6 wk post exposure; a third subject exposed to $6,400 \mathrm{ppm}$ $\mathrm{v} / \mathrm{v}$ R-227ea in air suffered rapid rise in pulse 3 min into exposure; report documents the details 
of these and additional tests as well as blood concentrations versus time during and following exposures; it discusses the unexpected results and anomalous finding of R-134a in the blood 1 hr post exposure; report concludes that no plausible mechanism is apparent for the adverse effects [Subsequent investigation of the results indicates that the reported responses probably were not due to the chemical exposures. No adverse effects were seen in other human exposures to R-134a and R-227ea at lower or higher concentrations. See RDB7C81 - RDB7C82 for further information.]

A. Vinegar, G. W. Jepson (ManTech Environmental Technology, Incorporated), and J. H. Overton (U.S. Environmental Protection Agency, EPA), PBPK Modeling of Short-Term (0-5 min) Human Inhalation Exposures to Halogenated Hydrocarbons, Inhalation Toxicology, 10:411429, 1998 (19 pages, RDB9950)

toxicity of fluorochemicals and other candidate fire suppressants also used as refrigerants); physiologically-based pharmacokinetic (PBPK) modeling

A. Vinegar and G. W. Jepson (ManTech Environmental Technology, Incorporated), Cardiac Sensitization Thresholds of Halon Replacement Chemicals Predicted in Humans by Physiologically-Based Pharmacokinetic Modelling, Risk Analysis, 16(4)571-579, 1996 (9 pages, RDB8194)

toxicity of fluorochemicals and other candidate fire suppressants also used as refrigerants); physiologically-based pharmacokinetic (PBPK) modeling

W. F. von Oettingen, The Halogenated Hydrocarbons: Their Toxicity and Potential Dangers, Joumal of Industrial Hygiene and Toxicology, 19:422 ff, 1937; republished as publication 414, Public Health Service, U.S. Department of Health and Human Services, Washington, DC, 1955 (RDB5933)

refrigerants, health effects, toxicity: asserts that phosgene is formed when R-1120 (trichloroethylene) passes through burning tobacco in smoking

H. Wada (Daikin Industries, Limited), Anesthetic Activity and Fatality ( $F-123,123 a, 124$, and 11), attachment to 19 May 1977 correspondence to $M$. B. Berenbaum (Allied Chemical Corporation), Daikin Kogyu Company Limited Chemical Division, Settsu-shi, Osaka, Japan, 16 May 1977 (3 pages with 2 tables, RDBA309) inhalation toxicity tests on adult male rats; provides results of tests for anesthesia for animals unable to maintain an upright position for 15 seconds in a bottle rotating at $14 \mathrm{rpm}$ for 10 $\mathrm{min}$; indicates calculated "approximate anesthetic concentration (AAC)" that appear to be $E_{50}$ values of $54,000 \mathrm{ppm}$ for $\mathrm{R}-11,130,000$ $\mathrm{ppm}$ for R-123, 190,000 ppm for R-123a, and 150,000 for R-124; also provides approximate lethal concentration (ALC) and estimated LC $_{50}$ (incorrectly identified as ALC in the document based on comparison to tabulated fatality counts) concentrations for $10 \mathrm{~min}$ exposures; $10 \mathrm{~min}$ ALC followed by (number of fatalities / sample size) and $L C_{50}$ rat, respectively, were $120,300(3 / 4)$ and $120,000 \mathrm{ppm}$ for $R-11$, $72,500(1 / 4)$ and $90,000 \mathrm{ppm}$ for R-123, 106,000 (3/4) and $100,000 \mathrm{ppm}$ for R-123a, $425,000(1 / 4)$ and 440,000 ppm for R-124; concludes that $R-123$ and $R-123$ a were more toxic than R-11 while R-124 was less toxic; transmittal letter raises questions about unidentified positive results of mutagenicity tests with $R$ 152a in Europe [Note: This document is referenced in the 1994 ECETOC JACC assessment report for R-124, see RDB4C02.]

Y. Wang, M. J. Olson (General Motors Research Laboratories), and M. T. Baker, Interaction of Fluoroethane Chlorofluorocarbon (CFC) Sub. stitutes with Microsomal Cytochrome P450 Stimulation of P450 Activity and Chlorodifluoroethene Metabolism, Biochemical Pharmacology, UK, 46(1):87-94, 6 July 1993 (6 pages, RDB6545)

$\mathrm{R}-123, \mathrm{R}-123 \mathrm{~B} 1, \mathrm{R}-133 \mathrm{a}, \mathrm{R}-124, \mathrm{R}-134 \mathrm{a}$ and R-125 metabolism and toxicity: ability to stimulate cytochrome P450 activities; R-1122a (CDE) defluorination in hepatic microsomes from phenobarbital-treated rabbits; $10,000 \mathrm{ppm}$ V/V R-123 doubled oxygen consumption; R-124, $R-134 a$, and $R-125$ were successively less effective, increasing oxygen consumption by 50 $100 \%$

R. S. Waritz (E. I. duPont de Nemours and Company, Incorporated). The Toxicology of Some Commercial Fluorocarbons, paper 7, Proceedings of the 2nd Annual Conference on Environmental Toxicology (31 August - 2 September 1971), report AMRL-TR-71-120, Aerospace Medical Research Laboratory, U.S. Air Force, WrightPatterson Air Force Base, $\mathrm{OH}, 85-99$, December 1971 (15 pages with 11 tables, available from NTIS as document AD-751426, RDB65H1) 
R-11, R-12, R-22, R-113, R-114, R-115, R-116, R-152a: ALC, clinical signs from inhalation, $A L D$, acute eye toxicity, chronic inhalation toxicity, chronic oral toxicity, chronic skin and eye toxicity, human studies; acute inhalation toxicity data for R-C318 and comparisons to the isolog hexafluorocyclobutene; acute inhalation toxicity comparison of octafluorobutene and octafluoroisobutene

S. Wason, W. B. Gibler, and M. Hassan, Ventricular Tachycardia Associated with Non-Freon Aerosol Propellants, Journal of the American Medical Association (JAMA), 256(1):78-80, July 1986 (3 pages, RDB6198)

case history of a nonfatal accident, involving cardiac sensitization and ventricular tachycardia in a 2-yr-old female patient, from inhalation of a commercial deodorant containing the aerosol propellants (also used as refrigerants) $R$ 290 (propane), $R-600$ (n-butane), and $R-600 a$ (isobutane): describes the incident and treatment, noting resolution of the arrythmia within $48 \mathrm{hr}$, health effects and toxicity of human exposures

T. Watanabe, H. Ratcliffe, G. E. Thompson, and D. M. Aviado (University of Pennsylvania School of Medicine), Bronchopulmonary Lesions Following Subacute Inhalation of Fluorocarbons, Propellant Supplement: Journal of Clinical Pharmacology, $67 \mathrm{ff}, 1977$ (RDB7246)

fluorocarbon refrigerants, health effects, toxicity

T. Watanabe, H. Ratcliffe, G. E. Thompson, and D. M. Aviado (University of Pennsylvania School of Medicine), Fluorinated Propellants for Aerosols, CRC Press, Cleveland, OH, 1977 (RDB7293)

fluorochemical propellants (many also used as refrigerants), health effects, toxicity

T. Watanabe and D. M. Aviado (University of Pennsylvania School of Medicine), Toxicity of Aerosol Propellants in the Respiratory and Circulatory Systems, VII. Influence of Pulmonary Emphysema and Anesthesia in the Rat, Toxicology, 3:225-240, 1975 (16 pages, RDB5C64)

R-11, R-12, R-152a, and others; cardiac sensitization, health effects, toxicity

T. Watanabe, and D. M. Aviado (University of Pennsylvania School of Medicine), Toxicity of Aerosol Propellants on the Respiratory and Circulatory Systems, IV. Influence of Pulmonary Emphysema and Anesthesia in the Rat,
Toxicology, 3:225-236, 1975 (12 pages, RDB8188)

cardiac sensitization, health effects, toxicity

T. Watanabe and D. M. Aviado (University of Pennsylvania School of Medicine), Subacute Inhalational Toxicity of Aerosol Propellants, abstract 097, Pharmacologist, 17(2):192, 1975 (1 page, RDB5A74)

$\mathrm{R}-12, \mathrm{R}-114$, and others; reduced pulmonary compliance in rats with 30 minute exposures to $40,000 \mathrm{ppm} \mathrm{R}-114$ in air for $6 \mathrm{~d} / \mathrm{wk}$ for 3 weeks as reported in RDB5141 and RDB5855

W. Weigand (Farbwerke Hoechst AG, Germany), Untersuchungen über die Inhalationstoxizität von Fluoroderivaten des Methan, Äthan, und Cyclobutan [Studies of the Inhalation Toxicity of Fluorinated Derivatives of Methane, Ethane and Cyclobutane], Zentralblatt für Arbeitsmedizin und Arbeitsschutz [Occupational Medicine and Safety], 21(5):149-156, 1971 (8 pages with 5 tables, in German, RDB5365)

R-12B1, R-13, R-21, R-22, R-115, R-C318, and others, toxicity: 2 -hr acute inhalation toxicity tests in rats and guinea pigs; subchronic inhalation toxicity tests in rats, guinea pigs, dogs, and cats; anesthetic effect tests in rats; 4 rats and 2 guinea pigs exposed to $600,000 \mathrm{ppm}$ R-C318 for $2 \mathrm{hr}$ showed normal behavior during and for the subsequent 7-day observation period; 5 rats, 2 guinea pigs, 2 dogs, and 2 cats exposed to $200,000 \mathrm{ppm}$ for $3 \frac{1}{2} \mathrm{hr} / \mathrm{d}, 5 \mathrm{~d} / \mathrm{wk}$ for 4 weeks showed no adverse effects by observation or pathological examination

M. Wilkenfeld, Simple Asphyxiants, Environmental and Occupational Medicine (second edition), edited by $W$. N. Rom, Little, Brown and Company, Boston, MA, 535-538, 1992 (4 pages, RDB8423)

reviews simple asphyxiants including $\mathrm{R}-50$ (methane), R-170 (ethane), R-702 (hydrogen), R-704 (helium), R-728 (nitrogen), R-740 (argon), R-744 (carbon dioxide), and R-744A (nitrous oxide): describes simple asphyxiants as gases that cause asphyxiation when present in concentrations high enough to lower oxygen levels, resulting in inadequate delivery of oxygen to tissues, but that are physiologically inert and do not suppress cardiac output or alter the function of hemoglobin; summarizes the physical and chemical characteristics of these substances; presents the concentrations known to produce asphyxiation and the occupational environments in which incidents and even fatali- 
ties have been reported; discusses case histories to illustrate the need for safety checks when simple asphyxiants are present and the use of a self-contained breathing apparatus (SCBA) as a protective measure

J. H. Wills, Sensitization of the Heart to Catecholamine-Induced Arrhythmia, Proceedings of the 3rd Annual Conference on Environmental Toxicology (Fairborn, OH, 25-27 October 1972), National Technical Information Service (NTIS), Springfield, VA, 249 ff, 1972 (available from NTIS as document AD-773766, RDB8187)

cardiac sensitization, toxicity

J. H. Wills, P. Bradley, H. Kao, H. Grace, W. Hull, T. B. Griffin, F. Coulston, and E. S. Harris (Albany Medical College and NASA Manned-Spacecraft Center), Sensitization of the Heart to Catecholamine-Induced Arrhythmia by Haloalkanes, abstract 82 (11th Annual Meeting of the Society of Toxicology, SOT), Toxicology and Applied Pharmacology (TAP), 22:305-306, 1972 (2 pages, RDB6118)

R-11, R13B1, R-114B2 or R-114aB2, R-116, RC318, cardiac sensitization via tracheal cannulae in anesthetized guinea pigs, cats, and dogs at concentrations of up to $200,000 \mathrm{ppm}$, all resulted in sensitization with R-11 the most potent followed by R-114B2 or R-114aB2

C. R. Wolf, D. Mansuy, W. Nastainczyk, G. Deutschmann, and $V$. Ullich, The Reduction of Polyhalogenated Methanes by Liver Microsomal Cytochrome P-450, Mol. Pharmacol., 13(4):698-705, 1977 (8 pages, RDB8186)

$\mathrm{R}-11, \mathrm{R}-12$, and others: biochemistry, health effects, metabolism, pharmacokinetics, toxicity

W. P. Yant (U.S. Bureau of Mines), Toxicity of Organic Fluorides, American Journal of Public Health, 23:930-934, 1933 (5 pages, RDB5A73)

R-114 and others, health effects, toxicity

D. S. Young et al., Journal of the American Chemical Society, 62:1171-1173, 1940 (3 pages, RDB5944)

\section{$\mathrm{R}-125$ and others}

W. Young and J. Parker, Effect of Freons on Acetylcholinesterase Activity and Some Counter Measures, Proceedings of the 3rd Annual Conference on Environmental Toxicology (Fairborn, OH, 25-27 October 1972), National Technical Information Service (NTIS), Springfield, VA, 259 ff, 1972 (available from NTIS as docu- ment AD-773766); republished in Combustion Toxicology, 2:286-297, 1975 (?/12 pages, RDB8190)

biochemistry, toxicity

J. A. Zapp, Jr., Fluorocarbons, Encyclopedia of Occupational Health and Safety (third revised edition), 1:895-897, 1983 (3 pages, RDB5951)

R-125 and others, health effects, toxicity

S. Zakhari, Cardiovascular Toxicology of Halogenated Hydrocarbons and Other Solvents, Cardiovascular Toxicology (second edition), Raven Press Limited, New York; NY, 1992 (RDB6685)

addresses possible mechanisms responsible for cardiac sensitization of refrigerants and other chemicals

S. Zakhari and D. M. Aviado, Cardiovascular Toxicology of Aerosol Propellants, Refrigerants, and Related Solvents, III. Toxicodynamics, Target Organ Toxicology Series, Cardiovascular Toxicology, edited by E. W. Van Stee, Raven -Press, New York, NY, 281-326, 1982 (46 pages, RDB6132)

R-10B4, R-12B1, R-31, R-40B2, R-41, R-113a, R-114, R-114a, R-114B2, R-130a, R-150, R150B2, R-151a, R-152a, R-260da, R-280fa, R280faB1, R-C318, R-31-10, R-1110, R-1114, $\mathrm{R}-1130 \mathrm{c}, \mathrm{R}-1130 \mathrm{t}, \mathrm{R}-1140 \mathrm{B1}$, and others; toxicity

E. Zeiserl, Hydrocarbon Ingestion and Poisoning. Compr. Ther., 5:35-42, 1979 (8 pages, RDB8122)

hydrocarbons, health effects, toxicity in humans: direct aspiration into the tracheobronchial system, rather than gastrointestinal absorption, is the major toxic mechanism - as reported in RDB7B43

C. Zenz, Fluorides, Occupational Medicine, Principles and Practical Applications (second edition), Year Book Medical Publishers, Incorporated, Chicago, IL, chapter 35, 540-544, 1987 (5 pages, RDB5945)

$\mathrm{R}-125$ and others, personal protective equipment, skin contact

P. S. Zurer, Liver Damage Tied to CFC Substitute, Chemical and Engineering News, 25 August 1997 (5 pages with 2 figures, limited copies available from JMC as RDB7C72)

provides further information on the underlying incident and subsequent investigations in re- 
sponse to an article in The Lancet (see RDB7C70) on exposures of workers to R-124/123 [actually R-290/124/123]: quotes R. Rubenstein (a senior staff toxicologist with the U.S. Environmental Protection Agency, EPA), on the hepatotoxicity mechanisms for R-123 and that it has "always been considered very safe when used properly"; quotes P. Hoet (one of the coauthors of the cited article) that the chronic concentrations involved were not determined but must have been high since "the workers said they were always refilling the airconditioning system"; quotes L. R. Pohl (another coauthor of the cited article) on the metabolic processes for R-123 and R-124 on the possibility of further liver damage through an autoimmune reaction; quotes G. R. Rusch (director of toxicology for AlliedSignal Incorporated) that no "chemical has ever been more extensively evaluated than HCFC-123" and W. J. Brock (staff toxicologist for DuPont Chemicals) that tested primates (monkeys) developed liver damage, which was reversible, only when exposed to doses 20 times higher than recommended exposure limits; quotes $D$. Hufford (acting director of the EPA Stratospheric Ozone Protection Division) that R-123 and R-124 are well studied and can be safe if industry standards are followed, but also can be misused

E. Zuskin, Z. Skuric, and V. Valic, Effects of Common Aerosols on the Ventilatory Lung Capacity, II, Acta Medica Yugoslavia, 28:247-259, 1974 (13 pages in Croatian with English summary, RDB8185)

fluorochemical aerosol propellants (also used as refrigerants), health effects, toxicity

2000 Threshold Limit Values for Chemical Substances in the Work Environment, 2000 TLVs ${ }^{\circledR}$ and BEIs ${ }^{\circledR}$ : Threshold Limit Values for Chemical Substances and Physical Agents and Biological Exposure Indices, publication 0100, American Conference of Governmental Industrial Hygienists (ACGIH), Cincinnati, OH, 2000 (105 pages with 2 tables, available from ACGIH for $\$ 27.95$, RDBA936)

This book provides data for use as guidelines or recommendations in the control of potential health hazards. Threshold limit values (TLVs) are tabulated for airborne concentrations of chemical substances to which workers may be exposed. These chemicals include some commonly or historically used as refrigerants, based on their chronic and/or acute toxicity. The TLV data refer to concentrations under which it is believed that nearly all workers may be repeatedly exposed without adverse health effects subject to identified considerations. The TLVs are categorized as time-weighted average (TWA or TLV-TWA), short-term exposure limit (STEL or TLV-STEL), and ceiling (TLV-C). The document indicates the molecular weights and bases or critical effects underlying the adopted TLV concentrations. Notes are provided for interpretation, but the information is not intended for use without training in industrial hygiene. The document flags TLVs and other data for compounds included in the Notice of Intended Changes (NIC), to flag data for which future revision has been proposed. It also provides appendices addressing carcinogens, substances of variable composition, mixtures, and sampling criteria for airborne particulate matter. The refrigerants addressed include R-11, R-12, R-13B1, R-20, R-21, R-22, R-30, R-40, R-113, R-114, R-115, R-160, R160B1, R-170 (ethane), R-290 (propane), R600 (butane), R-601 (pentane), R-610, R-611, R-630, R-631, R-717 (ammonia), R-744 (carbon dioxide), R-764 (sulfur dioxide), R-1130, R1150, R-1270, R-7146 (sulfur hexafluoride), and others. R-20B1, R-20B3, R-30, R-30B1, R-160B1, R-600a (isobutane), R-602 (nheptane), $R-1216, R-1250$, and others are identified as chemical substances under study.

An Investigation of Possible Cardiotoxic Effects of the Aerosol Propellants Arctons 11 and 12, Allen and Hanburys Limited, UK, 1971 (RDB8127)

cardiac sensitization of fluorochemical aerosols 11 and 12 (also used as refrigerants): toxicity of $\mathrm{R}-11$ and $\mathrm{R}-12$

C. F. Reinhardt, Cardiac Arrhythmias Induced by Epinephrine During Inhalation of Certain Halogenated Hydrocarbons: Cardiac Sensitization, report 14-69, Haskell Laboratory for Toxicology and Industrial Medicine, E. I. duPont de Nemours and Company, Incorporated, Newark, $D E, 14$ January 1969 (35 pages with 7 figures and 4 tables, RDB6A04)

toxicity tests to determine the cardiac sensitization potential in male beagle dogs with intravenous injection of epinephrine five minutes before and, with a challenge injection, midway into a 10 minute exposure of $R-11, R-13 B 1, R-$ 113 , and R-1120; for R-11 and R-113, LOEL at $5,000 \mathrm{ppm} v / \mathrm{v}$ as reported in RDB6467; for $\mathrm{R}-$ 13B1, LOEL at $75,000 \mathrm{ppm} \mathrm{v} / \mathrm{v}$ as reported in RDB6527; R-C318 and others 
Compendium of Abstracts from Long-Term Cancer Studies Reported by the National Toxicology Program from 1976-1992, Environmental Health Perspectives (EHP), publication NIH93218, National Institute of Health, U.S. Department of Health and Human Services (DHHS), Research Triangle Park, NC, 101(supplement 1), April 1993 (220 pages, RDB7744)

abstracts of toxicity studies from long-term cancer studies by the NTP from 1976-1992 for R10B2, R-11, R-20B1, R-20B2, R-20B3, R-2013, R-30, R-40B1, R-110, R-120, R-130, R-130a, R-140, R-140a, R-150, R-150a, R-160, R160B1, R-260da, R-260dab2, R-270da, R1110, R-1120, R-1130a, R-1270, and others

Compendium of Abstracts from Long-Term Cancer Studies Reported by the National Toxicology Program, Addendum to EHP Supplements, Vol. 101, National Institute of Health, U.S. Department of Health and Human Services (DHHS), Research Triangle Park, NC, November 1996 (116 pages with no figures or tables, RDB7745)

abstracts of toxicity studies

Criteria for a Recommended Standard - Occupational Exposure to Alkanes (C1-C8), publication 77-151, National Institute of Occupational Safety and Health (NIOSH), U.S. Department of Health and Human Services (DHS, then the U.S. Department of Health, Education, Welfare, DHEW), Cincinnati, OH, March 1977 (RDBA539)

toxicity of hydrocarbons $(\mathrm{HC})$

Documentation of the Threshold Limit Values and Biological Exposure Indices (sixth edition), publication 0206, American Conference of Governmental Industrial Hygienists (ACGIH), Cincinnati, OH, 1991 (RDB5141)

This book documents the bases for threshold limit values (TLVs) for airborne concentrations of chemical substances to which workers may be exposed. These chemicals include some commonly or historically used as refrigerants, based on their chronic and/or acute toxicity. The TLV data refer to concentrations under which it is believed that nearly all workers may be repeatedly exposed without adverse health effects subject to identified considerations. The TLVs are categorized as time-weighted average (TWA or TLV-TWA), short-term exposure limit (STEL or TLV-STEL), and ceiling (TLV-C). The refrigerants addressed include $R-11, R-12$, R-12B2, R-21, R-22, R-30, R-40, R-113, R-114, $\mathrm{R}-115, \mathrm{R}-170$ (ethane), R-290 (propane), R-
600 (butane), R-611, R-630, R-631, R-717 (ammonia), R-744 (carbon dioxide), R-764 (sulfur dioxide), R-1130, R-7146 (sulfur hexafluoride), and others.

Encyclopedia of Occupational Health and Safety, International Labour Office, Geneva, Switzerland, 1983 (RDB5140)

toxicity, flammability

Guide to Occupational Exposure Values 1999, publication 0377, American Conference of Governmental Industrial Hygienists (ACGIH), Cincinnati, OH, 1999 (145 pages, RDB9505)

This reference document compares the most recent concentrations for widely cited toxicity exposure limits. It includes Threshold Limit Values (TLV) from the American Conference of Governmental Industrial Hygienists (ACGIH), Permissible Exposure Limits (PEL) from the Occupational Safety and Health Administration (OSHA), the Recommended Exposure Limits (REL) from the National Institute for Occupational Safety and Health (NIOSH), and the 1989 maximale Arbeitsplatz Konzentration [maximum workplace concentration] (MAK) and biologischer Arbeitsstoff-Toleranz-Wert [biological tolerance value] (BAT) from the Deutsche Forschungsgemeinschaft [German Research Association] (DFG) Commission for the Investigation of Health Hazards of Chemical Compounds in the Workplace. It also identifies carcinogens found in the workplace that are identified by the above organizations, the International Agency for Research on Cancer (IARC), the U.S. National Toxicology Program (NTP), and the U.S. Environmental Protection Agency (EPA). The refrigerants addressed include $R-11, R-12, R-$ 12B2, R-21, R-22, R-30, R-40, R-114, R-115, $\mathrm{R}-170$ (ethane), R-290 (propane), R-600 (butane), R-611, R-630, R-631, R-717 (ammonia), $R-744$ (carbon dioxide), R-764 (sulfur dioxide), R-1130, R-7146 (sulfur hexafluoride), and others.

Hydrofluorocarbons and Hydrochlorofluorocarbons - Interim Report, external review draft, U.S. Environmental Protection Agency (EPA), Washington, DC, 15 November 1990 (84 pages with 20 tables, RDB7234)

draft support document to assess the toxicity of chlorofluorocarbon (CFC) replacements; summarizes toxicity studies for R-22, R-123, R-124, R-125, R-134a, R-141b, R-142b, and R-152a; discusses the potential for occupational and consumer exposures to these chemicals as re- 
frigerants and in other uses; briefly addresses their environmental fate and potential impacts on the general population; concludes as an interim finding based incomplete information and preliminary analyses that HCFCs and HFCs can be used in a manner safe to workers, consumers, and the general public given appropriate safeguards [Note: EPA staff indicate that this report has not been finalized (it remains a draft) as of February 1997, and there are no plans to publish a final version.]

IARC Monographs on the Evaluation of the Carcinogenic Risk of Chemicals to Humans, International Agency for Research on Cancer (IARC), Lyon, France, 1972-present (list of monographs as of 17 July 1998: 14 pages with no figures or tables, available from JMC as RDB8802)

The International Agency for Research on Cancer (IARC) / Centre International de Recherche sur le Cancer (CIRC) was established in 1965 by the United Nations (UN) World Health Organization (WHO). Its mission is to coordinate and conduct research on the causes of human cancer and to develop strategies for cancer control. The agency is involved in both epidemiological and laboratory research. IARC publishes a series of monographs summarizing evaluations of chemicals. IARC classifications indicated in the Refrigerant Database as from RDB8802 are as of 15 August 1998.

Inhalation Toxicity of Chlorotrifluoromethane (Freon 13) and Perfluorocyclobutane (Freon C318), report 38-56, Haskell Laboratory for Toxicology and Industrial Medicine, E. I. duPont de Nemours and Company, Incorporated, Newark, DE, 31 August 1956 (RDB7634)

subchronic toxicity tests for R-13 and R-C318

Kälteanlagen - Sicherheitstechnische Grundsätze für Gestaltung, Ausrüstung, Aufstellung, und Betrieben [Refrigerating Plants - Safety Principles for their Construction, Equipment, Installation, and Operation], DIN Standard 8975, Deutsches Institut für Normung e.V., Berlin, Germany (RDB4680)

Merck Index (eleventh edition), edited by S. Budavari, M. J. O'Neil, A. Smith, and P. E. Heckelman, Merck Company, Rahway, NJ, 1989 (RDB6A05)

widely cited reference for toxicity and other safety data

NEHC Recommends Submarine Atmosphere Limits for New Ozone-Friendly Refrigerants, CFC-Halon News, Navy CFC \& Halon Clearing- house, Arlington, VA, 5(3):6, September 1995 (1 page with 1 table, copies available from JMC as RDB5A11)

preliminary limits for submarine atmospheres, recommended by the Navy Environmental Health Center (NEHC), based on toxicity and potential decomposition by and/or corrosive effects on catalytic oxygen replenishment systems; recommended limits for $1 \mathrm{hr}, 24 \mathrm{hr}$, and 90 days are $5,000,1,000$, and $900 \mathrm{ppm}$ for $\mathrm{R}$ $134 \mathrm{a}$ and $2,000,1,000$, and $100 \mathrm{ppm}$ for R236fa; article indicates that Naval Sea Systems Command (NAVSEA) plans to reduce the R$134 \mathrm{a}$ limits to $2,000,1,000$, and $100 \mathrm{ppm}$, respectively, based on the concentrations used to test the atmosphere control system

NIOSH Pocket Guide to Chemical Hazards, publication 95-115, National Institute for Occupational Safety and Health (NIOSH), U.S. Department of Health and Human Services, Cincinnati, $\mathrm{OH}, 1994$ (available from GPO or NTIS, RDBA670)

This document lists safety information in tabular form for 677 chemicals found in the work environment. Many of them are regulated by the U.S. Occupational Safety and Health Administration (OSHA). Each entry identifies the chemical by name, synonym(s), trade name(s), structural or empirical chemical formula, Chemical Abstract Service (CAS) and Registry of Toxic Effects of Chemical Substances (RTECS) numbers, and assigned U.S. Department of Transportation identification and guide numbers. It then gives the National Institute for Occupational Safety and Health (NIOSH) Recommended Exposure Limit (REL), Short Term Exposure Limit (STEL), ceiling REL (REL-C), carcinogenic identification or cautions, and/or Immediately Dangerous to Life or Health (IDLH) value. It also indicates the OSHA Permissible Exposure Limit (PEL) or PEL ceiling value (PEL-C). The entries also provide physical descriptions and summary chemical and physical properties, as available. They include concentration conversion factors, molecular weight, normal boiling point, solubility in water, flash point, ionization potential, representative vapor pressure, melting or freezing point temperature, flammability limits, minimum explosive concentration, representative specific gravity, and relative density of gases referenced to air. Entries list incompatibilities, reactivities, and measurement methods for the chemicals along with recommendations for personal protection, sanitation, and respirators. The entries conclude with health hazard summaries that identify body en- 
try routes, symptoms, first aid measures, and impacted target organs. An introduction briefly describes these data and the codes used in the listings. Appendices provide recommendations on potential occupation carcinogens, identify 13 regulated carcinogens, discuss supplementary exposure limits, discuss exposure limits for certain substances without established RELs, discuss respirator recommendations for selected chemicals, and provide miscellaneous notes. They also list more protective OSHA PELs that were vacated by a court decision and provide indices by synonym and trade names, by CAS registry numbers, and by DOT identification numbers. A number of historical, current, and candidate refrigerants are covered. They include R-10, R-11, R-12, R-12B2, R13B1, R-21, R-22, R-30, R-30B1, R-40, R-112, R-113, R-114, R-115, R-290 (propane), R-600 (butane), R-600a (isobutane), R-601 (pentane), R-610, R-630, R-631, R-717 (ammonia), R-744 (carbon dioxide), R-764 (sulfur dioxide), R1120, R-1130, R-7146 (sulfur hexafluoride), and others.

PAFT Member Companies Respond to Lancet Report, press release, Programme for Alternative Fluorocarbon Toxicity Testing (PAFT), Washington, DC, 27 August 1997 (3 pages with no figures or tables, limited copies available from JMC as RDB7C73)

provides further information on the underlying incident and subsequent investigations in response to an article in The Lancet (see RDB$7 \mathrm{C70}$ ) on exposures of workers to R-124/123 [actually R-290/124/123]: describes the article as "incomplete and unsubstantiated"; quotes $G$. $R$. Rusch (director of toxicology and risk assessment for AlliedSignal Incorporated and chairman of the PAFT Toxicology Committee) that overexposure to any chemical can cause a toxic response; notes that $\mathrm{R}-123$ and $\mathrm{R}-124$ have undergone extensive toxicity testing and "have been found to not pose any adverse health effects, including acute liver toxicity, when used properly in their intended applications"; notes that the cited article attempted to correlate clinical evidence of hepatitis with exposure to refrigerants, but that exposure duration and concentration were never addressed; quotes W. J. Brock (staff toxicologist for DuPont Chemicals) that linkage of liver injury to exposure to R-123 or R-124 is premature since the authors made no attempt to assess exposure concentrations or to rule out other chemicals in the smelting plant; quotes R. Rubenstein (a toxicologist with the U.S. Environmental Protection Agency, EPA) that all of the alternative refrigerants can be used safely when industry standards are followed, but that they were not adhered to in this incident

PAFT Statement on Wright Patterson/Armstrong Laboratory Study Involving HFC-134a and HFC-227ea, press release, Programme for Alternative Fluorocarbon Toxicity Testing (PAFT), Washington, DC, 24 September 1997; posted on the Internet (http://thor.he.net/paft), 3 October 1997 (2 pages with no figures or tables, RDB7C81)

provides further information on inhalation toxicity test responses reported in an Air-Force study (see RDB7C80) for exposures with R13B1, R-134a, and R-227ea: notes that the wealth of experience from use of R-134a and $\mathrm{R}-227$ ea contradicts the results initially reported in that study; describes tests by the International Pharmaceutical Aerosol Consortium for Toxicology Testing (IPACT) and independent pharmaceutical companies at lower and higher concentrations in which no effect on blood pressure or pulse were seen; also notes animal tests relevant to human cardiovascular responses at concentrations at least 12 times higher with no adverse effects; concludes that the effects "were most likely not a direct result of the chemical exposure"; describes a planned clinical study, with independent oversight, to investigate the effects of prolonged exposures to $\mathrm{R}-134 \mathrm{a}$ and R-227ea

PAFT Update, document H-45950, DuPont Chemicals, Wilmington, DE, November 1992 (4 pages with 2 tables, available from JMC as RDB3909)

This bulletin outlines the Programme for Alternative Fluorocarbons Toxicity Testing (PAFT), a cooperative research effort sponsored by chlorofluorocarbon producers. PAFT is designed to expedite the development of toxicology data for possible substitute fluorocarbons. The programs integrate past and present toxicological information to perform a careful risk assessment. The bulletin outlines the six main types of studies conducted by PAFT. They include acute toxicity primarily by inhalation, genotoxicity, subchronic toxicity by inhalation, developmental toxicity by teratology and inhalation, chronic toxicity and carcinogenicity by inhalation, and environmental toxicity. PAFT has initiated more than 100 individual toxicology studies. A table gives the schedule for 
testing, broken into phases the longest of which requires four years. Five programs are identified: PAFT I, which began in 1987, covers R123 and R-134a. PAFT II, initiated in 1988, addresses R-141b. PAFT III, begun in late 1989, is studying $R-124$ and $R-125$. PAFT IV, established in May 1990, will examine R-225ca and $R-225 \mathrm{cb}$ for solvent cleaning. PAFT $V$, established in early 1992, will examine R-32. A table summarizes key results as of November 1992 for the fluids names as well as for R-11 and R-12 for comparison. The data include $A L C / L C_{50}$, oral $L D_{50}$ cardiac sensitization thresholds, Ames assay, in vivo mutagenicity, subchronic no observed effect level (NOEL), teratogenic, chronic inhalation, and flammability data. The completion schedule and recommended exposure limits are included.

Patty's Industrial Hygiene and Toxicology (fourth edition), edited by G. D. Clayton and F. E. Clayton, John Wiley and Sons, Incorporated, New York, NY, 1993-1995 (11 books totalling more than 9000 pages, RDB5704)

detailed reference for toxicity and other safety data; comprises volumes on "General Principles", "Toxicology", and "The Theory and Rationale of Industrial Hygiene Practice

Permissible Levels of Toxic Substances in the Working Environment, Proceedings of the 6th Session of the Joint ILOMHO Committee on Occupational Health (Geneva, Switzerland, 4-10 June 1968), International Labour Office (ILO) of the World Health Organization (WHO), Geneva, Switzerland, 1970 (405 pages, RDB8409)

R-717 and others, health effects, toxicity

Preliminary Environmental Hazard Assessment of Chlorinated Naphthalenes, Silicones, Fluorocarbons, Benzenepolycarboxylates, and Chlorophenols, U.S. Environmental Protection Agency (EPA), Washington, DC, 1973 (96 pages, available from NTIS as document PB-238074, RDB7241)

chlorinated refrigerants, BCFC, BHCFC, CFC, HCFC

Programme for Alternative Fluorocarbon Toxicity Testing (PAFT) Toxicology Summaries, PAFT, Washington, DC, September 1995 (16 pages, available from JMC as RDB5C39)

Eight data sheets summarize the status and findings of the Programme for Alternative Fluorocarbon Toxicity Testing (PAFT). They outline studies of acute toxicity (short-term exposures to high concentrations, such as accidental leakages), genotoxicity (effects on genetic material, an early screen for possible carcinogenic activity), sub-chronic toxicity (repeated exposure to determine any overall toxicological effect), reproductive and developmental toxicity (teratology, assessment of the effects on the reproductive system and of the potential for causing birth defects), chronic toxicity/carcinogenicity (lifetime testing to assess late-in-life toxicity or increased evidence of cancer), and ecotoxicity (assessment of potential to affect living organisms in the environment). Toxicology summaries for R-32, R-123, R-124, R-125, R-134a, and $R-141 b$ as well as a combined summary for R-225ca and R-225cb indicate that all have low or very low toxicity. Test results show them not to be genotoxic and, except for the R-225 isomers, also not developmental toxicants. The findings show that $R-225 \mathrm{ca}$ produced effects on the liver of rodents and a minor effect in a primate at $650-1,000 \mathrm{ppm}$. Exposures to R-225cb in the range of 1,000 to $5,000 \mathrm{ppm}$ resulted in only marginal effects in rodents or a primate. Additionally, R-123 was found to have low acute dermal and inhalation toxicity, but caused an increased incidence of benign - but not life threatening - tumors in animals following longterm exposures. $\mathrm{R}-134 \mathrm{a}$ and $\mathrm{R}-141 \mathrm{~b}$ also caused an increased incidence of benign - but not life threatening - tumors in animals following long-term exposure to high concentrations. A Glossary of Terms is provided.

Registry of Toxic Effects of Chemical Substances $\left(\right.$ RTECS $\left.^{\circledR}\right)$, computerized database, $\mathrm{Na}$ tional Institute of Occupational Safety and Health (NIOSH), U.S. Department of Health and Human Services, Cincinnati, $\mathrm{OH}$, updated quarterly since June 1971 (see synopsis below for availability, RDB4450)

The Registry of Toxic Effects of Chemical Substances, more widely known by the trademarked acronym RTECS ${ }^{\circledR}$, is a database of toxicologic information originally identified as the "Toxic Substances List" (TSL). RTECS is compiled, maintained, and updated by NIOSH pursuant to the Occupational Safety and Health Act of 1970. The database is a compendium of data extracted from the open scientific literature on more than 120,000 substances. They include most chemicals previously and currently used as refrigerants. Six types of toxicity data are included, namely primary irritation, mutagenic effects, reproductive effects, tumorigenic effects, acute toxicity, and other multiple-dose 
toxicity. Specific numeric toxicity values such as $L C_{50}, L D_{50}, T C_{L 0}$ are noted with the species studied, exposure duration, and route of administration. A coded bibliographic source is included for most data to enable access to the cited studies. The actual data have not been evaluated by NIOSH. RTECS also includes chemical identification data and special reviews, including exposure limits and carcinogenicity assessments. It also includes information on regulatory requirements, $\mathrm{NIOSH}$-generated data, and notations on U.S. Government program data tracking and sources. The database records consist of coded data that can be expanded, by the accessing software, to readable form. Quarterly updates to the database can be leased from the National Technical Information Service (NTIS). RTECS is more commonly accessed through a number of on-line services, among them the National Library of Medicine (NLM) TOXNET System, the Canadian Centre for Occupational Health and Safety (CCOHS) CCINFOLine system, and the European Space Agency (ESA) Informational Retrieval Service (IRS), and commercial sources. They include the Chemical Information Service (CIS), DIALOG, Science and Technology Network (STN, available in North America through the Chemical Abstract Service, CAS), and others. It also is available on the CHEM-BANK ${ }^{\text {TM }}$ compact disk from SilverPlatter Information Limited (Norwood, MA, USA, and London, UK) and TOMES-PLUS $^{\text {is }}$ from Micromedex Incorporated (Denver, CO, USA).

Registry of Toxic Effects of Chemical Substances (RTECS ${ }^{\circledR}$ ) - Comprehensive Guide to the RTECS ${ }^{\circledR}$, publication $93-1330$, edited by D. V. Sweet, National Institute of Occupational Safety and Health (NIOSH), U.S. Department of Health and Human Services, Cincinnati, $\mathrm{OH}$, September 1993 (80 pages with 1 figure, 19 tables, and a microfiche with the bibliographic citations, available from GPO, RDB4449)

This guide provides a detailed description of the format of the RTECS ${ }^{\circledR}$ database and tabulates the codes used and their meanings. The code tables and text define the measures, abbreviations, and qualitative descriptors contained in RTECS. The guide also summarizes data sources and record formats. It also reviews sources to obtain or access RTECS.

Report of Safety Concerns, press release, The Trane Company, La Crosse, WI, 25 August 1997
(3 pages with no figures or tables, limited copies available from JMC as RDB7C71)

provides further information on the underlying incident and subsequent investigations in response to an article in The Lancet (see RDB7 C70) on exposures of workers to R-124/123 [actually R-290/124/123]: notes that all of the workers fully recovered; quotes $R$. Rubenstein, a toxicologist with the U.S. Environmental Protection Agency (EPA), that the alternative refrigerants involved can be used safely and suggestion that incorrect handling practices resulted in hazardous conditions is "outlandish"; quotes W. R. Brock, manager of fluorochemical toxicology for DuPont Chemicals, that the recommended exposure limits were exceeded for a long period of time and that any chemical can be "dangerous" if not handled properly; quotes J. M. Calm, and independent engineering consultant, that numerous violations of accepted safety practices were involved and that the conditions were not typical; documents further details from these individuals; concludes that all refrigerants can be used safely and that all pose risks without regard to appropriate practices, as appears to have been the case in the reported exposures

Safety, Toxicity, and Biological Importance of Organofluorine Compounds, Organic Fluorine Chemistry, edited by W. A. Sheppard and C. M. Sharts, W. A. Benjamin Incorporated, New York, NY, 447-461, 1969 (25 pages, RDB7255)

reference for toxicity and other safety data for fluorinated refrigerants and other chemicals

Standard Test Method for Estimating Sensory Irritancy of Airborne Chemicals, standard E98184, American Society of Testing and Materials (ASTM), West Conshoshocken, PA, May 1994. reapproved in 1992 (10 pages with 9 figures, RDB6567)

This standard prescribes a laboratory test method for rapid determination of the sensory irritant potential for a wide range of airborne chemicals, including refrigerants and refrigerant blends. The procedure quantifies the concentration of airborne irritant that produces a $50 \%$ decrease in the respiratory rate $\left(R D_{50}\right)$ in mice, based on a dose-response curve constructed from tests conducted for a range of concentrations. The $R_{50}$ may be used to estimate the threshold limit value (TLV) for man, but does not address discomfort from odors. The standard outlines the significance and use of the method; details the required apparatus, rea- 
gents, and test animals; and identifies associated hazards and limitations. It outlines and schematically illustrates the procedure, including required steps for sample preparation, calibration, pretest conditioning, and selection of test parameters. It then presents the actual test procedure and methods to interpret the results. It also identifies the minimum information to be recorded and concludes with a nonmandatory list of accepted apparatus and suppliers.

Submarine Atmosphere Habitability Data Book, report AVSHIPS 250-649-1 revision 1, U.S. Bureau of Ships and U.S. Department of the Navy, Washington, DC, 1962 (RDB72A4)

maximum allowable concentration (MAC) for R717 (25 ppm v/v for 60 days continuous exposure and $400 \mathrm{ppm}$ for 1 hour) and others as reported in RDB5340

Tabulae Biologicae Per., 3:231-296, 1933 (65 pages in German, RDB6283)

LCLo human of 5,000 ppm v/v for R-717 (ammonia); $L C_{L o}$ human of $90,000 \mathrm{ppm} v / \mathrm{v}$ for $R$ 744 (carbon dioxide) - as reported in RDB4450 and RDB5340

The AlHA 2000 Emergency Response Planning Guidelines and Workplace Environmental Exposure Level Guides Handbook, document 358EA-00, American Industrial Hygiene Association (AlHA), Fairfax, VA, 2000 (RDBA948)

summarizes development and intended uses for Emergency Response Planning Guideline (ERPG) concentrations, to "assist emergency response personnel planning for catastrophic chemical releases to the community;" discuses other occupational and emergency exposure guides; summarizes the rationales for ERPG values based on "the maximum airborne concentration below which it is believed nearly all individuals could be exposed for 1 hour" without specified responses; identifies three levels, namely the ERPG-1 ("without experiencing more than mild transient adverse health effects or without perceiving a clearly defined objectionable odor"), ERPG-2 ("without experiencing or developing irreversible or other serious health effects or symptoms that could impair an individual's ability to take protective action"), and ERPG-3 ("without experiencing or developing life-threatening health effects"); tabulates ERPGs for R-10, R-20, R-30, R-40, R-40B1, R4011, R-140a, R-142b, R-630, R-631a, R-717 (ammonia), R-764 (sulfur dioxide), R-1110, R1113, R-1114, R-1120, R-1216, and others; describes Workplace Environmental Exposure Level (WEEL) Guides as "the workplace exposure levels to which it is believed that nearly all employees could be exposed repeatedly without adverse health effects;" tabulates WEELs for R-32, R-123, R-124, R-125, R-134a, R141b, R-142b, R-143a, R-152a, R-E170, R236fa, R-1113, and others; provides related definitions to assist users of ERPG and WEEL values in applying them

Two-Week Inhalation Toxicity Study of FC-132b and FC-124 in Rats, TSCAT report OTS0530590, Haskell Laboratory for Toxicology and Industrial Medicine, E. I. duPont de Nemours and Company, Incorporated, Newark, DE, 8 October 1976 (RDB7266)

$\mathrm{R}-124, \mathrm{R}-132 \mathrm{~b}$, health effects, toxicity

report 23-45, Haskell Laboratory for Toxicology and Industrial Medicine, E. I. duPont de Nemours and Company, Incorporated, Newark, DE, 1945 (RDB6511)

$\mathrm{R}-12, \mathrm{R}-23$, and others, acute inhalation toxicity: guinea pigs were exposed to $30,000 \mathrm{ppm}$ $\mathrm{R}-23$ for $6 \mathrm{hr}$ and no signs toxicity or gross or microscopic pathological changes were noted as reported in RDB5604

\section{R-10}

E. M. Adams, H. C. Spencer, V. K. Rowe, D D. McCollister, and D. D. Irish, Vapor Toxicity of Carbon Tetrachloride Determined by Experiments on Laboratory Animals, Archives of industrial Hygiene and Occupational Medicine, 6:5066, 1952 (7 pages, RDB6509)

$\mathrm{R}-10$, health effects, toxicity, $\mathrm{LC}_{50}$

Emergency Response Planning Guidelines Carbon Tetrachloride, American Industrial $\mathrm{Hy}$ giene Association (AlHA), Fairfax, VA, 1995 (RDB4B82)

R-10, tetrachloromethane, ERPG-1, ERPG-2, ERPG-3, toxicology data, toxicity, safety classification

\section{R-11}

J. Adir et al., Journal of Clinical Pharmacology, 15:116-128, 1975 (13 pages, RDB6552)

$\mathrm{R}-11$, health effects, toxicity 
J. Adir et al., Toxicology and Applied Pharmacology (TAP), 29(1):142-143, 1974 (2 pages, RDB6553)

$R-11$, health effects, toxicity

Y. M. Amin, E. B. Thompson, And W. L. Chiou, Fluorocarbon Aerosol Propellants. XIII. Correlation of Blood Levels of Trichloromonofluoromethane to Cardiovascular and Respiratory Responses in Anesthetized Dogs, Journal of Pharmaceutical Sciences, 68(2):160-163, 1979 (4 pages, RDB6554)

$\mathrm{R}-11$, cardiac sensitization, health effects, toxicity

J. Angerer, B. Schroder, and R. Heinrich, Exposure to Fluorotrichloromethane (R-11), International Archives of Occupational and Environmental Health, Germany, 56(1):67-72, 1985 (6 pages, RDB6493)

$\mathrm{R}-11$, health effects, toxicity

D. M. Aviado (University of Pennsylvania School of Medicine), Toxicity of Aerosol Propellants in the Respiratory and Circulatory Systems, IX. Summary of the Most Toxic: Trichlorofluoromethane (FC 11), Toxicology, 3:311-319, 1975 (9 pages, RDB5C69)

$\mathrm{R}-11$, cardiac sensitization, circulatory abnormalities, health effects, toxicity

C. E. Barras, Acute Inhalation Test [of Trichloromonofluoromethane (Freon ${ }^{\circledR} 11$ )], report 648-74, Haskell Laboratory for Toxicology and Industrial Medicine, E. I. duPont de Nemours and Company, Incorporated, Newark, DE, 29 October 1974 (2 pages with 1 table, RDB5C53)

tests of the acute inhalation toxicity of R-11 in groups of 6 albino ChR-CD rats at concentrations of $5,100,7,700,9,600,24,900,30,000$, 31,700 , and $35,300 \mathrm{ppm} \mathrm{v} / \mathrm{v}$ in air determined by gas chromatography of samples as timeweighted averages; no fatalities at $\mu 9,600 \mathrm{ppm}$; 3 of 6 animals died during exposures or recovery at $24,900 \mathrm{ppm}$; calculated $4-\mathrm{hr} \mathrm{LC}_{50}$ rat $=$ $26,200 \mathrm{ppm}$; at concentrations $\mu 9,600 \mathrm{ppm}$, mild hyperactivity for the first $2 \mathrm{hr}$ of exposure and rapid respiration throughout exposure; occasional weight loss for 1 day following exposure; at concentrations of $\cdot 24,900 \mathrm{ppm}$, initial mild hyperactivity followed by tremors, inactivity, irregular respiration, and death in 1-4 hr; surviving animals showed mild to moderate initial weight loss for 1 day post exposure
D. A. Blake and G. W. Mergner, Inhalation Studies on the Biotransformation and Elimination of $\left[{ }^{14} \mathrm{C}\right]-T$ richlorofluoromethane (FC-11) and $\left[{ }^{14} \mathrm{C}\right]$-Dichlorodifluoromethane (FC-12) in Beagles, Toxicology and Applied Pharmacology (TAP), 30(3):396-407, 1974 (12 pages, RDB5C70)

toxicity tests of $R-11$ and $R-12$ biochemistry and metabolism in dogs: very little of the inhaled dose appeared to be metabolized as reported in RDB5C72

P. J. Cox, L. J. King, and D. V. Parke, A Study of the Possible Metabolism of Trichlorofluoromethane, Biochemical Journal, 130(1):13P-14P, 1972 (2 pages, RDB8134)

R-11, biochemistry, health effects, metabolism, pharmacokinetics, toxicity

C. W. Eddy, Eye Irritation in Rabbits [of Trichloromonofluoromethane (Freon ${ }^{\circledR} 11$ )], report 423-70, Haskell Laboratory for Toxicology and Industrial Medicine, E. I. duPont de Nemours and Company, Incorporated, Newark, DE, 1 October 1970 (RDB5C54)

$\mathrm{R}-11$, dermal contact toxicity, irritation as reported in RDB5141

M. Haj, Z. Burstein, E. Horn, and B. Stamler, Perforation of the Stomach Due to Trichlorofluoromethane (Freon 11) Ingestion, Israeli Journal of Medical Science, 16(5):392-394, 1980 (3 pages, RDB7422)

$\mathrm{R}-11$, health effects, toxicity: nonfatal accident involving human exposure; healthy man mistook a bottle of R-11 in a refrigerator for water and drank it, resulting in freezing, tissue necrosis, and multiple perforations of the stomach as reported in RDB7414

D. B. Hood, Eye Irritation, report 107-64, Haskell Laboratory for Toxicology and Industrial Medicine, E. I. duPont de Nemours and Company, Incorporated, Newark, DE, 4 September 1964 (RDB5C55)

$\mathrm{R}-11$, dermal contact toxicity, irritation as reported in RDB5141

L. J. Jenkins, Jr., R. A. Jones, R. A. Coon, and J. Siegel, Repeated and Continuous Exposures of Laboratory Animais to Trichlorofluoromethane, Toxicology and Applied Pharmacology (TAP), 16:133-142, 1970 (RDB5C60)

$\mathrm{R}-11$, chronic inhalation toxicity as reported in RDB5141

A. Koops, Mutagenicity Activity of Methane, Trichlorofluoro- in the Salmonella/Microsome 
Assay, report 568-77, Haskell Laboratory for Toxicology and Industrial Medicine, E. I. duPont de Nemours and Company, Incorporated, Newark, DE, 5 August 1977 (RDB5C58)

R-11, mutagenicity, Ames Assay, toxicity as reported in RDB5141

D. M. Krentz, P. J. Lardear, and R. C. Graham, untitled toxicity review for Freon ${ }^{\circledR} 11$ (methane, trichlorofluoro-), Haskell Laboratory for Toxicology and Industrial Medicine, E. I. duPont de Nemours and Company, Incorporated, Newark, DE, 17 February 1992 (RDB5873)

$\mathrm{R}-11$, summary of published and unpublished toxicity literature

S. D. Morrison, Inhalation Toxicity of "Freon 11" and of "Freon 11 " $+0.3 \%$ Nitromethane, report 53-61, Haskell Laboratory for Toxicology and Industrial Medicine, E. I. duPont de Nemours and Company, Incorporated, Newark, DE, 25 October 1961 (5 pages with 2 tables, RDB65H3)

acute inhalation toxicity tests of R-11 and of mixtures for possible aerosol propellant use of R-11 with nitromethane: 0 of 4,1 of 4 , and 4 of 4 animals died in exposures to $35,600,65,680$, and $118,370 \mathrm{ppm} \mathrm{R}-11$ in air; found the 4-hr ALC rat to be $66,000 \mathrm{ppm} v / \mathrm{v}$ probably by interference with the central nervous control of respiration; also summarizes repeat exposure tests at $12,000 \mathrm{ppm}$ in groups of 4 male ChR$C D$ rats; exposed animals exhibited increased rate and depth of respiration, slight twitching, marked licking, and chewing movements, but apparent recovery was complete within a few minutes following exposures; no body weight gain or changes in gross appearance were observed, though one animal killed after the last R-11 exposure had kidney and liver weights slightly below those of a control group

National Cancer Institute ( $\mathrm{NCl}$ ), Bioassay of Trichlorofluoromethane for Possible Carcinogenicity, report 78-1356 (also identified as NCI-CGTR-106), National Institute of Health (NIH), U.S. Department of Health, Education, and Welfare, Bethesda, MD, 1978 (84 pages, RDB7427)

$\mathrm{R}-11$, toxicity: bioassays in mice and rats found R-11 to not be carcinogenic when administered orally as reported in RDB7414

S. Niazi and W. L. Chiou, Fluorocarbon Aerosol Propellants IV: Pharmacokinetics of Trichloromonofluoromethane Foliowing Single and Multiple Dosing in Dogs, Journal of Pharmaceutical
Sciences, 64(5):763-769, 1975 (7 pages, RDB8167)

R-11, biochemistry, elimination, health effects, toxicity

S. Nick, Inhalation Toxicity of Freon ${ }^{\circledR} 11$, report 71-64, Haskell Laboratory for Toxicology and Industrial Medicine, E. I. duPont de Nemours and Company, Incorporated, Newark, DE, 17 June 1964 (RDB5C56)

$\mathrm{R}-11$, inhalation toxicity as reported in RDB5141

R. Panson, J. Galvin, J. Figard, and S. Cragg, Nine Light Petroleum Hydrocarbons: Toxicology, Environmental Fate, Ecological Effects, Phillips Petroleum Company, Bartlesville, OK, 1996 (RDB7621)

summary of toxicity, ecotoxicity, and environmental fate, and ecological effects of R-600a (isobutane), R-601 (n-pentane), R-601a (isopentane), R-601b (neopentane), cyclopentane, 2-methylpentane, 2,2-dimethylbutane, and 2,3-methylbutane based on a review of published and unpublished studies

J. W. Sarver and D. A. Snee, Washout Time of Freon ${ }^{\circledR} 11$, report 349-70, Haskell Laboratory for Toxicology and Industrial Medicine, E. I. duPont de Nemours and Company, Incorporated, Newark, DE, 11 August 1970 (RDB5C59)

$R-11$, inhalation toxicity as reported in RDB5141

H. Sherman, 90-Day Feeding in Rats and Dogs with Freon ${ }^{\circledR} 11$, report 63-72, Haskell Laboratory for Toxicology and Industrial Medicine, E. I. duPont de Nemours and Company, Incorporated, Newark, DE, 18 February 1972 (RDB5C57)

$R-11$, ingestion toxicity by ingastric administration: no abnormality in results of tests of hematologic, hepatic, or renal values; a slight increase in urinary fluorides was statistically not significant - as reported in RDB5141 and RDB5C72

R. D. Stewart et al. (Medical College of Wisconsin), Acute and Repetitive Human Exposure to Fluorotrichloromethane [trichlorofluoromethane], report PB-279203/LLC, National Technical Information Service (NTIS), Springfield, VA, 1 December 1975 (available from NTIS, RDB6598)

reports an inhalation toxicity test of R-11 in male volunteers; notes that acute exposures to 250 , 500 , or $1000 \mathrm{ppm} \mathrm{v/v}$ for $1 \mathrm{~min}$ to $8 \mathrm{hr}$ "did not 
produce any untoward physiological effects"; also reports that groups of male volunteers repetitively exposed to $1,000 \mathrm{ppm} \mathrm{v} / \mathrm{v}$ for $1-10 \mathrm{hr} / \mathrm{d}, 5 \mathrm{~d} / \mathrm{wk}$, for 2-4 wk showed minor decrements in several cognitive tests, but no change in pulmonary function or alteration of in cardiac rhythm - as reported in RDB6115

G. J. Taylor and R. T. Drew, Toxicology and Applied Pharmacology (TAP), 32:177 ff, 1975 (RDB5C67)

$\mathrm{R}-11$, cardiac sensitization, circulatory abnormalities, health effects, toxicity

G. J. Taylor, Archives of Environmental Health, 30:349 ff, 1975 (RDB5C68)

$R-11$, cardiac sensitization, circulatory abnormalities, health effects, toxicity

G. Thomas, Narcotic Effects of Acute Exposures to Trichloromonofluoromethane (Freon 11), Transactions of the Association of Indian Medical Officers, 15(3):105-106, 1965 (2 pages, RDB8178)

$R-11$, anesthesia, narcosis, health effects, toxicity

F. M. Williams, G. H. Draffan, C. T. Dollery, J. C Clark, A. J. Palmer, and P. Vernon, Use of ${ }^{18} \mathrm{~F}$. Labelled Fluorocarbon-11 to Investigate the Fate of Inhaled Fluorocarbons in Man and in the Rat, Thorax, 29:99-103, 1974 (5 pages, RDB7429)

$R-11$, health effects, toxicity: inhaled $R-11$ is eliminated from the body rapidly as reported in RDB7414

C. R. Wolf, L. J. King, and D. V. Parke, The Anaerobic Dechlorination of Trichlorofluoromethane by Rat Liver Preparations in Vitro, Chemical-Biological Interactions, 21(2-3):277-288, 1978 (12 pages, RDB8189)

R-11: biochemistry, health effects, metabolism, pharmacokinetics, toxicity

$\mathrm{NCl}$ Carcinogenesis Bioassay Experimental Design Status Report, Contract Number 723278, Agent Number CO4637, National Cancer Institute (NCI), Bethesda, MD, 53, 22 February 1978 (RDB5C61)

$\mathrm{R}-11$, tumor incidence, toxicity as reported in RDB5141

Toxicity Summary - Genetron ${ }^{\circledR} 11$ (Trichloromonofluoromethane), Acute Toxicity and Cardiotoxicity, AlliedSignal Incorporated (then Allied
Corporation), Morristown, NJ, March 1978 (RDB5144)

R-11

Trichlorofluoromethane (CFC 11) Health Effects Information, CAS\# 75-69-4, Elf Atochem North America, Incorporated, Philadelphia, PA, undated circa 1995 (3 pages, RDB5B47)

R-11, summary of toxicological and ecological information

\section{$\mathrm{R}-12$}

T. Astrom et al., Exposure to Fluorocarbons During the Filling and Repair of AirConditioning Systems in Cars - A Case Report, Scandinavian Journal of Work Environment and Health, 13(6):527-528, 1987 (2 pages, RDB7529)

R-12, health effects, toxicity: human occupational exposures involving R-12

A. Azar, C. F. Reinhardt, M. E. Maxfield, P. E. Smith, Jr., and L. S. Mullin (E. I. duPont de Nemours and Company, Incorporated), Experimental Human Exposure to Fluorocardon [sic, Fluorocarbon] 12 (Dichlorodifluoromethane), presented at the AlHA Conference (Toronto, Canada, May 1971); republished in AlHA Journal, 33(4):207-216, April 1972 (10 pages with 4 figures and 7 tables, RDB5358)

tests in humans of the acute toxicity of R-12 by inhalation to determine safe hygienic standards for single, brief exposures; two volunteers were exposed whole body to concentrations of 0 (control), 1,000 and 10,000 ppm v/v (mean actual 1000 and 1027, 9855 and $10173 \mathrm{ppm} \mathrm{v} / \mathrm{v}$ ) $\mathrm{R}-12$ in air for $2 \frac{1}{2} \mathrm{hr}$; no adverse effects were found at $1,000 \mathrm{ppm}$ by clinical observations, laboratory tests, subjective impressions, electrocardiogram monitoring, and tests of psychomotor skills; exposures to $10,000 \mathrm{ppm} \mathrm{v} / \mathrm{v}$ evoked a $7 \%$ reduction in standardized psychomotor test scores; paper note that this finding is not indicative of a serious health threat; breath samples showed that R-12 is quickly eliminated from the lungs

A. Azar, J. A. Zapp, C. F. Reinhardt, and G. J. Stopps (E. I. duPont de Nemours and Company, Incorporated), Cardiac Toxicity of Aerosol Propellants, Journal of the American Medical Association (JAMA), 215(9):1501-1502, 1971 (2 pages, RDB8128) 
cardiac sensitization of fluorochemicals used as aerosol propellants and also as refrigerants, toxicity

M. Bass, Sudden Sniffing Death, Journal of the American Medical Association (JAMA), 212(12):2075-2079, 1970 (5 pages, RDB6722)

fatal human exposure involving $\mathrm{R}-12$ from abusive use of aerosol products

C. Brenner, Note on the Action of Dichlorodifluoromethane on the Nervous System of the Cat, Journal of Pharmacology and Experimental Therapeutics (JPET), 59:176-181, 1937 (6 pages, RDB7532)

R-12, health effects, acute inhalation toxicity, and anesthetic effect; examines the convulsant effects of R-12 in cats and concludes that the tremors see were the result of release phenomena, indicating a type of depressant with low toxicity rather than a true convulsant - as reported in RDB5828

R. F. Carlton, Fluorocarbon Toxicity: Aerosol Deaths and Anesthetic Reactions, Annals of Clinical and Laboratory Science, 6:411-414, 1976 (4 pages, RDB6723)

fatal human exposure involving $R-12$, sudden sniffing death from abusive use of aerosol products

I. K. Chernen'kii and V. A. Shugaev, Toxicity of Freon 12 with Consideration of Its Decomposition Products, Gigiena Truda i Professional'nye Zabolevaniya [Labor Hygiene and. Occupational Diseases], Russia (then USSR), (7):52 ff, 1974 (in Russian, RDB7528)

$R-12$, health effects, toxicity

J. Cheymol, Un réfrigérant industriel récent: le dichlorodifluorométhane [A New Industrial Refrigerant: Dichlorodifluoromethane], Presse Med., 44:1123 ff, 1936 (in French, RDB7219)

R-12, toxicity, historical perspective

R. Culik and $H$. Sherman, Teratogenic Study in Rats with Dichlorodifluoromethane (Freon 12), report 206-73, Haskell Laboratory for Toxicology and Industrial Medicine, E. I. duPont de Nemours and Company, Incorporated, Newark, DE, 1973 (RDB8135)

$R-12$, teratogenicity, toxicity - as reported in RDB5367

C. W. Eddy and F. D. Griffith (E. I. duPont de Nemours and Company, Incorporated), Metabolism of Dichlorodifluoromethane $\mathrm{c}^{14}$ (Freon $^{\circledR} 12$ $\mathrm{C}^{14}$ ) by Rats, presented at the AlHA Conference (Toronto, Canada, May 1971), American Industrial Hygiene Association (AlHA), Fairfax, VA, 1971 (RDB65H9)

$\mathrm{R}-12$, metabolism, toxicity: radio-labeled $\mathrm{R}-12$ study; approximately $2 \%$ of oral dose metabolized to carbon dioxide and $1 / 2 \%$ excreted in urine; R-12 and its matabolites were eliminated within 30 minutes - as reported in RDB65H1

I. G. Jefferson, Accidental Death of Child Playing with Deodorant Aerosol, Lancet, UK, 1:779, 1978 (1 page, RDB6727)

fatal human exposure involving R-12 from abusive use of aerosol products, sudden sniffing death

Joint FAONHO Expert Committee on Food Additives, WHO Food Additive Series, Report 8, World Health Organization (WHO), Geneva, Switzerland, 1975 (RDB7533)

R-12 and others, health effects, oral toxicity: dietary level of R-12 that causes no toxicological effect in rats is estimated to be $0.3 \%$ as reported in RDB5141

R. A. Kehoe, Report on Human Exposure to Dichlorodifluoromethane in Air, The Kettering Laboratory, University of Cincinnati, $\mathrm{OH}$, June 1943 (RDB5C45)

R-12, toxicity: acute exposures of humans, for 11-80 minutes to concentrations of 40,000 $110,000 \mathrm{ppm}$ as reported (with summary) in RDB5906 and RDB7414

S. M. Kilen and W. S. Harris, Effect of Hypoxia and Freon 12 on the Mechanics of Cardiac Contractions, American Journal of Physiology, 230(6):1701-1707, 1976 (7 pages, RDB8154)

cardiac effects of R-12 aerosol propellant (also used as a refrigerant), health effects, toxicity

S. M. Kilen and W. S. Harris, Direct Depression of Myocardial Contractility by the Aerosol Gas Dichlorodifluoromethane, Journal of Pharmacology and Experimental Therapeutics (JPET), 183(2):245-255, 1972 (11 pages, RDB8155)

cardiac effects of R-12 aerosol propellant (also used as a refrigerant), health effects, toxicity

M. A. Lee and R. C. Graham, untitled toxicity review for Freon ${ }^{\circledR} 12$ (methane, dichlorodifluoro-), Haskell Laboratory for Toxicology and Industrial Medicine, E. I. duPont de Nemours and Company, 
Incorporated, Newark, DE, 19 February 1992 (RDB7525)

$\mathrm{R}-12$, summary of published and unpublished toxicity literature

Y. Lessard and G. Paulet, A Proposed Mechanism for Cardiac Sensitization: Electrophysiological Study of Effects of Dichlorodifluoromethane and Adrenaline on Different Types of Cardiac Preparations Isolated from Sheep Hearts, Cardiovascular Research, 20:807-815, 1986 (9 pages, RDB8156)

\section{$\mathrm{R}-12$, cardiac sensitization}

Y. Lessard and G. Paulet, Mechanism of Liposoluble Drugs and General Anesthetic's Membrane Action: Action of Dichlorodifluoromethane (FC 12) on Different Types of Cardiac Fibers Isolated from Sheep Hearts, Cardiovascular Research, 19(8):465-473, 1985 (8 pages, RDB8157)

\section{R-12, cardiac sensitization}

Y. Lessard, S. Desbrousses, and G. Paulet, Action du dichlorodifluorométhane (FC 12) sur l'activité électrique cardiaque du mammifère au niveau cellulaire [Effect of R-12 on the cardiac electrical activity in the cellular system of mammals], Compte Rendu des Séances de la Societé Biol. [Proceedings of the Meeting of the Biological Society], Paris, France, 174(1):52-57, 1980 (6 pages in French, RDB8160)

$R-12$, cardiac sensitization, toxicity as reported in RDB5367

Y. Lessard, S. Desbrousses, and G. Paulet, Rôle de l'adrénaline endogène dans le déclenchment de l'arythmie cardiaque par le dichlorodifluorométhane (FC 12) chez les mammifères [Role of Endogenous Adrenaline in Initiating Cardiac Arrhythmia by R-12 in Mammals], Compte Rendu des Séances de la Société Biol. [Proceedings of the Meeting of the Biological Society], Paris, France, 172(2):337-347, 1978 (11 pages in French, RDB7432)

$\mathrm{R}-12$, cardiac sensitization with endogenous adrenaline, toxicity as reported in RDB7414

Y. Lessard, S. Desbrousses, and G. Paulet, Arythmie cardiaque chez le chien sous l'action de l'adrénaline et du dichlorodifluorométhane (FC 12) [Cardiac Arrhythmia in the Dog by the ACtion of Adrenaline and R-12], Compte Rendu des Séances de la Societé Biol. [Proceedings of the Meeting of the Biological Society], Paris, France,
171(6):1270-1282, 1977 (13 pages in French, RDB8158)

$\mathrm{R}-12$, cardiac sensitization with adrenaline, toxicity as reported in RDB5367

Y. Lessard, S. Desbrousses, and G. Paulet, Arythmie cardiaque chez le lapin sous l'action de l'adrénaline et du dichlorodifluorométhane (FC 12) [Cardiac Arrhythmia in the Rabbit by the Action of Adrenaline and R-12], Compte Rendu des Séances de la Société Biol. [Proceedings of the Meeting of the Biological Society], Paris, France, 171(4):883-895, 1977 (13 pages in French, RDB8159)

$\mathrm{R}-12$, cardiac sensitization with adrenaline, toxicity as reported in RDB5367

T. Midgley, Jr., and A. L. Henne (Ohio State University), Decomposition of DifluoroDichloromethane by Flames, presentation at the 83rd Meeting (New Orleans, LA, 28 March - 1 April 1932), American Chemical Society (ACS), Columbus, $\mathrm{OH}$; republished in Industrial and Engineering Chemistry, ACS, 24:641-645, June 1932 (5 pages with 11 figures and 6 tables, RDB2530)

$R-12$, toxicity resulting from decomposition in open flames; presents analytical and experimental data for the acid concentration generation as an indication of decomposition in a room, patterned after a kitchen; concludes that "even under the worst possible circumstances [of decomposition] life is not endangered"

S. Niazi and W. L. Chiou, Fluorocarbon Aerosol Propellants XI: Pharmacokinetics of Dichlorodifluoromethane in Dog Following Single and Multiple Dosing, Journal of Pharmaceutical Sciences, 66:49-53, 1977 (5 pages, RDB8166)

$\mathrm{R}-12$, biochemistry, elimination, health effects, toxicity

G. M. Rusch, Literature Review: An Interpretive Review of the Toxicity and Human Exposure Data on Dichlorodifluoromethane, Department of Toxicology report MA-250-82-2, AlliedSignal Incorporated (then Allied Corporation), Morristown, NJ, 11 February 1983 (11 pages with no figures or tables, RDB6138)

review of published literature on the health effects and toxicity of R-12: $30-\mathrm{min} \quad \mathrm{LC}_{50}$ is $760,000-800,000 \mathrm{ppm}$ in guinea pigs, mice, rabbits, and rats; one study with anesthetized rats exposed at 360,000 or 390,000 (conflict in document) ppm resulted in apnea for exposures of $15.6 \mathrm{~min}$ and death for exposures of $30 \mathrm{~min}$; this study is discussed with indication 
that the death may have been due to the anesthetic; cardiac sensitization level with exogenous epinephrine is $70,000-80,000 \mathrm{ppm}$ in dogs and $370,000 \mathrm{ppm}$ in cats; arrhythmias were not seen in mice even at $400,000 \mathrm{ppm}$; reviews subchronic, chronic, reproductive, and biochemistry studies in animals as well as studies with humans; concludes that R-12 "has shown a low level of toxicity," "has a long, satisfactory history of use in consumer products and immersion freezing of food," and "its presence as a low-level contaminant in food-grade hydrochloric acid should not represent a health hazard"

R. R. Sayers, W. P. Yant, J. Chornyak, and H. W. Shoaf, Toxicity of Dichlorodifluoromethane: A New Refrigerant, Report of Investigations 3013, U.S. Bureau of Mines, Washington, DC, May 1930 (18 pages with 1 figure and 3 tables, available from JMC as RDB5356)

Dogs, monkeys, and guinea pigs were exposed to air containing $200,000 \mathrm{ppm} R-12$ by volume for 7-8 hr/d, $5 \mathrm{~d} / \mathrm{wk}$ and $4 \mathrm{hr} / \mathrm{d}, 1 \mathrm{~d} / \mathrm{wk}$ for a 12week period. No fatalities occurred among the dogs and monkeys; two of 16 treated guinea pigs and one of 16 controls died, from which the report concludes that there was "no effect of exposure on fatality." The document identifies symptoms observed during exposure, which included generalized tremor in dogs and mild to moderate tremor in monkeys. The maximum severity was reached in the first 1020 minutes, suggesting development of tolerance during and for subsequent exposures. The report documents the test methods and observed responses, including slight to moderate inhibition of weight and growth in the first 23 weeks, which were subsequently regained and maintained similar to control animals. Like patterns were identified in red blood cell increases. Autopsies showed no treatmentrelated gross pathology. No differences were observed in the pregnancies and offspring of the guinea pigs. The report concludes that "the possibility of public health and accident hazards resulting from exposure to [R-12] when used as a refrigerant are remote."

E. H. Schjötz, Freezing Agent Dichlorodifluoromethane from Point of View of Industrial Hygiene, Nordisk Hygienisk Tidskrift, 27:230 ff, 1946; republished in French as L'agent frigorifique freon 12 (dichlorodifluorométhane) du point de vue de l'hygiene industrielle, Chimie Ind., 52:459 ff, 1947 (RDB7220)

\section{R-12, health effects, toxicity}

$H$. S. Sherman, Long-Term Feeding Studies in Rats and Dogs with Dichlorodifluoromethane (Freon ${ }^{\circledR}-12$ Food Freezant), report 24-74, Haskell Laboratory for Toxicology and industrial Medicine, E. I. duPont de Nemours and Company, Incorporated, Newark, DE, 1974 (21 pages, RDB7433)

$R-12$, ingestion toxicity: as reported in RDB7414

H. S. Sherman, J. R. Barnes, and E. F. Stula (E. I. duPont de Nemours and Company, Incorporated), The Oral Toxicology of Dichlorodifluoromethane, Toxicology and Applied Pharmacology (TAP), Academic Press, Incorporated, UK, 29:152, 1974 (1 page, RDB6711)

$\mathrm{R}-12$, chronic and reproductive toxicity: not teratogenic to rats, did not interfere with reproduction and lactation, did not effect survival or clinical laboratory parameters (hematology, urinalysis, and liver function; no evidence of toxicity in dogs after administered in feed at $10 \mathrm{mg} / \mathrm{kg}$ and $3,000 \mathrm{mg} / \mathrm{kg}$ for two years - as reported in RDB5143

H. S. Sherman and J. R. Barnes, Feeding Studies with Dichlorodifluoromethane (Freon ${ }^{\circledR}-12$ Food Freezant), report 12-66, Haskell Laboratory for Toxicology and Industrial Medicine, E. I. duPont de Nemours and Company, Incorporated, Newark, DE, 1966 (18 pages, RDB65H8)

R-12, ingestion toxicity: 90-day feeding study in rats and dogs; no clinical or histopathologic signs of toxicity; urinary fluoride and plasma alkaline phosphatase levels were higher than for controls but within normal ranges; blood and urine analytical results were comparable between treated animals and controls in dogs as reported in RDB65H1 and RDB7414

V. A. Shugaev, The Toxicology of Freon-12, Gigiena $i$ Sanitariya, Russia (then USSR), 28(6):95-97, 1963 (3 pages with no figures or tables, in Russian); translation included in U.S.S.R. Literature on Air Pollution and Related Occupational Diseases, A Survey, edited by B. S. Levine, 12:161-163, 1966 (3 pages, available from NTIS as document $T$ 66-61429, Rdb7434)

$\mathrm{R}-12$, health effects, toxicity: $3-\mathrm{hr}$ ALC rat $=$ $620,000 \mathrm{ppm} \mathrm{v} / \mathrm{V}$ as reported in RDB7414

R. D. Stewart, A. A. Herrmann, E. D. Baretta, H. V. Forster, J. H. Crespo, P. E. Newton, and R. J. Soto (Medical College of Wisconsin), Acute and Repetitive Human Exposure to Difluorodichlo- 
romethane [dichlorodifluoromethane], report PB279204/LLC, National Technical Information Service (NTIS), Springfield, VA, April 1976 (available from NTIS, RDB6486)

$R-12$, health effects, toxicity

Acute Oral Toxicity Of Freon ${ }^{\circledR} 12$, Dichlorodifluoromethane, report on project MR-312-7, Haskell Laboratory for Toxicology and Industrial Medicine, E. I. duPont de Nemours and Company, Incorporated, Newark, DE, 1955 (RDB7527)

toxicity tests of $R-12$ in rats for determine ingestion toxicity: oral ALD rat $>1,000 \mathrm{mg} / \mathrm{kg}$ (based on the maximum feasible dose dissolved in peanut oil) as reported in RDB7414 and RDB7525

Dichlorodifluoromethane (CFC 12) Health Effects Information, CAS\# 75-71-8, Elf Atochem North America, Incorporated, Philadelphia, PA, undated circa 1995 (3 pages, RDB5B48)

$\mathrm{R}-12$, summary of toxicological and ecological information

Report on Dichlorodifluoromethane, Miscellaneous Hazard Report Number 2256, Underwriters Laboratories Incorporated, Northbrook, IL (then Chicago, IL), 10 October 1931 (RDB6671)

$\mathrm{R}-12$, flammability, toxicity, decomposition

\section{R-12B1}

P. S. Beck, D. G. Clark, and D. J. Tinston (Imperial Chemical industries Limited, $\mathrm{ICl}, \mathrm{UK}$ ), The Pharmacologic Actions of Bromochlorodifluoromethane (BCF), Toxicology and Applied Pharmacology (TAP), 24:20-29, 1973 (10 pages, RDB5A26)

$\mathrm{R}-12 \mathrm{~B} 1$, health effects, toxicity

J. A. Styles et al. ( $\mathrm{ICl}$ Chemicals and Polymers, UK), Activity of Bromochlorodifluoromethane (BCF) in Three Mutation Tests, Mutation Research, The Netherlands, 142:187-192, 1985 (6 pages, RDB65G7)

R-12B1, mutagenicity, toxicity: mixed positive and negative results depending on test as reported in RDB65G1

\section{R-13B1}

L. S. Mullin, C. F. Reinhardt, and R. E. Hemingway (E. I. duPont de Nemours and Company, Incorporated), Cardiac Arrhythmias and Blood Levels
Associated with Inhalation of Halon 1301, AlHA Journal, 40:653-658, 1979 (6 pages, RDB6B47)

$R-13 B 1$, cardiac sensitization, health effects, toxicity

G. Paulet, Archives des Maladies Professionelles de Médecine du Travail et de Securité Sociale, France, 23:341 ff, 1962 (in French, RDB6588)

$$
\text { R-13B1, toxicity }
$$

C. F. Reinhardt and R. Reinke (E. I. duPont de Nemours and Company, Incorporated), Toxicity of Halogenated Fire Extinguishing Agents: Halon 1301 (Bromotrifluoromethane), An Appraisal of Halogenated Fire Extinguishing Agents, National Academy of Sciences (NAS), Washington, DC, 67-78, 1972 (12 pages, RDB5A69)

R-13B1, health effects, toxicity

E. W. Van Stee and K. C. Back (U.S. Air Force Aerospace Medical Research Laboratory, AMRL), The Hypotensive Response in Dogs Exposed to Bromotrifluoromethane, abstract 85 (11th Annual Meeting of the Society of Toxicology, SOT), Toxicology and Applied Pharmacology (TAP), 22:306-307, 1972 (2 pages, RDB6559)

R-13B1, cardiac sensitization, toxicity

E. W. Van Stee and K. C. Back (U.S. Air Force Aerospace Medical Research Laboratory, AMRL), Brain and Heart Accumulation of Bromotrifluoromethane, report AMRL-TR-70-139, Aerospace Medical Research Laboratory, U.S. Air Force, Wright-Patterson Air Force Base, OH, February 1971 (RDB7244)

$\mathrm{R}-13 \mathrm{B1}$, health effects, toxicity

report 45-71, Haskell Laboratory for Toxicology and Industrial Medicine, E. I. duPont de Nemours and Company, Incorporated, Newark, DE, 1971 (RDB7261)

toxicity tests to determine the cardiac sensitization potential in male beagle dogs with intravenous injection of epinephrine five minutes before and, with a challenge injection, midway into a 10 minute exposure; LOEL at 75,200 $\mathrm{ppm} v / \mathrm{v}$ R-13B1 in air as reported in RDB6527

\section{R-1311}

D. E. Dodd (ManTech Environmental Technology, Incorporated), R. E. Kinkead, R. E. Wolfe, H. F. Leahy (ManTech), J. H. English (U.S. Army Medical Research Unit), and A. Vinegar (ManTech), Acute and Subchronic Inhalation Studies on 
Trifluoroiodomethane Vapor in Fischer 344 Rats, Fundamental and Applied Toxicology, 35:64-77, 1997 (14 pages, RDB8417)

toxicity of R-13l1

D. E. Dodd (ManTech Environmental Technology, Incorporated), Trifluoroiodomethane - Subchronic Toxicity and Genotoxicity in Mice and Bacteria, Food and Chemical Toxicology (FCT), 35(5):30, May 1997 (1 page, RDBA625)

summarizes genotoxicity and mutagenicity studies of R-1311; mice exposed to concentrations of 50,000 or $75,000 \mathrm{ppm} v / \mathrm{v}$ for $6 \mathrm{hr} / \mathrm{d}$ for 3 days showed slight, dose-related increases in chromosome damage (micronuclei) in the blood; mutagenicity was seen in bacteria in an Ames test; it was not evident in mouse cells in culture with or without metabolic activation when tested up to cytotoxic concentrations

D. E. Dodd (ManTech Environmental Technology, Incorporated), Trifluoroiodomethane - Subchronic Toxicity and Genotoxicity in Rats, Food and Chemical Toxicology (FCT), 35(5):30, May 1997 (1 page, RDBA626)

13-week, subchronic inhalation toxicity and genotoxicity studies of R-13l1 in rats did not establish a no observable adverse effect level (NOAEL); the lowest exposure concentration tested, 20,000 ppm $\mathrm{v} / \mathrm{v}$, resulted in increased follicular colloid content in the thyroid, altered thyroid hormone levels, and degeneration of the testes; this concentration also produced chromosome damage in bone marrow; exposures to 40,000 and $80,000 \mathrm{ppm} \mathrm{v} / \mathrm{v}$ resulted in impaired growth and nasal inflammation

E. R. Kinkead et al. (ManTech Environmental Technology, Incorporated), 90-Day Nose Only Inhalation Toxicity Study of Trifluoroiodomethane $\left(\mathrm{CF}_{3} \mathrm{l}\right)$ in Male and Female Fischer Rats, report AL/OE-TR-1996-0024, Armstrong Laboratory, U.S. Air Force, Wright-Patterson Air Force Base, OH, March 1996 (RDB65G8)

R-13l1, subchronic inhalation toxicity: exposures at 20,000,40,000, and 80.000 ppm for 2 $\mathrm{hr} / \mathrm{d}, 5 \mathrm{~d} / \mathrm{wk}$, for 90 days: found alteration in thyroid hormone levels indicated as specific to rats; no precancerous cell changes were noted - as reported in RDB65G1

A. D. Mitchell, Genetic Toxicity Evaluation of lodotrifluoromethane $\left(\mathrm{CF}_{3} \mathrm{l}\right)$, Results of In Vivo Mouse Bone Marrow Erythrocyte Micronucleus Testing, Genesys Research Incorporated, Research Triangle Park, NC, 1995 (RDB65HO)
R-13l1, trifluoroiodomethane, mutagenicity, toxicity: no significant changes were seen in the male's premature red blood cells (PRBCs) at $25,000,50,000$, or $75,000 \mathrm{ppm}$; significant changes were observed in the results were seen in the female's PRBCs exposed to 25,000 ppm and above - as reported in RDB65G9

A. D. Mitchell, In Vitro Forward Mutation Assay of lodotrifluoromethane $\left(\mathrm{CF}_{3} \mathrm{l}\right)$ Using the L5178Y/tk+/- Mouse Lymphoma Cell Mutagenesis Assay (MLA) With Colony Sizing, With and Without Metabolic Activation, report on project 94036, Genesys Research Incorporated, Research Triangle Park, NC, July 1994 (RDB65G4)

$\mathrm{R}-13 \mathrm{l1}$, trifluoroiodomethane, mutagenicity, toxicity: mixed positive and negative results for potential for changes in genetic material depending on test as reported in RDB65G1

A. D. Mitchell, In Vivo Bone Marrow Erythrocyte Micronucleus Testing of lodotrifluoromethane $\left(\mathrm{CF}_{3} \mathrm{l}\right)$, report on project 94037 , Genesys Research Incorporated, Research Triangle Park, NC, July 1994 (RDB65G5)

R-13|1, trifluoroiodomethane, mutagenicity, toxicity: mixed positive and negative results for potential for changes in genetic material depending on test as reported in RDB65G1

A. D. Mitchell, Mutagenesis Testing of lodotrifluoromethane $\left(\mathrm{CF}_{3} \mathrm{l}\right)$ Using the Ames Salmonella Typhimurium Histidine Reversion Assay for Volatile Chemicals, With and Without Metabolic Activation, report on project 94036 , Genesys Research Incorporated, Research Triangle Park, NC, July 1994 (RDB65G6)

$\mathrm{R}-13 \mathrm{I} 1$, trifluoroiodomethane, mutagenicity, toxicity: mixed positive and negative results for potential for changes in genetic material depending on test as reported in RDB65G1

D. E. Dodd (ManTech Environmental Technology, Incorporated), H. F. Leahy (ManTech), M. L. Feldman, J. H. English (U.S. Army Medical Research Unit), and A. Vinegar (ManTech), Reproductive Toxicity Screen of Trifluoroiodomethane $\left(\mathrm{CF}_{3} \mathrm{l}\right)$ in Sprague-Dawley Rats, Inhalation Toxicology, 11(11):1041-1055, 1999 (15 pages, RDBA564)

reproductive toxicity tests in groups of $16 \mathrm{fe}-$ male rats exposed by whole body exposures at target concentrations of 0 (control), 2,000, 7,000 , and $20,000 \mathrm{ppm} v / \mathrm{v} \mathrm{R}-1311$ in air for 5 $\mathrm{d} / \mathrm{wk}$ before mating and then $7 \mathrm{~d} / \mathrm{wk}$ for $8 \mathrm{wk}$ during mating, gestation, and lactation; pups were not exposed; indicates that there were no 
clinical signs of toxicity except a minimal decrease in mean body weight in female rats at $20,000 \mathrm{ppm} \mathrm{v/v}$; indicates no statistically significant, treatment-related findings by observation or examination at necropsy in the dams or pups; concludes that the no observed adverse effect level (NOAEL) was $20,000 \mathrm{ppm} \mathrm{v} / \mathrm{v}$ on the basis of reproductive indices and parameters

A. Vinegar (ManTech Environmental Technology, Incorporated), G. W. Jepson (ManTech), S. J. Hammann (McDonnell Douglas Corporation), G. Harper (McDonnell Douglas), D. S. Dierdorf (Universal Technical Services), and J. H. Overton (U.S. Environmental Protection Agency, EPA), Simulated Blood Levels of $\mathrm{CF}_{3} \mathrm{l}$ in Personnel Exposed During Its Release from an F-15 Jet Engine Nacelle and During Intentional Inhalation, AlHA Journal, 60:403-408, May-June 1999 (6 pages with 5 figures, RDB9935)

summarizes tests of $R-13 l 1$ use as a fire suppressant for aircraft engine fires to assess potential hazards to ground crews during discharges; presents concentration measurements at locations near the discharge and physiologically-based pharmacokinetic (PBPK) modeling to simulate blood levels in exposed individuals; compares blood levels reached in three exposures to the lowest observed adverse affect level (LOAEL) for cardiac sensitization; simulations showed that the highest blood concentrations were twice the cardiac sensitization level, but that the simulated blood concentration for one individual peaked at 100 times the target level without adverse effect; suggests that actual human data may supersede findings of animal tests; discusses findings in context of anecdotal accounts of voluntary but unmonitored human exposures [note that no mention is made of experimental verification of adrenaline levels in exposed individuals, and tests did not include epinephrine challenges]

A. Vinegar, D. E. Dodd, G. W. Jepson, and E. R. Kinkead (ManTech Environmental Technology, Incorporated), Acute Toxicity, Genotoxicity, and Cardiac Sensitization Potential of CF $_{3}$ I (Trifluoroiodomethane), The Toxicologist, 15(1):190 ff, 1995 (RDB7C86)

toxicity of $R-1311$

Acute Inhalation Toxicity Study of lodotrifluoromethane in Rats, report on project 1530-001 study 2, ManTech Environmental Technology, Incorporated, March 1994 (RDB65G2)
R-13/1, trifluoroiodomethane, toxicity: 15-min $\mathrm{LC}_{50}$ rat $274,000 \mathrm{ppm}$; animals showed drowsiness, drooling, and heavy breathing at concentrations $>100,000 \mathrm{ppm}$ - as reported in RDB6208 and RDB65G1

Comparing the Toxicology of Triodide ${ }^{T M}$ and Halon 1211, HTL/KIN-Tech Division, Pacific Scientific, Duarte, CA, May 1996 (6 pages with 4 tables, RDB65G9)

$\mathrm{R}-12 \mathrm{~B} 1, \mathrm{R}-13 / 1$, toxicity: acute inhalation toxicity, cardiac sensitization, mutagenicity, decomposition product toxicity in fires with comparisons of $R-1311$ to $R-12 B 1, R-13 B 1, R-23, R-$ 227ea, R-31-10, and R-51-14

Toxicology of DuPont FE1301 Fire Extinguishing Agent, bulletin S-35A, E. 1. duPont de Nemours and Company, Incorporated, Wilmington, DE, 1971 (RDB7563)

toxicity of R-13B1 and its decomposition products in fires; safety

Toxicology of Triodide ${ }^{\text {TM }}$, HTL/KIN-Tech Division, Pacific Scientific, Duarte, CA, April 1996 (3 pages with no figures or tables, RDB65G1)

R-13/1, toxicity: $15-\mathrm{min} \mathrm{LC}_{50}$ rat $274,000 \mathrm{ppm}$; cardiac sensitization NOAEL at $2,000 \mathrm{ppm}$ and LOAEL at $4,000 \mathrm{ppm}$; mixed positive and negative results for potential for changes in genetic material depending on test; alteration in thyroid hormone levels, but no pre-cancerous cell changes were noted in subchronic inhalations tests

unpublished data, Huntingdon Research Centre Limited (HRC), Cambridgeshire, UK, July 1994 (RDB65G3)

R-13l1, cardiac sensitization study, toxicity: NOAEL at 2,000 ppm; LOAEL at 4,000 ppm as reported in RDB65G1

\section{R-14}

\section{R-20}

A. G. Levy, The Exciting Causes of Ventricular Fibrillation in Animals Under Chloroform Anes. thesia, Heart, 4:319-378, 14 June 1913 (60 pages, RDB6560)

discusses the evidence for and mechanisms of cardiac sensitization resulting from inhalation of R-20 (chloroform); discusses inducement of ventricular fibrillation (very rapid and irregular contractions of the ventricles of the heart re- 
sulting in loss of synchronization between the heartbeat and pulse beat)

A. G. Levy and T. Lewis, Heart Irregularities, Resulting from the Inhalation of Low Percentages of Chloroform Vapor, and their Relationship to Ventricular Fibrillation, Heart, 3:99-112, 1911 1912 (14 pages, RDB6561)

presents findings of cardiac sensitization resulting from inhalation of R-20 (chloroform); discusses inducement of ventricular fibrillation (very rapid and irregular contractions of the ventricles of the heart resulting in loss of synchronization between the heartbeat and pulse beat) [this paper is cited in toxicity literature as the initial, historic identification of cardiac sensitization by halocarbon inhalation]

\section{R-20B3}

Occupational Safety and Health Guideline for Bromoform; National Institute of Occupational Safety and Health (NIOSH), U.S. Department of Health and Human Services, Cincinnati, $\mathrm{OH}$; Occupational Safety and Health Administration (OSHA), U.S. Department of Labor, Washington, DC, 1992 (6 pages with no figures or tables, available from GPO, also available from JMC as RDB5C47)

R-20B3, toxicity; chemical and physical properties, reactivity, flammability, exposure limits, health hazard information, exposure sources and control methods, medical monitoring, workplace monitoring and measurement, personal hygiene, storage, spills and leaks, special requirements, respiratory protection, personal protective equipment, references

\section{R-21}

L. S. Mullin, Cardiac Sensitization with Freon21, report 707-75, Haskell Laboratory for Toxicology and Industrial Medicine, E. I. duPont de Nemours and Company, Incorporated, Newark, DE, 1975 (2 pages, RDB7435)

toxicity tests to determine the cardiac sensitization potential of R-21 in beagle dogs with intravenous injection of epinephrine five minutes before and, with a challenge injection, midway into a 10 minute exposure; marked response in 2 of 12 dogs at $10,000 \mathrm{ppm} v / \mathrm{v}$ in air [deemed a LOEL] as reported RDB7414

C. H. Tappan and R. S. Waritz, Acute Inhalation Toxicity of Freon ${ }^{\circledR}-21$ (Fluorodichlorometh- ane), report 128-64, Haskell Laboratory for Toxicology and Industrial Medicine, E. I. duPont de Nemours and Company, Incorporated, Newark, DE, 1964 (2 pages, RDB7436)

$R-21$, dichlorofluoromethane, health effects, toxicity: 4-hr ALC rat $=49,900 \mathrm{ppm}$ v/v as reported in RDB7414

\section{R-22}

D. Anderson and C. R. Richardson, Arcton 22: A Second Cytogenetic Study in the Rat, report CTLP/445, Central Toxicology Laboratory, imperial Chemical Industries Limited ( $\mathrm{ICl})$, Cheshire, UK, 3 May 1979 (RDB5924)

$\mathrm{R}-22$, health effects, toxicity

D. Anderson, C. R. Richardson, C. Howard, R. A. Riley, and T. M. Weight, Arcton 22: Cytogenetic Study in the Rat, report CTL/R/429, Central Toxicology Laboratory, Imperial Chemical Industries Limited (ICI), Cheshire, UK, 6 December 1977 (RDB5925)

R-22, health effects, toxicity

D. Anderson, E. C. M. Hodge, T. M. Weight, and R. A. Riley, Arcton 22: Dominant Lethal Study in the Mouse, report CTL/R/430, Central Toxicology Laboratory, Imperial Chemical Industries Limited (ICI), Cheshire, UK, 6 December 1977 (RDB5926)

\section{$\mathrm{R}-22$, health effects, toxicity}

B. E. Butterworth, In Vitro Microbiol Mutagenicity Studies of Methane, Chlorodifluoro, report 398-76, Haskell Laboratory for Toxicology and Industrial Medicine, E. I. duPont de Nemours and Company, Incorporated, Newark, DE, 1976 (RDB6478)

R-22, health effects, toxicity, mutagenicity, positive Ames assay as reported in RDB5923

I. F. Carney, Arcton 22 (Chlorodifluoromethane). Relationship Between Blood Levels and inhaled Concentrations in Anesthetized, Artificially-Ventilated Rats, report CTL/R/422, Central Toxicology Laboratory, Imperial Chemical Industries Limited (ICI), Cheshire, UK, 1977 (RDB6479)

R-22, health effects, toxicity, uptake and metabolism studies

B. Checket-Hanks, R-22 Leak at Ice Rink Kills One, Injures 34, Air Conditioning, Heating, and 
Refrigeration News, 1-2, 27 May 1991 (2 pages with 1 figure, RDB5118)

accident from escape of $0.9-1.8$ tonnes $(2,000$ $4,000 \mathrm{lb}) \mathrm{R}-22$ resulted in death to a 24 year old male by cardiac arrest; victim was the assistant manager for a skating rink located in the 150-store, Dimond Center Shopping Mall in Anchorage, Alaska; victim was wearing a respirator that was ineffective; two coworkers also were hospitalized, one in critical condition, for oxygen deprivation; a total of 34 people were taken to the hospital for treatment; they included a number of swimmers rescued from a pool in the mall, where the refrigerant cloud "hovered close to the pool water" and reportedly looked like steam; responding personnel found refrigerant escaping from a $9.5 \mathrm{~mm}$ (3/8") high pressure line; article includes an annotated floor plan of the accident site

R. Culik and C. D. Crowe, Embryotoxic and Teratogenic Studies in Rats with Inhaled Chlorodifluoromethane (FC-22) - Third Study, report 314-78, Haskell Laboratory for Toxicology and Industrial Medicine, E. I. duPont de Nemours and Company, Incorporated, Newark, DE, 1976 (RDB6580)

developmental and reproductive toxicity of R-22

R. Culik, D. P. Kelly, and B. A. Burgess, Embryotoxic and Teratogenic Studies in Rats with Inhaled Chlorodifluoromethane (FC-22), report 970-76, Haskell Laboratory for Toxicology and Industrial Medicine, E. I. duPont de Nemours and Company, Incorporated, Newark, DE, 1976 (RDB5B67)

developmental and reproductive toxicity of R-22

P. W. Grube and R. C. Graham, untitled toxicity review for chlorodifluoromethane, Haskell Laboratory for Toxicology and Industrial Medicine, E. I. duPont de Nemours and Company, Incorporated, Newark, DE, 26 March 1990 (28 pages, RDB5856)

$R-22$, summary of published and unpublished toxicity literature

E. C. M. Hodge, D. Anderson, I. P. Bennett, and T. $M$. Weight, Arcton 22: Second Dominant Lethal Study in the Mouse, report CTL/R/450 (revised), Central Toxicology Laboratory, Imperial Chemical Industries Limited (ICI), Cheshire, UK, 9 November 1979 (RDB5927)

$\mathrm{R}-22$, health effects, toxicity
B. C. Karpov, The Chronic Toxicity of 'Freon' 22 , Tr. Leningrad Sanit. Gigien. Med. Inst., 75:231240, 1963 (10 pages in Russian, RDB6166)

$\mathrm{R}-22$, health effects, toxicity

D. F. Krahn (possibly A. Koops), Mutagenic Activity of Methane, Chlorodifluoro- in the Salmonella Microsome Assay, report 577-77, Haskell Laboratory for Toxicology and Industrial Medicine, E. I. duPont de Nemours and Company, Incorporated, Newark, DE, 1977 (RDB6481)

R-22, health effects, toxicity, mutagenicity, positive Ames assay as reported in RDB5923

I. P. Lee and K. Suzuki, Studies on the Male Reproductive Toxicity of Freon 22, Fundamental and Applied Toxicology, 1(3):266-270, 1981 (5 pages, RDB6482)

outlines a reproductive study of $\mathrm{R}-22$; toxicity

M. A. Lee, P. W. Grube, and R. C. Graham, untitled toxicity review for chlorodifluoromethane, Haskell Laboratory for Toxicology and Industrial Medicine, E. I. duPont de Nemours and Company, Incorporated, Newark, DE, 15 February 1996 (35 pages, RDB6940)

summarizes published and unpublished toxicity information on R-22

M. H. Litchfield and E. Longstaff (Imperial Chemical Industries (ICl) plc, UK), The Toxicological Evaluation of Chlorofluorocarbon 22 (CFC 22) [sic, HCFC-22], Fundamentals of Chemical Toxicology, 22(6):465-475, 1984 (11 pages with 1 figure and 7 tables, RDB5923)

R-22, detailed review of acute, subchronic, and chronic toxicity studies: inhalation, mortality, uptake and metabolism, mutagenicity (R-22 "can be assessed as a bacteria-specific mutagen on the evidence of the Ames test, but it did not exhibit any consistent mutagenic activity in other assay systems"), reproductive toxicity, carcinogenicity

E. Longstaff and D. B. McGregor (Imperial Chemical Industries (ICl) plc, UK), Mutagenicity of the Halocarbon Refrigerant Monochlorodifluoromethane (R-22) in Salmonella Typhimurium, Toxicology Letters, 2(1):1-4, 1978 (4 pages, RDB6275)

$\mathrm{R}-22$, health effects, toxicity, positive Ames assay as reported in RDB5923

R. L. McCarthy, Ecology and Toxicity of Fluorocarbons, E. I. duPont de Nemours and Company, Incorporated, Newark, DE, 1973 (RDB8150) 
fluorochemical refrigerants, safety, impacts

K. T. McCooey, Methane, Chlorodifluoro-, Chinese Hamster Ovary Cell Assay for Mutagenicity, report 149-80, Haskell Laboratory for Toxicology and Industrial Medicine, E. I. duPont de Nemours and Company, Incorporated, Newark, DE, 1980 (RDB6483)

$\mathrm{R}-12$ and $\mathrm{R}-22$, health effects, toxicity, mutagenicity in both the presence and absence of metabolic activation at $330,000,670,000$, and $1,000,000$ ppm R-22, no mutagenic activity was found as reported in RDB5923

A. K. Palmer, D. D. Cozens, R. Clark, and G. C. Clark, Effect of Arcton 22 on Pregnancy of the New Zealand White Rabbit, report ICl 177/78505, Huntingdon Research Centre Limited (HRC), Cambridgeshire, UK, 1 August 1978 (RDB5928)

R-22, health effects, toxicity

A. K. Palmer, D. D. Cozens, R. Clark, and G. C. Clark, Effect of Arcton 22 on Pregnant Rats: Relationship to Anophthalmia and Microphthalmia, report $\mathrm{ICl}$ 174/78208, Huntingdon Research Centre Limited (HRC), Cambridgeshire, UK, 12 June 1978 (RDB5929)

$\mathrm{R}-22$, health effects, toxicity

M. Sakata, H. Kazama, A. Miki, A. Yoshida, M. Haga, and M. Morita, Acute Toxicity of Fluorocarbon-22: Toxic Symptoms, Lethal Concentration, and lts Fate in Rabbit and Mouse, Toxicology and Applied Pharmacology (TAP), 59(1):64-70, 1981 (7 pages, RDB5739)

$R-22$, inhalation toxicity, uptake and metabolism studies in the rabbit: 30 -min MLC (ALC) rabbit $=300,000 \mathrm{ppm}, 30-\mathrm{min} L_{50}$ mouse $=$ 280,000 ppm as reported in RDB5923

A. G. Salmon, S. K. Basu, M. Fitzpatrick, and J. A. Nash, Arcton 22: Metabolism Study in Vivo in Rats and in Vitro, report CTL/P/438, Central Toxicology Laboratory, Imperial Chemical Industries Limited (ICl), Cheshire, UK, 1979 (RDB6484)

$\mathrm{R}-22$, health effects, toxicity

M. Smidt, O. Axelson, and C. Edling, Kohortstudie av Freonexponerade, Acta Soc. Med. Suec. Hung., 90:77 ff, 1981 (RDB6485)

$R-22$, health effects, toxicity, epidemiological study of workers exposed to chlorofluorochemicals including R-22 found no increase in mortality due to heart, circulatory, or tumor disorders as reported in RDB5923
D. J. Tinston, I. S. Chart, M. J. Godley, C. W. Gore, B. A. Gaskell, and M. H. Litchfield, Chlorodifluoromethane (CFC 22): Long-Term Inhalation Study in the Mouse [HCFC-22], report CTL/P/547, Central Toxicology Laboratory, Imperial Chemical Industries Limited (ICl), Cheshire, UK, 1981 (RDB6487)

R-22, chronic health effects, toxicity as reported in RDB5923

D. J. Tinston, I. S. Chart, M. J. Godley, C. W. Gore, M. H. Litchfield, and M. Robinson, Chlorodifluoromethane (CFC 22): Long-Term Inhalation Study in the Rat [HCFC-22], report CTLP/548, Central Toxicology Laboratory, Imperial Chemical Industries Limited (ICl), Cheshire, UK, 1981 (RDB6488)

$\mathrm{R}-22$, chronic health effects, toxicity as reported in RDB5923

D. J. Tinston, unpublished data, Central Toxicology Laboratory, Imperial Chemical Industries Limited (ICl), Cheshire, UK, 1976 (RDB6127)

$\mathrm{R}-22$, acute toxicity as reported in RDB5856 and RDB5923

E. W. Van Stee and E. E. McConnell, Studies of the Effects of Chronic Inhalation Exposure of Rabbits to Chlorodifluoromethane, Environmental Health Perspectives (EHP), 20:246-247, 1977 (2 pages, RDB6490)

$\mathrm{R}-22$, health effects, toxicity

Assistant Manager at Ice Rink Asphyxiated by an Oxygen-Deficient Atmosphere, Alaska, May 20, 1991, Fatal Accident Circumstances and Epidemiology (FACE) Report, 1991 (available from NTIS as PB92-193697, RDB6A95)

This report summarizes an accident from an R22 leak in which a 24 year old male was killed by asphyxiation. The victim was the assistant manager for a skating rink located in the 170store, Dimond Center Shopping Mall in Anchorage, Alaska. The refrigeration system had a long history of leaks, one of which had been plugged in a "makeshift fashion." While performing a routine check, a maintenance worker noticed oil oozing from under the doors of the compressor room. He entered the machinery room with a maintenance supervisor and the victim to stop the leak. The victim was wearing a cartridge type respirator, which was inadequate in an oxygen deficient atmosphere. Also, the machinery room had self-closing doors. A coworker saw the maintenance worker and supervisor lying on the floor and called 911, the 
emergency assistance number in most of the USA, for help. They were rescued by the responding paramedics. The victim was not seen immediately and was not removed from the room until later, by which time he had suffocated. Two swimmers in a pool, located in the mall, and rescue personnel also were affected by the R-22 vapor, which spread into adjacent areas. The accident investigation found that there was no safety policy or program in place and identified a number of safety deficiencies, including an absence of leak detectors.

Chlorodifluoromethane (CAS No. 75-45-6), Joint Assessment of Commodity Chemicals (JACC) report 9, European Chemical Industry Ecology and Toxicology Centre (ECETOC), Brussels, Belgium, 19 October 1989 (80 pages with 1 figure and 11 tables, RDB3718)

This assessment reviews the environmental impacts and toxicity of R-22, described as a nonflammable, colorless, and nearly odorless gas under normal conditions. It notes that a large amount is used as a chemical intermediate, and not emitted to the atmosphere, whereas that used for air conditioning and refrigeration will eventually be released under current practices. Most released R-22 is destroyed by reaction with $\mathrm{OH}$-radicals in the troposphere or $\mathrm{OH}$ - and excited oxygen atoms in the stratosphere, but not to any major extent by photodecomposition. The calculated ozone depletion potential (ODP) for R-22 is 0.05 , described as low compared to fully halogenated chlorofluorocarbons. The report indicates that R-22 has a negligible bioaccumulation potential, based on its moderate water solubility and low octanol-water partition coefficient. Contamination of food or water should not be expected, and has not been reported. R-22 is rapidly equilibrated in tissues after inhalation, and is eliminated from the blood in expired air, with a half life of only a few minutes. Blood concentrations are linearly correlated with inhalation concentrations over a wide range, but metabolism occurs in only minor amounts if at all. The report indicates that R-22 has an extremely low order of acute toxicity, and that concentrations exceeding $200,000 \mathrm{ppm} \mathrm{v} / \mathrm{v}$ are required to produce a lethal effect. The report reviews cardiovascular studies and cites a cardiac sensitization NOEL $(0 / 12)$ to adrenaline for male beagles of $24,700 \mathrm{ppm}$ and a LOEL (2/12) of $50,000 \mathrm{ppm}$ with. R-22 showed no adverse effects on fertility in animal studies and was not teratogenic in rabbits at doses up to 50,000 ppm. A low incidence of anophthalmia (congenital absence of eyes) and microphthalmia (abnormally small eyes) combined (10 fetuses in 383 litters) was found when groups of 400 pregnant rats were exposed to $50,000 \mathrm{ppm}$. Although statistically significant, these findings and those of subsequent studies were deemed to not threaten the health of human beings occupationally exposed at or below recommended exposure limits. Most in vitro tests of $\mathrm{R}-22$ are negative and a positive response in the Ames test is consistent with occurrence of bacteria-specific metabolic pathways. Only minimal or no effects were observed chronic exposures of rats and mice for 4-131 weeks. A slight decrease in body weight gain and a small increase in liver and kidney weight occurred with an exposure level of $50,000 \mathrm{ppm}$. The only exception was a limited study in rats, mice and rabbits which reported changes in the blood, liver, lung and nervous system in animals exposed to $14,000 \mathrm{ppm}$ over a ten month period; these findings were not confirmed in subsequent studies. R-22 did not produce neoplastic changes in female rats and male and female mice exposed to concentrations as high as $50,000 \mathrm{ppm}$. In one study in male rats, a concentration of $50,000 \mathrm{ppm}$ was associated with an increase in the number of fibrosarcomas and Zymbal gland tumors; they occurred late in the study and were not considered relevant to humans. Lower concentrations $(10,000$ ppm and below) did not increase tumor rates in any study. The assessment concludes that R22 does not constitutes a carcinogenic hazard to man. The toxicological activities described are unlikely to be due to the formation of reactive intermediates. Although not a skin sensitizer, liquid R-22 in contact with skin causes local freezing. Reports on adverse health effects from R-22 are rare and are consistent with findings in experimental animals. Permissible occupational exposure limits vary between 500 1000 ppm ( 8 hr TWA) by country; the assessment judged these limits to provide adequate health protection.

Chlorodifluoromethane (Group 3), IARC Monographs on the Evaluation of the Carcinogenic Risk of Chemicals to Humans, International Agency for Research on Cancer (IARC). (supplement 7):149150, 1987 (2 pages, RDB8420)

concludes that the evidence for carcinogenicity of R-22 to humans is inadequate based on a study of 539 refrigeration workers exposed to various refrigerants, including R-22, for at least 
six months with follow up for up to 30 years: six deaths occurred from cancer and two of them from lung cancer, compared to statistical expectation of 5.7 and 2 , respectively; summarizes tests for carcinogenicity in rats by oral administration and in mice and rats by inhalation of R-22; one rat study yielded no increase in tumor incidence while a mouse study gave inconclusive results for males and negative results for females; a third study in rats was deemed inadequate; a fourth found that males exposed to the highest concentration had a marginal increase in the incidence of subcutaneous fibrosarcomas and Zymbal-gland tumors, but obtained negative results for females; data examined on the genetic and related effects of R-22 found insufficient indications in humans; R-22 did not induce dominant lethal mutations in rats, chromosomal aberrations in bone-marrow cells of mice treated in vivo, unscheduled DNA synthesis in human cells in vitro, mutation in cultured Chinese hamster V79 cells, or mutation or mitotic gene conversion in yeast, either after direct exposure or in a hostmediated assay; R-22 was found to be mutagenic to plants and bacteria

Chlorodifluoromethane, IARC Monographs on the Evaluation of the Carcinogenic Risk of Chemicals to Humans, International Agency for Research on Cancer (IARC), 41:237-252, 1986 (15 pages, RDB8421)

identifies potential routes of exposure to R-22 from use in refrigeration and air conditioning as releases into the atmosphere, resulting in widespread, low-level human exposures, and from occupational exposure during its production and use; summarizes R-22 tests for carcinogenicity; no increase in tumor incidence was observed in rats after oral administration by gavage; results were inconclusive for males and negative for females for mice exposed in an inhalation study; male rats receiving the highest dose in an inhalation study showed increase incidence of fibrosarcomas and Zymbal-gland tumors; negative results were obtained for female rats; R-22 causes malformations of the eyes of fetal rats, but has no reproductive effect in male rats and does not cause prenatal toxicity in rabbits following exposure by inhalation; R-22 was found to be mutagenic to salmonella typhimurium in the presence and absence metabolic activation; it does not induce mutation or gene conversion in yeast, DNA damage or mutation in cultured mammalian cells, or chromosomal damage in bone marrow; R-22 does not induce dominant lethal mutations in mice or rats treated in vivo; no data were available to evaluate the reproductive effects or prenatal toxicity of R-22 to humans; a study of workers exposed to R-22 and other fluorochemicals was deemed to be uninformative with regard to the carcinogenic hazard of this chemical; report concludes that there is limited evidence of the carcinogenicity of R-22 to experimental animals, but inadequate evidence of carcinogenicity to humans

Health Hazard Report for Chlorodifluoromethane CAS 75-45-6: Refrigeration Workers, Salt Lake City, Utah, Hazard Evaluation Technical Assistance report HETA-81-043-1207, National Institute for Occupational Safety and Health (NIOSH), U.S. Department of Health and Human Services, Cincinnati, OH, 1982 (13 pages, available from NTIS as document PB-84-172998, RDB7236)

R-22, safety, toxicity

Occupational Safety and Health Guideline for Chlorodifluoromethane, U.S. Department of Health and Human Services and U.S. Department of Labor, Washington, DC, 1995 (7 pages, available from JMC as RDB6286)

R-22, Occupational Health Guideline (OHG), Occupational Safety and Health Guideline (OSHG), physical data, reactivity, flammability, exposure limits, health hazard information, toxicity, emergency procedures, medical monitoring, workplace monitoring and measurement, personal hygiene, storage, spills and leaks, emergency planning and community right-toknow requirements, hazardous waste management requirements, respiratory protection, personal protective equipment, references

\section{R-23}

R. Atkinson, Chem. Review, 85:69-201, 1985 (133 pages, RDB5B61)

R-23, toxicity

R. Atkinson and W. P. L. Carter, Chem. Review, 84:437-470, 1984 (34 pages, RDB5825)

R-23, toxicity

C. A. Branch, D. A. Goldberg, J. R. Ewing, S. C. Fagan, S. S. Butt, and J. Gayner (Henry Ford Hospital), Evaluation of the Acute Cardiac and Central Nervous System Effects of the Fluorocarbon Trifluoromethane in Baboons, Journal of 
Toxicology and Environmental Health, 43(1):2535. September 1994 (11 pages, RDB5158)

This study reports tests of R-23 for acute cardiotoxic, anesthetic, and central nervous system effects in primates (papio anubis). A doseresponse effect was established for respiratory rate, electroencephalogram, and cardiac sinus rate, which exhibited a stepwise decrease from $10 \%$ R-23. No spontaneous arrhythmias were noted, and arterial blood pressure remained unchanged at any inspired level. Intravenous epinephrine infusions $(1 \mu \mathrm{g} / \mathrm{kg})$ induced transient cardiac arrhythmia in one animal with $70 \%$ R23 by volume. R-23 appears to induce mild dose-related physiological changes at inspired levels of $30 \%$ or more, indicative of an anesthetic effect. These data suggest that R-23 may be safe to use in humans, without significant adverse acute effects, at an inspired level of $30 \%$.

C. A. Branch, J. R. Ewing, S. C. Fagan, D. A. Goldberg, and K. M. A. Welch (Henry Ford Hospital), Acute Toxicity of a Nuclear Magnetic Resonance Cerebral Blood Flow Indicator in Cats, Stroke, 21(8):1172-1177, 1990 (6 pages, RDB5818)

R-23, acute inhalation toxicity: tests of blood flow, cardiac sensitization with epinephrine, and cerebral electrical activity tests in cats at 600,000 and 700,000 ppm R-23 as reported in RDB5604

J. C. Edwards, Arcton 23 - Documentation for Hygiene Standard, $\mathrm{ICl}$ Chemicals and Polymers Limited, Cheshire, UK, 17 June 1985 (1 page, RDB5A75)

R-23, toxicity, rationale for recommended hygiene standard (occupational exposure limit, OEL) of 1,000 ppm, 8-hr TWA

J. R. Ewing, C. A. Branch, S. C. Fagan, J. A. Helpern, R. T. Simkins, S. M. Butt, and K. M. A. Welch (Henry Ford Hospital), Fluorocarbon-23 Measure of Cat Cerebral Blood Flow by Nuclear Magnetic Resonance, Stroke, 21:100-106, 1990 (7 pages, RDB5736)

R-23, toxicity

S. C. Fagan, A. A. Rahill, G. Balakrishnan, J. R. Ewing, C. A. Branch, and G. G. Brown (Henry Ford Hospital), Neurobehavioral and Physiological Effects of Trifluoromethane in Humans, Journal of Toxicology and Environmental Health, 44:101-109, 1995 (9 pages with 1 table, RDB5737)
$\mathrm{R}-23$, toxicity: investigation of $\mathrm{R}-23$ as a nuclear magnetic resonance indicator in human subjects, discontinued based on slight anesthetic effects, light headedness, tingling and numbness of the extremities, and hyperacusis (increased hearing) at $300,000 \mathrm{ppm}$; heart rate and rhythm as well as blood pressure not affected at concentrations studied

D. M. Krentz, M. A. Lee, and R. C. Graham, untitled toxicity review for trifluoromethane, Haskell Laboratory for Toxicology and Industrial Medicine, E. 1. duPont de Nemours and Company, Incorporated, Newark, DE, 3 August 1992 (RDB5604)

R-23, summary of published and unpublished toxicity literature

S. M. Munley, An Inhalation Developmental Toxicity Study in Rats, report 995-96, Haskell Laboratory for Toxicology and Industrial Medicine, E. I. duPont de Nemours and Company, Incorporated, Newark, DE, 27 February 1997 (142 pages with 1 figure and 8 tables, Rdb7631)

tests of maternal and developmental toxicity of R-23 by inhalation in groups of 25 mated Crl:CD(SD)BR rats on days 7-21 of gestation at whole-body exposure levels of 0 (control), $5,000,20,000$, or $50,000(0,5,600 \pm 45,21,000$ \pm 48 , or $51,000 \pm 72$ actual) $\mathrm{ppm} v / \mathrm{v}$ in air for 6 $\mathrm{hr} / \mathrm{d}$ : no evidence of maternal or developmental toxicity was found at any exposure concentration tested; no compound-related effects were observed for clinical signs, food consumption, maternal body weights, weight changes, or postmortem findings; concludes that R-23 "was not uniquely toxic to the rat conceptus" and that "the maternal and developmental no-observedeffect level (NOEL) was 50,000 ppm"

S. D. Nash, Inhalation Approximate Lethal Concentration (ALC) of Trifluoromethane, report 641-80, Haskell Laboratory for Toxicology and Industrial Medicine, E. I. duPont de Nemours and Company, Incorporated, Newark, DE, 30 September 1980 ( 2 pages with 1 table, RDB6513)

$4 \mathrm{hr}$ acute inhalation toxicity tests of R-23 in groups of six male Charles River $C D$ albino rats by whole body exposures: mortality ratios and corresponding concentrations were $0 / 6$ at $18,900,0 / 6$ at 186,000 , and $0 / 6$ at $663,000 \mathrm{ppm}$ $\mathrm{v} / \mathrm{V}$ in air with oxygen enrichment (to maintain $19.7-21 \%$ levels); rats showed slight weight loss $24-48 \mathrm{hr}$ after exposure at $18,900 \mathrm{ppm}$ followed by normal weight gain in a 14-day recovery period; an unspecified number of rats showed reduced response to sound at 186,000 
$\mathrm{ppm}$; an unspecified number of rats showed no response to sound, gasping, labored breathing, sluggishness, and - for 1 of 6 rats - compulsive gnawing on basket at $663,000 \mathrm{ppm}$; an unspecified number exhibited slight weight loss $24 \mathrm{hr}$ after exposure at $663,000 \mathrm{ppm}$, but normal weight gain thereafter; report concludes that the 4-hr ALC rat for R-23 exceeds 663,000 $\mathrm{ppm} v / \mathrm{v}$

O. Schaumann, Archiv für Experimentelle Pathologie und Pharmakologie, 181:144-145, 1936 (2 pages, RDB5606)

R-23, inhalation toxicity: exposure (species not indicated) at $900,000 \mathrm{ppm}$ resulted in moderate, but not complete narcosis

Hazardous Substance Fact Sheet - Trifluoromethane, New Jersey Department of Health, Trenton, NJ, August 1988 (RDB5138)

R-23, toxicity

Toxicology Summary for FC 23 (Trifluoromethane), $\mathrm{ICl}$ Chemicals and Polymers Limited, Runcorn, UK, 21 September 1994 (1 page, RDB5168)

R-23, toxicity: references a 2-hr ALC rat $>200,000 \mathrm{ppm}$, cardiac sensitization $E_{50}$ $>500,000 \mathrm{ppm}$, and Ames test (not mutagenic); cites an $\mathrm{ICl}$ recommended exposure limit of 1,000 ppm, $8 \mathrm{hr}$ TWA

Trifluoromethane (HFC 23) Health Effects Information, CAS\# 75-46-7, Elf Atochem North America, Incorporated, Philadelphia, PA, undated circa 1995 (3 pages, RDB5B49)

R-23, summary of toxicological and ecological information

report 25-60, Haskell Laboratory for Toxicology and Industrial Medicine, E. I. duPont de Nemours and Company, Incorporated, Newark, DE, 1945 (RDB6512)

$\mathrm{R}-23$, acute inhalation toxicity: 12 guinea pigs were exposed to $200,000 \mathrm{ppm} R-23$ for $2 \mathrm{hr}$; no animals died and neither clinical signs of toxicity nor pathological changes were noted - as reported in RDB5604

\section{R-30}

M. E. Andersen, H. J. Clewell III, M. L. Gargas, F. A. Smith, and R. H. Reitz, Physiologically Based Pharmacokinetics and the Risk Assessment Process for Methylene Chloride, Toxicology and Applied Pharmacology (TAP), 87:185-205, 1987 (21 pages, RDB65E5) toxicity of R-30; pharmacokinetics; uptake, metabolism and elimination; health effects

G. D. Di Vincenzo and C. J. Kaplan, Uptake, Metabolism and Elimination of Methylene Chloride Vapor by Humans, Toxicology and Applied Pharmacology (TAP), 59:130-140, 1989 (11 pages, RDBA529)

toxicity of R-30; pharmacokinetics; uptake, metabolism and elimination; health effects

E. C. Riley, D. W. Fassett, and W. L. Sutton, Methylene Chloride Vapor in Expired Air of Human Subjects, AlHA Journal, 27:341 ff, 1966 (RDBA630)

toxicity of R-30

Methylene Chloride (Dichloromethane): An Overview of Experimental Work Investigating Species, Differences in Carcinogenicity, and their Relevance to Man, technical report 34, European Chemical Industry Ecotoxicology and Toxicology Centre (ECETOC), Brussels, Belgium, March 1989 (RDBA530)

toxicity of R-30; acute, subchronic, and chronic studies; carcinogenicity; mutagenicity; health effects

Methylene Chloride (Dichloromethane): Human Risk Assessment Using Experimental Animal Data, technical report 32, European Chemical Industry Ecotoxicology and Toxicology Centre (ECETOC), Brussels, Belgium, May 1988 (RDBA531)

toxicity of R-30; acute, subchronic, and chronic studies; carcinogenicity; mutagenicity; health effects

The Assessment of Carcinogenic Hazard for Human Beings Exposed to Methylene Chloride, technical report 26, European Chemical Industry Ecotoxicology and Toxicology Centre (ECETOC), Brussels, Belgium, January 1987 (RDBA532)

toxicity of R-30; carcinogenicity; mutagenicity; health effects

\section{R-30B1}

T. R. Torkelson, F. Oyen, and V. K. Rowe, The Toxicity of Bromochloromethane as Determined on Laboratory Animals, AlHA Journal, 21:275-286, 1960 (12 pages, RDB6510)

toxicity of R-30B1, health effects, $L_{50}$ 


\section{R-31}

W. B. Coate, R. W. Zapp, J. Anderson, and J. Charm, Inhalation Toxicity of Monochloromonofluoromethane Toxicology and Applied Pharmacology (TAP), 48(A109):79 ff, 1979 (RDB5563)

\section{R-31, toxicity}

R. C. Graham and C. D. VerNooy, untitled review of toxicity literature for methane, chlorofluoro- (R31), Haskell Laboratory for Toxicology and Industrial Medicine, E. I. duPont de Nemours and Company, Incorporated, Newark, DE, 6 April 1992 (10 pages with no figures or tables, RDB6778)

summary of published and unpublished information on the toxicity of R-31

\section{R-32}

M. Asakura (Japan Bioassay Laboratory, JBL, Japan), Report on a Chromosomal Aberration Test of Difluoromethane on Cultured Mammalian Cells (CHL), report 5918 for the Program for Alternative Fluorocarbon Toxicity Testing (PAFT), Japan Industrial Safety and Health Association, Kanagawa, Japan, 1993 (RDB5750)

R-32, toxicity

R. L. Bornschein, L. Hastings, and J. M. Manson, Behavioral Toxicity in the Offspring of Rats Following Maternal Exposure to Dichloromethane, Toxicology and Applied Pharmacology (TAP), 52:29-37, 1980 (9 pages, RDBA527)

toxicity of R-32; teratogenicity

R. D. Callander, HFC 32: An Evaluation of Mutagenic Potential Using S. Typhimurium and $E$. Coli, report CTL/P/3351 for the Program for Alternative Fluorocarbon Toxicity Testing (PAFT), Zeneca Central Toxicology Laboratory (then part of $\mathrm{ICl}$ Chemicals and Polymers Limited), Cheshire, UK, 10 June 1992 (RDB5745)

R-32, toxicity: negative result for mutagenicity as reported in RDB6686

M. K. Ellis, R. Trebicock, J. L. Naylor, K. Tseung, et al. (Zeneca Central Toxicology Laboratory, UK), The Inhalation Toxicology, Genetic Toxicology and Metabolism of Difluoromethane in the Rat, Fundamental and Applied Toxicology, 31(2):243251, 1996 (9 pages, RDB6A98)

R-32: toxicity, developmental toxicity, and genotoxicity; metabolism and disposition; physiologically-based pharmacokinetic (PB-PK) mod- el; approximately $2.1 \%$ of inhaled R-32 is absorbed; steady-state blood levels are achieved within $2 \mathrm{hr}$ and are proportional to dose; carbon dioxide is the major metabolite at all exposure levels; carbon monoxide was not detected; approximately $63 \%(1.4 \%$ of the R-32 entering the airways) was metabolized at all doses; inhalation of R-32 (up to 50,000 ppm) caused no organ-specific effects, but resulted in slight maternal toxicity in pregnant rats and rabbits and some fetotoxicity in rats; $50,000 \mathrm{ppm}$ did not sensitize the heart to adrenaline; the overall results indicate that $R-32$ is of very low toxicity

M. K. Ellis, R. Trebicock, and T. Green, Hydrofluorocarbon 32: Pharmacokinetics and Metabolism in the Rat, report CTL/R/1220 for the Program for Alternative Fluorocarbon Toxicity Testing (PAFT), Zeneca Central Toxicology Laboratory (then part of $\mathrm{ICl}$ Chemicals and Polymers Limited), Cheshire, UK, 1994 (RDB5979)

$$
\mathrm{R}-32 \text {, toxicity }
$$

M. K. Ellis, J. L. Naylor, and T. Green, Hydrofluorocarbon 32: Pharmacokinetics and Metabolism in Male Rats and Mice Following a Single Exposure by Inhalation, report CTL/R/1137 for the Program for Alternative Fluorocarbon Toxicity Testing (PAFT), Zeneca Central Toxicology Laboratory (then part of $\mathrm{ICl}$ Chemicals and Polymers Limited), Cheshire, UK, 23 December 1992 (RDB5749)

\section{$\mathrm{R}-32$, toxicity}

J. Ferguson-Smith, HFC 32 Hygiene Standard Documentation, ICl Chemicals and Polymers Limited, Cheshire, UK, 3 June 1996 (6 pages, RDB6686)

R-32, toxicity, literature review, rationale for recommended hygiene standard (occupational exposure limit, OEL) of 1,000 ppm, 8-hr TWA; cites a 10-min CNS EC 50 rat of $370,000 \mathrm{ppm}$ $v / v$, but this information could not be verified from the references provided

C. J. Hardy and P. C. Kieran, Hydrofluorocarbon 32: Assessment of Cardiac Sensitization Potential in Beagle Dogs, report ISN 276/920493 (also identified as ICl report CTUC/2723) for the Program for Alternative Fluorocarbon Toxicity Testing (PAFT), Huntingdon Research Centre Limited (HRC), Cambridgeshire, UK, 18 September 1992 (RDB5748)

R-32, health effects, toxicity: exposures as high as $350,000 \mathrm{ppm}$ v/v did not produce a cardiac sensitization response in beagle dogs with 
an intravenous epinephrine challenge as reported in RDB5C14 and RDB6686

K. Kawahara, Test on 1-Octanol/Water Partition Coefficient of HFC-32, report 80678, Kurume Research Laboratories (KRL), Fukuoka, Japan, 1992 (RDB5C29)

ecotoxicity of R-32; environmental impacts and fate; groundwater and soil

D. M. Krentz, M. A. Lee, and R. C. Graham, untitled toxicity review for difluoromethane, Haskell Laboratory for Toxicology and Industrial Medicine, E. I. duPont de Nemours and Company, incorporated, Newark, DE, 15 July 1992 (RDB5862)

$\mathrm{R}-32$, summary of published and unpublished toxicity literature

J. M. Mackay and C. A. Howard, HFC 32: An Evaluation in the In Vitro Cytogenetic Assay in Human Lymphocytes, report CTL/P/3465 for the Program for Alternative Fluorocarbon Toxicity Testing (PAFT), Zeneca Central Toxicology Laboratory (then part of $\mathrm{ICl}$ Chemicals and Polymers Limited), Cheshire, UK, 5 June 1992 (RDB5744)

R-32, toxicity, mutagenicity: no evidence of genotoxicity at $50,000-1,000,000 \mathrm{ppm}$ as reported in RDB6686

B. L. Moore, Acute Inhalation Toxicity (FC-32), report 698-75, Haskell Laboratory for Toxicology and Industrial Medicine, E. I. duPont de Nemours and Company, Incorporated, Newark, DE, 18 November 1975 (2 pages with 1 table, RDB6171)

reports an acute inhalation toxicity study of $R$ 32 in groups of six rats; indicates that no mortality resulted from 4-hr exposures to 111,000 , $235,000,270,000,315,000,432,000,455,000$,

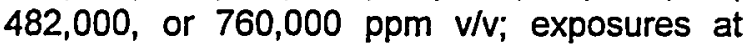
$315,000 \mathrm{ppm} v / \mathrm{v}$ and higher were oxygen enriched to maintain $\sim 20 \%$ levels; notes that rats exhibited lethargy and loss of mobility in hind legs at all exposure concentrations; also notes that they also exhibited spasms and gnawing at the bottom of the cage at $315,000 \mathrm{ppm} \mathrm{v/v} \mathrm{and}$ higher; notes quick recoveries, with no abnormal post-exposure signs, and no gross pathological changes in autopsies 14 days after exposures; concludes that the 4-hr ALC exceeds $760,000 \mathrm{ppm}$ v/v

M. E. Moxon, HFC-32: Developmental Toxicity Study in the Rat, report CTL/P/3835 for the Program for Alternative Fluorocarbon Toxicity Testing (PAFT), Zeneca Central Toxicology Laboratory (then part of $\mathrm{ICl}$ Chemicals and Polymers Limited), Cheshire, UK, 20 May 1992 (RDB5747)

R-32, toxicity: not teratogenic to the rat at $5,000-50,000 \mathrm{ppm}$ for $6 \mathrm{hr} / \mathrm{d}$; maternal food consumption was slightly decreased at 50,000 $\mathrm{ppm}$; minimal fetal toxicity at $50,000 \mathrm{ppm}$ - as reported in RDB6686

M. E. Moxon, HFC-32: Modified ChernoffKavlock Assay in the Rat, report CTL/P/3695 for the Program for Alternative Fluorocarbon Toxicity Testing (PAFT), ICl Central Toxicology Laboratory, $\mathrm{ICI}$ Chemicals and Polymers Limited, Cheshire, UK, 29 June 1992 (45 pages with 3 figures and 14 tables, RDB5C31)

modified Chernoff-Kavlock assay by wholebody exposure of groups of 10 female, Wistar derived rats to concentrations of 0 (control), 10,000 , or $50,000(0,9,930$, and 49,600 actual) ppm v/v R-32 in air for $6 \mathrm{hr} / \mathrm{d}$ on days 7-16 of gestation: exposures produced no clinical effects or changes in bodyweight gain and did not reduce litter size at birth or pup survival; a slight reduction in pup weight gain was observed at $50,000 \mathrm{ppm}$; report concludes that $\mathrm{R}-32$ is neither potentially teratogenic or fetotoxic at the concentrations tested

L. S. Mullin and H. J. Trochimowicz, Cardiac Sensitization Potential of Difluoromethane, report 257-73, Haskell Laboratory for Toxicology and Industrial Medicine, E. I. duPont de Nemours and Company, Incorporated, Newark, DE, 8 June 1973 (3 pages with 1 table, RDB6170)

toxicity tests to determine the cardiac sensitization potential of R-32 in male beagle dogs with intravenous injection of epinephrine five $\mathrm{min}$ utes before and, with a challenge injection, midway into a 10 minute exposure: marked response in 1 of 12 dogs at $250,000 \mathrm{ppm} \mathrm{v} / \mathrm{v}$ in air [deemed a LOEL]; no sensitization was found in the dog that reacted at $250,000 \mathrm{ppm}$ when tested several days later at $200,000 \mathrm{ppm}$; two dogs tested at $400,000 \mathrm{ppm}$ showed no response; concludes that R-32 is "a very weak cardiac sensitizer"

D. P. Myres (Huntingdon Research Centre Limited HRC, UK), HFC-32: A Study of the Effect on Pregnancy of the Rabbit, report ALS 19/942190 for the Program for Alternative Fluorocarbon Toxicity Testing (PAFT), AlliedSignal Incorporated, Morristown, NJ, 12 August 1994 (RDB5743)

$\mathrm{R}-32$, toxicity: not teratogenic to the rabbit at concentrations of $5,000-50,000 \mathrm{ppm}$ for $6 \mathrm{hr} / \mathrm{d}$; animals exposed to $50,000 \mathrm{ppm}$ had slight loss 
of body weight during days 8-10 of pregnancy; no evidence of fetal or embryo toxicity - as reported in RDB6686

R. J. Parr-Dobrzanski, HFC 32: 90-Day Inhalation Toxicity Study in the Rat, report CTL/P/4064, Zeneca Central Toxicology Laboratory (then part of $\mathrm{ICl}$ Chemicals and Polymers Limited), Cheshire, UK, 4 October 1993 (RDB5742)

\section{R-32, toxicity}

R. J. Parr-Dobrzanski, HFC-32: 28-Day Subacute Inhalation Toxicity Study in the Rat, report CTL/P/3746, ICl Central Toxicology Laboratory, $\mathrm{ICl}$ Chemicals and Polymers Limited, Cheshire, UK, 24 August 1992 (290 pages, RDB5741)

toxicity tests of groups of 5 female and 5 male Wistar-derived rats by whole body exposures for $6 \mathrm{hr} / \mathrm{d}, 5 \mathrm{~d} / \mathrm{wk}$, for $4 \mathrm{wk}$ to concentrations of 0 (control), $2,000,10,000$, or $50,000(0,2,010$, 9,870 , or 49,500 actual) $\mathrm{ppm} v / \mathrm{v}$ R-32 in air: no deaths or treatment-related effects on clinical condition, bodyweights, food consumption, organ weights, liver biochemistry, or pathology were observed; small changes in clinical chemistry and hematological parameters were noted; report concludes that the NOEL for this study was $49,500 \mathrm{ppm}$ v/v

R. J. Parr-Dobrzanski, HFC 32: 4-Hour Acute Inhalation Toxicity Study in the Rat, report CTL/P/3456 for the Program for Alternative Fluorocarbon Toxicity Testing (PAFT), Central Toxicology Laboratory, $\mathrm{ICl}$ Chemicals and Polymers Limited, Cheshire, UK, 5 June 1992 (50 pages with 1 figure and 27 tables, RDB5740)

nose-only inhalation tests with four groups of 5 female and 5 male albino rats exposed to 0 , $7,510,85,900$, and $520,000 \mathrm{ppm}$ v/v R-32 in air (oxygen-enriched for the highest concentration) for $4 \mathrm{hr}$. no deaths or severe toxicity resulted; minor clinical signs were observed during and immediately after exposures at the two highest concentrations, but the affected animals showed a rapid reversal of effects; no significant effects on body or lung weights resulted; no toxicologically significant effects were found at necropsy; report concludes the 4-hr $\mathrm{LC}_{50}$ rat exceeds $520,000 \mathrm{ppm} v / \mathrm{v}$, but indicates a reduced response to sound in $10 / 10$ rats at $85,900 \mathrm{ppm}$ that was not observed at 7,510 ppm

V. M. Randall and J. M. Mackay, HFC 32: An Evaluation of the Mouse Micronucleus Test, report CTL/P/3927, Zeneca Central Toxicology Laboratory (then part of $\mathrm{ICl}$ Chemicals and Polymers Limited), Cheshire, UK, 23 December 1992 (RDB5746)

R-32, toxicity, mutagenicity: no evidence for clastogenicity as reported in RDB6686

Y. Tobeta, Test on Biodegradability of HFC-32 by Microorganisms (Closed-Bottle Method), report 12121, Kurume Research Laboratories (KRL), Fukuoka, Japan, 1992 (RDB5C33)

ecotoxicity of R-32; environmental impacts and fate; groundwater and soil

Difluoromethane (HFC-32, CAS No. 75-10-5), Joint Assessment of Commodity Chemicals (JACC) report 32, European Chemical industry Ecotoxicology and Toxicology Centre (ECETOC), Brussels, Belgium, May 1995 (32 pages, RDB5C13)

This assessment reviews the environmental impacts and toxicity of R-32, described as a flammable, colorless, and odorless gas under normal conditions.

It notes that a large amount is used as a chemical intermediate, and not emitted to the atmosphere, whereas that used for air conditioning and refrigeration will eventually be released under current practices. Most released $\mathrm{R}-22$ is destroyed by reaction with $\mathrm{OH}$ - radicals in the troposphere or $\mathrm{OH}$ - and excited oxygen atoms in the stratosphere, but not to any major extent by photodecomposition. The calculated ozone depletion potential (ODP) for R-22 is 0.05 , described as low compared to fully halogenated chlorofluorocarbons. The report indicates that R-22 has a negligible bioaccumulation potential, based on its moderate water solubility and low octanol-water partition coefficient. Contamination of food or water should not be expected, and has not been reported. R-22 is rapidly equilibrated in tissues after inhalation, and is eliminated from the blood in expired air, with a half life of only a few minutes. Blood concentrations are linearly correlated with inhalation concentrations over a wide range, but metabolism occurs in only minor amounts if at all. The report indicates that R-22 has an extremely low order of acute toxicity, and that concentrations exceeding 200,000 $\mathrm{ppm} v / v$ are required to produce a lethal effect. The report reviews cardiovascular studies and cites a cardiac sensitization NOEL $(0 / 12)$ to adrenaline for male beagles of $24,700 \mathrm{ppm}$ and a LOEL (2/12) of $50,000 \mathrm{ppm}$ with. R-22 
showed no adverse effects on fertility in animal studies and was not teratogenic in rabbits at doses up to $50,000 \mathrm{ppm}$. A low incidence of anophthalmia (congenital absence of eyes) and microphthalmia (abnormally small eyes) combined (10 fetuses in 383 litters) was found when groups of 400 pregnant rats were exposed to $50,000 \mathrm{ppm}$. Although statistically significant, these findings and those of subsequent studies were deemed to not threaten the health of human beings occupationally exposed at or below recommended exposure limits. Most in vitro tests of R-22 are negative and a positive response in the Ames test is consistent with occurrence of bacteria-specific metabolic pathways. Only minimal or no effects were observed chronic exposures of rats and mice for 4-131 weeks. A slight decrease in body weight gain and a small increase in liver and kidney weight occurred with an exposure level of $50,000 \mathrm{ppm}$. The only exception was a limited study in rats, mice and rabbits which reported changes in the blood, liver, lung and nervous system in animals exposed to $14,000 \mathrm{ppm}$ over a ten month period; these findings were not confirmed in subsequent studies. R-22 did not produce neoplastic changes in female rats and male and female mice exposed to concentrations as high as $50,000 \mathrm{ppm}$. In one study in male rats, a concentration of $50,000 \mathrm{ppm}$ was associated with an increase in the number of fibrosarcomas and Zymbal gland tumors; they occurred late in the study and were not considered relevant to humans. Lower concentrations (10,000 ppm and below) did not increase tumor rates in any study. The assessment concludes that R-22 does not constitutes a carcinogenic hazard to man. The toxicological activities described are unlikely to be due to the formation of reactive intermediates. Although not a skin sensitizer, liquid R-22 in contact with skin causes local freezing. Reports on adverse health effects from R-22 are rare and are consistent with findings in experimental animals. Permissible occupational exposure limits vary between $500-1000$ ppm ( 8 hr TWA) by country; the assessment judged these limits to provide adequate health protection.

Difluoromethane (HFC 32) Health Effects Information, CAS\# 75-10-5, Elf Atochem North America, Incorporated, Philadelphia, PA, undated circa 1995 (3 pages, RDB5B50)

R-32, summary of toxicological and ecological information
Hydrofluorocarbon 32: Physiologically Based Pharmacokinetic Modelling to Predict Uptake and Metabolism in the Rat, report CTL/R/1137, Zeneca Central Toxicology Laboratory (then part of $\mathrm{ICl}$ Chemicals and Polymers Limited), Cheshire, UK, 1992 (RDB6689)

R-32, biochemistry, health effects, toxicity

Workplace Environmental Exposure Level Guide - Difluoromethane, American Industrial Hygiene Association (AIHA), Fairfax, VA, 1997 (3 pages with no figures or tables, RDB5C14)

R-32: identification, summary chemical and physical properties, uses, animal toxicology data, toxicity, human uses and experience (no reports of adverse effects), recommended WEEL guide $\left(1,000 \mathrm{ppm}, 2,200 \mathrm{mg} / \mathrm{m}^{3}\right.$, for $8-\mathrm{hr}$ TWA) and rationale, references

report 57-15, Jackson Laboratory / Haskell Laboratory for Toxicology and Industrial Medicine, E. I. duPont de Nemours and Company, Incorporated, Newark, DE, date unknown (RDB6169)

$R-32$, toxicity, subchronic exposure of guinea pigs as reported in RDB5862

report 159-76, Haskell Laboratory for Toxicology and Industrial Medicine, E. I. duPont de Nemours and Company, Incorporated, Newark, DE, 1976 (RDB6172)

$\mathrm{R}-32$, toxicity, subchronic exposures in rats as reported in RDB5862

\section{$R-40$}

Emergency Response Planning Guidelines Methyl Chloride, American Industrial Hygiene Association (AlHA), Fairfax, VA, 1995 (RDB4B83)

R-40, chloromethane, ERPG-1, ERPG-2, ERPG-3, toxicology data, toxicity, safety classification

\section{R-4011}

Emergency Response Planning Guidelines Methyl lodide, American Industrial Hygiene Association (AlHA), Fairfax, VA, 1991 (RDB4B80)

R-4011, iodomethane, ERPG-1, ERPG-2, ERPG-3, toxicology data, toxicity, safety classification 


\section{R-50 (METHANE)}

F. G. Carpenter, Anesthetic Action of Inert and Unreactive Gases on Intact Animals and Isolated Tissues, American Journal of Physiology, 178:505-509, 1954 (5 pages, RDB7421)

anesthetic effect of R-50 (methane) and others in mice, toxicity

Toxicity Study Summary: Methane, Pure Grade, Phillips Petroleum Company, Bartlesville, OK, February 1990 (1 page with no figures or tables, RDB7622)

tests of the acute inhalation toxicity in 5 female and 5 male albino, Sprague-Dawley rats by 4 $\mathrm{hr}$, whole body exposures to $34,149 \mathrm{ppm}$ v/v R50 (methane) in air: no deaths resulted and animals appeared normal throughout the exposures and subsequent 14-day observation period; no gross signs or symptoms of intoxication were noted; treated females showed a slight reduction in mean body weight on days 2-7 following exposures; animals appeared normal at necropsy, with the exception of 1 female; an enlarged mandibular lymph node and scattered white foci on the lung surface were found in pathological examination of this animal; concludes that the 4-hr $\mathrm{LC}_{50}$ rat is $>34,149 \mathrm{ppm}$ $\mathrm{v} / \mathrm{V}$; respiratory tract irritancy studies in male mice by head-only exposures to 34,276 ppm $v / v$ R-50 in air for $1 \mathrm{~min}$, repeated 10 minutes later, while monitored by a plethysmograph: no patterns of respiratory pause were evident; concludes that R-50 failed to produce upper respiratory irritancy in mice at $34,276 \mathrm{ppm} \mathrm{v} / \mathrm{v}$

\section{R-112 AND R-112a}

J. W. Clayton, Jr., H. Sherman, S. D. Morrison, J. R. Barnes, and D. B. Hood (E. I. duPont de Nemours and Company, Incorporated), Toxicity Studies on 1,2-Difluorotetrafluoroethane (Freon 112) and 1,1-Difluorotetrafluoroethane (Freon 112a), Toxicology and Applied Pharmacology (TAP), 6:342 ff, 1964 (RDB5562)

$R-112$ and $R-112 a$, health effects, toxicity

L. A. Greenberg and D. Lester (Yale University), The Toxicity of the Tetrachlorodifluoroethanes, Archives of Industrial Hygiene and Occupational Medicine, 2:345-347, 1950 (3 pages, RDB8145)

$\mathrm{R}-112$ and $\mathrm{R}-112 \mathrm{a}$, health effects, toxicity

\section{R-113}

T. K. Bodganowicz, Acute Inhalation Toxicity, report 179-73, Haskell Laboratory for Toxicology and Industrial Medicine, E. I. duPont de Nemours and Company, Incorporated, Newark, DE, 1973 (1 page, RDB7437)

$\mathrm{R}-113$, health effects, toxicity: 4-hr ALC rat as reported in RDB7414

V. L. Carter, P. M. Chikos, J. D. MacEwen, and K. C. Back, Effects of Inhalation of Freon 113 on Laboratory Animals, paper 20, Proceedings of the 1st Annual Conference. on Environmental Toxicology, report AMRL-TR-70-102, Aerospace Medical Research Laboratory, U.S. Air Force, Wright-Patterson Air Force Base, $\mathrm{OH}, 309-314$, 1970 (6 pages, available from NTIS as document AD-727524, RDB7438)

R-113, health effects, toxicity

R. Carchman and M. M. Greenberg, Summary Review of Health Effects Associated with 1,1,2Trichloro-1,2,2-trifluoroethane (Chlorofluorocarbon 113): Health Issue Assessment, report EPA 600/8-82-002F, Office of Health and Environmental Assessment, U.S. Environmental Protection Agency (EPA), Washington, DC, January 1983 (available from NTIS as document PB84118843, RDB6494)

$\mathrm{R}-113$, health effects, toxicity

E. E. Christofano, J. B. Bryant, J. M. Trowell, Human Exposures to 1,1,2-Trichloro-1,2,2-trifluoroethane, abstract 91 , proceedings of the AlHA conference (Denver, CO, 10-16 May 1969), American Industrial Hygiene Association (AlHA), Fairfax, VA, 30:134, 1969 (1 page, RDB5C02)

$R-113$, health effects, toxicity

O. L. Dashiell, 1,1,2-Trifluoro-1,2,2-trichloroethane: Acute Inhalation Toxicity, report 46-71, Haskell Laboratory for Toxicology and Industrial Medicine, E. I. duPont de Nemours and Company, Incorporated, Newark, DE, 1971 (2 pages, RDB7439)

R-113, 1,1,2-trichloro-1,2,2-trifluoroethane, toxicity, health effects: 4 -hr ALC rat as reported in RDB7414

H. Desoille, L. Truffert, A. Bourguignon, P. Delavierre, M. Philbert, and C. Girard-Wallon, Experimental Study on the Toxicity of Trichlorotrifluoroethane (Freon 113), Archives des Maladies Professionelles de Médecine du Travail et de Se- 
curité Sociale, France, 29(7-8):381-388, 1968 (8 pages, in French, RDB6B56)

R-113, toxicity: 2-hr ALC rat $110,000 \mathrm{ppm}$ as reported in $\mathrm{RDB65H} 1$

G. T. Hall, P. W. Grube, and R. C. Graham, untitled toxicity review for CFC-113 (ethane, 1,1,2trichloro-1,2,2-trifluoro-), Haskell Laboratory for Toxicology and Industrial Medicine, E. I. duPont de Nemours and Company, Incorporated, Newark, DE, 4 April 1993 (38 pages, RDB5874)

$\mathrm{R}-113$, summary of published and unpublished toxicity literature

Industrial Test Laboratory (ITL), Toxicity Tests of Non-Inflammable Solvent Cleaner for Insulated Electrical Motor Parts, report ITL 2335/NS 041 001. Philadelphia Naval Shipyard, U.S. Navy, Philadelphia, PA, 1952 (8 pages, RDB7456)

$\mathrm{R}-113$, health effects, toxicity

H. R. Imbus and C. Adkins, Physical Examinations of Workers Exposed to Trichlorotrifluoroethane, Archives of Environmental Health, 24(4):257-261, 1972 (5 pages, RDB6733)

R-113, 1,1,2-trichloro-1,2,2-trifluoroethane, human exposures, toxicity

T. Kawakami, T. Takano, and R. Araki (Japan Bioassay Laboratory, JBL, Japan), Enhanced Arrhythmogenicity of Freon 113 by Hypoxia in the Perfused Rat Heart, Toxicology Ind. Health, 6:493-506, 1990 (8 pages, RDB6B45)

R-113, cardiac sensitization, health effects, toxicity

G. Limperos, Inhalation Toxicity of 'Freon 113' (1,1,2-Trichlorotrifluoroethane), report 3-54, Haskell Laboratory for Toxicology and Industrial Medicine, E. I. duPont de Nemours and Company, Incorporated, Newark, DE, 1954 (9 pages, RDB7440)

$\mathrm{R}$-113, 1,1,2-trichloro-1,2,2-trifluoroethane, toxicity, health effects: rats exposed to an average of 2,520 ppm v/v for $7 \mathrm{hr} / \mathrm{d}, 5 \mathrm{~d} / \mathrm{wk}$ for 6 weeks showed no signs of toxicity as reported in RDB7414

D. C. May and M. J. Blotzer, A Report of Occupational Deaths Attributed to Fluorocarbon-113, Archives of Environmental Health, 39(5):352-354, 1984 (3 pages, RDB6734)

R-113, human exposures, toxicity

J. E. McKnight and J. L. McGraw, Ultrastructural Study of the Effects of Trichlorotrifluoroethane on the Liver of Hairless Mice, Journal of Submicroscopic Cytology, 15(2):447-451, 1983 (5 pages, RDB8165)

$\mathrm{R}-113$, mutagenicity, toxicity

J. B. Michaelson and D. J. Huntsman, Oral Toxicity Study of 1,2,2-Trichloro-1,1,2-trifluoroethane, Journal of Med. Pharm. Chem., 7:378-379, 1964 (2 pages, RDB7441)

$\mathrm{R}$-113, 1,1,2-trichloro-1,2,2-trifluoroethane, toxicity, health effects: $L D_{50}$ rat $=43 \mathrm{~g} / \mathrm{kg}$ as reported in RDB7414

G. B. Raffi and F. S. Violante, Is Freon 113 Neurotoxic? A Case Report, International Archives of Occupational and Environmental Health, 49:125-127, 1981 (RDB6735)

case history of human exposures to R-113; toxicity

C. F. Reinhardt, M. E. McLaughlin, M. E. Maxfield, L. S. Mullin, and P. E. Smith, Human Exposures to Fluorocarbon 113 (1,1,2-trichloro-1,2,2trifluoroethane), AlHA Joumal, 32:143-152, March 1971 (10 pages, RDB6496)

examination of human exposures to R-113; toxicity

C. F. Reinhardt and L. R. Schultze, Human Skin Absorption Studies with Trichlorotrifluoroethane, F-113, report 84-68, Haskell Laboratory for Toxicology and Industrial Medicine, E. I. duPont de Nemours and Company, Incorporated, Newark, DE, 1968 (7 pages, RDB7443)

R-113, dermal contact toxicity, health effects: skin exposures result in local numbness followed by transient erythema after exposure stopped as reported in RDB7414

R. E. Reinke, Freon 113 (1,1,2-trichlorotrifluoroethane). Eye Irritation Experiment, report 39-62, Haskell Laboratory for Toxicology and Industrial Medicine, E. I. duPont de Nemours and Company, Incorporated, Newark, DE, 1962 (1 page, RDB6B57)

dermal contact toxicity and eye irritation in rabbits of R-113 (1,1,2-trichloro-1,2,2-trifluoroethane); results in mild conjunctivitis (inflammation of the mucous membrane inside the eyelids) and corneal dullness - as reported in $\mathrm{RDB65H} 1$

H. Savolainen and P. Pfaffli, Dose Dependent Neurochemical Effects of 1,1,2-Trichloro-1,2,2trifluoroethane Inhalation Exposure in Rats, Toxicology Letters, 6:43-49, 1980 (RDB6497) 
inhalation toxicity of R-113

J. W. Sarver, 1,1,2-Trifluoro-1,2,2-trichloroethane: Four-Hour Inhalation Toxicity, report 10471, Haskell Laboratory for Toxicology and Industrial Medicine, E. I. duPont de Nemours and Company, Incorporated, Newark, DE, 7 April 1971 (2 pages with 1 table, RDB6804)

toxicity tests by acute inhalation of $\mathrm{R}-113$ in male, Manor Farm rats at $32,000,40,500$, 45,000 , and $53,000 \mathrm{ppm} \mathrm{v/v}$ and for 10 repeated, subacute exposures at $1,000 \mathrm{ppm}$; record of clinical signs shows anesthetic/central nervous system (CNS) effects as well as slight weight loss, followed by normal weight gain, at $32,000 \mathrm{ppm}$; 4-hr $\mathrm{LC}_{50}$ rat $=52,500 \mathrm{ppm}$; pathologic examination of exposed animals revealed no compound-related changes in the respiratory tract; comparison to prior test results with Charles River rats, which showed the 4-hr $\mathrm{LC}_{50}$ to be $56,000 \mathrm{ppm}$, concludes that "there are basically no differences ... in the two strains of rats ${ }^{n}$ within $95 \%$ confidence limits

M. B. Steinberg, R. E. Boldt, R. A. Renne, and M. $H$. Weeks, Inhalation Toxicity of 1,1,2-Trifluoro1,2,2-trichloroethane (TCTFE), report on study 33-18-68/69, Environmental Hygiene Agency, U.S. Army, Edgewood Arsenal, MD, 1969 (20 pages, available from NTIS as document AD-854705, RDB7444)

inhalation toxicity of $R-113 \quad(1,1,2$-trichloro1,2,2-trifluoroethane)

G. J. Stopps and M. McLaughlin, Psychophysiological Testing of Human Subjects Exposed to Solvent Vapors, AIHA Journal, 28:43-50, 1967 (8 pages, RDB7445)

toxicity tests of R-113 and others to humans

G. Triebig and K. Burkhardt, Occupational Medicine Investigations into Occupational Exposure to FC113, International Archives of Occupational and Environmental Health, Germany, 42:129-135, 1978 (7 pages, RDB6498)

toxicity of R-113

H. J. Trochimowicz (E. I. duPont de Nemours and Company), G. M. Rusch (AlliedSignal Incorporated), T. Chiu, and C. K. Wood, Chronic Inhalation Toxicity/Carcinogenicity Study in Rats Exposed to Fluorocarbon 113 (FC-113), Fundamental and Applied Toxicology, 11:68-75, 1988 (8 pages, RDB8179)

toxicity of R-113
H. J. Trochimowicz, The Toxicology of Fluorocarbon 113, presentation to the Swedish Board of Occupational Safety and Health (Stockholm, Sweden), E. I. duPont de Nemours and Company, Incorporated, Newark, DE, 19 January 1984 (RDB8180)

\section{toxicity of R-113}

H. Vainio, J. Nickels, and T. Heinonen, Dose Related Hepatotoxicity of 1,1,2-Trichloro-1,2,2-trifluoroethane in Short-Term Intermittent Inhalation Exposures in Rats, Toxicology, 18(1):17-35, 1980 (19 pages, RDB6499)

R-113, health effects, toxicity

C. K. Wood, Two-Year Inhalation Toxicity Study with 1,1,2-Trichloro-1,2,2-trifluoroethane in Rats, report 488-84, Haskell Laboratory for Toxicology and Industrial Medicine, E. I. duPont de Nemours and Company, Incorporated, Newark, DE, 5 March 1985 (63 pages, RDB6730)

$\mathrm{R}-113$, two year inhalation study in rats, as reported in RDB5143

B. H. Woollen, E. A. Guest, W. Howe, J. R. Marsh (ICl Central Toxicology Laboratory, UK), H. K. Wilson (Occupational Medicine and Hygiene Laboratories, UK), T. R. Auton, and P. G. Blain (ICl Central Toxicology Laboratory, UK), Human Inhalation Pharmacokinetics of 1,1,2-Trichloro-1,2,2trifluoroethane (FC113), International Archives of Occupational and Environmental Health, Germany, 62:73-78, 1990 (6 pages with 5 figures and 3 tables, RDB6492)

R-113, health effects, toxicity: tests of absorption and elimination in experimental human exposures by seven male volunteers at concentrations of 254,526 , or $980 \mathrm{ppm} v / \mathrm{v}$ for $4 \mathrm{hr}$

NIOSH Alert: Preventing Death from Excessive Exposure to Chlorofluorocarbon 113, publication 89-109, National Institute for Occupational Safety and Health (NIOSH), U.S. Department of Health and Human Services, Cincinnati, OH, 1989 (available from GPO; RDB6B48)

$R-113$, health effects, toxicity

Occupational Health Guideline for 1,1,2Trichloro-1,2,2-trifluoroethane, U.S. Department of Health and Human Services and U.S. Department of Labor, Washington, DC, September 1978 (4 pages, available from JMC a RDB6291)

R-113, Occupational Health Guideline (OHG), exposure limits, health hazard information, toxicity, chemical and physical data, reactivity, flammability, monitoring and measurement, res- 
pirators, personal protective equipment, emergency first aid procedures, spills and leaks, references

Teratogenicity Study of 1,1,2-Trichloro-1,2,2-trifluoroethane in Rats, report CTL/P/731, ICl Central Toxicology Laboratory, Cheshire, UK, 19 November 1982 (RDB6732)

R-113, teratogenicity study in rats, as reported in RDB5143 and RDB5367

Toxicity Studies with 1,1,2-Trichloro-1,2,2trifluoroethane, bulletin S-24, E. I. duPont de Nemours and Company, Incorporated, Wilmington, DE, 1980 (RDB8139)

$R-113$, health effects, safety, toxicity

\section{1,1,2-Trichloro-1,2,2-trifluoroethane}

(Freon

113), chemical infogram 22, Canadian Centre for Occupational Health and Safety (CCOHS), Hamilton, ON, Canada, December 1989 (1 page with no figures or tables, RDB7457)

$R-113$, safety, toxicity, flammability, occupational exposure guidance

report 3-54, Haskell Laboratory for Toxicology and Industrial Medicine, E. I. duPont de Nemours and Company, Incorporated, Newark, DE, 1954 (RDB7541)

toxicity tests to determine the acute dermal toxicity of R-113 applied to the clipped skin of rabbits at $>11,000 \mathrm{mg} / \mathrm{kg}$ : no signs indicating systemic toxicity were found, though the treatment caused local irritation, as reported in RDB5874

report 724-81, Haskell Laboratory for Toxicology and Industrial Medicine, E. I. duPont de Nemours and Company, Incorporated, Newark, DE, 11 December 1981 (RDB6731)

$\mathrm{R}-113,90$-day inhalation study in rats, as reported in RDB5143

\section{R-114}

D. W. D. Collure et al, Journal of the American College of Emergency Physicians, 5(10):814 ff, 1976 (RDB7544)

R-114, dermal effects and toxicity: experiments on human volunteers

H. Desoille et al., Archives des Maladies Professionelles de Médecine du Travail et de Securité Sociale, France, 36(12):697-706, 1975 (10 pages, in French, RDB7545)

\section{R-114, health effects, toxicity}

H. Desoille, L. Truffert, C. Gierard-Wallon, J. Ripault, and M. Philbert, Recherche Expérimentale de la Toxicité Chronique Éventuelle du Dichlorotetrafluoroethane [Experimental Research on the Long-Term Chronic Toxicity of Dichlorotetrafluoroethane], Archives des Maladies Professionelles de Médecine du Travail et de Securité Sociale, France, 34(3):117-125, 1973 (9 pages, in French, RDB5362)

$R-114$, health effects, toxicity

Z. Durakovic, L. Stilinovic, and I. Bakran, Jr., Electrocardiographic Changes in Rats After Inhalation in Dichlorotetrafluoroethane, Arcton 114, $\mathrm{C}_{2} \mathrm{Cl}_{2} \mathrm{C}_{4}$, Japan Heart Journal, 17(6):753-759, 1976 (7 pages, RDB6738)

$\mathrm{R}-114$, toxicity

F. D. Griffith and $H$. Sherman, The Effect of Freon ${ }^{\circledR} 114$ (Ethane-1,2-dichlorotetrafluoro) on Growth Rate, Urinary Fluoride, and Bone Fluoride in Rats, report 381-69, Haskell Laboratory for Toxicology and industrial Medicine, E. I. duPont de Nemours and Company, Incorporated, Newark, DE, December 1969 (RDB5A70)

$R-114$, toxicity, three-week intubation exposure study with rats, possibility of some metabolism as reported in RDB5141 and RDB7414

D. B. Hood, Skin Tests with Rexall Facial Cleanser Containing 'Freon-114', report 110-67, Haskell Laboratory for Toxicology and Industrial Medicine, E. I. duPont de Nemours and Company, Incorporated, Newark, DE, 1967 (RDB65H6)

R-114, dermal toxicity: no irritation observed when sprayed on shaved backs of guinea pigs as reported in $\mathrm{RDB} 65 \mathrm{H} 1$

D. B. Hood and M. Ward, Acute Oral Toxicity Of Freon ${ }^{\circledR}$ 114, Dichlorotetrafluoroethane, report on project MR-312-7, Haskell Laboratory for Toxicology and Industrial Medicine, E. I. duPont de Nemours and Company, Incorporated, Newark, DE, 1955 (RDB65H5)

toxicity tests of R-114 in rats for determine ingestion toxicity: oral ALD rat $>2,250 \mathrm{mg} / \mathrm{kg}$ (based on the maximum feasible dose dissolved in corn oil); comparison to R-12 ALD rat $>1,000 \mathrm{mg} / \mathrm{kg}$ (maximum feasible dose dissolved in corn oil) as reported in RDB65H1

D. B. Hood and M. Ward, Eye Irritation of Freon ${ }^{\circledR}$ 114 in Rabbits, report on project MR-312-7, Haskell Laboratory for Toxicology and Industrial 
Medicine, E. I. duPont de Nemours and Company, incorporated, Newark, DE, 1955 (RDB7447)

toxicity tests of R-114 in rabbits: mild conjunctivitis (inflammation of the mucous membrane inside the eyelids), persisting less than $48 \mathrm{hr}$, resulted from acute eye exposure tests with a mineral oil solution - as reported in RDB65H1

H. Kuebler, Aerosol Age, 9:44 ff., 1964 (RDB6870) indicates an anesthetic effect in rats exposed to $500,000 \mathrm{ppm} \mathrm{v/v} \mathrm{R-114} \mathrm{for} 2 \mathrm{hr}$ - as reported in RDB5855

R. R. Montgomery, M. A. Lee, R. C. Graham, and P. J. Lardear, untitled toxicity review for 1,2Dichloro-1,1,2,2-tetrafluoroethane, Haskell Laboratory for Toxicology and Industrial Medicine, E. I. duPont de Nemours and Company, Incorporated, Newark, DE, 11 June 1992 (RDB5855)

$\mathrm{R}-114$, summary of published and unpublished toxicity literature

S. Niazi and W. L. Chiou, Fluorocarbon Aerosol Propellants X: Pharmacokinetics of Dichlorotetrafluoroethane in Dogs, Journal of Pharmaceutical Sciences, 65:60-64, 1976 (5 pages, RDB7253)

$\mathrm{R}-114$, biochemistry, elimination, health effects, toxicity

G. Paulet and S. Desbrousses, Le Dichlorotetrafluoroethane: Toxicité Aiguë et Chronique à Moyen Terme [Dichlorotetrafluoroethane: Acute and Mid-Test Chronic Toxicityl, Archives des Maladies Professionelles de Médecine du Travail et de Securité Sociale, France, 30(9):477-492, 1969 (16 pages in French, RDB5172)

R-114, toxicity

J. F. Russell, report 19-78, Haskell Laboratory for Toxicology and Industrial Medicine, E. I. duPont de Nemours and Company, Incorporated, Newark, DE, January 1978 (RDB7446)

toxicity tests to determine the mutagenicity of $\mathrm{R}-114$ in Salmonella typhimurium bacteria (Ames assay) both in the presence and absence of a metabolic activation system (S-9) derived from rat livers: finding was negative as reported in RDB5141 and RDB5855

H. Sherman, Ninety-Day Feeding Study in Rats and Dogs with Dichlorotetrafluoroethane (Freon $\left.{ }^{\circledR} 114\right)$, report 5-72, Haskell Laboratory for Toxicology and Industrial Medicine, E. I. duPont de Nemours and Company, Incorporated, Newark, DE, January 1972 (RDB5A71)
R-114, toxicity, 90-day intubation exposure study with dogs and rats as reported in RDB5141

W. P. Yant, H. H. Schrenk, and F. A. Patty, The Toxicity of Dichlorotetrafluoroethane, Report of Investigations 3185 , U.S. Bureau of Mines, Washington, DC, October 1932 (12 pages with 1 table, available from JMC as RDB5359)

R-114; 12-week exposures of dogs, guinea pigs, and monkeys to $200,000 \mathrm{ppm} \mathrm{v} / \mathrm{v}$, reduced to $150,000 \mathrm{ppm} v / \mathrm{v}$ and then 141,600 $\mathrm{ppm} \mathrm{v} / \mathrm{v}$ (equivalent fluorine content on a weight basis to $200,000 \mathrm{ppm} v / \mathrm{v} \mathrm{R}-12$ ) following observation of effects; documents observed symptoms including tremors and convulsions, changes in weight, changes in blood picture, fatality, and pathology; report concludes that "the toxicity of [R-114] on a vapor-volume basis is somewhat greater than [R-12], but on a weight basis is of the same order as the latter, and is an organic vapor of remarkably low toxicity"

1,2-Dichloro-1,1,2,2-tetrafluoroethane (CFC 114) Health Effects Information, CAS\# 76-14-2, Elf Atochem North America, Incorporated, Philadelphia, PA, undated circa 1995 (8 pages, RDB5B51)

$R-114$, summary of toxicological and ecological information

Bioassay Report - Acute Toxicity - Fish, TSCAT report OTS-0520348, Bionomics Incorporated, 12 June 1989 (11 pages, RDB7252)

ecotoxicity of R-114; toxicological and ecological information; environmental impacts; toxicity

Occupational Health Guideline for Dichlorotetrafluoroethane (Refrigerant 114), U.S. Department of Health and Human Services and U.S. Department of Labor, Washington, DC, September 1978 (4 pages, available from JMC as RDB6287)

R-114, Occupational Health Guideline (OHG), exposure limits, health hazard information, toxicity, physical data, reactivity, flammability, monitoring and measurement, respirators, personal protective equipment, emergency first aid procedures, spills and leaks, references

\section{R-115}

R. F. Bopp et al., Organic Geochemistry, 3:9-14, 1981 (6 pages, RDB7613) 
R-115, aquatic and environmental toxicity, ecotoxicity: most $\mathrm{R}-115$ will volatize and bioconcentration in aquatic organisms is not expected to be important - as reported in RDB6776

J. F. Breen and J. E. Wallis, Report on Refrigerant, Miscellaneous Hazard Report Number 2256, Underwriters Laboratories Incorporated, Northbrook, IL (then Chicago, IL), 20 August 1963 (RDB7614)

toxicity tests of R-115 to guinea pigs by inhalation exposures at concentrations of 200,000 $\mathrm{ppm}$ for up to $2 \mathrm{hr}$ : no signs of toxicity, weight change, or gross or microscopic pathological abnormalities were found - as reported in RDB6776

J. W. Clayton, Jr., D. B. Hood, M. S. Nick, and R. S. Waritz (E. I. duPont de Nemours and Company, Incorporated), Inhalation Studies of Chloropentafluoroethane, AIHA Journal, 27(3):234-238, May-June 1966 (5 pages, RDB65H4)

R-115, toxicity: $4-h r$ ALC rat $>800,000 \mathrm{ppm}$ v/v as reported in $\mathrm{RDB} 65 \mathrm{H} 1$.

G. T. Hall, P. W. Grube, M. A. Lee, and R. C. Graham, untitled toxicity review for chloropentafluoroethane, Haskell Laboratory for Toxicology and Industrial Medicine, E. I. duPont de Nemours and Company, Incorporated, Newark, DE, 16 March 1993 (14 pages with no figures or tables, RDB6776)

R-115, summary of published and unpublished toxicity literature

H. S. Sherman, report 146-64, Haskell Laboratory for Toxicology and Industrial Medicine, E. I. duPont de Nemours and Company, Incorporated, Newark, DE, 1964 (RDB65H7)

R-115, ingestion toxicity: intragastric intubation of R-115 in cottonseed oil to rats on a daily level of $140-172 \mathrm{mg} / \mathrm{kg}$ for 10 days produced no clinical or histopathologic changes related to $\mathrm{R}-115$ as reported in RDB65H1

J. B. Terrill (E. I. duPont de Nemours and Company, Incorporated), Arterial Venous Blood Levels from Chlorofluoroethane Inhalation versus Oral Exposure, AlHA Journal, 35(5):269-275, May 1974 (7 pages, RDB7239)

R-115, health effects, toxicity: no deaths resulted in dogs that consumed up to 1,200 $\mathrm{mg} / \mathrm{kg}$ of $\mathrm{R}-115$ in a food topping, suggesting an $L D_{50}$ oral dog of $>1,200 \mathrm{mg} / \mathrm{kg}$
J. B. Terrill (E. I. duPont de Nemours and Company, incorporated), paper 12, Proceedings of the Fourth Annual Conference on Environmental Toxicology (Fairborn, OH, 16-18 October 1973), report AMRL-TR-73-125, Aerospace Medical Research Laboratory, U.S. Air Force, Wright-Patterson Air Force Base, OH, 151-174, December 1973 (24 pages, available from NTIS as document AD781031, RDB7615)

R-115 and others, health effects, toxicity

Chloropentafluoroethane (CFC 115) Health Effects Information, CAS\# 76-15-3, Elf Atochem North America, Incorporated, Philadelphia, PA, undated circa 1995 (4 pages, RDB5B52)

$R-115$, summary of toxicological and ecological information

Occupational Safety and Health Guideline for Chloropentafluoroethane, U.S. Department of Health and Human Services and U.S. Department of Labor, Washington, DC, 1995 (7 pages, available from JMC as RDB6288)

R-115, Occupational Health Guideline (OHG), Occupational Safety and Health Guideline (OSHG), physical data, reactivity, flammability, exposure limits, health hazard information, toxicity, emergency procedures, medical monitoring, workplace monitoring and measurement, personal hygiene, storage, leaks, emergency planning and community right-to-know requirements, hazardous waste management requirements, respiratory protection, personal protective equipment, references

report 143-64, Haskell Laboratory for Toxicology and Industrial Medicine, E. I. duPont de Nemours and Company, Incorporated, Newark, DE, 1964 (RDB7618)

$\mathrm{R}-115$, acute inhalation toxicity

\section{R-116}

J. Carson et al., abstract 133, Veterinary and Human Toxicology, 31(4):363 ff, 1989 (RDB6575)

accidents resulting in injection of $R-116$ into the fingers of workers, treatment and result

J. Ferguson-Smith, Hygiene Standard Documentation: Perfluoroethane document ESC/PFC $116, \mathrm{ICl}$ Chemicals and Polymers Limited, Cheshire, UK, 22 January 1996 (5 pages, RDB6687)

R-116, toxicity, literature review, rationale for recommended hygiene standard (Health Based Standard) of 1,000 ppm, 8-hr TWA 
T. B. Griffin et al., Toxicology and Applied Pharmacology (TAP), 29:92 ff, 1974 (RDB6577)

\section{$\mathrm{R}-116$, toxicity}

M. A. Lee and R. C. Graham, untitled toxicity review for hexafluoroethane, Haskell Laboratory for Toxicology and Industrial Medicine, E. I. duPont de Nemours and Company, Incorporated, Newark, DE, 26 July 1991 (RDB5605)

R-116, summary of published and unpublished toxicity literature

M. Hughes, unpublished data, Atlantic Research Corporation, 1973 (RDB6119)

R-116, cardiac sensitization, toxicity: indicates a cardiac sensitization NOEL dog at 600,000 ppm, but the test protocol (e.g., whether an epinephrine challenge was used, whether the test mixture was oxygen enriched, and the duration of exposure) is not clear - as reported in RDB5605

S. D. Morrison, report 41-62, Haskell Laboratory for Toxicology and Industrial Medicine, E. 1. duPont de Nemours and Company, Incorporated, Newark, DE, 1962 (RDB6580)

R-116, inhalation, acute toxicity: 2-hr ALC rat $>200,000 \mathrm{ppm}, 4-\mathrm{hr}$ ALC rat $>800,000 \mathrm{ppm}$ as reported in RDB5605 and RDB65H1

report CTL/L6119, ICl Central Toxicology Laboratory, Cheshire, UK, 22 November 1994 (RDB6691)

R-116, toxicity, mutagenicity: Ames test with and without metabolic activation to Salmonella typhimurium and Escherichia coli: not mutagenic as reported in RDB6687

report CTL/L6908, ICl Central Toxicology Laboratory, Cheshire, UK, 31 October 1995 (RDB6690)

$\mathrm{R}-116$, toxicity, mutagenicity: not clastogenic to human lymphocytes with and without metabolic activation as reported in RDB6687

\section{R-123}

F. C. Barsky, In Vitro Microbial Mutagenicity Studies of 2,2-Dichloro-1,1,1-Trifluoroethane, report 581-76, Haskell Laboratory for Toxicology and Industrial Medicine, E. I. duPont de Nemours and Company, Incorporated, Newark, DE, 1989 (RDB6164)

$\mathrm{R}-123$, toxicity
W. E. Brewer and S. Smith (Industrial Bio-Test Laboratories, Incorporated), Teratogenic Study via Inhalation with Genetron 123 in Albino Rats, report IBT 8562-09344, AlliedSignal Incorporated (then Allied Chemical Corporation), Morristown, NJ, 8 November 1977 (32 pages with 10 tables, RDB6143) [included in RDB6142 as appendix C]

toxicity test of the teratogenicity of R-123 in groups of 20 female (16 pregnant) Charles River albino rats by inhalation exposures at 0 (control) or $5,000 \mathrm{ppm}$ for $5 \mathrm{hr} / \mathrm{d}$ on gestation days 6-15: bodyweight gains were lower in the treated animals; both treated and control animals had red nasal discharges; no grossly apparent uterine anomalies attributed to exposures were noted; pathological findings of the sacrificed dams and fetuses were essentially the same for the test and control groups; report concludes that $\mathrm{R}-123$ was not teratogenic as tested

W. E. Brewer and S. Smith (Industrial Bio-Test Laboratories, Incorporated), 90-Day Subacute Inhalation Toxicity Study with Genetron 123 in Albino Rats, report IBT 8562-09344, AlliedSignal Incorporated (then Allied Chemical Corporation), Morristown, NJ, 2 September 1977 (19 pages with 2 tables, Rdb6144) [included in RDB6142 as appendix A]

R-123, subchronic inhalation toxicity: groups of 35 male and 25 female rats were exposed to concentrations of 0 (control), 500 (actual 487), 1000 (actual 1009), and 5000 (actual 4836) $\mathrm{ppm}$ for $6 \mathrm{hr} / \mathrm{d}$ for $5 \mathrm{~d} / \mathrm{wk}$; lower body weight gains were noted at $5000 \mathrm{ppm}$ in both sexes and at $1000 \mathrm{ppm}$ in females with full recovery post exposure; no remarkable effects were seen in hematology, clinical chemistry, or urinalysis parameters; increases were seen in liver weight to body weight ratios in males in the $5000 \mathrm{ppm}$ group and in the females in all three exposure groups; kidney and heart weight decreases were noted, but may have been related to depressed body weight; only the liver showed treatment-related microscopic changes, namely focal necrosis and multifocal atrophy of hepatocytes, but these findings also were seen in the control groups and neither their incidence nor severity followed a treatment-related pattern; summarized and discussed in RDB6142 and, as study A-77, in RDB65D8 [prepared from partial report with missing pages] 
M. R. Brittelli, Eye Irritation Test in Rabbits, report 747-75 (FYI-OTS-0889-0695), Haskell Laboratory for Toxicology and Industrial Medicine, E. I. duPont de Nemours and Company, Incorporated, Newark, DE, 1975 (RDB6160)

$R-123$, health effects, toxicity

W. J. Brock, Acute Dermal Toxicity Study of HCFC-123 in Rabbits, report 578-88 (FYI-OTS0889-0695), Haskell Laboratory for Toxicology and Industrial Medicine, E. I. duPont de Nemours and Company, Incorporated, Newark, DE, 1988 (RDB6148)

R-123, health effects, toxicity, 24-hr ALD skin rabbit $0 / 10>2,000 \mathrm{mg} / \mathrm{kg}$ as reported in RDB5861

W. J. Brock, Acute Dermal Toxicity Study of HCFC-123 in Rats, report 577-88, Haskell Laboratory for Toxicology and Industrial Medicine, E. I. duPont de Nemours and Company, Incorporated, Newark, DE, 1988 (RDB6147)

R-123, health effects, toxicity, 24-hr ALD skin rat $0 / 5>2,000 \mathrm{mg} / \mathrm{kg}$ as reported in RDB5861

W. J. Brock, Primary Dermal Irritation Study with HCFC-123 in Rabbits, report 535-88 (FYIOTS-0889-0695), Haskell Laboratory for Toxicology and Industrial Medicine, E. I. duPont de Nemours and Company, Incorporated, Newark, DE, 1988 (RDB6149)

$\mathrm{R}-123$, health effects, toxicity

D. Brusick (Litton Bionetics Incorporated, LBI), Mutagenicity Evaluation of Genetron 123, report on LBI project 2547, AlliedSignal Incorporated (then Allied Chemical Company), Morristown, NJ, 30 July 1976 (RDB6135)

$R-123$, health effects, toxicity

R. D. Callander, HCFC 123: An Evaluation Using the Salmonella Mutagenicity Assay, report CTL/P/2421 for the Program for Alternative Fluorocarbon Toxicity Testing (PAFT) \#88-01, ICl Central Toxicology Laboratory, Cheshire, UK, 15 December 1989 (RDB6136)

R-123, mutagenicity, toxicity

C. P. Chengelis (WIL Research Laboratories, Incorporated), Acute Pharmacokinetic Study of HCFC-123 in Dogs by Inhalation, Great Lakes Chemical Corporation, West Lafayette, IN, 5 July 1994 (82 pages with 5 figures and 18 tables, RDB8114)

R-123, biochemistry, health effects, toxicity; a copy of this report is appended to RDB6573
J. W. Clayton, Preliminary Studies on the Inhalation Toxicity of Technical (70.9 per cent) 1,1Dichloro-2,2,2-Trifluoroethane [2,2-dichloro1,1,1-trifluoroethane], report 151-64, Haskell Laboratory for Toxicology and Industrial Medicine, E. !. duPont de Nemours and Company, Incorporated, Newark, DE, 1966 (RDB6151)

$R-123$, toxicity

W. B. Coate (Hazleton Laboratories America, Incorporated), $\mathrm{LC}_{50}$ of $\mathbf{G 1 2 3}$ in Rats, report M165162 (FYI report OTS-06890695), AlliedSignal Incorporated (then Allied Chemical Company), Morristown, NJ, 4 October 1976 (RDB6137)

$\mathrm{R}-123$, acute inhalation toxicity, $\mathrm{LC}_{50}$ rat, $6 \mathrm{hr}=$ $52,640 \mathrm{ppm}$ as reported in RDB3719, RDB5371, RDB5861, and RDB65E8

D. W. Coombs (Huntingdon Research Centre Limited, HRC, UK), HCFC-123: 13-Week Inhalation Neurotoxicity Study in the Rat, report ALS 3/931038 for the Program for Alternative Fluorocarbon Toxicity Testing (PAFT), AlliedSignal Incorporated, Morristown, NJ, 21 June 1994 (RDB6217)

R-123, health effects, subchronic toxicity

C. A. Dance, In Vitro Assessment of the Clastogenic Activity of HCFC-123 in Cultured Human Lymphocytes, report 91/PFE 003/0093 for the Program for Alternative Fluorocarbon Toxicity Testing (PAFT), Life Science Research Limited (LSR), Suffolk, UK, 1991 (RDB6219)

R-123, toxicity, mutagenicity

R. W. Darr, An Acute Inhalation Toxicity Study of Fluorocarbon 123 in the Chinese Hamster, Corporate Medical Affairs report MA-25-78-15, AlliedSignal Incorporated (then Allied Corporation), Morristown, NJ, 10 June 1981 (25 pages with 1 figure and 1 table, RDB5149)

test of the acute inhalation toxicity of R-123 in groups of 5 male Chinese hamsters exposed to $10,000,14,000,22,000,26,000$, or $31,000 \mathrm{ppm}$ v/v R-123 in air: $4-h r L_{50}=28,400 \mathrm{ppm}$ v/v

W. Dekant, Metabolism of 1,1-Dichloro-2,2,2-trifluoroethane (HCFC-123), report MA-RR-931972a, Universität Würzburg, Germany, 1994 (RDB65C2)

toxicity of R-123 (2,2-Dichloro-1,1,1-trifluoroethane

C. Doleba-Crowe, Ninety-Day Inhalation Exposure of Rats and Dogs to Vapors of 2,2Dichloro-1,1,1-trifluoroethane (FC-123), report 
229-78, Haskell Laboratory for Toxicology and industrial Medicine, E. I. duPont de Nemours and Company, Incorporated, Newark, DE, 19 May 1978 (RDB6155)

R-123, subchronic inhalation toxicity: groups of 27 male and 27 female rats and 4 male beagle dogs were exposed to concentrations of 0 (control), 1000 (actual 900), and 10,000 (9800 ppm) for $6 \mathrm{hr} / \mathrm{d}, 5 \mathrm{~d} / \mathrm{wk}$ for 4 weeks; narcosis was seen at 10,000 , but not at $1000 \mathrm{ppm}$ and was reversible upon cessation of exposures; decreased rates of body weight gain and increased absolute and relative liver weight gains were seen in the rats; higher relative adrenal and testes weights were seen in the male rats and in the kidney and stomachs of the female rats at both exposure levels, while lower absolute heart and kidney weights were seen at $10,000 \mathrm{ppm}$; no remarkable hematology findings were reported, though sporadic increases in serum total protein levels were noted in both the males and females; there were no noteworthy histopathological findings from evaluation of tissues from rats; dogs exposed to $10,000 \mathrm{ppm}$ had lower erythrocyte counts, hemoglobin concentrations, and hematotcrits; dogs exposed to $10,000 \mathrm{ppm}$ also exhibited degenerative changes in the liver, overall, $1000 \mathrm{ppm}$ was a NOAEL for the dogs - as reported in RDB65D8 which summarizes this (identified as D-78) and three related studies

C. N. Edwards, HCFC-123 (Vapour Phase): In Vitro Assessment of Clastogenic Activity in Cultured Human Lymphocytes, report 91/PFE 002/0125 for the Program for Alternative Fluorocarbon Toxicity Testing (PAFT), Life Science Research Limited (LSR), Suffolk, UK, 1991 (RDB6220)

\section{$\mathrm{R}-123$, toxicity, mutagenicity}

J. Ferguson-Smith, Hygiene Standard Documentation - 1,1-Dichloro-2,2,2-trifluoroethane [2,2dichloro-1,1,1-trifluoroethane], document HSP/92/14, ICl Chemicals and Polymers Limited, Cheshire, UK, 1992 (14 pages with 3 tables, RDB5A77)

$\mathrm{R}-123$, toxicity, literature review, rationale for recommended hygiene standard (occupational exposure limit, OEL) of $10 \mathrm{ppm}, 8$-hr TWA, provisional

R. Ferrara (University of Padova Medical School, Italy), R. Tolando (University of Surrey, UK), L. J. King (Surrey), and M. Manno (Padova), Cytochrome P450 Inactivation During Reductive
Metabolism of 1,1-Dichloro-2,2,2trifluoroethane (HCFC-123) by Phenobarbitaland Pyridine-Induced Rat Liver Microsomes, Toxicology and Applied Pharmacology (TAP), 143(2):420-428, April 1997 (9 pages with 9 figures and 1 table, RDB7C67)

toxicity of R-123 by in vitro tests in rats of the reductive metabolic activation of $\mathrm{R}-123$; doseand time-dependent depletion of added exogenous glutathione was observed during incubation of liver microsomes under anaerobic conditions; R-133a and R-142 were detected as products of reductive metabolism of HCFC-123 under similar incubation conditions except for the absence of glutathione; results indicate that $R-123$, like hepatoxic R-123B1 (halothane) which is a close structural analog, is activated reductively to reactive metabolites by at least two P450 isoforms, namely P4502E1 and P4502B1/2; these metabolites, probably free radicals and/or carbene species, may attack the enzyme resulting in modification of the microsomal heme group and subsequent loss of catalytic activity

R. Ferrara, R. Tolando, L. J. King, and M. Manno (University of Padova Medical School, Italy), Reductive Activation of 2,2-Dichloro-1,1,1trifluoroethane (HCFC-123) by Rat Liver Microsomal Cytochrome P-450, Proceeding of the Fifth International Symposium on Biological Reactive Intermediates, Munich, Germany, 4-8 January 1995 (RDB65C5)

toxicity of R-123

K. M. Gerber, M. A. Lee, R. C. Graham, and S. W. Snyder, untitled review of toxicity literature for ethane, 2,2-dichloro-1,1,1-trifluoro- (HCFC-123), Haskell Laboratory for Toxicology and Industrial Medicine, E. I. duPont de Nemours and Company, Incorporated, Newark, DE, 28 February 1996 (25 pages, RDB6941)

summary of published and unpublished information on the toxicity of R-123

C. S. Godin, J. M. Drerup, and A. Vinegar, Conditions Influencing the Rat Liver Microsomal Metabolism of 2,2-Dichloro-1,1,1-Trifluoroethane (HCFC-123), Drug Metabolism and Disposition, 21(3):551-553, May 1993 (3 pages, RDB6213)

R-123, toxicity

N. C. Goodman, Primary Skin Irritation and Sensitization Tests on Guinea Pigs, report 678-75, Haskell Laboratory for Toxicology and Industrial Medicine, E. I. duPont de Nemours and Company, 
Incorporated, Newark, DE, 31 December 1975 (RDB6161)

$\mathrm{R}-123$, health effects, toxicity: produced no irritation or sensitization on shaved skin of albino guinea pigs in 10 and $50 \%$ solutions in propylene glycol as reported in RDB6941

G. T. Hall and B. L. Moore, 1,1-Dichloro-2,2,2-trifluoroethane: Acute Inhalation Toxicity, report 426-75, Haskell Laboratory for Toxicology and Industrial Medicine, E. I. duPont de Nemours and Company, Incorporated, Newark, DE, 16 July 1975 (2 pages with 1 table, RDB6139)

$4 \mathrm{hr}$ acute inhalation toxicity tests of R-123 in groups of six male Charles River CD rats: mortality ratios and corresponding concentrations were $0 / 6$ at $20,700,3 / 6$ at $32,000,3 / 6$ at $33,700,4 / 6$ at 42,100 , and $6 / 6$ at 52,500 and $55,000 \mathrm{ppm}$ v/v in air, rats showed loss of mobility, lethargy, prostration, unresponsiveness to sound, and dyspnea (difficult or labored breathing) within 5 minutes at all concentrations; rats that survived showed no noticeable clinical signs 30 minutes following exposures; no pathologic examinations were performed; concludes that the 4-hr $\mathrm{LC}_{50}$ rat for $\mathrm{R}-123$ is approximately $32,000 \mathrm{ppm} v / \mathrm{v}$, confirming an earlier study, and therefore "practically non-toxic on an acute inhalation basis," but that R-123 does cause an adverse central" nervous system (CNS) effect at 20,700 ppm

J. W. Harris, L. R. Pohl, J. L. Martin, and M. W. Anders, Tissue Acylation by the Chlorofluorocarbon Substitute 2,2-Dichloro-1,1,1-Trifluoroethane, Proceedings of the National Academy of Science (NAS), Washington, DC, 88(4):1407-1410 or 1407-1416, 15 February 1991 (4 or 10 pages, RDB6222)

toxicity of R-123; biochemistry

J. E. Henry and A. M. Kaplan, Acute Oral Test on FC-123, report 638-75 (FYI-OTS-0889-0695), Haskell Laboratory for Toxicology and Industrial Medicine, E. I. duPont de Nemours and Company, Incorporated, Newark, DE, 30 October 1975 (RDB6140)

toxicity of R-123; ALD as reported in RDB5861

E. W. Hughes (Huntingdon Research Centre Limited, HRC, Cambridgeshire, UK), A Study of the Effect on the Reproductive Function of Two Generations in the Rat, report ALS 5/932336 for the Program for Alternative Fluorocarbon Toxicity Testing (PAFT), AlliedSignal Incorporated, Morristown, NJ, 1994 (RDB6218) toxicity of R-123; health effects

E. W. Hughes, D. P. Myers, and D. W. Coombs (Huntingdon Research Centre Limited, HRC, UK), Range Finding Study in Mature Male and 'Female Rats and their Offspring, report ALS 4/920200 for the Program for Alternative Fluorocarbon Toxicity Testing (PAFT), AlliedSignal incorporated, Morristown, NJ, 28 February 1992 (RDB6574)

reproductive toxicity of $R-123$

J. Huwyler, D. Aeschlimann, U. Christen, and J. Gut, The Kidney as Novel Target Tissue for Protein Adduct Formation Associated with Metabolism of Halothane and the Candidate Chlorofluorocarbon Replacement 2,2-Dichloro1,1,1-trifluoroethane, European Joumal of Biochemistry, 207(1):229-238, 1 July 1992 (10 pages, RDB65C7)

toxicity of R-123 and R-123B1 (halothane); metabolism; health effects

J. Huwyler and J. Gut, Exposure to the Chlorofluorocarbon Substitute 2,2-Dichloro-1,1,1trifluoroethane and the Anesthetic Agent Halothane is Associated with Transient Protein Adduct Formation in the Heart, Biochemical and Biophysical Research Communications, 184(3):1344-1349, 15 May 1992 (6 pages, RDB65C8)

R-123, R-123B1 (halothane), health effects, toxicity

A. M. Jarabek (U.S. Environmental Protection Agency, EPA), J. W. Fisher (U.S. Air Force Armstrong Laboratory, USAF-AL), R. Rubenstein (EPA), J. C. Lipscomb (USAF-AL), R. J. Williams (USAF-AL), A. Vinegar (ManTech Environmental Technology, Incorporated), and J. N. McDougal (USAF), Mechanistic Insights Aid the Search for CFC Substitutes: Risk Assessment of HCFC-123 as an Example, Risk Analysis, 14(3):231-250, June 1994 (20 pages with 9 figures and 14 tables, RDB6B52)

brief review of stratospheric ozone depletion and the Montreal Protocol; discussion and table of ODP and GWP values; use of the former in setting production quotas; phaseout schedules; search for substitutes and structural similarities in candidates; summary of the Programme for Alternative Fluorocarbon Toxicity Testing (PAFT) and study sequences; risk characterization under the Significant New Alternatives Program (SNAP); use of the Workplace Guidance Level (WGL, described as essentially analogous to the Permissible Exposure Level, PEL) 
gous to the Permissible Exposure Level, PEL) and Emergency Guidance Level (EGL, described as similar in intent to the Immediately Dangerous to Life and Health concentration, IDLH) as screening tools; EGL is based on the no-observed-adverse-effect-level (NOAEL) or lowest-observed-adverse effect level (LOAEL) for cardiotoxicity in the dog; assessment by comparison of the WGL and EGL to a reference concentration (RfC) with tabular data for R-22, R-123, R-124, R-134a, R-141b, R-142b, and R-152a; review of R-123 toxicity and doseresponse analyses; risk assessment by physiologically-based, pharmacokinetic (PBPK) modeling for $R-123$ by analogy to R-123B1 (halothane) data, to decrease the need for extensive laboratory animal testing

C. A. Jenkins, HCFC 123: Acute Toxicity to Daphnia Magna, report 91/PFE006/0972 for the Program for Alternative Fluorocarbon Toxicity Testing (PAFT), Life Science Research Limited (LSR), Suffolk, UK, 1992 (RDB65C9)

$R-123$, effects on organisms in the environment, aquatic toxicity, mutagenicity

C. A. Jenkins, HCFC 123: Acute Toxicity to Rainbow Trout, report 91/PFE004/0939 for the Program for Alternative Fluorocarbon Toxicity Testing (PAFT), Life Science Research Limited (LSR), Suffolk, UK, 1992 (RDB65D0)

$R-123$, effects on organisms in the environment, aquatic toxicity, mutagenicity

C. A. Jenkins, HCFC 123 (Liquid): Biotic Degradation Closed Bottle Test, report 91/PFE008/0477 for the Program for Alternative Fluorocarbon Toxicity Testing (PAFT), Life Science Research Limited (LSR), Suffolk, UK, 1992 (RDB65D1)

$R-123$, effects on organisms in the environment, aquatic toxicity, mutagenicity

C. A. Jenkins, HCFC 123: Determination of its $E_{50}$ to Selenastrum Capricornutum, report 91/PFE007/0935 for the Program for Alternative Fluorocarbon Toxicity Testing (PAFT), Life Science Research Limited (LSR), Suffolk, UK, 1992 (RDB65D2)

$R-123$, effects on organisms in the environment, aquatic toxicity, mutagenicity

D. A. Keller, P. H. Lieder, W. J. Brock, and J. C. Cook (E. I. duPont de Nemours and Company), 1,1,1-Trifluoro-2,2-dichloroethane (HCFC-123) and 1,1,1-Trifluoro-2-bromo-2-chloroethane (Halothane) Cause Similar Biochemical Effects in Rats Exposed by Inhalation for Five Days, Drug and Chemical Toxicology, 21(4):405-415, November 1998 (11 pages, RDBA654)

summarizes comparative toxicity tests in groups of male rats by exposure to air (control) or to $5,000 \mathrm{ppm} \mathrm{v/v} \mathrm{R-123} \mathrm{or} \mathrm{R-123B1} \mathrm{(halo-}$ thane) for $6 \mathrm{hr} / \mathrm{d}$ for 5 consecutive days; indicates that rats exposed to either compound gained little or no weight during the study, their liver weights were slightly lower, serum triglycerides were decreased to approximately $20 \%$ of control level, and serum cholesterol was decreased to less than $80 \%$ of control; both compounds increased hepatic beta-oxidation by approximately a factor of three over the control animals, and R-123 caused a significant increase in hepatic cytochrome P450 content; results indicate that $R-123$ and halothane share not only common metabolic pathways, but also several common biological effects, specifically those associated with peroxisome proliferation; these data indicate that human experience with halothane may be useful in the risk assessment of $\mathrm{R}-123$

D. P. Kelly, Four-Week Inhalation Study with HCFC-123 in Rats, report 229-89 for the Program for Alternative Fluorocarbon Toxicity Testing (PAFT), Haskell Laboratory for Toxicology and Industrial Medicine, E. I. duPont de Nemours and Company, Incorporated, Newark, DE, 1989 (RDB6157)

R-123, subchronic inhalation toxicity: groups of 10 male and 10 female rats were exposed to concentrations of 0 (control), 1000,5000, and $10,000 \mathrm{ppm}$ for $6 \mathrm{hr} / \mathrm{d}, 5 \mathrm{~d} / \mathrm{wk}$ for 4 weeks; rats exposed to $5000 \mathrm{ppm}$ and higher showed exposure-related narcosis that was reversible upon cessation of exposures; decreased rates of body weight gain were seen in females at 1000 ppm and higher and in males at 10,000 and $20,000 \mathrm{ppm}$; this effect was not exposure related in females, but was in males; an exposure-related increase in relative liver weights was seen in the females, but only at 20,000 $\mathrm{ppm}$ in the males; no hematological effects were observed and the only noteworthy clinical chemistry effects were increases in SGOT and SGPT in the male rats at 20,000 ppm; a doserelated increase in cytochrome $P-450$ was seen in the female rats at $1000 \mathrm{ppm}$ and higher and in males at $10,000 \mathrm{ppm}$ and higher; urinary fluoride and volume increased with complimentary decrease in osmolarity, reported to be statistically significant only at the high concentrations; no treatment-related effects were seen 
in histopathological examination of the livers for either the male or female rats - as reported in RDB65D8 which summarizes this (identified as P-89) and three related studies

J. C. Kennelly, HCFC 123: Assessment for the Induction of Unscheduled DNA Synthesis in Rat Liver after Inhalation Exposure, report CTL/P/3807 for the Program for Alternative Fluorocarbon Toxicity Testing (PAFT), Zeneca Central Toxicology Laboratory (then part of $\mathrm{ICl}$ Chemicals and Polymers Limited), Cheshire, UK, 4 October 1993 (RDB6215)

$$
R-123 \text {, toxicity }
$$

M. A. Lee, R. C. Graham, and S. W. Snyder, untitled toxicity review for ethane, 2,2-dichloro-1,1,1trifluoro- (HCFC-123), Haskell Laboratory for Toxicology and Industrial Medicine, E. I. duPont de Nemours and Company, Incorporated, Newark, DE, 12 August 1991 (16 pages, RDB5861)

$\mathrm{R}-123$, summary of published and unpublished toxicity literature

R. W. Lewis, HCFC 123: 28-Day Inhalation Study to Assess Changes in Rat Liver and Plasma, report CTLT/2706, $1 \mathrm{Cl}$ Central Toxicology Laboratory, Cheshire, UK, 1990 (RDB6214)

$$
\text { R-123, toxicity }
$$

P. H. Lieder, J. C. Cook, and D. A. Keller (E. I. duPont de Nemours and Company), Similarities in Peroxisome Proliferation and Biochemical Effects between HCFC-123 and Halothane (HCFC-123B1) [sic HBCFC-123B1] in Rats, abstract 1552, The Toxicologist, 13(1):396 ff, 1993 (RDB6224)

toxicity of R-123; mechanistic studies based on structural similarity to R-123B1

G. D. Loizou (University of Rochester), G. Urban, W. Dekant (Universität Würzburg), and M. W. Anders (University of Rochester), Gas-Uptake Pharmacokinetics of 2,2-Dichloro-1,1,1-Trifluoroethane (HCFC-123), Drug Metabolism and Disposition, 22(4):511-517, July 1994 (7 pages with 5 figures and 3 tables, RDB6223)

in vivo rate constants for metabolism of $\mathrm{R}-123$ in rats, by physiologically-based, pharmacokinetic (PBPK) modeling with validation based on 6-hr inhalation exposures at $500-5,000 \mathrm{ppm}$ $\mathrm{V} / \mathrm{V}$; toxicity of R-123; concludes that uptake involves a single, saturable process, with no differences in rate constants between male and female rats; the model fails to simulate the reduction in R-123 uptake at 2,000-5,000 ppm; inhibition of uptake and a decrease in trifluoroacetic acid (TFA, the primary urinary metabolite of R-123) excretion by a selective, mechanismbased inhibitor (diallyl sulfide) indicate that cytochrome P450 2E1 catalyzes the biotransformation of R-123

J. M. Mackay, HCFC 123: An Evaluation in the In Vitro Cytogenetic Assay Using Human Lymphocytes, report CTL/P/2978 for the Program for Alternative Fluorocarbon Toxicity Testing (PAFT), Zeneca Central Toxicology Laboratory (then part of $\mathrm{ICl}$ Chemicals and Polymers Limited), Cheshire, UK, January 1992 (RDB6572)

R-123, mutagenicity, toxicity

G. Malinverno (Ausimont S.p.A., Italy), HCFC-123 and Reproductive Toxicity, Food and Chemical Toxicology (FCT), 35(2):305, February, 1997 (1 page, RDBA624)

summarizes a study of the reproductive toxicity of R-123 in rabbits; animals exposed to 5,000 $\mathrm{ppm} \mathrm{v} / \mathrm{v}$ in air for $6 \mathrm{hr} / \mathrm{d}$ on days 6-18 of pregnancy did not exhibit fetal malformations; mild maternal toxicity was evidenced by slight decreases in body weight gain and food consumption; also summarizes one- and two-generation rat studies; exposures to 5,000 and $1,000 \mathrm{ppm} \mathrm{v} / \mathrm{v}$, respectively, for $6 \mathrm{hr} / \mathrm{d}, 7 \mathrm{~d} / \mathrm{wk}$ did not adversely affect reproduction; the offspring in the two-generation study showed reduced body weight gain during lactation in all treatment groups, the lowest of which was 30 $\mathrm{ppm} v / \mathrm{v}$

G. Malinverno (Ausimont S.p.A., Italy), G. M. Rusch (AlliedSignal Incorporated, USA), R-J. Millischer (Elf Atochem S.A., France), E. W. Hughes (Huntingdon Research Centre, HRC, UK), R. E. Schroeder (Pharmaco-LSR, Incorporated, USA), and D. W. Coombs (HRC), Inhalation Teratology and Reproduction Studies with 1,1-Dichloro2,2,2-trifluoroethane (HCFC-123), article 0197, Fundamental and Applied Toxicology, 34(2):276287, December 1996 (12 pages with 10 figures and 2 tabies, RDB7233)

R-123, health effects, toxicity: summarizes an inhalation teratology study in rabbits; pregnant specimens were exposed to 0 (control), 500 , 1500 , and $5000 \mathrm{ppm} v / \mathrm{v}$ for $6 \mathrm{hr} / \mathrm{d}$ on days $6-18$ of gestation; reduced food consumption and slight body weight losses were observed at 500 ppm and higher in an exposure-related pattern, but there were no other signs of maternal toxicity or treatment-related effects on the kits; a one-generation reproduction study in rats is 
summarized; four groups of 24 male and female rats were exposed to concentrations of 0 (control), 300, 1000, and 5000 ppm R-123 for 6 $\mathrm{hr} / \mathrm{d}, 7 \mathrm{~d} / \mathrm{wk}$ for 4 weeks prior to mating and through weaning of the pups; no effects on mating, fertility, pub survival, or birth weight were found; a two-generation study also is summarized; five groups of 32 male and female rats were exposed to R-123 from the age of 6 weeks through weaning; groups of their offspring were exposed from weaning (4 weeks of age) through weaning of the following generation for $6 \mathrm{hr} / \mathrm{d}, 7 \mathrm{~d} / \mathrm{wk}$; exposures were at 0 (control), 30, 100, 300, and $1000 \mathrm{ppm} \mathrm{v} / \mathrm{v}$; no treatment-related effects were found on fertility or reproduction, but decreases were measured in serum triglyceride levels; pup survival and birth weight were not affected, but body-weight gain was lower in all treatment groups during nursing in an exposure-related pattern; liver weights of adult rats exposed at $100 \mathrm{ppm}$ and above were higher than in the controls; histopathologic examination found hepatic enlargement and vacuolation; the study concluded that exposure to R-123 at concentrations as high as 5000 ppm did not cause reproductive, survival, or viability effects, but did affect the body weight gain of offspring during lactation and liver weights

L. A. Malley (E. I. duPont de Nemours and Company, Incorporated), M. C. Carakostas (SmithKline Beecham Animal Health), J. F. Hansen (DuPont), G. M. Rusch (AlliedSignal Incorporated), D. P. Kelly (DuPont), and H. J. Trochimowicz (DuPont), Two-Year Inhalation Toxicity Study in Rats with Hydrochlorofluorocarbon 123, Fundamental and Applied Toxicology, 25(1):101-114, April 1995 (14 pages with 3 figures and 10 tables, RDB65D4)

$\mathrm{R}-123$, combined chronic toxicity and oncogenicity study: four groups of 80 male and 80 female rats were exposed to 0 (control), 300, 1000 , and $5,000 \mathrm{ppm} \mathrm{R-23}$ in air for $6 \mathrm{hr} / \mathrm{d}, 5$ $\mathrm{d} / \mathrm{wk}$, for up to two years (10 males and 10 females were subjected to clinical pathological examination at $6,12,18$, and 24 months); males and females exposed to $5000 \mathrm{ppm}$ and females exposed to 300 and $1000 \mathrm{ppm}$ had lower body weights and body weight gains; serum triglyceride and cholesterol were lower in both sexes at $300 \mathrm{ppm}$ except the latter was only seen in males at $5000 \mathrm{ppm}$; serum protein concentrations were altered at $300 \mathrm{ppm}$ and higher, but survival was notably higher in both sexes, and dramatically so in females, at 1000 and $5000 \mathrm{ppm}$; relative liver weight increases and absolute kidney weight decreases were observed at the high concentrations; benign hepatocellular adenomas increased in males at $5000 \mathrm{ppm}$ and in females at $300 \mathrm{ppm}$ and higher; hepatic cholangiofibromas also increased in females exposed to $5000 \mathrm{ppm}$; pancreatic acinar cell adenomas increased for all male test groups and acinar cell hyperplasia was increased in the $1000 \mathrm{ppm}$ and $5000 \mathrm{ppm}$ males and females; benign testicular interstitial adenomas and focal interstitial cell hyperplasia were higher in exposed males, and diffused retinal atrophy was increased in all male and female test groups, though found to be an indirect compound-related effect; hepatic peroxisome proliferation (beta oxidation activity) was higher at $300 \mathrm{ppm}$ and above in males and at $1000 \mathrm{ppm}$ and above in females; statistical differences in hepatic cell proliferation were not observed, and decreased incidences of agerelated lesions occurred with exposures at 1000 and 5000 ppm; a NOAEL was not achieved in this study, and recommendations are made for further mechanistic studies particularly with respect to relevance to humans

L. A. Malley, Combined Chronic Toxicity/Oncogenicity Study with HCFC-123: Two-Year Inhalation Toxicity Study in Rats, report 669-91 for the Program for Alternative Fluorocarbon Toxicity Testing (PAFT), Haskell Laboratory for Toxicology and Industrial Medicine, E. I. duPont de Nemours and Company, Incorporated, Newark, DE, 1992 (RDB6152)

$$
\text { R-123, toxicity - see RDB65D4 for summary }
$$

L. A. Malley (E. I. duPont de Nemours and Company, Incorporated), M. C. Carakostas (SmithKline Beecham Animal Health), J. F. Hansen (DuPont), H. J. Trochimowicz (DuPont), and G. M. Rusch (AlliedSignal Incorporated), Chronic Toxicity of Hydrochlorofluorocarbon HCFC-123, The Toxicologist, 11:103 ff, 1991 (RDB65F0)

\section{$\mathrm{R}-123$, health effects, toxicity}

L. A. Malley, Combined Chronic Toxicity/Oncogenicity Study with HCFC-123: Two-Year Inhalation Toxicity Study in Rats (One-Year Interim Report), report 260-90 for the Program for Alternative Fluorocarbon Toxicity Testing (PAFT), Haskell Laboratory for Toxicology and Industrial Medicine, E. I. duPont de Nemours and Company, Incorporated, Newark, DE, 1990 (RDB5B62)

R-123, toxicity - see RDB65D4 for summary 
L. A. Malley, Subchronic Inhalation Toxicity Study: 90-Day Study with HCFC-123 in Rats, report 594-89 for the Program for Alternative Fluorocarbon Toxicity Testing (PAFT), Haskell Laboratory for Toxicology and Industrial Medicine, E. I. duPont de Nemours and Company, Incorporated, Newark, DE, January 1990 (RDB6154)

R-123, subchronic inhalation toxicity: groups of 10 male and 10 female rats were exposed to concentrations of 0 (control), 300,1000, and $5000 \mathrm{ppm}$ for $6 \mathrm{hr} / \mathrm{d}, 5 \mathrm{~d} / \mathrm{wk}$ for 13 weeks; rats exposed to $5000 \mathrm{ppm}$ showed what might have been slight narcosis that disappeared upon cessation of exposures; no changes were seen in body weights compared to controls; serum triglyceride levels were lower in both males and females at $300 \mathrm{ppm}$ and higher; serum cholesterol levels were significantly lower in females exposed at 1000 and $5000 \mathrm{ppm}$; the mean lymphocyte count for females in the 5000 ppm group decreased; exposure related increases in urinary fluoride was seen in males exposed to $5000 \mathrm{ppm}$ and in females at $300 \mathrm{ppm}$ and higher, but these increases were described as minimal; absolute liver weights for males were significantly higher in the $5000 \mathrm{ppm}$ group and relative liver weights were higher in males and females at $1000 \mathrm{ppm}$ and higher, no morphological changes were found in tissues with microscopic examination, but hepatic peroxisomal activity increased in treated males and females; this increase was nearly double in high-dose males and females, suggesting a conclusion of very mild peroxisome proliferation - as reported in RDB65D8 which summarizes this (identified as P-90) and three related studies

G. B. Marit (U.S. Air Force Armstrong Laboratory, USAF-AL), D. E. Dodd (ManTech Environmental Technology, Incorporated), M. E. George (USAF$A L)$, and $A$. Vinegar (ManTech), Hepatotoxicity in Guinea Pigs Following Acute Exposure to 1,1Dichloro-2,2,2-Trifluoroethane, Toxicologic $\mathrm{Pa}$ thology, 22(4):404-414, July-August 1994 (11 pages with 5 figures and 4 tables, RDB6216)

acute inhalation toxicity tests of R-123 (2,2dichloro-1,1,1-trifluoroethane) for concentrations of $0,1,000,10,000,20,000$, and 30,000 $\mathrm{ppm} \mathrm{v/v}$ and of $10,000 \mathrm{ppm} \mathrm{v} / \mathrm{v}$ R-123B1 (halothane) in groups of 10 male Hartley guinea pigs for $4 \mathrm{hr}$. gross and histopathologic examination of the liver, heart, and kidney as well as routine hematology and clinical chemistry analyses were performed after sacrifice of the animals $48 \mathrm{hr}$ after the exposures; lesions were observed in the livers of $90-100 \%$ of the ex- posed animals, but were absent in the controls; the lesions included centrolobular vacuolar (fatty) change, multifocal random degeneration and necrosis, and centrolobular degeneration and necrosis; significant variation in susceptibility was observed, but there was similarity in the response between $\mathrm{R}-123$ and halothane; the paper concludes that humans susceptible to halothane-induced hepatitis may be susceptible to R-123 by a common mechanism; the paper also notes that all guinea pigs exposed to $10,000 \mathrm{ppm}$ v/v R-123B1 or $20,000-30,000$ $\mathrm{ppm} v / \mathrm{v}$ were rendered unconscious within $10-$ 15 minutes of exposure and that some animals exposed to lower concentrations were uncoordinated or lethargic

R. R. Marshall, Evaluation of Chromosomal Aberrations Frequencies in Cultured Peripheral Blood Lymphocytes from Rats Treated with HCFC-123, report ALS/4-R (also identified as ASU 1/RLC or 1RLRE ASU.001) for the Program for Alternative 'Fluorocarbon Toxicity Testing (PAFT), Hazleton Microtest, Harrogate, North Yorkshire, UK, 1992 (RDB6221)

$$
\mathrm{R}-123 \text {, health effects, toxicity }
$$

J. L. Martin, J. W. Harris, M. W. Anders, and L. R. Pohl (Johns Hopkins Medical Institute), Trifluoroacetylated Proteins in Livers of Rats Treated with the Potential Chlorofluorocarbon Replacement 1,1,1-Trifluoro-2,2-dichloroethane (HCFC123), abstract $A 348$, Anesthesiology, $73(3 A)$, September 1990 (1 page with no figures or tables, RDB7232)

inhalation toxicity tests for metabolism of $R-123$ (2,2-dichloro-1,1,1-trifluoroethane) in groups of male, Fischer 344 rats exposed for $2 \mathrm{hr}$ to 0 (control), 7,000 , or $11,000 \mathrm{ppm} \mathrm{v/v}$ and, for comparison, another group to $13,000 \mathrm{ppm} \mathrm{v} / \mathrm{v}$ R-123B1: both compounds were found to produce identical patterns of trifluoroacetyl (TFA) labeled proteins; concludes that further metabolic and toxicologic studies of R-123 are needed based on the possibility (not established) of sensitization and increased risk for hepatic damage from repeated exposures or following anesthesia with enflurane or halothane (R123B1) [see RDB6213, RDB6223, RDB65C2, RDB6A99, RDB65C7, RDB65E1, RDB65E2, and others for further investigations of this topic]

Meridian Research, Incorporated, Results of Employee Exposure Monitoring for HCFC-123 at Centrifugal Chiller Installations, U.S. Environ- 
mental Protection Agency (EPA), Washington, DC, 26 November 1991 (82 pages, RDB2529)

$\mathrm{R}-123$, toxicity, machine room concentrations, occupational exposures

MPI Research, HCFC-123: Inhalation Study in Pregnant Monkeys to Assess Milk Transfer and Composition Following Postpartum Exposures, report HLO-197-00152 (also identified as MPI study 125-042), Haskell Laboratory for Toxicology and Industrial Medicine, E. I. duPont de Nemours and Company, Incorporated, Newark, DE, 1997 (RDB7611)

mechanistic toxicity tests in Rhesus monkeys to investigate the transfer of $R-123$ and its major metabolite, trifluoroacetic acid (TFA), in milk from exposed mothers to nursing infants: groups of 4 mothers and infants were exposed to 0 (control) or $1,000 \mathrm{ppm} \mathrm{v} / \mathrm{v}$ in air by inhalation for $6 \mathrm{hr} / \mathrm{d}, 7 \mathrm{~d} / \mathrm{wk}$ for $3 \mathrm{wk}$ beginning 6-8 days following birth; no changes were observed in maternal weight gain, clinical signs deemed adverse, or blood chemistry (including triglyceride, cholesterol, and glucose levels); no effect was found on milk production or milk fat and protein content, but both R-123 and TFA were detected in the milk and TFA was found in maternal blood; slight, microscopic evidence of liver toxicity (focal cytotoxicity) was found in the mothers at study termination, but was deemed to be reversible; no increases were found in $B$ oxidation activity; infants showed neither adverse clinical signs nor body weight changes compared to control animals; TFA measurements in the blood of infants were three times higher than in the mothers; study concluded that there was no adverse consequence to the infant monkeys because of lactation exposure to either R-123 or its metabolite - as reported in RDB8611 [WEEL documentation notes that the absence of changes in infant weight gains with monkeys is significant; this finding supports the hypothesis that decreases in rat pup weight gains, previously observed in other studies, is due to peroxisome proliferation, to which primates do not respond]

L. S. Mullin, Fluorocarbon 123 (2,2-Dichloro1,1,1-trifluoroethane): Behavioral Toxicity Testing, report 941-76, Haskell Laboratory for Toxicology and Industrial Medicine, E. I. duPont de Nemours and Company, Incorporated, Newark, DE, 10 December 1976 (4 pages with 2 tables, RDB6153)

toxicity tests to determine the concentrations at which behavioral effects occur for whole-body exposures of groups of 6 male Charles River $C D$ rats to 0 (control), $1,000,2,500,5,000$, or $10,000(0,975,2,697,5,237$, and 9,950 actual) $\mathrm{ppm}$ v/v R-123 in air for $1 \mathrm{hr}$ : effects were measured on a pass-fail basis for a suite of tests to evaluate unconditioned reflexes, simple behavioral patterns, locomotor activity, and observation of unusual signs or symptoms; effects were measured before exposures, at 15, 30, and $60 \mathrm{~min}$ during exposures, and at $15 \mathrm{~min}$, $60 \mathrm{~min}$, and $24 \mathrm{hr}$ after exposures; $\mathrm{EC}_{50}$ values were calculated by probit analysis; results for the most sensitive tests - grip, lift, vertical bar, ataxia (inability to coordinate voluntary muscular movements), and pinna tactile (response to touching of an ear) were $>10,000 \mathrm{ppm}$ before exposures, 7,080-9,710 ppm at $15 \mathrm{~min}, 5,790$ 8,660 at $30 \mathrm{~min}, 3,930-8,830$ at $60 \mathrm{~min}$, and $>10,000$ after exposures; report notes that ataxia and catatonia (decrease in spontaneous activity) were evident $15 \mathrm{~min}$ into exposures and loss of exploratory activity was evident at $30 \mathrm{~min}$ for exposures at 9,950 ppm; mean body temperature of rats exposed to 5,237 and 9,950 $\mathrm{ppm}$ were lower than controls (by approximately $1^{\circ} \mathrm{C}, 1.8^{\circ} \mathrm{F}$, at $9,950 \mathrm{ppm}$ at $60 \mathrm{~min}$ of exposure); study concluded that behavioral test failures were produced consistently at and above 5,237 ppm during exposure, that occasional failures were seen $15 \mathrm{~min}$ after exposure at 9,950 ppm, and that recovery was rapid and complete in all cases

W. Müller and T. Hofmann (Pharma Research Toxicology and Pathology Laboratory), HCFC 123 - Micronucleus Test in Male and Female NMRI Mice After Inhalation, report 88.1340 (on study 88.0372) for the Program for Alternative Fluorocarbon Toxicity Testing (PAFT), Hoechst Aktiengesellschaft, Frankfurt am Main, Germany, 1988 (RDB6141)

\section{$\mathrm{R}-123$, mutagenicity, toxicity}

J. D. Obourn (E. l. duPont de Nemours and Company, Incorporated), S. R. Frame (DuPont), R. H. Bell, Jr. (University of Washington School of Medicine, Seattle Veterans Affairs Medical Center), D. S. Longnecker (Dartmouth Medical School), G. S. Elliott (DuPont), J. C. Cook (DuPont), Mechanisms for the Pancreatic Oncogenic Effects of the Peroxisome Proliferator Wyeth-14,643, Toxicology and Applied Pharmacology (TAP), 145(2):425-436, August 1997 (12 pages, RDBA649)

toxicity of R-123; examines the role of peroxisome proliferation in producing pancreatic aci- 
nar cell hyperplasia and adenocarcinomas in rats as detected in 2-yr bioassays; summarizes in vitro and in vivo approaches used to investigate mechanisms of pancreatic tumorigenesis by peroxisome proliferating compounds

T. L. Pravecek (U.S. Air Force, USAF) and S. R. Channel (Tri-Service Toxicology Consortium), A Volatile Exposure Method for Precision Cut Tissue Slices: HCFC-123 Study, In Vitro Toxicology, 8(3):283-289, 1995 (7 pages with 5 figures and 1 table, RDB6A99)

R-123 toxicity: application of precision-cut tissue slice cultures to study the metabolic fate and toxicologic effects of chemicals; data describe a saturable metabolic pathway for $R-123$ in Srague-Dawley rats; analyses of R-123 metabolism in rat livers suggest significant strain differences and that R-123 may inhibit its own metabolism at higher concentrations

G. M. Rusch (AlliedSignal Incorporated), H. J. Trochimowicz (E. I. duPont de Nemours and Company, Incorporated), L. A. Malley (DuPont), D. P. Kelly (DuPont), J. Peckham (Experimental Pathology Laboratory), J. Hansen (DuPont), and J. B. Charm (AlliedSignal), Subchronic Inhalation Toxicity Studies with Hydrochlorofluorocarbon 123 (HCFC 123), Fundamental and Applied Toxicology, 23(2):169-178, August 1994 (10 pages with 11 tables, RDB65D8)

summarizes four subchronic inhalation toxicity studies in rats and dogs for $R-123$ : increases in liver weight were seen at concentrations of $1000 \mathrm{ppm}$ and higher; one study showed this effect at $500 \mathrm{ppm}$; histopathological findings are described as minimal and include focal necrosis (death of a portion of tissue) in the liver of dogs, induction of peroxisomal activity, lowering of serum cholesterol and triglyceride levels, and an increase in urinary fluoride levels; paper observes that while the 4-hr $L^{-C_{50}}$ rat is $35,000 \mathrm{ppm}$, repeated exposures at 20,000 ppm (almost the same daily dose for the product of concentration and time) for $6 \mathrm{hr} / \mathrm{d}$ in a 4week study yielded no mortality or marked signs of toxicity; concludes that R-123 "appears to have a low level of toxicity by the inhalation route" and that there is "no evidence for cumulative toxicity from multiple exposures in these studies"

G. M. Rusch, A Ninety-Day Inhalation Toxicology Study and an Inhalation Teratology Study of Genetron 123 in the Rat, report MA-127-80-1 (also identified as MA-RR-85-993), AlliedSignal
Incorporated (then Allied Corporation), Morristown, NJ, 19 September 1985 (>61 pages with $\cdot 12$ tables, Rdb6142) [includes RDB6143 and RDB6144 as appendices $A$ and $C$ ]

examination and summary of the findings of two R-123 toxicity studies (IBT reports 856209344 dated 2 September 1977 and 8 November 1977, see RDB6143 and RDB6144) conducted by Industrial Bio-Test Laboratories, Incorporated: outlines the earlier findings, describes a review of the raw data, and compares earlier interpretations with those from reexamination of the original tissue specimen slides by a pathologist; report concludes that $500(487$ $\mathrm{ppm}$ actual) $\mathrm{ppm} \mathrm{v} / \mathrm{v}$ is a no-effect level (NOEL) for rat exposures to R-123 by inhalation for 6 $\mathrm{hr} / \mathrm{d}, 5 \mathrm{~d} / \mathrm{wk}$, for 3 months while 1,000 (1,009 actual) $\mathrm{ppm}$ represents a minimal biological effect level; notes that hepatic alterations identified in the earlier reports did not follow an exposure-related pattern by either severity or frequency and that they probably were not related to the exposures; also concludes that primary effect was depression of body weight gain in the female rats; addressing the teratology study, the report concludes that exposure of pregnant rats for $6 \mathrm{hr} / \mathrm{d}$ for days $6-15$ of gestation to a concentration of $5,000(4,495-6,065$ actual) $\mathrm{ppm} \mathrm{v} / \mathrm{v}$ produced a response of depressed weight gain in the dams, but did not result in a teratogenic response [prepared from partial report with missing pages]

J. Sandow, W. von Rechenberg, and G. JerabekSandow (Hoechst AG Pharma Research), HCFC123: Effect of HCFC-123 and Androgen Biosynthesis and Gonadotropin Secretion in Rats, report MA-RR-95-2196 (also identified as Hoechst report 1/94 and as "Hoechst study Exp. 8-210 as part of Huntingdon Toxicology Study ALS $/ 5^{n}$ ) for the Program for Alternative Fluorocarbon Toxicity Testing (PAFT), AlliedSignal Incorporated, Morristown, NJ, 30 April 1995 (34 pages with 4 figures and 2 tables, RDB65D9)

toxicity tests to investigate the mechanism of testicular changes (Leydig cell hyperplasia) found in adult male Wistar rats after long-term (22-week) exposures to R-123: tests examined the serum luteinising hormone (LH) response, testosterone secretion, and testosterone biosynthesis ex vivo to develop understanding of the endocrine mechanism, which if deemed to be reversible in rodents would be of no concern for human safety; report concludes that there was no qualitative change in androgen biosynthesis and "the small decrease in testosterone 
secretion observed at $1000 \mathrm{ppm}$ was not of concern for further compound development because Leydig cell hyperplasia is a rodentspecific finding in rats and mice rarely observed in humans;" report also notes that $R-123$ poses "a low risk for human exposure in industrial application"

R. Schroeder (Bio/dynamics Incorporated), An Inhalation Developmental Toxicity Study in Rabbits with HCFC-123, report 88-3304 for the Program for Alternative Fluorocarbon Toxicity Testing (PAFT), Haskell Laboratory for Toxicology and Industrial Medicine, E. I. duPont de Nemours and Company, Incorporated, Newark, DE, September 1989 (RDB6162)

study of the potential for $R-123$ to produce developmental and reproductive toxicity in New Zealand rabbits by exposure of pregnant dams to 0 (control), $500,1,500$, or $5,000 \mathrm{ppm}$ on gestation days 6-18; maternal toxicity was evident at all concentrations with decreased food consumption and bodyweight gain - as reported in RDB6B52

R. Schroeder (Bio/dynamics Incorporated), An Inhalation Range-Finding Study to Evaluate the Toxicity of HCFC-123 in the Pregnant Rabbit, report 88-3303, Haskell Laboratory for Toxicology and Industrial Medicine, E. I. duPont de Nemours and Company, Incorporated, Newark, DE, 1989 (RDB6163)

study of the potential for R-123 to produce developmental and reproductive toxicity in New Zealand rabbits by exposure of pregnant dams

H. W. Sibley, A Study for Determining Refrigerant Exposure Levels While Servicing an HCFC123 Centrifugal Chiller, publication 819-061, Carrier Corporation, Syracuse, NY, April 1992 (8 pages with 4 figures and 1 table, RDB2916)

This report summarizes an investigation to determine the exposure levels encountered while working around or on chillers using R-123. It also addresses whether there are reliable instruments to measure that low, and whether a machine room can be adequately monitored and ventilation controlled to stay below a recommended exposure limit of $10 \mathrm{ppm}$ [subsequently raised by some refrigerant manufacturers]. The single test involved a $700 \mathrm{~kW}(200$ ton) hermetic centrifugal chiller at the manufacturer's plant. The report outlines steps to select and qualify refrigerant sensors, develop a decision model for the test, prepare a team for use of the refrigerant monitors and safety equip- ment, develop service practices, and execute the plan. It was based on removal of R-123 from the machine and recharge with $R-11$. Additional steps were scheduled between charge removal and recharging, to determine the effects on components exposed to R-123 in a year of operation. For purposes of this test, the exhaust fans in the machinery room were reset from $10 \mathrm{ppm}$ to operation when concentrations exceeded $500 \mathrm{ppm}$. A table lists the R-123 monitors used, and a figure outlines the safety decision model developed. A figures shows measured exposure concentrations, with two excursions to approximately $500 \mathrm{ppm}$, and time-weighted average (TWA) exposures of 2-5 $\mathrm{ppm}$. The report notes that once R-123 was released, "it tended to drop to floor level, hang together, and move as an invisible cloud." One part of the plan included a deliberate spill of 1 litre ( $1 / 4$ gallon), deemed the maximum that would be lost during a typical accident. A plot shows the resulting concentrations. The report concludes that new methods and procedures will have to be employed to minimize exposures. A brief addendum to the report notes concentrations in field restoration of three other chillers from R-123 back to R-11 use.

E. Sullivan, Refrigerant Safety, Worker Health, and HCFC-123, Building Operating Management, 46-48, August 1997 (3 pages with no figures or tables, RDB7B47)

reviews new occupational exposure limits recommended by the American Industrial Hygiene Association (AlHA) and DuPont Chemicals for $\mathrm{R}-123$ in layman's terms; a sidebar summarizes the mechanistic tests that led to the AlHA Workplace Environmental Exposure Limit (WEEL) and DuPont Acceptable Exposure Limit (AEL) of $50 \mathrm{ppm} \mathrm{v} / \mathrm{v}$ on a time-weightedaverage basis; quotes a number of toxicologists and manufacturers representatives on the safety implications and recommended procedures for use of refrigerants and R-123 in particular, contrasts the new limits to concentrations normally encountered; compares the safety of R-123 to R-11 which it replaces; concludes that all refrigerants can be used safely if recommended procedures are followed

T. Takebayashi (Keio University School of Medicine, Japan), I. Kabe, Y. Endo, S. Tanaka (Kitasato University, Japan), H. Miyauchi, K. Nozi, S. Imamiya, K. Takahashi, and K. Omae, Exposure to 2,2-Dichloro-1,1,1-trifluoroethane (HCFC123) and Acute Liver Dysfunction: A Causal 
Inference, Journal of Occupational Health, Japanese Society of Occupational Health, Tokyo, Japan, 40(4):334-338, October 1998 (2 pages, RDBA927)

describes an investigation of reversible liver toxicity from chronic occupational exposures by inhalation in an industrial setting; reports acute liver dysfunction among workers repeatedly exposed to R-123; estimates levels of exposure by reproducing working conditions and by a job record survey; describes health surveillance, including liver function and subjective symptoms, initiated when two workers first complained of ill health; indicates that the mean R-123 concentration in air exceeded $200 \mathrm{ppm}$ v/v with a peak of approximately $1,000 \mathrm{ppm} \mathrm{v} / \mathrm{v}$; notes that concentrations of 18-24 ppm v/v were detected in the adjacent areas; describes classification of workers into groups according to their estimated exposure levels, confirmed by determination of urinary trifluoroacetic acid (TFA, a metabolite of R-123), to examine causality; notes that some workers also reported symptoms involving the central nervous system, digestive organs, and mucous membranes; indicates that the degree and prevalence of these health effects were higher in the high-exposure group suggesting an exposure-effect and exposure-response relationship; discusses the consistency and temporality of observed health effects in relation to R-123 exposure levels; concludes that repeated exposure to high concentrations of R-123 for no more than five weeks causes acute liver dysfunction in humans that can be severe and manifest itself in various symptoms; recommends that biological plausibility should be clarified to confirm the causality; case report of hepatotoxicity

T. Takebayashi (Keio University School of Medicine, Japan) I. Kabe, Y. Endo, S. Tanaka (Kitasato University, Japan), H. Miyauchi, K. Nozi, K. Takahashi, and K. Omae, Acute Liver Dysfunction among Workers Exposed to 2,2-Dichloro-1,1,1trifluoroethane (HCFC-123): A Case Report, Journal of Occupational Health, Japanese Society of Occupational Health, Tokyo, Japan, 40(3):169170, July 1998 (2 pages, RDBA928)

case report of reversible liver toxicity from chronic occupational exposures by inhalation of R-123 in an industrial setting; hepatotoxicity

S. Tanaka (Kitasato University, Japan), I. Kabe, T. Takebayashi (Keio University School of Medicine, Japan), Y. Endo, H. Miyauchi, K. Nozi, K. Takahashi, Y. Seki, and K. Omae, Environmental and
Biological Monitoring of 2,2-Dichloro-1,1,1trifluoroethane (HCFC-123), Journal of Occupational Health, Japanese Society of Occupational Health, Tokyo, Japan, 40(4):348-349, October 1998 (2 pages, RDBA929)

case report of reversible liver toxicity from chronic occupational exposures by inhalation of $\mathrm{R}-123$ in an industrial setting; investigation of exposure levels; hepatotoxicity

H. J. Trochimowicz (E. I. duPont de Nemours and Company, Incorporated), The Toxicology of HCFC-123 (2,2-Dichloro-1,1,1-Trifluoroethane), Proceedings of the European Meeting of the Toxicology Forum (Toulouse, France, 18-22 September 1989), Société Française de Toxicologie, France, 182-190, 1989; republished in the Journal of the American College of Toxicology (JACT), 8(6):1220 ff, 1989 (9 pages comprising an abstract and presentation outline, RDB6145)

R-123, toxicity: ALD oral rat $=9,000 \mathrm{mg} / \mathrm{kg}$; $\mathrm{LD}_{50}$ rat $=2,000 \mathrm{mg} / \mathrm{kg} ; 4-\mathrm{hr} L C_{50}$ rat $=32,000$ ppm; cardiac sensitization $\mathrm{EC}_{50}$ dog $=19,000$ $\mathrm{ppm}$; dose-related, anesthetic effects observed in rats at $5,000 \mathrm{ppm}$ during 15 -minute inhalation exposures in a glove box, NOEL at 2,500 ppm; not mutagenic, embryotoxic, fetotoxic, or teratogenic; low in toxicity on a subchronic basis; chronic inhalation toxicity study underway

H. J. Trochimowicz and L. S. Mullin, Cardiac Sensitization Potential $\left(E_{50}\right)$ of Trifluorodichloroethane, report 132-73 (FYI-OTS-0889-0695), Haskell Laboratory for Toxicology and Industrial Medicine, E. I. duPont de Nemours and Company, Incorporated, Newark, DE, 30 March 1973 (8 pages with 1 table, RDB6146)

summarizes tests of the cardiac sensitization potential of $R-123$ in healthy, male, beagle dogs with control injection of adrenalin before exposure and a challenge injection after inhalation for five minutes; target concentrations of $5,000,10,000,20,000$ and $80,000 \mathrm{ppm} \mathrm{v/v}$ bracketed the estimated median effective concentration $\left(E C_{50}\right)$ of $40,000 \mathrm{ppm} v / \mathrm{v}$ using a "staircase method," by which tests at 80,000 $\mathrm{ppm} \mathrm{v} / \mathrm{v}$ were eliminated based on results from the lower concentrations; actual concentrations were determined using by gas chromatography (GC) by samples taken at $75 \mathrm{sec}$ intervals; 7 of the 12 dogs tested exhibited marked responses, 6 of which resulted in ventricular fibrillation (very rapid and irregular contractions of the ventricles of the heart resulting in loss of synchronization between the heartbeat and pulse beat); and death; notes that obvious cen- 
tral nervous system depression (decreased motor reflexes and lassitude) as well as tachycardia were observed prior to adrenalin injection in the 6 dogs that died; reports marked responses in 0 of 3 dogs tested at 10,300 ppm $\mathrm{v} / \mathrm{v}, 4$ of $6=20,900 \mathrm{ppm} \mathrm{v} / \mathrm{v}$, and 3 of 3 at $40,600 \mathrm{ppm} v / \mathrm{v}$; reports NOEL $0 / 3$ as 10,300 $\mathrm{ppm} \mathrm{v} / \mathrm{v}$, LOEL $4 / 6$ as $20,900 \mathrm{ppm} \mathrm{v/v}$, and a calculated $\mathrm{EC}_{50}$ as $19,000 \mathrm{ppm}$; notes an estimated cardiac sensitization $E_{50}$ for $R-11$ of $12,400 \mathrm{ppm} v / \mathrm{v}$; toxicity data

G. Urban and W. Dekant (Universität Würzburg), Metabolism of 1,1-Dichloro-2,2,2trifluoroethane in Rats, Xenobiotica, 24(9):881892, September 1994 (12 pages, RDB8404)

toxicity of R-123: metabolism tests with two male rats individually exposed to radiolabeled $\mathrm{R}-123$ in air for $6 \mathrm{hr}$; $14 \%$ was recovered in urine within $48 \mathrm{hr}$; trifluoroacetic acid (TFA) was the major metabolite and $\mathrm{N}$-trifluoroacetyl-2aminoethanol and $\mathrm{N}$-acetyl-S-(2,2-dichloro-1,1difluoroethyl)-L-cysteine were identified as minor urinary metabolites; urinary excretion of these metabolites was very slow; examination of the sacrificed animals showed covalent binding of the metabolites to protein to be highest in the liver followed by the kidneys and lungs; no covalent binding above background levels was observed in the pancreas or testis, noted as the target organs of R-123 tumorigenicity; results suggest that the biotransformation of R-123 in rodents follows a pathway identical to that of R-123B1 (halothane), an extensively studied structural analog

G. Urban, P. Speerschneider, and W. Dekant (Universität Würzburg), Metabolism of the Chlorofluorocarbon Substitute 1,1-Dichloro-2,2,2trifluoroethane by Rat and Human Liver Microsomes: The Role of Cytochrome P450 2E1, Chemical Research in Toxicology, 7(2):170-176, March 1994 ( 7 pages with 8 figures and 3 tables, RDB65E1)

toxicity of R-123 (2,2-dichloro-1,1,1-trifluoroethane; shows that trifluoroacetic acid (TFA) is a major metabolite of R-123; also indicates that chlorodifluoroacetic acid and inorganic fluoride also are products of the enzymatic oxidation of $\mathrm{R}-123$ in rat and human liver microsomes; concludes that cytochrome $\mathrm{P} 450 \mathrm{2E} 1$ plays a major role in metabolism of R-123 and its analog $R-123 B 1$ (halothane) in vitro

G. Urban and W. Dekant (Universität Würzburg), The CFC Substitute 2,2-Dichloro-1,1,1-
Trifluoroethane is Primarily Oxidized by Cytochrome P450 2E1, abstract 1704, The Toxicologist, 13:431, 1993 (1 page, RDB65E4)

$$
\mathrm{R}-123 \text {, toxicity }
$$

A. Vinegar, D. E. Dodd, D. L. Pollard (ManTech Environmental Technology, Incorporated), R. J. Williams, and J. N. McDougal (Armstrong Laboratory, U.S. Air Force), Pharmacokinetics of HCFC-123 in Dogs, report AL/OE-TR-1995-0025, Armstrong Laboratory, U.S. Air Force (USAF), Wright-Patterson Air Force Base, OH, 1995 (104 pages with 8 figures and 20 tables, available from JMC as RDB6573)

\section{R-123, biochemistry, health effects, toxicity}

A. Vinegar, R. J. Williams, J. W. Fisher (ManTech Environmental Technology, Incorporated), and J. N. McDougal (Armstrong Laboratory, U.S. Air Force), Dose-Dependent Metabolism of 2,2Dichloro-1,1,1-trifluoroethane: A Physiologically Based Pharmacokinetic Model in the Male Fischer 344 Rat, Toxicology and Applied Pharmacology (TAP), 129(1):103-113, November 1994 (11 pages, RDB65E2)

$$
\mathrm{R}-123 \text {, toxicity }
$$

D. B. Warheit, Mechanistic Studies with HCFC123, report 828-92, Haskell Laboratory for Toxicology and Industrial Medicine, E. I. duPont de $\mathrm{Ne}$ mours and Company, Incorporated, Newark, DE, 1993 (RDB6159)

$$
\mathrm{R}-123 \text {, toxicity }
$$

R. S. Waritz and J. W. Clayton, Acute Inhalation Toxicity on 1,1-Dichloro-2,2,2-trifluoroethane (FC-123, Commercial Grade), report 16-66, Haskell Laboratory for Toxicology and Industrial Medicine, E. I. duPont de Nemours and Company, Incorporated, Newark, DE, 4 February 1966 with correction dated 12 May 1966 (two pages with no figures or tables, RDB6150)

acute inhalation toxicity tests of R-123 (2,2dichloro-1,1,1-trifluoroethane) in six male Charles River CD rats by $4 \mathrm{hr}$, whole body exposures; actual concentrations were determined by gas chromatography; notes that rats showed immediate unresponsiveness followed by loss of balance, unconsciousness, hyperemia, irregular breathing, and lacrimation before death, but that surviving rats regained consciousness within $5 \mathrm{~min}$ of the exposures; also notes slight weight loss on the day following exposures, but normal growth and behavior thereafter; also notes that rats exposed to sublethal concentrations of $16,480-18,900 \mathrm{ppm} v / \mathrm{v}$ 
showed the same clinical signs seen at lethal levels, but coordination returned within $10 \mathrm{~min}$ following exposures; gross examinations at necropsy revealed no treatment-related effects; microscopic examination showed marked congestion in the lungs, kidney, and liver as well as edema in the lungs of the rats that died during an exposure suggesting respiratory irritation at high concentrations; none of the survivors had microscopically observable tissue effects that were attributed to the exposures; indicates a 4 $\mathrm{hr} L \mathrm{C}_{50}$ rat of $35,000 \mathrm{ppm}$; warns that the $L C_{50}$ is less than the saturated vapor concentration and that anesthetic effects make self rescue doubtful at high concentrations

R. J. Williams (U.S. Air Force Armstrong Laboratory, USAF-AL), A. Vinegar (ManTech Environmental Technology, Incorporated), J. N. McDougal (USAF), A. M. Jarabek (U.S. Environmental Protection Agency, EPA), and J. W. Fisher (USAF$A L)$, Rat to Human Extrapolation of HCFC-123 Kinetics Deduced from Halothane Kinetics: A Corollary Approach to Physiologically Based Pharmacokinetic Modeling, Fundamental and Applied Toxicology, 30(1):55-66, March 1996 (12 pages, RDB7C68)

development and of a human, physiologically based pharmacokinetic (PBPK) model for R123 and its major metabolite, trifluoroacetic acid (TFA): corollary approach was developed in the absence of available human kinetic data for R-123, noted as a structural analog of the common anesthetic agent R-123B1 (halothane); both follow a common pathway of oxidative biotransformation, resulting in formation of TFA; models were developed and validated for halothane for rats and humans; a corollary approach then was used to develop the human R-123 model; this strategy was implemented by using a previously validated PBPK model for $\mathrm{R}$ 123 and TFA in the Fisher 344 rat as a template; metabolic constants for halothane in the rat were used in the human model, and specific parameters describing the kinetics of TFA were estimated by optimization; the model was validated against published human-exposure data for halothane; a similar approach was then used to derive the R-123 model; use of this PBPK model is proposed for deriving doseresponse estimates of health risks in the absence of human kinetic data

Crossover Study with HCFC-123 in Lactating Sprague-Dawley Rats Including Additional Studies on Milk Production and Metabolites in
Offspring Urine, report $95 / 9$ for the Program for Alternative Fluorocarbon Toxicity Testing (PAFT), Fraunhofer Institute of Toxicology and Aerosol Research, Hannover, Germany, 1996 (RDB7610)

tests of R-123 developmental and reproductive toxicity by exposure of pregnant and/or lactating rats to 0 (control) or $1,000 \mathrm{ppm} v / \mathrm{v}$ for 6 $\mathrm{hr} / \mathrm{d}$ on days 6-19 of gestation and 5-21 post partum, to evaluate the effects on offspring with cross fostering: control pups (from mothers in the control group) nursed by treated mothers showed reduction in body weight gain during the nursing period; pups from treated mothers nursed by control mothers did not; no effect was found on milk production or the nutritional composition of the milk from exposed mothers, but the milk contained the major metabolite of $\mathrm{R}-123$, trifluoroacetic acid (TFA); report concludes that the adverse effects occurred by transfer of the TFA in maternal milk, and were not attributable to developmental toxicity - as reported in RDB8611

1,1-Dichloro-2,2,2-trifluoromethane (HCFC-123, CAS No. 306-83-2), Joint Assessment of Commodity Chemicals (JACC) report 33, European Chemical Industry Ecotoxicology and Toxicology Centre (ECETOC), Brussels, Belgium, February 1996 (68 pages with 2 figures and 11 tables, RDB65E8)

R-123, 2,2-dichloro-1,1,1-trifluoroethane, environmental impacts, toxicity

1,1-Dichloro-2,2,2-trifluoroethane (HCFC 123) Health Effects Information, CAS\# 306-83-2, Elf Atochem North America, Incorporated, Philadelphia, PA, undated circa 1995 (5 pages, RDB5B53)

$\mathrm{R}$-123, 2,2-dichloro-1,1,1-trifluoroethane, summary of toxicological and ecological information

2,2-Dichlor-1,1,1-trifluorethan Toxikologischarbeitsmedizinische Begrundung von MAKWerten (Maximale Arbeitsplatz Konzentrationen) [Toxicological and Occupational Medical Basis for the 2,2-Dichloro-1,1,1-trifluoroethane MAK Values (Maximum Workplace Concentrations), Deutsche Forschungsgemeinschaft [German Research Association] (DFG), Bonn, Germany, 1994 (18 pages, RDB6AA0)

$\mathrm{R}-123$, documentation for listing as a III $\mathrm{B}$ compound [substances suspected of having carcinogenic potential and subject to reevaluation, for assignment to category III A (unequivocally carcinogenic substances) or dismissal from the category] in the German occupational exposure limits for chemicals 
2,2-Dichloro-1,1,1-trifluoroethane (HCFC-123) - Priority Existing Chemical 4, full public report, National Industrial Chemicals Notification and Assessment Scheme (NICNAS), Worksafe Australia, Sidney Australia, March 1996 (134 pages, available from the Australian Government Publishing Service, RDBA403)

toxicity and environmental risk assessment of $\mathrm{R}-123$

2,2-Dichloro-1,1,1-trifluoroethane (HCFC-123) - Priority Existing Chemical 4 Secondary Notification Assessment, full public report, National Industrial Chemicals Notification and Assessment Scheme (NICNAS), Worksafe Australia, Sidney Australia, July 1999 (142 pages, RDBA404)

toxicity and environmental risk assessment of R-123

Report on Testing and Analysis of the Concentration of HCFC-123 in Field Installations with General Machinery Rooms Containing Hermetic Centrifugal Chillers, report CFC-1, The Trane Company, La Crosse, WI, October 1991 (34 pages with 14 figures and 15 tables, limited copies available from JMC as RDB2246)

This report provides measured data on R-123 concentrations in equipment rooms housing hermetic centrifugal chillers. Twelve sites, representing a broad range of applications, were tested during normal operation. Additional data were taken at two sites during refrigerant transfers. Measurements were made by gas chromatography, both on site and using activated charcoal tubes (subsequently analyzed in laboratories). All of the sites except one were determined to have concentrations substantially less than $1 \mathrm{ppm}$, if any. Concentrations above minimum quantifiable levels, $0.33-0.56 \mathrm{ppm}$, were detected in only two cases. Leakage from empty, but improperly sealed, refrigerant drums was identified as the probable cause for one site. The sources in the other were improperly sealed drums and a leak from the purge vent line. The latter was ascribed to improper field installation and use of incompatible piping materials. A retest of this site, after corrections, found concentrations in line with the others. Reports on the individual sites and summary discussion describe the tests and safety considerations for refrigerants. The findings emphasize the importance of following proper refrigerant handling and storage procedures as well as installation recommendations.
Report of Worker Exposure to HCFC-123 During Servicing of Hermetic Centrifugal Chillers, report CFC-2, The Trane Company, La Crosse, WI, May 1992 (16 pages with 3 figures and 9 tables, limited copies available from JMC as RDB2908)

This report addresses the safety aspects of exposure of service personnel to R-123 during the full range of service procedures for centrifugal chiller applications. Earlier toxicity findings and implications are reviewed as background. The report then documents the measured concentrations during refrigerant transfer, routine maintenance, and major service from three different installations. Chronic (long-term), acute (short-term intermittent), and emergency acute exposure considerations are outlined. The measurement procedures are described; they used activated charcoal tubes subsequently analyzed by gas chromatography in laboratories, and on-site infrared vapor analyzers. Timeweighted average (TWA) exposures are tabulated for the chronic exposures, ranging from less than the limit of quantification (LOQ) to 1.9 ppm. Peak concentrations and durations are tabulated for the intermittent, acute exposures. Test reports are included to describe each site and provide detailed measured data. The report concludes that the long-term concentrations observed were more than five times below the allowable exposure limit (AEL) of $10 \mathrm{ppm}$ [subsequently raised by some refrigerant manufacturers]. The instantaneous concentrations were well within the $30-50 \mathrm{ppm}$ limits based on guidelines developed by the American Conference of Governmental Industrial Hygienists (ACGIH). The findings emphasize the importance of following proper handling and storage procedures for refrigerants and installation recommendations of ASHRAE Standard $15 R$ [now 15-1992].

Workplace Environmental Exposure Level Guide - 1,1,1-Trifluoro-2,2-dichloroethane, American Industrial Hygiene Association (AlHA), Fairfax, VA, 1998 (11 pages with 2 tables, RDB8611)

documents determination of a Workplace Environmental Exposure Level (WEEL) Guide for R123; summarizes identifiers, chemical and physical properties, uses, production quantities, toxicology tests conducted in animals (acute, subchronic, chronic, carcinogenicity, reproductive, developmental, genotoxicity, mutagenicity, metabolism, pharmacokinetics, and mechanistic); reviews human uses and exposures expe- 
rience; discusses a rationale for setting the occupational exposure limit; indicates that R-123 induces a slight anesthetic effect at $5000 \mathrm{ppm}$ $\mathrm{v} / \mathrm{v}$; notes that increased incidence of microscopic tumors in rats, found to be both benign (nonmalignant) and not life threatening, were due to induction of peroxisome proliferation and thus of little relevance to humans; discusses reversible hepatic lesions observed in monkeys, dogs, and guinea pigs at 1,000,10,000, and $5,000 \mathrm{ppm} v / \mathrm{v}$, respectively; allowing an interand intraspecies safety factor, recommends a WEEL guide of $50 \mathrm{ppm}\left(310 \mathrm{mg} / \mathrm{m}^{3}\right)$ for $8-\mathrm{hr}$ on a time-weighted average (TWA) basis; includes 64 references

Workplace Guidelines for Suva Centri-LP (HCFC-123) in Refrigeration and Air Conditioning Applications, document AS-5 (H-53018), DuPont Chemicals, Wilmington, DE, November 1993 (4 pages with 2 tables, RDB4C50)

This bulletin presents recommended workplace guidelines for use of R-123 in air-conditioning and refrigeration applications. It notes that DuPont has concluded that R-123 poses no acute (short-term, single exposure) or chronic (long-term, repeated exposure) hazard when handled in accordance with recommendations and when exposures are maintained at or below its recommended Acceptable Exposure Limit (AEL). The bulletin indicates that the AEL for $R-123$ has been set at $30 \mathrm{ppm}$ for exposures of 8 or 12 hours daily or 40 hours weekly on a time-weighted average (TWA) basis. It also notes that DuPont has set an Emergency Exposure Limit (EEL) of $1000 \mathrm{ppm}$ for up to 1 hour, with a 1 minute ceiling of $2500 \mathrm{ppm}$. The bulletin recommends that users read and understand the Material Safety Data Sheet (MSDS) and presents guidance for storage, handling, and use of R-123. It notes that ASHRAE Standard 15 requires area monitors to detect refrigerant concentrations and additional guidance on system and machinery room design. A table recommends alarm levels and appropriate actions for increasing detection levels. A second table outlines minimum respirator protection for concentrations exceeding the cited AEL. The bulletin identifies prohibited uses for R-123, personal protective equipment, and storage and handling procedures. It also recommends safety measures for system charging, maintenance, and sampling as well as responses to leaks or spills. DuPont's product names for R-123 are Suva ${ }^{\circledR}$ Centri-LP and Suva ${ }^{\circledR} 123$. report 57-95, Haskell Laboratory for Toxicology and Industrial Medicine, E. I. duPont de Nemours and Company, Incorporated, Newark, DE, 1995 (RDB7235)

presents a subchronic inhalation toxicity studyo $f$ in male rats; examines biochemical changes and peroxisome proliferation for exposures to $5,000 \mathrm{ppm} v / \mathrm{v}$ for $6 \mathrm{hr} / \mathrm{d}$ for 5 days

\section{R-123B1}

K. D. Hall and F. H. Norris, Jr., Fluothane Sensitization of Dog Heart to Epinephrine, Anesthesiology, 19:631-641, September-October 1958 (11 pages, RDB6565)

identifies R-123B1 as a cardiac sensitizer - as reported in RDB5644

J. Raventós, The Action of Fluothane - A New Volatile Anaesthetic, British Journal of Pharmacology, 11:394-410, 1956 (17 pages, RDB7365)

toxicity tests of R-123B1 with focus on its anesthetic effects

\section{R-124}

F. C. Barsky, In Vitro Microbial Mutagenicity Studies of 1-Chloro-1,2,2,2-tetrafluoroethane, report 349-76 (TSCAT report OTS-0530589), Haskell Laboratory for Toxicology and Industrial Medicine, E. I. duPont de Nemours and Company, Incorporated, Newark, DE, 4 May 1976 (RDB6178)

$\mathrm{R}-124$, genotoxicity, health effects, mutagenic potential, toxicity as reported in RDB5863 and RDB5B54

Bio/dynamics Incorporated, report 89-3513, AlliedSignal Incorporated, Morristown, NJ, 1991 (RDB6177)

$\mathrm{R}-124$, health effects, toxicity as reported in RDB5863

W. E. Brewer and S. Smith (Industrial Bio-Test Laboratories, Incorporated), Teratogenic Study via Inhalation with Genetron 124 in Albino Rats, report MA-RR-89-1493 (also identified as IBT 8562-09345 and as TSCAT report OTS0000695-2), AlliedSignal Incorporated (then Allied Chemical Corporation), Morristown, NJ, 8 November 1977 (32 pages with 10 tables, RDB6173)

toxicity test of the teratogenicity of R-124 in groups of 20 female Charles River albino rats by inhalation exposures at 0 (control) or 5,000 
(4,424-5,527 actual) ppm for $5 \mathrm{hr} / \mathrm{d}$ on gestation days 6-15: no difference was found in the maternal body weights and body weight gains between the test and control groups; some animals in both groups had red nasal discharges; the number of resorption sites were slightly higher and the number of pregnancies was 20 in the test group versus 16 in the control group; no other reaction or grossly apparent uterine anomalies attributed to exposures were noted; pathological findings of the sacrificed dams and fetuses were essentially the same for the test and control groups; report concludes that R124 was not teratogenic as tested [a letter from the study sponsor indicates that the quality of the R-124 used in this study was not certain and that further studies (see RDB5A22, RDB65F5, RDB7268, RDB7270, and others) were performed]

W. E. Brewer and S. Smith (Industrial Bio-Test Laboratories, Incorporated), 90-Day Subacute Inhalation Toxicity Study with Genetron 124 in Albino Rats, report IBT 8562-09345, AlliedSignal Incorporated (then Allied Chemical Corporation), Morristown, NJ, 2 September 1977 (RDB6174)

R-124, health effects, subchronic toxicity [detailed summary provided in RDB3720 and discussed in RDB4C02; a letter from the study sponsor indicates that the quality of the R-124 used in this study was not certain and that further studies (see RDB65F5 and others) were performed]

D. J. Brusick (Litton Bionetics Incorporated, LBI), Mutagenicity Evaluation of Genetron 124, report MA-RR-89-1451 (also identified as LBI project 2547), AlliedSignal Incorporated (then Allied Chemical Company), Morristown, NJ, 30 July 1976 (42 pages with 1 figure and 18 tables, RDB5807)

$\mathrm{R}-124$, health effects, toxicity: concluded that R-124 did not exhibit genetic activity in any of the in vitro assays evaluated: tests were performed with Saccharomyces cerevisiae (yeast) and Salmonella typhimurium (bacteria) with and without metabolic activation

C. N. Edwards and G. Hodson-Walker (Life Science Research Limited, LSR, UK), In Vitro Assessment of the Clastogenic Activity of HCFC 124 in Cultured Chinese Hamster Ovary (CHOK1) Cells, report MA-RR-92-1788a (also identified as LSR report 91/PAR002/0885a and 91/0885a, also identified as TSCAT report OTS-0535094, Japanese version identified as 91/PAR002/0885b) for the Program the Alternative Fluorocarbon Tox- icity Testing (PAFT), AlliedSignal Incorporated, Morristown, NJ, 20 December 1991 (52 pages with 1 figure and 11 tables, RDB6176)

toxicity tests of R-124 to assess mutagenic potential in cultured Chinese hamster ovary cells at concentrations of $150,000,300,000$, and $600,000 \mathrm{ppm} \mathrm{v/v}$; concludes that R-124 showed no evidence of clastogenic (chromosome-damaging) activity either in the presence or absence of a metabolic activation system, derived from rat livers, under the conditions tested

Gleason and W. B. Coate (Hazleton Laboratories America, Incorporated), LC $_{50}$ of $\mathbf{G 1 2 4}$ in Rats, report MA-RR-89-1450 (also identified as project 165-163), AlliedSignal Incorporated (then Allied Chemical Company), Morristown, NJ, 4 October 1976 (6 pages with 2 tables, RDB5806)

R-124, acute inhalation toxicity: tests of 10 male albino rats at 0 (control), 100,000, and 360,000 ppm R-124 nominal concentrations (actual concentrations not indicated) in air: no deaths occurred, leading to the conclusion that the $6-\mathrm{hr} \mathrm{LC}_{50}$ rat exceeds $360,000 \mathrm{ppm}$; hyperactivity was observed for the first 30 min during exposures at $100,000 \mathrm{ppm}$ followed by inactivity; compound awareness also was observed at $360,000 \mathrm{ppm}$, at which animals appeared inactive and anesthetized after $1 \mathrm{hr}$; animals appeared normal during the post-exposure observation period; animals exposed to $360,000 \mathrm{ppm}$ showed a lower rate of weight gain on days 1-8 following exposures, but the report suggests that it may have been due to their higher initial weight; one animal had no gross pathology, but discoloration was found in the cervical lymph nodes, lungs, liver, kidneys of one or more animals and one had unusually dark medullas while another had enlarged cervical lymph nodes; the report describes these pharmacotoxic effects as not serious

D. P. Kelly, Four-Hour Inhalation Approximate Lethal Concentration (ALC) of HCFC-124 in Rats, report 71-90 (possibly TSCAT report OTS0530585), Haskell Laboratory for Toxicology and Industrial Medicine, E. I. duPont de Nemours and Company, Incorporated, Newark, DE, 19 June 1990 (11 pages with 1 table, RDB6180)

tests of the acute inhalation toxicity of R-124 in groups of 6 male rats by $4-\mathrm{hr}$, nose-only exposures at mean concentrations based on measurements by gas chromatography (GC) of $48,000,160,000,230,000$, and $300,000 \mathrm{ppm}$ $\mathrm{v} / \mathrm{v}$ : no rats died at concentrations of 230,000 ppm and lower, but all six died at 300,000 ppm 
leading to a conclusion that the 4-hr ALC rat = 230,000-300,000 ppm; no clinical effects were observed at $48,000 \mathrm{ppm}$; animals exposed to 160,000 and $230,000 \mathrm{ppm}$ and higher showed weight loss the day after exposure followed by normal weight gain; animals exposed to 160,000 and 230,000 ppm exhibited decreased response to startle and tail pinches, prostration, lethargy, and incoordination; report indicates Good Laboratory Practice (GLP) compliance

D. M. Krentz, R. C. Graham, and M. A. Lee, untitled toxicity review for 2-chloro-1,1,1,2tetrafluoroethane, Haskell Laboratory for Toxicology and Industrial Medicine, E. I. duPont de Nemours and Company, Incorporated, Newark, DE, 8 June 1992 (RDB5863)

R-124, summary of published and unpublished toxicity literature

Life Science Research Limited (LSR, UK), report 91/PAR001/0925 for the Program the Alternative Fluorocarbon Toxicity Testing (PAFT), AlliedSignal Incorporated, Morristown, NJ, 20 December 1991 (RDB6175)

in vitro toxicity tests with Salmonella typhimurium and Escherichia coli to assess the mutagenicity of R-124

G. D. Loizou and M. W. Anders (University of Rochester), Gas-Uptake Pharmacokinetics and Metabolism of 2-Chloro-1,1,1,2tetrafluoroethane (HCFC-124) in the Rat, Mouse, and Hamster, Drug Metabolism and Disposition, 23(8):875-880, 1995 (6 pages, RDBA934)

reports on an investigation of the gas-uptake pharmacokinetics and metabolism of R-124 in rats, mice, and hamsters; notes that species differences were observed in the rate of R-124 uptake and in urinary trifluoroacetic acid (TFA, the major urinary metabolite of R-124) excretion; the uptake of R-124 was described by both saturable and first-order components in rats and mice whereas only first-order uptake was observed in hamsters; presents in vivo metabolic rate constants determined from computer simulation of the gas-uptake data; also presents simulations of the production and excretion of TFA in rats and mice, but not in hamsters, by a physiologically based pharmacokinetic (PBPK) model using the new metabolic rate constants; notes that the blood to air partition coefficient of R-124 in hamsters was lower than in mice or rats; indicates that a low coefficient may limit the pulmonary uptake of volatile chemicals, so species that show coefficients much lower than in humans may not be suitable for chemical hazard evaluation and human risk assessment

L. A. Malley (E. I. duPont de Nemours and Company, Incorporated), S. R. Frame (DuPont), G. S. Elliott (DuPont), K. S. Bentley (DuPont), W. J. Brock (DuPont), H. J. Trochimowicz (DuPont), and G. M. Rusch (AlliedSignal Incorporated), Chronic Toxicity, Oncogenicity, and Mutagenicity Studies with Chlorotetrafluoroethane (HCFC124), Drug and Chemical Toxicology, 21(4):417447, November 1998 (31 pages, RDBA665)

summarizes studies of the chronic toxicity, oncogenicity, and mutagenicity of R-124; in the chronic toxicity and oncogenicity study, groups of male and female rats were exposed whole body to target concentrations of 0 (control), $2,000,10,000$, or $50,000 \mathrm{ppm}$ v/v R-124 in air for $6 \mathrm{hr} / \mathrm{d}, 5 \mathrm{~d} / \mathrm{wk}$, for $2 \mathrm{yr}$, no compoundrelated, adverse effects were observed on body weight, food consumption, survival, clinical signs of toxicity, ophthalmoscopically observable ocular lesions, serum hormone concentrations, or clinical pathology parameters at any exposure concentration; compared to controls, urine fluoride was increased in both males and females at all exposure concentrations, and plasma fluoride was increased in females at all exposure concentrations; excretion of fluoride represents conversion of the R-124 parent molecule and was not considered an adverse effect; there were no toxicologically significant, compound-related organ weight changes or gross or microscopic findings at any of the exposure concentrations; R-124 was deemed not toxic or carcinogenic in rats of either sex after inhalation exposure at concentrations of up to $50,000 \mathrm{ppm}$; this concentration was deemed a no-observed-adverse-effect level (NOAEL); paper also summarizes a series of mutagenicity studies; R-124 was considered not mutagenic in Salmonella typhimurium with and without activation at concentrations up to $600,000 \mathrm{ppm}$ $\mathrm{v} / \mathrm{v}$ for $48 \mathrm{hr}$, no evidence of clastogenic activity was observed in cultured human lymphocytes at concentrations up to $1,000,000 \mathrm{ppm}$ v/v R124 for $3 \mathrm{hr}$, with and without metabolic activation; no micronuclei were induced in vivo in mouse bone marrow cells following exposure of mice to concentrations of 99,000 ppm R-124 for $6 \mathrm{hr} / \mathrm{d}$ for 2 days; overall, R-124 was found to be nontoxic for chronic exposures up to 50,000 $\mathrm{ppm} v / \mathrm{v}$, not mutagenic, and not oncogenic as tested 
L. A. Malley (E. I. duPont de Nemours and Company, Incorporated), M. C. Carakostas (DuPont), G. S. Elliott (DuPont), L. Alvarez (DuPont), R. E. Schroeder (Pharmaco-LSR, Incorporated), S. R. Frame (DuPont), C. Van Pelt (DuPont), H. J. Trochimowicz (DuPont), and G. M. Rusch (AlliedSignal Incorporated), Subchronic Toxicity and Teratogenicity of 2-Chloro-1,1,1,2-tetrafluoroethane (HCFC-124), article 0102, Fundamental and Applied Toxicology, 32(1):11-22, 1996 (12 pages with 1 figure and 8 tables, RDB65F5)

tests of subchronic toxicity of R-124 in groups of rats and mice by inhalation exposures for 6 $\mathrm{hr} / \mathrm{d}, 5 \mathrm{~d} / \mathrm{wk}$, for 13 weeks at 0 (control), 5,000, 15,000 , or $50,000 \mathrm{ppm} v / \mathrm{v}$; R-124 caused minimal compound-related effects; hepatic betaoxidation activity was significantly higher in male mice exposed at $5,000,15,000$, or 50,000 $\mathrm{ppm}$, but there were no compound related effects on beta-oxidation activity in rats; rats, mice, and rabbits exposed to $50,000 \mathrm{ppm}$ were less responsive to auditory stimuli or less active compared to controls; male rats exposed to 15,000 or $50,000 \mathrm{ppm}$ for 13 -weeks had decreased arousal; no compound-related effects on mortality, clinical signs, ocular tissues, hematology parameters, organ weights, or tissue morphology were found at any concentration in rats or mice; female rats showed a significant decrease in weight gain and food consumption at 50,000 ppm; pregnant rabbits also consumed less food at $50,000 \mathrm{ppm}$; no evidence of fetal toxicity was found at any concentration

L. A. Malley, Subchronic Inhalation Toxicity: 90-Day Study with HCFC-124 in Mice, report 695-91 for the Program for Alternative Fluorocarbon Toxicity Testing (PAFT), Haskell Laboratory for Toxicology and Industrial Medicine, E. I. duPont de Nemours and Company, Incorporated, Newark, DE, 21 October 1992 (459 pages, RDB7B41)

$R-124$, health effects, toxicity

L. A. Malley, L. Alvarez, S. R. Frame, M. C. Carakostas, H. J. Trochimowicz (E. I. duPont de $\mathrm{Ne}-$ mours and Company, Incorporated), and G. M. Rusch (AlliedSignal incorporated), Subchronic Toxicity and Teratogenicity of HCFC-124 in Rats, abstract 1388 (SOT 31st Annual Meeting, February 1992) for the Program for Alternative Fluorocarbon Toxicity Testing (PAFT), The Toxicologist, 12(1):355, 1992 (1 page with no figures or tables, RDB7268)

90-day inhalation toxicity study for R-124 in rats exposed to 0 (control), 5,000, 15,0000, or
$50,000 \mathrm{ppm} \mathrm{v} / \mathrm{v}$ for $6 \mathrm{hr} /$ day, $5 \mathrm{~d} / \mathrm{wk}$ : anesthetic effect observed at $50,000 \mathrm{ppm}$ based on reduced response to sound; slight differences observed in clinical chemistry parameters and decreased arousal in males at 15,000 and 50,000 ppm; no compound-related morphological changes were observed; in a related inhalation teratology study, groups of 24 pregnant rats were exposed to $50,000 \mathrm{ppm}$; no compound-related morphological changes were observed in the dams and no teratogenic changes were observed in the fetuses

L. A. Malley, Subchronic Inhalation Toxicity: 90-Day Study with HCFC-124 in Rats, report 7991 (TSCAT report OTS-0529977) for the Program for Alternative Fluorocarbon Toxicity Testing (PAFT), Haskell Laboratory for Toxicology and Industrial Medicine, E. I. duPont de Nemours and Company, Incorporated, Newark, DE, 8 August 1991 (602 pages, Rdb5C06)

subchronic inhalation study in groups of 20 male and 20 female Crl:CD-BR rats exposed to R-124 for $6 \mathrm{hr} / \mathrm{d}, 5 \mathrm{~d} / \mathrm{wk}$, for $13 \mathrm{wk}$ at target concentrations of 0 (control), 5,000, 15,000, and $50,000(0,5,033,15,143,49,987$ actual) $\mathrm{ppm} \mathrm{v} / \mathrm{v}$; males exposed to 15,000 and 50,000 $\mathrm{ppm}$ had lower serum triglyceride concentrations at the 45-day clinical evaluation; females exposed to $50,000 \mathrm{ppm}$ had higher alkaline phosphatase activity; plasma fluoride, urinary fluoride, and fractional clearance of free fluoride were increased compared to controls in both sexes at all exposure concentrations; plasma fluoride levels remained higher at the end of the recovery period following exposures for males exposed to 15,000 and $50,000 \mathrm{ppm}$ as did urinary fluoride in males and females at all exposure levels; these differences are described as expected and not considered adverse; males exposed to 15,000 and 50,000 ppm also exhibited mild diuresis attributed to osmotic activity from excreted fluoride ions; rats exposed to $50,000 \mathrm{ppm}$ showed anesthetic effects during exposures based on reduced response to sound; males had decreased arousal on the day after the final exposures $4 / 10$ at 15,000 $\mathrm{ppm}$ and $6 / 10$ at $50,000 \mathrm{ppm}$; there were no treatment-related effects on body weight, food consumption, mortality, clinical signs, ocular tissues, hematology parameters, organ weights, or tissue morphology at any tested concentration; study deemed the NOEL to be $5,000 \mathrm{ppm}$ for males and $15,000 \mathrm{ppm}$ for females 
L. A. Malley, Subchronic Inhalation Toxicity: Four-Week Study with HCFC-124, Inhalation Study in Rats, report 257-90 (TSCAT report OTS0000695-9) for the Program for Alternative Fluorocarbon Toxicity Testing (PAFT), Haskell Laboratory for Toxicology and industrial Medicine, E. I. duPont de Nemours and Company, Incorporated, Newark, DE, 24 October 1990 (340 pages, Rdb5A14)

tests of the subchronic inhalation toxicity of R124 in five groups of ten male and five groups of ten female rats exposed to target concentrations of 0 (control), $500,2,000,10,000$, or $50,000 \mathrm{ppm} \mathrm{v/v;} \mathrm{six} \mathrm{additional} \mathrm{female} \mathrm{rats} \mathrm{per}$ group were exposed concurrently for a probe teratology study: anesthetic LOEC rat $=50,000$ $\mathrm{ppm} ;$ NOEL rat $=10,000 \mathrm{ppm}$

K. May, D. Watson, and G. Hodson-Walker (Life Science Research Limited, LSR, UK), HCFC 124 in Gaseous Phase: Assessment of Mutagenic Potential in Amino-Acid Auxotrophs of Salmonella Typhimurium and Escherichia Coli (The Ames Test), report MA-RR-92-1787a (also identified as LSR report 91/PAR001/0842 and 91/0842 and as TSCAT report OTS-0535123) for the Program the Alternative Fluorocarbon Toxicity Testing (PAFT), AlliedSignal Incorporated, Morristown, $\mathrm{NJ}$, 20 December 1991 (51 pages with 1 figure and 23 tables, RDB5809)

toxicity tests to assess the mutagenicity of R124 in bacteria, both with and without an activating system derived from rat livers, at 31,250 , $62,500,125,000,250,000$, and $500,000 \mathrm{ppm}$ $\mathrm{V} / \mathrm{V}$; concludes that R-124 "was devoid of mutagenic activity under the conditions of the tests"

L. S. Mullin, Fluorocarbon 124 (1-Chloro-1,2,2,2tetrafluoroethane): Cardiac Sensitization, report 220-76 (TSCAT report OTS-0530588), Haskell Laboratory for Toxicology and Industrial Medicine, E. I. duPont de Nemours and Company, Incorporated, Newark, DE, 12 March 1976 (2 pages with 1 table, RDB5808)

toxicity tests to determine the cardiac sensitization potential of R-124 in male beagle dogs with intravenous injection of epinephrine five minutes before and, with a challenge injection, midway into a 10 minute exposure; no marked response in 10 dogs at $10,100 \mathrm{ppm} \mathrm{v} / \mathrm{v}$ in air [deemed a NOEL]; marked response in 4 of 10 dogs at 25,000 ppm [deemed a LOEL] with 1 death; marked response in 2 of 2 dogs at $50,000 \mathrm{ppm}$
M. J. Olson, J. T. Johnson, J. F. O'Gara, and S. E. Surbrook, Jr. (General Motors Research Laboratories), Metabolism In Vivo and In Vitro of the Refrigerant Substitute 1,1,1,2-Tetrafluoro-2chloroethane, Drug Metabolism and Disposition, 19(5):1004-1011, 1991 (8 pages, RDB6183)

$\mathrm{R}$-124, 2-chloro-1,1,1,2-tetrafluoroethane, toxicity, metabolism in rats

L. B. Rickard, Mouse Bone Marrow Micronucleus Assay of HCFC-124, report 52-90 for the Program for Alternative Fluorocarbon Toxicity Testing (PAFT), Haskell Laboratory for Toxicology and Industrial Medicine, E. I. duPont de Nemours and Company, Incorporated, Newark, DE, 26 February 1990 (24 pages, RDB5A21)

$\mathrm{R}-124$, genotoxicity, health effects, mutagenic potential, toxicity as reported in RDB4B88 and RDB5B54

An Inhalation Developmental Toxicity Study in Rabbits with HCFC-124, report 89-3514 (OTS0535094) for the Program for Alternative Fluorocarbon Toxicity Testing (PAFT), Bio/dynamics Incorporated, East Millstone, NJ, 19 December 1991 (RDB5A12)

$R-124$, health effects, toxicity as reported in RDB4B88

1-Chloro-1,2,2,2-tetrafluoroethane (HCFC 124, CAS No. 2837-89-0), Joint Assessment of Commodity Chemicals (JACC) report 25, European Chemical Industry Ecotoxicology and Toxicology Centre (ECETOC), Brussels, Belgium, July 1994 (34 pages, RDB4C02)

summarizes toxicity and environmental data for R-124 (2-chloro-1, 1, 1,2-tetrafluoroethane)

2-Chloro-1,1,1,2-Tetrafluoroethane (HCFC 124) Health Effects Information, CAS\# 2837-89-0, Elf Atochem North America, Incorporated, Philadelphia, PA, undated circa 1995 (5 pages, RDB5B54) summarizes toxicological and ecotoxicological information for R-124

Biodegradability of HCFC-124 by Microorganisms, report for the Program the Alternative Fluorocarbon Toxicity Testing (PAFT), Kurume Research Laboratories (KRL), Fukuoka, Japan, 1992 (RDB7B38)

documents an ecotoxicity study of R-124; environmental impacts and fate; groundwater and soil

Dissociation Constant of Flon 124, report for the Program the Alternative Fluorocarbon Toxicity 
Testing (PAFT), Kurume Research Laboratories (KRL), Fukuoka, Japan, 1991 (RDB7B39)

documents an ecotoxicity study of R-124; environmental impacts and fate; groundwater and soil

Octanol-Water Partition Coefficient of Flon 124, report for the Program the Alternative Fluorocarbon Toxicity Testing (PAFT), Kurume Research Laboratories (KRL), Fukuoka, Japan, 1992 (RDB7B40)

documents an ecotoxicity study of R-124; environmental impacts and fate; groundwater and soil

In Vitro Evaluation of HCFC-124 for Chromosome Aberrations in Human Lymphocytes, report 367-90 for the Program the Alternative Fluorocarbon Toxicity Testing (PAFT), Haskell Laboratory for Toxicology and Industrial Medicine, E. I. duPont de Nemours and Company, Incorporated, Newark, DE, 24 October 1990 (RDB5A20)

$\mathrm{R}-124$, mutagenicity, toxicity, potential clastogenic (chromosome-damaging) activity as reported in RDB4B88 and RDB5863

Mutagenicity Testing of HCFC-124 in the Salmonella Typhimurium Plate Incorporation Assay, report 191-90 (TSCAT report OTS-0530587), Haskell Laboratory for Toxicology and Industrial Medicine, E. I. duPont de Nemours and Company, Incorporated, Newark, DE, 14 September 1990 (RDB6182)

R-124, health effects, genotoxicity, mutagenic potential, toxicity as reported in RDB5863 and RDB5B54

Pilot Teratogenicity Study of HCFC-124 in the Rat, TSCAT report OTS-0530407, Haskell Laboratory for Toxicology and Industrial Medicine, E. I. duPont de Nemours and Company, Incorporated, Newark, DE, 9 March 1991 (RDB7270)

$R-124$, health effects, toxicity as reported in RDB5B54

Subchronic Inhalation Toxicity: 90-Day Study with 2-Chloro-1,1,1,2-Tetrafluoroethane in Mice, TSCAT report OTS-0543411, Haskell Laboratory for Toxicology and Industrial Medicine, E. I. duPont de Nemours and Company, Incorporated, Newark, DE, volumes I and II, 21 October 1992 (RDB7267)

$\mathrm{R}-124$, health effects, toxicity

Teratogenicity Study of HCFC-124 in Rats, report 482-90 (TSCAT report OTS-0535088) for the
Program for Alternative Fluorocarbon Toxicity Testing (PAFT), Haskell Laboratory for Toxicology and Industrial Medicine, E. I. duPont de Nemours and Company, Incorporated, Newark, DE, 25 October 1991 (RDB5A22)

$\mathrm{R}-124$, health effects, toxicity as reported in RDB4B88 and RDB5B54

\section{Workplace Environmental Exposure Level} Guide - 2-chloro-1,1,1,2-tetrafluoroethane, American Industrial Hygiene Association (AlHA), Fairfax, VA, 1992 (2 pages with no figures or tables, RDB4B88)

R-124: identification, summary chemical and physical properties, uses, animal toxicology data, toxicity, human uses and experience (no reports of adverse effects), recommended WEEL (1,000 ppm for 8-hr TWA) guide and rationale, references

report 108-90, Haskell Laboratory for Toxicology and Industrial Medicine, E. I. duPont de Nemours and Company, Incorporated, Newark, DE, 1990 (RDB6181)

R-124, health effects, toxicity, developmental and reproductive toxicity, teratogenicity as reported in RDB5863

\section{R-125}

M. W. Anders, Pharmacokinetics of HFC-125 (Pentafluoroethane) in Rats, report for the Program for Alternative Fluorocarbon Toxicity Testing (PAFT), University of Rochester Medical Center, Rochester, NY, 16 December 1993 (RDB5751)

$$
\mathrm{R}-125 \text {, toxicity }
$$

R. Atkinson et al., Advances in Photochemistry, 11:375-488, 1979 (114 pages, RDB5936)

\section{R-125}

A. J. Brooker, P. J. Brown, D. M. John, and D. W. Coombs (Huntingdon Research Center Limited HRC, UK), A Study of The Effect of HFC-125 on Pregnancy of the Rabbit, report ALS 10/920856 for the Program for Alternative Fluorocarbon Toxicity Testing (PAFT), AlliedSignal Incorporated, Morristown, NJ, 14 or 24 November 1992 (RDB5752)

$\mathrm{R}-125$, developmental toxicity, teratogenicity: neither embryotoxic nor teratogenic in the rabbit at exposures up to $50,000 \mathrm{ppm} \mathrm{v/v}$ as reported in 5B55 and 65E6 
C. A. Dance and G. Hodson-Walker, In Vitro Assessment of the Clastogenic Activity of HFC125 in Cultured Chinese Hamster Ovary (CHO) Cells, report 91/PAR006/1015a for the Program for Alternative Fluorocarbon Toxicity Testing (PAFT), Life Science Research Limited (LSR), Suffolk, UK, 18 May 1992 (RDB5759)

$R-125$, toxicity, mutagenicity: no evidence of clastogenic activity as reported in RDB65E6

C. A. Dance, K. E. Beach, and G. Hodson-Walker, In Vitro Assessment of the Clastogenic Activity of HFC-125 in Cultured Human Lymphocytes, report 91/PAR005/1014a for the Program for Alternative Fluorocarbon Toxicity Testing (PAFT), Life Science Research Limited (LSR), Suffolk, UK, 18 May 1992 (RDB5760)

R-125, toxicity, mutagenicity: no evidence of clastogenic activity as reported in RDB65E6

C. N. Edwards, G. Hodson-Walker, and S. Cracknell, HFC-125: In Vitro Assessment of Clastogenic Action on Bone Marrow Erthrocytes in the Micronucleus Test, report 92/PAR004/0148 for the Program for Alternative Fluorocarbon Toxicity Testing (PAFT), Life Science Research Limited (LSR), Suffolk, UK, 7 July 1992 (RDB5758)

R-125, toxicity, mutagenicity: no evidence of clastogenic activity in the mouse micronucleus assay as reported in RDB65E6

J. Ferguson-Smith, Hygiene Standard Documentation - HFC 125, document HSP/92/20, ICl Chemicals and Polymers Limited, Cheshire, UK, 27 January 1993 (4 pages, RDB5A78)

$R-125$, toxicity, literature review, rationale for recommended hygiene standard (occupational exposure limit, OEL) of 1,000 ppm, 8-hr TWA, provisional

T. Kawano (Daikin Industries, Limited, Japan), $H$. J. Trochimowicz (E. I. duPont de Nemours and Company, Incorporated, USA), G. Malinverno (Ausimont S.p.A., Italy), and G. M. Rusch (AlliedSignal Incorporated, USA), Toxicological Evaluation of 1,1,1,2,2-Pentafluoroethane (HFC-125), Fundamental and Applied Toxicology, 28(2):223231, 1995 (9 pages with 7 tables, RDB65E6)

summarizes the Program for Alternative Fluorocarbon Toxicity Testing (PAFT) studies of R125; indicates that the 4-hr ALC and $L C_{50}$ rat is $>800,000 \mathrm{ppm} v / \mathrm{v}$, the 5-min cardiac sensitization NOEL dog is $75,000 \mathrm{ppm} v / \mathrm{v}$, the LOEL is $100,000 \mathrm{ppm}$, and the $\mathrm{EC}_{50}$ is $100,000-150,000$ ppm; reports that subchronic exposures of rats for $6 \mathrm{hr} / \mathrm{d}, 5 \mathrm{~d} / \mathrm{wk}$ for 4 and 13 weeks showed no evidence of toxicity at $50,000 \mathrm{ppm} v / \mathrm{v}$, the highest concentration tested; indicates that the developmental tests found slight, reversible toxicity in the rabbit and rat dams, but no convincing evidence of embryotoxicity or teratogenicity; also indicates that in vitro tests in Salmonella typhimurium and Escherichia coli bacteria (Ames Assay) found no clear evidence of clastogenic activity in Chinese hamster ovary (CHO) cells or human lymphocytes; similarly indicates that in vivo tests found R-125 not mutagenic in the mouse micronucleus test, even at concentrations as high as $600,000 \mathrm{ppm} v / \mathrm{v}$ for $6 \mathrm{hr}$; concludes that R-125 is "very low in biological reactivity," "is not acutely toxic, not a cumulative toxin, not a developmental toxin, not a mutagen," and is "low or lower in toxicity than any of the CFCs it will eventually replace"

R. E. Masters, R. J. Brown, D. M. John, and D. W. Coombs (Huntingdon Research Center Limited HRC, UK), A Study of the Effect of HFC-125 on Pregnancy of the Rat (Inhalation Exposure), report ALS 9/920434 for the Program for Alternative Fluorocarbon Toxicity Testing (PAFT), AlliedSignal Incorporated, Morristown, NJ, 6 November 1992 (RDB5753)

$R-125$, developmental toxicity: neither embryotoxic nor teratogenic in the rat at exposures up to $50,000 \mathrm{ppm} \mathrm{v} / \mathrm{v}$ as reported in 65E6

K. May, D. Watson, and G. Hodson-Walker, HFC125 in Gaseous Phase: Assessment of Mutagenic Potential in Amino-Acid Auxotrophs of Salmonella Typhimurium and Escherichia Coli (The Ames Test), report 91/PAR003/1152a for the Program for Alternative Fluorocarbon Toxicity Testing (PAFT), Life Science Research Limited (LSR), Suffolk, UK, 12 or 28 May 1992 (RDB5757)

$\mathrm{R}-125$, toxicity, mutagenicity, Ames assay: R125 deemed not mutagenic as reported in RDB5B55 and RDB65E6

E. Nakayama, K. Nagano, M. Chrishi, S. Katagiri, and O. Motegi (Japan Bioassay Laboratory, JBL, Japan), Acute Inhalation Toxicity Study of 1,1,1,2,2-Pentafluoroethane (HFC-125) in Rats, report on study 0184 (also identified as MA-RR92-1801) for the Program for Alternative Fluorocarbon Toxicity Testing (PAFT), Japan Industrial Safety and Health Association, Kanagawa, Japan, 18 March 1992 (84 pages with 11 figures and 22 tables, RDB5754)

tests of the acute inhalation toxicity in groups of 5 female and 5 male Crj:CD(SD) rats exposed (whole body) to concentrations of 0 and 
$800,000(769,000$ actual with a brief excursion to 398,000$) \mathrm{ppm} v / \mathrm{v} R-125$ in oxygen: no deaths resulted, but "almost all" animals exhibited ataxic (uncoordinated) gait, decreases in locomotor movement and sound response [suggesting an anesthetic LOEC at 769,000 $\mathrm{ppm} \mathrm{v} / \mathrm{v}$ ], abnormal respiration, prone or lateral, up and down movement of the neck during exposures; mean body weight gain of the treated male rats was $3-5 \%$ lower postexposure than the controls; no other clinical signs were noted during a 14 day observation period and no toxic effects were found at necropsy; concludes that $\mathrm{R}-125$ has very low toxicity by acute inhalation and that the 4-hr ALC rat is $>800,000 \mathrm{ppm} v / \mathrm{v}$ as tested

E. Nakayama, K. Nagano, M. Ohnishi, S. Katagiri, and O. Motegi (Japan Bioassay Laboratory, JBL, Japan), Four-Week Inhalation Toxicity Study of 1,1,1,2,2-Pentafluoroethane (HFC-125) in Rats, report 0182 for the Program for Alternative Fluorocarbon Toxicity Testing (PAFT), Japan Industrial Safety and Health Association, Kanagawa, Japan, 23 October 1992 (RDB5756)

R-125, inhalation toxicity: 13-wk NOAEL rat at $0,5,000,15,000$, or $50,000 \mathrm{ppm} v / \mathrm{v}$ as reported in RDB65E6

E. Nakayama, K. Nagano, M. Ohnishi, and $O$. Motegi (Japan Bioassay Laboratory, JBL, Japan), Thirteen-Week Inhalation Toxicity Study of 1,1,1,2,2-Pentafluoroethane (HFC-125) in Rats, report 0197 for the Program for Alternative Fluorocarbon Toxicity Testing (PAFT), Japan Industrial Safety and Health Association, Kanagawa, Japan, 31 August 1993 (918 pages, RDB5761)

documents a study to determine the inhalation toxicity of R-125; 20 male and 20 female rats were exposed for $6 \mathrm{hr} / \mathrm{d}, 5 \mathrm{~d} / \mathrm{wk}$, for $13 \mathrm{wk}$ to target concentrations of $0,5,000,15,000$, or $50,000 \mathrm{ppm}$ v/v in air; actual mean concentrations determined from measurements by an automatic gas chromatograph (GC) were 0, 4995, 14,891 , and $50,113 \mathrm{ppm} \mathrm{v/v}$; half were then sacrificed for necropsy and the remainder were necropsied after an a 4 wk recovery period; all rats survived to the sacrifice and there were no treatment-related effects on body weight, food consumption, clinical signs, hematological parameters, blood biochemical parameters, urinalysis parameters, organ weights, gross findings, pathological findings, plasma fluoride concentrations, or urinary fluoride excretion at any exposure level; there also were no signs of peroxisomal B-oxidation activity of the livers and ophthalmoscopic findings; concludes that the no observable effect level (NOEL) is 50,000 $\mathrm{ppm} v / \mathrm{v}$

S. Nick, Pentafluoroethane (FC-125): Acute Inhalation Toxicity, report 54-64, Haskell Laboratory for Toxicology and Industrial Medicine, E. I. duPont de Nemours and Company, Incorporated, Newark, DE, 28 May 1964 (1 page with 1 table, RDB6456)

tests of the acute inhalation toxicity in groups of 4 male ChR-CD rats exposed whole body to concentrations of 50,000 and $100,000 \mathrm{ppm} \mathrm{v/v}$ R-125 in air for $4 \mathrm{hr}$ : no deaths resulted; exposed animals exhibited slightly deep respiration and inactivity during exposure, but recovered immediately thereafter; rats showed a weight gain within one day following exposures but appeared normal throughout the remainder of the 14-day observation period; gross examination of the lungs at necropsy revealed slight pulmonary changes; concludes that the 4-hr ALC rat exceeds $100,000 \mathrm{ppm} v / \mathrm{v}$ and that $R$ 125 appears to have no deleterious effect as a contaminant of R-115 food propellant (also used as a refrigerant) if present in the expected concentration of approximately $50 \mathrm{ppm}$

A. S. Panepinto, Four-Hour Inhalation Approximate Lethal Concentration (ALC) of HFC-125 in Rats, report 582-90 (TSCAT report 0530584), Haskell Laboratory for Toxicology and Industrial Medicine, E. I. duPont de Nemours and Company, Incorporated, Newark, DE, 4 December 1990 (12 pages with 1 table, RDB6457)

tests of the acute inhalation toxicity in groups of 6 male $C r j: C D(B R)$ rats exposed nose only to concentrations of 503,000 and $709,000 \mathrm{ppm} v / \mathrm{v}$ $\mathrm{R}-125$ in air, with oxygen added to maintain concentrations of $20-22 \%$, for $4 \mathrm{hr}$ : no deaths resulted and no clinical signs of toxicity were observed during exposures or immediately thereafter; animals showed transient weight losses of $1-9 \%$ on the first day following exposures, but gained weight during the 14-day recovery period; two rats exposed at the higher concentration also exhibited transient weight loss later in the recovery period; concludes that the 4-hr ALC exceeds 709,000 ppm and that R125 is considered to have a very low toxicity

R. L. Swann et al., Research Review, 85:17-28, 1983 (12 pages, RDB5943)

$\mathrm{R}-125$, toxicity 
V. Thompson and R. C. Graham, untitled toxicity review for pentafluoroethane, Haskell Laboratory for Toxicology and Industrial Medicine, E. I. duPont de Nemours and Company, Incorporated, Newark, DE, 10 December 1993 (16 pages, RDB5871)

R-125, summary of published and unpublished toxicity literature

Pentafluoroethane (HFC-125, CAS No. 354-336), Joint Assessment of Commodity Chemicals (JACC) report 24, European Chemical Industry Ecotoxicology and Toxicology Centre (ECETOC), Brussels, Belgium, May 1994 (36 pages with 2 tables, RDB4C01)

$R-125$, environmental impacts, toxicity

Pentafluoroethane (HFC 125) Health Effects Information, CAS\# 354-33-6, Elf Atochem North America, Incorporated, Philadelphia, PA, undated circa 1995 (3 pages, RDB5B55)

$\mathrm{R}-125$, summary of toxicological and ecological information

Test on Biodegradability of HFC-125 $(1,1,1,2,2-$ Pentafluoroethane) by Microorganisms (closed-Bottle Method), Haskell Laboratory for Toxicology and Industrial Medicine, E. I. duPont de Nemours and Company, Incorporated, Newark, DE, 19 January 1993 (RDB7271)

ecotoxicity of R-125; environmental impacts and fate; groundwater and soil

Test on 1-Octanol/Water Partition Coefficient of HFC-125, Kurume Research Laboratories (KRL), Fukuoka, Japan, 28 April 1992 (RDB7273)

ecotoxicity of R-125; environmental impacts and fate; groundwater and soil

Test Report HFC 125 Dissociation Constant, Kurume Research Laboratories (KRL), Fukuoka, Japan, 28 April 1992 (RDB7272)

ecotoxicity of R-125; environmental impacts and fate; groundwater and soil

Workplace Environmental Exposure Level Guide - 1,1,1,2,2-pentafluoroethane, American Industrial Hygiene Association (AIHA), Fairfax, VA, 1996 (4 pages, RDB5C15)

occupational exposure limit for R-125; WEEL; toxicology data; toxicity; safety

report 726-90, Haskell Laboratory for Toxicology and Industrial Medicine, E. I. duPont de Nemours and Company, Incorporated, Newark, DE, 1990 (RDB6455)
$R-125$, health effects, toxicity, mutagenicity

\section{R-132b}

M. R. Brittelli, Eye Irritation Test in Rabbits, report 13-76, Haskell Laboratory for Toxicology and Industrial Medicine, E. I. duPont de Nemours and Company, Incorporated, Newark, DE, 1976 (RDB5373)

$\mathrm{R}-132 \mathrm{~b}$, health effects, toxicity as reported in RDB5371

N. C. Goodman, Primary Skin Irritation and Sensitization Tests on Guinea Pigs, report 14-76, Haskell Laboratory for Toxicology and Industrial Medicine, E. I. duPont de Nemours and Company, Incorporated, Newark, DE, 1976 (RDB5374)

$R-132 b$, health effects, toxicity as reported in RDB5371

J. W. Harris and M. W. Anders, Metabolism of the Hydrochlorofluorocarbon 1,2-Dichloro-1,1difluoroethane, Chemical Research in Toxicology, 4:180-186, 1991 (7 pages, RDB5375)

$\mathrm{R}-132 \mathrm{~b}$, health effects, metabolism, toxicity

P. J. M. Janssen and T. E. Pot (Duphar B.V., The Netherlands), Acute Dermal Toxicity Study with FC 132b in Rats, report S.8807, Solvay et Cie S.A., Brussels, Belgium, 1989 (RDB5379)

toxicity tests for acute dermal toxicity of R-132b in rats as reported in RDB5371

P. J. M. Janssen (Duphar B.V., The Netherlands), Acute Inhalation Toxicity Studies on FC 132b in Rats, report S.8811, Solvay et Cie S.A., Brussels, Belgium, 1989 (RDB5380)

tests for acute inhalation toxicity of R-132b in rats as reported in RDB5371

P. J. M. Janssen (Duphar B.V., The Netherlands), Acute Inhalation Study to Investigate the Respiratory Irritancy Properties of FC 132b in Male Rats, report S.8907, Solvay et Cie S.A., Brussels, Belgium, 1989 (RDB-5381)

toxicity tests for acute inhalation toxicity of R$132 \mathrm{~b}$ in rats as reported in RDB5371

P. J. M. Janssen and T. E. Pot (Duphar B.V., The Netherlands), Acute Oral Toxicity Study with FC $132 b$ in Rats, report S.8816, Solvay et Cie S.A., Brussels, Belgium, 1988 (RDB5382)

toxicity tests for acute ingestion toxicity of R$132 \mathrm{~b}$ in rats as reported in RDB5371 
E. M. Johnson, B. E. G. Gabel, P. H. Lieder, and R. E. Staples, An in Vitro Evaluation of the Developmental Hazard Potential of DuPont compound H16469, report 643-86, Haskell Laboratory for Toxicology and Industrial Medicine, E. I. duPont de Nemours and Company, Incorporated, Newark, DE, 1986 (RDB5383)

R-132b, health effects, toxicity, mutagenicity, teratogenicity as reported in RDB5371

D. P. Kelly, HFA 132b: 90-Day Inhalation Toxicity Study in Rats, report 20-88, Haskell Laboratory for Toxicology and Industrial Medicine, E. I. duPont de Nemours and Company, Incorporated, Newark, DE, 1976 (RDB5384)

$R-132 b$, health effects, toxicity as reported in RDB5371

\section{R-133a}

B. R. Brown, Jr., I. G. Sipes, and R. K. Baker, Halothane Hepatoxicity and the Reduced Derivative, 1,1,1-Trifluoro-2-chloroethane, Environmental Health Perspectives (EHP), 21:185-188, 1977 (4 pages, RDB8813)

toxicity of R-133a; health effects

R. Culik and D. P. Kelly, Embryotoxic and Teratogenic Studies in Rats with Chlorotrifluoroethane (FC 133a), report 127-79, Haskell Laboratory for Toxicology and Industrial Medicine, E. I. duPont de Nemours and Company, Incorporated, Newark, DE, 1979 (RDB5376)

R-133a, health effects, developmental and reproductive toxicity as reported in RDB5371

E. C. M. Hodge, D. Anderson, I. P. Bennett, and T. M. Weight, Arcton 133a: Dominant Lethal Study in the Mouse, report CTL/P/467, Central Toxicology Laboratory, Imperial Chemical Industries Limited (ICl), Cheshire, UK, 1979 (RDB5377)

$\mathrm{R}-133 \mathrm{a}$, health effects, toxicity

E. C. M. Hodge, D. Anderson, I. P. Bennett, T. M. Weight, and J. Wilson, Arcton 133a: First Combined Dominant Lethal and Fertility Study in the Mouse, report CTL/P/461, Central Toxicology Laboratory, Imperial Chemical Industries Limited (ICl), Cheshire, UK, 1979 (RDB5378)

R-133a, health effects, toxicity

M. Kilmartin, D. Anderson, I. P. Bennett, D. Richards, T. M. Weight, and J. Wilson, Arcton 133a: Second Combined Dominant Lethal and Fertility Study in the Mouse, report CTL/P/544, Cen- tral Toxicology Laboratory, Imperial Chemical Industries Limited (ICl), Cheshire, UK, 1980 (RDB5875)

$\mathrm{R}-133 \mathrm{a}$, health effects, toxicity as reported in RDB5137

M. Shulman and M. S. Sadove (University of Illinois Research and Education Hospitals), Safety Evaluation and Anesthetic Properties of 1,1,1Trifluoroethyl Chloride, Toxicology and Applied Pharmacology (TAP), 7:473-477, 1965 (5 pages, RDB8812)

toxicity of R-133a; 10 minute $A D_{50}=$ approximately 80,000 ppm as reported in RDB8811

Summary of Toxicity Information: HCFC-133a, AlliedSignal Incorporated, Morristown, NJ, undated (after 1984) (5 pages, RDB8811)

toxicity of R-133a; health effects

\section{R-134}

M. A Collins, Toxicology Summary for 1,1,2,2Tetrafluoroethane (HFC 134), ICl Chemicals and Polymers Limited, Runcorn, UK, 9 June 1993 (2 pages, RDB6688)

R-134, summary of published and unpublished toxicity literature; $\mathrm{ICl}$ Hygiene Standard $=10$ ppm Occupational Exposure Band; no metabolism data available, but absorption is expected to be poor; reported to be of low acute inhalation toxicity, but no data are available; no chronic exposure data, but R-134 was administered to rats at $7 \mathrm{ppm}$ for $1 \mathrm{yr}$, as an impurity in refrigerant $134 a$, with no effect; mutagenicity tests (Ames test in human lymphocyte in vitro cytogenetics study) negative for genotoxic effects; concludes that $R-134$ is probably of low overall toxicity

Huntingdon Research Centre Limited (HRC), HFC 134: Assessment of Cardiac Sensitization Potential in Dogs, report DPT 300/932252, Haskell Laboratory for Toxicology and Industrial Medicine, E. I. duPont de Nemours and Company, Incorporated, Newark, DE, 21 February 1994 (29 pages, RDB6802)

$\mathrm{R}-134$, cardiac sensitization in beagle dogs for exposures with epinephrine challenge; NOEL at 50,000 and $75,000 \mathrm{ppm} \mathrm{v/v,} \mathrm{LOEL} 33 \%$ at $100,000 \mathrm{ppm}$

D. M. Krentz, M. A. Lee, and R. C. Graham, untitled toxicity review for 1,1,2,2-tetrafluoroethane, Haskell Laboratory for Toxicology and Industrial 
Medicine, E. I. duPont de Nemours and Company, Incorporated, Newark, DE, 6 October 1992 (RDB5864)

R-134, summary of published and unpublished toxicity literature

D. B. Warheit, Four-Hour Inhalation Approximate Lethal Concentration (ALC) of HFC-134 in Rats, report 452-91, Haskell Laboratory for Toxicology and Industrial Medicine, E. I. duPont de Nemours and Company, Incorporated, Newark, DE, 22 July 1991 revised 7 August 1991 (12 pages with 2 tables, RDB6188)

$4 \mathrm{hr}$ acute inhalation toxicity tests in groups of six male Charles River Crl:CD BR rats by noseonly exposures to R-134: no mortalities resulted at concentrations of $120,000,200,000$, 380,000 , and $460,000 \mathrm{ppm} v / \mathrm{v}$ in air with oxygen enrichment (to maintain $20-22 \%$ levels); no clinical signs were observed at $120,000 \mathrm{ppm}$; rats exposed to $200,000 \mathrm{ppm}$ showed no response to tapping on the chamber; rats exposed to 380,000 and $460,000 \mathrm{ppm}$ showed rapid breathing, but no response to a tail pinch or tapping on the chamber; all rats lost 1-6\% body weight 1 day following exposures but resumed weight gain thereafter; report concludes that the 4-hr ALC rat for R-134 exceeds $460,000 \mathrm{ppm}$ v/v and that $R-134$ is "considered to have very low toxicity" on an acute inhalation basis

report 725-90, Haskell Laboratory for Toxicology and Industrial Medicine, E. I. duPont de Nemours and Company, Incorporated, Newark, DE, 1990 (RDB6186)

$\mathrm{R}-134$, health effects, toxicity, mutagenicity, potential clastogenic (chromosome-damaging) activity as reported in RDB5864

report 729-90, Haskell Laboratory for Toxicology and Industrial Medicine, E. I. duPont de Nemours and Company, Incorporated, Newark, DE, 1990 (RDB6187)

R-134, health effects, toxicity, mutagenic potential, Ames Assay negative as reported in RDB5864

\section{$\mathrm{R}-134 \mathrm{a}$}

D. J. Alexander (Glaxo Research and Development Limited, UK), S. E. Libretto, M. J. Adams, E. $W$. Hughes, and $M$. Bannerman, HFA-134a (1,1,1,2-Tetrafluoroethane): Effects of Inhalation Exposure upon Reproductive Perform- ance, Development and Maturation of Rats, Human and Experimental Toxicology, 15(6):508517, 1996 (10 pages, RDB8416)

two series of toxicity tests of rats to R-134a: chronic exposures at $2,500,10,000$, or 50,000 $\mathrm{ppm}$ to assess effects on reproduction and development; exposures from days 17 to 20 of pregnancy and days 1 to 21 post partum to atmospheres of $1,800,9,900$, or $64,400 \mathrm{ppm}$; the only treatment-related effect was a slight reduction in body weight gain of males of the treated parental generation at $50,000 \mathrm{ppm}$; no adverse effects were observed in either study on the reproductive performance of treated animals or on the development, maturation, or reproductive performance of up to two successive generations

D. J. Alexander and S. E. Libretto (Glaxo Research and Development Limited, UK), An Overview of the Toxicology of HFA-134a $(1,1,1,2$ Tetrafluoroethane), Human and Experimental Toxicology, 14(9):715-720, 1995 (6 pages, RDBA931)

reviews the results of preclinical toxicology studies of R-134a for use as a pharmaceutical propellant for metered-dose inhaler (MDI) use [and also used as refrigerant]; outlines a comprehensive range of studies conducted in animal models selected for the individual investigations; indicates that exposures were by inhalation route in all in vivo studies except those for local tolerance and sensitization; reports that there were no incidences of mortality in rodents or dogs at extremely high vapor concentrations of $810,000 \mathrm{ppm} v / \mathrm{v}$; also reports that $R$ $134 a$ was deemed not toxic or oncogenic and be safe for use as an MDI propellant

D. J. Alexander (Glaxo Research and Development Limited, UK), S. E. Libretto, H. J. Chevalier, T. Imamura, et al., HFA-134a $(1,1,1,2-$ Tetrafluoroethane): Lack of Oncogenicity in Rodents after Inhalation, Human and Experimental Toxicology, 14(9):706-714, 1995 (6 pages, RDBA932)

reviews chronic toxicity studies of R-134a for use as a pharmaceutical propellant for metered-dose inhaler (MDI) use [and also used as refrigerant]; groups of 60 male and 60 female mice or Wistar rats were exposed snout-only to $\mathrm{R}-134 \mathrm{a}$ for $1 \mathrm{hr} / \mathrm{d}$ for at least 104 weeks at concentrations of $2,500,15,000$, and $75,000 \mathrm{ppm}$ $\mathrm{v} / \mathrm{V}$ for mice and at $2,500,10,000$, and 50,000 $\mathrm{ppm} \mathrm{v} / \mathrm{v}$ for rats; absorption was evident at each exposure and was dose related; no treat- 
ment-related effects on survival, clinical signs, body weights, hematology, or on the type, incidence, site, or severity of gross lesions was observed in either species; there was no effect of treatment on the type, incidence, site or severity of neoplasms in mice or rats; there were no non-neoplastic findings related to treatment in mice; concludes that R-134a is considered not to be oncogenic and to be safe for use in pharmaceutical MDIs

D. J. Alexander (Glaxo Research and Development Limited, UK), Safety of Propeliants, Journal of Aerosol Medicine, 8(supplement 1):S29-S34, 1995 (6 pages with 1 table, RDB8111)

summarizes a toxicological assessment of fluorochemical 134a for use as an aerosol propellant in metered-dose inhalers (MDIs, compound also is used as a refrigerant identified as R134a): test samples were prepared by adding "essentially all the manufacturing impurities" to a stock that was in excess of $99.9 \%$ pure; results showed that R-134a has exceptionally low acute toxicity and is devoid of genotoxicity and, in rats and mice, oncogenic potential; tests showed no toxicity in rabbits, rats, mice, or dogs exposed to maximum concentrations of $40,000,50,000,75,000$, or $120,000 \mathrm{ppm} v / \mathrm{v}$, respectively, for up to one year; no fetotoxicity, effects on reproductive performance, peri- or post-natal development, sensitization or local irritation to the eyes or skin was found; mice and rats exposed to concentrations of $810,000 \mathrm{ppm}$ $\mathrm{v} / \mathrm{v}$ with oxygen supplementation for $1 \mathrm{hr}$ showed no evidence of clinical reactions or acute toxicity; dogs were essentially unaffected by $R-134$ a during acute inhalation exposures of up to $80,000 \mathrm{ppm}$ but showed intolerance and minor motor disturbances at concentrations of 160,000 and $320,000 \mathrm{ppm} v / \mathrm{v}$ without oxygen enrichment; all species tested demonstrated high systemic concentrations with half-lives of 4-7 minutes following a 1-hr exposure; metabolism was very limited in rats with the main metabolites being carbon dioxide and trifluoroacetic acid (TFA) in the urine, amounting to less than $0.67 \%$ of the administered dose; TFA was detected at trace levels in the urine of humans exposed to high doses of R-134a; article concludes that $R-134 a$ is suitable for MDI use

P. Graepel and D. J. Alexander (Glaxo Research and Development Limited, UK), CFC Replacements: Safety Testing, Approval for Use in Metered Dose Inhalers, Journal of Aerosol Medicine, 4(3):193-200, 1991 (8 pages, RDB8193) toxicity of R-134a and others tested as metered-dose inhaler (MDI) propellants

D. Anderson and C. R. Richardson, Arcton 134a: A Cytogenetic Study in the Rat, report CTL/P/444 (SR0002, FYI-OTS-0689-0698), Central Toxicology Laboratory, Imperial Chemical Industries Limited (ICl), Cheshire, UK, 17 May 1979 (RDB5B33)

R-134a, mutagenicity, toxicity: no chromosomal damage in rat bone marrow cells as reported in RDB5150, RDB5870, and RDB6533

A. Araki (Japan Bioassay Laboratory, JBL, Japan), Report on Reverse Mutation Assay in Bacteria on Tetrafluoroethane, report 5292/5312, Japan Industrial Safety and Health Association, Kanagawa, Japan, 11 October 1990 (RDB6534)

mutagenicity test of R-134a; health effects; toxicity

M. Asakura (Japan Bioassay Laboratory, JBL, Japan), Report on a Chromosomal Aberration Test of 1,1,1,2-Tetrafluoroethane in Cultured Mammalian Cells, report 5879 for the Program for Alternative Fluorocarbon Toxicity Testing (PAFT), Japan Industrial Safety and Health Association, Kanagawa, Japan, 14 June 1990 (RDB6535)

mutagenicity test of R-134a; health effects; toxicity

R. D. Callander and K. P. Priestley, HFC 134a: An Evaluation Using the Salmonella Mutagenicity Assay, report CTL/P/2422 for the Program for Alternative Fluorocarbon Toxicity Testing (PAFT), Central Toxicology Laboratory, Imperial Chemical Industries Limited (ICI), Cheshire, UK, 16 March 1990 (RDB5B37)

$$
R-134 a \text {, toxicity }
$$

C. A. Coleman and R. S. Thompson, HFC 134a: Determination of the Acute Toxicity to Pseudomonas Putida, report CTL/BL3980/B, Central Toxicology Laboratory, Imperial Chemical Industries Limited (ICl), Cheshire, UK, 1990 (RDB65BO)

$\mathrm{R}-134 \mathrm{a}$, toxicity as reported in RDB6533

M. A. Collins ( $\mathrm{ICl}$ Chemicals and Polymers Limited, UK), G. M. Rusch (AlliedSignal Incorporated, USA), F. Sato (Showa Denko K.K., Japan), P. M. Hext (Zeneca Central Toxicology Laboratory, UK), and R-J. Millischer (Elf Atochem S.A., France), 1,1,1,2-Tetrafluoroethane: Repeat Exposure Inhalation Toxicity in the Rat, Developmental Toxicity in the Rabbit, and Genotoxicity in Vitro and in Vivo, Fundamental and Applied Toxicol- 
ogy, 25(2):271-280, May 1995 (10 pages with 1 figure and 6 tables, RDB5610)

This paper summarizes toxicity studies for animal exposures to R-134a by inhalation. It presents the findings for subchronic and chronic rat exposures to $0,2,500,10,000$, and 50,000 ppm for 13,52 , and 104 weeks. No statistically significant effects were observed on clinical condition, growth, and survival or on hematological, clinical chemistry, and urinary parameters. The only treatment-related pathological changes seen were increased incidence of Leydig cell hyperplasia (abnormal or unusual increase) and adenoma (benign tumor of glandular structure or origin) in male rats exposed to $50,000 \mathrm{ppm}$. The tumors, which also were found in control animals, were benign and not life threatening. The survival rates are plotted and key data are tabulated for these chronic inhalation studies. The paper also summarizes a battery of "in vivo" and "in vitro" tests for genotoxic activity. They include mouse micronucleus, unscheduled DNA synthesis assays, Ames assay, cytogenic assay in cultured mammalian cells, and cytogenic assay in human lymphocytes. Pregnant rabbits exposed to $\mathrm{R}-134 \mathrm{a}$ showed minimal maternal toxicity at high concentrations, but there were no effects on fetal development. The paper presents the test methods and conditions, statistical analysis approaches, and results. Data are tabulated for the developmental toxicity and in vivo genotoxicity studies. The paper concludes that $R-134 a$ is of very low toxicity.

M. A. Collins, HFC 134a: Acute Toxicity in Rats to Tetrafluoroethane, report CTL, Central Toxicology Laboratory, Imperial Chemical Industries Limited (ICI), Cheshire, UK, 1984 (RDB65A9)

$\mathrm{R}-134 \mathrm{a}$, inhalation toxicity as reported in RDB6533

K. M. Cooper, S. F. Chang, and L. I. Harrison, Determination of the Chlorofluorocarbon Substitute 1,1,1,2-Tetrafluoroethane (HFA-134a) in Human and Animal Blood Using Gas Chromatography with Headspace Analysis, Journal of Chromatography, 667:85-93, 1995 (9 pages, RDB8118)

R-134a: biochemistry, pharmacokinetics, toxicity

D. Donnell, L. I. Harrison, S. Ward, N. M. Klinger, B. P. Ekholm, K. M. Cooper, I. Porietis, and J. McEwen, Acute Safety of the CFC-Free Propellant HFA-134a from a Pressurized Metered-
Dose Inhaler, European Journal of Clinical Pharmacology, 48:473-477, 1995 (5 pages, RDB8117)

acute inhalation toxicity tests of R-134a for use as a metered-dose inhaler (MDI) aerosol propellant (compound also is used as a refrigerant identified as R-134a): health effects

M. K. Ellis, L. A. Gowans, T. Green, and R. J. N. Tanner (Zeneca Central Toxicology Laboratory, then part of $\mathrm{ICl}$ Chemicals and Polymers Limited, UK), Metabolic Fate and Disposition of 1,1,1,2Tetrafluoroethane (HFC-134a) in Rat Following a Single Exposure by Inhalation, Xenobiotica, 23(7):719-729, July 1993 (11 pages, RDB6531)

R-134a, metabolism, toxicity: tests in rats following single $1-\mathrm{hr}$ inhalation exposures at $10,000 \mathrm{ppm}$; approximately $1 \%$ was recovered in urine, feces, and expired air after exposures, indicating low absorption of R-134a by the lungs; of the $1 \%$, approximately two-thirds were exhaled within $1 \mathrm{hr}$ of the cessation of exposure as unchanged R-134a; remainder was exhaled as carbon dioxide and excreted in urine and feces as trifluoroacetic acid; carbon dioxide was the major metabolite accounting for 0.22 and $0.27 \%$ of the inhaled dose in male and female rats, respectively; urinary excretion accounted for $0.09 \%$ of the dose and fecal excretion $0.04 \%$ of the dose by both sexes; total metabolism in urine, feces and as carbon dioxide amounted to 0.34 and $0.40 \%$ of the inhaled dose in males and females, respectively; no evidence for a specific uptake of R-134a or metabolites into any organ or tissue analyzed, including fat

M. K. Ellis, L. A. Gowans, and T. Green, Hydrofluorocarbon 134a: Pharmacokinetics and Metabolism in Rats Following a Single Exposure by Inhalation, report CTLR/1090 (TSCAT report OTS-0536297), Central Toxicology Laboratory, Imperial Chemical Industries Limited (ICI), Cheshire, UK, circa 1985 (RDB6581)

R-134a, biochemical study, toxicity

H. H. Emmens and E. M. G. Hoogendijk, Report on an Ascending Dose Safety Study Comparing HFA-134a with CFC-12 and Air, Administered by Whole-Body Exposure to Healthy Volunteers, report V98.754 - volume 1 (also identified as Honeywell, the AlliedSignal, report MA-RR-992539 and MA-250B-82-306) for the Program for Alternative Fluorocarbon Toxicity Testing (PAFT) and the International Pharmaceutical Aerosol Consortium for Toxicology Testing OF HFA-134a (IPACT-I), TNO Nutrition and Food Research In- 
stitute, Zeist, The Netherlands, 23 February 1999 (154 pages, RDBA535)

documents a study to investigate the effect of $\mathrm{R}-134 \mathrm{a}$ on electrocardiogram (ECG), pulse rate, blood pressure, lung function, and pharmacokinetic data in relation to exposure levels in 8 ( 4 male and 4 female) healthy human volunteers; exposures were for $1 \mathrm{hr}$ each to ascending (subject to termination if lower concentrations indicated adverse affect) target concentrations of $1000,2000,4000$, and 8000 $\mathrm{ppm} \mathrm{v/v} \mathrm{in} \mathrm{air;} \mathrm{comparative} \mathrm{exposures} \mathrm{were}$ administered to air and to 1000 and $4000 \mathrm{ppm}$ $\mathrm{v} / \mathrm{v} \mathrm{R}-12$ in air as controls; introduction notes that the study was designed to evaluate responses associated with inhalation of R-134a at levels achieved in industrial, medical (pharmaceutical propellant in metered-dose inhalers, MDI), or emergency (fire suppression) uses; it cites motivation to respond to questions arising from an earlier pharmacokinetic study (see RDB7C80) halted based on observed effects, subsequently deemed to have been caused by factors other than R-134a exposure such as physical or psychological factors; concludes that there were no treatment-related effects of inhalation exposure to R-134a on ECG, pulse rate, systolic and diastolic blood pressure, or lung function for maximum concentrations of $8000 \mathrm{ppm} \mathrm{v} / \mathrm{v}$ target $(7600 \mathrm{ppm} \mathrm{v} / \mathrm{v}$ mean actual) R-134a compared to air or up to $4000 \mathrm{ppm}$ $\mathrm{v} / \mathrm{v}$ target (3913 ppm v/v mean actual) $\mathrm{R}-12$

H. H. Emmens and E. M. G. Hoogendijk, Report on an Ascending Dose Safety Study Comparing HFA-134a with CFC-12 and Air, Administered by Whole-Body Exposure to Healthy Volunteers, report V98.754 - volume 2 (also identified as Honeywell, the AlliedSignal, report MA-RR-992539 and MA-250B-82-306) for the Program for Alternative Fluorocarbon Toxicity Testing (PAFT) and the International Pharmaceutical Aerosol Consortium for Toxicology Testing OF HFA-134a (IPACT-I). TNO Nutrition and Food Research Institute, Zeist, The Netherlands, 23 February 1999 (99 pages, RDBA536)

documents the protocol including safety precautions for a study to investigate the effect of $\mathrm{R}-134 \mathrm{a}$ on electrocardiogram (ECG), pulse rate, blood pressure, lung function, and pharmacokinetic data in relation to exposure levels in 8 ( 4 male and 4 female) healthy human volunteers (see RDBA535); exposures were for 1 $\mathrm{hr}$ each to ascending (subject to termination if lower concentrations indicated adverse affect) target concentrations of $1000,2000,4000$, and
$8000 \mathrm{ppm} \mathrm{v/v}$ in air; comparative exposures were administered to air and to 1000 and 4000 ppm v/v R-12 in air as controls

J. Ferguson-Smith, Hygiene Standard Documentation - HFC 134a, document $\mathrm{HSP} / 93 / 02, \mathrm{ICl}$ Chemicals and Polymers Limited, Cheshire, UK, 1993 (9 pages with 2 tables, RDB5A79)

$\mathrm{R}-134 \mathrm{a}$, toxicity, literature review, rationale for recommended hygiene standard (occupational exposure limit, OEL) of 1,000 ppm, 8-hr TWA

E. L. Frederickson and C. Nielson, Progr. Anaesthesiology (proceedings of the 4th World Congress of Anaesthesiology, 1968), 723-729, 1970 (7 pages, RDB6532)

$\mathrm{R}-134 \mathrm{a}$, cardiac study, health effects, toxicity

P. W. Grube, D. M. Krentz, M. A. Lee, and R. C. Graham, untitled toxicity review for ethane, 1,1,1,2-tetrafluoro-, Haskell Laboratory for Toxicology and Industrial Medicine, E. I. duPont de Nemours and Company, Incorporated, Newark, DE, 14 February 1994 (18 pages, RDB5870)

$R-134 a$, summary of published and unpublished toxicity literature

C. J. Hardy (Huntingdon Research Centre Limited, HRC, UK), HFC-134a: Cardiac Sensitization Study in Dogs, report ISN 250/91169, Central Toxicology Laboratory, Imperial Chemical Industries Limited (ICI), Cheshire, UK, 1991 (RDB6B44)

R-134a, toxicity

L. I. Harrison (3M Pharmaceuticals), Pharmacokinetics of HFA-134a: A Preliminary Report, American Journal of Therapeutics, 3(11):763-766, November 1996 ( 3 pages with 1 figure and 2 tables, RDB7537)

combines the accumulated data from four clinical studies to provide a preliminary description of the pharmacokinetics of R-134a: notes that $\mathrm{R}-134 \mathrm{a}$ is absorbed and eliminated quickly, with an estimated half-life 5.1 minutes based on a population pharmacokinetics approach; subjects were healthy humans and, in one study, mild asthmatic patients; a total of 34 subjects were exposed and there were 24 controls

P. M. Hext and J. R. Parr-Dobrzanski, HFC 134a: 2-Year Inhalation Toxicity Study in the Rat, report CTL/P/3841, Zeneca Central Toxicology Laboratory (then part of $\mathrm{ICl}$ Chemicals and Polymers Limited), Cheshire, UK, 19 March 1993 (RDB6546) 
R-134a, health effects, $2-y r$ inhalation toxicity study in rats exposed to $0,2,500,10,000$, and $50,000 \mathrm{ppm}$ for $6 \mathrm{hr} / \mathrm{d}, 5 \mathrm{~d} / \mathrm{wk}$ : no significant differences in survival, body weight, clinical chemistry (except occasional small increases in urinary fluoride levels at 10,000 and 50,000 ppm), hematology, or organ weight (with exception of testes); mean testicular weight was higher at 50,000 ppm; tumor incidence comparable between control and exposed groups except at $50,000 \mathrm{ppm}$, at which the incidence of Leydig cell hyperplasia and benign Leydig cell tumors was statistically higher, low incidence of generalized large granular lymphocyte leukemia was seen across all groups, and while slightly higher in the control group and at $50,000 \mathrm{ppm} ; \mathrm{NOEL}=10,000 \mathrm{ppm}$ - as reported in RDB5870; see RDB 6A01 for discussion, which notes that "Leydig-cell tumors are not considered indicative of adverse effects in humans because they are not applicable to humans"

P. M. Hext, HFC 134a: 90-day Inhalation Toxicity Study in the Rat, report CTL/P/2466 for the Program for Alternative Fluorocarbon Toxicity Testing (PAFT), Central Toxicology Laboratory, Imperial Chemical Industries Limited (ICl), Cheshire, UK, 15 November 1989 (RDB5B38)

$\mathrm{R}-134 \mathrm{a}$, toxicity study in rats exposed to 2,000 , 10,000 , and $50,000 \mathrm{ppm}$ for $6 \mathrm{hr} / \mathrm{d}, 5 \mathrm{~d} / \mathrm{wk}$ : NOEL $=49,500$ ppm as reported in RDB5870

M. C. Hodge, M. Kilmartin, R. A. Riley, T. M. Weight, and J. Wilson, Arcton 134a: Teratogenicity Study in the Rat, report CTL/P/417 (FYIOTS-0689-0698), Central Toxicology Laboratory, Imperial Chemical Industries Limited (ICI), Cheshire, UK, 9 January 1980 (RDB5B31)

$\mathrm{R}-134 \mathrm{a}$, developmental and reproductive toxicity based on tests at 0 (control), 1,000,10,000, and $50,000 \mathrm{ppm}$; concludes that R-134a is neither teratogenic nor embryotoxic at the levels tested as reported in RDB5150

M. C. E. Hodge, D. Anderson, I. P. Bennett, and T. $M$. Weight, Arcton 134a: Dominant Lethal Study in the Mouse, report CTL/R/437 (FYI-OTS0689-0698), Central Toxicology Laboratory, Imperial Chemical Industries Limited (ICI), Cheshire, UK, 8 January 1979 (RDB5B32)

$\mathrm{R}-134 \mathrm{a}$, mutagenicity (negative), toxicity as reported in RDB5150 and RDB5870

IPACT Data Review Committee, Interpretation of Brain Weight Data from the Study 'HFC 134a: 90-day Inhalation Toxicity Study in the Rat (ICI Report Number CTL/P/2466, November 15, 1989)', International Pharmaceutical Aerosol Consortium for Toxicology Testing (IPACT), 1990 (RDB7B42)

$\mathrm{R}-134 \mathrm{a}$, interpretation of findings from a prior toxicity study (see RDB5B38) in rats exposed to $2,000,10,000$, and $50,000 \mathrm{ppm}$ for $6 \mathrm{hr} / \mathrm{d}, 5$ $\mathrm{d} / \mathrm{wk}$

Litton Bionetics Incorporated (LBI), Kensington, MD, 1976 (RDB6536)

$\mathrm{R}-134 \mathrm{a}$, health effects, extended oral toxicity study in rats administered $300 \mathrm{mg} / \mathrm{kg} / \mathrm{d}, 5 \mathrm{~d} / \mathrm{wk}$, for 52 weeks: no significant increase in the incidence of neoplasms was observed in any organ a reported in RDB5870

M. Lu and R. Staples, 1,1,1,2-Tetrafluoroethane (FC 134a): Embryo-Fetal Toxicity and Teratogenicity Study by Inhalation in the Rat, report 317-81 (TSCAT report OTS-0530569 and OTS0535914), Haskell Laboratory for Toxicology and Industrial Medicine, E. I. duPont de Nemours and Company, Incorporated, Newark, DE, 1981 (RDB6530)

$\mathrm{R}-134 \mathrm{a}$, developmental and reproductive toxicity

J. M. Mackay, HFA 134a: An Evaluation in the In Vitro Cytogenetic Assay in Human Lymphocytes, report CTL/P/2977 for the Program for Alternative Fluorocarbon Toxicity Testing (PAFT), Central Toxicology Laboratory, Imperial Chemical Industries Limited $(\mathrm{ICl})$, Cheshire, UK, 10 July 1990 (RDB5B41)

$\mathrm{R}-134 a$, mutagenicity, toxicity

T. A. Mahoney, Test Subject Faints After Inhaling HFC-134a in Botched Air Force Exposure Experiments, The Air Conditioning, Heating, and Refrigeration News, 202(8):1-2, 20 October 1997

(2 pages with no figures or tables, RDB7C82)

provides further information on the inhalation toxicity test responses reported in an Air-Force study (see RDB7C80) on exposures with R13B1, R-134a, and R-227ea: describes the tests in which "one subject lost consciousness and had to be revived" following exposure to R$134 \mathrm{a}$ at $4,000 \mathrm{ppm} \mathrm{v} / \mathrm{v}$ as "conducted somewhat haphazardly"; quotes D. Hufford (director of the U.S. Environmental Protection Agency, EPA, Stratospheric Ozone Protection Division) that the EPA believes "R-134a is safe and we are not urging any changes to its use"; quotes A. Vinegar (ManTech Environmental Technol- 
ogy, Incorporated, and coauthor of the original report) that the reported results were not a direct effect based on newer evidence; quotes a Program for Alternative Fluorocarbon Toxicity Testing (PAFT) statement (see RDB7C81) that the responses were "likely not a direct result of the chemical exposure"; quotes R. Rubenstein (an EPA toxicologist) that the first test subject reacted to a stress reaction from the manner of blood sampling and that similar causes are suspected in the other anomalous reactions

R-J. Millischer (Elf Atochem S.A., France), The Toxicology of HFC 134a, Journal of the American College of Toxicology (JACT), 8(6):1220 ff, 1989 (RDB6549)

summarizes toxicity assays of the acute, subacute, subchronic, and chronic toxicity as well as genotoxicity, teratogenicity and carcinogenicity of R-134a; notes that some tests are still in progress or planning, but the findings thus far point to a low order of toxicity; indicates a 4-hr $\mathrm{LC}_{50}$ in the rat of $>500,000 \mathrm{ppm} \mathrm{v/v}$ with acute exposure symptoms indicative of central nervous system (CNS) depression and anesthetic effects induced at sub-lethal levels; describes the cardiac sensitization potential as very low with a LOEL of $75,000 \mathrm{ppm}$ in dogs; indicates a NOEL at $50,000 \mathrm{ppm} \mathrm{v} / \mathrm{v}$ in subacute and subchronic inhalation studies in rats exposed for $6 \mathrm{ht} / \mathrm{d} 5 \mathrm{~d} / \mathrm{wk}$; describes a 90-day study, inhalation teratogenicity assays in the rat and rabbit; notes that no teratogenic effect was found at any of the exposure levels, although minimal maternal toxicity occurred at 40,000 $\mathrm{ppm} \mathrm{v} / \mathrm{v}$ in rabbits and maternal and embryofoetal toxicity was observed at $100,000 \mathrm{ppm} v / \mathrm{v}$ in rats; indicates that $R-134$ a was not genotoxic in a battery of short term tests in vitro studies including by Ames and human lymphocyte assays; notes that no carcinogenic potential was found in vivo in a micronucleus, dominant lethal, and similar studies as well as in a limited in vivo bioassay in rats receiving daily oral doses of $300 \mathrm{mg} / \mathrm{kg}$ in corn oil for $1 \mathrm{yr}$; notes that a 2-yr inhalation combined chronic toxicity and carcinogenicity bioassay in rats is in progress; describes a metabolic study in rats with radiolabelled material that found that $\mathrm{R}-134 \mathrm{a}$ is virtually not transformed in the body, is quickly eliminated via the respiratory route, and does not accumulated in any particular organ

L. S. Mullin, Ethane, 1,1,1,2-tetrafluoro-: Cardiac Sensitization, report 42-79, Haskell Laboratory for Toxicology and Industrial Medicine, E. I.
duPont de Nemours and Company, Incorporated, Newark, DE, 26 January 1979 (3 pages with 1 table, RDB6527)

toxicity tests to determine the cardiac sensitization potential of $R-134 a$ in male beagle dogs with intravenous injection of epinephrine five minutes before and, with a challenge injection, midway into a 10 minute exposure: no marked response in 10 dogs at $49,800 \mathrm{ppm} v / \mathrm{v} \mathrm{R}-134 \mathrm{a}$ in air [deemed a NOEL]; marked response in 2 of 10 dogs at $75,200 \mathrm{ppm}$ [deemed a LOEL] and in 2 of 4 dogs with one death at 101,900 ppm; concludes that R-134a is "a weak cardiac sensitizer similar to Halon 1301"

W. Müller and T. Hofmann (Pharma Research Toxicology and Pathology Laboratory), report 89.0115 (on study 88.1244), CFC 134a Micronucleus Test in Male and Female NMRI Mice After Inhalation, Hoechst Aktiengesellschaft, Frankfurt am Main, Germany, 16 March 1989 (36 pages, RDB6584)

\section{$\mathrm{R}-134 \mathrm{a}$, mutagenicity, toxicity}

M. J. Olson and S. E. Surbrook, Jr. (General Motors Research Laboratories), Human Metabolism of the CFC-Substitute HFC-134a: Role of Cytochrome P450IIE1, abstract 148, The Toxicologist, 12:62, 1992 (1 page with no figures or tables, RDB7538)

toxicity of R-134a: concludes that R-134a is "essentially nontoxic" based on rates of defluorination in human microsomes, with focus on the role of P450lIE1; defluorination rates were similar in humans, rabbits, and rats; suggests that potentiation of toxicity may be possible by P450lIE 1 induction for more-readily metabolized fluorochemicals, such as R-123

M. J. Olson (General Motors Research Laboratories), C. A. Reidy, J. T. Johnson, and T. C. Pederson, Oxidation of 1,1,1,2-Tetrafluoroethane in Rat Liver Microsomes is Catalyzed Primarily by Cytochrome P450lIE1, Drug Metabolism and Disposition, 19(2):298-303, 1991 (6 pages, RDB6537)

R-134a, health effects, metabolism, toxicity

M. J. Olson and S. E. Surbrook, Jr. (General Motors Research Laboratories), Defluorination of the CFC-Substitute 1,1,1,2-Tetrafluoroethane: Comparison in Human, Rat and Rabbit Hepatic Microsomes, Toxicology Letters, 59(1-3):89-99, December 1991 (11 pages, RDB6538)

$\mathrm{R}-134 \mathrm{a}$, toxicity: compares metabolism by hepatic microsomes in human tissue to that from rats and rabbits; defluorination of R-134a in a 
cytochrome-P450 catalyzed reaction was similar; maximal rate of $R-134 a$ metabolism was very low and showed little interindividual variation; findings indicate that laboratory animals are an adequate surrogate for humans to characterize R-134a toxicity, especially that which may be mediated by products of halocarbon metabolism

M. J. Olson (General Motors Research Laboratories), C. A. Reidy, J. T. Johnson, and T. C. Pederson, Oxidative Defluorination of 1,1,1,2Tetrafluoroethane by Rat Liver Microsomes, Drug Metabolism and Disposition, 18(6):992-998, 1990 (7 pages, RDB6539)

R-134a, health effects, metabolism, toxicity

M. J. Olson (General Motors Research Laboratories) C. A. Reidy, and J. T. Johnson, Defluorination of 1,1,1,2-Tetrafluoroethane (R-134a) by Rat Hepatocytes, Biochemical and Biophysical Research Communications, 166(3):1390-1397, 1990 (8 pages, RDB6540)

$\mathrm{R}-134 \mathrm{a}$, health effects, toxicity

M. J. Olson (General Motors Research Laboratories), C. A. Reidy, and J. T. Johnson, Modulation of Glucose Metabolism in Isolated Rat Hepato-

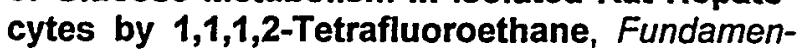
tal and Applied Toxicology, 15(2):270-280, August 1990 (11 pages, RDB6541)

R-134a, toxicity: effects on cell viability and functional competence at concentrations of $\mu 750,000 \mathrm{ppm}$ by glucose metabolism measurements in cultures of hepatocytes derived from fed or fasted rats; R-134a did not produce evidence of cytolethality following a 2-hr exposure; by contrast, R-123B1 caused cell death at 1250 ppm; $125,000-750,000$ ppm R-134a increased glycolysis (production of lactate and pyruvate) in a concentration-dependent manner in hepatocytes from fed rats; no effect was observed at 50,000 ppm; R-12, R-114, and R134 a were equal in potency to stimulate glycolysis at 250,000 ppm; R-115 depressed glycolysis slightly; R-123B1 markedly increased glycolysis rates at concentrations as low as 300 ppm; glucose production by hepatocytes of fed rats was decreased by R-12, R-114, and R$134 \mathrm{a}$ only at concentrations of $\cdot 250,000 \mathrm{ppm}$; R-123B1 potently decreased glucose production by hepatocytes at $\cdot 300$ ppm; R-134a exposure inhibited gluconeogenesis in a concentration-dependent manner in cells isolated from livers of fasted rats, although this effect was not significant until concentrations reached 125,000 ppm; comparative potency studies showed that $\mathrm{R}-12, \mathrm{R}-114$, and R-134a inhibited gluconeogenesis about equally at $250,000 \mathrm{ppm}$ while $R$ $123 \mathrm{~B} 1$ was effective at $300 \mathrm{ppm}$ and R-115 was without effect at $250,000 \mathrm{ppm}$; paper concludes that toxicologically significant alteration of glucose-linked bioenergetics is unlikely at anticipated levels of R-134a exposure in the workplace or environment

M. J. Olson (General Motors Research Laboratories) et al., abstract 202, The Toxicologist, 10(1):51 ff, 1990 (RDB6542)

$\mathrm{R}-134 \mathrm{a}$, health effects, toxicity

V. W. Pike, F. I. Aigbirhio, C. A. J. Freemantle, B. C. Page, C. G. Rhodes, S. L. Waters, T. Jones, P. Olsson, G. P. Ventresca (Glaxo Research and Development Limited, UK), R. J. N. Tanner, M. Hayes, and M. B. Hughes, Deposition of Inhaled 1,1,1,2-Tetrafluoroethane (HFA-134a) in Healthy Subjects and in Patients with Chronic Airflow Limitation, Drug Metabolism and Disposition, 23:832-839, 1995 (8 pages, RDB8116)

R-134a health effects, pharmacokinetics, toxicity

C. A. Reidy, J. T. Johnson, and M. J. Olson (General Motors Research Laboratories), Metabolism In Vitro of Fluorocarbon R-134a, abstract 1295, The Toxicologist, 10(1):324 ff, 1990 (RDB6543)

R-134a, biochemical study, health effects, toxicity

R. A. Riley, I. P. Bennett, I. S. Chart, C. W. Gore, M. Robinson, and T. M. Weight, Arcton 134a: Subacute Toxicity to the Rat by Inhalation, report CTLIP/463 (FYI-OTS-0689-0698), Central Toxicology Laboratory, Imperial Chemical Industries Limited (ICl), Cheshire, UK, 6 December 1979 (RDB5B34)

inhalation toxicity study in rats exposed to $1,000,10,000$, or $50,000 \mathrm{ppm} v / \mathrm{v} \mathrm{R}-134 \mathrm{a}$ in air for $6 \mathrm{hr} / \mathrm{d}, 5 \mathrm{~d} / \mathrm{wk}$, for 4 weeks: gas was absorbed into the blood; liver weights and plasma potassium levels were higher for 10,000 and $50,000 \mathrm{ppm}$ exposures; changes were noted in liver, kidney, and gonad weights of males at $50,000 \mathrm{ppm}$, but there were no pathology changes in these tissues; pathological change was observed in the lungs of several male rats at the $50,000 \mathrm{ppm}$ dose as reported in RDB5150 and RDB5870

S. B. Rissolo, 1,1,1,2-Tetrafluoroethane (Freon $^{\circledR}$ 134a): Acute Inhalation Toxicity, report 190-67, 
Haskell Laboratory for Toxicology and Industrial Medicine, E. I. duPont de Nemours and Company, Incorporated, Newark, DE, 2 November 1967 (2 pages with no figures or tables, RDB6525)

tests of the acute inhalation toxicity of R-134a in Charles River CD rats exposed to 750,000 $\mathrm{ppm} \mathrm{v/v} \mathrm{in} \mathrm{oxygen-enriched} \mathrm{air} \mathrm{for} 30 \mathrm{~min} ; 2$ of 4 rats died, one each at 20 and 29 minutes; rats showed incoordination, pumping respiration, darkening of the eyes, unresponsiveness, cyanosis (blue or purple discoloration of the skin or mucous membranes due to blood-oxygen deficiency), and convulsions; survivors were limp, but still showed a righting reflex after exposure and appeared to recover within $5 \mathrm{~min}$; one had a moderate weight loss the first day, but growth was normal for the remainder of the 14-day observation period; both rats that died had discolored and hyperinflated lungs with some areas of edema; the survivors were found in microscopic pathology to have pulmonary vascular congestion and edema, but no other compound related effects; recommends that $R$ $134 a$ be handled with care and in wellventilated areas in light of the findings and apparent lack of preliminary warning signs; findings imply a $30-\mathrm{min}$ ALC $2 / 4$ of $750,000 \mathrm{ppm}$ $\mathrm{v} / \mathrm{v}$

G. M. Rusch, Review of the ICI Toxicology Studies on Fluorocarbon 134a, report MA-141-80-1 (also identified as MA-RR-83-428), AlliedSignal Incorporated (then Allied Corporation), Morristown, NJ, 3 March 1983 (2 pages with no figures or tables, RDB5150)

review of teratogenicity; excretion, tissue distribution, and metabolism; subchronic toxicity; cytogenetic; and dominant lethal toxicity studies [see RDB5B31, RDB5B35, RDB5B34, RDB5B33, and RDB5B32, respectively]; concludes that $R-134$ a has a low order of toxicity showing only slight effects at an exposure level of $50,000 \mathrm{ppm}$; notes that R-134a is not teratogenic, embryotoxic, or mutagenic and is rapidly eliminated and poorly metabolized

A. G. Salmon, J. A. Nash, M. F. S. Oliver, and A. Reeve, Arcton 134: Excretion, Tissue Distribution, and Metabolism in the Rat, report CTL/P/513 (FYI-OTS-0689-0698), Central Toxicology Laboratory, Imperial Chemical Industries Limited (ICl), Cheshire, UK, 6 March 1980 (RDB5B35)

R-134a, biochemical study, toxicity: concludes that $\mathrm{R}-134 \mathrm{a}$ is rapidly eliminated and only poorly metabolized in the rat as reported in RDB5150
M. Shulman and M. S. Sadove (University of Illinois Research and Education Hospitals), 1,1,1,2Tetrafluoroethane. An Inhalation Anesthetic Agent of Intermediate Potency, Anesthesia and Analgesia, 46(5):629-635, September-October 1967 ( 7 pages with 3 figures and 3 tables, RDB7274)

examination of R-134a as an anesthetic: discusses effect levels in the mouse, $\left(E C_{50}\right.$ $270,000 \mathrm{ppm} \mathrm{v} / \mathrm{v}$ ), dog, cat, and rhesus monkey; notes cardiac sensitization, and specifically ventricular fibrillation (very rapid and irregular contractions of the ventricles of the heart resulting in loss of synchronization between the heartbeat and pulse beat), by R$134 a$ to catecholamines and infusions of epinephrine or isoproterenol; includes discussion by J. F. Artusio, Jr. (New York Hospital, Cornell University Medical Center)

L. S. Silber, Acute Inhalation Toxicity Study of Tetrafluoroethane, report 422-79, Haskell Laboratory for Toxicology and Industrial Medicine, E. I. duPont de Nemours and Company, Incorporated, Newark, DE, 3 August 1979 (3 pages with 1 table, RDB6529)

$4 \mathrm{hr}$ acute inhalation toxicity tests of R-134a in groups of six male Charles River $C D$ rats by whole body exposures: mortality ratios and corresponding concentrations were $0 / 6$ at $81,100,0 / 6$ at $205,000,0 / 6$ at $359,300,5 / 6$ at $566,700,0 / 6$ at 646,700 , and $2 / 6$ at 652,700 $\mathrm{ppm} \mathrm{V} / \mathrm{v}$ in air with oxygen enrichment; rats showed lethargy and rapid respiration at $205,200 \mathrm{ppm}$ and additionally trembling and tearing at $359,300 \mathrm{ppm} ; 4$ of 6 showed weight loss within $24 \mathrm{hr}$ of exposures at $359,300 \mathrm{ppm}$ followed by weight gain; the report does not comment on the high fatality occurrence at 566,700 ppm compared to the two higher concentrations tested, but the range of measured concentrations for this group is reported as $494,400-668,800 \mathrm{ppm}$, the high end of which is similar $(664,300-685,300)$ for the three highest concentrations tested

L. S. Silber and G. L. Kennedy, Subacute Inhalation Toxicity of Tetrafluoroethane (FC 134a), report 228-79, Haskell Laboratory for Toxicology and Industrial Medicine, E. I. duPont de Nemours and Company, Incorporated, Newark, DE, August 1979 (RDB6528)

$\mathrm{R}-134 \mathrm{a}$, toxicity study in rats: no exposure-related hematological, chemical analysis, or pathologic changes were observed; significant in- 
crease in fluoride excretion indicates metabolism of R-134a as reported in RDB5870

S. E. Surbrook, Jr., and M. J. Olson (General Motors Research Laboratories), Dominant Role of Cytochrome P450 2E1 in Human Hepatic Microsomal Oxidation of the CFC-Substitute 1,1,1,2Tetrafluoroethane, Drug Metabolism and Disposition, 20(4):518-524, 1992 (7 pages, RDB6544)

$\mathrm{R}-134 \mathrm{a}$, health effects, metabolism, toxicity

J. D. Taylor, 1,1,1,2-Tetrafluoroethane: Mutagenic Activity in the Salmonella/Microsome Assay, report 534-78, Haskell Laboratory for Toxicology and Industrial Medicine, E. I. duPont de $\mathrm{Ne}-$ mours and Company, Incorporated, Newark, DE, 1978 (RDB6526)

summarizes a mutagenicity study of R-134a by Ames assay; indicates that the results were negative; toxicity - as reported in RDB5870

R. W. Trueman, HFC 134a: Assessment for the Induction of Unscheduled DNA Synthesis in Rat Hepatocytes in Vivo, report CTL/P/2550 (SR0337) for the Program for Alternative Fluorocarbon Toxicity Testing (PAFT), Central Toxicology Laboratory, Imperial Chemical Industries Limited (ICl), Cheshire, UK, June 1990 (RDB5B40)

summarizes a mutagenicity study of $R-134 a$; toxicity

G. P. Ventresca (Glaxo Research and Development Limited, UK), Clinical Pharmacology of HFA134a, Journal of Aerosol Medicine, 8(supplement 1):S35-S39, 1995 (5 pages with 4 figures and 1 table, RDB8112)

summarizes an assessment in healthy human males of the safety, tolerability, and pharmacokinetics of fluorochemical 134a for use as an aerosol propellant in metered-dose inhalers (MDIs, compound also is used as a refrigerant identified as R-134a): absorption and disposition also were investigated in both healthy subjects and patients with severe chronic obstructive pulmonary disease (COPD) using radiolabeled R-134a; no serious adverse events were reported and there were no clinically significant changes in vital signs, electrocardiogram (ECG), pulmonary function tests, and laboratory parameters; R-134a was mainly eliminated by exhalation within several minutes; it was rapidly absorbed into the blood after inhalation, reaching maximum concentrations in 30-60 seconds, and distributed throughout the body with no obvious accumulation in any region; peak blood concentrations were highly variable, but generally dose dependent; blood concentrations declined rapidly after dosing, with an apparent half-life of 31 minutes; metabolism was not found to be significant; article, concludes that inhalation grade $\mathrm{R}-134 \mathrm{a}$ is safe and well tolerated in healthy subjects and that it is rapidly eliminated by exhalation without significant metabolism in by healthy subjects and COPD patients; no accumulation was found with repeat dosing

G. A. Wickramratne (possibly Wickramaratne), Fluorocarbon 134a: Embryotoxicity Inhalation Study in the Rabbit, report CTL/P/2380 for the Program for Alternative Fluorocarbon Toxicity Testing (PAFT), Central Toxicology Laboratory, Imperial Chemical Industries Limited (ICI), Cheshire, UK, 23 November 1989 (RDB5B36)

$\mathrm{R}-134 \mathrm{a}$, developmental and reproductive toxicity

G. A. Wickramratne (possibly Wickramaratne), HFC 134a: Teratogenicity Inhalation Study in the Rabbit, report CTL/P/2504 for the Program for Alternative Fluorocarbon Toxicity Testing (PAFT), Central Toxicology Laboratory, Imperial Chemical Industries Limited (ICI), Cheshire, UK, 23 November 1989 (RDB5B39)

$\mathrm{R}-134 \mathrm{a}$, developmental and reproductive toxicity

A. Woodcock (Wythenshawe Hospital, UK), Continuing Patient Care with Metered-Dose Inhalers, Journal of Aerosol Medicine, 8(supplement 2):S5-S10, 1995 (6 pages with 1 figure, RDB8113)

outlines clinical tests of fluorochemical 134a as an aerosol propellant in metered-dose inhalers (MDIs, compound also is used as a refrigerant identified as $R-134 a)$ : notes very few adverse affects from the R-134a inhalers, generally comparable to those from R-12/11 inhalers; paper discusses replacement of R-12/11 blends and the transition from to alternatives for MDI use

A Seven Day Multiple Dose Tolerability and Pharmacokinetic Study of HFA-134a in Healthy Volunteers at Two Dose Levels $(4 \times 100 \mu \mathrm{L}$ and $8 \times 100 \mu L$ ) Given Four Times Daily, report 7928, ICR, 1993 (RDBA537)

toxicity of R-134a; tests in humans

Criteria Group for Occupational Standards: Consensus Report for 1,1,1,2Tetrafluoroethane, Arbete och Hisa, 19:86-88, 1995 (3 pages, RDB8422) 
toxicological review of R-134a: concludes that the critical toxicity of R-134a is its effect on heart rhythm based on limited data from animal studies

HFC 134a: 2-Year Inhalation Toxicity Study in the Rat. Interim Report after 52 Weeks, TSCATS report 421398 (FYI-OTS-1091-0695) for the Program for Alternative Fluorocarbon Toxicity Testing (PAFT), Central Toxicology Laboratory, Imperial Chemical Industries Limited (ICI), Cheshire, UK, 30 September 1991 (RDB7C78)

$\mathrm{R}-134 a$, chronic toxicity study [also see RDB6582]

HFC 134a: 2-Year Inhalation Toxicity Study in the Rat. Interim Report after 52 Weeks, report CTL/P/3317 for the Program for Alternative Fluorocarbon Toxicity Testing (PAFT), Central Toxicology Laboratory, Imperial Chemical Industries Limited (ICI), Cheshire, UK, 21 August 1991 (RDB6582)

$\mathrm{R}-134 \mathrm{a}$, chronic toxicity study

Measurement of Dissociation Constant in Water of 1,1,1,2-Tetrafluoroethane, Kurume Research Laboratories (KRL), Fukuoka, Japan, 28 August 1990 (RDB7276)

ecotoxicity of R-134a; environmental impacts and fate; groundwater and soil

Measurement of 1-Octanol/Water Partition Coefficient of 1,1,1,2-Tetrafluoroethane, report 80678, Kurume Research Laboratories (KRL), Fukuoka, Japan, 22 August 1992 (RDB7277)

ecotoxicity of R-134a; environmental impacts and fate; groundwater and soil

Test on Biodegradability of HFC-134a by Microorganisms (Closed-Bottle Method), report 11598, Kurume Research Laboratories (KRL), Fukuoka, Japan, 22 August 1990 (RDB7275)

ecotoxicity of R-134a; environmental impacts and fate; groundwater and soil

1,1,1,2-Tetrafluoroethane (HFC-134a, CAS No. 811-97-2), Joint Assessment of Commodity Chemicals (JACC) report 31, European Chemical Industry Ecology and Toxicology Centre (ECETOC), Brussels, Belgium, February 1995 (42 pages with 1 figure and 3 tables, RDB6533)

R-134a, environmental impacts, toxicity

1,1,1,2-Tetrafluoroethane (HFC 134a) Health Effects Information, CAS\# 811-97-2, Elf Atochem
North America, Incorporated, Philadelphia, PA, undated circa 1995 (6 pages, RDB5B56)

$\mathrm{R}-134 \mathrm{a}$, summary of toxicological and ecological information

Workplace Environmental Exposure Level Guide - 1,1,1,2-Tetrafluoroethane, American Industrial Hygiene Association (AIHA), Fairfax, VA, 1991 (4 pages, RDB4B86)

R-134a, WEEL, toxicology data, toxicity, safety classification

\section{R-E134}

R. C. Graham and P. J. Lardear, untitled review of toxicity literature for bis(difluoromethyl) ether, Haskell Laboratory for Toxicology and Industrial Medicine, E. I. duPont de Nemours and Company, Incorporated, Newark, DE, 15 April 1994 (5 pages, RDB6206)

summary of published and unpublished information on the toxicity of R-E134

D. A. Keller et al., HFOC-134E, report for project MR-9563-1, Haskell Laboratory for Toxicology and Industrial Medicine, E. I. duPont de Nemours and Company, Incorporated, Newark, DE, 25 March 1994 (1 page with no figures or tables, RDB6207)

summarizes acute inhalation toxicity tests of RE134 in rats; indicates that rats exposed to approximately $100,000 \mathrm{ppm} v / \mathrm{v}$ for $4 \mathrm{hr}$ showed narcosis, but no other effects; notes that they recovered quickly and that no adverse effects were apparent three days later; indicates that rats exposed to approximately $45,000 \mathrm{ppm} \mathrm{v} / \mathrm{v}$ for $4 \mathrm{hr}$ showed light narcosis, but no other effects, and also recovered quickly; indicates that rats exposed to $18,000 \mathrm{ppm}$ v/v for $4 \mathrm{hr}$ showed no effects; concludes that the 4-hr ALC exceeds $100,000 \mathrm{ppm}$ v/v [a Haskell summary of this study elaborates that the tests were conducted in groups of 3 rats, cites the high exposure concentration as $95,000 \mathrm{ppm} \mathrm{v} / \mathrm{v}$; indicates the resulting 4-hr ALC rat as $>95,000$ ppm, and describes the behavior as incoordination and loss of response to sound - as reported in RDB6206]

C. W. Simons, G. J. O'Neill, and J. A. Gribens, New Aerosol Propellants for Personal Products, U.S. patent 4,041,148, 9 August 1977 (4 pages, RDBA944)

claims invention of dimethyl ethers, and specifically bis(difluoromethyl) ether [R-E134] and perfluorodimethyl ether [R-E116], as aerosol 
propellants for cosmetic, hygienic, pharmaceutical, and other personal products; provides limited toxicity data on them of potential relevance for their use as refrigerants; indicates no deaths or recognizable biological effects during or after exposures in six mice exposed to concentrations as high as $750,000 \mathrm{ppm}$ v/v R-E116 for 1 $\mathrm{hr}$; indicates no deaths but anesthetic effects in ten mice exposed to slightly more than 200,000 $\mathrm{ppm}$ V/V R-E134 for $2 \mathrm{hr}$; also indicates no deaths or signs of convulsions in an unspecified quantity of rats exposed to $50,000 \mathrm{ppm} \mathrm{v/v}$ R-E134 for $3 \mathrm{hr} / \mathrm{d}$ for 5 days

\section{R-141b}

M. Bazzon and G. Hervouet (Institut National de Recherche Chimique Appliquée, IRCHA, France), Determination of Acute Toxicity of HCFC 141b to Brachydanio Rerio, report B.7073 (FYI report OTS-5900695) for the Program for Alternative Fluorocarbon Toxicity Testing (PAFT), LevalloisPerret, France, 7 September. 1989 (29 pages with 3 figures and 8 tables, RDB6475)

tests for acute aquatic toxicity to brachydanio rerio (zebra fish) exposed for $96 \mathrm{hr}$ to concen-

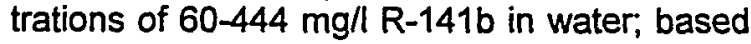
on observed mortalities, the $96-\mathrm{hr} \mathrm{LC}_{0}$ and $\mathrm{LC}_{50}$ were found to be $<60$ and $126 \mathrm{mg} / \mathrm{l}$, respectively

J. Bootman, G. Hodson-Walker, and J. M. Lloyd, CFC 141b: Investigation of Mutagenic Activity at the HGPRT Locus in a Chinese Hamster V79 Cell Mutation System, report 88/PSV005/257, Life Science Research Limited (LSR), Suffolk, UK, July 1988 (RDB59A1)

mutagenicity of R-141b (HCFC-141b); genotoxicity; genotoxic and mutagenic potential; toxicity

J. Bootman, G. Hodson-Walker, S. Cracknell, and C. A. Dance, HCFC 141b: Assessment of Clastogenic Action on Bone Marrow Erythrocytes in the Micronucleus Test, report 88/PSV029, Life Science Research Limited (LSR), Suffolk, UK, 1988 (RDB7746)

mutagenicity of R-141b; genotoxicity; genotoxic and mutagenic potential; toxicity

J. Bootman and G. Hodson-Walker, In Vitro Assessment of Clastogenic Activity of CFC 141 in Cultured Chinese Hamster Ovary (CHO-K1) Cells, report 88/PSV007/214, Life Science Research Limited (LSR), Suffolk, UK, 1988 (RDB7286) mutagenicity of R-141b (HCFC-141b); genotoxicity; genotoxic and mutagenic potential; toxicity - as reported in RDB5B57

D. Briand and G. Hervouet (Institut National de Recherche Chimique Appliquee, IRCHA, France), Determination of Acute Toxicity of HCFC 141b to Daphnia Magna, report B.7072 (FYI report OTS-5900695) for the Program for Alternative Fluorocarbon Toxicity Testing (PAFT), Elf Atochem S.A., Levallois-Perret, France, 7 September 1989 (31 pages with 3 figures and 9 tables, RDB6474)

tests for acute aquatic toxicity to daphnia magna exposed for $48 \mathrm{hr}$ to concentrations of 25.4-114.3 mg/l R-141b in water; based on observed immobilizations, the 24- and 48-hr $E C(I)_{50}$ (median effective inhibiting concentrations) were found to be 33.3 and $31.2 \mathrm{mg} / \mathrm{l}$, respectively

W. J. Brock (E. I. duPont de Nemours and Company, USA), H. J. Trochimowicz (DuPont), R-J. Millischer (Elf Atochem S.A., France), C. H. Farr (Elf Atochem North America, USA), T. Kawano (Daikin Industries, Japan), and G. M. Rusch (AlliedSignal Incorporated, USA), Acute and Subchronic Toxicity of 1,1-Dichloro-1-fluoroethane (HCFC-141b), Food and Chemical Toxicology (FCT), 33(6):483-490, June 1995 (8 pages with 3 tables, RDB65A4)

This paper summarizes acute and subchronic toxicity test methods and findings for R-141b, to assist in establishing proper handling guides. It describes the sample material used, acute oral toxicity tests in rats administered in corn oil, dermal toxicity tests in rats and rabbits, occular and dermal irritation tests in rabbits, and dermal sensitization studies in guinea pigs. It also outlines acute, two-week, four-week, and 90-day inhalation tests in rats, cardiac sensitization tests in beagle dogs and monkeys, and neurobehavioral toxicity tests in rats. The paper summarizes results including an oral $L D_{50}$ rat of $>5,000 \mathrm{mg} / \mathrm{kg}$ and oral $L D_{50}$ rat of $>2,000$ $\mathrm{mg} / \mathrm{kg}$, since none of the specimens died in these tests. It also provides inhalation lethality data by sex, concluding that the 4-hr and 6-hr $\mathrm{LC}_{50}$ rat is 61,647 and $56,700 \mathrm{ppm}$, respectively. It notes that reduced motor activity, rapid breathing, and anesthesia were observed at all concentrations above $29,000 \mathrm{ppm}$. The cardiac sensitization study showed a LOEL at 5,000 and $9,000 \mathrm{ppm}$ in the dog and monkey, respectively, the lowest concentrations tested; reference is made to earlier tests that showed 
an LOEL of $5,000 \mathrm{ppm}$ in dogs exposed for 5 minutes, also with epinephrine challenges. The NOAEL for subchronic inhalation studies was considered to be $8,000 \mathrm{ppm}$. No changes were found to indicate neurotoxicity. The data indicate that R-141b has low acute oral and inhalation toxicity potential, but is a mild skin and eye irritant.

W. J. Brock, Acute Dermal Toxicity Study of FC141b in Rabbits, report 501-88, Haskell Laboratory for Toxicology and Industrial Medicine, E. I. duPont de Nemours and Company, Incorporated, Newark, DE, 1988 (RDB6471)

toxicity of $R-141 \mathrm{~b}$ by skin contact; $L D_{50}$ dermal rabbit > 2,000 $\mathrm{mg} / \mathrm{kg}$ - as reported in RDB5868 and RDB5B57

W. J. Brock, Primary Eye Irritation Study with FC-141b in Rabbits, report 318-88, Haskell Laboratory for Toxicology and Industrial Medicine, E. I. duPont de Nemours and Company, Incorporated, Newark, DE, 1988 (RDB6470)

toxicity of R-141b; health effects; eye irritation in rabbits as reported in RDB5868 and RDB5B57

W. J. Brock, Skin Irritation Test in Rabbits of FC-141b, report 312-88, Haskell Laboratory for Toxicology and Industrial Medicine, E. I. duPont de Nemours and Company, Incorporated, Newark, DE, 1988 (RDB6469)

$R-141 b$, health effects, toxicity, dermal irritation in rabbits, not a skin irritant as reported in RDB5868 and RDB5B57

CIVO, report V89.214, Netherlands Organization for Applied Scientific Research (TNO), Apeldoorn, The Netherlands, 1989 (RDB5973)

$\mathrm{R}-141 \mathrm{~b}$, toxicity

CIVO Institute, report V88.156/271236, Netherlands Organization for Applied Scientific Research (TNO), Apeldoorn, The Netherlands, 1988 (RDB5974)

$\mathrm{R}-141 \mathrm{~b}$, health effects, toxicity

CIVO Institute, Chromosome Analysis of Chinese Hamster Ovary Cells Treated in Vitro with FC 141b, report 56645/50/88, Netherlands Organization for Applied Scientific Research (TNO), Apeldoorn, The Netherlands, 1988 (RDB7281)

R-141b, HCFC-141b, genotoxicity, health effects, toxicity
D. W. Coombs et al., Potential Neuropathological Effects of Exposure of Rats to the Vapour (6 Hours a day, 5 Days a Week Over a 16-Week Period) of HCFC-141b, report for the Program for Alternative Fluorocarbon Toxicity Testing (PAFT), Huntingdon Research Center Limited (HRC), Cambridgeshire, UK, 9 January 1992 (RDB7279)

$R-141 b$, health effects, subchronic toxicity as reported in 5B57

D. E. Coombs, C. J. Hardy, D. Crook, P. A. Mullins, and C. Gopinath (Huntingdon Research Centre Limited, HRC, UK), FC-141b: Two-Week Inhalation Study in the Rat, report PWT 76/8893, Elf Atochem North America (then Pennwalt Corporation), King of Prussia, PA, December 1988 (RDB5996)

$R-141 b$, health effects, subchronic toxicity

C. H. de Rooij (Solvay et Cie S.A., Belgium), The Toxicology of HFA-141b, Proceedings of the European Meeting of the Toxicology Forum (Toulouse, France, 18-22 September 1989), Société Française de Toxicologie, France, 204-216, 1989 (13 pages, RDB65A7)

R-141b, health effects, toxicity

C. Doleba-Crowe, Acute Inhalation Toxicity - 6 Hours (FC 141b), report 61-77, Haskell Laboratory for Toxicology and Industrial Medicine, E. I. duPont de Nemours and Company, incorporated, Newark, DE, 17 February 1977 (2 pages with 1 table, RDB6465)

reports acute inhalation toxicity tests for groups of six male rats exposed to R-141b; indicates 0 , $0,1,2,6$, and 6 deaths at $31,730,42,800$, $50,200,55,270,72,400$, and $95,900 \mathrm{ppm} \mathrm{v} / \mathrm{v}$, respectively; concludes that the 6-hr ALC rat is $50,200 \mathrm{ppm} v / \mathrm{v}$, described as "practically nontoxic"; notes shallow, rapid respiration and mild anesthesia in the test animals within the first hour of exposures at all levels; further notes tremors, incoordination, and some convulsions, indicative of a central nervous system response, at concentrations of $50,200 \mathrm{ppm} \mathrm{v/v}$ and higher; also notes that animals seemed to develop a tolerance to the compound at 31,730 and $42,800 \mathrm{ppm}$ after $4 \mathrm{hr}$, indicates that mild weight loss occurred one day post exposure followed by normal weight gain at all exposure levels; also indicates that no histopathologic examinations were conducted

F. J. Dwyer et al., CFC Alternatives in Thermoset Foam Production, Proceedings of the 33rd Annual Polyurethane Technical/Marketing Confer- 
ence (20 September - 3 October 1990), 400-406, 1990 (7 pages, RDB59A5)

$$
R-141 b \text {, toxicity }
$$

ENVIRON Corporation, Evaluation Of Potential Risks Associated with Substitution of HCFC $141 \mathrm{~b}$ for CFCs Used in the Manufacture of Insulating Foam, Elf Atochem North America, Incorporated, Philadelphia, PA, April 1992 (94 pages with 20 tables, RDB7288)

review of toxicity data for $R-141 b$ and its primary degradation products (R-151a, R-1131a, $R-142 b$, and R-143a), toxicity criteria and doseduration responses, exposure assessment, and risk characterization; concludes that "the potential health hazard ... of exposure is negligible for workers manufacturing the foam insulation and for residents (both aduits and children) living in homes insulated with foam boards"

J. W. Gardner (Huntingdon Research Centre Limited, HRC, UK), Acute Dermal Toxicity to Rats of 4874-89, report 881114D/PWT 91/AC, Elf Atochem North America (then Pennwalt Corporation), King of Prussia, PA, 12 October 1988 (16 pages with 4 tables, RDB5991)

test to assess the dermal toxicity of R-141b (identified in the study as 4874-89) by a single exposure to the skin of five male and five female CFY (Sprague-Dawley) rats: no signs of systemic reaction were observed and deaths resulted; rats achieved normal bodyweight gains and terminal autopsy findings were normal; concluded that the ALD dermal rat $>2000$ $\mathrm{mg} / \mathrm{kg}(1.63 \mathrm{~mL} / \mathrm{kg})$

C. J. Hardy, G. C. Jackson, R. S. Rao, D. J. Lewis, and C. Gopinath (Huntingdon Research Centre Limited, HRC, UK), HCFC 141b Acute Inhalation Toxicity Study in Rats 4-Hour Exposure, report PWT 95/881676, Elf Atochem North America (then Pennwalt Corporation), King of Prussia, PA, 17 July 1989 (69 pages with 2 figures and 8 tables including 1 page sponsor summary, RDB5995)

tests of acute inhalation toxicity to groups of five male and five female Sprague-Dawley, albino rats by whole-body exposures at 0 (control), $29,674,45,347,67,499$, or $76,485 \mathrm{ppm}$ v/v $\mathrm{R}-141 \mathrm{~b}$ in air for $4 \mathrm{hr}$ : 5 of 10 and 10 of 10 animals died at the two highest concentrations, leading to findings of 4-hr $\mathrm{LC}_{50}$ rat of 58,931 for the males, 64,991 for the females, and 61,647 $\mathrm{ppm} \mathrm{v} / \mathrm{v}$ overall; treated animals showed increased respiration rates, wetness around the mouth, reduced motor activity, restless behavior, and abnormal body carriage at all concen- trations except the control group; rats showed reduced bodyweight or bodyweight gain for one day following exposure; the lung to body weight ratios were high for those that died, but no treatment-related macroscopic or microscopic abnormalities were found in pathological examinations

J. H. Harris and M. W. Anders, In Vivo Metabolism of the Hydrochlorofluorocarbon 1,1Dichloro-1-fluoroethane (HCFC-141b), Biochemical Pharmacology, UK, 41(9):R13-R16, 1991 (4 pages, RDB5975)

R-141b, toxicity

Y. Hino, K. Yamasaki, and K. Shiraishi, TwentyEight Day Repeated Dose Inhalation Toxicity Study of HCFC-141b in Rats, report T-2966, Hita Research Laboratories Chemical Biotesting Center, Chemicals Inspection and Testing Institute, Japan, February 1992 (RDB5984)

R-141b, toxicity

G. Hodson-Walker, HCFC-141b: Lymphocyte Cytogenetic Study Using the Methodology Recommended by the OECD (1983), report 89/PFG002/1029 (FYI report OTS-006900695), Life Science Research Limited (LSR), Suffolk, UK, 1990 (RDB5998)

$R-141 b$, genotoxicity, health effects, mutagenic potential, toxicity

G. Hodson-Walker, In Vitro Assessment of Clastogenic Activity of HCFC-141b in Cultured Chinese Hamster Ovary (CHO-K1) Cells, report 89/PFG001/0971a (FYI report OTS-0690-0695), Life Science Research Limited, Suffolk, UK, 1990 (Rdb5997)

R-141b, genotoxicity, health effects, mutagenic potential, toxicity

G. Hodson-Walker and K. May, CFC 141b: Assessment of Mutagenic Potential in Histidine Auxotrophs of Salmonella Typhimurium (The Ames Test), report 88/PSV004/237, Life Science Research Limited (LSR), Suffolk, UK, 1988 (RDB59A0)

R-141b, HCFC-141b, genotoxicity, health effects, mutagenic potential, toxicity

G. Hodson-Walker and K. May, CFC 141b: Assessment of Its Ability to Cause Lethal DNA Damage in Strains of Escherichia Coli, report 88/PSV008/258, Life Science Research Limited (LSR), Suffolk, UK, June 1988 (RDB59A2) 
R-141b, HCFC-141b, genotoxicity, health effects, toxicity

W. Howe, Hygiene Standard Documentation HCFC 141b, document MC001, ICI Chemicals and Polymers Limited, Cheshire, UK, 14 October 1991 (3 pages, RDB5A80)

$\mathrm{R}-141 \mathrm{~b}$, toxicity, rationale for recommended hygiene standard (occupational exposure limit, OEL) of $500 \mathrm{ppm}, 8-\mathrm{hr}$ TWA, and for a shortterm exposure limit of $1,250 \mathrm{ppm}$ for $10 \mathrm{~min}$ utes

E. W. Hughes, B. A. Homan, D. M. John, T. J. Kenney, D. W. Coombs, and C. J. Hardy (Huntingdon Research Centre Limited (HRC, UK), Study of the Effect of PWC 4874-89 on the Pregnancy of the Rabbit, report PWT 82/89243, October 1989 (RDB5986)

$R-141 b$, health effects, reproduction and developmental study, toxicity

E. W. Hughes, B. A. Homan, T. J. Kenney, D. W. Coombs, C. J. Hardy, and S. A. Tesh (Huntingdon Research Centre Limited, HRC, UK), Effects of HCFC-141b on Pregnancy of the Rabbit, report PWT 77/88952, Elf Atochem North America (then Pennwalt Corporation), King of Prussia, PA, November 1988 (RDB5985)

$R-141 b$, developmental study, health effects, reproduction, toxicity

E. W. Hughes, B. A. Homan, T. J. Kenney, D. W. Coombs, and C. J. Hardy, The Effect of HCFC$141 \mathrm{~b}$ on Pregnancy of the Rat, Huntingdon Research Centre Limited (HRC), Cambridgeshire, UK, 1988 (RDB5A82)

R-141b, developmental study, health effects, reproduction, toxicity

Huntingdon Research Centre Limited (HRC, UK), HCFC-141b: Potential Tumorigenic and Toxicological Effects During Prolonged Inhalation Administration in Rats, report ALS 1/911274, AlliedSignal Incorporated, Morristown, NJ, 1992 (RDB7285)

$R-141 b$, health effects, chronic toxicity

Huntingdon Research Centre Limited (HRC, UK), HCFC-141b: Potential Tumorigenic and Toxicological Effects During Prolonged Inhalation Administration in Rats, report ALS 1/921032, AlliedSignal Incorporated, Morristown, NJ, volumes 1-8, 1993 (RDB5989)

$\mathrm{R}-141 \mathrm{~b}$, health effects, chronic toxicity
P. J. M. Janssen (Duphar B.V., The Netherlands), Acute Inhalation Toxicity Studies on FC 141b in Rats, report $S .8820$ (also identified as $55645 / 61 / 88$ ), Solvay et Cie S.A., Brussels, Belgium, October 1988 with June 1989 supplement (76 pages with 3 figures and 37 tables, RDB6463)

toxicity tests for acute inhalation toxicity of R$141 \mathrm{~b}$ in groups of 5 male and 5 female Wistarderived rats by nose-only exposures to 0 (control), $3,000,6,000$, or $10,000 \mathrm{ppm} v / \mathrm{v}$ averages of $0,2,946,5,992$, or $11,096 \mathrm{ppm}$ by analysis) $\mathrm{R}-141 \mathrm{~b}$ in air for $6 \mathrm{hr}$ : tests included observation of clinical signs, analysis of post-exposure blood and urine, and macroscopic and histopathologic examinations at necropsy; no irritation, anesthesia, or other clinical signs were observed during or after exposures; slight decreases were observed in body-weight gain for males exposed to 3,000 and $11,000 \mathrm{ppm}$ and females exposed to 6,000 and $11,000 \mathrm{ppm}$; slight effects were observed in the inorganic phosphate levels in blood plasma and in the and kidney function of treated animals; absolute increases were found in the lung weights of animals exposed to $3,000 \mathrm{ppm}$ and relative increases in kidney weights were observed in males exposed to 6,000 ppm; kidney is suggested as a possible target organ with prolonged exposure; no deaths resulted leading to interpretation that the 6-hr ALC rat exceeds $11,096 \mathrm{ppm} ; \mathrm{R}-141 \mathrm{~b}$ used is identified as 95.5$97.1 \%$ pure with $2.6-3.8 \% \mathrm{R}-365$ as the major impurity; supplement documents compliance with Good Laboratory Practice regulations

P. J. M. Janssen (Duphar B.V., The Netherlands), Acute Inhalation Study to Investigate the Respiratory Irritancy Properties of FC 141b in Male Rats, report 56645/41/89, Solvay et Cie S.A., Brussels, Belgium, 1989 (RDB7749)

tests for acute inhalation toxicity of R-141b in rats as reported in RDB5371

P. J. M. Janssen (Duphar B.V., The Netherlands), Acute Oral Toxicity Study with FC 141b in Rats, report S.8812 (also identified as 55645/36/88), Solvay et Cie S.A., Brussels, Belgium, January 1989 with June 1989 supplement (Rdb6461)

toxicity tests for acute ingestion toxicity of R$141 \mathrm{~b}$ in groups of 5 male and 5 female rats by ingastric intubation of 0 (control) or $2000 \mathrm{mg} / \mathrm{kg}$ bodyweight R-141b in corn oil: no deaths resulted and the report concludes that the $L_{50}$ oral rat exceeds $2000 \mathrm{mg} / \mathrm{kg}$ as tested; clinical signs were mainly indicative of an effect on the autonomic nervous system, on the central ner- 
vous system, on motor coordination, an motor activity, and on muscle tone; macroscopic examination at necropsy found a higher incidence of liver changes (swollen hydropic degeneration of the hepatocytes and cloudy swelling of the renal tubular epithelium) in treated animals compared to no males and 2 females in the control groups

P. J. M. Janssen and T. E. Pot (Duphar B.V., The Netherlands), Acute Dermal Toxicity Study with FC 141b in Rats, report S.8801 (also identified as 56645/23/88), Solvay et Cie S.A., Brussels, Belgium, May 1988 (13 pages with 5 tables, RDB6460)

toxicity tests for acute dermal toxicity of R-141b in groups of 5 male and 5 female Wistar rats by application under an occlusive dressing to the shaved, intact skin of 0 (control) or $2,000 \mathrm{mg} / \mathrm{kg}$ bodyweight R-141b: no deaths resulted and the report concludes that the $L D_{50}$ dermal rat exceeds $2,000 \mathrm{mg} / \mathrm{kg}$ as tested; slight vocalization was observed in 3 females of the treated group for the first 30 minutes of application; the treated males and 1 male from the control group were somewhat quiet for the corresponding period; macroscopic examination of the animals at necropsy revealed 5 males and 3 females with swollen or slightly swollen livers

R. J. Kenny (Huntingdon Research Centre Limited, HRC, UK), HCFC-1717: Assessment of Cardiac Sensitization Potential in Dogs, report ALS 57/942811 (also identified as MA-RR-95-2156), AlliedSignal Incorporated, Morristown, NJ, USA, 22 December 1994 ( 38 pages with 4 figures and 7 tables; 3 page cover and summary available from JMC as RDB7206)

toxicity tests of R-141b [identified with a truncated CAS number as a "blind control study"], to determine the cardiac sensitization level in male beagle dogs: 6 animals were exposed snout-only for 10 minutes following adrenaline administration, by intravenous injection, and with a second, challenge injection midway through the exposure; tests were repeated, on separate days, at concentrations of 0 (control), 10,000 , and $20,000 \mathrm{ppm} v / \mathrm{v} R-141 \mathrm{~b}$ in air: results were negative and no abnormal clinical signs were observed at $10,000 \mathrm{ppm} ; 1$ dog $(17 \%)$ responded positively, resulting in fatal ventricular fibrillation (very rapid and irregular contractions of the ventricles of the heart resulting in loss of synchronization between the heartbeat and pulse beat), at $20,000 \mathrm{ppm}$; report concludes that "HCFC-1717" [R-141b] has cardiac sensitization potential at a concentration in air of "up to 20,000 ppm"

J. C. Koorn (Duphar B.V., The Netherlands), Study to Examine the Possible Mutagenic Activity of the Volatile Liquid FC 141b in the Salmonella/Microsome Assay, report S.8819 (also identified as 55645/60/88 and 56645/41/89), Solvay et Cie S.A., Brussels, Belgium, 1988 (RDB6462)

R-141b, genotoxicity, mutagenic potential, toxicity

S. R. Kynoch and B. I. Parcell (Huntingdon Research Centre Limited, HRC, UK), Delayed Contact Hypersensitivity in the Guinea Pig with 4874-89, report PWT 94/881236D/SS, Elf Atochem North America (then Pennwalt Corporation), King of Prussia, PA, 1989 (RDB5994)

$\mathrm{R}-141 \mathrm{~b}$, health effects, toxicity as reported in RDB5371 and RDB5B57

T. D. Landry, F. S. Cieszlak, J. R. Szabo, and B. L. Yano, 1,1-Dichloro-1-fluoroethane: 13-Week Toxicity Study with Fischer 344 Rats, report DR-0006-8553-002B, Dow Chemical Company, Midland, MI, July 1989 (RDB5983)

summarizes a subchronic toxicity study of R$141 b$

J. P. Lavelle and T. Doyle (Elf Atochem North America), Safety, Storage, and Handling Recommendations for HCFC-141b, Proceedings of the 33rd Annual Polyurethane Technical/Marketing Conference (20 September - 3 October 1990), 407-412, 1990 (6 pages, RDB6583)

$\mathrm{R}-141 \mathrm{~b}$, safety, toxicity

M. A. Lee, D. M. Krentz, and R. C. Graham, untitled toxicity review for ethane, 1,1-dichloro-1fluoro-, Haskell Laboratory for Toxicology and industrial Medicine, E. I. duPont de Nemours and Company, Incorporated, Newark, DE, 24 November 1993 (18 pages, RDB5868)

$R-141 b$, summary of published and unpublished toxicity literature

M. P. Liggett, S. Allan, and I. S. Dawe (Huntingdon Research Centre Limited, HRC, UK), Acute Oral Toxicity to Rats of PWC 4874-89, report 8913D/PWT 90/AC, Elf Atochem North America (then Pennwalt Corporation), King of Prussia, PA, 5 October 1989 (20 pages with 9 tables, RDB5988)

toxicity tests for acute toxicity by a single oral dose of PWC 4874-89 [subsequently identified 
by the study sponsor as R-141b] dissolved $50 \%$ $w / v$ (sic) in corn oil administered to groups of 5 male and 5 female $C D$ rats by a syringe and plastic catheter: no deaths resulted and the report concludes that the $L D_{50}$ oral rat exceeds $5,000 \mathrm{mg} / \mathrm{kg}$ bodyweight as tested; piloerection was observed in all rats within 5 min of dosing and throughout the remainder of the day; no other clinical signs were observed and recovery, as judged by external appearance and behavior, was complete by the next day; bodyweight gains were slightly lower for 1 male and 2 females during the second week of observation, but all animals achieved anticipated bodyweight gains during the 14-day observation period; terminal autopsy findings were normal

M. P. Liggett (Huntingdon Research Centre Limited, HRC, UK), Irritant Effects on Rabbit Skin of 4874-89, report PWT 92/881066D/SE, Elf Atochem North America (then Pennwalt Corporation), King of Prussia, PA, 1988 or 1989 (RDB5992)

$\mathrm{R}-141 \mathrm{~b}$, toxicity as reported in RDB5B57

M. P. Liggett (Huntingdon Research Centre Limited, HRC, UK), Irritant Effects on the Rabbit Eye of 4874-89, report PWT 93/881067D/SE, Elf Atochem North America (then Pennwalt Corporation), King of Prussia, PA, 1988 or 1989 (RDB5993)

\section{$\mathrm{R}-141 \mathrm{~b}$, toxicity as reported in RDB5B57}

G. D. Loizou and M. W. Anders (University of Rochester), Gas-Uptake Pharmacokinetics and Biotransformation of 1,1-Dichloro-1fluoroethane (HCFC-141b), Drug Metabolism and Disposition, 21:634-639, 1993 (6 pages, RDB65E3)

\section{R-141b, biochemistry, metabolism, toxicity}

K. May, HCFC 141b: Assessment of Mutagenic Potential in Amino Acid Auxotrophs of Salmonella Typhimurium and Escherichia Coli (The Ames Test), report 89/PFG003/0600, Life Science Research Limited, Suffolk, UK, 1989 (RDB5999)

mutagenicity of R-141b; genotoxicity; genotoxic and mutagenic potential; toxicity; deemed R$141 \mathrm{~b}$ not mutagenic in 5 histidine-dependent auxotrophs of Salmonella typhimurium and 1 strain of tryptophan dependent auxotroph of Escherichia coli using modified pour-plate assays with and without metabolic activation as reported in RDB5868

K. May, HCFC 141b: Assessment of Mutagenic Potential in Amino Acid Auxotrophs of Salmo- nella Typhimurium and Escherichia Coli (The Ames Test), report 88/PFG003/0823, Life Science Research Limited (LSR), Suffolk, UK, 1989 (RDB7287)

R-141b, genotoxicity, health effects, mutagenic potential, toxicity as reported in RDB5B57

R-J. Millischer (Elf Atochem S.A., France), C. H. de Rooij (Solvay S.A., Belgium), G. M. Rusch (misspelled "Rush" in the paper, AlliedSignal Incorporated), C. H. Farr (Elf Atochem North America, USA), R. Ben-Dyke (Ben-Dyke Associates Incorporated, USA), C. J. Hardy (Huntingdon Research Centre Limited, HRC, UK), D. J. Lewis (HRC), and G. Hudson-Walker (Pharmaco:LSR, UK), Evaluation of the Genotoxicity and Chronic Inhalation Toxicity of 1,1-Dichloro-1Fluoroethane (HCFC-141b), Food and Chemical Toxicology (FCT), 33(6):491-500, June 1995 (10 pages with 2 figures and 3 tables, RDB65A8)

summarizes a series of in vitro and in vivo tests of R-141b for genotoxicity; based on the data presented and prior testing, deems $R-141 \mathrm{~b}$ to be non-genotoxic; reports a no-observable adverse effect level (NOAEL) of $1500 \mathrm{ppm} \mathrm{v/v} \mathrm{for}$ a two-year chronic exposure study in rats

L. S. Mullin, Fluorocarbon 141b (Ethane, 1,1-dichloro-1-fluoro): Cardiac Sensitization, report 957-77, Haskell Laboratory for Toxicology and Industrial Medicine, E. I. duPont de Nemours and Company, Incorporated, Newark, DE, 23 November 1977 (3 pages with 1 table, RDB6467)

$\mathrm{R}-141 \mathrm{~b}$, toxicity tests to determine the cardiac sensitization potential in male beagle dogs with intravenous injection of epinephrine five minutes before and, with a challenge injection, midway into a 10 minute exposure; NOEL $(0 / 10)$ at $2,600 \mathrm{ppm} \mathrm{v} / \mathrm{v}$; LOEL $(1 / 10)$ at 5,200 ppm; lethal to 1 of 10 dogs at 10,200 ppm and to 2 of 2 at $21,600 \mathrm{ppm}$; concludes that R-141b is a strong cardiac sensitizer

T. K. Nikijenko, Toksikologiya Novykh Promyshlennykh Khimicheskikh Veshchestva [Toxicology of New Industrial Chemical Substances], 8:83-97, 1966 (11 pages in Russian, RDB5978)

toxicity of R-141b

G. M. Rusch (AlliedSignal Incorporated), R-J. Millischer (Elf Atochem S.A., France), C. H. de Rooij (Solvay \& Cie S.A., Belgium), A. J. Brooker (Huntingdon Research Centre, HRC, UK), E. W. Hughes (HRC), and D. W. Coombs (HRC), Inhalation Teratology and Two-Generation Reproduction Studies with 1,1-Dichloro-1-fluoroethane 
(HCFC-141b), Food and Chemical Toxicology (FCT), 33(4):285-300, April 1995 (16 pages with 1 figure and 17 tables, RDB7283)

teratology studies in rabbits and rats and a two generation reproductive toxicity in rats of $R$ $141 \mathrm{~b}$; notes significant effects in pups as low as $8,000 \mathrm{ppm} v / \mathrm{v}$, but deems $2,000 \mathrm{ppm} v / \mathrm{v}$ to be a NOEL

J. F. Russell, Mutagenic Activity of Ethane, 1,1Dichloro-1-fluoroethane) in the Salmonella/Microsome Assay), report 988-77, Haskell Laboratory for Toxicology and Industrial Medicine, E. I. duPont de Nemours and Company, Incorporated, Newark, DE, 1977 (RDB6468)

mutagenicity of $R-141 b$; toxicity; not mutagenic by Ames Assay - as reported in RDB5868

J. W. Sarver, Acute Oral Toxicity Study of FC141b in Male Rats, report 363-88, Haskell Laboratory for Toxicology and Industrial Medicine, E. I. duPont de Nemours and Company, Incorporated, Newark, DE, 1988 (RDB5977)

toxicity of $R-141 \mathrm{~b} ; L D_{50}$ oral rat $>5,000 \mathrm{mg} / \mathrm{kg}$ - as reported in RDB5868 and RDB5B57

J. A. Seckar (Pennwalt Corporation), The Toxicology of HCFC-141b (1,1-Dichloro-1-fluoroethane), Journal of the American College of Toxicology (JACT), 8(6):1221, 1989 (1 page, RDB7278)

briefly summarizes toxicity studies of $R-141 \mathrm{~b}$; indicates that it is a very slight skin and eye irritant in rabbits, but not a dermal sensitizer in the guinea pig, with an $\mathrm{LD}_{50}$ dermal rabbit exceeding $5,000 \mathrm{mg} / \mathrm{kg}$; indicates that the $L D_{50}$ oral rat exceeds $2,000 \mathrm{mg} / \mathrm{kg}$ and the 4-hr $\mathrm{LC}_{50}$ rat exceeds $62,000 \mathrm{ppm} \mathrm{v} / \mathrm{v}$; notes central nervous system (CNS) depression above $30,000 \mathrm{ppm} v / \mathrm{v}$, rapidly reversible upon termination of exposure; reports tests of cardiac sensitization to epinephrine in dogs with an effect at $10,000 \mathrm{ppm} v / \mathrm{v}$; discusses reproductive, teratogenic, and subchronic toxicity tests that found an unequivocal no-observed-effect level

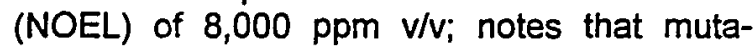
genicity, a combined chronic toxicity and oncogenicity, and ecotoxicity studies are planned

R. Tolando (University of Surrey, UK), R. Ferrara (University of Padova Medical School, Italy), N. I. Eldirdiri, A. Albores, L. J. King (Surrey), and M. Manno (Padova), Reductive Activation of 1,1-Dichloro-1-fluoroethane (HCFC-141b) by Phenobarbital- and Pyridine-Induced Rat Liver Microsomal Cytochrome P450, Xenobiotica, 26:425435, 1996 (11 pages, RDBA565) toxicity of R-141b; health effects

D. A. Viachos (possibly Vlachos), Mouse Bone Marrow Micronucleus Assay of FC-141b, report 746-88, Haskell Laboratory for Toxicology and Industrial Medicine, E. I. duPont de Nemours and Company, Incorporated, Newark, DE, 1988 (RDB6472)

sumarizes a mutagenicity study of R-141b; toxicity - as reported in RDB5868 and RDB5B57

B. L. Yano, F. S. Cieszlak, and T. D. Landry, 1,1Dichloro-1-fluoroethane: 4-Week Toxicity Study with Fischer 344 Rats, report DR-00068553-002A, Dow Chemical Company, 1989 (RDB5982)

subchronic toxicity of R-141b; health effects

4874-89: Assessment of Clastogenic Action on Bone Marrow Erythrocytes in the Micronucleus Test, report 88/0504, Life Science Research Limited (LSR), Suffolk, UK, 1989 or 1990 (RDB59A3)

toxicity of R-141b; genotoxicity; health effects; mutagenic potential - as reported in RDB5B57

CFC 141b: Investigation of Mutagenic Activity at the HGPRT Locus in a Chinese Hamster V79 Cell Mutation System, report 88/PSV005/257, Life Science Research Limited (LSR), Suffolk, UK, July 1988 (RDB59A1)

mutagenicity of R-141b; genotoxicity; toxicity

1,1-Dichloro-1-fluoroethane (HCFC-141b, CAS No 1717-00-6), Joint Assessment of Commodity Chemicals (JACC) report 29, European Chemical Industry Ecology and Toxicology Centre (ECETOC), Brussels, Belgium, December 1994 (RDB6585)

toxicity and environmental impacts of $R-141 \mathrm{~b}$

1,1-Dichloro-1-fluoroethane (HCFC 141b) Health Effects Information, CAS\# 1717-00-6, Elf Atochem North America, Incorporated, Philadelphia, PA, undated circa 1995 (6 pages, RDB5B57)

summary of toxicological and ecological information for R-141b; toxicity; environmental impacts

1,1-Dichloro-1-fluoroethane (HCFC-141b): Probe Study to Assess the Neuropharmacologic Effects of Acute Inhalation Exposure in Rats, Dow Chemical Company, 1989 (RDB7280) toxicity of R-141b; health effects 
Prehi. Prumysolve Toxikol. Org. Latky, $134 \mathrm{ff}$, 1986 (RDB5981)

toxicity of $R-141 b$

Workplace Environmental Exposure Level Guide - 1,1-dichloro-1-fluoroethane, American Industrial Hygiene Association (AlHA), Fairfax, VA, 1992 (RDB4B87)

Workplace Environmental Exposure Level (WEEL) Guide for R-141b; recommended occupational exposure limit; summary of toxicology data; toxicity

report 55635/49/90 [possibly 55645/49/90], Duphar B.V., The Netherlands, 1991 (RDB6464)

ecological toxicity of R-141b; aquatic environmental study,

report 715-77, Haskell Laboratory for Toxicology and Industrial Medicine, E. I. duPont de Nemours and Company, Incorporated, Newark, DE, 1977 (RDB6466)

subchronic inhalation toxicity tests of R-141b as reported in RDB5868

report ALS 1/901565, Huntingdon Research Centre (HRC), Cambridgeshire, UK, 17 December 1990 (RDB6473)

neurologic toxicity study of $R-141 \mathrm{~b}$ in rats; found no changes indicative of neurotoxic effect - as reported in RDB5868

report ALS 2/920766, Huntingdon Research Centre Limited (HRC), Cambridgeshire, UK, 1988 (RDB5990)

toxicity of $R-141 b$

study of the metabolic and biochemical effect of R$141 \mathrm{~b}$ in rats, AlliedSignal Incorporated, Morristown, NJ, 6 May 1992 (RDB6476)

toxicity of $R-141 b$

\section{R-142b}

F. C. Barsky and B. E. Butterworth, In Vitro Microbial Mutagenicity Studies of Ethane, Chlorodifluoro, report 322-76, Haskell Laboratory for Toxicology and Industrial Medicine, E. I. duPont de Nemours and Company, Incorporated, Newark, DE, 1976 (RDB6517)

$R-142 b$, health effects, toxicity as reported in RDB3721 and RDB5869

Bio/dynamics Incorporated, A Dominant-Lethal Inhalation Study with Fluorocarbon $142 b$ in
Rats, report 79-7288, Elf Atochem North America (then Pennwalt Corporation), King of Prussia, PA, 1980 (RDB5916)

$$
R-142 b \text {, toxicity }
$$

Bio/dynamics Incorporated, A Study in the Rat of the Cytogenetic Effect of Inhalation of Fluorocarbon 142b for 13 Weeks, report 79-7289, Elf Atochem North America (then Pennwalt Corporation), King of Prussia, PA, 1980 revised 16 June 1983 (RDB5917)

$R-142 b$, health effects, toxicity

Bio/dynamics Incorporated, A Two-Year Inhalation Study of Fluorocarbon 142b in the Rat, report 79-7287, Elf Atochem North America (then Pennwalt Corporation), King of Prussia, PA, 1983 (RDB5918)

$R-142 b$, health effects, toxicity

M. R. Brittelli, Eye Irritation Test in Rabbits, report 752-75, Haskell Laboratory for Toxicology and Industrial Medicine, E. I. duPont de Nemours and Company, Incorporated, Newark, DE, 1976 (RDB6521)

$R-142 b$, health effects, toxicity as reported in RDB5869

R. Culik and D. P. Kelly, Embryotoxic and Teratogenic Studies in Rats with Inhaled Chlorodifluoroethane (FC 142b), report 700-76, Haskell Laboratory for Toxicology and Industrial Medicine, E. I. duPont de Nemours and Company, Incorporated, Newark, DE, 1976 (RDB6520)

$R-142 b$, health effects, developmental and reproductive toxicity as reported in RDB3721, RDB5371, and RDB5869

D. R. Damske, F. J. Mecler, and R. P. Beliles, Teratology Study in Rats - Isotron 142b Monochlorodifluoroethane, report for project 20980 or 29890 , Litton Bionetics Incorporated (LBI), Kensington, MD, 1978 (RDB5914)

R-142b, health effects, toxicity

A. P. Filicheva, Changes in the Nervous System Following the Chronic Action of Fluorinated Aliphatic Carbohydrates, Gigiena Truda i Professional'nye Zabolevaniya [Labor Hygiene and Occupational Diseases], Moscow, Russia (then USSR), 10:14-16, 1975 (3 pages in Russian, RDB6523)

$R-142 b$, health effects, toxicity

A. H. C. Groeneveld and L. A. M. Kuijpers, The Acute Toxicity of 1-Chloro-1,1-difluoroethane 
(HCFC-142b) to Daphnia Magna, report 56635/32/90, Duphar B.V., The Netherlands, 1990 (RDB7747)

R-142b, aquatic environmental study, ecological toxicity

A. H. C. Groeneveld and L. A. M. Kuijpers, The Acute Toxicity of 1-Chloro-1,1-difluoroethane (HCFC-142b) to the Guppy Poecilia Reticulata, report 56635/33/90, Duphar B.V., The Netherlands, 1990 (RDB7748)

$\mathrm{R}-142 \mathrm{~b}$, aquatic environmental study, ecological toxicity

P. W. Grube, M. A. Lee, G. T. Hall, R. C. Graham, and $L . J$. Ver Nooy, untitled toxicity review for ethane, 1-chloro-1,1-difluoro-, Haskell Laboratory for Toxicology and Industrial Medicine, E. I. duPont de Nemours and Company, Incorporated, Newark, DE, 17 January 1990 (12 pages, RDB5869)

R-142b, summary of published and unpublished toxicity literature

D. G. Hutton, Flow-Through Acute 96-Hour $E_{50}$ of Chlorodifluoroethane to Rainbow Trout (Oncorhynchus Mykiss), report 543-89, Haskell Laboratory for Toxicology and Industrial Medicine, E. I. duPont de Nemours and Company, Incorporated, Newark, DE, 1989 (RDB7752)

ecotoxicity of R-42b; environmental impacts and fate; aquatic impacts - as reported in RDB5371

D. G. Hutton and P. H. Lieder, Static Acute 48Hour $E_{50}$ of Chlorodifluoroethane to Daphnia Magna, report 542-89, Haskell Laboratory for Toxicology and Industrial Medicine, E. I. duPont de Nemours and Company, Incorporated, Newark, DE, 1989 (RDB7751)

ecotoxicity of R-42b; environmental impacts and fate; aquatic impacts - as reported in RDB5371

D. R. Jagannath and D. J. Brusick, Mutagenicity Evaluation of Isotron 142b, report for project 20838, Litton Bionetics Incorporated (LBI), Kensington, MD, 1977 (RDB5912)

$R-142 b$, health effects, toxicity

D. P. Kelly and H. J. Trochimowicz, Ninety Day Inhalation Exposure of Rats and Dogs to Vapors of 1-Chloro-1,1-difluoroethane, report 46976. Haskell Laboratory for Toxicology and Industrial Medicine, E. I. duPont de Nemours and Company, Incorporated, Newark, DE, 1976 (RDB6518)
$R-142 b$, health effects, toxicity: no adverse clinical hematological, blood chemical, urine analytical, or histopathologic evidence of effects attributable to exposures at 1,000 or $10,000 \mathrm{ppm} \mathrm{v} / \mathrm{v}$ as reported in RDB3721 and RDB5869

A. Koops and D. F. Krahn, Mutagenic Activity of Ethane, Chlorodifluoro- in the Salmonella/Microsome Assay, report 578-77, Haskell Laboratory for Toxicology and Industrial Medicine, E. I. duPont de Nemours and Company, Incorporated, Newark, DE, 1977 (RDB6519)

$\mathrm{R}-142 \mathrm{~b}$, health effects, toxicity as reported in RDB2721, RDB5371, and RDB5869

D. W. Matheson and D. Brusick, Mutagenicity Evaluation of Isotron 142b in the In Vitro Transformation of BALB/3T3 Cells Assay, report for project 20840, Litton Bionetics Incorporated (LBI), Kensington, MD, 1978 (RDB5913)

R-142b, toxicity

F. J. Mecler and P. J. Knapinsky, Acute Inhalation Toxicity Study in Rats. Isotron $142 b$, report for project 20888, Litton Bionetics Incorporated (LBI), Kensington, MD, 1978 (RDB59A8)

$6 \mathrm{hr}$ acute inhalation toxicity tests of R-142b in Charles River CD rats: mortality ratios and corresponding concentrations were $0 \%$ at 200,000 $\mathrm{ppm}$ in air and $20 \%$ at $400,000 \mathrm{ppm}$ [deemed an ALCl; rats showed rapid and labored breathing, lethargy, and discharges from the eyes and nose at 400,000 ppm; necropsy of the surviving rats after a 14 day observation period revealed dark red mottling of the lungs and kidneys, especially at the higher concentration as reported in RDB3721

B. L. Moore and H. J. Trochimowicz, Subacute (Two-Week) Inhalation Toxicity of 1-Chloro-1,1difluoroethane, report 145-76, Haskell Laboratory for Toxicology and Industrial Medicine, E. I. duPont de Nemours and Company, Incorporated, Newark, DE, 1976 (RDB6516)

$R-142 b$, health effects, toxicity, inhalation study in the rat: no clinical hematological, blood chemical, urine analytical, or histopathologic evidence of effects attributable to exposures at 20,000 ppm as reported in RDB3721 and RDB5869

J. A. Seckar (Pennwalt Corporation), H. J. Trochimowicz (E. I. duPont de Nemours and Company, Incorporated), and G. K. Hogan, Toxicological Evaluation of Hydrochlorofluorocarbon 142b, 
Fundamentals of Chemical Toxicology, 24(3):237240, 1986 (RDB5919)

$\mathrm{R}-142 \mathrm{~b}$, health effects, toxicity

J. D. Sterling, Aerosol Age, 12:48-52, 1982 (5 pages, RDB6524)

\section{$R-142 b$, health effects, toxicity}

Acute Toxicity Study in Rats - Isotron 142b, Monochlorodifluoroethane, report for project 20795, Litton Bionetics Incorporated (LBI), Kensington, MD, 1978 (RDB5911)

\section{$\mathrm{R}-142 \mathrm{~b}$, health effects, toxicity}

Chlorodifluoroethane (1-Chloro-1,1-difluoroethane; HFA-142b, CAS: 75-68-3), Joint Assessment of Commodity Chemicals (JACC) report 17. European Chemical Industry Ecology and Toxicology Centre (ECETOC), Brussels, Belgium, February 1991 (36 pages with 1 table, RDB3721)

$R-142 b$, environmental impacts, toxicity

Workplace Environmental Exposure Level Guide - 1-chloro-1,1-difluoroethane, American Industrial Hygiene Association (AlHA), Fairfax, VA, 1994 (RDB4B89)

$\mathrm{R}-142 \mathrm{~b}$, toxicity, safety classification: 4-hr ALC rat $=128,000 \mathrm{ppm}$, WEEL $=1,000 \mathrm{ppm}$

\section{R-143}

L. J. Sibinski, Two-Year Oral (Diet) Toxicity-Carcinogenicity Study of the Fluorochemical FC 143 in Rats, Riker Laboratories, Incorporated, 3M Company, Minneapolis, MN, 1987 (RDB65E0)

R-143 (possibly R-143a), toxicity

\section{R-143a}

W. J. Brock, H. J. Trochimowicz (E. I. duPont de Nemours and Company, USA), C. H. Farr (Elf Atochem North America, USA), R-J. Millischer (Elf Atochem S.A., France), and G. M. Rusch (AlliedSignal Incorporated, USA), article 0092, Acute, Subchronic, and Developmental Toxicity and Genotoxicity of 1,1,1-Trifluoroethane (HFC143a), Fundamental and Applied Toxicology, 31(2):200-209, June 1996 (10 pages with 7 tables, RDB6597)

acute, subchronic, and developmental inhalation toxicity and genotoxicity studies for R143a: no mortalities resulted in groups of 6 Crl:CDBR male rats exposed nose-only for 4 hours to 97,000 and 540,000 ppm v/v R-134a in air with added oxygen to maintain $20 \%$ levels; animals showed body weight loss after exposures, but recovered by the second day; concludes that the 4-hr $\mathrm{LC}_{50}$ rat exceeds $540,000 \mathrm{ppm}$; groups of 5-6 male beagle dogs were exposed to $50,000,100,000,150,000$ $200,000,250,000$, and 300,000 ppm with intravenous injection of epinephrine five minutes before and, with a challenge injection, midway into a 10 minute exposure; 1 of 6 dogs exhibited an equivocal response at $200,000 \mathrm{ppm}$, but none (including the same dog) did so at $250,000 \mathrm{ppm}$ [deemed a NOEL]; 2 of 5 dogs showed marked responses at $300,000 \mathrm{ppm}$ considered to be the cardiac sensitization LOEL; four groups of 10 male and 10 female rats were exposed nose-only to concentrations of 0 (control), 2,000, 10,000, and 40,000 ppm $\mathrm{v} / \mathrm{v} \mathrm{R}-143 \mathrm{a}$ in air for $6 \mathrm{hr} / \mathrm{d}, 5 \mathrm{~d} / \mathrm{wk}$ for 4 weeks; 3 premature deaths were determined not to be treatment-related; decreases were observed in male, but not female body weights, but no toxicologically significant clinical signs or pathology measurements were observed during or after the exposures; microscopic degenerative changes were found in the testes of male rats in each exposure group, ranging in severity from slight at $2000 \mathrm{ppm}$ to minimal or mild at $10,000 \mathrm{ppm}$ and $40,000 \mathrm{ppm}$; based on problems encountered with the restraining devices, excessive temperatures, and prior documentation of related stress effects, the study was repeated without restraints (whole-body exposures); no adverse testicular effects or adverse clinical signs were seen at any exposure level; based on these results, $40,000 \mathrm{ppm}$ was determined to be the 4-wK NOAEL rat for R-143a: 20 male and 20 female rats were exposed (whole body) to 0 (control), 2,000,10,000, and $40,1000 \mathrm{ppm} \mathrm{v} / \mathrm{v}$ for $6 \mathrm{hr} /$ day, $5 \mathrm{~d} / \mathrm{wk}$ for 90 days; sporadic changes in body weight were observed, but did not follow a dose-response relationship and were not considered to be treatment-related; no clinical signs of toxicity were observed during or after the exposures, and no pathology changes were found; no Boxidation activity (peroxisome proliferation), organ weight, adverse gross, or microscopic changes were observed; 90-day NOAEL rat was considered to be 40,000 ppm; developmental studies conducted in both rats and rabbits by whole-body exposures at $2,000,10,000$, and $40,000 \mathrm{ppm}$ for $6 \mathrm{hr} / \mathrm{d}$ on days 6 through 15 (rats) or 18 (rabbits) of gestation; no adverse clinical signs or biologically significant effects were identified; reproductive parameters 
and developmental variations were unaffected in rats, but visceral variations due to retarded development were observed; incidence of skeletal malformations was higher in exposed rabbits than in controls, but did not follow a dose-response relation; genotoxicity studies included the Ames Assay, in vitro tests with human lymphocytes, and in vivo micronucleus tests in mouse bone marrow; no increases in revertants were found in bacteria with 5 strains of Salmonella typhimurium and 2 strains of Esche-richia coli; no aberrations in clastogenic activity were found in human lymphocytes; no clinical signs of toxicity or statistically significant effects were found in the micronucleus study using male and female mice; paper concludes that R-143a is "able to induce a cardiac sensitization response at very high exposure levels," "has a low acute and subchronic toxicity potential," is not a developmental toxicant, and is not genotoxic

S. Cracknell (Life Science Research Limited, LSR, UK), Forane 143a: Acute Inhalation Toxicity in the Rat, report 91/ATH007/1159 (also identified as 91/1159), Elf Atochem S.A., Paris - La Défense, France, 4 February 1992 (72 pages with 3 figures and 5 tables, Rdb7450)

toxicity tests for acute inhalation toxicity of R$143 a$ in groups of 5 male and 5 female Sprague-Dawley rats by nose-only exposures to 0 (control), 300,000 , or $600,000 \mathrm{ppm}$ v/v (averages of $0,305,000$, or $591,000 \mathrm{ppm} \mathrm{v/v}$ by analysis) R-143a in air for 4 hours: tests included observation of clinical signs, analysis of post-exposure blood and urine, and macroscopic and histopathologic examinations at necropsy; no deaths resulted and report concludes that the 4-hr LC $_{50}$ rat exceeds 591,000 ppm as tested; minor changes were observed in the respiratory pattern of 3 treated animals; peripheral vasodilation was seen for 4 females and 1 male exposed to $591,000 \mathrm{ppm}$; no other clinical signs, changes in bodyweight, effects on organ weight, or macroscopic changes attributed to treatment were found

S. R. Frame, M. C. Carakostas, and D. B. Warheit (E. I. duPont de Nemours and Company, Incorporated), Two Four-Week Inhalation Toxicity Studies with HFC-143a in Rats, abstract 1391 (SOT 31st Annual Meeting, February 1992) for the Program for Alternative Fluorocarbon Toxicity Testing (PAFT), The Toxicologist, 12(1):355, 1992 (1 page, RDB8120) inhalation toxicity tests in four groups of ten male and ten female $C D$ rats exposed noseonly to 0 (control), 2,000, 10,000, and 39,000 ppm v/V R-143a in air for 31 days: males showed a trend toward decreased absolute testicular weight and microscopic degenerative changes in the testes in all exposure groups; the validity of these findings was suspect and the effects noted could have been due to excessive stress and heat; a second 4-wk study was initiated with four groups of ten male rats exposed whole body to the same concentrations; no testicular or other differences (body weights, gross and microscopic pathology, or clinical signs) were noted compared to the control group; abstract concludes that 4-wk exposures to R-143a produced no significant pathological effects in rats at the concentrations tested

C. J. Hardy and I. J. Sharman (Huntingdon Research Centre Limited, HRC, UK), HFC 143a: Assessment of Cardiac Sensitization Potential in Dogs, report DPT 281/930477, Haskell Laboratory for Toxicology and Industrial Medicine, E. I. duPont de Nemours and Company, Incorporated, Newark, DE, 3 June 1993 (30 pages with 7 figures and 8 tables, RDB5C26)

toxicity tests to determine the cardiac sensitization potential of $R-143 a$ in male beagle dogs with intravenous injection of adrenaline five minutes before and, with a challenge injection, midway into a. 10 minute exposure; no marked response in 6 dogs at 50,000, 100,000, $150,000,200,000$, and $250,000 \mathrm{ppm} v / \mathrm{v}$ in air [deemed a NOEL]; marked response in 2 of 5 dogs at $300,000 \mathrm{ppm} v / \mathrm{v}$ [deemed a LOEL] with test interrupted in a sixth dog due to severe clinical signs

D. P. Kelly, Four-Hour Acute Inhalation Toxicity Study with FC-143a in Rats, report 283-90, Haskell Laboratory for Toxicology and Industrial Medicine, E. I. duPont de Nemours and Company, Incorporated, Newark, DE, 6 August 1990 (11 pages with 2 tables, RDB5C17)

$4 \mathrm{hr}$ acute inhalation toxicity tests in groups of six male Charles River Crl:CD BR rats by noseonly exposures to R-143a: no mortalities resulted at concentrations of 97,000 and 540,000 $\mathrm{ppm} \mathrm{v} / \mathrm{v}$ in air with oxygen enrichment (to maintain 19.8-20.2\% levels); rats responded normally to a tail pinch during exposures; clinical signs observed at both concentrations were noted as common to rats held in restrainers plus wet perineums following exposures at 
$540,000 \mathrm{ppm}$; rats exposed to 97,000 and $540,000 \mathrm{ppm}$ showed slight and moderate to severe, respectively, weight loss on the day following exposure, but returned to normal weight gain in the 14-day recovery period; report concludes that the 4-hr ALC rat for R-143a exceeds 540,000 and that this compound "is considered to have low acute toxicity on an acute inhalation basis"

M. A. Lee, P. J. Lardear, and R. C. Graham, untitled toxicity review for 1,1,1-trifluoroethane, Haskell Laboratory for Toxicology and Industrial Medicine, E. I. duPont de Nemours and Company, Incorporated, Newark, DE, 10 February 1994 (RDB5867)

R-143a, summary of published and unpublished toxicity literature

Pharmaco-LSR Limited (UK), Forane ${ }^{\circledR}$ 143a in Vapour Phase: Assessment of Mutagenic Potential in Amino-Acid Auxotrophs of Salmonella Typhimurium and Escherichia Coli (the Ames Test), report 93/ATH008/0209, Elf Atochem S.A., Paris - La Défense, France, 1993 (RDB5C27)

$\mathrm{R}-143 \mathrm{a}$, health effects, toxicity as reported in RDB5C16

An Inhalation Developmental Toxicity Study of HFC-143a in Rabbits, report WIL-189005, WIL Research Laboratories, Incorporated, volumes I and II, 27 August 1993 (RDB5C28)

$R-143 a$, health effects, toxicity as reported in RDB5C16

Bacterial Mutagenicity Testing of HFC-143a in the Salmonella Typhimurium and Escherichia Coli: Plate Incorporation Assay, report 787-93, Haskell Laboratory for Toxicology and Industrial Medicine, E. I. duPont de Nemours and Company, !ncorporated, Newark, DE, 1993 (21 pages, RDB5C18)

$R-143 a$, health effects, toxicity as reported in RDB5B58 and RDB5C16

Developmental Toxicity Study of HFC-143a in Rats, report 700-92, Haskell Laboratory for Toxicology and Industrial Medicine, E. I. duPont de Nemours and Company, Incorporated, Newark, DE, 1992 (RDB5C19)

R-143a, health effects, toxicity as reported in RDB5C16

Forane ${ }^{\circledR}$ 143a - Assessment of Peroxisome Proliferation in Vitro in Rat Hepatocycte Pri- mary Cultures Assay of Palmitoyl-CoA-Oxidase Activity, report RS0006931126/03 (PN), Elf Atochem S.A., Paris - La Défense, France, 1994 (RDB7282)

R-143a, health effects, toxicity as reported in RDB5B58

Four-Week Inhalation Toxicity Study with HFC143a in Rats, report 6-92 (TSCAT report OTS0529920-2), Haskell Laboratory for Toxicology and Industrial Medicine, E. I. duPont de Nemours and Company, Incorporated, Newark, DE, 1992 (RDB5C20)

four groups of 10 male rats were exposed (whole body) to concentrations of 0 (control), $2,000,10,000$, and $40,000 \mathrm{ppm} v / \mathrm{v} R-143 a$ in air for $6 \mathrm{hr} / \mathrm{d}, 5 \mathrm{~d} / \mathrm{wk}$ for 4 weeks; no adverse clinical signs or testicular effects were observed at any exposure level; 40,000 ppm was determined to be the 4-wk NOAEL rat as reported in RDB5B58, RDB5C16, and RDB6597

Four-Week Inhalation Toxicity Study with HFC143a in Rats, report 99-91 (TSCAT reports OTS0529920 and OTS-0529920-1), Haskell Laboratory for Toxicology and Industrial Medicine, E. I. duPont de Nemours and Company, Incorporated, Newark, DE, 1991 (RDB5C21)

four groups of 10 male and 10 female rats were exposed nose-only to concentrations of 0 (control), 2,000, 10,000, and 40,000 ppm v/v R$143 \mathrm{a}$ in air for $6 \mathrm{hr} / \mathrm{d}, 5 \mathrm{~d} / \mathrm{wk}$ for 4 weeks; 3 premature deaths were determined to not be treatment-related; decreases were observed in male, but not female body weights, but no toxicologically significant clinical signs or pathology measurements were observed during or after the exposures; microscopic degenerative changes were found in the testes of male rats in each exposure group, ranging in severity from slight at $2000 \mathrm{ppm}$ to minimal or mild at $10,000 \mathrm{ppm}$ and $40,000 \mathrm{ppm}$, but might have stemmed from stress due to the restraining devices and excessive temperatures - as reported in RDB5B58, RDB6597, and RDB5C16; study was repeated (see RDB5C20) with whole-body exposures based on the suspect findings as reported in RDB6597

In Vitro Evaluation of HFC-143a for Chromosome Aberrations in Human Lymphocytes, report 788-93, Haskell Laboratory for Toxicology and Industrial Medicine, E. I. duPont de Nemours and Company, Incorporated, Newark, DE, 1994 (23 pages, RDB5C22) 
$\mathrm{R}-143 \mathrm{a}$, health effects, toxicity as reported in RDB5B58 and RDB5C16

Metabolism of HFC-143a in the Rat, report 3-94, Haskell Laboratory for Toxicology and Industrial Medicine, E. I. duPont de Nemours and Company, Incorporated, Newark, DE, 1994 (RDB5C23)

$R-143 a$, health effects, toxicity as reported in RDB5C16

Mouse Bone Marrow Micronucleus Assay of HFC-143a by Inhalation, report 770-92, Haskell Laboratory for Toxicology and Industrial Medicine, E. l. duPont de Nemours and Company, Incorporated, Newark, DE, 1993 (30 pages, RDB5C24)

R-143a, health effects, toxicity as reported in RDB5B58 and RDB5C16

Summary - Mutagenicity Testing with Salmonella Typhimurium Strains on Plates of Gases, Liquids, and Solids, TSCAT report OTS0520485, ICl Central Toxicology Laboratory, Cheshire, UK, 1 August 1976 (RDB7284)

R-143a, genotoxicity, mutagenicity, toxicity as reported in RDB5B58

Static Acute 48-Hour $\mathrm{EC}_{50}{ }^{\mathrm{a}}$ to Daphnia Magna, report 541-89, Haskell Laboratory for Toxicology and Industrial Medicine, E. I. duPont de Nemours and Company, Incorporated, Newark, DE, 1989 (RDB5C93)

ecotoxicity of R-143a; ecological toxicity; aquatic impacts: $48-\mathrm{hr} E \mathrm{CC}_{50}$ daphnia magna $=300$ $\mathrm{mg} / \mathrm{l}$ (practically nontoxic) as reported in RDB5B58 and RDB5867

Subchronic Inhalation Toxicity: 90-Day Study with HFC-143a in Rats, report 690-92, Haskell Laboratory for Toxicology and Industrial Medicine, E. I. duPont de Nemours and Company, Incorporated, Newark, DE, 1992 (498 pages, RDB5C25)

20 male and 20 female rats were exposed (whole body) to 0 (control), 2,000,10,000, and $40,1000 \mathrm{ppm}$ v/v for $6 \mathrm{hr} / \mathrm{d}, 5 \mathrm{~d} / \mathrm{wk}$ for 90 days; sporadic changes in body weight were observed, but did not follow a dose-response relationship and were not considered to be treatment-related; no clinical signs of toxicity were observed during or after the exposures, and no pathology changes were found; no $B$-oxidation activity (peroxisome proliferation), organ weight, adverse gross, or microscopic changes were observed; 90-day NOAEL rat was considered to be $40,000 \mathrm{ppm}$ - as reported in RDB5C16 and RDB6597
1,1,1-Trifluoroethane (HFC 143a) Health Effects Information, CAS\# 420-46-2, Elf Atochem North America, Incorporated, Philadelphia, PA, undated circa 1995 (4 pages, RDB5B58)

$R-143 a$, summary of toxicological and ecological information

Workplace Environmental Exposure Level Guide - 1,1,1-trifluoroethane, American Industrial Hygiene Association (AIHA), Fairfax, VA, 1996 (2 pages, RDB5C16)

$\mathrm{R}-143 \mathrm{a}, \mathrm{WEEL}$, toxicology data, toxicity, safety classification

report 540-89, Haskell Laboratory for Toxicology and Industrial Medicine, E. I. duPont de Nemours and Company, Incorporated, Newark, DE, 1989 (RDB5C92)

$\mathrm{R}-143 \mathrm{a}$, toxicity, aquatic effects, 96-hr $\mathrm{LC}_{50}$ rainbow trout as reported in RDB5867

\section{R-152a}

R. H. Bruner (Pathology Associates, Incorporated), Pathology Peer Review of a Two-Year Inhalation Study of FC-152a in CD Rats, Haskell Laboratory for Toxicology and Industrial Medicine, E. I. duPont de Nemours and Company, Incorporated, Newark, DE, 19 May 1992 (RDB5C90)

summarizes chronic inhalation toxicity tests of $R-152 a$ in rats - as reported in RDB4B90

R. Culik and D. P. Kelly, Embryotoxicity and Teratogenicity Studies in Rats with 1,1Difluoroethane (FC-152a), report 437-79, Haskell Laboratory for Toxicology and Industrial Medicine, E. I. duPont de Nemours and Company, Incorporated, Newark, DE, 10 March 1980 (RDB5C88)

summarizes reproductive and teratology toxicity tests of R-152a in rats - as reported in RDB4B90

J. C. Edwards, Arcton 152a - Documentation for Hygiene Standard, $\mathrm{ICl}$ Chemicals and Polymers Limited, Cheshire, UK, 17 June 1985 (2 pages, RDB5A81)

outlines the rationale for an occupational exposure limit (OEL) for R-152a; recommends a hygiene standard of 1,000 ppm on an 8-hr TWA basis; toxicity

A. Koops, Mutagenic Activity of Ethane, 1,1-Difluoro in the Salmonella/Microsome Assay, report 731-77, Haskell Laboratory for Toxicology and Industrial Medicine, E. I. duPont de Nemours and 
Company, Incorporated, Newark, DE, 16 September 1977 (RDB5C86)

mutagenicity test of R-152a; toxicity - as reported in RDB4B90

M. A. Lee, P. W. Grube, and R. C. Graham, untitled toxicity review for 1,1-difluoroethane, Haskell Laboratory for Toxicology and Industrial Medicine, E. I. duPont de Nemours and Company, Incorporated, Newark, DE, 4 May 1990 (10 pages, RDB5857)

summarizes published and unpublished information on the toxicity of R-152a

D. E. Malek, Approximate Lethal Dose (ALD) of HFC-152a in Rats, report 524-90, Haskell Laboratory for Toxicology and Industrial Medicine, E. I. duPont de Nemours and Company, Incorporated, Newark, DE, 26 September 1990 (RDB5C84)

summarizes an ingestion toxicity study of R$152 a$ in rats - as reported in RDB4B90

J. W. McAlack and P. W. Schneider, Two-Year Inhalation Study with Ethane, 1,1-Difluoro (FC152a) in Rats, report 8-82 (OTS-0520320), Haskell Laboratory for Toxicology and Industrial Medicine, E. I. duPont de Nemours and Company, Incorporated, Newark, DE, 30 November 1982 (RDB5C87)

summarizes chronic inhalation toxicity tests of $\mathrm{R}-152 \mathrm{a}$ in rats - as reported in RDB4B90

B. L. Moore, Difluoroethane: Acute Inhalation Toxicity, report 699-75, Haskell Laboratory for Toxicology and Industrial Medicine, E. I. duPont de Nemours and Company, incorporated, Newark, DE, 18 November 1975 (2 pages with 1 table, RDB5C85)

tests of the acute inhalation toxicity in groups of 6 male Charles River CD rats exposed whole body to concentrations of $66,400,175,200$, $319,000,383,000$, and $437,500 \mathrm{ppm} v / \mathrm{v}$ in air for $4 \mathrm{hr}$ : except for the $66,400 \mathrm{ppm}$ exposures, oxygen was added to maintain a level of approximately $20 \%$; mortality ratios for the cited concentrations were $0 / 6,0 / 6,0 / 6,1 / 6$, and $2 / 6$ respectively; shallow breathing and hyperemia (excess of blood in a body part) were observed at $66,400 \mathrm{ppm}$ and higher; lethargy and reduced, or no, response to sound were observed at concentrations of 175,200 and higher; labored breathing resulted as the concentrations increased above $66,400 \mathrm{ppm}$; no abnormal signs were noted post exposure; gross pathology was performed on surviving animals after a 14-day recovery period, but no compound-related changes were found; concludes that the $4-h r$ ALC rat for $R-152 a$ is $383,000 \mathrm{ppm}$ v/v

B. L. Moore, Subacute Two-Week Inhalation Toxicity, report 158-76, Haskell Laboratory for Toxicology and Industrial Medicine, E. I. duPont de Nemours and Company, Incorporated, Newark, DE, 2 March 1976 (RDB5C89)

summarizes a 2-wk inhalation toxicity study of $\mathrm{R}-152 \mathrm{a}$ in rats - as reported in RDB4B90

1,1-Difluoroethane (HFC 152a) Health Effects Information, CAS\# 75-37-6, Elf Atochem North America, Incorporated, Philadelphia, PA, undated circa 1995 (4 pages, RDB5B59)

$\mathrm{R}-152 \mathrm{a}$, summary of toxicological and ecological information

Workplace Environmental Exposure Level Guide - 1,1-Difluoroethane, American Industrial Hygiene Association (AIHA), Fairfax, VA, 1994 (4 pages, RDB4B90)

R-152a, WEEL, toxicology data, toxicity, safety classification

\section{R-160}

H. Hermann and J. Vial, Syncopes AdrénalinoMonochloroéthanique [Sensitization by Adrenaline and Chloroethane], Compte Rendu Société de Biol., France, 117:439-440, October 1934 (2 pages, RDB6566)

$\mathrm{R}-160$, cardiac sensitization, toxicity

\section{R-170 (ETHANE)}

Toxicity Study Summary: Ethane, Phillips Petroleum Company, Bartlesville, OK, February 1990 (1 page with no figures or tables, RDB7623)

tests of the acute inhalation toxicity in 5 female and 5 male albino, Sprague-Dawley rats by 4 $\mathrm{hr}$, whole body exposures to $24,838 \mathrm{ppm}$ v/v R170 (ethane) in air: no deaths resulted and animals appeared normal throughout the exposures and 14-day observation period; treated females showed a slight reduction in mean body weight on day 3 following exposures; gross pathological signs found at necropsy included irregularly shaped pituitary with two clear cysts on the surface, scattered red foci on the surface of the lung, and - in two animals - dilation of the "right pelvis of the kidney" (probably intended as "pelvis of the right kidney"); concludes that the $4-\mathrm{hr} L \mathrm{CC}_{50}$ rat is 
$>24,838 \mathrm{ppm} \mathrm{v} / \mathrm{v}$; respiratory tract irritancy studies in male mice by head-only exposures to $23,985 \mathrm{ppm}$ v/v R-170 in air for $1 \mathrm{~min}$, repeated 10 minutes later, while monitored by a plethysmograph: no patterns of respiratory pause were evident; concludes that R-170 failed to produce upper respiratory irritancy in mice at $23,985 \mathrm{ppm}$ v/V

\section{R-E170}

Workplace Environmental Exposure Level Guide - Dimethyl Ether, American Industrial Hygiene Association (AIHA), Fairfax, VA, 1980 (RDB4B84)

R-E170, E-170, DME, WEEL, toxicology data, toxicity, safety classification

\section{R-21711}

Acute Inhalation Toxicity Study of lodoheptafluoropropane in Rats report on project 1530001 study 3, ManTech Environmental Technology, Incorporated, 1994 (RDB6693)

R-21711 (either R-217bal1 or R-217cal1), heptafluoroiodopropane, toxicity: 15-min LC $_{50}$ rat $62,000 \mathrm{ppm}$ (also see RTECS) as reported in RDB6208

\section{R-218}

Huntingdon Research Centre Limited (HRC, UK), report MIN 68/930457, 3M, St. Paul, MN, May 1993 (RDB65F8)

R-218 (3M PF-5030), acute inhalation (whole body exposure) toxicity study in rats: $4-\mathrm{hr} L \mathrm{LC}_{50}$ rat $>110,000 \mathrm{ppm}$; no deaths or clinical signs; body weight gain of female rats was slightly less than in the control group; macroscopic pathological examination after a 15-day observation period following exposures revealed some lung congestion in 1 of 10 exposed rats - as reported in RDB5703

Huntingdon Research Centre Limited (HRC, UK), report MIN 101/930623, 3M, St. Paul, MN, 23 June 1993 (RDB65F9)

R-218 (3M PF-5030), cardiac sensitization study in dogs, toxicity: tests at 50,000, $100,000,200,000,300,000$, and $400,000 \mathrm{ppm}$ in air (oxygen enriched above $100,000 \mathrm{ppm}$ to maintain oxygen concentration at approximately $22 \%$; sensitization NOEL $0 / 6$ at 300,000 ppm, LOEL 2/8 (one of them a questionable re- sponse) at $400,000 \mathrm{ppm}$ - as reported in RDB5703

Huntingdon Research Centre Limited (HRC, UK), report MIN 67/920961, 3M, St. Paul, MN, 24 August 1992 (RDB65G0)

R-218 (3M PF-5030), mutagenicity, toxicity: in vitro assay in Salmonella typhimurium bacteria at $800,000 \mathrm{ppm} \mathrm{v} / \mathrm{v}$; concluded not mutagenic - as reported in RDB5703

Huntingdon Research Centre Limited (HRC, UK), Perfluoropropane: Mouse Micronucleus Test, Rhône-Poulenc Chemicạls, Bristol, UK, 1992 (RDB6556)

R-218, health effects, mutagenicity for $6 \mathrm{hr}$ exposure of mice (negative at necropsy), toxicity

M. A. Lee and R. C. Graham, untitled toxicity review for 1,1,1,2,2,3,3,3-octafluoropropane, Haskell Laboratory for Toxicology and Industrial Medicine, E. I. duPont de Nemours and Company, Incorporated, Newark, DE, 8 January 1986 (5 pages, RDB5858)

summarizes published and unpublished information on the toxicity of R-218

Product Toxicity Summary Sheet: PF-5030 3M Brand Performance Fluid, summary 25937, 3M, St. Paul, MN, 22 October 1993 (2 pages, RDB5703)

R-218, toxicity: 4-hr $L^{2} C_{50}$ rat $>110,000 \mathrm{ppm}$; cardiac sensitization NOEL $0 / 6$ dogs at 300,000 ppm and LOEL 2/8 at 400,000 ppm; not mutagenic by in vitro assay in Salmonella typhimurium bacteria at $800,000 \mathrm{ppm} \mathrm{v/v}$

\section{R-225ca AND R-225cb}

Y. Kurata, Takechi, N. Toyatas, M. Tsuchitani, M. Katoh (Asahi Glass, Japan), G. M. Rusch (AlliedSignal Incorporated, USA), H. J. Trochimowicz (E. I. duPont de Nemours and Company, USA), and S. Shin-ya, Four-Week Repeated Inhalation Study of HCFC-225ca and HCFC-225cb in the Common Marmoset, Toxicology Letters, 92:209219, 1997 (11 pages, RDBA528)

subchronic toxicity of R-225ca and R-225cb

Four-Week Repeated Inhalation Study of HCFC-225ca and HCFC-225cb in the Common Marmoset, report 3L029, Mitsubishi-Kasei Institute of Toxicological and Environmental Sciences (MITES), Japan, 1993 (RDB65D6)

subchronic toxicity of R-225ca and R-225cb 


\section{R-227ea}

Dr. Bury, ARW-Begründung für HFA-227 [Documentation for Exposure Limit for HFA-227], Hoechst Aktiengesellschaft, Frankfurt am Main, Germany, 26 August 1992 (3 pages in German, RDB7116)

summary of unreferenced toxicity data: R227 ea resulted in no mortalities at $110,000 \mathrm{ppm}$ $\mathrm{v} / \mathrm{v}$, but higher concentrations were not tested because the rats [animals are not identified for this test, identification is based on corroborating information in a Hoechst Material Safety Data Sheet] breathed irregularly and became uncoordinated; Wistar rats and mice exposed head and nose only to concentrations of $0,150,000$, 300,000 and $500,000 \mathrm{ppm}$ for $1 \mathrm{hr}$ found the maximum tolerable concentration to exceed $500,000 \mathrm{ppm}$ subject to effects of sedation, trembling, increased blood-concentrations of carbon dioxide, all reversible following exposures; 2 male and 2 female beagle dogs exposed to concentrations of $50,000,150,000$, $200,000250,000,300,000$, and $500,000 \mathrm{ppm}$ R-227ea by face masks for $1 \mathrm{hr}$ showed lethargy, sedation, and listlessness at $150,000 \mathrm{ppm}$ and difficulty in breathing, spasms, and vomiting at higher concentrations, all fully reversible; concludes that the maximum tolerance in dogs is $300,000 \mathrm{ppm}$; summaries of subchronic and chronic exposures in rats and mice suggest NOEL concentrations of $300,000 \mathrm{ppm}$ and in beagle dogs at $150,000 \mathrm{ppm}$ (the highest concentration tested); an incomplete 90-day study on six beagle dogs found strongly narcotic effects, reversible following exposures, in two dogs at 300,000 ppm study; Ames tests in salmonella typhimurium and Escherichia coli with and without metabolic activation found R-227ea to not be mutagenic; negative findings also were found in a "HGPRT" test and a mouse micronucleus test; a cardiac sensitization test in dogs [use of epinephrine is typical but not mentioned] resulted in a response in one dog at $300,000 \mathrm{ppm}$ and a NOEL at $50,000 \mathrm{ppm}$; recommends an ARW [Hoechst exposure limit] of 1000 ppm [CAUTION: Users should examine the source documentation carefully before use of this summary, which was prepared without a translation.]

C. P. Chengelis (WIL Research Laboratories, Incorporated), Toxicological Evaluation of HFC227ea (FM-200 ${ }^{T M}$ ) - Background Document, Great Lakes Chemical Corporation, West Lafayette, IN, 20 June 1994 (RDB65F1) toxicity of R-227ea

Japan Bioassay Laboratory (JBL, Japan), Reverse Mutation Assay of HFC-227ea (Fron 227ea) by Using Bacteria (English translation), report 5542, Japan Industrial Safety and Health Association, Kanagawa, Japan, 1993 (RDBA618)

test of the mutagenicity of R-227ea of Salmonella typhimurium and Escherichia coli with and without metabolic activation; health effects; toxicity; results indicate that R-227ea is not mutagenic as tested - as reported in RDBA612

M. A. Lee and R. C. Graham, untitled toxicity review for 1,1,1,2,3,3,3-heptafluoropropane, Haskell Laboratory for Toxicology and Industrial Medicine, E. I. duPont de Nemours and Company, Incorporated, Newark, DE, 8 January 1986 (5 pages, RDB5860)

R-227ea, summary of published and unpublished toxicity literature

D. J. Naas (WIL Research Laboratories, Incorporated), A 90-Day Inhalation Toxicity Screen of FM-200 ${ }^{T M}$ in Rabbits, report on project WIL12318, Great Lakes Chemical Corporation, West Lafayette, IN, 1994 (RDBA619)

toxicity of R-227ea

M. D. Nemec (WIL Research Laboratories, Incorporated), An Inhalation Developmental Toxicity Study of FM-200 ${ }^{\mathrm{TM}}$ in Rabbits, report on project WIL-12317, Great Lakes Chemical Corporation, West Lafayette, IN, 1993 (RDBA620)

tests of the developmental toxicity of R-227ea in rabbits at concentrations of 0 (control), $20,000,50,000$, and $105,000 \mathrm{ppm} v / \mathrm{v}$ in air with enrichment to maintain $19 \%$ oxygen at the highest concentration; indicates a no observable adverse effect level (NOAEL) for both maternal dn developmental toxicity of 731.60 $\mathrm{mg} / \mathrm{L}[105,000 \mathrm{ppm} \mathrm{v} / \mathrm{v}]$ for $6 \mathrm{~h} / \mathrm{d}$ - as reported in RDBA612

M. D. Nemec (WIL Research Laboratories, Incorporated), An Inhalation Developmental Toxicity Study of FM-200 ${ }^{\mathrm{TM}}$ in Rats, report on project WIL12314, Great Lakes Chemical Corporation, West Lafayette, IN, 1993 (RDBA667)

tests of the developmental toxicity of R-227ea in groups of 24 rats at concentrations of 0 (control), 20,000,50,000, and 105,000 ppm v/v in air with enrichment to maintain $19 \%$ oxygen at the highest concentration; indicates a no observable adverse effect level (NOAEL) for both 
maternal and developmental toxicity of 731.60 $\mathrm{mg} / \mathrm{L}[105,000 \mathrm{ppm} \mathrm{v} / \mathrm{v}]$ for $6 \mathrm{~h} / \mathrm{d}$ - as reported in RDBA612

WIL Research Laboratories, incorporated, Acute Cardiac Sensitization Study in Dogs with FM$200^{\text {TM }}$ by Inhalation, report on project WIL-12265, Great Lakes Chemical Corporation, West Lafayette, IN, 1993 (RDBA615)

tests in 10 beagle dogs of the potential of R227ea to sensitize the heart to adrenaline; animals were exposed to $0,35,000,70,000$, $90,000,105,000$, and $140,000 \mathrm{ppm} \mathrm{v} / \mathrm{v}$ R227 ea in air for a minimum of $30 \mathrm{~min}$ at each concentration with at least $36 \mathrm{hr}$ between exposures; animals were challenged with intravenous injections of adrenaline both before and immediately after each induction; positive responses were determined by the occurrence of one or more premature ventricular contractions; they were observed in 1,5 , and 6 of 10 animals after exposures to $90,000,105,000$, and $140,000 \mathrm{ppm} \mathrm{v/v}$, respectively, indicating a treatment-dependent effect; there were no treatment-related clinical findings; indicates that concentrations of $90,000 \mathrm{ppm} \mathrm{v} / \mathrm{v}$ and higher cause cardiac sensitization as tested - as reported in RDBA612 [also see RDB65F2 for a summary of what probably was the same test with a different interpretation of the results]

WIL Research Laboratories, Incorporated, An Acute Inhalational Toxicity Screen of FM-200 ${ }^{\text {TM }}$ in Rats, report on project WIL-12248, Great Lakes Chemical Corporation, West Lafayette, IN, 1992 (RDBA616)

tests of the acute inhalation toxicity of R-227ea in 5 female and 5 male Charles River rats by whole-body exposures to concentrations of $788,696 \mathrm{ppm} v / \mathrm{v}$ in oxygen for $4 \mathrm{hr}$; no deaths occurred; females showed a $1.4 \%$ decrease in mean body weight and males showed a reduction in body weight gain during the 14 day observation period following exposures; all animals showed signs of anesthesia including decreased motor activity, decreased respiration, ataxia (inability to coordinate voluntary muscular movements), and prostration during exposures; one animal exhibited lacrimation and another tail chewing; necropsy revealed red foci in the lung of one animal and a mottled lung in another; no significant lung-weight differences were found; concluded that the 4-hr $\mathrm{LC}_{50}$ rat was $>788,696 \mathrm{ppm} v / \mathrm{v}$ - as reported in RDBA612
WIL Research Laboratories, Incorporated, Acute Inhalational Toxicity Screen in Albino Rats with FM-200 ${ }^{\text {TM }}$, report on project WIL-12226, Great Lakes Chemical Corporation, West Lafayette, IN, 1991 (RDBA617)

tests of the acute inhalation toxicity of R-227ea in groups of 3 female and 3 male Charles River rats by whole-body exposures to concentrations of 0 and $121,267 \mathrm{ppm} \mathrm{v/v}$ for $4 \mathrm{hr}$ or $241,188 \mathrm{ppm} \mathrm{v} / \mathrm{v}$ for $4.5 \mathrm{hr}$, the latter with a 19 min break during the exposure period; no deaths occurred; no significant body weight changes, exposure-related findings at necropsy, or lung-weight differences were found; all animals exhibited anesthesia, hypoactivity (less than normal activity), bradypnea (slow breathing), and/or ataxia (inability to coordinate voluntary muscular movements) during the treatment, but signs of anesthesia diminished after exposures; two animals showed signs of salivation for one day; concluded that the $\mathrm{LC}_{50}$ rat was $>241,188 \mathrm{ppm}$ v/v $~-$ as reported in RDBA612

FM-200 ${ }^{\mathrm{TM}}$ Toxicological Study Summaries, Great Lakes Chemical Corporation, West Lafayette, IN, circa 1995 (RDB65F2)

R-227ea, toxicity: 4-hr $L^{-} C_{50}$ rat $=>788,698$ ppm, anesthetic LOEL rat $=800,000 \mathrm{ppm}$, not mutagenic by reverse mutation assay with Salmonella typhimurium or Escherichia coli, no induction of chromosomal aberrations in cultured Chinese hamster lung, NOAEL $=100,000$ $\mathrm{ppm}$ in both a 10-day range-finding inhalation study in the rabbit and in a 14-day inhalation screening study in the rat, NOEL rat $=105,000$ ppm in a 90-day inhalation study, NOAEL rat = $105,000 \mathrm{ppm}$ in a developmental toxicity study, 30 -min cardiac sensitization in 10 male beagle dogs with pre-exposure and 30-minute challenge injections of epinephrine showed NOAEL (effects described as "minimal or mild in nature (single or few monofocal premature ventricular contractions)" $)=90,000 \mathrm{ppm}$ v/v, LOAEL for an unspecified number of dogs $=105,000 \mathrm{ppm} \mathrm{v} / \mathrm{v}$, and $E C_{50}=140,000 \mathrm{ppm} \mathrm{v/v}$

Heptafluoropropane (HFC-227ea) - Priority Existing Chemical 4 Secondary Notification Assessment, full public report, file EX/5 (NA/64), National Industrial Chemicals Notification and Assessment Scheme (NICNAS), Worksafe Australia, Sidney Australia, 30 July 1999 (30 pages with 4 tables, RDBA612)

toxicity and environmental risk assessment of R-227ea 
Test on Biodegradability of HFC-227ea by Microorganisms (Closed-Bottle Method), report 12419, Kurume Research Laboratories (KRL), Fukuoka, Japan, December 1993 (RDBA613)

ecotoxicity of R-227ea; environmental impacts and fate; groundwater and soil

\section{R-236fa}

C. J. Hardy, P. C. Kieran, and I. J. Sharman (Huntingdon Research Centre Limited, HRC, UK), HFC 236fa: Assessment of Cardiac Sensitization Potential in Dogs, report DPT 293/931308, Haskell Laboratory for Toxicology and Industrial Medicine, E. I. duPont de Nemours and Company, Incorporated, Newark, DE, 3 February 1994 (34 pages with 5 figures and 4 tables, RDB65A5)

cardiac sensitization tests for R-236fa in male beagle dogs for 10-minute exposures with adrenaline challenge by intravenous injection at 0 (control with adrenaline), 50,000, 100,000, $150,000,200,000,250,000$, and $300,000 \mathrm{ppm}$ $\mathrm{v} / \mathrm{v}$; positive responses were found in $0 / 6,0 / 6$, $0 / 6,2 / 6$, and $2 / 5$ dogs at $0,50,000,100,000$, 150,000 , and $200,000 \mathrm{ppm} \mathrm{v} / \mathrm{v}$; the investigators interpreted the result at $200,000 \mathrm{ppm} \mathrm{v/v}$ as $3 / 6$ positive based on the eliminated animal's responses at lower concentrations; $0 / 3$ tested animals showed positive responses at 250,000 ppm, but 3 dogs that had shown positive responses at lower concentrations were eliminated; tests at $300,000 \mathrm{ppm} \mathrm{v} / \mathrm{v}$ were not completed due to animal struggling at 250,000 $\mathrm{ppm}$ and observed anesthesia in 3 dogs at $300,000 \mathrm{ppm} \mathrm{v/v}$; concludes that R-236fa has the potential to cause cardiac sensitization in dogs at $150,000 \mathrm{ppm} \mathrm{v/v} \mathrm{(2/6} \mathrm{positive)} \mathrm{in-}$ creasing to $3 / 6$ positive at $200,000 \mathrm{ppm} \mathrm{v} / \mathrm{v}$; toxicity [findings imply a NOEL $0 / 6$ at 100,000 $\mathrm{ppm} \mathrm{v} / \mathrm{v}$ and a LOEL $2 / 6$ at $150,000 \mathrm{ppm} \mathrm{v} / \mathrm{v}$ ]

R. J. Hilarski (International Research and Development Corporation, IR\&D), Nose Only Acute Inhalation Toxicity Evaluation on T-6195 in Rats, report 137-178, 3M Company, St. Paul, MN, 27 September 1995 (41 pages with 1 figure and 26 tables, RDB6692)

tests of the acute toxicity of R-236fa by noseonly, $4 \mathrm{hr}$ inhalation in 5 male and 5 female albino rats; exposures at mean vapor concentrations of 1,960 and $148,600 \mathrm{ppm} v / \mathrm{v}$; no mortalities at either concentration [4-hr ALC rat $>148,600 \mathrm{ppm}$ ]; post exposure weight gain was normal, at 7 and 14 days, and no significant macroscopic abnormalities were noted at ne- cropsy; no significant pharmacotoxic signs were observed at $1,960 \mathrm{ppm}$; animals exposed to $148,600 \mathrm{ppm}$ exhibited rapid respiration; no anesthetic effect indicated at either concentration

N. D. Smith, Summary of HFC-236fa Toxicity Studies, fax to D. Minday (3M Company), U.S. Environmental Protection Agency (EPA), Research Triangle Park, NC, 22 February 1996 (5 pages, RDB65A6)

R-236fa, toxicity: mutagenicity tests in the Salmonella typhimurium and Escherichia coli (negative); clastogenic (chromosome damaging) test in human lymphocytes (negative); mouse bone-marrow micronucleus test (negative); twoweek inhalation study in the rat at $0,5,000$, 20,000 , and $50,000 \mathrm{ppm}$ (NOAEL at $5,000 \mathrm{ppm}$ and anesthetic effect at 20,000 ppm); 90-day inhalation study in the rat for $6 \mathrm{hr} / \mathrm{d}, 5 \mathrm{~d} / \mathrm{wk}$, for 14 weeks (NOAEL at 20,000 ppm); inhalation developmental study in the rat at $0,5,000$, 20,000 , and $50,000 \mathrm{ppm}$ (maternal NOAEL at $5,000 \mathrm{ppm}$, developmental NOAEL at 50,000, anesthetic effect at 20,000 ppm); 5-day extended inhalation study in the rat at $50,000 \mathrm{ppm}$ (slight respiratory tract irritation, concluded that R-236fa has low acute inhalation toxicity at $50,000 \mathrm{ppm}$ )

R. Valentine, D. A. Keller, J. C. Cook, L. B. Biegel, G. S. Elliott, T. W. Slone, S. M. Munley, K. S. Bentley, W. J. Brock (E. I. duPont de Nemours and Company, Incorporated), and G. B. Briggs (GeoCenters, Incorporated), Toxicologic Profile of the Alternative Refrigerant HFC-236fa, abstract 1489 (35th Annual Meeting, 1996), The Toxicologist, republished in Fundamental and Applied Toxicology, 30(1/2):291, March 1996 (1 page, RDB6A96)

R-236fa, HFC-236fa: acute, subchronic, developmental, and genetic toxicity; cardiac sensitization; 4-hr ALC rat $>195,000$ ppm; narcosis was the predominant clinical sign observed during exposure; cardiac sensitization LOEL in beagle dogs was $150,000 \mathrm{ppm}$ with a NOEL of $100,000 \mathrm{ppm}$; no evidence of mutagenic or genotoxic activity was found in vitro in Ames or human lymphocyte assays or in vivo in the mouse micronucleus assay; subchronic inhalation studies with exposures of rats for $6 \mathrm{hr} / \mathrm{d}, 5$ $\mathrm{d} / \mathrm{wk}$, for 2 and 13 weeks at 5,000,20,000 and $50,000 \mathrm{ppm}$ yielded no evidence of body or organ weight effects, clinical pathology, organ pathology, or induction of hepatic peroxisomes; rats exposed to 20,000 or $50,000 \mathrm{ppm}$ had a 
transiently diminished acoustic startle response during exposures; rats exposed to $5,000 \mathrm{ppm}$ were unaffected and some adaptation to narcosis was noted as the study progressed for the higher concentrations; maternal weight gain was evident at $\cdot 20,000 \mathrm{ppm}$ in a rat developmental toxicity study; fetal toxicity was found at $.5,000$ ppm; the overall NOEL was $5,000 \mathrm{ppm}$, based on narcosis noted at concentrations of $\cdot 20,000 \mathrm{ppm}$

R. Valentine, Two-Week Inhalation Toxicity Study with HFC-236fa in Rats, report 596-94, Haskell Laboratory for Toxicology and Industrial Medicine, E. I. duPont de Nemours and Company, Incorporated, Newark, DE, 20 June 1995 (211 pages with 3 figures and 21 tables, Rdb6A93)

R-236fa, health effects, toxicity: four groups of 5 female and 5 male rats were exposed by inhalation to target concentrations of $0,5,000$, 20,000 , or $50,000 \mathrm{ppm}$ v/v R-236fa in air for 6 $\mathrm{hr} / \mathrm{d}, 5 \mathrm{~d} / \mathrm{wk}$, for two weeks; actual concentrations determined by gas chromatography (GC) were $0,5,020 \pm 10,20,000 \pm 35$, and 50,000 60 $\mathrm{ppm} \mathrm{v} / \mathrm{v}$ and daily means were within $\pm 10 \%$ of the targets; no body weight effects or abnormal clinical observations to exposed rats; NOAEL rat $=5,000 \mathrm{ppm}$; anesthetic NOEL at 5,000 ppm and LOEC rat at 20,000 ppm; slight decrease in hepatic $B$-oxidation activity at 50,000 ppm in rats killed after the tenth exposure, but this effect was deemed not biologically adverse since the change was not accompanied by relevant clinical, pathologic, organ weight, or liver histopathologic changes

A. Vinegar (ManTech Environmental Technology, Incorporated), G. W. Butler, M. C. Caracci (Geocenters Incorporated), and J. D. McCafferty (U.S. Air Force, USAF), Gas Uptake Kinetics of 1,1,1,3,3,3-Hexafluoropropane (HFC-236fa) and Identification of its Potential Metabolites report AL/OE-TR-1995-0177, Armstrong Laboratory, U.S. Air Force, Wright-Patterson Air Force Base, $\mathrm{OH}$, 1995 (RDB7C85)

pharmacokinetic toxicity test of R-236fa in rats exposed to concentrations up to $20,000 \mathrm{ppm}$ $\mathrm{v} / \mathrm{v}$ for $6 \mathrm{hr}$; indicates that $\mathrm{R}-236 \mathrm{fa}$ was readily absorbed with inhalation, but analyses of blood, urine, and feces showed that it was not metabolized and was excreted as the parent compound - as reported in RDB8612

90-Day Inhalation Toxicity: 90-Day Study with HFC-236fa in Rats, report 211-95, Haskell Laboratory for Toxicology and Industrial Medicine, E. I.
duPont de Nemours and Company, Incorporated, Newark, DE, 12 February 1996 (332 pages with 4 figures and 28 tables, RDbA306)

subchronic toxicity tests of R-236fa in groups of 10 male and 10 female Charles River $C D$ rats by inhalation for whole-body exposures at target concentrations of 0 (control), $5,000,20,000$, or $50,000 \mathrm{ppm} \mathrm{v/v}$ for $6 \mathrm{hr} / \mathrm{d}, 5 \mathrm{~d} / \mathrm{wk}$; actual mean concentrations were $0,4,980,20,000$, or $50,300 \mathrm{ppm} \mathrm{v/v}$; indicates that there were no compound-related effects except reduced alerting response at the highest concentration; notes that this anesthetic effect was observed in 6-8 of 10 rats in the first day and in $1-3$ of 10 rats for the first two weeks, but primarily in the last two hours of the exposures; further notes that the number of rats exhibiting diminished response decreased with successive exposures and that all rats exhibited normal alerting response by study day 18; concludes that the no observed adverse effect level (NOAEL) was $20,000 \mathrm{ppm} \mathrm{v} / \mathrm{v}$ based on the anesthetic effect; report indicates Good Laboratory Practice (GLP) compliance

Acute Inhalation Toxicity Study of HFC-236fa in Albino Rats, report WIL-189022, WIL Research Laboratories, Incorporated, circa 1996 (Rdb6A94)

tests of the acute inhalation toxicity of R-236fa by whole-body exposures of 5 female and 5 male rats at $457,000 \mathrm{ppm} \mathrm{v} / \mathrm{v}$; since no deaths occurred, reports the 4-hr ALC rat as $>457,000$ ppm; all of the animals exhibited hyperactivity and then prostration during exposures, but recovered in 1 day; 2 females exhibited slight body weight loss, but appeared normal by the end of the 14-day observation period; 4 animals had dark red lungs at necropsy; 2 females had cysts on the kidneys and 1 female had an enlarged pituitary gland

In Vitro Assay of HFC-236fa for Chromosome Aberrations in Human Lymphocytes, report 604-94, Haskell Laboratory for Toxicology and Industrial Medicine, E. I. duPont de Nemours and Company, Incorporated, Newark, DE, 1994 (RDBA303)

mutagenicity toxicity study of R-236fa for target exposures of $200,000,300,000,400,000$, $600,000,800,000$, or $1,000,000 \mathrm{ppm} \mathrm{v} / \mathrm{v}$; indicates R-236fa was not genotoxic in this in vitro test - as reported in RDB8612

Inhalation Developmental Toxicity Study of HFC-236fa in Rabbits, report 417-96, Haskell Laboratory for Toxicology and Industrial Medicine, 
E. I. duPont de Nemours and Company, Incorporated, Newark, DE, 1996 (RDBA307)

developmental toxicity of R-236fa in 20 white rabbits exposed by inhalation to target concentrations of 0 (control), $5,000,20,000$, or 50,000 ppm v/v in air for $6 \mathrm{hr} / \mathrm{d}$ on days 7-19 of gestation; indicates no evidence of maternal or developmental effects at any concentration - as reported in RDB8612

Inhalation Developmental Toxicity Study of HFC-236fa in Rats, report 66-95, Haskell Laboratory for Toxicology and Industrial Medicine, E. I. duPont de Nemours and Company, Incorporated, Newark, DE, 1996 (RDBA308)

developmental toxicity of R-236fa in 25 pregnant rats exposed by inhalation to target concentrations of 0 (control), 5,000, 20,000, or $50,000 \mathrm{ppm} v / \mathrm{v}$ for $6 \mathrm{hr} / \mathrm{d}$ on days $6-15$ of gestation; indicates a decrease in maternal body weight gain for the first two days of exposure at 20,000 and $50,000 \mathrm{ppm} \mathrm{v} / \mathrm{v}$, with accompanying reduction in food consumption and occasional diminished alerting response at the higher exposure level; indicates no evidence of maternal toxicity at $5,000 \mathrm{ppm}$ v/v or of reproductive toxicity at any concentration - as reported in RDB8612

Mouse Bone Marrow Micronucleus Assay of HFC-236fa by Inhalation, report 602-94, Haskell Laboratory for Toxicology and Industrial Medicine, E. I. duPont de Nemours and Company, Incorporated, Newark, DE, 1994 (RDBA304)

mutagenicity toxicity study of R-236fa by a mouse bone marrow micronucleus test for target exposures of $5,000,20,000$, or $50,000 \mathrm{ppm}$ $\mathrm{v} / \mathrm{v}$ for $6 \mathrm{hr} / \mathrm{d}$ for 2 consecutive days; indicates $\mathrm{R}-236 \mathrm{fa}$ was inactive in this in vivo test - as reported in RDB8612

Mutagenicity Testing of HFC-236fa in the Salmonella Typhimurium and Escherichia Coli: Plate Incorporation Assay, report 647-94, Haskell Laboratory for Toxicology and Industrial Medicine, E. I. duPont de Nemours and Company, Incorporated, Newark, DE, 1994 (RDBA305)

mutagenicity toxicity test of R-236fa by an Ames assay; indicates R-236fa was found to not be mutagenic with or without metabolic activation - as reported in RDB8612

Workplace Environmental Exposure Level Guide - 1,1,1,3,3,3-Hexafluoropropane, American Industrial Hygiene Association (AlHA), Fairfax,
VA, 1998 ( 3 pages with no figures or tables, RDB8612)

documents determination of a Workplace Environmental Exposure Level (WEEL) Guide for R236fa; summarizes identifiers, chemical and physical properties, uses, production quantities, toxicology tests conducted in animals (acute, subchronic, chronic, carcinogenicity, reproductive, developmental, genotoxicity, mutagenicity, metabolism, and pharmacokinetics); indicates no human use and exposures experience; discusses a rationale for setting the occupational exposure limit; indicates that R-236fa is low in acute toxicity with a 4-hr $L C_{50}$ rat of $>450,000$ $\mathrm{ppm} \mathrm{v} / \mathrm{v}$ and cardiac sensitization LOEL in dogs

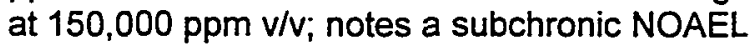
of 5,000 or $20,000 \mathrm{ppm} v / \mathrm{v}$ in rats based on a transient anesthetic effect; also notes absence of developmental toxicity up to $50,000 \mathrm{ppm}$ v/v and genotoxicity as tested; recommends a WEEL guide of $1,000 \mathrm{ppm}$ for 8 -hr on a timeweighted average (TWA) basis; includes 12 references

\section{R-245ca}

R. D. Bagnall, W. Bell, and K. Pearson (Imperial Chemical industries Limited, UK), New Inhalation Anaesthetics: IV - Fluorinated Propanes, Joumal of Fluorine Chemistry, 13(3):209-223, 1979 (15 pages with 6 figures and 4 tables, RDB5C76)

summarizes acute inhalation toxicity tests in mice of R-245ca and others for $30 \mathrm{~min}$ exposures of mixtures of the tested gases with oxygen; for R-245ca, indicates a 30-min ALC of $240,000 \mathrm{ppm} v / \mathrm{v}$ with minimal, unspecified side effects as well as an anesthetic LOEL at $100,000 \mathrm{ppm} \mathrm{v/v}$

P. W. Grube, untitled toxicity review for $1,1,2,2,3-$ pentafluoropropane, Haskell Laboratory for Toxicology and Industrial Medicine, E. I. duPont de Nemours and Company, Incorporated, Newark, DE, 24 July 1991 (5 pages, RDB5859)

summarizes published and unpublished toxicity information on R-245ca

report 33-60, Haskell Laboratory for Toxicology and Industrial Medicine, E. I. duPont de Nemours and Company, Incorporated, Newark, DE, 1960 (RDB5C77)

summarizes tests of the anesthetic capabilities of R-245ca in mice; animals were exposed to concentrations of $50,000,100,000,120,000$, 
and $150,000 \mathrm{ppm} v / \mathrm{v}$ for 5 or $10 \mathrm{~min}$ in a rotating apparatus; inability to maintain an upright position for a minimum of 15 consecutive seconds was deemed an anesthetic effect; the LOEC for an unspecified number of mice and unspecified number tested was $100,000 \mathrm{ppm}$ $\mathrm{V} / \mathrm{V}$; indicates no clinical evidence of permanent injury, but exposures at 120,000 and 150,000 $\mathrm{ppm} \mathrm{v} / \mathrm{v}$ caused severe, transient effects on respiration; toxicity - as reported in RDB5859

\section{R-245fa}

D. W. Coombs (Huntingdon Life Sciences Limited, HLS, UK), HFC-245fa (515-96A): A Study for Effects on Embryofoetal Development of the Rat (Inhalation Administration), report MA-RR-972374 (also identified as ALS 132/963712), AlliedSignal Incorporated, Morristown, NJ, USA, 20 June 1997 (137 pages with 2 figures and 12 tables, Rdb7556)

toxicity tests by whole-body exposures of pregnant rats to nominal concentrations of 0 (control), 500,2,000, 10,000, and 50,000 ppm R$245 \mathrm{fa}$ for $6 \mathrm{hr} / \mathrm{d}$ on days $6-19$ post coitum: food consumption and bodyweight gain were reduced on days 6-19 and 6-12, respectively, for animals exposed to $10,000 \mathrm{ppm}$ and on days 6 19 and 6-20, respectively, for animals exposed to $50,000 \mathrm{ppm}$; animals were sacrificed on day 20 for post mortem determination of litter values and examination for visceral and skeletal changes; no effects were found on fetal parameters; study concluded that the NOEL for maternal effects was $2,000 \mathrm{ppm}$ and for fetal effects was $50,000 \mathrm{ppm}$ [full report not obtained - prepared from report summary]

D. W. Coombs (Huntingdon Life Sciences Limited, HLS, UK), HFC-245fa (507-96A and 515-96A): 13-Week Repeat Dose Inhalation Toxicity Study in Rats, report MA-RR-97-2376 (also identified as ALS 131/963272), AlliedSignal Incorporated, Morristown, NJ, USA, 18 June 1997 (379 pages with 6 figures and 12 tables, Rdb7558)

subchronic inhalation toxicity tests of R-245a in rats exposed to concentrations of 0 (control), $500,2,000,10,000$, or $50,000(0,508,2,038$, 10,152 , and 50,555 actual) $\mathrm{ppm} v / \mathrm{v}$ in air for 6 $\mathrm{hr} / \mathrm{d}, 5 \mathrm{~d} / \mathrm{wk}$, for 13 weeks: exposures were reduced to approximately $3 \mathrm{hr}$ for exposure 40 due to apparatus failure; no treatment-related effects were seen in clinical signs, food consumption, bodyweight gains, macroscopically at necropsy, or in organ weight data; dose related increases were seen in urinary fluoride levels, water consumption, and urine volumes, suggesting some metabolism of R-245fa; some changes, possibly related to the increased urine volume, were observed in clinical chemistry parameters; they included increases in blood urea nitrogen and serum level enzyme activity, but they did not follow an exposurerelated pattern; trace to moderate inflammation of the myocardium (heart muscle) were found at necropsy in both the males and females; study suggests that this response resulted from long-term exposures, since myocarditis (inflammation of the heart muscle) was not observed in rats exposed for 28 days [full report not obtained - prepared from report summary and additional information from RDB7612]

D. W. Coombs (Huntingdon Life Sciences Limited, HLS, UK), HFC-245fa (507-96A): 28-Day Repeat Dose Inhalation Toxicity Study in Rats, report MA-RR-97-2346 (also identified as ALS 105/962523), AlliedSignal Incorporated, Morristown, NJ, USA, 3 March 1997 (350 pages with 6 figures and 20 tables, Rdb7557)

subchronic inhalation toxicity tests in groups of rats by whole-body exposures to concentrations of 0 (control), 500,2,000, 10,000, or $50,000(0,516,1,998,10,102$, and 49,600 actual) ppm v/v R-245fa in air for $6 \mathrm{hr} / \mathrm{d}$ for 28 consecutive days [RDB7612 indicates $5 \mathrm{~d} / \mathrm{wk}$ ]: 2 female rats exposed to $49,600 \mathrm{ppm}$ died during the study; absence of marked effects in other animals suggests that these deaths, considered to be agonal in origin, were not treatment related, but report notes that this possibility cannot be excluded; 4 females exposed to 49,600 ppm exhibited lethargy and unsteady gait following overnight collection of urine samples at week 4, but animals recovered when given access to water; increases were seen in urinary fluoride levels, water consumption, and urine volumes, suggesting some metabolism of $\mathrm{R}-245 \mathrm{fa}$; increases in blood urea nitrogen and serum level enzyme activity also were seen, but did not follow an exposure-related pattern; report concludes that the NOEL was $500 \mathrm{ppm}$ $\mathrm{v} / \mathrm{V}$; the no adverse effect level (NOAEL) is indicated as $50,000 \mathrm{ppm} \mathrm{v/v}$ based on lack of histopathological effects and significant recovery following withdrawal; study also concluded that the observed effects of treatment were considered of biological but not necessarily toxicological significance [full report not obtained - prepared from report summary and additional information from RDB7612] 
C. A. Dance (Pharmaco-LSR Limited, UK), HFC245fa (Vapour Phase): In-Vitro Assessment of Clastogenic Activity in Cultured Human Lymphocytes, report MA-RR-94-2014 (also identified as 93/ADL010/0986 and as 93/09086), AlliedSignal Incorporated, Morristown, NJ, USA, 18 February 1994 (53 pages with 10 tables, Rdb7551)

test for mutagenic potential of R-245fa by an in vitro mammalian cytogenetic study using cultured human lymphocytes with a metabolic activation system, derived from rat livers (S-9 mix), at nominal concentrations of $300,000,500,000$, and $700,000 \mathrm{ppm} \mathrm{v} / \mathrm{v}$ and without activation at $100,000,200,000$, and $300,000 \mathrm{ppm} \mathrm{v/v: \text {no }}$ biologically or statistically significant increases in aberrant cell frequencies were found with activation, but there was evidence of toxicity, based on a $25 \%$ reduction in mitotic activity, at 300,000 ppm without it; mean aberrant cell frequencies were $7.0,7.5$, and $12 \%$ at the three concentrations tested, respectively, without activation; study concluded that R-245fa showed weak clastogenic (chromosome-damaging) activity in the absence of metabolic activation [full report not obtained - prepared from report summary]

C. N. Edwards (Pharmaco-LSR Limited, UK), HFC-245fa: Mouse Micronucleus Test, report MA-RR-94-2016 (also identified as 93/ADL013/1219 and as 93/1219), AlliedSignal Incorporated, Morristown, NJ, USA, 18 February 1994 (40 pages with 1 figure and 2 tables, Rdb7552)

test for mutagenic potential of R-245fa by an in vivo mouse micronucleus study in bone marrow following a 4-hr acute inhalation exposure at 101,300 ppm: exposed animals were underactive in their restraint tubes during the exposures, but no depression of bone marrow proliferation that would indicate toxicity was found 24 or $48 \mathrm{hr}$ after exposure; report concludes that there was no evidence of induced chromosomal or other damage [full report not obtained - prepared from report summary and additional information from RDB7612]

Huntingdon Life Sciences Limited (HLS, UK), A Two-Week Dose Range-Finding Inhalation Toxicity Study with HFC-245fa in Dogs, report ALS 174/982608, AlliedSignal Incorporated, Morristown, NJ, USA, 1998 (RDBA474)

toxicity tests of R-245fa

Huntingdon Life Sciences Limited (HLS, UK), HFC-245fa (Vapour Phase): In-Vitro Assess- ment of Clastogenic Activity in Cultured Human Lymphocytes, report 96/ADL040/0605, AlliedSignal Incorporated, Morristown, NJ, USA, 19 November 1996 (RDB7554)

test for mutagenic potential of R-245fa

G. C. Jackson (Huntingdon Life Sciences Limited, HLS, UK), HFC 245fa (513-96A): Acute Inhalation Toxicity in Rats (4-Hour Exposure), report ALS 125/961468 (also identified as MA-RR-972342), AlliedSignal Incorporated, Morristown, NJ, USA, 27 January 1997 (46 pages with 2 figures and 12 tables; 4 page cover and summary available from JMC as RDB7115)

acute inhalation toxicity tests with two groups of 5 male and 5 female albino rats exposed (snout only) to 0 and $116,000 \mathrm{ppm} v / \mathrm{v}$ R-245fa in air for 4 hours: no deaths resulted; animals exhibited irregular respiration, restless behavior, and intermittent muscular contractions during exposure at $116,000 \mathrm{ppm}$ as well as piloerection (involuntary erection or bristling of fur) immediately following the exposure; no reported clinical signs indicate anesthesia or central nervous system (CNS) response, suggesting an anesthetic effect NOEL in rats of 116,000 ppm; no effects were noted on the rate of bodyweight gain, lung weight to bodyweight ratio, and food and water consumption; appearance and behavior were normal from the day following exposure; slight congestion was observed in the lungs of one male test rat, but deemed to be unrelated to the test treatment; no other macroscopic pathology abnormalities were found; report concludes that the 4-hr $L_{50}$ rat exceeds $116,000 \mathrm{ppm}$ v/v

G. C. Jackson (Huntingdon Life Sciences Limited, HLS, UK), HFC 245fa (493-95A): Acute Inhalation Toxicity in Rats 4-Hour Exposure, report ALS 104/951746 (also identified as MA-RR-962270), AlliedSignal Incorporated, Morristown, NJ, USA, 22 February 1996 (47 pages; 4 page cover and summary available from JMC as RDB7111)

acute inhalation toxicity tests with three groups of 5 male and 5 female Sprague-Dawley $C D$ rats exposed to 0 (control), 143,000, and 203,000 ppm v/v R-245fa in air for 4 hours: no deaths resulted; all animals exposed to 143,000 and $203,000 \mathrm{ppm} v / \mathrm{v}$ exhibited exaggerated respiratory movements, adoption of an abnormal posture, and reduced response to external stimuli during exposures; 3 female rats experienced occasional clonic (spasms of alternating contractions and partial relaxations) convulsions at $143,000 \mathrm{ppm}$; no clinical signs 
were observed during a subsequent 14-day observation period; food consumption was reduced for 1 day following exposures, but water consumption and the rate of bodyweight gain were the same for treated animals and controls; no macroscopic abnormalities were found; report concludes that the $4-\mathrm{hr} \quad \mathrm{LC}_{50}$ rat is $>203,000 \mathrm{ppm} v / \mathrm{v}$ and that there was evidence of an anesthetic effect at both 143,000 and $203,000 \mathrm{ppm} v / \mathrm{v}$ [full report not obtained - prepared from summary]

I. R. Johnson (Pharmaco-LSR Limited, UK), HFC. 245fa: Acute Percutaneous Toxicity Study in the Rabbit, report 93/ADL011/1086 (also identified as MA-RR-94-2015), AlliedSignal Incorporated, Morristown, NJ, USA, 21 February 1994 (37 pages; 3 page cover and summary available from JMC as RDB7113)

dermal toxicity of R-245fa to 5 male and 5 female New Zealand White rabbits at a dosage of $2 \mathrm{~mL} / \mathrm{kg}(0.031 \mathrm{oz} / \mathrm{lb})$ bodyweight for $24 \mathrm{hr}$. there were no deaths or signs of reaction to treatment; animals achieved expected bodyweight gains; organ weights were unremarkable and there was no sign of macroscopic lesion at necropsy; report concludes that the $\mathrm{LD}_{50}$ dermal was $>2 \mathrm{~mL} / \mathrm{kg}(>2,600 \mathrm{mg} / \mathrm{kg})$ [full report not obtained - prepared from summary]

T. J. Kenny (Huntingdon Research Centre Limited, HRC, UK), HFC 245fa: Assessment of Cardiac Sensitization Potential in Dogs, report ALS 52/942695 (also identified as MA-RR-94-2151), AlliedSignal Incorporated, Morristown, NJ, USA, 29 November 1994 (30 pages; 3 page cover and summary available from JMC as RDB7112)

toxicity for cardiac sensitization in 6 beagle dogs with intravenous injection of adrenaline before and during inhalation of 10,000 and $20,000 \mathrm{ppm}$ v/v R-245fa in air: no dogs responded positively at either concentration, leading to a finding that $20,000 \mathrm{ppm}$ is a cardiac sensitization NOEL [full report not obtained - prepared from summary]

K. May (Pharmaco-LSR Limited, UK), HFC-245fa: Assessment of Mutagenic Potential in AminoAcid Auxotrophs of Salmonella Typhimurium and Escherichia Coli (the Ames Test), report MA-RR-95-2205 (also identified as 95/ADL035/0004 and as 95/0004), AlliedSignal Incorporated, Morristown, NJ, USA, 30 June 1995 (49 pages with 6 tables, Rdb7553)

test for mutagenic potential of R-245fa by an Ames assay using five histidine-dependent strains of Salmonella typhimurium and one tryptophen-dependent auxotroph of E. coli exposed to R-245fa vapor, both with and without a metabolic activation system derived from rat livers (S-9 mix), at nominal concentrations of 0 (control), 25,000, 50,000, 100,000, 200,000, and $400,000 \mathrm{ppm} \mathrm{v} / \mathrm{v}$ : no increases in reversion to prototrophy were found; study concluded that R-245fa vapor is not mutagenic as tested [full report not obtained - prepared from report summary]

D. P. Meyers (Huntingdon Life Sciences Limited, HLS, UK), HFC-245fa: A Dose Range Finding Study by Inhalation Administration in the Pregnant Rat, report MA-RR-97-2339 (also identified as ALS 130/961798), AlliedSignal Incorporated, Morristown, NJ, USA, 15 January 1997 (87 pages with 2 figures and 4 tables, Rdb7555)

pilot developmental toxicity study in groups of 12 pregnant rats by whole body exposures to nominal concentrations of 0 (control), 500 , $2,000,10,000$, and 50,000 ppm R-245fa: no effects were observed at concentrations as high as $10,000 \mathrm{ppm}$; food intake was lower on days 9-10 and body weight gains were reduced on days 9-13 of pregnancy for animals exposed to $50,000 \mathrm{ppm}$, but there were no treatmentrelated effects on litter parameters or macroscopic external fetal structure; concluded that the tested concentrations would be appropriate for a future embryotoxicity study in the rat [full report not obtained - prepared from report summary]

G. M. Rusch (AlliedSignal Incorporated, USA), D. W. Coombs, and C. J. Hardy (Huntingdon Life Sciences Limited, HLS, UK), The Acute, Genetic, Developmental, and Inhalation Toxicity of 1,1,1,3,3-Pentafluoropropane, Toxicological Sciences, 52(2):289-301, December 1999 (13 pages with 14 tables, RDB9707)

summary of toxicity tests of R-245fa: there were no effects on body weight or observable changes at necropsy in screening inhalation tests with five male and five female mice at $101,300 \pm 12,900$ ppm $v / v$ by snout-only exposures for $4 \mathrm{hr}$; subsequent tests in groups of five male and five female Sprague Dawley CD rats at $143,000 \pm 1,800$ and $203,000 \pm 1,000$ ppm for $4 \mathrm{hr}$ yielded no mortalities or effects on body weight; food consumption was slightly reduced for the day following exposures, but rebounded; exposed animals showed signs of central nervous system (CNS) depression including irregular respiration, restless behavior, 
intermittent muscular contractions, abnormal posture, and reduced response to external stimuli, but these symptoms disappeared following exposures; tests of cardiac sensitization in six beagle dogs led to a fatal ventricular fibrillation (very rapid and irregular contractions of the ventricles of the heart resulting in loss of synchronization between the heartbeat and pulse beat) in one dog at $73,000 \mathrm{ppm} v / \mathrm{v}$ and disqualification of a second because it did not accept the procedure; concludes that the noobserved-effect level (NOEL) and the lowestobserved-effect level (LOEL) for cardiac sensitization were 34,100 and $44,000 \mathrm{ppm}$, respectively; tests of acute dermal toxicity of R-245fa with 5 male and 5 female rabbits yielded no adverse reactions at a dose of $2 \mathrm{ml} / \mathrm{kg}$ for $24 \mathrm{hr}$; no developmental effects were found in a rat study at 10,000 and $50,000 \mathrm{ppm}$, though there was a slight reduction in pup weight at the higher concentration; subchronic and chronic inhalation exposures of rats yielded no treatment related effects on survival, clinical signs, food consumption, or body weight gain at $50,000 \mathrm{ppm}$ v/v by snout-only exposures for 6 $\mathrm{hr} / \mathrm{d}$ for two weeks, by whole-body exposures for four weeks, or by whole-body exposures for $5 \mathrm{~d} / \mathrm{wk}$ for 13 weeks; urine volumes increased, urinary fluorides were elevated, and small changes were seen in red blood counts and blood chemistry, but histopathological examination showed no effects on the kidneys, liver, or lungs; there was an increased incidence of mild myocarditis (inflammation of the heart muscle) in all animals exposed at $50,000 \mathrm{ppm}$ and in the majority exposed at $10,000 \mathrm{ppm}$; the authors suggest a NOEL level of $2,000 \mathrm{ppm}$ $v / v ; R-245 f a$ was found to be inactive in reverse mutation genetic assays (Ames tests) with five strains of Salmonella typhimurium and one strain of Escherichia coli with and without S-9 metabolic activation at concentrations up to $400,000 \mathrm{ppm} \mathrm{v/v}$ in air and at $1,000,000 \mathrm{ppm}$ $\mathrm{v} / \mathrm{v}$ (no air); weak evidence of clastinogenic activity was seen in an in vitro cytogenetics study with cultured human lymphocytes in the absence of metabolic activation for exposures of 300,000 and $400,000 \mathrm{ppm} \mathrm{v} / \mathrm{v}$, but not at $200,000 \mathrm{ppm}$ v/v or for $6 \mathrm{hr}$ exposures; no evidence of clastogenicity was found with S-9 activation; tests for chromosomal damage in polychromatic erythrocytes of mice at $100,000 \mathrm{ppm}$ for $4 \mathrm{hr}$ were negative; paper concludes that R$245 \mathrm{fa}$ appears to show only minimal toxicity, that exposures up to $5,000 \mathrm{ppm} v / \mathrm{v}$ yielded no clear signs of toxicity, and exposures up to $10,000 \mathrm{ppm} v / \mathrm{v}$ resulted in only mild effects

G. M. Rusch, Toxicology Testing of a New Hydrofluorocarbon, FYI notification to the U.S. Environmental Protection Agency (EPA), AlliedSignal Incorporated, Morristown, NJ, 16 December 1996

(4 pages with no figures or tables, RDB7612)

summary of toxicity test data for R-245fa: mutagenicity tests include Ames (negative), human lymphocyte chromosome aberration (slight response, but only at high concentrations in the absence of metabolic activation), and mouse micronucleus genetic assays (negative); concludes that the weight of evidence indicates that R-245fa is not a mutagen; screening tests in beagle dogs with adrenaline injections show no potential for cardiac sensitization up to $20,000 \mathrm{ppm} \mathrm{v} / \mathrm{v}$ in air; tests in female rabbits indicate an acute dermal $L D_{50}$ of $>2 \mathrm{~mL} / \mathrm{kg}$; preliminary data from a developmental toxicity study in rats indicate that concentrations as high as $50,000 \mathrm{ppm} v / \mathrm{v}$ did not cause adverse effects on the development of pups; acute inhalation studies in mice and rats result in 4-hr $\mathrm{LC}_{50}$ determinations of $>101,300$ in mice and $>203,000$ in rats; treated mice showed underactivity and treated rats exhibited abnormal posture, exaggerated respiratory movements, reduced response to external stimuli, and - at high concentrations - evidence of an anesthetic effect; these effects were transient and all of the animals recovered rapidly following exposures; rats exposed for $6 \mathrm{hr} / \mathrm{d}, 5 \mathrm{~d} / \mathrm{wk}$, for 2 wh at concentrations up to $50,000 \mathrm{ppm}$ showed no evidence of treatment related effects, but were found to have increased levels of blood urea nitrogen and serum liver enzyme activities; the latter are described as not representing an adverse finding, since there was no marked dose response and since no histopathological changes were found in the kidneys, liver, or other organs; the finding leading to this "for your information" (FYl) notification was an increased frequency of slight to minimal myocarditis (inflammation of the heart muscle) in both male and female rats exposed to 10,000 and 50,000 ppm in a 13-week inhalation study; dose related increases were seen in urinary fluoride levels, water consumption, and urine volumes after 4 weeks, suggesting some metabolism of R-245fa; increases in blood urea nitrogen and serum level enzyme activity also were seen; the document describes R-245fa as of low toxicity based on the preliminary findings 
G. M. Rusch, Preliminary Results of an Acute Inhalation Study of Propane, 1,1,1,3,3Pentafluoro- In Mice, submission to the U.S. Environmental Protection Agency (EPANOTS 88940000354S, TSCATS 441482), AlliedSignal Incorporated, Morristown, NJ, 13 July 1994 (RDB8364)

acute inhalation toxicity of R-245fa

\section{R-C270}

H. L. Price, A. A. Lurie, R. E. Jones, M. L. Price, and H. W. Linde, Cyclopropane Anesthesia. II. Epinephrine and Nonepinephrine in Initiation of Ventricular Arrhythmias by Carbon Dioxide Inhalation, Anesthesiology, 19:619-630, September-October 1958 (12 pages, RDB5575)

toxicity tests for cardiac sensitization by $\mathrm{R}$ C270 (cyclopropane) with inhalation of R-744 (carbon dioxide)

J. W. Stutzman, Q. Murphy, C. R. Allen, and W. J. Meek, Further Studies on Production of Cyclopropane-Epinephrine Tachycardia, Anesthesio/ogy, 8:579 ff, November 1947 (RDB65B8)

cardiac sensitization of R-C270 (cyclopropane); toxicity

R. M. Waters, Present Status of Cyclopropane, British Medical Journal, UK, 2:1013-1017, 21 November 1936 (5 pages, RDBA673)

cardiac sensitization of R-C270 (cyclopropane); toxicity

\section{R-290 (PROPANE)}

L. Ambrosio, A. Inserra, and C. Sfogliano, Cases of Occupational Poisoning among Workers Engaged in Bottling Commercial Liquid Gas (Butane-Propane), Folia Med., Italy, 51:14 ff, 1968 (RDB7409)

R-290, R-600, R-290/600, toxicity, accidents involving human exposure as reported in RDB6569

L. L. Anderson and R. C. Graham, untitled toxicity review for propane, Haskell Laboratory for Toxicology and Industrial Medicine, E. I. duPont de Nemours and Company, Incorporated, Newark, DE, 6 March 1990 (RDB6107)

R-290, summary of published and unpublished toxicity literature

O. Ishikawa, S. Warita, M. Shimaoka, and Y. Miyachi, Atypical Scleroderma with Centrifugal Skin
Sclerosis, European Journal of Dermatology, 4(8):618-620, 1994 (3 pages, RDBA930)

presents a case history in which R-290 (propane) exposures were suggested as the eliciting factor in skin sclerosis (hardening from overgrowth of fibrous or interstitial tissue); case involved a 53 yr old male who had worked in a liquid propane company for $8 \mathrm{yr}$ and who responded to therapy with oral prednisone; dermal contact toxicity

F. A. Patty and W. P. Yant, The Toxicity of Propane or Odor Intensity and Symptoms Produced by Commercial Propane, Butane, Pentane, Hexane, and Hexane Vapor, Report of Investigations 2979, U.S. Bureau of Mines, Washington, DC, 1929 (RDB6114)

R-290; human exposures

S. Tsukamoto et al., Nippon Hoijaku Zasshi, Japan, 39(2):124-130, 1985 (7 pages, RDB6117)

R-290, metabolism, toxicity

Occupational Health Guideline for Propane, U.S. Department of Health and Human Services and U.S. Department of Labor, Washington, DC, September 1978 (4 pages with 1 table, RDB5608)

R-290, safety, toxicity, flammability, OHG, occupational exposure guidance

Propane, chemical infogram 87, Canadian Centre for Occupational Health and Safety (CCOHS), Hamilton, ON, Canada, October 1990 (1 page, RDB5609)

R-290, safety, toxicity, flammability, occupational exposure guidance

Toxicity Study Summary: Propane (Hydrocarbon Propellant A-108), Phillips Petroleum Company, Bartlesville, OK, February 1990 (1 page with no figures or tables, RDB7624)

tests of the acute inhalation toxicity in 5 female and 5 male albino, Sprague-Dawley rats by 4 $\mathrm{hr}$, whole body exposures to $12,190 \mathrm{ppm}$ v/v R290 (propane) in air: no deaths resulted and animals appeared normal throughout the exposures and subsequent 14-day observation period; no gross signs or symptoms of intoxication were noted; no exposure-related trend was evident in body weights following exposures; no mention is made of pathological examinations following the observation period; concludes that the $4-h r L_{50}$ rat is $>12,190 \mathrm{ppm}$ v/v; respiratory tract irritancy studies in male mice by head-only exposures to $11,472 \mathrm{ppm}$ v/v R-290 in air for $1 \mathrm{~min}$, repeated 10 minutes later, while 
monitored by a plethysmograph: no patterns of respiratory pause were evident; concludes that R-290 failed to produce upper respiratory irritancy in mice at $11,472 \mathrm{ppm} \mathrm{v/v}$

\section{R-C318}

S. Brubaker, G. A. Peyman, and C. Vygantas (University of Illinois Eye and Ear Infirmary), Toxicity of Octafluorocyclobutane After Intracameral Injection, Archives of Ophthalmology, 92:324-328, October 1974 ( 5 pages with 9 figures and 2 tables, RDB7223)

R-C318, comparisons to R-7146, toxicity: use for surgical injection to treat retinal detachment

J. W. Clayton, Jr., M. A. Delaplane, and D. B. Hood (E. I. duPont de Nemours and Company, Incorporated), Toxicity Studies with Octafluorocyclobutane, presented at the 21st Annual Meeting of the American Industrial Hygiene Association (AlHA, Rochester, NY 27 April 1960); republished in the American Industrial Hygiene Journal, 21:382-388, 1960 (7 pages with 5 figures and 2 tables, RDB5A25)

R-C318 (identified as OFCB), health effects, toxicity: 4-hr ALC rat $>800,000 \mathrm{ppm}$ v/v and 4$\mathrm{hr}$ anesthetic NOEL rat $=800,000 \mathrm{ppm} ; 10-\mathrm{min}$ $E_{50}$ mouse for anesthesia $>800,000$; exposures of 10 male and 10 female rats, 10 female mice, 2 male and 2 female rabbits, and 4 male dogs for $6 \mathrm{hr} / \mathrm{d}, 5 \mathrm{~d} / \mathrm{wk}$ for 90 days to 100,000 ppm revealed no harmful effects; paper concludes that R-C318 "is a material possessing an extremely low order of acute toxicity"

J. W. Clayton, Jr., M. A. Delaplane, E. E. Hurlbrink, G. L. Lescanec, L. B. Roemer, L. Wells, J. Zerbe, A. R. Borgmann, and D. M. Gay, Toxicity Studies with Octafluorocyclobutane (OFCB), report 15-59, Haskell Laboratory for Toxicology and Industrial Medicine, E. I. duPont de Nemours and Company, Incorporated, Newark, DE, 17 June 1959 (128 pages with 8 figures and 10 tables, RDB7736)

toxicity studies and literature review for $R$ C318, then a candidate food propellant; 4-hr acute inhalation toxicity test of R-C318 in 2 rats by exposures to $800,000 \quad(792,000-804,000$ actual, 798,000 midpoint) $\mathrm{ppm} \mathrm{v} / \mathrm{v}$ in oxygen; rats showed red ears, mild lacrimation (secretion of tears), and hyperpnea (abnormally rapid or deep breathing) during exposure and mild mydriasis (dilation of the pupil of the eye) afterward, but appeared normal 15 minutes after ex- posure; 4-hr ALC 0/2 rat $>798,000$ ppm; tests for anesthetic properties at $800,000 \mathrm{ppm} v / \mathrm{v}$ in oxygen in a jar rotated at $14 \mathrm{rpm}$ for 10 minutes found that 6 rats were able to maintain their balance; describes subchronic and chronic toxicity tests in rats, mice, dogs, and rabbits; concludes that animals showed no harmful effects at $100,000 \mathrm{ppm} v / \mathrm{v}$

S. W. D'Amico and R. C. Graham, untitled toxicity review for octafluorocyclobutane, Haskell Laboratory for Toxicology and Industrial Medicine, E. I. duPont de Nemours and Company, Incorporated, Newark, DE, 31 October 1997 (17 pages with 1 table, RDBA302)

R-C318, summary of published and unpublished toxicity literature

A. I. Evdokimov and V. V. Kerekesha, Tr. Leningrad Sanit. Gigien. Med. Inst., (III):39-41, 1975 (3 pages in Russian, RDB7217)

R-C318, health effects, toxicity: chronic exposure test at 100,000 ppm found no differences compared to a control group as reported in RDB7215

R. C. Graham, untitled toxicity review for octafluorocyclobutane, Haskell Laboratory for Toxicology and Industrial Medicine, E. I. duPont de Nemours and Company, Incorporated, Newark, DE, 27 April 1982 (13 pages with 1 table, RDB7215)

R-C318, summary of published and unpublished toxicity literature

C. H. Tappan, Freon-C318 ${ }^{\circledR}$ - JLNB-8580-70: Inhalation Toxicity, report 98-65, Haskell Laboratory for Toxicology and Industrial Medicine, E. I. duPont de Nemours and Company, Incorporated, Newark, DE, 9 July 1965 (1 page with 1 table, RDB7216)

4-hr acute inhalation toxicity test of R-C318, with approximately $40 \mathrm{ppm}$ of an identified impurity, in six rats by whole-body exposures to $800,000 \mathrm{ppm} \mathrm{v/v}$ in oxygen: rats showed red ears, mild lacrimation (secretion of tears, 2/6), and hyperpnea (abnormally rapid or deep breathing, 2/6) during exposure and mild mydriasis (dilation of the pupil of the eye, 6/6) afterward, but appeared normal 15 minutes after exposure; no examination was performed for pathology; 4-hr ALC rat $>800,000$ ppm 


\section{R-401A, R-401B, R-401C}

Safety of Suva MP Refrigerants, document AS-3 (H-35814-1), DuPont Chemicals, Wilmington, DE, May 1994 (4 pages with 1 table, RDB4C49)

This bulletin reviews considerations for safe use of R-401A, R-401B, and R-401C - ternary blends of R-22, R-152a, and R-124 - by providing answers to common questions. It outlines the uses of these as replacements for R12 in medium-temperature stationary refrigeration, stationary refrigeration with evaporator temperatures below $-23^{\circ} \mathrm{C}\left(-10^{\circ} \mathrm{F}\right)$ and some transport refrigeration, and retrofit of mobile airconditioning systems, respectively. It briefly reviews the collaborative Programme for Alternative Fluorocarbon Toxicity Testing (PAFT) and partial findings for R-124. The document discusses the flammability of R-22/152a/124 blends, noting that they are neither flammable nor will become so with leakage, but can become flammable when mixed with gases that are. The document notes that mixtures of R$134 a$ with more than $60 \%$ air (by volume) can become flammable at raised pressures and temperatures. It counsels that $\mathrm{R}-22 / 152 \mathrm{a} / 124$ blends may behave similarly and counsels against use of refrigerant-air mixtures for leak testing. The document then addresses inhalation toxicity. It explains the DuPont Acceptable Exposure Limit ( $A E L$ ), a time-weighted average (TWA) concentration for an 8 or 12 hour day or 40 hour week, to which nearly all workers may be repeatedly exposed without adverse effects. The compositions, average boiling points, and AELs are tabulated for R-401A, R-401B, and R401C. The document discusses symptoms of exposure to high refrigerant concentrations, cardiac sensitization, and special charging procedures these near-azeotropic blends. It also discusses suffocation, safety measures for enclosed areas, guidance when a large spill or leak occurs, dangers of deliberate inhalation, and detection by odor. The bulletin provides general advice on skin and eye contact with refrigerants, frostbite, pressure hazards, handling and disposal of cylinders, brazing or welding of pipes in air-conditioning and refrigeration systems, and decomposition. It concludes with specific cautions. The document recommends familiarization with the Material Safety Data Sheet (MSDS) available for these refrigerants. DuPont's product names R-401A, R-401B, and R-401C are Suva ${ }^{\circledR}$ MP39, Suva ${ }^{\circledR}$ MP66, and Suva ${ }^{\circledR}$ MP52, respectively.

\section{R-416A}

T. J. Kenny and C. J. Hardy (Huntingdon Research Centre Limited, HRC, UK), FRIGC ${ }^{\text {TM }}$ FR$12^{\mathrm{TM}}$ : Assessment of Cardiac Sensitization Potential in Dogs, report HZL 6/942384, Hazleton Washington, Incorporated, Vienna, VA, 31 May 1994 (33 pages with 7 figures and 9 tables, RDB7407)

toxicity tests to determine the cardiac sensitization potential of R-416A (R-134a/124/600 (59/37/4) by analysis) in male beagle dogs with intravenous injection of adrenaline five minutes before and, with a challenge injection, midway into a 10 minute exposure in 6 dogs at 50,000 and $70,000 \mathrm{ppm} v / \mathrm{v}, 5$ dogs at $85,000 \mathrm{ppm} \mathrm{v/v}$, and 2 dogs at $100,000 \mathrm{ppm} \mathrm{v} / \mathrm{v}$ in air; no positive responses were observed at $50,000 \mathrm{ppm}$ $\mathrm{v} / \mathrm{v}$ [deemed a NOEL], but two of the dogs were reported to be struggling; one dog died during testing at $70,000 \mathrm{ppm} v / \mathrm{v}$ before adrenaline administration and 1 (a replacement animal) of 6 dogs showed a positive response at 70,000 $\mathrm{ppm} \mathrm{v/v;} 1$ of 5 and 2 of showed positive responses at 85,000 and $100,000 \mathrm{ppm} \mathrm{v/v}$, respectively; the LOEL and $\mathrm{EC}_{50}$ were determined to be 70,000 and $90,000 \mathrm{ppm} \mathrm{v/v}$, respectively; comparative tests with 2 dogs for $R$ 11 indicate a LOEL at $20,000 \mathrm{ppm}$ v/v (lowest tested concentration); report cites prior studies that determined the $\mathrm{EC}_{50}$ for $\mathrm{R}-124$ and $\mathrm{R}-134 \mathrm{a}$ as 40,000 and $205,000 \mathrm{ppm} \mathrm{v} / \mathrm{v}$, respectively [Note: the referenced study could not be obtained, but a draft from the referenced source does not mention these data]; concludes that the cardiac sensitization level of the R$134 a / 124 / 600$ blend is predictable from the R124 content and that there is little evidence of a synergistic effect from the other components; the report indicates Good Laboratory Practice (GLP) compliance [Note: The cardiac sensitization level for R-416A may be slightly lower than indicated here; the fraction of R-124 in the tested sample was below that of the nominal formulation and outside the tolerance for this blend.]

\section{R-502}

J. F. Breen and J. E. Wallis, Report on Refrigerant, Miscellaneous Hazard Report, Underwriters Laboratories Incorporated, Northbrook, IL (then Chicago, IL), 19 November 1963 (RDB7654)

summarizes acute inhalation toxicity tests of $R$ 502 at 100,000 and $200,000 \mathrm{ppm} v / \mathrm{v}$ in groups 
of three guinea pigs; indicates no clinical signs after five min at $100,000 \mathrm{ppm} v / \mathrm{v}$, but all three treated animals breathed more rapidly after 30 min; also indicates that all three showed occasional chewing movements and slight shaking of their heads at $90 \mathrm{~min}$, but appeared normal and gained weight during a subsequent 10-day observation period; describes more pronounced responses at $200,000 \mathrm{ppm} \mathrm{v} / \mathrm{v}$ at which the animals appeared uneasy and showed occasional chewing movements and rapid breathing at $30 \mathrm{~min}$; indicates that one animal showed tremors and rubbed its nose while all three showed reduced activity at 60 min; notes that all had difficulty standing and their rear limbs were weak at 90 min progressing to loss of equilibrium and difficulty walking at $2 \mathrm{hr}$; reports that no gross abnormalities were found in autopsies after a ten-day observation period during which animals appeared normal and gained weight, but that microscopic examination revealed some focal, acute inflammation in the lungs - as reported in RDB5866

J. W. Clayton, Jr., Freon-502 Refrigerant (Azeotrope of F-22, 48.8\% and F-115, 51.2\%): Acute Testing, report 156-63, Haskell Laboratory for Toxicology and Industrial Medicine, E. I. duPont de Nemours and Company, Incorporated, Newark, DE, 27 December 1963 (1 page with 1 table, RDB6273)

2-hr acute inhalation toxicity tests of R-502 in four male Charles River CD rats by whole-body exposure to $200,000 \mathrm{ppm} \mathrm{v} / \mathrm{v}$ in air: this report expands on an earlier one [see RDB6272] with addition of pathology findings from histology studies; no deaths occurred, but animals showed discomfort, tremors, and rapid respiration during exposures; two rats showed slight weight loss during the 14-day observation period following exposures; gross examination of the sacrificed animals revealed some pulmonary congestion at necropsy; no compoundrelated changes were found in the brain, lung trachea, liver, kidneys, alimentary tract, spleen, thymus, pancreas, testes, or bone marrow; concludes that the 2-hr ALC rat for R-502 exceeds $200,000 \mathrm{ppm} v / \mathrm{v}$, which indicates a low order of toxicity by acute inhalation

J. W. Clayton, Jr., Freon-502 Refrigerant (Azeotrope of F-22, 48.8\% and F-115, 51.2\%): Acute Testing, report 91-62, Haskell Laboratory for Toxicology and Industrial Medicine, E. I. duPont de Nemours and Company, Incorporated, Newark, DE,
13 December 1962 (1 page with 1 table, RDB6272)

2-hr acute inhalation toxicity tests of R-502 in four male Charles River $C D$ rats by whole-body exposure to $200,000 \mathrm{ppm}$ v/v in air: no deaths occurred, but animals showed discomfort, tremors, and rapid respiration during exposures; two rats showed slight weight loss during the 14-day observation period following exposures; gross examination of the sacrificed animals revealed some pulmonary congestion at necropsy; histological examination had not been completed at report issuance [see RDB6273]; concludes that the 2-hr ALC rat for R-502 exceeds $200,000 \mathrm{ppm} v / \mathrm{v}$, which indicates a low order of toxicity by acute inhalation

M. A. Lee, untitled toxicity review for Freon ${ }^{\circledR} 502$, Haskell Laboratory for Toxicology and Industrial Medicine, E. I. duPont de Nemours and Company, Incorporated, Newark, DE, 22 May 1992 (RDB5866)

summarizes published and unpublished toxicity information for R-502

\section{R-600 (n-BUTANE)}

T. Berzins, Health Effects of Selected Chemicals 3. Butane, NORD, 28:10-26, 1995 (17 pages, RDBA933)

summarizes toxicity data for R-600 (n-butane) based on a literature search; indicates that the 4-hr $L C_{50}$ rat was $272,000 \mathrm{ppm}$ v/v [based on conversion from $658 \mathrm{mg} / \mathrm{l}$, [see RDB5363] and the 2-hr LC 50 mouse was 281,000 ppm v/v (680 $\mathrm{mg} / \mathrm{l}$ ) [see RDB5363] by inhalation exposures; indicates that the lethal concentrations in dogs ranged from $196,000-245,000$ (474 to $592 \mathrm{mg} / \mathrm{l}$ ) [200,000-250,000, see RDB6197]; notes that a concentration of $127,000 \mathrm{ppm} \mathrm{v/v} \mathrm{(308} \mathrm{mg/l)}$ caused light anesthesia in mice in an average of 25 minutes [see RDB6197], and an exposure to $215,000 \mathrm{ppm}$ v/v $(521 \mathrm{mg} / \mathrm{l})$ had similar effect within one minute [see RDB6197]; indicates that $n$-butane induced cardiac sensitization to epinephrine in dogs after inhalation; explains that no reports addressing the acute toxicity of R-600 by administration routes other than inhalation were located; summarizes a 21-day inhalation toxicity study in Sprague-Dawley rats exposed $6 \mathrm{hr} / \mathrm{d}$ for a total of 15 exposures over 3 wk to a mixture of R-600 (n-butane), R-600a (isobutane), R-601 (n-pentane), R-601a (isopentane) containing $25 \%$ of each, namely R-600a/600/601a/601 (25.0/25.0/25.0/25.0); in- 
dicates that no toxicity was evident up to 4,880 $\mathrm{ppm} \mathrm{v} / \mathrm{v}(11.8 \mathrm{mg} / \mathrm{l})$, which was the highest concentration tested; reports that no studies of long-term toxicity, carcinogenicity, reproduction toxicity, teratogenicity, immunotoxicity, or allergic sensitivity were found for pure n-butane; indicates that no mutagenic activity was observed in several tests in Salmonella typhimurium with or without metabolic activation; summarizes several reports of injuries and deaths from human exposures to R-600, among them accounts suggesting increasing abuse of volatile substances ["sniffing"], including of R-600; notes that use of an oven cleaner containing nbutane as propellant caused transient myoclonus in one patient; cites an incident in which an aerosol spray contained $n$-butane caused deep frostbite when sprayed directly on skin; cites a study of truck drivers and terminal operators from different loading facilities and service stations were examined for exposure to gasoline vapors containing $90-92 \% n$-butane, isobutane, $n$-pentane, and isopentane; reports that the concentrations were substantially lower than the established ACGIH TLV-TWA of $300 \mathrm{ppm}$ $\mathrm{v} / \mathrm{v}$ for gasoline and $800 \mathrm{ppm} \mathrm{v} / \mathrm{v}$ for $\mathrm{n}$-butane; also reports that 53 male workers exposed to nbutane for an average of 11 years in a refinery to concentrations of 0.17-7.4 ppm v/v (0.0004 $\mathrm{mg} / \mathrm{l}$ to $0.0178 \mathrm{mg} / \mathrm{l})$ without observed symptoms; notes that some case reports indicate central nervous system (CNS) effects in nbutane abusers including visual hallucinations, increasing irritability and tendency to become easily provoked; also indicates incidence of rapid and severe liver damage potentially attributable to long-term, heavy n-butane abuse by a 17-year-old male; concludes that exposures to low concentrations of $R-600$ has not been reported to cause adverse effects in humans, but that it causes anesthetic effects and sudden death if inhaled at high concentrations; notes that the safety margin between anesthetic and lethal concentrations appears to be very narrow

T. R. Cartwright, E. D. Brown, and R. E. Brashear (Indiana University School of Medicine), Pulmonary Infiltrates Following Butane 'Fire-Breathing', Archives of Internal Medicine, 143:2007-2008, October 1983 ( 2 pages with 3 figures, RDB7B43)

deliberate inhalation of ordinary R-600 (butane) for a stage trick identified as 'fire-breathing' resulted in rapidly progressive pulmonary infiltrates in a 19-yr old male leading to weakness, malaise, a nonproductive cough, and mild dys- pnea (difficult or labored breathing); article concludes that this misuse of chemicals can have a serious or even fatal outcome due to the pulmonary toxic reaction in humans

A. C. Evans and D. Raistrick, British Journal of Psychiatry, 150:769-773, June 1987 (5 pages, RDB6A84)

R-600, health effects, toxicity; study of the effects of misuse by direct inhalation; compared effects for 12 youths to 31 inhaling toluene and resultant marked changes of mental state; most users reported elevation of mood and hallucinations; nearly one-fourth believed they were able to fly or swim - as reported in RDB6A83

F. Fernandez, A. Perez-Higueras, R. Hernandez et al., Hydraencephaly After Maternal Butane Gas Intoxication During Pregnancy, Dev. Med. child. Neurology, 28:361-363, 1986 (3 pages, RDB7B49)

health effects of R-600 (n-butane); toxicity

R. Fytizas-Danielidou, Ann. Inst. Phytopathol. Benaki, 10(4):327-332, 1973 (6 pages, RDB6A85)

R-600, health effects, toxicity; liquified butane causes burns or frostbite to the eyes upon direct contact as reported in RDB6A83

M. Y. Gray and J. H. Lazarus (Llandough Hospital, UK), Butane Inhalation and Hemiparesis, Clinical Toxicology, 31(3):483-485, 1993 (3 pages with no figures or tables, RDB7406)

reports a case of hemiparesis (muscular weakness or paralysis on one side of the body) following inhalation of an unknown concentration of R-600 (butane) in a human; acute inhalation toxicity; health effects, substance abuse

T. J. Hughes et al., report EPA 600/S1-84-005, Office of Health and Environmental Assessment, U.S. Environmental Protection Agency (EPA), Washington, DC, 1984 (RDB6A86)

R-600, health effects, mutagenicity, toxicity: not mutagenic in Salmonella typhimurium or Escherichia coli in the absence and presence of rat microsomal activation as reported in RDB6A83

M. A. Lee and R. C. Graham, untitled toxicity review for butane, Haskell Laboratory for Toxicology and Industrial Medicine, E. I. duPont de Nemours and Company, Incorporated, Newark, DE, 29 January 1990 (RDB6A83)

$\mathrm{R}-600$, summary of published and unpublished toxicity literature 
W. W. Marsh, Butane Firebreathing in Adolescents: A Potentially Dangerous Practice, Journal of Adolescent Health Care, 5(1):59-60, 1984 (2 pages, RDB7408)

$\mathrm{R}-600$, abuse, health effects in humans, toxicity

B. Mathew, E. Kapp, and T. R. Jones, Commercial Butane Abuse: A Disturbing Case, British Joumal of Addiction, 84(5):563-564, 1989 (2 pages, RDB6A91)

health effects from "sniffing" R-600 (n-butane); abuse; toxicity in humans

A. S. Mclntyre and R. G. Long, Fatal Fulminant Hepatic Failure in a 'Solvent Abuser', Postgraduate Medical Journal, 68:29-30, 1992 (2 pages, RDB7B46)

health effects from "sniffing" R-600 (n-butane); abuse; toxicity in humans

H. Shimizu et al., Sangyo Igaku, 27(6):400-419, 1985 (20 pages, RDB6A88)

$\mathrm{R}-600$, health effects, toxicity; not mutagenic in Salmonella typhimurium or in Escherichia coli in the absence or presence of rat microsomal activation as reported RDB6A83

M. Vr'cevi'c and R. Stani'c, Butane Gas Poisoning - Current Problems in Emergency Internal Medicine, Med. Pregl., 26(5):173-179, 1973 (7 pages, RDB6A92)

$\mathrm{R}-600$, health effects, toxicity

Occupational Safety and Health Guideline for n-Butane; National Institute of Occupational Safety and Health (NIOSH), U.S. Department of Health and Human Services, Cincinnati, $\mathrm{OH}$; Occupational Safety and Health Administration (OSHA), U.S. Department of Labor, Washington, DC, 1992 (5 pages with no figures or tables, available from GPO, also available from JMC as RDB5C48)

$\mathrm{R}-600$, toxicity; chemical and physical properties, reactivity, flammability, exposure limits, health hazard information, exposure sources and control methods, medical monitoring, workplace monitoring and measurement, personal hygiene, storage, leaks, special requirements, respiratory protection, personal protective equipment, references

\section{R-600a (ISOBUTANE)}

M. A. Lee and R. C. Graham, untitled toxicity review for isobutane, Haskell Laboratory for Toxicol- ogy and Industrial Medicine, E. I. duPont de Nemours and Company, Incorporated, Newark, DE, 22 February 1990 (RDB5865)

$\mathrm{R}-600 \mathrm{a}$, summary of published and unpublished toxicity literature

T. Litovitz, Occupational Medicine - State of the Art Review, 3(3):567-568, 1988 (2 pages, RDB6193)

R-600, R-600a, cardiac sensitization, health effects, toxicity

R. D. Stewart (Medical College of Wisconsin), A. A. Herrmann (S. C. Johnson \& Son, Incorporated), E. D. Baretta (Johnson), H. V. Forster (Medical College of Wisconsin), J. J. Sikora (Johnson), P. E. Newton (Medical College of Wisconsin), and R. J. Soto (Johnson), Acute and Repetitive Human Exposure to Isobutane, Scandinavian Journal of Work Environment and Health, 1977(3):234-243, 1977 (10 pages with 4 tables, RDB5364)

summarizes acute and subchronic toxicity tests in healthy human volunteers by whole-body exposures; indicates that acute exposures to 250 , 500 , or $1000 \mathrm{ppm} \mathrm{v/v}$ for $1 \mathrm{~min}$ to $8 \mathrm{hr}$ "did not produce any untoward physiological effects" as determined by observation, routine hematology, clinical chemistry, EKG, and pulmonary function as well as by cognitive skill and other neurological measurements; further notes that repetitive exposures to $500 \mathrm{ppm}$ v/v for 1,2 , or 8 $\mathrm{hr} / \mathrm{d}, 5 \mathrm{~d} / \mathrm{wk}$, for $2 \mathrm{wk}$ also showed no adverse effects

Industrial Hygiene and Toxicology Bulletin, Phillips Petroleum Company, Bartlesville, OK, November 1976 (RDB6196)

R-600a, health effects, toxicity

Toxicity Study Summary: Isobutane (A-31 Hydrocarbon Propellant), Phillips Petroleum Company, Bartlesville, OK, February 1990 (1 page with no figures or tables, RDB7625)

tests of the acute inhalation toxicity in 5 female and 5 male albino, Sprague-Dawley rats by 4 $\mathrm{hr}$, whole body exposures to $13,023 \mathrm{ppm} v / \mathrm{v} R$ 600a (isobutane) in air: no deaths resulted and no symptoms of intoxication were noted; animals appeared normal throughout the exposures and 14-day observation period; treated males showed a slight reduction in mean body weight on days 2-3 following exposures and in females for the entire period; animals appeared normal in pathological examination at necropsy with the exception of 2 animals; one male exhibited dilation of the pelvis of the left kidney and 1 female had a dilated pelvis of the left kid- 
ney that was white and hard; concludes that the gross pathological findings did not appear related to treatment and that the 4-hr $\mathrm{LC}_{50}$ rat is $>13,023 \mathrm{ppm} \mathrm{V/v}$; respiratory tract irritancy studies in male mice by head-only exposures to $12,640 \mathrm{ppm}$ V/V R-600a in air for $1 \mathrm{~min}$, repeated 10 minutes later, while monitored by a plethysmograph: no patterns of respiratory pause were evident; concludes that R-600a failed to produce upper respiratory irritancy in mice at $12,640 \mathrm{ppm} v / \mathrm{v}$

\section{R-601 (n-PENTANE)}

Toxicity Study Summary: n-Pentane, Commercial, Phillips Petroleum Company, Bartlesville, OK, February 1990 (1 page with no figures or tables, RDB7626)

tests of the acute inhalation toxicity in 5 female and 5 male albino, Sprague-Dawley rats by 4 $\mathrm{hr}$, whole body exposures to 6,107 ppm v/v R601 (n-pentane) in air: while no deaths resulted during exposures or a subsequent 14day observation period; one female was observed to be wheezing with a bloody crust around the nose on days 10-11 following exposures; 3 females were found to have a grey depressed area on the left lung lobe, a pinpoint red spot on the left lobe surface of the liver, a $\tan$ area at the cleft of the medium lobe of the liver, a dilated kidney pelvis filled with white fluid, and enlarged peribronchial lymph nodes; concludes that the gross pathological findings were not consistent and that the 4-hr $\mathrm{LC}_{50}$ rat is $>6,107 \mathrm{ppm} \mathrm{v} / \mathrm{v}$; respiratory tract irritancy studies in male mice by head-only exposures to $5,559 \mathrm{ppm} \mathrm{v/v}$ pentane in air for $1 \mathrm{~min}$, repeated 10 minutes later, while monitored by a plethysmograph: no patterns of respiratory pause were evident; concludes that pentane failed to produce upper respiratory irritancy in mice at 5,559 ppm v/v

\section{R-601a (IOSPENTANE)}

M. A. Lee and R. C. Graham, untitled review of toxicity literature for isopentane, Haskell Laboratory for Toxicology and Industrial Medicine, E. I. duPont de Nemours and Company, Incorporated, Newark, DE, 19 August 1986 (6 pages with no figures or tables, RDB6777)

summary of published and unpublished information on the toxicity of R-601a (isopentane)

\section{CYCLOPENTANE}

Toxicity Study Summary: Cyclopentane (70\%), Phillips Petroleum Company, Bartlesville, OK, February 1990 (3 pages with no figures or tables, RDB7627)

cyclopentane, summary of acute oral toxicity, acute inhalation toxicity, respiratory tract irritan$c y$, eye irritancy, and primary dermal irritancy studies

\section{R-602 (n-HEXANE)}

Toxicity Study Summary: n-Hexane, Phillips Petroleum Company, Bartlesville, OK, February 1990 (5 pages with no figures or tables, RDB7629)

R-602 (n-hexane), summary of acute oral toxicity, acute dermal toxicity, acute inhalation toxicity, respiratory tract irritancy, eye irritancy, primary. dermal irritancy, and mutagenicity tests (Ames, mouse lymphoma forward mutational assay, and in vitro sister chromoid exchange) studies

\section{R-630}

Emergency Response Planning Guidelines Monomethylamine, American Industrial Hygiene Association (AlHA), Fairfax, VA, 1990 (RDB4B77)

R-630, ERPG-1, ERPG-2, ERPG-3, toxicology data, toxicity, safety classification

\section{R-717 (AMMONIA)}

J. S. Alabaster and D. W. M. Herbert, Influence of Carbon Dioxide on the Toxicity of Ammonia, Nature, UK, 174(4426):404 ff, 1954 (RDB8405)

R-717, toxicity

I. M. Alpatov, A Study of Gaseous Ammonia Toxicity, Gigiena Truda i Professional'nye Zabolevaniya [Labor Hygiene and Occupational Diseases], Russia (then USSR), 2:14-18, 1964 (5 pages in Russian, RDB6232)

$$
\text { R-717, toxicity }
$$

1. M. Alpatov and V. I. Mikhailov, Inquiries into Gaseous Ammonia Toxicity, Gigiena Truda i Professional'nye Zabolevaniya [Labor Hygiene and Occupational Diseases], Moscow, Russia (then USSR), 12:51-53, 1963 (3 pages in Russian, RDB6233)

$$
\mathrm{R}-717 \text {, toxicity }
$$


J. Andanson, J. Berenger, R. Castela, J. Catoir, $P$. Delmon, M. Graille, B. Guidoni, J. P. d'Ortoli, H. Raulot-Lapointe, and J. J. Vigneau, L'Intoxication Chronique par l'Ammoniaque: Est-Elle Observable en Service Médical Interentreprises? [Chronic Ammonia Poisoning: is it Detectable in Joint Plant Medical Services?], Revue de Médecine du Travail, France, 4(4):293-300, 1976 (in French, RDB6A36)

R-717, health effects, toxicity: workers exposed to low levels of ammonia for prolonged periods have reported symptoms of headache, drowsiness, asthma, laryngitis, and skin irritation as reported in RDB6270

L. M. Appelman (TNO-CIVO Toxicology and Nutrition Institute, The Netherlands), W. F. ten Berge (DSM, The Netherlands), and P. G. J. Reuzel (TNO-CIVO), Acute Inhalation Toxicology Study of Ammonia in Rats with Variable Exposure Periods, AlHA Journal, 43(9):662-665, September 1982 (4 pages with 4 tables, RDB5152)

presents acute inhalation toxicity data and a correlation between exposure concentrations and duration for R-717 (ammonia): summarizes lethality tests in male and female rats for varied concentrations and durations of 10,20 , 40 , and $60 \mathrm{~min}$; presents a probit relationship for concentrations, exposure periods, and mortality rates; indicates $\mathrm{LC}_{50}$ values in rats for $10,20,40$, and $60 \mathrm{~min}$ of $40,300,28,595$, 20,300 , and $16,600 \mathrm{ppm}$, respectively; demonstrates a higher mortality rate for male rats compared to females for tested concentrations as illustrated by $1-\mathrm{hr} L \mathrm{LC}_{50}$ values of 14,071 and $19,671 \mathrm{ppm}$ v/v respectively

R. Arwood, J. Hammond, and G. G. Ward, Ammonia Inhalation, Journal of Trauma, 25:444-447, 1985 (4 pages, RDB6257)

$R-717$, health effects, toxicity

C. S. Barrow and W. H. Steinhagen (University of Pittsburgh), $\mathbf{N H}_{3}$ Concentrations in the Expired Air of the Rat: Importance to Inhalation Toxicology, Toxicology and Applied Pharmacology (TAP), 53:116-121, 1980 (6 pages, RDB6A45)

R-717, health effects, toxicity

C. S. Barrow, Y. Alarie, and M. F. Stock (University of Pittsburgh), Sensory Irritation and Incapacitation Evoked by Thermal Decomposition Products of Polymers and Comparisons with Known Sensory Irritants, Archives of Environmental Health, 33(2):79-88, March-April 1978 (10 pages with 6 figures and 1 table, RDB6231)
R-717 (ammonia), health effects, toxicity, source-study for widely cited $R_{50}$ mouse of $303 \mathrm{ppm}$; the maximal response was achieved within the first two minutes of exposure for tested concentrations of R-717

S. P. Bessman and N. Pal, Ammonia Intoxication: Energy Metabolism and Brain Protein Synthesis, Israeli Journal of Medical Science, 18(1):171-175, 1982 (5 pages, RDB5762)

$\mathrm{R}-717$, health effects, toxicity

G. A. Birken, P. J. Fabri, and L. C. Carey, Acute Ammonia Intoxication Complicating Multiple Trauma, Journal of Trauma, 21(9):820-822, 1981 (3 pages, RDB6258)

R-717, health effects, toxicity

G. Bittersohl and H. Heberer, Toxicity of Aliphatic Amines, Zeitschrift für die gesamte $\mathrm{Hy}$ giene und Ihre Grenzgebiete, 24(7):529-534, 1978 (6 pages, RDB6A39)

R-717 and others, health effects, toxicity

G. Bittersohl, presentation at the XVIth International Congress of Occupational Health (Tokyo, Japan, 1969), Japanese Industrial Health Association, 250-251, 1971 (RDB6A57)

R-717, carcinogenic potential, health effects, toxicity: evaluation of neoplasms (new benign or malignant growths resembling the tissues from which they arise, but serving no physiologic functions) in East German manufacturing facilities; workers in ammonia plants exhibited a $500-600 \%$ higher rate of tumors than others; $80 \%$ of the workers were exposed for more than 10 years to exposures of $60-100 \mathrm{ppm}$ as reported in RDB1106

G. Bittersohl, Epidemiologische Untersuchungen über Krebserkrankungen in der chemischen Industrie [Epidemiological Study of Cancer Incidence in the Chemical Industry], Archiv für Geschwulstforschung, 38:198-209, 1971 (9 pages in German, RDB6A58)

R-717, carcinogenic potential, health effects, toxicity: expands on study in RDB6A57, noting that known carcinogens were present; reports a $200 \%$ increase in cancers for workers exposed to ammonia and asbestos compared to asbestos alone - as reported in RDB1 106 which discusses limitations to the study and concludes that the influence of ammonia on the formation of tumors by other agents, such as asbestos, cannot be determined 
E. M. Boyd, M. L. McLachlan, and W. F. Perry, Experimental Ammonia Gas Poisoning in Rabbits and Cats, Journal of Industrial Hygiene and Toxicology, 26:29-34, 1944 (6 pages, RDB6245)

R-717, toxicity: $1-\mathrm{hr} \quad \mathrm{LC}_{50}$ cat and rabbit $=$ 9,859 ppm v/v as reported in RDB5151 (with summary) and RDB5340

L. A. Buckley, X. Z. Jiang, R. A. James, K. T. Morgan, and C. S. Barrow (Chemical Industry Institute of Toxicology, CIIT), Respiratory Tract Lesions Induced by Sensory Irritants at the $\mathrm{RD}_{50}$ Concentration, Toxicology and Applied Pharmacology (TAP), 74(3):417-429, 1984 (13 pages with 8 figures and 2 tables, RDB6227)

toxicity; health effects; study to determine if pathologic changes occur in the respiratory tracts of mice after inhalation exposure at their $\mathrm{RD}_{50}$ concentration for 10 sensory irritants including R-717 (ammonia, RD 50 mouse $=303$ $\mathrm{ppm}$ ) and $\mathrm{R}-764$ (sulfur dioxide, $\mathrm{RD}_{50}$ mouse $=$ $117 \mathrm{ppm}$ ); found no or slight changes of doubtful significance in the squamous epithelium for all 10; found slight to minimal changes but moderate inflammation to the respiratory epithelium and no change to the olfactory epithelium or lungs for R-717; found moderate to severe changes and moderate inflammation to the respiratory epithelium and minimal to severe changes to the olfactory epithelium for $R$ 764; comments that ionization of R-717 in water is comparatively limited resulting in less damage than for hydrogen chloride, for which the $\mathrm{RD}_{50}$ and water solubility are similar; concludes that "the value of the mouse sensory irritant model for setting occupational guidelines to sensory irritants is strengthened in that these studies demonstrate the $R D_{50}$ concentration is associated with respiratory tract damage and is not an acceptable concentration for occupational exposures"; safe levels are predicted at 0.01-0.1 times the $\mathrm{RD}_{50}$ concentration

T. R. Burns, M. L. Mace, S. D. Greenberg, et al., Ultrastructure of Acute Ammonia Toxicity in the Human Lung, American Journal of Forensic Medical Pathologists, 6:204-210, 1985 (RDB6A41)

$\mathrm{R}-717$, health effects, toxicity

T. R. Burns, S. D. Greenberg, M. L. Mace, and J. A. Jachimczyk, Pulmonary Ultrastructure in Acute Ammonia Toxicity in Humans, Environmental and Occupational Health, 127:180 ff, 1983 (RDB6259)

R-717, health effects, toxicity
M. Caplin, Ammonia Gas Poisoning - FortySeven Cases in a London Shelter, Lancet, UK, 235(2):95-96, 1941 (2 pages, RDB6A06)

R-717, accident involving human exposure, health effects, toxicity: 13 of 47 people exposed to ammonia in an air raid shelter died within $48 \mathrm{hr}$ of initial exposure; paper documents affects including symptoms similar to pulmonary edema and bronchopneumonia

B. L. Carson, C. M. Beall, H. V. Ellis III, and L. H. Baker, Ammonia Health Effects, report EPA 460/3-81/027, Office of Mobile Source Air Pollution Control, U.S. Environmental Protection Agency (EPA), Ann Arbor, MI, 1981 (available from NTIS as document PB82-116047, RDB6260)

R-717, health effects, toxicity

E. Chico et al., Ammonia Overloading in Hepatocytes Isolated from Liver of Fetal and Adult Rats, Biochim. Biophys. Acta., 586(3):481-491. 1979 (11 pages, RDB6A40)

$R-717$, health effects, toxicity

Clement Associates, Incorporated, Health Effects Assessment for Ammonia, The Fertilizer Institute, Washington, DC, February 1990 (148 pages, RDB1106)

This document reviews data relevant to assessing the health effects of inhalation exposure to ammonia. The focus is on chronic exposures; information on subchronic and acute exposures also is reviewed to acquire a complete picture of the toxicity of ammonia. Physical and chemical properties, sources, environmental fate, environmental effects, and other related topics are discussed to provide context and perspective.

L. G. Close, F. I. Catlin, and A. M. Cohn, Acute and Chronic Effects of Ammonia Burns of the Respiratory Tract, Archives of Otolaryngology, 106(3):151-158, 1980 (8 pages, RDB5763)

R-717, health effects, toxicity

T. J. Cole, J. E. Cotes, G. R. Johnson, et al., Ventilation, Cardiac Frequency and Pattern of Breathing During Exercise in Men Exposed to o-Chlorobenzylidene Malonitrile and (CS) and Ammonia Gas in Low Concentrations, Quartenly Journal of Experimental Physiol. Cogn. Medical Science, 62:341-351, 1977 (11 pages, RDB6A07)

R-717, health effects, toxicity

R. A. Coon, R. A. Jones, L. J. Jenkins, Jr., and J. Siegel, Animal Inhalation Studies on Ammonia, 
Ethylene Glycol, Formaldehyde, Dimethylamine, and Ethanol, Toxicology and Applied Pharmacology (TAP), 646-655, 1970 (10 pages, RDB6234)

R-717 (ammonia) and five other gases, health effects, toxicity as reported in RDB5151 (with summary)

L. V. Cralley, The Effect of Irritant Gases Upon the Rate of Ciliary Activity, Journal of Industrial Hygiene and Toxicology, 24:193-198, 1942 (6 pages, RDB6235)

R-717, toxicity

T. A. Czuppon, S. A. Knez, and J. M. Rovner, Ammonia, Kirk-Othmer Encyclopedia of Chemical Technology, John Wiley and Sons, Incorporated, New York, 2:638-691, 1982 (54 pages, RDB9514)

R-717, properties, applications, safety (flammability and toxicity)

T. Dalhamn, Effect of Ammonia Alone and Combined with Carbon Particles on Ciliary Activity in the Rabbit Trachea in Vivo with Studies of the Absorption Capacity of the Nasal Cavity, International Journal of Air and Water Pollution, 7:531-539, 1963 (9 pages, RDB6A42)

$\mathrm{R}-717$, health effects, toxicity

M. L. Dalton, Jr., and D. L. Bricker, Anhydrous Ammonia Burn of the Respiratory Tract, Tex. Med., 74(9):51-54, 1978 (4 pages, RDB5764)

R-717, corrosivity, health effects, toxicity

K. T. Dodd and D. R. Gross, Ammonia Inhalation Toxicity in Cats: A Study of Acute and Chronic Respiratory Dysfunction, Archives of Environmental Health, 35:6-14, 1980 (RDB6236)

R-717, toxicity

G. D. Duda and P. Handler, Kinetics of Ammonia Metabolism in Vivo, Journal of Biol. Chem., 232:303-314, 1958 (12 pages, RDB8406)

toxicity of R-717: biochemistry, metabolism, pharmacokinetics

J. A. Duke, Phytotoxin Tables, Critical Reviews in Toxicology, 5:191 ff, 1977 (RDB5153)

R-717, health effects, toxicity

J. L. Egle, Jr., Retention of Inhaled Acetone and Ammonia in the Dog, AlHA Journal, 34:533-539, 1974 (7 pages, RDB6A09)

$\mathrm{R}-717$, health effects, toxicity
H. B. Elkins, Ammonia, $\mathrm{NH}_{3}$, The Chemistry of Industrial Toxicology (second edition), John Wiley and Sons, Incorporated, New York, NY, 1959 (86 pages, RDB8407)

toxicity of R-717

A. Z. El-Sewefy and S. Awad, Chronic Bronchitis in an Egyptian Ice Factory, Journal of the Egyptian Medical Association, 54:304-310, 1971 (7 pages, RDB7452)

$\mathrm{R}-717$, health effects, toxicity: effects on workers exposed to R-717, frost, and dampness in an ice-manufacturing plant; observed that $20 \%$ of the exposed workers had chronic bronchitis compared to $14 \%$ in a control group, but concludes that the difference is not statistically significant as reported in RDB7419

W. S. Ferguson, W. C. Koch, L. B. Webster, and J. R. Gould, Human Physiological Response and Adaptation to Ammonia, Journal of Occupational Medicine (JOM), 19:319-326, 1977 (RDB6237)

R-717, health effects, toxicity: people regularly exposed to ammonia may develop tolerance to its effects, as demonstrated in exposures of 6 male and female volunteers exposed to 25,50 , and $100 \mathrm{ppm}$ for 6 weeks; acclimatized subjects experienced eye tearing and some discomfort at $150-200 \mathrm{ppm}$, but no lasting health effects were observed as reported in RDB6270

A. J. Finkel, Ammonia, Hamilton and Hardy's Industrial Toxicology of Commercial Products (fourth edition), John Wright PSG Incorporated, 150-152, 1983 (3 pages, RDB6A35)

R-717, health effects, toxicity: anecdotal report of long-term exposure effect on hurnans; man working in a walk-in refrigerator developed a persistent cough and dyspnea (difficult or labored breathing); test showed lung damage even 3 yr later - as reported in RDB6270 and RDB6A37

K. E. Flury, D. E. Dines, J. R. Rodarte, and R. Rogers, Airway Obstruction Due to Inhalation of Ammonia, Mayo Clinic Proceedings, 58:389393, 1983 (5 pages, RDB6238)

R-717, health effects, toxicity

M. J. Gerald And M. D. Slot, Ammonia Gas Burns (An Account of Six Cases), Lancet, UK, 1356-1357, 1938 (2 pages, RDB6A48)

$\mathrm{R}-717$, health effects, toxicity, accident involving human exposure 
R. E. Gosselin, R. P. Smith, and H. C. Hodge, Ammonia, Clinical Toxicology of Commercial Products (fifth edition), Williams and Wilkins, Baltimore, MD, III-21 - III-26, 1984 (6 pages, RDB6A34)

\section{R-717, health effects, toxicity}

R. F. Griffiths and L. C. Megson, The Effect of Uncertainties in Human Toxic Response on Hazard Range Estimation of Ammonia and Chlorine, Atmospheric Environment, 18(6):11951206, 1984 (12 pages, RDB6261)

R-717, health effects, toxicity

H. W. Haggard, Action of Irritant Gases upon the Respiratory Tract, Joumal of Industrial Hygiene, 5:390-398, 1924 (9 pages, RDB8408)

toxicity of R-717 (ammonia) and others

D. V. Hatton and C. S. Leach, Collagen Breakdown and Ammonia Inhalation, Archives of Environmental Health, 34:83-87, 1979 (5 pages, RDB6A10)

$\mathrm{R}-717$, health effects, toxicity

R. A. Hawkins, A. L. Miller, R. C. Nielson, and R. L. Veech, The Acute Action of Ammonia on Rat Brain Metabolism In Vivo, Biochemical Journal, 134(4):1001-1008, 1973 (8 pages, RDB5765)

R-717, health effects, toxicity

Y. Henderson and H. W. Haggard, Noxious Gases and the Principles of Respiration Influencing their Action, American Chemical Society Monograph 35 (second edition), Reinhold Publishing Corporation, New York, NY, 1943 (RDB72AO)

reports that the maximum short exposure tolerance for R-717 (ammonia) is $300-500 \mathrm{ppm} \mathrm{v/v}$ for 30-60 minutes - as reported in RDB5340

C. Heymans and J. J. Es, Blood Changes Due to Ammonia Inhalation, Science, 109:121-122, 1949 ( 2 pages, RDB6A50)

$\mathrm{R}-717$, health effects, toxicity

C. J. Hilado, H. G. Cumming, A. M. Machado, C. J. Casey, and A. Furst, Effect of Individual Gaseous Toxicants on Mice, Proceedings of the Western Pharmacology Society, 21:159-160, 1978 (2 pages, RDB6A11)

R-717, health effects, toxicity

C. J. Hilado, C. J. Casey, and A. Furst, Effect of Ammonia on Swiss Albino Mice, Journal of
Combustion Toxicology, 41:385-388, 1977 (4 pages, RDB6251)

R-717, health effects, toxicity: 30-min $L_{50}$ mouse $=21,000 \mathrm{ppm}$ as reported in RDB5151 (with summary)

H. B. Hoeffler, H. I. Schweppe, and S. D. Greenberg, Bronchiectasis Following Pulmonary Ammonia Burn, Archives of Pathology and Laboratory Medicine, 106(13):686-687, 1982 (2 pages, RDB5766)

R-717, health effects, toxicity; accident in Houston involving a tanker truck, carrying 17.2 tonnes $(38,000 \mathrm{lb})$ of anhydrous ammonia, resulted in dispersion $1500 \mathrm{~m}\left(5000^{\prime}\right)$ downwind and $550 \mathrm{~m}$ (1800') wide; resulted in 5 deaths and 178 injuries; the fatalities and most disabling injuries occurred within approximately 70 $\mathrm{m}\left(230^{\circ}\right)$ of the accident; bronchiectasis is a a late-stage from of chronic bronchitis in which permanent dilation of bronchi due to destruction of the elastic and muscular components of the airway walls

H. Hofer, E. Reindle, and E. Hruby, Acute Inhalation Toxicity of Ammonia - Evaluation of an Apparatus, publication unknown, Austria, 1981 (RDB6246)

R-717, toxicity: $L C_{50}$ mouse, $30 \mathrm{~min}=9,085$ ppm as reported in RDB5151 (with summary)

D. L. Hoiness, J. T. Purdham, and J. R. Nethercott, Acute and Chronic Respiratory Effects of Occupational Exposure to Ammonia, AlHA Journal, 50(12):646-650, December 1989 (4 pages with 7 tables, RDB3238)

R-717, health effects, toxicity

International Programme on Chemical Safety (IPCS), Ammonia, Environmental Health Criteria (EHC) report 54, World Health Organization (WHO), Geneva, Switzerland, 1986 (214 pages with 21 tables, Rdb6241)

$R-717$, health effects, toxicity

N. I. Jarudi and B. Golden, Ammonia Eye Injuries, Journal of the lowa Medical Society, 63(6):260-263, 1973 (4 pages, RDB5767)

\section{$\mathrm{R}-717$, health effects}

J. Johnston, L. Legters, and R. Dailey (Enviro Control, Incorporated), Biological Effects of Short, High-Level Exposure to Gases: Ammonia, draft final report under contract DMD 17-79-C9086, U.S. Army Medical Research and Develop- 
ment Command, Fort Detrick, MD, 1979 (RDB6262)

R-717, health effects, toxicity: This draft report is widely cited in the literature, though no reference has been found to the final version. subjects experienced decreased respiratory tidal volume in exposures to $205-335 \mathrm{ppm} v / \mathrm{v}$ for 30 min and temporary changes in respiratory physiology at $500 \mathrm{ppm} \mathrm{v/v} \mathrm{for} 30 \mathrm{~min}$

S. R. Jones, Ammonia and the Nervous System, Journal of the American Medical Association (JAMA), $620 \mathrm{ff}, 1945$ (RDB6A51)

$\mathrm{R}-717$, health effects, toxicity

L. E. Kane, C. S. Barrow, and Y. Alarie (University of Pittsburgh), A Short-Term Test to Predict Acceptable Levels of Exposure to Airborne Sensory Irritants, AlHA Journal, 40:207-229, March 1979 (23 pages with 2 figures and 16 tables, RDB6249)

presents a model to predict human sensory irritation responses based on sensory irritation data from animal tests; provides $R D_{50}$ data and TLV comparisons for 11 chemicals including $303 \mathrm{ppm}$ for R-717 (ammonia) and $117 \mathrm{ppm}$ for R-764 (sulfur dioxide); compares predicted responses to published effects; proposes use of the $\mathrm{RD}_{50}$ measure to set guidelines for exposure limits; concludes that the threshold limit value (TLV) should be set at $1-10 \%$ of the $\mathrm{RD}_{50}$, the short-term exposure limit (STEL) at $20 \%$ of the $R_{50}$, and the emergency exposure limit (EEL) at $30 \%$ of the $R D_{50}$

J. C. Kapeghian, A. B. Jones, and I. W. Waters (Mississippi University), Effects of Ammonia on Selected Hepatic Microsomal Enzyme Activity in Mice, Bulletin of Environmental Contamination and Toxicology, 35:15-22, 1985 (8 pages, RDB6A14)

R-717, health effects, toxicity

J. C. Kapeghian, H. H. Mincer, A. B. Jones, A. J. Verlangieri, and I. W. Waters (Mississippi University). Acute Inhalation Toxicity of Ammonia in Mice, Bulletin of Environmental Contamination and Toxicology, 29(3):371-378, 1982 (8 pages, RDB5768)

R-717, toxicity: $30-\mathrm{min} L_{50}$ mouse $=4,230$ and $60-\mathrm{min} \mathrm{LC}_{50}$ mouse $=5,981 \mathrm{ppm}$, depending on calculation method as reported in RDB5151 (with summary) RDB5155

J. C. Kapeghian, A. B. Jones, H. H. Mincer, A. J. Verlangieri, and I. W. Waters (Mississippi Universi- ty), The Toxicity of Ammonia Gas in the Mouse, abstract 7586, Federation Proceedings, Federation of the American Society of Experimental Biologists, 41:1568, 1982 (1 page, RDB72A1)

$1-\mathrm{hr} \mathrm{LC}_{50}$ mouse $=4,230 \mathrm{ppm} \mathrm{v} / \mathrm{v}$ for $\mathrm{R}-717$ (ammonia) as reported in RDB5340

1. Kass, N. Zamel, C. A. Bobry, and M. Holzer, Bronchiectasis Following Ammonia Burns of the Respiratory Tract, Chest, 62:282-285, 1972 (4 pages, RDB6A13)

R-717, health effects, toxicity: high concentrations of ammonia dissolve in moisture on the skin, leading to skin corrosion as reported in RDB6270

A. Kimmerle, JFF/Combustion Technology, 1:4-51, 1974 (48 pages, RDB6250)

reviews toxicity parameters and data, from other sources, on the inhalation toxicity of R717 (ammonia) in animals; characterizes concentrations of $480-570 \mathrm{ppm}$ and $800-1070 \mathrm{ppm}$ as "irritation" for 4-hr exposures and "strong irritation" for 7-hr exposures, respectively; the latter overlaps with dyspnea (difficult or labored breathing) and traechitis from $820-1430 \mathrm{ppm}$; indicates that a 4-hr exposures of rats to 2,000 $\mathrm{ppm} \mathrm{v} / \mathrm{v}$ was not fatal while another at 4,000 $\mathrm{ppm} v / \mathrm{v}$ was, but does not indicate the quantities of animals involved; notes reports of $2-\mathrm{hr}$ $\mathrm{LC}_{50} \mathrm{~S}$ of $3,420,4,760$, and $10,930 \mathrm{ppm} \mathrm{v} / \mathrm{v}$ in mice, mice, and rats respectively - as reported (with summary) in RDB5151

V. Kirhov, Neuroautonomic Response of Workers in the Ammonia Industry, Suvrem Med, Bulgaria, 28(10):10-13, 1977 (in Bulgarian, RDB6A55)

R-717, health effects, toxicity: anecdotal study of extended occupational exposures; compares beginning and end-of-day symptoms for 45 male and 28 female workers, exposed to $13-51$ ppm, for 5-10 years in an ammonia production plant; subjects reported increases in headaches, vertigo, staggering, and tremors to interviewers; findings were dismissed by others as "not meaningful due to major methodological flaws" as reported in RDB1106

V. Kondrasuov, Gigiena Truda i Professional'nye Zabolevaniya [Labor Hygiene and Occupational Diseases], Russia (then USSR), 2:34-38, 1978 (5 pages in Russian, RDB6252)

$R-717$, toxicity: $L C_{50}$ rat comparison between inhalation and skin absorption as reported (with summary) in RDB5151 
Kujawska, Analysis of Lung Function in Workers Exposed to Irritating Dusts and Gases, Med. Pr., Poland, 28(5):419-426, 1977 (in Polish, RDB6A56)

R-717, health effects, toxicity: anecdotal study of extended occupational exposures; 143 workers exposed to 7-41 ppm of ammonia, as well as other irritants, had lowered forced expiratory volume than workers from the same facility not exposed to ammonia; critiqued for methodological flaws and other confounding factors as reported in RDB1106

D. Leduc, P. Gris, P. Lheureux, et al., Acute and Long Term Respiratory Damage Following Inhalation of Ammonia, Thorax, 47:755-757, 1992 (3 pages, RDB6A15)

$\mathrm{R}-717$, health effects, toxicity

K. B. Lehmann (Universität Wärzburg), Experimental Studies on the Acquisition of Tolerance to Technically and Hygienically Important Gases and Vapors: Ammonia, Archiv für $\mathrm{Hy}$ giene, 6, 1889 (126 pages in German, RDB6247)

R-717, toxicity: early toxicity testing with cats, dogs, guinea pigs, rabbits, and rats; distinctions among species as reported in RDB5151 (with summary)

K. B. Lehmann (Universität Würzburg), Experimental Studies on the Effect of Technically and Hygienically Important Gases and Vapors on the Organism (Part I, Ammonia), Archiv für Hygiene, 5:1-126, 1886 (126 pages in German, RDB6248)

R-717, toxicity: early toxicity testing with cats, dogs, guinea pigs, rabbits, and rats; distinctions among species - as reported in RDB5151 (with summary)

G. Leonardos, D. Kendall, and N. Barnard, Odour Threshold Determination of 52 Oderant Chemicals, Journal of the Air Pollution Control Association, 19:91-95, 1969 (5 pages, RDB8410) odor detection threshold of R-717 (ammonia)

D. M. Levy, M. B. Divertie, T. J. Litzow, and J. W. Henderson, Ammonia Burns of the Face and Respiratory Tract, Journal of the American Medical Association (JAMA), 190:95-98 (possibly 190:873-876), 1964 (4 pages, RDB6243)

R-717, health effects, toxicity: accidental exposure to high concentrations may cause severe eye injury, including fluid accumulation in the eyelids, scarring of the cornea, and even loss of vision as reported in RDB6270
H. Lonsdale, Ammonia Tank Failure - South Africa, Ammonia Plant Safety, American Institute of Chemical Engineers (AlChE), New York, NY, 17:126-131, 1975 (6 pages, RDB6A16)

$R-717$, health effects, toxicity, accident involving human exposure: failure of a pressurized ammonia tank in Potchefstroom, South Africa, released 38 tonnes (42 tons) of anhydrous ammonia resulting in 18 deaths and multiple injuries

J. D. MacEwen and E. H. Vernot (University of California, Irvine, UCl), Annual Technical Report, report NTIS AD 3555 358, Toxic Hazards Research Unit Technical, National Technical Information Service (NTIS), Springfield, VA, 1972 (RDB6230)

$\mathrm{R}-717$, toxicity, $1-\mathrm{hr} \mathrm{LC}_{50}$ rat, $=7,338 \mathrm{ppm}$ as reported in RDB1106 and (with summary) RDB5151

C. A. Mangold (U.S. Navy Industrial Hygiene Division of Investigation), Investigation of Occupational Exposure to Ammonia, Puget Sound Naval Shipyard, U.S. Navy, Puget Sound, WA, 1971 (RDB8412)

R-717 (ammonia), health effects, toxicity

A. Manninen, S. Antilla, and $H$. Savolainen, Rat Metabolic Adaptation to Ammonia Inhalation, Proceedings of the Society of Experimental Biol. Medicine, 187:278-281, 1988 (4 pages, RDB6263)

$\mathrm{R}-717$, health effects, toxicity

R. S. Marknam, A Review of Damage from Ammonia Spills, Proceedings of the Ammonia Symposium (Boston, MA, August 1986), American Institute of Chemical Engineers (AIChE), New York, NY, 1986 (RDB6A17)

R-717, health effects, toxicity, accident involving human exposure

J. L. Martin, Review of Literature on the Toxicity of Ammonia, The Fertilizer Institute, Washington, DC, 1979 (RDB6A54)

R-717, health effects, toxicity; anecdotal report of the potential effects of long-term, human occupational exposure to ammonia; physician reviewed the results of physical examinations of employees of a fertilizer manufacturer prior to employment and every two years thereafter for up to 15 years; concludes that there is no longterm toxicity due to ammonia exposure as reported in RDB1106 
M. H. Mayan and C. P. Merilan, Effects of Ammonia Inhalation on Young Cattle, New Zealand Veterinary Journal, 24:221-224, 1976 (4 pages, RDB6A18)

R-717, health effects, toxicity

M. H. Mayan and C. P. Merilan, Effects of Ammonia Inhalation on Respiration Rate of Rabbits, Journal of Animal Science, 34:448-452, 1972 (RDB6A19)

R-717, health effects, toxicity

S. E. A. McCallan and C. Setterstrom, Toxicity of Ammonia, Chlorine, Hydrogen Cyanide, Hydrogen Sulfide, and Sulfur Dioxide. I. General Methods and Correlations, Contrib. Boyce Thompson Institute, 11:325-330, 1940 (6 pages, RDB8411)

R-717, health effects, toxicity

J. A. McLean, K. P. Mathews, W. R. Solomon, P. R. Brayton, and N. K. Bayne, Effect of Ammonia on Nasal Resistance in Atopic and Nonatopic Subjects, Annals of Otol. Rhinol. Laryngol., 88:228-234, 1979 (7 pages, RDB7453)

R-717, health effects, toxicity

R. A. Michaels, Acute Inhalation Risk Potentially Posed by Anhydrous Ammonia, RAM TRAC Corporation, Schenectady, NY, 1996 (99 pages, RDB6A25)

$\mathrm{R}-717$, health effects, toxicity

W. Moeller, Zur chronischen Ammoniak-Exposition aus Opthalmologischer Sicht [Chronic Ammonia Exposure from an Ophthalmologic Point of View], Zeitschrift für die gesamte Hygiene und Ihre Grenzgebiete, 20(9):579-581, 1974 (3 pages, RDB5769)

R-717, health effects, toxicity

T. J. Montague and A. R. Macneil, Mass Ammonia Inhalation, Chest, 77(4):496-498, 1980 (3 pages, RDB5770)

R-717, health effects, toxicity

K. Mudan and K. Mitchell, Report on the Potchefsroom, South Africa Ammonia Incident, Four Elements, Incorporated, Columbus, $\mathrm{OH}$, 1996 (14 pages, RDB6A20)

$\mathrm{R}-717$, health effects, toxicity, accident involving human exposure

J. S. Mulder and $H$. O. Van der Zalm, Een gavel van dodelijke ammoniakvergiftiging [A Fatal Case of Ammonia Poisoningl, Tijdschrift Voor So- ciale Geneeskunde, Rotterdam, The Netherlands, 45:458-460, 1967 (3 pages in Dutch, RDB6A21)

describes a fatal accident involving human exposure to R-717 (ammonia); indicates that a worker exposed to a very high, but unknown concentration of ammonia vapor immediately experienced coughing, dyspnea (difficult or labored breathing), and vomiting; notes that the victim developed conjunctivitis (inflammation of the mucous membrane inside the eyelids) $3 \mathrm{hr}$ after exposure, and died of cardiac arrest $6 \mathrm{hr}$ after exposure; indicates that the autopsy revealed marked respiratory irritation, denudation of the tracheal epithelium (surface layer of tissue lining the air passages connecting the nose and mouth to the lungs), and pulmonary edema

H. Musehenborn, The Physiological Action of Ammonia on Man and Means of Protection Against It, Dräger Hefte, Germany, 190:3503 ff, 1937 (RDBA947)

toxicity study of R-717 (ammonia) in humans

National Research Council (NRC) Committee on Medical and Biological Effects of Environmental Pollutants, Ammonia, University Park Press, Baltimore, MD, 1979 (RDB5771)

$\mathrm{R}-717$, health effects, toxicity

National Research Council (NRC) Committee on Toxicology, Guideline for Short Term Exposures of the Public to Air Pollutants, IV, Guide for Ammonia, National Academy of Sciences (NAS), National Academy Press, Washington, DC, 1987 (RDB5154)

$\mathrm{R}-717$, health effects, toxicity

F. Navazio, T. Gerritsen, and G. J. Wright, Relationship of Ammonia Intoxication to Convulsions an Coma in Rats, Journal of Neuorochemistry, 8:146-151, 1961 (6 pages, RDB8413)

$\mathrm{R}-717$, health effects, toxicity

A. Niden, Effect of Ammonia Inhalation on the Terminal Airways, Proceedings of the 11th Aspen Emphysema Conference, II:41-44, 1968 (4 pages, RDB6253)

R-717, health effects, toxicity: histological changes of the lung after acute exposure to ammonia, dose-dependent effects in mice as reported in RDB5151 (with summary)

G. J. O'Kane, Inhalation of Ammonia Vapour: A Report of Eight Patients During the Acute Stages, Anesthesia, 38:1208-1213, 1983 (6 pages, RDB6A22) 
R-717, health effects, toxicity, accident involving human exposure

F. Pedersen and R. S. Selig, Predicting the Consequences of Short-Term Exposure to High Concentrations of Gaseous Ammonia, Journal of Hazardous Materials, 21:143-159, 1989 (17 pages, RDB6A23)

\section{$\mathrm{R}-717$, health effects, toxicity}

J. O. Pierce, Ammonia, Patty's Industrial Hygiene and Toxicology (fourth edition), edited by G. D. Clayton and F. E. Clayton, John Wiley and Sons, Incorporated, New York, NY, 2A:756-782, 1994 (27 pages, RDB6A24)

$\mathrm{R}-717$, health effects, toxicity

A. S. Propkop'eva and G. C. Yushkov, Toxicology of Ammonia in Animals Repeatedly Exposed for Short Lengths of Time, Gigiena Truda i Professional'nye Zabolevaniya [Labor Hygiene and Occupational Diseases], Russia (then USSR), 9:54-55, 1975 (2 pages in Russian, RDB6228)

R-717, health effects, toxicity: subchronic exposures of rats as reported in RDB5151 (with summary)

A. S. Propkop'eva, G. C. Yushkov, and I. O. Ubasheev, Materials for a Toxicological Characteristic of the Long-Term Effect of Ammonia on the Organisms of Animals After Brief Exposure, Gigiena Truda i Professional'nye Zabolevaniya [Labor Hygiene and Occupational Diseases], Russia (then USSR), 6:56-57, 1973 (2 pages in Russian, RDB6229)

-R-717, toxicity, $L_{50}$ rat $=26,704 \mathrm{ppm}$ for 5 $\min , 17,371 \mathrm{ppm}$ for $15 \mathrm{~min}, 10,050 \mathrm{ppm}$ for 30 $\mathrm{min}$, and $11,341 \mathrm{ppm}$ for $60 \mathrm{~min}$ as reported in RDB1106, RDB5106, and (with summary) RDB5151

P. K. Raj, J. H. Hagopian, A. S. Kalelkar, and J. Cece, Predicting Health Hazards from Ammonia Spills, Ammonia Plant Safety, 17:102-108, 1975 (7 pages, RDB6A52)

R-717, health effects, toxicity

D. Richard, J. Jouany, and C. L. Bondène, Acute Toxicity of Ammonia in Rabbits by Inhalation, Compte Rendu de l'Académie de Science [Proceedings of the Academy of Science], France, series $D, 287(4): 375-378,1978$ (4 pages, RDB5772)

R-717, health effects, toxicity: acute exposures of rabbits at $1000-2000 \mathrm{ppm}$ as reported in RDB5151 (with summary)
D. Richard, G. Bouley, and C. L. Bondène, Effets de I'Inhalation Continué d'Ammoniac Chez le Rat et la Souris [Effect of Continuous Inhalation of Ammonia in the Rat and Mouse], Bulletin of the European Physiopathol. Respir., 14(5):573-582, 1978 (10 pages in French, RDB6244)

R-717, health effects, toxicity: chronic exposures of rats at $250-300 \mathrm{ppm}$, without general toxic effect, and 500 ppm as reported RDB5151 (with summary)

K. J. Schadeberg, J. W. Goode, M. L. Keplinger, and J. C. Calandra (Industrial Bio-Test Laboratories, Incorporated), Irritation Threshold Evaluation Study with Ammonia, report IBT 663-03161, International Institute of Ammonia Refrigeration (IIAR), Washington, DC, 23 March 1973 (12 pages with 2 tables, RDB6A12)

R-717, health effects, toxicity; study to determine the threshold for irritation by R-717 in humans: irritation was taken to be any annoyance to eyes, nose, mouth, throat, or chest that persisted throughout a five-minute, head-only exposure for a panel of ten subjects; 1 of 10 reported dryness of the nose (deemed not an uncomfortable irritation in the report) at $32 \mathrm{ppm}$ and 2 of 10 did so at $50 \mathrm{ppm}$; irritation was reported by 3 or more of 10 subjects at 72 and $134 \mathrm{ppm}$; effects were greater with increasing concentrations; report concludes that concentrations of $50 \mathrm{ppm}$ or less did not cause irritation or discomfort

M. M. Saifutdinov, Maximum Permissible Concentration of Ammonia in the Atmosphere, Hyg. Sanitariya, 31:171-176, 1966 (6 pages in Russian, RDB6A53)

R-717, health effects, exposure limit, toxicity

A. D. Schaerdel, W. J. White, C. M. Lang, et al., Localized and Systemic Effects of Environmental Ammonia in Rats, Laboratory Animal Science, 33:40-45, 1983 (16 pages, RDB6A26)

$\mathrm{R}-717$, health effects, toxicity

M. B. Shimkin, A. A. de Lorimier, J. R. Mitchell, and T. P. Burroughs, Appearance of Carcinoma Following Single Exposure to a Refrigeration Ammonia-Oil Mixture: Report of a Case and Discussion of the Role of Co-Carcinoginesis, Archives of Industrial Hygiene and Occupational Medicine, 9:186-193, 1954 (8 pages, RDB6264)

R-717, carcinogenic potential, health effects, toxicity: technician splattered with R-717 and lubricant on his nose and lip developed a small blister, which became a persistent, chronically 
inflamed lump; biopsy confirmed presence of epidermoid carcinoma 6 months later as reported in RDB1106; RDB1106 discusses confounding factors and concludes that it is impossible to draw conclusions concerning the role of $\mathrm{R}-717$ in the observed response

S. D. Silver and F. P. McGrath, A Comparison of Acute Toxicities of Ethylene Amine and Ammonia to Mice, Journal of Industrial Hygiene and Toxicology, 30:7-9, 1948 (3 pages, RDB6254)

R-717, health effects, toxicity: acute exposures of mice at high concentrations, $10-\mathrm{min} \mathrm{LC}_{50}$ mouse $=10,152 \mathrm{ppm}$ as reported in RDB5151 (with summary)

L. Silverman, J. L. Whittenberger, and J. Muller (Harvard School of Public Health), Physiological Response of Man to Ammonia in Low Concentrations, American Public Health Association Meeting (session of the Industrial Hygiene Section, Boston MA, 10 November 1948), 1948; republished in the Journal of Industrial Hygiene and Toxicology, 31(2):74-78, March 1949 (5 pages with 1 figure and 1 table, RDB6226)

summarizes an inhalation toxicity study of R717 (ammonia) in human volunteers to determine the physiological effects; describes controlled exposures of six male subjects at rest to $500 \mathrm{ppm} \mathrm{v/v}$ for $30 \mathrm{~min}$ and a seventh for 15 min; reports that subjects exhibited hyperventilation (abnormally high breathing rate) with cyclic variation during exposures and transient hypoventilation (abnormally low breathing rate) during recovery following the exposures; notes that one subject showed a slight increase in blood pressure and pulse rate; also reports that the subjects exhibited irritation of the nose and throat, hypo-esthesia (reduced capacity for sensation and feeling) of the exposed skin, and lacrimation (abnormal or excessive secretion of tears); notes that subjects reported respiratory irritation with some effects persisting for $24 \mathrm{hr}$ after exposures; notes that the subjective reactions varied considerably, but were present in all subjects and severe in two

H. F. Smyth, Jr., Improved Communication: Hygienic Standards for Daily Inhalation, AlHA Quarterly, 17(2):129-185, 1956 (RDB72A2)

exposures to R-717 (ammonia) of 2,500-6,000 $\mathrm{ppm} v / \mathrm{v}$ for $30 \mathrm{~min}$ are considered dangerous to life as reported in RDB5340

H. F. Smyth, Jr., J. Seaton, and L. Fischer (Carnegie-Mellon Institute, then the Mellon Institute), The
Single Dose Toxicity of Some Glycols and Derivatives, Journal of Industrial Hygiene and OCcupational Medicine, 23(6):259-268, June 1941 (10 pages with 3 tables, RDB6267)

R-717 and others, health effects, toxicity: widely referenced $L D_{50}$ oral, rat $=350 \mathrm{mg} / \mathrm{kg}$ based on tests using ammonium hydroxide (ammonia-water solution, $\mathrm{NH}_{4} \mathrm{OH}$ ) with calculated result for ammonia

R. Sobonya, Fatal Anhydrous Ammonia Inhalation, Human Pathology, 8(3):293-299, 1977 (7 pages, RDB5773)

R-717, health effects, toxicity, accident involving human exposure: 4 fatalities following exposure to ammonia in a farming accident; 3 workers died immediately; the fourth, a 25-yr old man, died from secondary effects 60 days later

S. Stroud, Ammonia Inhalation - A Case Report, Critical Care Nurse, 1(2):23-26, 1981 (4 pages, RDB5774)

R-717, health effects, toxicity

M. Stupfel et al., Compte Rendu Société Biol., France, 165:1869-1872, 1971 (4 pages, RDB6255)

R-717, health effects, toxicity: acute exposures of mice at $12,000 \mathrm{ppm}$ for $20 \mathrm{~min}$ and at 11,000 ppm for $40 \mathrm{~min}$; males mortality found to be higher as reported in RDB5151 (with summary)

R. B. Swotinsky and K. H. Chase, Health Effects of Exposure to Ammonia: Scant Information, American Journal of Industrial Medicine, 17:515521, 1990 (7 pages, RDB6A27)

$\mathrm{R}-717$, health effects, toxicity

Syracuse Research Corporation, Toxicological Profile for Ammonia, report TP-90-03, Agency for Toxic Substances and Disease Registry (ATSDR), Public Health Service, U.S. Department of Health and Human Services, Washington, DC, December 1990 (available from NTIS as document PB91180315, RDB5156)

R-717, health effects, toxicity

S. P. Targowski W. Klucinski, S. Babiker, et al., Effect of Ammonia on in Vivo and in Vitro Immune Responses, Infect. Immunology, 43:289293, 1984 (5 pages, RDB6A43)

R-717, health effects, toxicity

J. S. Tepper, B. Weiss, and R. W. Wood, Alterations in Behavior Produced by Inhaled Ozone 
or Ammonia, Fundamental and Applied Toxicology, 5:1110-1118, 1985 (7 pages, RDB6A28)

R-717, health effects, toxicity

Y. C. Ting, The Toxicity of Ammonia, Science, American Association for the Advancement of Science (AAAS), Washington, DC, 112:91 ff, 1950 (RDB6265)

$R-717$, health effects, toxicity

M. M. Verberk, Effect of Ammonia in Volunteers, International Archives of Occupational and Environmental Health, Germany, 39:73-81, 1977 (9 pages, RDB6A29)

reports exposures of 16 volunteers to $50-140$ $\mathrm{ppm} \mathrm{v/v} \mathrm{R-717} \mathrm{(ammonia)} \mathrm{for} 2 \mathrm{hr}$; indicates that subjects experienced nasal irritation, but no changes to their ventilation capacity; notes that eight of the subjects found $140 \mathrm{ppm} \mathrm{v} / \mathrm{v}$ to be intolerable; toxicity - as reported in RDB4B76.

E. H. Vernot, J. D. MacEwen, C. C. Haun, and E. R. Kinkead (University of California, Irvine, UCl), Acute Toxicity and Skin Corrosion Data for Some Organic and Inorganic Compounds and Aqueous Solutions, Toxicology and Applied Pharmacology (TAP), 42:417-423, 1977; republished in report AMRL-TR-77-9, Aerospace Medical Research Laboratory, U.S. Air Force, WrightPatterson Air Force Base, OH, 1977 (7 pages with 1 table, RDB5157)

R-717 (ammonia), health effects, toxicity: LC $_{50}$ rat, $1 \mathrm{hr}=7338 \mathrm{ppm} ; L_{50}$ and/or $L D_{50}$ data for 110 organic compounds and aqueous solutions thereof (except for ammonia, the others are not commonly used as refrigerants)

D. P. Wallace, Atmospheric Emissions and Control, Ammonia Plant Safety, 21:51-56, 1979 (6 pages, RDBA672)

summarizes subjective observations by seven human volunteers accustomed to working with R-717 (ammonia) to informal exposures for $1-3$ min; indicates that concentrations of 150-200 $\mathrm{ppm} v / \mathrm{v}$ affected their eyes to a limited extent after approximately $1 \mathrm{~min}$, but their vision was not seriously impaired and breathing was not affected; $440 \mathrm{ppm} \mathrm{v} / \mathrm{v}$ affected their eyes more quickly, but not sufficiently to impair vision; 600 $\mathrm{ppm} \mathrm{v} / \mathrm{v}$ caused their eyes to stream after 30 seconds, but was still breathable; at $1,000 \mathrm{ppm}$ $\mathrm{V} / \mathrm{V}$, their eyes streamed instantly and vision was impaired but not lost, breathing was deemed intolerable to most of those exposed, and skin irritation followed several minutes of exposure; and 1,500 ppm v/v resulted in an instant reaction to get out of the area - as rreported in RDB4236

M. Walton, Industrial Ammonia Gassing, British Journal of Industrial Medicine, 30:78-86, 1973 (9 pages, RDB6A30)

R-717, health effects, toxicity: 1 of 7 workers exposed to ammonia in an industrial accident died; autopsy showed marked laryngeal edema, acute congestion, pulmonary edema (evidence of damage to alveolar region), and denudation of the bronchial epithelium

R. C. Wands, Alkaline Materials, Patty's Industrial Hygiene and Toxicology (third revised edition), edited by G. D. Clayton and F. E. Clayton, John Wiley and Sons, Incorporated, New York, NY, chapter 42, 2B(Toxicology):3045-3052, 1981 (8 pages, RDB6A38)

R-717 and others, health effects, toxicity

K. Ward, B. Murray, and G. P. Costello, Acute and Long Term Pulmonary Sequelae of Acute Ammonia Inhalation, Irish Medical Joumal, 76:279-281, 1983 (3 pages, RDB6A44)

R-717, health effects, toxicity

J. H. Weatherby, Chronic Toxicity of Ammonia Fumes by Inhalation, Proceedings of the Society of Experimental Biol. Medicine, 81:300-301, 1952 (2 pages, RDB6239)

$\mathrm{R}-717$, health effects, toxicity

F. R. Weedon, A. Hartzell, and C. Setterstrom, Toxicity of Ammonia, Chlorine, Hydrogen Cyanide, Hydrogen Sulfide, and Sulfur Dioxide. V. Animals, Contrib. Boyce Thompson Institute, $11: 475-482,1940$ (8 pages, RDB6256)

R-717, health effects, toxicity: acute exposures of rats, mice, and flies at $1,000 \mathrm{ppm}$ for $16 \mathrm{hr}$ as reported in RDB5151 (with summary)

E. S. White, Case Report - A Case of Near Fatal Ammonia Gas Poisoning, Journal of Occupational Medicine, 13:549-550, 1971 (2 pages, RDB7454)

R-717, toxicity: accident involving human exposure, health effects, treatment

J. Withers (LUT, UK), W. F. ten Berge (DSM, The Netherlands), J. Gordon (ICl), C. Harris (Technica), I. Hymes (HSE), and J. Jolley (HSE), The Lethal Toxicity of Ammonia - A Report to the MHAP [Major Hazards Advisory Panel], North Western Branch Papers, Institution of Chemical 
Engineers, UK, 1986(1):6.1-6.27, 1985 (27 pages with 3 figures and 9 tables, RDB6A31)

$\mathrm{R}-717$, health effects, toxicity

K. L. Wong, Ammonia, Maximum Allowable Concentrations for Selected Airborne Contaminants in Spacecraft, National Research Council (NRC), Committee on Toxicology, 39-59, 1995 (21 pages, RDB6A32)

R-717, health effects, toxicity

R. W. Wood, Determinants of Irritant Termination Behavior, Toxicology and Applied Pharmacology (TAP), 61:260-268, 1981 (9 pages, RDB6266)

R-717 (ammonia), health effects, toxicity

R. W. Wood, Behavioral Evaluation of Sensory Irritation Evoked by Ammonia, Toxicology and Applied Pharmacology (TAP), 50:157-162, 1979 (6 pages, RDB6240)

R-717 (ammonia), health effects: tolerance and respiratory depression (5\% and $500 \mathrm{ppm}$ ) as reported in RDB5151 (with summary)

D. Zissu (Institut National de Recherche et de Sécurité, France), Histopathological Changes in the Respiratory Tract of Mice Exposed to Ten Families of Airborne Chemicals, Journal of Applied Toxicology, 15(3):207-213, 1995 (7 pages with 3 figures and 3 tables, RDB6A33)

study to identify and compare the histopathological changes induced in the respiratory tract of mice by repeated exposures at targets of $0.3,1.0$, and 3.0 times the $R D_{50}$ concentrations of airborne chemicals, among them R-717 (ammonia); toxicity; health effects: only 11 of the 15 tested irritants induced lesions, among them R-717 which injured the respiratory epithelium; no pathological changes were observed from $\mathrm{R}-717$ at $257 \mathrm{ppm}\left(\mathrm{RD}_{50} \times 0.85\right)$, but lesions were found following exposures at $711 \mathrm{ppm}$ $\left(R D_{50} \times 2.35\right)$; injuries from $R-717$ progressed from moderate for 4 days to severe for 9 days to very severe for 14 days based on exposures of $6 \mathrm{hr} / \mathrm{d}$ for $5 \mathrm{~d} / \mathrm{wk}$; the authors suggest that a repair mechanism may moderate further injury for the majority of tested chemicals, for which the most severe injuries occurred within the first 4 days, but the intensity of lesions induced by R-717 became more severe with further exposures

Ammonia, chemical infogram 73, Canadian Centre for Occupational Health and Safety (CCOHS),
Hamilton, ON, Canada, October 1990 (1 page, RDB6269)

R-717, safety, toxicity, flammability, occupational exposure guidance

Ammonia, Industrial Exposure and Control Technologies for OSHA Regulated Hazardous Substances, Occupational Safety and Health Administration (OSHA), U.S. Department of Labor, Washington, DC, 104-111, March 1989 (8 pages, available from GPO, RDB6A37)

R-717, health effects, toxicity, exposure and use statistics, controls and protective equipment

Ammonia (CAS 7664-41-7), chemical hazard summary 37, report C88-1E, Canadian Centre for Occupational Health and Safety (CCOHS), Hamilton, ON, Canada, 1988 (18 pages with 1 table, RDB6270)

occupational exposure guidance for R-717 (ammonia); safety; toxicity; flammability

Ammonia: Environmental and Technical Information for Problem Spills, Environment Canada, Ottawa, Ontario, Canada, 1984 (RDB6271)

occupational exposure guidance for R-717 (ammonia); safety; toxicity; flammability; environmental impact

Ammonia Health Effects Information, CAS\# 7664-41-7, Elf Atochem North America, Incorporated, Philadelphia, PA, undated circa 1995 (2 pages, RDB5B60)

occupational exposure guidance for R-717 (ammonia); safety; toxicity; flammability; environmental impact; ecological information

Ammonia Toxicity Monograph, The Institution of Chemical Engineers, Warwickshire, UK, 1988 (RDB5151)

R-717, health effects: review and statistical analysis of $L C_{50}$ studies, regression for $L_{50}$ for the rat by time ( $30 \mathrm{~min}=11,502 \mathrm{ppm}, 1 \mathrm{hr}=$ $8,133 \mathrm{ppm}, 4 \mathrm{hr}=4,067 \mathrm{ppm}$ )

Criteria for a Recommended Standard - Occupational Exposure to Ammonia, publication 74136. National Institute of Occupational Safety and Health (NIOSH), U.S. Department of Health and Human Services, Cincinnati, OH, 1974 (RDB5369)

R-717, toxicity

Critical Reviews in Toxicology, Chemical Rubber Company (CRC), Cleveland, $\mathrm{OH}, 5: 189 \mathrm{ff}$, 1977 (RDB6591) 


\section{R-717, toxicity}

Emergency First Aid Treatment Guide for Ammonia (7664-41-7), U.S. Environmental Protection Agency (EPA), Washington, DC, undated circa 1990 (RDB6268)

R-717, health effects, toxicity

Emergency Response Planning Guidelines Ammonia, American Industrial Hygiene Association (AlHA), Fairfax, VA, 1988 (4 pages, RDB4B76)

R-717, ERPG-1, ERPG-2, ERPG-3, toxicology data, toxicity, safety classification

Hygienic Guide Series - Anhydrous Ammonia, American Industrial Hygiene Association (AlHA), AlHA Journal, 32:139-142, 1971 (3 pages, RDB5775)

R-717, health effects, toxicity: maximum shortexposure tolerance is $300-500 \mathrm{ppm} v / \mathrm{v}$ for 30 60 minutes, $2,500-6000 \mathrm{ppm}$ are considered dangerous to life, and $5,000-10,000 \mathrm{ppm}$ are reported to be fatal - as reported in RDB5340

Occupational Safety and Health Guideline for Ammonia; National Institute of Occupational Safety and Health (NIOSH), U.S. Department of Health and Human Services, Cincinnati, $\mathrm{OH}$; Occupational Safety and Health Administration (OSHA), U.S. Department of Labor, Washington, DC; 1992 (7 pages with no figures or tables, available from GPO, also available from JMC as RDB$5 \mathrm{C} 46$ )

R-717, toxicity; chemical and physical properties, reactivity, flammability, exposure limits, health hazard information, exposure sources and control methods, medical monitoring, workplace monitoring and measurement, personal hygiene, storage, spills and leaks, special requirements, personal protective equipment, references

Summary Review of Health Effects Associated with Ammonia: Health Issue Assessment, report EPA 600/8-89/052F, Office of Health and Environmental Assessment, U.S. Environmental Protection Agency (EPA), Washington, DC, June 1989 (72 pages with 1 figure and 5 tables, RDB5155)

assesses the toxicity R-717 (ammonia); provides an extensive list of references

\section{R-744 (CARBON DIOXIDE)}

M. R. Banerjee, International Journal of Biometeorology, 16(3):259-267, 1972 (9 pages, RDB6454)

effects on rats of acute exposure to increased R-744 (carbon dioxide) in the air; toxicity

P. Brighten, A Case of Industrial Carbon Dioxide Poisoning, Anaesthesia, UK, 31(3):406-407. 1976 (2 pages, RDB5788)

toxicity of R-744 (carbon dioxide)

E. B. Brown Jr. and F. Miller, Ventricular Fibrillation Following a Rapid Fall in Alveolar Carbon Dioxide Concentration, American Journal of Physiology, 169:56-60, 1952 (5 pages, RDBA936)

indicates that 15 of 17 dogs exposed to $400,000 \mathrm{ppm}$ v/v R-744 (carbon dioxide) for 2 hr or more died upon sudden reexposure to air; toxicity - as reported in RDB5787

E. W. Brown (Edgewood Arsenal, Medical Corps), The Physiological Effects of High Concentrations of Carbon Dioxide, U.S. Navy Medical Bulletin, 28:721-734, 1930 (15 pages with foldout and 1 figure and 1 table, RDBA655)

reports an inhalation toxicity study in human volunteers to determine the physiological effects of inhalation of R-744 (carbon dioxide); indicates that seven male subjects were exposed via face masks to concentrations of $55,000-124,000 \mathrm{ppm}$ v/v with oxygen maintained between 14.4 and $39.7 \%$; the concentrations tested were deemed tolerable for only short periods of time, ranging from $22 \mathrm{~min}$ for $60,000 \mathrm{ppm} \mathrm{v} / \mathrm{v}$ to $30 \mathrm{sec}$ for $124,000 \mathrm{ppm} \mathrm{v/v}$; notes that exposed subjects showed negligible to slight increases in respiratory rate, moderate increases in pulse rate, and dose-dependent increases in both diastolic and systolic blood pressure; notes that subjects showed varying degrees of increase in minute-expiration volume and the severity of subjective symptoms including dyspnea (difficult or labored breathing), dizziness, flushing and sweating of the face, feeling of stupefaction (reduced sensation, perception, and understanding), apprehension of impending collapse, and limited to moderately severe headache; indicates that despite marked differences in individual tolerance to the exposures, the author did not think any of the subjects could have withstood $100,000 \mathrm{ppm} v / \mathrm{v}$ for more than $10 \mathrm{~min}$ without loss of consciousness; notes that increasing the oxygen concentration did not alleviate the 
toxic effects of high carbon dioxide concentrations

E. S. Cooper et al., The Relation Between Cardiac Function and Cerebral Blood Flow in Stroke Patients 1: Effect of $\mathrm{CO}_{2}$ Inhalation, Stroke, 1(5):330-347, 1970 (18 pages, RDB5789)

toxicity of R-744 (carbon dioxide)

Council on Pharmacy and Chemistry of the American Medical Association (AMA), Reports of the Council: Oxygen-Carbon Dioxide Mixtures, Journal of the American Medical Association (JAMA), 114(12):1077-1079, 23 March 1940 (3 pages with no figures or tables, RDBA657)

toxicity of R-744 (carbon dioxide) in humans; cautions those administering oxygen-carbon dioxide therapy at $50,000 \mathrm{ppm}$ v/v carbon dioxide for longer than 30 min to look for signs of intoxication; further recommends careful observation from the start for concentrations of $100,000 \mathrm{ppm} \mathrm{v/v;} \mathrm{notes} \mathrm{that} \mathrm{carbon} \mathrm{dioxide}$ toxicity must be kept in mind after 10-15 min [by inference, the AMA cautions must have been predicated on incidents in which the anesthetic and toxic affects suggested were observed - see RDB72A5 for related information]

D. J. Cullen and E. L. Eger, Cardiovascular Effects of Carbon Dioxide in Man, Anesthesiology, 41:345-349, 1974 (5 pages, RDB5790)

$R-744$, health effects, toxicity

R. D. Dripps and J. H. Comroe, Jr. (University of Pennsylvania and the Hospital of the University of Pennsylvania), The Respiratory and Circulatory Response of Normal Man to Inhalation of 7.6 and 10.4 Per Cent $\mathrm{CO}_{2}$ with a Comparison to the Maximal Ventilation Produced by Severe Muscular Exercise, Inhalation of $\mathrm{CO}_{2}$, and Maximal Voluntary Hyperventilation, American Journal of Physiology, 149:43-51, 1947 (9 pages with 1 figure and 4 tables, RDBA658)

toxicity of R-744 (carbon dioxide) in humans: 1 of 42 subjects who breathed $76,000 \mathrm{ppm} v / \mathrm{v}$ for an average of $7.4 \mathrm{~min}$ lost consciousness while 9 of the subjects indicated dizziness; 3 of 31 subjects who breathed $104,000 \mathrm{ppm} \mathrm{v} / \mathrm{v}$ for an average of $3.8 \mathrm{~min}$ lost consciousness while 7 indicated faintness and 13 indicated dizziness leach subject may have reported more than one symptom]

F. Flury and F. Zernik, Schädliche gase, dämpfe, nebel, rauch- und staubarten [Dangerous
Gases, Fumes, Mists, Smokes, and Dusts], Springer-Verlag (then Verlag von Julius Springer), Berlin, Germany, 1931 (in German, RDB72A6)

exposure at $70,000-100,000 \mathrm{ppm} v / \mathrm{v}$ R-744 (carbon dioxide) produces unconsciousness in humans - as reported in RDB5340

W. J. Friedlander and T. Hill, EEG Changes During Administration of Carbon Dioxide, Diseases of the Nervous System, 15:71-75, 1954 (5 pages, RDBA663)

inhalation tests in humans exposed to 300,000 $\mathrm{ppm}$ v/v R-744 (carbon dioxide) in oxygen via a face mask for 50-52 sec; average patient lost consciousness within $24-28 \mathrm{sec}$, but regained it in 110 seconds after exposures; toxicity - as reported in RDB5787; subjects also had abnormal electroencephalograms (EEGs) suggesting cardiac irritability - as reported in RDB5787

P. M. Gramenitskii et al., Long Term Exposure of Rats to High Concentration of Carbon Dioxide, Biull. Eksp. Biol. Med., 86(9):285-287, 1978 (3 pages, RDB5791)

R-744, health effects, toxicity

E. Griez and M. A. Van den Hout, Carbon Dioxide and Anxiety: Cardiovascular Effects of a Single Inhalation, Journal of Behavioral Therapy and Experimental Psychiatry, 14(4):297-304, 1983 (8 pages, RDB8419)

toxicity of R-744 (carbon dioxide): effects of acute exposures on the cardiovascular system; notes that inhalation of carbon dioxide produces sensations usually associated with anxiety; investigation concluded that anxiety-like responses are largely sympathomimetic

J. Haldane and J. L. Smith, The Physiological Effects of Air Vitiated by Respiration, Journal of Pathology and Bacteriology, 1:168-186, 1893 (19 pages with 6 figures and 7 tables, RDBA659)

toxicity of R-744 (carbon dioxide) in humans: reports an observation from 1892 that breathing $186,000 \mathrm{ppm} \mathrm{v} / \mathrm{v}$ for $94-110$ seconds caused "confusion of the mind" in two subjects - as reported in RDB72A5

A. Hendrik and F. Muller, Hyperglycemic Effect of Acute Carbon Dioxide Intoxication, Zeitschrift für die gesamte Innre Medizin, Germany, 28(7):209-211, 1973 (3 pages, RDB5792)

$\mathrm{R}-744$, health effects, toxicity 
F. Herles et al., Influence of Acute Respiratory Acidosis, Induced by Carbon Dioxide Inhalation, on Pulmonary Circulation, Vnitr. Lek., 13(7):692-699, 1967 (8 pages, RDB5793)

$R-744$, health effects, toxicity

D. Hunter, The Diseases of Occupations (fifth edition), Hodder and Stoughton, London, UK, 618 ff, 1975 (RDB72A7)

exposure to $100,000 \mathrm{ppm}$ v/v R-744 (carbon dioxide) for only a few minutes can cause loss of consciousness in humans as reported in RDB5340

I. Juzwiak and K. Brodziak-Krzesiek, Clinical Studies on Persons Subjected to the Effect of Carbon Dioxide in Comparison to the Environmental Studies in Cellulose and Plastic Industry, Postepy Hig. Med. Dosw., 22(2):353-356, 1968 (4 pages, RDB5794)

toxicity of R-744 (carbon dioxide)

B. G. King (Medical Service, Civil Aeronautics Administration), High Concentration-Short Time Exposures and Toxicity, presented to the Greater New York Safety Council, 1 April 1949; republished in the Journal of Industrial Hygiene and Occupational Medicine, 31(6):365-375, November 1949 (11 pages with 3 figures and 3 tables, RDBA660)

examines the toxicity dependence on time and concentration for R-10 (carbon tetrachloride), R-40B1 (methyl bromide), and R-744 (carbon dioxide) in humans

S. Kwiatowski et al., Permanent Sequelae of Acute Carbon Dioxide Poisoning, Pol. Tyg Lek., 36(31):1165-1168, 1981 (4 pages, RDB5795)

toxicity of R-744 (carbon dioxide)

A. A. LaVerne et al., Occupational, Accidental, Explorational Carbon Dioxide Inhalation Poisonings and Prevention, Behavioral Neuropsychiatry, 4(12):33-48, 1973 (16 pages, RDB5796)

toxicity of R-744 (carbon dioxide)

C. D. Leake, A. E. Guedal, and M. E. Botsford, The Stimulating Effect of Carbon Dioxide Inhalations in Dementia Praecox Catatonia, California West. Med., 31:20-23, 1929 (4 pages, RDBA662)

use of R-744 (carbon dioxide) as an anesthetic for animal surgery; toxicity - as reported in RDB5787
F. M. MacDonald and E. Simonson, Human Electrocardiogram During and After Inhalation of Thirty Percent Carbon Dioxide, Journal of Applied Physiology, 6:304-310, 1953 (7 pages, RDBA940)

toxicity of R-744 (carbon dioxide); indicates that 17 male volunteers exposed to $300,000 \mathrm{ppm}$ $V / v$ carbon dioxide for $38 \mathrm{sec}$ experienced narcosis and electrocardiogram (ECG) abnormalities in a broader tests - as reported in RDB5787

H. Mitsuda et al., Effects of Carbon Dioxide on Serum Biochemical Patterns and on Histopathological Changes of Organ in Rats, Journal of Nutritional Science and Vitaminology, 28(2):105115, 1982 (11 pages, RDB5797)

R-744, health effects, toxicity based on studies of male rats anesthetized with a 40/60 mixture of carbon dioxide and oxygen for $3 \mathrm{hr}$

R. R. Montgomery, untitled toxicity review for carbon dioxide, Haskell Laboratory for Toxicology and Industrial Medicine, E. I. duPont de Nemours and Company, Incorporated, Newark, DE, 9 August 1983 (RDB6453)

R-744, summary of published and unpublished toxicity literature

D. P. Mukherjee and S. P. Singh, Effect of Increased Carbon Dioxide in Inspired Air on the Morphology of Spermatozoa and Fertility of Mice, Journal of Reproduction and Fertility, 13:165-167, 1967 (3 pages, RDBA938)

toxicity of R-744 (carbon dioxide); indicates that 10 male mice exposed to $350,000 \mathrm{ppm} v / \mathrm{v}$ for 6 $\mathrm{hr}$ experienced changes in their sperm development resulting in smaller litters - as reported in RDB5787

W. C. Petty and T. S. Sulkowski, Narcosis in the Rat - II. Effects on the ECG, Aerospace Medicine, 42:553-558, 1971 (6 pages, RDBA937)

toxicity of R-744 (carbon dioxide); indicates that rats exposed to concentrations of carbon dioxide exceeding $400,000 \mathrm{ppm} \mathrm{v} / \mathrm{v}$ for $20 \mathrm{~min}$ experienced arrhythmias 10-55 sec after sudden withdrawal — as reported in RDB5787

K. E. Schaefer, Studies of Carbon Dioxide Toxicity, report 181, Submarine Medical Research Laboratory, Bureau of Medicine and Surgery, U.S. Department of the Navy, New London, CT, 10:156-189, 1951 (34 pages, RDB72A8)

submarine personnel continuously exposed to 30,000 ppm R-744 were only slightly affected 
provided the oxygen content of the air was maintained at normal concentrations as reported in RDB5340

K. E. Schaefer, The Effects of $\mathrm{CO}_{2}$ and Electrolyte Shifts on the Central Nervous System, Selective Vulnerability of the Brain in Hypoxemia, edited by J. P. Schade and W. H. McMehemy, Blackwell Scientific Publications, Oxford, UK, 101123, 1963 (23 pages, RDBA939)

toxicity of R-744 (carbon dioxide); indicates that 8 rhesus monkeys exposed to $300,000 \mathrm{ppm} v / \mathrm{v}$ for 30 min showed increases in cortical and hypothalamic activities as well as increased tolerance for carbon dioxide after exposures; reports a range of central nervous system (CNS) effects in 42 human volunteers exposed to $75,000 \mathrm{ppm} \mathrm{v} / \mathrm{v}$ carbon dioxide for 15 minutes including restlessness for ten subjects, loss of balance in seven, loss of limb control for four, and mental disorientation for two; indicates that exposure to $33,000 \mathrm{v} / \mathrm{v}$ and higher depressed the autonomic nervous system - as reported in RDB5787

P. H. Sechzer, L. D. Egbert, H. W. Linde, D. Y. Cooper, R. D. Dripps, and H. L. Price, Effect of $\mathrm{CO}_{2}$ Inhalation on Arterial Pressure, ECG and Plasma Catecholamines and 17-OH Corticosteroids in Normal Man, Journal of Applied Physiology, 13:454-458, 1960 (5 pages, RDBA941)

toxicity of R-744 (carbon dioxide); indicates that 12 human male volunteers exposed to concentrations of carbon dioxide of 70,000-140,000 $\mathrm{ppm} \mathrm{v} / \mathrm{v}$ for 10-20 min experienced physiological effects, among them loss of consciousness at concentrations above $100,000 \mathrm{ppm} \mathrm{v/v}$ and headaches and sweating above $70,000 \mathrm{ppm}$ $\mathrm{v} / \mathrm{v}$ - as reported in RDB5787

D. Sevel and A. Freedman, Cerebro-Retinal Degeneration Due to Carbon Dioxide Poisoning, British Journal of Opthalmology, 51(7):475-482, 1967 (8 pages, RDB5798)

toxicity of R-744 (carbon dioxide)

J. M. Stinson and J. L. Mattsson, Cardiac Depression in the Detection of the High Environmental $\mathrm{CO}_{2}$ : A Comparative Study in Rhesus Monkeys and Chimpanzees, Aerospace Medicine, 42(1):78-80, 1971 (3 pages, RDB5815)

toxicity of R-744 (carbon dioxide): indicates that three chimpanzees exposed to concentrations up to $510,000 \mathrm{ppm}$ v/v survived if the con- centration was increased and decreased slowly - as reported in RDB5787

J. M. Stinson and J. L. Mattsson, Tolerance of Rhesus Monkeys to Graded Increase in Environmental $\mathrm{CO}_{2}$ : Serial Changes in Heart Rate and Cardiac Rhythm, Aerospace Medicine, 41(4):415-418, 1970 (4 pages, RDB5816)

toxicity of R-744 (carbon dioxide)

W. Storm and C. L. Giannetta, Effects of Hypercapnia and Bedrest on Psychomotor Performance, Aerospace Medicine, 45:431-433, 1974 (3 pages, RDBA943)

toxicity of R-744 (carbon dioxide); describes tests psychomotor impairment in six healthy male volunteers exposed to $40,000 \mathrm{ppm} v / \mathrm{v}$ for 2 wk; notes that the test series measured complex tracking performance, eye-hand coordination, and problem-solving ability; concludes that performance was not impaired - as reported in RDB5787

C. J. S. Thompson, Henry Hill Hickman - A Forgotten Pioneer of Anaesthesia, British Medical Journal, 1:843-845, 1912 (3 pages, RDBA661)

use of R-744 (carbon dioxide) as an anesthetic for animal surgery; toxicity - as reported in RDB5787

N. L. Vandemark, B. D. Schanbacher, and W. R. Gomes, Alterations in Testes of Rats Exposed to Elevated Atmospheric Carbon Dioxide, Journal of Reproduction and Fertility, 28(3):457-459, 1972 ( 3 pages, RDB5826)

toxicity of R-744 (carbon dioxide); indicates that 8 rhesus monkeys exposed to $300,000 \mathrm{ppm} \mathrm{v} / \mathrm{v}$ for $30 \mathrm{~min}$ showed increases in cortical and hypothalamic activities as well as increased tolerance for carbon dioxide after exposures; reports a range of central nervous system (CNS) effects in 42 human volunteers exposed to $75,000 \mathrm{ppm} v / \mathrm{v}$ carbon dioxide for 15 minutes including restlessness for ten subjects, loss of balance in seven, loss of limb control for four, and mental disorientation for two - as reported in RDB5787

E. B. Weybrew, An Exploratory Study of the Psychological Effects of Intermittent Exposure to Elevated Carbon Dioxide Levels, report 647. Submarine Medical Research Laboratory, Bureau of Medicine and Surgery, U.S. Navy, Groton, CT, 1970 (9 pages, RDBA942)

describes a test of human exposure to R-744 (carbon dioxide) to investigate its effects of on 
psychological function; indicates that a 24-yrold male was exposed to a concentration that increased from 300 to $30,000 \mathrm{ppm} \mathrm{v} / \mathrm{v}$ during 15 -hr periods for 6 days in a $30 \mathrm{~m}^{3}(1000 \mathrm{cf})$ recompression chamber; summarizes tests of psychologic efficiency including addition of single-digit numbers, cancellation of letters, response analysis testing (RATER), and selection of adjectives from a list to describe mood and interests; indicates that the tests did not show any remarkable changes related to the carbon dioxide exposure; concludes that the experiment produced emotional changes in the subject based on the adjective-choice test, but attributes these changes to the confinement; toxicity - as reported in RDB5787

H. I. Williams, Carbon Dioxide Poisoning - Report of Eight Cases, with Two Deaths, British Medical Journal, 2:1012-1014, 1958 (3 pages, RDB6281)

toxicity of R-744 (carbon dioxide)

J. Winter, Arbeitsschutz [Occupational Safety], 104-105, 1937 (RDB6282)

toxicity of R-744 (carbon dioxide); fatalities

Criteria for a Recommended Standard - Occupational Exposure to Carbon Dioxide, publication 76-194, National Institute of Occupational Safety and Health (NIOSH), U.S. Department of Health and Human Services, Cincinnati, $\mathrm{OH}$, August 1976 (184 pages with 1 figure and 11 tables, available from NTIS as publication PB266597, RDB5787)

review of toxicity data for R-744 (carbon dioxide) to recommend an occupational exposure limit; recommends that occupational exposures not exceed $10,000 \mathrm{ppm} \mathrm{v} / \mathrm{v}$ as a time-weighted average (TWA) for up to a 10-hr work shift in a 40-hr work week with a ceiling concentration of $30,000 \mathrm{ppm} \mathrm{v/v}$; notes that carbon dioxide is a normally present in air at approximately 300 $\mathrm{ppm} v / \mathrm{v}$, is widely used in direct-contact food refrigeration and preservation, is common in beverages for carbonation, and was used as an anesthetic for animal surgery in the early 1800 s

Hygienic Guide Series - Carbon Dioxide, AlHA Journal, 25:519-521, 1964 (3 pages, RDB5783)

toxicity of R-744 (carbon dioxide)

Occupational Health Guideline for Carbon Dioxide; National Institute of Occupational Safety and Health (NIOSH), U.S. Department of Health and Human Services, Cincinnati, $\mathrm{OH}$; Occupa- tional Safety and Health Administration (OSHA), U.S. Department of Labor, Washington, DC; September 1978 (available from GPO as part of publication PB83-154609; RDB6280)

R-744, toxicity; identification, exposure limits, health hazard information, chemical and physical properties, monitoring and measuring procedures, respirators and respiratory protection, personal protective equipment, common operations and controls, emergency first aid procedures, leak procedures, references

\section{R-744A}

B. D. Greenberg, R. Moore, R. Letz, and E. L. Baker, Computerized Assessment of Human Neurotoxicity: Sensitivity to Nitrous Oxide Exposure, Clinical Pharmacology and Therapeutics, 38:656-660, 1985 (6 pages, RDB5738)

\section{$\mathrm{R}-744 \mathrm{a}$}

\section{R-764}

H. Salem and D. M. Aviado (University of Pennsylvania School of Medicine), Inhalation of Sulfur Dioxide, Archives of Environmental Health, 2:656662, 1961 (RDB6939)

R-764, health effects, toxicity

Emergency Response Planning Guidelines Sulfur Dioxide, American Industrial Hygiene Association (AlHA), Fairfax, VA, 1990 (RDB4B78)

R-764, ERPG-1, ERPG-2, ERPG-3, toxicology data, toxicity, safety classification

\section{R-7146}

G. Kimmerle, Vergleichende Untersuchungen der inhalations Toxicität von Schwefelselenund Tellurhexafluorid, Archiv. für Toxik., 18:140144, 1960 (5 pages, RDB7367)

$\mathrm{R}-7146$ and others, toxicity

D. Lester and L. A. Greenberg (Yale University), The Toxicity of Sulfur Hexafluoride, Archives of Industrial Hygiene and Occupational Medicine, 2:348-349, 1950 (2 pages, RDB5A39)

R-7146, toxicity

$H$. Specht and H. F. Brubach, Inhalation of Sulfur Hexafluoride, Science, 114:662-663, 1951 (2 pages, RDB7368)

R-7146, toxicity 


\section{R-1110}

E. Guberan and J. Fernandez, Control of Exposure to Tetrachloroethylene by Measuring Alveolar Concentrations: Theoretical Approach Using a Mathematical Model, British Journal of Industrial Medicine, 31:159-167, 1974 (9 pages, RDB6495)

R-1110, health effects, toxicity

V. K. Rowe, D D. McCollister, H. C. Spencer, E. M. Adams, and D. D. Irish, Vapor Toxicity of Tetrachloroethylene for Laboratory Animals and Human Subjects, Archives of Industrial Hygiene and Occupational Medicine, 5:566-579, 1952 (14 pages, RDB6507)

$\mathrm{R}-1110$, health effects, toxicity, $\mathrm{LC}_{50}$

\section{R-1113}

Emergency Response Planning Guidelines Chlorotrifluoroethylene, American Industrial Hygiene Association (AlHA), Fairfax, VA, 1992 (RDB4B81)

R-1113, 1-chloro-1,2,2-trifluoroethene, ERPG1, ERPG-2, ERPG-3, toxicology data, toxicity, safety classification

Workplace Environmental Exposure Level Guide - Chlorotrifluoroethylene, American Industrial Hygiene Association (AlHA), Fairfax, VA, 1986 (RDB4B85)

R-1113, 1-chloro-1,2,2-trifluoroethene, WEEL, toxicology data, toxicity, safety classification

\section{R-1114}

R. Lafaux, Practical Toxicology of Plastics, CRC Press, Incorporated, Cleveland, OH, 205 ff, 1968 (RDB8124)

R-1114 toxicity: small amounts of fluorochemicals R-C318, R-1216, and R-1318 (perfluoroisobutylene, PFIB) are formed by thermal decomposition of R-1114 above $400{ }^{\circ} \mathrm{C}(752$ ${ }^{\circ} \mathrm{F}$ ) when air is present

Emergency Response Planning Guidelines Tetrafluoroethylene, American Industrial Hygiene Association (AlHA), Fairfax, VA, 1991 (RDB4B79)

R-1114, tetrafluoroethene, ERPG-1, ERPG-2, ERPG-3, toxicology data, toxicity, safety classification

\section{R-1120}

E. M. Adams, H. C. Spencer, V. K. Rowe, D D. McCollister, and D. D. Irish, Vapor Toxicity of Trichloroethylene Determined by Experiments on Laboratory Animals, Archives of Industrial Hygiene and Occupational Medicine, 4:469-481, 1951 (13 pages, RDB6508)

$R-1120$, health effects, toxicity, $L_{50}$

C. R. Elcombe, Species Differences in Carcinogenicity and Peroxisome Proliferation Due to Trichloroethylene: A Biochemical Human Hazard Assessment. Receptors and Other Targets for Toxic Substances, Archives of Toxicology Supplement, 8:6-17, 1985 (12 pages, RDB65C4)

$\mathrm{R}-1120$, health effects, toxicity

C. Maltoni, G. Lefermine, and G. Cotti, Experimental Research on Trichloroethylene Carcinogenesis, Archives of Research on Industrial Carcinogenesis, edited by C. Maltoni and M. A. Mohlman, Princeton Scientific Publishing Company, Princeton, NJ, 5:1-393, 1986 (393 pages, RDB$65 \mathrm{CO})$

$\mathrm{R}-1120$, health effects, toxicity

J. H. Mennear, Toxicology and Carcinogenensis Studies of Trichloroethylene (CAS No. 79-01-6) in Four Strains of Rats (ACl, August, Marshall, Osborne-Mendel) (Gavage Studies), report TR273 (also identified as NIH publication 88-2529) for the National Toxicology Program (NTP), U.S. Department of Health and Human Services, research Triangle Park, NC, April 1988 (available from NTIS as publication PB88-218896/AS, RDB65D5)

R-1120, carcinogenicity, health effects, toxicity

R. M. Waters, O. S. Orth, and N. A. Gillespie, Trichloroethylene Anesthesia and Cardiac Rhythym, Anesthesiology, 4:1 ff, 1943 (RDB65A1)

$\mathrm{R}-1120$, cardiac sensitization, health effects, toxicity

J. F. White and G. P. Carlson, Epinephrine-Induced Cardiac Arrhythmias in Rabbits Exposed to Trichloroethylene: Roie of Trichloroethylene Metabolites, Toxicology and Applied Pharmacology (TAP), 60:458-465, 1981 (8 pages, RDB6B49)

$\mathrm{R}-1120$, cardiac sensitization, health effects, toxicity: chemically-induced arrhythmias did not occur when epinephrine was administered 15-30 minutes after exposure to a sensitizing level of chemical 


\section{R-1140}

Occupational Safety and Health Guideline for Vinyl Chloride - Potential Human Carcinogen, National Institute of Occupational Safety and Health (NIOSH), U.S. Department of Health and Human Services, Cincinnati, OH, 1988 (available from GPO as document 1993-750-004/80503, RDB4A73)

R-1140, toxicity; properties, exposure limits, health hazard information, recommended medical practices, monitoring and measurement procedures, personal protective equipment, sanitation, common operations and controls, emergency first aid procedures, spills and leaks, waste removal and disposal, respiratory protection, references

\section{R-1150 (ETHYLENE)}

A. B. Luckhardt and J. B. Carter (University of Chicago), Journal of the American Medical Association (JAMA), 80:765-770, 1923 (6 pages, RDB7740)

toxicity tests of the anesthetic effects of R-1150

\section{R-1216}

Acute Inhalation Toxicity, report EPAOTS878220382 TSCATS 019448, Haskell Laboratory for Toxicology and Industrial Medicine, E. I. duPont de Nemours and Company, Incorporated, Newark, DE, circa 1987 (RDB8431)

acute inhalation toxicity of R-1216, hexafluoropropene oxide (HFPO), and others

Hexafluoropropylene, Evaluation in the in Vitro Assay for Chromosome Aberrations in Chinese Hamster Ovary (CHO) Cells, report EPANOTS09860510, Haskell Laboratory for Toxicology and Industrial Medicine, E. I. duPont de Nemours and Company, Incorporated, Newark, DE, 1986 (RDB8126)

toxicity tests of R-1216 mutagenicity in both the presence and absence of metabolic activation: significant increases were observed in the number of aberrations, percent abnormal cells, and percentage of cells with more than one aberration compared to controls in both activated and nonactivated cultures at $2900 \mathrm{ppm}, 5500 \mathrm{ppm}$, and above; positive dose-related trends were observed

\section{R-1270 (PROPYLENE)}

A. P. Altshuller et al., Preprints, American Chemical Society (ACS), Washington, DC, 5(1):183-184, 1965 (2 pages, RDB6A60)

$\mathrm{R}-1270$, health effects, toxicity: irradiated (to induce decomposition) mixture of $1 \mathrm{ppm} R$ 1270 and nitric oxide produced slight eye irritation, increased to moderate with 2-3 ppm R1270 as reported in RDB6A59

B. Beije (Nordic Expert Group), Propene, report 117, Arbete och Hlsa, 1995 (36 pages, RDB8427)

toxicity of R-1270: metabolizes to propylene oxide, which binds to hemoglobin and DNA; chronic inhalation of R-1270 on a long-term causes non-neoplastic toxic changes in the nasal cavity of rats, but not of mice; increases the incidence of chronic focal renal inflammation in male mice and of uterine endometrial stromal polyps and, to a lesser extent, hemangiosarcoma and combined hemangioma in female mice; data on the potential health hazard to humans are limited; because the metabolite, propylene oxide, is carcinogenic to experimental animals, it is not possible at the present time to rule out $R-1270$ as a human carcinogen

A. M. Caine and C. Reynolds (Tulane University of Louisiana), Electrocardiographic Studies of the Action of Propylene and Some Other Anesthetic Gases, Proceedings of the Society of Experimental Biology and Medicine, New York, 23:488, 1925-1926 (1 page, RDB7739)

$\mathrm{R}-1270$, and others, health effects, toxicity: examination of propylene as a general anesthetic agent

A. Ciliberti, C. Maltoni, and G. Perino (Institute of Oncology F. Addarii, Italy), Long-Term Carcinogenicity Bioassays on Propylene Administered by Inhalation to Sprague-Dawley Rats and Swiss Mice, Annals of the New York Academy of Science, 534:235-245, 1988 (11 pages, RDB8425)

toxicity of R-1270: tests with Sprague-Dawley rats and Swiss mice by inhalation of 0,200 , 1000 or $5,000 \mathrm{ppm} v / \mathrm{v}$ R-1270 in air for $7 \mathrm{hr} / \mathrm{d}$, $5 \mathrm{~d} / \mathrm{wk}$; groups of 120 male and 120 female rats (high-dose and controls) or 100 male and 100 female rats (mid- and low-dose) were treated for 104 weeks, and groups of 100 male and 100 female mice (each dose and controls) for 78 weeks; animals were kept under observation until spontaneous death; concludes that R1270 was not carcinogenic as tested 
R. B. Conolly and T. G. Osimitz, Mixed Function Oxidase System Inducers and Propylene Hepatoxicity, The Toxicologist, 1:406 ff, 1981 (RDB7741)

acute toxicity tests of R-1270 in rats with concentrations up to $65,000 \mathrm{ppm}$ for $4 \mathrm{hr}$ produced no observable toxic effects, but only the liver was examined - as reported in RDB6A72

E. F. Darley, J. T. Middleton, and M. J. Garber (University of California at Riverside), Plant Damage and Eye Irritation from OzoneHydrocarbon Reactions, Journal of Agriculture and Food Chemistry, 8(6):483-485, 1960 (3 pages with 1 table, RDB6A63)

toxicity tests of R-1270 and others used as fumigants: no appreciable plant damage or eye irritation from propylene-ozone reaction

B. M. Davidson (University of Aberdeen), Study of Intoxication IV. The Action of Propylene, Journal of Pharmacology and Experimental Therapeutics (JPET), 26:33-36, 1925 (4 pages with 1 figure and 1 table, RDB6A64)

toxicity tests of R-1270 in humans by acute inhalation exposures: inhalation of $64,000 \mathrm{ppm}$ $v / v$ with $26 \%$ oxygen for $21 / 4$ minutes yields mild intoxication, parasthesia, and inability to concentrate; the same symptoms are accentuated at $128,000 \mathrm{ppm}$ in 1 minute; unconsciousness results at 240,000 ppm in 3 minutes; concludes that R-1270 is more potent as an anesthetic than acetylene or R-1150 (ethylene) and appears more promising for intended use as an anesthetic

K. Golka, H. Peter, B. Denk, and J. G. Filser (Universität Dortmund, Germany), Pharmacokinetics of Propylene and its Reactive Metabolite Propylene Oxide in Sprague-Dawley Rats, Archives of Toxicology, 13(supplement):240-242, 1989 (3 pages, RDB8426)

$\mathrm{R}-1270$, health effects, metabolism, toxicity

J. T. Halsey, C. Reynolds, and W. A. Prout (Tulane University of Louisiana), A Study of the Narcotic Action of Propylene, Journal of Pharmacology and Experimental Therapeutics (JPET), 26:479-490, 1926 (12 pages with 3 tables, RDB$6 \mathrm{A65)}$

toxicity tests of anesthetic and central nervous system (CNS) effects in cats, dogs, mice, and humans: minimal anesthetic dose (MAD) or minimal narcotic dose (MND) is defined as loss of righting reflex; for R-1150, MAD mouse = $850,000-900,000 \mathrm{ppm} v / \mathrm{v}$ and the 1-hr approxi- mate lethal concentration (ALC) is inexplicably indicated as $1,400,000-1,460,000 \mathrm{ppm} \mathrm{v/v}$; for a commercial (E. R. Squibb and Sons) preparation of R-1270, indicated as containing an unidentified impurity, MAD $=400,000 \mathrm{ppm} \mathrm{v} / \mathrm{v}$ and $1-\mathrm{hr}$ ALC $=600,000-650,000 \mathrm{ppm} \mathrm{v} / \mathrm{v}$ in mice; anesthetic LOEL $=400,000-500,000 \mathrm{ppm} \mathrm{v/v}$ and 1 -hr ALC $=750,000-800,000$ ppm in dogs; effects are similar in cats and dogs; anesthetic effect to the point of unconsciousness in humans at $350,000-400,000 \mathrm{ppm}$

J. T. Halsey, C. Reynolds, and W. A. Prout (Tulane University of Louisiana), Anesthetic Properties of Propylene, Anesthesia and Analgesia, 4:195-198, 1925 (4 pages, RDB6A78)

$R-1270$, anesthetic and central nervous system (CNS) effects, health effects, toxicity

J. T. Halsey, C. Reynolds, and H. B. Cook (Tulane University of Louisiana), Some Observations on a New Anesthetic Gas, New Orleans Medical and Surgical Journal, 77:29-30, 1924-1925 (2 pages, RDB6A79)

$\mathrm{R}-1270$ and others, anesthetic and central nervous system (CNS) effects, health effects, toxicity

T. J. Hughes, Environ. Mutagen., 6:411-412, 1984 (2 pages, RDB6A66)

R-1270, mutagenic potential, health effects, toxicity: not mutagenic in Salmonella typhimurium as reported in RDB6A59

M. H. Kahn and L. K. Riggs, Electrocardiographic Studies of the Effects of Propylene as a General Anesthetic in Man, Annals of Internal Medicine, 5:651-658, 1931 (2 pages with 1 figure and 9 tables, RDB6A67)

$\mathrm{R}-1270$, health effects, toxicity: experimental human exposures at $400,000-750,000 \mathrm{ppm}$ v/v caused reddening of the eyelids, flushing of the face, lacrimation, coughing, and sometimes flexing of the legs in a few minutes when used as a general anesthetic; progress of the anesthesia was otherwise gradual and complete in an average of ten breaths

M. A. Lee, R. C. Graham, R. R. Montgomery, G. T. Hall, and L. J. Ver Nooy, untitled toxicity review for propylene, Haskell Laboratory for Toxicology and Industrial Medicine, E. I. duPont de Nemours and Company, Incorporated, Newark, DE, 5 January August 1993 (RDB6A59)

$R-1270$, summary of published and unpublished toxicity literature 
D. B. McGregor et al., Environmental and Molecular Mutagenicity, 17(2):122-129, 1991 (8 pages, RDB6A69)

$R-1270$, health effects, mutagenicity, toxicity

K. H. Meyer and H. Hopff, Theorie der Narkose durch Inhalationsanästhetika. II. Mitteilung. Narkose durch indifferente Gase under Druck [Theory of Narcosis by Inhaled Anesthetics. II. Announcement. Narcosis by Pressurized Inert Gases] Zeitschrift für Physiologische Chemie [Journal of Physiological Chemistry], Germany, $126: 280-298,1923$ (19 pages with 1 figure and 8 tables, in German, RDB6A70)

toxicity of R-20 (chloroform), R-40 (methyl chloride), R-50 (methane), R-160B1 (ethyl bromide), R-E170 (dimethyl ether), R-601 (pentane), R-610 (diethyl ether), R-728 (nitrogen), R-744A (nitrous oxide), R-1130 (dichloroethylene, specific isomer not indicated), R-1150 (ethylene), R-1270, acetylene, benzene, carbon disulphide, ether, ethylene oxide, and others

T. G. Osimitz and R. B. Conolly, Mixed-Function Oxidase System Induction and Propylene Hepatotoxicity, Journal of Toxicology and Environmental Health, 15(1):39-49, 1985 (11 pages, RDB8424)

toxicity tests of $R-1270$ in male rats: concludes that R-1270 is hepatoxic to rats based on $4-\mathrm{hr}$ inhalation exposures at $50,000 \mathrm{ppm} \mathrm{v} / \mathrm{v}$; liver weight to body weight ratios increased and serum enzyme activities were elevated in rats pretreated with polychlorinated biphenyls (PCB); concludes that PCB pretreatment is a prerequisite for $\mathrm{R}-1270$ hepatotoxicity; cytochrome P-450-dependent bioactivation of R1270 is associated with this hepatotoxicity, but further studies are needed to characterize the mechanism of the PCB-propene interaction

K. Pernov, Suvrem Med, Bulgaria, 30(1):27-31, 1979 (5 pages, in Bulgarian, RDB6A71)

R-1270, health effects, toxicity: irritation as a component of smog

J. A. Quest, J. E. Tomaszewski, J. K. Haseman, G. A. Boorman, J. F. Douglas (National Institute of Environmental Health Sciences), and W. J. Clarke (Battelle Pacific Northwest Laboratories), TwoYear Inhalation Toxicity Study of Propylene in F344/N Rats and B6C3F 1 Mice, Toxicology and Applied Pharmacology (TAP), 76(2):288-295, 1984 (8 pages with 2 tables, Rdb6A72)

chronic inhalation toxicity tests of R-1270 in groups of 50 male and 50 female Charles River
F344/N rats and 50 male and 50 female Charles River $\mathrm{B}_{6 \mathrm{C}} 3 \mathrm{~F}_{1}$ mice by whole-body exposures to 0 (control), 5,000 , and $10,000(4,985$ and 9,891 actual for rats and 4,999 and 9,957 actual for mice) ppm $v / v$ for $6 \mathrm{~h} / \mathrm{d}, 5 \mathrm{~d} / \mathrm{wk}$, for 103 wk: no compound-related clinical signs were observed in rats or mice, though the latter showed $6 \%$ reduced body weights during exposures to $10,000 \mathrm{ppm}$; survival among all groups was similar; increased incidences of nonneoplastic lesions in the nasal cavity; they consisted of epithelial hyperplasia in female rats exposed to $10,000 \mathrm{ppm}$, squamous metaplasia in female rats exposed to both treatment concentrations, and squamous metaplasia in male rats exposed to $5,000 \mathrm{ppm}$; inflammatory changes including an influx of lymphocytes, macrophages, and granulocytes into the submucosa and granulocytes into the lumen in male rats at both treatment concentrations; no signs of lesions were found in the mice; no treatment-related increases or decreases in tumor incidence was detected in either the rats or mice or rats; study concludes that chronic inhalation of R-1270 induces nasal cavity toxicity in rats but not mice, but that the chemical is not carcinogenic to either species as tested

L. K. Riggs (E. R. Squibb and Sons), Further Studies on the Physiologic Action of Propylene, Journal of the American Pharmaceutical Association, 16:635-639, 1925 (5 pages, RDB6A81)

R-1270 and others, anesthetic and central nervous system (CNS) effects, health effects, toxicity: anesthetic effect in white rats at 40,000 $\mathrm{ppm}$ v/v as reported in RDB6A59

L. K. Riggs and H. D. Goulden (E. R. Squibb and Sons), Studies of Physiologic Action of Propylene, Anesthesia and Analgesia, 4:299-307, 1925 (9 pages, RDB6A75)

$R-1270$, health effects, inhalation toxicity in humans as reported in RDB6A59 and RDB6A67

L. I. Sofina, Tr. Ufim. Nauch.-Issled. Inst. Gigiena i Professional'nye Zabolevaniya, 5:64-68, 1969 (5 pages in Russian, RDB6A76)

R-1270, health effects, chronic inhalation toxicity in rats

T. S. Sunargulov and E. T. Akhmetova, Vopr. Reakt. Org. Norme Patol. Mater. Nauchn. Konf., 118-120, 1974 (3 pages, RDB6A77)

R-1270, health effects, inhalation toxicity in humans 
Properties and Essential Information for Safe Handling and Use of Propylene Chemical Safety Data Sheet SD-59, Manufacturing Chemists Association (MCA), Washington, DC, 1974 (RDB6A68)

\section{$\mathrm{R}-1270$}

Propylene, IARC Monographs on the Evaluation of the Carcinogenic Risk of Chemicals to Humans, International Agency for Research on Cancer (IARC), 60:161-180, 1994 (20 pages, RDB8428)

toxicity of R-1270: data on occupational exposures are limited; no relevant human carcinogenicity data were found; R-1270 was tested by inhalation in two studies each in mice and rats, but two of them were inconclusive; a slight increase in the incidence of vascular tumors was observed in female mice in one study; no treatment-related increase in tumor incidence was observed in a rat study; a metabolism study in rats exposed to $50 \mathrm{ppm}$, approximately onesixth of the inhaled material was absorbed of which nearly half was later exhaled unchanged; the remainder was eliminated metabolically, through oxidation to propylene oxide; alkylation products of this metabolite were found in hemoglobin and in DNA from mice exposed to propylene by inhalation; although insufficient data are available to evaluate the genetic and related effects of propylene, the metabolite propylene oxide is genotoxic in a broad range of assays; concludes that R-1270 is not classifiable as to its carcinogenicity to humans (Group 3)

Propylene Toxicity Profile, BIBRA Toxicology International, British Industrial Biological Research Association (BIBRA), 1993 (5 pages, RDB8429)

$\mathrm{R}-1270$ vapor is not irritating to the skin, but may be an irritant to the eyes and mucous membranes; the liquified form causes skin and eye burns; inhalation induces rapid anesthesia and other central nervous system (CNS) effect5; nasal inflammation and mild kidney injury were observed in chronic inhalation studies in rats and mice, respectively; shorter exposures yielded liver effects in rodents; evidence of carcinogenicity was insufficient in rats or mice, but DNA binding was detected in mice; the results of mutagenicity assays were inconclusive

Toxicity Study Summary: Propylene, Phillips Petroleum Company, Bartlesville, OK, February 1990 (1 page with no figures or tables, RDB7630)

tests of the acute inhalation toxicity in 5 female and 5 male albino, Sprague-Dawley rats by 4 $\mathrm{hr}$, whole body exposures to $7,217 \mathrm{ppm} v / \mathrm{v}$ R-
1270 (propylene) in air: no deaths resulted, but animals were hyperactive for $5 \mathrm{~min}$ after exposure initiation; all animals showed a body weight increase after exposures, but females showed a slight weight decline on days 2-7 postexposure; no other gross signs or symptoms of intoxication are noted, and no gross pathological changes were found at necropsy; concludes that the 4-hr $L_{50}$ rat is $>7,217 \mathrm{ppm}$ $\mathrm{v} / \mathrm{v}$; respiratory tract irritancy studies in male mice by head-only exposures to $7,228 \mathrm{ppm} \mathrm{v} / \mathrm{v}$ $\mathrm{R}-1270$ in air for 1 min, repeated 10 minutes later, while monitored by a plethysmograph: half of the test animals showed decreases in respiratory rates up to $25 \%$; concludes that R1270 failed to produce upper airway irritancy in mice at $7,228 \mathrm{ppm} v / \mathrm{v}$ [implies that $R D_{50}$ exceeds $7,200 \mathrm{ppm}]$

National Toxicology Program (NTP), Toxicology and Carcinogenesis Studies of Propylene (CAS No. 115-07-1) in F344/N Rats and B6C3F 1 Mice (Inhalation Studies), report TR-272 (also identified as NIH publication 88-2528), U.S. Department of Health and Human Services (DHHS), Research Triangle Park, NC, November 1985 (146 pages with 11 figures and 16 tables available from NTIS as document PB86-145521/AS, also available from JMC as RDB7534)

$2-y r$ chronic inhalation toxicity tests of R-1270 in groups of 50 male and 50 female Charles River F344/N rats and 50 male and 50 female Charles River B6C3F, mice by whole-body exposures to 0 (control), 5,000 , or $10,000(4,985$ and 9,891 actual for rats and 4,999 and 9,957 actual for mice) ppm v/v for $6 \mathrm{~h} / \mathrm{d}, 5 \mathrm{~d} / \mathrm{wk}$, for 103 wk: report also summarizes 2 wk and 14wk pilot studies in groups of male and female rats exposed to concentrations of 0 (control), $625,1,250,2,500,5,000$, or $10,000 \mathrm{ppm} v / v$ for $6 \mathrm{hr} \mathrm{d}, 5 \mathrm{~d} / \mathrm{wk}$, for 2 or $14 \mathrm{wk}$; no compoundrelated clinical signs were observed in rats or mice in the 2-yr study, though the mice showed $6 \%$ reduced body weights during exposures to $10,000 \mathrm{ppm}$; survival among all groups was similar; increased incidences of nonneoplastic lesions in the nasal cavity; they consisted of epithelial hyperplasia in female rats exposed to 10,000 ppm, squamous metaplasia in female rats exposed to both treatment concentrations, and squamous metaplasia in male rats exposed to $5,000 \mathrm{ppm}$; inflammatory changes including an influx of lymphocytes, macrophages, and granulocytes into the submucosa and granulocytes into the lumen in male rats at both treatment concentrations; no signs of lesions 
were found in the mice; no treatment-related increases or decreases in tumor incidence was detected in either the rats or mice or rats; study concludes that chronic inhalation of R-1270 induces nasal cavity toxicity in rats but not mice, but that the chemical is not carcinogenic to either species as tested

\section{R-1316}

R. Truhaut, C. Boudene, J. M. Jouany, A. Bouant, Experimental Study of the Toxicity of a Fluoroalkene Derivative, Hexafluorodichlorobutene (HFCB), Fluoride, 5:4 ff, 1972 (RDB8181)

$R-1316$, health effects, toxicity

\section{HEXAFLUOROPROPENE OXIDE (HFPO)}

Acute Inhalation Exposure (Six-Hours) of a Dog To Hexafluoropropylene Epoxide (HFPO), Preliminary Report, report EPANOTS-878220383 TSCATS 019449, Haskell Laboratory for Toxicology and Industrial Medicine, E. I. duPont de Nemours and Company, incorporated, Newark, DE, circa 1987 (RDB8432)

test of the acute inhalation toxicity of hexafluoropropene oxide (HFPO) in a single male beagle dog exposed to $897 \mathrm{ppm}$ v/v (1000 ppm nominal) for $6 \mathrm{hr}$ : animal died within $24 \mathrm{hr}$ following exposure; no irritation was observed during the exposure, but convulsions were noted $10 \mathrm{~min}$ post-exposure; gross necropsy revealed severe pulmonary edema, dark red fluid in the pericardial sac, numerous subepicardial and subendocardial petechial and ecchymotic hemorrhages in the heart, scattered subserosal ecchymotic hemorrhages, and dark red kidneys

Acute Inhalation Studies On Hexafluoropropylene Epoxide (CAS 428-59-1), report EPANOTS878220381 TSCATS 019447, Haskell Laboratory for Toxicology and Industrial Medicine, E. I. duPont de Nemours and Company, Incorporated, Newark, DE, circa 1987 (RDB8430)

tests of the acute inhalation toxicity of hexafluoropropene oxide (HFPO) in groups of 2 male $\mathrm{ChR}-\mathrm{CH}$ albino rats by exposures to $500 \mathrm{ppm}$ $\mathrm{v} / \mathrm{v}$ for $4 \mathrm{hr}, 1000 \mathrm{ppm}$ for $4 \mathrm{hr}, 2,000 \mathrm{ppm}$ for 21/2-3 hr, or 4,000 ppm for $1 \mathrm{hr}$ : exposure was lethal to all animals in the 2,000 and $4000 \mathrm{ppm}$ groups; clinical signs were normal; gross necropsy revealed acute pulmonary edema and congestion in decedents, but no treatment-related effects in the survivors; concludes that the $4-\mathrm{hr}$ ALC rat $=2000 \mathrm{ppm}$ v/v

Subacute Inhalation Toxicity (CAS 428-59-1), report EPANOTS-878220386 TSCATS 019452, Haskell Laboratory for Toxicology and Industrial Medicine, E. I. duPont de Nemours and Company, Incorporated, Newark, DE, circa 1987 (RDB8433)

test of the subchronic inhalation toxicity of hexafluoropropene oxide (HFPO) in 4 male ChR$C D$ rats exposed to $400 \mathrm{ppm} v / \mathrm{v}$ nominal for 4 $\mathrm{hr} / \mathrm{d}$ for 10 exposures: there were no mortalities; clinical observations included deep respiration and inactivity; necropsy revealed no gross or microscopic effects 
Toxicity Data to Determine Refrigerant Concentration Limits

James M. Calm, Engineering Consultant 


\section{APPENDIX 5: BACKGROUND PAPER}

J. M. Calm (Engineering Consultant), The Toxicity of Refrigerants, Proceedings of the 1996 International Refrigeration Conference at Purdue (23-26 July 1996), edited by J. E. Braun and E. A. Groll, Purdue University, West Lafayette, IN, 157-162, July 1996; republished as report DOE/CE/23810-72E, AirConditioning and Refrigeration Technology Institute (ARTI), Arlington, VA, 1996 (RDB6832)

This paper presents toxicity data and exposure limits for refrigerants. The data address both acute (short-term, single exposure) and chronic (long-term, repeated exposure) effects, with emphasis on the former. The refrigerants covered include those in common use for the last decade, those used as components in alternatives, and selected candidates for future replacements. Specifically addressed are R-11, R-12, R-22, R-23, R-32, R-113, R-114, R-115, R-116, R-123, R-124, R-125, R-134a, R141b, R-142b, R-143a, R-152a, R-218, R-290, R-C318, R-600, R-600a, R-717, R-744, and R-1270. The paper also reviews the toxicity indicators used in both safety standards and building, mechanical, and fire codes. Data are tabulated for the $\mathrm{LC}_{50}$, cardiac sensitization NOEL and LOEL, anesthetic/CNS $E_{50}$, respiratory $\mathrm{RD}_{50}$, IDLH (both from the Standards Completion Program and from the 1994 revisions), PEL, UL group, and Standard 34 safety classifications. The paper outlines current classification methods for refrigerant safety and relates them to standard and code usage. 
Toxicity Data to Determine Refrigerant Concentration Limits

James M. Calm, Engineering Consultant 


\title{
THE TOXICITY OF REFRIGERANTS
}

\author{
James M. Calm, P.E. \\ Engineering Consultant \\ 10887 Woodleaf Lane \\ Great Falls, VA 22066-3003 USA
}

This paper presents toxicity data and exposure limits for refrigerants. The data address both acute (short-term, single exposure) and chronic (long-term, repeated exposure) effects, with emphasis on the former. The refrigerants covered include those in common use for the last decade, those used as components in alternatives, and selected candidates for future replacements. The paper also reviews the toxicity indicators used in both safety standards and building, mechanical, and fire codes. It then outlines current classification methods for refrigerant safety and relates them to standard and code usage.

\section{INTRODUCTION}

Most of the dominant refrigerants for the past fifty years have been or are being replaced, to protect the stratospheric ozone layer or as a precaution to address global warming. Much to the credit of the air-conditioning and refrigeration industry, both chemical and equipment manufacturers have resisted compromise to either safety or performance in developing replacements. None of the alternative refrigerants that have been commercialized are highly toxic or even toxic, as classified by federal regulations. ${ }^{1}$ Scrutiny of the new refrigerants shows them to be as safe or safer than those they replace. ${ }^{2}$ Still, safety concerns have surfaced as significant factors in regulations for the new refrigerants. These concerns do not arise from increased hazard levels, but from lack of familiarity and necessary information. The rapid phaseout schedule for chlorofluorocarbon refrigerants required introduction of new chemicals before complete data were available.

Most of the early refrigerants - before the 1930s - were flammable, toxic, or both. The advent of fluorochemicals ushered in a new era of safety, as illustrated by the dramatic demonstration by Thomas Midgley in April $1930 .^{3}$ In announcing the development of fluorochemicals to the American Chemical Society, he inhaled R-12 and blew out a candle with it. Although this dramatic performance suggested that the new refrigerant was neither toxic nor flammable, it would clearly violate current safety practices.

As subsequent testing established the low toxicity of the new refrigerants, recognition evolved that the primary safety risks were the pressure hazards inherent to any compressed gas, asphyxiation from possible displacement of air, and frostbite with skin contact at low temperatures. These concerns were, however, common to the volatile compounds used before fluorochemicals. As the level of safety improved, so did expectations. Rules evolved to also address acute exposure hazards under emergency conditions, for example potential decomposition in fires into carbonyl halides as well as hydrochloric and hydrofluoric acids. Likewise, safety provisions also addressed the potential for cardiac sensitization and the effects of chronic exposures for both technicians and building occupants. The resulting regulations restricted the use of refrigerants, set quantity limits in occupied areas, imposed isolation requirements for refrigerant-containing components and machinery rooms, and prescribed a range of detection, ventilation, pressure relief, emergency discharge, and other safety provisions.

More recent focus on the effects of refrigerants on the environment spawned two significant safety measures, namely system tightening and modification of service practices to reduce venting. While their motivation was environmental protection, to curtail avoidable emissions, the result also lowers the likelihood and concentrations of refrigerant exposures.

\section{CODE ACCEPTANCE OF ALTERNATIVE REFRIGERANTS}

International treaties, most noticeably the Montreal Protocol and Framework Convention on Climate Change, have focused on the global issues of environmental protection, information sharing, and assistance to developing countries. These treaties, and revisions to them, have fostered scientific assessments and set phaseout schedules for substances of concern. While federal laws govern the production and trade of alternative chemicals, most ordinances for application of refrigerants are adopted and enforced at the local level. They are included in building, mechanical, and fire safety codes, which govern building construction, system installation, equipment operation and maintenance, system modification including refrigerant conversion, and ultimate demolition. Although most are based on national or regional model codes, the introduction of alternative refrigerants occurred so rapidly that the cautious process of regulatory revision has not caught up yet. One cause of delay has been the time needed to complete toxicity tests, publish the findings, modify impacted standards, develop and adopt code revisions, and prepare design professionals, contractors, technicians, and building and fire prevention officials. 


\section{Toxicity Testing}

Facing unprecedented testing and phaseout requirements, the chemical industry formed an international consortium to accelerate the development of toxicology data for substitute fluorocarbons, both for refrigerant and other uses. The cooperative effort, named the Programme for Alternative Fluorocarbon Toxicity Testing (PAFT), was sponsored by the major producers of chlorofluorocarbons (CFCs). The PAFT research entailed more than 200 individual toxicology tests, by more than a dozen laboratories in Europe, Japan, and the United States. The first tests were launched in 1987, to address R-123 and R-134a (PAFT I). Subsequent programs were initiated for R-141b (PAFT II), R-124 and R-125 (PAFT III), R-225ca and R-225cb (PAFT IV), R-32 (PAFT V), and - still underway the mechanistic, metabolic, and pharmacokinetic aspects of the toxicology of fluorocarbons. ${ }^{4,5}$ Extensive additional data were developed, or contributed from prior tests, by individual chemical manufacturers.

The tasks of assembling and interpreting the resultant data were expanded by the need, in some cases, to collect comparative information for the refrigerants being replaced. Whereas their introduction largely preceded the more rigorous, current testing and classification requirements of the codes, the amount of information needed was significant.

\section{Safety Standards}

Most code provisions for refrigerant and refrigeration-system safety can be traced to either ASHRAE Standard 15. Safety Code for Mechanical Refrigeration, ${ }^{6}$ or to general code provisions developed for occupancies where more hazardous materials are used. Standard 15 prescribes safeguards for design, construction, installation, and operation of refrigerating systems. ${ }^{7.8}$ Many of the specific requirements are based on safety classifications from Standard 34, Designation and Safety Classification of Refrigerants. ${ }^{9.10}$ This standard is the definitive source for assignment of refrigerant number designations. It also provides a safety classification system and assigned classifications.

An effort is underway, by the committees responsible for the two standards, to move determination of data needed for Standard 15 into Standard 34, and to rewrite the application requirements in Standard 15 parametrically, based on the referenced data. In doing so, the committees are refining the methods to determine refrigerant quantity limits for occupied areas, both to increase consistency and to take advantage of the new data and understanding. They also are addressing the toxicity, flammability, and fractionation concerns arising from use of zeotropic and azeotropic refrigerant blends.

\section{Parameters}

Building, fire, and mechanical codes vary throughout the United States. They are based on state or local amendments to regional model codes or, in a few locations, locally-developed codes. Nevertheless, the data needed for compliance are fairly consistent. They include:

$\mathrm{LC}_{50}$ : The "lethal concentration for $50 \%$ of tested animals," sometimes referred to as the median lethal concentration, is a primary measure of acute toxicity by inhalation of gases. It most commonly is measured with rats for exposures of four hours. A number of federal regulations (e.g., reference 1) and most building, fire, and mechanical codes deem substances to be toxic for one-hour $L_{50}$ concentrations of $200-2000 \mathrm{ppm}$ and highly toxic for less than 200 ppm. $^{*}$ Typical $L_{50}$ concentrations for one hour are double those for four hours. ${ }^{11,12}$ Multipliers of 1.6-4 have been suggested, ${ }^{13,14}$ and one study found a range of 1.5-5.7 for 20 tested chemicals. $^{15}$ None of the refrigerants identified in table 1 , or blends of them, qualify as toxic or highly toxic based on the $\mathrm{LC}_{50}$ data and the stated criteria.

IDLH: The concentration deemed to be "immediately dangerous to life and health," set by the National Institute for Occupational Safety and Health (NIOSH). This measure was initially developed as a criterion for respirator selection in the 1970s as part of the Standards Completion Program (SCP). The SCP definition for IDLH was "the maximum concentration from which, in the event of a respirator failure, one could escape within 30 minutes without a respirator and without experiencing any escape-impairing (e.g., severe eye irritation) or irreversible health effects." The 1994 revision defines an IDLH condition as one "that poses a threat of exposure to airborne contaminants when that exposure is likely to cause death or immediate or delayed permanent adverse health effects or prevent escape from such an environment." The revised and added IDLHs in 1994 are based on additional toxicity criteria and data. Whereas the IDLHs derived for the SCP were set at

\footnotetext{
*The definitions for toxic and highly toxic also include $\mathrm{LD}_{50}$ (median lethal dosage) criteria for mortality by ingestion and contact. $\mathrm{LD}_{50}$ values generally are not determined for or applicable to gases and volatile substances, such as refrigerants, since the standard test methods are not suited for them. Similarly, the likelihood of ingestion of or prolonged contact with the quantities involved is remote. Some $L_{50}$ data, mostly based on solutions of refrigerants in liquids, are provided in reference 20.
} 
$100 \%$ of the lower-flammability limit (LFL), if there were no known health hazards below those concentrations, the 1994 IDLHs are reduced to $10 \%$ of the LFL. Most fire codes use the IDLH, based on the SCP definition, as a criterion for ventilation rates and emergency discharge treatment. Standard 15 also uses the SCP IDLH as one of several criteria to determine refrigerant quantity limits for occupied areas. Use of the IDLH concentrations for these purposes has been challenged as inconsistent with their definition.

PEL: The "permissible exposure limit" is the concentration level established by the Occupational Safety and Health Administration (OSHA). Without qualification, the PEL implies a time weighted average (TWA) for an 8-hour work shift in a 40-hour work week. Consistent data include similarly-defined occupational exposure limits set by manufacturers (such as the Allowable Exposure Limit, AEL), the Threshold Limit Value (TLV) adopted by the American Conference of Governmental Industrial Hygienists (ACGIH), ${ }^{16}$ and the Workplace Environmental Exposure Level (WEEL) guides developed by the American Industrial Hygiene Association (AIHA). ${ }^{17}$ Where designated as a PEL-C (e.g., for R-11), the PEL is a ceiling concentration that shall not be exceeded. Most codes use either PEL or TLV-TWA values as the maximum activation levels for leak-detector alarms; Standard 15 and the new International Mechanical Code (IMC) use the TLV-TWA. While the PEL and TLV-TWA are similarly defined, and the original PELs were based on TLVs, the PEL values have not been revised since 1971. More protective limits published in 1989 were vacated by a court order in 1992. ACGIH publishes annual TLV updates. Still, neither PELs nor TLVs have been set for most alternative refrigerants, for which the primary recourse is use of WEELs or other consistent measures.

UL group: The Underwriters Laboratories classification reflects the comparative life hazard of refrigerants in the absence of flames or surfaces at high temperatures. Group 1 is the most toxic (e.g., R-764) and group 6 the least. ${ }^{18}$ This measure is used to classify refrigerants in older codes, still in effect in some jurisdictions.

Standard 34 safety group: This classification consists of a letter ( $A$ or $B$ ), which indicates the toxicity class, followed by a number $(1,2$, or 3$)$, which indicates the flammability class. Toxicity classes A and B signify refrigerants with lower toxicity and higher toxicity, respectively, based on prescribed measures of chronic (longterm, repeated exposures) toxicity. Flammability class 1 indicates refrigerants that do not show flame propagation in air when tested by prescribed methods at specified conditions. Classes 2 and 3 signify refrigerants with lower flammability and higher flammability, respectively; the distinction depends on both the LFL and heat of combustion (HOC). Some of the mechanical codes written before 1993 used an older safety classification system from earlier editions of Standard 34. They included groups 1 (no flame propagation and low degree of toxicity), 2 (TLV-TWA less than $400 \mathrm{ppm}$ ), 3a (flammable with low LFL or high HOC), 3b (flammable with high LFL and low HOC), $4 a$ (mixtures of groups 1 and $3 a$ that are nonflammable as formulated, but could become flammable upon fractionation), and $4 \mathrm{~b}$ (mixtures of groups 1 and $3 \mathrm{~b}$ that are nonflammable as formulated, but could become flammable upon fractionation). Excluding the group 2 refrigerants, the ranked order from the lowest to highest flammability hazard was $1,4 b, 4 a, 3 b$, and $3 a$. One motive for the current classification system, introduced in 1992, was to provide a more rational system. Based on current understanding and usage, however, the author questions whether two toxicity classes provide sufficient distinction and whether the classification criterion should be a measure of acute, rather than chronic, toxicity or a combination of acute and chronic toxicity.

quantity limits for occupied areas: The primary criterion to determine whether refrigeration systems, or refrigerant-containing components, are allowed in occupied areas of buildings are quantity limits set by the codes or Standard 15. Nearly all limits set in the codes were transcribed from Standard 15, though a few intended and unintended revisions were made in the process.

As discussed above, efforts are underway to develop consensus quantity limits for new refrigerants, including blends. The following additional data are likely to be needed:

cardiac sensitization: An acute effect in which the heart is rendered more sensitive to the body's own catecholamine compounds or administered drugs, such as epinephrine, possibly resulting in irregular heart beat (cardiac arrhythmia), which could be fatal. ${ }^{10}$ LOEL is the "lowest-observed effect level," the lowest concentration at which sensitization occurs in tests, normally to beagle dogs treated with epinephrine to simulate the effects of stress. NOEL is the "no-observed effect level," the highest exposure concentration at which no sensitization is observed.

anesthetic $\mathrm{EC}_{50}$ : The concentration of a substance that caused the temporary loss of ability to perceive pain and other sensory stimulation to $50 \%$ of test animals, normally measured for 10 minute exposures. $E C_{50}$ refers to the "effective concentration for $50 \%$ of specimens."

$\mathrm{RD}_{50}$ : The concentration that resulted in $50 \%$ decrease in respiratory rate, normally measured in mice. A maximal effect generally occurs in less than 30 minutes; the response to R-717 (ammonia), as an example, is reported to take approximately two minutes. 
Table 1: Refrigerant Toxicity Data, Exposure Limits, and Classifications ${ }^{a}$ (data and limits in ppm v/v)

\begin{tabular}{|c|c|c|c|c|c|c|c|c|c|c|}
\hline \multirow{2}{*}{$\begin{array}{l}\text { refrig b } \\
\text { erant- }\end{array}$} & \multirow[b]{2}{*}{$\mathrm{LC}_{50} \mathrm{C}$} & \multicolumn{2}{|c|}{$\begin{array}{c}\text { cardiac } \\
\text { sensitization d }\end{array}$} & \multirow{2}{*}{$\begin{array}{l}\text { anes- } \\
\text { thetic } \\
E^{E C_{50}}\end{array}$} & \multirow[b]{2}{*}{$\mathrm{RD}_{50}{ }^{f}$} & \multicolumn{2}{|c|}{ IDLH } & \multirow[b]{2}{*}{ PEL h } & \multirow{2}{*}{$\begin{array}{l}\text { UL } \\
\text { group }\end{array}$} & \multirow{2}{*}{$\begin{array}{l}\text { safety } \\
\text { group }\end{array}$} \\
\hline & & & NOEL & & & SCP & 1994 & & & \\
\hline $\begin{array}{l}11 \\
12 \\
22 \\
23 \\
32\end{array}$ & $\begin{array}{r}26200 \\
>800000 \\
220000 \\
>663000 \\
>760000 n\end{array}$ & $\begin{array}{r}5000 \\
50000 \\
50000 \\
>800000 \\
250000\end{array}$ & $\begin{array}{r}1100 \\
25000 \\
25000 \\
300000 \\
200000\end{array}$ & $\begin{array}{l}00 \\
00 \\
00 \\
00 \mathrm{~m} \\
00^{\mathrm{m}}\end{array}$ & $\begin{array}{l}- \\
- \\
- \\
-\end{array}$ & $\begin{array}{c}5000^{j} \\
50000 \\
50000^{k} \\
-- \\
--\end{array}$ & $\begin{array}{r}2000 \\
15000 \\
-- \\
- \\
-\end{array}$ & $\begin{array}{l}1 \\
1 \\
1 \\
1\end{array}$ & $\begin{array}{c}5(a) \\
6 \\
5(a) \\
6 k \\
--\end{array}$ & $\begin{array}{l}\mathrm{A} 1 \\
\mathrm{~A} 1 \\
\mathrm{~A} 1 \\
\mathrm{~A} 1 \\
\mathrm{~A} 2\end{array}$ \\
\hline $\begin{array}{l}113 \\
114 \\
115 \\
116 \\
123\end{array}$ & $\begin{array}{r}52000^{n} \\
>600000^{\circ} \\
>800000^{n} \\
>800000^{n} \\
32000^{\circ}\end{array}$ & $\begin{array}{r}5000 \\
25000 \\
150000 \\
q \\
20000\end{array}$ & $\begin{array}{r}2500 \\
- \\
200000 \\
10300\end{array}$ & $\begin{array}{r}28 \\
250 \\
28 \\
800 \\
40\end{array}$ & $\begin{array}{l}- \\
- \\
- \\
-\end{array}$ & $\begin{array}{r}4500 \\
50000 \\
- \\
\overline{-} \\
4000^{k}\end{array}$ & $\begin{array}{r}2000 \\
15000 \\
- \\
- \\
-\end{array}$ & & $\begin{array}{c}4-5 \\
6 \\
6 \\
6 k \\
-\end{array}$ & $\begin{array}{l}\mathrm{A} 1 \\
\mathrm{~A} 1 \\
\mathrm{~A} 1 \\
\mathrm{~A} 1 \\
\mathrm{~B} 1\end{array}$ \\
\hline $\begin{array}{l}124 \\
125 \\
134 a \\
141 b \\
142 b\end{array}$ & $\begin{array}{r}262500 \\
>800000 \mathrm{n} \\
567000^{n} \\
61647 \\
128000^{n}\end{array}$ & $\begin{array}{r}25000 \\
100000 \\
75000 \\
5000 \\
50000\end{array}$ & $\begin{array}{r}10000 \\
75000 \\
50000 \\
2500 \\
25000\end{array}$ & $\begin{array}{r}140 \\
>10 \\
205 \\
25 \\
250\end{array}$ & $\begin{array}{l}- \\
- \\
- \\
-\end{array}$ & $\begin{array}{c}\overline{-} \\
50000^{k} \\
\overline{-}\end{array}$ & $\begin{array}{l}- \\
- \\
- \\
-\end{array}$ & & $\begin{array}{l}6 \\
- \\
- \\
- \\
-\end{array}$ & $\begin{array}{l}\mathrm{A} 1 \\
\mathrm{~A} 1 \\
\mathrm{~A} 1 \\
\overline{\mathrm{A} 2}\end{array}$ \\
\hline $\begin{array}{c}143 a \\
152 a \\
218 \\
290 \\
\text { C318 }\end{array}$ & $\begin{array}{r}>540000^{n} \\
383000^{n} \\
>800000^{\circ} \\
>800000^{\circ} \\
>800000^{\circ}\end{array}$ & $\begin{array}{l}300000 \\
150000 \\
400000 \\
100000 \\
100000\end{array}$ & $\begin{array}{r}250000 \\
50000 \\
300000 \\
50000\end{array}$ & $\begin{array}{r}>540000 \mathrm{~m} \\
200000 \\
>113000 \mathrm{~m} \\
280000 \\
>600000^{m}\end{array}$ & $\begin{array}{l}- \\
- \\
- \\
-\end{array}$ & $2000 \overline{-}$ & $210 \overline{-}$ & $\begin{array}{c}1000 p \\
1000 p \\
1000 p \\
1000 \\
-\end{array}$ & $\begin{array}{c}\overline{-} \\
\overline{5(b)} \\
6 k\end{array}$ & $\begin{array}{l}\mathrm{A} 2 \\
\mathrm{~A} 2 \\
\mathrm{~A} 1 \\
\mathrm{~A} 3 \\
\mathrm{~A} 1\end{array}$ \\
\hline $\begin{array}{c}600 \\
600 a \\
717 \\
744 \\
1270\end{array}$ & $\begin{array}{r}280000 \\
570000^{\circ} \\
2000^{\mathrm{t}} \\
\mathrm{v} \\
650000^{\mathrm{w}}\end{array}$ & $\begin{array}{r}5000 \\
50000 \\
- \\
100000\end{array}$ & $\begin{array}{r}25000 \\
- \\
- \\
-\end{array}$ & $\begin{array}{c}130000 \\
200000 \mathrm{~s} \\
- \\
\overline{-} \\
400000\end{array}$ & $\begin{array}{r}\overline{-} \\
303 \\
- \\
-\end{array}$ & $\begin{array}{r}\overline{-} \\
500 \\
50000 \\
-\end{array}$ & $\begin{array}{r}-\overline{-} \\
30 \overline{0} \\
40000 \\
-\end{array}$ & $\begin{array}{r}800 \mathrm{l} \\
600 \mathrm{k} \\
50 \mathrm{u} \\
5000 \\
1000 \mathrm{k}\end{array}$ & $\begin{array}{c}5(b) \\
5(b) \\
2 \\
5(a) \\
-\end{array}$ & $\begin{array}{l}\mathrm{A} 3 \\
\mathrm{~A} 3 \\
\mathrm{~B} 2 \\
\mathrm{~A} 1 \\
\mathrm{~A} 3\end{array}$ \\
\hline
\end{tabular}

a Please see the source publications (identified in reference 20 , to verify these data and examine their limitations.

b

c

d

e

f

g

h

s $17-$ min $\mathrm{EC}_{50}$ mouse

$\mathrm{t}$ wide dispersion found in the literature: $6586-19671$ for $1 \mathrm{hr}$ and $2000-4067$ for $4 \mathrm{hr}$

u ACGIH Threshold Limit Value - Time-Weighted Average (TLV-TWA) $=25 \mathrm{ppm}$

$\checkmark 5$-min $L C_{L 0}$ human $=90000 \mathrm{ppm}$

w 2 -hr ALC rat ( 4 -hr ALC rat $\approx 460,000 \mathrm{ppm}$ using the conversion from reference 11) 
Working drafts of the proposed method to determine quantity limits, identified as Recommended Quantity Limits (RQLS), use the preceding acute-toxicity data to determine an intermediate limit, identified as the Acute Toxicity Exposure Limit (ATEL). The RQL is then set at the lowest of the ATEL, the oxygen deprivation level (ODL, the calculated concentration that will reduce the oxygen concentration in normal air to below $191 / 2 \%$ by volume) of 69100 $\mathrm{ppm}$, and $25 \%$ of the LFL. One proposal to establish RQLs for blends uses the same calculation method as for single compounds, after determining a mole-weighted average for each parameter based on the values for the blend components.

\section{Findings}

Table 1 summarizes data for common, single-compound refrigerants. The quantity limits for occupied areas are not included, since the calculation method still is being revised. Those limits and corresponding data and limits for blends will be presented in a subsequent paper.

Multiple values were located for approximately half of the data in the table. In general, those shown are the most conservative found in the published literature, except that the highest published concentrations are included for noeffect levels and where a study found a lower bound to, but did not actually establish, an end point. Space limitations in this paper prevent inclusion of the more than 200 pertinent references from which the data in table 1 were obtained. Specific or multiple corroborating sources and additional data are identified in reference 20.

Comparison of the acute-toxicity data for R-123 to those for R-11 show it to be as safe, or safer, with respect to acute toxicity. The same conclusion results from comparison of the data for R-134a to those for R-12. An earlier paper showed that chronic exposures can be maintained well below recommended limits. ${ }^{2}$

Another point that is evident with the assembled data is that the hydrocarbons proposed as replacements for fluorochemicals are generally more toxic. The $L C_{50}$ values for $R-32, R-125, R-134 a, R-290$ (propane), $R-600$ (butane), R-600a (isobutane), and R-1270 (propene) all indicate very low acute toxicity. The cardiac sensitization and anesthetic effect indicators, however, suggest that R-600 and R-600a pose higher risks than R-134a and that the inhalation lethality of R-1270, while very low, is higher than for either R-32 or R-125. Although not addressed herein, these hydrocarbons also introduce much higher explosivity, flammability, and heat release concerns.

The compiled data result from a fairly extensive data search. Two caveats accompany the data presented. First, the table constitutes a work in progress, to provide data for interim use. Some of the values may be superseded as further information is located or new data become available. Second, users must satisfy themselves with the suitability and appropriateness of the data for specific uses. The data or resultant determinations also must be approved by the code official having jurisdiction where required. While care has been taken in assembly of the data, the effort cannot be viewed as exhaustive and no attempt was made to verify the data. They are intended for use by knowledgeable professionals, and offered without warranty of any kind.

\section{Additional Data Sources}

Other sources for the data include the PAFT summaries ${ }^{5}$ published scientific literature, manufacturers, and material safety data sheets. A number of databases are available to assist in finding the data, among them the Chemical Abstract Service (CAS), Hazardous Substances Data Base (HSDB), and Registry of Toxic Effects of Chemical Substances (RTECS). Additional sources, including a number of compilations, are identified in reference 20.

\section{CONCLUSIONS}

The toxicity data presented herein provide an interim means to address code requirements for use of alternative refrigerants, subject to the caveats indicated. These data also may be useful to evaluate proposed changes to safety standards for refrigerants and refrigeration. While the data show the alternative refrigerants to be of comparable or lower toxicity than those they replace, and especially so for acute effects, safe use depends on adherence to proper application, handling, and service procedures.

\section{ACKNOWLEDGMENTS}

This paper was prepared as an account of work supported in part by the U.S. Department of Energy under grant number DE-FG02-91CE23810, Materials Compatibility and Lubricant Research (MCLR) on CFC-Refrigerant Substitutes, managed by the Air-Conditioning and Refrigeration Technology Institute (ARTI). Additional program funding was provided by air-conditioning and refrigeration manufacturers through the Air-Conditioning and Refrigeration Institute (ARI). The ARTI Project Manager guiding the toxicity data project is Mr. Glenn C. Hourahan. Support by the 
cited parties does not constitute an endorsement, warranty, or assumption of liability for the data and views expressed herein.

The author appreciates the assistance of Mr. Hourahan and other ARTI staff in this work. Numerous individuals contributed to the underlying assembly and interpretation of safety data; notable among them are William J. Brock and Clem L. Warrick (DuPont Fluoroproducts), Susan G. Cairelli and Elaine Mann (NIOSH), Michael Collins and Paul H. Dugard (ICl Klea), Sandra R. Murphy (Elf Atochem North America), and George M. Rusch and David P. Wilson (AlliedSignal Incorporated). Any fault in this paper is the author's; the acknowledged individuals had no advance opportunity to review it.

\section{REFERENCES}

1 Occupational Safety and Health Administration (OSHA), U.S. Department of Labor, Health Hazard Definitions in Air Contaminants, 29 CFR $\$ 1910.1200$, Appendix A, Government Printing Office, Washington, DC, 1 July 1995

2 J. M. Calm, Refrigerant Safety, ASHRAE Journal, American Society of Heating, Refrigerating, and AirConditioning Engineers (ASHRAE), Atlanta, GA, 36(7):17-26, July 1994

3 R. C. Downing, Fluorocarbon Refrigerants Handbook, Prentice Hall, Englewood Cliffs, NJ, 1988

4 Programme for Alternative Fluorocarbon Toxicity Testing (PAFT), program description, PAFT, Washington, DC, September 1995

5 Programme for Alternative Fluorocarbon Toxicity Testing (PAFT) Toxicology Summaries, PAFT, Washington, DC, September 1995: The full texts of these summaries are included in reference 20.

6 Safety Code for Mechanical Refrigeration, ASHRAE Standard 15-1994, ASHRAE, August 1994

7 L. W. Burgett, Revised Standards for Mechanical Refrigeration, ASHRAE Journal, ASHRAE, 35(8):31-35, August 1993

8 T. E. Watson, ASHRAE Standards and Alternative Refrigerants, paper 7.4, Proceedings of the International Symposium on R22 \& R502 Alternative Refrigerants, Japan Refrigeration and Air-Conditioning Industry Association (JRAIA), Tokyo, Japan, 187-192, December 1994

9 Number Designation and Safety Classification of Refrigerants, ANSI/ASHRAE Standard 34-1992, ASHRAE, February 1992

10 Addenda to Number Designation and Safety Classification of Refrigerants, ANSI/ASHRAE 34a-o and 34q-s, ASHRAE, 1995. Additional addenda are in publication or under review.

11 Uniform Fire Code, International Fire Code Institute (IFCI), Whittier, CA, appendix VI-A, table A-VI-A-1, page 460, 1994, as amended by the Accumulative Supplement to the Uniform Fire Code, International Conference of Building Officials (ICBO), Whittier, CA, page 291, 1996

12 Class 6.1 - Poisons, International Maritime Dangerous Goods Code, International Maritime Organization, London, \$2.1.5.2.2, page 6007, 1986

13 H. R. Ludwig, S. G. Cairelli, and J. J. Whalen, Documentation for Immediately Dangerous to Life or Health Concentrations (IDLHS), National Institute for Occupational Safety and Health (NIOSH), U.S. Department of Health and Human Services, Cincinnati, OH, pages 5-6, May 1994

14 Haber's law, a tenet of toxicology, states that different concentrations have the same lethal effect when the product of the exposure concentration and duration - for small differences in time - is constant. Application to the example discussed yields a factor of four (see references 13,15 , and 17).

15 W. F. ten Berge, A. Zwart, and L. M. Appelman, Concentration-Time Mortality Response Relationship of Irritant and Systematically Acting Vapours and Gases, Journal of Hazardous Materials, Elsevier Science Publishers B.V., Amsterdam, The Netherlands, volume 13, pages 301-309, 1986: The range cited herein was calculated with correlations from this reference.

16 1995-1996 Threshold Limit Values for Chemical Substances and Physical Agents and Biological Exposure Indices, American Conference of Governmental Industrial Hygienists (ACGIH), Cincinnati, OH, 1995

17 The AlHA 1996 Emergency Response Planning Guidelines and Workplace Environmental Exposure Level Guides Handbook, American Industrial Hygiene Association (AlHA), Fairfax, VA, 1996

$18 \mathrm{~A}$. H. Nuckolls, The Comparative Life, Fire, and Explosion Hazards of Common Refrigerants, Miscellaneous Hazard Report Number 2375, Underwriters' Laboratories Incorporated, Chicago, IL, pages 106-107, 13 Novernber 1933

19 Refrigerant Safety Data - Recommendations of the Air-Conditioning and Refrigeration Industry, Air-Conditioning and Refrigeration Institute (ARI), Arlington, VA, October 1993

20 J. M. Calm, ARTI Refrigerant Database, Air-Conditioning and Refrigeration Technology Institute (ARTI), Arlington, VA, April 1996 (updated quarterly, see http://www.ari.org on the Internet for database information) 